

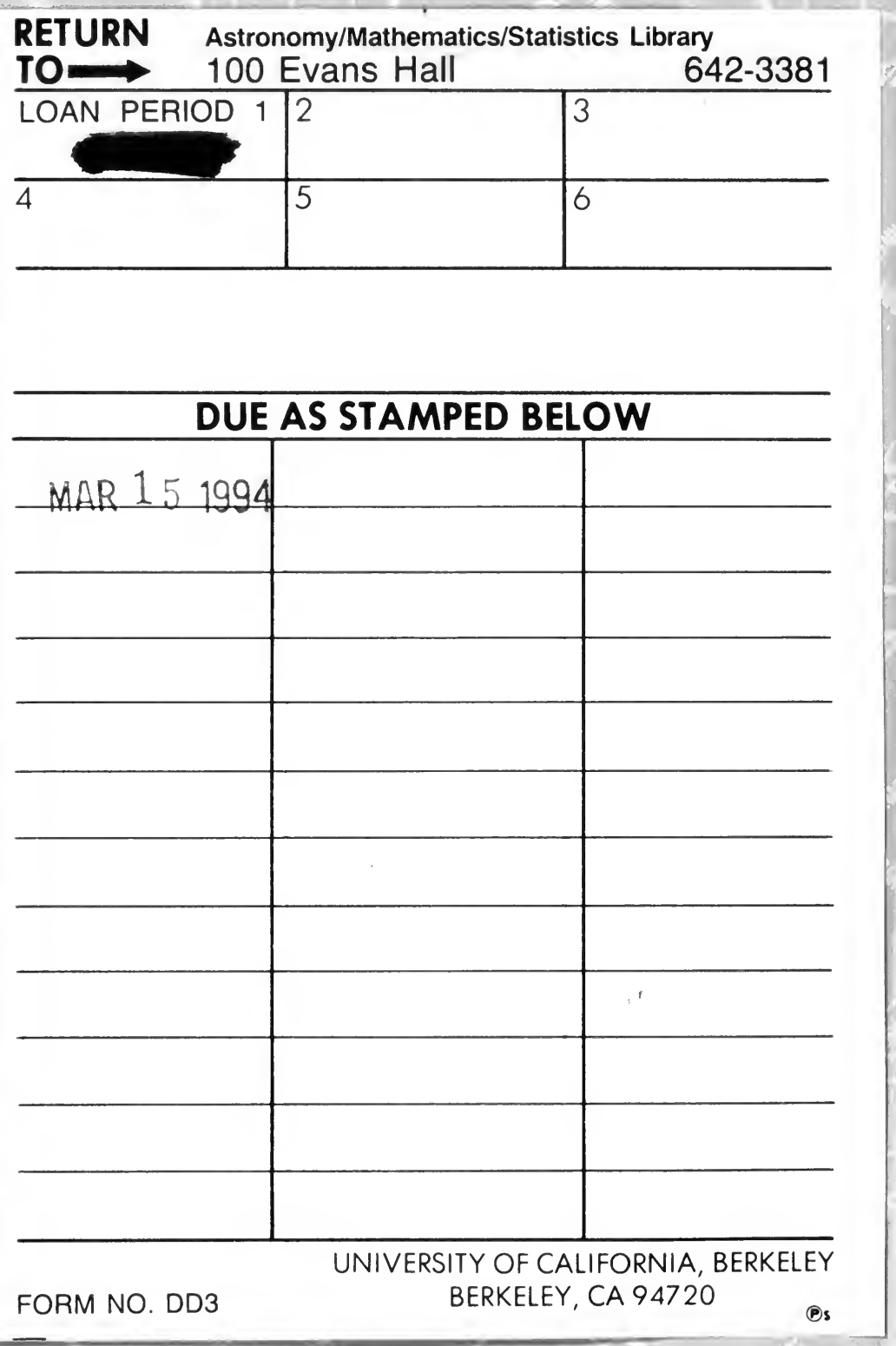
14 



\%. Bruder Otho

als evien keygliken Gruss ans

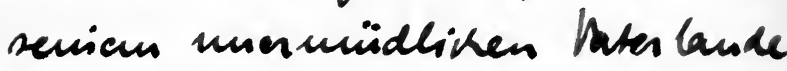
Koinigsberg, K/rr.2r. Kourad. 
DIE GRUNDLEHREN DER

\section{MATHEMATISCHEN WISSENSCHAFTEN}

IN EINZELDARSTELLUNGEN MIT BESONDERER BERÜCKSICHTIGUNG DER ANWENDUNGSGEBIETE

GEMEINSAM MIT

\begin{tabular}{|c|c|}
\hline $\begin{array}{l}\text { W. B LASCHKE } \\
\text { HAMBURG }\end{array}$ & $\begin{array}{l}\text { M. BOR O R N } \\
\text { GÖTTINGEN }\end{array}$ \\
\hline \multicolumn{2}{|c|}{ HERAUSGEGEBEN VON } \\
\hline & $\begin{array}{l}\text { OURANT } \\
\text { OUTTINGEN }\end{array}$ \\
\hline
\end{tabular}
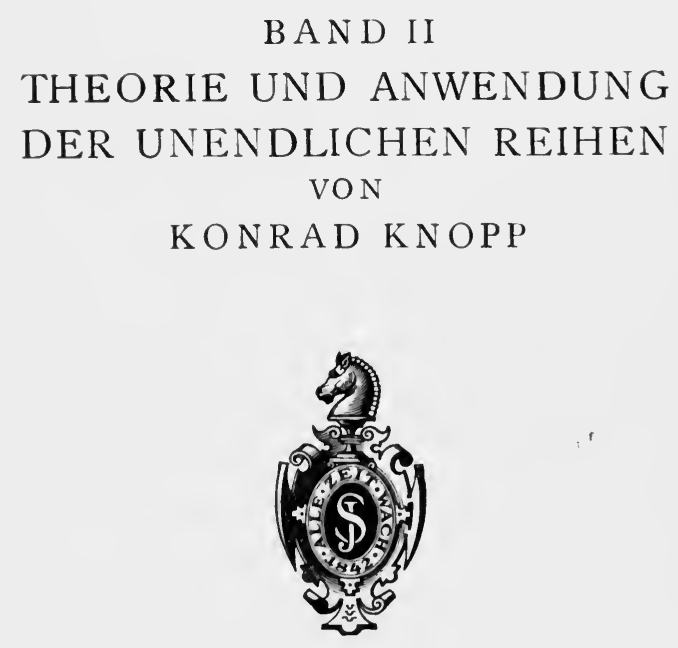

BERLIN

VERLAG VON JULIUS SPRINGER 


\title{
THEORIE UND ANWENDUNG DER UNENDLICHEN REIHEN
}

\author{
VON \\ DR. KONRAD KNOPP \\ ORD. PROFESSOR DER MATHEMATIK AN DER \\ UNIVERSITÄT KÖNIGSBERG
}

MIT I2 TEXTFIGUREN

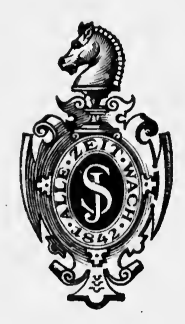

BERLIN

VERLAG VON JULIUS SPRINGER I 922 


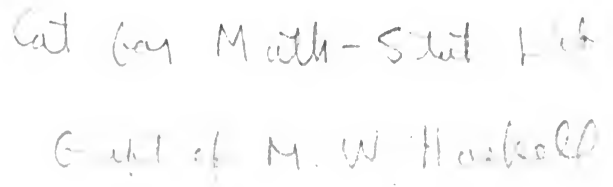

\section{MATH-STAR.}

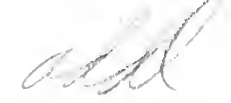

ALLE RECHTE, INSBESONDERE

DAS DER ÜBERSETZUNG IN FREMDE SPRACHEN, VORBEHALTEN. COPYRIGHT 1922 BY JULIUS SPRINGER IN BERLIN 


\section{QA295 155 i. ITH.- STAT. LIBRARY}

\section{Vorwort.}

Ausgangspunkt, Umrisse und Ziel einer Lehre von den unendlichen Reihen liegen nicht fest. Auf der einen Seite kann die gesamte höhere Analysis als ein Anwendungsfeld ihrer Theorie angesehen werden, weil alle Grenzprozesse - einschließlich Differentiation und Integration - auf die Untersuchung unendlicher Zahlenfolgen oder Reihen zurückgehen; auf der andern Seite, in einem strengsten, aber darum auch engsten Sinne, gehören in ein Lehrbuch der unendlichen Reihen nur deren Definition, die Handhabung der damit verbundenen Symbolik und die Konvergenztheorie.

Unter Beschränkung auf diese Teile behandeln die „Vorlesungen über Zahlen- und Funktionenlehre", Band I, Abteilung 2, von A. Pringsheim unsern Gegenstand. Es konnte nicht die Absicht sein, mit dem vorliegenden Buche etwas Ähnliches zu bieternth

Meine Ziele waren andere: AfTe Betrachtungen und Untersuchungen der höheren Analysis zusammenzufassen, bei denen die unendlichen Reihen im Vordergrund des Interesses stehen, möglichst voraussetzungsfrei, von den ersten Anfängen an, aber fortführend bis an die ausgedehnte Front der gegenwärtigen Forschung, und alles dies möglichst lebendig und leicht faßlich, doch selbstverständlich ohne den geringsten Verzicht auf Exaktheit, dargestellt, um so dem Studierenden eine bequeme Einführung und einen reichen Einblick in das vielgestaltige und fesselnde Stoffgebiet zu geben, - das schwebte mir vor.

Aber der Stoff wuchs unter den Händen und widersetzte sich der Gestaltung. Um etwas Handlich-Brauchbares zu schaffen, mußte darum das Gebiet beschränkt werden. Dabei setzte unvermeidlich eine gewisse Willkür ein, deren Walten nun die Form des Buches und damit seinen Wert bestimmt. Doch leiteten mich immer die Erfahrungen, die ich im Unterricht gesammelt - das gesamte Stoffgebiet habe ich mehrfach in Vorlesungen und Übungen an den Universitäten Berlin und Königsberg behandelt -, und die Zwecke, für die das Buch bestimmt sein soll: Es soll dem Studierenden bei den Vorlesungen eine zuverlässige und gründliche Hilfe bieten und gleichzeitig zur Durcharbeitung des ganzen Stoffes im Selbststudium geeignet sein. 
Dies letzte lag mir besonders am Herzen und mag die Form der Darstellung begreiflich machen. $\mathrm{Da}$ es, besonders für die jüngeren Semester, im allgemeinen leichter ist, eine rein mathematische Deduktion nachzuprüfen, als das Zwangsläufige des Gedankenzusammenhanges zu erkennen, habe ich mich stets bei den begriflichen Schwierigkeiten länger aufgehalten und sie durch mannigfache Erläuterungen zu beheben versucht. Und ist mir dadurch auch viel Raum für Sachliches verloren gegangen, so hoffe ich doch, daß es der Lernende mir danken wird.

Für unumgänglich habe ich es gehalten, mit einer Einführung in die Lehre von den reellen Zahlen $\mathrm{zu}$ beginnen, damit die ersten Konvergenztatsachen auf einem soliden Boden wachsen. Hieran schließt sich eine schon ziemlich weitführende Theorie der Zahlenfolgen und endlich die eigentliche Lehre von den unendlichen Reihen, die dann gleichsam in zwei Stockwerken aufgebaut wird, einem Unterbau, in dem mit den bescheidensten Mitteln gearbeitet und doch schon der klassische Teil der ganzen Lehre, etwa bis zu Cauchys Analyse algébrique, dargelegt wird, und einem Oberbau, der dann von ihrer weiteren Entwicklung im I9. Jahrhundert ein Bild zu geben versucht.

Aus den schon genannten Gründen fehlen viele Gegenstände, denen ich an und für sich gern noch Aufnahme gewährt hätte. Die halbkonvergenten Reihen, die Eulersche Summenformel, Eingehenderes über die Gammafunktion, der Problemenkreis der hypergeometrischen Reihe, die Theorie der Doppelreihen, die neueren Untersuchungen über Potenzreihen und besonders eine gründlichere Ausgestaltung des letzten Kapitels über divergente Reihen, alles dies mußte ich schweren Herzens - beiseite lassen. Dagegen habe ich Zahlenfolgen und Reihen mit komplexen Gliedern unbedingt aufnehmen zu müssen geglaubt. Da aber ihre Theorie derjenigen im Reellen fast parallel läuft, habe ich von Anfang an alle hierfür in Betracht kommenden Definitionen und Sätze so formuliert und bewiesen, daß sie ungeändert in Gültigkeit bleiben, mögen die auftreténden „beliebigen“ Zahlen reell oder komplex sein. Das Zeichen $\circ$ soll diese Definitionen und Sätze noch besonders kenntlich machen.

Bei der Auswahl der Aufgaben - die übrigens auf Originalität keinerlei Anspruch machen, bei deren Zusammenstellung vielmehr die vorhandene Literatur ausgiebig benutzt worden ist - habe ich mich bemüht, die praktische Verwendung in den Vordergrund zu stellen und ein Spiel mit theoretischen Finessen beiseite zu lassen. Darum findet man z. B. besonders zahlreiche Aufgaben zum VIII. Kapitel und nur ganz wenige zum IX. Kapitel. Die Lösungen der Aufgaben oder auch nur Anleitungen dazu beizufügen, verbot leider der Raum. 
Die wichtigsten Abhandlungen, zusammenfassenden Darstellungen und Lehrbücher über unendliche Reihen sind am Schlusse des Buches vor dem Register aufgeführt.

Bei der Korrektur haben mich die Herren R. Courant, E. Jacobsthal, H.Rademacher und $H$. Vermeil in freundlicher Weise unterstützt. Den Herren Jasobsthal und Rademacher verdanke ich ganz besonders viele Ratschläge und Verbesserungen sachlicher und formaler Art. Ihnen allen sei hier noch einmal der herzlichste Dank ausgesprochen.

Auch dem Verlage, der sich in diesen schwierigen Zeiten den mathematischen Wissenschaften in so weitherziger Weise zur Verfügung gestellt hat und auch bei der Drucklegung dieses Buches auf alle Wünsche bereitwillig eingegangen ist, möchte ich an dieser Stelle meinen Dank sagen.

Königsberg, September 1921.

Konrad Knopp. 


\section{Inhaltsverzeichnis.}

Einleitung ...........

Erster Teil.

\section{Reelle Zahlen und Zahlenfolgen.}

I. Ka pitel.

Grundsätzliches aus der Lehre von den reellen Zahlen.

$\$$ 1. Das System der rationalen Zahlen und seine Liicken . . . . . . 3

2. Rationale Zahlenfolgen . . . . . . . . . . . . . 12

3. Die irrationalen Zahlen .. . . . . . . . . . . . . . 21

\$ 4. Vollständigkeit und Einzigkeit des Systems der reellen Zahlen . . 32

5. Die Systembrïche und der Dedekindsche Schnitt . . . . . . . 35

A ufgaben zum I. Kapitel (1-8) . . . . . . . . . . 40

I1. K a pitel.

Reelle Zahlenfolgen.

6. Beliebige reelle Zahlenfolgen und Nullfolgen . . . . . . . . 41

7. Potenz, Wurzel und Logarithmus. Spezielle Nullfolgen . . . . 45

S. Konvergente Zahlenfolgen . . . . . . . . . . . . 60

9. Die beiden Hauptkriterien . . . . . . . . . . . . . . . 74

$\S 10$. Häufungswerte und Häufungsgrenzen . . . . . . . . . . 84

$\$ 11$. Unendliche Reihen, Produkte und Kettenbrüiche . . . . . . . . 93

A ufgaben zum II. Ka pitel (9-33) . . . . . . . . . 101

\section{Zweiter Teil.}

\section{Grundlagen der Theorie der unendlichen Reihen.}

III. Kapitel.

Reihen mit positiven Gliedern.

\$ 12. Das erste Hauptkriterium und die beiden Vergleichskriterien . . 105

13. Das Wurzel- und das Quotientenkriterium . . . . . . . . 111

$\S 14$. Reihen mit positiven monoton abnehmenden Gliedern . . . . . 114 A ufgaben zum III. Kapitel (34-44) . . . . . . . . . 119

IV. Kapitel.

Reihen mit beliebigen Gliedern.

$\$$ 15. Das zweite Hauptkriterium und das Rechnen mit konvergenten Reilıen 121

$\S 16$. Absolute Konvergenz. Umordnung von Reihen . . . . . . . . . 129

17. Multiplikation unendlicher Reihen . . . . . . . . . . . 139

Aufgaben zum IV. Kapitel (45-63) . . . . . . . . 142 
V. K a pitel.

Potenzreihen.

Seite

§ 18. Der Konvergenzradius . . . . . . . . . . . . . . . 145

$\S 19$. Funktionen einer reellen Veränderlichen . . . . . . . . . . 151

$\S 20$. Haupteigenschaften der durch Potenzreihen dargestellten Funktionen 162

$\S 21$. Das Rechnen mit Potenzreihen . . . . . . . . . . . . . . 171

Aufgaben zum V. Kapitel (64-73) . . . . . . . . . 179

VI. Ka pitel.

Die Entwicklungen der sog. elementaren Funktionen.

$\S 22$. Die rationalen Funktionen . . . . . . . . . . . . . 181

§ 23. Die Exponentialfunktion ................. . . 183

$\$$ 24. Die trigonometrischen Funktionen . . . . . . . . . . . 190

§ 25. Die binomische Reihe . . . . . . . . . . . . . . . . 200

§ 26. Die logarithmische Reihe ................ . 203

§ 27. Die zyklometrischen Funktionen . . . . . . . . . . . 205

A ufgaben zum VI. Kapitel (74-84) . . . . . . . . 208

VII. Ka p itel.

\title{
Unendliche Produkte.
}

$\S 28$. Produkte mit positiven Gliedern . . . . . . . . . . . . 211

$\S$ 29. Produkte mit beliebigen Gliedern. Absolute Konvergenz . . . . 215

$\S 30$. Zusammenhang zwischen Reihen und Produkten. Bedingte und unbedingte, Konvergenz ............. . . 219

Aufgaben zum VII. Kapitel (85-99).......... . . . 221

VIII. Ka pitel.

Geschlossene und numerische Auswertung der Reihensumme.

§ 31. Problemstellung . . . . . . . . . . . . . . . . . 223

$\S$ 32. Geschlossene Auswertung der Reihensumme . . . . . . . . . . . 226

$\S 33$. Reihentransformationen . . . . . . . . . . . . . . . 234

$\S 34$. Numerische Berechnungen . . . . . . . . . . . . . . 241

$\S 35$. Anwendung der Reihentransformationen bei numerischen Berechnungen . . . . . . . . . . . . . 253

Aufgaben zum VIII. Kapitel $(100-132)$. . . . . . . . 260

\section{Dritter Teil.}

\section{Ausbau der Theorie.}

\author{
IX. Ka pitel.
}

\section{Reihen mit positiven Gliedern.}

$\S 36$. Genauere Untersuchung der beiden Vergleichskriterien . . . . . 267

§ 37. Die logarithmischen Vergleichsskalen . . . . . . . . . . . 271

$\S 38$. Spezielle Kriterien . . . . . . . . . . . . . . . . . . 277

$\S 39$. Die Sätze von Abel, Dini und Pringsheim und neue Herleitung der

logarithmischen Vergleichsskalen aus ihnen . . . . . . 282

$\S 40$. Reihen mit positiven monoton abnehmenden Gliedern . . . . . 286

$\S 41$. Allgemeine Bemerkungen zur Konvergenztheorie der Reihen mit positiven Gliedern................ . 290

$\S 42$. Systematisierung der allgemeinen Konvergenztheorie . . . . . . . 297 Aufgaben zum IX. Kapitel $(133-141)$. . . . . . . . . 302 


\section{Kapitel. \\ Reihen mit beliebigen Gliedern.}

$\S 43$. Konvergenzkriterien für Reihen mit beliebigen Gliedern . . . . . 304

$\S 44$. Umordnung nur bedingt konvergenter Reihen . . . . . . . . . 310

$\S 45$. Multiplikation nur bedingt konvergenter Reihen . . . . . . . . . 312

A ufgaben zum X. Ka pitel (142-153) . . . . . . . . . . 314

XI. Kapitel.

\section{Reihen mit veränderlichen Gliedern (Funktionenfolgen).}

§ 46. Gleichmäßige Konvergenz . . . . . . . . . . . . . 316

$\S$ 47. Gliedweise Grenzüibergänge . . . . . . . . . . . . . . . . 326

$\$$ 48. Kriterien für gleichmäß乃ige Konvergenz . . . . . . . . . . . 332

§49. Fouriersche Reihen . . . . . . . . . . . . . . . 337

A. Die Eulerschen Formeln . . . . . . . . . . . 337

B. Das Dirichletsche Integral ............. 344

C. Kon vergenzbedingungen . . . . . . . . . . . . 352

§50. Anwendungen der Theorie der Fourierschen Reihen . . . . . . 359

$\$ 51$. Produkte mit veränderlichen Gliedern . . . . . . . . . . 366

A ufgaben zum XI. Kapitel (154-173) . . . . . . . . . 371

XII. Kapitel.

\section{Reihen mit komplexen Gliedern.}

§ 52. Komplexe Zahlen und Zahlenfolgen . . . . . . . . . . . . . . 374

§53. Reihen mit komplexen Gliedern . . . . . . . . . . . . . 382

§54. Potenzreihen. Analytische Funktionen . . . . . . . . . . . . . 386

\$55. Die elementaren analytischen Funktionen . . . . . . . . . . . 395

I. Die rationalen Funktionen . . . . . . . . . . . . 395

II. Die Exponentialfunktion . . . . . . . . . . . . . 396

III. $\cos z$ und $\sin z$. . . . . . . . . . . . . . . . 399

IV. $\operatorname{ctg} z$ und $\operatorname{tg} z$. . . . . . . . . . . . . . . . . 402

V. Die logarithmische Reihe . . . . . . . . . . 404

VI. Die arcsin-Reihe . . . . . . . . . $\therefore 406$

VII. Die $\operatorname{arctg}-$ Reihe . . . . . . . . . . . 406

VIII. Die Binomialreihe . . . . . . . . . . . . . 407

§56. Reihen mit veränderlichen Gliedern. Gleichmäßige Konvergenz.

Weierstrafsscher Doppelreihensatz . . . . . . . . . 411

$\S 57$. Produkte mit komplexen Gliedern . . . . . . . . . . . . 417

$\$ 58$. Spezielle Klassen von Reihen analytischer Funktionen . . . . . . 425

A. Dirichletsche Reihen . . . . . . . . . . . . 425

B. Fakultätenreihen .. . . . . . . . . . . . 430

C. Lambertsche Reihen . . . . . . . . . . . . 432

A ufgaben zum XII. Kapitel $(174-199)$. . . . . . . . 437

XIII. Ka pitel.

\section{Divergente Reihen.}

§ 59. Allgemeine Bemerkungen über divergente Reihen ........ 441

$\S 60$. Die Summierung durch arithmetische Mittel . . . . . . . 453

$\S 61$. Anwendung der (C 1$)$-Summierbarkeit auf die Theorie der Fourierschen Reihen . . . . . . . . . . . . . . 456

$\S 62$. Anwendungen der $(\mathrm{C} \mathrm{r})$-Summierbarkeit . . . . . . . . . 461

Aufgaben zum XIII. Kapitel (200-208) . . . . . . . . 467

Literatur . . . . . . . . . . . . . . . 468

Autoren- und Sachverzeichnis ............. . . 469 


\section{Einleitung.}

undoultly

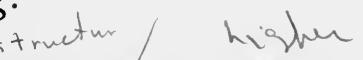

Das Fundament, auf dem das Gebäude der höheren Analysis ruht, ist die Lehre von den reellen Zahlen. Unausweichlich hat jede strenge Behandlung der. Grundlagen der Differential- und Integralrechnung, und der anschließenden Gebiete, ja selbst schon die strenge Behandlung etwa der Wurzel- oder Logarithmenrechnung, hier ihren Ausgangspunkt zu nehmen./Sie erst schafft das Material, in dem dann Arithmetik und Analysis fast ausschließlich arbeiten, mit dem sie bauen können.

Nicht von jeher war das Gefühl für diese Notwendigkeit vorhanden. Die großen Schöpfer der Infinitesimalrechnung - Leibniz und ewton $^{\mathbf{1}}$ ) — und die nicht weniger großen Ausgestalter derselben, unter denen vor allem Euler ${ }^{2}$ ) zu nennen ist, waren zu berauscht von dem gewaltigen Erkenntnisstrom, der aus den neu erschlossenen Quellen floß, als daß sie sich zu einer Kritik der Grundlagen veranlaßt fühlten. Der Erfolg der neuen Methode war ihnen eine hinreichende Gewähr für die Tragfestigkeit ihres Fundamentes. Erst als jener Strom abzuebben begann, wagte sich die kritische Analyse an die Grundbegriffe: etwa um die Wende des 18. Jahrhunderts, vor allem unter dem mächtigen Einfluß von $G x u \beta^{3}$ ) wurden solche Bestrebungen stärker und stärker. Aber es währte noch fast ein Jahrhundert, ehe hier die wesentlichsten Dinge als völlig geklärt angesehen werden durften.

Heute gilt die Strenge gerade in bezug auf den zugrunde liegenden Zahlbegriff als die wichtigste Forderung, die an die Behandlung jedweden mathematischen Gegenstandes $\mathrm{zu}$ stellen ist, und seit den letzten Jahrzehnten des vergangenen Jahrhunderts — in den 60 er Jahren wurde won Weierstra $\beta^{4}$ ) in seinen Vorlesungen und im Jahre

1) Gottfried Wilhelm Leibniz, geb. 1648 in Leipzig, gest. 1716 in Hannover. Isaac Newton, geb. 1643 in Woolsthrope, gest. 1727 in London. Beide sind unabhängig voneinander zur Entdeckung der Grundlagen der Infinitesimalrechnung gelangt.

2) Leonhard Euler, geb. 1707 in Basel, gest. 1783 in Petersburg.

$\left.{ }^{3}\right)$ Karl Friodrich Gauß, geb. 1777 in Braunschweig, gest. 1855 in Göttingen.

$\left.{ }^{4}\right)$ Karl Weierstraß, geb. 1815 in Ostenfelde, gest. 1897 in Berlin. Die von ihm in seinen Vorlesungen seit 1860 vorgetragene Lehre von den reellen Zahlen hat erst neuerdings durch einen seiner Schüler, G. Mittag-Leffler, eine sorgfältige Darstellung gefunden in dessen Abhandlung: Die Zahl, Einleitung zur Theorie der analytischen Funktionen, The Tohoku mathematical Journal, Bd. 17 (1920), S. 157 -209. 
1872 von Cantor $^{\mathbf{1}}$ ) und von Dedekind ${ }^{2}$ ) sozusagen das letzte Wort in der Sache gesprochen - kann keine Vorlesung, kein Werk, das die grundlegenden Kapitel der höheren Analysis behandelt, Anspruch auf Gültigkeit machen, wenn es nicht von dem gereinigten Begriff der, reellen Zahl seinen Ausgangspunkt nimmt.

Seit jenen Jahren ist darum die Lehre von den reellen Zahlen so oft und in so mannigfacher Art dargestellt worden, daß es überflüssig erscheinen könnte, eine erneute und in alle Einzelheiten gehende Darlegung derselben ${ }^{3}$ ) zu geben; denn mit dem vorliegenden Buche (wenigstens seinen späteren Kapiteln) möchten wir uns nur an solche wenden, die mit den Anfangsgründen der Differential- und Integralrechnung schon vertraut sind. Indessen dürfen wir uns doch nicht bloß mit einem solchen Hinweis auf anderweitige Darstellungen begnügen. Denn eine Theorie der unendlichen Reihen würde, wie aus den späteren Entwicklungen hinlänglich klar werden wird, durchaus in der Luft schweben, wollte man ihr nicht in dem System der reellen Zahlen das feste Fundament geben, auf dem sie sich allein gründen kann. Darum und um bezüglich der Voraussetzungen, auf denen wir aufbauen wollen, nicht die geringste Unklarheit zu lassen, wollen wir im folgenden diejenigen Begriffe und Tatsachen aus der Lehre von den reellen Zahlen durchsprechen, deren wir weiterhin benötigen. Aber es soll sich dabei keineswegs um einen nur auf knapperen Raum zusammengedrängten, sonst lückenlosen Aufbau jener Lehre handeln, sondern lediglich um eine möglichst deutliche Hervorhebung der Hauptgedanken, der wesentlichsten Fragestellungen und ihrer Antworten. In bezug auf diese freilich wollen wir durchaus lückenlos und ausführlich sein und uns nur bei allen weniger prinzipiell wichtigen Einzelheiten, wie auch bei den nicht mehr im Rahmen dieses Buches liegenden Fragen nach Vollständigkeit und Einzigkeit des Systems der reellen Zahlen, mit kürzeren Andeutungen begnügen.

1) Georg Cantor, geb. 1845 in Petersburg, gest. 1918 in Halle. - Vgl. Mathem. Annalen, Bd. 5 (1872), S. 123.

2) Richard Dedekind, geb. 1831 in Braunschweig, gest. ebenda 1916. - Vgl. dessen Schrift: Stetigkeit und irrationale Zahlen, Braunschweig 1872.

3) Eine besonders leicht fafliche und alle Hauptsachen bringende Darstellung findet sich in H.v. Mangoldt, Einführung in die höhere Mathematik, Bd. I, 2. Aufl., Leipzig 1919. - Scharf und knapp (aber gerade darum für den Anfänger etwas schwerer zugänglich) ist die Darstellung in G. Kowalewski, Grundzüge der Differential- und Integralrechnung, Leipzig 1909. - Ein strenger und bis in die letzten Einzelheiten ausführlicher Aufbau des Systems der reellen Zahlen findet sich in $A$. Loewy, Lehrbuch der Algebra, I. Teil, Leipzig 1915, und in $A$. Pringsheim, Vorlesungen über Zahlen- und Funktionenlehre, I. Band, I. Abteilung, Leipzig 1916 (vgl. auch die Besprechung des letzteren Werkes durch H. Hahn, Gött. gel. Anzeigen 1919, S. 321/47). 


\title{
Erster Teil. \\ Reelle Zahlen und Zahlenfolgen.
}

\author{
I. Kapitel.
}

\section{Grundsätzliches aus der Lehre von den reellen Zahlen.}

\section{§1. Das System der rationalen Zahlen und seine Lücken.}

Was heißt es, wenn wir sagen, daß wir eine bestimmte Zahl „kennen“, oder daß sie uns "gegeben" sei, oder daß wir sie „berechnen“ können? Was heißt es, wenn jemand sagt, er kenne $\sqrt{2}$ oder die Zahl $\pi$, oder er könne $\sqrt{5}$ berechnen? Solche und ähnliche Fragen sind leichter gestellt als beantwortet. Sage ich, es sei $\sqrt{\mathbf{2}}=\mathbf{1 , 4 1 4}$, so ist das offenber falsch, denn $1,414 \cdot 1,414$ ist nicht $=2$, wie man durch Ausrechnen sofort, bestätigt. Sage ich vorsichtiger, es sei $\sqrt{2}=1,4142135 u s w$., so ist auch das keine stichhaltige Antwort und zunächst völlig sinnlos; denn es handelt sich doch zum mindesten darum, wie es weiter geht. Und das ist nicht ohne weiteres gesagt. Auch wird dieser Übelstand nicht beseitigt, wenn man noch mehr Ziffern des angefangenen Dezimalbruchs angibt, mögen es auch einige hundert sein. In diesem Sinne mag man wohl sagen, daß noch niemand den Wert von $\sqrt{2}$ ganz vor Augen, sozusagen vollständig in Händen gehabt hat, - während uns etwa die Aussage, daß $\sqrt{9}=3$ oder daß $35: 7=5$ ist, eine restlos vollständige und befriedigende dünkt. Nicht besser steht es ersichtlich mit der Zahl $\pi$ oder irgendeinem Logarithmus, einem sin oder einem cos aus den Tafeln. Trotzdem haben wir das sichere Gefühl: $\sqrt{2}$ oder $\pi$ oder $\log 5$ usw. haben einen ganz bestimmten Wert und wir kennen ihn auch schon. Nur über die exakte Bedeutung solcher Aussagen fehlt uns die klare Vorstellung. Wir wollen versuchen, sie uns zu verschaffen.

Nachdem wir eben über die Berechtigung einer Aussage wie „ich kenne $\sqrt{2}$ “ oder ähnlichen zweifelhaft geworden sind, müssen wir folgerichtigerweise auch die Berechtigung einer Aussage wie „ich kenne die Zahl $-\frac{23}{7}$ “, oder ,,mir wird (zum Zweck irgendeiner Rechnung) 
I. Kap. Grundsätzliches aus der Lehre von den reellen Zahlen.

die Zahl $\frac{9}{4}$ gegeben" nachprüfen. Ja, auch der Sinn einer Aussage wie ,ich kenne die Zahl 97 “ oder ,,zu irgendeiner Berechnung wird mir $a=2$ und $b=5$ gegeben ", wäre nachzuprüfen; es wäre also auch die Frage nach dem Sinn oder dem Begriff der natürlichen Zahlen $1,2,3, \ldots$ zu stellen.

Bei dieser letzten Frage fühlt man aber schon deutlich, daß sie über das Gebiet der Mathematik hinauslangt, daß sie in eine andere Gedankenordnung gehört, als die, die wir hier entwickeln wollen. Dem ist in der Tat so.

Keine Wissenschaft ruht ausschlieBlich in sich selbst. Die Tragfähigkeit ihrer letzten Grundlagen entlehnt eine jede aus anderen Schichten, die über ihr oder unter ihr liegen, - der Erfahrung, der Erkenntnistheorie, der Logik, der Metaphysik oder anderen. Irgend etwas muß jede Wissenschaft schlechthin als gegeben hinnehmen, um dann von da aus weiterzubauen. Und eine Kritik der Grundlagen und ein daran ansetzender strenger Aufbau einer Wissenschaft hat lediglich die Vorfrage zu erledigen, was in diesem Sinne als "gegeben“ angenommen werden soll, oder besser: welches Mindestma $B$ an Voraussetzungen unbedingt gemacht werden muß, um aus ihnen alles übrige zu entwickeln.

Für unseren Fall, den Aufbau des Systems der reellen Zahlen, sind diese Untersuchungen langwierig und mühsam; ja es muß eingestanden werden, daß sie in restlos befriedigender Weise überhaupt noch nicht zu Ende geführt worden sind. Es würde daher den Rahmen des vorliegenden Buches bei weitem überschreiten, wenn hier alles nötige nach dem heutigen Stande der Wissenschaft ausgeführt werden sollte. Wir wollen uns daher nicht zwingen, alles auf einem Minimum von Voraussetzungen aufzubauen, sondern wollen sofort einen Kreis von Tatsachen als bekannt (oder ,gegeben“, „gesichert“, ...) hinnehmen, dessen Herleitung aus einem geringeren Maß von Voraussetzungen jedem geläufig ist. Ich meine das System der rationalen Zahlen, also der ganzen und gebrochenen, positiven und negativen Zahlen einschließlich der Null. Jedem ist auch in der Hauptsache geläufig, wie dies System aufzubauen ist, falls man - als geringeres $\mathrm{Ma} B$ von Voraussetzungen - nur die geordnete Folge der natürlichen Zahlen 1, 2, 3... und deren Verknüpfungen durch Addition und Multiplikation als ,gegeben“ ansieht. Denn jeder weiß, - wir deuten dies nur flüchtig an, - wie aus dem Bedürfnis, die Multiplikation umzukehren, die gebrochenen Zahlen entstehen, und aus dem Bedürfnis, die Addition umzukehren, die negativen Zahlen und die Null ${ }^{1}$ ).

1) Eine ausführliche Darstellung eines solchen Aufbaus findet sich aufer in den in der Einleitung (S. 2) genannten Werken von Loewy und Pringsheim noch bei $O$. Hölder, Die Arithmetik in strenger Begriindung, Leipzig 1914, und O. Stolz und J. A. Gmeiner, Theoretische Arithmetik, 3. Aufl., Leipzig 1911. 
Die Gesamtheit der solchergestalt geschaffenen Zahlen wird als das System der rationalen Zahlen bezeichnet. Eine jede derselben kann mit Hilfe höchstens zweier natürlicher Zahlen, eines Bruchstriches und ev. eines Minuszeichens vollständig oder ziffernmäßig „gegeben" oder ",hingeschrieben", ,zur Kenntnis gebracht" werden. Wir bezeichnen sie zur Abkürzung mit kleinen lateinischen Buchstaben: $a, b, \ldots, x, y, \ldots$ Die wesentlichsten Eigenschaften dieses Systems sind nun diese:

1. Die rationalen Zahlen bilden eine geordnete Menge, d. h. zwischen irgend zweien von ihnen, etwa $a$ und $b$, besteht stets eine und nur eine der drei Beziehungen

$$
\left.a<b, \quad a=b, \quad b<a^{1}\right) ;
$$

und diese Anordnung der rationalen Zahlen gehorcht einer Anzahl von ganz einfachen Gesetzen, den sogenannten Anordnungssätzen.

Von diesen Sätzen, die wir im übrigen als bekannt ansehen wollen, $\mathbf{1}$. sind die folgenden Grundgesetze der Anordnung allein wesentlich:

1. Es ist stets $a=a^{2}$ ).

2. Aus $a=b$ folgt $b=a$.

3. Aus $a=b, b=c$ folgt $a=c$.

4. Aus $a \leqq b, b<c$ oder aus $a<b, b \leqq c$ folgt $a<c$.

2. Je zwei der rationalen Zahlen können auf vier verschiedene, als die vier Spezies Addition, Subtraktion, Multiplikation und Division bezeichnete Arten miteinander verknüpft werden. Diese Rechenoperationen sind stets und mit eindeutigem Ergebnis ausführbar, mit alleiniger Ausnahme der Division durch 0, welche nicht definiert ist und als schlechtweg unausführbare oder sinnlose Operation anzusehen ist. Sie gehorchen außerdem einer Anzahl einfacher Rechengesetze, den sog. Grundgesetzen der Arithmetik und den daraus hergeleiteten weiteren Regeln.

Auch diese sehen wir als jedem bekannt an und stellen hier nur ganz kurz diejenigen Grundgesetze der Arithmetik zusammen, aus denen mit Sicherheit alle uibrigen rein formal (d. $h$. nach den Gesetzen der reinen Logik) hergeleitet werden können.

I. Addition. $1 . Z \mathrm{u}$ je $z$ wei Zahlen $a$ und $b$ gibt es stets eine dritte $Z a h l c$, die die Summe von $a$ und $b$ genannt und mit $a+b$ bezeichnet wird.

2. Aus $a=a^{\prime}, b=b^{\prime}$ folgt stets $a+b=a^{\prime}+b^{\prime}$.

3. Es ist stets $a+b=b+a$. (Kommutationsgesetz.)

4. Es ist stets $(a+b)+c=a+(b+c)$. (Assoziationsgesetz.)

5. Aus $a<b$ folgt stets $a+c<b+c$. (Monotoniegesetz.)

II. Subtraktion. $Z$ u je zwei Zahlen $a$ und $b$ gibt es stets eine dritte Zahl $c$, fïr die $a+c=b$ ist.

1) In der Beziehung $a>b$ ist nur eine andere Schreibweise für die Beziehung $b<a$ zu sehen. Man käme also prinzipiell mit dem einen Zeichen $"<$ " aus.

2) Über dieses trivial anmutende "Gesetz" vgl. die Bemerkung S. 27. 
III. Multiplikation. 1. $\mathrm{Zu}$ je zwei Zahlen $a$ und $b$ gibt es stets eine dritte Zahl $c$, die das Produkt von $a$ und $b$ genannt und mit $a b$ bezeichnet wird.

2. Aus $a=a^{\prime}, b=b^{\prime}$ folgt stets $a b=a^{\prime} b^{\prime}$.

3. Es ist stets $a b=b a$. (Kommutationsgesetz.)

4. Es ist stets $(a b) c=a(b c)$. (Assoziationsgesetz.)

5. Es ist stets $(a+b) c=a c+b c$. (Distributionsgesetz.)

6. Aus $a<b$ folgt, falls $c$ positiv ist, stets $a c<b c$. (Monotoniegesetz.)

IV. Division. $Z u$ je zwei Zahlen $a$ und $b$, deren erste nicht gleich 0 ist, gibt es stets eine dritte Zahl $c$, für die $a c=b$ ist.

Aus diesen wenigen Grundgesetzen ergeben sich, wie betont, alle bekannten Rechenregeln und vollziehen sich weiterhin alle mathematischen Schlüsse ausschliefilich nach den Gesetzen der reinen Logik. Unter diesen nimmt eines wegen seines von Haus aus mathematischen Charakters eine besondere Stellung ein, nämlich

V. das sog. Induktionsgesetz, das auch als Grundgesetz der natürlichen Zahlen den Grundgesetzen der Arithmetik selbst zugerechnet werden kain und das wir so formulieren wollen:

Wenn irgendeine Aussage von einer natïrlichen Zahl $n$ abhängt (z. B. „Für $n \geqq 10$ ist stets $2^{n}>n^{34}$ oder älinliches), und wenn

a) diese Aussage für $n=p$ richtig ist und

b) wenn, unter $k$ irgendeine natürliche $Z$ ahl $\geqq p$ verstanden, aus ihrer Richtigkeit für $n=p,=p+1, \ldots,=k$ stets auch ihre Richtigkeit für $n=k+1$ gefolgert werden kann,

so ist jene Aussage für jede natürliche Zahl $n \geqq p$ richtig. (Bei den Anwendungen dieses Gesetzes ist meist $p=1$; auch empfiehlt es sich oft, $p=0$ zuzulassen.)

Endlich nennen wir noch einen Satz, der im Bereich der rationalen Zahlen sofort beweisbar ist, der aber bald nachher einen prinzipiellen Charakter gewinnen wird, nämlich den

VI. Satz des Eudoxus. Ist $a$ eine beliebige rationale Zahl, so gibt es stets eine natürliche Zahl $n$, für die $-n<a<+n$ ist $\left.^{1}\right)$.

Diese vier Verknüpfungen zweier rationaler Zahlen führen als Ergebnis stets wieder zu einer rationalen Zahl. Und in diesem Sinne bildet das System der rationalen Zahlen eine geschlossene Gesamtheit, den sogenannten natürlichen Rationalitätsbereich oder Zahlkörper. Eine solche Geschlossenheit in bezug auf die vier Spezies besitzt offenbar die Gesamtheit aller natürlichen oder die aller ganzen (positiven und negativen) Zahlen noch nicht. Diese sind sozusagen zu spärlich gesät, um allen Anforderungen zu genügen, die die vier Spezies an sie stellen können.

Diesen natürlichen Rationalitä'sberoich und die in ihm geltenden Gesetze also - und nur diese - sehen wir als gegeben, bekannt, gesichert an.

3. Nur das Rechnen mit Ungleichungen und absoluten Beträgen pflegt manchen etwas weniger geläufig zu sein. Wir stellen darum die wichtigsten Regeln kurz und ohne Beweis zusammen:

1) Dieser Satz wird meist (doch mit Unrecht) nach Archimedes benannt; er findet sich schon bei Euklid, Elemente V, Def. 4. 
I. Ungleichungen. Hier folgt alles aus den Anordnungs- und den Monotoniegesetzen. Es gilt speziell:

1. Die Monotoniesätze sind umkehrbar, d. h. aus $a+c<b+c$ folgt stets, daf $a<b$ ist; und dies folgt auch aus $a c<b c$, falls $c>0$ ist.

2. Aus $a<b, c<d$ folgt stets $a+c<b+d$.

3. Aus $a<b, c<d$ folgt, falls $b$ und $c$ positiv sind, daf $a c<b d$ ist.

4. Aus $a<b$ folgt stets $-b<-a$, und falls $a$ positiv ist, auch $\frac{1}{b}<\frac{1}{a}$. Und diese Sätze, sowie die Anordnungs- und Monotoniegesetze gelten (mit sinngemäfen Einschränkungen) auch mit den Zeichen,$\leqq,>, \geqq$ und $\neq$ “ an Stelle von " $<$ ".

II. Absolute Beträge. Definition: Unter $|a|$, dem absoluten Betrag von $a$, versteht man die positive unter den Zahlen $+a$ und $-a$, falls $a \neq 0$ ist, und die Zahl 0 , falls $a=0$ ist. (Es ist also $|0|=0$ und für $a \neq 0$ stets $|a|>0$.)

Es gelten u. a. die Sätze:

1. $|a|=|-a|$. $|a b|=|a| \cdot|b|$.

3. $\left|\frac{1}{a}\right|=\frac{1}{|a|}$ und $\left|\frac{b}{a}\right|=\frac{|b|}{|a|}$, falls $a \neq 0$.

4. $|a+b| \leqq|a|+|b| ;|a+b| \geqq|a|-|b|$ und sogar $|a+b| \geq|a|-|b| \mid$.

5. Die beiden Beziehungen $|a|<r$ und $-r<a<+r$ sind völlig gleichbedeutend; ebenso die Beziehungen $|x-a|<r$ und $a-r<x<a+r$.

6. Es bedeutet $|a-b|$ den Abstand der Punkte $a$ und $b$ bei der gleich nachher beschriebenen Veranschaulichung der Zahlen auf der Zahlengeraden.

Desgleichen sehen wir es als bekannt an, daß und wie man sich die Größenbeziehungen der rationalen Zahlen durch die Lage von Punkten auf einer Geràden, der Zahlengeraden, veranschaulichen kann: Man markiert auf ihr ganz beliebig zwei verschiedene Punkte $O$ und $E$ als Nullpunkt $(0)$ und Einheitspunkt $(+1)$ und ordnet nun allgemein der Zahl $a=\frac{p}{q}(q>0, p \gtrless 0$, ganzzahlig) denjenigen Punkt $P$ zu, den man èrhält, wenn man den (elementar-geometrisch sofort zu konstruierenden) $q^{\text {ten }}$ Teil der Strecke $O E$ von $O$ aus $|p|$-mal hintereinander abträgt, und zwar in der Richtung $O E$, falls $p>0$ ist, in der entgegengesetzten Richtung, falls $p$ negativ ist. Den gewonnenen Punkt nennen wir kurz den Punkt $a$, und die Gesamtheit der auf diese Weise den rationalen Zahlen entsprechenden Punkte wollen wir kurz die rationalen Punkte der Zahlengeraden nennen. - Diese Gerade denkt man sich gewöhnlich von links nach rechts gezogen und $E$ rechts von $O$ gewählt. Dann bedeuten die Worte positiv und negativ offenbar soviel wie rechts von $O$ und links von $O$; und allgemein bedeutet $a<b, \mathrm{daB} a$ links von $b, b$ rechts von $a$ gelegen ist. Mit Hilfe dieser Ausdrucksweise können wir den abstrakten Beziehungen zwischen den Zahlen oft eine durch die Anschauung leichter zu erfassende Form geben.

Das ist nun in kurzen Strichen das Fundament, das wir als gesichert annehmen wollen. Durch die Beschreibung desselben wollen 
wir nun auch den Zahlbegriff selbst als charakterisiert ansehen, d. $h$. wir wollen ein System von begrifflich wohl unterschiedenen Dingen (Elementen, Zeichen) als ein Zahlensystem, seine Elemente als Zahlen ansprechen, wenn man — zunächst ganz kurz gesagt - mit ihnen im wesentlichen ebenso operieren kann, wie mit den rationalen Zahlen.

Diese noch etwas ungenaue Aussage wollen wir so präzisieren: 4. Es liege ein System $S$ von wohlunterschiedenen Dingen vor, die wir mit $\iota, \beta, \ldots$ bezeichnen. Dann wollen wir $S$ als ein Zahlensystem, seine Elemente $\iota, \beta, \ldots$ als Zahlen ansprechen, wenn die Zeichen $\alpha$, $\beta, \ldots$ zunächst einmal irgendwie ausschließlich mit Hilfe der rationalen - also letzten Endes der natürlichen - Zahlen hergestellt sind $^{1}$ ), und wenn das System überdies den folgenden vier Bedingungen genügt:

1. Zwischen je zwei Elementen $\&$ und $\beta$ aus $S$ besteht stets eine und nur eine der drei Beziehungen

$$
\left.\leftrightarrow<\beta, \quad \alpha=\beta, \quad \beta<\alpha^{2}\right)
$$

(man sagt kurz: $S$ ist geordnet); und diese Anordnung der Elemente von $S$ gehorcht denselben Grundgesetzen 1, wie die gleichbenannten Beziehungen im System der rationalen Zahlen ${ }^{3}$ ).

2. Es sind vier verschiedene, als Addition, Subtraktion, Multiplikation und Division bezeichnete Verknüpfungen je zweier Elemente $\alpha$ und $\beta$ aus $S$ erklärt; diese sind - mit einer einzigen, sogleich zu nennenden Ausnahme (s. 3) - stets und mit eindeutigem Ergebnis ausführbar und gehorchen dabei denselben Grundgesetzen 2, I-IV, wie die gleichbezeichneten Verknüpfungen im System der rationalen Zahlen ${ }^{4}$ ).

3. Jeder rationalen Zahl läßt sich ein Element aus $S$ (und alle ihm gleichen) so zuordnen, daß, wenn etwa den rationalen Zahlen $a$ und $b$ die Elemente $c$ und $\beta$ aus $S$ entsprechen, nun

1) Beispiele werden wir in $\S 3$ und $\S 5$ kennen lernen; im Augenblick denke man an Dezimalbrïche oder ähnliche aus rationalen Zahlen aufgebaute Zeichen. - Im übrigen vgl. hierzu die Fufnote 2 auf S. 31.

2) $\alpha>\beta$ ist nur als eine andere Schreibweise für $\beta<\alpha$ anzusehen; $\alpha \neq \beta$ bedeutet, dafs $\alpha$ nicht gleich $\beta$, also $<\beta$ oder $>\beta$ ist.

$\left.{ }^{3}\right)$ Uber den sozusagen praktischen Inhalt dieser Beziehungen ist dabei nichts gesagt; $\propto<\beta$ kann das übliche „kleiner" bedeuten, es kann aber auch "früher", "links von", „höher", "tiefer", "später", ja schlieflich jedwede Anordnungsbeziehung (also etwa auch "gröfier") bedeuten. Diese Bedeutung muf nur eindeutig festgelegt sein.

4) Auch bezüglich des praktischen Inhalts dieser 4 Verkniipfungsarten gilt eine analoge Bemerkung wie soeben bei den Zeichen $=$ und $<$. - Man wird noch bemerkt haben, dafs die Subtraktion schon vollständig mit Hilfe der Addition, die Division schon vollständig mit Hilfe der Multiplikation erklärt werden kann. Es sind also letzten Endes nur zwei Verknüpfungen, die schlechtweg als bekannt angesehen werden müssen. 
a) zwischen $\alpha$ und $\beta$ dieselbe der drei Beziehungen 1. besteht, wie zwischen $a$ und $b$; und daß

b) das Ergebnis der Verknüpfungen $\alpha+\beta, \alpha-\beta, \alpha \cdot \beta, \alpha: \beta$ auch stets dem Ergebnis der Verknüpfungen $a+b, a-b, a \cdot b, a: b$ zưgeordnet ist. [Hierfür sagt man wohl auch kürzer: $S$ enthält ein Teilsystem $S^{\prime}$ - nämlich die Gesamtheit aller Elemente aus $S$, die einer rationalen Zahl zugeordnet sind -, welches dem System der rationalen Zahlen ähnlich und isomorph ist $\left.{ }^{1}\right)$ ]. Ein hierbei der rationalen Zahl 0 entsprechendes Element aus $S$ (und alle ihm gleichen) kann man dann kurz als die „Null“ aus $S$ bezeichnen. Die unter 2. genannte Ausnahme bezieht sich dann auf die Division durch die Null $^{2}$ ).

4. 'Ist a ein beliebiges Element aus $S$, so soll es in $S$ auch ein Element $\boldsymbol{\nu}$ geben, das auf Grund von 3 einer natürlichen Zahl $n$ zugeordnet ist und für das $\alpha<v$ ist. (Postulat des Eudoxus; vgl. 2, VI.)

An diese abstrakte Charakterisierung des Zahlbegriffs knüpfen wir noch die folgende Bemerkung ${ }^{3}$ ): Enthält das System $S$ außer den

1) Man nennt zwei geordnete Systeme ähnlich, wenn sich ihre Elemente einander so zuordnen lassen, dafi zwischen zwei Elementen des einen Systems dieselbe der drei Beziehungen 4, 1 besteht wie zwischen den ihnen entsprechenden Elementen des andern. Und man nennt zwei Systeme in bezug auf die mit ihren Elementen möglichen vier Verknüpfungen isomorph, wenn das Resultat der Verknüpfung $z$ weier Elemente des einen Systems wiederum dem Resultat der gleichnamigen Verknüpfung der entsprechenden Elemente des andern Systems zugeordnet ist.

2) Die 3te der Forderungen, durch die wir den Zahlbegriff hier charakterisieren, ist übrigens schon eine Folge der 1 ten und 2ten. Diese Bemerkung ist für unsere $Z$ wecke nicht wesentlich; da sie aber vom methodischen Standpunkt aus bedeutungsvoll ist, deuten wir den Beweis kurz an: Nach 4, 2 gibt es ein Element $\zeta$, für das $\alpha+\zeta=\alpha$ ist. Aus den Grundgesetzen 2 , I folgt dann ganz leicht, daß́ dasselbe Element $\zeta$ für jedes $\alpha$ aus $S$ die Gleichung $\alpha+\zeta=\alpha$ erfüllt. Dieses Element $\zeta$ (und alle ihm gleichen) nennt man die "Null" aus $S$. Ist dann $\alpha$ von dieser "Null" verschieden, so gibt es weiter ein Element $\varepsilon$, für das $\alpha \varepsilon=\alpha$ ist; und es zeigt sich wieder, daf dieses selbe Element $\varepsilon$ dieser Gleichung für jedes $\alpha$ aus $S$ gentigt. Man nennt $\varepsilon$ (und alle ihm gleichen Elemente) die „Eins“ aus $S$. Die durch wiederholte Additionen und Subtraktionen dieser "Eins" erzeugten Elemente aus $S$ (und alle ihnen gleichen) wird man dann als die "ganzen Zahlen" aus $S$ bezeichnen. Die aus diesen durch beliebige Divisionen entstehenden weiteren Elemente (und alle ihnen gleichen) bilden dann das in Rede stehende Teilsystem $S^{\prime}$ von $S$, von dem hiernach unmittelbar klar ist, daf es zum System der rationalen Zahlen ahnlich und isomorph ist. - Tatsächlich ist also unser Zahlbegriff schon durch die Forderungen 4, 1, 2 und 4 festgelegt.

3) Wir haben den Zahlbegriff durch eine Anzahl ihn charakterisierender Eigenschaften festgelegt. Eine kritische Grundlegung der Arithmetik, von der in dem Rahmen dieses Buches nicht die Rede sein kann, müite nun genau untersuchen, inwieweit diese Eigenschaften voneinander unabhängig sind, ob also eine von ihnen als beweisbare Tatsache aus den anderen gefolgert werden 
auf Grund der Zuordnung 3 den rationalen Zahlen entsprechenden Elementen keine weiteren davon verschiedenen, so ist unser System $S$ überhaupt nicht wesentlich von dem System der rationalen Zahlen verschieden; sondern es unterscheidet sich von ihm letzten Endes nur durch die (rein äußerliche) Bezeichnung der Elemente oder durch die (rein praktische) Bedeutung, die wir diesen Zeichen geben, also im Grunde nicht viel wesentlicher, als wenn wir die Ziffern einmal mit arabischen, ein andermal mit römischen oder chinesischen Zeichen schreiben, und als wenn sie einmal Temperaturen, ein andermal Geschwindigkeiten oder elektrische Ladungen bedeuten. Wenn wir also von der äußerlichen Bezeichnung und der praktischen Bedeutung absehen, könnten wir geradezu sagen, das System $S$ sei mit den rationalen Zahlen identisch, und können in diesem Sinne geradezu $a=\iota, b=\beta, \ldots$ setzen.

Enthält aber das System $S$ außer den obengenannten noch weitere davon verschiedene Elemente, so werden wir sagen, $S$ umfasse das System der rationalen Zahlen, es sei eine Erweiterung desselben. Ob es überhaupt solche umfassenderen Systeme gibt, steht natürlich im Augenblick noch ganz offen; doch werden wir im System der reellen Zahlen nun sehr bald ein solches kennen lernen.

Nachdem wir uns so über das Maß von Voraussetzungen geeinigt haben, über das nicht mehr gestritten werden soll, werfen wir noch einmal die Frage auf: Was heißt es, wenn wir sagen, wir kennten die Zahl $\sqrt{2}$ oder die Zahl $\pi$ od. ähnl.?

Es muß zunächst als durchaus paradox bezeichnet werden, daß es eine Zahl, deren Quadrat $=2$ ist, in dem bisherigen System noch nicht gibt $^{1}$ ), oder geometrisch gesprochen, daß der Punkt $A$ der

kann oder nicht. Ferner müßte gezeigt werden, daßs keines jener Grundgesetze mit einem der andern in Widerspruch steht, - und noch manches andere. Diese Untersuchungen sind mühsam und können auch heute noch nicht als abgeschlossen angesehen werden. Näheres darüber bei $A$. Loewy, 1. c. Als eine besonders interessante Tatsache heben wir hervor, dafs z. B. das Kommutationsgesetz der Addition àls beweisbarer Satz aus den übrigen Grundgesetzen gefolgert werden kann (Hilbert, Jahresber. d. Dtsch. Math. Ver., Bd. 8, 1900, S. 180). Das Gesetz 2, I, 3 dürten wir also streichen, ohne dafs dadurch der Aufbau der Arithmetik eine Änderung erführe.

1) Beweis: Eine natürliche Zahl gibt es jedenfalls nicht, deren Quadrat $=2$ ist, da $1^{2}=1$ ist und die Quadrate aller übrigen natürlichen Zahlen $\geqq 4$ sind. Es käme also für $\sqrt{2}$ nur eine (positive) gebrochene Zahl $\frac{p}{q}$ in Betracht, bei der also $q \geq 2$ und $z \mathbf{u} p$ teilerfremd (der Bruch also in gekürzter Form) gedacht werden kann. Läßt sich aber $\frac{p}{q}$ nicht weiter kürzen, so ist dasselbe mit dem Bruch $\left(\frac{p}{q}\right)^{2}=\frac{p \cdot p}{q \cdot q}$ der Fall, der also nicht gleich der ganzen Zahl 2 sein kann. Oder etwas anders gefafit: Aus $\left(\frac{p}{q}\right)^{2}=2$ folgte $p^{2}=2 q^{2}$, wonach 
Zahlengeraden, dessen Entfernung von $O$ gleich der Diagonale des Quadrates mit der Seite $O E$ ist, mit keinem der oben eingeführten rationalen Punkte zusammenfällt. Denn einerseits liegen die rationalen Zahlen dicht, d. h. zwischen irgend zwei verschiedenen von ihnen lassen sich noch beliebig viele weitere angeben (denn ist $a<b$, so liegen die $n$ rationalen Zahlen, die die Formel $a+v \frac{b-a}{n+1}$ für $v=1,2, \ldots, n$ liefert, offenbar alle zwischen $a$ und $b$ und sind voneinander und von $a$ und $b$ verschieden). Andererseits aber liegen sie sozusagen noch nicht dicht genug, um alle denkbaren Punkte zu bezeichnen. Vielmehr, wie sich die Gesamtheit aller ganzen Zahlen als zu spärlich erwiesen hat, um allen durch die vier Spezies an sie gestellten Forderungen zu genügen, so erweist sich jetzt wieder die Gesamtheit aller rationalen Zahlen als zu lückenhaft ${ }^{1}$ ), um den weitergehenden Forderungen, die die Wurzelrechnung an sie stellt, zu genügen. Trotzdem hat man das Gefühl, daß auch diesem Punkte $A$ oder also dem Zeichen $\sqrt{2}$ ein ganz bestimmter Zahlenwert zukommt. Welche greifbaren Tatsachen liegen diesem Gefühl zugrunde?

Es ist zunächst offenbar dies: Man weiß zwar genau, daß für $\sqrt{2}$ die Angaben 1,4 oder 1,41 oder 1,414 usw. falsch sind, daß diese (rationalen) Zahlen zum Quadrat erhoben vielmehr $<2$ bleiben, also zu klein sind. Man weiß aber gleichzeitig, daß die Angaben 1,5 oder 1,42 oder 1,415 usw. in demselben Sinne zu groß wären, daß also der zu erfassende Wert zwischen den entsprechenden zu kleinen und zu großen Angaben liegen müßte. Und was uns trotz dieser „Falschheit“ der Angaben die Überzeugung gibt, hiermit den Wert $\sqrt{2}$ irgendwie doch erfaßt zu haben, kann nur dies sein: Wir besitzen ein Verfahren, um die obigen Angaben soweit fortzusetzen, wie wir wollen; wir können also Paare von Dezimalbrüchen mit je $1,2,3, \ldots$ Stellen angeben, die sich jeweils nur um eine Einheit in der letzten Stelle, also bei $n$ Stellen um $\left(\frac{1}{10}\right)^{n}$, unterscheiden, und von denen der eine Bruch zu klein, der andere zu groß ist. Da dieser Unterschied,

$p$ eine gerade Zahl, etwa $=2 r$ wäre. Aus $p^{2}=4 r^{2}=2 q^{2}$ folgte aber $q^{2}=2 r^{2}$, wonach auch $q$ eine gerade Zahl sein müiste, - im Gegensatz zu der Annahme, dafs $p$ und $q$ zueinander teilerfremd sein sollten. Es ist also niemals $\left(\frac{p}{q}\right)^{2}=2$. Diese Tatsache soll schon Pythagoras bekannt gewesen sein (vgl. M. Cantor, Gesch. d. Mathem, Bd. 1, 2. Auf1., 1894, S. 142 u. 169).

1) Gerade dies ist das Paradoxe und der unmittelbaren Anschauung schwer zugängliche, daßs auf der Zahlengeraden schon eine (im eben definierten Sinne) dichte Menge von Punkten markiert ist, und dak dies doch nicht alle Punkte der Geraden sind. - Vergleichsweise kann man dies so beschreiben: Die ganzen Zahlen bilden eine erste grobe Einteilung in Fächer; die rationalen Zahlen füllen diese Fächer wie mit feinem Sande aus, der aber für den schärferen Blick notwendig noch Lücken lassen mußs. Diese nun auszufüllen, wird unsere nächste Aufgabe sein. 
wenn wir nur die Anzahl $n$ der Stellen groß genug machen, so klein gemacht werden kann, wie wir wollen, da das Verfahren also den zu erfassenden Wert zwischen zwei Zahlen einzuklemmen lehrt, die so eng beieinander liegen, wie wir wollen, so sagen wir mit einer zunächst etwas kühnen Metapher: durch dies Verfahren sei uns $\sqrt{2}$ selber „gegeben“, auf Grund dieses Verfahrens „kennten“ wir $\sqrt{2}$ selbst, wir könnten $\sqrt{2}$,berechnen“ usw.

Genau so liegen die Dinge bei jedem andern Wert, der nicht durch eine rationale Zahl selbst bezeichnet werden kann, also z. B. bei $\pi, \log 2, \sin 10^{\circ}$ usw. Wenn wir sagen, wir kennten diese Zahlen, so liegt dem jedesmal nichts anderes zugrunde als dies: Wir kennen ein (in den meisten Fällen sehr beschwerliches) Verfahren, um in ähnlicher Weise, wie eben bei $\sqrt{2}$ genauer gezeigt, den zu erfassenden Wert zwischen immer dichter und schließlich beliebig dicht sich zusammenschließenden (rationalen) Zahlen einzuspannen.

Um diese Dinge etwas allgemeiner und schärfer zu erfassen, schalten wir eine vorläufige, aber doch für alles folgende durchaus grundlegende Betrachtung über Folgen rationaler Zahlen ein.

\section{§ 2. Rationale Zahlenfolgen ${ }^{1}$ ).}

Bei der vorhin angedeuteten Berechnung von $\sqrt{2}$ bildeten wir nacheinander wohlbestimmte rationale Zahlen. Von der speziellen Dezimalbruchform wollen wir uns hierbei freimachen und beginnen mit folgender

5. Definition: Läßt sich auf Grund irgendeines gesetzmäßigen Bildungsverfahrens der Reihe nach eine 1., eine 2., eine 3.,... (rationale) $Z$ ahl bilden, und entspricht somit jeder natürlichen Zahl $n$ eine und nur cine wohlbestimmte (rationale) Zahl $x_{n}$, so sagt man, daß diese Zahlen

$$
x_{1}, \quad x_{2}, x_{3}, \ldots, x_{n}, \ldots
$$

(in dieser den natürlichen Zahlen entsprechenden Anordnung) eine Zahlenfolge bilden. Wir bezeichnen sie kurz mit $\left(x_{n}\right)$ oder mit $\left(x_{1}, x_{2}, \ldots\right)$.

Beispiele.

6. $1 . x_{n}=\frac{1}{n}$, also die Folge $\left(\frac{1}{n}\right)$ oder $1, \frac{1}{2}, \frac{1}{3}, \ldots, \frac{1}{n}, \ldots$

2. $x_{n}=2^{n}$, also die Folge $2,4,8,16, \ldots$ sein soll.

3. $x_{n}=a^{n}$, also die Folge $a, a^{2}, a^{3}, \ldots$, bei der $a$ eine gegebene Zahl

4. $x_{n}=\frac{1}{2}\left[1-(-1)^{n}\right]$, also die Folge $1,0,1,0,1,0, \ldots$

5. $x_{n}=$ dem nach $n$ Ziffern abgebrochenen Dezimalbruch für $\sqrt{2}$.

$\left.{ }^{1}\right)$ Auch in diesem Paragraphen bedeuten alle vorkommenden Buchstabengrößen noch stets rationale Zahlen. 
6. $x_{n}=\frac{(-1)^{n-1}}{n}$, also die Folge $1,-\frac{1}{2},+\frac{1}{3},-\frac{1}{4}, \ldots$

7. Es soll $x_{1}=1, x_{2}=1, x_{3}=x_{1}+x_{2}=2$ und allgemein soll für $n \geq 3$ stets $\dot{x}_{n}=x_{n-1}+x_{n-2}$ sein. Man erhält so die Folge $1,1,2,3,5,8,13,21, \ldots$

8. $1,2, \frac{1}{2},-2,-\frac{1}{2}, 3, \frac{1}{3},-3,-\frac{1}{3}, \ldots$

9. $2, \frac{3}{2}, \frac{4}{3}, \frac{5}{4}, \ldots, \frac{n+1}{n}, \ldots$

10. $0, \frac{1}{2}, \frac{2}{3}, \frac{3}{4}, \frac{4}{5}, \ldots, \frac{n-1}{n}, \ldots$

11. $x_{n}=$ der $n$ ten Primzahl, also die Folge $2,3,5,7,11,13,17, \ldots$

12. Die Folge $1, \frac{3}{2}, \frac{11}{6}, \frac{25}{12}, \frac{137}{60}, \ldots$, bei der allgemein $x_{n}=\left(1+\frac{1}{2}+\cdots+\frac{1}{n}\right)$.

\section{Bemerkungen.}

1. Das Bildungsgesetz kann ganz beliebig sein. Es braucht insbesondere nicht in einer expliziten Formel $\mathrm{zu}$ bestehen, die es gestattet, bei gegebenem $n$ das zugeordnete $x_{n}$ direkt $z u$ berechnen. Bei Beispiel $\mathbf{6}, 5,7 \mathrm{u} .11$ ist dies offenbar nicht ohne weiteres möglich. Und bei einer zahlenmäßig vorgelegten Folge braucht weder das Bildungsgesetz (vgl. 6, 5 u. 12) noch sonst irgendeine Regelmäfigkeit unter den aufeinanderfolgenden Zahlen in die Augen zu fallen (vgl. 6, 11).

2. Manchmal ist es vorteilhaft, die Folge mit einem „0 ten "i Gliede $x_{0}$, oder gar einem $(-1)$ ten oder $(-2)$ ten Gliede $x_{-1}, x_{-2}$ beginnen $z u$ lassen. Wir wollen dann trotzdem als $n$ tes Glied stets dasjenige bezeichnen, welches den Index $n$ trägt. Bei 6, 2, 3 u. 4 kann man z. B. ohne weiteres ein 0 tes Glied oder auch ein $(-1)^{\text {tes }}$ oder $(-2)^{\text {tes }}$ voranstellen. In solchem Falle ist also das „erste" Glied nicht das Anfangsglied der Folge. Die Bezeichnung ist dann $\left(x_{0}, x_{1}, \ldots\right)$ bzw. $\left(x_{-1}, x_{0}, \ldots\right)$, und nur wenn über den Anfang der Numerierung kein $Z$ weifel besteht, oder wenn sie ganz belanglos ist, schreiben wir kurz $\left(x_{n}\right)$ zur Bezeichnung der Folge.

3. Eine Zahlenfolge wird oft besonders als unendliche bezeichnet. Dies Beiwort soll dann nur betonen, daßs auf jedes Glied noch weitere folgen. Man sagt dann auch, daß es sich um unendlich viele Glieder handle. Allgemein spricht man von endlich vielen oder unendlich vielen Dingen, je nachdem diese Dinge eine durch eine bestimmte natürliche Zahl angebbare Anzahl haben oder nicht. Auch weiterhin wird - wie wir gleich jetzt betonen wollen das Wort ,unendlich" immer nur eine symbolische Bedeutung haben, durch die ein wohlbestimmter (meist sehr einfacher) Sachverhalt abkürzend bezeichnet wird.

4. Haben alle Glieder einer Folge ein und denselben Wert $c$, so sagt man, die Folge sei identisch glerch $c$ und schreibt wohl $x_{n} \equiv c$. Allgemein schreiben wir $\left(x_{n}\right) \equiv\left(x_{n}^{\prime}\right)$, wenn die beiden Folgen $\left(x_{n}\right)$ und $\left(x_{n}{ }^{\prime}\right)$ Glied für Glied ubereinstimmen, wenn also für jeden in Betracht kommenden Index $x_{n}=x_{n}^{\prime}$ ist.

5. Oft ist es zur Veranschaulichung einer Zahlenfolge bequem, sich ihre Glieder als Punkte auf der Zahlengeraden $\mathrm{zu}$ markieren oder markiert $\mathrm{zu}$ denken. Wir haben dann eine Punktfolge vor uns. Doch ist hierbei $z u$ beachten, dafi in einer Zahlenfolge ein und dieselbe Zahl mehrmals, ja ,unendlich oft" auftreten kann (vgl. 6, 4). Dann ist der entsprechende Punkt mehrmals, evtl. unendlich oft zu zählen, d. h. als Glied der Punktfolge zu betrachten. 
6. Eine andere Art der Veranschaulichung besteht darin, daf man in einem rechtwinkligen Achsenkreuz die Punkte mit den Koordinaten $\left(n, x_{n}\right)$, für $n=1,2,3, \ldots$, markiert und der Reihe nach durch geradlinige Strecken verbindet. Der entstehende geradlinig-gebrochene Linienzug gibt dann ein Bild der Zahlenfolge.

Die Untersuchung der hiermit eingeführten Zahlenfolgen nach den mannigfachsten Gesichtspunkten wird nun den Hauptgegenstand aller folgenden Kapitel bilden. Insbesondere wird es sich dabei um Feststellungen oder Aussagen handeln, die für alle Glieder der Folge gelten, oder wenigstens für alle Glieder, die hinter einem bestimmten stehen ${ }^{1}$ ). Im Hinblick auf diese letztere Einschränkung sagt man wohl, daß es bei der betreffenden Feststellung ,auf endlich viele Glieder nicht ankäme" oder daß sie sich nur auf das infinitäre Verhalten der Folge bezöge. Als erste Beispiele solcher Feststellungen führen wir folgende Definitionen ein:

S. Definitionen. I. Einc Folge soll beschränkt ${ }^{2}$ ) heißen, wenn es. eine positive Zahl $K$ gibt, so daß für alle Glieder die Ungleichung

$$
\left|x_{n}\right| \leqq K \quad \text { oder } \quad-K \leqq x_{n} \leqq K
$$

gilt. K heißt dann eine Schranke für die Beträge der Glieder der Folge.

Bemerkungen und Beispiele.

$>1 . \mathrm{Ob}$ in der Definition $\mathbf{S}, \leq K$ " oder,$<K$ " steht, ist ziemlich gleichgültig. Denn ist stets (d. h. für alle in Betracht kommenden Indizes $n$ ) $x_{n} \leq K$, so gibt es auch eine Konstante $K^{\prime}$, so daf stets $\left|x_{n}\right|<K^{\prime}$ ist; denn offenbar kann jedes $K^{\prime}>K$ dafür gewählt werden. Ist umgekehrt stets $x_{n} \mid<K$, so ist erst recht $\left|x_{n}\right| \leq K$. In jedem Einzelfall kann der Unterschied natiirlich trotzdem sehr wesentlich werden.

2. Eine Schranke $K$ von $\left(x_{n}\right)$ kann also durch jede größere Zahl ersetzt werden.

3. Die Folgen $\mathbf{6}, 1,4,5,6,9,10$ sind offenbar beschränkt; $\mathbf{6}, 3$ ist es auch, falls $|a| \leq 1$ ist. Die Folgen 6, 2, 7, 8, 11 sind es sicher nicht. Ob die Folge 6, 12 und ob $\mathbf{6}, 3$ für ein jedes $|a|>1$ beschränkt ist oder nicht, ist nicht ohne weiteres zu sehen.

4. Weif man nur, daf3 es eine Konstante $K_{1}$ gibt $_{3}{ }^{\prime}$ für die stets $x_{n}<K_{1}$ bleibt, so soll die Folge nach rechts (oder: nach oben) beschränkt, $K_{1}$ selbst eine obeve (oder: rechte) Schranke der Folge heifen. Gibt es eine Konstante $K_{2}$, für die stets $x_{n}>K_{2}$ bleibt, so soll $\left(x_{n}\right)$ nach links beschränkt, $K_{2}$ eine untere Schranke der Folge heifien. Hierbei brauchen $K_{1}$ und $K_{2}$ nicht positiv zu sein.

5. Ist eine Folge nach rechts beschränkt, so braucht es doch unter ihren Zahlen keine größste zu geben. So ist z. B. 6, 10 nach rechts beschränkt, und doch wird jede Zahl dieser Folge von jeder folgenden libertroffen, so daf3.

1) Z.B.: Alle Glieder der Folge $\mathbf{6}, 9$ sind $>1$. Oder: Alle Glieder der Folge (i, 2, die hinter dem 6 ten stehen, sind $>100$ (kuirzer: für $n>6$ ist $x_{n}>100$ ).

-) Diese Bezeichnung scheint von C. Jordan, Cours d'analyse, Bd. 1, Paris 1893, S. 22, eingefuihrt worden zu sein. 
keine die gröfte sein $\left.k^{2} n^{1}\right)$. Entsprechend braucht eine nach links beschränḳte Folge kein kleinstes Glied zu enthalten; vgl. 6, 1 und 9. - Mit dieser zunächst paradox anmutenden Tatsache mache sich der Anfänger wohl vertraut! - Unter endlich vielen Größsen gibt es natürlich stets sowohl eine größíte als eine kleinste.

II. Eine Folge soll monoton wachsend oder zunehmend heißen, wenn stets

$$
x_{n} \leqq x_{n+1}
$$

ist; dagegen monoton fallend oder abnehmend, wenn stets

$$
x_{n} \geqq x_{n+1}
$$

ist. Beide Arten werden auch kurz als monotone Folgen bezeichnet.

Bemerkungen und Beispiele.

1. Eine Folge braucht natiirlich weder monoton wachsend, noch monoton 9. fallend zu sein; vgl. 6, 4, 6, 8. Doch sind monotone Folgen sehr häufig und im allgemeinen bequenier $\mathrm{zu}$ uiberschauen als die nicht monotonen. Darum ist es zweckmäfig, sie durch eine besondere Benennung auszuzeichnen.

2. Statt wachsend müiste man genauer ,nicht fallend“", statt fallend genauer "nicht wachsend" sagen; doch ist das meist nicht üblich. Soll in besonderen Fällen das Gleichheitszeichen ausgeschlossen werden, soll also stets $x_{n}<x_{n+1}$ bzw. $x_{n}>x_{n+1}$ sein, so nennt man die Folge im engeren Sinne monoton wachsend bzw. fallend.

3. Die Folgen 6, 2, 5, 7, 10, 11, 12 und 6, 1, 9 sind monoton; die erstgenannten steigend, die andern fallend. $\mathbf{6}, 3$ fällt monoton, falls $0 \leqq a \leqq 1$ ist, steigt dagegen monoton für $a \geqq 1$; für $a<0$ ist sie nicht monoton.

4. Die Bezeichnung "monoton" rührt von C. Neumann her (Über die nach Kreis-, Kugel- und Zylinderfunktionen fortschreitenden Entwicklungen. Leipzig 1881, S. 26/27).

Wir kommen nun zu einer Definition, der die größte Aufmerksamkeit zu schenken ist, und bei der man nicht müde werden darf, sich ihren Inhalt bis in die letzten Konsequenzen klarzumachen.

III. Eine Folge soll als Nullfolge bezeichnet werden, wenn sie 10. folgende Eigenschaft besitzt: Wenn eine beliebige positive Zahl $\varepsilon$ gegeben wird, so soll die Ungleichung

$$
\left|x_{n}\right|<\varepsilon
$$

durch alle Glieder, von höchstens endlich vielen ${ }^{2}$ ) Ausnahmen abgesehen, erfüllt werden. Oder anders ausgedrückt: Wenn eine beliebige positive Größe \& gewählt wird, so soll sich stets eine Zahl $n_{0}$ so angeben lassen, daß

ist.

$$
\text { für alle } \quad n>n_{0} \quad \text { stets } \quad\left|x_{n}\right|<\varepsilon
$$

1) Der Anfänger lasse sich nicht durch oft gehörte Redewendungen wie diese beirren: „Für unendlich großes $n$ sei $x_{n}=1$ und es sei also 1 die größte Zahl der Folge". So etwas ist barer Unsinn (vgl. hierzu auch 7,3 ). Denn die Glieder der Folge sind die Zahlen $0, \frac{1}{2}, \frac{2}{3}, \frac{3}{4}, \ldots$ und von diesen ist keine

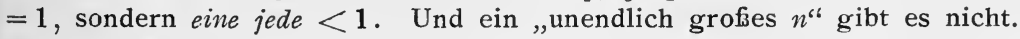

2) Vgl. 7, 3 . 
Bemerkungen und Beispiele.

1. Sind diese Bedingungen bei einer vorgelegten Folge für ein bestimmtes $\varepsilon$ erfüllbar, so sind sie um so eher für jedes größsere $\varepsilon$ erfüllbar (vgl. S, 1), nicht aber für jedes kleinere. (Bei 6, $10 \mathrm{z}$. B. sind die Bedingungen für $\varepsilon=1$, und also jedes größsere $\varepsilon$ erfüllt, wenn man $n_{0}=0$ nimmt; für $\varepsilon=\frac{1}{2}$ dagegen sind sie nicht erfüllbar.) Bei einer Nullfolge sollen aber die Bedingungen für jedes positive $\varepsilon$, insbesondere also auch für jedes sehr kleine $\varepsilon>0$ erfuillbar sein. Daher formuliert man die Definition etwas nachdrücklicher gewöhnlich so: $\left(x_{n}\right)$ ist eine Nullfolge, wenn jedem noch so kleinen $\varepsilon>0$ eine Zahl $n_{0}$ so entspricht, dafis nun

ist.

$$
\text { für alle } n>n_{0} \text { stets }\left|x_{n}\right|<\varepsilon
$$

2. Die Folge 6, 1 ist offenbar eine Nullfolge; denn für

$$
n>\frac{1}{\varepsilon} \text { ist stets }\left|x_{n}\right|<\varepsilon,
$$

wie auch $\varepsilon>0$ gegeben wird. Es genügt also $n_{0}=\frac{1}{\varepsilon}$ zu nehmen.

3. Die Stelle, von der an die Beträge der Glieder einer vorgelegten Folge bei gegebenem $\varepsilon$ stets $<\varepsilon$ bleiben, wird natiirlich im allgemeinen von der Größse der Zahl $\varepsilon$ abhängen und im großsen und ganzen um so weiter rechts liegen (d. h. $n_{0}$ wird um so größser sein), je kleiner das $\varepsilon$ gegeben wird (vgl. 2). Um diese Abhängigkeit der Zahl $n_{0}$ von $\varepsilon$ zu betonen, sagt man oft deutlicher "dem gegebenen $\varepsilon$ solle eine Zahl $n_{0}=n_{0}(\varepsilon)$ so entsprechen, daf $\ldots$...".

4. Es braucht $n_{0}$ keine ganze Zahl zu sein.

5. Die Vorzeichen der $x_{n}$ spielen hierbei keine Rolle, da $\left|-x_{n}\right|=\left|x_{n}\right|$ ist, und also keine oder beide $\langle\varepsilon$ sind. Demnach ist auch 6, 6 eine Nullfolge.

6. Bei einer Nullfolge braucht kein Glied gleich 0 zu sein. Alle. Glieder aber, deren Index sehr gro $\beta$ ist, werden sehr klein sein miissen. Denn wähle ich etwa $\varepsilon=\frac{1}{1000000}$, so muf ja doch für alle $n$, die eine gewisse Zahl $n_{0}$ iibersteigen, $\left|x_{n}\right|<\frac{1}{1000000}$ sein. Ebenso für $\varepsilon=10^{-10}$ und jedes andere $\varepsilon$.

7. Auch die Folge 6, 3 ist, falls $|a|<1$ ist, eine Nullfolge.

Beweis. Ist $a=0$, so ist die Behauptung trivial, da dann für jedes $\varepsilon>0$ stets $\left|x_{n}\right|<\varepsilon$ ist. Ist nun $0<|a|<1$, so ist (nach 3, I, 4) $\frac{1}{|a|}>1$. Setzen wir also

Dann ist aber für jedes $n \geqq 2$

$$
\frac{1}{|a|}=1+p, \text { so ist } p>0 \text {. }
$$

$$
(\mathbf{1}+\boldsymbol{1}))^{n}>\mathbf{1}+\boldsymbol{n} \boldsymbol{1} \text {. }
$$

Denn für $n=2$ ist $(1+p)^{2}=1+2 p+p^{2}>1+2 p$, die Beziehung also sicher richtig. Ist schon für $n=k \geqq 2$ festgestellt, dafs

ist, so folgt nach $\boldsymbol{2}$, III, 6 , dafi

$$
(1+p)^{k}>1+k p
$$

$$
(1+p)^{k+1}>(1+k p)(1+p)=1+(k+1) p+k p^{2}>1+(k+1) p
$$

ist, dafs also unsere Beziehung auch für $n=k+1$ richtig ist. Nach $2, \mathrm{~V}$ ist sie also für jedes $n \geq 2$ richtig $^{1}$ ).

1) Der Beweis lehrt sogar, daf3 (a) für $n \geq 2$ richtig ist, wofern nur $1+p>0$ also $p>-1$, aber $\neq 0$ ist. - Die Beziehung (a) nennt man die Bernoullische Ungleichung. (Jak. Bernoulli, Pos. arithm. de seriebus, 1689, Satz 4). 
Danach ist nun

$$
\left|x_{n}\right|=\left|a^{n}\right|=|a|^{n}=\frac{1}{(1+p)^{n}}<\frac{1}{1+n p}<\frac{1}{n p},
$$

also, wie auch $\varepsilon>0$ gegeben sein mag

$$
\left|x_{n}\right|=\left|a^{n}\right|<\varepsilon \quad \text { für alle } n>\frac{1}{p \varepsilon} .
$$

8. Nach 7. sind aufer der unter 2. genannten Folge $\left(\frac{1}{n}\right)$ insbesondere auch die Folgen $\left(\frac{1}{2^{n}}\right),\left(\frac{1}{3^{n}}\right),\left(\frac{1}{10^{n}}\right),\left(\left(\frac{4}{5}\right)^{n}\right)$ usw. Nullfolgen.

9. Ähnlich wie in 8,1 gilt hier die Bemerkung, daßs es bei der Definition 10 ziemlich gleichgiiltig ist, ob dort $"<\varepsilon^{*}$ oder $" \leqq \varepsilon^{"}$ steht. Denn ist für $n>n_{0}$ stets $\left|x_{n}\right|<\varepsilon$, so ist um so mehr $\left|x_{n}\right| \leqq \varepsilon$." Ist umgekehrt für jedes $\varepsilon>0$ zunächst $n_{0}$ nur so bestimmbar, daf für $n>n_{0}$ stets $\left|x_{n}\right| \leqq \varepsilon$ ist, so wähle man eine positive Zahl $\varepsilon_{1}<\varepsilon$; ihr muf dann eine Zahl $n_{1}$ so entsprechen, dafi für $n>n_{1}$ stets $\left|x_{n}\right| \leqq \varepsilon_{1}$ ist. Dann ist aber

$$
\text { für alle } n>n_{1} \text { stets }\left|x_{n}\right|<\varepsilon \text {; }
$$

die Bedingungen sind also auch in der alten Form erfüllbar. - Ganz ähnlich erkennt man, dafi es ziemlich gleichgültig ist, ob in der Definition $10 ">n_{0}$ " oder $" \geqq n_{0}$ " steht. - In jedem Einzelfall dagegen mufs dieser Unterschied natürlich wieder beachtet werden.

10. Obgleich in einer Zahlenfolge jedes Glied völlig für sich dasteht, einen festbestimmten Wert hat und keinerlei Beziehung $z u$ den vorangehenden oder nachfolgenden Gliedern $z u$ haben braucht, so pflegt man doch irgendwelche beim Durchgehen der Folge (von links nach rechts) beobachteten Eigentümlichkeiten "den Gliedern $x_{n}$ " oder "dem allgemeinen Gliede der Folge" zuzusprechen. So sagt man etwa bei 6, 1: die Glieder werden kleiner; bei 6, 2: die Glieder werden größser; bei $\mathbf{6}, 4$ oder $\mathbf{6}, 6$ : die Glieder schwanken auf und ab: bei 6, 11: das allgemeine Glied kann nicht durch eine explizite Formel erhalten werden usw. usw. - In diesem Sinne wollen wir das eigentümliche Verhalten der Glieder einer Nullfolge dadurch umschreiben, daf wir sagen: Die Glieder werden beliebig klein, oder: sie werden unendlich klein ${ }^{1}$ ), womit nicht mehr und nicht weniger gesagt werden soll, als was die Definition 10 enthält, nämlich daß̧ bei jedem noch so kleinen $\varepsilon>0$ die Beträge der Glieder doch schließlich (d. h. für alle Indizes $n$, die eine passende Zahl $n_{0}$ übersteigen; oder kürzer: von einer Stelle an) kleiner als $\varepsilon$ sind ${ }^{2}$ ).

11. Eine Nullfolge ist eo ipso beschränkt. Denn wählen wir $\varepsilon=\mathrm{I}$, so mufs es eine Zahl $n_{1}$ geben, so daß für $n>n_{1}$ stets $\left|x_{n}\right|<1$ ist. Unter den endlich vielen Beträgen $\left|x_{1}\right|,\left|x_{2}\right|, \ldots,\left|x_{n_{1}}\right|$ gibt es aber (vgl. 8, 5) einen größten, der $=M$ sei. Dann ist für $K=M+1$ ersichtlich stets $\left|x_{n}\right|<K$.

12. Wir betonen noch ausdrücklich, daf es zum Nachweis, ob eine vorgelegte Folge eine Nullfolge ist, unbedingt erforderlich ist, daf bei vorgeschriebenem $\varepsilon>0$ das zugehörige $n_{0}$ wirklich angegeben werden kann. Ebenso: wenn von einer Folge $\left(x_{n}\right)$ vorausgesetzt wird, dafis sie eine Nullfolge ist, so wird eben damit als möglich vorausgesetzt, daß $z u$ jedem $\varepsilon$ das zugehörige $n_{0}$ wirklich angegeben werden kann. Im Gegensatz hierzu mache

1) Diese Ausdrucksweise rührt von A. L. Cauchy her (Anal.alg., S. 4 u. 26).

$\left.{ }^{2}\right)$ Von einem monotonen Verhalten braucht dabei natïrlich keine Rede zu sein (vgl. 6, 6); es können also auch schon einige $\left|x_{n}\right|<\varepsilon$ sein, deren Index $<n_{0}$ ist.

Knopp, Unendliche Reihen. 
man sich genau klar, was es heift, daf eine Folge $\left(x_{n}\right)$ keine Nullfolge ist. Es heißst dies: Nicht bei jeder positiven Zahl $\varepsilon$ ist von einer Stelle ab immer $x_{n} \mid<\varepsilon$; - sondern also: Bei Wahl einer speziellen positiven Zahl, etwa der Zahl $\varepsilon_{0}$, ist von keiner Stelle an stets $\left|x_{n}\right|<\varepsilon_{0}$; es ist vielmehr hinter jeder Stelle immer wieder einmal (und also für unendlich viele Indizes) $\left|x_{n}\right| \geq \varepsilon_{0}$.

13. Endlich deuten wir noch an, wie man sich den Charakter einer Folge als Nullfolge geometrisch anschaulich machen kann:

Bei der Veranschaulichung $\boldsymbol{7}, 5$ haben wir es mit einer Nullfolge zu tun, wenn die Glieder der Folge von einer Stelle ab (für $n>n_{0}$ ) alle dem Intervall ${ }^{1}$ ) $-\varepsilon \ldots+\varepsilon$ angehören. Nennen wir ein solches Intervall kurz eine $\varepsilon$-Umgebung des Nullpunktes, so können wir sagen: $\left(x_{n}\right)$ ist eine Nullfolge, wenn in jeder (noch so kleinen) $\varepsilon$-Umgebung des Nullpunktes doch alle Glieder der Folge, von höchstens endlich vielen Ausnahmen abgesehen, gelegen sind.

Bei der Veranschaulichung $\boldsymbol{7}, 6$ ziehen wir durch die beiden Punkte $(0, \pm \varepsilon)$ Parallelen zur Abszissenachse und können sagen: $\left(x_{n}\right)$ ist eine Nullfolge, wenn das ganze Bild der Folge $\left(x_{n}\right)$ - von einem endlichen Anfangsstück abgesehen in jedem solchen (noch so schmalen) $\varepsilon$-Streifen um die Abszissenachse gelegen ist.

14. Der Begriff der Nullfolge, die „beliebig klein gegebene positive Größe $\varepsilon^{\text {", }}$, die uns von nun an ein nicht mehr zu entbehrendes Hilfsmittel sein wird und die darum einen Grundpfeiler für den Aufbau der gesamten Analysis bildet, scheint zuerst 1655 von $J$. Wallis, s. Opera I, S. 382/3 benutzt worden $z u$ sein. Der Sache nach findet sie sich aber schon bei Euklid, Elemente V.

Nun sind wir schon eher in der Lage, den Sachverhalt zu erfassen, der der oben erörterten Bedeutung von $\sqrt{2}$ oder $\pi$ od. ähnl. zugrunde liegt. Indem wir - wir bleiben bei dem Beispiel $\sqrt{2}-$ einerseits die Zahlen

$$
x_{1}=1,4 ; \quad x_{2}=1,41 ; \quad x_{3}=1,414 ; \quad x_{4}=1,4142 ; \ldots
$$

und andrerseits die Zahlen

$$
y_{1}=1,5 ; \quad y_{2}=1,42 ; \quad y_{3}=1,415 ; \quad y_{4}=1,4143 ; \ldots
$$

bilden, stellen wir offenbar zwei (rationale) Zahlenfolgen $\left(x_{n}\right)$ und $\left(y_{n}\right)$ nach einem ganz bestimmten (wenn' auch sehr mühsamen) Verfahren auf. Und zwar sind beide monoton, die Folge $\left(x_{n}\right)$ wachsend, die Folge $\left(y_{n}\right)$ fallend. Überdies ist stets $x_{n}<y_{n}$, aber der Unterschied beider, also die Zahlen

$$
y_{n}-x_{n}=d_{n}
$$

bilden nach 10, 8 eine Nullfolge, da ja $d_{n}=\frac{1}{10^{n}}$ ist. Diese Tatsachen sind es offenbar, die uns das Gefühl geben, daß wir $\sqrt{2}$,kennen“, daß wir es ,berechnen“ können usw., obgleich — wie wir oben sagten — noch niemand den Wert $\sqrt{2}$ sozusagen vollständig vor Augen gehabt hat. - Deuten wir diese Dinge noch etwas anschaulicher auf der Zahlengeraden, so haben wir offenbar dies (vgl. Fig. 1, S. 24): Die Punkte $x_{1}$ und $y_{1}$ begrenzen ein Intervall $^{1}$ ) $J_{1}$ von der

1) Als Intervall bezeichnen wir ein Stück der Zahlengeraden, das zwischen zwei bestimmten ihrer Punkte liegt. Je nachdem man diese Punkte selbst noch zum Intervall hinzurechnet oder nicht, nennt man es abgeschlossen oder offen. Wenn nichts besonderes gesagt ist, sollen im folgenden die Intervalle stets 
Länge $d_{1}$; die Punkte $x_{2}$ und $y_{2}$ ebenso ein Intervall $J_{2}$ von der Länge $d_{2}$. Dieses zweite Intervall liegt aber wegen

$$
x_{1} \leqq x_{2}<y_{2} \leqq y_{1}
$$

ganz in dem ersten. Ebenso begrenzen die Punkte $x_{3}$ und $y_{3}$ ein Intervall $J_{3}$ von der Länge $d_{3}$, das ganz in $J_{2}$ liegt; und allgemein begrenzen die Punkte $x_{n}$ und $y_{n}$ ein Intervall $J_{n}$ von der Länge $d_{n}$, das ganz in $J_{n-1}$ liegt. "Und die Längen dieser Intervalle bilden eine Nullfolge, es schnüren sich die Intervalle - wie man vermutet um eine ganz bestimmte Zahl zusammen, schrumpfen auf einen ganz bestimmten Punkt ein.

Wir haben nur noch zu prüfen, inwieweit diese Vermutung das richtige trifft. - Dazu geben wir allgemeiner die folgende

Definition. Liegt eine monoton steigende Folge $\left(x_{n}\right)$ und eine mono- 11. ton fallende Folge $\left(y_{n}\right)$ vor, deren Glieder für jedes $n$ die Bedingung

$$
x_{n} \leqq y_{n}
$$

erfüllen, und bilden die Differenzen

$$
d_{n}=y_{n}-x_{n}
$$

eine Nullfolge, so wollen wir kurz sagen, es sei uns eine Intervallschachtelung gegeben. Das $n^{\text {te }}$ Intervall $J_{n}$ erstreckt sich von $x_{n}$ bis $y_{n}$, und $d_{n}$ ist seine Länge. Die Schachtelung selbst soll dann kurz durch $\left(J_{n}\right)$ oder durch $\left(x_{n} \mid y_{n}\right)$ bezeichnet werden.

Unsere oben ausgesprochene Vermutung findet nun ihre erste Bestätigung in dem folgenden

Satz. Es gibt höchstens eine (rationale) Zahl s, die allen Intervallen einer Intervallschachtelung angehört, die also für jedes $n$ die Ungleichung

erfüllt.

$$
x_{n} \leqq s \leqq y_{n}
$$

Beweis: Gäbe es neben $s$ noch die davon verschiedene Zahl $s^{\prime}$, die auch für alle $n$ die Ungleichung

erfüllte, so wäre für alle $n$ neben

$$
x_{n} \leqq s^{\prime} \leqq y_{n}
$$

noch (s. 3, I, 4)

$$
x_{n} \leqq s \leqq y_{n}
$$$$
-y_{n} \leqq-s^{\prime} \leqq-x_{n}
$$

so daß nach $\mathbf{3}, \mathrm{I}, 2$ und $\mathbf{3}, \mathrm{II}, 5$ stets

$$
-d_{n} \leqq s-s^{\prime} \leqq d_{n} \quad \text { oder } \quad\left|s-s^{\prime}\right|<d_{n}
$$

abgeschlossen gedacht werden. (Bei 10,13 ist das nach 10,9 gleichgiiltig.) Ist $a$ der linke, $b$ der rechte Endpunkt eines Intervalles, so nennen wir dieses kurz das Intervall $a \ldots b$. 
I. Kap. Grundsätzliches aus der Lehre von den reellen Zahlen.

wäre. Wählen wir also $\varepsilon=\left|s-s^{\prime}\right|$, so wäre niemals, also auch von keiner Stelle $n_{0}$ ab, $d_{n}<\varepsilon$, - was der Voraussetzung, $\left(d_{n}\right)$ sei eine Nullfolge, widerspricht ${ }^{1}$ ). Die Annahme, daß zwei verschiedene Punkte allen Intervallen angehören, ist also unzulässig - w. z. b.w.

Bemerkungen und Beispiele.

1. Es sei $x_{n}=\frac{n-1}{n}, y_{n}=\frac{n+1}{n} ; \quad$ also $J_{n}=\frac{n-1}{n} \ldots \frac{n+1}{n}, d_{n}=\frac{2}{n}$. Man iiberzeugt sich sofort, dafi hier wirklich eine Intervallschachtelung vorliegt, da ja stets $x_{n}<x_{n+1}<y_{n+1}<y_{n}$ und da für $n>\frac{2}{\varepsilon}$ anch stets $d_{n}<\varepsilon$ bleibt, wie auch $\varepsilon>0$ gewählt wird.

Die Zahl $s=1$ gehört hier allen $J_{n}$ an, da stets $\frac{n-1}{n}<1<\frac{n+1}{n}$ ist. Neben 1 kann also keine zweite Zahl allen Intervallen angehören.

2. $\left(J_{n}\right)$ sei folgendermafien definiert: $\left.J_{0}{ }^{2}\right)$ sei die Strecke $0 \ldots 1, J_{1}$ davon die linke Hälfte, $J_{2}$ die rechte Hälfte von $J_{1}, J_{3}$ wieder die linke Hälfte von $J_{2}$ usw. Diese Intervalle sind offenbar ineinander geschachtelt, und da $J_{n}$ die Länge $d_{n}=\frac{1}{2^{n}}$ hat und da diese Zahlen eine Nullfolge bilden, so liegt eine Intervallschachtelung vor. Nach leichter Überlegung findet man, dafi die Folge der $x_{n}$ aus den Zahlen

$$
0, \frac{1}{4}, \frac{1}{4}+\frac{1}{16}=\frac{5}{16}, \frac{1}{4}+\frac{1}{16}+\frac{1}{64}=\frac{21}{64}, \ldots
$$

besteht, deren jede zweimal hintereinander genommen werden mufs, und dafis die Folge der $y_{n}$ mit 1 beginnt und dann aus den je zweimal hintereinander zu nehmenden Zahlen

$$
1-\frac{1}{2}=\frac{1}{2}, \quad 1-\frac{1}{2}-\frac{1}{8}=\frac{3}{8}, \quad 1-\frac{1}{2}-\frac{1}{8}-\frac{1}{32}=\frac{11}{32}, \ldots
$$

besteht. Da nun

$$
\frac{1}{4}+\frac{1}{16}+\frac{1}{64}+\ldots+\frac{1}{4 k}=\frac{1}{3}\left(1-\frac{1}{4 k}\right)<\frac{1}{3}
$$

und

$$
1-\frac{1}{2}-\frac{1}{8}-\ldots-\frac{1}{2 \cdot 4^{k-1}}=\frac{1}{3}\left(1+\frac{2}{4^{k}}\right)>\frac{1}{3}
$$

ist $^{3}$, so ist für jedes $n$ stets $x_{n}<\frac{1}{3}<y_{n}$ und also $s=\frac{1}{8}$ die einzige Zahl, die allen diesen Intervallen angehört. Durch $\left(J_{n}\right)$ wird hiér also die Zahl $\frac{1}{3}$,erfafit" oder ,,bestimmt", $\left(J_{n}\right)$ schrumpft auf die Zahl $\frac{1}{3}$ zusammen.

1) Anschaulich gesprochen sagt der Beweis: Wenn $s$ und $s^{\prime}$ allen Intervallen angehören, so ist die Länge allev Intervalle mindestens gleich dem Abstande $\left|s-s^{\prime}\right|$ von $s$ und $s^{\prime}(\mathrm{s}$. 3, II, 6); diese Längen können also keine Nullfolge bilden.

2) Wir lassen den Index hier von 0 an laufen (vgl. $\boldsymbol{\gamma}, 2$ ).

3) Für irgend zwei Zahlen $a$ und $b$ und jede natürliche Zahl $k$ gilt bekanntlich die Formel

$$
a^{k}-b^{k}=(a-b)\left(a^{k-1}+a^{k-2} b+\ldots+a b^{k-2}+b^{k-1}\right),
$$

woraus speziell die für $a \neq 1$ gültigen Formeln

$$
1+a+\ldots+a^{k-1}=\frac{1-a^{k}}{1-a} \text { und } a+a^{2}+\ldots+a^{k}=\frac{1-a^{k}}{1-a} \cdot a
$$

folgen. 
3. Wenn eine Intervallschachtelung $\left(J_{n}\right)$ vorliegt und eine Zahl $s$ bekannt geworden ist, die allen $J_{n}$ angehört, so ist diese Zahl $s$ durch $\left(J_{n}\right)$ nach unserem Satze völlig eindeutig bestimmt. Wir sagen daher schärfer: die Schachtelung $\left(J_{n}\right)$ definiere oder erfasse die Zahl $s$, oder $s$ sei der durch die Schachtelung gegebene Wert; oder auch: $s$ sei der innerste Punkt aller Intervalle.

4. Ist $s$ irgendeine gegebene rationale $Z$ ahl und setzt man für $n=1,2, \ldots$ $x_{n}=s-\frac{1}{n}$ und $y_{n}=s+\frac{1}{n}$, so ist $\left(x_{n} \mid y_{n}\right)$ ersichtlich eine Intervallschachtelung, durch die die Zahl $s$ selbst erfafist wird. Aber auch wenn stets $x_{n}=s$ und $y_{n}=s$ gesetzt wird, ist $\left(x_{n} \mid y_{n}\right)$ eine Schachtelung, durch die die Zahl $s$ erfafit wird. Man kann hiernach also in der mannigfachsten Art eine Intervallschachtelung bilden, die eine gegebene Zahl definiert. -

Unser Satz erledigt nun aber sozusagen nur die eine Hälfte unserer oben ausgesprochenen Vermutung: Wenn überhaupt eine Zahl $s$ allen Intervallen einer Schachtelung angehört, so gibt es neben ihr keine zweite, sie ist vielmehr durch die Schachtelung eindentig erfaßt.

Die andere Hälfte der Vermutung aber, daß es nämlich auch immer eine (rationale) Zahl $s$ gäbe, die allen Intervallen einer Schachtelung angehörte, diese Vermutung ist - und gerade dies wird uns der Anlaß zur Erweiterung des Systems der rationalen Zahlen werden - irrig.

Das zeigt folgendes Beispiel: Ist wie oben S. $18 x_{1}=1,4 ; x_{2}=1,41, \ldots$; $y_{1}=1,5 ; \quad y_{2}=1,42 ; \ldots$, so gibt es keine rationale $Z a h l \cdot s$, für die stets $x_{n} \leqq s \leqq y_{n}$ wäre. Setzen wir nämlich

$$
x_{n}{ }^{\prime}=x_{n}{ }^{2} \text { und } y_{n}{ }^{\prime}=y_{n}{ }^{2} \text {, }
$$

so bilden auch die Intervalle $J_{n}{ }^{\prime}=x_{n}{ }^{\prime} \ldots y_{n}{ }^{\prime}$ eine Schachtelung $\left.{ }^{1}\right)$. Nun war aber stets $x_{n}{ }^{\prime}=x_{n}{ }^{2}<2$ und $y_{n}{ }^{\prime}=y_{n}{ }^{2}>2$ (denn so waren ja die Dezimalbrüche $x_{n}$ und $y_{n}$ gewählt), also $x_{n}{ }^{\prime}<2<y_{n}{ }^{\prime}$. Ebenso folgte aus $x_{n} \leqq s \leqq y_{n}$ durch (nach 3, I, 3 erlaubtes) Quadrieren, daß3 stets $x_{n}{ }^{\prime} \leqq s^{2} \leqq y_{n}{ }^{\prime}$ sein muifte. Nach unserm Satz 12 miißste also $s^{2}=\mathbf{2}$ sein, was aber nach dem Beweis S. 10, Fußnote, unmöglich ist. Es gibt also hier sicher keine (rationale) Zahl, die allen Intervallen angehörte.

Was in solchem Falle nun $\mathrm{zu}$ tun ist, wollen wir im folgenden Paragraphen untersuchen.

\section{$\S$ 3. Die irrationalen Zahlen.}

Mit der Tatsache, daß es keine rationale Zahl gibt, deren Quadrat $=2$ ist, daß also zur Lösung der Aufgabe $x^{2}=2$ das System der rationalen Zahlen zu lückenhaft, zu unvollkommen ist, müssen wir uns abfinden. Aber nicht nur in diesem einen Falle, sondern zur

1) Denn aus $x_{n} \leqq x_{n+1}<y_{n+1} \leqq y_{n}$ folgt - da alles positiv ist - nach 3, I, 3 durch Quadrieren, dafs auch $x_{n}^{\prime} \leqq x_{n+1}^{\prime}<y_{n+1}^{\prime} \leqq y_{n}^{\prime}$ ist; und weiter ist $y_{n}^{\prime}-x_{n}^{\prime}=\left(y_{n}+x_{n}\right)\left(y_{n}-x_{n}\right)$, also, da die $x_{n}$ und $y_{n}$ für alle $n$ stets $<2$ sind: $y_{n}^{\prime}-x_{n}{ }^{\prime}<\frac{4}{10^{n}}$, also $<\varepsilon$, sobald $\frac{1}{10^{n}}<\frac{\varepsilon}{4}$ ist, was nach 10 , 8 von einem gewissen $n_{0}$ ab sicher der Fall ist. 
Lösung vieler tausend andrer Aufgaben erweisen sich die rationalen Zahlen als ein unzureichendes Material. Fast alle die Werte, die wir durch $\sqrt[p]{n}$, durch $\log n, \sin \alpha, \operatorname{tg} \alpha$ usw. zu bezeichnen pflegen, sind im System der rationalen Zahlen nicht vorhanden und können daher ebensowenig glatt hingeschrieben, ebensowenig unmittelbar „erfaßt“ oder „ziffernmäßig gegeben“ werden wie $\sqrt{2}$. Das Material ist zu grob für diese feineren $Z$ wecke.

Die Betrachtungen des vorigen Paragraphen geben uns nun einen Fingerzeig, wie wir uns ein geeigneteres Material schaffen können: Auf der einen Seite sahen wir nämlich, daß hinter dem Gefühl, wir kennten $\sqrt{2}$, im wesentlichen nur die Kenntnis eines Verfahrens lag, eine ganz bestimmte Intervallschachtelung anzugeben, für deren Konstruktion natürlich die Lösung der Aufgabe $x^{2}=2$ die Veranlassung $\left.\mathrm{gab}^{\mathbf{1}}\right)$. Auf der andern Seite sahen wir, daß, wenn eine Intervallschachtelung $\left(J_{n}\right)$ überhaupt eine angebbare (d. h. also immer noch: rationale) Zahl $s$ enthält, diese Zahl $s$ völlig eindeutig durch die Schachtelung bestimmt ist, so eindeutig, daß es eigentlich ganz gleichgültig ist, ob ich die Zahl $s$ direkt gebe (hinschreibe, nenne), oder ob ich statt ihrer die Schachtelung $\left(J_{n}\right)$ angebe, - mit dem stillschweigenden Zusatz, daß ich mit der letzteren eben die dadurch eindeutig eingespannte oder definierte Zahl s meine. In diesem Sinne leisten beide Angaben (beide Zeichen) ganz dasselbe, beide können gewissermaßen als gleich angesehen werden, so daß wir also geradezu

$$
\left(J_{n}\right)=s \text { oder }\left(x_{n} \mid y_{n}\right)=s
$$

schreiben können. Und demgemäß werden wir nicht nur sagen „Die

1) Der Kern dieses Verfahrens ist doch dieser: Man stellt fest, daß $1^{2}<2$, $2^{2}>2$ ist und setzt demgemäf $x_{0}=1, y_{0}=2$. Man teilt nun das Intervall $J_{0}=x_{0} \ldots y_{0}$ in 10 gleiche Teile und prüft für die Teilpunkte $1+\frac{k}{10}$, $k=0,1,2, \ldots, 9,10$, ob ihr Quadrat $<2$ oder $>2$ ist. Man findet, daf $k=0,1,2,3,4$ ein zu kleines, $k=5,6, \ldots, 10$ ein zu großes Quadrat liefern und setzt demgemäß $x_{1}=1,4$ und $y_{1}=1,5$, teilt das Intervall $J_{1}=x_{1} \ldots y_{1}$ wieder in 10 gleiche Teile, stellt die entsprechende Prüfung für' die Teilpunkte an usw. Das bekannte Verfahren, $\sqrt{2}$ auszuziehen, soll lediglich die jedesmalige Prüfung zu einer möglichst mechanischen machen. - Ebenso führt z. B. die Aufgabe $10^{x}=2$ (also die Bestimmung des Briggschen $\log 2$ ) auf folgende Schachtelung: Da $10^{\circ}<2,10^{1}>2$, so setzt man hier $x_{0}=0, y_{0}=1$ und teilt $J_{0}=x_{0} \ldots y_{0}$ in 10 gleiche Teile. Für die Teilpunkte $\frac{k}{10}$ prüft man nun, ob $10^{k: 10}<2$ oder $>2$, d. h. ob $10^{k}<2^{10}$ oder $>2^{10}$ ist. Auf Grund dieser Prüfung wird man $x_{1}=0,3, y_{1}=0,4$ setzen. Das Intervall $J_{1}=x_{1} \ldots y_{1}$ teilt man erneut in 10 gleiche Teile, stellt die entsprechende Prüfung für die Teilpunkte $\frac{3}{10}+\frac{k}{100}$ an, wird auf Grund derselben $x_{2}=0,30$ und $y_{2}=0,31$ setzen usw. - Dieses naheliegende Verfahren ist für die praktische Berechnung natürlich viel $z u$ miihsam. 


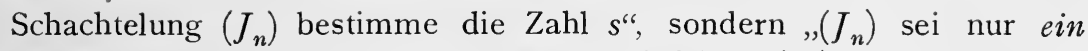
anderes Zeichen für die Zahl s", oder schließlich , $\left(J_{n}\right)$ ist die Zahl s", - genau wie wir in dem Dezimalbruch 0,333... nur ein anderes Zeichen für die Zahl $\frac{1}{3}$, oder eben die Zahl $\frac{1}{3}$ selbst zu sehen gewöhnt sind.

Es ist nun außerordentlich naheliegend, auch bei denjenigen Intervallschachtelungen, die keine rationale $Z$ ahl $s$ enthalten, versuchsweise eine ähnliche Ausdrucksweise einzuführen. Bedeuten also z. B. $x_{n}$ und $y_{n}$ die vorhin im Anschluß an die Aufgabe ' $x^{2}=2$ konstruierten Zahlen, so könnte man sich — da es doch im System der rationalen Zahlen keine einzige Zahl gibt, deren Quadrat $=2$ ist nun entschließen, zu sagen: diese Schachtelung $\left(x_{n} \mid y_{n}\right)$ bestimme den „,wahren“, nur eben mit Hilfe der rationalen Zahlen nicht bezeichenbaren „Wert von $\sqrt{2}$ ", sie spanne unzweideutig diese Zahl ein, also schließlich: „, sie sei ein neugeschaffenes Zeichen für diese Zahl“ oder kurz: „sie sei diese Zahl selbst“. Und entsprechend in jedem andern Falle. Ist $\left(J_{n}\right)=\left(x_{n} \mid y_{n}\right)$ irgendeine Schachtelung und gibt es keine rationale Zahl $s$, die allen ihren Intervallen angehört, so könnte man sich doch entschließen zu sagen: Diese Schachtelung erfasse einen ganz bestimmten - nur eben mit Hilfe der rationalen Zahlen nicht unmittelbar bezeichenbaren - Wert, sie definiere zwar eine ganz bestimmte, aber im System der rationalen Zahlen leider nicht vorhandene $Z a h l$, sie sei ein neu geschaffenes Zeichen für diese Zahl, oder kurz: sie sei diese Zahl selbst, - die dann im Gegensatze zu den rationalen eine irrationale $Z$ ahl genannt werden müßte.

Da erhebt sich nun allerdings die Frage: Geht denn das ohne weiteres an? Darf man das tun? Darf man diese neuartigen Symbole, also die Intervallschachtelungen $\left(x_{n} \mid y_{n}\right)$, ohne weiteres als Zahlen bezeichnen? Die folgenden Betrachtungen sollen zeigen, daß dem keinerlei Bedenken entgegenstehen.

Zunächst läßt uns die Veranschaulichung dieser Dinge auf der Zahlengeraden (s. Fig. 1) unsern Entschluß durchaus berechtigt erscheinen. Wenn wir durch irgendeine Konstruktion auf der Zahlengeraden einen Punkt $P$ markiert haben (z. B. indem wir von $O$ nach rechts die Diagonale des Quadrates mit der Seite $O E$ abtragen, od. ä.), so kann man auf mannigfache Art eine Intervallschachtelung angeben, die den Punkt $P$ erfaßt; z. B. so: Man denke sich zunächst die sämtlichen ganzen Zahlen $\gtreqless 0$ markiert. Unter ihnen wird es genau eine geben, sie heiße $p$, so daß $P$ auf der Strecke von $p$ einschließlich bis $(p+1)$ ausschließlich liegt. Wir setzen demgemäß $x_{0}=p$, $y_{0}=p+1$ und teilen das Intervall $J_{0}=x_{0} \ldots y_{0}$ in 10 gleiche Teile $^{\mathbf{1}}$ ).

1) Statt 10 kann man natürlich auch irgendeine andere natürliche Zahl $>2$ nehmen. Genaueres darüber s. §. 5 . 
Die Teilpunkte sind $p+\frac{k}{10}$ (mit $k=0,1,2, \ldots, 10$ ) und unter ihnen muß es wieder genau einen geben, etwa $p+\frac{k_{1}}{10}$, so daß $P$ zwischen $x_{1}=p+\frac{k_{1}}{10}$ einschließlich und $y_{1}=p+\frac{k_{1}+1}{10}$ ausschließlich gelegen ist. Das Intervall $J_{1}=x_{1} \ldots y_{1}$ teilen wir erneut in 10 gleiche Teile usw. Denken wir uns dieses Verfahren ohne Ende fortgesetzt, so erhalten wir eine ganz bestimmte Schachtelung $\left(J_{n}\right)$, deren sämtliche Intervalle $J_{n}$ den Punkt $P$ enthalten. Außer $P$ kann auch kein zweiter Punkt $P^{\prime}$ in allen Intervallen $J_{n}$ gelegen sein; denn dann müßten ja alle Intervalle die ganze Strecke $P P^{\prime}$ enthalten. Das ist aber unmöglich, da die Längen der Intervalle $\left(J_{n}\right.$ hat die Länge $\left.\frac{1}{10^{n}}\right)$ eine Nullfolge bilden.

$\mathrm{Zu}$ jedem willkürlich auf der Zahlengeraden gegebenen Punkt $P$ (mag es ein rationaler Punkt sein oder nicht) gibt es also — offenbar

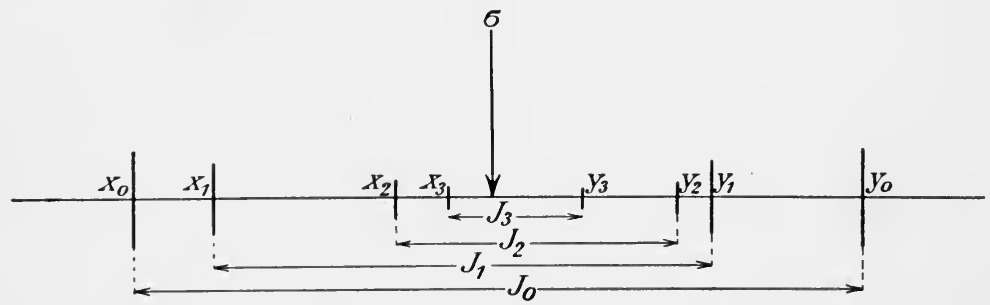

Fig. 1.

sogar viele verschiedene - Intervallschachtelungen, die diesen Punkt und keinen andern enthalten. Und hier - d. h. bei der geometrischen Veranschaulichung auf der Zahlengeraden - erscheint uns auch das umgekehrte durchaus plausibel: Wenn wir irgendeine Schachtelung haben, so scheint es immer einen (und also nach dem eben gegebenen Beweise auch nur diesen einen) Punkt zu geben, der allen Intervallen derselben angehört, der also durch sie bestimmt wird. Wir glauben dies jedenfalls aus unserer Vorstellung von der Lückenlosigkeit der Gevaden unmittelbar erschließen zu können ${ }^{1}$ ).

Hier bei der geometrischen Veranschaulichung hätten wir also völlige Gegenseitigkeit: Jeder Punkt läßt sich durch eine passende Intervallschachtelung erfassen und jede Schachtelung erfaßt immer genau einen Punkt.

1) Der Satz, der diese Tatsache der "Lückenlosigkeit der Geraden“ ausdrücklich postuliert - denn nach einem Beweis darf man hier nicht fragen, da es sich ja lediglich um eine Beschreibung der Form unserer Anschaunng von der geraden Linie handelt - heifst das Cantor-Dedekindsche Axiom. 
Die Anschauung der sogenannten Lückenlosigkeıt einer Geraden ist aber zweifellos etwas sehr Verschwommenes, begrifflich kaum in voller Schärfe zu Erfassendes; sie darf daher — wie übrigens alle nur der Anschauung entnommenen Vorstellungen - bei der Grundlegung der Analysis nicht selbst das letzte Fundament, sondern höchstens einen Wegweiser zur Schaffung desselben bilden. Daher vermögen wir aus der besprochenen geometrischen Veranschaulichung der in Rede stehenden Dinge zwar nicht gleich einen befriedigenden Satz abzulesen, aber wir gewinnen aus ihr ein hohes Maß von Vertrauen in die Zweckmäßigkeit und Berechtigung unseres Entschlusses, die Intervallschachtelungen als Zah!en aufzufassen, den wir nun in der folgenden Definition präzisieren:

Definition: Wir wollen von jeder Intervallschachtelung $\left(J_{n}\right)$ oder 13. $\left(x_{n} \mid y_{n}\right)$ sagen, sie definiere, oder kurz: sie sei eine wohlbestimmte Zahl. Zur Bezeichnung derselben gebrauchen wir das Zeichen der Intervallschachtelung selbst, und nur zur Abkürzung ersetzen wir diese durch einen kleinen griechischen Buchstaben und schreiben in diesem Sinne etwa

$$
\left.\left(\boldsymbol{J}_{n}\right) \text { oder }\left(\boldsymbol{x}_{n} \mid \boldsymbol{y}_{n}\right)=\sigma^{1}\right) \text {. }
$$

Dies erscheint nun trotz allem als ein sehr eigenmächtiger und willkürlicher Schritt, und wir müssen nachdrücklichst die oben aufgeworfene Frage wiederholen: Geht das ohne weiteres an? Dürfen wir denn ohne weiteres die eben definierten rein begrifflichen Dinge nämlich die Intervallschachtelungen (bzw. das durch eine solche erfaßte oder eingespannte, noch durchaus fragwürdige Etwas) - als Zahlen ansprechen? Sind es denn Zahlen in demselben Sinne wie die rationalen Zahlen, - oder präziser: in dem Sinne, wie wir den Zahlbegriff durch die Bedingungen 4 festgelegt haben?

Die Antwort kann einzig darin bestehen, daß wir entscheiden, ob die Gesamtheit aller nur denkbaren Intervallschachtelungen bzw. der dafür eingeführten Symbole $\left(J_{n}\right)$ oder $\left(x_{n} \mid y_{n}\right)$ oder $\sigma$ ein System von Dingen bildet, das den genannten Bedingungen $\mathbf{4}$ genügt $^{2}$ ), dessen Elemente also - so können wir diese Bedingungen kurz rekapitulieren - aus den rationalen Zahlen abgeleitet sind und 1. sich ordnen, 2. sich nach vier Spezies verknüpfen lassen, hierbei den Grundgesetzen $\mathbf{1}$ und $\mathbf{2}$, I-IV gehorchen, deren System 3. ein zu den rationalen Zahlen ähnliches und isomorphes Teilsystem enthält und 4. das Postulat des Eudoxus erfüllt.

Erst wenn diese Entscheidung im günstigen Sinne ausgefallen ist, so wäre alles in Ordnung; denn dann hätten unsere neuen Zeichen ihren

1) D. h. $\sigma$ ist eine abkürzende Bezeichnung für die Intervallschachtelung $\left(J_{n}\right)$ oder $\left(x_{n} \mid y_{n}\right)$.

2) Man lese daraufhin diese Bedingungen nun noch einmal durch! 
Zahlcharakter bewiesen, es wäre festgestellt: es sind Zahlen, deren Gesamtheit wir dann als das System der reellen Zahlen bezeichnen werden.

Die genannte Entscheidung bietet nun nicht die geringsten Schwierigkeiten, und wir können uns daher bei der Ausführung der Einzelheiten kurz fassen:

Die Intervallschachtelungen - also unsere neuen Zeichen $\left(x_{n} \mid y_{n}\right)$ sind jedenfalls nur mit Hilfe der rationalen Zahlzeichen aufgebaut, und es bedarf also nur der Erledigung der Punkte 4, 1-4. Hierbei werden wir folgendermaßen zu Werke gehen: Gewisse unter den Schachtelungen definieren eine rationale $\mathrm{Zahl}^{\mathbf{1}}$ ), also etwas, dessen Bedeutung und dessen Verknüpfungsarten schon festgelegt sind. Man nehme nun zwei solche rationalwertigen Schachtelungen, etwa $\left(x_{n} \mid y_{n}\right)=s$ und $\left(x_{n}{ }^{\prime} \mid y_{n}{ }^{\prime}\right)=s^{\prime}$. Dann können wir an den rationalen $\therefore$ Zahlzeichen $s$ und $s^{\prime}$ sofort feststellen, ob $s<$, = oder $>s^{\prime}$ ist, und können sie auch mit Hilfe der vier Spezies miteinander verknüpfen. Man hat nun lediglich zu versuchen, dasselbe direkt an den Schachtelungen für $s$ und $s^{\prime}$ selbst zu erkennen bzw. vorzunehmen und endlich das Ergebnis auf die Gesamtheit aller Schachtelungen zu übertragen. Ein bei den rationalwertigen Schachtelungen beweisbarer Satz (A) wird uns also jedesmal Veranlassung zu einer entsprechenden Definition (B) sein. Wir stellen diese Paare je eines Satzes (A) und einer Definition (B) zunächst kurz zusammen ${ }^{2}$ ):

14. Gleichheit: A. Satz. Sind $\left(x_{n} \mid y_{n}\right)=s$ und $\left(x_{n}{ }^{\prime} \mid y_{n}{ }^{\prime}\right)=s^{\prime}$ zwei rationalwertige Intervallschachtelungen, so ist dann und nur dann $s=s^{\prime}$, wenn neben

auch stets

$\left.i s t^{3}\right)$.

$$
x_{n} \leqq y_{n} \quad \text { und } \quad x_{n}{ }^{\prime} \leqq y_{n}{ }^{\prime}
$$

$$
x_{n} \leqq y_{n}{ }^{\prime} \text { und stets } x_{n}^{\prime} \leqq y_{n}
$$

$\left.{ }^{1}\right)$ Wir wollen solche Schachtelungen kurz als rationalwertig bezeichnen.

2) Man deute sich jedesmal den Inhalt von Satz und Definition auf der Zahlengeraden.

$\left.{ }^{3}\right)$ Auf die sehr einfachen Beweise der Sätze 14 bis 19 wollen wir aus den allgemeinen, S. 2 dargelegten Gründen nicht eingehen. Sie werden dem Leser, sobald er die Gegenstände des II. Kapitels beherrscht, nicht die geringsten Schwierigkeiten machen, während sie jetzt befremdlich wirken würden; sie sollen uns überdies in dem dortigen Kapitel als Übungsaufgaben dienen. Nur als Stichprobe und Anleitung für die späteren Aufgaben wollen wir hier den Satz 14 beweisen:

a) Wenn $s=s^{\prime}$ ist, so ist neben $x_{n} \leqq s \leqq y_{n}$ auch stets $x_{n}{ }^{\prime} \leqq s \leqq y_{n}{ }^{\prime}$, woraus sofort folgt, daß auch stets $x_{n} \leqq y_{n}{ }^{\prime}$ und stets $x_{n}{ }^{\prime} \leqq y_{n}$ sein muf.

b) Wenn umgekehrt stets $x_{n} \leqq y_{n}{ }^{\prime}$ ist, so muf auch $s \leqq s^{\prime}$ sein. Denn wäre $s>s^{\prime}$, also $s-s^{\prime}>0$, so könnte man, da $\left(y_{n}-x_{n}\right)$ eine Nullfolge ist, einen Index $p$ so wählen, daf

$$
y_{p}-x_{p}<s-s^{\prime}
$$


Auf diesen Satz gründen wir nun die

B. Definition. Zwei beliebige Intervallschachtelungen $\sigma=\left(x_{n} \mid y_{n}\right)$ und $\sigma^{\prime}=\left(x_{n}{ }^{\prime} \mid y_{n}{ }^{\prime}\right)$ sollen dann und nur dann einander gleich heißen, wenn

ist.

$$
\text { stets } x_{n} \leqq y_{n}^{\prime} \quad \text { und stets } x_{n}^{\prime} \leqq y_{n}
$$

\section{Bemerkungen und Beispiele.}

1. Die Zahlen $x_{n}$ und $x_{n}^{\prime}$ einerseits und die Zahlen $y_{n}$ und $y_{n}^{\prime}$ andererseits brauchen natürlich gar nichts miteinander $z u$ tun $z u$ haben. Es ist das nicht verwunderlicher, als daf äuferlich so ganz verschiedene rationale Zahlen wie $\frac{3}{8}, \frac{21}{56}$ und 0,375 als "gleich" angesprochen werden. Die Gleichheit ist eben etwas, was nicht a priori feststeht, sondern was definitionsweise festgelegt werden mußs und was mit einer - rein äuferlich aufgefaßsten - starken Verschiedenheit durchaus verträglich ist.

2. Die Schachtelung $\left(\frac{n-1}{3 n} \mid \frac{n+1}{3 n}\right)$ und die Schachtelung 12, 2 sind nach unserer jetzigen Definition einander gleich.

3. Nach 14 ist z. B. $\left(s-\frac{1}{n} \mid s+\frac{1}{n}\right)=s=(s \mid s)$, wenn das letztere Zeichen eine Schachtelung bedeutet, bei der alle linken und alle rechten Intervallenden $=s$ sind. Speziell ist $\left(-\frac{1}{n} \mid+\frac{1}{n}\right)=(0 \mid 0)=0$.

4. Es muf nun noch festgestellt werden - was aber so einfach ist, dafi wir nicht weiter darauf eingehen, dak (vgl. Fufnote 3 der vorigen Seite) auf Grund dieser Definition a) stets $\sigma=\sigma$ ist ${ }^{1}$ ), b) aus $\sigma=\sigma^{\prime}$ stets $\sigma^{\prime}=\sigma$ und c) aus $\sigma=\sigma^{\prime}, \sigma^{\prime}=\sigma^{\prime \prime}$ stets $\sigma=\sigma^{\prime \prime}$ folgt.

Ungleichheit: A. Satz. Sind $\left(x_{n} \mid y_{n}\right)=s$ und $\left(x_{n}{ }^{\prime} \mid y_{n}{ }^{\prime}\right)=s^{\prime}$ zwei 15. rationalwertige Intervalls hachtelungen, so ist dann und mur dann $s<s^{\prime}$, wenn

$$
\text { zwar stets } x_{n} \leqq y_{n}{ }^{\prime} \text {, aber } n \text { icht stets } x_{n}{ }^{\prime} \leqq y_{n},
$$

sondern für mindestens eine natürliche Zahl $m$ also $y_{m}<x_{m}^{\prime}$ ist ${ }^{2}$ ).

ist. Da aber sicher $s \leqq y_{p}$ ist, so miifite auch noch $x_{p}-s^{\prime}>0$ sein. Daher könnte man weiter einen Index $r$ so wählen, daf

$$
y_{r}^{\prime}-x_{r}^{\prime}<x_{p}-s^{\prime}
$$

ist. Wegen $x_{r}^{\prime} \leqq s^{\prime}$ müfite hiernach auch $y_{r}^{\prime}<x_{p}$ sein. Wählt man nun eine natürliche Zahl $m$, von der sowohl $p$ als $r$ übertroffen werden, so wäre mit Rücksicht auf das Steigen und Fallen unserer Zahlenfolgen erst recht $y_{m}{ }^{\prime}<x_{m}$, entgegen der Voraussetzung, daß3 stets $x_{n} \leq y_{n}^{\prime}$ sein sollte. Es muf also wirklich $s \leq s^{\prime}$ sein.

Vertauscht man bei dem ganzen Beweise die gestrichenen und die ungestrichenen Größen, so ergibt sich ebenso: wenn stets $x_{n}{ }^{\prime} \leqq y_{n}$ ist, so muf $s^{\prime} \leqq s$ sein. - Ist also stets sowohl $x_{n} \leqq y_{n}^{\prime}$ als auch $x_{n}^{\prime} \leqq y_{n}$, so mufs $s=s^{\prime}$ sein, - w. z. b.w.

1) Hier erkennt man nun, daf dieses "Gesetz" keineswegs trivial ist: Es mußs eben erst gezeigt werden, daß auf Grund der Definition der Gleichheit tatsächlich jede Intervallschachtelung sich selber „gleich" ist, d. h. den Bedingungen jener Definition geniigt.

2) Und entsprechend für $s>s^{\prime}$, d. h. $s^{\prime}<s$. 
B. Definition. Sind $\sigma=\left(x_{n} \mid y_{n}\right)$ und $\sigma^{\prime}=\left(x_{n}{ }^{\prime} \mid y_{n}{ }^{\prime}\right)$ zwei beliebige Intervallschachtelungen, so soll dann und nur dann $\sigma<\sigma^{\prime}$ heißen, wenn zwar stets $x_{n} \leqq y_{n}{ }^{\prime}$, aber nicht stets $x_{n}{ }^{\prime} \leqq y_{n}$, sondern für mindestens eine natürliche Zahl $m$ also $y_{m}<x_{m}^{\prime}$ ist.

\section{Bemerkungen und Beispiele:}

1. Nach Def. $\mathbf{1 4}$ und $\mathbf{1 5}$ ist nun ersichtlich die Gesamtheit aller nur denkbaren Intervallschachtelungen geordnet. Denn zwischen irgend zweien von ihnen, etwa $\sigma$ und $\sigma^{\prime}$, besteht entweder Gleichheit oder es ist mindestens einmal $y_{p}<x_{p}^{\prime}-$ und dann ist $\sigma<\sigma^{\prime}$, oder mindestens einmal $y_{r}^{\prime}<x_{r}-$ und dann ist $\sigma^{\prime}<\sigma$ zu nennen. Die beiden letzten Fälle können auch nicht zugleich eintreten, da dann, wenn $m$ größer als $r$ und $p$ ist, um so mehr $y_{m}^{\prime}<x_{m}$ sein müfte, was doch keinesfalls möglich ist. Es besteht also zwischen $\sigma$ und $\sigma^{\prime}$ tatsächlich immer eine und nur eine der drei Beziehungen

$$
\sigma<\sigma^{\prime}, \quad \sigma=\sigma^{\prime}, \quad \sigma^{\prime}<\sigma
$$

die Gesamtheit unserer neuen Zeichen ist also durch 14 und 15 geordnet.

2. Auch hier müfte nun in allen Einzelheiten festgestellt werden, dafs die reinen Anordnungssätze $\mathbf{1}$ bei der eingeführten Definition der Gleichheit und Ungleichheit giiltig bleiben. Das bietet nach dem Muster des Beweises in der Fußnote zu Satz 14 so wenig prinzipielle Schwierigkeiten, daßs wir darauf nicht weiter eingehen: Die Anordnungssätze bleiben tatsächlich alle gültig.

3. Auf Grund von $\mathbf{1 4}$ und $\mathbf{1 5}$ ist nun auch für jedes $n$

$$
x_{n} \leqq \sigma \leqq y_{n} .
$$

Was heift dies? Es bedeutet, dasi jede der rationalen Zahlen $x_{n}$ gemärs 14 und 15 nicht größer als die Intervallschachtelung $\sigma=\left(x_{n} \mid y_{n}\right)$ ist. Oder: Greift man eine spezielle der Zahlen $x_{n}$, etwa $x_{p}$, heraus und bezeichnet sie kurz mit $x$, so kann (s. 14, Bem. 3)

$$
\left(x_{p}=\right) \quad x=\left(x-\frac{1}{n} \mid x+\frac{1}{n}\right) \text { oder }=(x \mid x)
$$

gesetzt werden, und unsere Behauptung lautet dann

$$
(x \mid x) \leqq\left(x_{n} \mid y_{n}\right) \text {. }
$$

Wäre das aber nicht der Fall, so wäre für mindestens einen Index $r$

$$
y_{r}<x, \text { d. h. } y_{r}<x_{p}
$$

was dann für ein $m$ oberhalb $r$ und $p$ um so mehr

$$
y_{m}<x_{m}
$$

zur Folge hätte, - was sicher nicht sein kann. Ebenso findet man, dafs stets $\sigma \leqq y_{n}$ ist. Hiernach ist also $\sigma$ für jedes $n$ als zwischen $x_{n}$ und $y_{n}$ oder also als im Intervall $J_{n}$ gelegen anzusehen!

4. Da nach dem vorigen $\sigma \leqq y_{n}$ ist, und da $y_{n}$ nach dem Satz des Eudoxus ( 2, VI) von einer passenden natürlichen Zahl $p$ übertroffen wird, so gilt das gleiche von $\sigma$ selbst: $\sigma<p^{1}$ ).

5. Nach 15 ist dann und nur dann $\sigma>0$, also positiv zu nennen, wenn $\left(x_{n} \mid y_{n}\right)>(0 \mid 0)$, wenn also für einen passenden Index $p$ einmal $x_{p}>0$ ist.

1) Auch dies ist im Sinne von $\mathbf{1 5}$ so $z \mathfrak{u}$ verstehen, daf etwa

$$
\left(x_{n} \mid y_{n}\right)<\left(p-\frac{1}{n} \mid p+\frac{1}{n}\right)
$$

ist. 
Dann ist aber, da die $x_{n}$ ansteigen, erst recht für alle $n>p$ stets $x_{n}>0$. Wir können also sagen: $\sigma=\left(x_{n} \mid y_{n}\right)$ ist dann und nur dann positiv, wenn von einem gewissen Index an alle Intervallenden positiv sind. - Genau Entsprechendes gilt natürlich für $\sigma<0$.

6. Ist nach 5. $\sigma>0$ und für alle $n \geqq p$ stets $x_{n}>0$, so bilde man nun eine neue Intervallschachtelung $\left(x_{n}{ }^{\prime} \mid y_{n}{ }^{\prime}\right)=\sigma^{\prime}$, indem man $x_{1}{ }^{\prime}=x_{2}{ }^{\prime}=\ldots \ldots=x_{p-1}^{\prime}$ sämtlich $=x_{p}$ setzt, sonst aber alle $x^{\prime}$ und $y^{\prime}$ den entsprechenden Größen $x$ und $y$ gleichmacht. Nach $\mathbf{1 4}$ ist offenbar $\sigma=\sigma^{\prime}$, und wir können sagen: Ist $\sigma$ positiv, so gibt es stets "gleiche" Intervallschachtelungen, bei denen alle Intervallenden positiv sind. Genau Entsprechendes gilt für $\sigma<0$.

In bezug auf die Ordnungsfähigkeit hätten also unsere Intervallschachtelungen durchaus ihren Zahlcharakter bewährt. Nicht schwerer ist dies bezüglich der Verknüpfungsmöglichkeiten festzustellen.

Addition: A. Satz. Sind $\left(x_{n} \mid y_{n}\right)$ und $\left(x_{n}{ }^{\prime} \mid y_{n}{ }^{\prime}\right)$ zwei beliebige Inter- 16. vallschachtelungen, so ist auch $\left(x_{n}+x_{n}^{\prime} \mid y_{n}+y_{n}^{\prime}\right)$ eine solche; und sind die ersten beiden rationalwertig und $\stackrel{=}{=}$ bzw. $=s^{\prime}$, so ist es auch die dritte und diese bestimmt die Zahl $\left.s+s^{\prime \mathbf{1}}\right)$.

B. Definition. Sind $\left(x_{n} \mid y_{n}\right)=\sigma$ und $\left(x_{n}{ }^{\prime} \mid y_{n}{ }^{\prime}\right)=\sigma^{\prime}$ zwei beliebige Intervallschachtelungen und bezeichnet man die daraus abgeleitete Schachtelung $\left(x_{n}+x_{n}^{\prime} \mid y_{n}+y_{n}^{\prime}\right)$ mit $\sigma^{\prime \prime}$, so sagt man, es sei

$$
\sigma^{\prime \prime}=\sigma+\sigma^{\prime}
$$

und nennt $\sigma^{\prime \prime}$ die Summe von $\sigma$ und $\sigma^{\prime}$.

Subtraktion: A. Satz. Mit $\left(x_{n} \mid y_{n}\right)$ ist auch $\left(-y_{n} \mid-x_{n}\right)$ eine 17. Intervallschachtelung; und ist die erste rationalwertig und $=s$, so ist es auch die zweite, und diese definiert die Zahl $-s$.

B. Definition. Ist $\sigma=\left(x_{n} \mid y_{n}\right)$ eine beliebige Intervallschachtelung, so sagt man von der Schachtelung $\left(-y_{n} \mid-x_{n}\right)=\sigma^{\prime}$, es sei

$$
\sigma^{\prime}=-\sigma
$$

und nennt $\sigma^{\prime} z u$ o entgegengesetzt. - Unter der Differenz zweier Intervallschachtelungen versteht man dann die Summe der ersten und der zur zweiten entgegengesetzten.

Multiplikation: A. Satz. Sind $\left(x_{n} \mid y_{n}\right)$ und $\left(x_{n}{ }^{\prime} \mid y_{n}{ }^{\prime}\right)$ zwei beliebige $1 S$. positive Intervallschachtelungen und ersetzt man sie nötigenfalls (nach 15,6) durch zwei ihnen gleiche, bei denen sämtliche Intervallenden positiv (oder wenigstens nicht negativ) sind, so ist auch $\left(x_{n} x_{n}{ }^{\prime} \mid y_{n} y_{n}{ }^{\prime}\right)$ eine solche; und sind die beiden ersten rationalwertig und $=s$ bzw. $s^{\prime}$, so ist es auch die dritte, und diese bestimmt die Zahl ss'.

B. Definition. Sind $\left(x_{n} \mid y_{n}\right)=\sigma$ und $\left(x_{n}{ }^{\prime} \mid y_{n}{ }^{\prime}\right)=\sigma^{\prime}$ zwei beliebige positive Intervallschachtelungen, bei denen sümtliche Intervallenden positiv sind - was nach 15,6 keine Einschränkung bedeutet - und

1) Bezüglich des Beweises vgl. die Fußnn. 3 auf S. 26. 
bezeichnet man die daraus abgeleitete Schachtelung $\left(x_{n} x_{n}{ }^{\prime} \mid y_{n} y_{n}{ }^{\prime}\right)$ mit $\sigma^{\prime \prime}$, so sagt man, es sei

$$
\sigma^{\prime \prime}=\sigma \cdot \sigma^{\prime}
$$

und nennt $\sigma^{\prime \prime}$ das Produkt von $\sigma$ und $\sigma$.

Die geringen Modifikationen, die an dieser Definition vorzunehmen sind, falls $\sigma$ oder $\sigma^{\prime}$ oder beide negativ oder 0 sind, überlassen wir dem Leser und betrachten von jetzt ab das Produkt irgend zweier Intervallschachtelungen als definiert.

19. Division: A. Satz. Ist $\left(x_{n} \mid y_{n}\right)$ eine positive Intervallschachtelung mit lauter positiven Intervallenden (vgl. 15,6), so ist auch $\left(\frac{1}{y_{n}} \mid \frac{1}{x_{n}}\right)$ eine solche; und ist die erste rationalwertig und $=s$, so ist es auch die zweite, und diese bestimmt den Wert $\frac{1}{s}$.

B. Definition. Ist $\left(x_{n} \mid y_{n}\right)=\sigma$ eine beliebige positive Intervallschachtelung mit lauter positiven Intervallenden (vgl. 15,6), so sagt man von der Schachtelung $\left(\frac{1}{y_{n}} \mid \frac{1}{x_{n}}\right)=\sigma^{\prime}$, es sei

$$
\sigma^{\prime}=\frac{1}{\sigma}
$$

und nennt $\sigma^{\prime} z u$ o resiprok. - Unter dem Quotienten einer ersten durch eine zweite, positive Intervallschachtelung versteht man dann das Produkt der ersten mit der reziproken zur zweiten.

Die geringen Modifikationen, die an dieser Definition vorzunehmen sind, falls $\sigma$ bzw. die in den Nenner tretende Intervallschachtelung negativ ist, können wir wieder dem Leser überlassen und betrachten von jetzt ab den Quotienten irgend zweier Schachtelungen, deren zweite von 0 verschieden ist, als definiert. - Ist $\left(x_{n} \mid y_{n}\right)=\sigma=0$, so kann man durch eine analoge Betrachtung nicht zu einer „reziproken" Schachtelung gelangen: Die Division durch 0 bleibt auch hier unmöglich.

Das Ergebnis der bisherigen Betrachtungen ist nun dieses:

Durch die Definitionen 14 bis $\mathbf{1 9}$ ist das System aller Intervallschachtelungen im Sinne von 4,1 geordnet, und im Sinne von 4,2 können seine Elemente nach den vier Spezies verknüpft werden. Auf Grund der jedesmal vorangestellten Sätze 14 bis 19 besitzt dies System außerdem in der Gesamtheit aller rationalwertigen Intervallschachtelungen ein Teilsystem, das im Sinne von 4,3 zum System der rationalen Zahlen ähnlich und isomorph ist, und auf Grund der Bemerkung 15, 4 erfüllt es auch das Postulat des Eudoxus.

Es wäre nun lediglich in allen Einzelheiten noch festzustellen (vgl. 14, 4 und 15, 2), daß die durch $\mathbf{1 6}$ bis $\mathbf{1 9}$ definierten vier Spezies zwischen den Intervallschachtelungen den Grundgesetzen $\mathbf{2}$ gehorchen. Diese Feststellung macht wieder nicht die geringsten 
Schwierigkeiten und wir wollen uns daher der Mühe der Ausführung entheben $\left.{ }^{1}\right)$. Die Grundgesetze der Arithmetik, und damit der ganze im System der rationalen Zahlen gültige Rechenapparat, bleiben tatsächlich in Gültigkeit.

Damit aber haben sich die Intervallschachtelungen nun in jeder Beziehung im Sinne von $\mathbf{4}$ als Zahlen bewährt: Das System aller Intervallschachtelungen ist ein Zahlensystem ${ }^{2}$ ), die Schachtelungen selbst sind Zahlen ${ }^{3}$ ).

Dieses System bezeichnen wir fortan als das System der reellen Zahlen. Es ist im Sinne der Ausführungen von S. 10 eine Erweiterung des Systems der rationalen Zahlen, weil es ja außer den rational- • wertigen Intervallschachtelungen auch noch andere gibt.

Dies System der reellen Zahlen entspricht überdies in umkehrbareindeutiger Weise den sämtlichen Punkten der Zahlengeraden. Denn auf Grund der Ausführungen von S. 23/24 können wir unmittelbar sagen: Jeder Schachtelung $\sigma$ entspricht ein und nur ein Punkt, -

1) Bezüglich der Addition wäre z. B. zu zeigen:

a) Die Addition ist stets ausführbar. (Das folgt unmittelbar aus der Definition.)

b) Die Addition ist eindeutig; d. h. aus $\sigma=\sigma^{\prime}, \tau=\tau^{\prime}$ (im Sinne von 14) folgt $\sigma+\tau=\sigma^{\prime}+\tau^{\prime}$, - wenn die Summen nach 16 gebildet werden und die Prüfung der Gleichheit wieder nach 14 geschieht. In entsprechendem Sinne wäre weiter zu zeigen:

c) Es ist stets $\sigma+\tau=\tau+\sigma$

d) Es ist stets $(\varrho+\sigma)+\tau=\varrho+(\sigma+\tau)$.

e) Aus $\sigma<\sigma^{\prime}$ folgt stets $\sigma+\tau<\sigma^{\prime}+\tau$. - Und ähnlich bei den drei anderen Verknüpfungen.

2) Die Art, wie wir den Zahlbegriff unter 4 festgelegt haben, ist natiurlich nicht die einzig mögliche. Vielfach werden auch Dinge, die der einen oder andern der dort aufgeführten Forderungen nicht genügen, doch noch als Zahlen bezeichnet. So kann man z. B. auf das konstruktive Hervorwachsen der in Rede stehenden Dinge aus den rationalen Zahlen verzichten und irgendwelche Dinge (z. B. Strecken oder Punkte od. ähnl.) schon als Zahlen ansprechen, falls sie den Bedingungen 4, 1-4 genügen, also kurz gesagt dem soeben von uns geschaffenen System ähnlich und isomorph sind. - Dieser vom mathematischen Standpunkt aus durchaus berechtigten Auffassung der Zahlbegriffs, nach der also allgemein isomorphe Systeme im abstrakten Sinne als identisch gelten, wird man aber erkenntnistheoretische Bedenken entgegenstellen mïssen. Eine andere Modifikation des Zahlbegriffs werden wir gelegentlich der komplexen Zahlen kennen lernen.

$\left.{ }^{3}\right) \mathrm{Ob}$ man, wie wir es tun, die Intervallschachtelungen $\sigma=\left(x_{n} \mid y_{n}\right)=\left(J_{n}\right)$ selbst als Zahlen anspricht, oder ob man sich ein hypothetisches Ding eingeführt denkt, das allen Intervallen $J_{n}$ angehört $(\operatorname{vgl} \mathbf{1 5}, 3)$ und das nun erst recht eigentlich die durch die Schachtelung erfafite $\mathrm{Z}$ a h 1 und somit das allen „gleichen" Schachtelungen Gemeinsame sei, ist schlieflich belanglos und reine Geschmackssache. - Die Gleichung $\sigma=\left(x_{n} \mid y_{n}\right)$ dürfen wir (vgl. S. 25, Fufn. 1) von nun an jedenfalls nach Belieben lesen: entweder „, $\sigma$ ist eine abkürzende Bezeichnung für die Schachtelung $\left(x_{n} \mid y_{n}\right)$ " oder " $\sigma$ ist die durch die Schachtelung $\left(x_{n} \mid y_{n}\right)$ definierte Zahl". 
nämlich der eine auf Grund des Cantor-Dedekindschen Axiomes allemal als vorhanden betrachtete, allen Intervallen $J_{n}$ gemeinsame Punkt. Und zwei Schachtelungen $\sigma$ und $\sigma^{\prime}$ entspricht dann und nur dann derselbe Punkt, wenn sie im Sinne von 14 einander gleich sind. Jeder reellen Zahl $\sigma$ (d. h. also: allen untereinander gleichen Intervallschachtelungen) entspricht genau ein Punkt und jedem Punkt genau eine Zahl. Den auf diese Weise einer bestimmten Zahl entsprechenden Punkt nennt man deren Bild und kann nun sagen: Das System der reellen Zahlen läßt sich umkehrbar eindeutig auf die Punkte einer Geraden abbilden.

\section{§ 4. Vollständigkeit und Einzigkeit des Systems der reellen Zahlen.}

Noch zwei letzte Bedenken sind zu zerstreuen ${ }^{2}$ ): Wir gingen in $\S 3$ ron der Tatsache aus, daß das System der rationalen Zahlen zu lückenhaft war, um allen Anforderungen zu genügen, die bei den dementaren Rechenoperationen an sie gestellt werden können. Unser neu geschaffenes Zahlensystem, das wir für das folgende kurz das System $Z$ nennen wollen, ist in dieser Beziehung gewiß leistungsfähiger, denn es enthält ja z. B. eine Zahl $\sigma$, für die $\sigma^{2}=2$ ist $^{1}$ ). Es bleibt aber doch die Möglichkeit, daß das neue System $Z$ noch in ähnlicher Weise Lücken aufwiese wie jenes oder in anderer Weise noch einer Erweiterung fähig wäre.

Wir werfen demgemäß die Frage auf: Ist ein System $\bar{Z}$ denkbar, das im Sinne von $\mathbf{4}$ als ein Zahlensystem anzusehen ist, welches sämtliche Zeichen des Systems $Z$, aber außer diesen noch weitere, von jenen verschiedene Elemente enthält ${ }^{3}$ )

Es ist leicht zu sehen, daß dies nicht möglich ist, daß vielmehr der folgende Satz gilt:

20. Vollständigkeitssatz. Das System $Z$ aller reellen Zahlen ist keiner mit den Bedingungen 4 verträglichen Erweiterung mehr fähig.

Beweis: Es sei $\bar{Z}$ ein System, das den Bedingungen 4 genügt und das sämtliche Elemente aus $Z$ enthält. Ist dann \& ein beliebiges Element aus $\bar{Z}$, so gibt es nach 4, 4 eine natürliche Zahl $p>\alpha$ und eine andre natürliche Zahl $p^{\prime}>-\alpha$. Dann ist $\left.--p^{\prime}<\alpha<p^{4}\right)$. Geht man die (endlichvielen) ganzen Zahlen von $-p^{\prime}$ bis $p$ durch,

1) Denn ist $\sigma=\left(x_{n} \mid y_{n}\right)$ die Schachtelung, die wir S. 18 im Anschluf an die Aufgabe $x^{2}=2$ gebildet haben, so ist nach $18 \sigma^{2}=\left(x_{n}^{2} \mid y_{n}^{2}\right)$. Wegen $x_{n}^{2}<2$ und $y_{n}^{2}>2$ ist dann aber $\sigma^{2}=2$, w. $z$. b. w.

2) Vgl. hierzu die Schlufworte der Einleitung (S. 2).

$\left.{ }^{3}\right) \bar{Z}$ müiste dann also in ähnlicher Weise eine Erweiterung von $Z$ sein, wie $Z$ selbst eine Erweiterung des Systems der rationalen Zahlen ist.

4) An dieser Stelle gewinnt das Postulat des Eudoxus seine prinzipielle Bedeutung. 
so muß es unter ihnen eine letzte geben - sie heiße $g$-, welche $\leqq e$ ist. Dann ist

$$
g \leqq \alpha<g+1
$$

Wendet man auf dieses Intervall, wie schon mehrfach, die 10-Teilungsmethode an, so erhält man eine ganz bestimmte Intervallschachtelung $\left(x_{n} \mid y_{n}\right)$. Und die durch diese erfaßte reelle Zahl kann nicht $\gtrless$ e sein, wie der Beweis des Satzes 12 gelehrt hat. Jedes Element von $\bar{Z}$ ist also einer reellen $Z$ ahl gleich, so daß $\bar{Z}$ keine von den reellen Zahlen verschiedenen Elemente enthalten kann.

Und ein letztes Bedenken wäre dieses: Wir sind zwar auf eine verhältnismäßig naheliegende, aber immerhin willkürliche Art zur Bildung des Systems $Z$ gelangt. Selbstverständlich wird man auch andere Wege einschlagen können, um die Lückenhaftigkeit des Systems der rationalen Zahlen zu überwinden. (Schon im nächsten Paragraphen werden wir andere, gleichfalls sehr gangbare Wege dieser Art kennen lernen.) Und es wäre nun denkbar, daß man auf einem anderen Wege auch zu anderen Zahlen gelangte, - d. h. zu Zahlsystemen, die sich in mehr oder minder wesentlicher Weise von dem von uns geschaffenen unterscheiden. - Die hiermit angedeutete Frage wäre so zu präzisieren:

Auf irgendeinem Wege sei man, vom System der rationalen Zahlen ausgehend, zur Konstruktion eines Systems 3 von Elementen gelangt, welches wieder, wie unser System $Z$, den Bedingungen 4 genügt (also als ein Zahlsystem anzusprechen ist), welches aber im Gegensatz zum System der rationalen Zahlen noch der folgenden weiteren Forderung genügt, die man (wegen des eben bewiesenen Satzes) gewöhnlich als das Vollständigkeitspostulat zu bezeichnen pflegt: Auf Grund von 4,3 enthält 3 Elemente, die den rationalen Zahlen entsprechen. Ist dann $\left(x_{n} \mid y_{n}\right)$ irgendeine Intervallschachtelung und sind $\mathfrak{x}_{n}$ und $\mathfrak{y}_{n}$ Elemente aus 3 , die nach 4,3 den rationalen Zahlen $x_{n}$ und $y_{n}$ : zugeordnet sind, so lautet die Forderung, daß 3 immer mindestens ein Element $\mathfrak{B}$ enthalten soll, das für jedes $n$ den Bedingungen $\mathfrak{x}_{n} \leqq \mathfrak{z} \leqq \mathfrak{y}_{n}$ genügt.

Dann lautet das aufgeworfene Problem genauer: Kann sich ein solches System 3 in irgend welchen wesentlichen Eigenschaften vom System $Z$ der reellen Zahlen unterscheiden, oder müssen sie vielmehr in dem ganz präzisen Sinn als wesentlich identisch angesehen werden (vgl. S. 9/10), daß sie ähnlich und isomorph aufeinander bezogen werden können?

Der folgende Satz, der dies Problem in dem zu erwartenden Sinne löst, bedeutet dann den Abschluß des Aufbaues des Systems der reellen Zahlen.

Einzigkeitssatz. Jedes derartige System 3 muß auf das von uns 21. geschaffene System $Z$ der reellen ähnlich und isomorph bezogen werden können. Es gibt also im wesentlichen nur ein solches System. 
Beweis: Nach 4, 3 enthält 3 ein Teilsystem $Z^{\prime}$, dessen Elemente sich den in $Z$ enthaltenen rationalen Zahlen ähnlich und isomorph zuordnen lassen und die wir darum kurz die rationalen Elemente aus 3 nennen können. Ist dann $\sigma=\left(x_{n} \mid y_{n}\right)$ irgendeine reelle Zahl, so enthält 3 gemäß unserer neuen Forderung ein Element $\mathfrak{z}$, das für jedes $n$ den Bedingungen $\mathfrak{x}_{n} \leqq \mathfrak{z} \leqq \mathfrak{y}_{n}$ genügt, wenn $\mathfrak{x}_{n}$ und $\mathfrak{y}_{n}$ die den rationalen Zahlen $x_{n}$ und $y_{n}$ entsprechenden Elemente aus 3 sind. Und eine dem Beweis von 12 wörtlich nachzubildende Betrachtung würde lehren, daß dieses Element $\mathfrak{z}$ das einzige ist, das diesen Bedingungen genügt. Ordnen wir nun dieses Element $\mathfrak{B}$ und die reelle Zahl $\sigma$ einander zu, so wird deutlich, daß 8 ein Teilsystem $3^{*}$ enthält, dessen Elemente sich denen des Systems $Z$ aller reellen Zahlen ähnlich und isomorph zuordnen lassen. Daß ein solches System $3^{*}$ aber keiner mit den Bedingungen 4 verträglichen Erweiterung mehr fähig ist, sondern also $3^{*}$ mit 3 selber identisch sein muß, war der Inhalt des vorhin bewiesenen Vollständigkeitssatzes. Damit ist gezeigt, daß 3 und $Z$ sich ähnlich und isomorph aufeinander beziehen lassen und also als wesentlich identisch anzusehen sind: Das von uns geschaffene System $Z$ aller reellen Zahlen ist im wesentlichen das einzig mögliche, das den Bedingungen 4 und dem Volls!ändigkeilspostulat genügt.

Fassen wir nach diesen etwas abstrakten Betrachtungen das Hauptergebnis unserer ganzen Untersuchung noch einmal zusammen, so können wir sagen:

Neben den uns vertrauten rationalen Zahlen gibt es noch andere, die sogenannten irrationalen Zahlen. Eine jede von ihnen läßt sich (und zwar auf viele Arten) durch eine geeignete Intervallschachtelung erfassen (bestimmen, geben, ...). Diese irrationalen Zahlen reihen sich widerspruchslos dem System der rationalen Zahlen ein und zwar derart, daß von dem System $Z$ aller rationalen und irrationalen Zahlen zusammen die unter 4 formulierten Bedingungen erfüllt sind, daß man - kurz gesagt - mit ihnen allen formal genau so, in der Wirkung aber erfolgreicher rechnen kann, wie mit den rationalen Zahlen allein. Dieses umfassendere System ist überdies keiner mit den Bedingungen 4 verträglichen Erweiterung mehr fähig, und wesentlich das einzige System von Zeichen, das diesen Bedingungen 4 und zugleich dem Vollständigkeitspostulat genügt. Wir nennen es das System der reellen Zahlen.

Seine Elemente, die reellen Zahlen, sind es, mit denen wir weiterhin und zunächst ausschließlich arbeiten. Eine spezielle reelle Zahl gilt uns dann und nur dann als gegeben (bekannt, bestimmt, definiert, berechenbar, ...), wenn sie entweder eine rationale Zahl ist und also mit Hilfe der natürlichen Zahlen, nötigenfalls unter Hinzuziehung eines Bruchstrichs und eines Minuszeichens vollständig hingeschrieben werden 
kann, oder wenn uns - und dies gilt in jedem Falle - eine sie definierende Intervallschachtelung gegeben ${ }^{\mathbf{1}}$ ) ist.

Sehr bald werden wir indessen sehen, daß es an Stelle der Intervallschachtelungen noch viele andere Wege und Arten gibt, durch die eine reelle $Z$ ahl definiert werden kann. In dem Maße, wie wir solche Wege kennen lernen werden, werden wir die eben genannten Bedingungen, unter denen uns eine Zahl als gegeben gilt, dann auch erweitern.

\section{§ 5. Die Systembrüche und der Dedeliindsche Schnitt.}

Einige von den Intervallschachtelungen abweichende, für Theorie und Praxis besonders wichtige Arten, reelle Zahlen zu definieren, wollen wir sogleich angeben.

Zunächst braucht die Schachtelung nicht immer in der von uns betrachteten Form $\left(x_{n} \mid y_{n}\right)$ gegeben zu sein. Oft läßt sie sich bequemer schreiben. So sahen wir schon, daß ein Dezimalbruch, z. B. 1,41421 ..., sofort als Intervallschachtelung aufgefaßt werden kann, indem man

$$
x_{1}=1,4 ; \quad x_{2}=1,41 ; \quad x_{3}=1,414 ; \ldots
$$

also allgemein $x_{n}$ gleich dem nach $n$ Stellen abgebrochenen Dezimalbruch setzt und nun $y_{n}$ aus $x_{n}$ durch Erhöhung der letzten Ziffer um eine Einheit herleitet.

In den Dezimalbrüchen haben wir also lediglich eine besonders bequeme und übersichtliche Angabe von Intervallschachtelungen zu $\left.\operatorname{sehen}^{2}\right)$.

Es ist klar, daß die Rolle, die die Grundzdhl 10 hierbei spielt, keine wesentliche ist. Ist $g$ irgendeine natürliche Zahl $\geqq 2$, so können wir ganz ebenso systcmatische Brüche (oder kürzer: Systembrüche) mit der Grundzahl g einführen: Eine gegebene reelle Zahl $\sigma$ bestimmt zunächst eindeutig eine ganze Zahl $p(\gtreqless 0)$ durch die Bedingung

$$
p \leqq \sigma<p+1 \text {. }
$$

Das ganzzahlige Intervall $J_{0}$ von $p$ bis $p+1$ werde nun in $g$ gleiche Teile geteilt. $\mathrm{Zu}$ jedem dieser Teile rechnen wir hierbei - und ebenso bei den folgenden Schritten - den linken, nicht aber den rechten Endpunkt. Dann gehört $\sigma$ einem und nur einem dieser Teile an, d. h. es gibt unter den Zahlen $0,1,2, \ldots, g-1$ eine und nur

1) Also durch die eben beschriebene vollständige Angabe ihrer (rationalen) Intervallenden.

2) Ihr Nachteil liegt darin, daß man nur selten die Gesetzmäßigkeit in der Aufeinanderfolge der Ziffern -- also das Bildungsgesetz der $x_{n}$ und $y_{n}$ durchschauen kann. 
eine - wir wollen sie kurz eine „Ziffer“ nennen und mit $z_{1}$ bezeichnen -, so daß

$$
p+\frac{z_{1}}{g} \leqq \sigma<p+\frac{z_{1}+1}{g}
$$

ist. Das hierdurch bestimmte Intervall $J_{\mathbf{1}}$ teilen wir nun erneut in $g$ gleiche Teile, und $\sigma$ wird wieder einem und nur einem derselben angehören, d. h. es wird wieder eine bestimmte ,Ziffer" $z_{2}$ geben, so daß

$$
p+\frac{z_{1}}{g}+\frac{z_{2}}{g^{2}} \leqq \sigma<p+\frac{z_{1}}{g}+\frac{z_{2}+1}{g^{2}}
$$

ist. Das hierdurch bestimmte Intervall $J_{2}$ teilen wir erneut in $g$ gleiche Teile usw. Die hierdurch bestimmte Intervallschachtelung $\left(J_{n}\right)=\left(x_{n} \mid y_{n}\right)$, für die

$$
\begin{aligned}
& x_{n}=p+\frac{z_{1}}{g}+\frac{z_{2}}{g^{2}}+\ldots+\frac{z_{n-1}}{g^{n-1}}+\frac{z_{n}}{g^{n}} \\
& y_{n}=p+\frac{z_{1}}{g}+\frac{z_{2}}{g^{2}}+\ldots+\frac{z_{n-1}}{g^{n-1}}+\frac{z_{n}+1}{g^{n}}
\end{aligned} \quad(n=1,2,3, \ldots)
$$

ist $\left.^{1}\right)$, definiert offenbar die Zahl $\sigma$, so daß $\sigma=\left(x_{n}, y_{n}\right)$ ist. Nach Analogie der Dezimalbrüche werden wir nun aber

$$
\sigma=\boldsymbol{p}+\boldsymbol{0}, \boldsymbol{z}_{1} \boldsymbol{z}_{2} \boldsymbol{z}_{3} \ldots
$$

schreiben dürfen, - wobei dann allerdings die Grundzahl $g$ dieses Systembruches aus dem Zusammenhange heraus bekannt sein muß.

Es gilt also der

Satz. Jede reelle Zahl läßt sich auf eine und wesentlich nur eine") Art durch einen Systembruch mit der Grundzahl g darstellen. -

Als bekannt betrachten wir den weiteren sich auf diese Darstellung beziehenden

Satz. Der Systembruch für eine reelle. Zahl o fält - welches auch die gewählte Grundzahl $g \geqq 2$ sein mag - dann und nur dann periodisch aus, wenn $\sigma$ rational ist ${ }^{3}$ ).

1) Daß eine Intervallschachtelung vorliegt ist ja unmittelbar klar, da stets $x_{n-1} \leqq x_{n}<y_{n} \leqq y_{n-1}$ ist und $y_{n}-x_{n}=\frac{1}{g^{n}}$ nach 10, 7 eine Nullfolge bildet.

2) Die geringe Änderung, die in unserm Verfahren eintritt, wenn wir den Intervallen jedesmal den rechten und nicht den linken Endpunkt zurechnen, wird sich der Leser wohl allein zurechtlegen können. Das Ergebnis ist dann und nur dann ein anderes, wenn die gegebene Zahl $\sigma$ eine rationale ist, die mit einer Potenz von $g$ als Nenner geschrieben werden kann, oder also, wenn der Punkt $\sigma$ einmal Endpunkt eines unserer Intervalle ist. - In der Tat sind dann die beiden Intervallschachtelungen $p+0, z_{1} z_{2} \ldots z_{r-1}\left(z_{r}-1\right)(g-1)(g-1) \ldots$ und $p+0, z_{1} z_{2} \ldots z_{r-1} z_{r} 00 \ldots$ gemän 14 einander gleich. In jedem andern Falle sind zwei nicht identische Systembrüche auch gemä́s 14 nicht gleich.

$\left.{ }^{3}\right)$ Wir sehen hier die "abbrechenden" Systembrïche der Einfachheit 
Besonders vorteilhaft ist oft die Wahl $g=2$; man nennt dann das Verfahren zur Erfassung der Zahl $\sigma$ kurz die Halbierungsmethode, den resultierenden Systembruch, dessen Ziffern dann nur 0 und 1 sein können, einen dyadischen Bruch. Diese Methode besteht also, etwas allgemeiner angesehen, darin, daß man von einem bestimmten Intervall $J_{0}$ ausgeht, won seinen beiden Hälften nach irgendeiner Regel oder unter irgendeinem Gesichtspunkt eine bestimmte auswählt und mit $J_{1}$ bezeichnet, - indem man von $J_{1}$ wieder eine bestimmte der beiden Hälften mit $J_{2}$ bezeichnet usw. Man erfaßt dann stets eine wohlbestimmte reelle Zahl, die durch die Art der jedesmaligen Auswahl zwischen den beiden Hälften völlig eindeutig definiert ist ${ }^{1}$ ).

In den Systembrüchen sehen wir hiernach, wie in den Dezimalbrüchen, lediglich eine besonders bequeme und übersichtliche Form der Angabe einer Intervallschachtelung. Sie sollen demgemäß zur Definition reeller Zahlen fortan ebenso zugelassen werden, wie jene.

Ein wenig tiefer liegt der Unterschied zu den Intervallschachtelungen bei der folgenden Methode, eine reelle Zahl zu erfassen.

Auf irgendeine Weise seien uns zwei Klassen $A$ und $B$ von Zahlen gegeben ${ }^{2}$ ), doch so, daß folgenden drei Bedingungen genügt ist:

1) Jede der beiden Klassen enthält mindestens eine Zahl.

2) Jede Zahl der Klasse $A$ ist $\leqq j e d e r ~ Z a h l$ der Klasse $B$.

3) Wenn eine beliebige positive (kleine) Zahl $\varepsilon$ vorgeschrieben wird, so soll sich aus $A$ und $B$ je eine Zahl - etwa $a^{\prime}$ aus $A$ und $b^{\prime}$ aus $B$ - so auswählen lassen, daß

ist $\left.^{3}\right)$. - Dann gilt der

$$
b^{\prime}-a^{\prime}<\varepsilon
$$

Satz. Es gibt stets eine und nur eine reelle Zahl $\sigma$ derart, daß 22. fiir jede Zahl a aus $A$ und für jede Zahl $b$ aus $B$ die Beziehung

gilt.

$$
a \leqq \sigma \leqq b
$$

halber als periodisch mit der Periode 0 an. - Daf jede rationale Zahl durch einen periodischen Dezimalbruch dargestellt werden kann, hat schon J. Wallis, De algebra tractatus, 1693, S. 364 bewiesen. Dafi umgekehrt jede irrationale Zahl stets und nur auf eine Weise durch einen nicht periodischen Dezimalbruch dargestellt werden kann, hat erst O. Stolz, (s. Allgemeine Arithmetik I, 1885, S. 119) allgemein bewiesen.

1) Ein Beispiel war schon unter 12, 2 gegeben worden.

2) Z. B.: $A$ enthält alle rationalen Zahlen, deren dritte Potenz $<5, B$ alle rationalen Zahlen, deren dritte Potenz $>5$ ist, - od. ähnl.

3) Man sagt kurz: Die Zahlen der beiden Klassen kommen einander beliebig nahe. Bei dem Beispiel der vorangehenden Fufnote sieht man sofort, dafs die Bedingungen 1) und 2) erfüllt sind; daf auch 3) erfüllt ist, erkennt man, wenn man sich (etwa nach der 10-Teilungsmethode) zwei nur in der letzten Ziffer um eine Einheit unterschiedene $n$-stellige Dezimalbrüche $x_{n}$ und $y_{n}$ herstellt, für die $x_{n}{ }^{3}<5, y_{n}{ }^{3}>5$ und bei denen $n$ so gewählt ist, daß $\frac{1}{10^{n}}<\varepsilon$ ist. 
Beweis: $\mathrm{DaB}$ es nicht zwei verschiedene solche Zahlen, etwa $\sigma$ und $\sigma^{\prime}$, geben kann, ist wieder sofort klar. Denn setzt man $\left|\sigma-\sigma^{\prime}\right|=\varepsilon$, so wäre entgegen der Bedingung 3) für jeles Paar von Elementen $a$ und $b$ aus $A$ und $B$ stets $b-a \geqq \varepsilon$.

Es gibt also höchstens eine solche Zahl $\sigma$. Man findet sie so: Nach Voraussetzung gibt es in 'A wenigstens eine Zahl $a_{1}$ und in $B$ wenigstens eine Zahl $b_{1}$. Ist hier $a_{1}=b_{1}$, so ist ihr gemeinsamer Wert ersichtlich die gesuchte Zahl $\sigma$. Ist aber $a_{\mathbf{1}} \neq b_{1}$, also nach 2 ), $a_{1}<b_{1}$, so wenden wir die Halbierungsmethode an. Das durch $a_{1}$ und $b_{1}$ bestimmte Intervall $J_{1}$ teilen wir in zwei gleiche Teile und bezeichnen nun die linke oder die rechte Hälfte mit $J_{2}$, je nachdem in der linken Hälfte (Endpunkte einschließlich) noch ein Punkt der Klasse $B$ liegt oder nicht. Nach derselben Regel bezeichnen wir wieder eine bestimmte Hälfte von $J_{2}$ mit $J_{3}$ usw.

Die Intervalle $J_{1}, J_{2}, \ldots, J_{n}, \ldots$ bilden, weil nach der Halbierungsmethode gewonnen, eine Schachtelung

$$
\left(J_{n}\right)=\left(x_{n} \mid y_{n}\right)=\sigma \text {. }
$$

Sie haben überdies gemäß ihrer Bildungsweise die Eigenschaft, daß weder links vom linken Endpunkt eine $Z$ ahl aus $B$ noch rechts rom rechten Endpunkt eine $Z$ ahl aus $A$ liegen kann.

Daraus folgt aber sofort, daß die erfaßte Zahl $\sigma$ die verlangte Eigenschaft hat. Wäre nämlich eine spezielle Zahl $\bar{a}$ aus $A$, entgegen der Behauptung, $>\sigma$, also $\vec{a}-\sigma>0$, so wähle man aus der Folge der Intervalle $J_{n}$ ein spezielles aus, etwa $J_{p}=x_{p} \ldots y_{p}$, dessen Länge $<\vec{a}-\sigma$ ist. Wegen $x_{p} \leqq \sigma \leqq y_{p}$ wäre dann

$$
y_{p}-\sigma \leqq y_{p}-x_{p}<\bar{a}-\sigma, \quad \text { d. h. } \quad y_{p}<\bar{a},
$$

während doch rechts rom rechten Endpunkt $y_{p}$ ron $J_{p}$ tatsächlich kein Punkt aus $A$ mehr liegt. Wäre andrerseits einmal $\bar{b}<\sigma$, so folgte analog für einen geeigneten Index $q$, daß $\bar{b}<x_{q}$ sein müßte, während doch links vom linken Endpunkt eines Intervalles tatsächlich kein Punkt aus $B$ mehr liegt. Es $m u \beta$ also stets $a \leqq \sigma \leqq b \operatorname{sein}, w . z$. b.w.

\section{Bemerkungen und Beispiele.}

1. Statt der Forderung 3) ist es oft bequemer zu fordern, dafi z. B. alle rationalen Zahlen entweder zu $A$ oder zu $B$ gehören (wie dies im Beispiel der letzten Fufnote der Fall war). Dann ist nämlich, da die rationalen Zahlen dicht liegen, die Forderung 3) von selbst erfüllt. Um dies einzusehen, braucht man sich nämlich nur die ganze Zahlengerade in gleiche Teile von einer Länge $\langle\varepsilon / 2$ geteilt zu denken. Einmal mußs es dann vorkommen, daßs in zwei Nachbarteilen aus beiden Klassen $A$ und $B$ gleichzeitig mindestens je eine Zahl 1 iegt. Deren Abstand ist dann, wie es 3 ) verlangt, sicher $<\varepsilon$.

2. Oft ist es noch bequemer, alle reellen Zahlen auf die Klassen $A$ und $B$ zu verteilen. Dann ist die Forderung 3) natürlich erst recht von selbst erfüllt.

3. Sind die Klassen $A$ und $B$ gemäß einer der eben besprochenen Arten gegeben, so sagt man, es sei ein Dedekindscher Schnitt im Bereich der 
rationalen bzw. reellen Zahlen gegeben; und auch die etwas allgemeinere Angabe von zwei Klassen, wie sie unserm Satze zugrunde liegt, wollen wir kurz einen Schnitt nennen und mit $(A \mid B)$ bezeichnen. - Unser Satz kann dann kurz dahin ausgesprochen werden: Ein Schnitt $(A \mid B)$ definiert stets eine wohlbestimmte reelle $Z a h l \sigma$. Und sein Beweis besteht einfach darin, dafis aus der Angabe des Schnittes die Angabe einer bestimmten Intervallschachtelung hergeleitet wird, die eine Zahl $\sigma$ mit den verlangten Eigenschaften liefert.

4. Da hiernach jeder Schnitt sofort eine bestimmte Intervallschachtelung liefert, wollen wir fortan auch die Schnitte zur Erfassung (Bestimmung, Definition, ....) von reellen Zahlen zulassen; und wir schreiben, wenn der Schnitt $(A \mid B)$ die Zahl $\sigma$ definiert, nun auch kurz

$$
(\boldsymbol{A} \mid \boldsymbol{B})=\sigma \text {. }
$$

5. Das Umgekehrte ist natürlich auch der Fall und noch einfacher zu sehen: Ist die Schachtelung $\left(x_{n} \mid y_{n}\right)=\sigma$ vorgelegt und bildet man aus allen linken Intervallenden $x_{n}$ eine Klasse $A$, aus allen rechten Enden eine Klasse $B$, so liefern diese Klassen ersichtlich einen Schnitt, durch den gleichfalls die Zahl $\sigma$ definiert wird. - Eine Schachtelung kann hiernach als ein spezieller Schnitt aufgefaßit werden.

6. Nach der letzten Bemerkung ist die Methode der Schnitte (zur Erfassung reeller Zahlen) an Allgemeinheit der der Schachtelungen überlegen. Sie ist auch anschaulich ebenso bequem zugänglich wie diese. Denn nehmen wir etwa den Schnitt $(A \mid B)$ in der etwas spezielleren unter 2) besprochenen Form als Schnitt im Bereich der reellen Zahlen, so besagt unser Satz: Wenn man alle Punkte der Zahlengeraden in zwei Klassen $A$ und $B$ verteilt, z. B. die einen schwarz, die andern weifs gefärbt denkt, und wenn dabei 1) von jeder Art wenigstens ein Punkt da ist, wenn 2) jeder schwarze Punkt links von jedem weifen Punkt liegt und 3) auch wirklich jeder Punkt entweder schwarz oder weifs gefärbt ist, so müssen die beiden Klassen an einer ganz bestimmten Stelle zusammenstoßen und links von dieser Stelle ist alles schwarz, rechts davon alles weif.

7. Man hüte sich aber, die eben gegebene Veranschaulichung für einen Beweis zu halten. Hätten wir uns nicht vorher mit Hilfe der Intervallschachtelungen die reellen Zahlen geschaffen, so wäre unser Satz überhaupt gar nicht zu beweisen, - ebensowenig wie wir beweisen konnten, daß jede Schachtelung eine Zahl definiert. Wir entschlossen uns nur - und der Erfolg gab uns vollauf recht - jede Schachtelung als Zahl anzusprechen. Genau so kann man sich - und das ist tatsächlich der Ausgangspunkt $R$. Dedekinds ${ }^{1}$ ) beim Aufbau des Systems der reellen Zahlen - entschliefien, jeden Schnitt im Bereich der rationalen Zahlen als ,veelle $Z a h l$ anzusprechen; und man hätte dann nur, ganz entsprechend unsern Untersuchungen in $\S 3$, auch hier zu prüfen, ob das erlaubt ist, ob „es denn angeht"; d. h. man hätte festzustellen, ob die Gesamtheit aller solcher Schnitte $(A \mid B)$ im Sinne der Bedingungen 4 ein Zahlensystem bildet, - was nicht schwieriger ist als unsere analogen Feststellungen in $\S 3$.

Von nun ab bilden die reellen Zahlen unser zunächst ausschlieBliches Arbeitsmaterial. Dabei werden wir den Zusatz „reell“ nach Belieben auch weglassen dürfen: Unter „Zahl“" verstehen wir bis auf weiteres stets eine reelle Zahl.

1) Stetigkeit und irrationale Zahlen, Braunschweig 1872. 


\section{Aufgaben zum I. Kapitel.}

1. Aus den Grundgesetzen $\mathbf{I}$ und $\boldsymbol{2}$ sollen die wichtigsten weiteren Rechenregeln hergeleitet werden, z. B. a) das Produkt zweier negativer Zahlen ist positiv; b) aus $a+c<b+c$ folgt stets $a<b$; c) für jedes $a$ ist $a \cdot 0=0$ usw.

2. Wann gilt in $\mathbf{3}$, II, 4 ein Gleichheitszeichen?

3. Man stelle die folgenden Zahlen als dyadische (triadische) Brüche dar

$$
\frac{1}{2}, \frac{3}{8}, \frac{1}{3}, \frac{1}{7}, \frac{10}{17} ;
$$

für $\sqrt{2}, \sqrt{3}, \pi$ und $e$ gebe man die Anfangsziffern der dyadischen (triadischen) Darstellung.

†. Für die Folge 6,7 ist $x_{n}=\frac{c^{n}-\beta^{n}}{\alpha-\beta}$, wenn $\alpha$ und $\beta$ die Wurzeln der quadratischen Gleichung $x^{2}=x+1$ bedeuten. (Anl.: Die Folgen $\left(c^{n}\right)$ und $\left(\beta^{n}\right)$ haben dasselbe Bildungsgesetz wie die Folge $\mathbf{6}, 7$.)

5. Man bilde die Folgen $\left(x_{n}\right)$, die für $n \geq 1$ durch die Formel

$$
x_{n+1}=a x_{n}+b x_{n-1}
$$

geliefert werden, in welcher $a$ und $b$ gegebene positive Zahlen und die Anfangsglieder $x_{0}, x_{1}=0,1 ;=1,0 ;=1, \alpha ;=1, \beta$ oder beliebig sind $(\alpha$ und $\beta$ sollen hierbei die positive und die negative Wurzel der Gleichung $x^{2}=a x+b$ bedeuten). Man gebe für jeden der Fälle eine explizite Formel für $x_{n}$.

6. Ist $J_{0}, J_{1}, J_{2}, \ldots$ eine Folge ineinander geschachtelter Intervalle, iiber deren Längen sonst nichts bekannt ist, so gibt es mindestens einen Punkt, der allen $J_{n}$ angehört.

7. Eine reelle Zahl $\sigma$ ist irrational, wenn man eine wachsende Folge ganzer Zahlen $\left(q_{n}\right)$ so finden kann, daßs $q_{n} \sigma$ niemals eine ganze Zahl ist, dafs aber $\left(q_{n} \sigma-p_{n}\right)$ eine Nullfolge wird, wenn $p_{n}$ die zunächst bei $q_{n} \sigma$ gelegene ganze Zahl bedeutet.

8. Man zeig $\epsilon$, daf in $\left(x_{n} \mid y_{n}\right)$ Intervallschachtelungen vorliegen, wenn

a) $x_{n}=\frac{1^{2}+2^{2}+\ldots+(n-1)^{2}}{n^{3}}, \quad y_{n}=\frac{1^{2}+2^{2}+\ldots+n^{2}}{n^{3}}, \quad(n=1,2, \ldots)$;

b) $0<x_{1}<y_{1}$ und für $n>1$ stets $x_{n+1}=\sqrt{x_{n} y_{n}}, \quad y_{n+1}=\frac{1}{2}\left(x_{n}+y_{n}\right)$;

c) $0<x_{1}<y_{1} \quad, \quad, \quad, \quad, \quad x_{n+1}-\frac{1}{2}\left(x_{n}+y_{n}\right), \quad y_{n+1}=\sqrt{x_{n+1} \cdot y_{n}}$;

d) $0<x_{1}<y_{1} \quad, \quad, \quad, \quad, \quad y_{n+1}=\frac{1}{2}\left(x_{n}+y_{n}\right), \quad x_{n+1}=\sqrt{x_{n} \cdot y_{n+1}}$;

e) $0<x_{1}<y_{1} \quad, \quad, \quad, \quad, \quad x_{n+1} \sqrt{x_{n} y_{n}}, \quad y_{n+1}-\frac{1}{3}\left(x_{n+1}+y_{n}\right)$;

f) $0<x_{1}<y_{1} \quad, \quad, \quad, \quad, \quad y_{n+1} \quad \sqrt{x_{n} y_{n}}, \quad x_{n+1}=\frac{1}{2}\left(x_{n}+y_{n+1}\right)$;

g) $0<x_{1}<y_{1} \quad, \quad, \quad, \quad, \quad y_{n+1}-\frac{1}{2}\left(x_{n}+y_{n}\right), \quad x_{n+1}=\frac{x_{n} \cdot y_{n}}{y_{n+1}}$

gesetzt wird und bestimme in den Fällen a) und g) die dadurch definierte Zahl. (Vgl. hierzu Aufgabe 91 u. 92.) 


\section{Kapitel.}

\section{Reelle Zahlenfolgen.}

\section{$\$ 6$. Beliebige reelle Zahlenfolgen und Nullfolgen.}

Wir greifen nun noch einmal auf unsere Betrachtungen in $\S 2$ zurück, verallgemeinern sie aber dadurch, daß jetzt alle auftretenden Zahlen beliebige reelle Zahlen sein dürfen. Da sich mit diesen formal genau so operieren läßt wie mit den rationalen Zahlen, so werden bei dieser Verallgemeinerung die Definitionen und Sätze des $§ 2 \mathrm{im}$ wesentlichen ungeändert bleiben. Wir können uns daher kurz fassen.

Definition $\left.{ }^{1}\right)$. Entspricht jeder natürlichen $Z$ ahl $n=1,2,3, \ldots$ je eine wohlbestimmte $Z$ ahl $x_{n}$, so bilden diese Zahlen

eine Zahlenfolge.

$$
x_{1}, x_{2}, x_{3}, \ldots, x_{n}, \ldots
$$

Die Beispiele 6, 1-12, sind natïrlich auch hier zulässig. Ebenso behalten die Bemerkungen 7, 1-6, volle Giiltigkeit. Wir geben noch ein paar Beispiele, bei denen es nicht ohne weiteres $z u$ sehen ist, ob die auftretenden Zahlen rational sind oder nicht.

Beispiele: 1. Es sei $a=0,3010 \ldots$, d. h. gleich dem Dezimalbruch, dessen erste Ziffern auf Grund der Aufgabe $10^{x}=2$ in der Fufinote von S. 22 bestimmt wurden, und nun

$$
x_{n}=a^{n} \quad \text { für } \quad n=1,2,3, \ldots
$$

2. Bei derselben Bedeutung von $a$ sei $x_{n}=\frac{1}{a+n}$.

3. Man wende auf das Intervall $J_{0}=0 \ldots 1$ die Halbierungsmethode an und nehme das erste Mal die linke Hälfte, die beiden folgenden Male die rechte Hälfte, bei den nächsten 3 Schritten wieder die linke, dann 4 mal die rechte Hälfte usw. Die definierte Zahl heiß̧e $b^{2}$ ) (welchen Wert hat sie ungefähr?), und es werde nun $x_{n}$ der Reihe nach

gesetzt.

$$
=+b,-b,+\frac{1}{b},-\frac{1}{b},+b^{2},-b^{2},+\frac{1}{b^{2}},-\frac{1}{b^{2}},+b^{3}, \ldots
$$

4. Bei derselben Bedeutung von $b$ werde $x_{n}$ der Reihe nach gesetzt.

$$
=1-b, 1+b, 1-b^{2}, 1+b^{2}, 1-b^{3}, 1+b^{3}, \ldots
$$

5. Bei derselben Bedeutung von $a$ und $b$ sei $x_{1}$ der Mittelpunkt der Strecke zwischen beiden, also $x_{1}=\frac{1}{2}(a+b) ; x_{2}$ sei der Mittelpunkt zwischen $x_{1}$ und $b$; $x_{3}$ der zwischen $x_{2}$ und $a ; x_{4}$ der zwischen $x_{3}$ und $b ;-$ es sei also allgemein $x_{n+1}$ der Mittelpunkt $z$ wischen $x_{n}$ und $a$ oder aber $b$, je nachdem $n$ gerade oder ungerade ist.

1) Über die Bedeutung des Zeichens $\bigcirc$ vgl. das Vorwort, sowie später

2) Als dyadischer Bruch geschrieben ist $b=0,01100011110 \ldots$ 
24.

- Definitionen: 1. Eine Zahlenfolge $\left(x_{n}\right)$ heißt beschränkt, falls es eine Konstante $K$ gibt, so daß für alle $n$ die Ungleichung

erfïllt ist.

$$
\left|x_{n}\right| \leqq K
$$

2. Eine Zahlenfolge $\left(x_{n}\right)$ heißt monoton wachsend, wenn stets $x_{n} \leqq x_{n+1}$ ist; monoton fallend, wenn ste's $x_{n} \geqq x_{n+1}$ is'.

Alle bei $\mathbf{S}$ und $\mathbf{9}$ gemachten Bemerkungen behalten ihre volle Gültigkeit.

Beispiele: 1. Die Folgen 23, 1, 2, 4 und 5 sind ersichtlich beschränkt. Die 3. ist es nicht und zwar weder nach rechts noch nach links; denn es ist sicher $b<\frac{1}{2}$ und also $\frac{1}{b^{m}}>2^{m}>m$ und somit $-\frac{1}{b^{m}}<-m$. Man kann also stets Glieder der Folge angeben, die $>K$ oder $<-K$ sind, wie grof auch die Konstante $K$ gewählt wird. - Bei 5. folgt die Beschränktheit daraus, dafs alle Glieder zwischen $a$ und $b$ liegen.

2. Die Folgen 23, 1 und 2 sind monoton fallend; die andern sind nicht monoton.

Auch die Definition der Nullfolge und die daran geknüpften Bemerkungen - man lese sie nochmals sorgfältig durch! — bleiben ungeändert.

25. $\bigcirc$ Definition. Eine Folge $\left(x_{n}\right)$ soll als Nullfolge bezzichnet werden, wenn nach Wahl einer beliebigen positiven Zahl $\varepsilon$ sich stets eine Zaht $n_{0}$ s) angeben läßt, daß für alle $n>n_{0}$ die Ungleichung

\section{crfiillt ist.}

$$
\left|x_{n}\right|<\varepsilon
$$

Beispiele. 1. Die Folge $\mathbf{2 3}, 1$ ist eine Nullfolge, denn der Beweis 10, 7 gilt für beliebige reelle $a$, für die $|a|<1$ ist.

2. Auch 23, 2 ist eine Nullfolge, denn hier ist $\left|x_{n}\right|<\frac{1}{n}$, also $<\varepsilon$, sobald $n>\frac{1}{\varepsilon}$.

Über die Nullfolgen - sie werden weiterhin eine vorherrschende Rolle spielen - beweisen wir noch eine Anzahl ganz einfacher, aber im folgenden unausgesetzt angewandter Sätze. Ganz naheliegend sind zunächst die folgenden beiden:

26. ${ }^{\circ}$ Satz 1. Ist $\left(x_{n}\right)$ eine Nullfolge und $\left(a_{n}\right)$ irgendeine beschränkte Zahlenfolge, so bilden auch die Zahlen

eine Nullfolge.

$$
x_{n}^{\prime}=a_{n} x_{n}
$$

Beweis. Es sei stets $\left|a_{n}\right|<K$ und $\varepsilon>0$ werde gegeben. Dann läßt sich nach Voraussetzung $n_{0}$ so angeben, daß für alle $n>n_{0}$ stets

$$
\left|x_{n}\right|<\frac{\varepsilon}{K}
$$

ist. Dann ist aber für dieselben $n$ auch stets

$$
\left|x_{n}^{\prime}\right|=\left|a_{n}\right| \cdot\left|x_{n}\right|<K \cdot \frac{\varepsilon}{K}=\varepsilon .
$$


Also ist $\left(x_{n}{ }^{\prime}\right)$ eine Nullfolge. - Wir sagen kurz: Eine Nullfolge „darf“ mit beschränkten Faktoren multipliziert werden ${ }^{\mathbf{1}}$ ).

Beispiele. 1. Mit $\left(x_{n}\right)$ ist auch $10 x_{1}, \frac{x_{2}}{10}, 10 x_{3}, \frac{x_{4}}{10}, 10 x_{5}, \ldots$ eine Nullfolge.

2. Bedeutet $\left(x_{n}\right)$ die Nullfolge $\mathbf{2 3}, 2$ und $\left(a_{n}\right)$ die Folge $\mathbf{2 3}, 5$, so ist $\left(a_{n} x_{n}\right)$ wieder eine Nullfolge.

3. Eine Folge, deren sämtliche Glieder denselben Wert, etwa den Wert $c$ haben, ist gewiß bęschränkt. Mit $\left(x_{n}\right)$ ist daher auch $\left(c x_{n}\right)$ eine Nullfolge. Speziell sind also $\left(\frac{c}{n}\right),\left(c a^{n}\right)$ für $|a|<1$, usw. Nullfolgen.

oSatz 2. Ist $\left(x_{n}\right)$ eine Nullfolge und genügen die Glieder der Folge $\left(x_{n}{ }^{\prime}\right)$ für alle $n$ von einer Stelle an der Bedingung $\left|x_{n}{ }^{\prime}\right| \leqq \mid x_{n}$ oder allgemeiner der Bedingung

$$
\left|x_{n}{ }^{\prime}\right| \leqq K \cdot\left|x_{n}\right|
$$

in der $K$ eine be'iebige (feste) positive Za'll beteutet, so ist auch $\left(x_{n}{ }^{\prime}\right.$ ) eine $N$ ullfolge.

Der Bewsis bedarf keiner Ausführung.

Etwas tiefer liegen schon die folgenden Sätze:

o Satz 1. Ist $\left(x_{n}\right)$ eine Nullfolge, so ist auch jede Teilfolge $\left(x_{n}{ }^{\prime}\right)$ ders?lben eine Nullfolge $\left.{ }^{2}\right)$.

Beweis. Ist für $n>n_{0}$ stèts $\left|x_{n}\right|<\varepsilon$, so ist für diese $n$ von selbst auch

$$
\left|x_{n}^{\prime}\right|=\left|x_{k_{n}}\right|<\varepsilon,
$$

da ja mit $n$ sicher auch $k_{n}>n_{0}$ ist.

oSatz 2. Eine beliebige Folge $\left(x_{n}\right)$ warle in zwii Teilfslgen $\left(x_{n}{ }^{\prime}\right)$ und $\left(x_{n}^{\prime \prime}\right)$ zer'egt, derar: daß also jodes Glied von $\left(x_{n}\right)$ einer und nur einer dieser beiden Teilfolgen angehört. Sind dann $\left(x_{n}{ }^{\prime}\right)$ und $\left(x_{n}{ }^{\prime \prime}\right)$ beides Nullfolgen, so ist, auch $\left(x_{n}\right)$ solbst eine solche.

Beweis. Wählt man eine Zahl $\varepsilon>0$, so gibt es nach Voraussetzung eine Zahl $n^{\prime}$, so daß für $n>n^{\prime}$ stets $\left|x_{n}{ }^{\prime}\right|<\varepsilon$ ist und ebenso eine Zahl $n^{\prime \prime}$, so daß für $n>n^{\prime \prime}$ stets $\left|x_{n}{ }^{\prime \prime}\right|<\varepsilon$ ausfällt. Die Glieder $x_{n}{ }^{\prime}$, deren Index $\leqq n^{\prime}$ ist, und die Glieder $x_{n}^{\prime \prime}$, deren Index $\leqq n^{\prime \prime}$ ist, haben innerhalb der ursprünglichen Folge $\left(x_{n}\right)$ wohlbestimmte Nummern. Ist $n_{0}$ die größte derselben, so ist für $n>n_{0}$ offenbar stets $\left|x_{n}\right|<\varepsilon$, w. z. b. w.

1) Das ',darf" sie natürlich auch ohne besondere Erlaubnis. Gemeint ist mit dieser Ausdrucksweise nur, daß, wenn wir es tun, ihre im Augenblick wesentliche Eigenschaft - nämlich eine Nullfolge zu sein - nicht gestört wird.

2) Bilden $k_{1}<k_{2}<k_{3}<\ldots<k_{n}<\ldots$ irgendeine Folge natiurlicher Zahlen, so sagt man, daßs die Zahlen

$$
x_{n}{ }^{\prime}=x_{k_{n}} \quad(n=1,2,3, \ldots)
$$

eine Teilfolge der gegebenen Folge bilden. 
- Satz 3. Ist $\left(x_{n}\right)$ eine Nullfolge und $\left(x_{n}{ }^{\prime}\right)$ eine beliebige Umordnung $\left.{ }^{1}\right)$ derselben, so ist auch $\left(x_{n}^{\prime}\right)$ eine Nullfolge.

Beweis. Für $n>n_{0}$ sei stets $\left|x_{n}\right|<\varepsilon$. Unter den Nummern, die die endlich vielen Glieder $x_{1}, x_{2}, \ldots, x_{n_{0}}$ in der Folge $\left(x_{n}{ }^{\prime}\right)$ tragen, sei $n^{\prime}$ die größte. Dann ist ersichtlich für $n>n^{\prime}$ stets $\left|x_{n}{ }^{\prime}\right|<\varepsilon$; also ist auch $\left(x_{n}{ }^{\prime}\right)$ eine Nullfolge.

- Satz 4. Ist $\left(x_{n}\right)$ eine Nullfolge und entsteht die Folge $\left(x_{n}^{\prime}\right)$ aus ihr durch irgendwelche endlich vielen Anderungen $\left.{ }^{2}\right)$, so ist auch $\left(x_{n}{ }^{\prime}\right)$ eine Nullfolge.

Der Beweis folgt unmittelbar aus der Tatsache, daß für ein passendes ganzzahliges $p \gtreqless 0$ von einer Stelle ab $x_{n}{ }^{\prime}=x_{n+p}$ sein muß. Denn sind die $x_{n}$ für $n \geqq n_{1}$ ungeändert geblieben und hat $x_{n_{1}}$ in der Folge $\left(x_{n}{ }^{\prime}\right)$ die Nummer $n^{\prime}$ bekommen, so ist in der Tat für $n>n^{\prime}$ stets

wenn $p=n_{1}-n^{\prime}$ gesetzt wird $\left.^{3}\right)$.

$$
x_{n}{ }^{\prime}=x_{n+p},
$$

Das Rechnen mit den Nullfolgen endlich wird durch die beiden folgenden Sätze begründet:

○ Satz 1. Sind $\left(x_{n}\right)$ und $\left(x_{n}{ }^{\prime}\right)$ zwei Nullfolgen, so ist auch

$$
\left(y_{n}\right)-\left(x_{n}+x_{n}^{\prime}\right) \text {, }
$$

d. h. die Folge, deren Glieder die Zahlen $y_{n}=x_{n}+x_{n}{ }^{\prime}$ sind, eine Nullfolge. - Kürzer: Zwei Nullfolgen, ,dürfen" gliedweis addiert werden.

Beweis. Wird $\varepsilon>0$ beliebig gewählt, so gibt es nach Voraussetzung (vgl. 10, 12) je eine Zahl $n_{1}$ und $n_{2}$, so daß

für $n>n_{1}$ stets $\quad\left|x_{n}\right|<\frac{\varepsilon}{2}$ und für $n>n_{2}$ stets $x_{n}{ }^{\prime}<\frac{{ }_{2}}{2}$ ist. Ist dann $n_{0}$ eine Zahl, die $\geqq n_{1}$ und $\geqq n_{2}$ ist, so ist für $n>n_{0}$

$$
\left.\left|y_{n}\right|=\left|x_{n}+x_{n}^{\prime} \leqq \leqq\right| x_{n}|+| x_{n}^{\prime}{ }^{4}\right)<\frac{\varepsilon}{2}+\frac{\varepsilon}{2}=\varepsilon .
$$

$\left(y_{n}\right)$ ist also eine Nullfolge.

1) Ist $k_{1}, k_{2}, \ldots, k_{n}, \ldots$ eine Folge natürlicher Zahlen von der Beschaffenheit, dafi jede natürliche Zahl in ihr ein- und nur einmal vorkommt, so sagt man, dafi die Folge der Zahlen

$$
x_{n}{ }^{\prime}=x_{k_{n}}
$$

eine Umordnung der gegebenen Folge sei.

2) Wir wollen diesen Begriff so präzisieren: Wenn man eine beliebige Folge dadurch verändert, daf man endlich viele Glieder wegläfst oder einfügt oder abändert (oder alles zusammen) und die so veränderte Folge neu numeriert als Folge $\left(x_{n}{ }^{\prime}\right)$, so wollen wir sagen, $\left(x_{n}{ }^{\prime}\right)$ sei durch endlich viele Anderungen aus $\left(x_{n}\right)$ hervorgegangen.

$\left.{ }^{3}\right)$ Gerade wegen dieses Satzes sagt man wohl, die Eigenschaft einer Folge, Nullfolge zu sein, beziehe sich nur auf das infinttäre Verhalten ihrer Glieder (vgl. S. 14).

4) Nach 3, II, 4 . 
Da mit $\left(x_{n}{ }^{\prime}\right)$ nach 26, 3 (oder 10,5) auch $\left(-x_{n}{ }^{\prime}\right)$ eine Nullfolge ist, so ist nach dem eben Bewiesenen auch $\left(y_{n}{ }^{\prime}\right)=\left(x_{n}-x_{n}{ }^{\prime}\right)$ eine solche, d. h. es gilt der

o Satz 2. Mit $\left(x_{n}\right)$ und $\left(x_{n}{ }^{\prime}\right)$ ist auch $\left(y_{n}{ }^{\prime}\right)=\left(x_{n}-x_{n}{ }^{\prime}\right)$ eine Nullfolge. Oder kürzer: Nullfolgen ,dürfen" gliedweis subtrahiert werden.

\section{Bemerkungen.}

1. Da man zwei Nullfolgen gliedweis addieren darf, so darf man es auch mit dreien oder mit irgendeiner bestimmten Anzahl von Nullfolgen tun. Denn ist dies schon für $(p-1)$ Nullfolgen $\left(x_{n}{ }^{\prime}\right),\left(x_{n}{ }^{\prime \prime}\right), \ldots,\left(x_{n}^{(p-1)}\right)$ bewiesen, ist also schon

$$
\left(x_{n}{ }^{\prime}+x_{n}{ }^{\prime \prime}+\ldots+x_{n}^{(p-1)}\right)
$$

als Nullfolge erkannt, so liefert Satz 1 dasselbe für die Folge $\left(x_{n}\right)$ für die

$$
x_{n}=\left(x_{n}{ }^{\prime}+\ldots+x_{n}^{(p-1)}\right)+x_{n}^{(p)}
$$

ist. Der Satz gilt also für jede feste Anzähl von Nullfolgen.

2. Dafi zwèi Nullfolgen auch gliedweis miteinander multipliziert werden „dürfen", ist nach $\mathbf{2 6}, 1$ ohne weiteres klar, da ja die Nullfolgen nach 10, 11 von selbst beschränkt sind.

3. Eine gliedweise Division dagegen ist im allgemeinen nicht erlaubt, was z. B. schon daraus $z u$ ersehen ist, daf ja stets $\frac{x_{n}}{x_{n}}=1$ ist. Nimmt man gar $x_{n}=\frac{1}{n}, x_{n}^{\prime}=\frac{1}{n^{2}}$, so liefern die Quotienten $\frac{x_{n}}{x_{n}{ }^{\prime}}$ nicht einmal eine beschränkte Folge!

4. Auch bei anderen Folgen $\left(x_{n}\right)$ kann man zunächst nur wenig über die Folge $\left(\frac{1}{x_{n}}\right)$ der reziproken Werte aussagen. Naheliegend, aber oft niitzlich ist der folgende

oSatz 3. Hat die Folge $\left(\left|x_{n}\right|\right)$ der absoluten Beträge der Glieder von $\left(x_{n}\right)$ eine noch positive untere Schranke, existiert also eine $Z a h l \gamma>0$, so daß stets bleibt. so ist die Folge $\left(\frac{1}{x_{n}}\right)$ der reziproken Werte beschränkt.

In der Tat folgt aus $\mid x_{n} \geq \gamma>0$ sofort, daf 3 mit $K=\frac{1}{\gamma}$ stets

$$
\left|\frac{1}{x_{n}}\right| \leqq K
$$

bleibt.

Um weiterhin in der Anwendung unserer Begriffe sowie in der Bildung und Durchführung von Beispielen weniger beengt zu sein, schalten wir einen Abschnitt über Potenzen, Wurzeln, Logarithmen und die Kreisfunktionen ein.

\section{$\S$ \%. Potenz, Wurzel und Logarithmus. Spezielle Nullfolgen.}

Wie es bei der Besprechung des Systems der reellen Zahlen nicht unsere Absicht war, alle Einzelheiten erschöpfend auszuführen, 
sondern nur die Grundgedanken klarzustellen und im übrigen den von jedem ja durchaus beherrschten Rechenapparat als bekannt hinzunehmen, so wollen wir uns auch jetzt bei der Besprechung von Potenz, Wurzel und Logarithmus nur auf eine scharfe Klarstellung der Definitionen beschränken, im übrigen aber die Einzelheiten ihres Gebrauchs als jedem bekannt annehmen.

\section{Potenzen mit ganzzahligen Exponenten.}

Ist $x$ eine beliebige Zahl, so ist das Zeichen $x^{k}$ für ganze positive Exponenten $k$ bekanntlich definiert als das Produkt von $k$ Faktoren, die sämtlich $=x$ sind. Es handelt sich hierbei also gar nicht um etwas sachlich Neues, sondern nur um eine abkürzende Schreibweise für etwas schon Bekanntes. Ist $x \neq 0$, so ist darüber hinaus noch die Festsetzung zweckmäßig, es soll

$$
x^{0} \text { die Zahl 1, } x^{-k} \text { den Wert } \frac{1}{x^{k}} \quad(k=1,2,3, \ldots)
$$

bedeuten, so daß dann $x^{p}$ für jedes ganzzahlige $p \gtreqless 0$ definiert ist. Bei diesen Potenzen mit ganzzahligen Exponenten betonen wir nur die folgenden Tatsachen:

29. 1. Für beliebige ganzzahlige Exponenten $p$ und $q(\geq 0)$ gelten die drei Grundregeln

$$
x^{p} \cdot x^{q}=x^{p+q} ; \quad x^{p} \cdot y^{p}=(x y)^{p} ; \quad\left(x^{p}\right)^{q}=x^{p q},
$$

aus denen sich alle übrigen Regeln herleiten lassen, die das Rechnen mit Potenzen beherrschen ${ }^{1}$ ),

2. Da es sich bei einer Potenz mit ganzzahligen Exponenten nur um eine wiederholte Multiplikation bzw. Division handelt, hat ihre Berechnung natürlich nach 18 und 19 zu geschehen. Wenn also $x$ positiv ist und etwa durch die Schachtelung $\left(x_{n} \mid y_{n}\right)$ erfaßt wird, deren Intervallenden sämtlich $\geqq 0$ sind (vgl. 15, 5 u. 6 ), so gilt neben

$$
x=\left(x_{n} \mid y_{n}\right) \text { sofort auch } x^{k}=\left(x_{n}^{k} \mid y_{n}^{k}\right)
$$

für jeden ganzzahligen positiven Exponenten; und ähnlich - bei sinngemäßen Einschränkungen - für $x \leqq 0$ oder $k \leqq 0$.

3. Für positive $x$ hat man außerdem

$$
x^{k+1} \gtreqless x^{k} \quad \text { je nachdem } \quad x \gtreqless 1
$$

ist, - wie dies sofort aus $x^{k}=x^{k}$ durch Multiplikation (s. 3, I, 3) mit $x \gtreqless 1$ folgt. - Und ebenso einfach findet man:

Sind $x_{1}, x_{2}$ und der ganzzahlige Exponent $k$ posiliv, so ist

$$
x_{1}^{k} \lesseqgtr x_{2}^{k} \text { je nachdem } x_{1} \lesseqgtr x_{2} .
$$

1) Hierbei ist für die Basis $x$ bzw, $y$ der Wert 0 nur zulässig, falls der zugehörige Exponent positiv ist. 
30 .

$\S 7$. Potenz, Wurzel und Logarithmus. Spezielle Nullfolgen.

4. Für ganzzahlige positive Exponenten $n$ und beliebige $a$ und $b$ gilt die Formel

$$
\begin{aligned}
(a+b)^{n}=a^{n}+\left(\begin{array}{l}
n \\
1
\end{array}\right) a^{n-1} b+ & \left(\begin{array}{l}
n \\
2
\end{array}\right) a^{n-2} b^{2}+\ldots \\
& +\left(\begin{array}{l}
n \\
k
\end{array}\right) a^{n-k} b^{k}+\ldots+\left(\begin{array}{l}
n \\
n
\end{array}\right) b^{n},
\end{aligned}
$$

in $\operatorname{der}\left(\begin{array}{l}n \\ k\end{array}\right)$ für $1 \leqq k \leqq n$ die Bedeutung

$$
\left(\begin{array}{l}
n \\
k
\end{array}\right)=\frac{n(n-1)(n-2) \ldots(n-k+1)}{1 \cdot 2 \cdot 3 \quad \cdots \quad k}
$$

hat und $\left(\begin{array}{l}n \\ 0\end{array}\right)=1$ gesetzt werden kann. (Binomischer Lehrsatz.)

\section{Wurzeln.}

Ist jetzt $a$ eine beliebige positive reelle und $k$ eine positive ganze Zahl, so soll unter

$$
\sqrt[k]{a}
$$

eine Zahl verstanden werden, deren $k^{\text {te }}$ Potenz $=a$ ist. Was uns hier interessiert, ist lediglich die Existenzfrage: Gibt es eine solche Zahl, und inwieweit ist sie durch das gestellte Problem bestimmt?

Darüber gilt der

Satz. Es gibt stets eine und nur eine positive Zahl $\xi$, die $\mathbf{3 0 .}$ die Gleichung

$$
\xi^{k}=a
$$

erfüllt. Man schreibt $\boldsymbol{\xi}=\sqrt[\boldsymbol{k}]{\boldsymbol{a}}$ und nennt $\boldsymbol{\xi}$ die $k^{\text {te }}$ Wurzel aus a.

Beweis. Eine solche Zahl kann unmittelbar durch eine Intervallschachtelung bestimmt und damit also auch ihre Existenz nachgewiesen werden: Wir benutzen die 10 -Teilungsmethode. Da $0^{k}=0<a$, dagegen, falls $p$ eine natürliche Zahl $>a$ bedeutet, $p^{k} \geqq p>a$ ist, so gibt es eine und nur eine ganze Zahl $g \geqq 0$, für die

$$
g^{k} \leqq a<(g+1)^{k}
$$

ist $^{1}$ ). Das durch $g$ und $g+1$ bestimmte Intervall $J_{0}$ teilen wir in 10 gleiche Teile und gelangen in der nun schon oft durchgeführten Art zu einer bestimmten der Ziffern $0,1,2, \ldots, 9$, die etwa $z_{1}$ heißen möge, und für die

$$
\left(g+\frac{z_{1}}{10}\right)^{k} \leqq a<\left(g+\frac{z_{1}+1}{10}\right)^{k}
$$

1) $g$ ist die letzte der Zahlen $0,1,2, \ldots, p$, deren $k^{\text {te }}$ Potenz $\leqq a$ ist. 
ist, usw. usw. Wir gelangen also zu einer Intervallschachtelung $\left(J_{n}\right)=\left(x_{n} \mid y_{n}\right)$, deren Intervallenden wohlbestimmte Werte der Form

$$
x_{n}=g+\frac{z_{1}}{10}+\frac{z_{2}}{10^{2}}+\ldots+\frac{z_{n-1}}{10^{n-1}}+\frac{z_{n}}{10^{n}} \quad(n=1,2,3, \ldots)
$$

und

$$
y_{n}=g+\frac{z_{1}}{10}+\frac{z_{2}}{10^{2}}+\ldots+\frac{z_{n-1}}{10^{n-1}}+\frac{z_{n}+1}{10^{n}}
$$

haben. Ist $\xi=\left(x_{n} \mid y_{n}\right)$ die dadurch bestimmte Zahl, so folgt, da hier alle Intervallenden $\geqq 0$ sind, nach $\mathbf{2 9}, 2$ sofort, daß

$$
\xi^{k}=\left(x_{n}^{k} \mid y_{n}^{k}\right)
$$

ist. Da aber nach Konstruktion stets $x_{n}^{k} \leqq a \leqq y_{n}^{k}$ ist, so muß nach 12

$$
\xi^{k}=a
$$

sein. - Daß diese Zahl $\xi$ überdies die einzige positive Lösung des Problems ist, folgt unmittelbar aus 29, 3, da hiernach für ein positives $\xi_{1} \neq \xi$ auch $\xi_{1}^{k} \neq \xi^{k}$, d. h. $\neq a$ ist.

Ist $k$ eine gerade Zahl, so ist auch $-\xi$ eine Lösung des Problems. Doch werden wir diese im folgenden niemals heranziehen, sondern unter der $\dot{k}^{\text {ten }}$ Wurzel aus einer positiven Zahl a nur die durch 30 völlig eindeutig bestimmte positive $Z a h l$ g verstehen $\left.{ }^{1}\right)$. Für $a=0$ setzt man auch $\sqrt[k]{a}=0^{2}$ ).

Auf die bekannten Regeln über das Rechnen mit Wurzelzeichen gehen wir nicht weiter ein, setzen sie vielmehr als jedem geläufig voraus, und beweisen nur noch die folgenden einfachen Sätze:

Aus 29, 3 folgt sofort der

31. Satz 1. Es ist $\sqrt[k]{a} \lesseqgtr \sqrt[k]{a_{1}}$, je nachdem $a \lesseqgtr a_{1}$ ist. - Ferner gilt der

Satz 2. Ist $a>0$, so ist $(\sqrt[n]{-a})$ eine monotone Zahlenfolge; und zwar ist

dagegen

$$
a>\sqrt{a}>\sqrt[3]{a}>\ldots>1, \text { wenn } a>1,
$$

$$
a<\sqrt{a}<\sqrt[3]{a}<\ldots<1 \text {, wenn } a<1
$$

ist. (Fïr $a=1$ ist die Folge natürlich - 1.)

1) Hiernach ist also z. B. $\sqrt{a^{2}}$ nicht stets $=a$, sondern stets $=|a|$; $\sqrt[k]{a^{k}}=a$.

2) Für negative $a$ wollen wir $\sqrt[k]{a}$ gar nicht definieren; doch kann man, falls $k$ ungerade ist, $\sqrt[k]{a}=-\sqrt[k]{a_{a}}$ setzen. 
31. § 7. Potenz, Wurzel und Logarithmus. Spezielle Nullfolgen.

Beweis. Aus $a>1$ folgt nach 29, $3: a^{n+1}>a^{n}>1$, und also nach dem vorigen Satze, indem man die $n(n+1)^{\text {te }}$ Wurzel zieht:

$$
\sqrt[n]{a}>\sqrt[n+1]{a}>1
$$

Da sich für $a<1$ sämtliche Ungleichheitszeichen umkehren, so ist schon alles bewiesen. - - Hieraus folgt endlich der

Satz 3. Ist $a>0$, so bilden die Zahlen.

$$
x_{n}=\sqrt[n]{a}-1
$$

eins (nach dem vorigen Satze monotone) Nullfolge.

Beweis. Für $a=1$ ist die Behauptung trivial, da dann $x_{n}=0$ ist. Ist aber $a>1$ und also auch $\sqrt[n]{a}>1$, d. h. $x_{n}=\sqrt[n]{a}-1>0$, so schließen wir folgendermaßen: Aus $\sqrt[n]{a}=1+x_{n}$ folgt nach der Bernoullischen Ungleichung (s. 10, 7), daß auch

$$
a=\left(1+x_{n}\right)^{n}>1+n x_{n}>n x_{n}
$$

ist. Folglich ist $x_{n}=\left|x_{n}\right|<\frac{a}{n}$, also $\left(x_{n}\right)$ nach $\mathbf{2 6}, 1$ und 2 eine Nullfolge.

Ist aber $0<a<1$, so ist $\frac{1}{a}>1$ und somit nach dem eben erhaltenen Ergebnis

$$
(\sqrt[n]{1}-1)
$$

eine Nullfolge. Multipliziert man diese gliedweise mit den Faktoren $\sqrt[n]{a}$, die wegen $a \leqq \sqrt[n]{a}<1$ jetzt sicher eine beschränkte Folge bilden, so ergibt sich nach $\mathbf{2 6}, 1$ sofort, daß auch

$$
(1-\sqrt[n]{a}) \text { und also auch }\left(x_{n}\right)
$$

eine Nullfolge ist, - w. z. b. w.

\section{Potenzen mit rationalen Exponenten.}

Wir sehen wieder im wesentlichen als bekannt an, wie von den Wurzeln mit ganzzahligen Exponenten zu den Potenzen mit beliebigen rationalen Exponenten übergegangen wird: Unter $a^{\frac{p}{q}}$ mit $p \gtreqless 0$ $q>0$ versteht man bei positivem $a$ die durch

$$
a^{\frac{p}{q}}=(\sqrt[q]{a})^{p}
$$

eindeutig definierte wieder positive Zahl. Falls $p>0$, darf auch noch $a=0$ sein; man hat dann unter $a^{\frac{p}{q}}$ den Wert 0 zu verstehen. 
Bei diesen Festsetzungen gelten unverändert die drei Grundregeln 29, 1, also die Formeln

$$
a^{r} \cdot a^{r^{\prime}}=a^{r+r^{\prime}} ; \quad a^{r} b^{r}=(a b)^{r} ; \quad\left(a^{r}\right)^{r^{\prime}}=a^{r r^{\prime}}
$$

für beliebige rationale Exponenten, so daß das Rechnen mit diesen Potenzen formal dasselbe ist wie bei ganzzahligen Exponenten. Diese Formeln enthalten dann auch gleichzeitig alle Regeln für das Rechnen mit Wurzeln, da ja jede Wurzel nun als Potenz mit rationalem Exponenten geschrieben werden kann. - Von den weniger bekannten Folgerungen beweisen wir noch, weil für das Folgende besonders wichtig, die Sätze:

32. Satz 1. Ist $a>1$, so ist dann und mur dann gleichzeitig $a^{r}>1$, wenn $r>0$ ist. Ebenso ist bei positivem $a<1$ dann und nur dann gleichzeitig $a^{r}<1$, wenn $r>0$ ist.

Beweis. Nach 31, 2 sind $a$ und $\sqrt[q]{a}$ entweder beide größer oder beide kleiner als 1 ; nach $\mathbf{2 9}, 3$ gilt für $a$ und $(\sqrt[q]{a})^{p}=a^{r}$ dann und nur dann dasselbe, wenn $p>0$ ist.

Satz 2. Ist $a>0$ und die rationale $Z a h l ~ r$ zwischen den rationalen Zahlen $r^{\prime}$ und $r^{\prime \prime}$ gelegen, so liegt auch $a^{r}$ stets zwischen $a^{r^{\prime}}$ und $\left.a^{r^{\prime \prime} 1}\right)$ und umgekehrt - mag $a<,=$ oder $>1, r^{\prime}<$, = oder $>r^{\prime \prime} \operatorname{sein}$.

Bew eis. Ist zunächst $a>1$ und $r^{\prime}<r<r^{\prime \prime}$, so ist

$$
a^{r}=a^{r^{\prime}} \cdot a^{r-r^{\prime}}=\begin{gathered}
a^{r^{\prime \prime}} \\
a^{r^{\prime \prime}-r}
\end{gathered} \text {. }
$$

Nach dem vorigen Satze ergibt sich hieraus schon die Richtigkeit unserer Behauptung für diesen Fall. Für die andern möglichen Fälle aber ist der Beweis genau ebenso leicht. - Aus diesem Beweise folgt sogar genauer der

Satz 2a. Ist $a>1$, so entspricht dem größeren (rationalen) Exponenten auch der größere Wert der Potenz. Ist dagegen $a<1$, so liefert der größere Exponent die kleinere Potenz. - Ganz speziell also: Ist die (positive) Basis $a \neq 1$, so liefern verschiedene Exponenten auch verschiedene Potenzen. - Hieraus folgt nun weiter der

Satz 3. Ist $\left(r_{n}\right)$ eine beliebige (rationale) Nullfolge, so bilden auch die Zahlen

$$
x_{n}=a^{r}-1
$$

eine Nullfolge. Ist $\left(r_{n}\right)$ monoton, so ist es auch $\left(x_{n}\right)$.

1) Das Wort „zwischen" kann nach Belieben stets mit oder stets ohne Einschluf 3 der beiderseitigen Gleichheit verstanden werden, - aufier wenn $a=1$ und also auch alle Potenzen $a^{r}=1$ sind. 
33. $\$ 7$. Potenz, Wurzel und Logarithmus. Spezielle Nullfolgen.

Beweis. Nach 31, 3 sind $(\sqrt[n]{a}-1)$ und $\left(\sqrt[n]{\frac{1}{a}}-1\right)$ Nullfolgen. Ist also $\varepsilon>0$ gegeben, so kann man $n_{1}$ und $n_{2}$ so wählen, daß für $n>n_{1}$ stets $|\sqrt[n]{a}-1|<\varepsilon$ und für $n>n_{2}$, stets $\left|\sqrt[n]{\frac{1}{a}}-1\right|<\varepsilon$

ist. Ist dann $m$ eine ganze Zahl, die sowohl $n_{1}$ als $n_{2}$ übertrifft, so liegen die Zahlen $\left(a^{\frac{1}{m}}-1\right)$ und $\left(a^{-\frac{1}{m}}-1\right)$ beide zwischen $-\varepsilon$ und $+\varepsilon$, oder also

$a^{\frac{1}{m}}$ und $a^{-\frac{1}{m}}$ beide zwischen $1-\varepsilon$ und $1+\varepsilon$.

Nach Satz 2 liegt dann auch $a^{r}$ zwischen denselben Grenzen, wenn $r$ zwischen $-\frac{1}{m}$ und $+\frac{1}{m}$ liegt. Nach Voraussetzung kann man aber $n_{0}$ so wählen, daß für alle $n>n_{0}$

$$
\text { stets }\left|r_{n}\right|<\frac{1}{m} \text { oder } \quad-\frac{1}{m}<r_{n}<+\frac{1}{m}
$$

ist; für $n>n_{0}$ ist dann also $a^{r_{n}}$ zwischen $1-\varepsilon$ und $1+\varepsilon$ gelegen. Für diese $n$ ist also

$$
\left|a^{r_{n}}-1\right|<\varepsilon
$$

$\left(a^{r} n-1\right)$ somit eine Nullfolge. - Daß dieselbe monoton ist, falls $\left(\boldsymbol{r}_{n}\right)$ es ist, ergibt sich unmittelbar aus Satz $2 \mathrm{a}$.

Diese Sätze bilden nun die Grundlage für die Definition der

\section{Potenzen mit beliebigen reellen Exponenten.}

Hier gilt zunächst der

Satz. Ist $\left(x_{n} \mid y_{n}\right)$ eine beliebige Intervallschachtelung (mit ratio-33. nalen Intervallenden), so ist

$$
\begin{array}{rlll}
\text { für } a \geqq 1 & \text { auch } & \sigma & =\left(a^{x_{n}} \mid a^{y_{n}}\right) \\
\text { und für } a \leqq 1 & \text { auch } & \sigma & =\left(a^{y_{n}} \mid a^{x_{n}}\right)
\end{array}
$$

eine Intervallschachtelung. Und ist $\left(x_{n} \mid y_{n}\right)$ rationalwertig und $=r$, so ist $\sigma=a^{r}$.

Beweis. Daß in beiden Fällen die linken Folgen monoton steigen, die rechten monoton fallen, folgt unmittelbar aus $\mathbf{3 2}, 2$ a. Nach demselben Satze ist auch stets $a^{x_{n}} \leqq a^{y_{n}}$ (für $a \geqq 1$ ) bzw. $a^{y_{n}} \leqq a^{x_{n}}$ (für $a \leqq 1$ ). Daß endlich in beiden Fällen die Längen der Intervalle eine Nullfolge bilden, folgt mit Hilfe von $\mathbf{2 6}$ sofort aus

$$
\left|a^{y_{n}}-a^{x_{n}}\right|=\left|a^{y_{n}-x_{n}}-1\right| a^{x_{n}}
$$


denn der erste Faktor ist hier nach $\mathbf{3 2}, 3$ eine Nullfolge, da ja $\left(y_{n}-x_{n}\right)$ nach Voraussetzung eine Nullfolge mit rationalen Gliedern ist. Der zweite Faktor aber ist beschränkt, weil stets

$$
0<a^{x_{n}} \leqq a^{y_{1}} \quad \text { bzw. } \leqq a^{x_{1}}
$$

ist, je nachdem $a \geqq 1$ oder $\leqq 1$ ist.

Da endlich, wenn $\left(x_{n} \mid y_{n}\right)=r$ ist, $r$ für jedes $n$ zwischen $x_{n}$ und $y_{n}$ liegt, so liegt nach $\mathbf{3 2}, 2$ auch $a^{r}$ stets zwischen $a^{x_{n}}$ und $a^{y_{n}}$, so daß $\sigma=a^{r}$ sein muß.

Auf Grund dieses Satzes entschließen wir uns zu der folgenden so soll

Definition: Ist $a>0$ und $\varrho=\left(x_{n} \mid y_{n}\right)$ eine beliebige reelle Zahl,

$$
\boldsymbol{a}^{o}=\sigma, \quad \text { d. h. }\left\{\begin{array}{lll}
=\left(a^{x_{n}} \mid a^{y_{n}}\right) & \text { für } \quad a \geqq 1 \\
=\left(a^{y_{n}}\right. & \left.a^{x_{n}}\right) & \text { für } \quad a \leqq 1
\end{array}\right.
$$

gesetzt werden ${ }^{1}$.

Diese Definition wird man natürlich nur dann als eine zweckmäßige bezeichnen können, wenn sich der dadurch festgelegte Begriff der allgemeinen Potenz wesentlich denselben Gesetzen unterordnet, denen die bisherige Potenz mit rationalem Exponenten gehorchte. $\mathrm{Daß}$ dies tatsächlich im weitesten Maße der Fall ist, wird durch die folgenden Feststellungen erwiesen.

34. 1. Für rationale Exponenten liefert die neue Definition dasselbe wie die bisherige.

2. Ist $\varrho=\varrho^{\prime}$, so ist $a^{\varrho}=a^{\varrho^{\prime}} 2$ ).

3. Für zwei beliebige reelle Zahlen $\varrho$ und $\varrho^{\prime}$ gelten bei positivem $a$ und $b$ die drei Grundregeln

$$
a^{\varrho} \cdot a^{\varrho^{\prime}}=a^{\varrho+e^{\prime}} ; \quad\left(a^{\varrho} \cdot b^{\varrho}\right)=(a b)^{\varrho} ; \quad\left(a_{*}^{\varrho}\right)^{\varrho^{\prime}}=a^{2 Q^{\prime}},
$$

so daß mit den jetzigen allgemeinen Potenzen formal genau so gerechnet werden kann, wie mit den bisherigen speziellen.

Auf die überaus einfachen Beweise dieser Tatsachen wollen wir

1) Dieses Paar 33 aus Satz und Definition ist methodisch ein Paar von genau gleicher Art wi? die unter 14-19 zusammengestellten: Was im Falle rationaler Exponenten beweisbar ist, wird im Falle beliebiger Exponenten zum Range einer Definition erhoben, - deren Zweckmäfigkeit dann geprüft werden mußs.

2) Diese formal sehr trivial erscheinende Aussage lautet etwas ausführlicher: Sind $\left(x_{n} \mid y_{n}\right)=\varrho$ und $\left(x_{n}{ }^{\prime} \mid y_{n}{ }^{\prime}\right)=\varrho^{\prime}$ zwei Intervallschachtelungen, die gemäß 14 als gleich anzusehen sind, so sind auch diejenigen Schachtelungen einander gleich (wieder gemäß 14), durch die nach Definition 33 die Potenzen $a^{\varrho}$ und $a^{\varrho^{\prime}}$ geliefert werden. 
nun, wie S. $45 / 46$ betont, nicht weiter eingehen ${ }^{1}$ ); desgleichen wollen wir uns bei der nun ohne weiteres möglichen Übertragung der Sätze 32, 1-3, auf allgemeine Potenzen mit der neuen Formulierung und ein paar Stichworten für den Beweis begnügen. Es gelten also die gegenüber $\mathbf{3 2}, \mathbf{1}-\mathbf{3}$ verallgemeinerten Sätze:

Satz 1. Ist $a>1$, so ist dann und nur dann gleichzeitig $a^{\prime \prime}>1, \mathbf{3 5}$. wenn $\varrho>0$ ist. Ebenso ist bei (positivem) $a<1$ dann und nur dann gleichzeitig $a^{0}<1$, wenn $\varrho>0$ ist.

Denn nach $\mathbf{3 2}, 1$ ist z. B. bei $a>1$ dann und nur dann $a^{x_{n}}>1$, wenn $x_{n}>0$ ist.

Satz 2. Ist $a>0$ und $\varrho$ zwischen $\varrho^{\prime}$ und $\varrho^{\prime \prime}$ gelegen, so liegt auch $a^{\text {Q }}$ stets zwischen $a^{\varrho^{\prime}}$ und $a^{\varrho^{\prime \prime}}$. - Der Beweis ist genau derselbe wie unter $\mathbf{3 2}, 2$. Er liefert genauer den

Satz 2a. Ist $a>1$, so entspricht dem größeren Exponenten auch der größere Wert der Potenz; ist $a<1$, so liefert der größere Exponent die kleinere Potenz. Speziell: Ist $a \neq 1$, so liefern verschiedene Exponenten auch verschiedene Potenzen. - Und aus diesem Satz folgt wieder genau wie unter $\mathbf{3 2}, 3$ der abschließende

Satz 3. Ist $\left(\underline{o}_{n}\right)$ eine beliebige Nulljolge, so bilden auch die Zahlen

$$
x_{n}=a^{\varrho_{n}}-1
$$

eine Nullfolge. Ist $\left(\varrho_{n}\right)$ monoton, so ist es auch $\left(x_{n}\right)$.

Als eine spezielle Anwendung erwähnen wir noch den

Satz 4. Ist $\left(x_{n}\right)$ eine Nullfolge mit lauter positiven Gliedern, so ist fiir jedes positive a auch

$$
x_{n}^{\prime}=x_{n}{ }^{\alpha}
$$

Glied einer Nullfolge. _ So ist $\left(\frac{1}{n^{\alpha}}\right)$ für jedes $a>0$ eine Nullfolge.

Beweis. Ist $\varepsilon>0$ beliebig gegeben, so ist auch $\varepsilon^{\frac{1}{a}}$ eine positive Zahl. Nach Voraussetzung kann man also $n_{0}$ so bestimmen, daß für $n>n_{0}$ stets

$$
x_{n} \mid=x_{n}<\varepsilon^{\frac{1}{\alpha}}
$$

1) Als Stichprobe skizzieren wir den Beweis der ersten der drei Grundregeln: Ist $\varrho=\left(x_{n} \mid y_{n}\right)$ und $\varrho^{\prime}=\left(x_{n}{ }^{\prime} \mid y_{n}{ }^{\prime}\right)$, so ist nach $16 \varrho+\varrho^{\prime}=\left(x_{n}+x_{n}{ }^{\prime} \mid y_{n}+y_{n}{ }^{\prime}\right)$ und also - wir denken uns $a \geqq 1-$ :

$$
a^{\varrho}=\left(a^{x_{n}} \mid a^{y_{n}}\right), \quad a^{\varrho^{\prime}}=\left(a^{x_{n}^{\prime}}: a^{y_{n}^{\prime}}\right), \quad a^{\varrho+\varrho^{\prime}}=\left(a^{x_{n}+x_{n}^{\prime}}: a^{y_{n}+y_{n}^{\prime}}\right) .
$$

Da nun alle Intervallenden (als Potenzen mit rationalen Exponenten) positiv sind, so ist nach 18

$$
a^{o} \cdot a^{\varrho^{\prime}}=\left(a^{x_{n}} \cdot a^{x_{n}^{\prime}} \mid a^{y_{n}} \cdot a^{y_{n}^{\prime}}\right) \text {. }
$$

Weil aber für rationale Exponenten die erste der 3 Grundregeln schon als gültig erwiesen ist, so ist diese letzte Schachtelung derjenigen für $a \varrho+\varrho^{\prime}$ nicht nur gemäß 11 gleich, sondern sogar Glied für Glied mit ihr übereinstimmend. 
ist. Dann ist aber für $n>n_{0}$ nach $\mathbf{3 5}, 1$ auch

$$
x_{n}{ }^{a}=\left|x_{n}{ }^{\prime}\right|<\varepsilon
$$

womit schon alles bewiesen ist.

Durch diese Entwicklungen ist das Rechnen mit den allgemeinen Potenzen in seinen Grundzügen festgelegt.

\section{Logarithmen.}

Die Grundlage für die Definition der Logarithmen bildet der

36. Satz. Sind $a>0$ und $b>1$ reelle, sonst ganz beliebige Zahlen, so gibt es stets eine und nur eine reelle Zahl $\xi$, für die

$$
b^{\xi}=a
$$

ist.

Beweis. Daß es höchstens eine solche Zahl geben kann, folgt schon aus $\mathbf{3 5}, 2 \mathrm{a}$, weil die Basis $b$ mit verschiedenen Exponenten potenziert nicht denselben Wert $a$ ergeben kann. Und $d a \beta$ es eine solche Zahl gibt, beweisen wir wieder konstruktiv durch Angabe einer sie bestimmenden Intervallschachtelung, - etwa durch 10-Teilung: Da $b>1$ ist, so ist $\left(b^{-n}\right)=\left(\frac{1}{b^{n}}\right)$ nach 10,7 eine Nullfolge, und es gibt also, weil $a$ und $\frac{1}{a}$ positiv sind, je eine natürliche Zahl $p$ und $q$, für die

$$
b^{-p}<a \quad \text { und } \quad b^{-q}<\frac{1}{a} \text {, oder } b^{q}>a
$$

ist. Geht man nun die ganzen Zahlen von $-p$ bis $+q$ durch, indem man sie sich in den Exponenten von $b$ gesetzt denkt, so muß es unter ihnen eine, und kann es nur eine geben - sie heiße $g$ für die

$$
b^{g} \leqq a, \text { aber } \quad b^{g+1}>a
$$

ist. Das hierdurch bestimmte Intervall $J_{0}=g \ldots(g+1)$ teilen wir in 10 gleiche Teile und gelangen ganz entsprechend wie S. 47/48 zu einer „Ziffer“ $z_{\boldsymbol{1}}$, für die

$$
b^{g+\frac{z_{1}}{10}} \leqq a, \text { aber } b^{g+\frac{z_{1}+1}{10}}>a
$$

ist, - und gelangen durch Wiederholung des Teilungsverfahrens zu einer ganz bestimmten Intervallschachtelung

$$
\xi=\left(x_{n} \mid y_{n}\right) \quad \text { mit }\left\{\begin{array}{l}
x_{n}=g+\frac{z_{1}}{10}+\ldots+\frac{z_{n-1}}{10^{n}-1}+\frac{z_{n}}{10^{n}} \\
y_{n}=g+\frac{z_{1}}{10}+\ldots+\frac{z_{n-1}}{10^{n-1}}+\frac{z_{n}+1}{10^{n}}
\end{array}\right.
$$

für die stets

$$
b^{x_{n}} \leqq a<b^{y_{n}}
$$


ist, für die also gemäß $\mathbf{3 3}$

$$
b^{\xi}=a
$$

ist. - Dieser Satz gibt uns die Berechtigung zu folgender durch

Definition: Sind $a>0$ und $b>1$ beliebig gegeben, so heißt die

$$
b^{\xi}=a
$$

eindeutig bestimmte reelle Zahl $\xi$ der zur Basis $b$ genommene Logarithmus von a; in Zeichen

$$
\boldsymbol{\xi}={ }^{b} \log \boldsymbol{a} \text {. }
$$

(g nennt man wohl auch die Kennziffer, die Gesamtheit der Ziffern $z_{1} z_{2} z_{3} \ldots$ die Mantisse des Logarithmus.)

Von einem Logarithmensystem spricht man, wenn man die Basis $b$ ein für allemal festgelegt denkt und nun die Logarithmen aller möglichen positiven Zahlen $a$ zu dieser Basis $b$ sich genommen denkt. Man läßt dann das $b$ bei ${ }^{b} \log$, weil überflüssig, gewöhnlich fort. Sehr bald wird sich als Basis für alle theoretischen Betrachtungen ganz naturgemäß eine bestimmte, gewöhnlich mit $e$ bezeichnete reelle Zahl empfehlen; das auf dieser Basis aufgebaute Logarithmensystem wird dann das natürliche genannt. Für praktische Zwecke dagegen ist bekanntlich die Basis 10 am bequemsten; die auf ihr aufgebauten Logarithmen werden die Briggschen genannt. Es sind die Logarithmen, die in den gewöhnlichen Tafeln zu finden $\operatorname{sind}^{1}$ ).

Die Regeln für das Rechnen mit Logarithmen setzen wir, wie bei den Potenzen, wieder als bekannt voraus und begnügen uns mit der bloßen Angabe der wichtigsten. Ist die Basis $b>1$ beliebig, aber im folgenden festliegend gedacht, und bedeuten $a, a^{\prime}, a^{\prime \prime} \ldots$ irgendwelche positive Zahlen, so ist

1. $\log \left(a^{\prime} a^{\prime \prime}\right)=\log a^{\prime}+\log a^{\prime \prime}$.

2. $\log 1=0 ; \quad \log \frac{1}{a}=-\log a ; \quad \log b=1$.

3. $\log a^{\varrho}=\varrho \log a \quad(\varrho$ beliebig, reell).

4. $\log a \lesseqgtr \log a^{\prime}$, je nachdem $a \lesseqgtr a^{\prime}$ ist; speziell:

5. $\log a \gtreqless 0$, je nachdem $a \gtreqless 1$.

6. Sind $b$ und $b_{1}$ zwei verschiedene Basen $(>1)$ und sind $\xi$ und $\xi_{1}$ die Logarithmen derselben Zahl $a$ zu diesen beiden Basen, also

$$
\xi={ }^{b} \log a, \quad \xi_{1}={ }^{b} \log a,
$$

1) Selbstverständlich kann man auch auf einer Basis $b$, die kleiner ist als 1, ein Logarithmensystem aufbauen. Doch ist das wenig üblich. Übrigens waren die ersten von Napier 1614 berechneten Logarithmen auf einer Basis $b<1$ aufgebaut, was besonders für die Logarithmen der trigonometrischen Funktionen kleine Vorteile bietet. 
so ist

$$
\xi=\xi_{1} \cdot{ }^{b} \log b_{1}
$$

— wie dies sofort aus $(a=) b^{\xi}=b_{1} \xi_{1}$ folgt, wenn man beiderseits die Logarithmen zur Basis $b$ nimmt und $\mathbf{3 7}, 2$ und 3 beachtet.

7. $\left(\begin{array}{c}1 \\ \log n\end{array}\right), n=2,3,4, \ldots$, ist eine Nullfolge. In der Tat ist $\frac{1}{\log n}<\varepsilon$, sobald $\log n>\frac{1}{\varepsilon}$ oder also $n>b^{\frac{1}{\varepsilon}}$ ist.

VI. Die Kreisfunktionen. Eine ebenso strenge, d. h. die geometrische Anschauung als Beweisgrund prinzipiell vermeidende und lediglich auf den Begriff der reellen Zahl gegründete Einführung der sogenannten Kreisfunktionen, also des sin eines gegebenen Winkels ${ }^{1}$ ), seines cos, tg, ctg usw. ist an dieser Stelle noch nicht möglich. IVir werden später $(\S 24)$ darauf zurückkommen. Trotzdem aber wollen wir diese Kreisfunktionen zur Bereicherung der Anwendungen und zur Belebung der Beispiele (aber selbstverständlich niemals zu Beweisen allgemeiner Sätze) unbedenklich heranziehen, soweit ihre Kenntnis aus den Elementen als bekannt angesehen werden kann.

So stellt man z. B. sofort die beiden einfachen Tatsachen fest:

38. 1. Sind $\alpha_{1}, \alpha_{2}, \ldots, \alpha_{n}, \ldots$ irgendwelche Winkel (d. h. also: irgendwelche Zalılen), so sind

$$
\left(\sin \alpha_{n}\right) \text { und }\left(\cos \alpha_{n}\right)
$$

beschränkte Zahlenfolgen; und

2. die Folgen

$$
\left(\frac{\sin \alpha_{n}}{n}\right) \text { und }\left(\frac{\cos \alpha_{n}}{n}\right)
$$

sind (nach :6) Nullfolgen, denn ihre Glieder entstehen aus denen der Nullfolge $\left(\frac{1}{n}\right)$ durch Multiplikation mit beschränkten Faktoren.

Als weitere Anwendung der nun festgelegten Begriffe wollen wir noch eine Anzahl spezieller Zahlenfolgen untersuchen:

3. Ist $\mid a^{\prime}<1$, so ist neben ( $\left.\boldsymbol{\iota}^{\prime \prime}\right)$ sogar $\left(\boldsymbol{n} \boldsymbol{(}^{n}\right)$ eine Nullfolge.

1) Die Winkel werden wir im allgemeinen nach dem sog. Bogenmaß, messen. Denkt man sich in einem Kreise vom Radius 1 den Radius von einer bestimmten Anfangslage aus gedreht, so mifst man den Drehungswinkel durch die Mafzahl des Weges, den dabei der Endpunkt des beweglichen Radius zurückgelegt hat, - und zwar positiv, wenn die Drehung im Gegensinne des Uhrzeigers geschieht, andernfalls negativ. - Ein Winkel ist also hiernach eine reine $Z a h 1$; ein gestreckter Winkel hat die Mafzzahl $+\pi$ oder $-\pi$, ein rechter Winkel die Mafszahl $+\frac{\pi}{2}$ oder $-\frac{\pi}{2}$. Allgemein kommen jedem bestimmt orientierten Winkel unendlich viele Mafzahlen $z \mathrm{u}$, die sich voneinander aber nur um ganzzahlige Vielfache von $2 \pi$, also um volle Drehungen, voneinander unterscheiden. Die Maßzzahl 1 kommt dem Winkel zu, dessen Bogen gleich dem Radius ist, der also im Gradmafs etwa $=57^{\circ} 17^{\prime} 44^{\prime \prime}, 8$ ist. 
Beweis. Ähnlich wie unter 10,7 schliefsen wir so $\left.{ }^{1}\right)$ : Für $a=0$ ist die Behauptung trivial; für $0<|a|<1$ kann

$$
a \mid=\frac{1}{1+\varrho}, \quad \text { also } \quad a^{n}=\frac{1}{1+\left(\begin{array}{l}
n \\
1
\end{array}\right) \varrho+\ldots+\left(\begin{array}{l}
n \\
n
\end{array}\right) \varrho^{n}}
$$

mit $\varrho>0$ gesetzt werden. Da hier im Nenner alle Summanden positiv sind, ist für $n>1$

$$
\left|a^{n}\right|<\frac{1}{\left(\begin{array}{l}
n \\
2
\end{array}\right) \varrho^{2}}, \text { also }\left|n a^{n}\right|<\frac{1 \cdot 2}{(n-1) \varrho^{2}}
$$

und es wird also

ist, d. h. fuir alle

$$
\left|n a^{n}\right|<\varepsilon \text { sein, sobald } \frac{1 \cdot 2}{(n-1) \varrho^{2}}<\varepsilon
$$

$$
n>1+\frac{2}{\varepsilon \cdot \varrho^{2}} .
$$

Das somit bewiesene Resultat ist sehr bemerkenswert: Es besagt doch, dafi für große $n$ der Bruch $\frac{n}{(1+\varrho)^{n}}$ sehr klein, also sein Nenner selır vielmal größer ist als der Zähler. Dieser Nenner ist aber für $\varrho=0$ konstant $=1$, und wenn $\varrho$ eine sehr kleine positive Zahl ist, so wächst er sehr langsam mit $n$. Unser Ergebnis aber zeigt, daf, wenn nur $n$ hinveichend grof genommen wird, der Nenner doch vielmal größer ist als der Zähler. Die Stelle $u_{0}$, von der an $\left|n a^{n}\right|=\frac{n}{(1+\varrho)^{n}}$ unterhalb eines gegebenen $\varepsilon$ liegt - es hatte sich $n_{0}=1+\frac{2}{\varepsilon \cdot Q^{2}}$ ergeben - liegt ja in der Tat sehr weit rechts, nicht nur wenn $\varepsilon$, sondern auch wenn $\varrho=\frac{1}{|a|}-1$ sehr klein (d. h. $|a|$ sehr nahe an 1 gelegen) ist. Wesentlich ist und bleibt nur: Wie auch $|a|<1$ und $\varepsilon>0$ gegeben sein mögen, stets ist von einer leicht angebbaren Stelle an sicher $\left.\left|n a^{n}\right|<\varepsilon^{2}\right)$.

Aus diesem Ergebnis kann man viele andere, z. B. die noch paradoxere Tatsache herleiten:

4. Ist $|a|<1$ und $\alpha>0$, so ist auch $\left(\boldsymbol{n}^{\alpha} \boldsymbol{a}^{\boldsymbol{n}}\right)$ eine Nullfolge.

Beweis. Setzt man $|a|^{\frac{1}{\alpha}}=a_{1}$, so ist nach $\mathbf{3 5}, 1$ auch die positive Zahl $a_{1}<1$. Nach dem vorigen Ergebnis ist also $\left(n a_{1}{ }^{n}\right)$ eine Nullfolge. Nach $\mathbf{3 5}, 4$ ist dann auch

$$
\left[n a_{1}^{n}\right]^{\alpha}, \text { d. h. } n^{\alpha} \mid a_{1}^{n} \text { oder }\left|n^{\alpha} a^{n}\right|
$$

also schliefilich (nach 10,5) auch $n^{\alpha} a^{n}$ selber Glied einer Nullfolge.

1) Nur dafi $a$ und $\varrho$ jetzt nicht rational zu sein brauchen.

$\left.{ }^{2}\right)$ Indem man wie oben $|a|=\frac{1}{1+\varrho},\left|n a^{n}\right|=\frac{n}{(1+\varrho)^{n}}$ setzt, kann man auch sagen: $(1+\varrho)^{n}$ wird - bei positivem $\varrho-$ stärker gro $\beta$ oder auch stärker unendlich groß als $n$ selbst, - womit wieder (vgl., , 3) nicht mehr und nicht weniger gesagt werden soll, als dafis unsere Folge eben eine Nullfolge ist. - Für später sei noch bemerkt, dafi die in 3 und 4 bewiesenen Ergebnisse auch für komplexe a gelten, wenn nur $|a|<1$ ist. 
(Wenn man sich $\alpha$ sehr grof gegeben denkt und $|a|$ nur sehr wenig unterhalb 1, so ist dieses Resultat natiirlich noch merkwïrdiger als das vorige ${ }^{1}$ ).)

5. Ist $\sigma>0$, so ist $\left(\frac{\log n}{n^{\sigma}}\right)$ eine Nullfolge $\left.{ }^{2}\right)$, zu welcher Basis $b>1$ die Logarithmen auch genommen sein mögen.

Beweis. Wegen $b>1, \sigma>0$ ist (nach $\mathbf{3 5}, 1$ ) auch $b^{\sigma}>1$. Also ist $\left(\frac{n}{\left(b^{\sigma}\right)^{n}}\right)$ nach 3. eine Nullfolge. Bei gegebenem $\varepsilon>0$ ist daher von einer Stelle an - etwa für alle $n>m$ - stets

Nun ist aber jedenfalls

$$
\frac{n}{\left(b^{\sigma}\right)^{n}}<\varepsilon^{\prime}=\frac{\varepsilon}{b^{\sigma}} \text {. }
$$

$$
\frac{\log n}{n^{\sigma}}<\frac{g+1}{\left(b^{g}\right)^{\sigma}}=b^{\sigma} \cdot \frac{g+1}{\left(b^{\sigma}\right)^{g+1}},
$$

wenn $g$ die Kennziffer von $\log n$ bedeutet (so dafi $g \leq \log n<g+1$ ist). Wird also $n>b^{m}$ genommen, so ist $\log n$ und also erst recht $g+1>m$, und folglich der letzte Wert gemäß der Wahl von $m$

$$
<b^{\sigma} \cdot \frac{\varepsilon}{b^{\sigma}}=\varepsilon, \quad \text { d. h. es ist } \quad \frac{\log n}{n^{\sigma}}<\varepsilon \quad \text { für } \quad n>b^{m} .
$$

6. Sind $\alpha$ und $\beta$ beliebige positive Zahlen, so ist

$$
\left(\frac{\log ^{\alpha} n}{n^{\beta}}\right)
$$

eine Nullfolge -, mag $\alpha$ noch so grofs und $\beta$ noch so klein $\operatorname{sein}^{3}$ ).

Beweis. Nach 5 . ist $\left(\frac{\log n}{n^{\beta: \alpha}}\right)$ wegen $\frac{\beta}{\alpha}>0$ eine Nullfolge; nach $\mathbf{3 5}, 4$ also auch die vorgelegte Folge.

7. $\left(\boldsymbol{x}_{n}\right) \equiv(\sqrt[n]{\boldsymbol{n}}-\mathbf{1})$ ist eine Nullfolge. (Auch dieses Resultat ist sehr merkwürdig. Denn für grobes $n$ ist auch der Radikand eine grołe Zahl; dafür allerdings auch der Wurzelexponent. Und es ist von vornherein nicht zu sehen, wer von beiden - Radikand oder Exponent - sozusagen der stärkere ist.)

Beweis. Es ist für $n>1$ jedenfalls $\sqrt[n]{n}>1$, also $x_{n}=\sqrt[n]{n}-1$ sicher $>0$. Daher sind in

$$
n=\left(1+x_{n}\right)^{n}=1+\left(\begin{array}{c}
n \\
1
\end{array}\right) x_{n}+\ldots+\left(\begin{array}{l}
n \\
n
\end{array}\right) x_{n}{ }^{\prime \prime}
$$

alle Summanden positiv. Folglich ist speziell

$$
n>\left(\begin{array}{l}
n \\
2
\end{array}\right) x_{n}{ }^{2}=\frac{n(n-1)}{1 \cdot 2} x_{n}{ }^{2}
$$

1) In entsprechender Umschreibung wie vorhin sagt man hier: $(1+\varrho)^{n}$ wird stäker unendlich gro $\beta$ als jede noch so grofe (feste) Potenz von $n$ selbst!

$\left.{ }^{2}\right) " \log n$ wird schwächer gro $\beta$ als jede noch so kleine (aber feste und positive) Potenz von $n$ selber."

3) "Jede noch so grofe (feste) Potenz von $\log n$ wird schwächer grof als jede noch so kleine (feste) Potenz von $n$ selber." 
oder

Hieraus folgt

$$
\left.x_{n}^{2}<\frac{2}{n-1} \leqq \frac{2}{n-\frac{n}{2}}=\frac{4}{n} \quad 1\right) \text {. }
$$

$$
\left|x_{n}\right|<\frac{2}{n^{\frac{1}{2}}},
$$

so daß $\left(x_{n}\right)=(\sqrt[n]{n}-1)$ nach $\mathbf{2 6}, 3$ und $\mathbf{3 5}, 4$ in der Tat eine Nullfolge ist.

8. Ist $\left(x_{n}\right)$ eine Nullfolge, deren Glieder sämtlich $>-1$ sind, so bilden auch die Zahlen

eine Nullfolge ${ }^{2}$ ).

$$
x_{n}^{\prime}=\sqrt[k]{1}+x_{n}-1
$$

Beweis. Nach der S. 20, Fufn. 3, erwähnten Formel folgt, wenn darin $a=\sqrt[k]{1+x_{n}}$ und $b=1$ gesetzt wird, daf

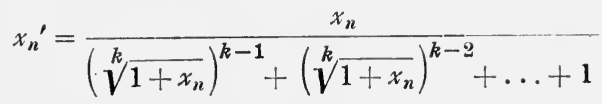

also, da im Nenner alles positiv, und der letzte Summand $=1$ ist:

$$
\left|x_{n}^{\prime}\right| \leqq\left|x_{n}\right|
$$

woraus nach $\mathbf{2 6}$ sofort die Behauptung folgt.

9. Ist $\left(x_{n}\right)$ eine Nullfolge von derselben Art wie in 8., so bilden auch die Zahlen

eine Nullfolge.

$$
y_{n}=\log \left(1+x_{n}\right)
$$

Beweis. Ist $b>1$ die Basis der Logarithmen und $\varepsilon>0$ gegeben, so setzen wir

$$
b^{\varepsilon}-1=\varepsilon_{1}, \quad 1-b^{-\varepsilon}=\varepsilon_{2}
$$

und die kleinere der beiden (eo ipso positiven) Zahlen $\varepsilon_{1}$ und $\varepsilon_{2}$ gleich $\varepsilon^{\prime}$. Nun wählen wir $n_{0}$ so grof 3 , daf für $n>n_{0}$ stets $\left|x_{n}\right|<\varepsilon^{\prime}$ ist. Dann ist für diese $n$ erst recht

$$
-\varepsilon_{2}<x_{n}<\varepsilon_{1}, \quad \text { d. h. } \quad b^{-\varepsilon}<1+x_{n}<b^{+\varepsilon},
$$

also (nach $\mathbf{3 5}, 2$ oder $\mathbf{3 7}, 4$ )

womit alles bewiesen ist.

$$
\left|y_{n}\right|=\left|\log \left(1+x_{n}\right)\right|<\varepsilon,
$$

10. Ist $\left(x_{n}\right)$ nochmals eine Nullfolge wie in 8 ., so bilden auch die Zahlen

$$
\approx_{n}=\left(1+x_{n}\right)^{\circ}-1
$$

eine Nullfolge, wenn $\varrho$ eine beliebige reelle Zahl bedeutet.

1) Daf3 man, sobald $n>2$, für $n-1$ verkleinernd $n-\frac{n}{2}$ schreibt, ist ein oft nützlicher Kunstgriff, um die Rechnung zu vereinfachen.

2) Durch die Voraussetzung, dafs alle $x_{n}>-1$ sind, soll nur bewirkt werden, dafs die Zahlen $x_{n}^{\prime}$ für alle $n$ definiert sind. Von einer gewissen Stelle an ist dies eo ipso der Fall, da $\left(x_{n}\right)$ eine Nullfolge sein soll und also von einer Stelle an sicher $\left|x_{n}\right|<1$, mithin $x_{n}>-1$ sein muf. 
Beweis. Nach 9. und $\mathbf{2 6}, 3$ bilden die Zahlen

$$
\varrho_{n}=\underline{o} \cdot \log \left(1+x_{n}\right)
$$

eine Nullfolge. Nach $\mathbf{3 5}, 3$ gilt dann dasselbe von den Zahlen

$$
b^{2} n-1=\left(1+x_{n}\right)^{e}-1=z_{n},
$$

w. z. b. w.

\section{§ 8. Konvergente Zahlenfolgen.}

\section{Definitionen.}

Bei der Betrachtung des Verhaltens einer vorgelegten Zahlenfolge haben wir bisher in der Hauptsache darauf geachtet, ob sie eine Nullfolge ist oder nicht. Indem wir diesen Gesichtspunkt in naheliegender Weise ein wenig erweitern, gelangen wir zu dem wichtigsten Begriff, mit dem wir uns überhaupt zu beschäftigen haben, nämlich dem der Konvergenz einer Zahlenfolge.

Die Eigenschaft einer Zahlenfolge $\left(x_{n}\right)$, Nullfolge zu sein, haben wir (vgl. 10,10) schon dahin beschrieben, daß wir sagten: Ihre Glieder werden klein, werden beliebig klein mit wachsendem $n$. Wir könnten auch sagen: Ihre Glieder nähern sich mit wachsendem $n$ dem Werte 0 - allerdings ohne ihn im allgemeinen jemals zu erreichen; aber sie nähern sich diesem Werte in dem Sinn beliebig, als die Beträge der Glieder (also doch ihre Abstände von 0 ) unter jede noch so kleine Zahl $\varepsilon(>0)$ herabsinken. Ersetzen wir bei dieser Auffassung den Wert 0 durch irgendeine andere reelle Zahl $\xi$, so würde es sich um eine Zahlenfolge $\left(x_{n}\right)$ handeln, bei der die Abstände der Glieder von der bestimmten Zahl $\xi$ - also nach $\mathbf{3 , I I , 6}$. die Werte $\left|x_{n}-\boldsymbol{\xi}\right|-$ mit wachsendem $n$ unter jede (noch so kleine) Zahl $\varepsilon>0$ herabsinken. Wir präzisieren diesen Sachverhalt zu folgender

39. Definition. Ist $\left(x_{n}\right)$ eine vorgelegte Zahlenfolge und steht sie zu einer bestimmten Zahl $\xi$ in der Beziehung, daß

$$
\left.\left(x_{n}-\xi\right){ }^{1}\right)
$$

eine Nullfolge bildet, so sagt man, die Folge $\left(x_{n}\right)$ koncergiere gegen $\xi$, oder sie sei konvergent mit dem Grenzwerte $\xi$, oder sie (bzw. ihre Glieder) näherten sich dem (Grenz-) Werte $\xi$, sie strebten gegen $\xi$, hätten den Limes $\xi$. Und man drïckt diese Tatsache durch die Symbole

$$
\boldsymbol{x}_{n} \rightarrow \quad \text { oder } \quad \lim \boldsymbol{x}_{n}=\boldsymbol{\xi}
$$

aus. Man schreibt auch, um deutlicher zu machen, daß die Annäherung an $\xi$ dadurch vor sich geht, daß der Index $n$ immer größer und größer genommen wird, vielfach

$$
\left.x_{n \rightarrow \xi} \text { für } n \rightarrow \infty \quad \text { oder } \quad \lim _{n \rightarrow \infty} x_{n}=\xi 2\right)
$$

1) Oder, was nach 10,5 auf genau dasselbe hinausläuft $\left(\xi-x_{n}\right)$ oder $x_{n}-\xi$.

2) Sprich: " $x_{n}$ (strebt) gegen $\xi$ für $n$ gegen $\infty$ " bzw. "Limes $x_{n}$ für $n$ gegen $\infty$ ist gleich $\xi "$. 
Indem wir die Definition der Nullfolge in die neue Definition mit hineinnehmen, können wir auch sagen:

Es strebt $x_{n} \rightarrow \xi$ für $n \rightarrow \infty$ (oder $\left.\lim _{n \rightarrow \infty}=\xi\right)$, wenn nach Wahl von $\varepsilon>0$ sich stets eine Zahl $n_{0}=n_{0}(\varepsilon)$ so angeben läßt, daß für alle $n>n_{0}$ immer

ausfällt.

$$
\left|x_{n}-\xi\right|<\varepsilon
$$

\section{Bemerkungen und Beispiele.}

1. Statt zu sagen $n\left(x_{n}\right)$ ist eine Nullfolge", können wir jetzt kürzer " $x_{n} \rightarrow 0^{*}$ schreiben. Die Nullfolgen sind konvergente Zahlenfolgen mit dem speziellen Grenzwerte 0.

2. Sinngemäßs gelten daher alle unter 10 gemachten Bemerkungen auch jetzt, da es sich ja nur um eine naheliegende Verallgemeinerung des Begriffs der Nullfolge handelt.

3. Nach 31, 3 und 38,7 strebt

$$
\sqrt[n]{a} \rightarrow 1 \quad \text { und } \quad \sqrt[n]{n} \rightarrow 1 \text {. }
$$

4. Ist $\left(x_{n} y_{n}\right)=\sigma$, so strebt $x_{n} \rightarrow \sigma$ und auch $y_{n} \rightarrow \sigma$. Denn es ist

$$
\left|x_{n}-\sigma\right| \text { und auch }\left|y_{n}-\sigma\right| \leqq y_{n}-x_{n} \mid
$$

so daf beide nach $\mathbf{2 6}, 2$ zugleich mit $\left(y_{n}-x_{n}\right)$ eine Nullfolge bilden.

5. Für $x_{n}=1-\frac{(-1)^{n}}{n}$, also für die Folge $2, \frac{1}{2}, \frac{4}{3}, \frac{3}{4}, \frac{6}{5}, \frac{5}{6}, \ldots$ strebt $x_{n} \rightarrow 1$, denn $\left|x_{n}-1\right|=\frac{1}{n}$ bildet eine Nullfolge.

6. Geometrisch gesprochen bedeutet $x_{n} \rightarrow \xi$, dafi alle Glieder mit hinreichend hohem Index in der Nähe des festen Punktes $\xi$ gelegen sind. Oder schärfer (vgl. 10,13), daf in jeder $\varepsilon$-Umgebung von $\xi$ alle Glieder mit höchstens endlich vielen Ausnahmen gelegen sind $\left.{ }^{1}\right)$. - Bei der Veranschaulichung $\boldsymbol{7}, 6$ ziehen wir durch die beiden Punkte $(0, \xi \pm \varepsilon)$ Parallelen zur Abszissenachse und können sagen: Es strebt $x_{n} \rightarrow \xi$, falls das ganze Bild der Folge $\left(x_{n}\right)$, von einem endlichen Anfangsstïck abgesehen, in jedem solchen (noch so schmalen) $\varepsilon$-Streifen gelegen ist.

7. Auf das entschiedenste $\mathrm{zu}$ verwerfen ist eine oft gehörte laxe Ausdrucksweise, die für $x_{n} \rightarrow \xi$ sagt "für $n=\infty$ sei $x_{n}=\xi^{\prime \prime}$. Denn eine natürliche Zahl $n=\infty$ gibt es nicht und $x_{n}$ braucht niemals $=\xi$ zu sein. Es handelt sich durchaus nur um einen, durch alles vorangehende nun wohl hinreichend geklärten Annäherungsprozef, von dem es gar keinen Zweck hat, sich ihn in irgendeiner Form vollendet $\mathrm{zu}$ denken. (In älteren Lehrbüchern und Abhandlungen findet man jedoch häufig die symbolische Schreibweise " $\lim _{n=\infty} x_{n}=\xi^{*}$, gegen die sich, weil sie ja nur symbolisch gemeint ist, nichts sagen läfit, - aufer eben, dafi sie ungeschickt ist und daf die Schreibweise "n= $n$ " notwendig Verwirrungen uiber den Begriff des Unendlichen in der Mathematik stiften muf.)

1) Vielfach sagt man dafuir auch kürzer: In jeder $\varepsilon$-Umgebung von $\xi$ sollen "fast alle" Glieder der Folge gelegen sein. Doch hat die Aussage "fast alle" auch noch andere Bedeutungen, z. B. in der Lehre von den Punktmengen. 
8. Strebt $x_{n} \rightarrow \xi$, so bezeichnet man die einzelnen Glieder der Folge $\left(x_{n}\right)$ auch wohl als Näherungswerte von $\xi$ und die Differen $z \xi-x_{n}$ als den Fehler, der dem Näherungswerte $x_{n}$ anhaftet.

9. Die Bezeichnung, "konvergent" scheint zuerst von J. Gregory (Vera circuli et hyperbolae quadratura, Padua, 1667), die Bezeichnung "divergent" (10) von Bernoulli (Brief an Leibniz vom 7.4.1713) gebraucht zu sein.

An die Definition von Konvergenz schließen wir sogleich diejenige der Divergenz:

10. Definition 1. Jede Zahlenfolge, die nicht im Sinne der Definition 39 konvergiert, heißt divergent.

Hiernach sind z. B. die Folgen (6, 2, 4, 7, 8, 11 sicher divergent.

Unter den divergenten Folgen zeichnet sich eine Art durch besondere Einfachheit und Durchsichtigkeit des Verhaltens aus, z. B. die Folgen $\left(n^{2}\right),(n),\left(a^{n}\right)$ mit $a>1$, $(\log n)$, u. a. Das ihnen Gemeinsame ist ersichtlich, daß die Glieder mit wachsendem $n$ jede noch so hohe Schranke übersteigen. Man sagt darum wohl auch, sie strebten nach $+\infty$, sie (bzw. ihre Glieder) würden unendlich groß. Wir präzisieren den Sachverhalt in folgender

Definition 2. Hat die Folge $\left(x_{n}\right)$ die Eigenschaft, daß nach Wahl einer beliebigen (großen) positiven Zahl G sich immer noch eine Zahl $n_{0}$ so angeben läßt, daß für alle $n>n_{0}$ stets

$$
x_{n}>G \text {, }
$$

(nicht $\mid x_{n}:>G$ )

so wollen wir sagen, $\left(x_{n}\right)$ divergiere gegen $+\infty$, strebe nach $+\infty$, oder sei bestimmt divergent mit dem Grenzwert $+\infty^{1}$ ); und wir schreiben dann

$x_{n} \rightarrow+\infty$ (für $\left.n \rightarrow \infty\right)$ oder $\lim x_{n}=+\infty$ bzw. $\lim _{n \rightarrow \infty} x_{n}=+\infty$.

Es bedeutet nur eine Vertauschung von rechts und links, wenn wir weiter definieren:

Definition 3. Hat die Folge $\left(x_{n}\right)$ die Eigenschaft, daß nach Wahl einer beliebigen (absolut großen) negativen Zahl $-G$ sich immer noch eine Zahl $n_{0}$ so angeben läßt, daß für alle $n>n_{0}$ stets

$$
x_{n}<-G
$$

ist, so wollen wir sagen, $\left(x_{n}\right)$ divergiere gegen - $\infty$, strebe nach - $\infty$ oder sei bestimmt divergent mit dem Grenzwert - $\infty$; und wir schreiben

$x_{n} \rightarrow-\infty \quad($ iür $n \rightarrow \infty)$ oder $\lim x_{n}=-\infty$ bzw. $\lim _{n \rightarrow \infty} x_{n}=-\infty$.

1) Manchmal sagt man sogar - in scheinbarer Verdrehung der Tatsachen -, die Folge konvergiere gegen $+\infty$. Es hat dies seinen Grund darin, daf das in Definition 2 beschriebene Verhalten in vieler Beziehung dem der Konvergenz (39) sehr nahe steht. Wir schliefen uns indessen dieser Ausdrucksweise $\mathrm{nicht}$ an, obwohl ein Mifsverständnis niemals zu befuirchten wäre. - Analog bei $-\infty$. 
Bemerkungen und Beispiele.

1. Die Folgen $(n),\left(n^{2}\right),\left(n^{\alpha}\right)$ für $\alpha>0,(\log n),\left(\log ^{\alpha} n\right)$ für $\alpha>0$ streben gegen $+\infty$, diejenigen, deren Glieder entgegengesetzte Werte haben, streben gegen $-\infty$.

2. Allgemein: Strebt $x_{n} \rightarrow+\infty$, so strebt $x_{n}{ }^{\prime}=-x_{n} \rightarrow-\infty$ und umgekehrt. - Darum genügt es weiterhin im wesentlichen, die Divergenz $\rightarrow+\infty$ zu betrachten.

3. In geometrischer Sprache bedeutet $x_{n} \rightarrow+\infty$ natürlich, daf, wie man auch (sehr weit rechts) einen Punkt $+G$ wählen möge, doch alle Punkte $x_{n}$, von höchstens endlich vielen Ausnahmen abgesehen, noch rechts von ihm gelegen sind. - Bei der Veranschaulichung $\mathbf{7 , 6}$ besagt es: Wie hoch oberhalb der Abszissenachse man auch eine Parallele zu ihr ziehen möge, es liegt doch das ganze Bild der Folge $\left(x_{n}\right)$ - von einem endlichen Anfangsstück abgesehen - noch oberhalb derselben.

4. Das Streben gegen $\pm \infty$ braucht kein monotones zu sein; so ist z. B. auch die Folge $1,2^{1}, 2,2^{2}, 3,2^{3}, 4,2^{4}, \ldots, k, 2^{k}, \ldots$ gegen $+\infty$ divergent.

5. Die Folge $1,-2,+3,-4, \ldots,(-1)^{n-1} n, \ldots$ ist weder nach $+\infty$ noch nach $-\infty$ divergent. - Dies veranlafit uns noch zu der

Definition 4: Von einer Folge $\left(x_{n}\right)$, die entweder im Sinne der Definition 39 konvergiert, oder im Sinne der Definition 40, 2 und 3 bestimmt divergiert, wollen wir sagen, daß sie sich $($ ür $n \rightarrow \infty)$ bestimmt verhält. Alle übrigen Folgen, die also weder konvergieren noch bestimmt divergieren, sollen unbestimmt divergent oder kurz unbestimmt heißen ${ }^{1}$.

Beispiele und Bemerkungen.

1. Die Folgen $\left[(-1)^{n}\right],\left[(-2)^{n}\right],\left(a^{n}\right)$ für $a \leqq-1$, ebenso die Folgen $0,1,0,2$, $0,3,0,4, \ldots$ und $0,-1,0,-2,0,-3, \ldots$, ferner die Folgen $\mathbf{6}, 4,8$ sind ersichtlich unbestimmt divergent.

2. Dagegen weist die Folge $\left(\left|a^{n}\right|\right)$ für beliebiges reelles $a$, ferner die Folgen $\left(3^{n}+(-2)^{n}\right),\left(n+(-1)^{n} \log n\right),\left(n^{2}+(-1)^{n} n\right)$, trotz aller Unregelmärigkeiten im einzelnen, doch ein bestimmtes Verhalten auf.

3. Die geometrische Deutung des unbestimmten Verhaltens ergibt sich unmittelbar daraus, dafs weder Konvergenz (s. 39, 6) noch bestimmte Divergenz (s. 40, 3, Bem. 3) stattfinden soll.

1) Es handelt sich also um drei typische Verhaltungsweisen einer Zahlenfolge, nämlich: a) Konvergenz gegen eine Zahl $\xi$, gemäf 39 ; b) Divergenz gegen $\pm \infty$; gemäß $\mathbf{1 0}, 2$ u. 3 ; c) keins von beiden. - Da das Verhalten b) manche Analogien mit a) und manche mit c) aufweist, schwankt hier der Sprachgebrauch. Man rechnet b) zwar allgemein zur Divergenz (die Ausdrucksweise, die in der vorigen Fufnnote erwähnt wurde, läßst sich nicht konsequent durchhalten), spricht aber andrerseits doch von den "Grenzwerten" $+\infty$ oder $-\infty$. - Wir sprechen also in den Fällen a) und b) von einem bestim m ten, im Falle c) von einem unbestimmten Verhalten; im Falle $a$ ), und nur in diesem, von Konvergenz, in den Fällen b) und c) von Divergenz. - Statt ,,bestimmt und unbestimmt divergent" sagt man auch „eigentlich und uneigentlich divergent". Da aber, wie betont, die bestimmte Divergenz noch viele Analogien mit der Konvergenz aufweist und bei ihr noch von einem Grenzwert gesprochen wird, erscheint es nicht ratsam, diesen Fall gerade als den der eigentlichen Divergenz zu bezeichnen. 
4. Aus $x_{n} \rightarrow+\infty$ und aus $x_{n} \rightarrow-\infty$ folgt, falls alle Glieder $\neq 0$ sind $^{\mathbf{1}}$ ), stets $\frac{1}{x_{n}} \rightarrow 0$; denn aus $\left|x_{n}\right|>G=\frac{1}{\varepsilon}$ folgt ja sofort $\left|\frac{1}{x_{n}}\right|<\varepsilon$. - Dagegen braucht aus $x_{n} \rightarrow 0$ sich kein bestimmtes Verhalten fuir $\left(\frac{1}{x_{n}}\right)$ zu ergeben. Beispiel: Für $x_{n}=\frac{(-1)^{n}}{n}$ strebt $x_{n} \rightarrow 0$, aber $\left(\frac{1}{x_{n}}\right)$ ist unbestimmt divergent. Dagegen gilt ersichtlich der leicht $z u$ beweisende

Satz: Ist $\left(x_{n}\right)$ eine Nullfolge, deven Glieder einerlei Vorzeichen haben, so ist die Folge $\left(\frac{1}{x_{n}}\right)$ bestimmt divergent; - und natürlich gegen $+\infty$ oder gegen $-\infty$, je nachdem alle $x_{n}$ positiv oder alle negativ sind.

5. Zur bequemeren Verständigung in häufig vorkommenden Fällen fuihren wir endlich noch die folgende Ausdrucksweise ein:

Definition 5. Stehen zwei Zahlenfolgen $\left(x_{n}\right)$ und $\left(y_{n}\right)$, die nicht zu konvergieren brauchen, zueinander in der Beziehung, daß der Quotient

$$
\frac{x_{n}}{y_{n}}
$$

fïr $n \rightarrow+\infty$ einem bestimmten endlichen und von 0 verschiedenen Grenzwert zustrebt ${ }^{2}$ ), so wollen wir sagen, beide Folgen seien asymptotisch proportional und schreiben dafür kurz

$$
x_{n} \sim y_{n} .
$$

Ist dieser Grenzwert speziell gleich 1, so nennen wir die Folgen asymptotisch gleich und schreiben prägnanter

So ist $z$. B.

$$
x_{n} \simeq y_{n} .
$$

$$
\begin{gathered}
\sqrt{n^{2}+1} \simeq n, \quad \log \left(5 n^{9}+23\right) \sim \log n, \quad \sqrt{n+1}-\sqrt{n} \sim \frac{1}{\sqrt{n}} \\
1+2+\ldots+n \sim n^{2}, 1^{2}+2^{2}+\ldots+n^{2} \simeq \frac{1}{3} n^{3} .
\end{gathered}
$$

Diese Bezeichnungsweisen ruhren im Prinzip von $P$. $d u$ Bois-Reymond her (Annali di matematica pura ed appl. (2) IV, S.338, 1870/71).

An diese Definitionen schließen wir nun sogleich eine Anzahl einfacher, aber durchaus grundlegender

\section{Sätze iiber konvergente Zahlenfolyen.}

11. Satz 1. Eine konvergente Zahlenfolge bestimmt ihren Grenzwert völlig eindeutig $\left.{ }^{3}\right)$.

$\left.{ }^{1}\right)$ Von einer Stelle an ist dies von selbst der Fall. .

2) Die $x_{n}$ und $y_{n}$ müssen dann von selbst von einer Stelle $a b \neq 0$ sein. Fïr alle $n$ soll dies durch die obige Definition nicht gefordert sein.

3) Durch eine konvergente Zahlenfolge ist also ihr Grenzwert ebenso eindeutig definiert (erfafit, bestimmt, gegeben,....), wie durch eine Intervallschachtelung oder einen Dedekindschen Schnitt die dort eingespannte Zahl. Daher können wir von nun an eine reelle Zahl auch als gegeben ansehen, wenn wir cine gegen sie konvergievende Zahlenfolge kennen. Und sagten wir früher kurz, eine Intervallschachtelung $\left(x_{n} \mid y_{n}\right)$ oder ein Dedekindscher Schnitt $(A B)$ oder ein Systembruch sei eine reelle Zahl, so können wir jetzt mit demselben Rechte sagen, eine gegen $\xi$ konvergierende Zahlenfolge $\left(x_{n}\right)$ sei die reelle Zahl $\xi$, in Zeichen: $\left(x_{n}\right)=\xi$. Näheres über diese Auffassung s. S. 74 und 90 . 
Beweis. Hat $\operatorname{man} x_{n} \rightarrow \xi$ und zugleich $x_{n} \rightarrow \xi^{\prime}$, so sind $\left(x_{n}-\xi\right)$ und $\left(x_{n}-\xi^{\prime}\right)$ Nullfolgen. Nach 28, 2 ist dann auch

$$
\left(\left(x_{n}-\xi\right)-\left(x_{n}-\xi^{\prime}\right)\right)=\left(\xi^{\prime}-\xi\right)
$$

eine Nullfolge, d. h. es ist $\xi=\xi^{\prime}$, w. z. b. w. $\left.{ }^{1}\right)$

-Satz 2. Eine konvergente Zahlenfolge $\left(x_{n}\right)$ ist stets beschränkt. Und ist stets $\left|x_{n}\right| \leqq K$, so gilt für den Grenzwert $\xi$, daß $|\xi| \leqq K$ ist $^{2}$ ).

Beweis. Strebt $x_{n} \rightarrow \xi$, so läßt sich nach Wahl von $\varepsilon>0$ die ganze Zahl $m$ so angeben, $\mathrm{da} B$ für $n>m$ stets

$$
\xi-\varepsilon<x_{n}<\xi+\varepsilon .
$$

Ist also $K$ eine Zahl, die die endlich vielen Werte $\left|x_{1}\right|,\left|x_{2}\right|, \ldots$, $\left|x_{m}\right|,|\xi-\varepsilon|$ und $|\xi+\varepsilon|$ übertrifft, so ist ersichtlich stets

$$
\left|x_{n}\right|<K \text {. }
$$

Und wäre nun $|\xi|>K$, so wäre $|\xi|-K>0$ und also von einer Stelle an

$$
|\xi|-\left|x_{n}\right| \leqq\left|x_{n}-\xi\right|<|\xi|-K,
$$

also $\left|x_{n}\right|>K$, entgegen der Bedeutung von $K$.

- Satz 3. Hat die konvergente Zahlenfolge $\left(x_{n}\right)$ lauter von 0 verschiedene Glieder und ist auch ihr Grenzwert $\xi \neq 0$, so ist auch die Folge $\left(\frac{1}{x_{n}}\right)$ beschränkt, oder: so gibt es eine Zahl $\gamma>0$, so daß stets $\left|x_{n}\right| \geqq \gamma>0$ ist; die Zahlen $\left|x_{n}\right|$ besitzen also eine noch positive untere Schranke:

Beweis: Nach Voraussetzung ist $\frac{1}{2}|\dot{\xi}|=\varepsilon>0$, und es gibt also eine ganze Zahl $m$, so daß für $n>m$ stets $\left|x_{n}-\xi\right|<\varepsilon$ und also $\left.\left|x_{n}\right|>\frac{1}{2} \mid \xi^{3}\right)$. Bezeichnet man die kleinste der endlich vielen positiven Zahlen $\left|x_{1}\right|,\left|x_{2}\right|, \ldots,\left|x_{m}\right|$ und $\frac{1}{2}|\xi|$ mit $\gamma$, so ist auch noch $\gamma>0$ und stets $\left|x_{n}\right| \geqq \gamma,\left|\frac{1}{x_{n}}\right| \leqq K=\frac{1}{\gamma}$, w. z. b.w.

1) Der hier zuletzt benutzte, im ersten Augenblick vielleicht verblüffende Schluf ist einfach der: Wenn man von einem bestimmten Zahlenwert $\alpha$ weifs, daß für jedes $\varepsilon>0$ stets $|\alpha|<\varepsilon$ ist, so muß notwendig $\alpha=0$ sein. Denn wäre $\alpha \neq 0$, so wäre $|\alpha|>0$. Wählt man für $\varepsilon$ nun die Zahl $\frac{1}{2}|\alpha|$, so ist doch nun die Beziehung $|\alpha|<\varepsilon$ gewif nicht richtig. Es mußs also doch $\alpha=0$ sein. Ebenso: weif man von einem bestimmten Zahlenwert $\alpha$, dafi für jedes $\varepsilon>0$ stets $a \leq K+\varepsilon$ ist, so mufs sogar die Ungleichung $a \leq K$ erfüllt sein. Das hier in Rede stehende Schlufverfahren "Ist fïr jedes $\varepsilon>0$ stets $|\alpha|<\varepsilon$, so ist notwendig $\alpha=0$ " ist genau das schon von den griechischen Mathematikern (vgl. Euklid, Elemente X) ständig benutzte und später als Exhaustionsmethode bezeichnete Verfahren.

$\left.{ }^{2}\right)$ Hier ist auf das Gleichheitszeichen in,$\xi \mid \leq K^{*}$ auch dann nicht $z u$ verzichten, wenn für alle $n$ stets $\left|x_{n}\right|<K$ ist.

$\left.{ }^{3}\right)$ Für $n>m$ sind also von selbst alle $x_{n} \neq 0$.

Knopp, Unendliche Reihen. 
Wendet man, wenn eine gegen $\xi$ konvergierende Zahlenfolge $\left(x_{n}\right)$ vorliegt, auf die Nullfolge $\left(x_{n}-\xi\right)$ die Sätze $\mathbf{2 7 , 1}$ bis 4 an, so hat man unmittelbar die Sätze:

○ Satz 4. Ist $\left(x_{n}{ }^{\prime}\right)$ eine Teilfolge von $\left(x_{n}\right)$, so folgt

$$
\text { aus } \quad x_{n} \rightarrow \xi \text { stets } x_{n}{ }^{\prime} \rightarrow \xi \text {. }
$$

- Satz 5. Läßt sich eine Folge $\left(x_{n}\right)$ so in zwei Teilfolgen ${ }^{1}$ zer. legen, daß jede derselben gegen $\xi$ konvergierl, so ist das auch mit $\left(x_{n}\right)$ selbst der Fall.

- Satz 6. Ist $\left(x_{n}{ }^{\prime}\right)$ eine beliebige Umordnung von $\left(x_{n}\right)$, so folgt

$$
\text { aus } \quad x_{n} \rightarrow \xi \text { stets } x_{n}{ }^{\prime} \rightarrow \xi \text {. }
$$

- Satz 7. Strebt $x_{n} \rightarrow \xi$ und entsteht $\left(x_{n}{ }^{\prime}\right)$ aus $\left(x_{n}\right)$ durch endlich viele Anderungen, so strebt aush $x_{n}{ }^{\prime} \rightarrow \xi$.

Das Rechnen endlich mit den konvergenten Zahlenfolgen wird durch die folgenden vier Sätze begründet:

○ Satz 8. Aus $x_{n} \rightarrow \xi$ und $y_{n} \rightarrow \eta$ folgt ste's $\left(x_{n}+y_{n}\right) \rightarrow \xi+\eta$, und das entsprechende gilt für die gliedweise Addition irgendeiner festen Anzahl - etwa $p$ - konvergenter Zahlenfolgen.

Beweis. Mit $\left(x_{n}-\xi\right)$ und $\left(y_{n}-\eta\right)$ ist nach 28,1 auch $\left(\left(x_{n}+y_{n}\right)-(\xi+\eta)\right)$ eine Nullfolge. - Ebenso folgt nach 28, 2 der

$\circ$ Satz 9. Aus $x_{n} \rightarrow \xi$ und $y_{n} \rightarrow \eta$ folgt stots $\left(x_{n}-y_{n}\right) \rightarrow \xi-\eta$.

$\circ$ Satz 10. Aus $x_{n} \rightarrow \xi$ und $y_{n} \rightarrow \eta$ folot stets $x_{n} y_{n} \rightarrow \xi \eta$ und das entsprechende gilt für die gliedweise Multiplikation irgendeiner festen Anzahl - etwa $p$ - konvergenter Zahlenfolgen.

Speziell: Aus $x_{n} \rightarrow \xi$ folgt stets $c x_{n} \rightarrow c \xi$, welche Zahl auch $c$ bedeuten möge.

Beweis. Es ist

$$
x_{n} y_{n}-\xi \eta=\left(x_{n}-\xi\right) y_{n}+\left(y_{n}-\eta\right) \xi
$$

und da hier rechterhand zwei Nullfolgen gliedweise mit beschränkten Faktoren multipliziert und dann addiert sind, so ist der Ausdruck selbst Glied einer Nullfolge, w. z. b. w.

○ Satz 11. Aus $x_{n} \rightarrow \xi$ und $y_{n} \rightarrow \eta$ folgt, falls alle $x_{n} \neq 0$ sind und auch $\xi \neq 0$ ist, stets

Beweis. Es ist

$$
\frac{y_{n}}{x_{n}} \rightarrow \frac{\eta}{\xi}
$$

$$
\frac{y_{n}}{x_{n}}-\frac{\eta}{\xi}=\frac{y_{n} \xi-x_{n} \eta}{x_{n} \cdot \xi}=\frac{\left(y_{n}-\eta\right) \xi-\left(x_{n}-\xi\right) \eta}{x_{n} \cdot \xi},
$$

1) Oder drei oder irgendeine bestimmte Anzahl. 
Hier steht im Zähler aus denselben Gründen wie soeben eine Nullfolge, und die Faktoren $\frac{1}{\xi \cdot x_{n}}$ sind nach Satz 3 beschränkt. Also ist der ganze Ausdruck wieder Glied einer Nullfolge. - Nur ein Spezialfall hiervon ist der

${ }^{\circ}$ Satz $11 \mathrm{a}$. Aus $x_{n} \rightarrow \xi$, folgt, falls alle $x_{n}$ und auch $\xi$ von 0 verschieden sind, daß stets

strebt ${ }^{1}$.

$$
\frac{1}{x_{n}} \rightarrow \frac{1}{\xi}
$$

Diese grundlegenden Sätze 8-11 führen nach wiederholter Anwendung zu dem folgenden umfassenderen

- Satz 12. Es sei $R=R\left(x^{(1)}, x^{(2)}, x^{(3)}, \ldots, x^{(p)}\right)$ ein Ausdruck, der durch endlich viele Additionen, Subtraktionen, Multiplikationen und Divisionen aus den Buchstaben $x^{(1)}, x^{(2)}, \ldots, x^{(p)}$ unter Hinzunahme beliebiger Zahlenkoeffizienten gebildet ist ${ }^{2}$ ) und es seien

$$
\left(x_{n}^{(1)}\right), \quad\left(x_{n}^{(2)}\right), \quad \ldots, \quad\left(x_{n}^{(p)}\right)
$$

p gegebene Zahlenfolgen, die der Reihe nach gegen $\xi^{(1)}, \xi^{(2)}, \ldots, \xi^{(p)}$, kon. vergieren. Dann strebt die Folge der Zahlen

$$
R_{n}=R\left(x_{n}^{(1)}, x_{n}^{(2)}, \ldots, x_{n}^{(p)}\right) \rightarrow R\left(\xi^{(1)}, \xi^{(2)}, \ldots, \xi^{(p)}\right),
$$

falls weder bei der Berechnung der Glieder $R_{n}$ noch der des Wertes $R\left(\xi^{(1)}, \xi^{(2)}, \ldots, \xi^{(p)}\right)$ die Division durch 0 verlangt wird.

Auf Grund dieser Sätze beherrschen wir die formale Handhabung konvergenter Zahlenfolgen. Wir geben noch einige weitere

\section{Beispiele.}

1. Aus $x_{n} \rightarrow \xi$ folgt bei $a>0$ stets

Denn

$$
\boldsymbol{a}^{x} \rightarrow \boldsymbol{a}^{\xi} \text {. }
$$

$$
a^{x_{n}}-a^{\xi}=a^{\xi}\left(a^{x_{n}-\xi}-1\right)
$$

ist nach 35, 3 eine Nullfolge.

2. Aus $x_{n} \rightarrow \xi$ folgt, falls alle $x_{n}$ und auch $\xi>0$ sind, dafs auch

strebt.

$\log x_{n} \rightarrow \log \xi$

1) Bei den Sätzen 3, 11 und $11 \mathrm{a}$ ist an den Voraussetzungen nur wesentlich, daf der in die Nenner tretende Grenzwert $\neq 0$ ist, denn dann sind von einer Stelle ab die im Nenner stehenden Glieder von selbst $\neq 0$, und man brauchte nur ,endlich viele Änderungen" vorzunehmen, damit dies stets der Fall ist.

2) Kürzer: eine rationale Funktion der $p$ Variablen $x^{(1)}, x^{(2)}, \ldots, x^{(p)}$ mit beliebigen Zahlenkoeffizienten. 
Beweis. Es ist

$$
\log x_{n}-\log \xi=\log \frac{x_{n}}{\xi}=\log \left(1+\frac{x_{n}-\xi}{\xi}\right),
$$

was nach 38,9 eine Nullfolge ist, da mit $x_{n}>0$ auch $\frac{x_{n}-\xi}{\xi}>-1$ ist.

3. Unter denselben Voraussetzungen wie bei 2. strebt anch

bei beliebigem reellem $\varrho$.

$$
x_{n}^{\varrho} \rightarrow \xi^{\prime}
$$

Beweis. Es ist

$$
x_{n}^{\varrho}-\xi^{\varrho}=\xi^{\varrho}\left(\frac{x_{n}^{\varrho}}{\xi^{\varrho}}-1\right)=\xi^{\varrho}\left[\left(1+\frac{x_{n}-\xi}{\xi}\right)^{\varrho}-1\right] \text {, }
$$

was nach 3S, 10 eine Nullfolge ist, da $\frac{x_{n}-\xi}{\xi}>-1$ ist und fuir $n \rightarrow \infty$ gegen 0 strebt $^{1}$ ). (Hierzu bildet noch $\mathbf{3 5}, 4$ eine gewisse Ergänzung.)

4. Strebt $x_{n}^{\prime \prime} \rightarrow \xi$ und ebenso $x_{n}^{\prime \prime} \rightarrow \xi$ und steht die Folge $\left(x_{n}\right)$ zu den Folgen $\left(x_{n}{ }^{\prime}\right)$ und $\left(x_{n}{ }^{\prime \prime}\right)$ in der Beziehung, da $\beta$ von einer Stelle $m$ an stets

ist, so strebt auch $x_{n} \rightarrow \xi$.

$$
x_{n}^{\prime} \leq x_{n} \leq x_{n}{ }^{\prime \prime}
$$

Beweis. Nach Wahl von $\varepsilon>0$ kann $n_{0}>m$ so gewählt werden, daf für $n>n_{0}$ stets $\xi-\varepsilon<x_{n}{ }^{\prime}$ und $x_{n}{ }^{\prime \prime}<\xi+\varepsilon$ bleibt. Für diese $n$ ist dann von selbst $\xi-\varepsilon<x_{n}<\xi+\varepsilon$, d. h. $\left|x_{n}-\xi\right|<\varepsilon$, w. z. b. w.

\section{Der Cauchysche Grenzwertsatz und seine Verallgemeinerungen.}

Wesentlich tiefer und weiterhin von großer Bedeutung ist eine Gruppe von Grenzwertsätzen ${ }^{2}$ ), die in ihrer einfachsten Form von Cauch $y^{3}$ ) herrühren und in neuerer Zeit nach verschiedenen Richtungen hin erweitert wurden. Es gilt zunächst der einfache

43.

- Satz 1. Ist $\left(x_{0}, x_{1}, \ldots\right)$ eine Nullfolge, so bilden auch die arithmetischen Mittel

$$
x_{n}{ }^{\prime}=\frac{x_{0}+x_{1}+\ldots-x_{n}}{n+1}, \quad n=-=0,1,2, \ldots,
$$

eine Nullfolge.

Beweis. Wird $\varepsilon>0$ gegeben, so kann $\operatorname{man}^{\prime} m$ so wählen, daß für $n>m$ stets $\left|x_{n}\right|<\frac{\varepsilon}{2}$ ausfällt. Dann ist für diese $n$

$$
\left|x_{n}^{\prime}\right| \leqq \frac{\mid x_{0}+x_{1}+\cdots+x_{m}}{n+1}+\frac{*}{2} \frac{n-m}{n+1} \text {. }
$$

1) Die Bedeutung der Beispiele 1. bis 3. ist - in die Sprache der Funktionenlehre übertragen - die, daf die Funktion $a^{x}$ an jeder Stelle, die Funktionen $\log x$ und $x^{2}$ an jeder positiven Stelle stetig sind.

2) Das Studium dieser Sätze kảnn verschoben werden, bis in den späteren Kapiteln von ihnen Gebrauch gemacht wird. (Zuerst im IV. Kapitel.)

3) Augustin Louis Cauchy, geb. 1789 in Paris, gest. 1857 in Sceaux. In seinem Werke Analyse algébrique, Paris 1821 (deutsche Ausgabe: Berlin 1885, bei J. Springer), werden zum erstenmale in voller Strenge die Grundlagen der höheren Analysis, unter ihnen die Lehre von den unendlichen Reihen entwickelt. Wir werden es im folgenden oft zu nennen haben; der obige Satz '2 findet sich daselbst S. 59 . 
Und da nun im Zähler des ersten Bruches rechterhand eine feste Zahl steht, so kann man weiter $n_{0}>m$ so bestimmen, daß für $n>n_{0}$ dieser Bruch $<\frac{\varepsilon}{2}$ bleibt. Dann ist für $n>n_{0}$ aber stets $\left|x_{n}{ }^{\prime}\right|<\varepsilon$, und unser Satz schon bewiesen. - Ein wenig allgemeiner, aber doch eine unmittelbare Folgerung ist der

$\circ$ Satz 2. Strebt $x_{n} \rightarrow \xi$, so streben auch die arithmetischen Mittel

$$
x_{n}{ }^{\prime}=\frac{x_{n}+x_{1}+\ldots+x_{n}}{n+1} \rightarrow \xi .
$$

Beweis. Mit $\left(x_{n}-\xi\right)$ ist nach Satz 1 auch

$$
\frac{\left.\left(x_{0}-\xi\right)+\left(x_{1}-\xi\right)+\cdots+x_{n}-\xi\right)}{n+1}=x_{n}{ }^{\prime}-\xi
$$

eine Nullfolge, w. z.b.w.

Aus diesem Satz läßt sich nun ganz leicht das Entsprechende für die geometrischen Mittel folgern.

Satz 3. Die Folge $\left(y_{1}, y_{2}, \ldots\right)$ sirebe $\rightarrow \eta$ und es seien alle ihre Glieder $y_{n}$ sowie ihr Grenzwert $\eta$ positiv. Dann strebt auch die Folge der geometrischen Mittel

$$
y_{n}^{\prime}=\sqrt[n]{y_{1} y_{2} \ldots y_{n}} \rightarrow \eta \text {. }
$$

Beweis. Aus $y_{n} \rightarrow \eta$ folgt, weil alles positiv ist, nach $\$ \mathbf{2}, 2$, daß

$$
x_{n}=\log y_{n} \rightarrow \xi=\log \eta
$$

strebt. Nach Satz 2 folgt hieraus, daß auch

$$
x_{n}{ }^{\prime}={ }^{x_{1}+x_{2}+\cdots+x_{n}}=\log \sqrt[n]{y_{1} y_{2} \ldots y_{n}}=\log y_{n}{ }^{\prime}>\log \eta
$$

strebt. Nach 42, 1 liefert dies sofort die Richtigkeit unserer Behauptung.

Beispiele.

1. $\frac{1+\frac{1}{2}+\ldots+\frac{1}{n}}{n} \rightarrow 0$, weil $\frac{1}{n} \rightarrow 0$ strebt.

2. $\sqrt[n]{n}=\sqrt[n]{1 \cdot \frac{2}{1} \cdot \frac{3}{2} \cdots \frac{n}{n-1} \rightarrow 1}$, weil $\frac{n}{n-1} \rightarrow 1$ strebt.

3. $\frac{1+\sqrt{2}+\sqrt[3]{3}+\ldots+\sqrt[n]{n}}{n} \rightarrow 1$, weil $\sqrt[n]{n} \rightarrow 1$ strebt.

4. Wegen $\left(1+\frac{1}{n}\right)^{n} \rightarrow c($ s. $\mathbf{1 6}, 4$ im nächsten $\S)$ strebt nach Satz 3 auch

$$
\sqrt[n]{\left(\frac{2}{1}\right)^{1} \cdot\left(\frac{3}{2}\right)^{2} \cdot\left(\frac{4}{3}\right)^{3} \cdots\left(\frac{n+1}{n}\right)^{n}}=\sqrt[n]{\frac{(n+1)^{n}}{n !}}=\frac{n+1}{\sqrt[n]{n !}}>e
$$


oder also

$$
\frac{1}{n} \sqrt[n]{n !} \rightarrow \frac{1}{e}
$$

eine Beziehung, die man sich auch in der Form,$\sqrt[n]{n !} \simeq \frac{n}{e}$ " merken mag.

Wesentlich weitergehend und doch ebenso leicht beweisbar ist die folgende Verallgemeinerung der Cauchyschen Sätze 1 und 2, die von $O$. Toeplitz herrührt $\left.{ }^{1}\right)$ :

o Satz 4. Es sei $\left(x_{0}, x_{1}, \ldots\right)$ wieder eine Nullfolge und die 'Koeffizienten $a_{\mu \nu}$ des Schemas

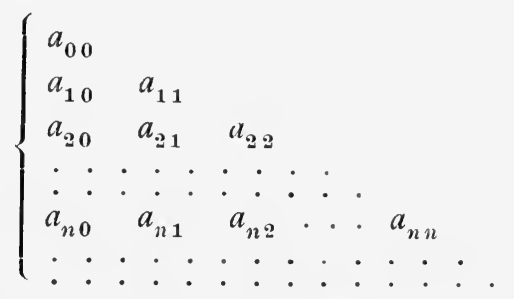

mögen den beiden Bedingungen geniigen:

(a) In jeder Spalte stehen Nullfolgen, d. h. bei festem $p \geqq 0$ strebl

$$
a_{n p} \rightarrow 0 \text { fïr } n \rightarrow+\infty \text {. }
$$

(b) Es gibt eine Konstante $K$, so daß die Summe der Beträge der Glieder einer jeden Zeile, also für jedes $n$ die Summe

$$
\left|a_{n 0}\right|+\left|a_{n 1}\right|+\ldots+\left|a_{n n}\right|<K
$$

bleibt. - Dann ist auch die Folge der Zahlen

eine Nullfolge.

$$
x_{n}{ }^{\prime}=a_{n 0} x_{0}+a_{n 1} x_{1}+a_{n 2} x_{2}+\ldots+a_{n n} x_{n}
$$

Beweis. Ist $\varepsilon>0$ gegeben, so bestimme man $m$ so, daß für $n>m$ stets $\left|x_{n}\right|<\frac{\varepsilon}{2 K}$ bleibt. Dann ist für diese $n$

$$
\left|x_{n}{ }^{\prime}\right|<\left|a_{n 0} x_{0}+\ldots+a_{n m} x_{m}\right|+\frac{\varepsilon^{\prime}}{2} .
$$

Nach Voraussetzung (a) kann man nun (da $m$ jetzt fest ist) $n_{0}>m$ so wählen, daß für $n>n_{0}$ stets $\left|a_{n 0} x_{0}+\ldots+a_{n m} x_{m}\right|<\frac{\varepsilon}{2}$ bleibt. Da für diese $n$ dann $\left|x_{n}{ }^{\prime}\right|<\varepsilon$ ist, so ist unser Satz schon bewiesen.

1) Der Cauchysche Satz 1 ist verschiedentlich verallgemeinert worden, so insbesondere von $J . L . W . V$. Jensen (Om en Sätning af Cauchy, Tidskrift for Mathematik, Bd. (5) 2, S.81-84) und O. Stolz (Úber eine Verallgemeinerung eines Satzes von Cauchy, Mathemat. Annalen, Bd. 33 (1889), S. 237). Die obige von $O$. Toeplitz (Über lineare Mittelbildungen, Prace matematyczno-fizyczne, Bd. 22 (1911), S. 113-119) herriihrende Fassung ist darum eine in gewissem Sinne äuferste Verallgemeinerung, weil er (a.a. O.) zeigt, dak die als hinreichend erkannten Bedingungen auch dazu notwendig sind, daf aus $x_{n} \rightarrow \xi$ stets $x_{n}^{\prime} \rightarrow \xi$ folgt. 
Für die Anwendungen handlich ist hierzu der folgende

- Zusatz. Ersetzt man die Koeffizienten $a_{\varkappa \lambda}$ durch andre Zahlen $a_{x \lambda}^{\prime}=a_{x \lambda} \cdot \alpha_{x \lambda}$, die aus den $a_{\varkappa \lambda}$ durch Hinzufügung von Faktoren $\alpha_{\varkappa \lambda}$ entstehen, die sämtlich unterhalb einer festen Konstanten a bleiben, so bilden auch die Zahlen

eine Nullfolge.

$$
x_{n}^{\prime \prime}=a_{n 0}^{\prime} x_{0}+a_{n 1}^{\prime} x_{1}+\ldots+a_{n n}^{\prime} x_{n}
$$

Beweis: Auch die $a_{\varkappa}^{\prime}$. erfüllen die Bedingungen (a) und (b) des Satzes 4 , denn bei festem $p$ strebt nach 26, $1, a_{n p}^{\prime} \rightarrow 0$ und es bleiben die Summen

$$
\left|a_{n 0}^{\prime}\right|+\left|a_{n 1}^{\prime}\right|+\ldots+\left|a_{n n}^{\prime}\right|<K^{\prime}=\alpha \cdot K .
$$

Aus Satz 4 folgt nun weiter der schärfere

- Satz 5. Strebt $x_{n} \rightarrow \xi$ und erfüllen die $a_{\mu \nu}$ außer den Bedingungen (a) und (b) des Satzes 4 noch die weitere, daß

$$
a_{n 0}+a_{n 1}+\ldots+a_{n n}=A_{n} \rightarrow 1
$$

strebt ${ }^{1}$ ), so konvergiert auch die Folge der Zahlen

$$
x_{n}^{\prime}=a_{n 0} x_{0}+a_{n 1} x_{1}+\ldots+a_{n n} x_{n} \rightarrow \xi .
$$

Beweis. Es ist jetzt

$$
x_{n}{ }^{\prime}=A_{n} \cdot \xi+a_{n 0}\left(x_{0}-\xi\right)+a_{n 1}\left(x_{1}-\xi\right)+\ldots+a_{n n}\left(x_{n}-\xi\right) \text {, }
$$

woraus sich nun infolge der neuen Bedingung (c) und nach Satz 4 sofort die neue Behauptung ergibt.

Ehe wir zu diesen wichtigen Sätzen Anwendungen und Beispiele geben, beweisen wir noch die folgende in eine neue Richtung weisende Verallgemeinerung.

- Satz 6. Evfüllen die Koeffizienten $a_{\mu \nu}$ des Schemas (A) außer den in Satz 4 und 5 genannten Bedingungen (a), (b) und (c) noch die weitere, daß

(d) auch die Zahlen jeder "Schräglinie" in (A) eine Nullfolge bilden, daß also bei festem $p$ für $n \rightarrow+\infty$ auch $a_{n-p} \rightarrow 0$ strebt, so folgt aus $x_{n} \rightarrow \xi$ und $y_{n} \rightarrow \eta$, daß die Zahlen streben $\left.^{2}\right)$.

$$
z_{n}=a_{n 0} x_{0} y_{n}+a_{n 1} x_{1} y_{n-1}+\ldots+a_{n n} x_{n} y_{0} \rightarrow \xi \cdot \eta
$$

Beweis. Wegen

ist

$$
x_{v} y_{n-v}=\left(x_{v}-\xi\right) y_{n-v}+\xi \cdot y_{n-v}
$$

$$
z_{n}=\sum_{\nu=0}^{n} a_{n \nu} y_{n-v}\left(x_{\nu}-\xi\right)+\xi \cdot \sum_{\nu=0}^{n} a_{n v} y_{n-v} .
$$

1) Bei den Anwendungen wird meist $A_{n} \equiv 1$ sein.

2) Für positive $a_{\mu \nu}$ findet sich dieser Satz in der Note des Verfassers "Über Summen der Form $a_{0} b_{n}+a_{1} b_{n-1}+\ldots+a_{n} b_{0}$ " (Rend. del circolo mat. di Palermo, Bd. 32, 1911, S. 95-110). 
Hier strebt der erste Summand nach Satz 4 und dessen Zusatz gegen 0, weil $\left(x_{v}-\xi\right)$ eine Nullfolge ist und die Faktoren $y_{n-v}$ beschränkt bleiben. Und schreibt man den zweiten Summanden in der Form

$$
\xi \cdot \sum_{\nu=0}^{n} a_{n n-v}, y_{v}=\xi \cdot \sum_{v=0}^{n} a_{n}^{\prime}, y_{v},
$$

so erkennt man nach Satz 5, daß derselbe, und somit auch $z_{n} \rightarrow \xi \eta$ strebt; denn wegen $(d)$ erfüllen die Zahlen $a_{n v}^{\prime}=a_{n n-v}$ genau die dortige Voraussetzung $(a)$.

Bemerkungen, Anwendungen, Beispiele.

1. Satz 1 ist ein Spezialfall von Satz 4; man hat in letzterem nur

$$
a_{n 0}=a_{n 1}=\ldots=a_{n n}=\frac{1}{n+1}, \quad(n=0,1,2, \ldots)
$$

$z$ u setzen. Satz 2 ist derselbe Spezialfall von Satz 5. Die Bedingungen (a), (b), (c) sind erfüllt.

2. Sind $\alpha_{0}, \alpha_{1}, \ldots$ irgendwelche positive Zahlen, für die die Summen

$$
\alpha_{0}+\alpha_{1}+\ldots+\alpha_{n}=\sigma_{n} \rightarrow+\infty
$$

streben, so folgt aus $x_{n} \rightarrow \xi$, dafi auch

$$
x_{n}^{\prime}=\frac{\alpha_{0} x_{0}+\alpha_{1} x_{1}+\ldots+\alpha_{n} x_{n}}{\alpha_{0}+\alpha_{1}+\ldots+\alpha_{n}} \rightarrow \varepsilon
$$

strebt $\left.^{1}\right)$. In der Tat hat man in Satz 4 nur

$$
a_{n v}=\frac{\alpha_{v}}{\sigma_{n}} \quad\left\{\begin{array}{l}
n=0,1,2, \ldots . \\
v=0,1, \ldots n .
\end{array}\right.
$$

zu setzen, um die Richtigkeit dieser Behauptung zu erkennen. Die Bedingungen (a), (b), (c) sind erfüllt. - Für $\alpha_{n}=1$ erhält man wieder Satz 2.

3. Statt die $\alpha_{n}$ positiv vorauszusetzen, gentigt es [nach (b)] zu verlangen, dafs nur $\left|\alpha_{0}\right|+\alpha_{1}|+\ldots+| \alpha_{n} \mid \rightarrow+\infty$ strebt, dafi aber eine Konstante $K$ existiert, so dafs für alle $n$

$$
\left|\alpha_{0}\right|+\left|\alpha_{1}\right|+\ldots+\left|\alpha_{n}\right| \leqq K \cdot \alpha_{0}+\alpha_{1}+\ldots+\alpha_{n} \mid
$$

bleibt ${ }^{2}$ ). (Für positive $\alpha_{n}$ steht die Existenz von $K$ außer Zweifel.)

4. Setzt man in 2. oder 3. zur Abkürzung $\alpha_{n} x_{n}=y_{n}$, so ergibt sich: Es strebt

$$
\frac{y_{0}+y_{1}+\ldots+y_{n}}{\alpha_{0}+\alpha_{1}+\ldots+\alpha_{n}} \rightarrow \xi, \text { falls auch } \frac{y_{n}}{\boldsymbol{\alpha}_{n}} \rightarrow \xi
$$

strebt, und falls die $\alpha_{n}$ die in 2. oder 3. genannten Bedingungen erfüllen.

5. Setzt man noch $y_{0}+y_{1}+\ldots+y_{n}=Y_{n}$ und $\alpha_{0}+\alpha_{1}+\ldots+\alpha_{n}=A_{n}$, so erhält das letzte Ergebnis die Form: es strebt

$$
\frac{Y_{n}}{A_{n}} \rightarrow \xi, \quad \text { falls auch } \frac{Y_{n}-Y_{n-1}}{A_{n}-A_{n-1}} \rightarrow \xi
$$

strebt und falls die Zahlen $\alpha_{n}=A_{n}-A_{n-1},\left(n \geq 1, \alpha_{0}=A_{0}\right)$, die in 2. oder 3 . genannten Bedingungen erfüllen.

6. So ist z. B. nach 5.:

$$
\lim \frac{1+2+\ldots+n}{n^{2}}=\lim \frac{n}{n^{2}-(n-1)^{2}}=\lim \frac{n}{2 n-1}=\frac{1}{2} .
$$

1) O. Stolz, a. a. O.

?) Jensen, a. a. O. 
Ebenso

und allgemein

$$
\lim \frac{1^{2}+2^{2}+\ldots+n^{2}}{n^{3}}=\lim \frac{n^{2}}{n^{3}-(n-1)^{3}}=\frac{1}{3}
$$

$$
\begin{aligned}
& \lim \frac{1^{p}+2^{p}+\ldots+n^{p}}{n^{p+1}}=\lim \frac{n^{p}}{n^{p+1}-(n-1)^{p+1}} \\
= & \lim \frac{n^{p}}{(p+1) n^{p}-\left(\begin{array}{c}
p+1 \\
2
\end{array}\right) n^{p-1}+\ldots}=\frac{1}{p+1},
\end{aligned}
$$

wenn $p$ eine natürliche $Z$ ahl bedeutet.

7. Ähnlich findet man, wenn man die unter $\mathbf{1 6}, 4$ bewiesene Konvergenz der Folge der Zahlen $\left(1+\frac{1}{n}\right)^{n+1}$ vorwegnimmt:

$$
\frac{\log 1+\log 2+\ldots+\log n}{n \log n}=\frac{\log n !}{\log n^{n}} \rightarrow 1, \quad \text { d. h. } \quad \log n ! \simeq \log n^{n}
$$

8. Auch die Zahlen

$$
a_{n v}=\frac{1}{2^{n}}\left(\begin{array}{c}
n \\
v
\end{array}\right) \quad\left\{\begin{array}{l}
n=0,1,2, \ldots \\
v=0,1, \ldots, n
\end{array}\right.
$$

genügen den Bedingungen (a), (b) und (c) der Sätze 4 und 5 ; denn bei festem $p$ strebt $a_{n p} \rightarrow 0$, weil es

ist, und es ist

$$
=\frac{1}{2^{n}}\left(\begin{array}{l}
n \\
p
\end{array}\right) \quad \text { und also }<\frac{n^{p}}{2^{n}} \quad(\text { s. 3S, } 4)
$$

$$
\left|a_{n 0}+\ldots+\right| a_{n n} \mid=a_{n 0}+\ldots+a_{n n}=1
$$

für jedes $n$. Also folgt aus $x_{n} \rightarrow \xi$ stets, dafi auch

strebt.

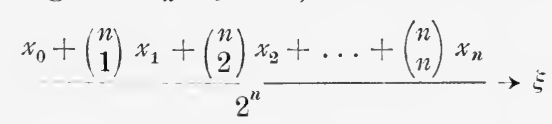

9. Dieselben Spezialisierungen, die in 1., 2., 3 und 8. für den Satz 5 gegeben wurden, lassen sich natürlich auch für Satz 6 durchführen. Es seien nur die beiden folgenden Sätze erwähnt:

a) Aus $x_{n} \rightarrow \xi$ und $y_{n} \rightarrow \eta$ folgt immer, daf

$$
\frac{x_{0} y_{n}+x_{1} y_{n-1}+x_{2} y_{n-2}+\ldots+x_{n} y_{0}}{n+1} \rightarrow \xi \eta
$$

strebt.

b) Sind $\left(x_{n}\right)$ und $\left(y_{n}\right)$ zwei Nullfolgen, deren zweite noch der Bedingung genïgt, dafs für alle $n$

$$
\left|y_{0}+y_{1}+\ldots+\right| y_{n} \mid
$$

unter einer festen Schranke $K$ bleibt, so bilden auch die Zahlen

$$
z_{n}=x_{0} y_{n}+x_{1} y_{n-1}+\ldots+x_{n} y_{0}
$$

eine Nullfolge. (Zum Beweis hat man in Satz 4 nur $a_{n v}=y_{n-v}$ zu setzen.)

10. Man wird bemerkt haben, daf3 es nicht wesentlich ist, dafi die Zeilen des Schemas (A) in Satz 4 gerade hinter dem $n$ ten Gliede abbrachen. Es dürfen vielmehr die Zeilen irgendeine Anzahl von Gliedern haben. Ja, wenn wir erst die Grundziige der Theorie der unendlichen Reihen besitzen werden, werden wir sehen, dafi diese Zeilen sogar unendlich viele Elemente $\left(a_{n 0}, a_{n 1}\right.$, $\left.\ldots, a_{n v}, \ldots\right)$ enthalten dürfen, sofern nur die sonst an das Schema gestellten Bedingungen erfüllt sind. Der hiermit angedeutete Satz wird S. 377 formuliert und bewiesen. 


\section{\$ 9. Die beiden Hauptkriterien.}

Wir sind nun hinlänglich vorbereitet, um die eigentlichen Konvergenzprobleme in Angriff zu nehmen. Zwei Gesichtspunkte sind es hauptsächlich, unter denen wir im folgenden die vorgelegten Zahlenfolgen untersuchen werden. Es ist vor allem das

Problem.A: Ist eine vorgelegte Zahlenfolge $\left(x_{n}\right)$ konvergent, bestimmt oder unbestimmt divergent? (Kürzer: Welches Konvergenzverhalten weist die Zahlenfolge auf?) - Und wenn sich nun eine Zahlenfolge als konvergent erwiesen hat, also die Existenz eines Grenzwertes gesichert ist, dann handelt es sich weiter um das

Problem B: Gegen welchen Grenzwert $\xi$ strebt die als konvergent erkannte Zahlenfolge $\left(x_{n}\right)$ ?

Ein paar Beispiele werden die Bedeutung dieser Probleme deutlicher machen: Sind z. B. die Folgen

$$
\begin{gathered}
(n(\sqrt[n]{2}-1)), \quad(n(\sqrt[n]{n}-1)), \quad\left(\left(1+\frac{1}{n}\right)^{n}\right), \quad\left(\left(1+\frac{1}{n^{2}}\right)^{n}\right), \\
\left(\frac{1+2^{2}+3^{3}+\ldots+n^{n}}{n^{n}}\right), \quad\left(\frac{1+\frac{1}{2}+\ldots+\frac{1}{n}}{\log n}\right. \text { usw. }
\end{gathered}
$$

vorgelegt, so erkennt man bei einiger Vertiefung in den Bau dieser Folgen, daß hier stets zwei (oder mehr) Kräfte sozusagen gegeneinander wirken und dadurch die Veränderlichkeit der Glieder hervorrufen. Die eine Kraft will sie vergrößern, die andere verkleinern, und es ist nicht ohne weiteres zu sehen, welche den Ausschlag gibt oder in welchem Maße dies der Fall ist. Jedes Mittel, das uns instand setzt, über das Konvergenzverhalten vorgelegter Zahlenfolgen zu entscheiden, nennen wir ein Konvergenz-bzw. Divergenzkriterium; sie dienen also zur Lösung des Problems A.

Das Problem B ist im allgemeinen viel schwieriger. Ja, man könnte fast sagen, daß es unlösbar - oder aber trivial ist. Letzteres, weil ja durch eine konvergente Zahlenfolge $\left(x_{n}\right)$ nach Satz 41, 1 der Grenzwert $\xi$ völlig eindeutig erfaßt ist und also durch die Folge selbst als ,gegeben“ angesehen werden kann (vgl. die Fußnote zu 41, 1). Wegen der unübersehbaren Vielgestaltigkeit der Zahlenfolgen ist diese Auslegung aber wenig befriedigend. Wir werden den Grenzwert $\xi$ vielmehr erst dann als „bekannt" ansehen wollen, wenn wir einen Dedekindschen Schnitt oder noch besser eine Intervallschachtelung, z. B. einen Systembruch, speziell einen Dezimalbruch vor uns haben. Besonders diese letzteren sind und bleiben uns die vertrautesten Darstellungsformen reeller Zahlen. Fassen wir das Pro- 
blem so auf, so können wir es als die Frage nach der numerischen Berechnung des Grenzwertes ansehen ${ }^{1}$ ).

Diese praktisch hoch bedeutungsvolle Frage ist theoretisch meist ziemlich gleichgültig, denn vom theoretischen Standpunkt aus sind alle Darstellungsformen einer reellen Zahl (Schachtelung, Schnitt, Zahlenfolge, ...) völlig gleichberechtigt. Beachtet man noch, daß die Darstellung einer reellen Zahl durch eine Zahlenfolge als die allgemeinste Darstellungsart angesehen werden kann, so kann nun das Problem B präzisiert werden zu dem

Problem $\mathrm{B}^{\prime}$ : Es sind zwei konvergente Zahlenfolgen $\left(x_{n}\right)$ und $\left(x_{n}{ }^{\prime}\right)$ vorgelegt, - wie läßt sich feststellen, ob beide denselben Grenzwert definieren, bzw. ob beide Grenzwerte in einer einfachen Beziehung zueinander stehen?

Ein paar Beispiele sollen auch diese Fragestellung noch erläutern:

1. Es sei

$$
x_{n}=\left(1+\frac{1}{n}\right)^{n} \text { und }{ }^{\circ} x_{n}{ }^{\prime}=\left(1+\frac{2}{n}\right)^{n} \text {. }
$$

Beide Folgen werden sich (s. u. S. 77 u. 185) ziemlich leicht als konvergent zu erkennen geben. Aber etwas tiefer liegt die Tatsache, daß, wenn $\xi$ der Grenzwert der ersten Folge ist, der der andern $=\xi^{2}$ ist.

2. Es sei die Folge

$$
\frac{1}{1}, \frac{3}{2}, \frac{7}{5}, \frac{17}{12}, \frac{41}{29}, \ldots
$$

vorgelegt, bei der der Zähler jedes neuen Bruches dadurch gebildet wird, dafs man zum verdoppelten Zähler des vorangehenden Bruches den Zähler des zweitvorangehenden Bruches addiert (z. B. $41=2 \cdot 17+7),-$ und ebenso für die Nenner. - Die Konvergenzfrage wird wieder keine Schwierigkeiten machen, auch die numerische Berechnung nicht, - wie aber erkennt man, dafs der Grenzwert $=\sqrt{2}$ ist?

3. Es sei

$$
x_{n}=\left(1-\frac{1}{3}+\frac{1}{5}-\frac{1}{7}+\ldots+\frac{(-1)^{n-1}}{2 n-1}\right) \quad(n=1,2, \ldots)
$$

und $x_{n}{ }^{\prime}$ sei der Umfang eines regulären $n$-Ecks, das einem Kreise mit dem Radius 1 einbeschrieben ist. Auch hier werden sich leicht beide Folgen als konvergent erkennen lassen. Sind nun $\xi$ und $\xi^{\prime}$ die Grenzwerte, - wie erkennt man, dafs hier $\xi^{\prime}=8 \cdot \xi$ ist?

Diese Beispiele machen es hinreichend wahrscheinlich, daß das Problem B bzw. $\mathrm{B}^{\prime}$ erheblich schwerer anzugreifen ist als das Problem A. Wir beschäftigen uns daher zunächst ausschließlich mit diesem, und wollen zunächst zwei Kriterien kennen lernen, aus denen sich dann alle andern werden herleiten lassen.

\section{Hauptkriterium (für monotone Folgen).}

Eine monotone und beschränkte Zahlenfolge ist stets kon. vergent; eine monotone und nicht beschränkte Zahlenfolge ist

1) Numerische Berechnung einer reellen Zahl = Darstellung derselben durch einen Dezimalbruch. Näheres darüber in VIII. Kapitel. 
stets bestimmt divergent. (Oder also: Eine monotone Folge verhält sich stets bestimmt, und zwar ist sie dann und nur dann konvergent, wenn sie beschränkt, dann und nur dann divergent, wenn sie nicht beschränkt ist. Und im letzteren Falle wird die Divergenz gegen $+\infty$ oder $-\infty$ stattfinden, je nachdem die monotone Folge steigt oder fällt.)

Beweis. a) Es sei $\left(x_{n}\right)$ monoton wachsend und nicht beschränkt. Da sie dann wegen $x_{n} \geqq x_{1}$ sicher nach links beschränkt ist, so kann sie nicht nach rechts beschränkt sein, und es gibt also nach Wahl einer beliebigen (großen) positiven Zahl $G$ immer noch einen Index $n_{0}$. für den

$$
x_{n_{0}}>G
$$

ausfällt. Dann ist aber, wegen des monotonen Wachsens, für alle $n>n_{0}$ um so mehr $x_{n}>G$, so daß nach Def. 40, 2 tatsächlich $x_{n} \rightarrow+\infty$ strebt. Durch Vertauschung von rechts und links erkennt man ganz ebenso, daß eine monoton fallende und nicht beschränkte Folge gegen $-\infty$ divergieren muß. Damit ist schon der zweite Teil des Satzes bewiesen.

b) Es sei jetzt $\left(x_{n}\right)$ monoton wachsend, aber beschränkt. Dann gibt es eine Zahl $K$, so daß stets $\left|x_{n}\right| \leqq K$, also auch stets

$$
x_{1} \leqq x_{n} \leqq K
$$

bleibt. In dem Intervall $J_{1}=x_{1} \ldots K$ liegen also alle Glieder von $\left(x_{n}\right)$; auf dieses Intervall wenden wir nun die Halbierungsmethode an: Wir bezeichnen die rechte oder die linke Hälfte von $J_{\mathbf{1}}$ mit $J_{\mathbf{2}}$, je nachdem in der rechten Hälfle noch Punkte von $\left(x_{n}\right)$ liegen oder nicht. Bei $J_{2}$ wählen wir nach derselben Regel eine Hälfte aus, nennen sie $J_{3}$, usw. Die Intervalle der so entstehenden Schachtelung haben dann die Eigenschaft ${ }^{1}$ ), daß rechts von ihnen kein Punkt der Folge mehr liegt, in ihnen aber mindestens noch einer gelegen ist. Oder: die (monoton nach rechts vorrückenden) Punkte der Folge dringen zwar noch in jedes Intervall hinein, aber nicht mehr aus ihm heraus; in einem jeden der Intervalle liegen also von einem passenden Index an alle Punkte der Folge. Wir können also, indem wir uns die Zahlen $n_{1}, n_{2}, \ldots$ geeignet gewählt denken, sagen:

In $J_{k}$ liegen alle $x_{n}$ mit $n>n_{k}$, aber rechts von $J_{k}$ liegt kein $x_{n}$ mehr.

Ist nun $\xi$ die durch diese Schachtelung $\left(J_{n}\right)$ bestimmte Zahl, so läßt sich sofort zeigen, daß $x_{n} \rightarrow \xi$ strebt. Ist nämlich $\varepsilon>0$ gegeben, so bestimme man den Index $p$, so daß die Länge von $J_{p}$ kleiner als $\varepsilon$ ist. Für $n>n_{p}$, liegen dann neben $\xi$ auch alle $x_{n}$ in $J_{p}$, so daß für diese $n$

$$
\left|x_{n}-\xi\right|<\varepsilon
$$

sein muß; $\left(x_{n}-\xi\right)$ ist also eine Nullfolge, es strebt $x_{n} \rightarrow \xi$, w. z. b.w.

1) Man veranschauliche sich den Sachverhalt auf der Zahlengeraden! 
Vertauscht man in diesem Beweise sinngemäß rechts und links, so ergibt sich, daß auch eine monoton fallende und beschränkte Folge stets konvergent sein muß. Damit ist dann der Satz in allen Teilen bewiesen.

\section{Bemerkungen und Beispiele.}

1. Wir erinnern zunächst daran, daf $(\operatorname{vgl} 1.41,2)$ trotz $\left|x_{n}\right|<K$ für den Grenzwert $\xi$ die Gleichung $|\xi|=K$ gelten kann.

2. Es sei

$$
x_{n}=\frac{1}{n+1}+\frac{1}{n+2}+\ldots+\frac{1}{2 n} \quad \cdot \quad(n=1,2, \ldots) \text {. }
$$

Diese Folge $\left(x_{n}\right)$ ist wegen

$$
x_{n+1}-x_{n}=\frac{1}{2 n+1}+\frac{1}{2 n+2}-\frac{1}{n+1}=\frac{1}{2 n+1}-\frac{1}{2 n+2}>0
$$

monoton wachsend und wegen $x_{n}<n \cdot \frac{1}{n+1}<1$ auch beschränkt. Sie ist also konvergent. Von ihrem Grenzwert $\xi$ weiß man zunächst nur, dafi für jedes $n$

$$
x_{n}<\xi \leqq 1
$$

ist, was für $n=3$ z. B. $\frac{37}{60}<\xi \leqq 1$ ergibt. Ob er einen rationalen Wert hat, oder ob $\xi \mathrm{zu}$ einer in irgendeinem andern Zusammenhang auftretenden Zahl in naher Beziehung steht - kurz: eine Antwort auf das Problem B - ist hier nicht ohne weiteres $\mathrm{zu}$ sehen. Später werden wir sehen, dafi gleich dem natürlichen Logarithmus von 2 ist.

3. Es sei $x_{n}=\left(1+\frac{1}{2}+\frac{1}{3}+\ldots+\frac{1}{n}\right)$, so dafi die Folge $\left(x_{n}\right)$ monoton wachsend ist (vg1. 6, 12). Ist sie beschränkt oder nicht? - Ist $G>0$ beliebig gegeben, so wähle man $m>2 G$; dann ist für $n>2^{m}$

$$
\begin{aligned}
x_{n} & >\left(1+\frac{1}{2}\right)+\left(\frac{1}{3}+\frac{1}{4}\right)+\left(\frac{1}{5}+\ldots+\frac{1}{8}\right)+\ldots+\left(\frac{1}{2^{m-1}+1}+\ldots+\frac{1}{2^{m}}\right) \\
& >\frac{1}{2}+2 \cdot \frac{1}{4}+4 \cdot \frac{1}{8}+8 \cdot \frac{1}{16}+\ldots+2^{m-1} \cdot \frac{1}{2^{m}}=\frac{m}{2}>G .
\end{aligned}
$$

Die Folge ist also nicht beschränkt und divergiert daher $\rightarrow+\infty$.

4. Besonders wichtig sind die beiden Folgen mit den Gliedern

$$
x_{n}=\left(1+\frac{1}{n}\right)^{n} \quad \text { und } \quad y_{n}=\left(1+\frac{1}{n}\right)^{n+1} \quad(n=1,2,3, \ldots) .
$$

Es ist nicht ohne weiteres $\mathrm{zu}$ sehen (vgl. die allgemeine Bemerkung S. 74), wie sich die Folgen bei zunehmendem $n$ verhalten.

Wir wollen zunächst zeigen, dafs die zweite Folge monoton fällt, daf also für $n \geq 2$

$$
y_{n-1}>y_{n} \quad \text { oder } \quad\left(1+\frac{1}{n-1}\right)^{n}>\left(1+\frac{1}{n}\right)^{n+1}
$$

ist. Diese Ungleichung ist nämlich gleichbedeutend mit

$$
\left(\frac{1+\frac{1}{n-1}}{1+\frac{1}{n}}\right)^{n}>1+\frac{1}{n}
$$

oder mit

$$
\left(\frac{n^{2}}{n^{2}-1}\right)^{n}>1+\frac{1}{n}, \quad \text { d. h. mit } \quad\left(1+\frac{1}{n^{2}-1}\right)^{n}>1+\frac{1}{n} \text {. }
$$


Die Richtigkeit dieser Ungleichung ist aber evident, da nach der Bernoullischen Ungleichung 10, 7 für $\alpha>-1$ und $n>1$ stets

also speziell

$$
(1+\alpha)^{n}>1+n \alpha \text {, }
$$

$$
\left(1+\frac{1}{n^{2}-1}\right)^{n}>1+\frac{n}{n^{2}-1}>1+\frac{n}{n^{2}}=1+\frac{1}{n}
$$

ist. Da uiberdies stets $y_{n}>1$ ist, so ist die Folge $\left(y_{n}\right)$ monoton fallend und beschränkt, also konvergent. Ihr Grenzwert wird weiterhin oft auftreten; er wird seit Euler mit dem besonderen Buchstaben $\boldsymbol{e}$ bezeichnet ${ }^{1}$ ). Über seinen Zahlenwert folgt zunächst nur, dafi $1 \leqq e<y_{n}$ ist, was für $n=5$ z. B.

liefert.

$$
1 \leq e<\frac{6^{6}}{5^{6}}<3
$$

Die erste unserer beiden Folgen dagegen ist monoton steigend. In der Tat bedeutet $x_{n-1}<x_{n}$, dafi

oder also dafis

$$
\left(1+\frac{1}{n-1}\right)^{n-1}<\left(1+\frac{1}{n}\right)^{n}
$$

d. h. daf

$$
\left(1+\frac{1}{n-1}\right)^{-1}<\left(\frac{1+\frac{1}{n}}{1+\frac{1}{n-1}}\right)^{n}
$$

$$
1-\frac{1}{n}<\left(\frac{n^{2}-1}{n^{2}}\right)^{n}=\left(1-\frac{1}{n^{2}}\right)^{n}
$$

sein soll. Nun ist aber, wieder nach 10,7, für $n>1$ tatsächlich stets

$$
\left(1-\frac{1}{n^{2}}\right)^{n}>1-\frac{n}{n^{2}}=1-\frac{1}{n} \text {. }
$$

Die Folge $\left(x_{n}\right)$ ist also monoton wachsend.

Da aber unter allen Umständen

$$
\left(1+\frac{1}{n}\right)^{n}<\left(1+\frac{1}{n}\right)^{n+1}, \quad \text { d. h. } \cdot x_{n}<y_{n}
$$

ist, so ist auch stets $x_{n}<y_{1}, \mathrm{~d} . \mathrm{h} .\left(x_{n}\right)$ ist auch beschränkt, und also konvergent. Da endlich die Zahlen

$$
y_{n}-x_{n}=\left(1+\frac{1}{n}\right)^{n} \cdot\left(1+\frac{1}{n}-1\right)=\frac{1}{n} \cdot x_{n}
$$

positiv sind und (nach $\mathbf{2 6}, 1$ ) eine Nullfolge bilden, so ergibt sich zunächst, dafs $\left(x_{n}\right)$ denselben Grenzwert wie $\left(y_{n}\right)$ hat. Es ist also

$$
\lim x_{n}=\lim y_{n}=e \text {. }
$$

Und für diese Zahl $e$ haben wir überdies nach allem Bewiesenen in

$$
e=\left(x_{n}: y_{n}\right)=\left(\left(1+\frac{1}{n}\right)^{n} \mid\left(1+\frac{1}{n}\right)^{n+1}\right)
$$

eine sie erfassende Intervallschachtelung. (Sie liefert z. B. für $n=3$ die Ungleichung $\frac{64}{27}<e<\frac{256}{81}$; doch werden wir später $(\S 23)$ andere gegen $e$ kon-

1) Euler benutzt diesen Buchstaben zur Bezeichnung des obigen Grenzwertes zuerst in einem Briefe an Goldbach (25. Nov. 1731) und im Jahre 1736 in seinem Werk: Mechanica sive motus scientia analytice exposita, II, S. 251. 
vergierende Zahlenfolgen kennen lernen, die für die numerische Berechnung geeigneter sind.)

5. Ist $\sigma=\left(x_{n} \mid y_{n}\right)$ eine beliebige Intervallschachtelung, so bilden die linken und die rechten Intervallenden je eine monotone, beschränkte und also konvergente Zahlenfolge. Es ist dann

$$
\lim x_{n}=\lim y_{n}=\left(x_{n} \mid y_{n}\right)=\sigma \text {. }
$$

Die Fruchtbarkeit des 1. Hauptkriteriums liegt vor allem darin begründet, daß es aus sehr wenigen und meist sehr leicht zu verifizierenden Voraussetzungen - nämlich allein aus der Monotonie und Beschränktheit — die Konvergenz einer Zahlenfolge zu erschließen gestattet. Andrerseits aber bezieht es sich doch nur auf eine spezielle, wenn auch besonders häufige und wichtige Art von Folgen, und erscheint darum theoretisch unzulänglich. Wir werden deshalb nach einem Kriterium fragen, welches ganz allgemein über das Konvergenzverhalten einer Zahlenfolge zu entscheiden gestattet. Das leistet das folgende

\section{OII. Hauptkriterium (1. Form).}

Eine beliebige Zahlenfolge $\left(x_{n}\right)$ ist dann und nur dann konvergent, wenn sich nach Wahl von $\varepsilon>0$ stets eine $Z$ ahl $n_{0}=n_{0}(\varepsilon)$ so angeben läßt, daß für irgend zwei Indizes $n$ und $n^{\prime}$, die beide oberhalb $n_{0}$ liegen, immer

$$
\left|\boldsymbol{x}_{\boldsymbol{n}}-\boldsymbol{x}_{\boldsymbol{n}^{\prime}}\right|<\varepsilon
$$

ausfällt. - Wir geben zunächst einige

\section{Erläuterungen und Beispiele.}

1. Die Bemerkungen 10, 1, 3, 4 und 9 gelten sinngemäßs auch hier, und es empfiehlt sich, sie daraufhin noch einmal durchzulesen.

2. Das Kriterium besagt - anschaulich gesprochen - : alle $x_{n}$ mit sehr hohen Indizes sollen sehr dicht beieinander liegen.

3. Es sei $x_{0}=0, x_{1}=1$ und nun jedes folgende Glied das arithmetische Mittel $\mathrm{zw}$ wischen den beiden vorausgehenden, also für $n \geqq 2$

$$
x_{n}=\frac{x_{n-1}+x_{n-2}}{2},
$$

so dafi $x_{2}=\frac{1}{2}, x_{3}=\frac{3}{4}, x_{4}=\frac{5}{8}, \ldots$ wird. Bei dieser ersichtlich nicht monotonen Folge ist einerseits klar, daf der Abstand zweier aufeinander folgender Glieder eine Nullfolge bildet; denn man bestätigt ganz leicht durch Induktion, daf $x_{n+1}-x_{n}=\frac{(-1)^{n}}{2^{n}}$ ist $\left.^{1}\right)$ und also $\rightarrow 0$ strebt. Andrerseits liegen zwischen diesen aufeinander folgenden Zahlen auch alle weiterhin folgenden. Wählt man

1) Für $n=0$ und 1 ist dies richtig. Aus $x_{k+2}-x_{k+1}=\frac{x_{k+1}-x_{k}}{2}+\frac{x_{k}-x_{k-1}}{2}$ folgt dann die Richtigkeit für $n=k+1$, falls sie schon für alle $n \leqq k$ festgestellt ist. 
also, nachdem nur $\varepsilon>0$ vorgeschrieben wurde, $p$ so grof, daf $\frac{1}{2^{p}}<\varepsilon$ ist, so ist

$$
\left|x_{n}-x_{n^{\prime}}\right|<\varepsilon
$$

wenn nur $n$ und $n^{\prime}>p$ sind. Nach dem 2. Hauptkriterium wäre die Folge $\left(x_{n}\right)$ also konvergent. Auch ihr Grenzwert $\xi$ ist hier zufällig ziemlich leicht anzugeben. Nach einiger Überlegung vermutet man nämlich, dafi $\xi=\frac{2}{3}$ ist. In der Tat beweist sich die Formel

$$
x_{n}-\frac{2}{3}=\frac{2}{3} \cdot \frac{(-1)^{n+1}}{2^{n}}
$$

sofort durch Induktion, und diese zeigt, da $3\left(x_{n}-\frac{2}{3}\right)$ wirklich eine Nullfolge ist.

Ehe wir uns nun weiter in die Bedeutung des 2. Hauptkriteriums vertiefen, geben wir seinen

Beweis. a) Daß die Bedingung des Satzes - wir wollen sie kurz seine $\varepsilon$-Bedingung nennen - notwendig ist, daß sie also, wenn $\left(x_{n}\right)$ konvergent ist, stets erfüllt werden kann, erkennt man so: Strebt $x_{n} \rightarrow \xi$, so ist $\left(x_{n}-\xi\right)$ eine Nullfolge; bei gegebenem $\varepsilon>0$ kann man also $n_{0}$ so wählen, daß für $n>n_{0}$ stets $\left|x_{n}-\xi\right|<\frac{\varepsilon}{2}$ ist. Ist dann neben $n$ auch $n^{\prime}>n_{0}$, so ist auch $\left|x_{n^{\prime}}-\xi\right|<\frac{\varepsilon}{2}$ und also $\left|x_{n}-x_{n^{\prime}}\right|=\left|\left(x_{n}-\xi\right)-\left(x_{n^{\prime}}-\xi\right)\right| \leqq\left|x_{n}-\xi\right|+\left|x_{n^{\prime}}-\xi\right|<\frac{\varepsilon}{2}+\frac{\varepsilon}{2}=\varepsilon$, womit dieser Teil des Satzes schon bewiesen ist.

b) Daß die $\varepsilon$-Bedingung auch hinreichend ist, ist nicht ganz so leicht zu erkennen. Wir beweisen es wieder konstruktiv, indem wir aus der Zahlenfolge $\left(x_{n}\right)$ eine Intervallschachtelung $\left(J_{n}\right)$ herleiten und dann zeigen, daß die dadurch bestimmte Zahl der Grenzwert der Zahlenfolge ist. Das geschieht so:

Nach Wahl eines jeden $\varepsilon>0$ soll immer $\left|x_{n}-x_{n^{\prime}}\right|<\varepsilon$ sein, wenn nur die Indizes $n$ und $n^{\prime}$ beide einen hinreichend hohen Wert ïbersteigen. Denkt man sich den einen von ihnen festgehalten und mit $p$ bezeichnet, so kann man auch sagen: Nach Wahl von $\varepsilon>0$ läßt sich immer noch ein Index $p$ (und zwar so weit rechts liegend wie wir wollen) so angeben, daß für alle $n>p$ stets

$$
\left|x_{n}-x_{p}\right|<\varepsilon
$$

ist. Wählen wir nun der Reihe pach $\varepsilon=\frac{1}{2}, \frac{1}{4}, \ldots, \frac{1}{2^{k}}, \ldots$ so haben wir:

1) Es gibt einen Index $p_{\mathbf{1}}$, so daß

für alle $n>p_{1}$ stets $\left|x_{n}-x_{p_{1}}\right|<\frac{1}{2} \quad$ ist.

2) Es gibt einen Index $p_{2}$, den wir $>p_{1}$ annehmen dürfen, so daß für alle $n>p_{2}$ stets $\left|x_{n}-x_{p_{2}}\right|<\frac{1}{2^{2}}$ ist. 
so daß

k) Es gibt einen Index $p_{k}$, den wir $>p_{k-1}$ annehmen dürfen, für alle $n>p_{k}$ stets $\left|x_{n}-x_{p_{k}}\right|<\frac{1}{2^{k}} \quad$ ist.

Demgemäß bilden wir die Intervalle $J_{k}$ :

1) Das Intervall $x_{p_{1}}-\frac{1}{2} \ldots x_{p_{1}}+\frac{1}{2}$ heiße $J_{1}$; es enthält alle $x_{n}$ mit $n>p_{1}$, also speziell den Punkt $x_{p_{2}}$. Es enthält daher ganz oder teilweis das Intervall $x_{p_{2}}-\frac{1}{4} \ldots x_{p_{2}}+\frac{1}{4}$, in welchem alle $x_{n}$ mit $n>p_{2}$ liegen. Da diese Punkte auch in $J_{1}$ liegen, so liegen sie in dem beiden Intervallen gemeinsamen Stück. Dieses gemeinsame Stück bezeichnen wir

2) mit $J_{2}$ und können sagen: $J_{2}$ liegt in $J_{1}$ und enthält alle Punkte $x_{n}$ mit $n>p_{2}$. Ersetzt man in dieser Herleitung sinngemäß $p_{1}$ und $p_{2}$ durch $p_{k-1}$ und $p_{k}$ und bezeichnet also

k) mit $J_{k}$ das Stück des Intervalles $x_{p_{k}}-\frac{1}{2^{k}} \ldots x_{p_{k}}+\frac{1}{2^{k}}$, das in $J_{k-1}$ liegt, so können wir sagen: $J_{k}$ liegt in $J_{k-1}$ und enthält alle Punkte $x_{n}$ mit $n>p_{k}$.

Dann ist aber $\left(J_{k}\right)$ eine Intervallschachtelung; denn jedes Intervall liegt im vorangehenden und die Länge von $J_{k}$ ist $\leqq \frac{2}{2^{k}}$.

Ist nun $\xi$ die hierdurch bestimmte Zahl, so behaupten wir endlich, daß

$$
x_{n} \rightarrow \xi
$$

strebt. In der Tat, ist jetzt ein beliebiges $\varepsilon>0$ gegeben, so wählen wir einen Index $r$ so groß, daß $\frac{2}{2^{r}}<\varepsilon$ ist. Dann ist aber

$$
\text { für } n>p_{r} \text { stets }\left|x_{n}-\xi\right|<\varepsilon,
$$

da ja neben $\xi$ selber auch alle $x_{n}$ mit $n>p_{r}$ in $J_{r}$ liegen und da dessen Länge $<\varepsilon$ ist. Damit ist alles bewiesen ${ }^{1}$ ).

Weitere Beispiele und Bemerkungen.

1. Die Folge 45, 3 wird nun leicht als konvergent erkannt. Denn es ist hier, wenn $n^{\prime}>n$ ist,

$$
x_{n^{\prime}}-x_{n}= \pm\left(\frac{1}{2 n+1}-\frac{1}{2 n+3}+\cdots \pm \frac{1}{2 n^{\prime}+1}\right) \text {. }
$$

Fafit man innerhalb der Klammer je zwei aufeinander folgende Summanden zusammen, so erkennt man (vgl. später $\mathbf{8 2}, 9$ ), daf ihr Wert positiv ist, daf also

$$
\left|x_{n^{\prime}}-x_{n}\right|=\frac{1}{2 n+1}-\frac{1}{2 n+3}+\ldots \pm \frac{1}{2 n^{\prime}+1} .
$$

1) Für dieses grundlegende 2. Hauptkriterium werden wir noch andere Beweise kennen lernen. Der vorstehende Beweis geht unmittelbar auf die Erfassung des Grenzwertes mit Hilfe einer Intervallschachtelung aus. - Eine Kritik früherer Beweise des Kriteriums findet man bei A. Pringsheim (Sitzungsber. d. Akad. München, Bd. 27 (1897), S. 303). 
Läfat man jetzt das erste Glied für sich stehen und fafit dann erst je zwei aufeinander folgende Summanden zusammen, so erkennt man weiter, daf

$$
\left|x_{n^{\prime}}-x_{n}\right|<\frac{1}{2 n+1}
$$

ist. Es ist also $x_{n^{\prime}}-x_{n}<\varepsilon$, sobald $n$ und $n^{\prime}$ beide $>\frac{1}{2 \varepsilon}$ sind. Die Folge ist also konvergent.

2. Ist $x_{n}=\left(1+\frac{1}{2}+\cdots+\frac{1}{n}\right)$, so sahen wir schon $\mathbf{1 6}, 3$, daf $\left(x_{n}\right)$ nicht konvergent ist. Mit Hilfe des 2. Hauptkriteriums folgt dies daraus, daf die $\varepsilon$-Bedingung für ein $\varepsilon<\frac{1}{2}$ nicht mehr stets erfüllbar ist. Denn wie man auch $n_{0}$ wählen mag, für $n>n_{0}$ und $n^{\prime}=2 n$ (also auch $>n_{0}$ ) ist

$$
x_{n^{\prime}}-x_{n}=\frac{1}{n+1}+\frac{1}{n+2}+\ldots+\frac{1}{2 n}>n \cdot \frac{1}{2 n}=\frac{1}{2} \text {, }
$$

also nicht $<\varepsilon$. Die Folge ist daher divergent.

3. Das vorige Beispiel lehrt zugleich, daf das Gegenteil der Erfüllbarkeit der $\varepsilon$-Bedingung dies ist (vgl. auch 10, 12): Nicht bei jeder Wahl von $\varepsilon>0$, läfst sich $n_{0}$ so angeben, dafs nun die $\varepsilon$-Bedingung erfiillt wäre; sondern bei einer speziellen Wahl dieser Zahl, etwa für den Wert $\varepsilon_{0}$ derselben, gibt es oberhalb jeder noch so großen $Z a h l n_{0}$ (also unendlich oft) zwei natiirliche Zahlen $n$ und $n^{\prime}$, für die ist.

$$
\left|x_{n}-x_{n}\right| \geq \varepsilon_{0}
$$

4. Das 2. Hauptkriterium wird im Anschlufi an P. du Bois-Reymond (Allgemeine Funktionentheorie, Tübingen 1882) jetzt meist als allgemeinstes Konvergenzprinzip bezeichnet. Es stammt der Sache nach von $B$. Bolzano (1817, vgl. O. Stolz, Mathem. Ann. Bd. 18, 1881, S.259), ist aber erst von A. L. Cauchy als formuliertes Prinzip an die Spitze gestellt worden (Analyse algébrique, S. 125).

Unserm Hauptkriterium kann man noch etwas andere Formen geben, die manchmal für die Anwendung bequemer sind. Wir denken uns dazu die Bezeichnung der Zahlen $n$ und $n^{\prime}$ so gewählt, daß $n^{\prime}>n$ ist und also $n^{\prime}=n+k$ gesetzt werden kann, wenn $k$ wieder eine natürliche Zahl bedeutet. Dann lautet das

\section{○II. Hauptkriterium (Form 1a).}

Die Folge $\left(x_{n}\right)$ ist dann und nur dann konvergent, wenn sich nach Wahl von $\varepsilon>0$ immer eine Zahl $n_{0}=n_{0}(\varepsilon)$ so angeben läßt, daß nun für jedes $n>n_{0}$ und jedes $k \geqq 1$ ste's

ausfällt.

$$
\left|\boldsymbol{x}_{\boldsymbol{n}+\boldsymbol{k}}-\boldsymbol{x}_{\boldsymbol{n}}\right|<\boldsymbol{\varepsilon}
$$

Aus dieser Fassung des Kriteriums können wir noch weitere Schlüsse ziehen. Denkt man sich nämlich ganz willkürliche natürliche Zahlen $k_{1}, k_{2}, \ldots, k_{n}, \ldots$ ausgewählt, so muß nach dem vorigen für $n>n_{0}$ doch stets

$$
\left|x_{n+k_{n}}-x_{n}\right|<\varepsilon
$$


sein. Dies bedeutet aber, daß die Folge der Differenzen

$$
d_{n}=\left(x_{n+k_{n}}-x_{n}\right) \text { 。 }
$$

eine Nullfolge bildet. - Um uns hier bequemer verständigen zu können, wollen wir die Folge $\left(d_{n}\right)$ kurz eine Differenzenfolge von $\left(x_{n}\right)$ nennen. In ihr ist also $d_{n}$ die Differenz von $x_{n}$ gegen irgendein bestimmtes späteres Glied. Dann können wir unser Kriterium auch so formulieren:

\section{$\circ$ II. Hauptkriterium (2. Form).}

Die Folge $\left(x_{n}\right)$ ist dann und nur dann konvergent, wenn jede ihrer Differenzenfolgen eine Nullfolge ist.

Beweis. Die Notwendigkeit dieser Bedingung haben wir eben schon gezeigt; es muß noch bewiesen werden, daß sie hinreicht. Wir setzen demgemäß voraus, daß jede Differenzenfolge gegen Null strebt, und haben zu zeigen, daß $\left(x_{n}\right)$ konvergiert. Wäre aber $\left(x_{n}\right)$ divergent, so hieße das nach 48,3, daß es bei Wahl einer speziellen Zahl $\varepsilon_{0}>0$ oberhalb jeder noch so großen Zahl $n_{0}$ immer noch (also unendlich oft) zwei Zahlen $n$ und $n^{\prime}=n+k$ gäbe, für die die Differenz

$$
\left|x_{n+k}-x_{n}\right| \geqq \varepsilon_{0}
$$

ausfällt. Da dies unendlich oft der Fall sein müßte, so gäbe es also entgegen der Voraussetzung - doch Differenzenfolgen, die nicht gegen 0 strebten $\left.{ }^{1}\right), \quad\left(x_{n}\right)$ mu $\beta$ also doch konvergieren, w. z. b. w.

Bemerkung. Ist $\left(x_{n}\right)$ konvergent und wählt man eine spezielle Differenzenfolge $\left(d_{n}\right)$, so strebt also sicher $d_{n} \rightarrow 0$. Dagegen sei ausdrücklich betont, dafs aus $d_{n} \rightarrow 0$ allein noch nicht die Konvergénz von $\left(x_{n}\right)$ zu folgen braucht. Vielmehr ist es dazu erst hinreichend, wenn sich jede beliebige Differenzenfolge (nicht nur eine spezielle) als Nullfolge erweist.

Ist $z$. B. die Folge $(1,0,1,0,1, \ldots)$ vorgelegt, so ist jede. Differenzenfolge derselben eine Nullfolge, bei der alle $k_{n}$ (von einer Stelle ab) gevade Zahlen sind. Trotzdem ist die vorgelegte Folge nicht konvergent.

Indem wir die zuletzt erhaltene Fassung des Kriteriums noch ein wenig erweitern, können wir es endlich so formulieren:

\section{○II. Hauptkriterium (3. Form).}

Ist $v_{1}, v_{2}, \ldots, v_{n}, \ldots$ irgendeine Folge natürlicher Zahlen ${ }^{2}$ ), die nur gegen $+\infty$ divergiert, und sind $k_{1}, k_{2}, \ldots, k_{n}, \ldots$ irgendwelche (also keinerlei Beschränkung unterworfene) natürliche Zahlen, und nennt man nun wieder die Folge der Differenzen

$$
d_{n}=x_{v_{n}+k_{n}}-x_{v_{n}}
$$

1) Denn bezeichnet man die unendlich vielen Werte von $n$, für die jene Ungleichung (bei jedesmal passender Wahl von $k$ ) möglich sein soll, mit $n_{1}, n_{2}, n_{3}, \ldots$, so gäbe es eine Differenzenfolge, deren $n_{1}{ }^{\text {tes }}, n_{2}$ tes, $n_{3}$ tes,.. Glied absolut genommen $\geqq \varepsilon_{0}>0$ wäre. Diese könnte dann keine Nullfolge sein.

2) Gleich oder ungleich, monoton oder nicht. 
kurz eine Differenzenfolge von $\left(x_{n}\right)$, so ist es für die Konvergenz von $\left(x_{n}\right)$ wieder notwendig und hinreichend, daß $\left(d_{n}\right)$ jedesmal eine Nullfolge ist.

Beweis. Daß diese Bedingung hinreichend ist, ist nach der vorigen Form des Kriteriums selbstverständlich, da $\left(d_{n}\right)$ nun auch immer eine Nullfolge sein muß, wenn $v_{n}=n$ gewählt wird. Und daß sie notwendig ist, ist auch sofort einzusehen. Denn ist $\varepsilon>0$ gewählt, so gibt es im Falle der Konvergenz von $\left(x_{n}\right)$ jedenfalls (s. Form 1a) eine Zahl $m$, so daß für jedes $n>m$ und jedes $k \geqq 1$ stets

$$
\left|x_{n+k}-x_{n}\right|<\varepsilon
$$

ist. Da nun $v_{n} \rightarrow+\infty$ divergiert, muß es eine Zahl $n_{0}$ geben, so daß für $n>n_{0}$ stets $\quad v_{n}>m$

ausfällt. Dann ist aber nach dem vorigen für $n>n_{0}$ stets

$$
\left|x_{\boldsymbol{v}_{n}+k_{n}}-x_{\boldsymbol{v}_{n}}\right|=\left|d_{n}\right|<\varepsilon
$$

d. h. $\left(d_{n}\right)$ ist eine Nullfolge, w. z. b. w.

\section{$\S 10$. Häufungswerte und Häufungsgrenzen.}

Der Begriff der Konvergenz einer Zahlenfolge, wie wir ihn in den beiden vorangehenden Paragraphen festgelegt haben, gestattet noch eine zweite, etwas allgemeinere Behandlungsart, bei der wir gleichzeitig einige weitere für alles Folgende höchst wichtige Begriffe kennen lernen werden.

Schon in 39, 6 veranschaulichten wir uns die Tatsache, daß eine gegen $\xi$ konvergierende Zahlenfolge $\left(x_{n}\right)$ vorliegt, dadurch, daß wir sagten, in jeder (noch so kleinen) $\varepsilon$-Umgebung von $\xi$ müßten alle Glieder der Folge - von höchstens endlich vielen Ausnahmen abgesehen - gelegen sein. Es liegen also in jeder noch so kleinen Umgebung von $\xi$ jedenfalls unendlich viele Glieder der Folge. Man nennt darum $\xi$ einen Häufungswert der vorgelegten Folge. Solche Punkte können, wie wir gleich sehen werden, auch bei divergenten Zahlenfolgen vorhanden sein, und wir definieren darum ganz allgemein:

- Definition. Eine Zahl $\xi$ soll Häufungswert, Häufungspunkt oder Häufungsstelle einer vorgelegten Zahlenfolge $\left(x_{n}\right)$ heißen, wenn in jeder noch so kleinen Umgebung von $\xi$ unendlich viele Glieaer der Zahlenfolge gelegen sind; oder also, wenn es nach Wahl einer beliebigen Zahl $\varepsilon>0$ immer noch unendlich viele Indizes $n$ gibt, für die

ist.

$$
\left|x_{n}-\xi\right|<\varepsilon
$$

Bemerkungen und Beispiele.

53. 1. Der Unterschied gegenüber der Definition 39 des Grenzwertes liegt, wie schon angedeutet, darin, daf $\left|x_{n}-\xi\right|<\varepsilon$ nicht für alle $n$ von einer Stelle an, sondern nur für irgendwelche unendlich vielen $n$ erfullt $z u$ sein braucht, also insbesondere jenseits jeder Stelle $n_{0}$ noch immer für mindestens ein $n$. 
Andererseits ist gemäf $\mathbf{3 9}$ der Grenzwert $\xi$ einer konvergenten Folge $\left(x_{n}\right)$ stets ein Häufungswert derselben.

2. Die Folge $\mathbf{6}, 1$ hat die Häufungsstelle $0 ; \mathbf{6}, 4$ hat die Häufungspunkte 0 und 1 . (Jede Zahl, die unendlich oft in einer Folge $\left(x_{n}\right)$ auftritt, ist also eo ipso ein Häufungswert derselben.) $\mathbf{6}, 2,7$ und 11 haben keinen Häufungswert; 6, 9 und 10 haben den Häufungswert 1 .

3. Wir bilden jetzt ein Beispiel von mehr als illustrativer Bedeutung: Ist $p$ eine natürliche $Z a h l \geq 2$, so gibt es ersichtlich nur endlich viele positive Brüche, für die die Summe aus Zähler und Nenner $=p$ ist, nämlich die Brüche $\frac{p-1}{1}, \frac{p-2}{2}, \ldots, \frac{1}{p-1}$. Von diesen denken wir uns alle diejenigen gestrichen, die sich kürzen lassen, und reihen nun die so für $p=2,3,4, \ldots$ zu bildenden Brïche aneinander. Dadurch erhält man eine mit

$$
1,2, \frac{1}{2}, 3, \frac{1}{3}, 4, \frac{3}{2}, \frac{2}{3}, \frac{1}{4}, \ldots
$$

beginnende Zahlenfolge, die alle positiven vationalen Zahlen enthält. Fïgen wir hinter einen jeden dieser Brüche den entgegengesetzten Wert ein und stellen die 0 voran, so treten in der entstehenden Zahlenfolge

$$
\begin{gathered}
\text { (b) } 0,1,-1,2,-2, \frac{1}{2},-\frac{1}{2}, 3,-3, \frac{1}{3},-\frac{1}{3}, 4,-4, \\
\frac{3}{2},-\frac{3}{2}, \frac{2}{3},-\frac{2}{3}, \frac{1}{4}, \ldots
\end{gathered}
$$

ersichtlich alle rationalen Zahlen und eine jede genau einmal auf.

Für diese merkwürdige Zahlenfolge ist nun jede reelle Zahl ein Häufungswert, denn in jeder Umgebung jeder reellen Zahl liegen unendlich viele rationale Zahlen (vgl. S. 11).

4. Das hier benutzte Anordnungsprinzip werden wir häufiger gebrauchen. Wir formulieren es darum etwas allgemeiner: Für $k=0,1,2, \ldots$ sei je eine Zahlenfolge

$$
x_{0}^{(k)}, x_{1}^{(k)}, x_{2}^{(k)}, \ldots \quad(k=0,1,2, \ldots)
$$

vorgelegt. Dann kann man auf mannigfache Weisen eine Zahlenfolge $\left(x_{n}\right)$ bilden, die jedes Glied einer jeden dieser Folgen und ein jedes genau einmal enthält.

Der Beweis besteht einfach darin, dafs man eine Zahlenfolge $\left(x_{n}\right)$ angibt, die das Verlangte leistet. Dazu schreiben wir die gegebenen Folgen zeilenweise untereinander:

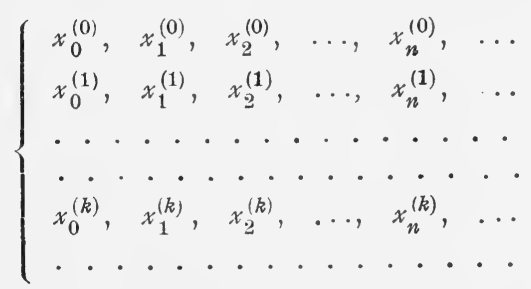

Dann stehen in derjenigen „Schräglinie" dieses Schemas, die das Element $x_{0}^{(p)}$ mit dem Element $x_{p}^{(0)}$ verbindet, alle Elemente $x_{n}^{(k)}$, für die $k+n=p$ ist, und nur diese. Ihre Anzahl ist $=p+1$. Diese Glieder reihen wir für $p=0,1,2, \ldots$ aneinander, jede Schräglinie etwa von unten nach oben durchlaufend. So entsteht die Folge

$$
x_{0}^{(0)}, \quad x_{0}^{(1)}, \quad x_{1}^{(0)}, \quad x_{0}^{(2)}, \quad x_{1}^{(1)}, x_{2}^{(0)}, \quad x_{0}^{(3)}, \quad x_{1}^{(2)}, \ldots
$$

die ersichtlich das Verlangte leistet. (Anordnung nach Schräglinien). 
Eine andere oft benutzte Anordnung ist die „nach Quadraten “. Hierbei schreibt man erst die Elemente $x_{0}^{(p)}, x_{1}^{(p)}, \ldots$, $x_{p}^{(p)} \operatorname{der} p^{\text {ten }}$ Zeile, dann die im obigen Schema senkrecht tiber $x_{p}^{(p)}$ stehenden Elemente $x_{p}^{(p-1)}, \ldots, x_{p}^{(0)}$ auf. Reiht man nun diese Gruppen von je $2 p+1$ Gliedern für $p=0,1,2, \ldots$ aneinander, so entsteht die mit

$$
x_{0}^{(0)}, \quad x_{0}^{(1)}, \quad x_{1}^{(1)}, \quad x_{1}^{(0)}, \quad x_{0}^{(2)}, \quad x_{1}^{(2)}, \quad x_{2}^{(2)}, x_{2}^{(1)}, x_{2}^{(0)}, x_{0}^{(3)}, \ldots
$$

beginnende Anordmung nach Quadraten.

Bestehen einige oder alle Zeilen des obigen Schemas nur aus je endlich vielen Gliedern, oder besteht das Schema nur aus endlich vielen Zeilen, so erfahren die beschriebenen Anordnungsarten eine leichte, sofort zu ubersehende Modifikation.

5. Ein zu 3. ähnliches Beispiel ist dieses: Für jedes $p \geq 2$ gibt es nur genau $p-1$ Zahlen der Form $\frac{1}{k}+\frac{1}{m}$, für die die Summe der natiirlichen Zahlen $k$ und $m$ gleich $p$ ist. Denken wir uns diese für $p=2,3,4, \ldots$ aneinandergereiht, so entsteht die Folge

$$
2, \quad \frac{3}{2}, \frac{3}{2}, \frac{4}{3}, 1, \frac{4}{3}, \frac{5}{4}, \frac{5}{6}, \frac{5}{6}, \ldots .
$$

Man findet, daß diese die Häufungswerte $0,1, \frac{1}{2}, \frac{1}{3}, \frac{1}{4}, \ldots$ und keine andern besitzt.

6. Wie der Grenzwert einer konvergenten Zahlenfolge, so brauchen auch die Häufungswerte einer beliebigen Folge nicht selbst in der Folge aufzutreten. So gehören in 3. die irrationalen Zahlen und in 5 . die 0 gewißs nicht zu der betreffenden Folge. Dagegen ist in beiden Fällen z. B. sowohl Häufungswert als auch Glied der Folge.

Grundlegend für unsere Zwecke ist nun der schon von B. Bolzano ${ }^{1}$ ) ausgesprochene, in seiner Bedeutung erst von $K$. Weierstra $\beta^{2}$ ) voll erkannte

- Satz. Jede beschränkle Zahlenfolge besitzt mindestens einen Häufungswert.

Beweis. Wir erfassen die fragliche Zahl wiedér durch eine passende Intervallschachtelung. Nach Voraussetzung gibt es ein Intervall $J_{0}$, in dem alle Glieder der gegebenen Folge $\left(x_{n}\right)$ gelegen sind. Auf dieses wenden wir die Halbierungsmethode an und nennen seine linke oder seine rechte Hälfte $J_{1}$, je nachdem schon in seiner linken Hälfte unendlich viele Glieder der Folge liegen oder nicht. Nach derselben Regel bezeichnen wir dann eine bestimmte Hälfte von $J_{1}$ mit $J_{2}$, usw. Die Intervalle der so entstehenden Schachtelung $\left(J_{n}\right)$ haben dann alle die Eigenschaft, daß in ihnen unendlich viele Glieder der Folge liegen, während links von ihrem linken Endpunkt jedesmal nur endlich viele Punkte derselben liegen können. Der erfaßte Punkt $\xi$

1) Rein analytischer Beweis des Lehrsatzes, daf ..., Prag, 1817.

2) In dessen Vorlesungen. 
ist offenbar Häufungswert; denn ist $\varepsilon>0$ beliebig gegeben, so wähle man aus der Folge der Intervalle $J_{n}$ eines aus, etwa $J_{p}$, dessen Länge $<\varepsilon$ ist. Die unendlich vielen Glieder der Folge $\left(x_{n}\right)$, die dem 。 Intervall $J_{p}$ angehören, liegen dann von selbst auch in der $\varepsilon$-Umgebung von $\xi$, - womit schon alles bewiesen ist.

Die Ähnlichkeiten in der Definition von Häufungswert und Grenzwert begründet natürlich trotz des in $\mathbf{5 3}, 1$ betonten Unterschiedes beider (,,jeder Grenzwert ist zugleich Häufungswert, aber nicht umgekehrt") auch eine gewisse Verwandtschaft beider. Diese findet ihre Klärung in dem folgenden

- Satz. Jeder Häufungswert einer Folge $\left(x_{n}\right)$ kann als Grenzwert $\mathbf{5 5}$. einer passenden Teilfolge derselben angesehen werden.

Beweis. Da bei jedem $\varepsilon>0$ für unendlich viele Indizes $\left|x_{n}-\xi\right|<\varepsilon$ ist, so ist für ein passendes $n=k_{1}$ insbesondere $\left|x_{k_{1}}-\xi\right|<1$; für ein passendes $n=k_{2}>k_{1}$, ebenso $\left|x_{k_{2}}-\xi\right|<\frac{1}{2}$, und allgemein für ein passendes $n=k_{v}>k_{v-1}$

$$
\left|x_{k_{v}}-\xi\right|<\frac{1}{v} \quad(v=2,3, \ldots) .
$$

Für die hierdurch herausgehobene Teilfolge $\left(x_{n}{ }^{\prime}\right)=\left(x_{k_{n}}\right)$ strebt dann $x_{n}{ }^{\prime} \rightarrow \xi$, weil $\left(x_{k_{n}}-\xi\right)$ nach $\mathbf{2 6}, 2$ eine Nullfolge bildet.

Der Beweis des Bolzano-Weierstraßschen Satzes gibt noch zu einer weiteren sehr wichtigen Bemerkung Anlaß: Die Intervalle $J_{n}$ der dort konstruierten Schachtelung hatten nicht nur die Eigenschaft, daß in ihnen stets unendlich viele Glieder der Folge $\left(x_{n}\right)$ lagen, sondern, wie betont, noch die weitere, daß links rom linken Endpunkt eines bestimmten der Intervalle immer nur endlich viele Glieder der Folge lagen. Hieraus folgt aber sofort, daß links von dem erfaßten Häufungswert $\xi$ kein weiterer Häufungswert gelegen sein ḳann. Wählt man nämlich irgendeine reelle $\mathrm{Zahl} \xi^{\prime}<\xi$, so wäre $\varepsilon=\frac{1}{2}\left(\xi-\xi^{\prime}\right)>0$; und wenn wir nun ein Intervall $J_{q}$ auswählen, dessen Länge $<\varepsilon$ ist, so läge die ganze $\varepsilon$-Umgebung des Punktes $\xi^{\prime}$ links vom linken Endpunkt des Intervalles $J_{q}$ und kann also nur endlich viele Glieder der Folge enthalten. Kein links von $\xi$ gelegener Punkt $\xi^{\prime}$ kann also noch ein Häufungspunkt der Folge $\left(x_{n}\right)$ sein, und wir haben den

Satz. Jede beschränkte Zahlenfolge besitzt einen wohlbesimmten 56. kleinsten Häufungswert.

Vertauscht man in diesen Betrachtungen sinngemäß rechts und links, so erhält $\operatorname{man}^{1}$ ) ganz entsprechend den

Satz. Jede beschränkte Zahlenfolge besitzt einen wohlbestimmten 5 \%. größten Häufungswert ${ }^{2}$ ).

1) Oder durch Spiegelung am Nullpunkt.

2) Diese Sätze sind wieder nur in dem Falle nicht selbstverstäindlich, daßs 
Diese beiden speziellen Häufungswerte wollen wir durch eine besondere Benennung auszeichnen:

58. Definition. Der kleinste Häufungswert einer (beschränkten) Zahlenfolge soll deren untere Häufungsgrenze, ihr unterer Limes oder ihr Limes inferior genannt werden. Bezeichnet man ihn mit $x$, so schreibt man

$$
\lim _{n \rightarrow \infty} x_{n}=\varkappa \quad \text { oder } \quad \liminf _{n \rightarrow \infty} x_{n}=\varkappa
$$

(ev. unter Fortlassung des Zusatzes , $n \rightarrow \infty$ "). Ist $\mu$ der größte Häufungswert der Folge, so schreibt man

$$
\varlimsup_{n \rightarrow \infty} x_{n}=\mu \quad \text { oder } \quad \limsup _{n \rightarrow \infty} x_{n}=\mu
$$

und nennt $\mu$ die obere Häufungsgrenze, den oberen Limes oder den Limes superior der Folge $\left(x_{n}\right)$. Es ist notwendig stets $x \leqq \mu$.

$\mathrm{Da}$ in jeder $\varepsilon$-Umgebung des Punktes $\varkappa$ unendlich viele Glieder der Folge $\left(x_{n}\right)$ liegen, da andererseits links vom linken Endpunkt einer solchen Umgebung nur endlich viele Glieder der Folge liegen dürfen, so ist $\varkappa$ (bezw $\mu$ ) auch durch folgende Bedingungen charakterisiert:

59. Satz. Die Zahl $\varkappa(b z w . \mu)$ ist dann und nur dann untere (bzw. obere) Häufungsgrenze dar Folge $\left(x_{n}\right)$, wenn nach Wahl eines beliebigen $\varepsilon>0$ doch noch für unendlich viole $n$ die Unleichung

$$
x_{n}<x+\varepsilon(b z w .>\mu-\varepsilon),
$$

dagegen für höchstens endlich viele $n$ die Ungleichung

erfïllt ist ${ }^{1}$ ).

$$
x_{n}<x-\varepsilon(b z w .>\mu+\varepsilon)
$$

Ehe wir hierzu noch einige Beispiele und Erläuterungen geben, ergänzen wir unsere Festsetzungen noch für den Fall nicht beschränkter Zahlenfolgen.

60. Definitionen. 1. Ist eine Folge nach links nicht beschränkt, so wollen wir sagen, es sei $-\infty$ ein Häufungswert derselben; und ist sie nach rechts nicht beschränk:, so wollen wir $+\infty$ einen Häufungswert derselben nennen. In diesen Fällen besitzt die Folge, wie groß auch die Zahl $G>0$ gewählt wird, immer noch unendlich viele Glieder unterhalb $-G$ bzw. oberhalb $+G^{2}$ ).

die Folge $\left(x_{n}\right)$ unendlich viele Häufungswerte hat, wie z. B. die Folge 53, 5 . Denn unter endlich vielen Werten müßte ja eo ipso ein kleinster und ein größter vorhanden sein.

1) Oder: von einer Stelle $n_{0} a b$ ist nie mehr $x_{n}<x-\varepsilon(>\mu+\varepsilon)$; dagegen ist jenseits jeder Stelle $n_{1}$ immer wieder einmal $x_{n}<x+\varepsilon(>\mu-\varepsilon)$.

$\left.{ }^{2}\right)$ Es spielt hier also - und ebenso bei den folgenden Festsetzungen der rechts von $+G$ gelegene Teil der Zahlengeraden die Rolle der $\varepsilon$-Umgebung von $+\infty$, der links von $-G$ gelegene Teil die der $\varepsilon$-Umgebung von $-\infty$. 
2. Ist also die Folge $\left(x_{n}\right)$ nach links nicht beschränkt, so ist $-\infty$ eo ipso ihr kleinster Häufungswert, so daß wir

$$
x=\lim _{n \rightarrow+\infty} x_{n}=-\infty
$$

setzen müssen. Ebenso müssen wir

$$
\mu=\varlimsup_{n \rightarrow+\infty} x_{n}=+\infty
$$

setzen, talls die Folge nach rechts nicht beschränkt ist. Es ist dann, wie auch $G>0$ gewählt wird, stets für unendlich viele Indizes $x_{n}<-G$ bzw. $x_{n}>+G$.

3. Wenn endlich die Folge nach rechts nicht beschränkt ist und (außer $+\infty$ ) sonst keinen Häufungspunkt hat, so ist $+\infty$ nicht nur ihr größter, sondern zugleich auch ihr kleinster Häufungswert, und wir werden daher auch ihren unteren Limes $=+\infty$ setzen:

$$
x=\lim _{n \rightarrow+\infty}=+\infty \text {; }
$$

und entsprechend wird man den oberen Limes $=-\infty$, also

$$
\mu=\varlimsup_{n \rightarrow+\infty}=-\infty
$$

setzen müssen, wenn die Folge nach links nicht beschränkt ist und (außer - $-\infty$ ) sonst keinen Häufungswert hat. Der erste (bzw. zweite) Fall liegt dann und nur dann vor, wenn nach Wahl einer beliebigen Zahl $G>0$ für unendlich viele $n$ die Ungleichung

$$
x_{n}>G\left(b z w . x_{n}<-G\right) \text {, }
$$

dagegen für höchstens endlich viele $n$ die Ungleichung

erfüllt ist.

$$
x_{n}<G\left(b z w . x_{n}>-G\right)
$$

Beispiele und Erläuterungen.

1. Auf Grund aller getroffenen Festsetzungen definiert nun jede Zahlenfolge von sich aus und in völlig eindeutiger Weise zwei wohlbestimmte Zeichen, $\varkappa$ und $\mu$, die nun allerdings auch die Bedeutung $+\infty$ oder $-\infty$ haben können und die in der Größenordnung $x \leqq \mu^{1}$ ) zueinander stehen. Und die nachfolgen den Beispiele lehren, daf $\varkappa$ und $\mu$ alle mit dieser Ungleichung $\varkappa \leqq \mu$ verträglichen endlichen oder unendlichen Werte auch tatsächlich haben können.
Denn für die Folge
$\left(x_{1}, x_{2}, x_{3}, \ldots\right)$
1. $(n) \equiv 1,2,3,4, \ldots$
2. $\left(a+n(-1)^{n}\right)=a+1, a+2, a+\frac{1}{3}, a+4, \ldots$
3. $a, b, a, b, a, b, \ldots \quad(a<b)$
4. $\left(a+\frac{(-1)^{n}}{n}\right)=a-1, a+\frac{1}{2}, a-\frac{1}{3}, a+\frac{1}{4}, \ldots$
5. $\left((-1)^{n} \cdot n\right) \equiv-1,+2,-3,+4, \ldots$
6. $\left(a-n^{(-1)^{n}}\right) \equiv a-1, a-2, a-\frac{1}{3}, a-4, \ldots$
7. $(-n) \equiv-1,-2,-3, \ldots$
ist

1) Von jeder reellen Zahl sagt man, sie sei $<+\infty$ und $>-\infty$, und bezeichnet sie darum gelegentlich ausdrücklich als eine "endliche". 
2. Man beachte besonders, dafi es mit dem Satze $\mathbf{5 9}$ nicht im Widerspruch steht, wenn links von $\varkappa$ oder rechts von $\mu$ unendlich viele Glieder der Folge liegen. So ist $z$. B. für die Folge $\left((-1)^{n} \frac{n+1}{n}\right)$, also für die Folge $-2,+\frac{3}{2},-\frac{4}{3},+\frac{5}{4},-\frac{6}{5}, \ldots$ ersichtlich $\varkappa=-1, \mu=+1$, und es liegen sowohl links von * als auch rechts von $\mu$ unendlich viele Glieder der Folge (und zwischen $*$ und $\mu$ liegt kein Glied derselben!). Es brauchen also keineswegs außerhalb des Intervalles $\varkappa \ldots \mu$ nur endlich viele Glieder der Folge zu liegen. Satz 59 besagt ja auch nur, daß links von $\varkappa-\varepsilon$ bzw. rechts von $\mu+\varepsilon$ (bei positivem $\varepsilon$ ) nur endlich viele Glieder der Folge liegen können.

3. "Endlich viele Änderungen" haben auf die Häufungswerte einer Folge, speziell auf deren beide Häufungsgrenzen $\psi$ und $\mu$ keinen Einflufi. Diese bezeichnen also eine infinitäre Eigenschaft der Folge.

4. Da durch die Folge $\left(x_{n}\right)$ auch jede der beiden Zahlen $*$ und $\mu$ völlig eindeutig bestimmt ist, und da ihr Wert ja auch im Anschluß an unsere Definition durch eine wohlbestimmte Intervallschachtelung erfaßt wurde, so haben wir hierin wieder ein neues legitimes Mittel, um reelle Zahlen zu definieren, zu bestimmen, zu geben: eine reelle Zahl ist hinfort auch als, ,gegeben" anzusehen, wenn sie die untere oder obere Häufungsgrenze einer gegebenen Zahlenfoige ist. Dieses Mittel zur Bestimmung reeller Zahlen ist ersichtlich noch allgemeiner als das bei 41,1 angegebene, weil jetzt die benutzte Zahlenfolge gar nicht konvergent zu sein braucht und überhaupt keiner Beschränkung mehr unterliegt ${ }^{1}$ ).

5. Wie man im Anschluf an $\mathbf{5 5}$ erkennt, gilt noch der folg€nde

Satz. Die obere Häufungsgrenze $\mu$ der Folge $\left(x_{n}\right), \mu=\varlimsup_{\lim } x_{n}$, ist auch durch folgende beide Bedingungen charakterisiert:

a) der Grenzwert $\xi^{\prime}$ jeder aus $\left(x_{n}\right)$ herausgehobenen konvergenten Teilfolge $\left(x_{n}{ }^{\prime}\right)$ ist stets $\leqq \mu$; aber es gibt

b) mindestens eine solche Teilfolge, deven Grenzwert gleich $\mu$ ist ; - und entsprechend für die untere Häufungsgrenzc.

6. Ein mit den Häufungsgrenzen verwandter, aber doch scharf von ihnen zu trennender Begriff ist der der oberen und unteren Grenze einer Zahlenfolge $\left(x_{\eta}\right)$, der sich durch folgende Bemerkung ergibt: Liegt rechts von $\mu=\lim x_{n}$ kein Glied der Folge, ist also steis $x_{n} \leq \mu$, so ist $\mu$ eine obere Schranke $(\mathbf{S}, 4)$ der Folge, - aber eine solche, die nun nicht mehr durch eine kleinere ersetzt werden kann. Es ist dann $\mu$ also die kleinste obere Schranke. Eine solche gibt es aber auch, wenn ein Glied der Folge $>\mu$ ist. Denn ist etwa $x_{p}>\mu$, so gibt es nach $\mathbf{5 9}$ sicher nur endlich viele Glieder in der Folge, die $\geq x_{p}$ sind, und unter diesen notwendig $(\mathbf{S}, 5)$ ein gröfstes, etwa $x_{\eta}$. Dann ist stets $x_{n} \leqq x_{\eta}$, d. h. $x_{q}$ ist eine obere Schranke der Folge, - aber wieder eine solche, die durch keine kleinere ersetzt werden kann. Jede nach rechts beschränkte Zahlenfolge besitzt also eine wohlbestimmte kleinste obere Schranke. Da ebenso jede nach links beschränkte Zahlenfolge eine wohlbestimmte größte untere Schranke haben mufs, so sind wir zu folgender Erklärung berechtigt:

1) Während uns also zunächst die Intervallschachtelung als das einzige Mittel zur Erfassung reeller Zahlen gelten sollte, haben wir jetzt eine ganze Anzahl anderer Mittel daraus hergeleitet, die nun auch als legitime Mittel zugelassen sind: Die Systembrüche, der Dedekindsche Schnitt, konvergente Zahlenfolgen, obere und untere Häufungsgrenze einer Folge. In allen diesen Fällen aber hatten wir gesehen, wie man sofort auch eine Intervallschachtelung angeben kann, welche die betreffende Zahl erfaßt. 
Definition. Als obere Grenze einer nach rechts beschränkten Zahlenfolge bezeichnet man die nach den Vorbemerkungen stets bestimmte kleinste ihrer oberen Schranken, ebenso als untere Grenze einer nach links beschränkten Zahlenfolge die größte unter ihren unteren Schranken. Von einer nach rechts nicht beschräkten Zahlenfolge sagt man, ihre obere Grenze sei $+\infty$, von einer nach links nicht beschränten Folge entsprechend, ihre untere Grenze sei $-\infty$.

7. Die Begriffe der unteren und oberen Häufungsgrenze rühren von A. L. Cauchy (Analyse algébrique, Paris 1821, S. 132) her, sind aber erst durch $P$. $d u$ Bois-Reymond (Allgemeine Funktionentheorie, Tübingen 1882) allgemein bekannt geworden. Die Benennung und Bezeichnung schwankt bis auf den heutigen Tag. Die besonders bequeme, im Text benutzte Bezeichnug durch lim und lim ist von A. Pringsheim (Sitzungeber. d. Akad. zu München, Bd. 28, 1898, S. 62) eingeführt worden, von dem auch die Benennungen als unterer und oberer Limes herrühren. Die im Texte. benutzte ausfuhrlichere Bezeichnung Häufungsgrenze soll nur den Unterschied zu der soeben definierten unteren und oberen Grenze stärker betonen.

Die bisherigen Untersuchungen dieses Paragraphen waren völlig unabhängig von den Konvergenzbetrachtungen der $\$ \S 8$ und 9 gehalten und geben uns eben dadurch ein neues Mittel an die Hand, das Konvergenzproblem $\mathrm{A}$ aus $\S 9$ anzugreifen. Es läßt sich zeigen, daß die Kenntnis der beiden Häufungsgrenzen $\varkappa$ und $"$ einer Zahlenfolge - also die Kenntnis zweier Zahlen, deren Existenz von vornherein feststeht! - vollständig ausreicht, um über die Konvergenz oder Divergenz dieser Zahlenfolge zu entscheiden. Es gelten nämlich die Sätze

Satz 1. Die Zahlenfolge $\left(x_{n}\right)$ ist dann und nur dann konvergent, $\mathbf{6 3 .}$ wenn ihre Häufungsgrenzen $x$ und " einander gleich und endlich sind. Ist $\lambda$ der gemeinsame (und also von $+\infty$ und $-\infty$ verschiedene) Wert von $\varkappa$ und $\mu$, so strebt $x_{n} \rightarrow \lambda$.

Beweis. a) Es sei $\varkappa=\mu$ und ihr gemeinsamer Wert $=\lambda$. Dann ist nach $\mathbf{5 9}$ bei gegebenem $\varepsilon$ für höchstens endlich viele $n$ die Ungleichung

$$
x_{n}<\varkappa-\varepsilon=\lambda-\varepsilon
$$

und ebenso für höchstens endlich viele $n$ die Ungleichung

$$
x_{n}>\mu+\varepsilon=\lambda+\varepsilon
$$

erfüllt. Von einer Stelle $n_{0}$ ab ist also stets

$$
\lambda-\varepsilon<x_{n}<\lambda+\varepsilon \text { oder }\left|x_{n}-\lambda\right|<\varepsilon,
$$

d. h. die Folge ist konvergent und $\lambda$ ihr Grenzwert.

b) Ist umgekehrt $\lim x_{n}=\lambda$, so ist nach Wahl von $\varepsilon>0$ für alle $n>n_{0}(\varepsilon)$ stets $\lambda-\varepsilon<x_{n}<\lambda+\varepsilon$. Es ist also für unendlich viele $n$ die Ungleichung

$$
x_{n}<\lambda+\varepsilon \text { bzw. }>\lambda-\varepsilon,
$$


dagegen nur für höchstens endlich viele $n$ die Ungleichung

$$
x_{n}<\lambda-\varepsilon \text { bzw. }>\lambda+\varepsilon
$$

erfüllt. Das eine besagt, $\mathrm{da} \beta \varkappa=\lambda$, das andere, $\mathrm{da} \beta \mu=\lambda$ ist. Damit ist alles bewiesen.

64. Satz 2. Die Zahlenfolge $\left(x_{n}\right)$ ist dann und nur dann bestimmt divergent, wenn ihre Häufungsgrenzen zwar einander gleich sind, ihr gemeinsamer Wert aber gleich $+\infty$ oder $-\infty$ ist $^{1}$ ). (Und im ersten Falle divergiert sie natürlich nach $+\infty$, im zweiten nach $-\infty$.)

Beweis. a) Ist $\varkappa=\mu=+\infty$ (bzw. $=-\infty)$, so heißt dies nach 60, 2 und 3, daß nach Wahl von $G>0$ von einer Stelle ab stets

$$
\left.x_{n}>+G \text { (bzw. }<-G\right)
$$

ist; es ist dann also $\lim x_{n}=+\infty$ (bzw. $=-\infty$ ).

b) Ist umgekehrt $\lim x_{n}=+\infty$, so ist nach Wahl von $G>0$ für alle $n$ von einer Stelle ab stets $x_{n}>+G$; es ist also

für höchstens endlich viele $n$ die Ungleichung $x_{n}<+G$ erfüllt, während

für unendlich viele $n$ die Ungleichung $x_{n}>+G$

gilt. Das besagt aber nach 60, daß $\varkappa=+\infty$ und also von selbst auch $\mu=+\infty$ ist. Es ist also $x=\mu=+\infty$. Und ganz entsprechend schließt man aus $\lim x_{n}=-\infty, \mathrm{da} \beta \quad x=\mu=-\infty$ ist.

65. Satz 3. Die Zahlenfolge $\left(x_{n}\right)$ ist dann und nur dann unbestimmt divergent, wenn ihre Häufungsgrenzen voneinander verschieden sind.

Beweis. a) Ist $x \neq \mu$, also $x<\mu$, so liegt weder die Situation des Satzes 1, noch die des Satzes 2 vor; es muß also $\left(x_{n}\right)$ dann notwendig unbestimmt divergieren. Und ebenso:

b) Ist $\left(x_{n}\right)$ unbestimmt divergent, so liegt gleichfalls weder die Situation des 1 . noch die des 2 . Satzes vor, und es, kann daher nicht $\varkappa=\mu$ sein.

Der Inhalt dieser drei Sätze liefert uns folgendes.

66. Drittes Hauptkriterium für das Konvergenzverhalten von Zahlenfolgen:

Die Folge $\left(x_{n}\right)$ verhält sich bestimmt oder unbestimmt, je nachdem ihre Häufungsgrenzen einander gleich oder voneinander verschieden sind. Im Falle bestimmten Verhaltens ist sie konvergent oder divergent, je nachdem der gemeinsame Wert der Häufungsgrenzen endlich oder unendlich ist.

1) Daß man die Symbole $+\infty$ und $-\infty$, die ja gewí̋ keine Zahlen sind, gelegentlich doch als „Werte" anspricht, darf nur als eine (nicht wichtig zu nehmende) sprachliche Freiheit angesehen werden. 
Über die Möglichkeiten im Konvergenzverhalten einer Zahlenfolge und über die dabei benutzten Bezeichnungen geben wir noch in folgender Tabelle eine Übersicht.

\begin{tabular}{|c|c|c|}
\hline$x=\mu$, beide $=\lambda \neq \pm \infty$ & $x=\mu=+\infty$ oder $-\infty$ & $x<\mu$ \\
\hline $\begin{array}{c}\text { konvergent (mit dem } \\
\text { Grenzwert } \lambda \text { ) } \\
\lim _{x_{n}}=\lambda \\
(n \rightarrow+\infty) \\
x_{n} \rightarrow \lambda \\
(\text { für } n \rightarrow+\infty)\end{array}$ & $\begin{array}{l}\text { divergent (ev. auch: konver- } \\
\text { gent) gegen (oder: mit dem } \\
\text { Grenzwert) }+\infty \text { bzw. }-\infty \text {; } \\
\text { beides: bestimmt divergent. } \\
\text { lim } x_{n}=+\infty \text { bzw. }-\infty \\
\quad x_{n} \rightarrow+\infty \text { bzw. }-\infty\end{array}$ & $\begin{array}{c}\text { unbestimmt } \\
\text { divergent }\end{array}$ \\
\hline konvergent & \multicolumn{2}{|c|}{ divergent } \\
\hline bestimmtes & Verhalten & $\begin{array}{c}\text { unbestimmtes } \\
\text { Verhalten }\end{array}$ \\
\hline
\end{tabular}

\section{\$ 11. Unendliche Reihen, Produkte und Kettenbrüche.}

Eine Zahlenfolge kann in der mannigfachsten Weise gegeben sein; die vielfachen bisher gegebenen Beispiele belegen dies hinreichend. Doch war hierbei in den überwiegend meisten Fällen das $n^{\text {te }}$ Glied $x_{n}$ der Bequemlichkeit halber durch eine explizite Formel gegeben, die es direkt zu berechnen gestattet. Das ist aber bei den Anwendungen der Zahlenfolgen in allen Teilen der Mathematik keineswegs die Regel. Hier bieten sich die zu untersuchenden Zahlenfolgen vielmehr meist indirekt dar; und zwar kommen dafür, neben mancherlei weniger wichtigen, vor'allem drei Arten in Betracht, die wir jetzt kurz besprechen wollen.

I. Unendliche Reihen. Das sind Zahlenfogen, die auf folgende Art gegeben werden. Es wird zunächst eine Zahlenfolge $\left(a_{0}, a_{1}, \ldots\right)$ auf irgendeine Weise (meist durch unmittelbare Angabe ihrer Glieder) vorgelegt, doch ohne daß sie selbst den Gegenstand der Untersuchung bilden soll. Aus ihr soll vielmehr dadurch eine neue Folge, deren Glieder wir nun mit $s_{n}$ bezeichnen, hergeleitet werden, daß

$$
s_{0}=a_{0} ; \quad s_{1}=a_{0}+a_{1} ; \quad s_{2}=a_{0}+a_{1}+a_{2}
$$

und allgemein

$$
\boldsymbol{s}_{\boldsymbol{n}}=\boldsymbol{a}_{\mathbf{0}}+\boldsymbol{a}_{\mathbf{1}}+\boldsymbol{a}_{\mathbf{2}}+\ldots+\boldsymbol{a}_{\boldsymbol{n}}, \quad(n=0,1,2, \ldots)
$$

gesetzt wird. Und erst die Folge $\left(s_{n}\right)$ dieser Zahlen soll den Gegenstand der Untersuchung abgeben. Man gebraucht für diese Folge $\left(s_{n}\right)$ die Symbolik

$$
a_{0}+a_{1}+a_{2}+\ldots+a_{n}+\ldots
$$

oder kürzer

$$
a_{0}+a_{1}+a_{2}+\ldots
$$


oder noch kürzer und prägnanter

c)

$$
\sum_{n=0}^{\infty} a_{n}
$$

und nennt dies neue Symbol eine unendliche Reihe; die Zahlen $s_{n}$ bezeichnet man als ihre Teil-oder Partialsummen. - Wir können daher sagen:

68. Definition. Eine unendliche Reihe ist ein neues Symbol für eine bestimmte aus ihm horzuleitende Zahlenfolge, nämlich für die Folge seiner Teilsummen.

Bemerkungen und Beispiele.

1. Mit $\sum_{n=0}^{x} a_{n}$ sind die Symbole

$$
a_{0}+\sum_{n=1}^{\infty} a_{n} ; \quad a_{0}+a_{1}+\sum_{n=2}^{\infty} a_{n} ; \quad a_{0}+a_{1}+\ldots+a_{m}+\sum_{n=m+1}^{\infty} a_{n}
$$

völlig gleichbedeutend. Der Index $n$ heißt hierbei der Summationsbuchstabe. Fïr ihn diurfen natiirlich auch beliebige andere Buchstaben genommen werden:

$$
\sum_{\nu=0}^{\infty} a_{\nu} ; a_{0}+a_{1}+a_{2}+\sum_{\varrho=3}^{\infty} a_{\varrho} ; \text { usw. }
$$

Die Zahlen $a_{n}$ heißsen die Glieder der Reihe. Sie brauchen nicht von 0 an indiziert zu sein. So bedeutet das Symbol

$$
\sum_{\lambda=1}^{\infty} a_{2} \text { die Zahlenfolge }\left(a_{1}, a_{1}+a_{2}, a_{1}+a_{2}+a_{3}, \ldots\right)
$$

und allgemeiner

$$
\sum_{k=p}^{\infty} a_{k}
$$

die Folge der Zahlen $s_{p}, s_{p+1}, s_{p+2}, \ldots$, die durch

$$
s_{n}=a_{p}+a_{p+1}+\ldots+a_{n} \text { für } n=p, p+1
$$

definiert sind. Hierbei darf $p \gtreqless 0$ irgendeine ganze Zahl sein. Schlieflich schreibt man auch ganz kurz

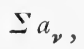

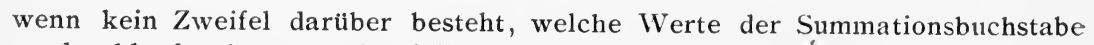
zu durchlaufen hat, - oder falls dies gleichgiiltig ist.

2. Für $n=0,1,2, \ldots$ sei $a_{n}$
a) $=\frac{1}{2^{n}}$;
b) $=\frac{1}{(n+1)(n+2)}$;
c) $=1$;
d) $=n$;
e) $=\frac{(-1)^{n}}{n+1}$;
f) $=(-1)^{n}$;
g) $=(-1)^{n}(2 n+1)$;
h) $=\frac{1}{(\alpha+n)(\boldsymbol{\alpha}+n+1)}, \alpha=$ reelle $\mathrm{Zahl} \neq 0,-1,-2, \ldots$

Dann handelt es sich um die unendliche Reihe
a) $\sum_{n=0}^{\infty} \frac{1}{2^{n}}$;
b) $\sum_{n=0}^{\infty} \frac{1}{(n+1)(n+2)}=\frac{1}{1 \cdot 2}+\frac{1}{2 \cdot 3}+\frac{1}{3 \cdot 4}+\ldots$
c) $1+1+1+\ldots$;
d) $0+1+2+3+\ldots$;
e) $\sum_{\nu=0}^{\infty} \frac{(-1)^{\nu}}{v+1}$ oder
$1-\frac{1}{2}+\frac{1}{3}-\frac{1}{4}+\ldots$; 

f) $\sum_{\lambda=0}^{\infty}(-1)^{\lambda}$ oder $1-1+1-1+\ldots$;
g) $1-3+5-7+9-+\ldots$
h) $\sum_{k=0}^{\infty} \frac{1}{(\alpha+k)(\alpha+k+1)}=\frac{1}{\alpha(\alpha+1)}+\frac{1}{(\alpha+1)(\alpha+2)}+\frac{1}{(\alpha+2)(\alpha+3)}+\cdots$

Und hierin ist lediglich ein neues - und, wie sich zeigen wird, sehr handliches - Symbol für die Zahlenfolge $\left(s_{0}, s_{1}, s_{2}, \ldots\right)$ zu sehen, bei der $s_{n}$

$$
\begin{aligned}
\text { a) } & =1+\frac{1}{2}+\frac{1}{4}+\ldots+\frac{1}{2^{n}}=2-\frac{1}{2^{n}} ; \\
\text { b) } & =\frac{1}{1 \cdot 2}+\frac{1}{2 \cdot 3}+\frac{1}{3 \cdot 4}+\ldots+\frac{1}{(n+1)(n+2)} \\
& =\left(1-\frac{1}{2}\right)+\left(\frac{1}{2}-\frac{1}{3}\right)+\ldots+\left(\frac{1}{n+1}-\frac{1}{n+2}\right)=1-\frac{1}{n+2} ; \\
\text { c) } & =n+1 ; \quad \text { d) }=\frac{n(n+1)}{2} ; \\
\text { e) } & =1-\frac{1}{2}+\frac{1}{3}-\ldots+\frac{(-1)^{n}}{n+1},(\operatorname{vgl} \cdot \mathbf{4 5}, 3 \text { und } \mathbf{4 S}, 1) ; \\
\text { f) } & \left.\left.=\frac{1}{2}\left[1-(-1)^{n+1}\right]^{1}\right) ; \quad \mathrm{g}\right)=(-1)^{n}(n+1) ; \\
\text { h) } & =\frac{1}{\alpha(\alpha+1)}+\frac{1}{(\alpha+1)(\alpha+2)}+\ldots+\frac{1}{(\alpha+n)(\alpha+n+1)} \\
& =\left(\frac{1}{\alpha}-\frac{1}{\alpha+1}\right)+\left(\frac{1}{\alpha+1}-\alpha+2\right)+\ldots+\left(\frac{1}{\alpha+n}-\frac{1}{\alpha+n+1}\right) \\
& =\frac{1}{\alpha}-\frac{1}{\alpha+n+1} \cdot
\end{aligned}
$$

ist. -

3. Wir betonen vor allem, daf die neuen Symbole von sich aus keinerlei Bedeutung haben. Zwar ist die Addition eine wohldefinierte Operation, die sich für zwei oder irgendeine bestimmte Anzahl von Zahlen stets und nur in einer Weise ausführen läft. Es haben also, wie auch die Glieder $a_{n}$ gegeben sein mögen, die Teilsummen $s_{n}$ unter allen Umständen wohlbestimmte Werte. Aber das Symbol $\sum_{n=0}^{\infty} a_{n}$ hat von sich aus keinerlei Bedeutung, - auch nicht in einem scheinbar so durchsichtigen Falle wie $2 \mathrm{a}$; denn eine Addition unendlich vieler Summanden ist etwas nicht Definiertes, etwas schlechthin Sinnloses. Es ist lediglich als eine Übereinkunft anzusehen, daf3 wir unter dem neuen Symbol die Folge seiner Teilsummen $z u$ verstehen haben.

4. Man beachte hiernach wohl den Unterschied zwischen einer Reihe und einer Folge $\left.{ }^{2}\right)$ : Eine Reihe ist ein neues Symbol für eine aus ihm nach einer bestimmten Regel abzuleitende Folge.

5. Die Symbolik mit dem Summenzeichen „ $\Sigma$ “ wird man natiurlich nur dann anwenden können, wenn die Glieder der Reihe nach einem explizit gegebenen Gesetz gebildet werden, oder wenn für sie eine besondere Bezeichnung zur Verfïgung steht. Sollen aber z. B. die Zahlen

$$
\frac{1}{2}, \frac{1}{3}, \frac{1}{5}, \frac{1}{7}, \frac{1}{11}, \frac{1}{13}, \frac{1}{17}, \ldots
$$

1) D. h. gleich 1 oder 0 , je nachdem $n$ gerade oder ungerade ist. werden.

2) Der Zusatz „unendlich“ kann, wenn selbstverständlich, weggelassen 
oder die Zahlen

$$
\frac{1}{3}, \frac{1}{7}, \frac{1}{8}, \frac{1}{15}, \frac{1}{24}, \frac{1}{31}, \ldots
$$

die Glieder der Reihe sein, so werden wir die ausführlichere Symbolik

und

$$
\frac{1}{2}+\frac{1}{3}+\frac{1}{5}+\frac{1}{7}+\frac{1}{11}+\frac{1}{13}+.
$$

$$
\frac{1}{3}+\frac{1}{7}+\frac{1}{8}+\frac{1}{15}+\frac{1}{24}+\frac{1}{31}+\ldots
$$

anwenden miissen und hierbei so viele Glieder hinschreiben, das man vom Leser annehmen kann, dafs er das Bildungsgesetz erkannt hat. Das mag man bei der ersten dieser beiden Reihen nach dem Gliede $\frac{1}{13}$ erwarten: die Glieder sollen die reziproken Werte der aufeinander folgenden Primzahlen sein. Beim zweiten Beispiel wird man auch nach dem Gliede $\frac{1}{31}$ noch nicht wissen, wie es weiter gehen soll: In den Nennern der Glieder sollen die ganzen Zahlen der Form

$$
p^{q}-1 \quad(p, q=2,3,4, \ldots
$$

der Größse nach geordnet stehen.

Wir treffen nun weiter die Festsetzung, daß wir alle bei der Beschreibung des Konvergenzverhaltens einer Zahlenfolge eingeführten Ausdrucksweisen von der Folge $\left(s_{n}\right)$ auf die unendliche Reihe $\Sigma a_{n}$ selbst übertragen. Dadurch gewinnen wir insbesondere die folgende

69. Definition. Eine unendliche Reihe $\Sigma a_{n}$ heißt konvergent, bestimmt oder unbestimmt divergent, je nachdem die Folge ihrer Teilsummen das gleichbenannte Verhalten aufweist. Strebt im Falle der Konvergenz $s_{n} \rightarrow s$, so sagt man, s sei der Wert oder die Summe der konvergenten unendlichen Reihe und man schreibt kurz

$$
\left.\sum_{v=0}^{\infty} a_{v}=s^{1}\right)
$$

im Falle der bestimmten Divergenz der Folge $\left(s_{n}\right)$, sagt man auch von der Reihe, daß sie bestimmt divergent sei, und daß sie, je nachdem $s_{n} \rightarrow+\infty$ oder $\rightarrow-\infty$ strebt, gegen $+\infty$ bzw. gegen $-\infty$ diver. giere $\left.^{2}\right)$. Sind endlich im Falle der unbestimmten Divergenz von $\left(s_{n}\right)$, $*$ und $\mu$ die beiden Häufungsgrenzen dieser Folge, so sagt man auch von der Reihe, sie sei unbestimmt divergent und oszilliere zwischen den (Häufungs)-Grenzen $x$ und $\mu$.

Bemerkungen und Beispiele.

1. Man iberblickt sofort, dafs die Reihen $\mathbf{6 5}, 2 \mathrm{a}, \mathrm{b}$ und h konvergieren und die Summe +2 bzw. 1 und $\frac{1}{\alpha}$ haben; $2 \mathrm{c}$ und d sind bestimmt divergent gegen $+\infty$; $2 \mathrm{e}$ ist konvergent und hat die Zahl $s$ zur Summe, die durch die Intervall-

1) Genau wie wir gemäßs Fußnote zu 41, 1 jetzt auch $\left(s_{n}\right)=s$ schreiben diirften.

2) Ev. auch: konvergiere. 
schachtelung $\left(s_{2 k-1} \mid s_{2 k}\right)$ definiert wird $\left.{ }^{1}\right) ; 2 \mathrm{f}$ endlich oszilliert zwischen 0 und 1 , $2 \mathrm{~g}$ zwischen $-\infty$ und $+\infty$.

2. Bezüglich der Bezeichnung Summe ist sogleich nachdrücklich vor einem Miß̋verständnis zu warnen: Die Zahl s ist nicht eine Summe in irgendeinem bisher üblichen Sinne, sondern nur der Grenzwert einer unendlichen Folge von Summen; es ist also die Gleichung

$$
\sum_{n=0}^{\infty} a_{n}=s \text { oder } a_{0}+a_{1}+\ldots+a_{n}+\ldots=s
$$

lediglich eine andre Schreibweise für

$$
\lim s_{n}=s \text { oder für } s_{n} \rightarrow s .
$$

Es wäre daher sinngemäßer, nicht von der Summe, sondern von dem Grenzwevt oder dem Wert der Reihe zu sprechen. Doch ist die Bezeichnung "Summe“ von den Zeiten her in Geb rauch geblieben, in denen Reihen zuerst in der Wissenschaft auftraten und in denen man von dem darin steckenden Grenzprozesse sowie allgemein von dem „Unendlichen“ noch keine klare Vorstellung hatte.

3. Die Zahl $s$ ist also keine Summe, sondern wird der Kürze halber nur so genannt. Insbesondere wird das Rechnen mit unendlichen Reihen keineswegs all den Regeln für das Rechnen mit Summen gehorchen. Z. B. darf man bei einer (wirklichen) Summe beliebige Klammern setzen oder fortlassen, so dafs etwa

$$
1-1+1-1=(1-1)+(1-1)=1-(1-1)-1=0
$$

ist. Es ist aber keineswegs

dasselbe wie

$$
\sum_{n=0}^{\infty}(-1)^{n}=1-1+1-1+\cdots
$$

oder wie

$$
(1-1)+(1-1)+(1-1)+\ldots \equiv 0+0+0+\ldots
$$

$$
1-(1-1)-(1-1)-(1-1)-\ldots \equiv 1-0-0-0-\ldots
$$

Immerhin wird das Rechnen mit Reihen viele Analogien mit dem Rechnen mit (wirklichen) Summen aufweisen. Doch ist das Bestehen einer solchen Analogie in jedem einzelnen Falle erst zu beweisen.

4. Es ist auch vielleicht nicht überflüssig, $\mathrm{zu}$ betonen, dafi es eigentlich etwas ganz Paradoxes ist, dafi eine unendliche Reihe, etwa $\sum_{n=0}^{\infty} \frac{1}{2^{n}}$, iiberhaupt so etwas hat, was man eine Summe nennen kann. Deuten wir es in Quartanerart mit Mark und Pfennigen: Ich gebe jemandem erst 1 M., dann $1 / 2$ M., dann $1 / 4$ M., dann $1 / 8$ M. usw. Höre ich nun mit diesen Schenkungen nie auf, so entsteht die Frage, ob der Reichtum des Beschenkten dabei notwendig jede Höhe überschreiten muf oder nicht. Zunächst hat man das Gefühl, daf notwendig das erstere eintreten muf; denn wenn ich immer wieder etwas hinzu-

1) In der Tat ist $s_{2 k-1}=\left(1-\frac{1}{2}\right)+\left(\frac{1}{3}-\frac{1}{4}\right)+\ldots+\left(\frac{1}{2 k-1}-\frac{1}{2 k}\right)$ $=\frac{1}{1 \cdot 2}+\frac{1}{3 \cdot 4}+\ldots+\frac{1}{(2 k-1) 2 k}$, so daf $s_{1}<s_{3}<s_{5}<\ldots$ ist; ebenso folgt aus $s_{2 k}=1-\left(\frac{1}{2}-\frac{1}{3}\right)-\ldots-\left(\frac{1}{2 k}-\frac{1}{2 k+1}\right)$, daf $s_{0}>s_{2}>s_{4}>\ldots$ ist. Endlich ist $s_{2 k}-s_{2 k-1}=+\frac{1}{2 k+1}$, also positiv und gegen 0 strebend. Nach 46, 5 strebt $s_{n} \rightarrow\left(s_{2 k-1} \mid s_{2 k}\right)$. Vgl. S2, 9 u. 10, wo diese Betrachtung verallgemeinert wird. 
füge, so müfte - scheint es - die Summe schliefilich jeden Betrag übersteigen. Im vorliegenden Falle ist dem nicht so, da für jedes $n$

$$
s_{n}=1+\frac{1}{2}+\frac{1}{4}+\ldots+\frac{1}{2^{n}}=2-\frac{1}{2^{n}}<2
$$

bleibt. Die Schenkung erreicht also niemals auch nur den Betrag von $2 \mathrm{M}$. Und wenn wir nun trotzdem sagen, $\perp \frac{1}{2^{n}}$ sei gleich 2 , so ist das lediglich ein kurzer Ausłruck dafür, daf die Folge der Teilsummen dem Grenzwert 2 zustrebt.

5. Auch im Falle der bestimmten Divergenz kann man noch im übertragenen Sinne von einer Summe der Reihe sprechen, die dann eben den "Wert" $+\infty$ oder $-\infty$ hat. So ist $z$. B. die Reihe

$$
\sum_{n=1}^{\infty} \frac{1}{n} \text { oder } 1+\frac{1}{2}+\frac{1}{3}+\frac{1}{4}+\ldots
$$

bestimmt divergent, hat die ,Summe" $+\infty$, weil nach $\mathbf{4 6}, 3$ ihre Teilsummen $\rightarrow+\infty$ streben $^{1}$ ). Man schreibt kurz

$$
\sum_{n=1}^{\infty} \frac{1}{n}=+\infty
$$

was nur eine andre Schreibweise für $\lim \left(1+\frac{1}{2}+\ldots+\frac{1}{n}\right)=+\infty$ ist.

6. Im Falle einer unbestimmt divergenten Reihe verliert dagegen das Wort "Summe“ jede Bedeutung. Ist in diesem Falle $\lim s_{n}=\varkappa$ und $\overline{\lim } s_{n}=\mu(>x)$, so sagten wir oben, die Reihe oszillieve zwischen $\bar{x}$ und $\mu$. Hierbei ist indessen $z u$ beachten (vgl. 61,2), daßs es sich nur um die Beschreibung eines infinitären Verhaltens handelt. Tatsächlich brauchen die Teilsummen $s_{n}$ nicht zwischen $\%$ und $\mu$ zu liegen. Ist z. B. $a_{0}=2$ und für $n>0$ stets

$$
a_{n}=(-1)^{n}\left[\frac{n+1}{n}+\frac{n+2}{n+1}\right] \text {, }
$$

so rechnet man sofort nach, daf

$$
s_{n}=a_{0}+a_{1}+\ldots+a_{n}=(-1)^{n} \frac{n+2}{n+1} \quad(n=0,1,2, \ldots)
$$

und also $\lim s_{n}=-1, \varlimsup \lim s_{n}=+1$ ist. Aber alle Glieder der Folge $\left(s_{n}\right)$ liegen außerhalb des Intervalles $-1 \ldots+1$, und zwar abwechselnd links und rechts, so daf auf beiden Seiten des Intervalles unendlich viele Glieder der Folge liegen.

7. Betonten wir oben, daf in einer Reihe $\Sigma a_{n}$ lediglich die Folge $\left(s_{n}\right)$ ihrer Teilsummen, - also lediglich eine andere Schreibweise für eine Folge zu sehen ist, so überzeugt man sich umgekehrt leicht, dafi auch jede Folge $\left(x_{0}, x_{1}, \ldots\right)$ als Reihe geschrieben werden kann. Man hat dazu nur

$$
a_{0}=x_{0}, \quad a_{1}=x_{1}-x_{0}, \quad a_{2}=x_{2}-x_{1}, \ldots, \quad a_{n}=x_{n}-x_{n-1}, \ldots \quad(n \geq 1)
$$

1) Wenn also die unter 4. besprochenen Schenkungen der Reihe nach 1 M. , $1 / 2$ M., $1 / 3$ M., $1 / 4$ M., . . betragen, so wächst der Reichtum des Beschenkten nun doch iiber alles Maßs. Aber es ist im Augenblick gar nicht zu erkennen, woran es liegt, dafi im Falle 4. die Summe einen bescheidenen Betrag nicht ïbersteigt, im jetzigen dagegen iaber jede Höhe hinauswächst. Die Divergenz dieser Reihe ist von Joh. Bernoulli entdeckt und von Jak. Bernoulli 1689 veröffentlicht worden; doch scheint sie auch schon Leibniz 1673 bekannt gewesen zu sein. 
zu setzen. In der Tat hat dann die Reihe

die Teilsummen

$$
\sum_{n=0}^{\infty} a_{n}=x_{0}+\sum_{k=1}^{\infty}\left(x_{k}-x_{k-1}\right)
$$

$$
s_{0}=x_{0} ; \quad s_{1}=x_{0}+\left(x_{1}-x_{0}\right)=x_{1} ;
$$

und allgemein für $n \geq 1$

$$
s_{n}=x_{0}+\left(x_{1}-x_{0}\right)+\left(x_{2}-x_{1}\right)+\ldots+\left(x_{n-1}-x_{n-2}\right)+\left(x_{n}-x_{n-1}\right)=x_{n},
$$

so dafi die hingeschriebene Reihe tatsächlich einfach die Folge $\left(x_{n}\right)$ bedeutet. Das neue Symbol der unendlichen Reihe ist also weder spezieller noch allgemeiner als das der unendlichen Folge. Sein Sinn liegt hauptsächlich darin, daf nicht so sehr die Glieder der zu untersuchenden Zahlenfolge $\left(s_{n}\right)$ selbst, als vielmehr die Unterschiede $a_{n}=s_{n}-s_{n-1}$ eines jeden gegen das vorangehende gegeben bzw. betont werden.

8. Über die Geschichte der unendlichen Reihen unterrichtet trefflich ein Büchlein von R. Reiff (Tübingen, 1889). Hier mag die Erwähnung folgender Tatsachen genuigen: Das erste Beispiel einer unendlichen Reihe pflegt man Archimedes zuzuschreiben (Opera, ed. J. L. Heiberg, Bd. 2, Leipzig 1913, S. 310 ff.). Doch zeigt er nur, daf $1+\frac{1}{4}+\ldots+\frac{1}{4^{n}}<\frac{4}{3}$ bleibt, welchen Wert auch $n$ haben mag, und daf der Unterschied $z$ wischen beiden Werten $=\frac{1}{3} \cdot \frac{1}{4^{n}}$ ist und folglich kleiner als eine gegebene positive Zahl ausfällt, sofern $n$ grof genug genommen wird. Eine allgemeinere Benutzung unendlicher Reihen setzt aber erst in der zweiten Hälfte des 17. Jahrhunderts ein, als N. Mercator und W. Brouncker 1668 bei der Quadratur der Hyperbel die logarithmische Reihe 120 entdeckten und J. Newton 1669 in dem Werke De analysi per aequationes numero terminorum infinitas ihren Gebrauch auf eine festere Grundlage gestellt hatte. Im 18. Jahrhundert wurden die prinzipiellen Gesichtspunkte z. T. zwar aufer acht gelassen, dafür aber die Praxis der Reihen, vor allem durch Euler, in grofiartiger Weise entfaltet. Im 19. Jahrhundert endlich wurde die Theorie durch A. L. Cauchy (Analyse algébrique, Paris 1821) in einwandfreier Weise begründet, - wenn man von den Unklarheiten absieht, die damals dem Zahlbegriff als solchem noch anhafteten. (Weitere historische Bemerkungen s. in der Einleitung zu §59.)

II. Unendliche Produkte. Hier handelt es sich um Ausdrücke der Form

$$
u_{1} \cdot u_{2} \cdot u_{3} \ldots u_{n} \ldots \quad \text { oder } \prod_{n=1}^{\infty} u_{n}
$$

und sie sind in ganz entsprechender Weise wie die eben behandelten unendlichen Reihen lediglich als eine neue Symbolik für die wohlbestimmte Zahlenfolge der Teilprołukte

$$
p_{1}=u_{1} ; \quad p_{2}=u_{1} \cdot u_{2} ; \quad \ldots ; \quad p_{n}=u_{1} \cdot u_{2} \ldots u_{n}
$$

anzusehen. Indessen werden wir später mit Rücksicht auf die Ausnahmerolle, die die Zahl 0 bei der Multiplikation spielt, hier noch einige besondere Festsetzungen zu machen haben. 
1. Ist z. B. für $n \geq 1$ stets $u_{n} \doteq \frac{(n+1)^{2}}{n(n+2)}$, so bedeutet das unendliche Produkt

$$
\prod_{n=1}^{\infty} \frac{(n+1)^{2}}{n(n+2)} \quad \text { oder } \quad \frac{2^{2}}{1 \cdot 3} \cdot \frac{3^{2}}{2 \cdot 4} \cdot \frac{4^{2}}{3 \cdot 5} \cdot \frac{5^{2}}{4 \cdot 6} \cdots \frac{(n+1)^{2}}{n(n+2)} \cdots
$$

die Folge der Zahlen

$$
p_{1}=\frac{4}{3} ; \quad p_{2}=\frac{2 \cdot 3}{4} ; \quad p_{3}=\frac{2 \cdot 4}{5} ; \ldots ; \quad p_{n}=\frac{2(n+1)}{n+2} ; \ldots
$$

2. Die soeben unter I gegebenen Zusätze und Bemerkungen behalten mutatis mutandis auch hier ihre Bedeutung. Auf alles Nähere werden wir später eingehen (VII. Kapitel).

III. Unendliche Kettenbrüche. Hier wird die zu untersuchende Zahlenfolge $\left(x_{n}\right)$ mit Hilfe zweier anderer Folgen $\left(a_{1}, a_{2}, \ldots\right)$ und $\left(b_{0}, b_{1}, \ldots\right)$ hergestellt, indem man

$$
\begin{gathered}
x_{0}=b_{0}, \quad x_{1}=b_{0}+\frac{a_{1}}{b_{1}}, \quad x_{2}=b_{0}+\frac{a_{1}}{b_{1}+\frac{a_{2}}{b_{2}}}, \\
x_{3}=b_{0}+\frac{a_{3}}{b_{1}+\frac{a_{2}}{b_{2}+\frac{a_{3}}{b_{3}}}}
\end{gathered}
$$

usw. setzt, indem man also allgemein $x_{n}$ dadurch aus $x_{n-1}$ herleitet, dafs man in $x_{n-1}$ den letzten Nenner $b_{n-1}$ durch $\left(b_{n-1}+\frac{a_{n}}{b_{n}}\right)$ ersetzt und so ohne Ende fortfährt. Für den hierdurch entstandenen „unendlichen Kettenbruch“ ist keine allgemein angenommene neue Symbolik in Gebrauch. Das naheliegendste wäre, ihn mit

$$
b_{0}+\sum_{n=1}^{\infty} \frac{a_{n}}{b_{n}}
$$

zu bezeichnen. Auch hier muf man noch durch einige besondere Festsetzungen dem Umstande Rechnung tragen, da 3 bei der Division wieder die Zahl 0 eine Ausnahmerolle spielt. Auf die Kettenbriiche werden wir in diesem Buche indessen nicht eingehen ${ }^{1}$ ).

Von den besprochenen drei Arten, eine Zahlenfolge vorzulegen, ist die durch unendliche Reihen bei weitem die wichtigste für alle Anwendungen in der höheren Mathematik. Mit ihnen werden wir uns daher vornehmlich zu beschäftigen haben. - Da in den Reihen nichts anderes als Folgen zu sehen sind, so liefern uns die einleitenden Ausführungen des $\S 9$ die Gesichtspunkte, unter denen eine vorgelegte Reihe zu untersuchen sein wird: Neben dem Problem $A$, das sich auf das Konvergenzverhalten einer vorgelegten Reihe bezieht, steht wieder das schwierigere Problem B, das nach der Summe einer als konvergent erkannten Reihe fragt. Und genau aus denselben Gründen wie damals wird sich das zweite Problem meist in der Form stellen:

1) Eine vollständige Darstellung ihrer Theorie und ihrer Anwendungen bietet O. Perron, Die Lehre von den Kettenbrüchen, Leipzig 1913. 
Eine Reihe $\Sigma a_{n}$ ist als konvergent erkannt; stimmt nun ihre Summe mit derjenigen einer anderen Reihe oder mit dem Grenzwert irgendeiner anderen Zahlenfolge überein oder steht sie zu solchen in einer irgendwie angebbaren Beziehung ? ${ }^{1}$ )

Da das Konvergenzproblem A das leichtere ist und da es - im Gegensatz zum Problem B - eine methodische Erledigung zuläßt, wollen wir uns diesem zunächst ausführlich zuwenden.

\section{Aufgaben zum II. Kapitel ${ }^{2}$ ).}

9. Man beweise die Sätze $\mathbf{1 5}$ bis $\mathbf{1 9}$ des I. Kapitels nach der zu $\mathbf{1 4}$ in der Fufinote gegebenen Anleitung.

10. Man beweise in allen Einzelheiten, daf die durch $\mathbf{1 4}$ und $\mathbf{1 5}$ defi- nierte Anordnung der Gesamtheit der Intervallschachtelungen den sämtlichen Anordnungssätzen 1 gehorcht. (Vgl. hierzu 14, 4 und 15,2).

11. Man führe die Einzelheiten der S. $30 / 31$ geforderten Beweise durch, zeige also, daf die durch $\mathbf{1 6}$ bis $\mathbf{1 9}$ definierten vier Verknüpfungsarten zwischen den Intervallschachtelungen allen Grundgesetzen $\boldsymbol{2}$ gehorchen.

12. Bei fest $\in \mathrm{m} \varrho$ mit $0<\varrho<1$ strebt

$$
x_{n}=(n+1)^{Q}-n^{\varrho} \rightarrow 0 \text {. }
$$

13. Bei beliebigen positiven $\alpha$ und $\beta$ strebt

$$
\frac{(\log \log n)^{\alpha}}{(\log n)^{\beta}} \rightarrow 0 .
$$

14. Welche der Zahlen $\left(\frac{e}{2}\right)^{\sqrt{3}}$ und $(\sqrt{2})^{\frac{\pi}{2}}$ ist die größsere?

15. Beweise die folgenden Grenzbeziehungen :

a)

$$
\begin{aligned}
\text { a) } & {\left[\frac{1}{n^{2}}+\frac{2}{n^{2}}+\ldots+\frac{n}{n^{2}}\right] \rightarrow \frac{1}{2} ; } \\
\text { b) } \quad & {\left[\log \left(1+\frac{1}{n^{2}}\right)+\log \left(1+\frac{2}{n^{2}}\right)+\ldots+\log \left(1+\frac{n}{n^{2}}\right)\right] \rightarrow \frac{1}{2} ; } \\
\text { c) } & {\left[\frac{1}{\sqrt{n^{2}}+1}+\frac{1}{\sqrt{n^{2}+2}}+\cdots+\frac{1}{\sqrt{n^{2}+n}}\right] \rightarrow 1 ; }
\end{aligned}
$$

1) So wird sich z. B. die Reihe $1+1+\frac{1}{2 !}+\frac{1}{3 !}+\ldots+\frac{1}{n !}+\ldots$ sehr bald als konvergent erweisen. Wie sieht man, daf ihre Summe mit der durch die Folge $\left(1+\frac{1}{n}\right)^{n}$ gelieferten Zahl $e$ identisch ist? Ebenso werden wir sehr bald die Reihen $1+\frac{1}{4}+\frac{1}{9}+\ldots+\frac{1}{n^{2}}+\ldots$ und $1-\frac{1}{3}+\frac{1}{5}-\frac{1}{7}+-\ldots$ als konvergent erkennen. Wie findet man, dafs, wenn $s$ und $s^{\prime}$ ihre Summen sind, $s=\frac{8}{3} s^{\prime 2}$ und daf $4 s^{\prime}=\pi$ (also gleich dem Grenzwert eines dritten, beim Kreise auftretenden Grenzprozesses; vgl. 192 u. 207) ist?

$\left.{ }^{2}\right)$ Bei mehreren der folgenden Aufgaben werden einige der einfachsten Ergebnisse tiber den Logarithmus und die Zahlen $e$ und $\pi$ als bekannt angenommen, obwohl sie im Text erst später hergeleitet werden. 
d)

$$
\begin{aligned}
& {\left[\frac{n}{n^{2}+1^{2}}+\frac{n}{n^{2}+2^{2}}+\ldots+\frac{n}{n^{2}+n^{2}}\right]>\frac{\pi}{4}} \\
& {\left[\left(\frac{n}{n}\right)^{n}+\left(\frac{n-1}{n}\right)^{n}+\ldots+\left(\frac{1}{n}\right)^{n}>c-1\right.} \\
& \frac{1}{n} \sqrt[n]{(n+1)(n+2) \ldots(n-n)>\frac{4}{e}} .
\end{aligned}
$$

Man beachte, daf 3 bei a) bis d) ein gliedwciser Grenzibergang ein falsches, bei e) dagegen ein richtiges Ergebnis liefert.

16. Es sei $a>0$ und $x_{1}>0$ und eine Folge $\left(x_{1}, x_{2}, \ldots\right)$ durch die Festsetzung definiert, dafi für $n>2$

$$
\begin{array}{cc}
x_{n} \quad \sqrt{a+x_{n-1}} \\
x_{n} \quad \frac{a}{1-x_{n}}
\end{array}
$$

sein soll. Man zeige, dafi im Falle a) die Folge monoton gegen die positive Wurzel von $x^{2}-x-a-0$; im Falle b) gegen diejenige von $x^{2}+x-a=0$ strebt; letzteres jedoch so, daf die $x_{n}$ abwechselnd links und rechts von ihrem Grenzwert liegen.

17. Man untersuche das Konvergenzerhalten der folgenden Zahlenfolgen:

a) $x_{0}, x_{1}$ beliebig, fuir $n>2$ stets $x_{n} \quad{ }_{2}^{1}\left(x_{n-1}-x_{n-2}\right)$;

b) $x_{0}, x_{1}, \ldots, x_{p-1}$ beliebig, fiir $n>p$ stets

$$
\begin{gathered}
x_{n}=a_{1} x_{n-1}+a_{2}, x_{n-2} \ldots \ldots, a_{p}, x_{n-1}, \\
\left(a_{1}, a_{2}, \ldots, a_{p} \text { gegebene Konstanten, \%. B. alle }=\frac{1}{p}\right) ;
\end{gathered}
$$

c) $x_{0}, x_{1}$ positiv, fuir $n>2$ stets $x_{n}=1^{\prime} x_{n-1} \cdot x_{n-2}$;

d) $x_{0}, x_{1}$ beliebig, fiir $n>2$ stets $x_{n} \quad \begin{aligned} & 2 x_{n-1} x_{n-2} \\ & x_{n-1}+x_{n-2}\end{aligned}$.

18. Wird in Aufgabe 17, c speziell $x_{0}-1, x_{1}-2$ gesetzt, so ist der Grenzwert der Folge $\sqrt[3]{4}$.

19. Es seien $a_{1}, a_{2}, \ldots, a_{p}$, beliebig gegebene positive Gröfien und es werde fiir $n=1,2, \ldots$

$$
a_{1}^{n}+a_{2}^{n}+\ldots+a_{p}^{n}=s_{n} \text { und } \quad \underline{V} s_{n} x_{n}
$$

gesetzt. Es soll gezeigt werden, daf $x_{n}$ stets monoton wächst und, falls unter den gegebenen Zahlen eine bestimmte, etwa $a_{1}$, die gröbte war, dieser gröbten als Grenzwert zustrebt: $x_{n}>a_{1}$.

(An1.: Man zeige zunaichst, daf

$$
\left.s_{1}<\frac{s_{2}}{s_{1}} \quad \frac{s_{3}}{s_{2}}<\ldots\right) .
$$

20. Man setze, ahnlich wie in der vorigen Aufgabe,

$$
a_{1}^{\frac{1}{n}} \cdot a_{2}^{\frac{1}{n}}+\ldots+a_{p}^{\frac{1}{n}} s_{n}{ }^{\prime} \text { und }\left(s_{n}{ }^{\prime}\right)^{n}=x_{n}{ }^{\prime}
$$

und zeige, daf $x_{*}^{\prime}$ monoton fallend $\rightarrow \stackrel{p}{V} a_{1} a_{2} \ldots a_{1}$, strebt. 
21. Das Intervall $a \ldots b(0<a<b)$, werde in $n$ gleiche Teile geteilt; $x_{0}=a, x_{1}, x_{2}, \ldots, x_{n}=b$ seien die Teilpunkte. Man zeige, daf3 deren geometrisches Mittel

$$
\sqrt[n+1]{\sqrt{x_{0}} \cdot x_{1} \cdot x_{2}} \cdots \overline{x_{n}} \rightarrow \frac{1}{e}\left(\frac{b^{b}}{a^{a}}\right)^{\frac{1}{b-a}}
$$

and ihr harmonisches Mittel $\rightarrow \frac{b-a}{\log b-\log a}$ strebt.

22. Man zeige, dafi im Falle der allgemeinen Folge in Aufgabe 5

strebt.

$$
\frac{x_{n}}{c^{n}} \rightarrow \frac{x_{1}-\beta x_{0}}{c(\alpha-\beta)}
$$

23. Es sei $x>0$ und dif Folge $\left(x_{n}\right)$ durch die Festsetzungen

$$
x_{1}=x, \quad x_{2}=x^{x_{1}}, \quad x_{3}=x^{x_{2}}, \ldots, x_{n}=x^{x_{n-1}}, \ldots
$$

definiert. Für welche $x$ fällt diese Folge konvergent aus?

24. Es sei $\lim x_{n}=\varkappa,\left\lceil\lim x_{n}=\mu, \lim x_{n}{ }^{\prime}=\varkappa^{\prime}\right.$ und $\left\lceil x_{n}{ }^{\prime}=\mu^{\prime}\right.$. Was läßst sich ẗber die Lage der Häufungsgrenzen der Folgen

$$
\left(-x_{n}\right), \quad\left(\frac{1}{x_{n}}\right), \quad\left(x_{n}+x_{n}{ }^{\prime}\right), \quad\left(x_{n}-x_{n}{ }^{\prime}\right), \quad\left(x_{n} \cdot x_{n}{ }^{\prime}\right), \quad\left(\frac{x_{n}}{x_{n}{ }^{\prime}}\right)
$$

aussagen? Man diskutiere alle möglichen Fälle!

25. Es sei $\left(\alpha_{n}\right)$ beschränkt und (nach etwaiger Überspringung einiger Anfangsglieder)

$$
\log \left(1+\frac{\epsilon_{n}}{n}\right)=\frac{\beta_{n}}{n}
$$

gesetzt. Dann ist haben $\left(c_{n}\right)$ und $\left(\beta_{n}\right)$ dieselben Häufungsgrenzen. Dasselbe gilt, wenn

gesetzt wird.

$$
\log \left(1+\frac{1}{n}+\frac{\alpha_{n}}{n \log n}\right)=\frac{1}{n}+\frac{\beta_{n}}{n \log n}
$$

26. Gilt Satz $\$ 3,2$ auch noch, falls $\xi=+\infty$ oder $-\infty$ ist? Und gilt 43, :) noch, falls $\eta=0$ oder $=+\infty$ ist?

27. Wenn die in $\mathbf{4 3}, 2$ und 3 , gegebenen Folgen $x_{n}$ und $y_{n}$ monoton sind, so sind es auch die dortigen Folgen $x_{n}{ }^{\prime}$ und $y_{n}{ }^{\prime}$.

28. Ist die Folge $\left(\frac{a_{n}}{b_{n}}\right)$ monoton und $b_{n}>0$, so ist es auch die Folge der Quotienten

$$
\frac{a_{1}+a_{2}+\ldots+a_{n}}{b_{1}+b_{2}+\ldots+b_{n}}
$$

29. Es ist

$$
\lim \frac{a_{n}}{b_{n}}=\lim \frac{a_{n}-a_{n+1}}{b_{n}-b_{n+1}},
$$

falls der rechtsstehende Grenzwert existiert und falls $\left(a_{n}\right)$, und $\left(b_{n}\right)$ Nullfolgen sind, deren zweite monoton ist. 
30. Bei positiven, monotonen $c_{n}$ kann aus

$$
\frac{x_{0}+x_{1}+\ldots+x_{n}}{n+1} \rightarrow \xi \quad \text { auf } \quad \frac{c_{0} x_{0}+c_{1} x_{1}+\ldots+c_{n} x_{n}}{c_{0}+c_{1}+\ldots+c_{n}} \rightarrow \xi
$$

geschlossen werden, falls $\left(\frac{n c_{n}}{C_{n}}\right)$ beschränkt ist und $C_{n} \rightarrow+\infty$ strebt. (Hierbei ist $C_{n}=c_{0}+c_{1}+\ldots+c_{n}$ gesetzt.)

31. Ist $b_{n}>0$, strebt $b_{0}+b_{1}+\ldots+b_{n}=B_{n} \rightarrow+\infty$ und ebenso $x_{n} \rightarrow+\infty$, so kann von

$$
\frac{B_{n}}{b_{n}}\left(x_{n}-x_{n-1}\right) \rightarrow \xi \quad \text { auf } \quad\left[x_{n}-\frac{b_{0} x_{0}+b_{1} x_{1}+\ldots+b_{n} x_{n}}{b_{0}+b_{1}+\ldots+b_{n}}\right] \rightarrow \xi
$$

geschlossen werden.

32. Es ist für jede Folge $\left(x_{n}\right)$ stets

(Vgl. hierzu Satz 161.)

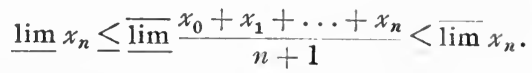

33. Man zeige, daf, wenn die Koeffizienten $a_{\lambda \mu}$ beim Toeplitzschen Satze 43, 5 positiv sind, für jede Folge $\left(x_{n}\right)$ die Beziehung

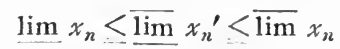

gilt, in der $x_{n}{ }^{\prime}=a_{n_{0}} x_{0}+a_{n_{1}} x_{1}+\ldots+a_{n n} x_{n}$ gesetzt ist. 
$Z_{\text {weiter Teil. }}$

\title{
Grundlagen der Theorie der unend- lichen Reihen.
}

\author{
III. Kapitel. \\ Reihen mit positiven Gliedern.
}

\section{$\$ 12$. Das erste Hauptkriterium und die beiden Vergleichs- kriterien.}

In diesem Paragraphen wollen wir uns ausschließlich mit Reihen beschäftigen, deren sämtliche Glieder positive oder wenigstens nicht negative Zahlen sind. Ist $\Sigma a_{n}$ eine solche Reihe, die wir kurz als Reihe mit positiven Gliedern bezeichnen wollen, so ist wegen $a_{n} \geqq 0$

$$
s_{n}=s_{n-1}+a_{n} \geqq s_{n-1}
$$

und also die Folge $\left(s_{n}\right)$ der Teilsummen eine monoton wachsende Zahlenfolge. Ihr Konvergenzverhalten ist daher besonders einfach, denn es ist dafür das 1. Hauptkriterium $\mathbf{4 6}$ maßgebend. Es liefert uns sofort das ebenso einfache wie grundlegende

I. Hauptkriterium. Eine Reihe mit positiven Gliedern kann nur 70. konvergieren oder gegen $+\infty$ divergieren. Und zwar ist sie dann und nur dann konvergent, wenn ihre Teilsummen beschränkt sind $\left.{ }^{1}\right)$.

Bevor wir die ersten Anwendungen dieses Hauptsatzes geben, wollen wir uns seinen Gebrauch noch durch die folgenden Zusätze erleichtern: Reihen

Satz 1. Ist $p$ eine beliebige natürliche Zahl, so haben die beiden

$$
\sum_{n=0}^{\infty} a_{n} \quad \text { und } \quad \sum_{n=p}^{\infty} a_{n}
$$

dasselbe Konvergenzverhalten ${ }^{2}$ ).

1) Es kommt nur Beschränktheit nach rechts in Frage, da eine wachsende Folge nach links von selbst beschränkt ist.

2) Kürzer: Man „darf" einen beliebigen Anfang weglassen. - Darum ist es häufig nicht nötig, die Summationsgrenzen anzugeben. 
Beweis. Die demselben Index des Reihengliedes entsprechenden Teilsummen beider Reihen unterscheiden sich nur um die feste Zahl $\left(a_{0}+a_{1}+\ldots+a_{p-1}\right)$ und sind also für beide Reihen beschränkt oder für beide nicht beschränkt.

Satz 2. Mit $\Sigma c_{n}$ is! auch $\Sigma_{{ }^{\prime}}{ }_{n} c_{n}$ eine konvergente Reihe mit positiven Gliedern, falls die Fakicren $\gamma_{n}$ irsendwelche positive, aber beschräakte Faktcren sind ${ }^{1}$ ).

Beweis. Bleiben die Teilsummen von $\Sigma c_{n}$ stets $<K$ und die Faktoren $\gamma_{n}$ stets $\left\langle\gamma\right.$, so bleiben die Teilsummen von $\Sigma \gamma_{n} c_{n}$ ersichtlich stets $\langle\gamma K$, womit nach dem Hauptkriterium schon alles bewiesen ist.

Satz 3. Mit $\Sigma d_{n}$ ist auch $\Sigma \delta_{n} d_{n}$ eine divergente Reihe mit positiven Gliedern, falls die $\delta_{n}$ irgenäwelche Zahlen sind, die eine noch positive untere Schranke $\delta$ besitzen.

Beweis. Wird $G>0$ beliebig gewählt, so sind nach Voraussetzung die Teilsummen von $\Sigma d_{n}$ doch von einem passenden Index an sämtlich $>G: \delta$. Von demselben Index an sind dann die Teilsummen von $\Sigma \delta_{n} d_{n}$ sämtlich $>G$. Also ist $\Sigma \delta_{n} d_{n}$ divergent.

Beide Sätze sind im wesentlichen enthalten in dem folgenden

Satz 4. Genügen die Faktcren " ${ }_{u}$ den Ungleichungen

$$
0<c^{\prime} \leqq c_{n} \leqq c^{\prime \prime},
$$

so weisen die leiden Reihen $\Sigma a_{n}$ und $\Sigma{ }^{\prime}{ }_{n} a_{n}$ mit positiven Gliedern dasselbe Konvergenzverhalten auf. Oder etwas anders ausgedrückt: Die beiden Reihen mit positiven Gliedern $\Sigma^{\prime} a_{n}$ und $\Sigma^{\prime} a_{n}{ }^{\prime}$ weisen dasselbe Konvergenzverhalten auf, falls es zwei. positive Zahlen $a^{\prime}$ und $a^{\prime \prime}$ gibt, so daß stets (oder wenigstens von einer Stẹlle an)

$$
\iota^{\prime} \leqq \frac{a_{n}{ }^{\prime}}{a_{n}} \leqq a^{\prime \prime}
$$

ist $\left.{ }^{2}\right)$, speziell also, wenn $a_{n}{ }^{\prime} \sim a_{n}$ oder gar $a_{n}{ }^{\prime} \simeq a_{n}^{\prime}$ ist (s. 40, 5).

1. Ist $K$ eine Schranke für die Teilsummen der Reihe $\stackrel{\prime}{\prime} a_{n}$ mit positiven Gliedern, so ist ihre Summe $s \leq K$ (s. 46, 1).

2. Die geometrische Reihe. Es sei $a>0$ und die sogenannte geometrische Reihe

$$
\underset{n=0}{\stackrel{\infty}{N}} a^{n}-1+a+a^{2}+\ldots+a^{n}+\cdots
$$

1) Wir werden im folgenden die Glieder einer als konvergent vorausgesetztcn Reihe meist mit $c_{n}$, die einer als divergent vorausgesetzten Reihe meist mit $d_{n}$ bezcichnen.

*) Da bei dieser Formulierung der Voraussetzungen mit $a_{n}$ dividiert wird, so steckt darin die selbstverständliche Voraussetzung, dafi die $a_{n}>0$ und niemals $=0$ sind. - Entsprechendes ist auch weiterhin des öfteren zu beachten: 
vorgelegt. Ist $a \geqq 1$, so ist $s_{n}>n$ und also $\left(s_{n}\right)$ sicher nicht beschränkt, die Reihe also divergent. Ist aber $a<1$, so hat man

$$
\left.s_{n}=1+a+a^{2}+\ldots+a^{n}=\frac{1-a^{n+1}}{1-a}, \quad \text { (vgl. S. 20, Fufn. }\right)
$$

und es ist also für jedes $n$

$$
s_{n}<\frac{1}{1-a},
$$

die Reihe also konvergent. Da iiberdies

$$
\left|s_{n}-\frac{1}{1-a}\right|=\frac{1}{1-a} \cdot a^{n+1}
$$

nach 10, 7 und 26, 1 eine Nullfolge ist, so ergibt sich hier - es ist dies ein seltener Fall - auch sogleich für die Summe der Reihe eine einfache Darstellung:

$$
\sum_{n=0}^{\infty} a^{n}=\frac{1}{1-a}
$$

3. Die Reihe $\sum_{n=1}^{\infty} \frac{1}{n(n+1)} \equiv \frac{1}{1 \cdot 2}+\frac{1}{2 \cdot 3}+\frac{1}{3 \cdot 4}+\ldots$ hat die Teilsummen

$$
s_{n}=\left(1-\frac{1}{2}\right)+\left(\frac{1}{2}-\frac{1}{3}\right)+\ldots+\left(\frac{1}{n}-\frac{1}{n+1}\right)=1-\frac{1}{n+1} .
$$

Diese sind stets $<1$, die Reihe also konvergent. Zufällig sieht man hier sofort, dafs $s_{n} \rightarrow 1$ strebt, daf also $s=1$ ist.

4. Die harmonischen Reihen. $\sum_{n=1}^{\infty} \frac{1}{n}=1+\frac{1}{2}+\ldots+\frac{1}{n}+\ldots$ ist $d i$ vergent, denn wir sahen schon in 46,3, daf ihre Teilsummen, also die Zahlen

$$
s_{n}=\left(1+\frac{1}{2}+\ldots+\frac{1}{n}\right) \text {. }
$$

gegen $+\infty$ divergieren ${ }^{1}$ ). Dagegen ist die Reihe

$$
\sum_{n=1}^{\infty} \frac{1}{n^{2}}=1+\frac{1}{4}+\frac{1}{9}+\frac{1}{16}+\ldots
$$

konvergent. Denn die $n$ te Teilsumme ist

$$
\begin{gathered}
s_{n}=1+\frac{1}{2 \cdot 2}+\frac{1}{3 \cdot 3}+\ldots+\frac{1}{n \cdot n}<1+\frac{1}{1 \cdot 2}+\frac{1}{2 \cdot 3}+\ldots+\frac{1}{(n-1) n} \\
=1+\left(1-\frac{1}{2}\right)+\left(\frac{1}{2}+\frac{1}{3}\right)+\ldots+\left(\frac{1}{n-1}-\frac{1}{n}\right)=2-\frac{1}{n}
\end{gathered}
$$

und es ist also stets $s_{n}<2$, die vorgelegte Reihe also konvergent. - Die Summe $s$ ist hier nicht so leicht anzugeben; es ist aber jedenfalls $s \leqq 2$. Wir werden später finden, daß $s=\frac{\pi^{2}}{6}$ ist. - Eine Reihe der Form $\sum \frac{1}{n^{\alpha}}$ nennt man eine harmonische Reihe.

1) Vgl. S. 98, Fufin. 
5. Die Reihe $\sum_{n=0}^{\infty} \frac{1}{n !} \equiv 1+1+\frac{1}{2 !}+\frac{1}{3 !}+\ldots$ hat die Teilsummen $s_{0}=1$, $s_{1}=2$, und für $n \geq 2$ ist

$$
\begin{gathered}
\left.s_{n}=2+\frac{1}{2}+\frac{1}{2 \cdot 3}+\ldots+\frac{1}{2 \cdot 3 \ldots n} \leqq 2+\frac{1}{2}+\frac{1}{2 \cdot 2}+\ldots+\frac{1}{2 \cdot 2 \ldots}{ }^{1}\right) \\
=2+\frac{1}{2}+\frac{1}{2^{2}}+\ldots+\frac{1}{2^{n-1}}=3-\frac{1}{2^{n-1}}<3 .
\end{gathered}
$$

Die Reihe ist also konvergent mit einer Summe $\leq 3$. Wir werden später sehen, dafs ihre Summe mit dem Grenzwert $e$ der Zahlen $\left(1+\frac{1}{n}\right)^{n}$ übereinstimmt.

6. Sagten wir oben, daf jede Reihe mit positiven Gliedern eine monoton wachsende Zahlenfolge bedeutet, so sieht man auch umgekehrt, daf jede monoton wachsende Folge $\left(x_{0}, x_{1}, \ldots\right)$ als Reihe mit positiven Gliedern geschrieben werden kann (vgl. hierzu 69, 7), falls $x_{0}$ positiv ist. Man hat hierzu nur

$$
a_{0}=x_{0}, \quad a_{1}=x_{1}-x_{0}, \ldots, \quad a_{n}=x_{n}-x_{n-1}, \ldots
$$

zu setzen; in der Tat ist dann

und alle $a_{n}$ sind $\geq 0$.

$$
s_{n}=x_{0}+\left(x_{1}-x_{0}\right)+\ldots+\left(x_{n}-x_{n-1}\right)=x_{n}
$$

Aus unserm Hauptsatz werden wir nun nach und nach immer speziellere, aber auch immer leichter zu handhabende und immer wirksamere Kriterien ableiten. Dies wird uns hauptsächlich durch Vermittlung der beiden folgenden „Vergleichskriterien“ gelingen.

\section{Vergleichskriterium 1. Art.}

Es sei $\Sigma c_{n}$ eine schon als konvergent und $\Sigma d_{n}$ eine schon als divergent erkannte Reihe mit positiven Gliedern. Genügen dann die Glieder einer vorgelegten Reihe $\Sigma a_{n}$ mit gleichfalls positiven Gliedern für alle $n$ von einer Stelle an, etwa für alle $n>m$

a) der Bedingung

$$
\boldsymbol{a}_{\boldsymbol{n}} \leqq \boldsymbol{c}_{\boldsymbol{n}}
$$

so ist auch $\sum a_{n}$ eine konvergente Reihe. - Ist dagegen für $n>m$

b) stets

$$
\boldsymbol{a}_{n} \geqq \boldsymbol{d}_{n}
$$

so muß auch die Reihe $\Sigma a_{n}$ divergieren²).

Beweis. Nach $\boldsymbol{\boldsymbol { \pi 0 }} 1$ genügt es, das Konvergenzverhalten der

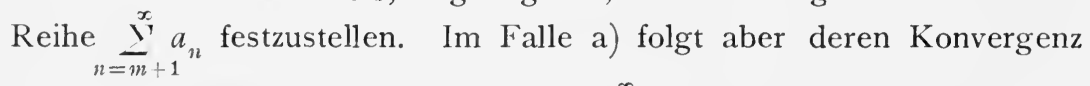
sofort nach $\mathbf{7 0}, 2$ aus derjenigen von $\sum_{n=m+1}^{\infty} c_{n}$, weil nach Voraussetzung

1) Es wurde im Nenner jeder Faktor durch den kleinsten von ihnen, also 2, ersetzt.

2) Gauß benutzt dies Kriterium schon im Jahre 1812 (s. Werke III, S. 140). Ausdrücklich als Konvergenzkriterium formuliert wurde es indessen, ebenso wie das nachfolgende Kriterium 2. Art, erst von Cauchy in seiner Analyse algébrique (Paris, 1821). 
73. § 12. Das erste Hauptkriterium u. d. beiden Vergleichskriterien. 109

für $n>m$ stets $a_{n}=\gamma_{n} c_{n}$ mit $\gamma_{n} \leqq 1$ gesetzt werden kann. Im Falle b) folgt ebenso ihre Divergenz aus derjenigen von $\sum_{n=m+1}^{\infty} d_{n}$, weil jetzt $a_{n}=\delta_{n} d_{n}$ mit $\delta_{n} \geqq 1$ gesetzt werden kann $^{1}$ ).

Vergleichskriterium 2. Art.

Es sei wieder $\Sigma c_{n}$ eine schon als konvergent und $\Sigma a_{n}$ eine schon als divergent erkannte Reihe mit positiven Gliedern. Genïgen dann die Glieder einer vorgelegten Reihe $\Sigma a_{n}$ mit gleichfalls positiven Gliedern für alle $n$ von einer Stelle an, etwa für alle $n \geqq m$

a) den Bedingungen

$$
\frac{a_{n+1}}{a_{n}} \leqq \frac{c_{n+1}}{c_{n}}
$$

so ist auch $\Sigma a_{n}$ eine konvergente Reihe. Ist dagegen für $n>m$

b) stets

$$
\frac{a_{n+1}}{a_{n}} \geqq \frac{d_{n+1}}{d_{n}}
$$

so $m u \beta$ auch $\Sigma a_{n}$ divergieren.

Beweis. Im Falle a) hat man für $n>m$ stets

$$
\frac{a_{n+1}}{c_{n+1}} \leqq \frac{a_{n}}{c_{n}}
$$

Die Folge des Quotienten $\gamma_{n}=\frac{a_{n}}{c_{n}}$ ist also von einer Stelle an monoton fallend und folglich, weil ihre Glieder sämtlich positiv sind, notwendig beschränkt. Satz $\mathbf{7 0 , 2}$ liefert nun die Konvergenz. Im Falle b) hat man analog $\frac{a_{n+1}}{d_{n+1}} \geqq \frac{a_{n}}{d_{n}}$, so daß die Quotienten $\delta_{n}=\frac{a_{n}}{d_{n}}$ von einer Stelle an monoton wachsen. Da sie aber stets positiv sind, so haben sie eine noch positive untere Schranke. Satz 70, 3 liefert nun die Divergenz.

Diese Vergleichskriterien können uns natürlich nur nützlich sein, wenn wir schon viele konvergente und divergente Reihen mit pasitiven Gliedern kennen. Wir werden uns dazu also sozusagen einen möglichst großen Vorrat an Reihen mit bekanntem Konvergenzverhalten anlegen müssen. Hierzu sollen die folgenden Beispiele den Grundstock liefern:

1) Oder - und fast noch kürzer - so: Im Falle a) ist jede Schranke der Teilsummen von $\Sigma c_{n}$ auch eine solche für die Teilsummen von $\Sigma a_{n}$; und im Falle b) müssen die Teilsummen von $\Sigma a_{n}$ schliefilich jede Schranke übersteigen, da schon diejenigen von $\Sigma d_{n}$ dies tun. 
74. 1. $\sum_{n=1}^{\infty} \frac{1}{n}$ hatte sich als divergent, $\sum_{n=1}^{\infty} \frac{1}{n^{2}}$ als konvergent erwiesen. Nach dem 1. Vergleichskriterium ist also die sogenannte harmonische Reihe

$$
\sum_{n=1}^{\infty} \frac{1}{n^{\prime \prime}}
$$

für $\alpha \leqq 1$ sicher divergent, für $\alpha \geqq 2$ sicher konvergent. (Ihre Summe weif man allerdings nur für den Fall, dä $\beta$ zugleich eine ganze und gerade Zahl ist, mit anderweitig vorkommenden Zahlen in Beziehung zu bringen; z. B. werden wir später sehen, dafis fiir $\alpha=4$ die Summe $=\frac{\pi^{4}}{90}$ ist.)

2. Nach dem vorigen bleibt das Konvergenzverhalten von $\ \frac{1}{n^{\alpha}}$ nur noch für $1<\boldsymbol{\alpha}<2$ fraglich. Folgendermaßen lïfst sich zeigen, daf die Reihe in diesen Fällen konvergiert: Um eine beliebige Teilsumme $s_{n}$ der Reihe nach oben abzuschätzen, wähle man $k$ so groß, dafa $2^{k}>n$ ausfällt. Dann ist sicher $s_{n} \leqq s_{2^{k}-1}=1+\left(\frac{1}{2^{\alpha}}+\frac{1}{3^{\alpha}}\right)+\left(\frac{1}{4^{\alpha}}+\frac{1}{5^{\alpha}}+\frac{1}{6^{\alpha}}+\frac{1}{7^{\alpha}}\right)+\ldots+\left(\frac{1}{\left(2^{k-1}\right)^{\alpha}}+\ldots+\frac{1}{\left(2^{k}-1\right)^{\alpha}}\right)$. Hier sind jedesmal die Glieder in einer Klammer vereinigt, deren Index von einer Poten $z$ von 2 (einschl.) bis zur nächsten Potenz von 2 (ausschl.) läuft. Ersetzt man nun in jeder Klammer alle Summanden durch den ersten von ihnen, so bedeutet dies eine Vergrößerung und man hat also

$$
s_{n}<1+\frac{2}{2^{(e)}}+\frac{4}{4^{\prime \prime}}+\ldots+\frac{2^{k-1}}{\left(2^{k-1}\right)^{\prime \prime}} .
$$

Setzt man nun zur Abkürzung $\frac{1}{2^{a-1}}=\vartheta$, - eine positive Zahl, die, wegen $\alpha>1$, sicher $<1$ ist - , so ist

$$
s_{n}<1+\vartheta+\vartheta^{2}+\ldots+\vartheta^{k-1}=\frac{1-\vartheta^{k}}{1-\vartheta}<\frac{1}{1-\vartheta} ;
$$

und da dies für jedes $n$ gilt, so sind die Teilsummen unserer Reihe beschränkt, die Reihe selbst also konvergent, w. z. b.w.

$$
\text { Die harmonische Reihe } \geq \frac{1}{n^{\prime \prime}} \text { ist also für } \alpha<1 \text { divergent, fïr } \alpha>1
$$

konvergent. Sie bildet zusammen mit der geometrischen'Reihe schon einen sehr nützlichen Schatz an Vergleichsreihen.

3. Auch die Reihen

$$
\sum_{n=1}^{\infty}(a n+b)^{\alpha}
$$

in denen $a$ und $b$ gegebene positive Zahlen sind, werden für $\%<1$ divergieren. fuir $x>1$ konvergieren. Denn wegen

$$
\frac{n^{\prime \prime}}{(a n-b)^{n}}=\left(\frac{1}{a+\frac{b}{n}}\right)^{\prime \prime} \rightarrow \frac{1}{a^{\prime \prime}} \quad \text { ist } \quad \frac{1}{(a n+b)^{\alpha}} \sim \frac{1}{n^{\alpha}} ;
$$

70, 4 liefert daher die Richtigkeit der ausgesprochenen Behauptung.

Hiernach sind ganz speziell die Reihen

$$
1+\frac{1}{3^{\alpha}}+\frac{1}{5^{\alpha}}+\ldots=\sum_{n=0}^{\infty} \frac{1}{(2 n+1)^{a}}
$$

für $\%>1$ konvergent, für $\alpha \leq 1$ divergent. 
4. Ist $\underset{n=0}{\stackrel{\infty}{N}} c_{n}$ eine konvergente Reihe mit positiven Gliedern und bildet man aus ihr dadurch eine neue Reihe $\Sigma c_{n}{ }^{\prime}$, dafs man irgendwelche Glieder streicht und die stehenbleibenden mit $c_{0}{ }^{\prime}, c_{1}{ }^{\prime}, \ldots$ bezeichnet, so ist auch die entstandene "Teilreihe" $\Sigma c_{n}{ }^{\prime}$ konvergent. Denn für ihre Teilsummen ist jede Zahl eine Schranke, die für die Teilsummen von $\Sigma c_{n}$ eine Schranke ist.

Hiernach ist $z$. B. die Reihe $\Sigma \frac{1}{p^{\alpha}}$, in der $p$ alle Primzahlen durchläuft, also die Reihe

$$
\frac{1}{2^{\alpha}}+\frac{1}{3^{\alpha}}+\frac{1}{5^{\alpha}}+\frac{1}{7^{\alpha}}+\frac{1}{11^{\alpha}}+\ldots
$$

für $\alpha>1$ sicher konvergent. (Dagegen darf man natürlich nicht ohne weiteres schliefen, daßs sie für $\alpha \leqq 1$ divergiert!)

5. Da $\Sigma a^{n}$ für $0 \leqq a<1$ schon als konvergent erkannt ist, so ist insbesondere

$$
\sum_{n=1}^{\infty} \frac{1}{10^{n}}=\frac{1}{10}+\frac{1}{10^{2}}+\ldots+\frac{1}{10^{n}}+\ldots
$$

konvergent. Bedeuten nun $z_{1}, z_{2}, \ldots, z_{n}, \ldots$ irgendwelche „Ziffern“, d. h. je eine der Zahlen $0,1,2, \ldots, 9$, und ist $z_{0}$ irgendeine ganze Zahl $\gtreqless 0$, so ist auch

$$
\sum_{n=0}^{\infty} \frac{z_{n}}{10^{n}}
$$

nach 70, 2 konvergent. - Man erkennt hieraus, daf3 man einen unendlichen Dezimalbruch auch als unendliche Reihe auffassen darf. In diesem Sinne können wir sagen: Jeder unendliche Dezimalbruch ist konvergent und stellt also eine wohlbestimmte reelle Zahl dar. - Bei dieser Form der Reihe haben wir auch durch Gewohnheit eine unmittelbare Vorstellung von dem Wert ihrer Summe.

\section{$\$ 13$. Das Wurzel- und das Quotientenkriterium.}

Eine systematischere Ausnutzung der beiden Vergleichskriterien bahnen wir nun durch die beiden folgenden Sätze an. Nehmen wir zunächst als Vergleichsreihe die geometrische Reihe $\sum a^{n}$ mit $0<a<1$, so ergibt sich unmittelbar der

Satz 1. Ist von einer Stelle an stets $a_{n} \leqq a^{n}$ oder also

$$
\sqrt[n]{\boldsymbol{a}_{\boldsymbol{n}}} \leqq \boldsymbol{a}<\mathbf{1}
$$

so ist die Reihe $\sum a_{n}$ konvergent; ist dagegen von einer Stelle ab stets

$$
\sqrt[n]{\boldsymbol{a}_{n}} \geqq 1
$$

so ist $\sum a_{n}$ divergent. (Cauchysches Wurzelkriterium ${ }^{1}$ ).)

$Z$ usatz. Für die Divergenz genügt es offenbar schon, wenn die Ungleichung $\sqrt[n]{a_{n}} \geqq 1$ für unendlich viele verschiedene Werte von $n$ erfüllt ist.

1) Analyse algebrique, S. $132 \mathrm{ff}$. 
Denn für dieselben unendlich vielen $n$ ist dann auch $a_{n} \geq 1$; und eine bestimmte Teilsumme $s_{m}$ wird daher eine gegebene (positive ganze) Zahl $G$ iiberschreiten, wenn man $m$ so groß wählt, daf diese Ungleichung für $0 \leqq n \leq m$ schon mindestens $G$-mal eingetreten ist. Die Folge $\left(s_{n}\right)$ ist also keinesfalls beschränkt.

Das 2. Vergleichskriterium liefert ebenso unmittelbar den

Satz 2. Ist von einer Stelle an stets

$$
\frac{\boldsymbol{a}_{\boldsymbol{n}+\mathbf{1}}}{\boldsymbol{a}_{\boldsymbol{n}}} \leqq \boldsymbol{a}<\mathbf{1}
$$

so ist die Reihe $\sum a_{n}$ konvergent. Ist dagegen von einer Stelle ab stets

$$
\frac{a_{n+1}}{a_{n}} \geqq 1,
$$

so ist die Reihe $\Sigma a_{n}$ divergent. (Cauchysches Quotienten. kriterium $\left.{ }^{1}\right)$.)

1. Bei den beiden in diesen Sätzen enthaltenen Konvergenzkriterien ist es wesentlich, daf $\sqrt[n]{a_{n}}$ bzw. $\frac{a_{n+1}}{a_{n}}$ einen festen echten Bruch $a$ von einer Stelle an nicht mehr ibersteigt. Es geniigt keineswegs zur Konvergenz, wenn stets

$$
\sqrt[n]{a_{n}}<1 \quad \text { bzw. } \quad \frac{a_{n+1}}{a_{n}}<1
$$

leibt. Ein Beispiel hierfür bietet schon die harmonische Reihe $\Sigma \frac{1}{n}$, für die gewißs für $n>1$ stets

$$
\sqrt[n]{\frac{1}{n}}<1 \quad \text { und auch } \quad \frac{1}{n+1}: \frac{1}{n}=1-\frac{1}{n+1}<1
$$

ist, die aber doch divergiert. Wesentlich ist eben, daß3 sich Wurzel und Quotient der Zahl 1 nicht beliebig nälern sollen.

2. Sind die Folgen $\left(\stackrel{n}{V} a_{n}\right)$ oder $\left(\frac{a_{n+1}}{a_{n}}\right)$ konvergent, etwa mit dem Grenzwert $\alpha$, so lehren die Sätze 1 und 2 , dafs für $\alpha<1$ Konvergenz, für $\alpha>1$ Divergenz stattfindet. Denn strebt z. B. $\sqrt[n]{a_{n}} \rightarrow \alpha<1$, so ist $\varepsilon=\frac{1-\alpha}{2}>0$ und also $m$ so bestimmbar, dafi für $n>m$ stets

$$
\sqrt[n]{a_{n}}<\alpha+\varepsilon=\frac{1+c}{2}=a
$$

ist. Und da dieser Wert $a<1$ ist, so lehrt Satz 1 die Konvergenz. Ist dagegen $\iota>1$, so ist $\varepsilon^{\prime}=\frac{\varepsilon-1}{2}>0$ und $m^{\prime}$ so bestimmbar, daß für $n>m^{\prime}$ stets

$$
\sqrt[n]{a_{n}}>a-\varepsilon^{\prime}=\frac{1+\epsilon}{2}=a
$$

ist. Und da jetzt dieser Wert $a>1$ ist, so lehrt Satz 1 die Divergenz. Ganz entsprechend schliefit man für den Quotienten.

Ist $\alpha=1$, so lehren diese Sätze noch gar nichts.

1) Analyse algébrique, S. 134 ff. 
3. Die unter 2. angewendeten Schlüsse sind offenbar schon erlaubt, falls $\varlimsup \sqrt[n]{a_{n}} \quad$ bzw. $\quad \varlimsup \lim \frac{a_{n+1}}{a_{n}}<1$

oder

$$
\underline{\lim } \sqrt[n]{a_{n}} \quad \text { bzw. } \quad \underline{\lim } \frac{a_{n+1}}{a_{n}}>1
$$

ist. Ist eine dieser Häufungsgrenzen $=1$, oder die obere $>1$, die untere $<1$, so lehren sie uns gar nichts iber das Konvergenzverhalten von $\Sigma a_{n}$. Der zu 75, 1 gemachte Zusatz lehrt aber, dafi beim Wurzelkriterium schon die Bedingung $\varlimsup \sqrt[n]{\lim } \sqrt{a_{n}}>1$ für die Divergenz hinreichend ist ${ }^{1}$ ).

4. Die unter 2. und 3. gemachten Bemerkungen sind so naheliegend, dab wir sie bei ähnlichen Fällen hinfort nicht mehr besonders erwähnen.

5. Das Wurzel- und das Quotientenkriterium sind für die Praxis weitaus die wichtigsten. Bei den meisten in den Anwendungen vorkommenden Reihen kann man mit ihrer Hilfe die Konvergenzfrage schon erledigen. Wir geben einige Beispiele, bei denen $x$ zunächst eine positive Zahl sein soll.

a) $n^{*} x^{n} \quad(\alpha$ beliebig $)$.

Hier strebt

$$
\frac{a_{n+1}}{a_{n}}=\left(\frac{n+1}{n}\right)^{\alpha} \cdot x \rightarrow x
$$

da ja $\frac{n+1}{n}=1+\frac{1}{n} \rightarrow 1$ strebt und dauernd positiv ist (s. $\mathbf{3 5}, 4$ und $\mathbf{3 5}, 10$ ). Die Reihe ist daher - und dies ohne Rücksicht auf den Wert von $\alpha$ - konvergent, falls $x<1$, divergent, falls $x>1$ ist. Für $x=1$ liefern unsere Kriterien keine Entscheidung, doch haben wir dann die uns bekannte harmonische Reihe vor uns.

$$
\text { b) } \sum_{n=0}^{\infty}\left(\begin{array}{c}
n+p \\
p
\end{array}\right) x^{n}=\sum_{n=0}^{\infty}(-1)^{n}\left(\begin{array}{c}
-p-1 \\
n
\end{array}\right) x^{n} \quad(p \geqq 1 \text { ganzzahlig }) .
$$

Hier strebt

$$
\frac{a_{n+1}}{a_{n}}=\frac{(n+p+1)(n+p) \ldots(n+2) \cdot p !}{(n+p)(n-1+p) \ldots(n+1) \cdot p !} \cdot x=\frac{n+p+1}{n+1} x \rightarrow x .
$$

Daher ist auch diese Reihe für $x<1$ konvergent, für $x>1$ divergent, welchen Wert auch $p$ haben mag. Fiir $x=1$ divergiert sie offenbar auch, da dann stets $\frac{a_{n+1}}{a_{n}}>1$ ist. Im Konvergenzfalle wird für ihre Summe später der Wert $\left(\frac{1}{1-x}\right)^{1+p}$ gefunden werden.

$$
\text { c) } \sum_{n=0}^{\infty} \frac{x^{n}}{n !}-1+x+\frac{x^{2}}{2 !}+\ldots+\frac{x^{n}}{n !}+\ldots .
$$

Hier strebt für jedes $x$

$$
\frac{a_{n+1}}{a_{n}}=\frac{x}{n+1} \rightarrow 0(<1) ;
$$

die Reihe ist also für jedes $x$ konvergent. Für ihre Summe wird später der Wert $e^{x}$ gefunden werden.

1) Dadurch erhält das Kriterium eine disjunktive Form. $\Sigma a_{n}$ ist konvergent oder divergent, je nach de.n $\lim \sqrt[n]{a_{n}}<1$ oder $>1$ ist. (Näheres in $\S 36$ und 42). 
d) $\sum \frac{1}{n^{n}}$ ist konvergent, weil $\sqrt[n]{\frac{1}{n^{n}}}=\frac{1}{n} \rightarrow 0$ strebt.

e) $\sum \frac{1}{(\log n)^{n}}$ ist konvergent, weil wieder $\sqrt[n]{a_{n}} \rightarrow 0$ strebt $\left.{ }^{1}\right)$.

f) $\sum \frac{1}{1+n^{2}}$ konvergent, weil $a_{n}<\frac{1}{n^{2}}$;

$\sum \frac{n !}{n^{n}}$ konvergent, weil für $n>2$ stets $a_{n}=\frac{1 \cdot 2 \ldots n}{n \cdot n \ldots n} \leqq \frac{2}{n^{2}}$;

$\sum \frac{1}{\sqrt{n(n+1)}}$ divergent, weil $a_{n}>\frac{1}{n+1}$;

$\sum \frac{1}{\sqrt{n\left(1+n^{2}\right)}}$ konvergent, weil $a_{n}<\frac{1}{n^{\frac{3}{2}}}$.

g) $\sum \frac{1}{(\log n)^{p}}, p>0$ fest, ist divergent, weil nach 38, 6 von einer Stelle an $(\log n)^{p}<n$ ist.

h) $\sum \frac{1}{n \sqrt[n]{n}}$ ist divergent, weil $\sqrt[n]{n} \rightarrow 1$ strebt.

i) $\sum \frac{1}{(\log n)^{\log n}}$ ist konvergent, wie man sofort erkennt, wenn man das allgemeine Glied in der Form

schreibt. Dagegen ist

$$
\frac{1}{n^{\log \log n}}
$$

$$
\sum \frac{1}{(\log n)^{\log \log n}}=\sum e^{-(\log \log n)^{2}}
$$

divergent, weil nach 38, 6 und Aufg. 13 von einer Stelle ab $(\log \log n)^{2}<\log n$, und also das allgemeine Glied der Reihe $>\frac{1}{n}$ ist.

\section{\$14. Reihen mit positiven monoton abnehmenden Gliedern.}

Bevor wir diese ganz elementar gehaltenen Betrachtungen verlassen, wollen wir unter den Reihen mit positiven Gliedern noch eine besonders einfache Klasse hervorheben, nämlich diejenigen, bei denen die Folge $\left(a_{n}\right)$ ihrer Glieder wenigstens von einer Stelle ab monoton ist. In diese Klasse gehören fast alle vorhin als Beispiele gegebenen Reihen und zu ihr gehören auch die Mehrzahl der in den Anwendungen vorkommenden Reihen. Für solche Reihen gilt folgender

1) Bei dieser Reihe darf die Summation erst bei $n=2$ beginnen, da $\log 1=0$ ist. Solche und ähnliche selbstverständliche Beschränkungen werden wir im folgenden nicht immer ausdrücklich betonen: es genügt für die Konvergenzfrage, wenn die hingeschriebenen Reihenglieder von einer Stelle an wohl bestimmte Werte haben. - Von jetzt ab bedeutet, wofern das Gegenteil nicht ausdrücklich gesagt wird, das Zeichen "log" stets den natürlichen Logarithmus, also den mit der Basis e (46, 4). 
Cauchyscher Konvergenzsatz. Ist $\sum_{n=1}^{\infty} a_{n}$ eine Reihe, deren Glieder 77. eine positive monotone Folge $\left(a_{n}\right)$ bilden, so hat sie dasselbe Konvergenzverhalten wie die Reihe

$$
\left.\sum_{k=0}^{\infty} 2^{k} a_{\mathrm{2} k}=a_{1}+2 a_{\mathrm{2}}+4 a_{4}+8 a_{8}+\cdots{ }^{1}\right) \text {. }
$$

Vorbemerkung. An diesem Satze ist besonders merkwürdig, dafi nach ihm für das Konvergenzverhalten der Reihe sich schon ein geringer Bruchteil aller Glieder als entscheidend erweist. Man bezeichnet ihn darum wohl auch als Verdichtungssatz.

Nach ihm ist z. B. die harmonische Reihe $\Sigma \frac{1}{n}$ sicher divergent, denn sie verhält sich ebenso wie die Reihe

$$
\sum \frac{2^{k}}{2^{k}} \equiv 1+1+1+\ldots,
$$

welche doch gewiß divergiert. Und allgemein verhält sich nach ihm die Reihe $\Sigma \frac{1}{i^{\alpha}}$ ebenso wie die Reihe

$$
\Sigma \frac{2^{k}}{\left(2^{k}\right)^{\alpha}} \equiv \Sigma\left(\frac{1}{2^{\alpha-1}}\right)^{k}
$$

Die letztere ist aber eine geometrische Reihe und konvergiert oder divergiert also je nachdem $\alpha>1$ oder $\leqq 1$ ist.

Diese Beispiele lehren zugleich, daf das Konvergenzverhalten von $\Sigma 2^{k} a_{2 k}$ oft leichter erkennbar ist als das der Reihe $\Sigma a_{n}$ selber; und gerade hierin liegt der Wert des Satzes.

Beweis. Wir bezeichnen die Teilsummen der gegebenen Reihe mit $s_{n}$, die der neuen Reihe mit $t_{k}$. Dann ist (vgl. $\left.\mathbf{7 4}, 2\right)$

a) für $n<2^{k}$

$$
\begin{aligned}
s_{n} & <a_{1}+\left(a_{2}+a_{3}\right)+\ldots+\left(a_{2^{k}}+\ldots+a_{2^{k+1}-1}\right) \\
& <a_{1}+2 a_{2}+4 a_{4}+\ldots+2^{k} a_{2^{k}}=t_{k},
\end{aligned}
$$

also

$$
s_{n}<t_{k}
$$

b) für $n>2^{k}$

also

$$
\begin{aligned}
s_{n} & >a_{1}+a_{2}+\left(a_{3}+a_{4}\right)+\ldots+\left(a_{2 k-1+1}+\ldots+a_{2}=t_{k}\right) \\
& >\frac{1}{2} a_{1}+a_{2}+2 a_{4}+\ldots+2^{k-1} a_{2 k}=\frac{1}{2} t_{k},
\end{aligned}
$$

$$
2 s_{n}>t_{k} \text {. }
$$

Die Abschätzung a) lehrt nun, daß mit der Folge $\left(t_{k}\right)$ auch die Folge $\left(s_{n}\right)$ beschränkt ist; die Abschätzung b) umgekehrt, daß mit $\left(s_{n}\right)$ auch $\left(t_{k}\right)$ beschränkt ist. Diese Folgen sind also entweder beide beschränkt oder beide nicht beschränkt, die in Rede stehenden Reihen also entweder beide konvergent oder beide divergent, w. z.b.w.

1) Analyse algébrique, S. 135. 
Ehe wir weitere Beispiele zu diesem Satze geben, wollen wir ihn noch etwas erweitern ${ }^{1}$ ); denn man sieht sofort, daß die Bevorzugung der Zahl 2 kein wesentlicher Teil des Satzes ist. In der Tat gilt allgemeiner der

78. Satz. Ist Lan wieder eine Reihe, deren Glieder eine positiv monoton abnehmende Folge $\left(a_{n}\right)$ bilden, und ist $\left(g_{0}, g_{1}, \ldots\right)$ irgendeine monolone wachsende Folge ganzer Zahlen, so sind die beiden Reihen

$$
\stackrel{\sum_{n=0}^{\infty}}{a_{n}} \quad \text { und } \quad \sum_{k=0}^{\stackrel{x}{N}}\left(g_{k+1}-g_{k}\right) a_{g_{k}}
$$

entweder beide konvergent oder beide divergent, falls die $g_{k}$ für jedes $k$ die Bedingungen

$$
g_{k}>g_{k-1} \geqq 0 \quad \text { und } \quad g_{k+1}-g_{k} \leqq M \cdot\left(g_{k}-g_{k-1}\right)
$$

erfüllen, in deren zweiter $M$ eine positive Konstante sein soll $\left.{ }^{2}\right)$.

Beweis. Ganz ähnlich wie vorhin hat man

a) für $n<g_{k}$, wenn $A$ die Summe der dem Gliede $a_{g_{0}}$ etwa vorangehenden Glieder (sonst 0 ) bedeutet,

$$
\begin{aligned}
s_{n}<s_{g_{k}} & \leqq A+\left(a_{g_{0}}+\ldots+a_{g_{1}-1}\right)+\ldots+\left(a_{g_{k}}+\ldots+a_{g_{k+1}-1}\right) \\
& <A+\left(g_{1}-g_{0}\right) a_{g_{0}}+\ldots+\left(g_{k+1}-g_{k}\right) a_{\boldsymbol{s}_{k}} .
\end{aligned}
$$

also

$$
s_{n}<A+t_{k}
$$

b) für $n>g_{k}$

$$
\begin{aligned}
s_{n}>s_{g_{k}} & >\left(a_{g_{0}+1}+\ldots+a_{g_{1}}\right)+\ldots+\left(a_{g_{k-1}}+1+\ldots+a_{g_{k}}\right) \\
& >\left(g_{1}-g_{0}\right) a_{g_{1}}+\ldots+\left(g_{k}-g_{k-1}\right) a_{g_{k}} \\
M s_{n} & >\left(g_{2}-g_{1}\right) a_{g_{1}}+\ldots+\left(g_{k+1}-g_{k}\right) a_{g_{k}} .
\end{aligned}
$$

also

$$
M s_{n}>t_{k}-t_{0} \text {. }
$$

Und aus diesen beiden Abschätzungen zieht man genau wie vorhin die in Rede stehenden Schlïsse.

\section{Bemerkungen.}

79. 1. Es genügt nattïlich, wenn die Bedingungen der beiden Sätze erst von einer gewissen Stelle ab erfüllt sind. Daher darf man in dem erweiterten Satze sich speziell

$$
g_{k}=3^{k},=4^{k} ; \ldots, \quad \text { oder } \quad=\left[g^{k}\right]
$$

gesetzt denken, wenn $g$ irgendeine reelle $Z$ ahl $>1$ und $[g k]$ die gröfste nicht oberhalb $g^{k}$ gelegene ganze Zahl bedeutet. Auch genügt man mit der Wahl

$$
g_{k}=k^{2},=k^{3},=k^{4}, \ldots
$$

1) O. Schlömilch, Zeitschrift für Math. u. Phys., Bd. 18 (1873), S. 425 .

2) Die zweite Bedingung bedeutet, dafi die Lücken, die die Folge $\left(g_{k}\right)$ gegenuiber der Folge aller natürlichen Zahlen aufweist, sich nicht zu stark vergröfern dïrfen. 
den Bedingungen des Satzes. Für $g_{k}=k^{2}$ erhält man z. B. den Satz, daß

$$
\sum_{n=0}^{\infty} a_{n} \quad \text { und } \quad \stackrel{\infty}{N}_{k=0}^{\infty}(2 k+1) a_{k^{2}}=a_{0}+3 a_{1}+5 a_{4}+7 a_{9}+\ldots
$$

entweder beide konvergieren oder beide divergieren, - falls $\left(a_{n}\right)$ eine positive monoton abnehmende Folge ist. Statt der letzten Reihe darf man nach $\boldsymbol{7 0}, 4$ auch die Reihe $\Sigma k a_{k^{2}}=a_{1}+2 a_{4}+3 a_{9}+\ldots$ nehmen.

2. $\sum_{n=2}^{\infty} \frac{1}{n \log n}$ ist divergent, - trotzdem ihre Glieder wesentlich kleiner sind als die der harmonischen Reihe; denn nach unserm Satze verhält sich diese Reihe ebenso wie die Reihe

$$
\sum_{k=1}^{\infty} \frac{2^{k}}{2^{k} \cdot \log \left(2^{k}\right)}=\sum_{k=1}^{\infty} \frac{1}{(\log 2) \cdot k}
$$

und ist also nach $\boldsymbol{7 0}, 2$ gleich der harmonischen Reihe $\perp_{k}^{1}$ divergent. Die Divergenz dieser Reihe und der in den folgenden Beispielen behandelten wurde von N.H. Abel entdeckt (s. Euvres II, S. 200).

3. $\sum_{n=3}^{\infty} \frac{1}{n \log n \cdot \log \log n}$ ist auch noch divergent, obwohl ihre Glieder wieder wesentlich kleiner sind als die der eben behandelten Abelschen Reihe. In der Tat verhält sie sich nach dem Cauchyschen Satze ebenso wie die Reihe

$$
\sum_{k=2}^{\infty} \frac{2^{k}}{2^{k} \cdot \log \left(2^{k}\right) \cdot \log \left(\log 2^{k}\right)}=\sum_{k=2}^{\infty} \frac{1}{k \cdot \log 2 \cdot \log (k \log 2)}
$$

und diese hat, da $\log 2<1$ ist, größsere Glieder als die eben behandelte Abclsche Reihe $\perp \frac{1}{k \log k}$ und mufs also gleich dieser divergieren.

4. So kann man beliebig fortfahren. Zur Abkürzung bezeichnen wir den $r$-fach iterierten Logarithmus einer positiven Zahl $x$ mit $\log _{r} x$, so daf

$$
\begin{gathered}
\log _{0} x=x, \quad \log _{1} x=\log x, \quad \log _{2} x=\log (\log x), \quad \ldots \\
\log _{r} x=\log \left(\log _{r-1} x\right)
\end{gathered}
$$

ist. Unter $\log _{-1} x$ können wir noch den Wert $e^{x}$ verstehen.

Diese iterierten Logarithmen haben erst einen Sinn, wenn $x$ hinreichend grof ist, $\log x$ nämlich erst für $x>0, \log _{2} x$ erst für $x>1, \log _{3} x$ erst für $x>e$, usw.; und in den Nenner unserer Reihenglieder wollen wir sie erst setzen, wenn sie positiv sind, also $\log x$ erst für $x>1, \log _{2} x$ erst fuir $x>e$, $\log _{3} x$ erst für $x>e^{e}$, usw. Setzen wir daher die Reihe an

$$
\frac{1}{n} n \log n \cdot \log _{2} n \ldots \log _{p} n \quad(p \geq 1 \text { ganz }) \text {, }
$$

so darf die Summation erst bei einem passenden hinreichend grofen Index beginnen, - auf dessen genauen Wert es aber (nach $\boldsymbol{7 0}, 1)$ nicht ankommt. Diese Reihe verhält sich nun, da die Logarithmen mit $n$ monoton wachsen, die Reihenglieder also monoton abnehmen, nach dem Cauchyschen Satze ebenso wie die Reihe

$$
\frac{1}{k} \frac{1}{\log 2^{k} \cdot \log _{2} 2^{k} \ldots \log _{\text {, }} 2^{k}}
$$

und diese wird, da $2<e$, sicher divergieren, wenn sogar die Reihe

$$
\frac{1}{k} \frac{1}{k \log k} \ldots \log _{p-1} k
$$


divergiert. Da nun diese Divergenz für $p=1$ (und $p=2$ ) schon festgestellt ist, so folgt hieraus durch vollständige Induktion $(\mathbf{2}, \mathbf{V})$ die Divergenz für jedes $p \geq 1$.

5. Die betrachteten Reihen gehen aber in konvergente Reihen über, falls man dem letzten Faktor des Nenners einen oberhalb 1 gelegenen Exponenten gibt. Daf3 $\Sigma \frac{1}{n^{\alpha}}$ für $\alpha>1$ konvergiert, ist uns schon geläufig. Nehmen wir nun an, es sei schon für ein bestimmtes (ganzes) $p \geq 1$ die Konvergenz der Reihe

$$
\left.\frac{1}{k} k \cdot \log k \ldots \log _{p-2} k \cdot\left(\log _{p-1} k\right)^{\alpha}{ }^{1}\right)
$$

bewiesen, so folgt daraus ganz ähnlich wie vorhin die Konvergenz der Reihe

$$
\frac{1}{n}-\frac{1}{n \cdot \log n \ldots \log _{p-1} n \cdot\left(\log _{p} n\right)^{\alpha}}
$$

Denn diese wird sich nach dem erweiterten Cauchyschen Satze oS ebenso verhalten wie die Reihe - wir wählen $g_{k}=3^{k}-$

$$
\sum_{k} \frac{3^{k+1}-3^{k}}{3^{k} \log 3^{k} \ldots\left(\log _{p} 3^{k}\right)^{\alpha}}
$$

Wegen $3>e$ hat diese aber kleineve Glieder als die soeben als konvergent vorausgesetzte Reihe $\left({ }^{*}\right)$, falls man deren Glieder vorher mit 2 multipliziert hat (was nach $\mathbf{7 0 , 2}$ die Konvergenz nicht stört).

Die in den beiden letzten Beispielen aufgestellten Reihen werden uns als Vergleichsreihen weiterhin die wertvollsten Dienste leisten.

Noch einen letzten bemerkenswerten Satz wollen wir über die Reihen mit positiven monoton abnehmenden Gliedern beweisen, obgleich er in gewisser Weise schon efwas von den allgemeinen Konvergenzbetrachtungen des folgenden Kapitels (s. 82,6) vorwegnimmt.

S0. Satz. Wenn die Reihe $\Sigma a_{n}$ mit positiven monoton abnehmenden Gliedern konvergieren soll, so muß nicht nur $a_{n} \rightarrow 0_{r}$ sondern sogar

streben $\left.^{2}\right)$.

$$
n \boldsymbol{a}_{n} \rightarrow()
$$

Beweis. Nach Voraussetzung ist die Folge der Teilsummen $a_{0}+a_{1}+\ldots+a_{n}=s_{n}$ konvergent. Nach Wahl von $\varepsilon>0$ kann man daher $m$ so wählen, daß für alle $\nu>m$ und alle $\lambda \geqq 1$ stets

d. h.

$$
\left|s_{\nu+\lambda}-s_{\nu}\right|<\frac{\varepsilon}{2}
$$

$$
a_{v+1}+a_{v+2}+\ldots+a_{v+\lambda}<\frac{\varepsilon}{2}
$$

1) Für $p=1$ soll dies die Reihe $\Sigma_{k^{u}}$ sein.

2) L. Olivier, Journ. f. d. reine u. angew. Math., Bd. 2 (1827), S. 34. 
bleibt. Wählen wir nun $n>2 m$, so ist, wenn $v=\left[\frac{1}{2} n\right]$ die größte ganze nicht oberhalb $\frac{1}{2} n$ gelegene Zahl bedeutet, $v \geqq m$ und also

$$
a_{v+1}+a_{v+2}+\ldots+a_{n}<\frac{\varepsilon}{2}
$$

um so mehr ist dann

und erst recht

$$
(n-v) a_{n}<\frac{\varepsilon}{2}
$$

$$
\frac{n}{2} \cdot a_{n}<\frac{\varepsilon}{2}, \quad \text { d. h. } \quad n a_{n}<\varepsilon .
$$

Es strebt also $n a_{n} \rightarrow 0$, w. z. b. w.

Bemerkung. Ausdrücklich mufi hervorgehoben werden, dafi die Beziehung $n a_{n} \rightarrow 0$ für unsere Reihenart nur eine notwendige, aber keine hinreichende Konvergenzbedingung ist, d. $\mathrm{h}$. wenn $n a_{n}$ nicht gegen 0 strebt, so ist die betreffende Reihe sicher divergent ${ }^{1}$ ), während aus $n a_{n} \rightarrow 0$ nichts über die etwaige Konvergenz der Reihe $z u$ folgen braucht. In der Tat ist ja die Abelsche Reihe $\Sigma \frac{1}{n \log n}$ divergent, obgleich sie monoton abnehmende Glieder hat und

strebt.

$$
n a_{n}=\frac{1}{\log n} \rightarrow 0
$$

\section{Aufgaben zum III. Kapitel.}

34. Man untersuche das Konvergenzverhalten einer Reihe $\Sigma a_{n}$, bei der $a_{n}$ von einer Stelle an einen der folgenden Werte hat:

$\frac{1}{n^{1+\frac{1}{n}}}, \quad \frac{n^{a}}{n !}, \quad \frac{n+\sqrt{n}}{n^{2}-n}, \quad \frac{(n !)^{2}}{(2 n) !}, \quad \frac{2^{n} \cdot n !}{n^{n}}, \quad \frac{3^{n} \cdot n !}{n^{n}}, \quad\left(\begin{array}{c}a+n \\ n\end{array}\right)$, $\frac{1}{1+a^{n}}, \quad(\sqrt[n]{a}-1), \quad\left(\sqrt[n]{a}-1-\frac{1}{n}\right), \quad(\sqrt{n+1}-\sqrt{n}), \quad(\sqrt[3]{n+1}-\sqrt[3]{n})$, $\frac{\sqrt{n+1}-\sqrt{n}}{n}, \quad \frac{1}{(\log \log n)^{\log n}}, \quad \frac{1}{\left(\log _{3} n\right)^{\log n}}, \quad a^{1 \bar{n}}, \quad a^{\log n} a^{\log \log n}$,

$$
\left(1-\frac{\log n}{n}\right)^{n}
$$

Bei denjenigen Reihen, in deren Gliedern die Konstante a auftritt, ist die Frage dahin zu verschärfen, für welche Werte von $a$ die Reihe konvergent ausfällt, für welch€ nicht.

35. Mit $\Sigma d_{n}$ divergiert auch $\sum \frac{d_{n}}{1+d_{n}}$. Wie verhalten sich $\sum \frac{d_{n}}{1+n d_{n}}$ und $\sum \frac{d_{n}}{1+n^{2} d_{n}} ?\left(d_{n}>0\right)$.

1) Es muf hiernach z. B. die harmonische Reihe $\sum \frac{1}{n}$ divergieren, weil sie monoton abnehmende Glieder hat, aber $n \cdot \frac{1}{n}$ nicht gegen 0 strebt. 
36. Wie verhält sich, wieder unter der Voraussetzung, dak $\beth_{n}$ divergiert und $d_{n}>0$ ist, die Reihe $\perp_{1+d_{n}{ }^{2}}$ ?

37. Es strebe $p_{n} \rightarrow+\infty$. Wie verhalten sich die Reihen

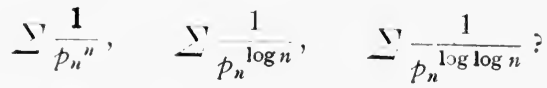

3S. Es strebe $p_{n} \rightarrow+\infty$, doch so, daf

$$
0<\lim \left(p_{n+1}-p_{n}\right)<+\infty
$$

ausfält. Welche Haiufungsgrenzen muf die Folge $\left(\varrho_{n}\right)$ haben, damit

konvergiert, bzw, divergicrt.

$$
\pm \frac{1}{p_{n}{ }^{2} n}
$$

39. Für jedes $n>1$ ist

10. Die Folge

$$
\frac{n}{2}<1+\frac{1}{2}+\frac{1}{3}+\frac{1}{4}+\cdots+\frac{1}{2^{n}-1}<n .
$$

fällt monoton.

$$
\left.x_{n}=1+\frac{1}{2}+\cdots+\frac{1}{n}-\log n\right]
$$

11. Wenn ${ }^{\prime} a_{n}$ positive Glieder hat und konvergiert, so konvergiert auch $\ \checkmark a_{n} a_{n+1}$. Man zeige an einem Beispiel, dafi die Umkelurung dieses Satzes nicht allgemein richtig ist, und beweise, dafi sie doch gilt, wenn $\left(a_{n}\right)$ monoton ist.

42. Wenn $\grave{L} a_{n}$ konvergiert, so konvergitrt auch $\perp \frac{\sqrt{ } a_{n}}{n}$, ja sogar für jedes $\delta>0$ die Reihe $\leq \frac{\sqrt{a_{n}}}{\sqrt{n}-1+\gamma}$.

43. Jede positive reelle Zahl $x_{1}$ kann auf eine und nur cine Weise in der Form

$$
x_{1}=a_{1}+\frac{a_{2}}{2 !}+\frac{a_{3}}{3 !}+\frac{a_{4}}{4 !}+\ldots
$$

dargestellt werden, wenn die $a_{n}$ ganze, nicht negative \%ahlen sind, die der Bedingung $a_{n} \leqq n-1$ genügen, ohne von einer Stelle an dauernd $=n-1 \quad \mathrm{zu}$ sein. Wenn $x_{1}$ rational ist, und nur in diesem Falle, bricht die Reihe ab.

14. Ist $0<x \leqq 1$, so gibt es eine und nur eine der Bedingung $1<k_{1}<k_{2}$ $\leqq k_{3} \leq \ldots$ genügende Folge natiurlicher Zahlen, für die

$$
x=\frac{1}{k_{1}}+\frac{1}{k_{1} k_{2}}+\ldots+\frac{1}{k_{1} k_{2} \ldots k_{n}}+\ldots
$$

ist. $x$ ist dann und nur dann rational, wenn die $k_{v}$ von einer stelle an sïmtlich einander gleich sind. 
IV. Kapitel.

\section{Reihen mit beliebigen Gliedern.}

\section{\$15. Das zweite Hauptkriterium und das Rechnen mit konvergenten Reihen.}

In einer unendlichen Reihe $\sum_{n=0}^{\infty} a_{n}$, deren Glieder nun keiner Beschränkung mehr unterworfen sein sollen, sondern beliebige reelle Zahlen bedeuten dürfen, sollte lediglich ein neues Symbol für die Folge $\left(s_{n}\right)$ ihrer Teilsummen

$$
s_{n}=a_{0}+a_{1}+\ldots+a_{n} \quad(n=0,1,2, \ldots)
$$

gesehen und die für das Konvergenzverhalten von $\left(s_{n}\right)$ eingeführten Bezeichnungen unmittelbar auf die Reihe selbst übertragen werden. Uns interessiert vor allem wieder der Fall der Konvergenz. Das II. Hauptkriterium (47-51), das die notwendige und hinreichende Bedingung für die Konvergenz aussprach, liefert hier sofort den folgenden

${ }^{\circ}$ Hauptsatz (1. Form). Die notwendige und hinreichende Be-81. dingung für die Konvergenz der Reihe $\Sigma_{a}$ besteht darin, daß nach Wahl von $\varepsilon>0$ sich eine Zahl $n_{0}=n_{0}(\varepsilon)$ so angeben lasse, da $\beta$ für alle $n>n_{0}$ und alle $k \geqq 1$ ste's

$$
\left|\boldsymbol{s}_{\boldsymbol{n}+\boldsymbol{k}}-\boldsymbol{s}_{\boldsymbol{n}}\right|<\varepsilon .
$$

d. h. also jelzt, daß stets

ausfällt.

$$
\left|a_{n+1}+a_{n+2}+\ldots+a_{n+k}\right|<\varepsilon
$$

Stützen wir uns auf die 2. Form des Hauptkriteriums, so erhalten wir auch für den jetzigen Hauptsatz die folgende

O2. Form. Die Reihe $\Sigma a_{n}$ ist dann und nur dann konvergent, wenn nach Wahl einer ganz be'iebigen Folge $\left(k_{n}\right)$ von natürlichen Zahlen sich die Folge der Zahlen

$$
\boldsymbol{T}_{\boldsymbol{n}}=\left(\boldsymbol{a}_{n+1}+\boldsymbol{a}_{n+2}+\ldots+\boldsymbol{a}_{\boldsymbol{n}+\boldsymbol{k}_{\boldsymbol{n}}}\right)
$$

stets als eine $N$ ullfolge erweist ${ }^{1}$ ). Und ähnlich wie damals können wir dies noch etwas erweitern zu der

$\circ$ 3. Form. Die Reihe $\mathcal{L}^{\prime} a_{n}$ ist dann und nur dann konvergent, wenn nach der Wahl zweier ganz beliebiger Folgen $\left(v_{n}\right)$ und $\left(k_{n}\right)$ von

1) Diese Form ist es im wesentlichen, in der $N$. H. Abel in seiner grund legenden Arbeit über die Binomialveihe (Journ. f. die reine u. angew. Math., Bd. 1, 1826, S. 311) das Kriterium aufstellt. 
natürlichen Zahlen, von denen wenigstens die erste $\rightarrow+\infty$ streben soll, sich die Folge der Zahlen

$$
\boldsymbol{T}_{\boldsymbol{n}}=\left(\boldsymbol{a}_{v_{n}+\mathbf{1}}+\boldsymbol{a}_{\boldsymbol{v}_{n}+\mathbf{2}}+\ldots+\boldsymbol{a}_{\boldsymbol{v}_{n}+\boldsymbol{k}_{\boldsymbol{n}}}\right)
$$

stets als eine Nullfolge erweist.

Bemerkungen und Beispiele.

1. Da es sich jetzt bei den Reihen lediglich um eine neue Schreibart für Zahlenfolgen handelt und insbesondere, wie betont, nicht nur jede Reihe eine Zahlenfolge darstellt, sondern auch jede Zahlenfolge als Reihe geschrieben werden kann, so gelten sinngemäß alle S. $79 \mathrm{ff}$. gemachten Bemerkungen und Beispiele.

2. Den Inhalt des Hauptsatzes kann man etwa folgendermafien in Worte kleiden: Nach Wahl von $\varepsilon>0$ muf jedcs beliebig lange Stück der Reihe, dessen Anfangsindex nur hinreichend grof ist, eine Summe haben, deren Betrag $<\varepsilon$ ist. Oder: Nach Wahl von $\varepsilon>0 \mathrm{mu}$ sich eine Stelle $m$ so angeben lassen, daf̧ für $n>m$ die Addition beliebig vieler weiterer Glieder $z u s_{n}$ den Wert dieser Teilsumme niemals um mehr als $\varepsilon$ ändern kann.

3. Ein beliebig herausgeschnittenes Stück der Reihe, wie das Stück

$$
a_{v+1}+a_{v+2}+\ldots+a_{v+\lambda}
$$

wollen wir im folgenden der Kürze halber ein Teilstïck der Reihe nennen und mit $T_{y}$ bezeichnen, wenn es hinter dem $v$ ten Gliede beginnt. Wenn nötig, kann man auch noch die Anzahl $\lambda$ der Glieder des Stückes deutlich machen, indem man es mit $T_{v, \lambda}$ bezeichnet. Haben wir eine beliebige Folge solcher Teilstücke vor uns, deren erster Index $\rightarrow+\infty$ strebt, so wollen wir kurz von einer Teilstïckfolge der vorgelegten Reihe sprechen. Die 2. und 3. Form des Hauptsatzes kann dann auch so ausgesprochen werden:

04. Form. Die Reihe $\Sigma a_{n}$ ist dann und nur dann konvergent, wenn jede Teilstückfolge derselben eine Nullfolge ist.

4. $\sum a_{n}$ ist also dann und nur dann divergent, wenn sich wenigstens eine Teilstückfolge angeben läft, die keine Nullfolge ist. Für die harmonische Reihe $\sum \frac{1}{n}$ ist z. B.

$$
T_{n}=T_{n, n}=\frac{1}{n+1}+\frac{1}{n+2}+\cdots+\frac{1}{2 n}>n \cdot \frac{1}{2 n}=\frac{1}{2} .
$$

Diese Folge $\left(T_{n}\right)$ ist also sicher keine Nullfolge, also $\Sigma \frac{1}{n}$ divergent.

5. Für $\unrhd \frac{1}{n^{2}}$ ist

$$
\begin{aligned}
T_{v} & =T_{v, \lambda}=\frac{1}{(v+1)^{2}}+\ldots+\frac{1}{(v+\lambda)^{2}} \\
& <\frac{1}{v(v+1)}+\frac{1}{(v+1)(v+2)}+\ldots+\frac{1}{(v+\lambda-1)(v+\lambda)} \\
& <\left(\frac{1}{v}-\frac{1}{v+1}\right)+\left(\frac{1}{v+1}-\frac{1}{v+2}\right)+\ldots+\left(\frac{1}{v+i-1}-\frac{1}{v+\lambda}\right)=\frac{1}{v}-\frac{1}{v+\lambda}
\end{aligned}
$$

also $T_{v}<\frac{1}{v}$, so dafi $T_{y} \rightarrow 0$ strebt, wenn $v \rightarrow+\infty$ strebt. Die Reihe ist konvergent. 
82. § 15. Das 2. Hauptkriterium u. d. Rechnen mit konvergenten Reihen.

6. Da nach der 2. Form, wenn man dort alle $k_{n}=1$ setzt, sich $a_{n+1} \rightarrow 0$ ergibt, so strebt (nach $\mathbf{2 7}, 4$ ) auch $a_{n} \rightarrow 0$, d. h. es gilt der

- Satz. Bei einer konvergenten Reihe müssen die Glieder eine Nullfolge bilden.

Dafs diese Bedingung für die Konvergenz nicht hinreichend ist, wissen wir schon von der harmonischen Reihe her.

Weif man andererseits schon, dafi $\Sigma^{*} a_{n}$ konvergiert, so konvergiert mit ihr auch die Reihe $a_{n+1}+a_{n+2}+a_{n+3}+\ldots \equiv \sum_{\nu=n+1}^{\infty} a_{\nu}$, deren Summe man als sogen. Rest der Reihe $\Sigma a_{n}$ gewöhnlich mit $\dot{r}_{n}$ bezeichnet (so daß also $s_{n}+v_{n}$ $=s=$ der Summe der ganzen Reihe ist). Man darf dann in der für $n>n_{0}$ und alle $k \geq 1$ gültigen Abschätzung

$$
\left|a_{n+1}+a_{n+2}+\ldots+a_{n+k}\right|<\varepsilon
$$

$k$ über alle Grenzen wachsen lassen, und findet, dafi für $n>n_{0}$ stets $\left|r_{n}\right| \leqq \varepsilon$ ausfällt. Es bilden also auch die Reste einer konvergenten Reihe $\sum_{n=0}^{\infty} a_{n}=s$, also die Zahlen

stets eine Nullfolge.

$$
\left(r_{-1}=s\right), r_{0}, r_{1}, r_{2}, \ldots, r_{n}, \ldots
$$

7. In 80 sahen wir, daf, wenn die Glieder einer konvergenten Reihe $\sum a_{n}$ (mit positiven Gliedern) monoton fallen, über den eben in 6 . bewiesenen Satz hinaus sogar die Beziehung $n a_{n} \rightarrow 0$ gilt. Daß dies bei Reihen mit beliebigen Gliedern nicht mehr der Fall zu sein braucht, lehrt schon die gleich hernach in 9. gegebene Reihe. Doch läßst sich zeigen, dafi jetzt

$$
\frac{a_{1}+2 a_{2}+\ldots+n a_{n}}{n} \rightarrow 0
$$

streben muß, dafi also die Glieder der Folge $\left(n a_{n}\right)$ im Durchschnitt kleine Werte ergeben. Es gilt sogar allgemeiner der

- Satz. Ist $\sum_{n=0}^{\infty} a_{n}$ eine konvergente Reihe mit beliebigen Gliedern und bedeutet $\left(p_{0}, p_{1}, \ldots\right)$ eine beliebige monoton $\rightarrow+\infty$ wachsende Folge positiver Zahlen, so strebt der Quotient

$$
\left.\frac{p_{0} a_{0}+p_{1} a_{1}+\ldots+p_{n} a_{n}}{p_{n}} \rightarrow 0^{1}\right) .
$$

Beweis. Nach 44, 2, folgt aus $s_{n} \rightarrow s$, dafi auch

$$
\frac{p_{1} s_{0}+\left(p_{2}-p_{1}\right) s_{1}+\ldots+\left(p_{n}-p_{n-1}\right) s_{n-1}}{p_{n}} \rightarrow s
$$

strebt. Wegen $\frac{p_{0} s_{0}}{p_{n}} \rightarrow 0, s_{n} \rightarrow s$, muf also

$$
s_{n}-\frac{\left(p_{1}-p_{0}\right) s_{0}+\left(p_{2}-p_{1}\right) s_{1}+\ldots+\left(p_{n}-p_{n-1}\right) s_{n-1}}{p_{n}} \rightarrow 0
$$

1) L. Kronecker, Comptes rendus de l'Ac. de Paris, Bd. 103 (1886), S. 980 . 
streben. Dies ist aber genau die zu beweisende Beziehung, wie man sofort erkennt, wenn man alles auf den Nenner $p_{n}$ bringt und nun der Reihe nach die Glieder mit $p_{0} . p_{1}, \ldots, p_{n}$ sammelt $^{1}$ ).

8. Unsere jetzigen Sätze und Bemerkungen gelten natiirlich auch für Reihen mit positiven Gliedern. Man prüfe das in jedem Falle nach.

9. Für die Reihe

$$
\sum_{n=1}^{\infty} \frac{(-1)^{n-1}}{n}=1-\frac{1}{2}+\frac{1}{3}-\frac{1}{4}+\frac{1}{5}-+\ldots
$$

ist

$$
T_{n}=T_{n, k}=(-1)^{n}\left[\frac{1}{n+1}-\frac{1}{n+2}+\frac{1}{n+3}+\ldots+\frac{(-1)^{k-1}}{n+k}\right] .
$$

Mag nun $k$ gerade oder ungerade sein, der Ausdruck in der Klammer ist jedenfalls positiv und $<\begin{gathered}1 \\ n+1\end{gathered}$. Denn faft man je ein positives und ein negatives Glied in eine Klammer zusammen, so ist deren Wert jedenfalls positiv. Bei geradem $k$ werden auf diese Weise alle Glieder anfgebraucht, bei ungeradem $k$ bleibt ein positives übrig, so dá in beiden Fällen der Ausdruck als positir erkannt wird. Schreibt man ihn andererseits in der Form

$$
n+1-\left(\frac{1}{n+2}-\frac{1}{n+3}\right)-\left(\begin{array}{cc}
1 & 1 \\
n+4 & n+5
\end{array}\right)-\ldots
$$

so werden jetzt bei ungeradem $k$ alle Glieder aufgebraucht, während bei geradem $k$ ein negatives Glied übrigbleibt, so dafi in beiden Fällen von $\begin{gathered}1 \\ n+1\end{gathered}$ nur abgezogen wird, der Ausdruck also $<\frac{1}{n-1}$ ist. Wegen

folgt nun aber, daf

$$
T_{n}=T_{n, k} \quad \begin{gathered}
1 \\
n+1
\end{gathered}
$$

$$
T_{n} \rightarrow 0
$$

strebt, unsere Reihe also konvergent ist. - Wir werden später (S. 205) sehell. dafs ihre Summe ïbereinstimmt mit dem Grenzwert der Folge $\mathbf{1 6}, 2$ und den Wert $\log 2$ hat.

10. Ist $\left(a_{0}, a_{1}, a_{2}, \ldots\right)$ eine positive monotone Nullfolgc', so zeigt man genat

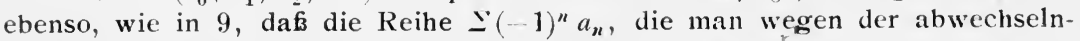
den Vorzeichen ihrer Glieder als alternievende Reihe bezeichnet, konvergiert. Denn jetzt ist

$$
T_{n} \mid=T_{n, k}<a_{n},
$$

was $T_{n}>0$ und also die Konvergenz der Reihe zur Folge hat. Es gilt also die folgende

Leibnizsche Regel $\left.{ }^{2}\right)$ : Eine alternierende Reihe, bei weliher die Beträge der Glied,r ine monotone Nullfolge bilden, ist stets konvergent.

1) Statt der positiven $p_{n}$ darf man auch (vgl. \$4,3 und 5) irgendeine Zahlenfolge $\left(p_{n}\right)$ nehmen, für die einerseits $\mid p_{n} \rightarrow+\infty$ strebt und andrerseits eine Konstante $K$ angegeben werden kann, so daf stets

bleibt.

$$
p_{0}+\left|p_{1}-p_{0}\right|+\ldots+p_{n}-p_{n-1}<K\left|p_{n}\right|
$$

2) Briefe an J. Hermann vom 26. VI. 1705, und an Joh. Bernoulli vom 10. I. 1714. 
S3. §15. Das 2. Hauptkriterium u. d. Rechnèn mit konvergenten Reihen. 125

11. An den Konvergenzbedingungen ist noch hervorzuheben, daf sich die darin geforderten Bedingungen immer nur auf diejenigen Glieder beziehen - oder zu beziehen brauchen -, die hinter einem bestimmten stehen, dessen Index übrigens durch jeden größeren ersetzt werden dürfte. Es kommt also bei der Entscheidung der Konvergenzfrage, wie man kurz zu sagen pflegt, auf den Anfang der Reihe nicht an. Wir präzisieren dies genauer in dem folgenden

○ Satz. Leitet man aus einer vorgelegten Reihe $\sum_{n=0}^{\infty} a_{n}$ dadurch eine neue Reihe $\sum_{n=0}^{\infty} a_{n}^{\prime}$ her, daß man bei jener endlich viele Glieder wegstreicht oder endlich viele Glieder voranstellt oder endlich viele. Glieder abändert (oder alles zugleich) und nun die Glieder der entstandenen Reihe neu mit $a_{0}{ }^{\prime}, a_{1}{ }^{\prime}, \ldots$ bezeichnet ${ }^{1}$ ), so sind beide Reihen konvergent oder beide divergent.

Beweis. Die Voraussetzungen besagen, daf es eine bestimmte ganze Zahl $q \gtreqless 0$ gibt, so dafs von einer Stelle ab, etwa für alle $n>m$ stets

$$
a_{n}^{\prime}=a_{n+q}
$$

ist. Jedes Teilstiick der einen Reihe ist daher auch ein Teilstiick der andern Reihe, wofern der Anfangsindex derselben nur $>m+|q|$ ist. Der Hauptsatz 81, 2 liefert nun unmittelbar die Richtigkeit unserer Behauptung.

12. Ausdrücklich sei noch hervorgehoben, dafs für Reihen mit beliebigen Gliedern die Vergleichskriterien aller Art vollständig außer Kraft gesetzt sind. Insbesondere kann von zwei Reihen $\Sigma a_{n}$ und $\Sigma a_{n}{ }^{\prime}$, deren Glieder asymptotisch gleich sind $\left(a_{n} \simeq a_{n}{ }^{\prime}\right)$, sehr wohl die eine konvergieren, die andre divergieren. Man setze z. B. $a_{n}=\frac{(-1)^{n}}{n}$ und $a_{n}{ }^{\prime}=a_{n}+\frac{1}{n \log n}$.

\section{Das Rechnen mit konvergenten Reihen.}

Schon S. 97 wurde betont, daß die Bezeichnung „Summe“ für den Grenzwert der Folge der Teilsummen einer Reihe insofern irreführend ist, als sie den Glauben erweckt, mit einer unendlichen Reihe lasse sich nach denselben Regeln rechnen, wie mit einer (wirklichen) Summe aus einer bestimmten Anzahl Summanden, also etwa wie mit Summen der Form $(a+b+c+d)$. Das ist aber keineswegs der Fall, jener Glaube also prinzipiell falsch, wenn auch einige jener Regeln tatsächlich für unendliche Reihen gültig bleiben. Die Hauptgesetze für das Rechnen mit (wirklichen) Summen sind (nach $\boldsymbol{2}$, I. u. III) das Assoziations-, das Distributions- und das Kommutationsgesetz. Die folgenden Sätze sollen lehren, inwieweit diese Gesetze auch noch für unendliche Reihen gelten und inwieweit nicht.

- Satz 1. Das Assoziationsgesetz gilt uneingeschränkt auch bei unendlichen Reihen, d. h. aus

$$
a_{0}+a_{1}+a_{2}+\ldots=s
$$

1) Also kurz: „... daf man bei der Folge $\left(a_{n}\right)$ der Glieder jener Reihe endlich viele Änderungen $(\mathbf{2 7}, 4)$ vornimmt, ...". 
folgt

$$
\left(a_{0}+a_{1}+\ldots+a_{v_{1}}\right)+\left(a_{v_{1}+1}+a_{v_{1}+2}+\ldots+a_{v_{2}}\right)+\ldots . .=s,
$$

wenn $v_{1}, v_{2}, \ldots$ irgendeine wachsende Folge verschiedener ganzer Zahlen $\geqq 0$ bedeutet und die Summe der durch eine Klammer zusammenge. schlossenen Glieder als ein Glied einer neuen Reihe

$$
\begin{gathered}
A_{0}+A_{1}+\ldots+A_{k}+\ldots \\
\left(A_{k}=a_{v_{k+1}}+a_{v_{k}+2}+\ldots+a_{v_{k+1}} ; k=0,1,2, \ldots ; \quad v_{0}=-1\right)
\end{gathered}
$$

angesehen wird.

Beweis. Die Folge der Teilsummen $S_{k}$ von $\Sigma A_{k}$ ist ersichtlich die Teilfolge $s_{\boldsymbol{v}_{1}}, s_{v_{2}}, \ldots, s_{\boldsymbol{v}_{\boldsymbol{k}}}, \ldots$ der Folge der Teilsummen $s_{n}$ von $\Sigma a_{n}$. Nach 41, 4 strebt daher $S_{n}$ demselben Grenzwert zu wie $s_{n}$.

Beispiel. Mit $\sum_{n=1}^{\infty} \frac{(-1)^{n-1}}{n} 1-\frac{1}{2}+\frac{1}{3}-\frac{1}{4}+\ldots$ ist also auch

$$
\sum_{k=1}^{\infty}\left(\frac{1}{2 k-1}-\frac{1}{2 k}\right)=\sum_{k=1}^{\infty} \frac{1}{(2 k-1) \cdot 2 k}=\frac{1}{1 \cdot 2}+\frac{1}{3 \cdot 4}+\frac{1}{5 \cdot 6}+\ldots
$$

und ebenso auch die Reihe

$$
1-\left(\frac{1}{2}-\frac{1}{3}\right)-\left(\frac{1}{4}-\frac{1}{5}\right)-\ldots=1-\frac{1}{2 \cdot 3}-\frac{1}{4 \cdot 5}-\frac{1}{6 \cdot 7}-\ldots
$$

konvergent und alle drei haben dieselbe Summe. Bezeichnen wir diese mit $s$, so lehrt die $z$ weite der Reihen, daß jedenfalls $s>\frac{1}{1 \cdot 2}+\frac{1}{3 \cdot 4}=\frac{7}{12}$, die dritte aber, dafs $s<1-\frac{1}{2 \cdot 3}=\frac{10}{12}$ sein mus. Es ist also

$$
\frac{7}{12}<s<\frac{10}{12} \text {. }
$$

Bemerkung. Ausdrücklich sei betont, daf3 man nach Satz 1 zwar Klammern setzen, nicht aber ohne weiteres weglassen darf, - wie folgendes einfache Beispiel zeigt: Die Reihe $0+0+0+\ldots$ ist gewiß konvergent mit der Summe 0 . Setzen wir überall $(1-1)$ statt 0 , so bekommen wir zunächst die richtige Gleichung

$$
(1-1)+(1-1)+\ldots \quad=\Sigma(1-1)=0 .
$$

Ohne die Klammern aber erhalten wir die divergente Reihe

$$
1-1+1-1+-\ldots,
$$

die also nicht $"=0^{\prime}$ gesetzt werden darf, denn sonst würde man durch eine erneute, aber nun etwas geänderte Zusammenfassung der Glieder die Reihe

$$
1-(1-1)-(1-1)-\ldots=1-0-0-0-\ldots
$$

erhalten, welche nun wieder konvergiert und die Summe 1 hat. Man würde also schließlich herausbekommen, daßs $0=1$ ist!! ${ }^{1}$ )

1) In früheren Zeiten - also vor der strengen Begründung des Rechnens mit unendlichen Reihen (s. Einl.) - stand man solchen Paradoxien ziemlich ratlos gegenüber. Und wemn auch die besseren Mathematiker sozusagen instinktiv solchen Schliissen, wie dem obigen, aus dem Wege gingen, so wurden sie den minderen Köpfen um so mehr Anlaß zu den kühnsten Spekulationen. So glaubte 
83. § 15. Das 2. Hauptkriterium u. d. Rechnen mit konvergenten Reihen. 127

Wir ergänzen daher den Satz 1 sogleich durch den folgenden

- Satz 2. Sind die Glieder einer konvergenten unendlichen Reihe $\sum_{k=0}^{\infty} A_{k}$ selbst wirkliche Summen (etwa, wie vorhin, $A_{k}=a_{v_{k}+1}+\ldots+a_{v_{k+1}}$; $k=0,1, \ldots ; v_{0}=-1$ ), so darf man die sie umschließenden Klammern nur dann weglassen, wenn die dadurch entstehende neue Reihe $\sum_{n=0}^{\infty} a_{n}$ wieder konvergiert. In der Tat ist ja dann nach dem vorigen Satze $\Sigma a_{n}=\Sigma A_{k}$, während im Falle der Divergenz von $\Sigma a_{n}$ diese Gleichung sinnlos wäre.

Ein meist ausreichendes Kennzeichen dafür, $o b$ nun die neue Reihe konvergiert, liefert der folgende

o Zusatz. Die nach dem vorigen Satz aus $\Sigma A_{k}$ hergeleitete neue Reihe $\Sigma a_{n}$ fällt sicher konvergent aus, wenn die Größen

eine Nullfolge bilden ${ }^{1}$ ).

$$
A_{k}^{\prime}=\left|a_{v_{k}+1}\right|+\left|a_{v_{k}+2}\right|+\ldots+\left|a_{v_{k+1}}\right|
$$

Beweis. Ist $\varepsilon>0$ gegeben, so wähle man $m_{1}$ so grofi, daß̧ für $k>m_{1}$ stets

$$
\left|S_{k-1}-s\right|<\frac{\varepsilon}{2}
$$

ausfällt; und $m_{2}$ so groß, dafi für $k>m_{2}$ stets $A_{k}^{\prime}<\frac{\varepsilon}{2}$ bleibt. Ist dann $m$ größier als jede der beiden Zahlen $m_{1}$ und $m_{2}$, so ist für $n>v_{m}$ stets

$$
\left|s_{n}-s\right|<\varepsilon \text {. }
$$

Denn jedem solchen $n$ entspricht eine ganz bestimmte Zahl $k$, für die

$$
v_{k}<n \leqq v_{k+1}
$$

ist und diese Zahl $k$ muf $\geq m$ sein. Dann ist aber

$$
s_{n}=S_{k-1}+a_{v_{k}+1}+\ldots+a_{n} \quad\left|s_{n}-S_{k-1}\right| \leqq A_{k}^{\prime}<\frac{\varepsilon}{2} .
$$

Und wegen

ist dann in der Tat

$$
s_{n}-s=\left(s_{n}-S_{k-1}\right)+\left(S_{k-1}-s\right)
$$

$$
\left|s_{n}-s\right|<\varepsilon, \quad \text { d. h. } \sum_{n=0}^{\infty} a_{n}=s, \quad \text { w. z. b. w. }
$$

Beispiel.

$$
\sum_{n=1}^{\infty} A_{k} \equiv\left(1+\frac{1}{3}-\frac{1}{2}\right)+\left(\frac{1}{5}+\frac{1}{7}-\frac{1}{4}\right)+\ldots+\left(\frac{1}{4 k-3}+\frac{1}{4 k-1}-\frac{1}{2 k}\right)+\ldots
$$

ist konvergent, denn $A_{k}$ ist positiv und für $k>1$ stets

$$
<\frac{2}{4 k-4}-\frac{1}{2 k}=\frac{1}{2(k-1) \cdot k} \leqq \frac{1}{k^{2}} \text {. }
$$

z. B. Guido Grandi (nach R. Reiff, s. 69, 8) durch die obige irrige Schlußkkette, die aus der Null eine Eins werden läßt, die Möglichkeit der Erschaffung der Welt aus dem Nichts mathematisch bewiesen $z u$ haben!

$\left.{ }_{1}^{1}\right)$ Wegen $A_{k} \rightarrow 0$ ist das von selbst der Fall, wenn die Summanden, aus denen ein jedes $A_{k}$ besteht, jedesmal unter sich gleiche Vorzeichen haben, insbesondere also, wenn alle $A_{k}$ und alle $a_{n} \geq 0$ sind. 
Da ebenso für $k>1$ stets

$$
A_{k^{\prime}}<\frac{2}{4 k-4}+\frac{1}{2 k}<\frac{1}{k-1}
$$

ist, so ist $\left(A_{k}{ }^{\prime}\right)$ eine Nullfolge. Also ist die Reihe

$$
1+\frac{1}{3}-\frac{1}{2}+\frac{1}{5}+\frac{1}{7}-\frac{1}{4}++-\ldots
$$

konvergent. - Thre Summe - sie heifie $S$ - ist, da die Reihe in der ersten Gestalt nur positive Glieder hatte, jedenfalls $>A_{1}+A_{2}>\frac{11}{12}$.

o Satz 3. Konvergente Reihen darf man gliedweis addieren. Genauer: Aus

folgt scwohl

$$
\stackrel{\infty}{N}_{n=0}^{\infty} a_{n}=s \quad \text { und } \underset{n=0}{\stackrel{\infty}{!}} b_{n}=t
$$

$$
\sum_{n=0}^{x}\left(a_{n}+b_{n}\right)=s+t
$$

als auch - ohne Klammern! -

$$
a_{0}+b_{0}+a_{1}+b_{1}+a_{2}+\ldots=s+t
$$

Beweis. Sind $s_{n}$ und $t_{n}$ die Teilsummen der ersten beiden Reihen, so sind $\left(s_{n}+t_{n}\right)$ diejenigen der dritten. Nach 41, $s$ folgt also sofort, daß $\left(s_{n}+t_{n}\right) \rightarrow s+t$ strebt. Daß man in der hiernach als konvergent erkannten Reihe die Klammern weglassen darf, folgt aber nach dem Zusatz zu Satz 2 sofort daraus, daß $a_{n} \mid$ und $b_{n}$ und also auch $\left(\left|a_{n}+b_{n}\right|\right)$ Nullfolgen sind.

o Satz 4. Konvergente Reihen darf man in demiselben Sinne sliedweis voneinander substrahieren. Beweis ebenso.

- Satz 5. Konvergente Reihen darf man mit einer Konstanten multiplizieren, d.h. aus $\sum a_{n}=s$ folgt, wenn $c$ eine beliebige Zahl ist,

$$
\Sigma\left(c a_{n}\right)=c s \text {. }
$$

Beweis. Die Teilsummen der neuen Reihe sin'd $c s_{n}$, wenn diejenigen der alten Reihe $s_{n}$ heißen. Satz 41, 10 liefert nun sofort die Behauptung. - Dieser Satz überträgt in gewissem Ausmaße das Distributionsgesetz der Addition auf unendliche Reihen.

Bemerkungen und Beispiele.

1. Diese einfachen Sätze sind darum so wichtig, weil sie jedesmal nicht nur die Konvergenz einer neuen Reihe liefern, sondern auch deren Summe mit denen der alten in Beziehung setzen. Sie bilden daher die Grundlage für ein wirkliches Rechnen mit unendlichen Reihen.

2. Die Reihe $\sum_{n=1}^{\infty}\left(-\frac{1)^{n-1}}{n}\right.$ war konvergent. Ihre Summe heifie s. Nach Satz 1 sind auch die Reihen

$$
\sum_{k=1}^{\infty}\left(\begin{array}{c}
1 \\
2 k-1
\end{array}-\frac{1}{2 k}\right) \text { und } \sum_{k=1}^{\infty}\left(\frac{1}{4 k-3}-\frac{1}{4 k-2}-\frac{1}{4 k-1}-\frac{1}{4 k}\right)
$$


konvergent mit der Summe $s$. Multipliziere ich die erste nach Satz 4 gliedweis mit $\stackrel{1}{2}-$ dies liefert

$$
\sum_{k=1}^{\infty}\left(\frac{1}{4 k-2}-\frac{1}{4 k}\right)=\frac{s}{2}
$$

- und addiere sie nach Satz 3 gliedweis zur zweiten, so erhalte ich

$$
\sum_{k=1}^{\infty}\left(\frac{1}{4 k-3}+\frac{1}{4 k-1}-\frac{1}{2 k}\right)=\frac{3}{2} s,
$$

d. h. genauer: es hat sich die Konvergenz der linksstehenden Reihe und der Wert ihrer Summe ergeben, - letzterer ausgedrikkt durch die Summe der Reihe, von der wir ausgingen. Die Konvergenz hatten wir oben bei Satz 2 schon direkt festgestellt; die jetzige Rechnung fuhrt aber erheblich weiter, da sie eine bestimmte Aussage über die Summe liefert.

Ehe wir die Gültigkeit des Kommutations- und des Distributionsgesetzes und mit letzterem die Möglichkeit der Multiplikation zweier Reihen untersuchen, bedürfen wir noch einer wichtigen Vorbereitung.

\section{'§ 16. Absolute Konvergenz. Umordnung von Reihen.}

Die Reihe $1-\frac{1}{2}+\frac{1}{3}-\frac{1}{4}+\ldots$ hatte sich $\mathbf{8 2}, 9$ als konvergent erwiesen. Ersetzt man aber jedes Glied derselben durch seinen absoluten Betrag, so geht sie in die divergente harmonische Reihe $1+\frac{1}{2}+\frac{1}{3}+\ldots$ über. Für alles folgende wird es meist einen sehr wesentlichen Unterschied machen, ob eine konvergente Reihe $\Sigma a_{n}$ auch noch konvergent bleibt, wenn man alle Glieder durch ihren absoluten Betrag ersetzt, oder ob sie dadurch divergent wird. Hier gilt zunächst der

- Satz. Eine Reihe $\Sigma a_{n}$ ist sicher konvergent, wenn die Reihe (mit $\mathbf{8 5 .}$ positiven Gliedern) $\Sigma\left|a_{n}\right|$ konvergiert $\left.^{1}\right)$. Und ist dann $\Sigma a_{n}=s$, $\Sigma\left|a_{n}\right|=S$, so ist $|s| \leqq S$.

Beweis. Wegen

$$
\left|a_{n+1}+a_{n+2}+\ldots+a_{n+k}\right| \leqq\left|a_{n+1}\right|+\ldots+\left|a_{n+k}\right|
$$

ist mit der rechten Seite auch die linke $<\varepsilon$, woraus nach dem Hauptsatz 81 sofort die erste Behauptung folgt. Da ferner

$$
\left|s_{n}\right| \leqq\left|a_{0}\right|+\left|a_{1}\right|+\ldots+\left|a_{n}\right|<S
$$

ist, so ist nach 41,2 auch $|s| \leqq S$.

Hiernach verteilen sich alle konvergenten Reihen $\Sigma a_{n}$ auf zwei verschiedene Klassen, je nachdem nämlich $\Sigma\left|a_{n}\right|$ auch noch konvergiert oder nicht. Wir definieren

1) Cauchy, Anal. alg., S. 142. (Der Beweis ist noch unzulänglich.) - Dagegen lehrte das eben gebrachte Beispiel, dafs aus der Konvergenz von $\Sigma a_{n}$ nicht diejenige von $\Sigma\left|a_{n}\right| \mathrm{zu}$ folgen braucht. 
86. ${ }^{-D e f i n i t i o n . ~ I s t ~ e i n e ~ k o n v e r g e n t e ~ R e i h e ~} \sum a_{n}$ so beschaffen, daß sogar $\Sigma\left|a_{n}\right|$ konvergiert, so soll die erste Reihe absolut konvergent, andernfalls nicht-absolut konvergent heißen $\mathbf{1}$ ).

Beispiele. Die Reihen

$$
\begin{gathered}
\sum_{n=1}^{\infty} \frac{(-1)^{n}}{n^{2}} ; \quad \sum_{n=1}^{\infty} \frac{(-1)^{n-1}}{n^{\alpha}}, \quad(\alpha>1) ; \quad \sum_{n=0}^{\infty} a^{n}, 0>a>-1 ; \\
\sum_{n=0}^{\infty} \frac{x^{n}}{n^{n}}, \quad x<0 ; \quad \sum_{n=1}^{\infty} \frac{x^{n}}{n !}, \quad x<0,
\end{gathered}
$$

sind absolut konvergent. - Jede konvergente Reihe mit positiven Gliedern ist eo ipso absolut konvergent.

Die große Bedeutung des Begriffs der absoluten Konvergenz wird sich einmal darin zeigen, daß die Konvergenz absolut konvergenter Reihen viel leichter als diejenige nicht-absolut konvergenter Reihen erkannt werden kann, nämlich meist durch Vergleich mit Reihen mit positiven Gliedern, so daß dafür die einfachen und weitreichenden Sätze des vorigen Kapitels zuständig sind. Sodann abèr wird diese Bedeutung sich darin zeigen, daß man mit absolut konvergenten Reihen im großen und ganzen so rechnen darf wie mit (wirklichen) Summen aus einer bestimmten Anzahl Summanden. während dies bei nicht-absolut konvergenten Reihen im allgemeinen nicht mehr der Fall ist. - Die folgenden Sätze werden dies im einzelnen zeigen.

87. $\quad$ Satz 1. Ist $\Sigma c_{n}$ eine konvergente Reihe mit positiven Gliedern und genügen die Glieder einer vorgelegten Reihe $\Sigma a_{n}$ für $n>m$ der Bedingung

$$
\left|a_{n}\right| \leqq c_{n} \quad \text { oder der Bedingung }\left|\frac{a_{n+1}}{a_{n}}\right| \leqq \frac{c_{n+1}}{c_{n}},
$$

so ist $\sum a_{n}($ absolut $)$ konvergent $\left.{ }^{2}\right)$.

Beweis. Nach dem 1. bzw. 2. Vergleichskritęrium 72 und 73 ist $\Sigma\left|a_{n}\right|$, also nach $\mathbf{8 5}$ auch $\sum a_{n}$ konvergent $^{3}$ ).

Infolge dieses einfachen Satzes ist der ganze Vorrat von Kriterien, die sich auf Reihen mit positiven Gliedern beziehen, nunmehr für Reihen mit beliebigen Gliedern nutzbar gemacht. Wir lesen aus ihm noch den folgenden Satz ab:

1) "Nicht-absolut konvergent" heifit also eine Reihe, wenn sie zwar konvergiert, aber nicht absolut. Die Bezeichnung "nicht-absolut konvergent" soll also nur auf konvergente Reihen angewendet werden.

$\left.{ }^{2}\right)$ Bei der $z$ weiten Bedingung wird wieder stillschweigend angenommen, dafi für $n>m$ stets $a_{n} \neq 0$ und $c_{n} \neq 0$ ist.

3) Die entsprechenden Divergenzkriterien , $\left|a_{n}\right| \geqq d_{n}$ " bzw. , $\left|\frac{a_{n+1}}{a_{n}}\right| \geqq \mid \frac{d_{n+1}}{d_{n}}$ ! fallen hier natürlich fort, da nach ihnen $z$ war $\Sigma\left|a_{n}\right|$, aber nicht notwendig $\Sigma a_{n}$ divergieren müßste. Vgl. die Fufnote auf der vorigen Seite. 
- Satz 2. Ist $\sum a_{n}$ eine absolut konvergente Reihe und bilden die Faktoren $\alpha_{n}$ eine beschränkte Zahlenfolge, so ist auch die Reihe

(absolut) konvergent.

$$
\sum \alpha_{n} a_{n}
$$

Beweis. Da mit $\left(\alpha_{n}\right)$ auch $\left(\left|\alpha_{n}\right|\right)$ eine beschränkte Folge ist, so ist nach $\mathbf{7 0}, 2$ mit $\Sigma\left|a_{n}\right|$ auch $\Sigma\left|\alpha_{n}\right| \cdot\left|a_{n}\right| \equiv \Sigma\left|\alpha_{n} a_{n}\right|$ konvergent.

Beispiele.

1. Ist $\Sigma c_{n}$ irgendeine konvergente Reihe mit positiven Gliedern und sind die $\alpha_{n}$ beschränkt, so ist auch $\Sigma \alpha_{n} c_{n}$ konvergent, - denn $\Sigma c_{n}$ ist auch absolut konvergent. Man darf also $\mathrm{z}$. B. die Glieder $c_{0}, c_{1}, c_{2}, \ldots$ statt stets durch +-Zeichen ganz beliebig durch +- und --Zeichen verbinden, - immer entsteht eine (absolut) konvergente Reihe; denn die Faktoren \pm 1 bilden gewis eine beschränkte Folge. So sind also z. B. die Reihen

$$
\Sigma(-1)^{n} c_{n}, \quad \Sigma(-1)^{\left[\sqrt{n}^{[}\right]} c_{n}, \quad \Sigma(-1)^{[\log n]} c_{n}, \ldots
$$

konvergent, bei denen $[z]$ wie immer die größste ganze nicht oberhalb $z$ gelegene Zahl bedeutet.

2. Ist $\Sigma a_{n}$ absolut konvergent, so entsteht durch beliebige Änderung der Vorzeichen der Glieder immer wieder eine absolut konvergente Reihe.

Wir wollen nun - und damit kehren wir zu den am Ende des vorigen Paragraphen verlassenen Fragen zurück - zeigen, daß für absolut konvergente Reihen die Grundgesetze für das Rechnen mit (wirklichen) Summen im wesentlichen erhalten bleiben, daß dies für nicht absolut konvergente Reihen dagegen nicht mehr der Fall ist.

So gilt schon das Kommutationsgesetz $\left(, a+b=b+a^{\prime \prime}\right)$ nicht mehr allgemein für unendliche Reihen. Der Sinn dieser Aussage ist der: Ist $\left(\boldsymbol{v}_{0}, \boldsymbol{v}_{1}, \boldsymbol{v}_{\mathbf{2}}, \ldots\right)$ irgendeine Umordnung $(\mathbf{2 7}, 3)$ der Folge $(0,1,2, \ldots)$, so wollen wir von der Reihe

$$
\sum_{n=0}^{\infty} a_{n}{ }^{\prime} \equiv \sum_{n=0}^{\infty} a_{v_{n}} \quad\left(\text { also } a_{n}{ }^{\prime}=a_{r_{n}} \text { für } n=0,1,2, \ldots\right)
$$

kurz sagen, sie sei durch Umordnung aus der gegebenen Reihe $\sum_{n=0}^{\infty} a_{n}$ entstanden. Bei (wirklichen) Summen aus einer bestimmten Anzahl Summanden bleibt der Wert derselben ungeändert, wie man auch die Reihenfolge der Summanden umordnen (permutieren) möge. Bei unendlichen Reihen ist dies nicht mehr der Fall ${ }^{1}$ ). Dies lehren schon die beiden oben bei $\mathbf{8 2}, 9$ und $\mathbf{8 3}, 1$ und 2 behandelten konvergenten Reihen

$$
1-\frac{1}{2}+\frac{1}{3}-\frac{1}{4}+-\ldots \text { und } 1+\frac{1}{3}-\frac{1}{2}+\frac{1}{5}+\frac{1}{7}-\frac{1}{4}++-\ldots \text {, }
$$

die ersichtlich Umordnungen voneinander sind, die aber verschiedene Summen haben. Die Summe $s$ der ersten war nämlich $<\frac{10}{12}$, die Summe $s^{\prime}$ der zweiten $>\frac{11}{12}$; und die Betrachtungen 84, 2 lehrten sogar genauer, $\mathrm{da} ß s^{\prime}=\frac{3}{2} s$ ist.

1) Zuerst von Cauchy bemerkt (Résumés analytiques, Turin 1833). 
Diese Tatsache zwingt uns natürlich große Vorsicht beim Rechnen mit unendlichen Reihen auf, da man - kurz gesagt - auf die Reihenfolge ihrer Glieder achten $m u B^{1}$ ). Um so wertvoller ist es darum zu wissen, in welchen Fällen diese Vorsicht etwa außer acht gelassen werden darf. Hierüber gilt der

- Satz 1. Bei absolut konvergenten Reihen gilt das Kommutationsgesetz uneingeschränkt ${ }^{2}$.

Beweis. a) Wir beweisen den Satz zunächst für Reihen mit positiven Gliedern. Es sei $\Sigma c_{n}$ mit den Teilsummen $s_{n}$ und der Summe $s$ eine solche, und $\Sigma c_{n}{ }^{\prime} \equiv \Sigma c_{v_{n}}$ mit den Teilsummen $s_{n}{ }^{\prime}$ eine Umordnung derselben. Dann ist

$$
s_{n}^{\prime}<s_{N}
$$

wenn $N$ größer genommen wird als jede der Zahlen $v_{0}, v_{1}, v_{2}, \ldots, v_{n}$; denn es treten dann rechterhand, neben andern, die sämtlichen Summanden der linken Seite auf. Wegen $s_{N}<s$ ist also für jedes $n$ stets $s_{n}{ }^{\prime}<s$ und folglich (nach $\boldsymbol{7 0}$ ) $\Sigma c_{n}{ }^{\prime}$ konvergent. Ist $s^{\prime}$ die Summe, so ist (nach 46,1) überdies $s^{\prime} \leqq s$. Da nun umgekehrt $\Sigma c_{n}$ als eine Umordnung der Reihe $\Sigma c_{n}{ }^{\prime}$ aufgefaßt werden kann, so folgt analog $s \leqq s^{\prime}$. Es muß also $s=s^{\prime}$ sein, - womit alles bewiesen ist.

b) Es sei jetzt $\sum a_{n}$ eine beliebige absolut konvergente Reihe, also auch $\Sigma\left|a_{n}\right|$ konvergent. Deuten wir wieder durch Anfügen eines Akzentes an die Reihenglieder eine vollzogene Umordnung an, so ist nach a) auch $\Sigma\left|a_{n}^{\prime}\right|$, also nach $\mathbf{8 5}$ auch $\Sigma a_{n}^{\prime}$ konvergent. Durch die Umordnung wird also jedenfalls die Tatsache der Konvergenz nicht zerstört. Es bleibt aber auch der Summenwert ungeändert. Denn ist $\varepsilon>0$ beliebig gegeben, so wähle man nach $\mathbf{9 1}, 1$ zunächst $m$ so groß, daß für jedes $k \geqq 1$

$$
\left|a_{m+1}\right|+\left|a_{m+2}\right|+\ldots+\left|a_{m+k}\right|<\varepsilon
$$

ausfällt, und nun $n_{0}$ so groß, daß unter den Zahlen $v_{0}, v_{1}, v_{2}, \ldots, v_{n_{0}}$ die Zahlen $0,1,2, \ldots, m$ sämtlich vorgekommen $\operatorname{sind}^{3}$ ). Dann heben sich für $n>n_{0}$ in der Differenz $s_{n}^{\prime}-s_{n}$ ersichtlich die Glieder $a_{0}, a_{1}, a_{2}, \ldots, a_{m}$ fort und es bleiben höchstens solche Glieder stehen,

1) Da $\Sigma a_{n}$ lediglich fuir die Folge $\left(s_{n}\right)$ der Teilsummen steht, und da die bei einer Umordnung von $\Sigma a_{n}$ entstehende neue Reihe $\Sigma a_{n}^{\prime}$ ganz andre Teilsummen $s_{n}^{\prime}$ hat - die $s_{n}^{\prime}$ bilden nicht etwa blos eine Umordnung der $s_{n}$, sondern sind ganz andre Zahlen!! - so war es a priori sehr wahrscheinlich, daß eine solche Umordnung nicht ohne Einflufs auf das Verhalten der Reihe sein würde.

2) G. Lejenne Dirichlet, Abh. Akad. Berlin 1837, S. 48. (Werke I, S. 319.) Hier findet sich auch schon das im Text gegebene Beispiel der Veränderung der Summe durch Umordnung. ordnung.

$\left.{ }^{3}\right)$ Dafs eine solche Zahl $n_{0}$ existiert, folgt ja aus der Definition der Um- 
deren Index $>m$ ist, - also nur (endlich viele) der Glieder $a_{m+1}, a_{m+2}, \ldots$ Da aber die Summe der Beträge beliebiger vieler von diesen $<\varepsilon$ bleibt, so ist für $n>n_{0}$ stets

$$
\left|s_{n}{ }^{\prime}-s_{n}\right|<\varepsilon,
$$

also $\left(s_{n}{ }^{\prime}-s_{n}\right)$ eine Nullfolge. Dann hat aber $s_{n}{ }^{\prime}=s_{n}+\left(s_{n}{ }^{\prime}-s_{n}\right)$ denselben Grenzwert wie $s_{n}$, d. h. $\sum a_{n}$ und $\sum a_{n}{ }^{\prime}$ haben dieselbe Summe, w. z. b. w. ${ }^{\mathbf{1}}$ ).

Diese Eigenschaft absolut konvergenter Reihen ist so wesentlich, $\mathrm{da} ß$ sie eine besondere Bezeichnung verdient:

- Definition. Eine konvergente unendliche Reihe, für die das Kommutationsgesetz uneingeschränkt gilt, die also bei jeder Umordnung konvergent bleibt und auch ihren Summenwert nicht ändert, soll unbedingt konvergent genannt werden. Eine konvergente Reihe dagegen, deren Konvergenzverhalten durch Umordnungen verändert werden kann, bei der es also auf die Reihenfolge der Glieder ankommt, soll bedingt konvergent heißen.

Der vorhin bewiesene Satz kann dann auch so ausgesprochen werden: "Jede absolut konvergente Reihe ist stets auch unbedingt konvergent." - Von diesem Satze gilt auch die Umkehrung, also der

-Satz 2. Jede nicht-absolut konvergente Reihe ist nur bedingt konvergent ${ }^{2}$ ). M. a. W.: Bei einer nicht-absolut konvergenten Reihe $\Sigma a_{n}$ mit der Summe $s$ hängt das Bestehen der Gleichung

$$
\sum_{n=0}^{\infty} a_{n}=s
$$

wesentlich von der Anordnung der Glieder der linksstehenden Reihe $\mathrm{ab}$ und kann also durch eine geeignete Umordnung gestört werden.

Beweis. Es genügt offenbar zu zeigen, daß aus $\Sigma a_{n}$ durch eine passende Umordnung eine divergierende Reihe $\Sigma a_{n}{ }^{\prime}$ hergestellt werden

1) Zweiter Beweis. Da $\Sigma a_{n}$ und $\sum\left|a_{n}\right|$ konvergieren, sind nach 83, 3,4 und 5 auch

$$
\sum \frac{\left|a_{n}\right|+a_{n}}{2} \text { und } \sum \frac{\left|a_{n}\right|-a_{n}}{2}
$$

konvergent; $P$ und $N$ seien ihre Summen. Dies sind aber Reihen mit positiven Gliedern. (Welche Bedeutung haben diese Reihen und ihre Summen?) Sie gestatten also nach a) eine beliebige Umordnung, so daf auch

$$
\sum \frac{\left|a_{n}{ }^{\prime}\right|+a_{n}{ }^{\prime}}{2}=P \quad \text { und } \quad \sum \frac{\left|a_{n}{ }^{\prime}\right|-a_{n}{ }^{\prime}}{2}=N
$$

ist. Subtrahiert man diese Reihen - was nach 83, 4 geschehen darf - voneinander, so findet man: $\sum a_{n}{ }^{\prime}=P-N$, während die ungestrichenen Reihen durch Subtraktion $\Sigma a_{n}=P-N$ liefern. $\sum a_{n}{ }^{\prime}$ konvergiert also und hat dieselbe Summe wie $\Sigma a_{n}$, w. z. b. w.

2) Vgl. hierzı den Hauptsatz aus § 44 . 
kann. Das gelingt so: Diejenigen Glieder der Reihe $\Sigma a_{n}$, die $\geqq 0$ sind, sollen in der Reihenfolge, wie sie in $\Sigma a_{n}$ auftreten, mit $p_{1}, p_{2}, p_{3}, \ldots$ bezeichnet werden; diejenigen, die $<0$ sind, ebenso mit $-q_{1},-q_{2},-q_{3}, \ldots$ Dann sind $\Sigma p_{n}$ und $\Sigma q_{n}$ Reihen mit positiven Gliedern. Von diesen muß mindestens eine divergent sein. Denn wären sie beide konvergent und $P$ und $Q$ ihre Summen, so wäre für jedes $n$ ersichtlich $\left|a_{0}\right|+\left|a_{1}\right|+\ldots+\left|a_{n}\right| \leqq P+Q$, also $\Sigma a_{n}$ entgegen der Annahme absolut konvergent ${ }^{1}$ ). Ist etwa $\Sigma p_{n}$ divergent, so setzen wir eine Reihe der Form

$p_{1}+p_{2}+\ldots+p_{m_{1}}-q_{1}+p_{m_{1}+1}+p_{m_{1}+2}+\ldots+p_{m_{2}}-q_{2}+p_{m_{2}+1}+\ldots$ an, in der also jedesmal auf eine Gruppe pasitiver Glieder ein einziges negatives Glied folgt. Diese Reihe ist offenbar eine Umordnung der gegebenen Reihe $\Sigma a_{n}$ und soll als solche mit $\Sigma a_{n}{ }^{\prime}$ bezeichnet werden. Da nun die Reihe $\Sigma p_{n}$ divergieren sollte und ihre Teilsummen also nicht beschränkt sind, so kann man hierbei zunächst $m_{1}$ so groß wählen, $\mathrm{da} B p_{1}+p_{2}+\ldots+p_{m_{1}}>1+q_{1}$ ist, dann $m_{2}>m_{1}$ so groß, daß

$$
p_{1}+p_{2}+\ldots+p_{m_{1}}+\ldots+p_{m_{2}}>2+q_{1}+q_{2}
$$

wird, und nun allgemein $m_{v}>m_{v-1}$ so gro $\beta$, daß

$$
p_{1}+p_{2}+\ldots+p_{m_{v}}>v+q_{1}+q_{2}+\ldots+q_{v}
$$

ausfällt (für $v=3,4, \ldots$ ). Dann ist aber $\Sigma a_{n}{ }^{\prime}$ offenbar divergent; denn diejenigen Teilsummen dieser Reihe, deren letzter Summand ein negatives Glied $-q_{v}$ ist, sind hiernach $>v(v=1,2, \ldots)$. Und da hierin $v$ jede natürliche Zahl sein darf, so sind die Teilsummen von $\sum a_{n}{ }^{\prime}$ keinesfalls beschränkt, $\sum a_{n}{ }^{\prime}$ selbst also divergent, w. z. b. w. $\left.{ }^{2}\right)$

Ist $\Sigma q_{n}$ divergent, so hat man im vorangehenden nur die Rollen der beiden Reihen $\Sigma p_{n}$ und $\Sigma q_{n}$ sinngemäß zu vertauschen, um zu demselben Ergebnis zu gelangen.

\section{Beispiel.}

$\sum_{n=1}^{\infty} \frac{(-1)^{n}}{n} \equiv-1+\frac{1}{2}-\frac{1}{3}+\frac{1}{4}-\frac{1}{5}+\ldots$ war als nicht-absolut konvergent erkannt. Da (vgl. 46, 3) fuir $\lambda=1,2, \ldots$

ist, so ist für $v=1,2, \ldots$

$$
\frac{1}{2}+\frac{1}{4}+\frac{1}{6}+\cdots+\frac{1}{2^{2}}>\frac{\lambda}{4}
$$

$$
\frac{1}{2}+\frac{1}{4}+\frac{1}{6}+\ldots+\frac{1}{2^{8 v}}>2 v
$$

Wendet man also das beschriebene Verfahren auf die Reihe $\sum \frac{(-1)^{n}}{n}$ an,

1) Es ist nicht schwer zu sehen, daf sogar beide Reihen $\Sigma p_{n}$ und $\Sigma q_{n}$ divergieren miissen (vgl. §44); doch ist das im Augenblick belanglos.

2) $\Sigma a_{n}^{\prime}$ divergiert ersichtlich $\rightarrow+\infty$. 
so hat man nur $m_{\nu}=8 v$ zu setzen, um aus ihr lediglich durch Umordnung die divergente Reihe

$$
\frac{1}{2}+\frac{1}{4}+\frac{1}{6}+\ldots+\frac{1}{2^{8}}-1+\frac{1}{2^{8}+2}+\ldots+\frac{1}{2^{16}}-\frac{1}{3}+\ldots
$$

herzuleiten. Denn die mit dem $v$ ten negativen Summanden endende Teilsumme ist größer als $2 v$ vermindert um $v$ echte Brüche, - also sicher $>v$.

Der Satz 88, 1 von der Umordnung absolut konvergenter Reihen läßt sich noch wesentlich verallgemeinern. Dazu beweisen wir zunächst den folgenden einfachen

- Satz 3. Ist $\sum a_{n}$ absolut konvergent, so ist auch iede "Teilreihe“ $\Sigma a_{\lambda_{n}}$ - bei der also die $\lambda_{n}$ irgendeine monoton wachsende Folge verschiedener natürlicher Zahlen bedeuten — wieder (absolut) konvergent.

Beweis. Nach $\boldsymbol{7 4}, 4$ ist mit $\sum\left|a_{n}\right|$ auch $\sum\left|a_{\lambda_{n}}\right|$ konvergent. Nach 85 folgt hieraus sofort die Behauptung.

Nunmehr können wir den Umordnungssatz 88, 1 folgendermaßen erweitern. Aus der absolut konvergenten Reihe $\sum a_{n}$ werde eine erste Teilreihe $\sum a_{\lambda_{n}}$ herausgegriffen, die wir jetzt mit

$$
a_{0}^{(0)}+a_{1}^{(0)}+\ldots+a_{n}^{(0)}+\ldots
$$

und deren nach dem letzten Satz vorhandene Summe wir mit $z^{(0)}$ bezeichnen wollen ${ }^{1}$ ). Bei ihr und den folgenden Teilreihen wollen wir auch zulassen, daß sie nur aus endlich vielen Gliedern besteht, also gar keine unendliche Reihe ist. Aus den übriggebliebenen Gliedern werde - soweit das noch möglich ist - wieder eine (endliche oder unendliche) Teilreihe herausgegriffen, die mit

$$
a_{0}^{(1)}+a_{1}^{(1)}+a_{2}^{(1)}+\ldots+a_{n}^{(1)}+\ldots
$$

bezeichnet werden und die Summe $z^{(1)}$ haben möge; aus den übrig gebliebenen Gliedern wieder eine neue Teilreihe usw. Wir bekommen dadurch im allgemeinen eine unendliche Folge endlicher oder (absolut) konvergenter unendlicher Reihen

$$
\begin{aligned}
& a_{0}{ }^{(0)}+a_{1}{ }^{(0)}+a_{2}{ }^{(0)}+\ldots+a_{n}{ }^{(0)}+\ldots=z^{(0)} \\
& a_{0}{ }^{(1)}+a_{1}{ }^{(1)}+a_{2}{ }^{(1)}+\ldots+a_{n}{ }^{(1)}+\ldots=z^{(1)} \\
& a_{0}{ }^{(2)}+a_{1}{ }^{(2)}+a_{2}{ }^{(2)}+\ldots+a_{n}{ }^{(2)}+\ldots=z^{(2)}
\end{aligned}
$$

Ist das Verfahren nun so beschaffen, da $\beta$ jedes Glied der gegebenen Reihe $\Sigma a_{n}$ in einer und nur einer der Teilreihen vorkommt, so kann man die Reihe $z^{(0)}+z^{(1)}+z^{(2)}+\ldots$ oder also die Reihe

$$
\left(\sum a_{n}{ }^{(0)}\right)+\left(\sum a_{n}{ }^{(1)}\right)+\left(\sum a^{(2)}\right)+\ldots
$$

1) Der Buchstabe $z$ soll an die Zeilen des nachfolgenden $z$ weifach-unendlichen Schemas erinnern. 
auch noch im weiteren Sinne eine Umordnung der gegebenen Reihe nennen ${ }^{1}$ ). Auch für eine solche gilt noch der dem Satz 88, 1 entsprechende

-Satz 4. Eine absolut konvergente Reihe ,darf“ auch in diesem erweiterten Sinne umgeordnet werden. Genauer: Auch die Reihe

$$
z^{(0)}+z^{(1)}+z^{(2)}+\ldots
$$

ist (absolut) konvergent und ikre Summe ist gleich derjenigen von $\Sigma a_{n}$. Beweis. 1. Nach $\mathbf{8 5}$ ist

$$
\begin{aligned}
& z^{(0)}|\leqq| a_{0}{ }^{(0)}|+| a_{1}{ }^{(0)}|+\ldots+| a_{n}{ }^{(0)} \mid+\ldots \\
& \left|z^{(1)} \leqq\right| a_{0}^{(1)}|+| a_{1}^{(1)}|+\ldots+| a_{n}^{(1)} \mid+\ldots \\
& z^{(k)}\left|\leqq a_{0}{ }^{(k)}\right|+\left|a_{1}{ }^{(k)}\right|+\ldots+\left|a_{n}{ }^{(k)}\right|+\ldots
\end{aligned}
$$

woraus durch Addition (nach 83, 3) folgt, daB $\left|z^{(\mathbf{0})}\right|+\left|z^{(\mathbf{1})}\right|+\ldots+\left|z^{k}\right|$ die Summe einer gewissen Teilreihe von $\Sigma\left|a_{n}\right|$ und also sicher nicht größer als die Summe $S$ dieser Reihe selbst ist. Da dies für $k=0,1,2, \ldots$ gilt, so sieht man, daß die Teilsummen der Reihe

$$
z^{(0)}|+| z^{(1)}|+| z^{(2)} \mid+\ldots
$$

beschränkt sind. Also ist $\Sigma\left|z^{(k)}\right|$ konvergent, $\Sigma^{(k)}$ absolut konvergent.

2. Ist nun $\varepsilon>0$ gegeben und $m$ wie auf S. 132 so bestimmt, daß für jedes $k \geqq 1$ das Teilstück $\left|a_{m+1}\right|+\left|a_{m+2}+\ldots+\right| a_{m+k} \mid<\varepsilon$ ausfällt, so wähle man jetzt $n_{0}$ so, daß in den ersten Teilreihen $\sum a_{n}{ }^{(r)}$, für $v=0,1, \ldots, n_{0}$, sicher die Glieder $a_{0}, a_{1}, a_{2}, \ldots, a_{m}$ der gegebenen Reihe auftreten. Dann enthält für $n>n_{0}$ die Reihe

$$
\left(z^{(0)}+z^{(1)}+\ldots+z^{(n)}\right)-s_{n}
$$

nur solche Glieder $\pm a_{n}$, die einen oberhalb $m$ liegenden Index haben. Daher ist, nach der Art wie $m$ bestimmt wurde, der Betrag dieser Differenz $\leqq \varepsilon$; sie strebt also mit wachsendem $n$ gegen 0 , so daß

$$
\lim _{n \rightarrow \infty}\left(z^{(\mathbf{0})}+z^{(\mathbf{1})}+\ldots+z^{(n)}\right)=\lim _{n \rightarrow \infty} s_{n}=s=\sum a_{n}
$$

ist, w. z. b. w.

Von diesem Satz gilt natürlich noch weniger als von Satz $\mathbf{8 3}, 1$ ohne weiteres die Umkehrung, d. h. wenn für $k=0,1,2, \ldots$ die konvergenten Reihen

$$
z^{(k)}=\sum_{n=0}^{\infty} a_{n}{ }^{(k)}
$$

1) In die erste Teilreihe tue man z. B. aufer $a_{0}$ und $a_{1}$ alle Glieder $a_{n}$, deren Index $n$ durch 2 teilbar ist, in die nächste alle diejenigen der übrig gebliebenen Glieder, deren Index durch 3 teilbar ist, in die nächste alle übrig gebliebenen Glieder, deren Index durch 5 teilbar ist; usw. unter Benutzung der Primzahlen $7,11,13, \ldots$ als Teiler. 
vorgelegt sind und wenn nun die sämtlichen Größen $a_{n}{ }^{(k)}$ irgendwie zu einer Folge $\left(a_{n}\right)$ angeordnet werden (vgl. 53,4), so braucht $\sum a_{n}$ nicht zu konvergieren, - selbst wenn $\sum_{k=0}^{\infty} z^{(k)}$ konvergent sein sollte. Schon ein so einfaches Beispiel, bei dem für jede der Reihen $z^{(k)}$ die Reihe $1-1+0+0+0+\ldots$ genommen wird, läßt dies erkennen. Und selbst wenn $\Sigma a_{n}$ konvergiert, so braucht ihre Summe nicht gleich derjenigen von $\Sigma z^{(k)}$ zu sein.

Die allgemeine Behandlung der Frage, unter welchen Umständen diese Umkehrung unseres Satzes nun doch erlaubt ist, gehört in die Lehre von den Doppelreihen. Doch können wir schon hier den folgenden für die Anwendungen besonders wichtigen Fall beweisen:

- Großer Umordnungssatz. Es seien die unendlich vielen kon-90. vergenten Reihen

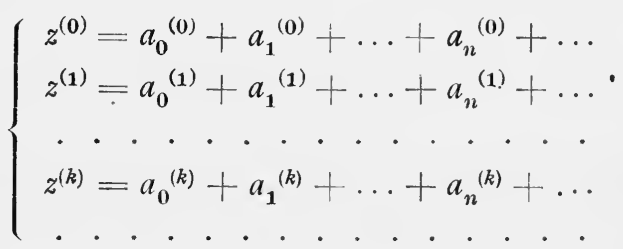

vorgelegt, und es seien diese Reihen nicht nur absolut konvergent, sondern es möge auch noch, wenn

gesetzt wird,

$$
\sum_{n=0}^{\infty}\left|a_{n}{ }^{(k)}\right|=\zeta^{(k)} \quad(k=0,1,2, \ldots, \text { fest })
$$

$$
\sum_{k=0}^{\infty} \zeta^{(k)}=\sigma
$$

eine konvergente Reihe sein. Dann bilden auch die spaltenweis untereinanderstehenden Glieder (absolut) konvergente Reihen; und wenn

$$
\sum_{k=0}^{\infty} a_{n}{ }^{(k)}=s^{(n)} \quad(n=0,1,2, \ldots, \text { fest })
$$

geselzt wird $\left.{ }^{1}\right)$, so ist auch noch $\sum s^{(n)}$ absolut konvergent und es besteht die Gleichheit

$$
\sum_{n=0}^{\infty} S^{(n)}=\sum_{k=0}^{\infty} z^{(k)}
$$

d. $h$. die Reihe aus den Zeilensummen und diejenige aus den Spaltensummen sind beide absolut konvergent und haben dieselbe Summe.

Der Beweis ist äußerst einfach: Die sämtlichen Glieder des Schema (A) denke man sich irgendwie (nach 53, 4) zu einer ein-

1) Hi er soll der Buchstabe $s$ an die Spalten von $(A)$ erinnern. 
fachen Folge angeordnet und als solche mit $a_{0}, a_{1}, a_{2}, \ldots$ bezeichnet. Dann ist $\sum a_{n}$ absolut konvergent. Eine jede Teilsumme von $\Sigma\left|a_{n}\right|$, z. B.

$$
\left|a_{0}\right|+\left|a_{1}\right|+\ldots+\left|a_{m}\right|
$$

muß nämlich noch $<\sigma$ sein; denn wählt man $k$ so groß, daß dic Glieder $a_{0}, a_{1}, a_{2}, \ldots, a_{m}$ sämtlich in den ersten $k$ Zeilen aufgetreten sind, so ist doch sicher $\left|a_{0}\right|+\left|a_{1}\right|+\ldots+\left|a_{m}\right| \leqq \zeta^{(0)}+\zeta^{(1)}+\ldots+\zeta^{(k)}$, also noch $<\sigma$. Eine andre Anordnung $\operatorname{der} a_{n}{ }^{(k)}$ zu einer einfachen Folge $a_{0}{ }^{\prime}, a_{1}{ }^{\prime}, a_{2}{ }^{\prime}, \ldots$ würde eine Reihe $\sum a_{n}{ }^{\prime}$ liefern, die nur eine Umordnung von $\sum a_{n}$ ist und die also gleichfalls absolut und mit derselben Summe konvergiert. Diese stets zum Vorschein kommende Summe heiße $s$.

Von $\Sigma a_{n}=s$ ist nun aber sowohl $\sum z^{(k)}$ als auch $\Sigma s^{(n)}$ eine Umordnung in dem erweiterten Sinne des eben bewiesenen Satzes 4 . Also sind auch diese beiden Reihen absolut konvergent und haben dieselbe Summe, w. z. b.w.

Dieser grofe Umordnungssatz kann folgendermaßen noch etwas allgemeiner gefafit werden:

○ Zusatz. Ist $M$ eine abzählbare Zahlenmenge und existicrt eine Konstante $K$, so daß die Summe der absoluten Beträge irgendwelcher endlich vieler Elemente aus $M$ stets $<K$ bleibt, so ist jede Reihe $\Sigma A_{k}$ absolut konvergent - und jedesmal mit derselben Summe s-, deren Glicder $A_{k}$ je eine Summe von endlich oder unendlich vielen Elementen aus $M$ darstellen (wofern jedes Element aus $M$ in einem und nur einem Gliede $A_{k}$ auftritt). Und dies gilt auch noch, wenn man ein mehrfaches Auftreten der Elemente in $M$ zuläpt, wofern dann jedes Element in den $A_{k}$ zusammengenommen genau ebenso oft auftritt wie in $M$ selber ${ }^{1}$ ).

Beispiele zu diesen wichtigen Sätzen werden im folgenden mehrfach an entscheidenden Stellen auftreten. Wir geben hier noch ein paar naheliegende Anwendungen:

1. Es sei $\Sigma a_{n}=s$ eine absolut konvergente Reihe und es werde

$$
\frac{a_{0}+2 a_{1}+4 a_{2}+\cdots+2^{n} a_{n}}{2^{n+1}}=a_{n}{ }^{\prime} \quad{ }^{\prime} \quad(n=0,1,2, \ldots)
$$

gesetzt. Dann ist auch $\Sigma_{n_{n}}{ }^{\prime}=s$. Der Beweis ergibt sich nach dem großen Umordnungssatz unmittelbar aus dem Schema

$$
\left\{\begin{array}{c}
a_{0}=\frac{a_{0}}{2}+\frac{a_{0}}{4}+\frac{a_{0}}{8}+\frac{a_{0}}{16}+\ldots \\
a_{1}=0+2 \frac{a_{1}}{4}+2 \frac{a_{1}}{8}+2 \frac{a_{1}}{16}+\ldots \\
a_{2}=0+0+4 \frac{a_{2}}{8}+4 \frac{a_{2}}{16}+\ldots \\
\ldots \ldots
\end{array}\right.
$$

1) Ein unendlich-maliges Auftreten eines von 0 verschiedenen Gliedes ist dabei von vornherein ausgeschlossen, da sonst die Konstante $K$ des Satzes sicher nicht vorhanden wäre. Und die Zahl 0 kann nicht stören. 
2. Ähnlich hat man wegen $\frac{1}{p(p+1)}+\frac{1}{(p+1)(p+2)}+\ldots=\frac{1}{p}($ s. 68, 2 h $)$ aus dem Schema

$$
\left\{\begin{array}{l}
a_{0}=\frac{a_{0}}{1 \cdot 2}+\frac{a_{0}}{2 \cdot 3}+\frac{a_{0}}{3 \cdot 4}+\ldots \\
a_{1}=0+2 \frac{a_{1}}{2 \cdot 3}+2 \frac{a_{1}}{3 \cdot 4}+\ldots \\
a_{2}=0+0+3 \frac{a_{2}}{3 \cdot 4}+\ldots \\
\ldots . . . . . .
\end{array}\right.
$$

die Gleichung

$$
\sum_{n=0}^{\infty} a_{n}=\frac{a_{0}}{1 \cdot 2}+\frac{a_{0}+2 a_{1}}{2 \cdot 3}+\frac{a_{0}+2 a_{1}+3 a_{2}}{3 \cdot 4}+\ldots
$$

3. Der große Umordnungssatz gilt offenbar stets, wenn alle $a_{n}{ }^{(k)} \geqq 0$ sind und wenigstens eine der beiden Reihen $\Sigma z^{(k)}$ und $\Sigma s^{(n)}$ konvergiert. Ferner wenn man dem Schema (A) ein analog gebautes Schema $\left(A^{\prime}\right)$ an die Seite stellen kann, dessen Zahlen positiv und $\geq$ den Beträgen der entsprechenden Zahlen in (A) sind, und wenn nun in $\left(\mathrm{A}^{\prime}\right)$ entweder die Summe der Zeilensummen oder die der Spaltensummen konvergiert.

\section{$\S 17$. Multiplikation unendlicher Reihen.}

Wir fragen endlich, inwieweit das Distributionsgesetz „a $a(b+c)$ $=a b+a c^{\prime \prime}$ für unendliche Reihen noch gültig ist. Daß eine konvergente unendliche Reihe $\sum a_{n}$ gliedweis mit einer Konstanten multipliziert werden darf, hatten wir schon $\mathbf{8 3}, 5$ gesehen. In der einfachsten Form

$$
c\left(\Sigma a_{n}\right)=\Sigma\left(c a_{n}\right)
$$

ist also das Gesetz für alle konvergenten Reihen richtig. Bei wirklichen Summen folgt aber aus dem Distributionsgesetz sofort weiter, $\mathrm{da} B(a+b)(c+d)=a c+a d+b c+b d$ und allgemein, daß

$$
\left(a_{0}+a_{1}+\ldots+a_{q}\right)\left(b_{0}+b_{1}+\ldots b_{r}\right)=a_{0} b_{0}+a_{0} b_{1}+\ldots+a_{q} b_{r}
$$

oder kürzer, daß

$$
\left(\sum_{\lambda=0}^{q} a_{\lambda}\right) \cdot\left(\sum_{\mu=0}^{r} b_{\mu}\right) \underset{\substack{\lambda=0, \ldots, q \\ \mu=0, \ldots, r}}{=} \sum_{\lambda} a_{\lambda} b_{\mu}
$$

ist, wobei die Symbolik rechterhand bedeuten soll, daß die Indizes $\lambda$ und $\mu$ unabhängig voneinander alle ganzen Zahlen von 0 bis $q$ bzw. 0 bis $r$ durchlaufen und alle $(q+1)(r+1)$ derartigen Produkte $a_{\lambda} b_{\mu}$ in irgendeiner Reihenfolge addiert werden sollen.

Bleibt diese Folgerung auch noch für unendliche Reihen bestehen? Ist also, wenn $\Sigma a_{n}=s$ und $\Sigma b_{n}=t$ zwei gegebene konvergente 
unendliche Reihen mit den Summen $s$ und $t$ sind, eine ähnliche Ausmultiplikation des Produktes

$$
s \cdot t=\left(\sum_{\lambda=0}^{\infty} a_{\lambda}\right) \cdot\left(\sum_{\mu=0}^{\infty} b_{\mu}\right)
$$

möglich, bzw. in welchem Sinne ist dies möglich? Oder genauer: Die Produkte

$$
a_{\lambda} b_{\mu} \quad\left(\begin{array}{l}
\lambda=0,1,2, \ldots \\
\mu=0,1,2, \ldots
\end{array}\right)
$$

mögen in irgendeiner Reihenfolge mit $p_{0}, p_{1}, p_{2}, \ldots$ bezeichnet werden $^{1}$ ); ist dann die Reihe $\Sigma p_{n}$ konvergent und hat sie im Falle der Konvergenz die Summe $s \cdot t$ ? - Auch in dieser Frage verhalten sich die absolut konvergenten Reihen ebenso wie wirkliche Summen. Es gilt nämlich der

91. Satz. Sind die Reihen $\sum a_{n}=s$ und $\sum b_{n}=t$ absolut konvergent, so konvergiert auch die Reihe $\Sigma p_{n}$ absolut und hat die Summe $\left.s \cdot t^{2}\right)$.

Beweis. 1. Es sei $n$ eine bestimmte natürliche Zahl, und unter den Indizes $\lambda$ und $\mu$ der mit $p_{0}, p_{1}, \ldots, p_{n}$ bezeichneten Produkte $a_{\lambda} b_{\mu}$ sei $m$ der höchste. Dann ist ersichtlich

$$
\left|p_{0}\right|+\left|p_{1}\right|+\cdots+\left|p_{n}\right| \leqq\left(\sum_{\lambda=0}^{m}\left|a_{\lambda}\right|\right) \cdot\left(\sum_{\mu=0}^{m}\left|b_{\mu}\right|\right)
$$

also $\left\langle\sigma \cdot \tau\right.$, wenn mit $\sigma$ und $\tau$ die Summen der Reihen $\Sigma\left|a_{\lambda}\right|$ und $\Sigma\left|b_{\mu}\right|$ bezeichnet werden. Die Teilsummen der Reihe $\Sigma\left|p_{n}\right|$ sind also beschränkt und $\Sigma p_{n}$ somit absolut konvergent.

2. Nachdem die absolute Konvergenz von $\Sigma p_{n}$ festgestellt ist, brauchen wir die Summe dieser Reihe - sie heiße $S$ - nur für eine spezielle Anordnung der Produkte $a_{\lambda} b_{\mu}$, etwa der „nach Quadraten" zu ermitteln. Bei dieser ist aber ersichtlich

$$
a_{0} b_{0}=p_{0}, \quad\left(a_{0}+a_{1}\right)\left(b_{0}+b_{1}\right)=p_{0}+p_{1}+p_{2}+p_{3}
$$

und allgemein

$$
\left(a_{0}+\ldots+a_{n}\right)\left(b_{0}+\ldots+b_{n}\right)=p_{0}+\ldots+p_{(n+1)^{2}-1},
$$

eine Gleichung, die nach $\mathbf{4 1}, 10$ und 4 für $n \rightarrow \infty$ in die behauptete Beziehung

übergeht.

$$
s \cdot t=S
$$

Bemerkungen und Beispiele.

1. Wie betont, ist es für das Bestehen der Gleichung $\Sigma p_{n}=s \cdot t$ unter den gemachten Voraussetzungen ganz gleichgültig, in welcher Weise wir die

1) Wir denken uns dazu die Produkte $a_{\lambda} b_{\mu}$ genau wie in 53,4 und 90 die Zahlen $a_{n}{ }^{(k)}$ oder $a_{2}^{(\mu)}$ in Form eines zweifach-unendlichen Schemas (A) hingeschrieben. Dann kann man sich die Numerierung der Produkte insbesondere wieder nach Schräglinien oder nach Quadraten vorgenommen denken.

2) Cauchy, Analyse alg., S. 147. 
Produkte $a_{\lambda} b_{\mu}$ abgezählt, d. h. zu der einfachen Folge der $p_{n}$ angeordnet haben. Besonders wichtig für die Anwendungen ist die Anordnung nach Schräglinien, die uns, wenn man alle in derselben Schräglinie stehenden Produkte (nach $\mathbf{8 3}, 1$ ) zusammenfafit, zu der folgenden Gleichung führt:

$$
\begin{gathered}
\sum_{n=0}^{\infty} a_{n} \cdot \sum_{n=0}^{\infty} b_{n}=a_{0} b_{0}+\left(a_{0} b_{1}+a_{1} b_{0}\right)+\left(a_{0} b_{2}+a_{1} b_{1}+a_{2} b_{0}\right)+\cdots \\
=\sum_{n=0}^{\infty} c_{n},
\end{gathered}
$$

wenn zur Abkürzung $a_{0} b_{n}+a_{1} b_{n-1}+a_{2} b_{n-2}+\ldots a_{n} b_{0}=c_{n}$ gesetzt wird. Deren Bestehen ist also gesichert, wenn die beiden linksstehenden Reihen absolut konvergieren.

Auf die so angeordnete "Produktreihe“, die man auch als das Cauchysche Produkt der gegebenen Reihen bezeichnet ${ }^{1}$ ), wird man auch durch die Multiplikation ganzer rationaler Funktionen und die im näclisten Kapitel zu besprechenden Potenzreihen geführt: Multipliziert man nämlich die ganzen rationalen Funktionen

$$
a_{0}+a_{1} x+a_{2} x^{2}+\ldots+a_{q} x^{q} \quad \text { und } \quad b_{0}+b_{1} x+b_{2} x^{2}+\ldots+b_{r} x^{r}
$$

miteinander und ordnet das Produkt wieder nach steigenden Potenzen von $x$, so beginnt es mit den Gliedern

$$
a_{0} b_{0}+\left(a_{0} b_{1}+a_{1} b_{0}\right) x+\left(a_{0} b_{2}+a_{1} b_{1}+a_{2} b_{0}\right) x^{2}+\ldots,
$$

so daf3 als Koeffizienten gerade die eben eingeführten Zahlen $c_{0}, c_{1}, c_{2}, \ldots$ auftreten. Und eben wegen dieses Zusammenhanges tritt das Cauchysche Produkt zweier Reihen besonders häufig auf.

2. Da $\Sigma x^{n}$ für $|x|<1$ absolut konvergent, so hat man für diese $x$

$$
\left(\frac{1}{1-x}\right)^{2}=\sum_{n=0}^{\infty} x^{n} \cdot \sum_{n=0}^{\infty} x^{n}=\sum_{n=0}^{\infty}(n+1) x^{n} .
$$

3. Die Reihe $\sum \frac{x^{n}}{n !}$ ist, vgl. $\boldsymbol{7 6}, 4 \mathrm{c}$, für jede reelle Zahl $x$ absolut konvergent. Sind also $x_{1}$ und $x_{2}$ irgend zwei reelle Zahlen, so dürfen wir

$$
\Sigma a_{n} \equiv \Sigma \frac{x_{1}{ }^{n}}{n !} \quad \text { und } \quad \sum b_{n} \equiv \sum \frac{x_{2}{ }^{n}}{n !}
$$

nach der Cauchyschen Regel ausmultiplizieren. Hier wird

$$
c_{n}=\sum_{\nu=0}^{n} a, b_{n-v}=\sum_{\nu=0}^{n} \frac{x_{1}{ }^{\prime} x_{2}{ }^{n-v}}{v !(n-v) !}=\frac{1}{n !} \sum_{v=0}^{n} \frac{n !}{v !(n-v) !} x_{i}{ }^{v} x_{2}{ }^{n-v}=\frac{\left(x_{1}+x_{2}\right)^{n}}{n !} .
$$

Es ist also - für beliebige $x_{1}$ und $x_{2}$ - stets

$$
\sum_{n=0}^{\infty} \frac{x_{1}{ }^{n}}{n !} \cdot \sum_{n=0}^{\infty} \frac{x_{2}{ }^{n}}{n !}=\sum_{n=0}^{\infty} \frac{x_{3}{ }^{n}}{n !}
$$

wenn $x_{1}+x_{2}=x_{3}$ gesetzt wird.

Durch unsern Satz ist nunmehr dargetan, daß das Distributionsgesetz auf unendliche Reihen sicher dann ohne weiteres ausgedehnt werden kann - und sogar bei beliebiger Anordnung der Produkte $a_{\lambda} b_{\mu}$ - wenn die gegebenen Reihen beide absolut konvergieren. Es wäre denkbar, daß diese einschränkende Voraussetzung unnötig hart ist. Daß der Satz aber ohne jede einschränkende Voraussetzung nicht

$\left.{ }^{1}\right)$ Cauchy untersucht 1. c. die Produktreihe nur in dieser speziellen Form. 
mehr gilt, lehrt das folgende schon von Cauchy $^{1}$ ) zu diesem Zwecke gegebene Beispiel: Es sei

$$
a_{0}=b_{0}=0 \quad \text { und } \quad a_{n}=b_{n}=\frac{(-1)^{n-1}}{\sqrt{n}^{n}} \text { für } n \geqq 1 .
$$

Dann ist $c_{0}=c_{1}=0$ und für $n \geqq 2$

$$
c_{n}=(-1)^{n}\left[\frac{1}{\sqrt{1} \sqrt{n-1}}+\frac{1}{\sqrt{2} \sqrt{n-2}}+\ldots+\frac{1}{\sqrt{n-1 \sqrt{1}}}\right] .
$$

Ersetzt man hierin alle Radikanden durch den größten von ihnen, also durch $n-1$, so folgt, daß für $n \geqq 2$

$$
\left|c_{n}\right| \geqq \frac{n-1}{\sqrt{n-1 \cdot \sqrt{n-1}}}=1
$$

ist und daß also die Produktreihe $\Sigma c_{n} \equiv \Sigma\left(a_{0} b_{n}+a_{1} b_{n-1}+\ldots+a_{n} b_{0}\right)$ nach $\mathbf{8 2}, 6$ sicher divergent ist. Sie muß es um so mehr sein, wenn man die Klammern wegläßt.

Immerhin bleibt die Frage offen, ob man nicht unter geringeren Voraussetzungen die Konvergenz der Produktreihe $\Sigma p_{n}$ - wenigstens bei spezieller Anordnung der Produkte $a_{k} b_{\mu}$, etwa wie in der Reihe $\Sigma c_{n}$ - beweisen kann. Auf diese Frage werden wir in $\S 45$ zurückkommen.

\section{Aufgaben zum IV. Kapitel.}

45. Man prïfe das Konvergenzverhalten der Reihe $\mathcal{Y}(-1)^{n} a_{n}$, bei der $a_{r}$ von einer Stelle an einen der folgenden Werte hat:

$$
\frac{1}{a+n}, \frac{1}{a n+b}, \frac{1}{\sqrt{n}}, \frac{1}{\log n}, \frac{1}{\log \log n}, \frac{1}{\sqrt[n]{n}}, \frac{1}{\sqrt{n}}+\frac{(-1)^{n}}{n}, \ldots
$$

16. Wie ändern sich die Antworten in Aufgabe 34, wenn nach dem Konvergenzverhalten von $\Sigma(-1)^{n} a_{n}$ gefragt wird.

17. Es sei

Dann ist die Reihe

$$
\varepsilon_{n}=\left\{\begin{array}{l}
+1 \text { fuir } 2^{2 k} \leqq n<2^{2 k+1}, \\
-1 \text { fuir } 2^{2 k+1} \leqq n<2^{2 k+2}
\end{array} \quad(k=0,1,2, \ldots)\right.
$$

$$
\sum_{k=2}^{\infty} \frac{\varepsilon_{n}}{n \log n}
$$

konvergent. Wie verhält sich $\sum \frac{\varepsilon_{n}}{n}$ ?

18. $\sum_{n=1}^{\infty}(-1)^{n-1} \frac{2 n+1}{n(n+1)}$ ist konvergent und hat 1 zur Summe.

49. Die Teilsummen der Reihe $1-\frac{1}{2}+\frac{1}{3}-\frac{1}{4}+-\ldots$ seien mit $s_{n}$ be-

1) Analyse algébrique, S. 149. 
zeichnet und $\frac{1}{n+1}+\frac{1}{n+2}+\cdots+\frac{1}{2 n}=x_{n}$ gesetzt. Man zeige, daß für jedes $n$ und also $\lim x_{n}=\sum_{n=1}^{\infty} \frac{(-1)^{n-1}}{n}=s(=\log 2)$ ist.

50. Es sei wie eben $s(=\log 2)$ die Summe der Reihe $1-\frac{1}{2}+\frac{1}{3}-+\ldots$ Man beweise die folgenden Gleichungen:
a) $\frac{1}{3}-\frac{1}{5}-\frac{1}{7}+\frac{1}{5}-\frac{1}{9}-\frac{1}{11}+\frac{1}{7}-\frac{1}{13}-\frac{1}{15}+--\ldots=\frac{1}{3}-\frac{1}{2} \log 2$;
b) $1-\frac{1}{2}-\frac{1}{4}+\frac{1}{3}-\frac{1}{6}-\frac{1}{8}+\frac{1}{5}-\frac{1}{10}-\frac{1}{12}+-\ldots=\frac{1}{2} \log 2$;
c) $1-\frac{1}{2}-\frac{1}{4}+\frac{1}{5}+\frac{1}{7}-\frac{1}{8}-\frac{1}{10}++-\ldots=\frac{2}{3} \log 2$;
d) $1+\frac{1}{3}+\frac{1}{5}-\frac{1}{2}-\frac{1}{4}+++-\ldots=\frac{1}{2} \log 6$;
e) $1+\frac{1}{3}+\frac{1}{5}-\frac{1}{2}-\frac{1}{4}-\frac{1}{6}+++--\ldots=\log 2$.

51. Im Anschluf an die beiden letzten Fragen zeige man allgemein, dafs die Reihe konvergent bleibt, wenn man stets auf $p$ positive Glieder $q$ negative folgen läft, und dafs dann ihre Summe $=\log 2+\frac{1}{2} \log \frac{p}{q}$ ist.

52. Die harmonische Reihe $1+\frac{1}{2}+\frac{1}{3}+\frac{1}{4}+\ldots$ bleibt divergent, wenn man die Vorzeichen so abändert, dafs auf je $p$ positive Glieder immer $q$ negative folgen und $p \neq q$ ist. Ist $p=q$, so ist die entstandene Reihe konvergent.

53. Man nehme mit der Reihe $\sum_{n=1}^{\infty} \frac{(-1)^{n-1}}{\sqrt{n}}$ genau dieselben Umordnungen vor wie in Aufgabe 50 und 51 mit der Reihe $\Sigma \frac{(-1)^{n-1}}{n}$. Wann erhält man eine konvergente Reihe, wann nicht? Wann läft sich ihre Summe durch die der gegebenen ausdrïcken?

54. Man nehme bei der Reihe $\geq \frac{1}{\sqrt{n}}$ dieselben Vorzeichenänderungen vor, wie in Aufgabe 52 bei der Reihe $\Sigma \frac{1}{n}$. Wann entsteht eine konvergente Reihe?

55. Für welche $\alpha$ konvergieren die Reihen

$$
\begin{gathered}
1-\frac{1}{2^{\alpha}}+\frac{1}{3}-\frac{1}{4^{\alpha}}+\frac{1}{5}-\frac{1}{6^{\alpha}}+-\ldots, \\
1+\frac{1}{3^{\alpha}}-\frac{1}{2^{\alpha}}+\frac{1}{5^{\alpha}}+\frac{1}{7^{\alpha}}-\frac{1}{4^{\alpha}}++-\ldots ?
\end{gathered}
$$

56. Die Summe der Reihe $1-\frac{1}{2^{\alpha}}+\frac{1}{3^{\alpha}}-\frac{1}{4^{\alpha}}+-\ldots$ liegt für jedes $\alpha>0$ zwischen $\frac{1}{2}$ und 1 . 
5\%. Es sei

$$
\sum_{n=1}^{\infty} \frac{1}{n^{2}} \quad s\left(=\frac{\pi^{2}}{6}\right) .
$$

Dann ist

$$
\begin{aligned}
& 1+\frac{1}{3^{2}}+\frac{1}{5^{2}}+\frac{1}{7^{2}}+\ldots=\frac{3}{4} s, \\
& 1+\frac{1}{5^{2}}+\frac{1}{7^{2}}-\frac{1}{11^{2}}+\frac{1}{13^{2}}+\ldots=\frac{2}{3} s, \\
& 1-\frac{1}{2^{2}}-\frac{1}{4^{2}}+\frac{1}{5^{2}}+\frac{1}{7^{2}}-\frac{1}{8^{2}}-\frac{1}{10^{2}}++\cdots . .=\frac{4}{9} s .
\end{aligned}
$$

(Mit der letzten Gleichung vgl. Aufgabe $50 \mathrm{c}$ ).

55. In jeder (bedingt) konvergenten Reihe kann man die Glieder so in Gruppen zusammenfassen, dafs die neue Reihe absolut konvergiert.

59. Leitet man aus $\stackrel{\Sigma}{\prime} a_{n}$ mit den Teilsummen $s_{n}$ durch Assoziation von Gliedern eine neue Reihe $\Sigma A_{k}$ mit den Teilsummen $S_{k}$ her, so ist stets

$$
\underline{\lim } s_{n} \leqq \underline{\lim } S_{k} \leqq \lim s_{n}
$$

mag $\mathcal{Y} a_{n}$ konvergieren oder divergieren.

60. Ist $\Sigma a_{n}$ mit den Teilsummen $s_{n}$ unbestimmt divergent und ist $s^{\prime}$ einer der Häufungswerte der Folge $\left(s_{n}\right)$, so kann man durch Assoziation von. Gliedern aus $\Sigma a_{n}$ eine zur Summe $s^{\prime}$ konvergierende Reihe $\Sigma A_{k}$ herleiten.

61. Ist $\Sigma a_{n}$ mit den Teilsummen $s_{n}$ unbestimmt divergent und strebt $a_{n} \rightarrow 0$, so ist jeder Punkt der Strecke zwischen den beiden Häufungsgrenzen von $\left(s_{n}\right)$ ein Häufungspunkt dieser Folge.

62. Wenn jede Teilreihe von $\Sigma a_{n}$ konvergiert, so ist diese Reihe absolut konvergent.

63. Das Cauchysche Produkt der beiden bestimmt divergenten Reihen

$$
1-\frac{3}{2}-\left(\frac{3}{2}\right)^{2}-\left(\frac{3}{2}\right)^{3}-\ldots
$$

und

$$
1+\left(2+\frac{1}{2^{2}}\right)+\frac{3}{2}\left(2^{2}+\frac{1}{2^{3}}\right)+\left(\frac{3}{2}\right)^{2}\left(2^{3}+\frac{1}{2^{4}} \cdot\right)+\cdots
$$

lautet

$$
1+\frac{3}{4}+\left(\frac{3}{4}\right)^{2}+\left(\frac{3}{4}\right)^{3}+-\ldots
$$

und ist also absolut konvergent. Wie ist dies Paradoxon zu erklären? 
V. Kapitel.

\section{Potenzreihen.}

\section{$\S 18$. Der Konvergenzradius.}

Die Glieder der Reihen, die wir bisher betrachtet haben, waren im allgemeinen wohlbestimmte Zahlen. Man spricht daher wohl schärfer von Reihen mit konstanten Gliedern. Indessen war das doch nicht durchweg der Fall. Bei der geometrischen Reihe $\sum a^{n}$ z. B. sind die Glieder erst dann bestimmte Zahlen, wenn der Wert der Größe a gegeben wird. Die Konvergenzuntersuchung dieser Reihe endete daher auch nicht einfach mit der Entscheidung über Konvergenz oder Divergenz, sondern ihr Ergebnis lautete: $\sum a^{n}$ ist konvergent, falls $|a|<1$ ist, dagegen divergent, falls $|a| \geqq 1$ ist. Die Entscheidung der Konvergenzfrage hängt also, wie die Reihenglieder selbst, von dem Wert einer noch nicht festgelegten Größe, einer Veränderlichen ab. Reihen, deren Glieder und bei denen somit das Konvergenzverhalten noch von einer veränderlichen Größe abhängt - wir werden eine solche dann meist mit $x$ bezeichnen und von Reihen mit veränderlichen Gliedern sprechen ${ }^{1}$ ) —, werden wir später genauer untersuchen. Für den Augenblick wollen wir, im Anschluß an die geometrische Reihe, nur solche Reihen dieser Art betrachten, deren allgemeines Glied nicht eine Zahl $a_{n}$ ist, sondern die Gestalt

hat, also Reihen der Form

$$
a_{n} x^{n}
$$

$$
a_{0}+a_{1} x+a_{\mathbf{2}} x^{2}+\ldots+a_{n} x^{n}+\ldots=\sum_{n=0}^{\infty} a_{n} x^{n} .
$$

Solche Reihen nennt man Potenzmeihen (in $x$ ), die Zahlen $a_{n}$ ihre Koeffizienten. Bei solchen Potenzreihen wird es sich also nicht einfach um die Alternative ,konvergent oder divergent" handeln, sondern um die genauere Frage: für welche $x$ ist sie konvergent, fur welche divergent?

Einfache Beispiele sind uns schon begegnet:

1. Die geometrische Reihe $\Sigma x^{n}$ ist für $|x|<1$ konvergent, für $|x| \geq 1$ divergent. Für $|x|<1$ ist die Konvergenz sogar eine absolute.

2. $\sum \frac{x^{n}}{n !}$ ist für jedes reelle $x$ (absolut) konvergent. Ebenso die Reihen

$$
\sum_{k=0}^{\infty}(-1)^{k} \frac{x^{2 k}}{(2 k) !} \quad \text { und } \quad \sum_{k=0}^{\infty}(-1)^{k} \frac{x^{2 k+1}}{(2 k+1) !} \text {. }
$$

1) Auch die harmonische Reihe $\Sigma \frac{1}{n^{x}}$ ist eine solche Reihe; sie ist für $x>1$ konvergent, für $x \leq 1$ divergent. 
3. $\sum \frac{x^{n}}{n}$ ist wegen $\left|\frac{x^{n}}{n}\right| \leqq x^{n}{ }^{n}$ fuir. $\quad x \mid<1$ absolut konvergent. Für $x>1$ ist die Reihe divergent, weil (nach 3S, 3 und 40) dann $\frac{x^{n}}{n} \rightarrow+\infty$ strebt. Fiir $x=1$ geht sie in die divergente harmonische Reihe, fiir $x=-1$ in eine $\mathbf{S 2}, 9$ als konvergent erkannte Reihe über.

4. $\sum_{k=1}^{\infty} \frac{x^{n}}{n^{2} \cdot 2^{n}}$ ist fuir $|x| \leqq 2$ (absolut) konvergent, fuir $|x|>2$ dagegen divergent.

5. $\sum_{n=1}^{\infty} n^{n} x^{n}$ ist für $x=0$ konvergent; für jeden von 0 verschiedenen Wert von $x$ dagegen divergent, denn für $x \neq 0$ strebt $|n x| \rightarrow+\infty$, um so mehr also auch $\left|n^{n} x^{n}\right| \rightarrow+\infty$, so dafi von Konvergenz (nach $\mathbf{8 2}, 6$ ) keine Rede sein kann.

Für $x=0$ ist offenbar jede Potenzreihe $\sum a_{n} x^{n}$ konvergent, was für Zahlen auch die Koeffizienten $a_{n}$ sein mögen. Der allgemeinere Fall ist augenscheinlich der, daß die Potenzreihe für gewisse Werte von $x$ konvergiert, für gewisse andere divergiert, wobei auch die beiden extremen Fälle eintreten können, daß sie für jedes $x$ konvergiert (Beispiel 2), oder daß sie für kein von Null verschiedenes $x$ konvergiert (Beispiel ら).

In dem ersten dieser besonderen Fälle sagt man, die Potenzreihe sei beständig konvergent, im zweiten - indem man den selbstverständlichen Konvergenzpunkt $x=0$ außer acht läßt - sie sei nirgends konvergent. Allgemein nennt man die Gesamtheit der Punkte $x$, für die die vorgelegte Reihe $\sum a_{n} x^{n}$ konvergiert, ihren Konvergenzbereich. Bei 2. besteht dieser also aus der ganzen $x$-Achse, bei 5. nur aus dem Punkte 0 ; bei den anderen Beispielen besteht er aus einer Strecke, die durch den Nullpunkt halbiert wird, - teils mit, teils ohne Einschluß eines oder beider Endpunkte.

Hierdurch ist schon das Verhalten im allgemeinsten Falle getroffen, denn es gilt der

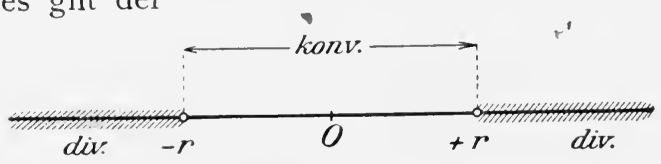

Fig. 2.

93. $\quad$ Hauptsatz. Ist $\Sigma a_{n} x^{n}$ eine beliebige Potenzreihe, die weder nirgends noch beständig konvergiert, so gibt es eine wohlbestiwimte Zahl $r$, derart, daß $\sum a_{n} x^{n}$ für alle $|x|<r$ konvergiert (und sogar absolut), während sic für alle $|x|>r$ divergiert $\left.{ }^{1}\right)$. Die Zahl $r$ bezeichnet man als den Konvergenzradius, die Strecke $-r \ldots+r$ als das Konvergenzintervall der vorgelegten Potenzreihe. - Fig. 2 veranschaulicht die durch diesen Satz festgestellte typische Situation.

1) In den beiden extremen Fällen sagt man wohl auch, der Konvergenzradius der Reihe sei $r=0$ bzw. $r=+\infty$. 
Die Grundlage des Beweises bilden die beiden Sätze:

- Satz 1. Ist eine vorgelegte Potenzreihe $\sum a_{n} x^{n}$ für $x=x_{0}\left(x_{0} \neq 0\right)$ konvergent oder auch nur die Folge $\left(a_{n} x_{0}{ }^{n}\right)$ ihrer Glieder beschränkt, so ist $\sum a_{n} x^{n}$ für jedes $x=x_{1}$ absolut konvergent, das näher am Nullpunkt liegt als $x_{0}$, für das also $\left|x_{1}\right|<\left|x_{0}\right|$ ist.

Beweis. Ist etwa stets $\left|a_{n} x_{0}{ }^{n}\right|<K$, so ist

$$
\left|a_{n} x_{1}{ }^{n}\right|=\left|a_{n} x_{0}{ }^{n}\right| \cdot\left|\frac{x_{1}}{x_{0}}\right|^{n} \leqq K \cdot \vartheta^{n},
$$

wenn der echte Bruch $\left|\frac{x_{1}}{x_{0}}\right|=\vartheta$ gesetzt wird. Nach 87,1 ergibt sich nun unmittelbar die Behauptung.

- Satz 2. Ist die vorgelegte Potenzreihe $\sum_{a_{n}} x^{n}$ für $x=x_{0}$ divergent, so divergiert sie um so mehr für jedes $x=x_{1}$, das weiter vom Nullpunkt abliegt als $x_{\mathbf{0}}$, für das also $\left|x_{\mathbf{1}}\right|>\left|x_{\mathbf{0}}\right|$ ist.

Beweis. Wäre die Reihe für $x_{1}$ konvergent, so müßte sie nach Satz 1 auch in dem näher am Nullpunkt gelegenen Punkt $x_{0}$ konvergieren, entgegen der Annahme.

Beweis des Hauptsatzes. Nach Voraussetzung soll mindestens ein Divergenzpunkt und ein von 0 verschiedener Konvergenzpunkt existieren. Wir können daher eine positive Zahl $x_{0}$ wählen, die näher am Nullpunkt liegt als der Konvergenzpunkt und eine positive Zahl $y_{0}$, die weiter vom Nullpunkt abliegt als der Divergenzpunkt. Nach den Sätzen 1 und 2 ist dann die Reihe $\sum a_{n} x^{n}$ für $x=x_{0}$ konvergent, für $x=y_{0}$ divergent, und es ist daher sicher $x_{0}<y_{0}$. Auf das Intervall $J_{0}=x_{0} \ldots y_{0}$ wenden wir nun die Halbierungsmethode an: wir bezeichnen seine linke oder seine rechte Hälfte mit $J_{1}$, je nachdem $\sum a_{n} x^{n}$ im Mittelpunkt von $J_{0}$ divergiert oder konvergiert. Nach derselben Regel bezeichnen wir eine bestimmte Hälfte von $J_{1}$ mit $J_{2}$, usw. Die Intervalle dieser Intervallschachtelung $\left(J_{n}\right)$ sind dann alle so beschaffen, daß $\sum a_{n} x^{n}$ in ihrem linken Endpunkt (er heiße $x_{n}$ ) konvergiert, im rechten dagegen (er heiße $y_{n}$ ) divergiert. Die durch die Schachtelung bestimmte (eo ipso positive) Zahl $r$ leistet nun das im Satz verlangte.

In der Tat, ist $x=x^{\prime}$ eine beliebige reelle Zahl, für die $\left|x^{\prime}\right|<r$ ist (mit Ausschluß der Gleichheit!), so wird $\left|x^{\prime}\right|<x_{k}$ sein, sobald wir $k$ so groß nehmen, daß die Länge des Intervalles $J_{k}$ kleiner als $r-\left|x^{\prime}\right|$ ist. Nach Satz 1 ist dann mit $x_{k}$ auch $x^{\prime}$ ein Konvergenzpunkt; und zwar ist die Konvergenz in $x^{\prime}$ eine absolute. Ist aber $x^{\prime \prime}$ eine Zahl, für die $\left|x^{\prime \prime}\right|>r$, so ist auch $\left|x^{\prime \prime}\right|>y_{m}$, sobald $m$ so groß gewählt wird, daß die Länge von $J_{m}$ kleiner als $\left|x^{\prime \prime}\right|-r$ ist. Mit $y_{m}$ ist dann nach Satz 2 auch $x^{\prime \prime}$ ein Divergenzpunkt. Damit ist alles bewiesen. 
Dieser gedanklich sehr einfache Beweis ist deshalb etwas unbefriedigend, als er nur lediglich die Existenz des Konvergenzradius $r$ nachweist, aber über seine Größe nichts aussagt. Wir wollen daher den Hauptsatz noch auf eine zweite Art und nun so beweisen, daß wir dabei die Größe des Radius selbst erhalten.

Dazu beweisen wir - ganz unabhängig vom vorigen - den genaueren

94. ${ }^{\circ}$ Satz $\left.{ }^{1}\right):$ Ist die Potenzreihe $\Sigma a_{n} x^{n}$ vorgelegt und $\mu$ die obere Häufungsgrenze der (positiven) Zahlenfolge

also

$$
\left|a_{1}\right|, \quad \sqrt{\left|a_{2}\right|}, \quad \sqrt[3]{\left|a_{3}\right|}, \ldots, \sqrt[n]{\left|a_{n}\right|}, \ldots
$$

so ist

$$
\mu=\varlimsup \sqrt[n]{\left|a_{n}\right|}
$$

a) für $\mu=0$ die Potenzreihe beständig konvergent;

b) für $\mu=+\infty$ die Potenzreihe nirgends konvergent;

c) für $0<\mu<+\infty$ die Potenzreihe für jedes

$$
\begin{aligned}
& |x|<\frac{1}{\mu} \quad \text { absolut konvergent, für jedes } \\
& |x|>\frac{1}{\mu} \quad \text { dagegen divergent, }
\end{aligned}
$$

Es ist also - bei sinngemäßer Auslegung -

$$
\boldsymbol{r}=\frac{1}{\boldsymbol{\mu}}=\frac{1}{\overline{\lim } \sqrt{\left|a_{n}\right|}}
$$

der Konvergenzraäius der vorgelegten Potenzreihe $\left.{ }^{2}\right)$.

Beweis. Ist im Falle a) $x_{0}$ eine beliebige reelle Zahl $(\neq 0)$, so ist $\frac{1}{2 \mid \overline{x_{0} \mid}}>0$ und also nach $\mathbf{5 9}$ für alle $n \gg m$

$$
\sqrt[n]{\left|a_{n}\right|}<\frac{1}{2\left|x_{0}\right|} \text { oder }\left|a_{n} x_{0}{ }^{n}\right|<\frac{1}{2^{n}}
$$

Nach $\mathbf{8 7}, 1$ ist also $\sum a_{n} x_{0}{ }^{n}$ absolut konvergent, - womit a) bewiesen ist.

1) Cauchy, Analyse algébrique, S. 151. - Dieser schöne Satz blieb indessen völlig unbeachtet, bis ihn J. Hadamard (J. de math. pures et appl. (4) Bd. 8 (1892), S. 107) wiederfand und zu wichtigen Anwendungen verwertete.

2) Hier soll also ei ımal der bequemeren Zusammenfassung halber ausnahmsweise $\frac{1}{0}=+\infty, \frac{1}{+\infty}=0$ gesetzt werden. - Übrigens sei noch bemerkt, daßs keineswegs $\frac{1}{\varlimsup \sqrt[n]{\left|a_{n}\right|}}$ etwa dasselbe ist wie $\varlimsup \frac{1}{\sqrt[n]{\left|a_{n}\right|}}$, wie man sich an naheliegenden Beispielen klarmachen kann. (Vgl. Aufgabe 24). 
Ist umgekehrt $\sum a_{n} x^{n}$ für $x=x_{1} \neq 0$ konvergent, so ist die Folge $\left(a_{\imath \imath} x_{1}{ }^{n}\right)$ und um so mehr die Folge $\sqrt[n]{\left|a_{n} x_{1}{ }^{n}\right|}$ beschränkt. Ist etwa stets

$$
\sqrt[n]{\left|a_{n} x_{1}{ }^{n}\right|}<K_{1}, \quad \text { so ist stets } \sqrt[n]{\left|a_{n}\right|}<\frac{K_{1}}{\left|x_{1}\right|}=K \text {, }
$$

d. h. es ist dann $\left(\sqrt[n]{\left|a_{n}\right|}\right)$ eine beschränkte Folge. Im Falle b), in dem diese Folge als nach rechts nicht beschränkt angenommen wird, kann daher die Reihe für kein $x \neq 0$ konvergieren.

Ist endlich im Falle c) $x^{\prime}$ eine Zahl, für die $\left|x^{\prime}\right|<\frac{1}{\mu}$ ist, so wähle man eine positive Zahl $\varrho$, für die $\left|x^{\prime}\right|<\varrho<\frac{1}{\mu}$ und also $\frac{1}{\varrho}>\mu$ ist. Der Bedeutung von $\mu$ entsprechend muß dann von einer Stelle an

$$
\sqrt[n]{\left|a_{n}\right|}<\frac{1}{\varrho} \quad \text { und folglich } \sqrt[n]{\left|a_{n} x^{\prime n}\right|}<\left|\frac{x^{\prime}}{\varrho}\right|<1
$$

sein. Nach 75, 1 ist daher $\sum a_{n} x^{\prime n}$ (absolut) konvergent.

Ist aber $\left|x^{\prime \prime}\right|>\frac{1}{\mu}$. also $\left|\frac{1}{x^{\prime \prime}}\right|<\mu$, so ist unendlich oft (immer wieder; s. 59)

$$
\sqrt[n]{\left|a_{n}\right|}>\left|\frac{1}{x^{\prime \prime}}\right| \text { oder }\left|a_{n} x^{\prime \prime n}\right|>1
$$

Nach 82, 6 kann also die Reihe sicher nicht konvergieren ${ }^{1}$ ).

Damit ist der Satz in allen Teilen bewiesen.

Bemerkungen und Beispiele.

1. Da die drei Teile a), b), c) des letzten Satzes sich gegenseitig ausschliefen, so ergibt sich, dafi ihre Bedingungen nicht nur hinreichend, sondern auch notwendig fur das Eintreten des betreffenden Konvergenzverhaltens der Reihe $\sum a_{n} x^{n}$ sind.

2. Insbesondere strebt also bei jeder beständig konvergenten Potenzreihe $\sqrt[n]{\left|a_{n}\right|} \rightarrow 0$. Denn nach dem oben Bemerkten ist $\mu=0$; und da es sich um eine Folge von positiven Zahlen handelt, so ist ihr unterer Limes $\varkappa$ notwendig $\geqq 0$. Weil andererseits $\varkappa \leqq \mu$ sein muf, ist $\varkappa=\mu=0$. Nach 63 ist die Folge $\left(\sqrt[n]{\left|a_{n}\right|}\right)$ also konvergent mit dem Grenzwert 0 . So strebt z. B.

$$
\sqrt[n]{\frac{1}{n !}} \rightarrow 0 \quad \text { oder } \quad \sqrt[n]{n !} \rightarrow+\infty
$$

weil $\sum \frac{x^{n}}{n !}$ beständig konvergiert.

1) Den Fall c) kann man etwas kürzer so abtun: Wenn

$\varlimsup \sqrt[n]{\left|a_{n}\right|}=\mu$ ist, so wird $\varlimsup \sqrt[n]{\left|a_{n}\right|} \cdot|x|=\varlimsup \lim \sqrt[n]{\left|a_{n} x^{n}\right|}=\mu \cdot|x|$ sein (warum?). Nach $\boldsymbol{7 6}, 3$ ist also die Reihe für $\mu \cdot|x|<\mathrm{I}$ absolut konvergent, fur $\mu \cdot|x|>1$ sicher divergent, w. z. b. w. 
3. Über das Verhalten der Potenzreihe für $x=+r$ und $x=-r$ sagen die Sätze 93 und 94 nichts aus; es ist von Fall $z u$ Fall verschieden: $\sum x^{n}, \sum \frac{x^{n}}{n}, \sum \frac{x^{n}}{n^{2}}$ haben alle drei den Radius 1 . Die erste konvergiert in keinem der Punkte \pm 1 , die zweite in nur einem von ihnen, die dritte in beiden.

4. Weitere Beispiele von Potenzreihen werden in den folgenden Paragraphen unausgesetzt auftreten, so dafs wir hier keine besonderen zu geben brauchen.

Wir sahen, daß die Konvergenz einer Potenzreihe im Innern des Konvergenzintervalles sogar eine absolute ist. Wir wollen weiter zeigen, daß die Konvergenz sogar so stark ist, daß sie durch das Hinzutreten beträchtlicher Faktoren noch nicht gestört wird. Es gilt nämlich der 95. $\quad$ Satz. Hat $\sum_{n=0}^{\infty} a_{n} x^{n}$ den Konvergenzradius r, so hat die Potenzreihe $\sum_{n=0}^{\infty} n a_{n} x^{n-1}$ oder, was dasselbe ist, die Reihe $\sum_{n=0}^{\infty}(n+1) a_{n+1} x^{n}$ genau denselben Radius.

Beweis. Man kann diesen Satz unmittelbar aus Satz 94 ablesen. Denn setzt man $n a_{n}=a_{n}{ }^{\prime}$, so ist

$$
\sqrt[n]{\left|a_{n}{ }^{\prime}\right|}=\sqrt[n]{\left|a_{n}\right|} \cdot \sqrt[n]{n}
$$

Da nun (nach 38, 7) $\sqrt[n]{n} \rightarrow 1$ strebt, so folgt nach $\mathbf{6 1}, 5$ unmittelbar, daß die Folgen $\left(\sqrt[n]{\left|a_{n}^{\prime}\right|}\right)$ und $\left(\sqrt[n]{\left|a_{n}\right|}\right)$ denselben oberen Limes haben. Denn hebt man aus beiden dieselbe Teilfolge heraus, so sind sie, da sich die entsprechenden Glieder nur durch den $\rightarrow+1$ strebenden Faktor $\sqrt[n]{n}$ unterscheiden, entweder beide divergent oder beide mit demselben Grenzwert konvergent ${ }^{\mathbf{1}}$ ).

\section{Beispiele.}

1. Wendet man den Satz mehrmals an, so ergibt sich, dafs die Reihen $\sum n a_{n} x^{n-1}, \quad \sum n(n-1) a_{n} x^{n-2}, \ldots \sum n(n-1) \ldots(n-k+1) a_{n} x^{n-k}$ oder, was ganz dasselbe ist, die Reihen

$$
\begin{gathered}
\Sigma(n+1) a_{n+1} x^{n}, \quad \Sigma(n+1)(n+2) a_{n+2} x^{n}, \ldots, \\
\Sigma(n+1)(n+2) \ldots(n+k) a_{n+k} x^{n}=k ! \Sigma\left(\begin{array}{c}
n+k \\
k
\end{array}\right) a_{n+k} x^{x^{n}}
\end{gathered}
$$

alle denselben Radius haben wie $\Sigma a_{n} x^{n}$, welche natürliche Zahl auch $k$ bedeuten möge.

1) 2. Beweis. Nach $\mathbf{7 6}, 5$ a oder 91, 2 ist $\sum n \vartheta^{n-1}$ für jedes $|\vartheta|<1$ konvergent. Ist nun $\left|x_{0}\right|<r$ und $\varrho$ so, daß $\left|x_{0}\right|<\varrho<r$ ist, so ist $\Sigma a_{n} \varrho^{n}$ konvergent, also $\left(a_{n} \varrho^{n}\right)$ beschränkt, etwa stets $\left|a_{n} \varrho^{n}\right|<K$. Dann ist $\left|n a_{n} x_{0}{ }^{n-1}\right|<\frac{K}{\varrho} \cdot n\left(\frac{x_{0}}{\varrho}\right)^{n-1}$, was nun wegen $\left|\frac{x_{0}}{\varrho}\right|<1$ die behauptete Konvergenztatsache lehrt. 
2. Dasselbe gilt natürlich auch von den Reihen

$\sum \frac{a_{n}}{n+1} x^{n+1}, \quad \sum \frac{a_{n}}{(n+1)(n+2)} x^{n+2}, \ldots, \quad \sum \frac{a_{n}}{(n+1)(n+2) \cdots(n+k)} x^{n+k}$

Wir haben bisher nur Potenzreihen der Form $\sum a_{n} x^{n}$ betrachtet. Es macht in den Betrachtungen nur einen geringen Unterschied, wenn wir die allgemeinere Form

$$
\sum_{n=0}^{\infty} a_{n}\left(x-x_{0}\right)^{n}
$$

zugrunde legen: Setzt man $x-x_{0}=x^{\prime}$, so erkennt man, daß diese Reihen für $\left|x^{\prime}\right|=\left|x-x_{0}\right|<r$ absolut konvergieren, für $\left|x-x_{0}\right|>r$ dagegen divergieren, wenn $r$ wieder die nach Satz 94 bestimmte Zahl ist. Der Konvergenzbereich der Reihe ist also - von den extremen Fällen, daß sie nur für $x=x_{0}$ oder aber für jedes $x$ konvergiert, abgesehen - eine Strecke, die durch den Punkt $x_{0}$ halbiert wird, teils mit, teils ohne Einschluß eines oder beider Endpunkte. Von dieser Verschiebung der Lage des Konvergenzintervalles abgesehen, bleiben alle unsere Betrachtungen in Gültigkeit. Den Punkt $x_{0}$ werden wir der Kürze halber den Mittelpunk: der Potenzreihe nennen. Ist $x_{0}=0$, so haben wir wieder die frühere Reihenform.

Im Konvergenzintervall hat die Potenzreihe $\sum a_{n}\left(x-x_{0}\right)^{n}$ für jedes $x$ eine wohlbestimmte Summe $s$, die natürlich für verschiedene $x$ im allgemeinen verschieden ausfällt. Um diese Abhängigkeit von $x$ zum Ausdruck zu bringen, setzen wir

$$
\sum_{n=0}^{\infty} a_{n}\left(x-x_{0}\right)^{n}=s(x)
$$

und sagen, die Potenzreihe definiere in ihrem Konvergenzintervall eine Funktion von $x$.

Die Grundlagen der Lehre von den reellen Funktionen, also die Grundlagen der Differential- und Integralrechnung setzen wir, wie schon in der Einleitung betont, im wesentlichen als bekannt voraus. Nur um über das $\mathrm{Maß}$ der aus diesen Gebieten benutzten Tatsachen keinerlei Unklarheit aufkommen zu lassen, wollen wir im folgenden Paragraphen alle Definitionen und Sätze, deren wir benötigen, kurz angeben, ohne auf genauere Erläuterungen und Beweise einzugehen.

\section{$\S 19$. Funktionen einer reellen Veränderlichen.}

Definition 1 (Funktion). Ist jedem Werte $x$ eines Intervalles der $x$-Achse durch irgendeine Vorschrift ein bestimmter Wert $y$ zugeordnet, so sagt man, $y$ sei eine in dem betreffenden Intervall definierte Funktion von $x$ und schreibt dafür kurz

$$
\boldsymbol{y}=\boldsymbol{f}(\boldsymbol{x})
$$


indem ${ }^{*}$ " die Vorschrift symbolisiert, auf Grund deren jedem $x$ das $z u-$ gehörige $y$ entspricht ${ }^{1}$ ).

Das Intervall, das abgeschlossen oder auch auf einer oder auf beiden Seiten offen, beschränkt oder nicht beschränkt sein darf, heifst dann das Definitionsintervall von $f(x)$.

Definition 2 (Beschränktheit). Gibt es eine Konstante $K_{1}$, so daf für alle $x$ des Definitionsintervalles

$$
f(x) \geq K_{1}
$$

bleibt, so heifst die Funktion $f(x)$ nach links (oder nach unten) beschränkt und $K_{1}$ eine linke (oder untere) Schranke von $f(x)$. Gibt es eine Kunstante $K_{2}$, so dafi für alle $x$ des Definitionsintervalles $f(x) \leqq K_{2}$ ist, so heifit $f(x)$ nach rechts (oben) beschränkt und $K_{2}$ eine rechte (obere) Schranke von $f(x)$. Eine beiderseits beschränkte Funktion wird schlechtweg als beschränkt bezeichnet. Es gibt dann eine Konstante $K$, so dafs für alle $x$ des Definitionsintervalles

bleibt.

$$
|f(x)|<K
$$

Definition 3 (Untere und obere Grenze, Schwankung). Unter allen unteren Schranken einer beschränkten Funktion gibt es stets eine größte und ebenso unter ihren obersten Schranken stets eine kleinste ${ }^{2}$ ). Jene bezeichnet man als die untere, diese als die obere Grenze, die Differenz beider als die Schwankung der Funktion $f(x)$ in ihrem Definitionsintervall. Entsprechende Bezeichnungen gebraucht man auch für ein Teilintervall $a^{\prime} \ldots b^{\prime}$ des Definitionsintervalles.

Definition 4 (Grenzwert einer Funktion). Ist $\xi$ ein Punkt des Definitionsintervalles einer Funktion $f(x)$ oder einer seiner Endpunkte, so bedeutet die Symbolik

oder

$$
\lim _{x \rightarrow \xi} f(x)=c
$$

$$
f(x) \rightarrow c \text { für } x \rightarrow \xi,
$$

a) dab für jede dem Definitionsintervall entnommene und gegen $\xi$ konvergierende Zahlenfolge $\left(x_{n}\right)$, deven Glieder sämtlich von $\xi$ verschieden sind, die Folge der zugehörigen Funktionswerte

gegen $c$ konvergiert; oder

$$
y_{n}=f\left(x_{n}\right) \quad \cdot \quad(n=1,2,3, \ldots)
$$

b) daf nach Wahl einer beliebigen positiven Zahl $\varepsilon$ sich stets eine andere positive Zahl $\delta=\delta(\varepsilon)$ so angeben läbt, daßs für alle dem Definitionsintervall angehörigen Werte von $x$, für die

ist, stets auch

$$
|x-\xi|<\delta, \text { aber } x \neq \xi
$$

$$
f(x)-c \mid<\varepsilon
$$

ausfällt $\left.{ }^{3}\right)$. - Die beiden Definitionsformen a) und b) besagen genau dasselbe.

1) Statt eines Intervalles der $x$-Achse kann man auch allgemein die Punkte einer bestimmt gegebenen Punktmenge $M$ auf der $x$-Achse zugrunde legen. Man sagt dann, die Funktion sei auf der Punktmenge $M$ definiert. Doch werden wir weiterhin ausschlieflich Intervalle zugrunde legen.

2) Vgl. 8, 2, sowie $\mathbf{6 2}$

3) Die ältere Schreibweise $\lim _{x=\xi} f(x)$ statt $\lim _{x \rightarrow \xi} f(x)$ ist durchaus zu verwerfen, weil gerade $x \neq \xi$ bleiben soll. 
Definition 5 (Rechts- und linksseitiger Grenzwert). Liegen im Falle der Definition 4 alle in Betracht gezogenen Punkte $x_{n}$ bzw. $x$ rechts von $\xi$, so spricht man von einem rechtsseitigen Grenzwert und schreibt

ebenso schreibt man

$$
\lim _{x \rightarrow \xi+0} f(x)=c
$$

$$
\lim _{x \rightarrow \xi-0} f(x)=c
$$

und spricht von einem linksseitigen Grenzwert, wenn man nur links von $\xi$ gelegene Punkte $x_{n}$ bzw. $x$ in Betracht zieht.

Definition 5a (Weitere Grenzwertformen). Neben den nunmehr festgelegten 3 Grenzwertformen kommen noch insgesamt die folgenden vor:

$$
\text { oder } \left.\left.\quad \begin{array}{l}
\lim f(x)= \\
f(x) \rightarrow
\end{array}\right\} c,+\infty,-\infty^{1}\right)
$$

mit einem der folgenden fünf Zusätze („Bewegungen von $\left.x^{“}\right)$ :

$$
\text { für } x \rightarrow \xi, \rightarrow \xi+0, \rightarrow \xi-0, \rightarrow+\infty, \rightarrow-\infty \text {. }
$$

Im Anschluf an 2 und 3 wird es keinerlei Schwierigkeiten machen, diejenigen Definitionen - in der Form a) oder b) - genau zu formulieren, die den eben behandelten entsprechen.

Da wir diese Dinge, wie betont, im wesentlichen als bekannt ansehen, unterdrücken wir alle ins einzelne gehenden Erläuterungen und Beispiele und betonen nur noch besonders, dafi der Wert $c$, gegen den eine Funktion z. B. für $x \rightarrow \xi$ strebt, gar nichts mit dem Werte der Funktion an der Stelle $\xi$ zu tun zu haben braucht. Nur hierfür wollen wir noch ein Beispiel ausführen: $f(x)$ sei für alle $x$ dadurch definiert, daf $f(x)=0$ gesetzt wird, falls $x$ eine irrationale $Z$ ahl ist, dafs aber $f(x)=\frac{1}{q}$ gesetzt wird, falls $x$ gleich der rationalen Zahl $\frac{p}{q}$ ist, wobei wir uns diese auf den kleinsten positiven Nenner $q$ gebracht denken. (Es ist also z. B. $f\left(\frac{3}{4}\right)=\frac{1}{4}, f(0)=f\left(\frac{0}{1}\right)=1, f(\sqrt{2})=0$, usw.)

Hier ist für jedes $\xi$

$$
\lim _{x \rightarrow \xi} f(x)=0
$$

Denn ist $\varepsilon$ eine beliebige positive Zahl und $m$ so grof, daß $\frac{1}{m}<\varepsilon$, so gibt es zunächst einmal in jedem beschränkten Intervalle nur endlich viele rationale Punkte, deren (kleinster positiver) Nenner $\leq m$ ist. Diese denke ich mir etwa in dem Intervall $\xi-1 \ldots \xi+1$ sämtlich markiert. $D a$ es nur endlich viele sind, muß einer von ihnen der Stelle $\xi$ am nächsten liegen; (ist $\xi$ selber eine dieser Stellen, so rechnet sie hierbei natürlich nicht mit). Deren (positiven) Abstand von $\xi$ nenne ich $\delta$. Dann ist jedes $x$, für das

$$
0<|x-\xi|<\delta
$$

1) Im 1. dieser drei Fälle sagt man $f(x)$ strebe oder konvergieve gegen $c$; im 2. und 3. Falle: $f(x)$ strebe oder divergiere (bestimmt) gegen $+\infty$ bzw. $-\infty$ und spricht in allen Fällen von einem bestimmten Verhalten oder auch von einem Grenzwert im weiteren Sinne. Weist $f(x)$ keine dieser 3 Verhaltungsweisen auf, so sagt man: $f(x)$ divergiere unbestimmt (bei der in Betracht gezogenen Bewegung von $x$ ). 
ist, entweder irrational oder eine rationale Zahl, deren kleinster positiver Nenner $q$ dann notwendig $>m$ ist. In einem Falle ist $f(x)=0$, im andern $=\frac{1}{q}<\frac{1}{m}<\varepsilon$. Es ist also für alle $0<\mid x-\xi<\delta$

d. h. wie behauptet

$$
f(x)-0<\varepsilon,
$$

$$
\lim _{x \rightarrow \xi} f(x)=0 \text {. }
$$

Ist also $\xi$ speziell eine rationale Zahl, so ist dieser Grenzwert von dem Funktionswert $f(\xi)$ selbst durchaus verschieden.

Das Rechnen mit Grenzwerten wird durch den folgenden Satz ermöglicht:

Satz 1. Sind $f_{1}(x), f_{2}(x), \ldots, f_{p}(x)$ gegebene Funktionen ( $p$ eine bestimmte natürliche $\mathrm{Zahl}$ ), die sämtlich bei ein und derselben der in Def. 5 a genannten Bewegungen von $x$ je einem endlichen Grenzwert zustreben, etwa $f_{1}(x) \rightarrow c_{1}, \ldots$, $f_{\nu}(x) \rightarrow c_{p}$, so strebt auch

a) die Funktion $f(x)=\left[f_{1}(x)+f_{2}(x)+\ldots+f_{p}(x)\right] \rightarrow c_{1}+c_{2}+\ldots+c_{p}$;

b) die Funktion $f(x)=\left[f_{1}(x) \cdot f_{2}(x) \ldots f_{p}(x)\right] \rightarrow c_{1} \cdot c_{2} \ldots c_{p}$;

c) speziell also die Funktion $a f_{1}(x)>a c_{1},(a=$ beliebige reelle Zahl) und die Funktion $f(x)_{1}-f_{2}(x) \rightarrow c_{1}-c_{2}$;

d) die Funktion $\frac{1}{f_{1}(x)} \rightarrow \frac{1}{c_{1}}$, falls $c_{1} \neq 0$ ist.

Satz 2. Ist $\lim _{x \rightarrow \xi} f(x)=c(\neq \pm \infty)$, so ist $f(x)$ in einer Umgebung von $\xi$ beschränt, d. h. existieren zwei positive Zahlen $\delta$ und $K$, so daf

$$
|f(x)|<K \text { bleibt, falls }|x-\xi|<\delta \text { ist, }
$$

und entsprechendes gilt bei (endlichem) $\lim f(x)$ fuir $x \rightarrow \xi+0, \xi-0,+\infty,-\infty$.

Definition 6 (Stetigkeit in einem Punkte). Ist $E$ ein Punkt des Definitionsintervalles von $f(x)$, so heifst $f^{\prime}(x)$ an dieser Stelle stetig, falls

$$
\lim _{x \rightarrow \xi} f(x)
$$

existiert und mit dem Funktionswerte $f(\xi)$ an der Stelle $\xi$ ibereinstimmt:

$$
\lim _{x \rightarrow \xi} f(x)=f(\xi) \text {. }
$$

Nimmt man die Definition des lim in diese neue Definition mit hinein, so kann man auch sagen:

Definition 6a. $f(x)$ heifst stetig an der Stelle $\xi$, falls für jede dem Definitionsintervall entnommene und gegen $\xi$ strebende Punktfolge $\left(x_{n}\right)$ die zugehörigen Funktionswerte

streben.

$$
y_{n}=f\left(x_{n}\right) \rightarrow f(\xi)
$$

Definition 6b. $f(x)$ heifit stetig an der Stelle $\xi$, falls nach Wahl eines beliebigen $\varepsilon>0$ sich $\delta=\delta(\varepsilon)>0$ so angeben läfst, dafi für alle $x$ des Definitionsintervalles, für die

ist, stets auch

ausfällt.

$$
0<\mid x-\xi<\delta
$$

$$
|f(x)-f(\xi)|<\varepsilon
$$

Definition 7 (Rechts- und linksseitige Stetigkeit). $f(x)$ heifit an der Stelle $\xi$ nach rechts bzw. nach links stetig, falls wenigstens für $x \rightarrow \xi+0$ bzw. $x \rightarrow \xi-0$ der Grenzwert $\lim f^{\prime}(x)$ vorhanden ist und mit $f(\xi)$ iibereinstimmt. 
Dem Satz 1 entsprechend gilt hier der

Satz 3. Sind $f_{1}(x), f_{2}(x), \ldots, f_{p}(x)$ gegebene Funktionen ( $p$ eine bestimmte natïliche Zahl), die sämtlich in $\xi$ stetig sind, so sind dort auch die Funktionen

a) $f_{1}(x)+f_{2}(x)+\ldots+f_{p}(x)$,

b) $\left.f_{1}(x) \cdot f_{2}(x) \ldots f_{p} x\right)$

c) $a f_{1}(x)$, ( $a$ eine beliebige reelle Zahl) $f_{1}(x)-f_{2}(x)$, und

d) falls $f_{1}(\xi) \neq 0$ ist, auch $\frac{1}{f_{1}(x)}$

im Punkte $\xi$ stetig. Das Entsprechende gilt, wenn man nur die rechtsseitige oder nur die linksseitige Stetigkeit voraussetzt.

Durch wiederholte Anwendung dieses Satzes auf die sicher iiberall stetige Funktion. $f(x) \equiv x$ (denn für $x \rightarrow \xi$ strebt eben $x \rightarrow \xi$ ) ergibt sich sofort:

Alle rationalen Funktionen sind überall stetig mit Ausnahme der (höchstens endlich vielen) Stellen, an denen der Nenner $=0$ ist. Speziell: Die ganzen rationalen Funktionen sind iiberall stetig.

Ebenso lehrten die Limesbeziehungen 12. 1-3, daf die Funktion $a^{x},(a>0)$, fuir jedes reelle $x$ stetig ist,

$\log x$ fuir jedes $x>0$ stetig ist,

$x^{\alpha},(\alpha$ beliebig reell) für jedes $x>0$ stetig ist.

Definition 8 (Stetigkeit in einem Intervall). Ist eine Funktion in jedem einzelnen Punkte eines Intervalles $J$ stetig, so sagt man, sie sei in diesem Intervall $J$ stetig. Die Endpunkte von $J$ können dabei je nach Lage des Falles hinzugerechnet werden oder auch nicht. Über Funktionen, die in einem $a b$ geschlossenen Intervall stetig sind, läfst sich eine Anzahl wichtiger Sätze aussprechen, von denen wir die folgenden erwähnen:

Satz 4. Ist $f(x)$ in dem abgeschlossenen Intervall $a \leqq x \leq b$ stetig und ist $f(a)>0$, aber $f(b)<0$, so gibt es $z$ wischen $a$ und $b$ mindestens einen Punkt $\xi$, fuir den $f(\xi)=0$ ist.

Satz 4 a. Ist $f(x)$ in dem abgeschlossenen Intervall $a \leq x \leq b$ stetig und ist $\eta$ irgendeine zwischen $f(a)$ und $f(b)$ gelegene reelle Zahl, so gibt es wieder zwischen $a$ und $b$ mindestens einen Punkt $\xi$, für den $f(\xi)=\eta$ ist. Oder: Die Gleichung $f(x)=\eta$ hat dort mindestens eine Lösung.

Satz 5. Ist $f(x)$ in dem abgeschlossenen Intervall $a \leq x \leq b$ stetig, so läfst sich nach Wahl von $\varepsilon>0$ stets eine Zahl $\delta>0$ so angeben, daßi für irgend $z$ wei Punkte $x^{\prime}$ und $x^{\prime \prime}$ des genannten Intervalles, deren Abstand $\left|x^{\prime \prime}-x^{\prime}\right|<\delta$ ist, die Differen $z$ der zugehörigen Funktionswerte $\left|f\left(x^{\prime \prime}\right)-f\left(x^{\prime}\right)\right|<\varepsilon$ ausfällt. (Man bezeichnet die durch diesen Satz festgelegte Eigenschaft einer Funktion als deren gleichmäßige Stetigkeit in dem zugrundegelegten Intervall.)

Definition 9 (Monotonie). Eine im Intervall $a . . b$ definierte Funktion heifst dort monoton wachsend bzw. fallend, wenn für zwei dem genannten Intervall entnommene Punkte $x_{1}$ und $x_{2}$, für die $x_{1}<x_{2}$ ist, stets $f\left(x_{1}\right) \leqq f\left(x_{2}\right)$ bzw. $f\left(x_{1}\right) \geq f\left(x_{2}\right)$ ausfällt. Man spricht von einem Wachsen bzw. Fallen im engeren * Sinne, wenn man die eben noch zugelassene Gleichheit zwischen den Funktionswerten ausschliefit.

Satz 6. Der unter den Voraussetzungen der Sätze 4 und 4 a sicher vorhandene Punkt $\xi$ ist notwendig der einzige seiner Art, wenn die benutzte Funktion $f(x)$ im Intervall $a \ldots b$ im engeren Sinne "monoton ist. Dann entspricht also jedem $\eta$ zwischen $f(a)$ und $f(b)$ stets ein und nur ein $\xi$, für das 
$f(\xi)=\eta$ ist. Man sagt in diesem Falle: Die Funktion $y=f(x)$ sei in jenem Intervall eindeutig umkehrbar.

Definition 10 (Differenzierbarkeit). Eine im Punkte $\xi$ und einer gewissen Umgebung desselben definierte Funktion $f(x)$ heifst im Punkt $\xi$ differenzierbar, wenn der Grenzwert

$$
\lim _{x \rightarrow \xi} \frac{f^{\prime}(x)-f(\xi)}{x-\xi}
$$

existiert. Sein Wert heifst die Ableitung oder der Differentialquotient von $f(x)$ in $\xi$ und wird mit $f^{\prime}(\xi)$ bezeichnet. Ist der genannte Grenzwert nur rechtsbzw. linksseitig (also nur für $x \rightarrow \xi+0$ bzw. $\rightarrow \xi-0$ ) vorhanden, so spricht man von rechts. bzw. linksseitiger Differenzierbarkeit, Ableitung usw.

Ist eine Funktion $f(x)$ in jedem einzelnen Punkte eines Intervalles $J$ differenzierbar, so sagt man kurz, sie sei in diesem Intervalle differenzisrbar.

Die Regeln für die Differentiation einer Summe oder eines Produktes einer bestimmten (festen) Anzahl von Funktionen, einer Differenz oder eines Quotienten zweier Funktionen, einer mittelbaren Funktion, sowie die Regeln für die Differentiation der elementaren und der aus ihnen zusammengesetzten Funktionen sehen wir als bekannt an.

Alle zu ihrer Aufstellung notwendigen Hilfsmittel sind im Vorangehenden entwickelt, wenn man noch die Kenntnis des in 112 genannten Grenzwertes vorweg nimmt, der dort ganz direkt bestimmt wird. Wird z. B. nach der Differenzierbarkeit und der Ableitung von $a^{x}, a>0$ und $\neq 1$, im Punkte $\xi$ gefragt, so hat man nach Def. 10 und 4 eine Nullfolge $\left(x_{n}\right)$ zu wählen, deren Glieder aber sämtlich von 0 verschieden sind, und die Zahlenfolge

$$
X_{n}=\frac{a^{\xi+x_{n}}-a^{\xi}}{x_{n}}=a^{\xi} \cdot \frac{a^{x_{n}}-1}{x_{n}}
$$

zu untersuchen. Setzt man den letzten Zähler $=y_{n}$, so wissen wir aus $\mathbf{3 5}, 3$ dafs auch $\left(y_{n}\right)$ eine Nullfolge ist, und zwar wieder eine solche, bei der kein Glied gleich 0 ist. Mit ihrer Hilfe kann man $X_{n}$ in der Form

$$
X_{n}=a^{\xi} \cdot \frac{y_{n} \cdot \log a}{\log \left(1+y_{n}\right)}
$$

schreiben. Da aber, wie bemerkt, $y_{n}$ eine Nullfolge ist, so strebt nach 112

$$
\frac{\log \left(1+y_{n}\right)}{y_{n}} \rightarrow 1 \text {. }
$$

Da dasselbe dann nach 41, 11 a auch für den reziproken Wert gilt, so strebt also $X_{n} \rightarrow a^{\xi} \cdot \log a$. Die Funktion $a^{x}$ ist also für jedes $x$ differenzierbar und hat die Ableitung $a^{x} \cdot \log a$.

Ebenso ergibt sich bezüglich der Differenzierbarkeit und der Ableitung von $\log x$ an einer Stelle $\xi>0$ durch Betrachtung der Folge

$$
X_{n}=\frac{\log \left(\xi+x_{n}\right)-\log \xi}{x_{n}}=\frac{\log \left(1+\frac{x_{n}}{\xi}\right)}{x_{n}}=\frac{1}{\xi} \cdot \log \left(1+\frac{x_{n}}{\xi}\right)^{\frac{\xi}{x_{n}}},
$$

daf dort die Ableitung existiert und $=\frac{1}{\xi}$ ist.

Trotz der grundlegenden Bedeutung dieser Begriffe für die gesamte Analysis werden wir von ihnen in den folgenden Kapiteln nur verhältnismäbig selten Gebrauch zu machen haben. Wir benutzen zunächst fast nur die folgenden einfachen Sätze: 
Satz 7. Ist eine Funktion $f(x)$ in einem Intervalle $J$ differenzierbar, und ist ihre Ableitung dort stets $=0$, so ist $f(x)$ in $J$ konstant, ist also $\equiv f\left(x_{0}\right)$, wenn $x_{0}$ irgendeinen Punkt aus $J$ bezeichnet.

Sind die beiden Funktionen $f_{1}(x)$ und $f_{2}(x)$ in $J$ differenzierbar und stimmen dort ihre Ableitungen stets überein, so ist die Differenz beider Funktionen in $J$ konstant; es ist also

$$
f_{2}(x)=f_{1}(x)+c=f_{1}(x)+\left[f_{2}\left(x_{0}\right)-f_{1}\left(x_{0}\right)\right],
$$

wenn $x_{0}$ irgendeinen Punkt aus $J$ bezeichnet.

Satz 8. (Erster Mittelwertsatz der Differentialrechnung). Ist $f(x)$ in dem abgeschlossenen Intervall $a \leqq x \leqq b$ stetig und wenigstens im offenen Intervall $a<x<b$ differenzierbar, so gibt es in letzterem mindestens einen Punkt $\xi$, für den

$$
f^{\prime}(\xi)=\frac{f(b)-f(a)}{b-a}
$$

ist. (In Worten: Der Differenzenquotient bezüglich der Enden des Intervalles ist gleich dem Differentialquotienten an einer passenden Stelle im Innern.)

Satz 9. Ist $f(x)$ in $\xi$ differenzierbar und ist $f^{\prime}(\xi)>0(<0)$, so "wächst" ("fällt") $f(x)$ im Punkte $\xi$, d. h. es hat die Differenz

$$
f(x)-f(\xi) \quad\left\{\begin{array}{c}
\text { dasselbe } \\
\text { (das entgegengesetzte })
\end{array}\right\} \quad \text { Vorzeichen wie }(x-\xi),
$$

solange $|x-\xi|<$ als eine passende Zahl $\delta$ gehalten wird.

Satz 10. Ist $f(x)$ in $\xi$ differenzierbar, so kann der Funktionswert $f(\xi)$ nur dann von keinem andern Funktionswert $f(x)$ in einer Umgebung von $\xi$ der Form $|\grave{x}-\xi|<\delta$ uibertroffen werden, d. h. $\xi$ kann nur dann eine Stelle des Maximums sein, wenn $f^{\prime}(\xi)=0$ ist. Ebenso ist die Bedingung $f^{\prime}(\xi)=0$ notwendig dafür, daf $\xi$ eine Stelle des Minimums ist, daf also $f(\xi)$ von keinem andern Funktionswert $f(x)$ untertroffen wird, solange $x$ in einer passenden Umgebung von $\xi$ verbleibt.

Definition 11 (Höhere Ableitungen). Ist $f(x)$ im Intervall $J$ differenzierbar, so ist (gemäf Def. 1) $f^{\prime}(x)$ wieder eine in $J$ definierte Funktion. Ist diese erneut in $J$ differenzierbar, so nennt man ihre Ableitung die zweite Ableitung von $f(x)$ und bezeichnet sie mit $f^{\prime \prime}(x)$. Entsprechend gelangt man zu einer dritten, und allgemein $z u$ einer $k^{\text {ten }}$ Ableitung, die dann mit $f^{(k)}(x)$. bezeichnet wird. Zur Existenz der $k^{\text {ten }}$ Ableitung in einem Punkte $\xi$ ist hiernach (s. Def. 10) erforderlich, dab die $(k-1){ }^{\text {te }}$ Ableitung im Punkte $\xi$ und in allen Punkten einer gewissen Umgebung derselben vorhanden ist. - Die $l^{\text {te }}$ Ableitung von $f^{(k)}(x)$ ist $f^{(k+l)}(x)$, $k \geqq 0, l \geqq 0$. (Als $0^{\text {te }}$ Ableitung von $f(x)$ sieht man hierbei die gegebene Funktion selbst an).

Von der Integralvechnung werden im folgenden nur die allereinfachsten Begriffe und Sätze gebraucht, aufer in $\S 49$ und 61 uber Fourierreihen, wo etwas tiefergehende Dinge herangezogen werden muissen.

Definition 12 (Unbestimmtes Integral). Ist im Intervall $a \ldots b$ eine Funktion $f(x)$ gegeben und läbt sich eine differenzierbare Funktion $F(x)$ finden, so daki für alle Punkte des genannten Intervalles $F^{\prime}(x)=f(x)$ ist, so sagt man, $F(x)$ sei dort ein unbestimmtes Integral von $f(x)$. Aufer $F(x)$ sind dann auch die Funktionen $F(x)+c$ unbestimmte Integrale von $f(x)$, wenn $c$ irgendeine reelle $Z$ ahl bedeutet. Aufer diesen aber gibt es keine weiteren. Man schreibt

$$
F(x)=\int f(x) d x
$$


In den einfachsten Fällen ergeben sich die unbestimmten Integrale durch Umkehrung der elementaren Formeln der Differentialrechnung. Z. B. folgt aus $(\sin \alpha x)^{\prime}=c \cos \alpha x$, daf $\int \cos \alpha x d x=\frac{\sin \alpha x}{\alpha}$, usw. Diese elementaren Regeln sehen wir als bekannt an. An speziellen Integralen dieser Art werden aufer den allereinfachsten nur wenige im folgenden gebraucht; wir erwähnen:

$$
\begin{aligned}
& \int \frac{d x}{1+x^{3}}=\frac{1}{3} \log (1+x)-\frac{1}{6} \log \left(1-x+x^{2}\right)+\frac{1}{\sqrt{3}} \operatorname{arctg} \frac{2 x-1}{\sqrt{3}} \\
& \int \frac{d x}{1+x^{4}}=\frac{\sqrt{2}}{8} \log \frac{x^{2}+x \sqrt{2}+1}{x^{2}-x \sqrt{2}+1}+\frac{\sqrt{2}}{4} \operatorname{arctg} \frac{x \sqrt{2}}{1-x^{2}} \\
& \int\left[\operatorname{ctg} x-\frac{1}{x}\right] d x=\log \frac{\sin x}{x} .
\end{aligned}
$$

Handelt es sich bei dem unbestimmten Integral lediglich um eine neue Schreibweise für Formeln der Differentialrechnung, so liegt im bestimmten Integral ein wesentlich neuer Begriff vor.

Definition 13 (Bestimmtes Integral). Eine im abgeschlossenen Intervall $a \ldots b$ definierte und dort beschränkte Funktion heifit über dieses Intervall integrierbar, wenn sie der folgenden Forderung genïgt:

Man teile das Intervall $a \ldots b$ irgendwie in $n$ gleiche oder ungleiche Teile $(n \geq 1$, natürliche Zahl), nenne die Teilpunkte von $a$ nach $b$ der Reihe nach $x_{1}, x_{2}, \ldots, x_{n-1}$ und setze noch $a=x_{0}, b=x_{n}$. Nun wähle man in jedem der $n$ Teile (zu denen man beide Endpunkte hinzurechnen darf) je irgend-

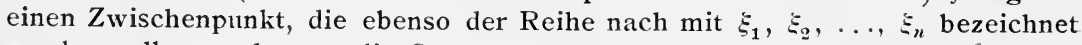
werden sollen, und setze die Summe

$$
S_{n}=\sum_{v=1}^{n}\left(x_{v}-x_{v-1}\right) f\left(\xi_{v}\right)
$$

an ${ }^{1}$ ). Solche Summenwerte $S_{n}$ berechne man für $n=1,2,3, \ldots$ und zwar einen jeden ganz unabhängig von den übrigen (d. h. also bei jedesmal ganz neuer Wahl der $x_{v}$ und $\xi_{v}$ ). Doch soll, wenn $l_{n}$ die Länge des längsten der $n$ Teile ist, in die das Intervall bei der Bildung von $S_{n}$ zerlegt wurde, $l_{n} \rightarrow 0$ streben $^{2}$ ).

Wenn dann die Folge der Zahlen $S_{1}, S_{2}, \ldots$, wie man sie auch hergestellt haben mag, immer konvergent ausfält und immer denselben Grenzwert $S$ liefert ${ }^{3}$ ), so soll $f^{\prime}(x)$ über das Intervall $a \ldots b$ im Riemannschen Sinne integrierbar heißen und der Grenzwert $S$ soll als bestimmtes Integral von $f(x)$, über $a \ldots b$ und mit

$$
S=\int_{a}^{b} f(x) d x
$$

1) Ist $f(x)>0, a<b$ und betrachtet man das Flächenstlick $S$, das von der Abszissenachse einerseits, den in $a$ und $b$ auf ihr errichteten Loten und der Kurve $y=f(x)$ andererseits begrenzt wird, so ist $S_{n}$ ein approximativer Wert des Inhaltes von $S$. Doch gibt dies nur dann ein anschauliches Bild, falls $y=f(x)$ eine Kurve im anschaulichen Sinne ist.

2) Man sagt dann wohl auch, daf die Einteilungen mit wachsendem $n$ unbegrenzt feiner werden.

$\left.{ }^{3}\right)$ Es läßst sich leicht zeigen, daf wenn die Folge $\left(S_{n}\right)$ immer konvergent ausfällt, sie auch von selbst immer denselben Grenzwert liefert. 
bezeichnet werden. $x$ heißst die Integrationsveränderliche und darf natürlich durch jeden anderen Buchstaben ersetzt werden. - Statt $f\left(\xi_{v}\right)$ darf in $S_{n}$ auch die untere oder auch die obeve Grenze aller Funktionswerte des Teilintervalles $x_{v-1} \ldots x_{v}$ gesetzt werden ${ }^{1}$.

Satz 11 (Riemannsches Integrabilitätskriterium). Die notwendige und hinreichende Bedingung dafür, daf $f(x)$ uiber $a \ldots b$ integrierbar ist, ist diese: Nach Wahl von $\varepsilon>0$ muf sich eine Wahl von $n$ und der Punkte $x_{1}, x_{2}, \ldots x_{n-1}$ so treffen lassen, dafi

$$
\sum_{v=1}^{n} i_{v} \sigma_{r}<\varepsilon
$$

ausfällt, wenn $i_{v}=\left|x_{v}-x_{v-1}\right|$ die Länge des $v^{\text {ten }}$ Teiles von $a \ldots b$ und $\sigma_{v}$ die Schwankung von $f(x)$ (s. Def. 3 ) in diesem Teilintervall bedeutet.

Aus diesem Kriterium leitet man die folgenden speziellen Sätze her:

Satz 12. Jede in $a<x<b$ monotone, sowie jede dort stetige Funktion ist iiber $a \ldots b$ integrierbar.

Satz 13. Die Funktion $f(x)$ ist iiber $a \ldots b$ integrierbar, falls sie dort beschränkt ist und nur endlich viele Unstetigkeiten besitzt.

Satz 14. Die Funktion $f(x)$ ist uiber $a \ldots b$ auch noch integrierbar, wenn sie dort beschränkt ist und unendlich viele Unstetigkeiten besitzt, wofern diese nach Annahme einer Zahl $\varepsilon>0$ doch noch in endlich oder unendlich viele Intervalle eingeschlossen werden können, deren Gesamtlänge $<\varepsilon$ ist $^{2}$ ).

Satz 15. Die Funktion $f(x)$ ist iiber $a \ldots b$ sicher nicht integrierbar, wenn sie in jedem Punkte des Intervalles unstetig ist.

Satz 16. Ist $f(x)$ uiber das Intervall $a \ldots b$ integrierbar, so ist $f(x)$ auch iiber jedes Teilintervall $a^{\prime} \ldots b^{\prime}$ desselben integrierbar.

Satz 17. Ist die Funktion $f(x)$ über $a \ldots b$ integrierbar, so ist auch jede Funktion $f_{1}(x)$ über $a \ldots b$ integrierbar und liefert denselben Integralwert, die aus $f(x)$ durch willkiirliche Abänderung irgendwelcher endlich vieler Funktionswerte entsteht.

Satz 18. Sind $f(x)$ und $f_{1}(x)$ zwei über $a \ldots b$ integrierbare Funktionen, so liefern sie denselben Integralwert, falls sie wenigstens in allen Punkten einer in $a \ldots b$ dicht gelegenen Punktmenge (z. B. nur in allen rationalen Punkten) übereinstimmen.

Für das Rechnen mit Integralen gelten die folgenden einfachen Sätze, bei denen $f(x)$ stets eine in dem Intervall $a \ldots b$ integrierbare Funktion bedeuten soll.

Satz 19. Es ist $\int_{b}^{a} f(x) d x=-\int_{a}^{b} f(x) d x$ und, wenn $a_{1}, a_{2}, a_{3}$ drei beliebige Punkte des Intervalles $a \ldots b$ sind, stets

$$
\int_{a_{1}}^{a_{2}} f(x) d x+\int_{a_{2}}^{a_{3}} f(x) d x+\int_{a_{3}}^{a_{1}} f(x) d x=0 \text {. }
$$

1) In diesen Fällen liefert $S_{n}$ den Inhalt eines dem Flächenstücke $S$ einbzw. umbeschriebenen („Treppen“-)Polygons.

2) Dieser Satz erfordert für den Fall, dafi unendlich viele Intervalle zur Einschliefung der Unstetigkeitspunkte von $f(x)$ notwendig sind, tiefer gelegene Hilfsmittel zu seinem Beweise. Doch wird dieser Teil des Satzes nur beiläufig auf S. 329 für einen Zusatz in der Fufnote gebraucht. 
Satz 20. Sind $f(x)$ und $g(x)$ zwei über $a \ldots b,(a<b)$, integrierbare Funktionen und ist in $a \ldots b$ stets $f(x) \leqq g(x)$, so ist auch

$$
\int_{a}^{b} f(x) d x \leq \int_{a}^{b} g(x) d x \text {. }
$$

Satz 20a. Mit $f(x)$ ist auch $\mid f(x)$ iuber $a \ldots b$ integrierbar und es ist, falls $a<b$,

$$
\int_{a}^{b} f(x) d x<\int_{a}^{b} f(x) d x
$$

Satz 21. (1. Mittelwertsatz der Integralrechnung). Es ist

$$
\int_{a}^{b} f(x) d x=u \cdot(b-a) \text {. }
$$

wenn $\mu$ eine passende, $z$ wischen der unteren Grenze $m$ und der oberen Grenze $M$ von $f(x)$ gelegene $Z$ ahl bedeutet $(m \leq \mu \leq M)$. Speziell ist

$$
\int_{a}^{b} f(x) d x \mid \leq K \cdot(b-a) \text {. }
$$

wenn $K$ eine Schranke von $f(x)$ bedeutet.

Satz 22. Sind die Funktionen $f_{1}(x), f_{2}(x), \ldots, f_{\nu}(x)$ sämtlich über $a \ldots b$ integrierbar $(p=f e s t e$, natürliche Zahl), so ist auch ihre Summe und ihr Produkt eine über $a: \ldots b$ integrierbare Funktion, und für das Integral der Summe gilt die Formel:

$$
\int_{a}^{b}\left(f_{1}(x)+\ldots+f_{p}(x)\right) d x=\int_{a}^{b} f_{1}(x) d x+\ldots+\int_{a}^{b} f_{p}(x) d x ;
$$

d. h. eine Summe aus einer festen Anzahl von Funktionen darf gliedweise integriert werden.

Satz 23. Ist $f(x)$ ïber $a \ldots b$ integrierbar, so ist die Funktion

$$
F(x)=\int_{a}^{x} f(t) d t
$$

im Intervall $a \ldots b$ stetig und an allen denjenigen Stellen dieses Intervalles auch differenzierbar, an denen $f(x)$ selber stetig ist. Ist $x_{0}$ eine solche Stelle, so ist dort $F^{\prime}\left(x_{0}\right)=f\left(x_{0}\right)$.

Satz 24. Ist $f(x)$ über $a \ldots b$ integrierbar, und ist dort $F(x)$ ein unbestimmtes Integral von $f(x)$, so ist

$$
\int_{a}^{b} f(x) d x=F(b)-F(a)
$$

(Hauptformel zur Berechnung bestimmter Integrale).

Satz 25 (Anderung der Integrationsveränderlichen). Ist $f(x)$ über $a \ldots b$ integrierbar und ist $x=\varphi(t)$ eine in $\alpha \ldots \beta$ stetige und differenzierbare Funktion, für die $\varphi(\alpha)=a$ und $\varphi(\beta)=b$ ist, die sich ïberdies, wenn $t$ sich von $\alpha$ bis $\beta$ bewegt, (im engeren Sinne) monoton von $a$ bis $b$ ändert, und deren Ableitung $p^{\prime}(t)$ ubber $\kappa \ldots \beta$ integrierbar ist $\left.{ }^{1}\right)$, so ist

$$
\int_{b}^{a} f(x) d x=\int_{\beta}^{\alpha} f(\varphi(t)) \cdot p^{\prime}(t) d t
$$

1) Die Ableitung einer differenzierbaren Funktion braucht nicht integrierbar zu sein. Beispiele, die diese Tatsache belegen, sind indessen nicht ganz leicht zu bilden. (Vgl. etwa H. Lebesgue, Leçons sur l'intégration, Paris 1904, S. $93-94)$. 
Satz 26 (Particlle Integration). Ist $f(x)$ über $a \ldots b$ integrierbar und $F(x)$ ein unbestimmtes Integral von $f(x)$, ist ferner $g(x)$ eine in $a \ldots b$ differenzierbare Funktion, deren Ableitung dort integrierbar ist, so ist

$$
\left.\int_{a}^{b} f(x) g(x) d x=[F(x) \cdot g(x)]_{a}^{b}-\int_{a}^{b} F(x) \cdot g^{\prime}(x) d x^{1}\right) \text {. }
$$

Wesentlich tiefer als alle diese einfachen Sätze liegt der folgende

Satz 27 (2. Mittelwertsatz der Integralrechnung). Sind $f(x)$ und $\varphi(x)$ iiber $a \ldots b$ integrierbar und ist $\varphi(x)$ eine dort monotone Funktion, so gibt es eine der Bedingung $a \leqq \xi \leqq b$ genügende $Z$ ahl $\xi$, so daßs die Gleichung

$$
\int_{a}^{b} \varphi(x) \cdot f(x) d x=\varphi(a) \int_{a}^{\xi} f(x) d x+\varphi(b) \int_{\xi}^{b} f(x) d x
$$

besteht. Fïr $\varphi(a)$ kann hierbei auch der nach den iibrigen Voraussetzungen sicher vorhandene Grenzwert $\varphi_{a}=\lim _{x \rightarrow a} \varphi(x)$ und ebenso für $\varphi(b)$ der Grenzwert $\varphi_{b}=\lim _{x \rightarrow b} \varphi(x)$ genommen werden ${ }^{2}$.

Von den Anwendungen des besprochenen Integralbegriffs erwähnen wir nur :

Satz 28 (Inhalt). Ist $f(x)$ in $a \ldots b,(a<b)$, integrierbar und etwa stets positiv $\left.^{3}\right)$, so hat das durch die Abszissenachse, die Ordinaten in $a$ und $b$ und durch die Kurve $y=f(x)$ begrenzte Flächenstück - genauer: die Menge der Punkte $(x, y)$ für die $a \leqq x \leqq b$ und bei jedem solchen $x$ zugleich $0 \leqq y \leqq f(x)$ ist - einen meßbaren Inhalt und dieser wird durch das Integral $\int_{a}^{b} f(x) d x$ gemessen.

Satz 29 (Länge). Sind $x=\varphi(t)$ und $y=\psi(t)$ zwei in $c \leqq t \leqq \beta$ stetige und differenzierbare Funktionen und sind $\varphi^{\prime}(t)$ und $\psi^{\prime}(t)$ ihrerseits stetig in $\alpha \ldots \beta$, so hat die Bahn, die vom Punkte $x=\varphi(t), y=\psi(t)$ in der Ebene eines rechtwinkligen $x y$-Kreuzes beschrieben wird, falls $t$ das Intervall von $\alpha$ bis $\beta$ durchläuft, eine meßbare Länge, und diese wird durch das Integral

geliefert. -

$$
\int_{\alpha}^{\beta} \sqrt{\varphi^{\prime}(t)^{2}+\psi^{\prime}(t)^{2}} d t
$$
gesagt :

Endlich seien noch ein paar Worte über sogenannte uneigentliche Integrale

Definition 14. Ist $f(t)$ für $t \geqq a$ definiert und für jedes $x>a$ über das Intervall $a \leqq t \leqq x$ integrierbar, so daf also auch die Funktion

$$
F(x)=\int_{a}^{x} f(t) d t
$$

für alle $x \geqq a$ definiert ist, so sagt man, das uneigentlicho Integral

$$
\int_{a}^{+\infty} f(t) d t
$$

sei konvergent und habe den Wert $c$, falls $\lim _{x \rightarrow+\infty} F(x)$ existiert und $=c$ ist.

1) Hierbei soll $[h(x)]_{a}^{b}$ die Differenz $h(b)-h(a)$ bedeuten.

2) Es handelt sich hier natürlich um einseitige Grenzwerte, da $x$ aus dem Innern des Intervalles $\rightarrow a$ bzw. $b$ rücken soll.

$\left.{ }^{3}\right)^{\prime}-$ was stets durch Addition einer passenden Konstanten erreicht werden kann.

Knopp, Unendliche Reihen. 
Satz 30. Ist für $t \geq a$ stets $f(t) \geq 0$, so ist $\int_{a}^{\infty} f(t) d t$ dann und nur dann konvergent, wenn die Funktion $F(x)$ der Def. 14 für $x>a$ beschränkt bleibt. Und ganz ähnlich

Definition 15. Ist $f(t)$ in dem links offenen Intervall $a<t \leqq b$ definiert und fuir jedes der Bedingung $a<x<b$ genligende $x$ über das Intervall $x \leqq t \leqq b$ (nicht aber über das ganze Intervall $a \ldots b$ ) integrierbar, so das auch die Funktion

$$
F(x)=\int_{x}^{b} f(t) d t
$$

für alle diese $x$ definiert ist, so sagt man, das uneigentliche Integral

$$
\int_{a}^{b} f(t) d t
$$

sei konvergent und habe den Wert $c$, falls $\lim F(x)$ existiert und $=c$ ist.

$$
x \rightarrow a+0
$$

Satz 31. Ist im Falle der Def. 15 noch stets $f(t) \geq 0$, so ist das dort genannte uneigentliche Integral dann und nur dann vorhanden, wenn $F(x)$ in $a<x<b$ beschränkt bleibt.

\section{$\S 20$. Haupteigenschaften der durch Potenzreihen dargestellten Funktionen.}

Wir knüpfen nun wieder an die Schlußbemerkung des $\S 18$ an, nach der durch die Summe einer Potenzreihe im Innern ihres Konvergenzintervalles eine Funktion definiert wird, die wir nun $f(x)$ nennen wollen:

$$
f(x)=\sum_{n=0}^{\infty} a_{n}\left(x-x_{0}\right)^{n}, \quad\left|x-x_{0}\right|<r .
$$

Das Konvergenzintervall wollen wir dabei, wenn nicht das Gegenteil ausdrücklich gesagt wird, beiderseits offen lassen, selbst dann, wenn die Potenzreihe noch in dem einen oder anderen Endpunkte konvergieren sollte.

Wird nun, wie hier, durch eine unendliche R'eihe in einem gewissen Intervall eine Funktion definiert, so ist die wichtigste Aufgabe im allgemeinen die, aus der Reihe die Haupteigenschaften - dieses Wort etwa im Sinne der Zusammenstellung des vorigen Paragraphen verstanden - der dargestellten Funktion abzulesen. Für Potenzreihen bietet das keine großen Schwierigkeiten. Wir werden im ganzen sehen, daß eine durch eine Potenzreihe dargestellte Funktion alle die Eigenschaften besitzt, die man überhaupt an Funktionen als besonders wichtig schätzt, und daß das Rechnen mit Potenzreihen sich besonders einfach gestaltet. Aus diesem Grunde spielen gerade die Potenzreihen eine hervorragende Rolle, und eben darum gehört ihre Behandlung durchaus in die Anfangsgründe der Reihentheorie.

Bei diesen Untersuchungen dürfen wir, ohne dadurch die Tragweite der Ergebnisse zu beeinträchtigen, nach Belieben auch $x_{0}=0$, 
also die Potenzreihe in der vereinfachten Form $\sum a_{n} x^{n}$ annehmen. Ihr Konvergenzradius $r$ soll natürlich positiv $(>0)$ sein, darf aber auch $+\infty$, die Reihe also beständig konvergent sein. - Dann gilt zunächst der

- Satz. Die durch die Potenzreihe $\sum_{n=0}^{\infty} a_{n}\left(x-x_{0}\right)^{n}$ im Konvergenz- 96. intervall derselben definierte Funktion $f(x)$ ist an der Stelle $x=x_{0}$ stetig; oder also: es ist

$$
\lim _{x \rightarrow x_{0}} f(x)=\lim _{x \rightarrow x_{0}} \sum_{n=0}^{\infty} a_{n}\left(x-x_{0}\right)^{n}=a_{0}=f\left(x_{0}\right) .
$$

Beweis. Ist $0<\varrho<r$, so ist nach $\mathbf{8 3}, 5$ mit

$$
\sum_{n=0}^{\infty}\left|a_{n}\right| \varrho^{n} \quad \text { auch } \quad \sum_{n=1}^{\infty}\left|a_{n}\right| \varrho^{n-1}
$$

konvergent. Setzen wir die Summe der letzten Reihe $=K(>0)$, so ist für $\left|x-x_{0}\right| \leqq \varrho$ stets

$$
\left|f(x)-a_{0}\right|=\left|\left(x-x_{0}\right) \cdot \sum_{n=1}^{\infty} a_{n}\left(x-x_{0}\right)^{n-1}\right| \leqq\left|x-x_{0}\right| \cdot K .
$$

Ist also $\varepsilon>0$ beliebig gegeben und ist $\delta>0$ kleiner als jede der beiden Zahlen $\varrho$ und $\frac{\varepsilon}{K}$, so ist für alle $\left|x-x_{0}\right|<\delta$ stets

$$
\left|f(x)-a_{0}\right|<\varepsilon
$$

womit nach $\S 19$, Def. $6 \mathrm{~b}$ alles bewiesen ist.

Aus diesem Satz folgt unmittelbar der sehr weitgehende und oft angewandte

Identitätssatz für Potenzreihen. Haben die beiden Potenzreihen 97.

$$
\sum_{n=0}^{\infty} a_{n} x^{n} \quad \text { und } \quad \sum_{n=0}^{\infty} b_{n} x^{n}
$$

beide einen Konvergenzradius, der $\geqq \varrho>0$ ist (diese Zahl @ darf im übrigen so klein sein wie sie will), und haben sie für alle $|x|<\varrho$ dieselbe Summe, so sind beide Reihen vollständig identisch, d.h. für jedes $n=0,1,2, \ldots$ ist dann stets

Beweis. Aus

$$
a_{n}=b_{n} .
$$

$$
a_{0}+a_{1} x+a_{2} x^{2}+\ldots=b_{0}+b_{1} x+b_{2} x^{2}+\ldots
$$

folgt nach dem vorigen Satz, indem wir beiderseits $x \rightarrow 0$ rücken lassen, daß

$$
a_{0}=b_{0}
$$

ist. Läßt man diese gleichen Glieder beiderseits weg und dividiert durch $x$, so folgt, daß für $0<|x|<\varrho$

$$
a_{1}+a_{2} x+a_{3} x^{2}+\ldots=b_{1}+b_{2} x+b_{3} x^{2}+\ldots
$$


ist, - eine Gleichung, aus der nun ganz ebenso ${ }^{1}$ ) folgt, da $B$

und

$$
a_{1}=b_{1}
$$

$$
a_{2}+a_{3} x+\ldots=b_{2}+b_{3} x+\ldots
$$

ist. Fährt man in dieser Weise fort, so ergibt sich der Reihe nach (schärfer: durch vollständige Induktion) für jedes $n$ die Richtigkeit der Behauptung.

\section{Beispiele und Erläuterungen.}

1. Dieser Identitätssatz wird uns in der Theorie sowohl wie in den Anwendungen oft begegnen. Man kann seinen Inhalt auch so deuten: wenn eine Funktion für eine Umgebung des Nullpunktes durch eine Potenzreihe dargestellt werden kann, so kann dies nur auf eine einzige Art geschehen. In dieser Form bezeichnet man den Satz wohl auch als Unitätssatz. Er gilt natiirlich entsprechend für die allgemeinen Potenzreihen $\Sigma a_{n}\left(x-x_{0}\right)^{n}$.

2. Da die Aussage des Satzes darin gipfelt, dafs in der Gleichung (a) die entsprechenden Koeffizienten auf beiden Seiten gleich sind, spricht man bei den Anwendungen des Satzes wohl auch von der Methode der Koeffizientenvergleichung.

3. Ein einfaches Beispiel für diese Anwendungsform ist dieses: Es ist gewifs für alle $x$

oder

$$
(1+x)^{k}(1+x)^{k}=(1+x)^{2 k}
$$

$$
\sum_{\nu=0}^{k}\left(\begin{array}{c}
k \\
v
\end{array}\right) x^{\nu} \cdot \sum_{\nu=0}^{k}\left(\begin{array}{c}
k \\
v
\end{array}\right) x^{\nu}=\sum_{i=0}^{2 k}\left(\begin{array}{c}
2 k \\
\lambda
\end{array}\right) x^{\lambda} \text {. }
$$

Multipliziert man linkerhand nach 91, Bem. 1 aus und vergleicht die entsprechenden Koeffizienten auf beiden Seiten, so ergibt sich z. B. durch Vergleich der Koeffizienten von $x^{k}$, daf

$$
\left(\begin{array}{l}
k \\
0
\end{array}\right)\left(\begin{array}{l}
k \\
k
\end{array}\right)+\left(\begin{array}{l}
k \\
1
\end{array}\right)\left(\begin{array}{c}
k \\
k-1
\end{array}\right)+\ldots+\left(\begin{array}{l}
k \\
k
\end{array}\right)\left(\begin{array}{l}
k \\
0
\end{array}\right) \equiv\left(\begin{array}{l}
k \\
0
\end{array}\right)^{2}+\left(\begin{array}{c}
k \\
1
\end{array}\right)^{2}+\ldots+\left(\begin{array}{l}
k \\
k
\end{array}\right)^{2}=\left(\begin{array}{c}
2 k \\
k
\end{array}\right)
$$

ist, - eine Beziehung zwischen Binomialkoeffizienten, die auf anderem Wege nicht ganz so leicht zu beweisen wäre.

4. Ist $f(x)$ für $|x|<r$ definiert und ist dort stets

$$
f(x)=f .(-x) \text {, }
$$

so nennt man $f(x)$ eine gerade Funktion. Ist sie durch eine Potenzreihe darstellbar, so ergibt die Koeffizientenvergleichung sofort, dak

$$
a_{1}=a_{3}=a_{5}=\ldots=a_{2 k+1}=\ldots=0
$$

sein muf, daßs also in der Potenzreihe von $f(x)$ nur gerade Potenzen von $x$ einen von 0 verschiedenen Koeffizienten haben können.

5. Ist dagegen $f(-x)=-f(x)$, so nennt man die Funktion eine ungerade. Ihre Potenzreihenentwicklung kann nur ungerade Potenzen von $x$ enthalten. Speziell ist $f(0)=0$.

1) Für $x=0$ ist die Gleichung (b) zunächst noch nicht gesichert, da sie ja durch eine Division mit $x$ erhalten wurde. Für den Grenzübergang $x \rightarrow 0$ ist das aber ganz gleichgültig (vgl. § 19, Def. 4). 
Wir gehen nun einen Schritt weiter und beweisen eine Anzahl Sätze, die in der Lehre von den Potenzreihen in jeder Beziehung als die wichtigsten bezeichnet werden müssen:

○ Satz 1. Ist

$$
\sum_{n=0}^{\infty} a_{n}\left(x-x_{0}\right)^{n}
$$

eine Potenzreihe mit dem (positiven) Radius $r$, so läßt sich die dadurch für $\left|x-x_{0}\right|<\boldsymbol{r}$ dargestellte Funktion $f(x)$ auch um jeden andern im Konvergenzintervall gelegenen Punkt $x_{1}$ als Mittelpunkt in eine Potenzreihe entwickeln; und zwar ist

wenn

$$
f(x)=\sum_{k=0}^{\infty} b_{k}\left(x-x_{1}\right)^{k}
$$

$$
b_{k}=\sum_{n=0}^{\infty}\left(\begin{array}{c}
n+k \\
k
\end{array}\right) a_{n+k}\left(x_{1}-x_{0}\right)^{n}
$$

gesetzt wird, und der Radius $r_{1}$ dieser neuen Reihe ist mindestens gleich der noch positiven Zahl $r-\left|x_{1}-x_{0}\right|$.

Beweis. Liegt $x_{1}$ im Konvergenzintervall der gegebenen Reihe, ist also $\left|x_{1}-x_{0}\right|<r$, so ist

$$
f(x)=\sum_{n=0}^{\infty} a_{n}\left[\left(x_{1}-x_{0}\right)+\left(x-x_{1}\right)\right]^{n}
$$

(a) $f(x)=\sum_{n=0}^{\infty} a_{n}\left[\left(x_{1}-x_{0}\right)^{n}+\left(\begin{array}{l}n \\ 1\end{array}\right)\left(x_{1}-x_{0}\right)^{n-1}\left(x-x_{1}\right)+\ldots\right.$

$$
\left.\cdots+\left(\begin{array}{l}
n \\
n
\end{array}\right)\left(x-x_{1}\right)^{n}\right]
$$

und alles, was wir zu zeigen haben, ist dies, daß wir hier alle Glieder mit derselben Potenz von $\left(x-x_{1}\right)$ zusammensuchen dürfen, daß also der große Umordnungssatz $\mathbf{9 0}$ angewendet werden darf. Ersetzt man aber, um dessen Anwendbarkeit zu prüfen, in der letzten Reihe jeden Summanden jedes Gliedes durch seinen absoluten Betrag, so erhält man die Reihe

$$
\sum_{n=0}^{\infty}\left|a_{n}\right|\left[\left|x_{1}-x_{0}\right|+\left|x-x_{1}\right|\right]^{n}
$$

und diese ist gewiß noch konvergent, wenn

$$
\left|x_{1}-x_{0}\right|+\left|x-x_{1}\right|<r \quad \text { oder } \quad\left|x-x_{1}\right|<r-\left|x_{1}-x_{0}\right|
$$

ist. Wenn also $x$ näher an $x_{1}$ liegt als jedes der Enden des ursprünglichen Konvergenzintervalles, so ist die geplante Umordnung erlaubt, und wir erhalten für $f(x)$, wie behauptet, ein? Darstellung der Form

$$
f(x)=\sum_{k=0}^{\infty} b_{k}\left(x-x_{1}\right)^{k} \quad\left(\left|x-x_{1}\right|<r-\left|x_{1}-x_{0}\right|\right) .
$$

Führt man das Zusammenfassen der Glieder mit $\left(x-x_{1}\right)^{k}$ im ein- 
zelnen durch, indem man die Glieder der Reihe (a) zeilenweis untereinander schreibt, so liefert die $k^{\text {te }}$ Spalte:

$$
\left.b_{k}=\left(\begin{array}{l}
k \\
k
\end{array}\right) a_{k}+\left(\begin{array}{c}
k+1 \\
k
\end{array}\right) a_{k+1}\left(x_{1}-x_{0}\right)+\ldots=\sum_{n=0}^{\infty}\left(\begin{array}{c}
n+k \\
k
\end{array}\right) a_{n+k}\left(x_{1}-x_{0}\right)^{n} \mathbf{1}\right)
$$

- womit dann alles bewiesen ist.

Aus diesem Satz ziehen wir die mannigfachsten Folgerungen. Zunächst ergibt sich der

- Satz 2. Eine durch eine Potenzreihe dargestellte Funktion

$$
f(x)=\sum_{n=0}^{\infty} a_{n}\left(x-x_{0}\right)^{n}
$$

ist in jedem inneren Punkte $x_{1}$ des Konvergenzintervalles stetig.

Beweis. Nach dem vorigen Satz darf für eine gewisse Um. gebung von $x_{1}$

mit

$$
f(x)=\sum_{n=0}^{\infty} a_{n}\left(x-x_{0}\right)^{n}=\sum_{n=0}^{\infty} b_{n}\left(x-x_{1}\right)^{n}
$$

$$
b_{0}=\sum_{n=0}^{\infty} a_{n}\left(x_{1}-x_{0}\right)^{n}=f\left(x_{1}\right)
$$

gesetzt werden. Für $x \rightarrow x_{1}$ liefert dann die zweite der Darstellungen von $f(x)$ nach 96 sofort die zu beweisende Beziehung (s. $§ 19$, Def. 6)

$$
\lim _{x \rightarrow x_{1}} f(x)=f\left(x_{1}\right) \text {. }
$$

- Satz 3. Eine durch eine Potenzreihe dargestellte Funktion

$$
f(x)=\sum_{n=0}^{\infty} a_{n}\left(x-x_{0}\right)^{n}
$$

ist in jedem inneren Punkte $x_{1}$ ihres Konvergenzintervalles differenzierbar (s. § 19, Def. 10) und die Ableitung daselbst, $f^{\prime}\left(x_{1}\right)$, kann durch gliedweise Differentiation gewonnen werden, d.h. es ist

$$
f^{\prime}\left(x_{1}\right)=\sum_{n=0}^{\infty} n a_{n}\left(x_{1}-x_{0}\right)^{n-1}=\sum_{n=0}^{\infty}(n+1) a_{n+1}\left(x_{1}-x_{0}\right)^{n} .
$$

Beweis. Wegen $f(x)=\sum_{n=0}^{\infty} b_{n}\left(x-x_{1}\right)^{n}$ ist für alle hinreichend nahe bei $x_{1}$ gelegenen $x$

$$
\frac{f(x)-f\left(x_{1}\right)}{x-x_{1}}=b_{1}+b_{2}\left(x-x_{1}\right)+\ldots
$$

woraus für $x \rightarrow x_{1}$ nach 96 und unter Berücksichtigung der Bedeutung von $b_{1}$ sofort die Behauptung folgt: $f^{\prime}\left(x_{1}\right)=b_{1}=\sum n a_{n}\left(x_{1}-x_{0}\right)^{n-1}$.

-Satz 4. Eine durch eine Potenzreihe dargestellte Funktion

$$
f(x)=\sum_{n=0}^{\infty} a_{n}\left(x-x_{0}\right)^{n}
$$

1) Es ergibt sich also noch einmal ganz nebenbei die schon in 95 festgestellte Konvergenz der für die $b_{k}$ erhaltenen Reihen. 
ist in jedem inneren Punkte $x_{1}$ ihres Konvergenzintervalles beliebig oft differenzierbar und es ist

$$
f^{(k)}\left(x_{1}\right)=k ! b_{k}=\sum_{n=0}^{\infty}(n+1)(n+2) \ldots(n+k) a_{n+k}\left(x_{1}-x_{0}\right)^{n} .
$$

Beweis. Für jedes $x$ des Konvergenzintervalles ist, wie eben gezeigt,

$$
f^{\prime}(x)=\sum_{n=0}^{\infty}(n+1) a_{n+1}\left(x-x_{0}\right)^{n} .
$$

$f^{\prime}(x)$ ist also seinerseits eine durch eine Potenzreihe dargestellte Funktion, - und zwar durch eine Potenzreihe, die nach $\mathbf{9 5}$ dasselbe Konvergenzintervall hat wie die ursprüngliche. Daher kann auf $f^{\prime}(x)$ noch einmal derselbe Schluß angewendet werden; dieser liefert $f^{\prime \prime}(x)=\sum_{n=0}^{\infty} n(n+1) a_{n+1}\left(x-x_{0}\right)^{n-1}=\sum_{n=0}^{\infty}(n+1)(n+2) a_{n+2}\left(x-x_{0}\right)^{n}$. Durch Wiederholung dieses einfachen Schlusses ergibt sich für jedes $k$

$$
f^{(k)}(x)=\sum_{n=0}^{\infty}(n+1)(n+2) \ldots(n+k) a_{n+k}\left(x-x_{0}\right)^{n},
$$

— gültig für jedes $x$ des ursprünglichen Konvergenzintervalles. Für den speziellen Wert $x=x_{1}$ folgt hieraus unmittelbar die Behauptung.

Setzen wir für die $b_{k}$ die nun erhaltenen Werte $\frac{1}{k !} f^{(k)}\left(x_{1}\right)$ in die Entwicklung des Satzes 1 ein, so ergibt sich aus allem vorangehenden schließlich die sogenannte

- Taylorsche Reihe ${ }^{1}$ ). Wenn für $\left|x-x_{0}\right|<r$

$$
f(x)=\sum_{n=0}^{\infty} a_{n}\left(x-x_{0}\right)^{n}
$$

ist und wenn $x_{1}$ ein innerer Punkt des Konvergenzintervalles ist, so ist für alle $\left|x-x_{1}\right|<r_{1}=r-\mid x_{1}-x_{0}$

$$
\begin{gathered}
f(x)=f\left(x_{1}\right)+\frac{f^{\prime}\left(x_{1}\right)}{1 !}\left(x-x_{1}\right)+\frac{f^{\prime \prime}\left(x_{1}\right)}{2 !}\left(x-x_{1}\right)^{2}+\ldots \\
\left.\ldots+\frac{f^{(k)}\left(x_{1}\right)}{k !}\left(x-x_{1}\right)^{k}+\ldots{ }^{2}\right) .
\end{gathered}
$$

1) Brook Taylor, Methodus incrementorum directa et inversa, London 1715 . - Vgl. dazu A. Pringsheim, Geschichte des Taylorschen Lehrsatzes, Bibl. mathem., Bd. (3) 1 (1900), S. 433.

2) Die im Satze angegebene Zahl $r_{1}=r-\left|x_{1}-x_{0}\right|$ braucht nicht der genaue Konvergenzradius der neuen Potenzreihe zu sein. Dieser kann vielmehr erheblich größser ausfallen. Für $f(x)=\Sigma x^{n}=\frac{1}{1-x}$ und $x_{1}=-\frac{1}{2}$ erhält man z. B. nach leichter Rechnung

$$
f(x)=\sum_{k=0}^{\infty}\left(\frac{2}{3}\right)^{k+1} \cdot\left(x+\frac{1}{2}\right)^{k}
$$

und der Radius dieser Reihe ist nicht $=r-x_{1}-x_{0} \mid=\frac{1}{2}$, sondern $=\frac{3}{2}$. 
Dem Satz 3 über die Differentiation unserer Reihen stellen wir noch den entsprechenden über die Integration an die Seite. Da eine durch eine Potenzreihe dargestellte Funktion im Innern ihres Konvergenzintervalles stetig ist, so ist sie nach $\S 19$, Satz 12 auch über jedes Intervall integrierbar, das einschließlich seiner Enden jenem Innern angehört. Hierüber gilt der

- Satz 5. Das Integral der durch $\sum_{n=0}^{\infty} a_{n}\left(x-x_{0}\right)^{n}$ im Konvergenzintervall dargestellten (stetigen) Funktion $f(x)$ darf durch gliedweise Integration gemäß der Formel

$$
\int_{x_{1}}^{x_{2}} f(t) d t=\sum_{n=0}^{\infty} \frac{a_{n}}{n+1}\left[\left(x_{2}-x_{0}\right)^{n+1}-\left(x_{1}-x_{0}\right)^{n+1}\right]
$$

gewonnen werden, wofern $x_{1}$ und $x_{\mathbf{2}}$ beide im Innern des Konvergenzintervalles gelegen sind.

Beweis. Wegen $\S 19$, Satz 19 genügt es zu zeigen, daß für jeden Punkt $x$ des Konvergenzintervalles

$$
\int_{x_{0}}^{x} f(x) d x=\sum_{n=0}^{\infty} \frac{a_{n}}{n+1}\left(x-x_{0}\right)^{n+1}
$$

ist. Nun steht aber rechterhand jedenfalls eine für $\left|x-x_{0}\right|<r$ konvergente Potenzreihe. Deren Ableitung ist nach 98, 3 ersichtlich $=\sum a_{n}\left(x-x_{0}\right)^{n}=f(x)$. Dieselbe Ableitung hat nach $\S 19$, Satz 23 auch die auf der linken Seite stehende Funktion, so daß auf beiden Seiten unserer Gleichung wohlbestimmte Funktionen stehen, die für $\left|x-x_{0}\right|<r$ definiert und differenzierbar sind, und deren Ableitungen dort übereinstimmen. Beide Seiten unterscheiden sich also nach $\S 19$, Satz 7 höchstens um eine Konstante. Setzt man aber $x=x_{0}$, so zeigt sich, $\mathrm{da} B$ die unterscheidende Konstante selbst $=0$ ist, daß also beide Seiten unserer Gleichung dieselbe Funktion darstellen, w. z. b.w.

Diesen Sätzen über Potenzreihen wollen wir noch nach einer besonderen Seite hin eine wichtige Ergänzung anfügen: Der Satz 2 von der Stetigkeit der durch eine Potenzreihe dargestellten Funktion galt, wie wir ausdrücklich noch einmal betonen, nur für das offene Konvergenzintervall. So läßt sich z. B. im Falle der geometrischen Reihe $\sum x^{n}$ mit der Summe $\frac{1}{1-x}$ aus unsern Betrachtungen weder unmittelbar die Unstetigkeit der Funktion $\frac{1}{1-x}$ an der Stelle $x=+1$ noch die Stetigkeit derselben an der Stelle $x=-1$ aus der Reihe ablesen. Auch wenn die Potenzreihe noch in einem der Endpunkte des Konvergenzintervalles konvergieren sollte (wie z. B. $\sum \frac{x^{n}}{n}$ in $x=-1$ ), ist ein solcher Schluß nicht ohne weiteres gestattet. 
$\mathrm{Da} B$ jedoch in diesem letzteren Falle die vermutete 'Tatsache selbst wenigstens in gewissem Umfange richtig ist, lehrt folgender

Abelscher Grenzwertsatz $\left.{ }^{1}\right)$. Die Potenzreihe $f^{\prime}(x)=\sum_{n=0}^{\infty} a_{n} x^{n}$ habe $\mathbf{1 0 0 .}$ den Konvergenzradius $r$ und sei noch im Punkte $x=+r$ konvergent.

Dann ist

$$
\lim _{x \rightarrow r-0} f(x) \quad \text { vorhanden und }=\sum_{n=0}^{x} \boldsymbol{a}_{n} \boldsymbol{p} \text {. }
$$

Oder m. a. W.: Ist $\sum_{n=0}^{\infty} a_{n} x^{n}$ noch in $x=+r$ konvergent, so ist die durch die Reihe in $-r<x \leqq+r$ definierte Funktion auch in dem Endpunkt $x=+r$ noch linksseitig stetig.

Beweis. Es bedeutet keine Einschränkung, $r=+1$ anzunehmen $^{2}$ ). Denn hat $\Sigma a_{n} x^{n}$ den Radius $r$, so hat die Reihe $\Sigma a_{n}{ }^{\prime} x^{n}$, in der $a_{n}^{\prime}=a_{n} r^{n}$ sein soll, ersichtlich den Radius +1 ; und die letztere Reihe ist dann und nur dann in +1 oder -1 konvergent, wenn es die vorige in $+r$ bzw. $-r$ war.

Wir nehmen daher weiterhin $r=+1$ an. Unsere Voraussetzung ist dann, daß $f(x)=\Sigma a_{n} x^{n}$ den Radius 1 hat und daß $\Sigma a_{n}=s$ konvergiert; und die Behauptung lautet, daß auch

$$
\lim _{x \rightarrow 1-0} f(x)=s\left(=\stackrel{\varliminf}{N}=0_{n}^{\infty} a_{n}\right)
$$

ist. Nun ist nach 91 (s. auch weiter unter 102) für $|x|<1$

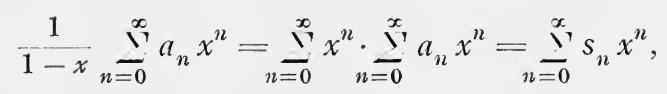

wenn mit $s_{n}$ die Teilsummen von $\Sigma a_{n}$ bezeichnet werden. Folglich ist $f(x)=(1-x) \Sigma s_{n} x^{n}$ und wegen $1=(1-x) \Sigma x^{n}$ hat man also für $|x|<1$

$$
s-f(x)=(1-x) \sum_{n=0}^{\infty}\left(s-s_{n}\right) x^{n}=(1-x) \sum_{n=0}^{\infty} r_{n} x^{n} .
$$

Hier wurden zuletzt die "Reste“ $s-s_{n}=r_{n}$ gesetzt; sie bilden nach 82, 6 eine Nullfolge.

1) Journal f. d. reine u. angew. Math., Bd. 1 (1826), S. 311. Vgl. hierzu $\S 54$, S. 390 und $\S 62$. - Der Satz wird schon von Gauß (Disquis. generales circa seriem ..., 1812, Werke III, S. 143) ausgesprochen und benutzt, und $z$ war genau in der nachher bewiesenen Form, daf aus $r_{n} \rightarrow 0$ stets $(1-x) \sum v_{n} x^{n} \rightarrow 0$ folgt, falls $x$ von links her $\rightarrow 1$ rïckt (s. o., Gl. (a)). Der von Gauß an der genannten Stelle angegebene Beweis ist indessen nicht richtig, da er ohne besondere Prüfung die beiden für diesen Satz in Frage kommenden Grenzübergänge vertauscht.

2) Diese Bemerkung gilt allgemein bei Untersuchungen über (nicht beständig konvergente) Potenzreihen mit positivem Radius $r$. 
Ist nun $\varepsilon>0$ beliebig gegeben, so wählen wir zunächst $m$ so groß, daß für $n>m$ stets $\left|r_{n}\right|<\frac{\varepsilon}{2}$ ist. Dann ist für $0 \leqq x<1$

$$
|s-f(x)| \leqq\left|(1-x) \sum_{n=0}^{m} r_{n} x^{n}\right|+\frac{\varepsilon}{2}(1-x) \cdot \sum_{n=m+1}^{\infty} x^{n},
$$

also, wenn $\left|r_{0}\right|+\left|r_{1}+\ldots+\right| r_{m} \mid=p$ gesetzt wird,

$$
\leqq p \cdot(1-x)+\frac{\varepsilon}{2}(1-x) \cdot \frac{x^{m+1}}{1-x} \text {. }
$$

Setzt man nun $\delta=\frac{\varepsilon}{2 p}$, so ist für $1-\delta<x<1$ stets

$$
|s-f(x)|<\frac{\varepsilon}{2}+\frac{\varepsilon}{2}=\varepsilon,
$$

womit nach $\S 19$, Def. 5 die Behauptung , $f(x) \rightarrow s$ für $x \rightarrow 1-0$ " schon bewiesen ist.

Ganz entsprechend gilt natürlich der Abelsche Grenzwertsatz für das linke Ende des Konvergenzintervalles:

Ist $\sum_{n=0}^{\infty} a_{n} x^{n}$ noch für $x=-r$ konvergent, so ist $\lim _{x \rightarrow-r+0} f(x) \quad$ vorhanden und $=\sum_{n=0}^{\infty}(-1)^{n} a_{n} r^{n}$.

Bemerkungen und Beispiele für diese und die folgenden Sätze dieses Paragraphen werden im nächsten Kapitel ausführlich gebracht werden.

Der Stetigkeitssatz 98, 2 und der Abelsche Grenzwertsatz 100 besagen zusammen, daß stets

$$
\lim _{x \rightarrow \xi}\left(\sum a_{n} x^{n}\right)=\sum a_{n} \xi^{n}
$$

ist, falls die rechtsstehende Reihe konvergiert und falls sich $x$ vom Nullpunkt her gegen die Stelle $\xi$ bewegt.

Divergiert die Reihe $\sum a_{n} \xi^{n}$, so kann man über das Verhalten von $\sum a_{n} x^{n}$ bei der Annäherung von $x \rightarrow \xi$ ohne besondere Voraussetzungen nichts aussagen. Doch gilt in dieser Hinsicht der folgende etwas engere

Satz. Ist $\Sigma a_{n}$ eine divergente Reihe mit positiven Gliedern und hat $\Sigma a_{n} x^{n}$ den Radius 1, so strebt

$$
f(x)=\sum_{n=0}^{\infty} a_{n} x^{n}>+\infty,
$$

wenn $x$ vom Nullpunkt her gegen +1 rückt. 
Beweis. Wird $\dot{G}>0$ beliebig gegeben, so kann man $m$ so groß wählen, daß $a_{0}+a_{1}+\ldots+a_{m}>G+1$ ist, und dann nach $\S 19$, Satz 3 $\delta$ so klein, daß für alle $1>x>1-\delta$

$$
a_{0}+a_{1} x+\ldots+a_{m} x^{m}>G
$$

bleibt. Dann ist für diese $x$ um so mehr

$$
f(x)=\sum_{n=0}^{\infty} a_{n} x^{n}>G,
$$

womit schon alles bewiesen ist.

\section{§ 21. Das Rechnen mit Potenzreihen.}

Ehe wir von den tiefgehenden und mitten in das große Anwendungsfeld der Reihenlehre hineinführenden Sätzen der vorigen Paragraphen Gebrauch machen, wollen wir noch auf einige Fragen eingehen, deren Beantwortung uns das Rechnen mit den Potenzreihen erleichtern soll.

$\mathrm{Da} B$ man Potenzreihen, solange sie konvergieren, gliedweis addieren und subtrahieren darf, folgt schon aus 83, 3 und 4 . Daß man auch ohne weiteres zwei Potenzreihen gliedweis ausmultiplizieren darf, solange wir im Innern der Konvergenzintervalle bleiben, folgt sofort aus 91, weil ja die Potenzreihen im Innern ihrer Konvergenzintervalle stets absolut konvergieren. Es ist also neben

auch

$$
\sum a_{n} x^{n} \pm \Sigma b_{n} x^{n}=\Sigma\left(a_{n} \pm b_{n}\right) x^{n}
$$

$$
\sum_{n=0}^{\infty} a_{n} x^{n} \cdot \sum_{n=0}^{\infty} b_{n} x^{n}=\sum_{n=0}^{\infty}\left(a_{0} b_{n}+a_{1} b_{n-1}+\ldots+a_{n} b_{0}\right) x^{n},
$$

solange $x$ im Innern des Konvergenzintervalles beider Reihen liegt ${ }^{\mathbf{1}}$ ).

Die Formeln 91, Bem. 2 und 3 waren schon eine erste Anwendung dieses Satzes. Ist die zweite Reihe speziell die geometrische Reihe, so hat man

d. h.

$$
\sum_{n=0}^{\infty} a_{n} x^{n} \cdot \sum_{n=0}^{\infty} x^{n}=\sum_{n=0}^{\infty} s_{n} x^{n}
$$

oder

$$
\frac{1}{1-x} \sum_{n=0}^{\infty} a_{n} x^{n}=\sum_{n=0}^{\infty} s_{n} x^{n}
$$

$$
\sum_{n=0}^{\infty} a_{n} x^{n}=(1-x) \sum_{n=0}^{\infty} s_{n} x^{n},
$$

wenn $s_{n}=a_{0}+a_{1}+\ldots+a_{n}$ gesetzt wird und $|x|<1$ und zugleich kleiner als der Radius von $\sum a_{n} x^{n}$ ist.

1) Hier tritt also die besondere Bedeutung der Cauchyschen Multiplikation (s. 91, 1) zutage. 
Ebenso einfach ergibt sich, daß man jede Potenzreihe mit sich selbst - und dies beliebig oft - multiplizieren darf. So ist

$$
\left(\sum_{n=0}^{\infty} a_{n} x^{n}\right)^{2}=\sum_{n=0}^{\infty}\left(a_{0} a_{n}+a_{1} a_{n+1}+\ldots+a_{n} a_{0}\right) x^{n} ;
$$

und allgemein läßt sich für jeden positiv-ganzen Exponenten $k$

103.

$$
\left(\sum_{n=0}^{\infty} a_{n} x^{n}\right)^{k}=\sum_{n=0}^{\infty} a_{n}^{(k)} x^{n}
$$

setzen, wo die Koeffizienten $a_{n}^{(k)}$ in ganz bestimmter - für größere $k$ allerdings nicht sehr durchsichtiger ${ }^{1}$ ) - Weise aus den $a_{n}$ gebildet sind. Und alle diese Reihen sind absolut konvergent, solange $\sum a_{n} x^{n}$ selber es ist.

Dies Ergebnis legt die Vermutung nahe, daß man auch durch Potenzreihen dividieren „darf“, daß man also z. B. auch

$$
\frac{1}{a_{0}+a_{1} x+a_{2} x^{2}+\cdots}=c_{0}+c_{1} x+c_{2} x^{2}+\cdots
$$

setzen darf, und daß die $c_{n}$ hier wieder in bestimmter Weise aus den $a_{n}$ zu bilden sind. Denn für den linken Quotienten kann man wenn $-\frac{a_{n}}{a_{0}}=a_{n}^{\prime}$ gesetzt wird (für $n=1,2,3, \ldots$ ) zunächst

und dann weiter

$$
\frac{1}{a_{0}} \cdot \frac{1}{1-\left(a_{1}^{\prime} x+a_{2}^{\prime} x^{2}+\ldots\right)}
$$

$$
\frac{1}{a_{0}}\left[1+\left(a_{1}^{\prime} x+a_{2}^{\prime} x^{2}+\ldots\right)+\left(a_{1}^{\prime} x+\ldots\right)^{2}+\left(a_{1}^{\prime} x+\ldots\right)^{3}+\ldots\right]
$$

schreiben, was dann, wenn man sich die Potenzen nach 103 entwickelt und die gleichen Potenzen von $x$ gesammelt denkt, in der Tat eine Potenzreihe der Form $\sum c_{n} x^{n}$ liefern würde.

Die Berechtigung eines solchen Ansatzes wollen wir sogleich von einem etwas allgemeineren Standpunkt aus prüfen:

Es sei eine Potenzreihe $\Sigma a_{n} x^{n}$ (im vorangehenden war es die Reihe $\left.\sum_{n=1}^{\infty} a_{n}{ }^{\prime} x^{n}\right)$ vorgelegt, deren Summe mit $f(x)$ oder kürzer mit $y$ bezeichnet werde. Es sei zweitens eine Potenzreihe in $y$, etwa $g(y)=\Sigma b_{n} y^{n}$ (im vorangehenden die geometrische Reihe $\Sigma y^{n}$ ) vorgelegt und.in diese werde für $y$ die erste Potenzreihe eingesetzt:

$$
b_{0}+b_{1}\left(a_{0}+a_{1} x+\ldots\right)+b_{2}\left(a_{0}+a_{1} x+\ldots\right)^{2}+\ldots
$$

Unter welchen Bedingungen führt hier die Ausführung aller Potenzen nach 103 und die Zusammenfassung der gleichen Potenzen von $x$

1) Rekursionsformeln zur Berechnung der $a_{n}^{(k)}$ findet man bei J.W.L.Glaisher, Note on Sylvester's paper: Development of an idea of Eisenstein (Quarterly Journal, Bd. 14, 1875, S. 79-84), in dem sich auch weitere Literaturangaben finden. Ferner bei B. Hansted, Tidskrift for Mathematik (4), Bd. 5, 1881, S. 12-16. 
zu einer neuen Potenzreihe $c_{0}+c_{1} x+c_{2} x^{2}+\ldots$, welche konvergiert und als Summe den mittelbaren Funktionswert $g(f(x))$ hat? Wir behaupten den

○ Satz. Dies gilt sicher für alle diejenigen $x$, für die $\sum_{n=0}^{\infty}\left|a_{n} x^{n}\right|$ 104. konvergiert und eine Summe hat, die kleiner ist als der Radius von $\Sigma b_{n} y^{n}$.

Beweis. Es liegt hier offenbar ein Fall des großen Umordnungssatzes 90 vor und wir haben nur zu prüfen, ob dessen Voraussetzungen hier erfüllt sind. Setzen wir dazu zunächst die nach $\mathbf{1 0 3}$ gebildeten Potenzen

$$
y^{k}=\left(a_{0}+a_{1} x+\ldots\right)^{k}=a_{0}^{(k)}+a_{1}^{(k)} x+a_{2}^{(k)} x^{2}+\ldots
$$

und denken uns diese Schreibweise auch für $k=0$ und $k=1$ angewendet ${ }^{1}$ ), so sind

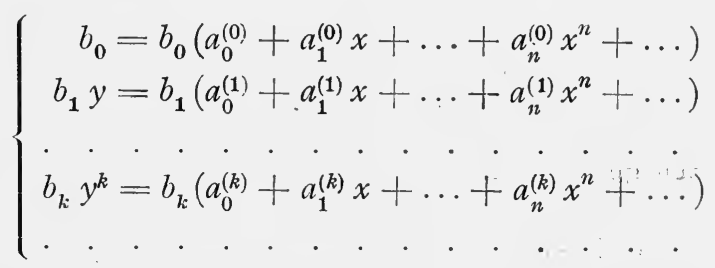

die im großen Umordnungssatz auftretenden Reihen $z^{(k)}$. Nehmen wir nun statt $y=\sum a_{n} x^{n}$ die Reihe $\eta=\Sigma\left|a_{n} x^{n}\right|$ und bilden, indem wir noch $|x|=\xi$ setzen, ganz analog

$$
\left\{\begin{array}{c}
\left|b_{0}\right|=\left|b_{0}\right|\left(\alpha_{0}^{(0)}+\alpha_{1}^{(0)} \xi+\ldots+\alpha_{n}^{(0)} \xi^{n}+\ldots\right) \\
\left|b_{1}\right| \eta=\left|b_{1}\right|\left(\alpha_{0}^{(1)}+\alpha_{1}^{(1)} \xi+\ldots+\alpha_{n}^{(1)} \xi^{n}+\ldots\right) \\
. b_{k}\left|\eta^{k}=\right| b_{k} \mid\left(\alpha_{0}^{(k)}+\alpha_{1}^{(k)} \xi+\ldots+\alpha_{n}^{(k)} \xi^{n}+\ldots\right) \\
. \quad . \quad . \quad . \quad . \quad . \quad . \quad . \quad . \quad . .
\end{array}\right.
$$

so sind in diesem Schema $\left(\mathrm{A}^{\prime}\right)$ alle Zahlen positiv; und da überdies $\Sigma\left|b_{k}\right| \eta^{k}$ noch konvergieren sollte, so ist auf (A') der große Umordnungssatz anwendbar. Da aber ersichtlich jede Zahl im Schema (A) absolut genommen $\leqq$ der entsprechenden $\mathrm{Zahl}$ in $\left(\mathrm{A}^{\prime}\right)$ ist, so ist dieser Satz um so mehr auf (A) selber anwendbar (vgl. 90, Bem. 3). Es liefern also insbesondere die in (A) spaltenweis untereinanderstehenden Koeffizienten stets (absolut) konvergente Reihen

$$
\sum_{k=0}^{\infty} b_{k} a_{n}^{(k)}=c_{n} \quad(\text { für jedes bestimmte } n=0,1,2, \ldots)
$$

und die mit diesen Zahlen als Koeffizienten angesetzte Potenzreihe

$$
\sum_{n=0}^{\infty} c_{n} x^{n}
$$

1) Es sind dann also $a_{0}^{(0)}=1, a_{1}^{(0)}=a_{2}^{(0)}=\ldots=0$ und $a_{n}^{(1)}=a_{n}$ zu setzen, letzteres für $n=0,1,2, \ldots$ 
ist ihrerseits wieder für die genannten $x$ (absolut) konvergent und ihre Summe ist gleich derjenigen von $\Sigma b_{n} y^{n}$. Es ist also, wie behauptet,

$$
g(f(x))=\sum_{n=0}^{\infty} c_{n} x^{n}
$$

mit der angegebenen Bedeutung der $c_{n}$.

105.

Bemerkungen und Beispiele.

1. Ist die „äufere" Reihe $g(y)=\Sigma b_{k} y^{k}$ beständig konvergent, so gilt unser Satz offenbar fuir jedes $x$, für das $\Sigma a_{n} x^{n}$ absolut konvergiert. Sind beide Reihen beständig konvergent, so gilt der Satz ohne Einschränkung für jedes $x$.

2. Ist $a_{0}=0$, so gilt der Satz sicher für alle, hinreichend" kleinen $x$, d. h. es gibt dann sicher eine positive $Z a h l \varrho$, so daß er für alle $|x|<\varrho$ gültig ist. Denn ist $y=a_{1} x+a_{2} x^{2}+\ldots$, so ist $\eta=\left|a_{1}\right| \cdot|x|+\left|a_{2}\right| \cdot|x|^{2}+\ldots$; und da nun für $x \rightarrow 0$ nach 96 auch $\eta \rightarrow 0$ strebt, so ist $\eta$ sicher $<$ als der Radius. von $\Sigma b_{k} y^{k}$ für alle $x$, deren Betrag $<$ als eine passende Zahl $\varrho$ bleibt.

3. In die Reihe $\sum \frac{y^{n}}{n !}$ "darf“ man z. B. $y=\Sigma x^{n}$ für $|x|<1$, oder $y=\Sigma \frac{x^{n}}{n !}$ für alle $x$ einsetzen und nach Potenzen von $x$ umordnen.

4. Der oben gemachte Ansatz

$$
\frac{1}{a_{0}+a_{1} x+a_{2} x^{2}+\ldots}=c_{0}+c_{1} x+c_{2} x^{2}+\ldots
$$

ist, wie man nun erkennt, sicher dann erlaubt, wenn zunächst $a_{0} \neq 0$ und wenn weiter $x$ seinem Betrage so klein gehalten wird, dafs

$$
\eta=\left|\frac{a_{1}}{a_{0}} x\right|+\left|\frac{a_{2}}{a_{0}} x^{2}\right|+\ldots<1
$$

bleibt, was nach Bem. 2 bei passender Wahl von $\varrho$ für alle $x \mid<\varrho$ der Fall ist. Wir können also sagen: Durch cine Potenzreihe "darf" dividiert werden, falls $i$ hr konstantes Glied $\neq 0$ ist und falls man sich auf hinreichend kleine Werte von $x$ beschränkt ${ }^{1}$ ).

Die Koeffizienten $c_{n}$ nach dem allgemeinen Ansatz zu ermitteln, wäre selbst für die ersten Indizes - sehr mühsam. Aber nachdem erst einmal die Möglichkeit der Entwicklung dargetan ist - die nun nach 97 zugleich die einzig mögliche ist - findet man die $c_{n}$ schneller aus der Bemerkung, daf

$$
\Sigma a_{n} x^{n} \cdot \Sigma c_{n} x^{n}=1
$$

ist, daßi also der Reihe nach (vgl. 97, 2)

$$
\begin{aligned}
& a_{0} c_{0}=1 \\
& a_{0} c_{1}+a_{1} c_{0}=0 \\
& a_{0} c_{2}+a_{1} c_{1}+a_{22} c_{0}=0 \\
& a_{0} c_{3}+a_{1} c_{2}+a_{2} c_{1}+a_{3} c_{0}=0
\end{aligned}
$$

ist, - Gleichungen, aus denen man, da $a_{0} \neq 0$ ist, der Reihe nach $c_{0}, c_{1}, c_{2}, \ldots$ in ganz eindeutiger Weise findet ${ }^{2}$ ).

1) Wie klein $x$ sein muf, ist meist gleichgultig. Wesentlich ist aber, dafs es überhaupt einen positiven Radius $\varrho$ gibt, so daf die Relation für alle $x \mid<\varrho$ gilt. - Die Feststellung des genauen Gültigkeitsbereiches erfordert die tieferen Hilfsmittel der Funktionentheorie.

2) Explizite Formeln für die Entwicklungskoeffizienten des Quotienten zweier Potenzreihen findet man z. B. bei J. Hagen, On division of series, Americ. Journ. of Math., Bd. 5 (1883), S. 236. 
5. Als ein für viele spätere Untersuchungen besonders wichtiges Beispiel geben wir dic folgende Divisionsaufgabe ${ }^{1}$ ):

Es soll

$$
\frac{1}{1+\frac{x}{2 !}+\frac{x^{2}}{3 !}+\ldots} \quad \text { oder } \frac{x}{\left(1+x+\frac{x^{2}}{2 !}+\frac{x^{3}}{3 !}+\ldots\right)-1}
$$

nach Potenzen von $x$ entwickelt werden. Hier wird die Berechnung der neuen Koeffizienten besonders elegant, wenn man sie nicht mit $c_{n}$, sondern mit $\frac{c_{n}}{n}$ ! oder, wie wir es aus historischen Gründen tun wollen, mit $\frac{B_{n}}{n !}$ bezeichnet. Dann lautet der Ansatz:

$$
\left(1+\frac{x}{2 !}+\frac{x^{2}}{3 !}+\ldots\right) \cdot\left(B_{0}+\frac{B_{1}}{1 !} x+\frac{B_{2}}{2 !} x^{2}+\ldots\right) \equiv 1
$$

und die Gleichungen zur Berechnung der $B_{n}$ sind der Reihe nach:

$$
B_{0}=1, \quad \frac{1}{2 !} \frac{B_{0}}{0 !}+\frac{1}{1 !} B_{1}=0
$$

und allgemein für $n=2,3, \ldots$

$$
\frac{1}{n !} \cdot \frac{B_{0}}{0 !}+\frac{1}{(n-1) !} \cdot \frac{B_{1}}{1 !}+\frac{1}{(n-2) !} \cdot \frac{B_{2}}{2 !}+\ldots+\frac{1}{1 !} \cdot \frac{B_{n-1}}{(n-1) !}=0 .
$$

Erweitert man diese Gleichung noch mit $n$ !, so kann man kürzer dafür schreiben

$$
\left(\begin{array}{l}
n \\
0
\end{array}\right) B_{0}+\left(\begin{array}{c}
n \\
1
\end{array}\right) B_{1}+\left(\begin{array}{l}
n \\
2
\end{array}\right) B_{2}+\ldots+\left(\begin{array}{c}
n \\
n-1
\end{array}\right) B_{n-1}=0 \text {. }
$$

Stünde hier überall $B^{v}$ statt $B_{v}$, so könnte man für diese Gleichung noch kiirzer

$$
(B+1)^{n}-B^{n}=\mathbf{0}
$$

schreiben, und in dieser bequemen Form kann man sich auch die vorstehende Rekursionsformel als sogenannte symbolische Gleichung merken, d. h. als eine Gleichung, die nicht wörtlich zu verstehen ist, sondern die erst auf Grund einer besonderen Verabredung guiltig ist, - hier also der Verabredung, daf nach Ausführung der $n$ ten Potenz des Binoms überall wieder $B^{v}$ durch $B_{\boldsymbol{v}}$ zu ersetzen ist. Unsere Formel liefert nun für $n=2,3,4,5, \ldots$ der Reihe nach die Gleichungen

$$
\begin{aligned}
& 2 B_{1}+1=0 \\
& 3 B_{2}+3 B_{1}+1=0, \\
& 4 B_{3}+6 B_{2}+4 B_{1}+1=0, \\
& 5 B_{4}+10 B_{3}+10 B_{2}+5 B_{1}+1=0,
\end{aligned}
$$

aus denen sich

und dann weiter

$$
B_{1}=-\frac{1}{2}, \quad B_{2}=\frac{1}{6}, \quad B_{3}=0, \quad B_{4}=-\frac{1}{30}
$$

$$
B_{5}=B_{7}=B_{9}=B_{11}=B_{13}=\ldots=0 \text {, sowie }
$$

$$
B_{6}=\frac{1}{42}, \quad B_{\times}=-\frac{1}{30}, \quad B_{10}=\frac{5}{66}, \quad B_{12}=-\frac{691}{2730}, \quad B_{14}=\frac{7}{6}, \ldots
$$

ergibt. Von diesen sogenannten Bernoullischen Zahlen wird später noch öfter die Rede sein. In Augenblick ist nur zu sehen, dafs die $B_{n}$ wohlbestimmte rationale. Zahlen sind. Sie folgen indessen keiner oberflächlichen Ge-

1) Euler, Institutiones calc. diff., Bd. 2 (1755), § 122. 
setzmäßigkeit und sind der Gegenstand vieler und tiefgelıender Untersuchungen gew orden ${ }^{1}$ ).

Endlich wollen wir noch ein letztes allgemeines Theorem über Potenzreihen beweisen: Wenn die für $x-x_{0}<r$ konvergente Potenzreihe $y=\sum_{n=0}^{\infty} a_{n}\left(x-x_{0}\right)^{n}$ vorgelegt ist, so entspricht jedem $x$ in der Umgebung von $x_{0}$ ein bestimmter Wert von $y$, speziell dem Werte $x=x_{0}$ der Wert $y=a_{0}$, den wir darum auch mit $y_{0}$ bezeichnen wollen. Dann ist

$$
y-y_{0}=a_{1}\left(x-x_{0}\right)+a_{2}\left(x-x_{0}\right)^{2}+\ldots
$$

Wegen der Stetigkeit der Funktion entspricht jedem nahe bei $x_{0}$ gelegenen $x$ auch ein nahe bei $y_{0}$ gelegener Wert von $y$. Wir wollen nun fragen, ob bzw, in wieweit jeder in der Nähe von $y_{0}$ gelegene Wert von y erhalten wird und ob er nur genau einmal erhalten wird. Wenn das letztere zutrifft, würde nämlich nicht nur $y$ durch $x$, sondern auch umgekehrt $x$ durch $y$ bestimmt, also $x$ eine Funktion von $y$ sein. Es wäre, wie man kurz sagt, die gegebene Funktion $y=f(x)$ in der Umgebung von $x_{0}$ umkehrbar (vgl. $§ 19$, Satz 6). Über die Möglichkeit solcher Umkehrung gilt nun folgender

107. OUmkehrsatz für Potenzreihen. Ist die für $x-x_{0}<r$ konvergente Entwicklung

$$
y-y_{0}=a_{1}\left(x-x_{0}\right)+a_{2}\left(x-x_{0}\right)^{2}+\ldots
$$

vorgelegt, so ist unter der alleinigen Voraussetzung, da $\beta a_{1} \neq 0$ ist, die hierdurch in der Umgebung von $x_{0}$ definierte Funktion $y=f(x) u m$. kehrbar, d. h. es gibt eine und nur eine Funktion $x=\varphi(y)$, die durch eine in einer gewissen Umgebung von $y_{0}$ konvergente Potenzreihe der Form

$$
x-x_{0}=b_{1}\left(y-y_{0}\right)+b_{2}\left(y-y_{0}\right)^{2}+\ldots
$$

darstellbar ist und für die dort (im Sinne von 104)

ist.

$$
f(\varphi(y))-y
$$

1) Die Numerierung der Bernoullischen Zahlen ist häufig eine etwas andre, indem $B_{0}, B_{1}, B_{3}, B_{5}, B_{7}, \ldots$ gar nicht bezeichnet werden und statt $B_{2 k}, k=1,2, \ldots$, nun $(-1)^{k-1} B_{k}$ gesetzt wird. Eine Tafel der Zahlen $B_{2}, B_{4}, \ldots$, bis $B_{124}$ findet man bei J. C. Adams. Journ. f. d. reine u. angew. Math., Bd. 85 (1878). Es sei beiläufig erwähnt, daf3 $B_{120}$ einen 113 -stelligen Zähler und den Nenner 2328255930 hat; $B_{12: 2}$ hat den Nenner 6 und einen 107-stelligen Zähler. - Die Zahlen $B_{2}, B_{4}, \ldots$ bis $B_{62}$ hatte schon Ohm, ibid., Bd. 20 (1840), S. 11 berechnet. - Die Zahlen $B_{p}$ treten zuerst bei Jak. Bernoulli, Ars conjectandi, 1713, S. 96 auf. - Eine zusammenfassende Darstellung gibt L. Saalschütz in seinen „Vorlesungen über die Bernoullischen Zahlen", Berlin (J. Springer) 1893. Neuere Untersuchungen, die besonders den arithmetischen Teil der Theorie beriicksichtigen, gibt G. Frobenius, Sitzgsber. d. Berl. Ak., 1910 , S. $809-847$. 
Beweis. Wie schon öfter nehmen wir - was keine Einschränkung bedeutet, beim Beweise an, daß $x_{0}$ und $y_{0}$ gleich $\left.=0 \operatorname{sind}^{1}\right)$. Dann aber wollen wir auch annehmen, daß $a_{1}=1$ ist, daß also die Entwicklung

$$
y=x+a_{2} x^{2}+a_{3} x^{3}+\cdots
$$

zur Umkehrung vorgelegt ist. Auch das bedeutet keine Einschränkung; denn da $a_{1} \neq 0$ sein sollte, können wir $a_{1} x+a_{2} x^{2}+\ldots$ in der Form

$$
\left(a_{1} x\right)+\frac{a_{2}}{a_{1}{ }^{2}}\left(a_{1} x\right)^{2}+\frac{a_{3}}{a_{1}{ }^{2}}\left(a_{1} x\right)^{3}+\cdots
$$

schreiben. Setzen wir dann zur Abkürzung $a_{1} x=x^{\prime}$ und für $n \geqq 2$

$$
\frac{a_{n}}{a_{1}{ }^{n}}=a_{n}^{\prime}
$$

und lassen nachträglich der Einfachheit halber die Akzente weg, so bekommen wir gerade die obige Entwicklungsform. Es genügt also diese zu behandeln. Dann können wir aber zeigen, daß eine in einem gewissen Intervall konvergente Potenzreihe der Form

$$
x=y+b_{2} y^{2}+b_{3} y^{3}+\ldots
$$

existiert, die die Umkehrung der vorigen bildet, für die also

(c) $\left(y+b_{2} y^{2}+\ldots\right)+a_{2}\left(y+b_{2} y^{2}+\ldots\right)^{2}+a_{3}\left(y+b_{2} y^{2}+\ldots\right)^{3}+\ldots$ identisch $=y$ ist, wenn diese Reihe gemäß 104 nach Potenzen von $y$ geordnet wird, - so da $B$ also alle Koeffizienten $=0$ sein müssen außer dem von $y^{1}$, welcher $=1$ ist.

Nehmen wir für den Augenblick die Richtigkeit dieser Behauptung an, so sind die Koeffizienten $b_{v}$ ganz eindcutig durch diese Bedingung bestimmt, daß nach der Umordnung der Reihe (c) die Koeffizienten von $y^{2}, y^{3}, \ldots$ alle $=0$ sein müssen. In der Tat liefert diese Forderung die Gleichungen

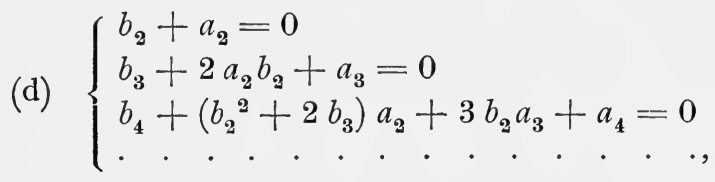

aus denen, wie unmittelbar zu sehen, der Reihe nach die Koeffizienten $b_{v}$ eindeutig berechnet werden können. So erhält man zunächst die Werte

$$
\text { (e) }\left\{\begin{array}{l}
b_{2}=-a_{2} \\
b_{3}=-2 b_{2} a_{2}-a_{3}=2 a_{2}{ }^{2}-a_{3} \\
b_{4}=-\left(b_{2}{ }^{2}+2 b_{3}\right) a_{2}-3 b_{2} a_{3}-a_{4} \\
b_{5}=\ldots \\
. . . . . . . . . . .
\end{array}\right.
$$

- eine Rechnung, die indes sehr bald undurchsichtig wird. Doch zeigt der Ansatz dieser Rechnung jedenfalls, daß, wenn es überhaupt

1) Oder: Wir setzen zur Abkïrzung $x-x_{0}=x^{\prime}$ und $y-y_{0}=y^{\prime}$ und lassen dann der Einfachheit halber die Akzente weg.

Kn op p, Unendliche Reihen. 
eine in eine Potenzreihe entwickelbare Umkehrung $x=\varphi(y)$ der Funktion $y=f(x)$ gibt, es nur eine einzige geben kann.

Diese soeben angedeutete Rechnung liefert nun aber, wie auch die Ausgangsreihe (a) gegeben sein mag, stets wohlbestimmte Werte $b_{\nu}$, so da $B$ man also stets eine Potenzreihe $y+b_{2} y^{2}+\ldots$ erhält, welche wenigstens formal den Bedingungen des Problems genügt, für die also die Reihe (c) identisch $=y$ wird. Fraglich bleibt nur, ob die Potenzreihe auch konvergiert. Können wir auch das nachweisen, so wäre die Umkehrung vollständig geleistet.

Dieser Konvergenzbeweis läßt sich nun, wie Cauchy zuerst gezeigt hat, in der Tat ganz allgemein folgendermaßen erbringen: Wählt man irgendwelche positive Zahlen $\alpha_{v}$, für die stets

$$
\left|a_{v}\right| \leqq \alpha_{v}
$$

ist, und macht genau den Ansatz, wie eben, für die Reihe

deren Umkehrung dann

$$
y=x-\alpha_{2} x^{2}-\alpha_{3} x^{3}-\ldots
$$

$$
\dot{x}=y+\beta_{2} y^{2}+\beta_{3} y^{3}+\ldots
$$

sein möge, so erhält man für die $\beta_{\nu}$, ganz entsprechend wie oben, die Gleichungen

$$
\begin{aligned}
& \beta_{2}=\alpha_{2} \\
& \beta_{3}=2 \beta_{2} \alpha_{2}+\alpha_{3} \\
& \beta_{4}=\left(\beta_{2}^{2}+2 \beta_{3}\right) \alpha_{2}+2 \beta_{2} \alpha_{3}+\alpha_{4}
\end{aligned}
$$

bei denen nun alle Summanden positiv sind. Es ist daher stets

$$
\beta_{\nu} \geqq\left|b_{v}\right| \text {. }
$$

Könnte man also die $\alpha_{v}$ noch so wählen, daß die Reihe $\sum \beta_{v} y^{v}$ konvergent wird, so würde daraus auch die Konvergenz der Reihe $\Sigma b_{v} y^{\nu}$ folgen und der Beweis wäre vollendet.

Die $\alpha_{v}$ wählen wir nun so: Es gibt jedenfalls eine positive Zahl $\varrho$, für die die Ausgangsreihe $x+a_{2} x^{2}+\ldots$ 'absolut konvergiert. Dann muß es aber (nach $\mathbf{8 2}, 6$ und 10, 11) eine positive Zahl $K$ geben, so daß für $\boldsymbol{v}=2,3, \ldots$ stets

$$
\left|a_{v}\right| \varrho^{v} \leqq K \quad \text { oder }\left|a_{\nu}\right| \leqq \frac{K}{\varrho^{v}}
$$

bleibt. Daraufhin setzen wir für $\boldsymbol{v}=2,3, \ldots$

$$
\alpha_{\nu}=\frac{K}{\varrho^{v}},
$$

so da $B$ es sich um die Umkehrung der für $|x|<\varrho$ konvergenten Reihe

$$
y=x-K \cdot \frac{x^{2}}{\varrho^{2}} \cdot\left(1+\frac{x}{\varrho}+\frac{x^{2}}{\varrho^{2}}+\ldots\right)=x-\frac{K \cdot x^{2}}{\varrho(\varrho-x)}
$$

handeln würde. Diese Funktion kann man aber ganz unmittelbar umkehren, denn aus

$$
y=x-\frac{K x^{2}}{\varrho(\varrho-x)} \text { oder }(K+\varrho) x^{2}-\varrho(\varrho+y) x+\varrho^{2} y=0
$$


folgt

$$
x=\frac{\varrho(\varrho+y) \pm \varrho \sqrt{(\varrho+y)^{2}-4 y(K+\varrho)}}{2(K+\varrho)} .
$$

Da aber die Werte $x=0$ und $y=0$ zusammengehören und kleinen Werten der einen Veränderlichen auch kleine Werte der andern entsprechen müssen, so haben wir hier vor der Quadratwurzel das + -Zeichen zu verwerfen, so daß völlig eindeutig

$$
x=\frac{\varrho}{2(K+\varrho)}\left[\varrho+y-\sqrt{y^{2}-2(2 K+\varrho) y+\varrho^{2}}\right]
$$

sich ergibt. Nun ist weiter

$$
y^{2}-2(2 K+\varrho) y+\varrho^{2} \equiv(y-A)(y-B),
$$

wenn zur Abkürzung

$$
\begin{aligned}
& A=2 K+\varrho+2 \sqrt{K(K+\varrho)} \\
& B=2 K+\varrho-2 \sqrt{K(\overline{K+\varrho)}}
\end{aligned}
$$

gesetzt wird, - zwei Größen, die beide $>0$ sind, weil es die erste ist und weil ihr Produkt $=\varrho^{2}$ ist. Dann ist aber

$$
x=\frac{\varrho^{2}}{2(K+\varrho)}\left[1+\frac{y}{\varrho}-\left(1-\frac{y}{A}\right)^{\frac{1}{2}} \cdot\left(1-\frac{y}{B}\right)^{\frac{1}{2}}\right] .
$$

Im nächsten Kapitel werden wir nun lernen, daß für $|z|<1$ die Potenz $(1-z)^{\frac{1}{2}}$ tatsächlich in eine Potenzreihe - beginnend mit $1-\frac{z}{2}+\ldots-$ entwickelt werden kann. Indem wir dieses Resultat vorwegnehmen, ergibt sich nun unmittelbar, daß auch $x$ in eine mindestens für $|y|<B$ konvergente Potenzreihe

$$
\begin{aligned}
x & =\frac{\varrho^{2}}{2(K+\varrho)}\left[1+\frac{y}{\varrho}-\left(1-\frac{y}{2 A}+\ldots\right)\left(1-\frac{y}{2 B}+\ldots\right)\right] \\
& =y+\beta_{2} y^{2}+\ldots
\end{aligned}
$$

entwickelt werden kann. Nach den vorausgegangenen Bemerkungen ist hiermit aber der Beweis in allen Teilen vollendet.

Die tatsächliche Herstellung der Reihe $y+b_{2} y^{2}+\ldots$ aus der Reihe $x+a_{2} x^{2}+\ldots$ ist auch hier meist mit erheblichen Schwierigkeiten verknüpft und erfordert von Fall zu Fall besondere Hilfsmittel ${ }^{1}$ ). Beispiele hierfür werden in den $\S \S 26$ und 27 auftreten.

\section{Aufgaben zum V. Kapitel.}

64. Man bestimme den Konvergenzradius der Potenzreihe $\Sigma a_{n} x^{n}$, wenn die Koeffizienten $a_{n}$ von einer Stelle an einen der in Aufgabe 34 oder 45 angegebenen Werte hat.

1) Die allgemeinen Werte der Entwicklungskoeffizienten $b_{n}$ findet man bis zu $b_{13}$ ausgerechnet bei C. E. van Orstrand, Reversion of power series, Philos. Magazine, (6) Bd. 19 (1910), S. 366. 
65. Man bestimme die Radien der Potenzreihen

$$
\begin{gathered}
\sum \vartheta^{n^{2}} \cdot x^{n}, \quad 0<\vartheta<1 ; \quad \sum\left(\frac{1 \cdot 2 \ldots n}{3 \cdot 5 \ldots(2 n+1)}\right)^{2} x^{n} \\
\sum \frac{n !}{n^{n}} x^{n}, \quad \sum \frac{n !}{a^{n^{2}}} x^{n}, \quad a>1 ; \quad \sum \frac{(n !)^{3}}{(3 n) !} x^{n} .
\end{gathered}
$$

66. Werden die Häufungsgrenzen von $\left|\frac{a_{n}}{a_{n+1}}\right|$ mit $x$ und $\mu$ b zeichnet, so gilt für den Radius $r$ der Potenzreihe $\Sigma a_{n} x^{n}$ stets die Beziehung $\varkappa \leqq r \leqq \mu$. Im besonderen: Wenn $\lim \left|\frac{a_{n}}{a_{n+1}}\right|$ existiert, so liefert sein Wert den Radius von $\Sigma a_{n} x^{n}$.

6\%. $\Sigma a_{n} x^{n}$ habe den Radius $r, \Sigma a_{n}^{\prime} x^{n}$ den Radius $r^{\prime}$. Was läßst sich über den Radius der Potenzreihen

$$
\sum\left(a_{n} \pm a_{n}{ }^{\prime}\right) x^{n}, \quad \sum a_{n} a_{n}{ }^{\prime} x^{n}, \quad \sum \frac{a_{n}}{a_{n}{ }^{\prime}} x^{n}
$$

aussagen?

67. Welchen Radius hat $\Sigma a_{n} x^{n}$, falls $0<\overline{\lim }\left|a_{n}\right|<+\infty$ ?

68. Die Potenzreihe $\sum_{n=2}^{\infty} \frac{\varepsilon_{n}}{n \log n} x^{n}$, in der $\varepsilon_{n}$ die Bedeutung aus Aufgabe 47 hat, konvergiert an beiden Enden des Konvergenzintervalles, aber in beiden nur bedingt.

69. Beweise im Anschlufs an 97, Beispiel 3, daf

$$
\sum_{v=0}^{n}\left(\begin{array}{c}
n \\
v
\end{array}\right)^{2}=(-1)^{n} \sum_{v=0}^{2 n}(-1)^{v}\left(\begin{array}{c}
2 n \\
v
\end{array}\right)^{2}=\left(\begin{array}{c}
2 n \\
n
\end{array}\right)
$$

ist.

70. In Ergänzung des Abelschen Grenzwertsatzes 100 läft sich zeigen, dafs in jedem Falle, wenn nur $\sum a_{n} x^{n}$ inen Radius $r \geq 1$ hat,

ist. $\left(s_{n}=a_{0}+a_{1}+\cdots+a_{n}\right)$.

$$
\underline{\lim } s_{n} \leqq \varlimsup_{x \rightarrow 1-0}\left(\sum_{n=0}^{\infty} a_{n} x^{n}\right) \leqq \varlimsup \lim s_{n}
$$

71. Die im allgemeinen nicht erlaubte Umkehrung des Abelschen Grenzwertsatzes 100 ist dennoch gestattet, wenn die Koeffizienten $a_{n} \geq 0$ sind; wenn dann

$$
\lim _{x \rightarrow r-0} \sum a_{n} x^{n}
$$

existiert, so ist also $\sum a_{n} \gamma^{n}$ konvergent mit demselben Werte.

72. Es sei

$$
\sum_{n=1}^{\infty} a_{n} x^{n}=f(x) \quad \text { und } \quad \sum_{n \equiv 1}^{\infty} b_{n} x^{n}=g(x)
$$

und beide Reihen für $|x|<\varrho$ konvergent. Dann ist (für welche $x$ ?)

$$
\sum_{n=1}^{\infty} b_{n} f\left(x^{n}\right)=\sum_{n=1}^{\infty} a_{n} g\left(x^{n}\right)
$$

(Die Spezialisierung der Kotffizienten ergibt viele interessante Identitäten. Man setze etwa $b_{n} \equiv 1,(-1)^{n-1}, \frac{1}{n}$ usw.).

73. Wie beginnen die durch Division gewonnenen Potenzreihen für

$$
\frac{1}{1-\frac{x^{2}}{2 !}+\frac{x^{4}}{4 !}-+\ldots}, \frac{1}{1+\frac{x}{2}+\frac{x^{2}}{3}+\ldots}
$$

(Weitere Aufgaben beim nächsten Kapitel.) 
VI. Kapitel.

\section{Die Entwicklungen der sog. elementaren Funktionen.}

Mit den Sätzen der vorigen beiden Paragraphen haben wir nun das Rüstzeug in Händen, mit dessen Hilfe wir eine große Zahl von Reihen nach jeder Richtung hin beherrschen. Dies wollen wir jetzt an den wichtigsten Beispielen ausführen.

Eine gewisse - übrigens ziemlich kleine - Zahl von Potenzreihen bzw. die. durch sie dargestellten Funktionen haben vor allen anderen eine große Bedeutung für die gesamte Analysis, und man bezeichnet sie darum gern als die elementaren Funktionen. Mit diesen wollen wir uns vorerst beschäftigen.

\section{$\S$ 22. Die rationalen Funktionen.}

Aus der geometrischen Reihe

$$
1+x+x^{2}+\ldots=\sum_{n=0}^{\infty} x^{n}=\frac{1}{1-x},
$$

die den Grundstock für viele der folgenden speziellen Untersuchungen bildet, findet man durch wiederholte Differentiationen nach 98, 4 der Reihe nach

$$
\sum_{n=0}^{\infty}(n+1) x^{n}=\frac{1}{(1-x)^{2}}, \quad \sum_{n=0}^{\infty}\left(\begin{array}{c}
n+2 \\
2
\end{array}\right) x^{n}=\frac{1}{(1-x)^{3}}, \ldots
$$

und allgemein für jede natürliche Zahl $p$

$$
\sum_{n=0}^{\infty}\left(\begin{array}{c}
n+p \\
p
\end{array}\right) \boldsymbol{x}^{n}=\frac{1}{(1-x)^{p+1}}, \quad|x|<1.108 .
$$

Multipliziert man nach 91 diese Gleichung noch einmal mit $\Sigma x^{n}=\frac{1}{1-x}$, so ergibt sich nach $\mathbf{9 1}$ und $\mathbf{1 0 S}$

$$
\sum_{n=0}^{\infty}\left[\left(\begin{array}{c}
p \\
p
\end{array}\right)+\left(\begin{array}{c}
p+1 \\
p
\end{array}\right)+\ldots+\left(\begin{array}{c}
p+n \\
p
\end{array}\right)\right] x^{n}=\sum_{n=0}^{\infty}\left(\begin{array}{c}
n+p+1 \\
p+1
\end{array}\right) x^{n}
$$

Durch Vergleich der Koeffizienten (nach 97) schlieft man hieraus, dafi

$$
\left(\begin{array}{c}
p \\
p
\end{array}\right)+\left(\begin{array}{c}
p+1 \\
p
\end{array}\right)+\ldots+\left(\begin{array}{c}
p+n \\
p
\end{array}\right)=\left(\begin{array}{c}
n+p+1 \\
p+1
\end{array}\right)
$$

sein muf, was man natürlich auch ganz leicht direkt (durch Induktion) beweist. Tut man dies, so kann man die Gleichung 108 auch durch mehrmaliges Multiplizieren von $\Sigma x^{n}=\frac{1}{1-x}$ mit sich selbst nach 103 herleiten.

Da

$$
\left(\begin{array}{c}
n+p \\
p
\end{array}\right)=\left(\begin{array}{c}
n+p \\
n
\end{array}\right)=(-1)^{n}\left(\begin{array}{c}
-p-1 \\
n
\end{array}\right)
$$


ist, so erhält man aus 108, wenn man dort noch $x$ durch $-x$ und $p+1$ durch $-k$ ersetzt, die Formel

109.

$$
(1+x)^{k}=\sum_{n=0}^{\infty}\left(\begin{array}{l}
k \\
n
\end{array}\right) x^{n}
$$

gültig für $|x|<1$ und negativ-ganzzahliges $k$. Diese Formel ist ersichtlich eine Erweiterung des binomischen Lehrsatzes $(\mathbf{2 9}, 4)$ auf negativ-ganzzahlige Exponenten; denn diesen Lehrsatz darf man auch für positiv-ganzzahlige $k$ (oder $k=0$ ) in der Form 109 schreiben, da dann für $n>k$ die Glieder der Reihe doch alle $=0$ sind.

Formeln wie die eben abgeleiteten haben - wir wollen dies gleich ein für allemal betonen - zweierlei Bedeutung: lesen wir sie von links nach rechts, so geben sie die Entwicklung oder Darstellung einer Funktion durch eine Potenzreihe, lesen wir sie von rechts nach links, so geben sie uns die Summe einer unendlichen Reihe in geschlossener Form. Je nach Lage des Falles kann die eine oder die andere Bedeutung im Vordergrund stehen.

Mit Hilfe dieser einfachen Formeln wird es oft gelingen, eine beliebig vorgelegte rationale Funktion

$$
f(x)=\frac{a_{0}+a_{1} x+\ldots+a_{m} x^{m}}{b_{0}+b_{1} x+\ldots+b_{k} x^{k}}
$$

in eine Potenzreihe zu entwickeln, nämlich immer dann, wenn man $f(x)$ in Partialbrüche, d. h. in eine Summe von Brüchen der Form

$$
\frac{A}{(x-a)^{p}}
$$

zu zerlegen vermag. Jeden einzelnen dieser Brüche wird man nach 108 durch eine Potenzreihe darstellen können, und somit auch die gegebene Funktion. Und zwar kann diese Entwicklung für die Umgebung eines jeden Punktes $x_{0}$ angesetzt werden, der von $a$ verschieden ist. Dazu hat man nur

$$
\left(\frac{1}{x-a}\right)^{p}=\frac{1}{\left(x_{0}-a\right)^{p}} \cdot\left(\frac{1}{1-\left(\frac{x-x_{0}}{a-x_{0}}\right)}\right)^{p}
$$

zu setzen und den letzten Bruch nach $\mathbf{1 0 8}$ zu entwickeln. Hierdurch erkennt man zugleich, daß die Entwicklung für $\left|x-x_{0}\right|<\left|a-x_{0}\right|$ konvergieren wird, und nur für diese Werte von $x$.

Prinzipielle Bedeutung gewinnt diese Methode allerdings erst bei Benutzung komplexer Größen.

110. Beispiele.
1. $\sum_{n=0}^{\infty} \frac{1}{2^{n}}=2$
2. $\sum_{n=0}^{\infty} \frac{n+1}{2^{n}}=4$
3. $\sum_{n=0}^{\infty} \frac{(n+1)(n+2)}{2^{n+1}}=8$
4. $\sum_{n=0}^{\infty}\left(\begin{array}{c}n+p \\ p\end{array}\right) \cdot\left(\frac{2}{3}\right)^{n}=3^{n+1}$ 


\section{$\$ 23$. Die Exponentialfunktion.}

1. Neben der geometrischen Reihe spielt vor allem die sogenannte Exponentialreihe

$$
\sum_{n=0}^{\infty} \frac{x^{n}}{n !} \equiv 1+x+\frac{x^{2}}{2 !}+\frac{x^{3}}{3 !}+\cdots+\frac{x^{n}}{n !}+\ldots
$$

weiterhin eine grundlegende Rolle. Mit der durch sie dargestellten Funktion wollen wir uns jetzt näher beschäftigen, Wir bezeichnen diese sog. Exponentialfunktion vorläufig mit $E(x)$. Da die Reihe beständig konvergiert, ist $E(x)$ nach 98 jedenfalls eine für alle $x$ definierte, stetige und beliebig oft differenzierbare Funktion. Für ihre Ableitung findet man unmittelbar

$$
E^{\prime}(x)=E(x),
$$

so daß auch alle höheren Ableitungen

sein müssen.

$$
E^{(v)}(x)=E(x)
$$

Wir wollen versuchen, allein aus der Reihe heraus ihre weiteren Eigenschaften abzulesen. In 91, 3 haben wir schon gezeigt, daß für irgend zwei reelle Zahlen $x_{1}$ und $x_{2}$ stets

$$
\boldsymbol{E}\left(x_{1}+x_{2}\right)=\boldsymbol{E}\left(x_{1}\right) \cdot \boldsymbol{E}\left(x_{2}\right)
$$

ist. Diese grundlegende Formel bezeichnet man kurz als das Additionstheorem der Exponentialfunklion $\left.{ }^{\mathbf{1}}\right)$. Nach ihm ist weiter

$$
E\left(x_{1}+x_{2}+x_{3}\right)=E\left(x_{1}+x_{2}\right) \cdot E\left(x_{3}\right)=E\left(x_{1}\right) \cdot E\left(x_{2}\right) \cdot E\left(x_{3}\right) ;
$$

und durch Wiederholung dieses Schlusses findet man, daß für irgendeine Anzahl reeller Zahlen $x_{1}, x_{2}, \ldots, x_{k}$ stets

(b) $\quad E\left(x_{1}+x_{2}+\ldots+x_{k}\right)=E\left(x_{1}\right) \cdot E\left(x_{2}\right) \ldots E\left(x_{k}\right)$

ist. Setzt man hierin alle $x_{v}=1$, so findet man speziell, daß die Gleichung *

$$
E(k)=[E(1)]^{k}
$$

für jede natürliche Zahl $k$ richtig ist. $\mathrm{Da} E(0)=1$, gilt sie auch für $k=0$. Setzt man jetzt in (b) alle $x_{\nu}=\frac{m}{k}$, unter $m$ eine zweite ganze $Z$ ahl $\geqq 0$ verstanden, so folgt, daß

$$
E\left(k \cdot \frac{m}{k}\right)=\left[E\left(\frac{m}{k}\right)\right]^{k}
$$

1) 2. Beweis. Die Taylorsche Reihe $\mathbf{9 9}$ für $E(x)$ lautet: $E(x)=E\left(x_{1}\right.$ $+\frac{E^{\prime}\left(x_{1}\right)}{1 !}\left(x-x_{1}\right)+\ldots$, gültig für alle Werte von $x$ und $x_{1}$. Beachtet man, dás $E^{(v)}\left(x_{1}\right)=E\left(x_{1}\right)$ ist, so folgt, wenn man noch $x$ durch $x_{1}+x_{2}$ ersetzt, unmittelbar $E\left(x_{1}+x_{2}\right)=E\left(x_{1}\right) \cdot\left[1+\frac{x_{2}}{1 !}+\frac{x_{2}^{2}}{2 !}+\cdots\right]=E\left(x_{1}\right) \cdot E\left(x_{2}\right), \quad$ w. z. b. w. 
oder also - wegen $E(m)=[E(1)]^{m}-\mathrm{da} B$

$$
E\left(\frac{m}{k}\right)=[E(\mathbf{1})]^{\frac{m}{k}} \text {. }
$$

Setzen wir noch zur Abkürzung $E(1)=E$, so ist bewiesen, daß die Gleichung

$$
E(x)=E^{x}
$$

für jedes rationale $x \geqq 0$ richtig ist.

Ist dann $\xi$ irgendeine positive irrationale Zahl, so können wir auf mannigfache Weise eine gegen $\xi$ konvergierende Folge $\left(x_{n}\right)$ mit positiven rationalen Gliedern bilden. Für jedes $n$ ist dann nach dem eben bewiesenen

$$
E\left(x_{n}\right)=E^{x_{n}} \text {. }
$$

Für $n \rightarrow+\infty$ strebt hierin die linke Seite (nach 98,2) gegen $E(\xi)$, die rechte Seite (nach $\mathbf{4 2}, 1$ ) gegen $E^{\xi}$, so daß wir das Ergebnis

$$
E(\xi)=E^{\xi}
$$

erhalten. Damit ist die Gleichung (c) für jedes reelle $x \geqq 0$ bewiesen.

Endlich ist aber nach (a)

$$
E(-x) \cdot E(x)=E(x-x)=E(0)=1,
$$

woraus zunächst folgt, daß für kein $x \geqq 0$ etwa $E(x)=0$ sein $\operatorname{kann}^{1}$ ), und $\mathrm{da} B$

$$
E(-x)=\frac{1}{E(x)}=\frac{1}{E^{x}}=E^{-x}
$$

ist. Dies bedeutet aber, daß auch für jedes negativ-reelle $x$ die Gleichung (c) besteht.

Damit ist bewiesen, daß diese Gleichung für alle reellen $x$ besteht; und zugleich hat damit die Funktion $E(x)$ ihren Namen als Exponentialfunktion gerechtfertigt: $E(x)$ ist die $x^{\text {te }}$ Potenz der festen Grundzahl

$$
E=E(1)=1+\frac{1}{1 !}+\frac{1}{2 !}+\frac{1}{3 !}+\ldots+\frac{r^{\prime} 1}{n !}+\cdots
$$

2. Es wird sich nun weiter darum handeln, über diese Grundzahl noch Näheres zu erfahren. Wir wollen zeigen, daß sie mit der schon in 46, 4 angetroffenen Zahl $e$ identisch, daß also

$$
\lim _{\Gamma n \rightarrow \infty}\left(1+\frac{1}{n}\right)^{n}=\sum_{\nu=0}^{\infty} \frac{1}{\nu !}
$$

ist $^{2}$ ). Wir führen den Beweis etwas umfassender, indem wir in Ergänzung der Untersuchung von $\mathbf{4 6}, 4$ sogleich den folgenden Satz beweisen:

1) Das ist natiirlich auch unmittelbar aus der Reihe abzulesen, die ja für $x \geq 0$ eine Reihe mit positiven Gliedern ist, deren 0 tes gleich 1 ist.

$\left.{ }^{2}\right)$ Es handelt sich hier also um ein prägnantes Beispiel zu Problem B. Vgl. die Einleitung zu § 9 . 
- Satz. Für jedes reelle $x$ existiert

$\lim _{n \rightarrow \infty}\left(1+\frac{x}{n}\right)^{n}$ und ist gleich der Summe der Reihe $\left.\sum_{\nu=0}^{\infty} \frac{x^{v}}{v !} \mathbf{1}\right)$.

Beweis. Wir setzen zur Abkürzung

$$
\left(1+\frac{x}{n}\right)^{n}=x_{n} \quad \text { und } \quad \sum_{\nu=0}^{n} \frac{x^{\nu}}{\nu !}=s_{n} .
$$

Dann genügt es, zu zeigen, daß $\left(s_{n}-x_{n}\right) \rightarrow 0$ strebt. Ist aber, nachdem für $x$ eine bestimmte Zahl gewählt ist, $\varepsilon>0$ gegeben, so können wir zunächst $p$ so groß nehmen, daß der Rest

$$
\frac{|x|^{p+1}}{(p+1) !}+\frac{|x|^{p+2}}{(p+2) !}+\ldots<\frac{\varepsilon}{2}
$$

ausfällt. Weiter ist für $n>2$

$$
\begin{gathered}
x_{n}=1+\left(\begin{array}{c}
n \\
1
\end{array}\right) \frac{x}{1}+\ldots+\left(\begin{array}{l}
n \\
k
\end{array}\right) \cdot \frac{x^{k}}{n^{k}}+\ldots+\left(\begin{array}{c}
n \\
n
\end{array}\right) \frac{x^{n}}{n^{n}} \\
=1+x+\frac{1}{2 !}\left(1-\frac{1}{n}\right) x^{2}+\ldots+\frac{1}{k !}\left[\left(1-\frac{1}{n}\right)\left(1-\frac{2}{n}\right) \ldots\left(1-\frac{k-1}{n}\right)\right] x^{k}+ \\
\ldots+\frac{1}{n !}\left[\left(1-\frac{1}{n}\right) \ldots\left(1-\frac{n-1}{n}\right)\right] x^{n} .
\end{gathered}
$$

Hier ist das $k^{\text {te }}$ Glied ersichtlich absolut genommen kleiner als das entsprechende Glied der Exponentialreihe, d. h.

$$
\left|\frac{1}{k !}\left[\left(1-\frac{1}{n}\right) \ldots\left(1-\frac{k-1}{n}\right)\right] x^{k}\right| \leqq \frac{\left|x^{k}\right|}{k !} .
$$

Folglich ist für $\left.n>p^{2}\right)$ - nach der Art wie wir $p$ gewählt haben -

$$
\begin{aligned}
\left|x_{n}-s_{n}\right| \leqq & \frac{1}{2 !}\left[1-\left(1-\frac{1}{n}\right)\right] \cdot|x|^{2}+\ldots \\
& \ldots+\frac{1}{p !}\left[1-\left(1-\frac{1}{n}\right) \ldots\left(1-\frac{p-1}{n}\right)\right] \cdot|x|^{p}+\frac{\varepsilon}{2} .
\end{aligned}
$$

Jeder einzelne der $(p-1)$ ersten Summanden rechter Hand ist nun ersichtlich Glied einer Nullfolge ${ }^{3}$ ); also strebt auch deren Summe -

1) Zuerst - wenn auch nicht in ganz einwandfreier Weise - von Euler (Introductio in analysin infinitorum, Lausanne 1748, S. 86) bewiesen. - Die Exponentialreihe und ihre Summe $e^{x}$ kannten schon Newton (1669) und Leibniz (1676).

2) Man denke sich von vornherein $p>2$ genommen.

3) Es strebt $\left(1-\frac{1}{n}\right) \rightarrow 1,\left(1-\frac{2}{n}\right) \rightarrow 1, \ldots,\left(1-\frac{p-1}{n}\right) \rightarrow 1$, also strebt auch (nach 41, 10) ihr Produkt $\rightarrow 1$, folglich $\left[1-\left(1-\frac{1}{n}\right) \ldots\left(1-\frac{p-1}{n}\right)\right] \rightarrow 0$ und ebenso strebt auch, da $x$ und $p$ feste Zahlen sind, das Produkt dieses letzten Ausdrucks mit $\frac{1}{p !}|x|^{p}$ gegen 0, - und entsprechend für die andern Summanden. - Auch kann man unmittelbar nach 41, 12 schliefien. 
VI. Kap. Die Entwicklungen der sog. elementaren Funktionen.

denn $p$ ist eine feste Zahl - gegen 0 , und wir können $n_{0}>p$ so wählen, daß diese Summe für $n>n_{0}$ auch $<\frac{\varepsilon}{2}$ bleibt. Dann ist aber für alle $n>n_{0}$

$$
\left|x_{n}-s_{n}\right|<\varepsilon
$$

womit unsere Behauptung bewiesen ist. - Für $x=1$ folgt ganz speziell, daß

$$
E=\sum_{v=0}^{\infty} \frac{1}{v !}=\lim _{n \rightarrow \infty}\left(1+\frac{1}{n}\right)^{n}=e
$$

ist; und allgemeiner ist für jedes reelle $x$

$$
\boldsymbol{E}(x)=\sum_{\nu=0}^{\infty} \frac{x^{\nu}}{\nu !}=\boldsymbol{e}^{x} .
$$

Die hiermit gewonnene neue Darstellung der Zahl $e$ durch die Exponentialreihe ist für die weitere Untersuchung dieser Zahl sehr viel handlicher. Zunächst kann man mit ihrer Hilfe nun leicht einen guten Annäherungswert der Zahl $e$ erhalten. Denn es ist, da alle Glieder der Reihe positiv sind, offenbar für jedes $n$

oder

$$
s_{n}<e<s_{n}+\frac{1}{(n+1) !}+\frac{1}{(n+1) !(n+1)}+\frac{1}{(n+1) !(n+1)^{2}}+\ldots
$$

$$
\begin{gathered}
s_{n}<e<s_{n}+\frac{1}{(n+1) !} \cdot \frac{n+1}{n} \\
s_{n}<e<s_{n}+\frac{1}{n ! n} .
\end{gathered}
$$

Rechnet man diese einfachen Werte z. B. für $n=9$ aus, so findet man

$$
2,718281<e<2,718282,
$$

- was schon eine gute Vorstellung von dem Werte der Zahl $e$ gibt $^{1}$ ). - Aus der Formel (a) kann man aber noch weitere wichtige Schlüsse ziehen. Eine Zahl hat man nur dann vollständig vor sich, wenn sie rational ist und in der Form $\frac{p}{q}$ geschrieben wird. Ist die Zahl e vielleicht rational? Die Ungleichungen (a) lehren ganz leicht, daß dem leider nicht so ist. Wäre nämlich $e=\frac{p}{q}$, so lieferte die Formel (a) für $n=q$ :

$$
s_{q}<\frac{p}{q}<s_{q}+\frac{1}{q ! q}
$$

mit $s_{q}=2+\frac{1}{2 !}+\ldots+\frac{1}{q !}$. Multiplizieren wir nun diese Unglei-

1) Die Zahl $e$ ist von J.M. Boormann auf 346 Dezimalen berechnet worden (Math. magazine, Bd. 1, Nr.12, 1884, S.204). Der Nutzen solcher langwierigen Rechnungen ist gering. 
chung mit $q$ !, so wird $q$ ! $s_{g}$ offenbar eine ganze Zahl, die wir für den Augenblick mit $g$ bezeichnen wollen, und es folgte

$$
g<p \cdot(q-1) !<g+\frac{1}{q} \leqq g+1,
$$

- und dies mit Ausschluß der beiderseitigen Gleichheit. Das ist aber unmöglich; denn zwischen den beiden aufeinanderfolgenden ganzen Zahlen $g$ und $g+1$ kann nicht eine von beiden verschiedene wieder ganze Zahl $p \cdot(q-1)$ ! gelegen sein: Die Zahl $e$ ist irrational.

3. Obgleich durch die vorangegangenen Untersuchungen über den Grenzwert von $\left(1+\frac{x}{n}\right)^{n}$ alles bekannt geworden ist, was man zunächst wissen will - die beiden Probleme $A$ und $B(\S 9)$ sind befriedigend erledigt - wollen wir wegen der grundlegenden Bedeutung dieser Dinge denselben Grenzwert noch einmal und auf andere Art - ganz unabhängig vom Vorangehenden - bestimmen.

Von früher her wissen wir nur, $\operatorname{da}\left(1+\frac{1}{n}\right)^{n} \rightarrow e$ strebt. Wir wollen dies zunächst dahin verallgemeinern, daß wir zeigen, daß auch

$$
\left(1+\frac{1}{y_{n}}\right)^{y_{n}} \rightarrow e
$$

strebt, wenn $\left(y_{n}\right)$ irgendeine gegen $+\infty$ strebende Folge positiver Zahlen ist. Sind hierbei die $y_{n}$ ganze positive Zahlen, so ist dies eine unmittelbare Folge des früheren Resultates ${ }^{1}$ ). Sind aber die $y_{n}$ nicht ganz, so wird es doch für jedes $n$ eine (und nur eine) ganze Zahl $k_{n}$ geben, so daß

$$
k_{n} \leqq y_{n}<k_{n}+1
$$

ist; und die Folge dieser ganzen Zahlen muß ersichtlich auch gegen $+\infty$ streben. Nun ist aber

$$
\left(1+\frac{1}{k_{n}+1}\right)^{k_{n}}<\left(1+\frac{1}{y_{n}}\right)^{y_{n}}<\left(1+\frac{1}{k_{n}}\right)^{k_{n}+1} .
$$

Und weil die $k_{n}$ ganze Zahlen sind, so strebt nach der Vorbemerkung die Folge

$$
\left(1+\frac{1}{k_{n}}\right)^{k_{n}+1}=\left(1+\frac{1}{k_{n}}\right)^{k_{n}} \cdot\left(1+\frac{1}{k_{n}}\right)
$$

ebenso wie die Folge

$$
\left(1+\frac{1}{k_{n}+1}\right)^{k_{n}}=\left(1+\frac{1}{k_{n}+1}\right)^{k_{n}+1} \cdot \frac{1}{1+\frac{1}{k_{n}+1}}
$$

gegen $e$. Nach 42,4 muß also auch

$$
\left(1+\frac{1}{y_{n}}\right)^{y_{n}} \rightarrow e
$$

1) Denn ist $\varepsilon>0$ gegeben und $n_{0}$ nach $\mathbf{4 6}, 4$ so bestimmt, daf $\left|\left(1+\frac{1}{n}\right)^{n}-e\right|<\varepsilon$ bleibt für $n>n_{0}$, so wird für $n>n_{1}$ auch $\left|\left(1+\frac{1}{y_{n}}\right)^{y_{n}}-e\right|<\varepsilon$ sein, sobald nämlich $n_{1}$ so bestimmt wird, dafi für $n>n_{1}$ stets $y_{n}>n_{0}$ ist. 
streben. - Jetzt läßt sich weiter zeigen, daß mit $y_{n}^{\prime} \rightarrow-\infty$ auch

$$
\left(1+\frac{1}{y_{n}^{\prime}}\right)^{y_{n}^{\prime}} \rightarrow e
$$

strebt, oder also, daß mit $y_{n} \rightarrow+\infty$, auch

$$
\left(1-\frac{1}{y_{n}}\right)^{-y_{n}} \rightarrow e
$$

konvergiert. Doch müssen wir uns dabei alle $y_{n}{ }^{\prime}<-1$ bzw. alle $y_{n}>1$ denken, damit die Basis der Potenz nicht negativ oder 0 wird; doch kann dies stets durch „endlich viele Änderungen“ erreicht werden. Da nun

$$
\left(1-\frac{1}{y_{n}}\right)^{-y_{n}}=\left(\frac{y_{n}}{y_{n}-1}\right)^{y_{n}}=\left(1+\frac{1}{y_{n}-1}\right)^{y_{n}-1} \cdot\left(1+\frac{1}{y_{n}-1}\right)
$$

ist und da mit $y_{n}$ auch $\left(y_{n}-1\right) \rightarrow+\infty$ strebt, so ist diese Behauptung eine unmittelbare Folge der vorangehenden.

Setzen wir $\frac{1}{y_{n}}=z_{n}$, so sagen unsere beiden Ergebnisse: Es strebt stets

$$
\left(1+z_{n}\right)^{\frac{1}{z_{n}}} \rightarrow \mathcal{e},
$$

wenn $\left(z_{n}\right)$ irgendeine Nullfolge mit lauter positiven oder lauter negativen Gliedern ist, - die im letzteren Falle nur alle $>-1$ sein sollen. Hieraus folgt nun schließlich der alles Bisherige zusammenfassende

112. Satz: Ist $\left(x_{n}\right)$ eine beliebige Nullfolge, deren Glieder aber alle von 0 verschieden und von Anfang an $>-1$ sein sollen ${ }^{1}$ ), so ist stets

$$
\left.\lim _{n \rightarrow \infty}\left(1+x_{n}\right)^{\frac{1}{x_{n}}}=e^{2}\right) .
$$

Beweis. Da alle $x_{n} \neq 0$ sind, so kann man die Folge $\left(x_{n}\right)$ in zwei Teilfolgen zerlegen, deren eine nur positive, deren andere nur negative Glieder enthält. Da für beide Teilfolgen der in Rede stehende Grenzwert, wie bewiesen, vorhanden und $=e$ ist $^{3}$ ), so ist er nach 41, 5 auch für die vorgelegte Folge vorhanden und $=e$. Nach 42, 2 kann das gewonnene Ergebnis auch in der Form

$$
\frac{\log \left(1+x_{n}\right)}{x_{n}} \rightarrow 1 \text {. }
$$

geschrieben werden, in der sie oft gebraucht werden wird.

1) Das letztere ist stets durch „endlich viele Änderungen" (vgl. 38,8) zu erreichen.

2) Cauchy, Résumé des leçons sur le calcul infinit., Paris 1823, S. 81 .

3) Bricht eine der beiden Teilfolgen ab, so können wir sie uns durch endlich viele Änderungen ganz ausgeschaltet denken. 
Nach $\S 19$, Def. 4, bedeutet dies Ergebnis auch: Es ist stets

$$
\lim _{x \rightarrow 0}(1+x)^{\frac{1}{x}}=e
$$

wenn $x$ nur von vornherein $>-1$ gehalten wird.

Aus diesen Ergebnissen folgt nun noch einmal — also völlig unabhängig von den Untersuchungen in 1. und 2. - daß

$$
\left(1+\frac{x}{n}\right)^{n} \rightarrow e^{x}
$$

strebt; denn $\left(\frac{x}{n}\right)$ ist jedenfalls eine Nullfolge $^{1}$ ), so daß nach dem letzten Satze

$$
\left(1+\frac{x}{n}\right)^{\frac{n}{x}} \rightarrow e \quad \text { und also } \quad\left(1+\frac{x}{n}\right)^{n} \rightarrow e^{x}
$$

strebt, - womit schon alles bewiesen ist ${ }^{2}$ ).

4. Ist $a>0$ und $x$ beliebig reell, so ist

$$
a^{x}=e^{x \log a}=1+\frac{\log a}{1 !} x+\frac{\log ^{2} a}{2 !} x^{2}+\frac{\log ^{3} a}{3 !} x^{3}+\ldots
$$

eine Potenzreihenentwicklung der beliebigen Potenz. Aus ihr lesen wir die Grenzwertbeziehung

$$
\frac{a^{x}-1}{x} \rightarrow \log a \quad \text { für } \quad x \rightarrow 0
$$

$\left.a b^{3}\right)$. Diese Formel liefert uns einen ersten schon einigermaßen gangbaren Weg zur Berechnung der Logarithmen. Denn nach ihr ist z. B. (vgl. § 9, S. 74)

$$
\begin{aligned}
\log a & =\lim _{n \rightarrow \infty} n(\sqrt[n]{a}-1) \\
& =\lim _{k \rightarrow \infty} 2^{k}\left(\sqrt[2^{k}]{a}-1\right) .
\end{aligned}
$$

Da man nun Wurzeln, deren Exponent eine Potenz von 2 ist, durch wiederholtes Quadratwurzelziehen direkt berechnen kann, so ist hiermit ein (allerdings noch sehr primitiver) Weg zur Berechnung der Logarithmen gegeben.

1) Wir betrachten diese Nullfolge erst für $n>|x|$, damit stets $\frac{x}{n}>-1$ ist.

$\left.{ }^{2}\right)$ Verbinden wir dieses Ergebnis mit dem in 1. hergeleiteten, daf der obige Limes denselben Wert hat wie die Exponentialreihe, so haben wir einen $z$ weiten Beweis dafür, daf auch die Summe der Exponentialreihe $=e^{x}$ ist.

3) Direkter Beweis: Bilden die $x_{n}$ eine Nullfolge, so tun es nach $\mathbf{3 5}, 3$ auch die Zahlen $y_{n}=a^{x_{n}}-1$; und folglich strebt nach 112(b)

$$
\frac{a^{x_{n}}-1}{x_{n}}=\frac{y_{n} \cdot \log a}{\log \left(1+y_{n}\right)} \rightarrow \frac{\log a}{1}=\log a
$$


5. Daß die Funktion $e^{x}$ durchweg stetig und beliebig oft differenzierbar ist - mit $e^{x}=\left(e^{x}\right)^{\prime}=\left(e^{x}\right)^{\prime \prime}=\ldots$ - hatten wir schon betont. Daß sie durchweg positiv ist und mit $x$ monoton wächst, teilt sie mit jeder Potenz $a^{x}$, deren Basis $a>1$ ist.

Bemerkenswerter sind eine Anzahl einfacher Ungleichungen, die wir in der Folge noch oft gebrauchen werden, und die sich meist durch Vergleich der Exponentialreihe mit der geometrischen Reihe ergeben, und deren Beweis wir dem Leser überlassen wollen:

114.
$\alpha)$ für jedes $x$ ist $e^{x}>1+x^{1}$ )
B) für $x<1$ ist $e^{x}<\frac{1}{1-x}$
$\gamma$ ) für $x>-1$ ist $\frac{x}{1+x}<1-e^{-x}<x$
ס) für $x<+1$ ist $x<e^{x}-1<\frac{x}{1-x}$
ह) für $x>-1$ ist $1+x>e^{\frac{x}{1+x}}$

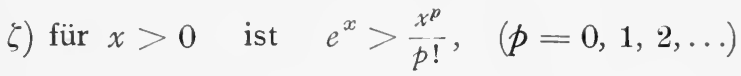
$\eta)$ für $x>0, y>0$ ist $e^{x}>\left(1+\frac{x}{y}\right)^{y}>e^{\frac{x y}{x+y}}$.

\section{§24. Die trigonometrischen Funktionen.}

Wir sind jetzt auch in der Lage, eine strenge, d. h. rein arithmetische Einführung der Kreisfunktionen zu geben. Wir betrachten dazu die nach $\mathbf{9 2}, 2$ beständig konvergenten Reihen

und

$$
C(x)=1-\frac{x^{2}}{2 !}+\frac{x^{4}}{4 !}-\ldots+(-1)^{k} \frac{x^{2 k}}{(2 k) !}+\ldots
$$

$$
S(x)=x-\frac{x^{3}}{3 !}+\ldots+(-1)^{k} \frac{x^{2 k+1}}{(2 k+1) !}+\cdots,
$$

deren jede eine durchweg stetige und beliebig oft differenzierbare Funktion darstellt. Wir werden die Eigenschaften dieser Funktionen aus ihrer Reihendarstellung heraus feststellen und schließlich finden, daß sie mit den aus den Elementen her bekannten Funktionen $\cos x$ und $\sin x$ identisch sind.

1. Zunächst findet man nach $\mathbf{9 8}, 3$, daß ihre Ableitungen die folgenden Werte haben:

$$
\begin{aligned}
& C^{\prime}=-S, \quad C^{\prime \prime}=-C, \quad C^{\prime \prime \prime}=S, \quad C^{\prime \prime \prime \prime}=C ; \\
& S^{\prime}=C, \quad S^{\prime \prime}=-S, \quad S^{\prime \prime \prime}=-C, \quad S^{\prime \prime \prime \prime}=S,
\end{aligned}
$$

1) Nur für $x=0$ gehen diese und die folgenden Ungleichungen in eine Gleichung über. - Man veranschauliche sich den Inhalt der Ungleichungen an den zugehörigen Kurven. 
- gültig für jedes $x$ (das wir der Kürze halber weggelassen haben). Da hiernach die $4^{\text {ten }}$ Ableitungen mit den ursprünglichen Funktionen übereinstimmen, wiederholen sich von hier an die Werte der Ableitungen in derselben Reihenfolge. Ferner sieht man sofort, daß $C(x)$ eine gerade und $S(x)$ eine ungerade Funktion ist:

$$
C(-x)=C(x), \quad S(-x)=-S(x) .
$$

Ähnlich der Exponentialfunktion besitzen diese Funktionen auch einfache Additionstheoreme, mit deren Hilfe sie dann weiter untersucht werden können. Man gewinnt sie am schnellsten durch die Taylorsche Reihenentwicklung (vgl. S. 183, Fußnote). Nach ihr hat man - und zwar, da die Reihen beständig (absolut) konvergieren, für irgend zwei reelle Zahlen $x_{1}$ und $x_{2}-$

$$
\mathrm{C}\left(x_{1}+x_{2}\right)=C\left(x_{1}\right)+\frac{C^{\prime}\left(x_{1}\right)}{1 !} x_{2}+\frac{C^{\prime \prime}\left(x_{1}\right)}{2 !} x_{2}^{2}+\ldots,
$$

und da diese Reihe absolut konvergiert, dürfen wir nach $\mathbf{8 9}, 4$ beliebig umordnen, dürfen also insbesondere alle die Glieder vereinigen, für die die darin auftretende Ableitung denselben Wert hat. Das ergibt

oder

$$
\begin{aligned}
C\left(x_{1}+x_{2}\right)=C\left(x_{1}\right)[1 & \left.-\frac{x_{2}{ }^{2}}{2 !}+\frac{x_{2}{ }^{4}}{4 !}-\ldots\right] \\
& -S\left(x_{1}\right)\left[x_{2}-\frac{x_{2}{ }^{3}}{3 !}+\frac{x_{2}{ }^{5}}{5 !}-\ldots\right]
\end{aligned}
$$

$$
C\left(x_{1}+x_{2}\right)=C\left(x_{1}\right) C\left(x_{2}\right)-S\left(x_{1}\right) S\left(x_{2}\right)
$$

und ganz ähnlich findet man

$$
\left.S\left(x_{1}+x_{2}\right)=S\left(x_{1}\right) C\left(x_{2}\right)+C\left(x_{1}\right) S\left(x_{2}\right)^{1}\right) .
$$

Aus diesen Theoremen, die der Form nach mit den aus den Elementen her bekannten Additionstheoremen der Funktionen cos und sin übereinstimmen, folgt dann leicht, daß für unsere Funktionen $C$ und $S$ auch alle andern sog. rein goniometrischen Formeln gelten. Wir heben insbesondere diese hervor:

Aus (a) folgt für $x_{2}=-x_{1}$, daß für jedes $x$

ist; und aus (a) und (b), indem man dort $x_{1}$ und $x_{2}$ beide $=x$ setzt:

$$
\begin{aligned}
& C(2 x)=C^{2}(x)-S^{2}(x) \\
& S(2 x)=2 C(x) S(x) .
\end{aligned}
$$

1) 2. Beweis. Die Ausmultiplikation und Zusammenfassung der Reihen

$$
C\left(x_{1}\right) C\left(x_{2}\right)-S\left(x_{1}\right) S\left(x_{2}\right)
$$

liefert die Reihe für $C\left(x_{1}+x_{2}\right)$, - ähnlich wie 91, 3 bei der Exponentialreihe.

3. Beweis. Die Ableitung der Funktion $f(x)=$

$$
\left[C\left(x_{1}+x\right)-C\left(x_{1}\right) C(x)+S\left(x_{1}\right) S(x)\right]^{2}+\left[S\left(x_{1}+x\right)-S\left(x_{1}\right) C(x)-C\left(x_{1}\right) S(x)\right]^{2}
$$

ist, wie man sofort findet, $\equiv 0$. Es ist also (nach $\S 19$, Def. 12) $f(x) \equiv f(0)=0$. Also muß jede eckige Klammer fuir sich $\equiv 0$ sein, was sofort beide Additionstheoreme liefert. 
2. Ein klein wenig mühsamer ist es, die Periodizitätseigenschaften unserer Funktionen direkt aus den Reihen abzulesen. Es gelingt so: Es ist

$$
C(0)=1>0 \text {. }
$$

Dagegen ist $C(2)<0$, denn es ist

$$
C(2)=1-\frac{2^{2}}{2 !}+\frac{2^{4}}{4 !}-\left(\frac{2^{6}}{6 !}-\frac{2^{8}}{8 !}\right)-\left(\frac{2^{10}}{10 !}-\frac{2^{12}}{12 !}\right)-\ldots
$$

und da hier die in den runden Klammern stehenden Größen alle positiv sind - denn für $n \geqq 2$ ist

$$
\frac{2^{n}}{n !}-\frac{2^{n+2}}{(n+2) !}>0 \quad-
$$

so ist $C(2)<1-\frac{4}{2}+\frac{16}{24}=-\frac{1}{3}$, also gewiß negativ. Nach $\S 19$, Satz 4 besitzt also die Funktion $C(x)$ mindestens eine zwischen 0 und 2 gelegene Nullstelle $\xi$. Da übrigens

$$
S(x)=x\left(1-\frac{x^{2}}{2 \cdot 3}\right)+\frac{x^{5}}{5 !}\left(1-\frac{x^{2}}{6 \cdot 7}\right)+\ldots
$$

zwischen 0 und 2, wie man ebenso leicht erkennt, dauernd positiv, also $C^{\prime}(x)=-S(x)$ dort dauernd negativ ist und die Funktion $C(x)$ somit in diesem Intervall monoton fällt, so ist $\xi$ die einzige dort gelegene Wurzel. Die kleinste positive Nullstelle von $C(x)$, also $\xi$, ist hiernach eine ganz bestimmte reelle Zahl. Wir werden gleich nachher sehen, daß sie dem vierten Teil des Umfanges eines Kreises vom Radius 1 gleich ist und wollen sie darum schon jetzt mit $\frac{\pi}{2}$ bezeichnen:

$$
\left.\xi=\frac{\pi}{2}, \quad C\left(\frac{\pi}{2}\right)=0^{1}\right) .
$$

Aus (c) folgt dann, daß $S^{2}\left(\frac{\pi}{2}\right)=1$, also, da $S(x)^{\prime}$ zwischen 0 und 2 als positiv erkannt war, daß

$$
S\left(\frac{\pi}{2}\right)=1
$$

ist. Die Formeln (d) lehren nun, daß

$$
C(\pi)=-1, \quad S(\pi)=0
$$

ist, und ihre nochmalige Anwendung, daß

$$
C(2 \pi)=1, \quad S(2 \pi)=0
$$

1) Die Situation ist also die, dafi hier $\pi$ zunächst lediglich eine $\mathbf{A b}$ kürzung für $2 \xi$ sein soll; erst nachher wird gezeigt werden, daf́ diese Zahl $\pi$ die bekannte Bedeutung für den Kreis hat. 
ist. Und nun folgt endlich aus den Additionstheoremen, daß für jedes $x$

$$
\begin{cases}C\left(x+\frac{\pi}{2}\right)=-S(x), & S\left(x+\frac{\pi}{2}\right)=C(x) \\ C(x+\pi)=-C(x), & S(x+\pi)=-S(x) \\ C(\boldsymbol{\pi}-x)=-C(x), & S(\boldsymbol{\pi}-x)=S(x) \\ \boldsymbol{C}(\boldsymbol{x}+\mathbf{2} \boldsymbol{x})=\boldsymbol{C}(\boldsymbol{x}), & \boldsymbol{S}(\boldsymbol{x}+\mathbf{2} \boldsymbol{\pi})=\boldsymbol{S}(\boldsymbol{x})\end{cases}
$$

ist. Unsere beiden Funktionen besitzen also die Periode $2 \pi^{\mathbf{1}}$ ).

3. Es bleibt nun allein noch zu zeigen übrig, daß die von uns rein arithmetisch eingeführte Zahl $\pi$ die bekannte geometrische Bedeutung für den Kreis hat. Damit wird sich dann auch die völlige Identität der Funktionen $C(x)$ und $S(x)$ mit $\cos x$ bzw. $\sin x$ ergeben.

Bewegt sich in der Ebene eines rechtwinkligen Koordinatensystems $O X Y$ ein Punkt $P$ derart, daß zur Zeit $t$ seine Koordinaten $x$ und $y$ durch die beiden Gleichungen

$$
x=C(t) \quad \text { und } \quad y=S(t)
$$

gegeben sind, so ist sein Abstand $|O P|=\sqrt{x^{2}+y^{2}}$ vom Koordinatenanfangspunkt nach (c) stets $=1$. Er bewegt sich also auf der Peripherie des Kreises mit dem Radius 1 um $O$. Läßt man speziell $t$ von 0 bis $2 \pi$ wachsen, so beginnt der Punkt $P$ seine Wanderung bei dem auf der positiven $x$. Achse gelegenen Punkt $A$ des Kreises und umläuft nun im mathematisch-positiven Sinne (d. h. im Gegensinne des Uhrzeigers) genau einmal die Peripherie. In der Tat, während $t$ von 0 bis $\pi$ zunimmt, fällt, wie nun ersichtlich, $x=C(t)$ monoton von +1

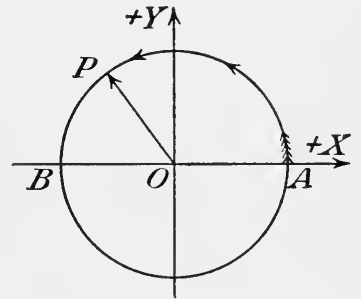

Fig. 3. bis -1 , die Abszisse von $P$ durchläuft also alle diese Werte und jeden genau einmal. Da gleichzeitig $S(t)$ dauernd positiv ist, so heißt dies, daß $P$ den oberen Halbkreis von $A$ nach $B$ genau einmal durchlaufen hat. Die Formeln (e) zeigen dann weiter, daß,

1) $2 \pi$ ist auch eine sogen. primitive Periode unserer Funktionen, d. h. eine Periode, von der nicht ein (ganzzahliger) Bruchteil auch schon Periode ist. Wegen der zweiten der Formeln (e) ist nämlich $\frac{2 \pi}{2}=\pi$ sicher keine Periode. Auch ein höherer Bruchteil $\frac{2 \pi}{m}(m>2)$ kann nicht Periode sein, da dann z. B. $S\left(\frac{2 \pi}{m}\right)=S(0)=0$ sein mübte. Das ist aber unmöglich, da $S(x)$ zwischen 0 und 2 als positiv erkannt wurde und wegen $S(\pi-x)=S(x)$ sogar zwischen 0 und $\pi$ stets $>0$ ist. Analog schliefit man für $C(x)$, dafi $\frac{2 \pi}{m}(m>1)$ keine Periode sein kann. 
wenn $t$ von $\pi$ bis $2 \pi$ wächst, genau ebenso der untere Halbkreis von $B$ nach $A$ durchlaufen wird. Diese Betrachtung lehrt zunächst den

Satz. Sind $x$ und $y$ irgend zwei reelle Zahlen, für die $x^{2}+y^{2}=1$ ist, so gibt es stets eine und nur eine zwischen 0 (einschl.) und $2 \pi$ (ausschl.) gelegene Zahl $t$, für die gleichzeitig

ist.

$$
C(t)=x \quad \text { und } \quad S(t)=y
$$

Fragen wir nun endlich nach der Länge des Weges, den der Punkt zurückgelegt hat, wenn $t$ von 0 bis zu einem Werte $t_{0}$ gewachsen ist, so ergibt die Formel $\S 19$, Satz 29 hierfür sofort den Wert

$$
\int_{0}^{t_{0}} \sqrt{C^{2}+S^{\prime 2}} d t=\int_{0}^{t_{0}} d t=t_{0}
$$

insbesondere ist der volle Umfang des Kreises

$$
=\int_{0}^{2 \pi} \sqrt{C^{\prime 2}+S^{\prime 2}} d t=\int_{0}^{2 \pi} d t=2 \pi .
$$

Hiermit ist der erstrebte Zusammenhang zwischen unsern anfänglichen Betrachtungen und der Kreisgeometrie hergestellt: $C(t)$ ist als Abszisse desjenigen Punktes $P$, für den der Bogen $\overparen{A P}=t$ ist, mit dem cos dieses Bogens bzw. des zugehörigen Zentriwinkels identisch, und $S(t)$ als Ordinate von $P$ mit dem sin dieses Winkels. Fortan dürfen wir also $\cos t$ statt $C(t)$ und $\sin t$ statt $S(t)$ schreiben. - Der Unterschied unserer Behandlungsart von der elementaren ist hauptsächlich der, daß dort diese beiden Funktionen auf Grund geometrischer Erwägungen, die die Strecken-, Winkel-, Bogenlängen- und InhaltMessungen sozusagen naiv benutzen, eingeführt und untersucht werden, und daß man erst als letztem Ergebnis zu der Potenzreihenentwicklung derselben gelangt. Wir gingen umgekehrt von diesen Potenzreihen aus, studierten die dadurch definierten Funktionen und stellten schließ: lich - unter Benutzung eines durch die Integralrechnung geläuterten Begriffs der Bogenlänge - fest, daß sie die bekannte Bedeutung für den Kreis haben.

4. Die Funktionen $\operatorname{ctg} x$ und $\operatorname{tg} x$ definieren wir nun wie üblich als Quotienten

$$
\operatorname{ctg} x=\frac{\cos x}{\sin x}, \quad \operatorname{tg} x=\frac{\sin x}{\cos x}
$$

sie bieten daher als Funktionen nichts prinzipiell Neues.

Die Potenzreihenentwicklungen dieser Funktionen sind indessen nicht so einfach. Nach dem 105, 4 beschriebenen Divisionsverfahren kann man natürlich leicht einige Koeffizienten der Entwicklung be- 
rechnen. Doch gewinnt man damit keinen Einblick in die Zusammenhänge. Wir gehen so vor: In $\mathbf{1 0 5}, 5$ hatten wir die Entwicklung

$$
\frac{x}{e^{x}-1}=\sum_{\nu=0}^{\infty} \frac{B_{\nu} x^{\nu}}{v !}=1-\frac{x}{2}+\frac{B_{2} x^{2}}{2 !}+\frac{B_{3} x^{3}}{3 !}+\ldots
$$

kennen gelernt, in der die Bernoullischen Zahlen $B_{v}$ zwar nicht explizit bekannt, aber mit Hilfe der sehr durchsichtigen Rekursionsformel 106 bequem berechenbar sind. Diese Zahlen können und wollen wir daher weiterhin als schlechtweg bekannt ansehen ${ }^{\mathbf{1}}$ ). Hiernach ist - gültig für alle "hinreichend“ kleinen $x$ (vgl. 105, 2, 4) -

$$
\frac{x}{e^{x}-1}+\frac{x}{2}=1+\frac{B_{2} x^{2}}{2 !}+\cdots
$$

Die linksstehende Funktion ist aber

$$
=\frac{x}{2}\left(\frac{2}{e^{x}-1}+1\right)=\frac{x}{2} \frac{e^{x}+1}{e^{x}-1}=\frac{x}{2} \frac{e^{\frac{x}{2}}+e^{-\frac{x}{2}}}{e^{\frac{x}{2}}-e^{-\frac{x}{2}}}
$$

und aus dieser Darstellung erkennt man, daß es sich um eine gerade Funktion handelt. Die Bernoullischen Zahlen $B_{3}, B_{5}, B_{7}, \ldots$ sind also sämtlich $=0$, und wir haben, indem wir für $e^{\frac{x}{2}}$ die Exponentialreihe benutzen und zur Abkürzung $\frac{x}{2}=z$ setzen:

$$
z \cdot \frac{1+\frac{z^{2}}{2 !}+\frac{z^{4}}{4 !}+\ldots}{z+\frac{z^{3}}{3 !}+\frac{z^{5}}{5 !}+\ldots}=1+\frac{B_{2}}{2 !}(2 z)^{2}+\frac{B_{4}}{4 !}(2 z)^{4}+\ldots
$$

Stünden hier im Zähler und Nenner linkerhand abwechselnde Vorzeichen, so hätten wir ersichtlich gerade die Funktion $z \operatorname{ctg} z$ vor uns. Darf man nun aus unserer Gleichung - in der wir uns noch links, um lauter gerade Potenzen zu bekommen, den Faktor $z$ gehoben denken - ohne weiteres schließen, daß sie auch mit abwechselnden Vorzeichen richtig bleibt, daß also auch

$$
z \operatorname{ctg} z=\frac{1-\frac{z^{2}}{2 !}+\frac{z^{4}}{4 !}-\ldots}{1-\frac{z^{2}}{3 !}+\frac{z^{4}}{5 !}-+\therefore}=1-\frac{B_{2}}{2 !}(2 z)^{2}+\frac{B_{4}}{4 !}(2 z)^{4}-+\ldots
$$

ist? Offenbar ja! Denn ist allgemein für alle hinreichend kleinen $z$

$$
\frac{1+a_{2} z^{2}+a_{4} z^{4}+\cdots}{1+b_{2} z^{2}+b_{4} z^{4}+\cdots}=1+c_{2} z^{2}+c_{4} z^{4}+\ldots,
$$

1) Wie aus ihrer Definition hervorgeht, sind sie jedenfalls alle rational. 
so besteht auch dieselbe Beziehung mit abwechselnden Vorzeichen. Denn in beiden Fällen sind gemäß 105, 4 die Koeffizienten $c_{2}$ v aus den Gleichungen

$$
\begin{gathered}
c_{2}+b_{2}=a_{2} ; \quad c_{4}+c_{2} b_{2}+b_{4}=a_{4} ; \ldots c_{6}+c_{4} b_{2}+c_{2} b_{4}+b_{6}=a_{6} ; \ldots \\
c_{2 v}+c_{2 v-2} b_{2}+\ldots+c_{2} b_{2 v-2}+b_{2 v}=a_{2 v}
\end{gathered}
$$

der Reihe nach zu bestimmen. Es ist also in der Tat - wir schreiben nun $x$ statt $z-$

115. $\left.x \operatorname{ctg} x=1-\frac{2^{2} B_{2}}{2 !} x^{2}+\frac{2^{4} B_{4}}{4 !} x^{4}-+\ldots+(-1)^{k} \frac{2^{2 k} B_{2 k}}{(2 k) !} x^{2 k}+\ldots 1\right)$.

Die Entwicklung für $\operatorname{tg} x$ erhält man nun am einfachsten mit Hilfe des Additionstheorems

aus dem

$$
2 \operatorname{ctg} 2 x=\frac{\cos ^{2} x-\sin ^{2} x}{\cos x \cdot \sin x}=\operatorname{ctg} x-\operatorname{tg} x,
$$

und also

$$
\operatorname{tg} x=\operatorname{ctg} x-2 \operatorname{ctg} 2 x
$$

\section{6.}

$$
\operatorname{tg} x=\sum_{k=1}^{\infty}(-1)^{k-1} \frac{2^{2 k}\left(2^{2 k}-1\right) B_{2 k}}{(2 k) !} x^{2 k-1}
$$

folgt. - Diese Entwicklungen sind an dieser Stelle noch unbefriedigend, da ihr Gültigkeitsintervall noch nicht angegeben werden kann; wir wissen nur, $d a \beta$ die Reihen einen positiven Konvergenzradius besitzen, nicht aber, wie groß er ist.

5. Noch von einer ganz anderen Seite her ist Euler zu einer interessanten Reihenentwicklung für die Funktion ctg gelangt, die wir jetzt, zugleich wegen ihrer großen Bedeutung für viele Reihenprobleme, herleiten wollen ${ }^{2}$ ). Aus ihr wird sich dann auch der Konvergenzradius der Reihen 115 und 116 ergeben (s. S. 402).

Es ist, wie eben gezeigt

oder also

$$
\operatorname{ctg} y=\frac{1}{2}\left(\operatorname{ctg} \frac{y}{2}-\operatorname{tg} \frac{y}{2}\right)
$$

$$
\operatorname{ctg} \pi x=\frac{1}{2}\left\{\operatorname{ctg} \frac{\pi x}{2}+\operatorname{ctg} \frac{\pi(x+1)}{2}\right\},
$$

eine Formel, bei der rechts ein beliebiges der Zeichen \pm genommen werden darf. Es sei nun $x$ eine beliebige von $0, \pm 1, \pm 2, \ldots$ ver-

1) Diese und die folgenden Reihenentwicklungen stammen fast sämtlich von Euler her und finden sich im 9. und 10. Kapitel seiner Introductio in analysin infinitorum, Lausanne 1748.

2) Die folgende gegenüber der Eulerschen stark vereinfachte Herleitung der Entwicklung rührt von Schröter her (Ableitung der Partialbruch- und Produktentwicklungen für die trigonometrischen Funktionen, Zeitschrift für Math. u. Phys., Bd. 13, 1868, S. 254). 
schiedene reelle $Z a h l$, deren Wert im folgenden festgehalten werden soll. Dann ist

$$
\pi x \operatorname{ctg} \pi x=\frac{\pi x}{2}\left\{\operatorname{ctg} \frac{\pi x}{2}+\operatorname{ctg} \frac{\pi(x \pm 1)}{2}\right\}
$$

und die nochmalige Anwendung derselben Formel auf die beiden Funktionswerte der rechten Seite ergibt

$\pi x \operatorname{ctg} \pi x=\frac{\pi x}{4}\left\{\operatorname{ctg} \frac{\pi x}{4}+\left[\operatorname{ctg} \frac{\pi(x+1)}{4}+\operatorname{ctg} \frac{\pi(x-1)}{4}\right]+\operatorname{ctg} \frac{\pi(x+2)}{4}\right\}$.

Ein dritter analoger Schritt ergibt für $\pi x \operatorname{ctg} \pi x$ den Wert

$$
\frac{\pi x}{8}\left\{\begin{array}{c}
\operatorname{ctg} \frac{\pi x}{8}+\operatorname{ctg} \frac{\pi(x+1)}{8}+\operatorname{ctg} \frac{\pi(x+2)}{8}+\operatorname{ctg} \frac{\pi(x+3)}{8} \\
+\operatorname{ctg} \frac{\pi(x-1)}{8}+\operatorname{ctg} \frac{\pi(x-2)}{8}+\operatorname{ctg} \frac{\pi(x-3)}{8}+\operatorname{ctg} \frac{\pi(x+4)}{8}
\end{array}\right\},
$$

da je zwei Summanden, die zu dem Mittelpunkt (•) des in der geschweiften Klammer stehenden Aggregates spiegelbildlich liegen, gemäß Formel (a) nach Hinzufügung des Faktors $\frac{1}{2}$ je einen Summanden des vorigen Aggregates bilden. Führt man in derselben Weise im ganzen $n$ solche Schritte aus, so erhält man

(b) $\pi x \operatorname{ctg} \pi x=\frac{\pi x}{2^{n}}\left\{\operatorname{ctg} \frac{\pi x}{2^{n}}+\sum_{\nu=1}^{2^{n-1}-1}\left[\operatorname{ctg} \frac{\pi(x+v)}{2^{n}}+\operatorname{ctg} \frac{\pi(x-v)}{2^{n}}\right]-\operatorname{tg} \frac{\pi x}{2^{n}}\right\}$.

Da nun nach $\mathbf{1 1 5}$

$$
\lim _{z \rightarrow 0} z \operatorname{ctg} z=1
$$

und also auch für jedes $\alpha \neq 0$

$$
\lim _{z \rightarrow \infty} \frac{1}{2^{n}} \operatorname{ctg} \frac{\alpha}{2^{n}}=\frac{1}{\alpha}
$$

ist, so würde man, wenn man in der lètzten Darstellung $n \rightarrow \infty$ streben läßt, und auf der rechten Seite, zunächst versuchsweise den Grenzübergang bei jedem Summanden einzeln ausführt, die Entwicklung $\pi x \operatorname{ctg} \pi x=1+x \cdot \sum_{v=1}^{\infty}\left(\frac{1}{x+v}+\frac{1}{x-v}\right)+0=1+2 x^{2} \cdot \sum_{v=1}^{\infty} \frac{1}{x^{2}-v^{2}}$ erhalten. Wir wollen nun zeigen, daß diese im allgemeinen fehlerhafte Form des Grenzüberganges uns hier doch zu einem richtigen Resultat geführt hat:

Zunächst ist die erhaltene Reihe für jedes $x \neq 0, \pm 1, \pm 2, \ldots$ nach 70, 4 absolut konvergent, da die Beträge ihrer Glieder denen der Reihe $\Sigma \frac{1}{\boldsymbol{v}^{2}}$ asymptotisch gleich sind. Nun werde eine beliebige ganze Zahl $k>6|x|$ gewählt und vorläufig ebenfalls festgehalten. Ist dann $n$ so groß, daß die Zahl $2^{n-1}-1$, die wir zur Abkürzung mit $m$ be- 
zeichnen wollen, $>k$ ist, so zerlegen wir die gewonnene Darstellung (b) von $\pi x \operatorname{ctg} x$ wie folgt:

$$
\pi x \operatorname{ctg} \pi x=\frac{\pi x}{2^{n}}\left\{\operatorname{ctg} \frac{\pi x}{2^{n}}-\operatorname{tg} \frac{\pi x}{2^{n}}+\sum_{r=1}^{k}[\ldots]\right\}+\frac{\pi x}{2^{n}}\left\{\sum_{v=k+1}^{m}[\ldots]\right\} .
$$

(In die eckige Klammer ist hier natürlich dasselbe wie in (b) einzusetzen). Die beiden Teile dieses Ausdrucks nennen wir $A_{n}$ und $B_{n}$. Da nun $A_{n}$ nur aus einer festen Anzahl von Summanden besteht, so ist hier der gliedweise Grenzübergang nach 41, 8 gewiß gestattet, und wir haben

$$
\lim _{n \rightarrow \infty} A_{n}=1+2 x^{2} \sum_{r=1}^{k} \frac{1}{x^{2}-v^{2}}
$$

und da $B_{n}$ nichts anderes ist als $\pi x \operatorname{ctg} \pi x-A_{n}$, so ist auch $\lim B_{n}$ vorhanden. Seinen Wert, der noch von der getroffenen Wahl von $k$ abhängt, wollen wir mit $r_{k}$ bezeichnen, so daß also

$$
\lim _{n \rightarrow \infty} B_{n}=r_{k}=\pi x \operatorname{ctg} \pi x-\left[1+2 x^{2} \sum_{r=1}^{k} \frac{1}{x^{2}-r^{2}}\right]
$$

ist. Die Zahlen $B_{n}$, folglich ihr Grenzwert $r_{k}$ und also auch die zuletzt stehende Differenz lassen sich nun leicht abschätzen:

Es ist

und also

$$
\operatorname{ctg}(a+b)+\operatorname{ctg}(a-b)=\frac{-2 \operatorname{ctg} a}{\frac{\sin ^{2} b}{\sin ^{2} a}-1}
$$

$$
\operatorname{ctg} \frac{\pi(x+v)}{2^{n}}+\operatorname{ctg} \frac{\pi(x-v)}{2^{n}}=\frac{-2 \operatorname{ctg} \alpha}{\frac{\sin ^{2} \beta}{\sin ^{2} \alpha}-1},
$$

wenn zur Abkürzung für den Augenblick $\frac{\pi x}{2^{n}}=\alpha$ und $\frac{\pi v}{2^{n}}=\beta$ gesetzt wird.

$$
\begin{aligned}
& \text { Wegen } 2^{n}>k>6|x| \text { ist sicher }\left|\alpha_{2}\right|=\left|\frac{\pi x}{2^{n}}\right|^{r^{\prime}}<1 \text { und also } \\
& \left.|\sin \alpha|=\left|\alpha-\frac{\alpha^{3}}{3 !}+\ldots\right| \leqq|\alpha|\left(1+\frac{1}{3 !}+\frac{1}{5 !}+\ldots\right)<2|\alpha|^{1}\right) .
\end{aligned}
$$

Da ferner $0<\beta<\frac{\pi}{2}<2$ ist, so ist

$$
\left.\sin \beta=\beta\left(1-\frac{\beta^{2}}{2 \cdot 3}\right)+\frac{\beta^{5}}{5 !}\left(1-\frac{\beta^{2}}{6 \cdot 7}\right)+\ldots>\frac{\beta}{3}{ }^{2}\right)
$$

und also

$$
\left|\frac{\sin \beta}{\sin \alpha}\right|>\frac{\beta}{6|\alpha|}=\frac{r}{6|x|}>1 \text {, }
$$

1) Späterer Anwendung zuliebe machen wir diese Abschätzung in der obigen groben Form.

2) Vgl. den Schlufs auf S. 192. 
letzteres, da $v>k>6|x|$ ist. Daher ist nun (für $v>k$ )

und also

$$
\left|\operatorname{ctg} \frac{\pi(x+v)}{2^{n}}+\operatorname{ctg} \frac{\pi(x-v)}{2^{n}}\right| \leqq \frac{2\left|\operatorname{ctg} \frac{\pi x}{2^{n}}\right|}{\frac{v^{2}}{36 x^{2}}-1}
$$

$$
\left|B_{n}\right| \leqq\left|\frac{\pi x}{2^{n}} \operatorname{ctg} \frac{\pi x}{2^{n}}\right| \cdot \sum_{\nu=k+1}^{m} \frac{72 x^{2}}{v^{2}-36 x^{2}} .
$$

Der vor der Summe stehende Faktor ist nun - ganz grob abgeschätzt - sicher $<3$, denn es war $\left|\frac{\pi x}{2^{n}}\right|<1$ und für $|z|<1$ ist

$$
\left.|z \operatorname{ctg} z|=\left|\frac{1-\frac{z^{2}}{2 !}+\frac{z^{4}}{4 !}-+\ldots}{1-\frac{z^{2}}{3 !}+\frac{z^{4}}{5 !}-+\ldots}\right|<\frac{1+\frac{1}{2 !}+\frac{1}{4 !}+\ldots}{1-\frac{1}{3 !}-\frac{1}{5 !}+\ldots}<3^{1}\right) .
$$

Daher ist

$$
\left|B_{n}\right|<216 x^{2} \cdot \sum_{\nu=k+1}^{m} \frac{1}{\nu^{2}-36 x^{2}}<216 x^{2} \cdot \sum_{\nu=k+1}^{\infty} \frac{1}{v^{2}-36 x^{2}} .
$$

Hier steht nun aber eine von $n$ ganz unabhängige Zahl, so daß auch

$$
\left|\lim B_{n}\right|=\left|\boldsymbol{r}_{k}\right| \leqq 216 x^{2} \cdot \sum_{\nu=k+1}^{\infty} \frac{1}{v^{2}-36 x^{2}}
$$

ist. Diese nun für $r_{k}$ gefundene obere Schranke ist aber gleich dem hinter dem $k^{\text {ten }}$ Gliede beginnenden Rest einer konvergenten Reihe ${ }^{2}$ ). Ist also $\varepsilon>0$ gegeben, so können wir uns $k_{0}$ so wählen, daß für $k>k_{0}$ stets

$$
\left|v_{k}\right|<\varepsilon
$$

ausfällt. Gehen wir auf die Bedeutung von $r_{k}$ zurück, so heißt dies, daß

$$
\lim _{k \rightarrow \infty}\left\{\pi x \operatorname{ctg} \pi x-\left[1+2 x^{2} \sum_{n=1}^{k} \frac{1}{x^{2}-v^{2}}\right]\right\}=0
$$

oder also daß, wie behauptet,

$$
\pi x \operatorname{ctg} \pi x=1+2 x^{2} \sum_{\nu=1}^{\infty} \frac{1}{x^{2}-v^{2}}
$$

ist, - eine Formel, die für jedes $x \neq 0, \pm 1 \pm 2, \ldots$ nun als gültig erwiesen ist.

6. Von dieser äußerst merkwürdigen sog. Partialbruchzerlegung der Funktion ctg werden wir gleich im nächsten Kapitel wichtige

1) Vgl. Fußnote 1 der vorigen Seite.

2) Die Konvergenz ergibt sich ebenso einfach wie weiter oben die der Reihe $\sum \frac{1}{x^{2}-v^{2}}$. 
Anwendungen zu machen haben. Aus ihr kann man natülich leicht viele weitere herleiten; wir nennen noch folgende:

Gemäß der Formel

$$
\pi \operatorname{ctg} \frac{\pi x}{2}-2 \pi \operatorname{ctg} \pi x=\pi \operatorname{tg} \frac{\pi x}{2}
$$

findet man zunächst

$$
\left.\pi \operatorname{tg} \frac{\pi x}{2}=\sum_{v=0}^{\infty} \frac{4 x}{(2 v+1)^{2}-x^{2}}, \quad x \neq \pm 1, \pm 3, \pm \tilde{5}, \ldots 1\right) .
$$

Die Formel

$$
\operatorname{ctg} z+\operatorname{tg} \frac{z}{2}=\frac{1}{\sin z}
$$

liefert dann weiter für $x \neq 0, \pm 1, \pm 2, \ldots$

$$
\begin{aligned}
\frac{\pi}{\sin \pi x} & =\frac{1}{x}+\frac{2 x}{1^{2}-x^{2}}-\frac{2 x}{2^{2}-x^{2}}+-\ldots \\
& =\frac{1}{x}+\left(\frac{1}{1-x}-\frac{1}{1+x}\right)-\left(\frac{1}{2-x}-\frac{1}{2+x}\right)+-\ldots
\end{aligned}
$$

Ersetzt man endlich hierin $x$ durch $\frac{1}{2}-x$, so folgt

$$
\frac{\pi}{\cos \pi x}=\frac{2}{1-2 x}+\left(\frac{2}{1+2 x}-\frac{2}{3-2 x}\right)-\left(\frac{2}{3+2 x}-\frac{2}{5-2 x}\right)+-\ldots
$$

Nach 83, 2 darf man hier die Klammern weglassen. Faßt man dann aber, vorn beginnend, wieder je zwei Glieder zusammen, so ergibt sich - gültig, falls $x \neq+\frac{1}{2}, \pm \frac{3}{2}, \pm_{2}^{5}, \ldots$ ist -

118.

$$
\frac{\pi}{\cos \pi x}=\frac{4 \cdot 1}{12-4 x^{2}}-\frac{4 \cdot 3}{32-4 x^{2}}+\frac{4 \cdot 5}{5^{2}-4 x^{2}}-+\ldots
$$

Mit diesen Partialbruchzerlegungen der Funktionen $\mathrm{ctg}, \operatorname{tg}, \frac{1}{\sin }$ und $\frac{1}{\cos }$ wollen wir unsere Untersuchungen über die trigonometrischen Funktionen abbrechen.

\section{§25. Die binomische Reihe.}

Schon in $\S 21$ hatten wir gesehen, daß der Binomialsatz für ganze positive Exponenten, wenn man ihm die Form

$$
(1+x)^{k}=\sum_{n=0}^{\infty}\left(\begin{array}{l}
k \\
n
\end{array}\right) x^{n}
$$

gibt, ungeändert auch für den Fall gültig bleibt, daß $k$ eine negative ganze Zahl ist ${ }^{2}$ ). Nur muß dann $|x|<1$ bleiben. Wir wollen jetzt

1) Die Formel ergibt sich zunächst nur für $x \neq 0, \pm 1, \pm 2, \ldots$, doch prüft man ihre Richtigkeit für $x=0, \pm 2, \pm 4, \ldots$, nachträglich ohne Schwierigkeit. (Die Reihe hat 0 zur Summe!).

2) Im ersten Falle ist die Reihe nur formal, im letzten tatsächlich eine unendliche. 
zeigen, daß unter dieser Einschränkung der Satz sogar für jeden reellen Exponenten $a$ gilt, daß also

$$
(\mathbf{1}+\boldsymbol{x})^{\alpha}=\sum_{n=0}^{\infty}\left(\begin{array}{l}
\alpha \\
n
\end{array}\right) \boldsymbol{x}^{n}, \quad\left\{\begin{array}{l}
|x|<1, \\
\alpha \text { beliebig reell, }
\end{array}\right.
$$

ist $^{1}$ ). Wie in den vorangehenden Fällen, wollen wir auch hier von der Reihe ausgehen und zeigen, daß sie die in Rede stehende Funktion darstellt.

Die Konvergenz der Reihe für $|x|<1$ ist sofort festzustellen, denn der Betrag des Quotienten des $(n+1)^{\text {ten }}$ zum $n^{\text {ten }}$ Gliede ist

$$
=\left|\frac{\alpha-n}{n+1} x\right| \text { und strebt also } \rightarrow|x|,
$$

womit nach $\mathbf{7 6}, 2$ schon gezeigt ist, daß +1 der genaue Konvergenzradius der binomischen Reihe ist. Nicht ganz so leicht ist es zu sehen, daß ihre Summe gleich dem - eo ipso positiven - Wert von $(\mathbf{1}+x)^{\alpha}$ ist. Bezeichnen wir die für $|x|<1$ durch die Reihe dargestellte Funktion vorläufig mit $f_{\alpha}(x)$, so kann man den Beweis folgendermaßen führen.

Da $\Sigma\left(\begin{array}{l}\alpha \\ n\end{array}\right) x^{n}$ bei beliebigem $\alpha$ für $|x|<1$ absolut konvergiert, ist (nach 91, Bem. 1) für beliebige $\alpha$ und $\beta$ und alle $|x|<1$

$$
\sum_{n=0}^{\infty}\left(\begin{array}{l}
\alpha \\
n
\end{array}\right) x^{n} \cdot \sum_{n=0}^{\infty}\left(\begin{array}{l}
\beta \\
n
\end{array}\right) x^{n}=\sum_{n=0}^{\infty}\left[\left(\begin{array}{l}
\alpha \\
0
\end{array}\right)\left(\begin{array}{l}
\beta \\
n
\end{array}\right)+\left(\begin{array}{l}
\alpha \\
1
\end{array}\right)\left(\begin{array}{c}
\beta \\
n-1
\end{array}\right)+\ldots+\left(\begin{array}{l}
\alpha \\
n
\end{array}\right)\left(\begin{array}{l}
\beta \\
0
\end{array}\right)\right] x^{n} \text {. }
$$

Nun ist aber stets

$$
\left(\begin{array}{l}
\boldsymbol{a} \\
\mathbf{0}
\end{array}\right)\left(\begin{array}{l}
\boldsymbol{\beta} \\
\boldsymbol{n}
\end{array}\right)+\left(\begin{array}{l}
\boldsymbol{a} \\
\mathbf{1}
\end{array}\right)\left(\begin{array}{c}
\boldsymbol{\beta} \\
\boldsymbol{n}-\mathbf{1}
\end{array}\right)+\ldots+\left(\begin{array}{l}
\boldsymbol{\alpha} \\
\boldsymbol{n}
\end{array}\right)\left(\begin{array}{l}
\boldsymbol{\beta} \\
\mathbf{0}
\end{array}\right)=\left(\begin{array}{c}
\boldsymbol{\alpha}+\boldsymbol{\beta} \\
\boldsymbol{n}
\end{array}\right)
$$

wie man z. B. durch Induktion ganz leicht beweist $\left.{ }^{2}\right)$. Daher ist bei festem $|x|<1$ - für beliebige $\alpha$ und $\beta$ stets

$$
f_{\alpha} \cdot f_{\beta}=f_{\alpha+\beta} \text {. }
$$

1) Das Symbol $\left(\begin{array}{l}\alpha \\ n\end{array}\right)$ ist für beliebige reelle $\alpha$ und ganze $n \geqq 0$ durch die beiden Festsetzungen

$$
\left(\begin{array}{c}
\alpha \\
0
\end{array}\right)=1, \quad\left(\begin{array}{l}
\alpha \\
n
\end{array}\right)=\frac{\alpha(\alpha-1) \ldots(\alpha-n+1)}{1 \cdot 2 \cdot \ldots} \text { für } n \geqq 1
$$

definiert und genügt für alle reellen $\alpha$ und alle $n \geqq 1$ der Beziehung

$$
\left(\begin{array}{l}
\alpha-1 \\
n-1
\end{array}\right)+\left(\begin{array}{c}
\alpha-1 \\
n
\end{array}\right)=\left(\begin{array}{l}
\alpha \\
n
\end{array}\right)
$$

deren Richtigkeit man sofort nachrechnet. die Form

2) Hierzu bringe man die Behauptung durch Multiplikation mit $n$ ! auf

$$
\begin{aligned}
& \left(\begin{array}{l}
n \\
0
\end{array}\right) \cdot \overline{\beta(\beta-1) \ldots(\beta-n+1)}+\ldots+\left(\begin{array}{l}
n \\
k
\end{array}\right) \alpha(\alpha-1) \ldots(\alpha-k+1) \cdot \overline{\beta(\beta-1) \ldots(\beta-n+k+1)}+\ldots \\
& +\left(\begin{array}{l}
n \\
n
\end{array}\right) \overline{\alpha(\alpha-1) \ldots(\alpha-n+1)}=(\alpha+\beta)(\alpha+\beta-1) \ldots(\alpha+\beta-n+1) .
\end{aligned}
$$

Multipliziert man nun die $(n+1)$ Summanden der linken Seite erst der Reihe 
Wörtlich ebenso nun, wie wir bei der Exponentialfunktion aus dem Additionstheorem $E\left(x_{1}\right) \cdot E\left(x_{2}\right)=E\left(x_{1}+x_{2}\right)$ gefolgert haben, daß für alle reellen $x$ stets $E(x)=(E(\mathbf{1}))^{x}$, folgt hier, daß für alle reellen $\alpha$ stets

$$
f_{\alpha}=\left(f_{1}\right)^{\alpha}
$$

ist. Da aber $f_{1}=1+x$ ist, so besagt dies, daß

ist, w. z. b. w. ${ }^{1}$ ).

$$
f_{\alpha}=(1+x)^{\alpha}
$$

Mit dem hiermit vollendeten Nachweis der Tatsache, daß sich $(1+x)^{a}$ in eine Potenzreihe in $x$ entwickeln läßt, ist nun auch die $\S 21$ beim Beweise des Umkehrsatzes noch gebliebene Lücke ausgefüllt.

Die Binomialreihe liefert, ähnlich wie die Exponentialreihe für $a^{\varrho}$, eine Entwicklung der allgemeinen Potenz: Man wähle, um $a^{\varrho} \mathrm{zu}$ entwickeln, eine (positive) Zahl $c$, für die einerseits $c^{\varrho}$ als bekannt

nach mit

dann mit

$$
\alpha,(\alpha-1), \ldots,(\alpha-k), \ldots,(\alpha-n)
$$

$$
(\beta-n),(\beta-n+1), \ldots,(\beta-n+k), \ldots, \beta
$$

und addiert, so hat man sie im ganzen mit $(\alpha+\beta-n)$ multipliziert und bekommt, wenn man die gleichartigen Glieder links zusammenfaßt, genau die für $n+1$ statt $n$ angesetzte Behauptungsgleichung. - Die obige Formel bezeichnet man gewöhnlich als Additionstheorem der Binominalkoeffizienten.

1) Ein zweiter, fast noch leichterer Beweis, der aber von der Differentialrechnung Gebrauch macht, geht so vor: Aus $f_{\alpha}=\sum_{n=0}^{\infty}\left(\begin{array}{l}\alpha \\ n\end{array}\right) x^{n}$ folgt

$$
f_{\alpha}^{\prime}(x)=\sum_{n=0}^{\infty} n\left(\begin{array}{l}
\alpha \\
n
\end{array}\right) x^{n-1}=\sum_{n=0}^{\infty}(n+1)\left(\begin{array}{c}
\alpha \\
n+1
\end{array}\right) x^{n} .
$$

Da aber $(n+1)\left(\begin{array}{c}\alpha \\ n+1\end{array}\right)=\alpha\left(\begin{array}{c}\alpha-1 \\ n\end{array}\right)$ ist, so ergibt sich weiter

Nun ist aber

$$
f_{\alpha}^{\prime}(x)=\alpha \cdot f_{\alpha-1}(x) \text {. }
$$

$$
\begin{aligned}
(1+x) f_{\alpha-1}(x) & =(1+x) \cdot \sum_{n=0}^{\infty}\left(\begin{array}{c}
\alpha-1 \\
n
\end{array}\right) x^{n} \\
& =1+\sum_{n=1}^{\infty}\left[\left(\begin{array}{c}
\alpha-1 \\
n
\end{array}\right)+\left(\begin{array}{l}
\alpha-1 \\
n-1
\end{array}\right)\right] x^{n}=\sum_{n=0}^{\infty}\left(\begin{array}{l}
\alpha \\
n
\end{array}\right) x^{n},
\end{aligned}
$$

so dás für alle $|x|<1$ die Gleichung

$$
(1+x) f_{\alpha}^{\prime}(x)-\alpha f_{\alpha}(x)=0
$$

besteht. Da aber $(1+x)^{\alpha}>0$ ist, so lehrt diese Gleichung, dafi der Quotient

$$
\frac{f_{\alpha}(x)}{(1+x)^{\alpha}}
$$

dort allenthalben die Ableitung 0 hat, dort also identisch ein und derselben Konstanten gleich ist. Da sich deren Wert für $x=0$ sofort als +1 ergibt, so ist die Behauptung $f_{\alpha}(x)=(1+x)^{\alpha}$ erneut bewiesen. 
angesehen werden kann, andererseits $0<\frac{a}{c}<2$ ist. Dann kann $\frac{a}{c}=1+x$ mit $|x|<1$ gesetzt werden und man hat in

$$
a^{\varrho}=c^{\varrho} \cdot(1+x)^{\varrho}=c^{\varrho}\left[1+\left(\begin{array}{l}
\varrho \\
1
\end{array}\right) x+\left(\begin{array}{l}
\varrho \\
2
\end{array}\right) x^{2}+\ldots\right]
$$

die gesuchte Entwicklung. So ist z. B.

$$
\begin{aligned}
\sqrt{2}=2^{\frac{1}{2}} & =\left(\frac{49}{25}\right)^{\frac{1}{2}} \cdot\left(\frac{50}{49}\right)^{\frac{1}{2}}=\frac{7}{5} \cdot\left(\frac{49}{50}\right)^{-\frac{1}{2}}=\frac{7}{5} \cdot\left[1-\frac{1}{50}\right]^{-\frac{1}{2}} \\
& =\frac{7}{5}\left[1-\left(\begin{array}{c}
-\frac{1}{2} \\
1
\end{array}\right) \frac{1}{50}+\left(\begin{array}{c}
-\frac{1}{2} \\
2
\end{array}\right) \frac{1}{50^{2}}-\left(\begin{array}{c}
-\frac{1}{2} \\
3
\end{array}\right) \frac{1}{50^{3}}+-\ldots\right]
\end{aligned}
$$

eine bequeme Entwicklung von $\sqrt{2}$.

Die Entdeckung der Binomialreihe durch Nereton ${ }^{\mathbf{1}}$ ) bildet einen der großen Marksteine in der Entwicklung der mathematischen Wissenschaften. Wie hoch sie von seinen Zeitgenossen bewertet wurde, mag man daraus ersehen, daß die Binomialreihe in den Sarkophag des Nerotonschen Grabes in der Westminster Abbey in London eingemeißelt steht. Später hat Abel diese Reihe erneut zum Gegenstande von Untersuchungen ${ }^{2}$ ) gemacht, die einen wohl gleich wichtigen Markstein in der Entwicklung der Reihentheorie bilden. (Vgl. weiter unten 170, 1 und $\mathbf{2 6 0}$ ).

\section{§ 26. Die logarithmische Reihe.}

Wie schon S. 55 betont, empfiehlt es sich für die Theorie, ausschließlich die sogenannten natürlichen Logarithmen, also diejenigen mit der Basis e zu benutzen. Im folgenden bedeutet also $\log x$ stets soviel wie ${ }^{e} \log x,(x>0)$.

Ist $y=\log x$, so ist $x=e^{y}$ oder

$$
x-1=y+\frac{y^{2}}{2 !}+\frac{y^{3}}{3 !}+\cdots
$$

Nach dem Umkehrsatz für Potenzreihen (107) ist hiernach $y=\log x$ für alle hinreichend nahe bei +1 gelegenen $x$ nach Potenzen von $(x-1)$ oder also $y=\log (1+x)$ für alle hinreichend kleinen $|x|$ in eine Potenzreihe in $x$

$$
y=\log (1+x)=x+b_{2} x^{2}+b_{3} x^{3}+\ldots
$$

entwickelbar. Es wäre wohl ein vergebliches Unterfangen, die Koeffizienten $b_{n}$ nach dem dortigen Verfahren allgemein berechnen zu wollen. Es muß uns genügen, durch den Umkehrsatz die Möglichkeit

1) Brief an Oldenburg vom 13. Juni 1676. - Newton besaß $\mathrm{B}$ damals noch keinen Beweis der Formel; dieser wurde erst 1774 von Euler gefunden,

2) J. f. d. reine u. angew. Math., Bd. 1 (1826), S. 311. 
dieser Entwicklung festgestellt $\mathrm{zu}$ haben. Zur Bestimmung der Koeffizienten $b_{n}$ müssen wir bequemere Wege suchen. Hier führt $z$. B. folgender Kunstgriff zum Ziel:

Es ist für $|x|<1$ und $z>0$

$$
(1+x)^{z}=e^{z \log (1+x)} \text {. }
$$

Benutzen wir nun links die Binomial-, rechts die Exponentialreihe, so erhalten wir

$$
\begin{gathered}
1+\left(\begin{array}{l}
z \\
1
\end{array}\right) x+\ldots+\left(\begin{array}{l}
z \\
k
\end{array}\right) x^{k}+\ldots \\
=1+z \log (1+x)+\ldots+\frac{z^{n} \log ^{n}(1+x)}{n !}+\ldots
\end{gathered}
$$

Die Glieder der linken Seite können wir nun durch Ausmultiplikation von

$$
\left(\begin{array}{l}
z \\
k
\end{array}\right)=\frac{z(z-1)(z-2) \ldots(z-k+1)}{k !}=\frac{(-1)^{k-1}}{k} z+\ldots+\frac{z^{k}}{k !}
$$

nach Potenzen von $z$ ordnen. Darf man dann den großen Umordnungssatz anwenden und die gleichen Potenzen von $z$ sammeln? Nach 90 sicher dann, wenn die Reihe $\Sigma\left(\begin{array}{l}z \\ k\end{array}\right) x^{k}$ noch konvergent bleibt, falls nach der eben beschriebenen Zerlegung ihrer Glieder jeder einzelne Summand durch seinen absoluten Betrag ersetzt wird. Das gibt abe $r$ da $z>0$ sein sollte, die Reihe

$$
\sum_{k=0}^{\infty} \frac{z(z+1)(z+2) \cdots(z+k-1)}{k !}|x|^{k} \text {. }
$$

Diese Reihe ist aber, da $|x|<1$ sein sollte, nichts andres als die Binomialentwicklung von $(1-|x|)^{-z}$ und ist also wirklich konvergent. Die demnach erlaubte Anwendung des großen Umordnungssatzes liefert nun

$$
1+\left[x-\frac{x^{2}}{2}+\frac{x^{3}}{3}-\ldots\right] z+\ldots=1+z \log _{r}(1+x)+\ldots
$$

Hier haben wir nun zwei Potenzreihen in $z$ vor uns, die für alle positiven $z$ - bei festem $|x|<1$ - übereinstimmen. Nach dem Identitätssatz für Potenzreihen 97 muß also speziell für jedes. $|x|<1$

$$
\text { 120. (a) } \log (1+x)=x-\frac{x^{2}}{2}+\frac{x^{3}}{3}-+\ldots+\frac{(-1)^{k-1}}{k} x^{k}+\ldots
$$

$\operatorname{sein}^{1}$ ). Damit ist die fragliche Entwicklung gewonnen, der man nun nachträglich auch ansieht, daß sie für $|x|>1$ nicht mehr gelten kann. Ersetzt man in dieser sogen. logarithmischen Reihe $x$ durch $-x$ und wechselt beiderseits das Vorzeichen, so erhalten wir gleichfalls für alle $|x|<1$ -

$$
\log \frac{1}{1-x}=x+\frac{x^{2}}{2}+\frac{x^{3}}{3}+\ldots+\frac{x^{n}}{n}+\ldots
$$

1) Vgl. hierzu die historischen Bemerkungen in $\mathbf{6 9}, 8$. 
und aus diesen durch Addition — wieder für alle $|x|<1$ gültig -

$$
\underbrace{\log \frac{1+x}{1-x}}=2\left[x+\frac{x^{3}}{3}+\frac{x^{5}}{5}+\ldots+\frac{x^{2 k+1}}{2 k+1}+\ldots\right] .
$$

Selbstverständlich führen noch mancherlei Wege zu diesen Reihenentwicklungen, doch schließen sich diese entweder nicht so unmittelbar an die Definition des log als Umkehrung der Exponentialfunktion an, oder sie machen von der Differential- und Integralrechnung ausgiebigeren Gebrauch ${ }^{\mathbf{1}}$ ).

Unsere Herleitung der logarithmischen Reihe - auch die beiden in der Fußnote genannten Arten - läßt nicht erkennen, ob die Darstellung auch noch für $x=+1$ und $x=-1$ gilt. Da wir aber aus 120 a für $x=+1$ die kọnvergente Reihe (s. 82,9)

$$
\mathbf{1}-\frac{\mathbf{1}}{\mathbf{2}}+\frac{\mathbf{1}}{\mathbf{3}}-\frac{\mathbf{1}}{4}+-\ldots
$$

erhalten, so ist nach dem Abelschen Grenzwertsatz der Wert dieser Reihe.

$$
=\lim _{x \rightarrow 1-0} \log (1+x)=\log 2 .
$$

Unsere Darstellung gilt also auch noch für $x=+1$; für $x=-1$ dagegen gilt sie sicher nicht mehr, da die Reihe dann divergiert.

\section{$\S 2 \%$ Die zyklometrischen Funktionen.}

$\mathrm{Da}$ die trigonometrischen Funktionen sin und tg in Potenzreihen entwickelbar sind, bei denen die erste Potenz der Veränderlichen den von 0 verschiedenen Koeffizienten +1 besitzt, so ist dies nach 107 auch mit ihren Umkehrungen, den sog. zyklometrischen Funktionen arcsin und arctg der Fall. Wir haben also für alle hinreichend kleinen Werte von $|x|$ die Ansätze

$$
\begin{aligned}
& y=\arcsin x=x+b_{3} x^{3}+b_{5} x^{5}+\ldots \\
& y=\operatorname{arctg} x=x+b_{3}{ }^{\prime} x^{3}+b_{5}{ }^{\prime} x^{5}+\ldots,
\end{aligned}
$$

1) Wir deuten noch die folgenden beiden Wege an:

1. Da wir aus dem Umkehrsatz wissen, daf

$$
\log (1+x)=x+b_{2} x^{2}+b_{3} x^{3}+\ldots
$$

gesetzt werden darf, hat man nach der Taylorschen Reihe $\mathbf{9 9}$

$$
b_{k}=\frac{1}{k !}\left(\frac{d^{k} \log (1+x)}{d x^{k}}\right)_{x=0}=\frac{(-1)^{k-1}}{k} .
$$

2. $\mathrm{Da} \frac{d \log (1+x)}{d x}=\frac{1}{1+x}=1-x+x^{2}-+\ldots+(-1)^{k} x^{k}+\ldots \equiv \sum_{n=0}^{\infty}(-1)^{k} x^{k}$ und da $\log 1=0$ ist, folgt nach 99, Satz 5 durch Integration sofort

$$
\log (1+x)=\sum_{k=0}^{\infty} \frac{(-1)^{k}}{k+1} x^{k+1}=\sum_{k=1}^{\infty} \frac{(-1)^{k-1}}{k} x^{k} .
$$

Der im Text beschrittene Weg ist insofern einfacher, als er vollständig ohne Benutzung der Differential- und Integralrechnung auskommt. 
bei denen wir die geraden Potenzen gleich fortgelassen haben, weil unsere Funktionen ungerade sind. Auch hier wäre es aussichtslos, die Koeffizienten $b$ und $b^{\prime}$ nach dem allgemeinen Verfahren von 107 bestimmen zu wollen. Wir gehen wieder bequemere Wege: Die Reihe für $\operatorname{arctg} x$ ist die Umkehrung von

$$
x=\operatorname{tg} y=\frac{\sin y}{\cos y}=\frac{y-\frac{y^{3}}{3 !}+\frac{y^{5}}{5 !}-+\cdots}{1-\frac{y^{2}}{2 !}+\frac{y^{4}}{4 !}-+\cdots},
$$

bzw. der nach Ausführung der letzten Division nach 105, 4 entstehenden Reihe. Stünden hier in Zähler und Nenner lauter +- Zeichen, so handelte es sich um die Umkehrung der Funktion

$$
x=\frac{e^{y}-e^{-y}}{e^{y}+e^{-y}}=\frac{e^{2 y}-1}{e^{2 y}+1} .
$$

Die Umkehrung dieser Funktion ist aber

$$
\frac{1}{2} \log \frac{1+x}{1-x}=x+\frac{x^{3}}{3}+\frac{x^{5}}{5}+\ldots
$$

Man überzeugt sich nun ganz ähnlich, wie in $\S 24$, S. 195/6 bei der Herleitung der Potenzreihe von $\pi x \operatorname{ctg} \pi x$, daß die Umkehrung der tatsächlich vorgelegten Reihe für $x=\operatorname{tg} y$ aus dieser letzten gewonnen wird, indem wir in ihr wieder alternierende Vorzeichen nehmen, so daß

$$
\operatorname{arctg} x=x-\frac{x^{3}}{3}+\frac{x^{5}}{5}-+\ldots
$$

wird $^{1}$ ). Diese Potenzreihe, die ersichtlich den Radius 1 hat, liefert also, wenn sie für $y$ in den rechterhand in $(a)$ stehenden Quotienten eingesetzt und die sicher erlaubte Umordnung vorgenommen wird, $\equiv x$. Ihre Summe ist also bei beliebig gegebenem $|x|<1$ eine Lösung der Gleichung $\operatorname{tg} y=x$ und zwar der sogenannte Hauptwert der hier-

1) Eine andere Herleitung ist diese: Es ist

$$
\frac{d \operatorname{arctg} x}{d x}=\frac{1}{\frac{d \operatorname{tg} y}{d y}}=\frac{1}{1+\operatorname{tg}^{2} y}=\frac{1}{1+x^{2}}=1-x^{2}+x^{4}-+\ldots,
$$

letzteres für $|x|<1$. Da $\operatorname{arctg} 0=0$ ist, folgt hieraus wieder nach 99, Satz 5, dafs für $|x|<1$

$$
\operatorname{arctg} x=x-\frac{x^{3}}{3}+\frac{x^{5}}{5}-+\cdots
$$

ist. - Eine Herleitung, die der in der vorigen Fufnote an erster Stelle gegebenen entspricht, ist hier etwas mühsamer, da die höheren Ableitungen von $\operatorname{arctg} x$ - selbst nur an der Stelle 0 - nicht leicht direkt zu finden sind. Die arctg-Entwicklung ist von J. Gregory im Jahre 1671 gefunden, aber erst 1712 bekannt geworden. 
durch erklärten Funktion $\operatorname{arctg} x, \mathrm{~d}$. h. derjenige Wert, der für $x=0$ selbst $=0$ ist und sich dann mit $x$ stetig ändert. Er liegt für

$$
-1<x<+1 \quad \text { zwischen } \quad-\frac{\pi}{4} \text { und }+\frac{\pi}{4}
$$

und ist innerhalb dieses Intervalles eindeutig bestimmt.

Für $|x|>1$ ist die gefundene Entwicklung sicher nicht mehr gültig; dagegen ergibt sich nach dem Abelschen Grenzwertsatz, daß sie auch noch für $x= \pm 1$ gilt. Denn die Reihe ist noch an beiden Endpunkten des Konvergenzintervalles konvergent und $\operatorname{arctg} x$ ist dort stetig. Wir haben also insbesondere die wegen ihrer Durchsichtigkeit und Einfachheit besonders bemerkenswerte Reihe

$$
\frac{\pi}{4}=1-\frac{1}{3}+\frac{1}{5}-\frac{1}{7}+-\ldots
$$

und damit zugleich einen ersten zur Berechnung von $\pi$ schon einigermaßen gangbaren Weg. Diese schöne Gleichung wird meist nach Leibni $\boldsymbol{z}^{\mathbf{1}}$ ) benannt; sie liefert sozusagen die Zahl $\pi$ der rein arithmetischen Behandlung aus. Es ist, als ob der Schleier, der über dieser seltsamen Zahl lag, durch diese Entwicklung fortgezogen sei.

Für die Herleitung der Reihe für $\arcsin x$ führt der Weg, der dem eben bei $\operatorname{arctg} x$ begangenen entspricht, nicht zum Ziel. Das in der letzten Fußnote angedeutete Verfahren aber liefert uns die gesuchte Reihe: Es ist

$$
\frac{d \arcsin x}{d x}=\frac{1}{\left(\frac{d \sin y}{d y}\right)}=\frac{1}{\cos y}=\frac{1}{\sqrt{1-x^{2}}}=\left(1-x^{2}\right)^{-\frac{1}{2}},
$$

letzteres, weil die Ableitung von $\arcsin x$ im Intervall $-1 \ldots+1$ stets positiv ist. Aus

$$
(\arcsin x)^{\prime}=1-\left(\begin{array}{c}
-\frac{1}{2} \\
1^{2}
\end{array}\right) x^{2}+\left(\begin{array}{c}
-\frac{1}{2} \\
2
\end{array}\right) x^{4}-+\ldots
$$

folgt aber wegen $\arcsin 0=0$ nach 99, Satz 5 sofort, daß für $|x|<1$

$$
\arcsin x=x+\frac{1}{2} \frac{x^{3}}{3}+\frac{1 \cdot 3}{2 \cdot 4} \cdot \frac{x^{5}}{5}+\frac{1 \cdot 3 \cdot 5}{2 \cdot 4 \cdot 6} \cdot \frac{x^{7}}{7}+\ldots
$$

ist. Auch diese Potenzreihe hat den Radius 1, und ganz entsprechende Gründe, wie eben, lehren, daß für $|x|<1$ ihre Summe der Hauptwert von $\arcsin x$ ist, also diejenige eindeutig bestimmte Lösung $y$ der Gleichung $\sin y=x$, die zwischen $-\frac{\pi}{2}$ und $+\frac{\pi}{2}$ gelegen ist.

Für $x= \pm 1$ ist diese Gleichung noch nicht gesichert. Sie wird dort nach dem Abelschen Grenzwertsatz dann und nur dann gelten, wenn die Reihe daselbst konvergiert. Diese Konvergenz findet

1) Er fand sie wahrscheinlich im Jahre 1673. 
tatsächlich statt. Wegen des einfachen Vorzeichenwechsels beim Übergang von $+x \mathrm{zu}-x$ brauchen wir dies nur für die Stelle $+1 \mathrm{zu}$ zeigen. Dort handelt es sich um eine Reihe mit lauter positiven Gliedern, und es genügt, die Beschränktheit der Teilsummen zu zeigen. Nun ist für $0<x<1$, wenn wir die Teilsummen von $123 \mathrm{mit}$ $s_{n}(x)$ bezeichnen,

$$
s_{n}(x)<\arcsin x<\arcsin 1=\frac{\pi}{2} .
$$

Und da dies (bei festem $n$ ) für jedes positive $x<1$ gilt, so ist auch

$$
\lim _{x \rightarrow 1} s_{n}(x)=s_{n}(1) \leqq \frac{\pi}{2}
$$

und da dies für jedes $n$ gilt, so ist damit schon alles bewiesen. Es ist also

$$
\frac{\pi}{2}=1+\frac{1}{2} \cdot \frac{1}{3}+\frac{1 \cdot 3}{2 \cdot 4} \cdot \frac{1}{5}+\frac{1 \cdot 3 \cdot 5}{2 \cdot 4 \cdot 6} \cdot \frac{1}{7}+\ldots
$$

Durch die $\$ \S 22-27$ sind wir in den Besitz der für alle Anwendungen wichtigsten Potenzreihen gelangt.

\section{Aufgaben zum VI. Kapitel.}

74. Man zeige, dafi die Potenzreihenentwicklungen der folgenden Funktionen die ang $€$ gebene Form haben:

a) $e^{x} \sin x=\sum_{n=0}^{\infty} \frac{s_{n}}{n !} x^{n}$ mit $s_{n}=\sqrt{2}^{n} \cdot \sin n \frac{\pi}{4}$, d. h. $s_{4 k}=0$,

$$
s_{4 k+1}=(-1)^{k} 2^{2 k}, s_{4 k+2}=(-1)^{k} 2^{2 k+1}, s_{4 k+3}=(-1)^{k} 2^{2 k+1} ;
$$

b) $\frac{1}{2}(\operatorname{arctg} x)^{2}=\sum_{k=1}^{\infty}(-1)^{k-1} b_{k} \cdot \frac{x^{2 k}}{2 k} \quad$ mit $\quad b_{k}=1+\frac{1}{3}+\frac{1}{5}+\ldots+\frac{1}{2 k-1}$

c) $\frac{1}{4} \operatorname{arctg} x \cdot \log \frac{1+x}{1-x}=\sum_{k=0}^{\infty} c_{k} \cdot \frac{x^{4 k+2}}{4 k+2}$ mit $c_{k}=1-\frac{1}{3}+\frac{1}{5}-\cdots+\frac{1}{4 k+1}$;

d) $\frac{1}{2} \operatorname{arctg} x \cdot \log \left(1+x^{2}\right)=\sum_{k=1}^{\infty}(-1)^{k-1} h_{2 k} \frac{x^{2 k+1}}{2 k+1}$ mit $h_{n}=1+\frac{1}{2}+\ldots+\frac{1}{n}$

e) $\frac{1}{2}\left[\log \frac{1}{1-x}\right]^{2}=\sum_{n=2}^{\infty} \frac{h_{n-1}}{n} x^{n}$, mit derselben Bedeutung von $h_{n}$, wie eben.

75. Man zeige, dafi die Potenzreihenentwicklungen der folgenden Funktionen in der angegebenen Weise beginnen:

a) $\frac{x}{\log \frac{1}{1-x}}=1-\frac{x}{2}-\frac{x^{2}}{12}-\frac{x^{3}}{24}-\ldots$; 

b) $(1-x) e^{x+\frac{x^{2}}{2}+\ldots+\frac{x^{m}}{m}}=1-\frac{x^{m+1}}{m+1}+\ldots,(m \geqq 1)$;
c) $\operatorname{tg}(\sin x)-\sin (\operatorname{tg} x)=\frac{1}{30} x^{7}+\frac{29}{756} x^{9}+\ldots$;
d) $\frac{1}{e}(1+x)^{\frac{1}{x}}=1-\frac{x}{2}+\frac{11}{24} x^{2}-\frac{7}{16} x^{3}+\frac{2447}{5760} x^{4}-\frac{959}{2304} x^{5}+\ldots$;
e) $\frac{x^{2}}{x-\log (1+x)}=2+\frac{4}{3} x-\frac{1}{9} x^{2}+\frac{8}{135} x^{3}+\ldots$

76. Man leite im Anschluf an 105, 5, 115 und 116 die Potenzreihenentwicklungen der folgenden Funktionen her:
a) $\log \cos x$;
b) $\log \frac{\sin x}{x}$
c) $\log \frac{\operatorname{tg} x}{x}$;
d) $\frac{x}{\sin x}$;
e) $\frac{x^{2}}{1-\cos x}$;
f) $\frac{1}{\cos x}$;
g) $\log \frac{x}{2 \sin \frac{1}{2} x}$;
h) $\frac{1}{e^{x}+1}$;
i) $\frac{e^{x}}{e^{x}+1}$;
k) $\frac{1}{\cos x-\sin x}$.

77. Es ist

$$
\begin{aligned}
\frac{1}{\sqrt{1-x}} \cdot\left[\frac{1}{a}+\frac{1}{2} \frac{x}{a+2}+\frac{1 \cdot 3}{2 \cdot 4} \cdot \frac{x^{2}}{a+4}+\ldots\right] & = \\
& =\frac{1}{2}\left[1+\frac{a+1}{a+2} x+\frac{(a+1)(a+3)}{(a+2)(a+4)} x^{2}+\ldots\right] .
\end{aligned}
$$

78. Es strebt $\left(\frac{2 n+1}{2 n-1}\right)^{n} \rightarrow e$. Geschieht dies monoton? Steigend oder fallend? Wie verhalten sich die Folgen

$$
\left(1+\frac{1}{n}\right)^{n+\alpha}, \quad 0<\alpha<1 \text { ? }
$$

79. Aus $x_{n} \rightarrow \xi$ folgt stets

$$
\left(1+\frac{x_{n}}{n}\right)^{n} \rightarrow e^{\xi}
$$

und, falls alle $x_{n}$ und $\xi$ positiv sind, auch

$$
n\left(\sqrt[n]{x_{n}}-1\right) \rightarrow \log \xi .
$$

80. Ist $\left(x_{n}\right)$ eine beliebige reelle Folge, für die $\frac{x_{n}{ }^{2}}{n} \rightarrow 0$ strebt, und wird $\left(1-\frac{x_{n}}{n}\right)^{n}=y_{n}$ gesetzt, so ist stets

$$
y_{n} \cong e^{-x_{n}}
$$


81. Man beweise die unter 114 aufgeführten Ungleichungen.

82. Man suche die Summe der folgenden Reihen durch die elementaren Funktionen geschlossen darzustellen:

a) $\frac{1}{2}+\frac{x}{5}+\frac{x^{2}}{8}+\frac{x^{3}}{11}+\ldots$

(Anleitung: Ist $f(x)$ die gesuchte Funktion, so ist ersichtlich

$$
\left(x^{2} \cdot f\left(x^{3}\right)\right)^{\prime}=\frac{x}{1-x^{3}},
$$

woraus sich $f(x)$ bestimmen läfit. Ähnlich bei den folgenden Beispielen.)

b) $\frac{x^{3}}{1 \cdot 3}-\frac{x^{5}}{3 \cdot 5}+\frac{x^{7}}{5 \cdot 7}-\frac{x^{9}}{7 \cdot 9}+\cdot-$;

c) $\frac{1}{1 \cdot 2 \cdot 3}+\frac{x}{2 \cdot 3 \cdot 4}+\frac{x^{2}}{3 \cdot 4 \cdot 5}+\cdots$;

d) $x+\frac{x^{3}}{3}-\frac{x^{5}}{5}-\frac{x^{2}}{7}++-\cdots$

83. Man ermittle die folgenden Reihensummen als spezielle Werte elementarer Funktionen:

a) $\frac{1}{2 !}+\frac{2}{3 !}+\frac{3}{4 !}+\frac{4}{5 !}+\ldots=1$;

b) $\frac{1}{2}+\frac{1}{2 \cdot 4}+\frac{1 \cdot 3}{2 \cdot 4 \cdot 6}+\frac{1 \cdot 3 \cdot 5}{2 \cdot 4 \cdot 6 \cdot 8}+\ldots=1$;

c) $\frac{1}{2}+\frac{1 \cdot 3}{2 \cdot 4 \cdot 6}+\frac{1 \cdot 3 \cdot 5 \cdot 7}{2 \cdot 4 \cdot 6 \cdot 8 \cdot 10}+\frac{1 \cdot 3 \cdot 5 \cdot 7 \cdot 9 \cdot 11}{2 \cdot 4 \cdot 6 \cdot 8 \cdot 10 \cdot 12 \cdot 14}+\ldots=\frac{1}{2} \sqrt{2}$

d) $\frac{1}{2}-\frac{1 \cdot 3}{2 \cdot 4 \cdot 6}+\frac{1 \cdot 3 \cdot 5 \cdot 7}{2 \cdot 4 \cdot 6 \cdot 8 \cdot 10}-\frac{1 \cdot 3 \cdot 5 \cdot 7 \cdot 9 \cdot 11}{2 \cdot 4 \cdot 6 \cdot 8 \cdot 10 \cdot 12 \cdot 14}+-\ldots=\sqrt{\frac{\sqrt{2}-1}{2}}$.

84. Aus den Partialbruchzerlegungen $117 \mathrm{ff}$. sollen die folgenden Darstellungen von $\pi$ hergeleitet werden:

$$
\begin{aligned}
& \pi=\alpha \cdot \operatorname{tg} \frac{\pi}{\alpha}\left[1-\frac{1}{\alpha-1}+\frac{1}{\alpha+1}-\frac{1}{2 \alpha-1}+\frac{1}{2 \alpha+1}-\ldots\right] \\
& \pi=\alpha \sin \frac{\pi}{\alpha}\left[1-\frac{1}{\alpha-1}-\frac{1}{\alpha+1}+\frac{1}{2 \alpha-1}+\frac{1}{2 \alpha+1}--++\ldots\right],
\end{aligned}
$$

in denen $\alpha \neq 0, \pm 1, \pm \frac{1}{2}, \pm \frac{1}{3}, \ldots$ sein soll. Man setze speziell $\alpha=3,4,6$. 
VII. Kapitel.

\section{Unendliche Produkte.}

\section{\$28. Produkte mit positiven Gliedern.}

Ein unendliches Produkt

$$
u_{1} \cdot u_{2} \cdot u_{3} \cdot \ldots \cdot u_{n} \cdot \ldots
$$

sollte nach $\S 11$, II lediglich ein anderes Symbol für die Folge der Teilprodukte

$$
p_{n}=u_{1} \cdot u_{2} \cdot \ldots \cdot u_{n}
$$

sein: Danach müßte man ein solches unendliches Produkt konvergent mit dem Werte $U$ nennen, also

$$
\prod_{n=1}^{\infty} u_{n}=U
$$

setzen, wenn die Folge $\left(p_{n}\right)$ gegen die Zahl $U$ als ihren Grenzwert strebt. Das bringt die Unzuträglichkeit mit sich, daß dann jedes Produkt konvergent genannt werden müßte, bei dem nur ein einziger Faktor $=0$ ist. Denn ist $u_{m}=0$, so würde auch $p_{n}=0$ sein für alle $n \geqq m$ und es würde $p_{n} \rightarrow U=0$ streben. Ebenso wäre ersichtlich jedes Produkt konvergent - und wieder mit dem Werte $0-$, bei dem von einer Stelle ab für alle $n$

$$
\left|u_{n}\right| \leqq \vartheta<1
$$

ist. Um diese nichtssagenden Fälle auszuschließen, beschreibt man das Konvergenzverhalten eines unendlichen Produktes nicht ohne weiteres durch dasjenige der Folge der Teilprodukte, sondern benutzt zweckmäßiger die folgende Definition, die der Sonderrolle der Null bei der Multiplikation Rechnung trägt:

- Definition. Das unendliche Produkt

$$
\prod_{n=1}^{\infty} u_{n} \equiv u_{1} \cdot u_{2} \cdot u_{3} \cdot \ldots
$$

soll dann und nur dann (im engeren Sinne) konvergent heißen, wenn von einer Stelle $a b-$ etwa fïr alle $n>m-$ kein Faktor mehr verschwindet und wenn die hinter dieser Stelle beginnenden Teilprodukte

$$
p_{n}=u_{m+1} \cdot u_{m+2} \cdot \ldots \cdot u_{n}
$$$$
(n>m)
$$ 
mit wachsendem $n$ gegen einen endlichen und von 0 verschiedenen Grenzwert streben. Setzt man diesen $=U_{m}$, so wird die Zahl

$$
U=u_{1} \cdot u_{2} \cdot \ldots \cdot u_{m} \cdot U_{m}
$$

als Wert des Produktes angesehen ${ }^{\mathbf{1}}$ ).

Dann gilt zunächst, wie für endliche Produkte der

- Satz 1. Ein konvergentes unendliches Produkt hat dann und nur dann den Wert 0 , wenn einer seiner Faktoren $=0$ ist.

Da ferner mit $p_{n} \rightarrow U_{m}$ auch $p_{n-1} \rightarrow U_{m}$ strebt und da $U_{m} \neq 0$ sein sollte, so strebt (nach $\mathbf{4 1}, 11)$

und wir haben den

$$
u_{n}=\frac{p_{n}}{p_{n-1}} \rightarrow 1
$$

- Satz 2. In einem konvergenten unendlichen Produkt strebt die Folge der Faktoren stets $\rightarrow 1$.

Aus diesem Grunde wird es zweckmäßiger sein, die Faktoren $u_{n}=1+a_{n}$ zu setzen und also die Produkte in der Form

$$
\prod_{n=1}^{\infty}\left(1+a_{n}\right)
$$

zugrunde zu legen. Für diese ist dann die Bedingung $a_{n} \rightarrow 0$ eine notwendige Konvergenzbedingung. Die Zahlen $a_{n}$ wollen wir - als die wesentlichsten Teile der Faktoren - als die Glieder des Produktes bezeichnen. Sind sie alle $\geqq 0$, so sprechen wir, ähnlich wie bei den unendlichen Reihen, von Produkten mit positiven Gliedern. Mit diesen wollen wir uns zunächst befassen.

Die Konvergenzfrage wird hier schon vollständig erledigt durch den

Satz 3. Ein Produkt $I\left(1+a_{n}\right)$ mit positiven Gliedern $a_{n}$ ist dann und nur dann konvergent, wenn die Reihe $\sum_{i} a_{n}$ konvergiert.

Beweis. Da die Teilprodukte $p_{n}=\left(1+a_{1}\right) \ldots\left(1+a_{n}\right)$ wegen $a_{n} \geqq 0$ monoton wachsen, so ist das 1 . Hauptkriterium $(\mathbf{4 6})$ zuständig und wir haben nur zu zeigen, daß die Teilprodukte $p_{n}$ dann und nur

1) Unendliche Produkte finden sich zuerst bei F. Vieta (Opera, Leyden 1646, S. 400), der das Produkt

$$
\frac{2}{\pi}=\sqrt{\frac{1}{2}} \cdot \sqrt{\frac{1}{2}+\frac{1}{2} \sqrt{\frac{1}{2}}} \cdot \sqrt{\frac{1}{2}+\frac{1}{2} \sqrt{\frac{1}{2}+\frac{1}{2} \sqrt{\frac{1}{2}}}} \ldots,
$$

(vgl. Aufg 89) und bei $J$. Wallis (Opera I, Oxford 1695, S.468), der 1656 das Produkt

$$
\frac{\pi}{4}=\frac{2}{3} \cdot \frac{4}{3} \cdot \frac{4}{5} \cdot \frac{6}{5} \cdot \frac{6}{7} \ldots
$$

angibt. Erst durch Euler aber erhielten die unendlichen Produkte Bürgerrecht in der Mathematik, indem er zahlreiche wichtige Produktenwicklungen aufstellte. Die ersten Konvergenzkriterien riihren von Cauchy her. 
dann beschränkt bleiben, wenn die Teilsummen $s_{n}=a_{1}+a_{2}+\ldots+a_{n}$ es tun. Nun ist aber nach $\mathbf{1 1 4} \alpha$ stets $1+a_{v} \leqq e^{a_{\nu}}$ und also

andererseits ist

$$
p_{n} \leqq e^{s_{n}}
$$

$p_{n}=\left(1+a_{1}\right) \ldots\left(1+a_{n}\right)=1+a_{1}+a_{2}+\ldots+a_{n}+a_{1} a_{2}+\ldots \geqq s_{n}$, letzteres, weil in dem ausgeführten Produkt außer den Summanden von $s_{n}$ noch viele andere, aber nur nichtnegative Summanden auftreten. Es ist also stets

$$
s_{n} \leqq p_{n} .
$$

Die erste Abschätzung lehrt nun, daß mit $s_{n}$ auch $p_{n}$ beschränkt bleibt, die zweite lehrt das umgekehrte, — womit schon alles bewiesen ist $^{1}$ ).

Beispiele.

1. Da wir schon viele Beispiele konvergenter Reihen $\Sigma a_{n}$ mit positiven Gliedern kennen, so ergeben sich auf Grund von Satz 3 ebensoviele Beispiele von konvergenten Produkten $\Pi\left(1+a_{n}\right)$. Wir erwähnen:

$$
\Pi\left(1+\frac{1}{n^{\alpha}}\right) \text { ist für } \alpha>1 \text { konvergent, für } \alpha \leqq 1 \text { divergent. - Das letz- }
$$

tere ist hier leichter $z \mathfrak{u}$ erkennen als bei der entsprechenden Reihe, da strebt $\left.{ }^{2}\right)$.

$$
\left(1+\frac{1}{1}\right)\left(1+\frac{1}{2}\right) \ldots\left(1+\frac{1}{n}\right)=\frac{2}{1} \cdot \frac{3}{2} \cdot \frac{4}{3} \ldots \frac{n+1}{n}=n+1 \rightarrow+\infty
$$

2. $\Pi\left(1+x^{n}\right)$ ist fuir $0 \leqq x<1$ konvergent; ebenso $\Pi\left(1+x^{2^{n}}\right)$.

3. $\prod_{n=2}^{\infty}\left(1-\frac{2}{n(n+1)}\right) \equiv \prod_{n=2}^{\infty} \frac{(n-1)(n+2)}{n(n+1)}=\frac{1}{3}$.

Dem Satz 3 stellen wir sogleich den folgenden sehr ähnlichen an die Seite:

Satz 4. Ist stets $a_{n} \geqq 0$, so ist auch das Produkt $\Pi\left(1-a_{n}\right)$ dann und nur dann konvergent, wenn $\sum a_{n}$ konvergiert.

Beweis. Strebt $a_{n}$ nicht gegen 0 , so sind Reihe und Produkt sicher divergent. Strebt aber $a_{n} \rightarrow 0$, so ist von einer Stelle an, etwa

1) Der erste Teilbeweis dieses elementaren Satzes benutzt die transzendente Exponentialfunktion. Folgendermaßen kann man sich davon frei machen: Ist $\Sigma a_{n}=s$ konvergent, so wähle man $m$ so, dafs für $n>m$ stets

bleibt. Da nun ersichtlich für diese $n$

$$
a_{m+1}+a_{m+2}+\ldots+a_{n}<\frac{1}{2}
$$

$$
\left(1+a_{m+1}\right) \ldots\left(1+a_{n}\right) \leqq 1+\left(a_{m+1}+\ldots+a_{n}\right)+\left(a_{m+1}+\ldots+a_{n}\right)^{2}+\ldots
$$

ist, so ist für alle $n$ stets

$$
+\left(a_{m+1}+\ldots+a_{n}\right)^{n}<2
$$

also $\left(p_{n}\right)$ beschränkt.

$$
p_{n}<2 \cdot\left(1+a_{1}\right) \ldots\left(1+a_{m}\right)=K,
$$

2) Hierin steckt also auf Grund von Satz 3 ein neuer Divergenzbeweis für die Reihe $\Sigma \frac{1}{n}$. 
für $n>m$, stets $a_{n}<\frac{1}{2}$ und also $1-a_{n}>\frac{1}{2}$. Wir betrachten Reihe und Produkt erst von dieser Stelle an.

Ist nun das Produkt konvergent, so streben also die monoton abnehmenden Teilprodukte $p_{n}=\left(1-a_{m+1}\right) \ldots\left(1-a_{n}\right)$ gegen eine noch positive Zahl $U_{m}$, und es ist für alle $n>m$ stets

$$
\left(1-\dot{a}_{m+1}\right) \ldots\left(1-a_{n}\right) \geqq U_{m}>0 .
$$

Da nun für $0 \leqq a_{\nu}<1$ stets

$$
\left(1+a_{v}\right) \leqq \frac{1}{1-a_{v}}
$$

ist (wie man durch Heraufmultiplizieren des Nenners sofort sieht), so ist

$$
\left(1+a_{m+1}\right)\left(1+a_{m+2}\right) \ldots\left(1+a_{n}\right) \leqq \frac{1}{U_{m}}
$$

Hiernach folgt also aus der Konvergenz von $\Pi\left(1-a_{n}\right)$ diejenige des Produktes $\Pi\left(1+a_{n}\right)$ und also die der Reihe $\Sigma a_{n}$. - Ist umgekehrt $\sum a_{n}$ konvergent, so konvergiert auch $\sum 2 a_{n}$ und wir benutzen nun die Tatsache, daß für $0 \leqq a_{n} \leqq \frac{1}{2}$ stets

$$
1-a_{\nu} \geqq \frac{1}{1+2 a_{v}}
$$

ist - wie man wieder durch Heraufmultiplizieren des Nenners erkennt. Da nun bei passender Wahl von $K$ die Produkte $\left(1+2 a_{m+1}\right) \ldots$ $\left(1+2 a_{n}\right)<K$ bleiben, so bleibt

$$
\left(1-a_{m+1}\right) \ldots\left(1-a_{n}\right)>\frac{1}{K}>0
$$

und die linksstehenden Teilprodukte streben also, weil monoton abnehmend, gegen einen noch positiven Grenzwert: das Produkt $\Pi\left(1-a_{n}\right)$ ist konvergent.

1. $\prod_{n=2}^{\infty}\left(1-\frac{1}{n^{\alpha}}\right)$ ist für $\alpha>1$ konvergent, für $\alpha \leq 1$ divergent.

2. Sind die $a_{n}<1$ und ist $\Sigma a_{n}$ divergent, so ist $I\left(1-a_{n}\right)$ gemäß unserer Bezeichnung nicht konvergent. Da aber die Teilprodukte $p_{n}$ monoton abnehmen und $>0$ bleiben, so haben sie einen Grenzwert, der aber nun notwendig $=0$ sein mufs. Man sagt: das Produkt divergiere gegen 0 . Die Sonderrolle der 0 zwingt uns hier also eine kleine Inkonsequenz in der Bezeichnung auf: Es wird ein Produkt divergent genannt, für das die Teilprodukte eine durchaus konvergente Folge, nämlich eine Nullfolge, $\left(p_{n}\right)$ bilden. An diese Inkonsequenz soll bei der Definition 125 der'Zusatz ,im engeren Sinne" bei "konvergent" erinnern, falls dies erforderlich ist.

leicht aus

3. Daf z. B. $\prod_{n=2}^{\infty}\left(1-\frac{1}{n}\right)$ gegen 0 divergiert, erkennt man wieder sehr

$$
p_{n}=\left(1-\frac{1}{2}\right)\left(1-\frac{1}{3}\right) \ldots\left(1-\frac{1}{n}\right)=\frac{1}{2} \cdot \frac{2}{3} \cdot \frac{3}{4} \cdot \ldots \cdot \frac{n-1}{n}=\frac{1}{n} \rightarrow 0 .
$$




\section{\$29. Produkte mit beliebigen Gliedern ${ }^{1}$ ). Absolute Konvergenz.}

Haben die Glieder des Produktes $a_{n}$ beliebige Vorzeichen, so gilt - dem 2. Hauptkriterium $\mathbf{8 1}$ bei Reihen entsprechend - der

- Satz 5. Das unendliche Produkt $\Pi\left(1+a_{n}\right)$ ist dann und nur dann konvergent, wenn nach Wahl von $\varepsilon>0$ ein $n_{0}$ so bestimmt werden kann, daß fïr alle $n>n_{0}$ und alle $k \geqq 1$ stets

ausfällt²).

$$
\left|\left(1+a_{n+1}\right)\left(1+a_{n+2}\right) \ldots\left(1+a_{n+k}\right)-1\right|<\varepsilon
$$

Beweis. a) Ist das Produkt konvergent, so ist von einer Stelle $\mathrm{ab}$, etwa für $n>m$, stets $a_{n} \neq-1$, und es streben die Teilprodukte

$$
p_{n}=\left(1+a_{m+1}\right) \ldots\left(1+a_{n}\right), \quad(n>m),
$$

gegen einen von 0 verschiedenen Grenzwert. Daher gibt es (s. 41, 3) eine positive Zahl $\beta$, so daß für $n>m$ stets $\left|p_{n}\right| \geqq \beta>0$ bleibt. Nach dem 2. Hauptkriterium $\mathbf{4 9}$ kann nun, wenn $\varepsilon>0$ gegeben wird, $n_{0}$ so bestimmt werden, $\mathrm{da} \beta$ für $n>n_{0}$ und alle $k \geqq 1$ stets

$$
\left|p_{n+k}-p_{n}\right|<\varepsilon \cdot \beta
$$

bleibt. Dann ist aber für dieselben $n$ und $k$

$$
\left|\frac{p_{n+k}}{p_{n}}-1\right|=\left|\left(1+a_{n+1}\right)\left(1+a_{n+2}\right) \ldots\left(1+a_{n+k}\right)-1\right|<\varepsilon,
$$

- und das ist gerade die erste unserer Behauptungen.

b) Ist umgekehrt die $\varepsilon$-Bedingung des Satzes erfüllt, so wähle man zunächst $\varepsilon=\frac{1}{2}$ und bestimme $m$ so, daß für $n>m$ stets

$$
\left|\left(1+a_{m+1}\right) \ldots\left(1+a_{n}\right)-1\right|=\left|p_{n}-1\right|<\frac{1}{2}
$$

bleibt. Dann ist für diese $n$ stets

$$
\frac{1}{2}<\left|p_{n}\right|<\frac{3}{2}
$$

und dies lehrt, daß für $n>m$ stets $1+a_{n} \neq 0$ sein muß; und weiter, daß, wenn $p_{n}$ gegen einen Grenzwert strebt, dieser sicher nicht 0 sein kann. Nun läßt sich aber bei gegebenem $\varepsilon>0$ die Zahl $n_{0}$ so bestimmen, $\mathrm{da} ß$ für $n>n_{0}$ und alle $k \geqq 1$

$$
\left|\frac{p_{n+k}}{p_{n}}-1\right|<\frac{\varepsilon}{2}
$$

1) Eine ausführliche und einheitliche Darstellung der Konvergenztheorie unendlicher Produkte findet man bei A. Pringsheim, Über die Konvergenz unendlicher Produkte, Math. Annalen Bd. 33 (1889), S. 119-154.

2) Oder - s. 81, 2. Form - wenn stets

$$
\left[\left(1+a_{n+1}\right)\left(1+a_{n+2}\right) \ldots\left(1+a_{n+k_{n}}\right)\right] \rightarrow 1
$$

strebt; oder - s. 81, 3. Form - wenn stets

strebt.

$$
\left[\left(1+a_{v_{n}+1}\right) \cdots\left(1+a_{v_{n}+k_{n}}\right)\right] \rightarrow 1
$$


oder also

$$
\left|p_{n+k}-p_{n}\right|<\left|p_{n}\right| \cdot \frac{\varepsilon}{2}<\varepsilon
$$

bleibt. Und dies lehrt, $d a \beta p_{n}$ wirklich einen Grenzwert hat. Damit ist die Konvergenz des Produktes bewiesen.

Ähnlich wie bei den unendlichen Reihen sind auch hier bei den Produkten diejenigen besonders leicht zu behandeln, die ,absolut“ konvergieren. Doch versteht man darunter nicht Produkte $\Pi u_{n}$, für die auch $\Pi\left|u_{n}\right|$ konvergiert - denn eine solche Festsetzung wäre nichtssagend, da ja dann jedes konvergente Produkt auch absolut konvergieren würde - , sondern man definiert:

127. ${ }^{\circ}$ Definition. Das Produkt $\Pi\left(1+a_{n}\right)$ soll absolut konvergent heißen, falls das Produkt $\Pi\left(1+\left|a_{n}\right|\right)$ konvergiert.

Ihre Bedeutung bekommt diese Definition erst durch den

- Satz 6. Die Konvergenz von $I\left(1+\left|a_{n}\right|\right)$ zieht diejenige von $I I\left(1+a_{n}\right)$ nach sich.

Beweis. Es ist stets

$$
\begin{aligned}
\mid\left(1+a_{n+1}\right) & \left(1+a_{n+2}\right) \ldots\left(1+a_{n+k}\right)-1 \mid \\
& \leqq\left(1+\left|a_{n+1}\right|\right)\left(1+\left|a_{n+2}\right|\right) \ldots\left(1+\left|a_{n+k}\right|\right)-1,
\end{aligned}
$$

wie man sofort erkennt, wenn man sich die Klammern ausmultipliziert denkt. Ist also für $\Pi\left(1+\left|a_{n}\right|\right)$ die notwendige und hinreichende Konvergenzbedingung aus Satz 5 erfüllt, so ist sie es von selbst auch für $I\left(1+a_{n}\right)$, w. z. b. w.

Auf Grund von Satz 3 können wir daher sogleich sagen:

- Satz \%. Ein Produkt II $\left(1+a_{n}\right)$ ist dann und nur dann absolut konvergent, wenn $\sum a_{n}$ absolut konvergiert.

Da wir zur Feststellung der absoluten Konvergenz einer Reihe schon eine hinreichend ausgebaute Theorie in Händén haben, erledigt dieser Satz 7 in befriedigender Weise die Konvergenzfrage bei absolut konvergenten Produkten. In allen anderen Fällen führt der folgende Satz die Konvergenzfrage für Produkte vollständig auf die entsprechende Frage bei Reihen zurück:

- Satz 8. Das Produkt II $\left(1+a_{n}\right)$ ist dann und nur dann konvergent, wenn die Glieder $a_{n}$ eine Nullfolge bilden und wenn die hinter einem passenden Index $m$ begonnene ${ }^{1}$ ) Reihe

$$
\sum_{n=m+1}^{\infty} \log \left(1+a_{n}\right)
$$

konvergiert. Und die Konvergenz des Produktes ist dann und nur

1) Es geniigt, $m$ so zu wählen, dafi für $n>m$ stets $\left|a_{n}\right|<1$ ist. 
dann eine absolute, wenn die der Reihe eine absolute ist. Hat diese die Summe $L$, so ist überdies

$$
\prod_{n=1}^{\infty}\left(1+a_{n}\right)=\left(1+a_{1}\right) \ldots\left(1+a_{m}\right) \cdot e^{L} .
$$

Beweis. a) Ist $\Pi\left(1+a_{n}\right)$ konvergent, so strebt $a_{n} \rightarrow 0$, und es ist also von einer Stelle ab $\left|a_{n}\right|<1$, etwa für alle $n>m$. Da ferner die Teilprodukte

$$
p_{n}=\left(1+a_{m+1}\right) \ldots\left(1+a_{n}\right), \quad n>m,
$$

gegen einen von 0 verschiedenen (also positiven) Grenzwert $U_{m}$ streben, so strebt (nach $\mathbf{4 2}, 2)$

$$
\log p_{n} \rightarrow \log U_{m} \text {. }
$$

$\log p_{n}$ ist aber die mit dem $n^{\text {ten }}$ Gliede endende Teilsumme der in Rede stehenden Reihe. Diese ist also konvergent mit der Summe $L=\log U_{m}$. Wegen $U_{m}=e^{L}$ ist dann also

$$
I I\left(1+a_{n}\right)=\left(1+a_{1}\right) \ldots\left(1+a_{m}\right) \cdot e^{L} .
$$

b) Ist umgekehrt die Reihe konvergent mit der Summe $L$, so strebt eben $\log p_{n} \rightarrow L$ und folglich (nach $\mathbf{4 2}, 1$ )

$$
p_{n}=e^{\log p_{n} \rightarrow e^{L}},
$$

womit auch der zweite Teil des Satzes schon bewiesen ist, da $e^{L} \neq 0$ ist.

$\mathrm{Da}$ endlich Reihe und Produkt stets beide zugleich absolut konvergieren, folgt durch Vermittlung des Satzes 7 sofort daraus, daß mit $a_{n} \rightarrow 0$ nach $\mathbf{1 1 2}(\mathrm{b})$

$$
\left|\frac{\log \left(1+a_{n}\right)}{a_{n}}\right| \rightarrow 1 \text {. }
$$

strebt. (Hierbei hat man diejenigen Glieder $a_{n}$, welche etwa $=0$ sind, einfach außer acht zu lassen.)

So vollständig dieser Satz nun auch das Konvergenzproblem der unendlichen Produkte auf dasjenige der unendlichen Reihen zurückführt, so läßt dieser Satz doch praktisch deswegen etwas unbefriedigt, weil die direkte Feststellung der Konvergenz einer Reihe der Form $\Sigma \log \left(1+a_{n}\right)$ meist mit Schwierigkeiten verknüpft sein wird. Diese Lücke wollen wir, wenigstens teilweise, durch den folgenden Satz ausfüllen:

- Satz 9. Die (bei einem passenden Index begonnene) Reihe $\Sigma \log \left(1+a_{n}\right)$ und mit ihr das Produkt $\Pi\left(1+a_{n}\right)$ ist sicher dann konvergent, wenn $\Sigma a_{n}$ konvergiert und $\Sigma a_{n}{ }^{2}$ absolut konvergiert $\left.{ }^{1}\right)$.

1) $\Sigma a_{n}^{2}$ ist, wenn überhaupt, so sicher absolut konvergent. Wir wählen den obigen Wortlaut, damit der Satz auch für komplexe $a_{n}$ richtig bleibt, für die $a_{n}{ }^{2}$ nicht $>0$ zu sein braucht (vgl. $§ 57$ ). 
Beweis. Wir wählen wieder $m$ so, daß für $n>m$ stets $\left|a_{n}\right|<\frac{1}{2}$ bleibt und betrachten $\Pi\left(1+a_{n}\right)$ und $\Sigma \log \left(1+a_{n}\right)$ erst vom $(m+1)^{\text {ten }}$ Gliede an. Setzt man dann

$$
\log \left(1+a_{n}\right)=a_{n}+\vartheta_{n} \cdot a_{n}{ }^{2} \quad \text { oder } \quad \frac{\log \left(1+a_{n}\right)-a_{n}}{a_{n}{ }^{2}}=\vartheta_{n},
$$

so bilden die hierdurch bestimmten Zahlen $\vartheta_{n}$ sicher eine beschränkte Folge. Denn wegen $a_{n} \rightarrow 0$ strebt $\left.\vartheta_{n} \rightarrow-\frac{1}{2} 1\right)$. Ist also $\sum a_{n}$ und $\Sigma\left|a_{n}\right|^{2}$ konvergent, so ist hiernach auch $\Sigma \log \left(1+a_{n}\right)$ und deshalb auch das Produkt $\Pi\left(1+a_{n}\right)$ konvergent.

Dieser einfache Satz legt noch den folgenden weiteren nahe:

- Satz 10. Ist $\sum a_{n}{ }^{2}$ absolut konvergent und für $n>m$ stets $\left|a_{n}\right|<1$, so stehen die Teilprodukte

$$
p_{n}=\prod_{m+1}^{n}\left(1+a_{v}\right) \text { und die Teilsummen } s_{n}=\sum_{m+1}^{n} a_{v},(n>m),
$$

zueinander in einer solchen Beziehung, daß

$$
\boldsymbol{p}_{n} \sim \boldsymbol{e}^{\boldsymbol{s}_{n}}
$$

ist, daß also der Quotient beider Seiten gegen einen bestimmten endlichen und von 0 verschiedenen Grenzwert strebt, - mag $\Sigma a_{n}$ konvergieren oder divergieren.

Beweis. Benutzen wir die Bezeichnungen des vorigen Beweises, so ist wegen $\log \left(1+a_{n}\right)=a_{n}+\vartheta_{n} \cdot a_{n}{ }^{2}$ für $n>m$

$$
\left(1+a_{m+1}\right) \cdots\left(1+a_{n}\right)=\prod_{v=m+1}^{n} e^{a_{v}+\vartheta_{v} a_{v}{ }^{2}}=e^{\Sigma a_{v}} \cdot e^{\Sigma \vartheta_{v} a_{v}{ }^{2}},
$$

wenn in den beiden letzten Exponenten die Summation ebenfalls von $\boldsymbol{\nu}=m+1$ bis $\boldsymbol{v}=n$ erstreckt wird.

Und da wegen der Beschränktheit der $\vartheta_{n}$ mit $\sum a_{n}{ }^{2}$ auch $\sum \vartheta_{n} a_{n}{ }^{2}$ absolut konvergiert, so kann aus dieser Gleichung sofort die Behauptung abgelesen werden.

Bemerkungen und Beispiele. ${ }^{\prime}$

125. 1. Die Bedingungen des Satzes 9 sind nur hinreichend; das Produkt $I I\left(1+a_{n}\right)$ kann konvergieren, auch wenn $\Sigma a_{n}$ divergiert. Dann mufs aber nach Satz 10 auch $\Sigma\left|a_{n}\right|^{2}$ divergieren.

2. Wendet man Satz 10 auf das (divergente) Produkt $\prod_{n=1}^{\infty}\left(1+\frac{1}{n}\right)$ an, so ergibt sich aus ihm, daf

ist.

$$
e^{1+\frac{1}{2}+\frac{1}{3}+\ldots+\frac{1}{n}} \sim n
$$

1) Denn für $0<|x|<1$ ist $\log (1+x)=x+x^{2}\left[-\frac{1}{2}+\frac{x}{3}-\frac{x^{2}}{4}+\ldots\right]$ oder

$$
\frac{\log (1+x)-x}{x^{2}}=-\frac{1}{2}+\frac{x}{3}-+\ldots
$$

Und diejenigen Glieder, welche etwa $=0$ sind, diirfen wir hier wieder einfach uiberspringen, da sie auf die vorliegende Frage keinen Einfluf haben. 
3. $\prod_{n=1}^{\infty}\left(1+\frac{(-1)^{n-1}}{n}\right)$ ist konvergent. Seinen Wert findet man zufällig sofort durch Bildung der Teilprodukte; er ist $=1$.

4. $\prod_{n=1}^{\infty}\left(1+\frac{x}{n}\right)$ divergiert. Aber der Satz 10 lehrt trotzdem, daß̉ $\prod_{\nu=1}^{n}\left(1+\frac{x}{v}\right) \sim e^{x\left(1+\frac{1}{2}+\ldots+\frac{1}{n}\right)}$ oder, was nach 2. auf dassselbe hinauskommt, $\sim n^{x}$ ist, daßs also (s. 40,5) der Quotient

$$
n^{-x} \cdot \prod_{\nu=1}^{n}\left(1+\frac{x}{v}\right)=\frac{(x+1)(x+2) \ldots(x+n)}{n ! n^{x}}
$$

für jedes (feste) $x$ mit wachsendem $n$ einen bestimmten (endlichen) Grenzwert hat, der auch von 0 verschieden ist, falls $x \neq-1,-2, \ldots$ genommen wird.

5. $\prod_{n=1}^{\infty}\left(1-\frac{x^{2}}{n^{2}}\right)$ ist für jedes $x$ absolut konvergent.

6. $\prod_{n=2}^{\infty}\left(1-\frac{1}{n^{2}}\right)=\frac{1}{2}$.

\section{\$30. Zusammenhang zwischen Reihen und Produkten.} Bedingte und unbedingte Konvergenz.

Wir haben mehrfach betont, daß eine unendliche Reihe $\Sigma a_{n}$ nur ein andres Symbol für die Folge $\left(s_{n}\right)$ ihrer Teilsummen ist. Abgesehen von der Berücksichtigung der Sonderrolle, die die 0 bei der Multiplikation spielt, gilt das entsprechende von den unendlichen Produkten. Daraus folgt, daß man - von jener Rücksicht auf die Rolle der 0 abgesehen - jede Reihe als Produkt und jedes Produkt wird als Reihe schreiben können. Im einzelnen hat dies so zu geschehen:

1. Ist $\prod_{n=1}^{\infty}\left(1+a_{n}\right)$ vorgelegt, so bedeutet dies Produkt, wenn

$$
\prod_{\nu=1}^{n}\left(1+a_{v}\right)=p_{n}
$$

gesetzt wird, im wesentlichen die Folge $\left(p_{n}\right)$. Diese Folge ihrerseits wird durch die Reihe

$p_{1}+\left(p_{2}-p_{1}\right)+\left(p_{3}-p_{2}\right)+\ldots \equiv p_{1}+\sum_{n=2}^{\infty}\left(1+a_{1}\right) \ldots\left(1+a_{n-1}\right) \cdot a_{n}$

dargestellt. Diese und das vorgelegte Produkt bedeuten also dasselbe, - falls das Produkt im Sinne unserer Definition konvergiert. Dagegen kann die Reihe auch einen Sinn haben, wenn dies beim Produkte nicht der Fall ist (z. B. wenn der Faktor $\left(1+a_{5}\right)=0$ ist und alle übrigen Faktoren $=2$ sind). 
2. Ist umgekehrt die Reihe $\sum_{n=1}^{\infty} a_{n}$ vorgelegt, so bedeutet sie die Folge der $s_{n}=\sum_{\nu=1}^{n} a_{\nu}$. Diese selbe Bedeutung hat das Produkt

$$
s_{1} \cdot \frac{s_{2}}{s_{1}} \cdot \frac{s_{3}}{s_{2}} \ldots-s_{1} \cdot \prod_{n=2}^{\infty} \frac{s_{n}}{s_{n-1}} \equiv a_{1} \cdot \prod_{n=2}^{\infty}\left(1+\frac{a_{n}}{a_{1}+a_{2}+\ldots+a_{n-1}}\right),
$$

- falls es überhaupt einen Sinn hat. Und hierzu ist ersichtlich nur nötig, daß alle $s_{n} \neq 0$ sind. Lediglich wenn $s_{n} \rightarrow 0$ strebt, nennen wir die Reihe konvergent mit der Summe 0, während wir das Produkt als gegen 0 divergierend bezeichnen.

So bedeuten z. B.

$$
\begin{array}{lll}
\sum_{n=1}^{\infty} \frac{1}{2^{n}} & \text { und } & \frac{1}{2} \cdot \prod_{n=2}^{\infty}\left(1+\frac{1}{2^{n}-2}\right) \\
\sum_{n=1}^{\infty} \frac{1}{n(n+1)} & \text { und } & \frac{1}{2} \cdot \prod_{n=2}^{\infty}\left(1+\frac{1}{n^{2}-1}\right)
\end{array}
$$

genau dasselbe.

Dieser Übergang vom einen zum andern Symbol wird jedoch nur selten für die Untersuchung von Vorteil sein. Der für die Theorie entscheidende Zusammenhang zwischen Reihen und Produkten wird vielmehr allein durch den Satz 8 hergestellt - bzw. durch den Satz 7, wenn es allein auf die Frage nach absoluter Konvergenz ankommt. Um den Wert dieser Sätze für allgemeine Fragen zu zeigen, beweisen wir noch - als Analogon zu Satz 89, 2 - den folgenden

130. ${ }^{-S a t z ~ 11 . ~ E i n ~ u n e n d l i c h e s ~ P r o d u k t ~} \Pi\left(1+a_{n}\right)$ ist dann und nur dann unbedingt konvergent, d. h. es bleibt dann und nur dann bei jeder Umordnung $($ s. 27, 3) der Faktoren konvergent und zwar mit ungeändertem Werte, wenn es absolut konvergiert ${ }^{1}$ ).

Beweis. Diejenigen sicher nur endlich vielęn Glieder $a_{n}$, für die $\left|a_{n}\right| \geqq \frac{1}{2}$ ist, ersetzen wir durch 0. Dann haben wir im Sinne von $\mathbf{2 7}, 4$ nur ,endlich viele Änderungen“ ausgeführt und haben erreicht, daß jetzt alle $\left|a_{n}\right|<\frac{1}{2}$ sind. Daher kann die beim Beweise des Satzes 8 benutzte Zahl $m=0$ genommen werden. Wir beweisen dann den Satz zunächst für das solchergestalt abgeänderte Produkt.

Jetzt sind aber

$$
\Pi\left(1+a_{n}\right) \quad \text { und } \quad \Sigma \log \left(1+a_{n}\right)
$$

gleichzeitig konvergent oder divergent, und im ersteren Falle gilt für ihre Werte $U$ und $L$ die Beziehung $U=e^{L}$. Daraus folgt: Eine Umordnung der Faktoren des Produktes wird dasselbe dann und nur

1) U. Dini, Sui prodotti infiniti, Annali di Matem., Bd. (2) 2 (1868), 
dann mit ungeändertem Werte $U$ konvergent lassen, wenn die entsprechende Umordnung der Reihe auch diese mit ungeändertem Werte konvergent läßt. Dies findet für eine Reihe (bei jeder beliebigen Umordnung) dann und nur dann statt, wenn sie absolut konvergiert. Nach Satz 8 gilt also dasselbe für das Produkt.

Wenn man nun vor der Umordnung endlich viele Änderungen vornimmt, diese aber nachher wieder rückgängig macht, so kann dies auf unsere jetzige Frage keinen Einfluß haben. Der Satz ist also für alle Produkte richtig.

Zusatz. Mit Benutzung des erst weiter unten bewiesenen Riemannschen Satzes (§44) kann man natürlich genauer sagen: Ist das Produkt nicht absolut konvergent, und ist kein Faktor $=0$, so kann man durch eine passende Umordnung stets erreichen, daß für das neue Produkt die Folge der Teilprodukte vorgeschriebene Häufungsgrenzen $\varkappa$ und $\mu$ hat, wofern sie beide das gleiche Vorzeichen haben wie der Wert des vorgelegten Produktes ${ }^{1}$ ). Dabei dürfen $\varkappa$ und $\mu$ auch 0 und $\pm \infty$ sein.

\section{Aufgaben zum VII. Kapitel.}

85. Man beweise, daßs die folgenden Produkte konvergieren und den angegebenen Wert haben:
a) $\prod_{n=2}^{\infty} \frac{n^{3}-1}{n^{3}+1}=\frac{2}{3}$
b) $\prod_{n=0}^{\infty}\left(1+\left(\frac{1}{2}\right)^{2^{n}}\right)=2$;
c) $\prod_{n=2}^{\infty}\left(1+\frac{2 n+1}{\left(n^{2}-1\right)(n+1)^{2}}\right)=\frac{4}{3}$.

86. Man stelle das Konvergenzverhalten der folgenden Produkte fest:
a) $\prod_{n=2}^{\infty}\left(1+\frac{(-1)^{n}}{\sqrt{n}}\right)$;
b) $\prod_{n=2}^{\infty}\left(1+\frac{(-1)^{n}}{\log n}\right)$;
c) $\left(1-\frac{1}{\sqrt{3}}\right)\left(1+\frac{1}{\sqrt{2}}\right)\left(1-\frac{1}{\sqrt{5}}\right)\left(1-\frac{1}{\sqrt{7}}\right)\left(1+\frac{1}{\sqrt{4}}\right)\left(1-\frac{1}{\sqrt{9}}\right) \ldots$;
d) $\left(1+\frac{1}{\alpha-1}\right)\left(1-\frac{1}{2 \alpha-1}\right)\left(1+\frac{1}{3 \alpha-1}\right)\left(1-\frac{1}{4 \alpha-1}\right) \ldots$, falls $\alpha \neq 1, \frac{1}{2}, \frac{1}{3}, \ldots$ ist.

87. Man zeige, daß $\Pi \cos x_{n}$ konvergiert, falls $\Sigma\left|x_{n}\right|^{2}$ konvergiert.

1) Denn ein konvergentes unendliches Produkt hat jedenfalls nur endlich viele negative Faktoren; und deren Anzahl wird durch die Umordnung nicht geändert. 
55. Das Produkt in Aufg. $86 \mathrm{~d}$ hat für positiv-ganzzahlige Werte von $\alpha$ den Wert $\sqrt[\alpha]{2}$. [Anl.: Das bis zum Faktor $\left(1-\frac{1}{2 k \alpha-1}\right)$ genommene Teilprodukt ist $\left.=\prod_{\nu=k+1}^{2 k}\left(1-\frac{1}{v \alpha}\right)^{-1}.\right]$

99. Im Anschlufs an Aufg. 87 zeige man, dafs

$$
\cos \frac{\pi}{4} \cdot \cos \frac{\pi}{8} \cdot \cos \frac{\pi}{16} \ldots=\frac{2}{\pi}
$$

ist. (Man erkennt hierin das S. 212, Fußnn., genannte Produkt von Vieta.)

90. Man zeige allgemeiner, dafi für jedes $x$

$$
\begin{gathered}
\cos \frac{x}{2} \cdot \cos \frac{x}{4} \cdot \cos \frac{x}{8} \cdot \cos \frac{x}{16} \ldots=\frac{\sin x}{x} . \\
\operatorname{ch} \frac{x}{2} \cdot \operatorname{ch} \frac{x}{4} \cdot \operatorname{ch} \frac{x}{8} \cdot \operatorname{ch} \frac{x}{16} \ldots=\frac{\operatorname{sh} x}{x},
\end{gathered}
$$

in welch letzterer Formel $\operatorname{ch} x=\frac{e^{x}+e^{-x}}{2}, \operatorname{sh} x=\frac{e^{x}-e^{-x}}{2}$ ist.

91. Mit Hilfe von Aufg. 90 zeige man, daf3 die durch die Intervallschachtelung in Aufg. $8 \mathrm{c}$ definierte Zahl $=\frac{\sin \vartheta}{\vartheta} y_{1}$ ist, wenn $\vartheta$ als positiver spitzer Winkel aus $\cos \vartheta=\frac{x_{1}}{y_{1}}$ bestimmt wird. Ebenso ist die durch Aufg. $8 \mathrm{~d}$ definierte $Z$ ahl $=\frac{\operatorname{sh} \vartheta}{\vartheta} x_{1}$, wenn $\vartheta$ aus $\operatorname{ch} \vartheta=\frac{y_{1}}{x_{1}}$ bestimmt wird.

92. Man zeige in ähnlicher Weise, daßs die in Aufg. 8 e und f definierten Zahlen die Werte haben

e) $\frac{\operatorname{sh} 2 \vartheta}{2 \vartheta} x_{1} \quad$ mit $\quad \operatorname{ch}^{2} \vartheta=\frac{y_{1}}{x_{1}}$,

f) $\frac{\sin 2 \vartheta}{2 \vartheta} y_{1} \quad$ mit $\quad \cos ^{2} \vartheta=\frac{x_{1}}{y_{1}}$.

93. Es ist

$$
\begin{aligned}
1-\frac{x}{a_{1}}+\frac{x\left(x-a_{1}\right)}{a_{1} \cdot a_{2}}- & +\ldots+(-1)^{n} \frac{x\left(x-a_{1}\right) \ldots\left(x-a_{n-1}\right)}{a_{1} \cdot a_{2} \ldots a_{n}} \\
& =\left(1-\frac{x}{a_{1}}\right)\left(1-\frac{x}{a_{2}}\right) \ldots\left(1-\frac{x}{a_{n}}\right) .
\end{aligned}
$$

Was folgt daraus für die Reihe und das Produkt, deren Anfänge hier stehen?

94. Man beweise mit Hilfe des Satzes 10 aus $\S 29$, daf3

$$
\frac{1 \cdot 3 \cdot 5 \ldots(2 n-1)}{2 \cdot 4 \cdot 6 \ldots 2 n} \sim \frac{1}{\sqrt{n}}
$$

ist.

95. Man zeige ebenso, dafs für $0 \leqq x<y$

strebt.

$$
\frac{x(x+1)(x+2) \ldots(x+n)}{y(y+1)(y+2) \cdots(y+n)} \rightarrow 0
$$


96. Man zeige ebenso, daf, wenn $a$ und $b$ positiv sind und $A_{n}$ und $G_{n}$ das arithmetische bzw. geometrische Mittel der $n$ Größen

$$
a, \quad a+b, a+2 b, \ldots, a+(n-1) b
$$

bedeuten $(n=2,3,4, \ldots)$,

strebt.

$$
\frac{A_{n}}{G_{n}} \rightarrow \frac{e}{2}
$$

97. Was läßt sich aus der Konvergenz von $\Pi\left(1+a_{n}\right)$ und $\Pi\left(1+b_{n}\right)$ iiber diejenige von

aussagen? (Vg1. 83, 3 und 4.)

$$
\Pi\left(1+a_{n}\right)\left(1+b_{n}\right) \quad \text { und } \quad \Pi \frac{1+a_{n}}{1+b_{n}}
$$

98. Es strebe monoton fallend $u_{n} \rightarrow 1$. Ist dann

$$
u_{1} \cdot \frac{1}{u_{2}} \cdot u_{3} \cdot \frac{1}{u_{4}} \cdot u_{5} \ldots
$$

immer konvergent? (Vg1. 82, 10).

99. In Ergänzung zu $§ 29$, Satz 9 , beweise man, dafi $\Pi\left(1+a_{n}\right)$ sicher konvergiert, wenn die beiden Reihen

$$
\Sigma\left(a_{n}-\frac{1}{2} a_{n}^{2}\right) \quad \text { und } \quad \Sigma\left|a_{n}\right|^{3}
$$

konvergieren. - Wie läßst sich dies verallgemeinern? - Andrerseits zeige man an dem Beispiel des Produktes

$$
\left(1-\frac{1}{2^{\alpha}}\right)\left(1+\frac{1}{2^{\alpha}}+\frac{1}{2^{2 \alpha}}\right)\left(1-\frac{1}{3^{\alpha}}\right)\left(1+\frac{1}{3^{\alpha}}+\frac{1}{3^{2 \alpha}}\right)\left(1-\frac{1}{4^{\alpha}}\right) \cdots
$$

bei dem $\frac{1}{3}<\alpha \leqq \frac{1}{2}$ sein soll, daß $\Pi\left(1+a_{n}\right)$ konvergieren kann, selbst wenn die Reihen $\Sigma a_{n}$ und $\Sigma a_{n}^{2}$ beide divergieren.

VIII. Kapitel.

\section{Geschlossene und numerische Auswertung der Reihensumme.}

\section{$\S$ 31. Problemstellung.}

Im III. und IV. Kapitel haben wir uns hauptsächlich mit dem Problem A, der Konvergenzfrage, beschäftigt und erst in den letzten Kapiteln haben wir gleichzeitig die Reihensumme mit in Betracht gezogen. Diesen letzteren Gesichtspunkt wollen wir nun in den Yordergrund stellen. Doch ist es in Ergänzung unserer Ausführungen von S. 74/75 und 100/101 nötig, sich noch einmal die Bedeutung der dabei in Betracht kommenden Fragen klarzumachen. Hat man z. B. die Gleichung $\mathbf{1 2 2}$

$$
\frac{\pi}{4}=1-\frac{1}{3}+\frac{1}{5}-\frac{1}{7}+\ldots
$$

bewiesen, so kann man ihren Inhalt auf zweierlei Art deuten. Einmal sagt sie uns, daß die Summe der rechtsstehenden Reihe den 
Wert $\frac{\pi}{4}$ hat, also der vierte Teil einer $Z$ ahl ist ${ }^{1}$ ), die in sehr vielen andern Zusammenhängen auftritt und von der. auch ein jeder angenäherte Werte kennt. In diesem Sinne mag man wohl sagen, da $B$ wir die Summe der obigen Reihe haben angeben können. Das gilt aber doch nur sehr bedingt; denn die Zahl $\pi$ kann auf keine Weise (vgl. S. 18) vollständig hingeschrieben werden, - außer durch eine Intervallschachtelung oder ein äquivalentes Symbol. Ein solches liegt aber gerade in der Reihe, also dem in der obigen Gleichung rechts stehenden Ausdruck vor. Daher können wir von ihr auch gerade umgekehrt sagen: Sie liefert eine (sehr einfache) Darstellung der Zahl $\pi$ durch eine Reihe, d. h. also durch eine konvergente Zahlenfolge, - die hier sogar besonders durchsichtig ist und (nach $\mathbf{6 9}, 1$ ) auch unmittelbar als. Intervallschachtelung geschrieben werden kann²).

Ganz anders liegt der Fall bei der Gleichung (vgl. 68, $2^{\text {b) }}$

$$
\frac{1}{1 \cdot 2}+\frac{1}{2 \cdot 3}+\frac{1}{3 \cdot 4}+\ldots=1 \text {. }
$$

Hier sind wir mit der Feststellung, daß die Summe der Reihe $=1$ ist, vollständig befriedigt, da uns eben die Zahl 1 (und ebenso jede rationale Zahl) restlos in die Hände gegeben werden kann. Hier werden wir also mit vollem Rechte sagen, daß wir die Reihensumme gesçhlossen ausgewertet haben. In allen andern Fällen aber, in denen also die Reihensumme keine rationale Zahl ist oder jedenfalls nicht als solche erkannt wird $^{3}$ ), kann von einer geschlossenen Auswertung der Reihensumme im strengen Sinne nicht geredet werden.' Hier wird umgekehrt die Reihe als ein (mehr oder weniger vollkommenes) Mittel angesehen werden müssen, eine Darstellung und also rationale Näherungswerte für die Reihensumme zu bekommen. Indem wir uns diese dann herstellen (gewöhnlich in Dezimalbruchform) und die íhnen noch anhaftenden Fehler abschätzen, spręchen wir von einer numerischen Auswertung der Reihensumme.

Wenn endlich, wie in dem obigen Falle der Reihe für $\frac{\pi}{4}$ festgestellt wird, daß die Reihensumme eine Zahl ist, die zu einer in andern Zusammenhängen auftretenden Zahl in einfacher oder jeden-

1) In älteren Zeiten, in denen man alle diese Dinge mehr geometrisch deutete, sah man in $\frac{\pi}{4}$ stets das Verhältnis der Kreisfläche zum umgeschriebenen Quadrat.

2) Nämlich: $\frac{\pi}{4}=\left(s_{2 k} \mid s_{2 k+1}\right)$, wenn $s_{n}=1-\frac{1}{3}+\frac{1}{5}+\ldots+\frac{(-1)^{n-1}}{2 n-1}$ gesetzt wird. $(n=2,3, \ldots)$

3) Haben wir z. B. festgestellt, daß eine Reihensumme $=2^{\sqrt{3}}$ ist, wissen wir nicht, ob das eine rationale Zahl ist oder nicht. 
falls angebbarer Beziehung steht - so folgt doch z. B. aus 122 und 124, daß

$$
1+\frac{1}{2} \cdot \frac{1}{3}+\frac{1 \cdot 3}{2 \cdot 4} \cdot \frac{1}{5}+\ldots=2\left[1-\frac{1}{3}+\frac{1}{5}-+\ldots\right]
$$

st, - so werden wir auch eine solche Feststellung im allgemeinen begrüßen, da sie eine Verbindung zwischen vorher getrennten Ergebnissen herstellt. Man pflegt auch in solchen Fällen - wenn auch in übertragenem Sinne - von einer geschlossenen Auswertung der Reihensumme zu sprechen; denn man pflegt aus dem andern $\mathrm{Zu}$ sammenhange her die betreffende Zahl dann als „bekannt" anzusehen und kann nun mit ihrer Hilfe auch die neue Reihensumme „geschlossen“ angeben. Doch hüte man sich da vor Selbsttäuschung. Hat man z. B. festgestellt (s. S. 203), daß die Summe der Reihe

$$
1+\frac{1}{2} \cdot \frac{1}{50}+\frac{1 \cdot 3}{2 \cdot 4} \cdot \frac{1}{50^{2}}+\frac{1 \cdot 3 \cdot 5}{2 \cdot 4 \cdot 6} \cdot \frac{1}{50^{3}}+\ldots
$$

gleich $\frac{5}{7} \sqrt{2}$ ist, so ist damit die Summe der Reihe doch nur in sehr bedingtem Sinne „geschlossen ausgewertet“. Denn $\sqrt{2}$ ist uns an und für sich nicht besser bekannt als die Summe irgendeiner konvergenten Reihe. Nur weil $\sqrt{2}$ noch in vielen hundert andern Zusammenhängen auftritt und für praktische Zwecke schon oft numerisch berechnet worden ist, pflegen wir ihren Wert als fast ebenso „,bekannt" anzusehen, wie irgendeine hingeschriebene rationale Zahl. Nehmen wir aber statt der obigen etwa die folgende binomische Reihe

$$
\frac{5}{2}\left[1+\frac{1}{5} \cdot \frac{24}{1000}-\frac{4}{5 \cdot 10} \cdot \frac{24^{2}}{1000^{2}}+\frac{4 \cdot 9}{5 \cdot 10 \cdot 15} \cdot \frac{24^{3}}{1000^{3}}-\ldots\right]
$$

und hat man gefunden, daß ihre Summe $=\sqrt[5]{100}$ ist, so werden wir schon weniger geneigt sein, hierdurch die Summe als festgestellt anzusehen; vielmehr werden wir die Reihe als ein recht brauchbares Mittel zur genaueren Berechnung von $\sqrt[5]{100}$ begrüßen, die auf andre Weise nicht so leicht durchzuführen wäre. M. a. W.: Von den wenigen Fällen abgeșehen, wo wir als Reihensumme eine bestimmte rationale Zahl angeben können, wird das Schwergewicht des Interesses an einer Gleichung der Form , $s=\Sigma a_{n}$ " je nach Lage des Falles auf der rechten oder auf der linken Seite der Gleichung liegen. Dürfen wir $s$ aus andern Zusammenhängen her als bekannt ansehen, so werden wir auch jetzt noch (in übertragenem Sinne) sagen: Wir hätten die Reihensumme geschlossen ausgewertet. Ist das nicht der Fall, so werden wir sagen: die Reihe sei ein Mittel zur Berechnung der (durch sie definierten) Zahl s. (Selbstverständlich können beide Gesichtspunkte auch bei einer und derselben Gleichung zur Geltung 
kommen.) In dem ersten der beiden Fälle sind wir sozusagen fertig, da dann auch das Problem B (s. S. 101) in einer uns befriedigenden Form gelöst ist. In dem zweiten Fall dagegen beginnt nun eine neue Aufgabe, nämlich die der tatsächlichen Herstellung der durch die Reihe gelieferten Näherungswerte ihrer Summe in übersichtlicher Form, also etwa - das wird uns das erwünschteste sein - in (endlichen) Dezimalbrüchen, und die Abschätzung der diesen Näherungswerten noch anhaftenden Fehler.

\section{§32. Geschlossene Auswertung der Reihensumme.}

1. Unmittelbare Auswertung. Es ist natürlich leicht, sich Reihen zu bilden, die eine vorgeschriebene Summe haben. Denn soll $s$ diese Summe sein, so konstruiere man sich nach einem der vielen Verfahren, die wir nun kennen, eine gegen $s$ konvergierende Zahlenfolge $\left(s_{n}\right)$ und setze die Reihe

$$
s_{0}+\left(s_{1}-s_{0}\right)+\left(s_{2}-s_{1}\right)+\ldots+\left(s_{n}-s_{n-1}\right)+\ldots
$$

an. Da deren $n^{\text {te }}$ Teilsumme ersichtlich gerade $=s_{n}$ ist, so ist diese Reihe konvergent mit der Summe $s$. In diesem einfachen Verfahren hat man eine unerschöpfliche Quelle zur Herstellung von Reihen, die man geschlossen summieren kann; z. B. braucht man nur eine der vielen uns bekannten Nullfolgen $\left(x_{n}\right)$ zu nehmen und $s_{n}=s-x_{n}$ zu setzen, $n=0,1,2, \ldots$.

Beispiele für Reihen mit der Summe 1.

1. $\left(x_{n}\right)=\left(\frac{1}{n+1}\right)$ liefert : $\frac{1}{1 \cdot 2}+\frac{1}{2 \cdot 3}+\frac{1}{3 \cdot 4}+\ldots=1$

2. $\left(x_{n}\right)=\left(\frac{(-1)^{n}}{n+1}\right) \quad$ " $\frac{3}{1 \cdot 2}-\frac{5}{2 \cdot 3}+\frac{7}{3 \cdot 4}-\frac{9}{4 \cdot 5}+\ldots=1$

3. $\left(x_{n}\right)=\left(\frac{1}{n+1}\right)^{2} \quad n \sum_{n=1}^{\infty} \frac{2 n+1}{n^{2}(n+1)^{2}}=1$

4. $\left(x_{n}\right) \equiv\left(\frac{1}{n+1}\right)^{3} \quad$ " $\sum_{n=1}^{\infty} \frac{3 n^{2}+3 n+1}{n^{3}(n+1)^{3}}=1$

5. $\left(x_{n}\right)=\left(\frac{1}{2^{n}}\right) \quad, \quad \sum_{n=1}^{\infty} \frac{1}{2^{n}}=1$

6. $\left(x_{n}\right)=\left(\frac{1}{\sqrt{n+1)}}\right) \quad \eta \quad \sum_{n=1}^{\infty} \frac{1}{\sqrt{n(n+1)}(\sqrt{n}+\sqrt{n+1})}=1$ usw.

7. Multipliziert man die Glieder einer dieser Reihen mit $s$, so erhält man eine konvergente Reihe mit der Summe $s$.

Die Herstellung solcher Beispiele ist nicht uberflissig, da wir sehen werden, das ein großer Vorrat an Reihen mit bekannter Summe für die Behandlung weiterer Reihen vorteilhaft ist. 
Kehren wir das eben behandelte Prinzip um, so haben wir den

○ Satz. Ist $\sum_{n=0}^{\infty} a_{n}$ vorgelegt und gelingt es, das Glied $a_{n}$ in der 131. Form $a_{n}=x_{n}-x_{n+1}$ darzustellen, in der $x_{n}$ das Glied einer konvergenten Folge mit dem bekannten Grenzwert $\xi$ ist, so läßt sich die Summe der Reihe angeben, denn es ist

Beweis. Es ist

$$
\sum_{n=0}^{\infty} a_{n}=x_{0}-\xi
$$

$$
s_{n}=\left(x_{0}-x_{1}\right)+\left(x_{1}-x_{2}\right)+\ldots+\left(x_{n}-x_{n+1}\right)=x_{0}-x_{n+1} \text {, }
$$

woraus wegen $x_{n} \rightarrow \xi$ sofort die Behauptung folgt.

$$
\text { Beispiele. }
$$

132. (s. 68, $2^{\text {h }}$

1. Ist $\alpha$ irgendeine von $0,-1,-2, \ldots$ verschiedene reelle $Z$ ahl, so ist

$$
\sum_{n=0}^{\infty} \frac{1}{(\alpha+n)(\alpha+n+1)}=\frac{1}{\alpha} \text {, da hier } a_{n}=\left[\frac{1}{\alpha+n}-\frac{1}{\alpha+n+1}\right] .
$$

2. Ebenso ist

da jetzt

$$
\sum_{n=0}^{\infty} \frac{1}{(\alpha+n)(\alpha+n+1)(\alpha+n+2)}=\frac{1}{2 \alpha(\alpha+1)},
$$

$$
a_{n}=\frac{1}{2}\left[\frac{1}{(\alpha+n)(\alpha+n+1)}-\frac{1}{(\alpha+n+1)(\alpha+n+2)}\right] .
$$

3. Allgemein findet man für jede natürliche Zahl $p$

$$
\sum_{n=0}^{\infty} \frac{1}{(\alpha+n)(\alpha+n+1) \cdots(\alpha+n+p)}=\frac{1}{p} \frac{1}{\alpha(\alpha+1) \ldots(\alpha+p-1)} .
$$

4. Für $\alpha=\frac{1}{3}$ findet man z. B. aus 2 .

$$
\frac{1}{1 \cdot 4 \cdot 7}+\frac{1}{4 \cdot 7 \cdot 10}+\frac{1}{7 \cdot 10 \cdot 13}+\ldots=\frac{1}{24} \text {. }
$$

5. Für $\alpha=1$ liefert uns das 3. Beispiel

$$
\frac{1}{1 \cdot 2 \ldots(p+1)}+\frac{1}{2 \cdot 3 \ldots(p+2)}+\ldots=\frac{1}{p \cdot p !} \quad \text { oder } \quad \sum_{n=1}^{\infty} \frac{1}{\left(\begin{array}{c}
p+n+1) \\
n
\end{array}\right)}=\frac{1}{p} \text {. }
$$

Etwas allgemeiner hat man den

- Satz. Ist in $\sum a_{n}$ das Glied $a_{n}$ in der Form $x_{n}-x_{n+q}$ dar-133. stellbar, in der $x_{n}$ das Glied einer konvergenten Folge mit dem bekannten Grenzwert $\xi$ ist und $q$ eine feste natürliche Zahl bedeutet, so ist

$$
\sum_{n=0}^{\infty} a_{n}=x_{0}+x_{1}+\ldots+x_{q-1}-q \xi \text {. }
$$

Beweis. Es ist für $n>q$

$$
\begin{gathered}
s_{n}=\left(x_{0}-x_{q}\right)+\left(x_{1}-x_{q+1}\right)+\ldots+\left(x_{q-1}-x_{2 q-1}\right)+\left(x_{q}-x_{2 q}\right) \\
+\ldots+\left(x_{n}-x_{n+q}\right) \\
=\left(x_{0}+x_{1}+\ldots+x_{q-1}\right)-\left(x_{n+1}+x_{n+2}+\ldots+x_{n+q}\right),
\end{gathered}
$$

woraus wegen $x_{v} \rightarrow \xi$ (nach $\mathbf{4 1}, 8$ ) sofort die Behauptung folgt. 
Beispiele.

da hier

1. $\sum_{n=0}^{\infty} \frac{1}{(\alpha+n)(\alpha+n+q)}=\frac{1}{q}\left(\frac{1}{\alpha}+\frac{1}{\alpha+1}+\cdots+\frac{1}{\alpha+q-1}\right)$,

$$
a_{n}=\frac{1}{q}\left(\frac{1}{\alpha+n}-\frac{1}{\alpha+n+q}\right) .
$$

2. Für $\alpha=1$ und $q=2$. hat man hiernach

$$
\frac{1}{1 \cdot 3}+\frac{1}{2 \cdot 4}+\frac{1}{3 \cdot 5}+\ldots=\frac{3}{4}
$$

für $\alpha=\frac{1}{2}$ und $q=3$ ebenso

$$
\frac{1}{1 \cdot 7}+\frac{1}{3 \cdot 9}+\frac{1}{5 \cdot 11}+\ldots=\frac{23}{90} \text {. }
$$

3. Etwas allgemeiner hat man, wenn neben $q$ auch $k$ eine feste naturliche Zahl bedeutet

$$
\sum_{n=0}^{\infty} \frac{1}{(\alpha+n)(\alpha+n+q) \ldots(\alpha+n+k q)}=\frac{1}{k q} \sum_{\nu=0}^{q-1} \ldots \frac{1}{(\alpha+v)(\alpha+v+q) \ldots(\alpha+v+\overline{k-1} q)} .
$$

4. Für $\alpha=\frac{1}{2}, q=2, k=2$ findet man so

$$
\frac{1}{1 \cdot 5 \cdot 9}+\frac{1}{3 \cdot 7 \cdot 11}+\frac{1}{5 \cdot 9 \cdot 13}+\ldots=\frac{13}{420} \text {. }
$$

Verallgemeinern wir die hierbei angewendeten Kunstgriffe ein wenig, so gelangen wir schließlich zu dem folgenden wesentlich weitergehenden

- Satz. Sind die Glieder einer Reihe $\Sigma a_{n}$ für jedes $n$ in der Form

$$
a_{n}=c_{1} x_{n+1}+c_{2} x_{n+2}+\ldots+c_{k} x_{n+k}, \quad(k \geqq 2, f e s t),
$$

darstellbar, bei der $\left(x_{n}\right)$ eine konvergente Zahlenfolge mit dem bekannten Grenzwert $\xi$ bedeuten soll und die Koeffizienten $c_{\lambda}$ der Bedingung $c_{1}+c_{2}+\ldots+c_{k}=0$ genügen, so ist $\Sigma a_{n}$ konvergent und

$$
\begin{gathered}
\sum_{n=0}^{\infty} a_{n}=c_{1} x_{1}+\left(c_{1}+c_{2}\right) x_{2}+\ldots+\left(c_{1}+c_{2}+\ldots+c_{k-1}\right) x_{k-1} \\
+\left(c_{2}+2 c_{3}+\ldots+\overline{k-1} c_{k}\right) \xi .
\end{gathered}
$$

Der Beweis ergibt sich unmittelbar, wenn man zeilenweise die Werte von $a_{0}, a_{1}, \ldots, a_{m}$ so untereinander schreibt, daß die Glieder mit demselben $x_{v}$ in ein und derselben Spalte zu stehen kommen. Addiert man dann spaltenweise - was hier auch ohne den großen Umordnungssatz sofort erlaubt ist - und berücksichtigt die Bedingung, der die $c_{\text {. }}$ genügen sollen, so ergibt sich

$$
\sum_{n=0}^{m} a_{n}=\sum_{\lambda=1}^{k-1}\left(c_{1}+c_{2}+\ldots+c_{\lambda}\right) x_{\lambda}+\sum_{\lambda=1}^{k-1}\left(c_{\lambda+1}+\ldots+c_{k}\right) x_{m+\lambda+1},
$$

also wieder ein Ausdruck mit einer festen Anzahl von Summanden. Für $m \rightarrow \infty$ erhält man dann sofort die behauptete Gleichung. 
Beispiele.

1. $x_{n}=\frac{n^{2}}{n^{2}+1}, \quad k=2, \quad c_{1}=-1, \quad c_{2}=+1$ liefert

$$
\frac{3}{2 \cdot 5}+\frac{5}{5 \cdot 10}+\frac{7}{10 \cdot 17}+\ldots+\frac{2 n+1}{\left(n^{2}+1\right)\left(\overline{n+1^{2}}+1\right)}+\ldots=\frac{1}{2},
$$

2. $\sum_{n=0}^{\infty} \frac{1}{(3 n+1)(3 n+10)}=\frac{1}{27} \sum_{n=0}^{\infty}\left(\frac{1}{-\frac{2}{3}+n+1}-\frac{1}{-\frac{2}{3}+n+4}\right)=\frac{13}{84}$,

und diese Beispiele lassen sich natürlich leicht beliebig vermehren.

2. Anwendung der elementaren Funktionen. Mit diesen wenigen soeben gegebenen Sätzen haben wir im großen und ganzen die Typen derjenigen Reihen kennen gelernt, die man ohne tiefer gehende Kunstgriffe geschlossen summieren kann.

Bei weitem die meisten der bei allen Anwendungen auftretenden Reihen sind solche, die sich für spezielle Werte von $x$ aus den Reihenentwicklungen der elementaren Funktionen und den verschiedensten Umformungen, Zusammensetzungen und anderweitigen Folgerungen aus ihnen ergeben. Die Fülle der Beispiele geschlossener Summation, die auf diese Weise gewonnen werden können, ist unübersehbar. Wir müssen uns damit begnügen, den Leser auf die Durchrechnung der für diesen Zweck besonders zahlreich zusammengestellten Aufgaben zum VIII. Kapitel hinzuweisen, die ihn mit den hauptsächlichsten dabei in Frage kommenden Kunstgriffen vertraut machen wird. Die weiteren Ausführungen dieses und des nächsten Paragraphen werden noch weitere Anleitung dazu geben. Hier bemerken wir nur generell, daß man einer vorgelegten Reihe $\Sigma a_{n}$ oft dadurch beikommt, daß man ihre Glieder in zwei oder mehr Summanden zerlegt, die einzeln wieder konvergente Reihen liefern; oder dadurch, daß man zu $\Sigma a_{n}$ eine andere Reihe mit bekannter Summe gliedweis addiert oder sie von ihr subtrahiert. Insbesondere wird, wenn $a_{n}$ eine rationale Funktion von $n$ ist, die Partialbruchzerlegung häufig gute Dienste leisten.

3. Anwendung des Abelschen Grenzwertsatzes. Ein andrer prinzipiell sehr wichtiger Weg zur Auswertung einer Reihensumme, der im Prinzip von den soeben angedeuteten Wegen abweicht, aber wegen 101 meist aufs engste mit ihnen verbunden bleibt, besteht in der Anwendung des Abelschen Grenzwertsatzes. Liegt die konvergente Reihe $\Sigma a_{n}$ vor, so ist die Potenzreihe $f(x)=\Sigma a_{n} x^{n}$ mindestens für $-1<x \leqq+1$ konvergent und nach $\mathbf{1 0 1}$ ist daher

$$
\sum a_{n}=\lim _{x \rightarrow 1-0} f(x) \text {. }
$$

Ist nun die durch die Potenzreihe dargestellte Funktion $f(x)$ soweit bekannt, daß man diesen letzteren Grenzwert angeben kann, so ist die Auswertung der Reihensumme geleistet. Hierzu bieten die Ent- 
wicklungen des VI. Kapitels eine breite Grundlage und dort haben wir auch schon mehrfach den $\mathrm{Abelschen} \mathrm{Grenzwertsatz} \mathrm{in} \mathrm{dem} \mathrm{jetzigen}$ Sinne benutzt.

Nachstehend geben wir nur noch einige naheliegende Beispiele und verweisen im übrigen auf die diesem Kapitel angehängten Aufgaben.

135.

Beispiele. Neben den uns schon bekannten Reihen

1. $\sum_{n=0}^{\infty} \frac{(-1)^{n}}{n+1}=\lim _{x \rightarrow 1-0} \sum_{n=0}^{\infty}(-1)^{n} \frac{x^{n+1}}{n+1}=\lim _{x \rightarrow 1-0} \log (1+x)=\log 2$,

2. $\sum_{n=0}^{\infty} \frac{(-1)^{n}}{2 n+1}=\lim _{x \rightarrow 1-0} \sum_{n=0}^{\infty}(-1)^{n} \frac{x^{2} n+1}{2 n+1}=\lim _{x \rightarrow 1-0} \operatorname{arctg} x=\frac{\pi}{4}$ hat man

3. $\sum_{n=0}^{\infty} \frac{(-1)^{n}}{3 n+1}=\lim _{x \rightarrow 1-0}\left(x-\frac{x^{4}}{4}+\frac{x^{7}}{7}-+\ldots\right)$. Die in der Klammer stehende Funktion hat aber die Ableitung $\left(1-x^{3}+x^{6}-+\ldots\right)=\frac{1}{1+x^{3}}$ und ist also die Funktion (s. §19, Def. 12)

$$
\int_{0}^{x} \frac{d x}{1+x^{3}}=\frac{1}{6} \log \frac{(x+1)^{2}}{x^{2}-x+1}+\frac{1}{\sqrt{3}} \operatorname{arctg} \frac{2 x-1}{\sqrt{3}} .
$$

Hiernach ist die Summe der vorgelegten Reihe $=\frac{1}{3} \log 2+\frac{\pi}{6 \sqrt{3}}$.

4. Ähnlich findet man (s. §19, Def. 12).

$$
\sum_{n=0}^{\infty} \frac{(-1)^{n}}{4 n+1}=1-\frac{1}{5}+\frac{1}{9}-\frac{1}{13}+\cdots=\frac{1}{8} \sqrt{2}[\pi+\log (3+\sqrt{2})] .
$$

Für die weiteren analog gebildeten Reihen werden die Formeln natiirlich immer verwickelter.

4. Anwendung des großen Umordnungssatzes. Theoretisch und praktisch gleich bedeutungsvoll ist die Anwendung des großen Umordnungssatzes für unsere jetzige Frage. Wir erläutern seine Anwendung gleich an einem der wichtigsten Fälle; weitere Beispiele bieten wieder die Aufgaben.

In 115 und $\mathbf{1 1 7}$ hatten wir zwei ganz verschiedene Entwicklungen der Funktion ctg erhalten, die beide jedenfalls für alle hinreichend kleinen $|x|$ gelten. Ersetzen wir in der ersten von ihnen $x$ durch $\pi x$, so muß also für solche $x$

$$
1+\sum_{n=1}^{\infty}(-1)^{n} \frac{2^{2 n} B_{2 n}}{(2 n) !}(\pi x)^{2 n}=1-\sum_{k=1}^{\infty} \frac{2 x^{2}}{k^{2}-x^{2}}
$$

sein. Jedes Glied der Reihe rechts läßt sich nun offenbar nach Potenzen von $x$ entwickeln

$$
-\frac{2 x^{2}}{k^{2}-x^{2}}=-\sum_{n=1}^{\infty} 2\left(\frac{x^{2}}{k^{2}}\right) \quad(k=1,2, \ldots \text { fes } !)
$$

Das wären die Reihen $z^{(k)}$ des großen Umordnungssatzes; und da sich dessen Reihen $\zeta^{(k)}$ hier ersichtlich nur durch das Vorzeichen 
von den $z^{(k)}$ unterscheiden, so sind die Voraussetzungen jenes Satzes alle erfüllt und wir können spaltenweis addieren. Dabei wird der Koeffizient von $x^{2 p}$ auf der rechten Seite

$$
=-2 \sum_{k=1}^{\infty} \frac{1}{k^{2 p}}
$$

und da er nach $\mathbf{9 7}$ mit dem auf der linken Seite übereinstimmen muß, so haben wir das wichtige Ergebnis, bei dem wir den Summationsbuchstaben nun wieder mit $n$ bezeichnen wollen

$$
\sum_{n=1}^{\infty} \frac{1}{n^{2 p}}=(-1)^{p-1} \frac{B_{2 p}(2 \pi)^{2 p}}{2(2 p) !}
$$

durch das die Reihen

$$
1+\frac{1}{2^{2 p}}+\frac{1}{3^{2 p}}+\ldots+\frac{1}{n^{2 p}}+\cdots
$$

geschlossen ausgewertet sind, denn die Zahl $\pi$ und die (rationalen) Bernoullischen Zahlen dürfen wir als bekannt ansehen. Speziell haben wir:

$$
\left.\sum_{n=1}^{\infty} \frac{1}{n^{2}}=\frac{\pi^{2}}{6}, \quad \sum_{n=1}^{\infty} \frac{1}{n^{4}}=\frac{\pi^{4}}{90}, \quad \sum_{n=1}^{\infty} \frac{1}{n^{6}}={\frac{\pi^{6}}{945}}^{1}\right) .
$$

Es ist nicht überflüssig, sich einmal genau zu vergegenwärtigen, was alles nötig war, um auch nur das erste dieser schönen Ergebnisse zu zeitigen ${ }^{2}$ ). Man wird finden, daß dazu ein sehr erheblicher Bruchteil unserer gesamten bisherigen Untersuchungen erforderlich ist.

1) Ganz nebenbei ergibt die Formel 136, daßs die Bernoullischen Zahlen $B_{2 n}$ abwechselnde Vorzeichen haben und daß $(-1)^{n-1} B_{2 n}$ positiv ist. Ferner auch, dafs die Bernoullischen Zahlen mit wachsendem Index außerordentlich stark wachsen; denn da für jedes $n$ der Wert $\Sigma \frac{1}{k^{2 n}}$ zwischen 1 und 2 gelegen ist, so ist

$$
2 \frac{2(2 n) !}{(2 \pi)^{2 n}}>(-1)^{n-1} B_{2 n}>\frac{2(2 n) !}{(2 \pi)^{2 n}},
$$

woraus noch weiter folgt, daf $\left|\frac{B_{2 n+2}}{B_{2 n}}\right| \rightarrow+\infty$ strebt. Da endlich die obige Umformung für $|x|<1$ galt, so ergibt sich aus dieser Betrachtung auch noch, das die Reihe 115 sicher für $|x|<\pi$ absolut konvergiert. Da sie für $|x|>\pi$ sicher nicht mehr konvergent sein kann (denn nach 98, 2 miibste dann $\operatorname{ctg} x$ in $x=\pi$ stetig sein, was doch nicht der Fall ist), so hat $\mathbf{1 1 5}$ genau den Radius $\pi$. Hiernach folgt dann, dafi 116 den Radius $\frac{\pi}{2}$ hat.

2) Um die Summe der Reihe $1+\frac{1}{4}+\frac{1}{9}+\frac{1}{16}+\ldots$ zu finden, haben sich Jak. und Joh. Bernoulli aufs äußerste bemüht. Ersterer hat die Lösung des Problems nicht mehr erlebt, die erst durch L. Euler im Jahre 1736 gefunden wurde. Joh. Bernoulli, dem sie dann bald bekannt wurde, sagt dazu (Werke, Bd. 4, S. 22): Atque ita satisfactum est ardenti desiderio Fratris mei, qui agnos- 
Damit hätten wir die Summen aller harmonischen Reihen mit geraden ganzzahligen Exponenten ermittelt; von den Summen dieser Reihen bei ungeraden Exponenten $(>1)$ weiß man zur Zeit nichts, d. h. es ist bisher nicht gelungen, diese Summen (also z. B. $\Sigma \frac{1}{k^{3}}$ ) mit irgendwelchen in anderen Zusammenhängen auftretenden Zahlen in Verbindung $\mathrm{zu}$ bringen. (Ihrer beliebig genauen numerischen Berechnung steht natürlich nichts im Wege; s. § $35^{1}$ ). Dagegen kann man aus unsern Ergebnissen noch leicht die folgenden herleiten: Es ist

$$
\sum_{n=1}^{\infty} \frac{1}{n^{2 p}}=\sum_{v=1}^{\infty} \frac{1}{(2 v-1)^{2 p}}+\sum_{\nu=1}^{\infty} \frac{1}{(2 v)^{2}} .
$$

Hier ist aber die letzte Reihe nichts anderes als $\frac{1}{2^{2 p}} \sum_{n=1}^{\infty} \frac{1}{n^{2 p}}$. Ziehen wir diese ab, so haben wir

oder also

$$
\sum_{n=1}^{\infty} \frac{1}{(2 n-1)^{2 p}}=\left(1-\frac{1}{2^{2 p}}\right) \sum_{n=1}^{\infty} \frac{1}{n^{2 p}}
$$

137.

$$
1+\frac{1}{3^{2 p}}+\frac{1}{5^{2 p}}+\ldots=(-1)^{p-1} \frac{2^{2 p}-1}{2(2 p) !} B_{2 p} \cdot \pi^{2 p} .
$$

Für $p=1,2,3, \ldots$ liefert dies ganz speziell die Summen

$$
\frac{\pi^{2}}{8}, \quad \frac{\pi^{4}}{96}, \frac{\pi^{6}}{960},
$$

Subtrahieren wir die eben abgezogene Reihe noch einmal, so erhalten wir

oder also

$$
\sum_{n=1}^{\infty} \frac{(-1)^{n-1}}{n^{2 p}}=\left(1-\frac{2}{2^{2 p}}\right) \sum_{2=1}^{\infty} \frac{1}{n^{2 p}}
$$

138. $1-\frac{1}{2^{2 p}}+\frac{1}{3^{2 p}}-\frac{1}{4^{2 p}}+-\ldots=(-1)^{p-1} \frac{2^{2 p-1}-1}{(2 p) !} B_{2, p} \cdot \pi^{2 p}$.

Für $p=1,2,3, \ldots$ liefert dies speziell die Summeń

$$
\frac{1}{12} \pi^{2}, \quad \frac{7}{720} \pi^{4}, \quad \frac{31}{30240} \pi^{6}, \quad \ldots
$$

Aber auch hier weiß man von den entsprechenden Reihen mit ungeraden Exponenten nichts. - Die beiden letzten Ergebnisse hätte

cens summae huius pervestigationem difficilorem quam quis putaverit, ingenue fassus est omnem suam industriam fuisse elusam ... Utinam Frater superstes esset! Ein zweiter, ganz andrer Beweis wird in 156, ein dritter in $\mathbf{2 1 0}$ gegeben.

1) T.J. Stieltjes (Tables des valeurs des sommes $S_{k}=\sum_{n=1}^{\infty} \frac{1}{n^{k}}$, Acta mathematica, Bd. 10 (1887), S. 299) hat die Summen dieser Reihen bis zum Exponenten 70 auf je 32 Dezimalen genau berechnet. 
man natürlich auch gewonnen, wenn man die Partialbruchzerlegung von $\operatorname{tg}$ oder $\frac{1}{\sin }$ ebenso behandelt hätte wie eben diejenige von ctg. $\mathrm{Zu}$ neuen Ergebnissen gelangen wir noch, wenn wir dasselbe mit der Partialbruchzerlegung 118 für $\frac{1}{\cos }$ machen, also mit

$$
\frac{\pi}{4 \cos \frac{\pi x}{2}}=\frac{1}{1^{2}-x^{2}}-\frac{3}{3^{2}-x^{2}}+\frac{5}{5^{2}-x^{2}}-\ldots=\sum_{v=0}^{\infty} \frac{(-1)^{v}(2 v+1)}{(2 v+1)^{2}-x^{2}} \text {. }
$$

Das $\boldsymbol{\nu}^{\text {te }}$ Glied liefert hier die Potenzreihe

$$
(-1)^{v} \sum_{k=0}^{\infty} \frac{x^{2 k}}{(2 v+1)^{2 k+1}},
$$

so daß nach der Umordnung der Koeffizient von $x^{2 p}$ lautet:

$$
\sum_{\nu=0}^{\infty} \frac{(-1)^{\nu}}{(2 v+1)^{2 p+1}}=1-\frac{1}{3^{2 p+1}}+\frac{1}{5^{2 p+1}}-\ldots
$$

Bezeichnen wir diese Summen für den Augenblick mit $\sigma_{2 p+1}$, so ist also

oder

$$
\frac{\pi}{4 \cos \frac{\pi x}{2}}=\sigma_{1}+\sigma_{3} x^{2}+\sigma_{5} x^{4}+\ldots
$$

$$
\frac{1}{\cos z}=\frac{4}{\pi}\left[\sigma_{1}+\sigma_{3}\left(\frac{2 z}{\pi}\right)^{2}+\sigma_{5}\left(\frac{2 z}{\pi}\right)^{4}+\ldots\right] .
$$

Diese Potenzreihe kann nun andrerseits unmittelbar durch Division gewonnen werden und ihre Koeffizienten also - genau wie in 105, 5 die Bernoullischen Zahlen - durch einfache Rekursionsformeln gewonnen werden. Man schreibt gewöhnlich

so $\mathrm{da} ß$

$$
\left.\frac{1}{\cos z}=\sum_{n=0}^{\infty}(-1)^{n} \frac{E_{2 n}}{(2 n) !} z^{2 n 1}\right) \text {, }
$$

$$
\left(1-\frac{x^{2}}{2 !}+\frac{x^{4}}{4 !}-+\ldots\right)\left(E_{0}-\frac{E_{2}}{2 !} x^{2}+\frac{E_{4}}{4 !} x^{4}-+\ldots\right) \equiv 1
$$

ist. Dies liefert $E_{0}=1$ und für $n \geqq 1$ die Rekursionsformeln, die man nach Multiplikation mit $(2 n)$ ! so schreiben kann:

$$
\boldsymbol{E}_{2 n}+\left(\begin{array}{c}
2 n \\
\mathbf{2}
\end{array}\right) \boldsymbol{E}_{2 n-2}+\left(\begin{array}{c}
\mathbf{2} \boldsymbol{4} \\
\mathbf{4}
\end{array}\right) \boldsymbol{E}_{2 n-4}+\ldots+\boldsymbol{E}_{\mathbf{0}}=\mathbf{0}
$$

oder noch kürzer in symbolischer Schreibweișe (vgl. 106)

$$
(E+1)^{k}+(E-1)^{k}=\mathbf{0},
$$

die nun für alle $k \geqq 1$ gilt.

1) Die hierdurch bestimmten (iibrigens ganzen rationalen) Zahlen bezeichnet man meist als Eulersche Zahlen. Die ersten 30 dieser Zahlen hat $W$. Scherk, Mathem. Abh., Berlin 1825, berechnet. 
Aus ihnen findet man mühelos

und

$$
E_{1}=E_{3}=E_{5}=\ldots=0
$$

$$
E_{0}=1, \quad E_{2}=-1, \quad E_{4}=5, \quad E_{6}=-61, \quad E_{8}=1385,
$$

Mit diesen Zahlen, die wir mit vollem Recht als bekannt ansehen dürfen, hat man dann endlich

d. h.

$$
\frac{E_{2 p}}{(2 p) !}=\frac{4}{\pi} \sigma_{2 p+1} \cdot \frac{2^{2 p}}{\pi^{2 p}},
$$

140 .

$$
1-\frac{1}{3^{2 p+1}}+\frac{1}{5^{2 p+1}}-+\ldots=(-1)^{p} \frac{E_{2 p}}{2^{2 p+2}(2 p) !} \pi^{2 p+1} .
$$

Für $p=0,1,2,3, \ldots$ liefert dies ganz speziell die Summen

$$
\frac{1}{4} \pi, \quad \frac{1}{32} \pi^{3}, \quad \frac{5}{1536} \pi^{5}, \quad \frac{61}{2^{12} \cdot 45} \pi^{7}, \quad \ldots
$$

\section{§33. Reihentransformationen.}

Im vorigen Paragraphen haben wir einige der wichtigsten Typen von Reihen kennen gelernt, die man geschlossen - im engeren und im weiteren Sinne - summieren kann. Bei den letzten schon recht tiefgehenden Auswertungen spielte der große Umordnungssatz eine wesentliche Rolle, da durch ihn die ursprünglich vorgelegte Reihe sozusagen in eine ganz andre verwandelt wurde, die uns dann weitere Aufschlüsse vermittelte. Es handelt sich also im wesentlichen um eine spezielle Reihentransformation ${ }^{\mathbf{1}}$ ). Solche Umformungen sind häufig von größtem Nutzen, und zwar für die im nächsten Paragraphen zu besprechenden numerischen Auswertungen noch mehr als für die geschlossene Summierung. Ihnen wollen wir uns jetzt zuwenden, und wir beginnen sogleich mit einer allgemeineren Auffassung der durch den großen Umordnungssatz bewirkten Reihentransformation, die wir im vorigen Paragraphen schon mit Vorteil mehrfach verwendeten:

Es sei die konvergente Reihe $\sum_{k=0}^{\infty} z^{(k)}$ vorgelegt und es werde auf irgendeine Weise (z. B. nach $\S 32$, S. 226) jedes Glied derselben durch eine unendliche Reihe dargestellt:

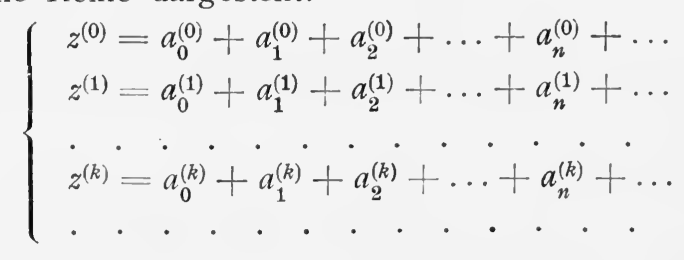

1) Solche Transformationen sind zuerst von J. Stivling (Methodus differentiales, London 1730) angegeben worden und beruhen bei ihm auf ähnlichen Grundgedanken wie die obigen, nur daßs die Prüfung der Gültigkeitsbedingungen noch fehlt. 
Wir wollen ferner annehmen, daß in diesem Schema auch die Spalten konvergente Reihen bilden, und ihre Summen mit $s^{(0)}, s^{(1)}, \ldots, s^{(n)}, \ldots$ bezeichnen. Unter welchen Bedingungen dürfen wir dann erwarten, daß die aus diesen Zahlen gebildete Reihe $\sum_{n=0}^{\infty} s^{(n)}$ konvergiert und daß

$$
\sum_{k=0}^{\infty} z^{(n)}=\sum_{n=0}^{\infty} s^{(n)}
$$

ist? Ist diese Gleichung richtig, so hätten wir jedenfalls eine Transformation der vorgelegten Reihe ausgeführt. Der große Umordnungssatz liefert sofort den

- Satz. Wenn die in den Zeilen des Schemas (A) stehenden Reihen 141. absolut konvergieren und wenn - unter $\zeta^{(k)}$ die Summe $\sum_{n=0}^{\infty}\left|a_{n}^{(k)}\right|$ der Beträge der Glieder einer Zeile verstanden - auch $\Sigma \zeta^{(k)}$ noch konvergiert, so ist $\Sigma s^{(n)}$ konvergent und $=\Sigma z^{(k)}$.

Das ist der Satz, den wir im vorigen Paragraphen mehrfach verwendet haben. Es entsteht aber die Frage, ob er nicht unnötig viel verlangt, und ob die Transformation nicht unter viel geringeren Voraussetzungen richtig ist.

A. A. Markof ${ }^{1}$ ) hat in dieser Richtung einen sehr weitgehenden Satz bewiesen. Er setzt zunächst nur voraus, daß außer der Ausgangsreihe auch die einzelnen Spalten im Schema (A) konvergieren, daß also die $s^{(k)}$ wohlbestimmte Werte haben. Mit

$$
\sum_{k=0}^{\infty} z^{(k)} \text { und } \sum_{k=0}^{\infty} a_{0}^{(k)} \quad \text { konvergiert dann auch } \sum_{k=0}^{\infty}\left(z^{(k)}-a_{0}^{(k)}\right),
$$

und ebenso konvergiert dann, wenn $m$ irgendeine feste Zahl ist, die Reihe

$$
\sum_{k=0}^{\infty}\left(z^{(k)}-a_{0}^{(k)}-a_{1}^{(k)}-\ldots-a_{m-1}^{(k)}\right)
$$

( $m$ fest).

Die Glieder dieser Reihe sind aber nichts anderes als die Reste der in den einzelnen Zeilen stehenden Reihen, jedesmal mit dem $m^{\text {ten }}$ Glied derselben beginnend $\left.{ }^{2}\right)$. Setzen wir diese Reste zur Abkürzung $=\boldsymbol{r}_{m}^{(k)}$, so daß also

ist, so ist die Reihe

$$
r_{m}^{(k)}=\sum_{n=m}^{\infty} a_{n}^{(k)}
$$

( $k$ und $m$ fest)

$$
\sum_{k=0}^{\infty} r_{m}^{(k)}=R_{m}
$$

1) Mémoire sur la transformation des séries (Mém. de l'Acad. Imp. de St. Pétersbourg, Bd. (7) 37 (1891).

2) Für $m=0$ wollen wir darunter natürlich die ganze Reihe, also $z^{(k)}$ selber, verstanden wissen. 
konvergent. Dann wird nun weiter verlangt, daß für $m \rightarrow \infty$

$$
R_{m} \rightarrow 0
$$

strebt. Unter diesen Voraussetzungen läßt sich zeigen, daß $\sum s^{(n)}$ konvergiert und $=\Sigma z^{(k)}$ ist. Es gilt also der folgende Satz:

142. Markoffsche Reihentransformation. Es sei die konvergente Reihe $\sum_{k=0}^{\infty} z^{(k)}$ vorgelegt und jedes ihrer Glieder seinerseits durch eine konvergente Reihe

$$
z^{(k)}=a_{0}^{(k)}+a_{1}^{(k)}+\ldots+a_{n}^{(k)}+\ldots, \quad(k=0,1,2, \ldots)
$$

dargestellt. Es seien die einzelnen Spalten $\sum_{k=0}^{\infty} a_{n}^{(k)}$ des hierdurch entstehenden Schemas (A) konvergent und $=s^{(n)}, n=0,1,2, \ldots$, so daß auch die Zeilenreste

eine konvergente Reihe

$$
r_{m}^{(k)}=\sum_{n=m}^{\infty} a_{n}^{(k)}
$$

$$
\sum_{k=0}^{\infty} r_{m}^{(k)}=R_{m}
$$

bilden, und es strebe endlich wit wachsendem $m$ diese Zahl

$$
\cdot R_{m} \rightarrow 0 \text {. }
$$

Dann bilden auch die Spaltensummen eine konvergente Reihe $\Sigma s^{(n)}$, und es ist

$$
\sum_{n=0}^{\infty} s^{(n)}=\sum_{k=0}^{\infty} z^{(k)}
$$

Der Beweis ist äußerst einfach. Es ist

$$
a_{n}^{(k)}=r_{n}^{(k)}-r_{n+1}^{(k)} \quad\left\{\begin{array}{l}
k=0,1,2, \ldots \\
n=0,1,2, \ldots
\end{array}\right.
$$

und also der Spaltenanfang der $n^{\text {ten }}$ Spalte $a_{n}^{(0)}+a_{n}^{(1)}+\ldots+a_{n}^{(q)}=\left(r_{n}^{(0)}+r_{n}^{(1)}+\ldots+r_{n}^{(q)}\right)-\left(r_{n+1}^{(0)}+r_{n+1}^{\prime(1)}+\ldots+r_{n+1}^{(q)}\right)$. Rechts stehen jetzt die Anfänge zweier nach Voraussetzung konvergenter Reihen. Lassen wir also $q$ über alle Grenzen wachsen, so folgt sofort für festes $n$

$$
\sum_{k=0}^{\infty} a_{n}^{(k)}=s^{(n)}=R_{n}-R_{n+1}
$$

Hiernach ist aber

$$
s^{(0)}+s^{(1)}+\ldots+s^{(n)}=R_{0}-R_{n+1},
$$

was nach der zweiten Voraussetzung die Konvergenz der Reihe $\Sigma s^{(n)}$ zur Folge hat und für ihren Wert

$$
\sum_{n=0}^{\infty} s^{(n)}=R_{0}
$$


liefert. $R_{0}$ ist aber nichts anderes als $\sum_{k=0}^{\infty} r_{0}^{(k)}=\sum_{k=0}^{\infty} z^{(k)}$, womit schon alles bewiesen ist.

B. Der Vorteil der Markoffschen Transformation gegenüber Satz 141 besteht natürlich darin, dafs bei ihr nirgends von absoluter Konvergenz die Rede ist, sondern allenthalben nur die Konvergenz schlechtweg vorausgesetzt wird. Sie gestattet viele fruchtbare Anwendungen; auf diejenigen zur numerischen Berechnung gehen wir in $\$ 35$ ein, und geben hier eine ihrer schönsten, die in der Herleitung einer schon von Euler ${ }^{1}$ ) - natürlich ohne Konvergenzbetrachtungen - angegebenen Transformation besteht.

Da man dabei mit Vorteil von den Bezeichnungen der Differenzenrechnung Gebrauch macht, wollen wir diese zunächst kurz erläutern. Ist $\left(x_{0}, x_{1}, x_{2}, \ldots\right)$ irgendeine Zahlenfolge, so nennt man

$$
x_{0}-x_{1}, x_{1}-x_{2}, \ldots, x_{k}-x_{k+1}, \ldots
$$

die Folge ihrer ersten Differenzen und bezeichnet sie mit

$$
\Delta x_{0}, \Delta x_{1}, \ldots, \Delta x_{k}, \ldots
$$

Die ersten Differenzen dieser Folge, also die Größen $\Delta x_{k}-\Delta x_{k+1}, k=0,1,2, \ldots$, nennt man die Folge der zweiten Differenzen von $\left(x_{n}\right)$ und bezeichnet sie mit $\Delta^{2} x_{0}, \Delta^{2} x_{1}, \ldots$ Allgemein setzt man für $n \geqq 1$

$$
\Delta^{n+1} x_{k}=\Delta^{n} x_{k}-\Delta^{n} x_{k+1} \quad(k=0,1,2, \ldots),
$$

und diese Formeln gelten auch schon für $n=0$, wenn man übereinkommt, unter $\Delta^{0} x_{k}$ die Zahl $x_{k}$ selber zu verstehen. Es ist vorteilhaft, eine Folge $\left(x_{k}\right)$ und ihre Differenzenfolge zeilenweis so untereinander zu schreiben, daf jede Differenz unter der Liicke der Glieder steht, deren Differenz sie ist. So entsteht das Schema

$$
\begin{gathered}
x_{0}, \Delta x_{0}, x_{1}, \Delta x_{1}, \quad \Delta x_{2},{ }^{x_{2}}, \Delta x_{3}, \ldots \ldots \\
\Delta^{2} x_{0}, \Delta^{2} x_{1}, \Delta^{2} x_{2}, \ldots \ldots \\
\Delta^{3} x_{0}, \Delta^{3} x_{1}, \ldots \ldots \\
\Delta^{4} x_{0}, \ldots \ldots
\end{gathered}
$$

Wir wollen noch $\Delta^{n} x_{k}$ durch die Glieder der gegebenen Folge ausdrücken. Man findet sofort

und ebenso leicht

$$
\begin{aligned}
\Delta^{2} x_{k}=\Delta x_{k}-\Delta x_{k+1} & =\left(x_{k}-x_{k+1}\right)-\left(x_{k+1}-x_{k+2}\right) \\
& =x_{k}-2 x_{k+1}+x_{k+2}
\end{aligned}
$$

so dafis die Formel

$$
\Delta^{3} x_{k}=x_{k}-3 x_{k+1}+3 x_{k+2}-x_{k+3},
$$

$$
\Delta^{n} x_{k}=x_{k}-\left(\begin{array}{l}
n \\
1
\end{array}\right) x_{k+1}+\left(\begin{array}{l}
n \\
2
\end{array}\right) x_{k+2}-+\ldots+(-1)^{n}\left(\begin{array}{l}
n \\
n
\end{array}\right) x_{k+n}
$$

bei festem $k$ für $n=1,2,3$ bewiesen ist. Hieraus folgt ihre Richtigkeit allgemein durch Induktion. Denn ist 143 schon für die natürliche Zahl $n$ bewiesen, so folgt für die nächstgrößere

$$
\begin{aligned}
\Delta^{n+1} x_{k}= & \Delta^{n} x_{k}-\Delta^{n} x_{k+1} \\
= & x_{k}-\left(\begin{array}{l}
n \\
1
\end{array}\right) x_{k+1}+\left(\begin{array}{l}
n \\
2
\end{array}\right) x_{k+2}-+\ldots+(-1)^{n}\left(\begin{array}{l}
n \\
n
\end{array}\right) x_{k+1} \\
& \quad-\left(\begin{array}{l}
n \\
0
\end{array}\right) x_{k+1}+\left(\begin{array}{l}
n \\
1
\end{array}\right) x_{k+2}-+\ldots+(-1)^{n}\left(\begin{array}{c}
n \\
n-1
\end{array}\right) x_{k+n}+(-1)^{n+1}\left(\begin{array}{l}
n \\
n
\end{array}\right) x_{k+n+1}
\end{aligned}
$$

1) Institutiones calculi differentialis, 1755, S. 281. 
und hieraus durch Addition - wegen $\left(\begin{array}{c}n \\ v\end{array}\right)+\left(\begin{array}{c}n \\ v-1\end{array}\right)=\left(\begin{array}{c}n+1 \\ v\end{array}\right)$ - sofort die für $n+1$ statt $n$ angesetzte Formel 143. Damit ist diese allgemein bewiesen. Unter Benutzung dieser einfachen Bezeichnungen und Tatsachen gilt nun der folgende Satz:

\section{4. $\circ$ Eulersche Reihentransformation. Ist}

$$
\sum_{k=0}^{\infty}(-1)^{k} a_{k} \equiv a_{0}-a_{1}+a_{2}-+\ldots
$$

eine beliebige konvergente Reihe ${ }^{1}$ ), so ist stets

$$
\sum_{k=0}^{\infty}(-1)^{k} a_{k}=\sum_{n=0}^{\infty} \frac{\Delta^{n} a_{0}}{2^{n+1}} ;
$$

- die neue Reihe konvergiert also stets und hat dieselbe Summe wie die vorgelegte $^{2}$.

Beweis. Wir setzen

$$
a_{n}^{(k)}=(-1)^{k}\left[\frac{1}{2^{n}} \Delta^{n} a_{k}-\frac{1}{2^{n+1}} \Delta^{n+1} a_{k}\right] \text {. }
$$

Dann ist, wenn wir bei festem $k$ über $n$ (also die Glieder der $k$ ten Zeile) summieren, (nach 131)

denn

$$
\sum_{n=0}^{\infty} a_{n}^{(k)}=(-1)^{k} \frac{1}{2^{0}} \Delta^{0} a_{k}=(-1)^{k} a_{k}
$$

$$
\lim _{n \rightarrow \infty} \frac{\Delta^{n} a_{k}}{2^{n}}=\lim _{n \rightarrow \infty} \frac{\left(\begin{array}{l}
n \\
0
\end{array}\right) a_{k}-\left(\begin{array}{l}
n \\
1
\end{array}\right) a_{k+1}+\ldots+(-1)^{n}\left(\begin{array}{l}
n \\
n
\end{array}\right) a_{k+n}}{2^{n}}
$$

ist nach 44, 8 gleich 0 , da ja $a_{k},-a_{k+1}, a_{k+2}, \ldots$ eine Nullfolge bildet. Es liefert also (a) die Darstellung der einzelnen Glieder der vorgelegten Reihe $\Sigma(-1)^{k} a_{k}$ durch unendliche Reihen. Summieren wir nun die $n$ te Spalte, so bekommen wir die Reihe

$$
\sum_{k=0}^{\infty}(-1)^{k}\left[\frac{1}{2^{n}} \Delta^{n} a_{k}-\frac{1}{2^{n+1}} \Delta^{n+1} a_{k}\right],
$$

deren allgemeines Glied kann aber, wegen $\Delta^{n+1} a_{k}=\Delta^{n} a_{k}-\Delta^{n} a_{k+1}$ in die Form

$$
\frac{(-1)^{k}}{2^{n+1}}\left[\Delta^{n} a_{k}+\Delta^{n} a_{k+1}\right]=\frac{1}{2^{n+1}}\left[(-1)^{k} \Delta^{n} a_{k}-(-1)^{k+1} \Delta^{n} a_{k+1}\right]
$$

gebracht werden, so daf nun die in Rede stehende Reihe wieder nach 131 ganz einfach summiert werden kann. Wir erhalten

$$
\sum_{k=0}^{\infty} a_{n}^{(k)}=\frac{1}{2^{n+1}}\left[\Delta^{n} a_{0}-\lim _{k \rightarrow \infty}(-1)^{k} \Delta^{n} a_{k}\right]
$$

1) Die Reihe braucht nicht alternierend, die $a_{n}$ brauchen also nicht positiv zu sein. Dafs wir die Reihe trotzdem alternierend schreiben, bietet kleine (durchaus nicht wesentliche) Vorteile bei der Durchführung der Transformation.

2) Daf die Transformation stets guiltig ist, wenn nur die Reihe $\Sigma(-1)^{k} a_{n}$ als konvergent vorausgesetzt wird, hat zuerst L. D. Ames (Annals of Math., Bd. (2) 3 (1901), S. 185) bewiesen. Vgl. auch E. Jacobsthal (Mathem. Zeitschr., Bd. 6 (1920), S. 100) und die anschliefende Note des Verf. (ebenda S. 118). 
Da aber $\left(a_{k}\right)$ eine Nullfolge bildet, so bildet auch die Folge der ersten Differenzen der $a_{k}$ eine solche, und allgemein auch - bei festem $n$ - die Folge der $n$ ten Differenzen. Somit erweisen sich die Spalten als konvergent und ihre Summen sind

$$
s^{(n)}=\frac{\Delta^{n} a_{0}}{2^{n+1}} .
$$

Die Gültigkeit der Eulerschen Transformation ist also dargetan, sobald wir noch zeigen, daf $R_{m} \rightarrow 0$ strebt. Für die Zeilenreste findet man aber, ganz ebenso wie kurz vorher für die vollen Zeilen die Werte

$$
r_{m}^{(k)}=(-1)^{k} \frac{\Delta^{m} a_{k}}{2^{m}},
$$

so dak

$$
R_{m}=\frac{1}{2^{m}} \sum_{k=0}^{\infty}(-1)^{k} \Delta^{m} a_{k}
$$

Diese Reihe kann aber, wenn wir zur Abkürzung die Reihenreste

$$
(-1)^{k}\left(a_{k}-a_{k+1}+a_{k+2}-+\ldots\right)=r_{k}
$$

setzen, durch gliedweise Addition der $(m+1)$ Reihen

$$
r_{0}, \quad\left(\begin{array}{c}
m \\
1
\end{array}\right) r_{1},\left(\begin{array}{c}
m \\
2
\end{array}\right) r_{2}, \ldots,\left(\begin{array}{c}
m \\
m
\end{array}\right) r_{m}
$$

entstanden gedacht werden. Daher ist

$$
R_{m}=\frac{r_{0}+\left(\begin{array}{c}
m \\
1
\end{array}\right) r_{1}+\left(\begin{array}{c}
m \\
2
\end{array}\right) r_{2}+\ldots+\left(\begin{array}{c}
m \\
m
\end{array}\right) r_{m}}{2^{m}}
$$

und bildet also wieder nach 44, 8 zugleich mit $\left(v_{m}\right)$ eine Nullfolge. Damit ist aber die Gültigkeit der Eulerschen Transformation allgemein bewiesen.

1. Es sei

$$
\text { Beispiele. }
$$

$$
s=1-\frac{1}{2}+\frac{1}{3}-\frac{1}{4}+\ldots
$$

vorgelegt, so daßs das Schema (4) hier die folgende Gestalt hat

$$
\begin{gathered}
1, \frac{1}{2}, \frac{1}{3}, \frac{1}{4}, \frac{1}{5}, \ldots \\
\frac{1}{1 \cdot 2}, \frac{1}{2 \cdot 3}, \frac{1}{3 \cdot 4}, \frac{1}{4 \cdot 5}, \ldots \\
\frac{1 \cdot 2}{1 \cdot 2 \cdot 3}, \frac{1 \cdot 2}{2 \cdot 3 \cdot 4}, \frac{1 \cdot 2}{3 \cdot 4 \cdot 5}, \ldots \\
\frac{1 \cdot 2 \cdot 3}{1 \cdot 2 \cdot 3 \cdot 4}, \frac{1 \cdot 2 \cdot 3}{2 \cdot 3 \cdot 4 \cdot 5}, \ldots
\end{gathered}
$$

und allgemein findet man

$$
\Delta^{n} a_{k}=\frac{n !}{(k+1)(k+2) \ldots(k+n+1)} \text {, also speziell } \Delta^{n} a_{0}=\frac{1}{n+1},
$$

was man auch leicht durch Induktion bestätigt. Daher ist

$$
s=\log 2=1-\frac{1}{2}+\frac{1}{3}-\frac{1}{4}+-\ldots=\frac{1}{1 \cdot 2^{1}}+\frac{1}{2 \cdot 2^{2}}+\frac{1}{3 \cdot 2^{3}}+\frac{1}{4 \cdot 2^{4}}+\ldots,
$$

eine Umformung, deren Bedeutung z. B. für die numerische Berechnung (§ 34) sofort erhellt. 
2. Ganz ebenso leicht findet man

$$
\frac{\pi}{4}=1-\frac{1}{3}+\frac{1}{5}-\frac{1}{7}+\ldots=\frac{1}{2}\left[1+\frac{1}{3}+\frac{1 \cdot 2}{3 \cdot 5}+\frac{1 \cdot 2 \cdot 3}{3 \cdot 5 \cdot 7}+\ldots\right] .
$$

In welchen Fällen diese Transformation für die numerische Berechnung besondere Vorteile bietet, werden wir im nächsten Paragraphen sehen.

C. Kummersche Reihentransformation. Eine andre sehr naheliegende Transformation besteht einfach darin, daß man von einer vorgelegten Reihe eine andere subtrahiert, die einerseits eine geschlossen angebbare Summe hat und deren Glieder andererseits denen der vorgelegten möglichst ähnlich gebaut sind. So entsteht z. B. aus der Reihe $s=\Sigma \frac{1}{n^{2}}$ durch Subtraktion der bekannten Reihe $(\mathbf{6 8}, 2 \mathrm{~b})$

die Transformation

$$
1=\sum_{n=1}^{\infty} \frac{1}{n(n+1)}
$$

$$
s=\sum_{n=1}^{\infty} \frac{1}{n^{2}}=1+\sum_{n=1}^{\infty} \frac{1}{n^{2}(n+1)},
$$

deren Vorteil für die Berechnung von $s$ sofort klar ist.

So einfach und naheliegend diese Transformation auch ist, so bildet sie doch in Wahrheit den Kern der Kummerschen Reihentransformation $^{\mathbf{1}}$ ), nur daß bei dieser auf eine sinngemäße Auswahl der zu subtrahierenden Reihe besonderes Gewicht gelegt wird. Das geschieht so: Es sei $\Sigma a_{n}=s$ die vorgelegte (natürlich als konvergent vorausgesetzte) Reihe und $\Sigma c_{n}=C$ eine konvergente Reihe mit der bekannten Summe $C$. Die Glieder beider Reihen seien asymptotisch proportional, etwa

Dann ist

$$
\lim _{n \rightarrow \infty} \frac{a_{n}}{c_{n}}=\gamma \neq 0
$$

145.

$$
s=\sum_{n=0}^{\infty} a_{n}=\gamma \boldsymbol{C}+\sum_{n=0}^{\infty}\left(1-\gamma \frac{c_{n}}{a_{n}}\right) a_{n}
$$

und es kann die hier auftretende neue Reihe als eine Transformation der vorgelegten angesprochen werden. Ihr Vorteil besteht hauptsächlich darin, daß die neue Reihe kleinere Glieder hat als die vorgelegte, da ja die Faktoren $\left(1-\gamma \frac{c_{n}}{a_{n}}\right) \rightarrow 0$ streben. Daher liegt ihr Anwendungsfeld hauptsächlich auf dem Gebiete dèr numerischen Berechnung und wir werden im nächsten Paragraphen Beispiele dazu kennen lernen.

1) E. E. Kummer, J. f. d. reine u. angew. Math., Bd. 16 (1837), S. 206. Vgl. hierzu auch Leclert und Catalan [Mémoires couronnés et de savants étrangers de l'Ac. Belgique, Bd. 33 (1865/67)]. 


\section{§34. Numerische Berechnungen.}

1. Allgemeines. Wir haben schon mehrfach hervorgehoben, daß die im eigentlichen Sinne geschlossenen Auswertungen von Reihen nur recht selten sind. Der allgemeinere Fall ist der, daß durch die konvergente Reihe, wie durch eine wohlbestimmte Zahlenfolge, die reelle Zahl, gegen die sie konvergiert, sozusagen erstmalig definiert (gegeben, erfaßt...) ist, in dem Sinne, wie nach den Auseinandersetzungen im I. und II. Kapitel überhaupt eine reelle Zahl allein gegeben sein kann ${ }^{1}$ ). In diesem Sinne können wir (genau wie S. 25) geradezu sagen: Die konvergente Reihe ist die Zahl, gegen die ihre Teilsummen konvergieren. Aber für praktische $Z$ wecke ist uns damit meistens wenig gedient. Hier wollen wir vielmehr eine deutlichere Vorstellung von der Größe der Zahl bekommen, wollen mehrere Zahlen untereinander bequem vergleichen können usw. Dazu ist erforderlich, daß wir alle durch irgendwelche Grenzprozesse definierten Zahlen auf eine und dieselbe als typisch angesehene Form bringen. Hierfür kommen in erster Linie die Darstellungen der Zahlen durch Dezimalbrüche als die uns heute vertrauteste Form in Betracht ${ }^{2}$ ). Doch mache man sich genau klar, daß man durch die Gewinnung dieser Darstellung im Grunde nichts anderes getan hat, als da $B$ man eine durch einen gewissen Grenzprozeß definierte Zahl durch einen andern Grenzprozeß noch einmal darstellt. Die Vorteile dieses letzteren, des Dezimalbruchs, liegen hauptsächlich in der bequemen Vergleichbarkeit der durch sie dargestellten Zahlen und in der bequemen Abschätzung des Fehlers, der dem irgendwo abgebrochenen Dezimalbruch anhaftet. Demgegenüber stehen aber beträchtliche Nachteile: die in den meisten Fällen völlig dunkle Aufeinanderfolge der Ziffern und die Mühe, die demgemäß ihre sukzessive Berechnung macht.

Man mache sich diese Vorteile und Nachteile etwa an den beiden folgenden Beispielen klar:

$$
\begin{gathered}
\left(\frac{\pi}{4}=\right) 1-\frac{1}{3}+\frac{1}{5}-\frac{1}{7}+-\ldots=0,785398 \ldots \\
(\log 2=) 1-\frac{1}{2}+\frac{1}{3}-\frac{1}{4}+-\ldots=0,693147 \ldots
\end{gathered}
$$

1) Die unendliche Reihe ist sogar - wie die bisherigen Betrachtungen wohl hinlänglich belegt haben - eine der vorteilhaftesten, theoretisch wie praktisch bedeutungsvollsten dieser Arten. Man vergleiche etwa die elementargeometrische Definition der Zahl $\pi$ (oder die von uns S. 192 benutzte rein analytische) und die Erfassung derselben durch die Reihe 122; ebenso die Definition 46, 4 von $e$ mit ihrer Erfassung durch die Reihe 111.

2) Daneben nur gelegentlich die Darstellung durch gewöhnliche Brüche. Der Grund ist immer der der bequemen Vergleichbarkeit: Ob $\frac{11}{17}$ oder $\frac{25}{39}$ die größere Zahl ist, sieht man nicht sofort, während dieselbe Entscheidung bei 0,647 und 0,641 keinerlei Rechnung erfordert.

Knopp, Unendliche Reihen. 
Die Reihen zeigen deutlich ein Bildungsgesetz der Zahl, lassen aber z.B. nicht einmal erkennen, welche der beiden Zahlen die größere ist, und um wieviel sie es etwa sein mag; die Dezimalbrüche zeigen gar kein Bildungsgesetz, geben aber ein unmittelbares Gefühl von der gegenseitigen und der absoluten Größe beider Zahlen.

Nach allem wollen wir also im folgenden unter numerischer Berechnung stets die Darstellung der Zahl durch einen Dezimalbruch verstehen.

Da wir diesen nicht vollständig hinschreiben können, müssen wir ihn nach einer bestimmten Anzahl von Ziffern abbrechen. Über die Bedeutung dieses Abbrechens sind noch ein paar Worte nötig. Will man z. B. die Zahl $e$ mit zwei Dezimalen angeben, so könnte man mit gleichem Rechte 2,71 als 2,72 schreiben, - das erste, weil die beiden ersten Dezimalen wirklich 7 und 1 lauten, das zweite, weil dieser Angabe der kleinere Fehler anzuhaften scheint. Wir wollen in dieser Beziehung folgende Vereinbarung treffen: Soll angedeutet werden, dafs die hingeschriebenen $n$ Ziffern hinter dem Komma eines Dezimalbruchs die wahren ersten $n$ Ziffern des (ohne Ende vorgestellten) Dezimalbruchs der betreffenden Zahl sind, so lassen wir hinter der $n$ ten Ziffer einige Punkte folgen und schreiben also in diesem Sinne $e=2,71 \ldots$ Soll dagegen mit Hilfe von $n$ Ziffern die in Rede stehende Zahl möglichst genau angegeben werden, soll also die $n$ te Ziffer um eine Einheit erhöht werden oder nicht, je nachdem die nächsten (wahren) Ziffern. mehr als eine halbe Einheit der $n$ ten Dezimale liefern oder nicht, so setzen wir keine Punkte. In diesem Sinne ist $e=2,72^{1}$ ).

Beide Angaben sind gleich ungenau, denn der Spielraum fur den wahren Wert beträgt beidemal $1: 10^{n}$, im ersten Falle nur nach oben, im zweiten halb nach oben und halb nach unten. Die erste Angabe kann man als die theoretisch klarere, die zweite als die praktisch brauchbarere bezeichnen. Auch die Schwierigkeit in der Ermittlung der betreffenden Ziffern ist in beiden Fällen wesentlich dieselbe. Denn beidemal kann es bei besonders ungünstig liegenden Fällen zur Feststellung der $n$ ten Ziffer nötig werden, den Fehler der Berechnung noch sehr erheblich unter $1: 10^{n}$ herabzudricken. Liegt z. B. eine Zahl $\alpha=5,27999999326 \ldots$ vor, so würde zur Entscheidung dariiber, ob mit 2 Dezimalen $\alpha=5,27 \ldots$ oder $5,28 \ldots$ ist, der Fehler in der Berechnung noch unter eine Einheit der 8 ten Dezimale herabgedrückt werden müssen. Ist andererseits eine Zahl $\beta=2,3850000026 \ldots$, so wïrde gle1chfalls 'auf die Alternative, ob $\beta \approx 2,38$ oder $\approx 2,39 \mathrm{zu}$ setzen ist, eine Unsicherheit um eine Einh 8 ten Dezimale noch von Einflufi sein ${ }^{2}$ ).

2. Fehler- oder Restabschätzungen. Ist nun die konvergente Reihe $\Sigma a_{n}=s$ vorgelegt, so nehmen wir natürlich an, daß die einzelnen Reihenglieder „bekannt" sind, daß also ihre Dezimalbruchentwicklungen bis zu jeder beliebigen Ziffer ohne Mühe hingeschrieben werden können. Durch Addition können wir daher auch

1) Oder besser $e \approx 2,72$, weil doch $e \neq 2,72$ ist. Bei $e=2,71 \ldots$ kann das Gleichheitszeichen im Sinne einer Limesbeziehung gerechtfertigt werden.

2) Die Wahrscheinlichkeit für das Eintreten solcher Fälle ist natürlich äuferst gering. Es sollte durch ihre Erwähnung nur auf die Bedeutung dieser Dinge aufmerksam gemacht werden. In Aufgabe 139 wird indessen ein besonders krasser Fall dieser Art angegeben. 
jede beliebige Teilsumme $s_{n}$ berechnen und die einzige Frage ist nun die: Wie groß ist der Fehler, der einem bestimmten $s_{n}$ noch anhaftet? ${ }^{1}$ ) Als Fehler bezeichnen wir dabei diejenige (positive oder negative) Zahl, die zu $s_{n}$ noch hinzugetügt werden muß, um den ge. suchten Wert $s$ zu ergeben. Da dieser Fehler $=s-s_{n}$, also gleich dem hinter dem $n^{\text {ten }}$ Gliede begonnenen Rest der Reihe ist, so wollen wir ihn mit $r_{n}$ bezeichnen und die Bestimmung des Fehlers auch eine Restabschätzung der Reihe nennen.

Die Restabschätzungen kommen in der Praxis fast immer auf eine der beiden folgenden Arten zurück:

A. Reste absolut konvergenter Reihen. Ist $s=\sum a_{n}$ absolut kon-) vergent, so suche man eine Reihe $\Sigma a_{n}^{\prime}$ mit positiven Gliedern, die bequem geschlossen summiert werden kann und deren Glieder mindestens so groß sind wie die Beträge derjenigen der gegebenen Reihe (und doch auch nur möglichst wenig größer). Dann ist offenbar

$$
\left|\boldsymbol{r}_{n}\right| \leqq\left|a_{n+\mathbf{1}}\right|+\left|a_{n+\mathbf{2}}\right|+\ldots \leqq a_{n+1}^{\prime}+a_{n+2}^{\prime}+\ldots=\boldsymbol{r}_{n}^{\prime}
$$

und die als bekannt anzusehende Zahl $r_{n}^{\prime}$ gibt uns eine Abschätzung von $\boldsymbol{r}_{n}$, nämlich $\left|\boldsymbol{r}_{n}\right| \leqq \boldsymbol{r}_{n}^{\prime}$, die um so besser sein wird, je weniger die $a_{n}^{\prime}$ die $\left|a_{n}\right|$ übertreffen.

Besonders häufig ist der Fall, daß̧ für ein gewisses $n$ und alle $k \geq 1$

$$
\left|a_{n+k}\right| \leqq\left|a_{n}\right| \cdot a^{k} \quad(0<a<1
$$

ist; dann ist natürlich

also ganz speziell

$$
\left|v_{n}\right| \leqq\left|a_{n}\right| \frac{a}{1-a},
$$

$$
\left|v_{n}\right| \leqq\left|a_{n}\right| \text {, }
$$

wenn $0<a \leqq \frac{1}{2}$ ist. In diesem Falle ist also der Betrag des Restes höchstens so grof wie der des letzten berechneten Gliedes ${ }^{2}$ ).

B. Reste alternievender Reihen. Liegt eine Reihe der Form $s=\Sigma(-1)^{n} a_{n}$ vor und nehmen die (positiven) $a_{n}$ monoton zu Null ab, so ist (vgl. $\mathbf{8 2}, 9$ u. 10)

$$
\begin{aligned}
0<(-1)^{n+1} r_{n} & =\left(a_{n+1}-a_{n+2}\right)+\left(a_{n+3}-a_{n+4}\right)+\ldots \\
& =a_{n+1}-\left(a_{n+2}-a_{n+3}\right)-\ldots<a_{n+1}
\end{aligned}
$$

und man kann sagen: der Fehler $r_{n}$ hat dasselbe Vorzeichen wie das erste vernachlässigte Glied, ist aber absolut genommen kleiner als dieses.

Sind diese beiden Methoden nicht anwendbar, so ist die Restabschätzung meist mühsamer und man ist von Fall zu Fall auf besondere Kunstgriffe angewiesen. Als gut oder schlecht konvergent werden wir dabei eine Reihe be-

1) Oder praktischer gefaft: In wieviel Dezimalen stimmt $s_{n}$ mit dem gesuchten Wert $s$ überein?

2) Bei diesen Abschätzungen ist zu beachten, daßs sie zwar über den Betrag des Restes $r_{n}$, nicht aber unmittelbar über sein Vorzeichen Aufschlufs geben. 
zeichnen, je nachdem $r_{n}$ schon für mäßige Werte von $n$ unter die gewünschte Genauigkeitsgrenze herabsinkt oder nicht ${ }^{1}$ ).

Einige weitere prinzipielle Bemerkungen endlich erläutern wir an der

3. Berechnung der Zahl e. Es war

$$
e=1+\frac{1}{1 !}+\frac{1}{2 !}+\frac{1}{3 !}+\ldots+\frac{1}{n !}+\ldots
$$

und wir hatten schon S. 186 gefunden, daß, hier der (eo ipso positive) Rest $r_{n}$ kleiner als der $n^{\text {te }}$ Teil des letzten berechneten Gliedes, daß also

$$
s_{n}<e<s_{n}+\frac{1}{n ! n}
$$

ist. Bei der Durchführung der nun einsetzenden Zahlenrechnung hat man aber noch folgenden Umstand zu beachten: Auch bei der Darstellung der einzelnen Reihenglieder durch Dezimalbrüche müssen wir irgendwo abbrechen, d. h. Fehler machen. Und wenn $n$ nicht gar zu klein ist, können sich diese Fehler so häufen, daß dadurch die ganze Restabschätzung illusorisch zu werden droht. Man muß dann so vorgehen: Es ist, wenn wir etwa nach 9 Ziffern abbrechen,

$$
\begin{aligned}
a_{0}+a_{1}+a_{2}=\frac{5}{2} & =2,500000000 \\
a_{3} & =0,166666667^{-} \\
\left.a_{4}{ }^{2}\right) & =0, .41666667^{-} \\
a_{5} & =0, \ldots 8333333^{+} \\
a_{6} & =0, \ldots 1388889^{-} \\
a_{7} & =0, \ldots 198413^{-} \\
a_{8} & =0, \ldots 24802^{-} \\
a_{9} & =0, \ldots \ldots 2756^{-} \\
a_{10} & =0, \ldots \ldots 276^{-} \\
a_{11} & =0, \ldots \ldots 25^{+} \\
a_{12} & =0, \ldots \ldots 2^{+} \\
r_{12} & <0, \ldots \ldots 0^{+}
\end{aligned}
$$

und hier sollen die kleinen \pm -Zeichen andeuten, ob der bei dem betreffenden Gliede gemachte Fehler positiv oder negativ ist. In beiden Fällen ist er absolut genommen $<$ als eine halbe Einheit der letzten Dezimale. Die Addition aller Zahlen ergibt

$$
2,718281830 \text {. }
$$

$s_{12}$ selber kann aber möglicherweise, nämlich wenn alle positiven Fehler nahe an 0 liegen, alle negativen Fehler dagegen nahezu eine

1) Eine schärfere Definition der Gute der Konvergenz werden wir in $\S 37$ geben.

$\left.{ }^{2}\right)$ Es entsteht $a_{n}$ aus $a_{n-1}$ einfach, indem dieses durch $n$ dividiert wird. 
halbe Einheit der letzten Dezimale ausmachen, noch bis zu $\frac{7}{2}$ Einheiten der letzten Dezimale kleiner sein, - kann dort aber auch bis zu $\frac{3}{2}$ Einheiten größer sein, denn wir haben 7 negative und 3 positive Fehler angemerkt. Berücksichtigt man noch den Rest, so folgt wegen $s_{n}<e=s_{n}+r_{n}$ mit Sicherheit aus unseren Zahlen nur, daß

$$
2,718281826<e<2,718281832
$$

ist. Durch unsere Berechnung sind also die wahren Dezimalen nur bis zur 7 ten einschließlich gesichert, während für den abgerundeten Wert sich bis zur $8^{\text {ten }}$ Stelle $e \approx 2,71828183$ ergeben hat ${ }^{1}$ ).

Für die Praxis wird es im allgemeinen genügen, wenn man die Berechnung der Reihenglieder ein paar Dezimalen (2 oder höchstens 3) weitertreibt, als für die Summe selbst gebraucht werden, und wenn man die Anzahl $n$ der Reihenglieder so groß nimmt, daß die für $r_{n}$ bekannte Restabschätzung höchstens eine Einheit in der letzten hingeschriebenen Dezimale liefert. Dann wird es auf die Fehler bei diesen Reihengliedern selbst im allgemeinen nicht mehr ankommen. Will man aber absolut gesicherte Ziffern haben, so wird man wie beschrieben vorgehen müssen; denn mag man die Berechnung der Glieder auch um viele Ziffern weiter treiben, - es haften den dann abgebrochenen Dezimalbrïchen doch immer noch Fehler an, die sich verstärken und bei besonders ungünstig liegenden Fällen (vgl. die Beisp. S. 242) ihren Einfluf bis in erheblich weiter vorn gelegene Dezimalen erstrecken können.

4. Berechnung der Zahl $\boldsymbol{\pi}$. Zur Berechnung der Zahl $\pi$ haben wir bisher vor allem die arctg- und die arcsin-Reihe zur Verfügung, von denen die erstere wegen ihrer einfacheren Bauart jetzt den Vorzug verdient. Aus ihr hatten wir die Reihe $\frac{1}{4} \pi=1-\frac{1}{3}+\frac{1}{5}-\ldots$ gewonnen, die für die numerische Verwendung so gut wie wertlos ist. Denn nach S. 243 können wir über den Rest $r_{n}$ zunächst nur sagen, da $\beta$ er das Vorzeichen $(-1)^{n+1}$ hat und absolut genommen $<\frac{1}{2 n+3}$ ist. Um also 6 Dezimalen zu sichern, müßte man mindestens $n>10^{6}$ nehmen. Eine Million Glieder zu berechnen, ist aber praktisch unmöglich. Eine wesentliche Verbesserung der Konvergenz erzielen wir hier durch die Eulersche Transformation 144,2. Auf den Nutzen solcher Umformungen für die numerische Berechnung wollen wir im nächsten Paragraphen eingehen. Hier wollen wir aus der arctg-Reihe selbst vorteilhaftere Reihen für $\pi$ ableiten.

Schon leidlich gut brauchbar ist die Reihe für $\operatorname{arctg} \frac{1}{\sqrt{3}}=\frac{\pi}{6}$; sie liefert

$$
\frac{\pi}{6}=\frac{1}{\sqrt{3}}\left[1-\frac{1}{3 \cdot 3}+\frac{1}{5 \cdot 3^{2}}-\frac{1}{7 \cdot 3^{3}}+\ldots\right] .
$$

1) Vg1. S. 242, Fuín. 1. 
Doch liefert das folgende Verfahren wesentlich günstigere Reihen ${ }^{1}$ ): Die Zahl

$$
\alpha=\operatorname{arctg} \frac{1}{5}=\frac{1}{5}-\frac{1}{3 \cdot 5^{3}}+\frac{1}{5 \cdot 5^{5}}-\frac{1}{7 \cdot 5^{7}}+-\ldots
$$

ist aus der Reihe leicht zu berechnen (s. u.). Für sie ist $\operatorname{tg} \alpha=\frac{1}{5}$, also

$$
\operatorname{tg} 2 \alpha=\frac{2 \operatorname{tg} \alpha}{1-\operatorname{tg}^{2} \alpha}=\frac{5}{12} \quad \text { und } \quad \operatorname{tg} 4 \alpha=\frac{120}{119} .
$$

Hiernach ist $4 \alpha$ nur wenig größer als $\frac{\pi}{4}$. Setzen wir $4 \alpha-\frac{\pi}{4}=\beta$, so ist

$$
\operatorname{tg} \beta=\frac{\operatorname{tg} 4 \alpha-\operatorname{tg} \frac{1}{4} \pi}{1+\operatorname{tg} 4 \alpha \cdot \operatorname{tg} \frac{1}{4} \pi}=\frac{1}{239} .
$$

Daher ist $\beta$ aus

$$
\beta=\operatorname{arctg} \frac{1}{239}=\frac{1}{239}-\frac{1}{3} \cdot \frac{1}{239^{3}}+-\ldots
$$

sehr bequem zu berechnen. Dann liefern $\alpha$ und $\beta$ zusammen

$$
\boldsymbol{\pi}=4(4 \alpha-\beta)
$$

146. $=16 \cdot\left[\frac{1}{5}-\frac{1}{3 \cdot 5^{3}}+\frac{1}{5 \cdot 5^{5}}-\ldots\right]-4\left[\frac{1}{239}-\frac{1}{3 \cdot 239^{3}}+\ldots\right]$.

Wollen wir nun etwa die ersten 7 wahren Dezmalen von $\pi$ berechnen, so versuchen wir es etwa mit 9 Dezimalen bei den Reihengliedern und dem Rest auszukommen ${ }^{2}$, - was knapp bemessen ist, da die den Zahlen $\alpha$ und $\beta$ anhaftenden Fehler ja zum Schlus noch mit 16 bzw. 4 multipliziert werden. Bezeichnen wir die erste Reihe mit $a_{1}-a_{3}+a_{5}-\ldots$, die zweite mit $a_{1}^{\prime}-a_{3}{ }^{\prime}+a_{5}{ }^{\prime}-+\ldots$ und die Teilsummen entsprechend mit $s_{v}$, und $s_{\boldsymbol{v}}{ }^{\prime}$, so geht nun die Berechnung so:

$$
\begin{aligned}
& a_{1}=0,200000000 \\
& a_{5}=0,000064000 \\
& a_{9}=0, \ldots \ldots 57^{-} \\
& a_{1}+a_{5}+a_{9}=0,200064057- \\
& \left.s_{11}=0,197395559 \pm++3\right) \text {, } \\
& a_{3}=0,002666667^{-} \\
& a_{7}=0,000001829^{-} \\
& \begin{aligned}
a_{11} & =0,000000002- \\
a_{3}+a_{7}+a_{11} & =0,002668498^{-}-
\end{aligned} \\
& 0<r_{11}<10^{-10}
\end{aligned}
$$

Hiernach ist

$$
3,158328936<16 \propto<3,158328970,
$$

denn nach der Multiplikation mit 16 müssen $\frac{16}{2}=8$ Einheiten in der 9 ten Dezimale abgezogen und $\frac{48}{2}=24$ Einheiten zugezählt werden, um eine untere bzw. obere Schranke für $16 s_{11} \mathrm{zu}$ bekommen. Wegen $0<16 r_{11}<2 \cdot 10^{-9}$ muissen wir endlich zur letzteren noch 2 Einheiten hinzufügen, um die entsprechenden Schranken für $16 \propto \mathrm{zu}$ bekommen. Weiter ist nun

$$
\begin{aligned}
a_{1}{ }^{\prime} & =0,004184100^{+} \\
a_{3}{ }^{\prime} & =0, \ldots \ldots 024^{+} \\
a_{1}{ }^{\prime}-a_{3}{ }^{\prime} & =0,004184076^{ \pm}
\end{aligned} \quad 0<r_{3}{ }^{\prime}<10^{-1: 2},
$$

1) J. Machin (in W. Jones, Synopsis, London 1706).

$\left.{ }^{2}\right)$ Erst der Erfolg kann lehren, ob dies genügt. Denn man weif̧ im voraus nicht, ob nicht einer der S. 242 beschriebenen besonders ungünstigen Fälle vorliegt.

s) Beim Subtrahieren wechseln die Fehler ihr Vorzeichen. 
also

$$
-0,016736307<-4 \beta<-0,016736302,
$$

was zusammen mit dem vorigen

$$
3,141592629<\pi<3,141592668
$$

ergibt. Unsere kurze Rechnung ergibt uns also wirklich die 7 ersten wahren Dezimalen für $\pi$ :

$$
\pi=3,1415926 \ldots
$$

(Dieselbe Rechnung würde uns für den abgerundeten Wert erst 6 Dezimalen sichern; vgl. dazu die Berechnung von $e$, wo es in dieser Beziehung gerade umgekehrt lag.)

Die benutzten Reihen für die Berechnung von $\pi$ gehören $z u$ den bequemsten; man kann mit ihrer Hilfe ohne allzu große Mühe eine viel höhere Anzahl von Dezimalen sichern ${ }^{1}$ ), und wir können daher mit vollem Recht die Zahl $\pi$ von nun an $z u$ den "bekannten" Zahlen rechnen.

5. Berechnung der Logarithmen. Schon in $\S 26$ sagten wir, daß die Reihe

$$
\log \frac{1+x}{1-x}=2\left[x+\frac{x^{3}}{3}+\frac{x^{5}}{5}+\ldots\right],
$$

den Ausgangspunkt für die Berechnung der (natürlichen) Logarithmen bildet. Für $x=\frac{1}{3}$ ist die Reihe schon gut konvergent und liefert sofort

$$
\log 2=2\left[\frac{1}{3}+\frac{1}{3 \cdot 3^{3}}+\frac{1}{5 \cdot 3^{5}}+\ldots\right] .
$$

Bezeichnen wir die Glieder der in der eckigen Klammer stehenden Reihe mit $a_{0}, a_{1}, \ldots$, so ist

und

$$
a_{n}=\frac{1}{(2 n+1) \cdot 3^{2 n+1}}
$$

also

$$
0<r_{n}<\frac{1}{(2 n+3) \cdot 3^{2 n+3}}\left[1+\frac{1}{9}+\frac{1}{9^{2}}+\ldots\right] \text {, }
$$

$$
0<r_{n}<\frac{1}{(2 n+1) \cdot 3^{2 n+1}} \cdot \frac{1}{3^{2}} \cdot \frac{9}{8}=\frac{a_{n}}{8} .
$$

Die Rechnung sieht nun, wenn wir die Glieder wieder mit 9 Dezimalen hinschreiben, so aus:

$$
\begin{gathered}
a_{0}=0,333333333^{+} \\
a_{1}=0,012345679^{+} \\
a_{2}=0,000823045^{+} \\
a_{3}=0, \ldots 065321^{+} \\
a_{4}=0, \ldots 005645^{+} \\
a_{5}=0, \ldots \ldots 513^{+} \\
a_{6}=0, \ldots \ldots 048^{+} \\
a_{7}=0, \ldots .005^{+} \\
{\left[r_{7}<0, \ldots .001\right]} \\
\hline 0,346573589
\end{gathered}
$$

1) Die Zahl $\pi$ ist auf über 700 Dezimalen berechnet worden. Vgl. indessen die Bemerkung S. 186, Fufnn. 1. 
Hieraus folgt nun, unter Beriicksichtigung des Restes und der kleinen +- Zeichen: $\log 2=0,6931471 \ldots$

mit 7 gesicherten Dezimalen ${ }^{1}$ ).

Hat man erst $\log 2$ in Händen, so macht die Berechnung der Logarithmen der übrigen Zahlen nur noch sehr viel geringere Mühe Denn unsere Reihe liefert für $x=\frac{1}{2 p+1}$

148. $\log (p+1)=\log p+2\left[\frac{1}{2 p+1}+\frac{1}{3(2 p+1)^{3}}+\frac{1}{5(2 p+1)^{5}}+\ldots\right]$ ist also $\log p$ bekannt $(p=2,3, \ldots)$, so liefert sie $\log (p+1)$, und zwar mit Hilfe einer Reihe, die wegen $\frac{1}{2 p+1}=\frac{1}{5}, \frac{1}{7}, \ldots$ sehr gut konvergiert. Denn für den Rest hat man (vgl. oben den Fall $p=1$ )

$$
0<r_{n}<\frac{1}{(2 n+3) \cdot(2 p+1)^{2 n+3}} \cdot \frac{1}{1-\frac{1}{(2 p+1)^{2}}}<\frac{a_{n}}{4 p(p+1)},
$$

also eine schon bei mäßigen Werten von $n$ sehr kleine Schranke. Und diese Güte der Konvergenz steigert sich natürlich, sobald $p$ schon etwas größer ist, sobald man also die Berechnung der ersten Logarithmen überwunden hat. - Hierbei ist noch die Bemerkung von Nutzen, daß man wegen $\mathbf{3 7}, 1$ nur die Logarithmen der Primzahlen $2,3,5,7,11,13, \ldots$ nötig hat, um daraus durch einfachste Kombinationen diejenigen aller übrigen Zahlen zu bilden.

Nehmen wir nun etwa an, wir hätten schon die Berechnung der Logarithmen der ersten vier Primzahlen 2, 3, 5, 7 durchgeführt, so ist die Arbeit zur Berechnung der übrigen nur gering. So ist z. B., indem wir $p=10$ setzen,

$$
\log 11=\log 2+\log 5+2\left[\frac{1}{21}+\frac{1}{3 \cdot 21^{3}}+\frac{1}{5 \cdot 21^{5}}+\ldots\right]
$$

mit

so daß schon für $n=3$

$$
0<r_{n}<\frac{a_{n}}{11 \cdot 40},
$$

$$
0<r_{n}<\frac{1}{7 \cdot 21^{7} \cdot 11 \cdot 40}<\frac{1}{20^{8} \cdot 2 \cdot 11 \cdot 7}<\frac{1}{10^{9} \cdot 2^{9} \cdot 7}<\frac{1}{10^{12}}
$$

ist, - ein Genauigkeitsgrad, der selbst für die feinsten wissenschaftlichen Zwecke ausreichend ist.

Hiernach möchte man nur für die Berechnung von $\log 2, \log 3, \log 5$ und allenfalls noch für $\log 7$ eine etwas bequemere Methode $z u$ besitzen wünschen. Mannigfache Kunstgriffe führen hier zum Ziel, die alle darauf

1) Die Reihe $1-\frac{1}{2}+\frac{1}{3}-\frac{1}{4}+\ldots$ für $\log 2$ ist zur Berechnung dieser Zahl natürlich ganz ungeeignet; auch ihre in 144, 1 vorgenommene Eulersche Transformation ist noch nicht so bequem wie die eben benutzte Reihe. 
ausgehen, eine rationale Zahl $\frac{k}{m} z u$ suchen, die dicht an 1 liegt und bei der Zähler und Nenner nur aus Potenzen dieser ersten Primzahlen bestehen. So kann man z. B. die Logarithmen von $\frac{16}{15}, \frac{25}{24}, \frac{81}{80}$ mit unserer Ausgangsreihe 147 äuß3erst schnell berechnen, da man dort $x=\frac{1}{31}, \frac{1}{49}, \frac{1}{161}$ setzen muß, also noch erheblich kleinere Werte als eben zur Berechnung von log 11. Hat man diese berechnet, so prüft man sofort, daßs nun

$$
\begin{aligned}
& \log 2=7 \log \frac{16}{15}+5 \log \frac{25}{24}+3 \log \frac{81}{80} \\
& \log 3=11 \log \frac{16}{15}+8 \log \frac{25}{24}+5 \log \frac{81}{80} \\
& \log 5=16 \log \frac{16}{15}+12 \log \frac{25}{24}+7 \log \frac{81}{80}
\end{aligned}
$$

ist. Hiermit wären diese drei Logarithmen durch äuferst schnell konvergierende Reihen dargestellt. - Noch bequemer für die tatsächliche Durchführung der Rechnung ist der folgende von $A$ dams $^{1}$ ) angegebene Weg: Man berechne die Logarithmen von $\frac{10}{9}, \frac{25}{24}, \frac{81}{80}$ nicht mit Hilfe der eben benutzten Reihe, sondern mit Hilfe der ursprünglichen Reihe 120, a und b, die hier die Entwicklungen

$$
\begin{aligned}
& \log \frac{10}{9}=-\log \left(1-\frac{1}{10}\right)=\frac{1}{10}+\frac{1}{2 \cdot 10^{2}}+\frac{1}{3 \cdot 10^{3}}+\ldots \\
& \log \frac{25}{24}=-\log \left(1-\frac{4}{100}\right)=\frac{4}{100}+\frac{1}{2} \cdot \frac{16}{100^{2}}+\frac{1}{3} \cdot \frac{64}{100^{3}}+\ldots \\
& \log \frac{81}{80}=\log \left(1+\frac{1}{80}\right)=\frac{1}{8 \cdot 10}-\frac{1}{2} \cdot \frac{1}{64 \cdot 10^{2}}+\frac{1}{3 \cdot 512 \cdot 10^{3}}+\ldots
\end{aligned}
$$

liefern. Wegen der im Nenner auftretenden Potenzen von 10 ist hier die Berechnung nun eben äuferst bequem. Mit Hilfe dieser Logarithmen hat man dann

$$
\begin{aligned}
& \log 2=7 \log \frac{10}{9}-2 \log \frac{25}{24}+3 \log \frac{81}{80} \\
& \log 3=11 \log \frac{10}{9}-3 \log \frac{25}{24}+5 \log \frac{81}{80} \\
& \log 5=16 \log \frac{10}{9}-4 \log \frac{25}{24}+7 \log \frac{81}{80} .
\end{aligned}
$$

Verschafft man sich nun noch den ebenso leicht $\mathrm{zu}$ berechnenden Wert

$$
\left.\log \frac{126}{125}=\log \left(1+\frac{8}{1000}\right)=\frac{8}{10^{3}}-\frac{1}{2} \cdot \frac{8^{2}}{10^{6}}+\frac{1}{3} \cdot \frac{8^{8}}{10^{9}}-\ldots{ }^{2}\right),
$$

1) Proc. of the Royal Soc. of London, Bd. 27 (1878), S. 88.

2) Wie bequem diese Berechnung ist, zeigt die folgende Ausführung, die uns nach 5 einfachen Zeilen 10 gesicherte Dezimalen von $\log \frac{126}{125}$ liefert:

$$
\left.\begin{array}{l}
+0,008000000000 \\
-0, \ldots 032000000 \\
+0, \ldots \ldots 10667- \\
-0, \ldots \ldots 001024 \\
+0, \ldots \ldots .007-\ldots .00
\end{array}\right\} \log \frac{126}{125}=0,0079681696 \ldots
$$


so hat man auch noch

$$
\log 7=19 \log \frac{10}{9}-4 \log \frac{25}{24}+8 \log \frac{81}{80}+\log \frac{126}{125} .
$$

Damit ist ein praktisch bequem gangbarer Weg zur tatsächlichen Berechnung der natürlichen Logarithmen eröffnet. Auf weitere Einzelheiten bei der Herstellung der Tafeln können wir hier nicht eingehen.

Mit $\log 2$ und $\log 5$ hat man auch $\log 10$ und also in

$$
M=\frac{1}{\log 10}=0,43429448190 \ldots
$$

den Modul des Briggschen Logarithmensystems mit der Basis 10, d. h. die Zahl, mit der alle natürlichen Logarithmen multipliziert werden müssen, um die Briggschen zu ergeben ${ }^{1}$ ).

6. Berechnung der Wurzeln. Nachdem man in den Besitz der Logarithmen gelangt ist, ist es für die Praxis nicht sehr wichtig, auch noch bequeme Mittel zur Berechnung der Wurzeln aus den natürlichen Zahlen zu besitzen. IVir fassen uns daher bei den folgenden Ausführungen ganz kurz: Auch die binomische Reihe

$$
(1+x)^{\alpha}=\sum_{n=0}^{\infty}\left(\begin{array}{l}
\alpha \\
n
\end{array}\right) x^{n}
$$

konvergiert um so besser, je kleiner $|x|$ ist. Man wird aber stets die Potenz $\sqrt[p]{q}=q_{\text {s. }}^{\frac{1}{p}}$ im wesentlichen auf die Form $(1+x)^{\frac{1}{p}}$ mit kleinem $|x|$ bringen können.

149. Einige Beispiele mögen zur Erläuterung dienen. Für $\sqrt{2}$ hatten wir schon S. 203 die Reihe für $\frac{7}{5}\left(1-\frac{1}{50}\right)^{-\frac{1}{2}}$ angegeben:

$$
\sqrt{2}=\frac{7}{5}\left[1+\frac{1}{2} \cdot \frac{1}{50}+\frac{1 \cdot 3}{2 \cdot 4} \cdot \frac{1}{50^{2}}+\frac{1 \cdot 3 \cdot 5}{2 \cdot 4 \cdot 6} \cdot \frac{1}{50^{3}}+\ldots\right] .
$$

Da $(-1)^{n}\left(\begin{array}{c}-\frac{1}{2} \\ n\end{array}\right)$ stets positiv ist und monoton fällt, hat mán für den Rest $r_{n}$ die Abschätzung

$$
0<r_{n}<a_{n} \cdot\left(\frac{1}{50}+\frac{1}{50^{2}}+\ldots\right)=\frac{a_{n}}{49},
$$

- also schon bei kleinem $n$ eine bedeutende Genauigkeit ${ }^{2}$ ).

1) Ganz nebenbei sei noch bemerkt, dafi die anfänglich befremdliche Bezeichnung der Logarithmen mit der merkwürdigen Basis $e$ als "natürliche" sich nun wohl hinlänglich gerechtfertigt hat.

2) Wie bequem hiernach die Rechnung ist, zeigt die folgende Ausführung:

$$
\left.\begin{array}{r}
a_{0}+a_{1}=1,010 \ldots \ldots \ldots 0 \\
a_{2}=0, \ldots 15 \ldots \ldots .0 \\
a_{3}=0, \ldots \ldots 25 \ldots \ldots 0 \\
a_{4}=0, \ldots \ldots .4375 .0 \\
a_{5}=0, \ldots \ldots \ldots 7875
\end{array}\right\} \begin{gathered}
s_{5}=1,0101525445375 \\
0<r_{5}<17 \cdot 10^{-12} \\
\sqrt{2}=1,4142135623 \ldots
\end{gathered}
$$

die uns also schon die ersten 10 Dezimalen sichert. 
Noch wirkungsvoller sind die Ansätze

$$
\sqrt{2}=\frac{99}{70}\left(1+\frac{1}{9800}\right)^{-\frac{1}{2}}, \quad \sqrt{2}=\frac{141}{100}\left(1-\frac{119}{20000}\right)^{-\frac{1}{2}},
$$

oder ähnliche, die man erhält, wenn man irgendeinen rohen Approximationswert $a$ von $\sqrt{2}\left(\right.$ im ersten Falle: $\frac{99}{70}$, im zweiten: 1,41) ausgeht und

$$
\sqrt{2}=a \sqrt{\frac{2}{a^{2}}}
$$

setzt. Da $a^{2}$ in der Nähe von 2 liegen sollte, hat der Radikand jetzt die Form $1+x$ mit kleinem $|x|$. - Weif man z. B. schon, dafi $\sqrt{3}=1,732 \ldots$ ist, so hat man nur

$$
\sqrt{3}=1,732 \sqrt{\frac{3}{(1,732)^{2}}}=1,732\left[1-\frac{176}{3000000}\right]^{-\frac{1}{2}}
$$

zu setzen, um aus dieser Entwicklung $\sqrt{3}$ mit größster Leichtigkeit auf 50 oder mehr Dezimalen berechnen zu können.

Wir geben noch ohne weitere Erläuterung die Beispiele:

$$
\begin{array}{ll}
\sqrt{11}=\frac{10}{3}\left(1-\frac{1}{100}\right)^{\frac{1}{2}}, & \sqrt{13}=\frac{18}{5}\left(1-\frac{1}{325}\right)^{-\frac{1}{2}} \\
\sqrt[3]{2}=\frac{5}{4}\left(1+\frac{3}{125}\right)^{\frac{1}{3}}, & \sqrt[3]{3}=\frac{10}{7}\left(1+\frac{29}{1000}\right)^{\frac{1}{3}} .
\end{array}
$$

\%. Berechnung der trigonometrischen Funktionen. Die Reihen 150. für $\sin x$ und $\cos x$ sind noch besser konvergent als die Exponentialreihe, da entweder nur gerade oder nur ungerade Potenzen von $x$ auftreten und da überdies die Vorzeichen abwechseln. Daher sind keine besonderen Kunstgriffe notwendig: Für nicht allzu große Winkel leisten die Reihen alles nur irgend Wünschenswerte.

Um z. B. $\sin 1^{0} \mathrm{zu}$ berechnen, haben wir zunächst $\mathbf{1}^{0} \mathrm{im}$ Bogenmaß auszudrücken. Es ist $1^{0}=\frac{\pi}{180}=0,017453292 \ldots$, also $<\frac{1}{50}$. Bezeichnen wir diese Zahl mit $\alpha$, so ist

$$
\sin 1^{0}=\alpha-\frac{\alpha^{3}}{3 !}+\frac{\alpha^{5}}{5 !}-+\ldots-a_{0}-a_{1}+a_{2}-+\ldots
$$

und für den Fehler $r_{n}$ hat man nach S. $243, \mathrm{~B}$

$$
0<(-1)^{n+1} r_{n}<\frac{\alpha^{2 n+3}}{(2 n+3) !}
$$

was schon für $n=2$ kleiner als $\frac{1}{4} \cdot 10^{-15}$ ist.

Ähnlich einfach liegen die Dinge bei $\cos 1^{0}$; doch kann man diesen Wert auch, da $\sin ^{2} 1^{0}<\frac{1}{2500}$ ist, sehr leicht aus

$$
\cos 1^{0}=\left(1-\sin ^{2} 1^{0}\right)^{\frac{1}{2}}
$$

mit Hilfe der binomischen Reihe berechnen. - $\operatorname{tg} x$ und $\operatorname{ctg} x$ ergeben sich nun durch Division, oder auch aus ihren Entwicklungen 116 und 115, die für kleine $|x|$ noch recht gut konvergieren. 
151.

Aus diesen letzten Reihen gewinnt man noch brauchbare Entwicklungen für die Logarithmen von $\sin x$ und $\cos x$, - Werte, die für die Praxis wichtiger sind, als $\sin x$ und $\cos x$ selber. Man hat nämlich (vgl. $\S 19$, Def. 12).

und ganz ähnlich aus $\mathbf{1 1 6}$

$$
\left.\log \sin x=\log x+\log \frac{\sin x}{x}=\log x+\int_{0}^{x}\left[\operatorname{ctg} x-\frac{1}{x}\right] d x^{1}\right)
$$

$$
=\log x+\sum_{k=1}^{\infty}(-1)^{k} \frac{2^{2 k} \cdot B_{2 k}}{2 k \cdot(2 k) !} x^{2 k}
$$

152.

$$
-\log \cos x=\int_{0}^{x} \operatorname{tg} x d x=\sum_{k=1}^{\infty}(-1)^{k-1} \frac{2^{2 k}\left(2^{2 k}-1\right) B_{2 k}}{2 k \cdot(2 k) !} x^{2 k}
$$

$\log \operatorname{tg} x$ und $\log \operatorname{ctg} x$ ergeben sich hieraus einfach durch Addition. -

Auf weitere Einzelheiten bei der Berechnung der trigonometrischen Tafeln wollen wir hier nicht eingehen, da sie reihentheoretisch nicht von Belang sind.

8. Verfeinerte Restabschätzungen. In den bisherigen Fällen haben wir die Summe einer vorgelegten Reihe stets ganz unmittelbar durch Berechnung einer passenden Teilsumme und Abschätzung des zugehörigen Restes gewonnen. Es ist klar, daß dieser Weg praktisch nur beschreitbar ist, wenn die Reihe einigermaßen gut konvergiert. Will man aber z. B. die Summe

$$
s=\sum_{n=1}^{\infty} \frac{1}{n^{2}}
$$

genauer berechnen, so ist dieser direkte Weg ziemlich hoffnungslos ${ }^{2}$ ). Für den Rest

$$
r_{n}=\frac{1}{(n+1)^{2}}+\frac{1}{(n+2)^{2}}+\ldots
$$

hat man nämlich bei vorsichtiger Vergrößerung nur

$$
r_{n}<\frac{\cdot 1}{n(n+1)}+\frac{1}{(n+1)(n+2)}+\ldots=\frac{1}{n},
$$

so daß, um nur 6 Dezimalen zu sichern, über eine Million Glieder berechnet werden müßten, was nicht angeht.

Dieser Übelstand kann nun häufig in etwas behoben werden, wenn man für den Rest auch eine untere Schranke zu finden sucht. Bei unserm Beispiel hat man nach demselben Prinzip auch

$$
r_{n}>\frac{1}{(n+1)(n+2)}+\frac{1}{(n+2)(n+3)}+\ldots=\frac{1}{n+1},
$$

1) Unter der in der eckigen Klammer stehenden Funktion soll hier die Reihe 115 nach Division durch $x$ und Abzug des vordersten Gliedes $1 / x$ verstanden werden. Die Funktion ist also auch für $x=0$ definiert und stetig.

2) Da wir hier zufällig wissen, daßs die Summe $=\frac{\pi^{2}}{6}$ ist, so ist ihre Berechnung auf dem Umwege über $\pi$ natürlich ganz einfach. Wir wollen aber fuir den Augenblick annehmen, daß wir von ihrer Summe ebenso wenig wüßsten wie etwa von der Reihe $\Sigma \frac{1}{n^{3}}$. 
so daß wir nun wissen, daß die gesuchte Summe $s$ den Bedingungen

$$
1+\frac{1}{2^{2}}+\cdots+\frac{1}{n^{2}}+\frac{1}{n+1}<s<1+\frac{1}{2^{2}}+\ldots+\frac{1}{n^{2}}+\frac{1}{n}
$$

für jedes $n$ genügt. Und um 6 Dezimalen zu sichern, wären nun vielleicht nur 1000 Glieder nötig. Doch wäre auch diese Anzahl für die praktische Durchführung noch zu groß. Trotzdem kann in besonderen Fällen diese Methode der Abschätzung des Restes nach oben und unten zum gewünschten Ziele führen. (Vgl. Aufgabe 131.)

Solche Fälle sind indessen doch so selten, daß sie für praktische Arbeit nicht in Betracht kommen. Wesentlicher, weil von größerer Anwendbarkeit, sind dagegen die Methoden, die eine schlecht konvergente und also für die numerische Berechnung zunächst unbrauchbare Reihe in eine besser konvergente verwandeln. Hierauf wollen wir nunmehr eingehen.

\section{§ 35. Anwendung der Reihentransformationen bei numerischen Berechnungen.}

In Fällen schlechter Konvergenz wird man versuchen, die Reihe durch passende Umformungen in eine besser konvergente zu verwandeln. Wir wollen die in $\S 33$ besprochenen Reihentransformationen daraufhin durchgehen, ob sie uns in dieser Beziehung nützen können.

A. Die Kummersche Transformation. Bei ihr ist am unmittelbarsten zu erkennen, ob und inwieweit eine Verbesserung der Konvergenz erzielt wird. Denn mit den Bezeichnungen von 145 haben wir

$$
\sum_{n=0}^{\infty} a_{n}=\gamma C+\sum_{n=0}^{\infty}\left(1-\gamma \frac{c_{n}}{a_{n}}\right) a_{n} ;
$$

und da $\left(1-\gamma \frac{c_{n}}{a_{n}}\right) \rightarrow 0$ strebt, so sind die Glieder der neuen Reihe (von einer Stelle an) kleiner als die der vorgelegten. Die Wirkung dieser Methode wird hiernach um so größer sein, je kleiner von Anfang an die Faktoren $\left(1-\gamma \frac{c_{n}}{a_{n}}\right)$ sind, d. h. also, je besser die Glieder der Reihe $\Sigma c_{n}$ denen der Reihe $\Sigma a_{n}$ angepaßt sind. Einige

$$
\text { Beispiele }
$$

sollen die Anwendung dieser Methoden erläutern.

1. Wir hatten schon S. 240 gefunden, dafs $\sum \frac{1}{n^{2}}=1+\sum \frac{1}{n^{2}(n+1)}$ gesetzt 153. werden kann. Die Glieder der neuen Reihe sind denen von

$$
\sum_{n=1}^{\infty} \frac{1}{n(n+1)(n+2)}=\frac{1}{2} \sum_{n=1}^{\infty}\left(\frac{1}{n(n+1)}-\frac{1}{(n+1)(n+2)}\right)=\frac{1}{4}
$$

asymptotisch gleich, d. h. es ist hier $C=\frac{1}{4}$ und $\gamma=1$, also

$$
\sum_{n=1}^{\infty} \frac{1}{n^{2}}=1+\frac{1}{4}+2 \sum_{n=1}^{\infty} \frac{1}{n^{2}(n+1)(n+2)} \text {. }
$$


Fährt man in entsprechender Weise fort, so erhält man nach $p$ Schritten

$$
\sum_{n=1}^{\infty} \frac{1}{n^{2}}=1+\frac{1}{2^{2}}+\frac{1}{3^{2}}+\ldots+\frac{1}{p^{2}}+p ! \sum_{n=1}^{\infty} \frac{1}{n^{2}(n+1)(n+2) \ldots(n+p)},
$$

was schon für mäßige Werte von $p$ eine gut konvergente Reihe liefert.

2. Etwas allgemeiner sei die Reihe

$$
\sum_{n=0}^{\infty} a_{n} \equiv \sum_{n=0}^{\infty} \frac{1}{(n+\alpha)^{2}(n+\alpha+1)^{2} \ldots(n+\alpha+p-1)^{2}},\left\{\begin{array}{l}
\alpha \neq 0,-1, \ldots \text { beliebig, } \\
p \geqq 1 \text { gan },
\end{array}\right.
$$

vorgelegt. Wir wählen hier

$$
c_{n}=(n+y) a_{n}-(n+1+y) a_{n+1},
$$$$
n=0,1,2, \ldots,
$$

und versuchen $y$ so festzulegen, daßs sich die $c_{n}$ den $a_{n}$ möglichst gut anpassen $\left.{ }^{1}\right)$. Hier wird $C=y \cdot a_{0}$, und eine leichte Rechnung ergibt $\gamma=\frac{1}{2 p-1}$. Daher erhalten wir

$$
\sum_{n=0}^{\infty} a_{n}=\frac{y a_{0}}{2 p-1}+\sum_{n=0}^{\infty}\left(1-\frac{(n+y) a_{n}-(n+1+y) a_{n+1}}{(2 p-1) a_{n}}\right) a_{n} .
$$

Die runde Klammer ist hier

$$
=1-\frac{(n+y)(n+\alpha+p)^{2}-(n+1+y)(n+\alpha)^{2}}{(2 p-1)(n+\alpha+p)^{2}},
$$

also, da sich bei der Vereinfachung die Glieder mit $n^{3}$ und $n^{2}$ von selbst wegheben müssen

$$
=\frac{(2 \alpha+3 p-2-2 y) p n+(2 p-1-y)(\alpha+p)^{2}+\alpha^{2}(1+y)}{(2 p-1)(n+\alpha+p)^{2}} .
$$

Wählt man nun $y$ so, dafs sich auch noch die Glieder mit $n$ wegheben, setzt man also $y=\alpha+\frac{3}{2} p-1$, so wird die runde Klammer weiter

und folglich

$$
=\frac{p^{3}}{2(2 p-1)} \cdot \frac{1}{(n+\alpha+p)^{2}}
$$

$$
\begin{gathered}
\sum_{n=0}^{\infty} \frac{1}{(n+\alpha)^{2} \ldots(n+\alpha+p-1)^{2}} \\
=\frac{\left(\alpha+\frac{3}{2} p-1\right) \cdot \frac{1}{2 p-1}}{\alpha^{2}(\alpha+1)^{2} \ldots(\alpha+p-1)^{2}}+\frac{p^{3}}{2(2 p-1)} \sum_{n=0}^{\infty} \frac{1}{(n+\alpha)^{2} \ldots(n+\alpha+p)^{2}} .
\end{gathered}
$$

Die Transformation hat also den Erfolg, daf ein quadratischer Faktor mehr im Nenner steht. - Spezialfälle:

a) $\alpha=1$ liefert

$$
\begin{aligned}
& \sum_{n=0}^{\infty} \frac{1}{(n+1)^{2}(n+2)^{2} \ldots(n+p)^{2}}=\frac{\frac{3}{2} p \cdot \frac{1}{2 p-1}}{1^{2} \cdot 2^{2} \ldots p^{2}}+ \\
& \quad+\frac{p^{3}}{2(2 p-1)} \cdot \sum_{n=0}^{\infty} \frac{1}{(n+1)^{2} \cdots(n+p)^{2}(n+p+1)^{2}}
\end{aligned}
$$

1) Die Wahl eines $c_{n}$ von der Form $x_{n}-x_{n+1}$ wird wegen 131 stets die bequemste sein, da man dann jedenfalls sofort $C$ angeben kann und sich die Wahl so einrichten kann, daßs die $c_{n}$ den $a_{n}$ gut angepafit sind. 
Setzt man zur Abkürzung

$$
\sum_{k=1}^{\infty} \frac{1}{k^{2}(k+1)^{2} \ldots(k+p-1)^{2}} \equiv \sum_{n=0}^{\infty} \frac{1}{(n+1)^{2} \cdots(n+p)^{2}}=S_{p},
$$

so hat man also

$$
S_{p}=\frac{3 p}{2(2 p-1) \cdot 1^{2} \cdot 2^{2} \cdots p^{2}}+\frac{p^{3}}{2(2 p-1)} \cdot S_{p+1},
$$

- eine Formel, mit deren Hilfe man sich leicht für $s=S_{1}=\sum \frac{1}{n^{2}}$ sehr gut konvergierende Reihen verschaffen kann.

b) $\alpha=\frac{1}{2}$ liefert ähnlich

$$
\begin{gathered}
\sum_{n=0}^{\infty} \frac{1}{(2 n+1)^{2}(2 n+3)^{2} \ldots(2 n+2 p-1)^{2}} \\
=\frac{3 p-1}{2(2 p-1)} \cdot \frac{1}{1^{2} \cdot 3^{2} \ldots(2 p-1)^{2}}+\frac{2 p^{3}}{2 p-1} \sum_{n=0}^{\infty} \frac{1}{(2 n+1)^{2} \ldots(2 n+2 p+1)^{2}},
\end{gathered}
$$

- eine Formel, aus der man sich in entsprechender Weise gut konvergierende Reihen für $\sum \frac{1}{(2 n+1)^{2}}$ verschaffen kann.

Bezïglich weiterer Beispiele verweisen wir auf die Aufgaben $127 \mathrm{ff}$.

\section{B. Die Eulersche Transformation.}

Mit der Eulerschen Transformation 144 braucht keineswegs immer eine Verbesserung ${ }^{\mathbf{1}}$ ) der Konvergenz verbunden $\mathrm{zu}$ sein.

So liefert z. B. die Transformation von $\sum_{n=0}^{\infty}\left(\frac{1}{2}\right)^{n}$ die Reihe $\frac{1}{2} \sum_{n=0}^{\infty}\left(\frac{3}{4}\right)^{n}, \mathbf{1 5 4}$. welche ersichtlich schlechter konvergiert. Aber auch bei alternierenden Reihen braucht sich keine Verbesserung der Konvergenz zu ergeben, vielmehr zeigen die drei folgenden Beispiele, dałs hier alle denkbaren Fälle wirklich eintreten können:

1. $\sum_{n=0}^{\infty}(-1)^{n} \frac{1}{2^{n}}$ liefert die besser konvergente Reihe $\frac{1}{2} \sum_{n=0}^{\infty} \frac{1}{4^{n}}$

2. $\sum_{n=0}^{\infty}(-1)^{n} \frac{1}{3^{n}} \quad " \quad$, gleichartig $\quad " \quad \quad \frac{1}{2} \sum_{n=0}^{\infty} \frac{1}{3^{n}}$

2. $\sum_{n=0}^{\infty}(-1)^{n} \frac{1}{4^{n}} \quad n \quad "$ schlechter $\quad \quad \quad \quad \frac{1}{2} \sum_{n=0}^{\infty}\left(\frac{3}{8}\right)^{n}$

Wir werden nun aber zeigen, daß in den praktisch allein wichtigen Fällen alternierender Reihen $\Sigma(-1)^{n} a_{n}, a_{n}>0$, in denen die

1) Die ausführliche Definition dessen, was unter besserer und schlechterer Konvergenz verstanden werden soll, wird in $\S 37$ gegeben werden: $\Sigma a_{n}^{\prime}$ wird besser oder schlechter konvergent genannt werden als $\Sigma a_{n}$, je nachdem

strebt.

$$
\left|\frac{r_{n}^{\prime}}{v_{n}}\right|=\left|\frac{a_{n+1}^{\prime}+a_{n+2}^{\prime}+\ldots}{a_{n+1}+a_{n+2}+\ldots}\right| \rightarrow 0 \text { oder } \rightarrow+\infty
$$


gegebene Reihe nicht besonders gut konvergiert, die Beträge $a_{n}$ ihrer Glieder aber in einer gleich zu nennenden regelmäßigen Art zu 0 abnehmen, - daß in diesen Fällen doch eine oft beträchtliche Verbesserung der Konvergenz erzielt wird.

Wir wollen nämlich nicht nur verlangen, daß die $a_{n}$ selber monoton abnehmen, daß also ihre ersten Differenzen $\Delta a_{n}$ sämtlich positiv sind, sondern wollen fordern, daß dies auch für alle höheren Differenzen der Fall ist. Man pflegt eine (positive) Zahlenfolge $a_{0}, a_{1}, a_{2}, \ldots$-fach monoton zu nennen ${ }^{1}$ ), wenn die Folge der 1., 2.,... und $p$. Differenzen lauter positive Glieder hat, und man nennt sie vollmonoton, wenn dies mit allen Differenzen $\Delta^{k} a_{n},(k, n,=0,1,2, \ldots)$, der Fall ist. Mit dieser Bezeichnungsweise lautet der angedeutete

155. Satz. Ist $\sum_{n=0}^{\infty}(-1)^{n} a_{n}$ eine alternierende Reihe, für die die (positiven) Zahlen $a_{0}, a_{1}, \ldots$ eine vollmonotone Nullfolge bilden, und

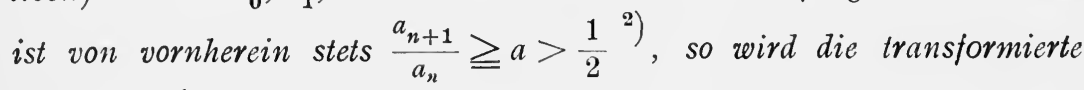
Reihe $\sum \frac{1}{2^{n+1}} \Delta^{n} a_{0}$ besser konvergieren als die gegebene.

Der Beweis ist äußerst einfach. Wegen $\frac{a_{n+1}}{a_{n}} \geqq a$ ist zunächst $a_{n} \geqq a_{0} \cdot a^{n}$. Und für den Rest $r_{n}$ der gegebenen Reihe hat man $(-1)^{n+1} r_{n}=a_{n+1}-a_{n+2}+-\ldots=\Delta a_{n+1}+\Delta a_{n+3}+\Delta a_{n+5}+\ldots$, also, da auch die $\Delta a_{v}$ monoton abnehmen

$$
\left|\boldsymbol{r}_{n}\right| \geqq \frac{1}{2}\left(\Delta a_{n+1}+\Delta a_{n+2}+\Delta a_{n+3}+\ldots\right)=\frac{1}{2} a_{n+1} \geqq \frac{1}{2} a_{0} \cdot a^{n+1} .
$$

Andererseits nehmen wegen $\Delta^{n} a_{0}-\Delta^{n+1} a_{0}=\Delta^{n} a_{1} \geqq 0$ die Zähler der transformierten Reihe ihrerseits monoton ab und sind also dauernd $\leqq a_{0}$. Infolgedessen hat man für den Rest $r_{n}^{\prime}$ der transformierten Reihe - welche jetzt übrigens eine Reihe mit positiven Gliedern ist -

$$
r_{n}^{\prime}=\frac{\Delta^{n+1} a_{0}}{2^{n+2}}+\ldots \leqq \frac{a_{0}}{2^{n+2}}\left(1+\frac{1}{2}+\ldots\right)=\frac{a_{0}}{2^{n+1}} .
$$

Folglich ist

$$
\left|\frac{r_{n}{ }^{\prime}}{r_{n}}\right| \leqq \frac{1}{a}\left(\frac{1}{2 a}\right)^{n}
$$

woraus nun die Behauptung in vollem Umfange abgelesen werden kann. Und man erkennt darüber hinaus noch, daß die Konvergenzverbesserung um so beträchtlicher sein wird, daß also $r_{n}^{\prime} / r_{n}$ um so schneller $\rightarrow 0$ strebt, je größer $a$ ist. Insbesondere werden alle diejenigen (alternierenden) Reihen, bei denen der Betrag des Quotienten

1) Vgl. die zu 144 genannte Arbeit von E. Jacobsthal.

2) Durch diese Voraussetzung soll der vorher gebrauchte Ausdruck, dafi die gegebene Reihe nicht besonders gut konvergiert, präzisiert sein. Die Reihe wird dann, wie der Beweis genauer zeigt, schlechter konvergieren als die Reihe $\Sigma\left(\frac{1}{2}\right)^{n}$. 
zweier aufeinanderfolgenden Glieder $\rightarrow 1$ strebt, die also im allgemeinen schlecht konvergieren, in Reihen verwandelt, die wesentlich ebenso gut konvergieren, wie die Reihe $\Sigma\left(\frac{1}{2}\right)^{n}$.

Beispiele. Die beiden markantesten Beispiele zur Eulerschen Transformation - diejenige von $\Sigma \frac{(-1)^{n}}{n+1}$ und $\sum \frac{(-1)^{n}}{2 n+1}$ haben wir schon zu 144 vorweg genommen. Für die weiteren Anwendungen ist es wesentlich, zu wissen, welche Nullfolgen vollmonoton sind. Hieriber beweist man durch wiederholte Anwendung des 1. Mittelwertsatzes der Differentialrechnung ( $(19$, Satz 8) leicht den

Satz 2. Die (positive) Folge $a_{0}, a_{1}, \ldots$ ist vollmonoton fallend, wenn es eine für $x \geq 0$ definierte und für $x>0$ beliebig oft differenzierbare Funktion $f(x)$ gibt, für die $f(n)=a_{n}$ ist und deren $k^{\text {te }}$ Ableitung das feste Vorzeichen $(-1)^{k}$ hat $(k=0,1,2, \ldots)$.

Hiernach sind z. B. die Folgen

$a^{n}, \quad(0<a<1), \frac{1}{(n+p)^{\alpha}}, \quad(p>0, \alpha>0), \quad \frac{1}{\log (n+p)}, \quad(p>1), \ldots$

vollmonoton fallend; und aus diesen leitet man viele neue her durch den

Satz 3. Sind die Folgen $a_{0}, a_{1}, \ldots$ und $b_{0}, b_{1}, \ldots$ vollmonoton fallend, so gilt das gleiche von der Folge $a_{0} b_{0}, a_{1} b_{1}, a_{2} b_{2}, \ldots$ Formel

Beweis. Es gilt die durch Induktion sofort als richtig zu erweisende

$$
\Delta^{k} a_{n} b_{n}=\sum_{\nu=0}^{k}\left(\begin{array}{l}
k \\
v
\end{array}\right) \Delta^{k-v} a_{n+v} \cdot \Delta^{v} b_{n}
$$

in der unter $\Delta^{0} a_{n}$ und $\Delta^{0} b_{n}$ natürlich $a_{n}$ bzw. $b_{n}$ selber zu verstehen sind. Nach ihr sind in der Tat alle Differenzen der Folge $\left(a_{n} b_{n}\right)$ positiv, wenn es diejenigen der Folgen $\left(a_{n}\right)$ und $\left(b_{n}\right)$ sind.

Als ein besonderes Zahlenbeispiel skizzieren wir noch das folgende:

Die Reihe

$$
\sum_{n=0}^{\infty}(-1)^{n} a_{n} \equiv \frac{1}{\log 10}-\frac{1}{\log 11}+\frac{1}{\log 12}-+\ldots
$$

konvergiert auferordentlich langsam, nämlich wesentlich ebenso langsam wie die Abelsche Reihe $\Sigma 1 / n \log ^{2} n$. Mit Hilfe der Eulerschen Transformation kann man trotzdem ihre Summe verhältnismäßig leicht genau berechnen. Benutzt man nur die ersten 7 Glieder $\left(\right.$ bis $\left.\frac{1}{\log 16}\right)$, so kann man mit ihrer Hilfe auch von der transformierten Reihe die ersten 7 Glieder berechnen. Benutzt man 7 stellige Logarithmen, so findet man schon mit 6 gesicherten Dezimalen für die Reihensumme den Wert $0,221840 \ldots{ }^{1}$ ).

\section{Die Markoff sche Transformation.}

Bei der großen Willkür, die wir für den Ansatz des Schema (A), S. 234, gelassen hatten, aus dem sich die Markoffsche Transformation ergab, ist es nicht verwunderlich, daß wir keine allgemeinen Sätze

1) Wir entnehmen dieses Resultat dem Werke „Differenzenrechnung“ von A. A. Markoff, S. 184, Leipzig 1896.

Knopp; Unendliche Reihen. 
über die Wirkung der Transformation auf die Güte der Konvergenz werden aussprechen können. Wir werden uns daher mit etwas allgemeiner gehaltenen Richtlinien zu ihrer vorteilhaften Verwendung und mit der Ausführung einiger Beispiele begnügen müssen:

Bezeichnen wir die gegebene (als konvergent vorausgesetzte) Reihe wieder mit $\Sigma z^{(k)}$, so wähle man die $0^{\text {te }}$ Spalte $a_{0}^{(0)}, a_{0}^{(1)}, \ldots, a_{0}^{(k)}, \ldots$ des Schema (A) so, daß sie, ähnlich wie bei der Kummerschen Transformation, der gegebenen Reihe möglichst gut angepaßt ist und andererseits eine bequem geschlossen angebbare Summe $s^{(0)}$ hat. Mit der Reihe $\Sigma\left(z^{(k)}-a_{0}^{(k)}\right)$, die nun schon besser konvergieren wird als $\Sigma z^{(k)}$, verfahre man genau ebenso für die Wahl der nächsten Spalte usw. Dann wird die Wirkung der Transformation eine ähnliche sein, als wenn man unbegrenzt oft eine Kummsrsche Transformation ausgeführt hätte, — eine Möglichkeit, die wir schon bei den Beispielen 153, 2 a angedeutet hatten (vgl. Aufg. 130).

156. Als Beispiel wählen wir die Reihe $\sum_{k=1}^{\infty} \frac{1}{k^{2}}$, die zur Berechnung ihrer Summe $\frac{\pi^{2}}{6}$ praktisch unbrauchbar ist. Hier denken wir uns die $0^{\text {te }}$ Zeile und die $0^{\text {te }}$ Spalte aus lauter Nullen bestehend, die wir nicht hinsch reiben. Dann liegt es nahe, als erste Spalte die schon S. 240 benutzte Reihe $\Sigma \frac{1}{k(k+1)}$ zu nehmen Dadurch wird

$$
\Sigma\left(z^{(k)}-a_{1}^{(k)}\right) \equiv \Sigma_{k^{2}(k+1)},
$$

und als zweite Spalte wird man nun, wieder wie damals, die Reihe

$$
\sum \frac{1}{k(k+1)(k+2)}
$$

nehmen, usw. Dann würde die $k^{\text {te }}$ Zeile des Schemas das folgende Bild bieten

$$
\frac{1}{k^{2}}=\frac{0 !}{k(k+1)}+\frac{1 !}{k(k+1)(k+2)}+\frac{2 !}{k(k+1)(k+2)(k+3)}+\ldots
$$

Die weitere Rechung ist nun etwas einfacher, wenn man, statt diese Zeilen nach rechts unbegrenzt fortzusetzen, sie nach $(k-1)$ Gliedern abbricht und als $k^{\text {tes }}$ Glied den fehlenden Rest $v_{k}$ hinzufügt, auf den dann lauter Nullen zu folgen hätten. Dann sieht die $k^{\text {te }}$ Zeile so aus:

$$
\frac{1}{k^{2}}=\frac{0 !}{k(k+1)}+\frac{1 !}{k(k+1)(k+2)}+\ldots+\frac{(k-2) !}{k(k+1) \ldots(2 k-1)}+r_{k} \text {. }
$$

Indem man hier die Glieder der rechten Seite sukzessive von der linken Seite abzieht, findet man ganz leicht, daf

$$
r_{k}=\frac{(k-1) !}{k^{2}(k+1) \cdots(2 k-1)}
$$

ist. Die Auflösung der Reihe $\Sigma 1 / k^{2}$ in ein Schema der Form (A) von S. 234 sieht dann in unserem Falle folgendermafen aus: 
$1=1$

$\frac{1}{2^{2}}=\frac{0 !}{2 \cdot 3} \quad+\frac{1 !}{2^{2} \cdot 3}$

$\frac{1}{3^{2}}=\frac{0 !}{3 \cdot 4} \quad+\frac{1 !}{3 \cdot 4 \cdot 5}+\frac{2 !}{3^{2} \cdot 4 \cdot 5}$

$\frac{1}{k^{2}}=\frac{0 !}{k(k+1)}+\frac{1 !}{k(k+1)(k+2)}+\ldots+\frac{(k-2) !}{k(k+1) \ldots(2 k-1)}+\frac{(k-1) !}{k^{2}(k+1) \ldots(2 k-1)}$.

Da hier alle Zahlen des Schemas $\geq 0$ sind, so lehrt schon der große Umordnungssatz 90, dafs wir nach Spalten summieren dürfen und als Endergebnis wieder $\frac{\pi^{2}}{6}$ erhalten. In der $n^{\text {ten }}$ Spalte steht nun die Reihe

$$
r_{n}+(n-1) !\left[\frac{1}{(n+1) \ldots(2 n+1)}+\frac{1}{(n+2) \ldots(2 n+2)}+\ldots\right], \quad(n \text { fest }) .
$$

Und da nach 132,3 die in der eckigen Klammer stehende Reihe die Summe

$$
\frac{1}{n(n+1) \cdots(2 n)}
$$

hat, so liefert die $n^{\text {te }}$ Spalte die Summe

Es ist also

$$
\begin{aligned}
s^{(n)} & =(n-1) !\left[\frac{1}{n^{2}(n+1) \ldots(2 n-1)}+\frac{1}{n(n+1) \ldots(2 n)}\right] \\
& =3 \frac{(n-1) !}{n(n+1) \ldots(2 n)}=3 \frac{(n-1) !^{2}}{(2 n) !} .
\end{aligned}
$$

$$
\sum_{k=1}^{\infty} \frac{1}{k^{2}}=3 \cdot \sum_{n=1}^{\infty} \frac{(n-1) !^{2}}{(2 n) !} .
$$

Diese Formel ist nicht nur für numerische Zwecke wegen der erheblichen Verbesserung der Konvergenz von Bedeutung, sondern fast mehr noch, weil sie ein neues Mittel zur geschlossenen Auswertung der Reihensumme $\Sigma 1 / k^{2}$ eröffnet, die uns bisher nür auf dem weiten Umwege über die Partialbruchund die Potenzreihenentwicklnng der Funktion ctg gelungen war. Man kann nämlich leicht direkt feststellen (vgl. Aufgabe 123), dafi aus 121 folgt, dás für $x \mid \leqq 1$ die Entwicklung

$$
(\arcsin x)^{2}=\frac{1}{2} \sum_{n=1}^{\infty} \frac{(n-1) !^{2}}{(2 n) !}(2 x)^{2 n}
$$

gültig ist. Setzt man hierin $x=\frac{1}{2}$, so ergibt sich sofort, dafis

$$
\sum_{k=1}^{\infty} \frac{1}{k^{2}}=3 \sum_{n=1}^{\infty} \frac{(n-1) !^{2}}{(2 n) !}=3 \cdot 2 \cdot\left(\frac{\pi}{6}\right)^{2}=\frac{\pi^{2}}{6}
$$

ist.

Eine weitere prinzipiell wichtige Anwendung der Markoffschen Transformation haben wir schon (s. 144) in der Eulerschen Transformation gegeben, welche ja aus jener gefolgert worden ist.

Bezüglich weiterer, meist nur auf Grund besonderer Kunstgriffe glückender, aber zum Teil überraschend wirkungsvoller Anwendungen der Markoffschen Transformation müssen wir auf die Darstellungen von Markoff selbst (s. vorige Fußn.) und von E. Fabry (Théorie des séries à termes constants, Paris, 1910) verweisen, wo sich zahlreiche Beispiele vollständig durchgeführt finden. 


\section{Aufgaben zum VIII. Kapitel.}

I. Direkte Bildung der Folge der Teilsummen.

100. a) $\frac{x}{1+x}+\frac{2 x^{2}}{1+x^{2}}+\frac{4 x^{4}}{1+x^{4}}+\frac{8 x^{8}}{1+x^{8}}+\ldots=\frac{x}{1-x}$ für $|x|<1$.

b) $\frac{x}{1-x^{2}}+\frac{x^{2}}{1-x^{4}}+\frac{x^{4}}{1-x^{8}}+\frac{x^{8}}{1-x^{16}}+\ldots=\left\{\begin{array}{cc}\frac{x}{1-x} \text { für }|x|<1 \\ -\frac{1}{x-1} \text { für }|x|>1 .\end{array}\right.$

101. $\sum_{n=1}^{\infty} \frac{a_{n}}{\left(1+a_{1}\right)\left(1+a_{2}\right) \ldots\left(1+a_{n}\right)}$ ist für positive $a_{n}$ stets konvergent. Wann konvergiert die Reihe noch bei beliebigen $a_{n}$, und welche Summe hat sie im Falle der Konvergenz?

102. a) $\sum_{n=1}^{\infty} \operatorname{arctg} \frac{2}{n^{2}}=\frac{3 \pi}{4}$;

(Anl.: $\left.\operatorname{arctg} \frac{1}{n-1}-\operatorname{arctg} \frac{1}{n+1}=\operatorname{arctg} \frac{2}{n^{2}} \cdot\right)$

b) $\sum_{n=1}^{\infty} \operatorname{arctg} \frac{1}{n^{2}+n+1}=\frac{\pi}{4}$.

103. a) $\sum_{n=1}^{\infty} \frac{1}{(x+1)(2 x+1) \ldots(n x+1)}=\frac{1}{x}$, falls $x \neq 0,-\frac{1}{2},-\frac{1}{3}, \ldots$

b) $\frac{1}{y}+\frac{x}{y(y+1)}+\frac{x(x+1)}{y(y+1)(y+2)}+\ldots=\frac{1}{y-x}$, falls $y>x>0$.

c) $1+\frac{a}{b}+\frac{a(a+1)}{b(b+1)}+\frac{a(a+1)(a+2)}{b(b+1)(b+2)}+\ldots=\frac{b-1}{b-a-1}$,

falls $b>a+1>1$.

104. $\frac{1}{k_{1}}+\frac{k_{1}-b}{k_{1}} \cdot \frac{1}{k_{2}}+\frac{\left(k_{1}-b\right)\left(k_{2}-b\right)}{k_{1} \cdot k_{2}} \cdot \frac{1}{k_{3}}+\ldots=\frac{1}{b}$,

falls $b \neq 0$, alle $k_{v}>0$ und $\sum \frac{1}{k_{v}}$ divergent ist.

105. a) $\sum_{n=0}^{\infty} \frac{1}{2^{n}} \operatorname{tg} \frac{\pi}{2^{n+2}}=\frac{4}{\pi}$;

b) $\sum_{n=1}^{\infty} \frac{1}{2^{n}} \operatorname{tg} \frac{x}{2^{n}}=\frac{1}{x}-\operatorname{ctg} x$;

(Anl.: $\operatorname{ctg} y-\operatorname{tg} y=2 \operatorname{ctg} 2 y$.)

106. In $\sum_{n=0}^{\infty} \frac{g(n)}{\left(\alpha+p_{1}+n\right)\left(\alpha+p_{2}+n\right) \ldots\left(\alpha+p_{k}+n\right)}$ seien $p_{1}, p_{2}, \ldots, p_{k}$ fest gegebene, voneinander verschiedene natürliche Zahlen, $\alpha \neq 0,-1,-2, \ldots$ beliebig reell und $g(x)$ eine ganze rationale Funktion eines Grades $\leqq k-2$. Es gelte die Partialbruchzerlegung

$$
\frac{g(x-\alpha)}{\left(x+p_{1}\right) \ldots\left(x+p_{k}\right)}=\frac{c_{1}}{x+p_{1}}+\ldots+\frac{c_{k}}{x+p_{k}} .
$$


156.

Dann hat die vorgelegte Reihe die Summe

$$
-\sum_{\nu=1}^{k} c_{\nu}\left[\frac{1}{\alpha}+\frac{1}{\alpha+1}+\ldots+\frac{1}{\alpha+p_{\nu}-1}\right] \text {. }
$$

107. a) $\frac{1}{1 \cdot 2 \cdot 6 \cdot 7}-\frac{1}{3 \cdot 4 \cdot 8 \cdot 9}+\frac{1}{5 \cdot 6 \cdot 10 \cdot 11}-\ldots=\frac{1}{60}\left(\pi-\frac{149}{60}\right)$;

b) $\frac{1}{1 \cdot 2 \cdot 4 \cdot 5}-\frac{1}{3 \cdot 4 \cdot 6 \cdot 7}+\frac{1}{5 \cdot 6 \cdot 8 \cdot 9}-\ldots=\frac{5}{36}-\frac{1}{6} \log 2$;

c) $\frac{1}{1 \cdot 2 \cdot 4 \cdot 5}+\frac{1}{3 \cdot 4 \cdot 6 \cdot 7}+\frac{1}{5 \cdot 6 \cdot 8 \cdot 9}+\ldots=\frac{1}{36}$;

d) $\frac{1^{3}}{1^{4}+4}-\frac{3^{3}}{3^{4}+4}+\frac{5^{3}}{5^{4}+4}-\ldots=0$;

e) $\frac{1}{1 \cdot\left(1^{4}+4\right)}-\frac{1}{3 \cdot\left(3^{4}+4\right)}+\frac{1}{5 \cdot\left(5^{4}+4\right)}-+\ldots=\frac{\pi}{16}$;

f) $\frac{1}{1 \cdot\left(4 \cdot 1^{4}+1\right)}-\frac{1}{2 \cdot\left(4 \cdot 2^{4}+1\right)}+\frac{1}{3 \cdot\left(4 \cdot 3^{4}+1\right)}-+\ldots=\log 2-\frac{1}{2}$;

g) $\frac{1}{1 \cdot 2 \cdot 3 \cdot 4}+\frac{1}{5 \cdot 6 \cdot 7 \cdot 8}+\ldots=\frac{1}{4} \log 2-\frac{\pi}{24}$;

h) $\frac{1}{1 \cdot 2 \cdot 3}+\frac{1}{4 \cdot 5 \cdot 6}+\frac{1}{7 \cdot 8 \cdot 9}+\ldots=\frac{\pi}{12} \sqrt{3}-\frac{1}{4} \log 3$.

II. Geschlossene Auswertungen mit Hilfe der Entwicklungen für die elementaren Funktionen.
108. a) $1-\frac{1}{5 \cdot 3^{2}}-\frac{1}{7 \cdot 3^{3}}+\frac{1}{11 \cdot 3^{5}}+\frac{1}{13 \cdot 3^{6}}-++\ldots=\log \sqrt{7}$;
b) $\frac{1}{2} \cdot \frac{1}{2}+\frac{1 \cdot 3}{2 \cdot 4} \cdot \frac{1}{4}+\frac{1 \cdot 3 \cdot 5}{2 \cdot 4 \cdot 6} \cdot \frac{1}{6}+\ldots=\log 2$;
c) $1+\frac{1}{3}-\frac{1}{5}-\frac{1}{7}+\frac{1}{9}+\frac{1}{11}--++\ldots=\frac{1}{4} \sqrt{2 \pi}$;
d) $\frac{1}{x}+\frac{1}{x-y}+\frac{1}{x+y}+\frac{1}{x-2 y}+\frac{1}{x+2 y}+\cdots=\frac{\pi}{y} \operatorname{ctg} \frac{\pi x}{y}$

liefert für $y=7$ nebst $x=1,2,3$

$$
1+\frac{1}{2}-\frac{1}{3}+\frac{1}{4}-\frac{1}{5}-\frac{1}{6}+\stackrel{7}{0}++-+-\frac{14}{0} \ldots=\frac{\pi}{\sqrt{7}} .
$$

109. a) $\frac{1}{1 \cdot 2 \cdot 3}+\frac{1}{3 \cdot 4 \cdot 5}+\frac{1}{5 \cdot 6 \cdot 7}+\ldots=\log 2-\frac{1}{2}$;
b) $\frac{1}{1 \cdot 2 \cdot 3}-\frac{1}{3 \cdot 4 \cdot 5}+\frac{1}{5 \cdot 6 \cdot 7}+\ldots \frac{1}{2}(1-\log 2)$;
c) $\frac{1}{2 \cdot 3 \cdot 4}-\frac{1}{4 \cdot 5 \cdot 6}+\frac{1}{6 \cdot 7 \cdot 8}+\ldots \frac{1}{4}(\pi-3)$;
d) $\sum_{n=1}^{\infty} \frac{1}{4 n^{2}-1}=\frac{1}{2}, \quad \sum_{n=1}^{\infty} \frac{1}{\left(4 n^{2}-1\right)^{2}}=\frac{\pi^{2}-8}{16}$,$$
\sum_{n=1}^{\infty} \frac{1}{\left(4 n^{2}-1\right)^{3}}=\frac{32-3 \pi^{2}}{64} \text {; }
$$
e) $1-\frac{1}{5}+\frac{1}{7}-\frac{1}{11}+\frac{1}{13}-\frac{1}{17}+-\ldots=\frac{\pi}{2 \sqrt{3}}$. 
110. a) $\frac{1 \cdot 2}{1 \cdot 3}+\frac{1 \cdot 2 \cdot 3}{1 \cdot 3 \cdot 5}+\frac{1 \cdot 2 \cdot 3 \cdot 4}{1 \cdot 3 \cdot 5 \cdot 7}+\ldots=\frac{\pi}{2}$;

b) $\frac{1}{2 \cdot 3}+\frac{1 \cdot 2}{3 \cdot 4 \cdot 5}+\frac{1 \cdot 2 \cdot 3}{4 \cdot 5 \cdot 6 \cdot 7}+\ldots=\frac{2 \pi}{3 \sqrt{3}}-1$;

c) $\frac{1}{2 \cdot 3 \cdot 4}+\frac{1 \cdot 2}{3 \cdot 4 \cdot 5 \cdot 6}+\frac{1 \cdot 2 \cdot 3}{4 \cdot 5 \cdot 6 \cdot 7 \cdot 8}+\ldots=\frac{\pi^{2}}{18}-\frac{1}{2}$;

d) $\sum_{n=1}^{\infty} \frac{1}{n\left(4 n^{2}-1\right)}=2 \log 2-1$;

e) $\sum_{n=1}^{\infty} \frac{1}{n\left(4 n^{2}-1\right)^{2}}=\frac{3}{2}-2 \log 2$;

f) $\sum_{n=1}^{\infty} \frac{1}{2^{n} \cdot n^{2}}=\frac{\pi^{2}}{12}-\frac{1}{2}(\log 2)^{2}$.

111. Wenn $\sum_{n=0}^{\infty}\left(\frac{n !}{(p+n) !}\right)^{2}=T_{p}$ gesetzt wird, so ist

$$
T_{2}=\frac{\pi^{2}}{3}-3, \quad T_{3}=\frac{\pi^{2}}{4}-\frac{39}{16}, \quad T_{4}=\frac{5}{54} \pi^{2}-\frac{192}{216} .
$$

112. Wenn $\sum_{n=1}^{\infty} \frac{n^{p}}{n !}=g_{p} \cdot e$ gesetzt wird $(p=1,2, \ldots)$, so sind die $g_{p}$ ganze Zahlen, die aus der symbolischen Rekursionsformel $g^{p+1}=(1+g)^{p}$ gewonnen werden können. Es ist $g_{1}=1, g_{2}=2, g_{3}=5, \ldots$.

113.

$$
\frac{1}{x}-\frac{1}{x+y}+\frac{1}{x+2 y}-\frac{1}{x+3 y}+-\cdots
$$

läft sich durch die elementaren Funktionen geschlossen summieren, falls $x: y$ rational ist. Speziell ist

$$
\begin{aligned}
& 1-\frac{1}{4}+\frac{1}{7}-\frac{1}{10}+-\ldots=\frac{1}{3}\left(\frac{\pi}{\sqrt{3}}+\log 2\right) \\
& \frac{1}{2}-\frac{1}{5}+\frac{1}{8}-\frac{1}{11}+-\ldots=\frac{1}{3}\left(\frac{\pi}{\sqrt{3}}-\log 2\right) \\
& 1-\frac{1}{5}+\frac{1}{9}-\frac{1}{13}+-\ldots=\frac{1}{4 \sqrt{2}}(\pi+2 \log (\sqrt{2}+1)) .
\end{aligned}
$$

114. Wird $\sum_{n=1}^{\infty} \frac{x^{n}}{1-x^{n}}=L(x)$ gesetzt $(x \mid<1)$ und bedeutet $\left(x_{n}\right)$ die Folge $\mathbf{6}, 7$, so ist

$$
\sum_{k=1}^{\infty} \frac{1}{x_{2 k}} \equiv 1+\frac{1}{3}+\frac{1}{8}+\frac{1}{21}+\ldots=\sqrt{5}\left[L\left(\frac{3-\sqrt{5}}{2}\right)-L\left(\frac{7-3 \sqrt{5}}{2}\right)\right],
$$

und, wenn $\sum_{k=1}^{\infty} \frac{1}{x_{2 k-1}^{2}}=s$ sowie $\sum_{k=1}^{\infty}(-1)^{k-1} \cdot k=x^{\prime}$ gesetzt wird, $\frac{s}{s^{\prime}}=\sqrt{5}$. 
III. Aufgaben zur Eulerschen Transformation.

115. Es ist (für welche $x$ ?)

a) $\sum_{n=0}^{\infty} \frac{(-1)^{n}}{x+n}=\sum_{k=0}^{\infty} \frac{1}{2^{k+1}} \cdot \frac{k !}{x(x+1) \ldots(x+k)}$;

b) $\sum_{n=0}^{\infty} \frac{(-1)^{n}}{\alpha+n} x^{n}=\frac{1}{\alpha(1+x)}\left[1+\frac{1}{\alpha+1}\left(\frac{x}{1+x}\right)+\frac{1 \cdot 2}{(\alpha+1)(\alpha+2)}\left(\frac{x}{1+x}\right)^{2}+\ldots\right]$;

c) $\sum_{n=0}^{\infty} \frac{(-1)^{n}}{(n+1)(n+2) \cdots(n+p+1)}=\frac{1}{p !} \sum_{k=0}^{\infty} \frac{1}{2^{k+1}(p+k+1)}$.

116. Wird

$$
e^{-x} \cdot \sum_{n=0}^{\infty} a_{n} \frac{x_{n}}{n !}=\sum_{n=0}^{\infty} b_{n} \frac{x^{n}}{n !}
$$

gesetzt, so ist $b_{n}=\Delta^{n} a_{0}$. Speziell also
a) $\left.e^{-x} \mid 1+x+\frac{\alpha+2}{\alpha+1} \cdot \frac{x^{2}}{2 !}+\frac{(\alpha+2)(\alpha+4)}{(\alpha+1)(\alpha+2)} \frac{x^{3}}{3 !}+\cdots\right]$

$$
=1+\frac{1}{\alpha+1} \frac{x^{2}}{2^{1} \cdot 1 !}+\frac{1}{(\alpha+1)(\alpha+3)} \frac{x^{4}}{2^{2} \cdot 2 !}+\cdots
$$
b) $e^{x} \cdot \sum_{n=1}^{\infty} \frac{(-1)^{n-1}}{n \cdot n !} x^{n}=\sum_{n=1}^{\infty} h_{n} \frac{x^{n}}{n !}, \quad\left(h_{n}=1+\frac{1}{2}+\cdots+\frac{1}{n}\right)$.

11\%. Ganz speziell ist:
a) $\left(\begin{array}{l}n \\ 1\end{array}\right)-\frac{1}{2}\left(\begin{array}{l}n \\ 2\end{array}\right)+\frac{1}{3}\left(\begin{array}{l}n \\ 3\end{array}\right)-+\cdots+\frac{(-1)^{n-1}}{n}\left(\begin{array}{l}n \\ n\end{array}\right)=h_{n}=1+\frac{1}{2}+\ldots+\frac{1}{n}$;
b) $1-\frac{1}{3}\left(\begin{array}{c}n \\ 1\end{array}\right)+\frac{1}{5}\left(\begin{array}{l}n \\ 2\end{array}\right)-+\ldots+(-1)^{n} \frac{1}{2 n+1}\left(\begin{array}{l}n \\ n\end{array}\right)=\frac{2 \cdot 4 \ldots(2 n)}{3 \cdot 5 \ldots(2 n+1)}$.

118. Ist $\Delta^{n} a_{0}=b_{n}$, so ist $\Delta^{n} b_{0}=a_{n}$. Wie lauten demgemäß die Umkehrungen der Gleichungen der vorigen Aufgabe?

119. Ist $\left(a_{n}\right)$ eine $p$-fach monoton fallende Nullfolge $(p \geq 1)$, so gilt für die Summe $s$ der Reihe $\sum_{n=0}^{\infty}(-1)^{n} a_{n}$ die Abschätzung

$$
\frac{a_{0}}{2}+\frac{\Delta a_{0}}{2^{2}}+\cdots+\frac{\Delta^{p-1} a_{0}}{2^{p}}<s<\frac{a_{0}}{2}+\frac{\Delta a_{0}}{2^{2}}+\cdots+\frac{\Delta^{p-1} a_{0}}{2^{p}}+\frac{\Delta^{p} a_{0}}{2^{p}} .
$$

Man beweise mit ihrer Hilfe die Gleichung

$$
\lim _{x \rightarrow 1-0}\left[\frac{1}{2}-\frac{x}{1+x}+\frac{x^{2}}{1+x^{2}}-\cdots\right]=\frac{1}{4} .
$$

120. Sind $s_{k}$ und $S_{n}$ die Teilsummen der beiden Reihen in 144, so ist

$$
S_{n}=\frac{\left(\begin{array}{c}
n+1 \\
1
\end{array}\right) s_{0}+\left(\begin{array}{c}
n+1 \\
2
\end{array}\right) s_{1},+\ldots+\left(\begin{array}{c}
n+1 \\
n+1
\end{array}\right) s_{n}}{2^{n+1}} .
$$

Man beweise mit Hilfe dieser Beziehung die Gültigkeit der Eulerschen Transformation. 
121. Es ist

a) $\Sigma(-1)^{k} a_{k} x^{k}=(1-y) \Sigma \Delta^{n} a_{0} \cdot y^{n} \quad$ mit $\quad(1+x)(1-y)=1$;

b) $\Sigma(-1)^{k} a_{2 k} x^{2 k}=\left(1-y^{2}\right) \Sigma \Delta^{n} a_{0} \cdot y^{2 n}$ mit $\left(1+x^{2}\right)\left(1-y^{2}\right)=1$;

c) $\Sigma(-1)^{k} a_{2 k+1} x^{2 k+1}=\sqrt{1-y^{2}} \cdot \Sigma \Delta^{n} a_{0} \cdot y^{2 n+1}$ mit $\left(1+x^{2}\right)\left(1-y^{2}\right)=1$, wenn die Summation stets bei 0 begonnen wird und die Differenzenbildung sich auf die linkerhand auftretenden Koeffizienten $a_{k}$ bzw. $a_{2 k}$ und $a_{2 k+1}$ bezieht.

122. So ist $z$. B.

$$
\operatorname{arctg} x=\frac{x_{i}}{1+x^{2}}\left[1+\frac{2}{3} \frac{x^{2}}{1+x^{2}}+\frac{2 \cdot 4}{3 \cdot 5}\left(\frac{x^{2}}{1+x^{2}}\right)^{2}+\ldots\right]
$$

was für $x=\frac{1}{2}, \frac{1}{3}, \frac{1}{7}, \frac{2}{11}, \frac{3}{79}, \ldots$ besonders bequeme numerische Reihen für $\pi$ liefert, wie

$$
\begin{aligned}
& \frac{\pi}{4}=\operatorname{arctg} \frac{1}{2}+\operatorname{arctg} \frac{1}{3}=\frac{4}{10}\left[1+\frac{2}{3}\left(\frac{2}{10}\right)+\ldots\right]+\frac{3}{10}\left[1+\frac{-2}{3}\left(\frac{1}{10}\right)+\ldots\right], \\
& \frac{\pi}{4}=2 \operatorname{arctg} \frac{1}{2}+\operatorname{arctg} \frac{1}{7}=5 \operatorname{arctg} \frac{1}{7}+8 \operatorname{arctg} \frac{3}{79}, \text { u. a. }
\end{aligned}
$$

123. Die vorige Reihe für $\operatorname{arctg} x$ kann auch in der Form

$$
\frac{\arcsin y}{\sqrt{1-y^{2}}}=y+\frac{2}{3} y^{3}+\frac{2 \cdot 4}{3 \cdot 5} y^{5}+\ldots
$$

geschrieben werden. Hieraus folgt die Entwicklung

$$
2(\arcsin y)^{2}=\sum_{n=1}^{\infty} \frac{(n-1) !^{2}}{(2 n) !}(2 y)^{2 n} .
$$

\section{Andere Reihentransformationen.}

124. Wird $\sum_{n=2}^{\infty} \frac{1}{n^{p}}=S_{p}$ gesetzt, so ist
a) $S_{2}+S_{3}+S_{4}+\ldots=1$;
b) $S_{2}+S_{4}+S_{6}+\ldots=\frac{3}{4}$;
c) $S_{3}+S_{5}+S_{7}+\ldots=\frac{1}{4}$;
d) $S_{2}+\frac{1}{2} S_{4}+\frac{1}{3} S_{6}+\cdots=\log 2$;
e) $S_{2}-S_{3}+S_{4}-+\cdots=\frac{1}{2}$;
f) $S_{2}-\frac{1}{2} S_{4}+\frac{1}{3} S_{6}-+\ldots=\log \frac{e^{\pi}-e^{-\pi}}{4 \pi}$;
g) $\frac{1}{2} S_{2}+\frac{1}{3} S_{3}+\frac{1}{4} S_{4}+\ldots=1-C$;
h) $\frac{1}{2} S_{2}-\frac{1}{3} S_{3}+-\ldots=\log 2+C-1$.

125. Hat $S_{\mu}$ die Bedeutung aus der vorigen Aufgabe und wird

$$
\left(\frac{1}{k+1}-\frac{1}{2 k}\right)=b_{k} \text { und } \lim \frac{n ! e^{n}}{n^{n+\frac{1}{2}}}=\lambda
$$


gesetzt, so ist

$$
b_{2} S_{2}+b_{3} S_{3}++\ldots=1-\log \lambda .
$$

(Die Existenz des Grenzwertes $\lambda$ ergibt sich hierbei durch die Konvergenz der Reihe. Es ist $\lambda=\sqrt{2 \pi}$.)

126. Es ist

a) $\sum_{n=0}^{\infty} \frac{1}{x(x+1) \cdots(x+n)}=e \cdot\left[\frac{1}{x}-\frac{1}{1 !} \frac{1}{x+1}+\frac{1}{2 !} \frac{1}{x+2}-\ldots\right]$;

b) $\sum_{n=0}^{\infty} \frac{1}{n !} \frac{a^{n}}{1+x^{2} a^{2 n}}=e^{a}-x^{2} e^{a^{3}}+x^{4} e^{a^{5}}-+\ldots$

127. a) $\frac{1}{\alpha}+\frac{1}{2} \frac{1 !}{\alpha(\alpha+1)}+\frac{1}{3} \frac{2 !}{\alpha(\alpha+1)(\alpha+2)}=\frac{1}{\alpha^{2}}+\frac{1}{(\alpha+1)^{2}}+\ldots$;

b) $\sum_{n=0}^{\infty} \frac{1}{(n+1)^{3}(n+2)^{3}}=10-\pi^{2}$.

128. Im Anschlufi an $\S 35 \mathrm{~A}$ stelle man die Beziehung zwischen $\sum_{n=1}^{\infty} \frac{1}{(n+\alpha)^{3}(n+\alpha+1)^{3} \cdots(n+\alpha+p-1)^{3}}$ und $\sum_{n=1}^{\infty} \frac{1}{(n+\alpha-1)^{3} \cdots(n+\alpha+p)}$ auf und beweise durch Spezialisierung von $\alpha$ und $p$ die folgenden Umformungen

a) $\sum \frac{1}{n^{3}}=\frac{9}{8}+\frac{25}{2^{2} \cdot 3^{4}}-\frac{4}{3} \sum_{n=1}^{\infty} \frac{1}{(n+1)^{3}(n+2)^{3}\left(n+3^{3}\right)}$

$$
=\frac{9}{8}+\frac{133}{2^{6} \cdot 3^{3}}+\frac{3 \cdot 4^{4}}{5} \sum_{n=1}^{\infty} \frac{1}{n^{3}(n+1)^{3}(n+2)^{3}(n+3)^{3}(n+4)^{3}} ;
$$

b) $\sum_{n=1}^{\infty} \frac{1}{n^{3}(n+1)^{3}}=\frac{2537}{2520}-\frac{2^{3} \cdot 3^{4}}{35} \sum_{n=1}^{\infty} \frac{1}{n^{3}(n+1)^{3}} \frac{1}{(n+2)^{3}(n+3)^{3}}$.

Man berechne die erste Reihensumme auf 6 Dezimalen.

129. Man beweise ähnlich die Umformungen

a) $\sum_{n=1}^{\infty} \frac{1}{n^{4}}=\frac{7}{6}-\sum_{n=1}^{\infty} \frac{7 n(n+1)+2}{6 n^{5}(n+1)^{5}}$;

b) $\sum_{n=1}^{\infty} \frac{1}{n^{5}}=\frac{7}{6}-\sum_{n=1}^{\infty} \frac{28 n^{2}\left(n+\frac{3}{2}\right)+24 n+5}{12 n^{6}(n+1)^{6}}$;

c) $\sum_{n=1}^{\infty} \frac{(-1)^{n-1}}{n^{2}(n+1)^{2} \cdots(n+p-1)^{2}}$

$$
=\frac{5 p+2}{4(p+1)} \frac{1}{(p !)^{2}}-\frac{p(p+1)^{3}}{4} \sum_{n=1}^{\infty} \frac{(-1)^{n}}{n^{2}(n+1)^{2} \cdots(n+p+1)^{2}} \cdot
$$

Man berechne die Reihensummen unter a) und b) auf 6 Dezimalen.

130. a) Bezeichnet man die Reihensumme unter c) der vorigen Aufgabe mit $S_{p}$, so liefert sie Beziehungen zwischen $S_{0}$ und $S_{2 k}$ bzw. zwischen $S_{1}$ und $S_{2 k+1}$. Wie lauten diese? Kann man in ihnen $k \rightarrow \infty$ streben lassen? Welche Transformationen erhält man dadurch? Kann man diese direkt als Markoffsche Transformation gewinnen? 
b) Ist $\log 2=\sum_{n=1}^{\infty} \frac{(-1)^{n-1}}{n}=\frac{1}{2}+\frac{1}{2} \sum_{n=1}^{\infty} \frac{(-1)^{n-1}}{n(n+1)}=\frac{3}{4}-\frac{1}{4} S_{2}$.

Wie lauten nun die unter a) angedeuteten Transformationen der Reilie für $\log 2$ ?

c) Führe dasselbe mit der Reihe 12: für $\frac{\pi}{4}$ aus.

131. Die Summe $s$ der Reihe $\sum \frac{1}{n \log n \log _{2} n\left(\log _{3} n\right)^{2}}$, bei der $n$ von der ersten ganzen Zahl an laufen soll, für die $\log _{3} n>1$ ist, ist auf 8 Dezimalen abgerundet genau $\approx 1,00000000$. - Wie läßst sich entscheiden, ob ihre wahre Dezimaldarstellung mit $0, \ldots$ oder $1, \ldots$ beginnt?

(Vgl. hierzu die Bemerkungen auf S. 242.)

132. Man ordne die natürlichen Zahlen der Form $p^{q}$ mit $p \geq 2, q \geq 2$ der Größe nach und bezeichne die entstehende Folge mit $\left(p_{n}\right)$, so daf $\left(p_{1}, p_{2}, \ldots\right) \equiv(4,8,9,16,25,27,32, \ldots)$. Dann ist

$$
2 \frac{1}{p_{n}-1}=1 \text {. }
$$




\section{Dritter Teil. \\ Ausbau der Theorie.}

IX. Kapitel.

\section{Reihen mit positiven Gliedern.}

\section{§36. Genauere Untersuchung der beiden Vergleichskriterien.}

In den vorangehenden Kapiteln haben wir uns mit der $\mathrm{Zu}$ sammenstellung der grundlegenden Tatsachen aus der Theorie der unendlichen Reihen begnügt. Von nun an stellen wir uns etwas weitere Ziele, wollen tiefer in die Theorie eindringen und zu vielseitigeren Anwendungen übergehen. Dazu nehmen wir zunächst noch einmal die ganz elementar gehaltenen Betrachtungen des III. und IV. Kapitels wieder auf und beginnen mit einer genaueren Untersuchung der beiden Vergleichskriterien I. und II. Art (72 und 73), die wir sofort aus dem ersten Hauptkriterium ( $\mathbf{7 0}$ ) für die Untersuchung des Konvergenzverhaltens von Reihen mit positiven Gliedern hergeleitet hatten. Diese und alle verwandten Kriterien wollen wir weiterhin durch eine etwas kürzere Schreibweise zum Ausdruck bringen: $\Sigma c_{n}$ und $\sum d_{n}$ sollen im folgenden irgendwelche Reihen mit positiven Gliedern bedeuten, deren Konvergenz bzw. Divergenz schon bekannt ist, $\sum a_{n}$ dagegen soll eine Reihe sein - und in diesem Kapitel auch stets mit positiven Gliedern -, die auf ihr Konvergenzverhalten hin untersucht werden soll. Dann schreiben wir das Kriterium 72 einfach in der Form

$$
\boldsymbol{a}_{\boldsymbol{n}} \leqq \boldsymbol{c}_{\boldsymbol{n}} \quad: \quad \mathfrak{C},
$$$$
\boldsymbol{a}_{n} \geqq \boldsymbol{d}_{n}
$$

womit genauer besagt werden soll: Wenn die Glieder $a_{n}$ der zu untersuchenden Reihe $\Sigma a_{n}$ von einer Stelle an die erste Ungleichung erfüllen, so wird die Reihe konvergieren; wenn sie dagegen von einer Stelle an die zweite Ungleichung erfüllen, so muß sie divergieren.

Das Kriterium

73 lautet dann, ebenso kurz geschrieben:

$$
\frac{\boldsymbol{a}_{n+1}}{\boldsymbol{a}_{n}} \leqq \frac{\boldsymbol{c}_{n+1}}{\boldsymbol{c}_{n}}
$$$$
\text { : }
$$

$$
\frac{a_{n+1}}{a_{n}} \geqq \frac{d_{n+1}}{d_{n}} \quad:
$$

An diese beiden Kriterien wollen wir nun zunächst noch einige Bemerkungen knüpfen. Vorweg sei aber noch einmal betont: Diese und 
alle ähnlichen im folgenden aufgestellten Kriterien brauchen für eine vorgelegte Reihe die Entscheidung in der Konvergenzfrage nicht zu bringen. Es sind nur hinreichende Kriterien und sie können daher in speziellen Fällen sehr wohl versagen. Ihre Wirksamkeit wird von der Auswahl der Vergleichsreihen $\sum c_{n}$ und $\Sigma d_{n}$ abhängen (s. u.). Und der Inhalt der folgenden Seiten wird gerade darin zu bestehen haben, durch Aufstellung möglichst vieler und möglichst wirksamer Kriterien die Aussicht auf eine tatsächliche Entscheidung in speziellen Fällen zu erhöhen.

Bemerkungen zum I. Vergleichskriterium (157).

1. Da für jede positive Zahl $g$ die Reihen $\Sigma g c_{n}$ und $\Sigma g d_{n}$ zugleich mit $\Sigma c_{n}$ und $\Sigma d_{n}$ konvergieren bzw. divergieren, so kann man das erste unserer Kriterien auch in der Form

$$
\frac{a_{n}}{c_{n}} \leqq g(<+\infty) \quad: \quad \mathcal{C}, \quad a_{n} \geqq g(>0) \quad:
$$

oder also noch prägnanter in der Form

$$
\varlimsup \quad: \quad \stackrel{e}{\lim } \frac{a_{n}}{c_{n}}<+\infty \quad \underline{\lim } \frac{a_{n}}{d_{n}}>0 \quad:
$$

aussprechen.

2. Hiernach mufs also stets

$$
\varlimsup \frac{d_{n}}{c_{n}}=+\infty, \quad \underline{\lim } \frac{c_{n}}{d_{n}}=0
$$

sein; oder anders ausgedrückt:

$\varlimsup \frac{a_{n}}{c_{n}}=+\infty$ ist eine notwendige Bedingung für die Divergenz von $\Sigma a_{n}$

$\lim \frac{a_{n}}{d_{n}}=0 \quad " \quad \quad \quad$ notwendige $\quad "$ Konvergenz von $\Sigma a_{n}$.

3. Die Limites selber brauchen hier wie in allen folgenden Fällen nicht $z u$ existieren. Das folgt schon, allgemein $z$ u reden, daraus, dafs das Konvergenzverhalten der Reihen mit positiven Gliedern nicht geändert wird, wenn wir sie beliebig umordnen (s. SS). Die Umordnung kann man aber stets so einrichten, dafs die in Rede stehenden Grenzwerte nicht existieren. Nimmt man z. B. fuir $\Sigma c_{n}$ die Reihe $1+\frac{1}{2}+\frac{1}{4}+\frac{1}{8}+\ldots$ und für $\Sigma a_{n}$ die hieraus durch Vertauschung je zweier Nachbarglieder entstehende Reihe

$$
\frac{1}{2}+1+\frac{1}{8}+\frac{1}{4}+\frac{1}{32}+\frac{1}{16}+\ldots .
$$

so strebt $\frac{a_{n}}{c_{n}}$ gewif́ keinem Grenzwert zu, die Häufungsgrenzen dieses Quotienten haben vielmehr die voneinander verschiedenen Werte $\frac{1}{2}$ und 2 . Nimmt man ebenso für $\Sigma d_{n}$ die Reihe $1+\frac{1}{2}+\frac{1}{3}+\frac{1}{4}+\ldots$ und für $\Sigma a_{n}$ die daraus durch Umordnung entstandene Reihe

$$
1+\frac{1}{3}+\frac{1}{2}+\frac{1}{5}+\frac{1}{7}+\frac{1}{4}+\frac{1}{9}+\frac{1}{11}+\frac{1}{6}+\ldots
$$

(bei der also auf je zwei ungerade Nenner ein gerader folgt), so hat $\frac{a_{n}}{d_{n}}$ die voneinander verschiedenen Häufungsgrenzen $\frac{3}{4}$ und $\frac{3}{2}$. Und in ähnlich einfacher Weise kann man sich in den andern Fällen an Beispielen klar machen, daf die Limites selber nicht zu existieren brauchen. Wenn aber der Limes vorhanden ist, so unterliegt er natürlich, weil er dann beiden Häufungsgrenzen gleich ist, den für diese ausgesprochenen Bedingungen. 
4. Im besonderen: Keine Bedingung der Form $\frac{a_{n}}{d_{n}} \rightarrow 0$ ist für die Konvergenz von $\Sigma a_{n}$ notwendig, - es sei denn, daf alle Glieder der divergenten Reihe $\Sigma d_{n}$ oberhalb einer festen positiven Zahl $\delta$ bleiben. Denn ist auch nur $\lim d_{n}=0$ und wählt man nun $k_{1}<k_{2}<\ldots<k_{v}<\ldots$ so, daf

$$
d_{\boldsymbol{k}_{\boldsymbol{v}}}<\frac{1}{2^{v}}
$$

ist und setzt $a_{k_{v}}=d_{k_{v}}$ und die andern $a_{n}$ entweder $=0$ oder gleich den entsprechenden Gliedern irgendeiner konvergenten Reihe $\Sigma c_{n}$, so ist offenbar $\Sigma a_{n}$ konvergent, aber ebenso offensichtlich strebt $\frac{a_{n}}{d_{n}}$ nicht $\rightarrow 0$.

\section{Bemerkungen zum II. Vergleichskriterium (158).}

1. Die Gültigkeit des Vergleichskriteriums II. Art erkennt man jetzt noch kürzer so: Da nach ihm im Falle (e) wegen $\frac{a_{n}}{c_{n}} \geqq \frac{a_{n+1}}{c_{n+1}}$ von einer Stelle an $a_{n} / c_{n}$ monton fällt und dauernd $>0$ bleibt, so strebt dieser Quotient gegen einen bestimmten Grenzwert $\gamma \geq 0$. Es ist also auch $\varlimsup \frac{a_{n}}{c_{n}}=\gamma<+\infty$ und also $\sum a_{n}$ nach $\mathbf{1 5 9}, 1$ konvergent. Im Falle (T) wächst $a_{n} / d_{n}$ von einer Stelle an monoton und strebt also gleichfalls gegen einen bestimmten Grenzwert $>0$ oder gegen $+\infty$. Da dann jedenfalls auch $\underline{\lim } \frac{a_{n}}{d_{n}}>0$ ist, so lehrt 159, 1 die Divergenz von $\Sigma a_{n}$.

2. Das Kriterium II. Art erweist sich hiernach als eine fast unmittelbare Folge des Kriteriums I. Art, d. h. wenn für eine Reihe $\mathcal{L} a_{n}$ das Konvergenzverhalten durch Vergleich mit den (bestimmt gewählten) Reihen $\Sigma c_{n}$ bzw. $\Sigma d_{n}$ nach 158 festgestellt werden kann, so ist dies stets auch nach 157 (bezw. 159, 1 ) möglich. Dies Verhältnis zwischen den beiden Kriterien ist aber keineswegs umkehrbar, d. h. wenn I zur Entscheidung führt, braucht II nicht dasselbe zu leisten.

Beispiele hierfür bieten schon die in 159, 3 angegebenen Reihenpaare: Für das erste Paar ist $\overline{\lim } \frac{a_{n}}{c_{n}}=2$, während $\frac{a_{n+1}}{a_{n}}$ abwechseind $=2$ und $=\frac{1}{8}$ ist, also teils größer teils kleiner als der entsprechende Quotient $\frac{c_{n+1}}{c_{n}}$ ausfällt, welcher ja stets $=\frac{1}{2}$ ist. - Ebenso einfach liegt es bei dem zweiten Paar.

3. Besonders interessant ist dieses Verhältnis der beiden Kriterienarten bei denjenigen beiden Kriterien, die wir als ihre unmittelbare Anwendung in $\S 13$ kennen gelernt hatten, dem Wurzel- und dem Quotientenkriterium, die sich aus I und II durch Benutzung der geometrischen Reihe als Vergleichsreihe ergaben:

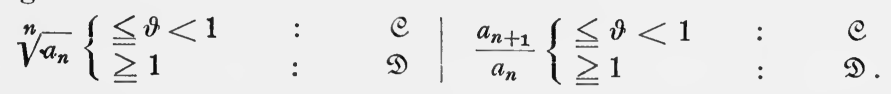

Nach den Bemerkungen unter 2. kann das Quotientenkriterium sehr wohl versagen, wenn das Wurzelkriterium noch entscheidet (die dort gegebenen Reihen $\Sigma a_{n}$ sind naheliegende Beispiele dafür); dagegen muf3 nach 1. das Wurzelkriterium stets entscheiden, wenn es das Quotientenkriterium schon tut. Seinen prägnanteren Ausdruck findet dieses gegenseitige Verhältnis beider Kriterien in dem folgenden Satz, der als eine Erweiterung von 43, 3 angesehen werden kann. 
Satz. Für erne beliebige Zahlenfolge $\left(x_{1}, x_{2}, \ldots\right)$ mit positiven Gliedern ist stets

$$
\underline{\lim } \frac{x_{n+1}}{x_{n}} \leqq \underline{\lim } \sqrt[n]{x_{n}} \leqq \varlimsup \sqrt[n]{x_{n}} \leqq \varlimsup_{\lim } \frac{x_{n+1}}{x_{n}}{ }^{1} \text {. }
$$

Beweis. Der mittlere Teil dieser Ungleichung ist selbstverständlich ${ }^{2}$ ), und linker und rechter Teil sind so ähnlich gebaut, daf wir uns mit dem Beweis der einen begniigen können. Wir wählen den rechten und setzen

$$
\varlimsup \sqrt[n]{x_{n}}=\mu, \quad \varlimsup \lim \frac{x_{n+1}}{x_{n}}=\mu^{\prime},
$$

so dak die Behauptung,$\mu \leqq \mu^{\prime \prime}$ lautet. Ist nun $\mu^{\prime}=+\infty$, so ist hier nichts zu beweisen. Ist aber $\mu^{\prime}<+\infty$, so läfit sich nach Wahl von $\varepsilon>0$ eine ganze Zahl $p$ so angeben, daßi für $v \geqq p$ stets

$$
\frac{x_{v+1}}{x_{\nu}}<\mu^{\prime}+\frac{\varepsilon}{2}
$$

bleibt. Denken wir uns diese Ungleichung für $v=p, p+1, \ldots, n-1$ hingeschrieben und alle miteinander multipliziert, so folgt, dafi für $n>p$

$$
x_{n}<x_{p} \cdot\left(\mu^{\prime}+\frac{\varepsilon}{2}\right)^{n-p}
$$

bleibt. Setzt man nun zur Abkürzung die feste Zahl $x_{p} \cdot\left(\mu^{\prime}+\frac{\varepsilon}{2}\right)^{-p}=A$, so ist für $n>p$ stets

$$
\sqrt[n]{x_{n}}<\sqrt[n]{A} \cdot\left(\mu^{\prime}+\frac{\varepsilon}{2}\right)
$$

Da nun $\sqrt[n]{A} \rightarrow 1$ und somit $\left(\mu^{\prime}+\frac{\varepsilon}{2}\right) \sqrt[n]{A} \rightarrow \mu^{\prime}+\frac{\varepsilon}{2}$ strebt, so kann man $n_{0}>p$ so wählen, daßi für $n>n_{0}$ stets $\left(\mu^{\prime}+\frac{\varepsilon}{2}\right) \sqrt[n]{A_{n}}<\mu^{\prime}+\varepsilon$ bleibt. Dann ist für $n>n_{0}$ um so mehr

$$
\sqrt[n]{x_{n}}<\mu^{\prime}+\varepsilon
$$

so daßi auch $\mu \leqq \mu^{\prime}+\varepsilon$, also wie behauptet, sogar $\mu \leqq \mu^{\prime}$ sein muß. (Vgl. S. 65, Fußnote 1.) Ubrigens lehren einfache Beispiele, dafs an keiner der drei Stellen der bewiesenen Beziehung 161 das Gleichheitszeichen zu gelten braucht.

4. Der vorige Satz lehrt insbesondere, daßs, wenn $\lim _{\frac{x_{n+1}}{x_{n}}}$ vorhanden ist, auch $\lim \sqrt[n]{x_{n}}$ existieren und denselben Wert haben muf. Also ganz speziell: Wenn das Quotientenkriterium in der $\boldsymbol{7 6}, 2$ gegebenen Form eine Entscheidung

$\left.{ }^{1}\right)$ Dieser Satz ist vom Charakter des Satzes 43,3. Denn setzt man die Quotienten $\frac{x_{1}}{1}, \frac{x_{2}}{x_{1}}, \frac{x_{3}}{x_{2}}, \ldots$ der Reihe nach $=y_{1}, y_{2}, y_{3}, \ldots$, so handelt es sich um die Folge $\left(y_{n}\right)$ einerseits und die Folge der $y_{n}^{\prime}=\sqrt[n]{y_{1} y_{2} \ldots y_{n}}$ andrerseits, deren Häufungsgrenzen hier verglichen werden.

2) Man schreibt darum wohl kürzer - und auch wir wollen eine solche Schreibweise im folgenden oft verwenden -

$$
\underline{\lim } \frac{x_{n+1}}{x_{n}} \leqq \varlimsup \sqrt[n]{x_{n}} \leqq \varlimsup \lim \frac{x_{n+1}}{x_{n}}
$$

wo nun in der Mitte nach Belieben der obere oder untere Limes genommen werden darf. 
bringt, so wird es das Wurzelkriterium sicher auch tun (aber nicht umgekehrt!). Alles in allem: Das Quotientenkriterium ist theoretisch weniger wirksam; es empfiehlt sich aber oft durch seine bequemere Verwendbarkeit.

5. Hierher gehören auch die schon unter $\boldsymbol{7 5}, 1$ und $\boldsymbol{7 6}, 3$ gemachten Bemerkungen.

\section{§3\%. Die logarithmischen Vergleichsskalen.}

Es ist schon betont worden, daß Kriterien, wie die zuletzt behandelten, nur hinveichende Kriterien sind und daher gegebenenfalls versagen können. Ihre Wirksamkeit wird von der Natur der benutzten Vergleichsreihen $\Sigma c_{n}$ und $\Sigma d_{n}$ abhängen; und ein $\mathcal{C}$-Kriterium wird, allgemein zu reden, um so mehr Aussicht auf Erfolg bieten, je größer die $c_{n}$-, ein $\mathfrak{D}$-Kriterium, je kleiner die $d_{n}$ sind. Zur Präzisierung dieser Verhältnisse wollen wir zunächst den hiermit schon angedeuteten Begriff der schnelleren und langsameren Konvergenz bzw. Divergenz festlegen: Wir werden eine konvergente Reihe um so besser konvergent nennen, je schneller ihre Teilsummen sich ihrem Summenwert nähern, und werden eine divergente Reihe um so schwächer divergent nennen, je langsamer ihre Teilsummen ansteigen. Oder genauer:

Definition. Sind $\Sigma c_{n}=s$ und $\Sigma c_{n}{ }^{\prime}=s^{\prime}$ zwei konvergente Reihen 162. mit positiven Gliedern, sind $s_{n}$ und $s_{n}{ }^{\prime}$ ihre Teilsummen und $s-s_{n}=r_{n}$, $s^{\prime}-s_{n}{ }^{\prime}=r_{n}{ }^{\prime}$ die zugehörigen Reste, so soll die zweite der ersten gegenüber als schneller oder langsamer (auch: besser oder schlechter) konvergent bezeichnet werden, je nachdem

$$
\lim \frac{r_{n}{ }^{\prime}}{r_{n}}=0 \quad \text { oder } \quad \lim \frac{r_{n}^{\prime}}{r_{n}}=+\infty
$$

ist. Ist der Grenzwert dieses Quotienten vorhanden und hat er einen positiven endlichen Wert, oder sind auch nur seine beiden Häufungsgrenzen $>0$ und $<+\infty$, so wird man die Konvergenz beider Reihen als eine gleichartige bezeichnen. Liegt auch dieser Fall nicht vor, so ist es nicht tunlich, die Güte ihrer Konvergenz zu vergleichen ${ }^{1}$ ). - Sind $\Sigma d_{n}$ und $\Sigma d_{n}^{\prime}$ zwei divergente Reihen mit positiven Gliedern und $s_{n}$ und ' $s_{n}{ }^{\prime}$ ihre Teilsummen, so soll die zweite gegenüber der

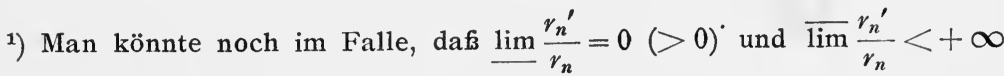
$(=+\infty)$ ist, die Reihe $\Sigma c^{\prime}{ }_{n}$ "nicht schlechter" („nicht besser") konvergent nennen als die Reihe $\Sigma c_{n}$; doch bietet das keine besonderen Vorteile. Im Falle endlich, dafs der untere Limes $=0$ und der obere $=+\infty$ ist, wäre die Güte der Konvergenz beider Reihen durchaus unvergleichbar. Und ähnliche Bemerkungen gelten für die Divergenz. (Man mache sich an Beispielen klar, dafis alle genannten Fälle wirklich eintreten können.) 
ersten als langsamer oder schneller divergent bezeichnet werden, je nachdem

$$
\lim \frac{s_{n}{ }^{\prime}}{s_{n}}=0 \quad \text { oder } \quad \lim \frac{s_{n}{ }^{\prime}}{s_{n}}=+\infty
$$

ist. Hat die Folge dieser Quotienten positive endliche Häufungsgrenzen, so wird man die Divergenz beider Reihen als gleichartig bezeichnen. Liegt auch dieser Fall nicht vor, so wird man sie hinsichtlich der Schnelligkeit ihrer Divergenz miteinander nicht vergleichen ${ }^{\mathbf{1}}$ ).

Die beiden folgenden Sätze zeigen, daß man die Schnelligkeit der Konvergenz oder Divergenz zweier Reihen schon oft ihren Gliedern (nicht erst ihren Resten bzw. Teilsummen) ansehen kann:

Satz 1. Strebt $\frac{c_{n}{ }^{\prime}}{c_{n}} \rightarrow 0(\rightarrow+\infty)$, so konvergiert $\Sigma c_{n}^{\prime}$ schneller (langsamer) als $\Sigma c_{n}$.

Beweis. Nach Wahl von $\varepsilon$ wird man im ersten Falle $n_{0}$ so bestimmen können, daß für $n>n_{0}$ stets $c_{n}{ }^{\prime}<\varepsilon c_{n}$ ist. Dann ist aber auch

$$
\frac{r_{n}^{\prime}}{r_{n}}=\frac{c_{n+1}^{\prime}+c_{n+2}^{\prime}+\cdots}{c_{n+1}+c_{n+2}+\cdots}<\varepsilon \frac{c_{n+1}+\cdots}{c_{n+1}+\cdots}=\varepsilon
$$

so daß dieser Quotient $\rightarrow 0$ streben wird. Und da der zweite Fall aus dem ersten durch Vertauschung der beiden Reihen hervorgeht (vgl. den Satz aus 40,4, Bem. 4,), so ist hiermit schon alles bewiesen.

Satz 2. Strebt $\frac{d_{n}^{\prime}}{d_{n}} \rightarrow 0(\rightarrow+\infty)$, so divergiert $\sum d_{n}^{\prime}$ langsamer (schneller) als $\Sigma d_{n}$.

Beweis. Nach 44, 4 folgt aus $\frac{d_{n}{ }^{\prime}}{d_{n}} \rightarrow 0$ unmittelbar, daB auch

$$
\frac{d_{1}^{\prime}+d_{2}^{\prime}+\ldots+d_{n}^{\prime}}{d_{1}+d_{2}+\ldots+d_{n}}=\frac{s_{n}^{\prime}}{s_{n}} \rightarrow 0
$$

strebt, - womit der Satz schon bewiesen ist.

163.

$$
\text { Einfache Beispiele. 1. Von den Reihen }
$$

$$
\sum \frac{1}{n \log ^{2} n}, \quad \sum \frac{1}{n^{2}}, \quad \sum \frac{1}{n^{3}}, \quad \sum \frac{1}{2^{n}}, \quad \sum \frac{1}{3^{n}}, \quad \sum \frac{1}{n !}, \quad \sum \frac{1}{n^{n}}
$$

konvergiert jede folgende besser als die vorangehende. Denn es ist z. B. für $n>4$

$$
\frac{1}{n !}: \frac{1}{3^{n}}=\frac{3^{n}}{n !}=\left(\frac{3 \cdot 3 \cdot 3}{1 \cdot 2 \cdot 3}\right) \frac{\cdot 3 \ldots 3}{\cdot 4 \ldots n}<\frac{9}{2} \cdot\left(\frac{3}{4}\right)^{n-3}
$$

1) Die in diesen Definitionen festgelegten Eigenschaften sind ersichtlich transitiv, d. h. wenn eine erste Reihe schneller konvergiert als eine zweite, diese ihrerseits schneller als eine dritte, so ist die erste Reihe auch schneller konvergent als die dritte. 
was gegen 0 strebt. Ebenso strebt $\frac{\log ^{2} n}{n} \rightarrow 0$ (nach $\left.\mathbf{3 8}, 6\right)$, - und noch einfacher liegt es bei den andern Reihenpaaren.

2. Von den Reihen

$$
\Sigma 2^{n}, \Sigma n, \Sigma 1, \Sigma \frac{1}{n}, \sum \frac{1}{n \log n}, \sum \frac{1}{n \log n \log _{2} n}, \ldots
$$

divergiert jede folgende schwächer als die vorangehende.

Neben diesen einfachen Beispielen bieten die Reihen, die wir schon in $\S 14$ antrafen, die wichtigsten Beispiele für Reihen von abgestufter Schnelligkeit ihrer Konvergenz bzw. Divergenz. Wir sahen dort, daß die Reihen

$\sum \frac{1}{n^{\alpha}}, \quad \leq \frac{1}{n \log ^{\alpha} n}, \quad \leq \frac{1}{n \log n \log _{2}^{\alpha} n}, \ldots, \quad \leq \frac{1}{n \log n \ldots \log _{p-1} n \cdot \log _{p}^{\alpha} n}$ für $\alpha>1$ konvergieren, für $\alpha \leqq 1$ divergieren. Jetzt lehren die Sätze 1 und 2 genauer: Bei festem $p$ wird eine jede dieser Reihen um so langsamer konvergieren bzw. divergieren, je näher der Exponent a $(>1 \mathrm{bzw} . \leqq 1)$ an $1 \mathrm{liegt}$; und ebenso wird eine jede dieser Reihen um so langsamer konvergieren bzw. divergieren, je größer $p$ ist, und dies, welchen positiven ${ }^{1}$ ) Wert bei einer jeden von ihnen der Exponent $\alpha(>1$ bzw. $\leqq 1)$ sonst auch haben mag.

Nur die zweite dieser Feststellungen bedarf vielleicht einer Begründung: Dividieren wir dazu das allgemeine Glied der $(p+1)^{\text {ten }}$ Reihe mit dem Exponenten $\alpha^{\prime}$ durch dasjenige der $p^{\text {ten }}$ Reile mit dem Exponenten $\alpha$, so ergibt sich

$$
\frac{\log _{p}^{\alpha} n}{\log _{p} n \cdot \log _{p+1}^{a^{\prime}} n} \text {. }
$$

Im Falle der divergenten Reihen sind nun $\alpha$ und $c^{\prime}$ positiv, aber $<1$; der Quotient strebt gegen 0, w. z.b.w. Im Falle der Konvergenz, wo also $\alpha$ und $\alpha^{\prime}>1$ sind, strebt er aber $\rightarrow+\infty$, weil - ganz ähnlich wie 38, $6-$ der Hilfssatz giit, dafi die Zahlen

$$
\frac{\log _{p+1} n}{\log _{p}^{\beta} n}=\frac{\log \left(\log _{p} n\right)}{\log _{p}^{\beta} n}
$$

eine Nullfolge bilden, wenn $\beta$ irgendein positiver Exponent und $p$ eine natürliche Zahl ist. Damit ist beides bewiesen.

Wegen dieser abgestuften Schnelligkeit der Konvergenz und Divergenz liefern uns diese Reihen ganze Skalen von Kriterien, wenn wir sie bei den Kriterien I und II (S. 267) als Vergleichsreihen be-

1) Für $\alpha=-\beta<0$ divergiert die Reihe natürlich schneller als die jeweils vorangehende mit dem Exponenten 1; z. B. $\Sigma \frac{\log ^{\beta} n}{n}$ mit $\beta>0$ divergiert schneller als $\Sigma \frac{1}{n}$. 
nutzen. Diese Kriterien erhalten wir zunächst ganz unmittelbar in der Form

$$
\left.a_{n} \leqq\right\} \frac{1}{a_{n} \geqq} \frac{1}{n \log n \ldots \log _{p-1} n \log _{p}^{\alpha} n} \text { mit }\left\{\begin{array}{lll}
\alpha>1 & : & \mathfrak{C} \\
\alpha \leqq 1 & : & \mathfrak{D}
\end{array}\right.
$$

164.

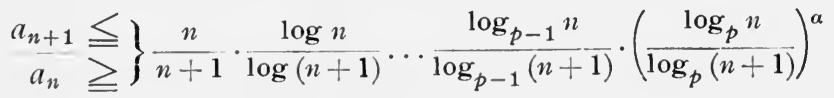

$$
\begin{aligned}
& \text { mit }\left\{\begin{array}{lll}
a>1 & : & \subset \\
a \leqq 1 & : & \mathbb{D} .
\end{array}\right.
\end{aligned}
$$

Und diese Kriterien, die wir in der Folge kurz als die logarithmischen Krittrien bezeichnen wollen - auch im Falle $p=0$-, können nach dem vorher Gesagten durch Wahl von $p$ und bei festem $p$ noch durch Wahl von $\alpha$ in ihrer Wirksamkeit gesteigert werden ${ }^{1}$ ).

Für den praktischen Gebrauch ist es vorteilhaft, diesen Kriterien noch etwas andere Gestalt zu geben. Wir wollen im folgenden einige solcher Umformungen nennen und einige Bemerkungen daran ankniipfen, ohne indessen die dazu nötigen Rechnungen vollständig hinzuschreiben.

\section{Umformung der logarithmischen Kriterien 164, I.}

1. Da bei positivem $a$ und $b$ die Ungleichungen $a \leqq b$ und $\log a \leqq \log b$ dasselbe besagen, so kann den Ungleichungen 164, (1) nach geringen Umformungen die Gestalt gegeben werden:

$$
\frac{\log a_{n}+\log n+\log _{2} n+\ldots \log _{p} n}{\log _{p} n}\left\{\begin{array}{lll}
\leqq-\beta<0 & : & \mathfrak{C} \\
\geq 0 & : & \mathfrak{D} .
\end{array}\right.
$$

2. Bezeichnet man für einen Augenblick den in diesem Kriterium ( $\mathbf{I}^{\prime}$ ) linksstehenden Ausdruck zur Abkürzung mit $A_{n}$, so hat man in

$$
\varlimsup \quad: \quad \text { e, } \quad \underline{\lim } A_{n}<0>0 \quad \text { : }
$$

ein wesentlich dasselbe leistendes Kriterium. Sein auf die Konvergenz bezüglicher Teil ist nämlich mit dem vorigen völlig inhaltgleich, der auf die Divergenz bezügliche nicht ganz. Denn hier wird jetzt nicht nur verlangt, dás der Ausdruck $A_{n}$ von einer Stelle an stets $\geq 0$ bleibt, sondern sogar, daßs er oberhalb einer festen positiven Zahl bleibt ${ }^{2}$ ).

1) Die Konvergenz und Divergenz der Reihen des obigen Typs war N. H. Abel schon 1827 bekannt, wurde aber von ihm nicht veröffentlicht (Euvres II, S. 200). Zur Kriterienbildung wurden diese Reihen zuerst von A. de Morgan benutzt (The differential and integral calculus, London 1842). Umformungen dieser ihrem Wesen nach stets auf 164, I und II, zuriickkommenden Kriterien wurden dann vielfach als besondere Kriterien veröffentlicht, so von J. Bertrand (J. de math. pures et appl., Bd. (1) 7 (1842), S. 35), O. Bonnet (ebenda, Bd. (1) 8 (1843), S. 78), U. Dini (Giornale di matematiche, Bd. 6 (1868), S. 166) u. a.

2) Das letzte $\mathfrak{D}$-Kriterium in $\operatorname{der}$ Form $\lim A_{n} \geq 0 \mathrm{zu}$ schreiben wäre aber ersichtlich falsch, da die untere Häufungsgrenze einer Zahlenfolge sehr wohl 0 sein kann, ohne daßs auch nur ein einziges Glied derselben positiv ist. 
3. Setzt man in etwas sorgfältigerer Schreibweise $A_{n}=A_{n}^{(p)}$ und betrachtet neben $A_{n}^{(p)}$ noch $A_{n}^{(p+1)}$, so ist ersichtlich

$$
A_{n}^{(p+1)}=1+\frac{\log _{p} n}{\log _{p+1} n} \cdot A_{n}^{(p)} .
$$

Und da nun $\frac{\log _{p} n}{\log _{p+1} n}=\frac{\log _{p} n}{\log \left(\log _{p} n\right)}$ wegen 38, 6 mit wachsendem $n$ gegen $+\infty$ strebt, so lehrt diese einfache Umformung folgendes: Wenn für ein bestimmtes $p$ eine der Häufungsgrenzen von $A_{n}=A_{n}^{(p)}$ nicht 0 ist, so ist sie für das nächstfolgende $p$, unter Beibehaltung des Vorzeichens, sicher $\infty$. Oder genauer: Bezeichnen wir für jedes $p$ die untere und die obere Häufungsgrenze von $A_{n}=A_{n}^{(p)}$ mit $\varkappa_{p}$ und $\mu_{p}$, und ist für ein bestimmtes $p$

und ist

$$
\varkappa_{p} \leqq \mu_{p}<0, \quad \text { so ist } \quad x_{p+1}=\mu_{p+1}=-\infty \text {, }
$$

$$
\mu_{p} \geqq x_{p}>0, \quad \text { so ist } \quad \mu_{p+1}=\varkappa_{p+1}=+\infty \text {. }
$$

Ist aber

$$
x_{p}<0 ; \quad \mu_{p}>0, \quad \text { so ist } x_{p+1}=-\infty, \mu_{p+1}=+\infty \text {. }
$$

Eine Erledigung der Konvergenzfrage ergibt sich also durch die Kriterienskalen (I) dann und nur dann, wenn für ein bestimmtes $p$ die beiden Werte $\varkappa_{p}$ und $\mu_{p}$ dasselbe Vorzeichen haben. Ist es negativ, so konvergiert die Reihe, ist es positiv, so divergiert sie. Haben die beiden Zahlen für ein $p$ verschiedene Vorzeichen, so ist für alle höheren $p$

$$
\underline{\lim } A_{n}^{(p)}=-\infty, \quad \quad \quad \quad \lim A_{n}^{(p)}=+\infty
$$

und die Skala liefert also keine Entscheidung. Desgleichen versagt sie, wenn eine der beiden Zahlen für jedes $p$ gleich 0 ist.

\section{Umformung der logarithmischen Kriterien 164, II.}

1. Hier gilt zunächst der folgende leicht beweisbare

Hilfssatz. Es besteht für jedes ganze $p \geqq 0$ und jedes reelle $\alpha$ eine Gleichung der Form

$$
\left(\frac{\log _{p}(n-1)}{\log _{p} n}\right)^{\alpha}=1-\frac{\alpha}{n \log n \ldots \log _{p} n}-\frac{\vartheta_{n}}{n^{2}}
$$

bei der die $\vartheta_{n}$ eine beschränkte Zahlenfolge bedeuten ${ }^{\mathbf{1}}$. Dabei soll $n$ erst von einem passenden, hinreichend großen Werte an laufen.

1) Eine Gleichung der hingeschriebenen Form besteht natürlich unter allen Umständen; denn die $\vartheta_{n}$ kann man ja geradezu durch die Gleichung selbst als definiert ansehen:

$$
\vartheta_{n}=n^{2}\left[1-\frac{\alpha}{n \log n \ldots \log _{p} n}-\left(\frac{\log _{p}(n-1)}{\log _{p} n}\right)^{\alpha}\right] .
$$

Der Schwerpunkt der Behauptung liegt also erst darin, daß diese $\vartheta_{n}$ eine beschränkte Zahlenfolge bilden. - Der Beweis ergibt sich induktiv unter Benutzung der beiden Bemerkungen, dafs die durch die Ansätze

$$
\left(1-x_{n}\right)^{\alpha}=1-\alpha x_{n}-\vartheta_{n}{ }^{\prime} x_{n}{ }^{2} \quad \text { und } \quad \log \left(1-\frac{1}{n y_{n}}\right)=-\frac{1}{n y_{n}}-\frac{\vartheta_{n}{ }^{\prime \prime}}{n^{2}}
$$

von einer Stelle an definierten Folgen $\left(\vartheta_{n}{ }^{\prime}\right)$ und $\left(\vartheta_{n}{ }^{\prime \prime}\right)$ beschränkt sind, falls die $x_{n}$ eine Nullfolge bilden und die $y_{n}$ etwa alle absolut genommen $\geqq 1$ sind. 
Und aus ihm ergibt sich unmittelbar, daßs für jedes ganze $p \geqq 0$, jedes reelle $\alpha$ und alle $n$ von einer Stelle an

$$
\begin{gathered}
\frac{n-1}{n} \cdot \frac{\log (n-1)}{\log n} \ldots \frac{\log _{p-1}(n-1)}{\log _{p-1} n} \cdot\left(\frac{\log _{p}(n-1)}{\log _{p} n}\right)^{\alpha} \\
=1-\frac{1}{n}-\frac{1}{n \log n}-\ldots-\frac{1}{n \log n \ldots \log _{p-1} n}-\frac{\alpha}{n \log n \ldots \log _{p} n}-\frac{\eta_{n}}{n^{2}}
\end{gathered}
$$

gesetzt werden kann, wenn die $\eta_{n}$ wieder eine passende, jedenfalls beschränkte Zahlenfolge bezeichnen.

Endlich kann dies auch

$$
=1-\frac{1}{n}-\frac{1}{n \log n}-\ldots-\frac{1}{n \log n \ldots \log _{p-1} n}-\frac{\alpha+\varepsilon_{n}}{n \log n \ldots \log _{p} n}
$$

gesetzt werden, wenn $\left(\varepsilon_{n}\right)$ eine passende Nullfolge bedeutet ${ }^{1}$ ).

2. Hiernach können den logarithmischen Kriterien zweiter Art z. B. die folgenden Formen gegeben werden ${ }^{2}$ ):

$$
\begin{aligned}
& \text { 16\%. } \left.\quad \frac{a_{n+1}}{a_{n}} \lesseqgtr\right\} 1-\frac{1}{n}-\frac{1}{n \log n}-\ldots-\frac{1}{n \log n \ldots \log _{p-1} n}-\frac{\alpha+\varepsilon_{n}}{n \log n \ldots \log _{p} n} \\
& \operatorname{mit}\left\{\begin{array}{lll}
\alpha>1 & : & e \\
\alpha \leqq 1 & : & \mathfrak{D}
\end{array}\right.
\end{aligned}
$$

oder auch

$$
\begin{aligned}
& \text { 168. } \quad \frac{a_{n+1}}{a_{n}} \geqq \int 1-\frac{1}{n}-\frac{1}{n \log n}-\ldots-\frac{1}{n \log n \ldots \log p-1 n}-\frac{\alpha^{\prime}}{n \log n \ldots \log p n} \\
& \operatorname{mit}\left\{\begin{array}{lll}
\alpha^{\prime}>1 & : & \mathbb{C} \\
\alpha^{\prime} \leqq 1 & : & \mathfrak{D}
\end{array}\right. \text {, }
\end{aligned}
$$

nach leichter Umformung auch die Form:

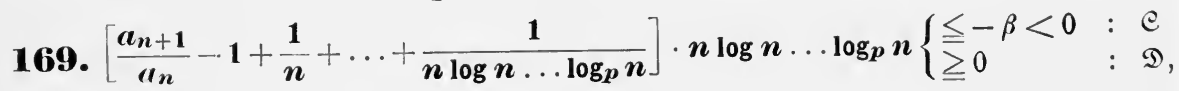
oder endlich, indem man den hier linksstehenden Ausdruck abkürzend mit $B_{n}$ bezeichnet und bezüglich des $\mathfrak{D}$-Kriteriums eine geringe Einbußse seiner Tragweite mit in Kauf nimmt (vgl. 165, 2)

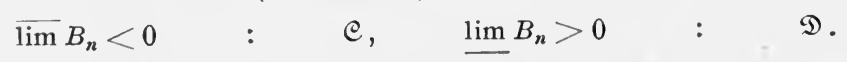

Die hieran in 165, 2 angeknüpften Bemerkungen bleiben sinngemäß bestehen.

5. Auch die Ausführungen in $\mathbf{1 6 5}, 3$ bleiben hier mit ganz unwesentlichen Änderungen gültig. Denn setzt man, in etwas sorgfältigerer Schreibweise, $B_{n}=B_{n}^{(p)}$, so ist ersichtlich

$$
B_{n}^{(p+1)}=1+B_{n}^{(p)} \cdot \log _{p+1} n .
$$

Und an diese Beziehung knüpfen sich nun in der Tat wegen $\log _{p+1} n \rightarrow+\infty$ genau dieselben Ausführungen wie in 165, 3 an die dort benützte Gleichung. Es erubrigt sich, dies im einzelnen auszufuhren.

6. Man stellt nun sofort noch allgemeiner fest, daßs eine Reihe von der Form

$$
\sum \frac{1}{e^{(\alpha-1) n} \cdot n^{\alpha_{0}} \log ^{\alpha_{1}} n \log _{2}^{\alpha_{2}} n \ldots \log _{q}^{\alpha_{q} n}}
$$

1) Wie dies alles für $p=0 \mathrm{zu}$ verstehen ist, ist ja unmittelbar klar.

2) Wir stellen hier das $n^{\text {te }}$ Glied der zu untersuchenden Reihe $\Sigma a_{n}$ dem $(n-1)^{\text {ten }}$ Glied der Vergleichsreihe gegenuiber, was nach $\mathbf{8 2}, 11$ geschehen darf. 
dann und nur dann konvergieren kann, wenn der erste der Exponenten $\alpha, \alpha_{0}$, $\alpha_{1}, \ldots, \alpha_{q}$, der von 1 verschieden ist, $>1$ ausfällt. Auf die Werte der folgenden Exponenten kommt es dann gar nicht mehr an. - Bei dieser Form der Vergleichsreihe sieht man noch deutlich, da $\$$ das in $\$ 38$ gegebene Raabesche und das Cauchysche Quotientenkriterium als $0^{\text {tes }}$ bzw. $(-1)^{\text {tes }}$ Glied der logarithmischen Skala angesehen werden können.

\section{§ 38. Spezielle Kriterien.}

Die logarithmischen Kriterien, die wir im vorigen Paragraphen abgeleitet haben, besitzen zweifellos mehr ein theoretisches als ein praktisches Interesse, da sie zwar eine vertiefte Einsicht in eine systematische Konvergenztheorie der Reihen mit positiven Gliedern gewähren, aber für die tatsächliche Prüfung der Konvergenz von Reihen, wie sie in den Anwendungen der Theorie auftreten, wenig in Frage kommen. (Darum haben wir die darauf bezüglichen Betrachtungen auch nur skizzenhaft durchgeführt.) Hierzu eignen sich höchstens ihre ersten zwei oder drei Glieder, aus denen wir nun durch Spezialisierung eine Anzahl einfacherer Kriterien herleiten wollen, die im Laufe der Zeiten mehr zufällig entdeckt und jeweils auf besonderen Wegen bewiesen wurden, und die sich nun alle in einen größeren Zusammenhang einordnen.

Für $p=0$ liefert die logarithmische Skala ein Kriterium 2. Art, das schon von J.L. Raabe $e^{1}$ ) herrührt. Wir erhalten es zunächst in der Form

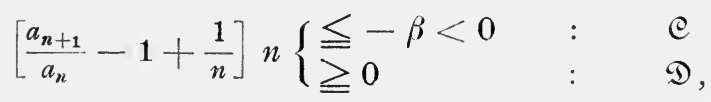

was man nun vorteilhafter so schreibt:

$$
\left[\frac{a_{n+1}}{a_{n}}-1\right] n\left\{\begin{array}{lll}
\leqq-a<-1 & : & \mathcal{e} \\
\geqq-1 & : & \mathscr{D}^{2}
\end{array}\right) .
$$

1) Zeitschrift für Phys. u. Math. von Baumgarten u. Ettinghausen, Bd. 10 (1832), S. 63. Vgl. auch J.M.C. Duhamel (J. de math. pures et appl. Bd. (1) 4 (1839), S. 214.)

2) Wegen des elementaren Charakters und der grofen Brauchbarkeit dieses Kriteriums lohnt es sich, einen direkten Beweis für seine Gültigkeit zu geben: Die C-Bedingung besagt, daf von einer Stelle an

$\frac{a_{n+1}}{a_{n}} \leqq 1-\frac{\alpha}{n} \quad$ oder $\quad n a_{n+1} \leqq(n-1) a_{n}-\beta a_{n}$ ist, mit $\beta=\alpha-1>0$.

Danach ist aber

$$
(n-1) a_{n}-n a_{n+1} \geqq \beta a_{n}>0
$$

und also die Folge $\left(n a_{n+1}\right)$ von dieser Stelle an monoton fallend. Da sie positiv bleibt, strebt sie einem Grenzwert $\gamma \geqq 0 \mathrm{zu}$, so daf die Reihe $\Sigma c_{n}$ mit $c_{n}=(n-1) a_{n}-n a_{n+1}$ nach 131 konvergent ist. Wegen $a_{n} \leqq \frac{1}{\beta} c_{n}$ liefert dies aber auch unmittelbar die Konvergenz von $\Sigma a_{n}$. - Ebenso hat man, wenn 
Hat der linksstehende Ausdruck für $n \rightarrow+\infty$ einen Grenzwert $l$, so ergibt die schon mehrfach angewandte Betrachtung (s. $\boldsymbol{7 6}, 2$ ), daß $l<-1$ die Konvergenz und $l>-1$ die Divergenz von $\sum a_{n}$ zur Folge hat, während $l=-1$ uns keinen unmittelbaren Aufschluß gibt.

\section{Beispiele.}

1. In $\S 25$ hatten wir die Binomialreihe untersucht und es fehlte dort noch die Entscheidung darüber, ob diese Reihe auch noch in den Endpunkten des Konvergenzintervalles konvergiert oder nicht, ob also bei gegebenem reellem $\alpha$ die Reihen

$$
\sum_{n=0}^{\infty}\left(\begin{array}{l}
\alpha \\
n
\end{array}\right) \quad \text { und } \quad \sum_{n=0}^{\infty}(-1)^{n}\left(\begin{array}{l}
\alpha \\
n
\end{array}\right)
$$

konvergieren oder nicht. Diese Entscheidung können wir jetzt fällen.

Für die zweite der Reihen hat man

$$
\frac{a_{n+1}}{a_{n}}=-\frac{\alpha-n}{n+1}=\frac{(n+1)-(\alpha+1)}{n+1} .
$$

Da dieser Quotient von einer Stelle an positiv ist, so haben die Glieder von da ab alle dasselbe Vorzeichen. Dies können wir positiv annehmen, da es auf ein gemeinsames Vorzeichen aller Glieder natürlich nicht ankommt. Weiter ist nun hiernach

$$
\left(\frac{a_{n+1}}{a_{n}}-1\right) n=-(\alpha+1) \cdot \frac{n}{n+1} \rightarrow-(\alpha+1)
$$

und das Raabesche Kriterium liefert nun sofort, dab die zweite unserer Reihen für $\alpha>0$ konvergiert, für $\alpha<0$ divergiert. Für $\alpha=0$ reduziert sich die Reihe auf das Anfangsglied 1.

Für die erstc der Reihen ist

$$
\frac{a_{n+1}}{a_{n}}=-1+\frac{\alpha+1}{n+1}
$$

und da dieser Wert von einer Stelle an negativ ist, so hat diese Reihe von $\mathrm{da}$ ab alternierende Vorzeichen. Ist nun $\alpha+1 \leqq 0$, so ist hiernach

$$
\left|\frac{a_{n+1}}{a_{n}}\right| \geqq 1 \text {, }
$$

und dies lehrt, daß̧ die Glieder $a_{n}$ schlief̧lich nie mehr abnehmen. Die Reihe mufs also divergieren. Ist aber $\alpha+1>0$, so ist $\left|\frac{a_{n+1}}{a_{n}}\right|<1$ und die Glieder nehmen jetzt von jener Stelle an ihrem Betrage nach monoton ab. Nach dem Leibnizschen Kriterium fuir Reihen mit alternierenden Gliedern mus die unsere also konvergieren, wenn wir noch zeigen könnten, dafi jetzt $\left(\begin{array}{l}\alpha \\ n\end{array}\right) \rightarrow 0$ strebt. Ist aber die Ungleichung

$$
\left|\frac{a_{n+1}}{a_{n}}\right| \leqq 1-\frac{\alpha+1}{n+1}
$$

die $\mathfrak{D}$-Bedingung erfüllt ist,

$\frac{a_{n+1}}{a_{n}} \geq 1-\frac{1}{n}$ oder $(n-1) a_{n}-n a_{n+1} \leqq 0$. Hiernach ist $n a_{n+1}$ monoton wachsend und bleibt daher größrer als eine feste positive Zahl $\gamma$. Aus $a_{n+1}>\frac{\gamma}{n}$, $\gamma>0$, folgt nun aber unmittelbar die Divergenz. 
etwa für $n \geqq m$ erfüllt, so folgt durch Multiplikation dieser für $m, m+1$, $\ldots, n-1$ angesetzten Beziehungen, daßs für $n>m$

$$
\left|a_{n}\right| \leqq\left|a_{m}\right| \cdot \prod_{\nu=m+1}^{n}\left(1-\frac{\alpha+1}{v}\right)
$$

ist. Da aber das Produkt $\Pi\left(1-\frac{\alpha+1}{v}\right)$ nach 126, 2, 3 gegen 0 divergiert, so strebt auch $a_{n} \rightarrow 0$ und $\Sigma\left(\begin{array}{l}\alpha \\ n\end{array}\right)$ mus also konvergieren. Zusammenfassend haben wir also bezüglich der binomischen Reihe:

$\sum_{n=0}^{\infty}\left(\begin{array}{l}\alpha \\ n\end{array}\right) x^{n}$ ist dann und nur dann konvergent, wenn $|x|<1$ oder wenn $x=-1$ und gleichzeitig $\alpha>0$ oder wenn $x=+1$ und gleichzeitig $\alpha>-1$ ist. Ihre Summe ist dann stets $(1+x)^{\alpha}$. In allen andern Fälen ist sie divergent. (Eine wesentliche Ergänzung hierzu bildet noch 247, S. 410.)

2. Nicht wesentlich verschieden von dem Raabeschen Kriterium ist das folgende, das von $O$. Schlömilch herrührt:

$$
n \log \frac{a_{n+1}}{a_{n}}\left\{\begin{array}{lll}
\leqq-\alpha<-1 & : & \mathfrak{C} \\
\geqq-1 & : & \mathfrak{D} .
\end{array}\right.
$$

In der Tat ist im Falle (D) nach 114

$$
\frac{a_{n+1}}{a_{n}} \geqq e^{-\frac{1}{n}}>1-\frac{1}{n}
$$

was nach dem Raabeschen Kriterium die Divergenz zur Folge hat; und im Falle (e) ist von einer Stelle an

$$
\frac{a_{n+1}}{a_{n}} \leqq e^{-\frac{\alpha}{n}} \leqq 1-\frac{\alpha^{\prime}}{n},
$$

wenn $\alpha>\alpha^{\prime}>1$ ist. Dies zieht nach 170 die Konvergenz nach sich.

Wählen wir in der logarithmischen Skala $p=1$, so erhalten wir ein Kriterium 2. Art, das wir unter Weglassung des Grenzfalles $\alpha=1$ so schreiben können

$$
\frac{a_{n+1}}{a_{n}}=1-\frac{1}{n}-\frac{\alpha_{n}}{n \log n} \text { mit }\left\{\begin{array}{lll}
\alpha_{n} \geqq \alpha>1 & : & \mathcal{C} \\
\alpha_{n} \leqq \alpha<1 & : & \mathfrak{D} .
\end{array}\right.
$$

Auch hier gilt der schon mehrfach gemachte Zusatz, daß falls $a_{n}$ gegen einen Grenzwert $l$ strebt, für $l>1$ Konvergenz, für $l<1$ Divergenz stattfinden wird, während aus $l=1$ zunächst noch gar nichts geschlossen werden kann ${ }^{\mathbf{1}}$ ).

1) Ein direkter Beweis für die Gültigkeit dieses Kriteriums kann ähnlich wie beim Raabeschen Kriterium folgendermaken gegeben werden: Wie dort geben wir ihm zunächst die Form:

$$
[(n-1) \log n-1] a_{n}-[n \log n] a_{n+1}\left\{\begin{array}{lll}
\geqq \beta a_{n} & \text { mit } \quad \beta>0 \\
\leqq-\beta^{\prime} a_{n} & \text { mit } \quad \beta^{\prime}>0
\end{array} \quad: \quad\right. \text { : }
$$

Ist nun die $\mathcal{C}$-Bedingung evfüllt, so ist wegen

$$
(n-1) \log (n-1)>(n-1) \log n-1
$$

- eine Beziehung, die man nach 114, $\alpha$ sofort als richtig erkennt - erst recht

$$
(n-1) \log (n-1) \cdot a_{n}-n \log n \cdot a_{n+1} \geqq \beta a_{n} .
$$


Selbst dieses erste (wirklich logarithmische) Kriterium der Skala wird nur selten in der Praxis zur Anwendung gelangen; denn die Reihen, die auf dieses Kriterium reagieren und nicht schon auf ein einfacheres (das Raabesche oder das Quotientenkriterium) treten äußerst selten auf; und da ihre Konvergenz eine so langsame ist wie bei der Reihe $I_{n} \frac{1}{\log ^{\alpha} n},(\omega>1)$, so sind sie auch für numerische Zwecke völlig unbrauchbar.

Mit seiner Hilfe lassen sich aber nun leicht noch einige andere Kriterien herleiten; wir erwähnen nur noch folgendes

172. Gaußisches Kriterium ${ }^{1}$ ): Kann man den Quotienten $\frac{a_{n+1}}{a_{n}} i n$ der Form

$$
\frac{a_{n+1}}{a_{n}}=1-{ }_{n}-\frac{\vartheta_{n}}{n^{2}}
$$

schreiben und ist hicrin $\lambda>1$ und $\left(\vartheta_{n}\right)$ eine beschränkte $\left.{ }^{2}\right)$ Zahlenfolge, so ist $\sum a_{n}$ für $a>1$ konvergent, für $" \leqq 1$ divergent.

Der Beweis ist unmittelbar: für $" \gtrless 1$ lehrt schon das Raabesche Kriterium die Richtigkeit der Behauptung. Für $\alpha=1$ setzen wir

$$
\frac{a_{n+1}}{a_{n}}=1-\frac{1}{n}-\frac{1}{n \log n}\left(\frac{\vartheta_{n} \cdot \log n}{n^{\lambda-1}}\right) ;
$$

und da nun der letzte in Klammern gesetzte Faktor wegen $(\lambda-1)>0$ eine Nullfolge bildet, so ist die Reihe nach 171 gewiß divergent.

Gau $\beta$ hat dies Kriterium in noch etwas speziellerer Form folgendermaßen ausgesprochen: ,Kann der Quotient $a_{n+1} / a_{n}$ in der Form

$$
\frac{a_{n+1}}{a_{n}}=\frac{n^{k}+b_{1} n^{k-1}+\ldots+b_{k}}{n^{k}+b_{1}{ }^{\prime} n^{k-1}+\ldots+b_{k}^{\prime}}
$$

Es ist also $n \log n \cdot a_{n+1}$ monoton fallend und strebt daher gegen einen Grenzwert $\gamma \geq 0$. Nach 131 muf also die Reihe mit dem Gliede

$$
c_{n}=(n-1) \log (n-1) \cdot a_{n}-n \log n \cdot a_{n+1}
$$

konvergieren. Wegen $a_{n} \leq \frac{1}{\beta} c_{n}$ gilt dann das gleiche für $\Sigma a_{n}$. Ist andrerseits die $\mathfrak{D}$-Bedingung erfüllt, so ist

$$
\left.(n-1) \log (n-1) \cdot a_{n}-n \log n \cdot a_{n+1} \leqq-\beta^{\prime}+1-(n-1) \log \left(1+\frac{1}{n-1}\right)\right] a_{n} .
$$

Für $n \rightarrow+\infty$ strebt aber (nach 11: b) die in der eckigen Klammer stehende Folge $\rightarrow-\beta^{\prime}$, und ist also von einer Stelle an negativ. Daher nimmt jetzt die Folge $n \log n \cdot a_{n+1}$ von dieser Stelle an monoton $z u$ und bleibt also oberhalb einer gewissen positiven Zahl $\gamma$. Wegen $a_{n+1}>\frac{\gamma}{n \log n}, \gamma>0$, muf nun aber $\Sigma a_{n}$ divergieren. worden.

1) Werke, Bd. 3, S. 140. - Dies Kriterium ist von Gauß 1812 aufgestellt

2) Vgl. die Fufnote auf S. 275. 
geschrieben werden, so wird $\sum a_{n}$ tür $b_{1}-b_{1}{ }^{\prime}<-1$ konvergieren, für $b_{1}-b_{1}{ }^{\prime} \geqq-1$ divergieren." - Der Beweis liegt nach dem Vorangehenden auf der Hand.

Beispiele.

1. Dieses Kriterium hatte $\operatorname{Gau} \beta$ hergeleitet, um iiber die Konvergenz der sogen. hypergeometrischen Reihe

$$
\begin{aligned}
& 1+\frac{\alpha \cdot \beta}{1 \cdot \gamma} x+\frac{\alpha(\alpha+1)}{1 \cdot 2} \cdot \frac{\beta(\beta+1)}{\gamma(\gamma+1)} x^{2}+\ldots \\
& =\sum_{n=0}^{\infty} \frac{\alpha(\alpha+1) \ldots(\alpha+n-1)}{1 \cdot 2 \ldots n} \cdot \frac{\beta(\beta+1) \ldots(\beta+n-1)}{\gamma(\gamma+1) \ldots(\gamma+n-1)} x^{n}
\end{aligned}
$$

zu entscheiden, bei der $\alpha, \beta, \gamma$ irgendwelche von $0,-1,-2, \ldots$ verschiedene reelle Zahlen bedeuten ${ }^{1}$ ). Hier ist

$$
\frac{a_{n+1}}{a_{n}}=\frac{(\alpha+n)(\beta+n)}{(1+n)(\gamma+n)} x
$$

und dies lehrt zunächst, das die Reihe für $|x|<1$ (absolut) konvergiert, für $|x|>1$ divergiert. Es bleiben also (ähnlich wie bei der binomischen Reihe, in die die vorgelegte ja übergeht, wenn man $\beta=\gamma(=1)$ wählt und zugleich $\alpha$ und $x$ durch $-\alpha$ und $-x$ ersetzt) allein die beiden Werte $x=1$ und $x=-1$ zur Untersuchung. Für $x=+1$ ist

$$
\frac{a_{n+1}}{a_{n}}=\frac{n^{2}+(\alpha+\beta) n+\alpha \beta}{n^{2}+(\gamma+1) n+\gamma}
$$

und dies zeigt, dafi die Glieder der Reihe von einer Stelle ab alle das gleiche Vorzeichen haben, das wir wieder positiv annehmen dürfen. Und nun lehrt das Gau $\beta$ sche Kriterium, dafs die Reihen für $\alpha+\beta-\gamma-1<-1$, d. h. für $\alpha+\beta<\gamma$ konvergieren, für $\alpha+\beta \geqq \gamma$ divergieren werden.

Für $x=-1$ ist die Reihe (von einer Stelle $a b$ ) alternierend, weil

$$
\frac{a_{n+1}}{a_{n}}=-\frac{n^{2}+(\alpha+\beta) \dot{n}+\alpha \beta}{n^{2}+(\gamma+1) n+\gamma}=-\left[1+\frac{\alpha+\beta-\gamma-1}{n}+\frac{\vartheta_{n}}{n^{2}}\right]
$$

schliefilich negativ wird ${ }^{2}$ ). Fast wörtlich dieselben Erwägungen wie in 170, 1 bei der binomischen Reihe lehren nun, daf

$$
\begin{array}{ll}
\text { für } \alpha+\beta-\gamma>1 & \text { Divergenz, } \\
\text { fuir } \alpha+\beta-\gamma<1 & \text { Konvergenz }
\end{array}
$$

der hypergeometrischen Reihe eintreten wird $^{3}$ ).

1) Für diese Werte würde die Reihe entweder abbrechen oder sinnlos werden. Für $n=0$ soll das allgemeine Glied der Reihe $=1$ gesetzt werden.

2) Die $\vartheta_{n}$ bedeuten hier wieder eine beschränkte Zahlenfolge.

3) Nur dafs auch Divergenz eintritt, wenn $\alpha+\beta-\gamma=1$ ist, muf hier ein wenig anders bewiesen werden wie damals. Man schliefit etwa so: Ist für $n>p>1$

$$
\frac{a_{n+1}}{a_{n}}=-\left(1+\frac{\vartheta_{n}}{n^{2}}\right) \text { und hierbei stets }\left|\vartheta_{n}\right| \leqq \vartheta,
$$

so ist, wenn wir uns $p$ gleich so groß gewählt denken, daß $p^{2}>\vartheta$ ist,

$$
\left|a_{n}\right|>\left|a_{p}\right|\left(1-\frac{\vartheta}{p^{2}}\right)\left(1-\frac{\vartheta}{(p+1)^{2}}\right) \ldots\left(1-\frac{\vartheta}{(n-1)^{2}}\right) .
$$

Und da hier das Produkt konvergiert und nur positive Faktoren hat, so bleiben alle $\left|a_{n}\right|$ oberhalb einer noch positiven Schranke. Die Reihe kann nicht konvergieren. 
2. Das Raabesche C-Kriterium versagt, falls in

$$
\frac{a_{n+1}}{a_{n}}=1-\frac{\alpha_{n}}{n}
$$

die Zahlen $\alpha_{n}$ zwar ständig $>1$ bleiben, aber den Wert $1^{\circ}$ zur unteren Häufungsgrenze haben. Setzt man in solchem Falle $\alpha_{n}=1+\beta_{n}$, so ist die Bedingung

$$
\varlimsup_{\lim } n \beta_{n}=+\infty
$$

notwendig zur Konvergenz von $\left.\Sigma a_{n}{ }^{1}\right)$; denn wäre $n \beta_{n}=\vartheta_{n}$ beschränkt, so wäre

$$
\frac{a_{n+1}}{a_{n}}=1-\frac{1}{n}-\frac{\vartheta_{n}}{n^{2}}
$$

und folglich $\Sigma a_{n}$ nach dem Gaußischen Kriterium divergent.

\section{§ 39. Die Sätze von Abel, Dini und Pringsheim und neue Herleitung der logarithmischen Vergleichsskalen aus ihnen.}

Die logarithmischen Kriterien, die wir als die bisher weitestgehenden erhalten haben, sind insofern von etwas zufälligem Charakter, als sie auf der Benutzung der Abelschen Reihen als Vergleichsreihen beruhten, die uns ihrerseits als zufällige Anwendungen des Cauchyschen Verdichtungskriterium begegnet waren. Dieser Charakter der Zufälligkeit wird ihnen in etwas genommen werden, wenn wir auch von ganz andrer Seite her mit einer gewissen Notwendigkeit zu ihnen gelangen. Den Ausgangspunkt hierzu bildet der folgende

173. Satz von Abel und $\boldsymbol{D i n i}^{2}$ ): Ist $\sum_{n=1}^{\infty} d_{n}$ eine beliebige divergente Reihe mit positiven Gliedern und sind $D_{n}=d_{1}+d_{2}+\ldots+d_{n}$ ihre Teilsummen, so ist die Reihe

$$
\sum_{n=1}^{\infty} a_{n}=\sum_{n=1}^{\infty} \frac{d_{n}}{D_{n}^{\alpha}} \quad \text { für } \quad\left\{\begin{array}{l}
\alpha>1 \text { konvergent, } \\
\alpha \leqq 1 \text { divergent. }
\end{array}\right.
$$

Beweis. Da im Falle, daß $\alpha=1$ ist,

$$
\frac{d_{n+1}}{D_{n+1}}+\ldots+\frac{d_{n+k}}{D_{n+k}} \geqq \frac{d_{n+1}+\ldots+d_{n+k}}{D_{n+k}}=1-\frac{D_{n}}{D_{n+k}}
$$

ist, und da nach Voraussetzung $D_{r} \rightarrow+\infty$ strebt, so kann man zu jedem $n$ sich $k=k_{n}$ so gewählt denken, daß

$$
\frac{D_{n}}{D_{n+k_{n}}}<\frac{1}{2} \text {, also } a_{n+1}+a_{n+2}+\ldots+a_{n+k_{n}}>\frac{1}{2}
$$

1) E. Cahen, Nouv. Annales de Math., Bd. (3) 5, S. 535.

2) N. H. Abel hatte (J. f. d. reine u. angew. Math., Bd. 3 (1828), S. 81) nur die Divergenz von $\sum \frac{d_{n}}{D_{n-1}}$ bewiesen; $U$. Dini (Sulle serie a termini positivi, Annali Univ. Toscana, Bd. 9 (1867), bewies den Satz im obigen Umfange. Erst 1881 wurden Schriften von $\mathrm{Abel}$ bekannt (Euvres II, S. 197), die auch den auf die Konvergenz bezüglichen Teil des obigen Satzes enthalten. 
ist; nach 81, 2 muß also $\sum a_{n}$ für $\alpha=1$ und daher um so mehr für $\alpha \leqq 1$ divergieren.

Der Konvergenzbeweis im Falle $\alpha>1$ ist ein klein wenig mühsamer. Wir beweisen ihn sogleich in einer von Pringsheim ${ }^{1}$ ) herrührenden Verallgemeinerung:

Satz von Pringsheim: Haben $d_{n}$ und $D_{n}$ dieselbe Bedeutung wie 174. soeben, so ist die Reihe

$$
\sum_{n=2}^{\infty} \frac{d_{n}}{D_{n} \cdot D_{n-1}^{\varrho}} \equiv \sum_{n=2}^{\infty} \frac{D_{n}-D_{n-1}}{D_{n} \cdot D_{n-1}^{o}}
$$

für jedes $\varrho>0$ konvergent.

Beweis. Man wähle eine natürliche Zahl $p$ so, daß $\frac{1}{p}<\varrho$ ist. Dann genügt es, die Konvergenz unserer Reihe für den Fall zu beweisen, daß in ihr der Exponent $\varrho$ durch $\tau=\frac{1}{p}$ ersetzt wird. Da ferner die Reihe

$$
\sum_{n=2}^{\infty}\left(\frac{1}{D_{n-1}^{\tau}}-\frac{1}{D_{n}^{\tau}}\right)
$$

wegen $D_{n-1}<D_{n} \rightarrow+\infty$ nach 131 konvergent ist und positive Glieder hat, so würde es ausreichen, das Bestehen der Ungleichung

$$
\frac{D_{n}-D_{n-1}}{D_{n} \cdot D_{n-1}^{\tau}} \leqq \frac{1}{\tau}\left(\frac{1}{D_{n-1}^{\tau}}-\frac{1}{D_{n}^{\tau}}\right)
$$

oder also der Ungleichung

$$
\left(1-x^{p}\right) \leqq p(1-x), \quad\left[x=\left(\frac{D_{n-1}}{D_{n}}\right)^{\frac{1}{p}}\right]
$$

für alle $0<x \leqq 1$ zu beweisen. Die Richtigkeit dieser letzten Ungleichung ist aber wegen $\left(1-x^{p}\right)=(1-x)\left(1+x+\ldots+x^{p-1}\right)$ sofort einzusehen. Damit ist der Satz schon bewiesen.

Zusätze und Beispiele.

1. Bei dem Abel-Dinischen Satze darf man natürlich die Größen $D_{n}$ durch irgendwelche Zahlen $D_{n}{ }^{\prime}$ ersetzen, die ihnen asymptotisch gleich sind oder für die der Quotient $D_{n}^{\prime} / \mathrm{D}_{n}$ für alle $n$ (wenigstens von einer Stelle an) $z$ wischen zwei festen positiven Zahlen bleibt. Das kann nach $\boldsymbol{7 0}, 4$ das Konvergenzverhalten nicht ändern.

2. Mit $\Sigma d_{n}$ ist nach dem Abel-Dinischen Satze auch

$$
\Sigma d_{n}^{\prime}=\Sigma \frac{d_{n}}{D_{n}}
$$

divergent. Man könnte fragen, in welchem Größsenverhältnis die entsprechenden Teilsummen beider Reihen zueinander stehen. Hierüber gilt folgender schöne

1) Math. Annalen, Bd. 35 (1890), S. 329. 
Satz $\left.^{1}\right)$. Falls $\frac{d_{n}}{D_{n}} \rightarrow 0$ strebt $\left.{ }^{2}\right)$, gilt für die Teilsummen der neuen Reihe die Abschätzung

$$
\frac{d_{1}}{D_{1}}+\frac{d_{2}}{D_{2}}+\ldots+\frac{d_{n}}{D_{n}} \simeq \log D_{n}
$$

sie wachsen also wesentlich genau so, wie die Logarithmen der alten Teilsummen.

Beweis. Wenn $x_{n}=\frac{d_{n}}{D_{n}} \rightarrow 0$ strebt, so strebt nach $112, \mathrm{~b}$

$$
\frac{x_{n}}{\log \frac{1}{1-x_{n}}} \text { oder also } \frac{d_{n} / D_{n}}{\log \frac{D_{n}}{D_{n-1}}} \rightarrow 1 \text {. }
$$

Die nicht definierte Zahl $D_{0}$ denken wir uns dabei $=1$ und für diejenigen Indizes, für die $x_{n}=0$ sein sollte, auch den eben genannten Quotienten $=1$ gesetzt. Nach dem Grenzwertsatz 44, 4 strebt dann (wegen $\log D_{n} \rightarrow+\infty$ ) auch

$$
\frac{\frac{d_{1}}{D_{1}}+\frac{d_{2}}{D_{2}}+\ldots+\frac{d_{n}}{D_{n}}}{\log D_{1}+\log \frac{D_{2}}{D_{1}}+\ldots+\log \frac{D_{n}}{D_{n-1}}}=\frac{1}{\log D_{n}}\left[\frac{d_{1}}{D_{1}}+\frac{d_{2}}{D_{2}}+\ldots+\frac{d_{n}}{D_{n}}\right] \rightarrow 1,
$$

womit der Satz schon bewiesen ist.

Man erkennt auch noch sofort, daf3 bei der Behauptung dieses Satzes links und rechts die $D_{n}$ durch irgendwelche Größen $D_{n}^{\prime}$ ersetzt werden dürfen, die ihnen asymptotisch gleich sind.

3. Mit Hilfe dieser Bemerkungen lassen sich nun in einfachster Weise die zu Eingang des Paragraphen angedeuteten Betrachtungen durchfuhren:

a) Die Reihe $\sum_{n=1}^{\infty} d_{n}$, bei der stets $d_{n}=1$ und also $D_{n}=n$ ist, mufs als die einfachste divergente Reihe angesprochen werden, denn die Folge $D_{n} \equiv n$ der natürlichen Zahlen ist der Prototyp einer $\rightarrow+\infty$ divergierenden Folge. Der Abel-Dinische Satz lehrt dann sofort, dafis die harmonischen Reihen

$$
\sum_{n=1}^{\infty} \frac{1}{n^{\alpha}} \quad \text { für } \quad\left\{\begin{array}{l}
\alpha>1 \text { konvergieren, } \\
\alpha \leqq 1 \text { divergieren, }
\end{array}\right.
$$

und der Satz aus 2. lehrt weiter, daf im letzteren Falle

$$
1+\frac{1}{2}+\frac{1}{3}+\ldots+\frac{1}{n} \simeq \log n
$$

ist. - b) Wählen wir nunmehr im Abel-Dinischen Satze für $\Sigma d_{n}$ die nach a) erneut als divergent erkannte Reihe $\Sigma \frac{1}{n}$, so dürfen wir nach 1. und 2. $D_{n}$ durch $D_{n}^{\prime}=\log n$ ersetzen und finden, daß auch

$$
\sum_{n=1}^{\infty} \frac{1}{n \log ^{\alpha} n} \quad \text { für } \quad\left\{\begin{array}{l}
\alpha>1 \text { konvergiert, } \\
\alpha \leqq 1 \text { divergiert. }
\end{array}\right.
$$

Und der Satz aus 2. lehrt weiter, daf

$$
\frac{1}{2 \log 2}+\frac{1}{3 \log 3}+\ldots+\frac{1}{n \log n} \simeq \log \log n=\log _{2} n
$$

1) s. E. Cesaro, Nouv. Annales de Math., Bd. (3) 9 (1890), S. 353.

2) Diese Forderung ist sicher schon dann erfullt, wenn die $d_{n}$ beschränkt bleiben, - also bei allen in der Folge auftretenden Reihen. 
ist. - c) Durch Wiederholung dieser äußerst einfachen Schlüsse ergibt sich nun erneut, aber völlig unabhängig von den früheren Resultaten: Die bei einem hinveichend hohen Index ${ }^{1}$ ) begonnenen Reihen

$$
\sum_{n} \frac{1}{n \log n \ldots \log _{p-1} n\left(\log _{p} n\right)^{\alpha}} \quad \text { sind für }\left\{\begin{array}{l}
\alpha>1 \text { konvergent, } \\
\alpha \leqq 1 \text { divergent, }
\end{array}\right.
$$

welche natürliche $Z$ ahl auch $p$ bedeuten mag. Und für die Teilsummen der mit $\alpha=1$ angesetzten Reihe gilt die asymptotische Gleichung

$$
\sum_{\nu=e_{p}+1}^{n} \frac{1}{v \log v \ldots \log _{p-1} v \cdot \log _{p} v} \simeq \log _{p+1} n .
$$

4. Ein zu $\mathbf{1 7 3}$ analoger, aber von einer konvergenten Reihe ausgehender Satz ist der folgende

Satz von Dini $^{2}$ ). Ist $\Sigma c_{n}$ eine konvergente Reihe mit positiven Gliedern und sind $r_{n-1}=c_{n}+c_{n+1}+\ldots$ deren Reste, so ist

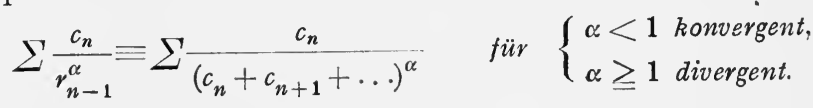

Beweis. Der Divergenzfall erledigt sich wieder ganz leicht, denn für $\alpha=1$ ist

$$
\frac{c_{n}}{r_{n-1}}+\ldots+\frac{c_{n+k}}{r_{n+k-1}} \geq \frac{c_{n}+\ldots+c_{n+k}}{r_{n-1}}=1-\frac{r_{n+k}}{r_{n-1}}
$$

und da dieser Wert bei jedem (festen) $n$ wegen $r_{\lambda} \rightarrow 0$ durch Wahl von $k$ noch $>\frac{1}{2}$ gemacht werden kann, so mufs die Reihe nach \$1, 2 divergieren. Für $\alpha>1$ wird dies dann, da von einer Stelle ab $r_{n}<1$ ist, erst recht der Fall sein.

Ist aber $\alpha<1$, so kann man die natürliche Zahl $p$ so wählen, daß auch noch $\alpha<1-\frac{1}{p}$ ist, und es genügt nun - wieder wegen $r_{n}<1$ für $n>n_{1}$ die Konvergenz der Reihe

$$
\sum \frac{c_{n}}{\left(r_{n-1}\right)^{1-\tau}} \equiv \sum \frac{r_{n-1}-r_{n}}{r_{n-1}} \cdot r_{n-1}^{\tau}
$$

zu beweisen, bei der zur Abkïrzung wieder $\frac{1}{p}=\tau$ gesetzt wurde.

Da nun $r_{n}$ monoton $\rightarrow 0$ strebt und also $\Sigma\left(r_{n-1}^{\tau}-r_{n}^{\tau}\right)$ eine sicher konvergente Reihe mit positiven Gliedern ist, so genügt es zu zeigen, daf

oder also, daf

$$
\frac{\dot{r}_{n-1}-r_{n}}{r_{n-1}} \cdot r_{n-1}^{\tau} \leqq \frac{1}{\tau}\left(r_{n-1}^{\tau}-r_{n}^{\tau}\right)
$$

$$
\left(1-y^{p}\right) \leqq p(1-y)
$$

$$
\left[y=\left(\frac{r_{n}}{r_{n-1}}\right)^{\frac{1}{p}}\right]
$$

ist, eine Beziehung, deren Richtigkeit wegen $0<y \leqq 1$ nun wieder evident ist.

1) Setzt man $e=e^{\prime}, e^{e^{\prime}}=e^{\prime \prime}$, und für $v \geqq 1$ allgemein $e^{e^{(v)}}=e^{(v+1)}$ und setzen wie die größste ganze in $e^{(v)}$ enthaltene $Z$ ahl $\left[e^{(v)}\right]=e_{v}$, so sind in dem Nenner unserer Reihe alle Faktoren $>1$, falls $n$ etwa bei $e_{p}+1$ zu laufen beginnt.

2) s. S. 282 , Furnote 2. 


\section{§ 40. Reihen mit positiven monoton abnehmenden Gliedern.}

Die bisherigen Betrachtungen bezogen sich meist auf ganz beliebige Reihen mit positiven Gliedern. Die Vergleichsreihen aber, die wir zur Bildung der Kriterien benutzten, waren fast immer von viel einfacherer Bauart; insbesondere nahmen ihre Glieder monoton ab. Es ist anzunehmen, daß für solche Reihen überhaupt einfachere Gesetze gültig sein werden und vielleicht auch einfachere Kriterien sich werden aufstellen lassen.

Schon S. 118 hatten wir z. B. den Satz bewiesen, daf3 bei einer konvergenten Reihe $\Sigma c_{n}$ mit monoton zu 0 abnehmenden Gliedern notwendig $n c_{n} \rightarrow 0$ streben müsse, was bei andern konvergenten Reihen (auch solchen mit nur positiven Gliedern) nicht der Fall zu sein braucht. Auch das Cauchysche Verdichtungskriterium $7 \boldsymbol{7}$ gehört hierher.

Im folgenden wollen wir einige weitere Untersuchungen dieser Art anstellen, und zunächst einige ebenso einfache wie weittragende Kriterien für solche Reihen herleiten. Ihre Konvergenz ist, wie wir sehen werden, oft sehr viel leichter zu erkennen als die allgemeinerer Reihen.

176. 1. Das Integralkriterium $\left.{ }^{1}\right)$. Ist ${ }^{\prime} a_{n}$ eine vorgelegte Reihe mit positiven monoton abnehmenden Gliedern und gibt es eine für $x \geqq 1$ positive und gleichfalls monoton fallende Funktion $f(x)$, für die bei jedem $n$

$$
f(n)=a_{n}
$$

ist, so ist $\Sigma a_{n}$ dann und nur dann konvergent, wenn die Integralwerte

$$
J_{n}=\int_{1}^{n} f(t) d t
$$

cine beschränkte Zahlenfolge bilden ${ }^{2}$.

Beweis. Da für $k-1 \leqq t \leqq k$ stets $f(t) \geqq a_{k}$ und für $k \leqq t \leqq k+1$ stets $f(t) \leqq a_{k}$ ist $(k \geqq 2$, ganz), so ist (nach $\S 19$, Satz 20)

$$
\int_{k}^{k+1} f(t) d t \leqq a_{k} \leqq \int_{k-1}^{k} f(t) d t, \quad(k=2,3, \ldots),
$$

und wenn man diese Ungleichung für $k=2,3, \ldots, n$ ansetzt und addiert, so folgt

$$
\int_{2}^{n+1} f(t) d t \leqq a_{2}+a_{3}+\ldots+a_{n}=s_{n}-a_{1} \leqq \int_{1}^{n} f(t) d t .
$$

1) Cauchy, Exercices mathém., Bd. 2, Paris 1827, S. 221.

?) Nach $\mathbf{7 0}, 4$ genügt es natürlich, wenn $f(x)$ den Gliedern $a_{n}$ asymptotisch proportional ist, oder wenn $f(x)=\alpha_{n} a_{n}$ gesetzt werden kann und hierbei die Faktoren $\alpha_{n}$ positive Häufungsgrenzen haben. - Statt zu fordern , $J_{n}$ bleibt beschränkt" kann man natiirlich auch fordern, dafis $\int_{i}^{\infty} f(t) d t$ konvergiert. Beides besagt (nach §19, Def. 14) genau dasselbe. 
Nach der rechten Häifte dieser Ungleichung folgt aus der Beschränktheit der Integrale $J_{n}$ die der Teilsummen der Reihe, nach der linken das umgekehrte, womit nach $\boldsymbol{7 0}$ schon alles bewiesen ist.

Zusatz. Hierbei nähert sich $\left(s_{n}-J_{n}\right)$ monoton fallend einem zwischen 0 und $a_{1}$ gelegenen Grenzwert. - In der Tat ist

$$
\left(s_{n}-J_{n}\right)-\left(s_{n+1}-J_{n+1}\right)=\int_{n}^{n+1} f(t) d t-a_{n+1} \geqq 0,
$$

woraus sofort alles folgt, da $a_{1} \geqq s_{n}-J_{n} \geqq a_{n}>0$ ist.

\section{Beispiele und Erläuterungen.}

1. Das Kriterium lehrt nicht nur das Konvergenzverhalten vieler Reihen erkennen, sondern gestattet auch oft, die Geschwindigkeit von Konvergenz und Divergenz bequem abzuschätzen. So sieht man sofort, daßi für $\alpha>1$

$$
\sum_{n=1}^{\infty} \frac{1}{n^{\alpha}} \text { wegen } J_{n}=\int_{1}^{n} \frac{d t}{t^{\alpha}}=\frac{1}{\alpha-1}\left(1-\frac{1}{n^{\alpha-1}}\right)<\frac{1}{\alpha-1}
$$

konvergiert, daßs dagegen

$$
\sum_{n=1}^{\infty} \frac{1}{n} \text { wegen } J_{n}=\int_{1}^{n} \frac{d t}{t}=\log n \rightarrow+\infty
$$

divergiert. Aber wir erfahren hier noch genauer, daf für $\alpha>1$

also

$$
\int_{n+1}^{n+k+1} \frac{d t}{t^{\alpha}} \sum_{\nu=n+1}^{n+k} \frac{1}{v^{\pi t}}<\int_{n}^{n+k} \frac{d t}{t^{\alpha}}
$$

$$
\frac{1}{\alpha-1} \cdot \frac{1}{(n+1)^{\alpha-1}}<r_{n}<\frac{1}{\alpha-1} \cdot \frac{1}{n^{\alpha-1}}
$$

ist, - eine Restabschätzung, die wir für $\alpha=2$ schon S. 252 gefunden hatten. Ebenso erfahren wir jetzt durch den Zusatz zu 176, dafi

$$
\left[1+\frac{1}{2}+\ldots+\frac{1}{n}-\log n\right]
$$

eine monoton fallende Zahlenfolge ist, die einem positiven, zwischen 0 und 1 gelegenen Grenzwert zustrebt. Diesen Zahlenwert bezeichnet man gewöhnlich mit $\mathrm{C}$ und nennt ihn die Eulersche oder Mascheronische Konstante. Es ist $C=0,57721566 \ldots$

Für $0<\alpha<1$ lehrt dieser Zusatz ebenso, dafi

$$
1+\frac{1}{2^{\alpha}}+\frac{1}{3^{\alpha}}+\ldots+\frac{1}{n^{\alpha}}-\int_{1}^{n} \frac{d t}{t^{\alpha}}
$$

eine monoton fallende Zahlenfolge ist, die einem zwischen 0 und 1 gelegenen Grenzwert zustrebt. Speziell ist also (vgl. 14,6) für $0<\alpha<1$

$$
1+\frac{1}{2^{\alpha}}+\ldots+\frac{1}{n^{\alpha}} \simeq \frac{n^{1-\alpha}}{1-\alpha}
$$

ist, und man stellt leicht fest, dafs diese Beziehung auch noch für $\alpha \leqq 0$ gilt. 
2. Allgemein folgt aus

$$
\int \frac{d t}{t \log t \ldots \log _{p-1} t \cdot \log _{p}^{\alpha} t}=\left\{\begin{array}{cl}
-\frac{1}{\alpha-1} \frac{1}{\log _{p}^{\alpha-1} t}, & \text { falls } \alpha \neq 1 \\
\log _{p+1} t, & \text { falls } \alpha=1
\end{array}\right.
$$

ebenso unmittelbar - nunmehr also auf einem dritten wieder gänzlich neuen Wege - das uns schon bekannte Konvergenzverhalten der Abelschen Reihen. Und der Zusatz zu 176 liefert wieder gute Abschätzungen der Reste (im Falle der Konvergenz) bzw. der Teilsummen (im Falle der Divergenz).

3. Ist $f(x)$ eine für alle $x$ von einer Stelle an positive Funktion, die dort eine monoton zu 0 abnehmende (also auch positive) Ableitung $f^{\prime}(x)$ besitzt, so nimmt auch $f^{\prime}(x) / f(x)$ monoton ab, und wegen

werden die beiden Integrale

$$
\int \frac{f^{\prime}(t)}{f(t)} d t=\log f(t)
$$

$$
\int^{x} f^{\prime}(t) d t \quad \text { und } \quad \int^{x} \frac{f^{\prime}(t)}{f(t)} d t
$$

entweder beide beschränkt oder beide nicht beschränkt sein. Daraus folgt: Die Reihen

$$
\Sigma f^{\prime}(n) \quad \text { und } \quad \Sigma \frac{f^{\prime}(n)}{f(n)}
$$

werden entweder beide konvergieren oder beide divergieren. Im Falle der Divergen $z$, in dem also notwendig $f(n) \rightarrow+\infty$ strebt, wird

Denn es ist jetzt

$$
\sum \frac{f^{\prime}(n)}{[f(n)]^{\alpha}} \quad \text { für } \alpha>1 \text { konvergieren. }
$$

$$
\int \frac{f^{\prime}(t)}{[f(t)]^{\alpha}} d t=-\frac{1}{\alpha-1} \frac{1}{[f(t)]^{\alpha-1}},
$$

woraus die Richtigkeit der Behauptung abgelesen werden kann. - Diese Sätze stehen in naher Beziehung zum Abel-Dinischen Satze.

2. Im wesentlichen denselben Wirkungsbereich hat das folgende in seiner Formulierung von der Integralrechnung freie

Steht $f(x) z u$ der Reihe $\Sigma a_{n}$ mit positiven monoton abnehmenden Gliedern in derselben Beziehung wie beim Integralkriterium und genügt es auch sonst den dort gemachten Voraussetzungen, so ist $\Sigma a_{n} \equiv \Sigma f(n)$

$$
\left.\begin{array}{l}
\text { konvergent, } \\
\text { divergent, }
\end{array}\right\} \quad \text { falls } \quad \frac{e^{x} f\left(e^{x}\right)}{f(x)}\left\{\begin{array}{l}
\leqq \vartheta<1 \\
\geqq 1
\end{array}\right.
$$

ausfält, - wenigstens von einer Stelle an.

Beweis. Ist die erste Ungleichung etwa für alle $x \geqq x_{0}$ erfüllt, so ist für diese $x$

$$
\int_{e^{x_{0}}}^{e^{x}} f(t) d t=\int_{x_{0}}^{x} e^{t} f\left(e^{t}\right) d t \leqq \vartheta \int_{x_{0}}^{x} f(t) d t
$$

$\left.{ }^{1}\right)$ Bulletin des sciences mathém., Bd. (1) 2 (1871), S. 250. 
und folglich

$$
\begin{aligned}
(1-\vartheta) \int_{e^{x_{0}}}^{e^{x}} f(t) d t & \leqq \vartheta\left[\int_{x_{0}}^{x} f(t) d t-\int_{e^{x_{0}}}^{e^{x}} f(t) d t\right] \\
& \leqq \vartheta\left[\int_{x_{0}}^{e^{x_{0}}} f(t) d t-\int_{x}^{e^{x}} f(t) d t\right] \\
& \leqq 9 \int_{x_{0}}^{e^{x_{0}}} f(t) d t
\end{aligned}
$$

Hiernach bleibt das linksstehende Integral und folglich auch $\int_{x_{0}}^{x} f(t) d t$ für alle $x>x_{0}$ unterhalb einer festen Schranke, so daß $\Sigma a_{n}$ nach dem Integralkriterium konvergieren muß.

Ist umgekehrt die zweite Ungleichung des Satzes für alle $x \geqq x_{1}$ erfüllt, so ist für diese $x$

$$
\int_{e^{x_{1}}}^{e^{x}} f(t) d t=\int_{x_{1}}^{x} e^{t} f\left(e^{t}\right) d t \geqq \int_{x_{1}}^{x} f(t) d t
$$

and der Vergleich des ersten und dritten Integrales lehrt weiter, daß

$$
\int_{x}^{e^{x}} f(t) d t \geqq \int_{x_{1}}^{e^{x_{1}}} f(t) d t
$$

Hier steht rechts eine feste Zahl $\gamma>0$, und die Ungleichung besagt, daß man zu jedem $n\left(>x_{1}\right)$ ein $k_{n}$ so angeben kann, daß, wenn $J_{n}$ dieselbe Bedeutung wie in $\mathbf{1 7 6}$ hat,

$$
J_{n+k_{n}}-J_{n}=\int_{n}^{n+k_{n}} f(t) d t \geqq \gamma>0
$$

bleibt. Nach 46 und $\mathbf{5 0}$ bleiben daher die $J_{n}$ nicht beschränkt und $\sum a_{n}$ kann daher nicht konvergieren ${ }^{1}$ ).

\section{Bemerkungen.}

1. Das Ermakoffsche Kriterium hat eine gewisse Ähnlichkeit mit dem Cauchyschen Verdichtungskriterium. Es enthält, insbesondere wie dieses, die ganze logarithmische Vergleichsskala, zu der wir damit einen vierten Zugang gewonnen hätten: Das Konvergenzverhalten der Reihe

$$
\perp \frac{1}{n \log n \ldots \log _{p-1} n \log _{p}^{\alpha} n}
$$

wird durch das Verhalten des Quotienten

$$
\frac{\log _{p-1} x \cdot \log _{p}^{\alpha} x}{\log _{p-1}^{\alpha} x}
$$

1) Es ist nicht schwer, den Beweis von dem Hilfsmittel des Integrals zu befreien; doch wird er dadurch etwas schwerfälliger. 
bestimmt. Da aber diese Quotienten $\rightarrow 0$ streben, wenn $\alpha>1$ ist, dagegen $\rightarrow+\infty$ streben, wenn $\alpha \leqq 1$ ist, so liefert in der Tat das Ermakoffsche Kriterium das uns bekannte Verhalten dieser Reihen ${ }^{1}$ ).

2. Statt $e^{x}$ darf man natürlich auch andre Funktionen verwenden: Ist $\varphi(x)$ irgendeine mit $x$ monoton wachsende positive und differenzierbare Funktion, für die stets $\varphi(x)>x$ ist, so wird $\Sigma a_{n}$ wieder konvergieren oder divergieren, je nachdem von einer Stelle ab stets

bleibt.

$$
\frac{\varphi^{\prime}(x) f(\varphi(x))}{f(x)}\left\{\begin{array}{l}
\leqq \vartheta<1 \quad \text { oder stets } \\
\geqq 1
\end{array}\right.
$$

Mit dem Ermakoffschen und dem Integralkriterium haben wir die wichtigsten Kriterien für unsere jetzigen Reihen in Händen.

\section{$\$$ 41. Allgemeine Bemerkungen zur Konvergenztheorie der Reihen mit positiven Gliedern.}

Die Aufstellung der in den vorangehenden Paragraphen mitgeteilten Konvergenzkriterien und die Klärung ihrer Bedeutung hat fast das ganze 19. Jahrhundert in Anspruch genommen. Erst gegen Ende desselben, insbesondere durch die Untersuchungen von Pringsheim haben die prinzipiellen Fragen einen gewissen Abschluß erreicht. Durch diese, sich in den mannigfachsten Richtungen bewegenden Untersuchungen sind auch eine ganze Reihe von Fragen erledigt, an die vordem nur zaghaft herangetreten wurde, die uns jetzt aber so einfach und durchsichtig erscheinen, daß wir es kaum noch begreifen, daß sie einstmals Schwierigkeiten gemacht haben, oder gar in völlig verkehrter Weise beantwortet oder abgetan wurden ${ }^{2}$ ). Wie weit aber der Weg bis hierher war, wird einem klar, wenn man bedenkt, daß Euler überhaupt noch nicht um Konvergenzfragen sich kümmerte, sondern einer Reihe, wo immer sie auftrat, ohne weiteres den Wert des Ausdrucks beilegte, der zu ihrer Entstehung Anlaß $\left.\mathrm{gab}^{3}\right)$; und daß Lagrange noch $1770^{4}$ ) meintę, eine jede Reihe stelle einen bestimmten Wert dar, wenn ihre Glieder zu 0 abnehmen ${ }^{5}$ ).

1) Das gilt sogar noch für $p=0$, wenn man unter $\log _{-1} x$ die Exponentialfunktion $e^{x}$ versteht.

2) Als Kuriosum sei erwähnt, dafs noch im Jahre 1885 und 1889 mehrere Abhandlungen veröffentlicht wurden, deren Ziel es war, die Existenz von konvergenten Reihen $\Sigma c_{n}$ zu beweisen, bei denen $\frac{c_{n+1}}{c_{n}}$ keinem Grenzwerte zustrebt!

$\left.{ }^{3}\right)$ So schrieb er als Folgerung aus $\frac{1}{1-x}=1+x+x^{2}+\ldots$ unbekiimmert

oder

$$
\frac{1}{2}=1-1+1-1+-\ldots
$$

Vgl. hierzu auch die ersten Absätze des $\$ 59$.

4) s. Oewvres, Bd. 3, S. 61 .

$\left.{ }^{5}\right)$ Hier sind immerhin Spuren eines Konvergenzgefühles zu sehen. 
Diese letzte Annahme etwa durch den Hinweis auf die (übrigens schon damals bekannte) Divergenz der Reihe $\Sigma \frac{1}{n}$ ausdrücklich zu widerlegen, erscheint uns heute überflüssig, und ähnlich liegt es bei vielen anderen Annahmen und Beweisversuchen aus früherer Zeit. Sie haben daher meist nur für den Historiker unserer Theorie ein Interesse. Ein Teil von ihnen ist aber, mögen die Antworten bejahend oder verneinend ausgefallen sein, doch noch von solchem Interesse, daß wir kurz darüber berichten möchten. Viele von diesen sind auch von der Art, daß jeder, der sich unbefangen mit den Reihen beschäftigt, ganz natürlich zu ihnen geführt wird.

Die Quelle aller dieser Fragen, die wir besprechen wollen, liegt in dem unzureichenden Charakter der Kriterien: Diejenigen, die für die Konvergenz notwendig und hinreichend sind (also die beiden Hauptkriterien 70 und 81) sind von so allgemeiner Art, daß in speziellen Fällen mit ihrer Hilfe die Konvergenz nur selten wird festgestellt werden können; die übrigen aber (es waren alles Vergleichskriterien und Umformungen derselben) waren nur hinreichende Kriterien, und lehrten nur dann die Konvergenz einer Reihe erkennen, wenn sie mindestens ebenso gut konvergierte wie die zum Vergleich benutzte. Hiertaucht aber sofort die Frage auf:

1. Gibt es eine am langsamsten konvergierende Reihe? Diese 178. Frage ist schon durch Satz 175, 4 verneinend entschieden. Denn mit $\sum c_{n}$ konvergiert auch noch $\Sigma c_{n}^{\prime}=\sum \frac{c_{n}}{r_{n-1}^{\frac{1}{2}}}$, aber offenbar langsamer als die vorige, weil $c_{n}: c_{n}^{\prime}=r_{n-1}^{\frac{1}{2}} \rightarrow 0$ strebt. - Fast noch einfacher hat $J$. Hadamard ${ }^{1}$ ) diese Frage durch Angabe der Reihe $\sum c_{n}$ $\equiv \Sigma\left(\sqrt{r_{n-1}}-\sqrt{r_{n}}\right)$ beantwortet. Da $c_{n}=r_{n-1}-r_{n}$, so strebt $c_{n}: c_{n}^{\prime}$ $=\sqrt{r_{n-1}}+\sqrt{r_{n}} \rightarrow 0$, so daß auch hier die gestrichene Reihe langsamer konvergiert als die ungestrichene.

Ebenso einfach erledigt sich die Frage

2. Gibt es eine am langsamsten divergierende Reihe? Auch hier lehrt schon der Abel-Dinische Satz 173, nach dem mit $\Sigma d_{n}$ auch noch $\Sigma d_{n}^{\prime} \equiv \sum \frac{d_{n}}{D_{n}}$ divergiert, daß die Frage zu verneinen ist. Denn da $d_{n}: d_{n}^{\prime}=D_{n} \rightarrow+\infty$ strebt, so läßt sich nach ihm jeder divergenten Reihe eine langsamer divergierende an die Seite stellen. - Diese Tatsachen, zusammen mit den Vorbemerkungen, lehren:

3. Kein Vergleichskriterium kann für alle Reihen wirksam sein.

Eng hängt damit die folgende von $A b e l^{2}$ ) aufgeworfene und beantwortete Frage zusammen:

1) Acta mathematica, Bd. 18 (1894), S. 319.

2) J.f. d. reine u. angew. Math., Bd. 3 (1828), S. 80. 
4. Gibt es eine positive Zahlenfolge $\left(p_{n}\right)$, so daß gleichzeitig $\left.\begin{array}{l}\text { a) } p_{n} a_{n} \rightarrow 0 \quad \text { für die Konvergenz } \\ \text { b) } p_{n} a_{n} \geqq \alpha>0, \text {, Divergenz }\end{array}\right\}$ jederReihe $\Sigma a_{n}$ hinreichend ist? Hier folgt wieder aus dem Abel-Dinischen Satze, daß dem nicht so ist. Setzen wir nämlich $a_{n}=\frac{\alpha}{p_{n}}$, so müßte diese Reihe $\Sigma a_{n}$ und also auch noch $\Sigma a_{n}^{\prime} \equiv \sum \frac{a_{n}}{s_{n}}$ divergieren, bei der $s_{n}=a_{1}+\ldots+a_{n}$ gesetzt ist. Für diese strebt aber $p_{n} a_{n}^{\prime}=\frac{\alpha}{s_{n}} \rightarrow 0$.

Das Ziel der Vergleichskriterien war es in gewisser Hinsicht, möglichst umfassende Bedingungen zu finden, die für die Entscheidung über das Konvergenzverhalten einer Reihe hinreichend sind. Als Gegenfrage kann man die nach möglichst engen Bedingungen aufstellen, die für die Konvergenz (oder Divergenz) einer Reihe $\Sigma a_{n}$ notwendig sind. Nach dieser Richtung wissen wir bisher nur, daß $a_{n} \rightarrow 0$ für die Konvergenz notwendig ist, und man wird daher sofort fragen:

5. Müssen die Glieder $a_{n}$ einer konvergenten Reihe mit einer besonderen Geschwindigkeit gegen 0 abnehmen? Pringsheim ${ }^{1}$ ) zeigte, $\mathrm{da} B$ dem nicht so ist. Vielmehr: Wie schwach auch die positiven Zahlen $p_{n} \rightarrow+\infty$ wachsen mögen, - es gibt stets konvergente Reihen $\Sigma c_{n}$, für die doch

$$
\lim p_{n} c_{n}=+\infty
$$

ist. Ja, sogar jede konvergente Reihe $\Sigma c_{n}^{\prime}$ kann durch passende Umordnung in eine als Beleg für diese Behauptung brauchbare Reihe $\Sigma c_{n}$ verwandelt werden ${ }^{2}$ ).

Beweis. Es sei also die positive gegen $+\infty$ wachsende Folge $\left(p_{n}\right)$ und die konvergente Reihe $\Sigma c_{n}^{\prime}$ gegeben. Dann wähle man eine wachsende Folge ungerader natürlicher Zahlen $n_{1}, n_{2},{ }_{i}, \ldots, n_{v}, \ldots$ für die

$$
\frac{1}{p_{n_{v}}}<\frac{c_{2 v-1}^{\prime}}{v}
$$$$
(v=1,2, \ldots)
$$

ist und setze $c_{n_{\nu}}=c_{2 v-1}^{\prime}$; und für die übrigen $c_{n}$ nehme man die Glieder $c_{2}^{\prime}, c_{4}^{\prime}, \ldots$ in ihrer natürlichen Reihenfolge. Dann ist $\Sigma c_{n}$ offenbar eine Umordnung von $\Sigma c_{n}^{\prime}$; aber es ist.

$$
p_{n} c_{n}>v
$$

wenn $n$ gleich einer der Zahlen $n_{v}$ ist. Hiernach ist wirklich

$$
\lim _{n} p_{n}=+\infty \text {. }
$$

Der Grund dieser Erscheinung ist einfach der, daß das Kon-

1) Math. Annalen, Bd. 35 (1890), S. 344.

2) Vgl. demgegenüber den Satz $\mathbf{8 2}, 7$, der sozusagen die durchschnittliche Abnahme der $a_{n}$ berïcksichtigt. 
vergenzverhalten einer Folge der Form $\left(p_{n} c_{n}\right)$ nichts Wesentliches mit dem Verhalten der Reihe $\Sigma c_{n}$ - also doch mit dem der Folge ihrer Teilsummon! - zu tun haben kann, da zwar das erstere, nicht aber das letztere durch eine Umordnung der Reihenglieder von Grund aus geändert werden kann.

6. Ebensowenig kann eine Bedingung der Form $\lim _{p_{n}} d_{n}>0$, in der $\left(p_{n}\right)$ eine, wenn auch noch so schnell gegen $+\infty$ wachsende positive Folge bedeutet, für die Divergenz von $\Sigma d_{n}$ notwendig $\left.\operatorname{sein}^{1}\right)$. Vielmehr kann jede divergente Reihe $\sum d_{n}^{\prime}$, deren Glieder nur zu 0 abnehmen müssen, durch eine passende Umordnung in eine solche (natürlich wieder divergente) Reihe $\Sigma d_{n}$ verwandelt werden, für die $\underline{\lim } p_{n} d_{n}=0$ ist. - Der Beweis ist nach dem Muster der vorigen Nummer leicht zu führen.

Noch etwas weiter geht die Frage:

7. Kann es eine Vergleichsskala geben, die für alle Fälle ausreicht? Genauer: Es seien $\Sigma c_{n}^{(1)}, \Sigma_{n}^{(2)}, \ldots, \Sigma_{n}^{(k)}, \ldots$ konvergente Reihen, von denen jede folgende etwa in der Weise schlechter konvergiert als die vorangehende, daß bei festem $k$

$$
\frac{c_{n}^{(k+1)}}{c_{n}^{(k)}} \rightarrow+\infty
$$

strebt. (Die logarithmische Skala bietet ein Beispiel für solche Reihen.) Kann es dann eine Reihe geben, die langsamer konvergitrt als eine jede dieser Reihen? Die Antwort lautet bejahend ${ }^{2}$ ), und es ist nicht schwer, eine solche Reihe wirklich zu konstruieren. Bei passender Wahl der ganzen Zahlen $n_{1}, n_{2}, \ldots, n_{k}, \ldots$ wird schon die Reihe

$\Sigma c_{n} \equiv c_{1}^{(1)}+c_{2}^{(1)}+\ldots+c_{n_{1}}^{(1)}+c_{n_{1}+1}^{(2)}+\ldots+c_{n_{2}}^{(2)}+c_{n_{3}+1}^{(3)}+\ldots+c_{n_{3}}^{(3)}+c_{n_{3}+1}^{(4)}+\ldots$, die aus aneinanderpassenden Stücken der Reihen $\Sigma c_{n}^{(k)}$ zusammengesetzt ist, das verlangte leisten. Wir wählen nämlich $n_{1}, n_{2}, \ldots$ so groß, daß

$$
\begin{aligned}
& \text { für } n \geqq n_{1} \quad \text { stets } r_{n}^{(2)}<\frac{1}{2} \text { und zugleich } c_{n}^{(2)}>2 c_{n}^{(1)} \\
& , n \geqq n_{2}>n_{1} \quad, \quad r_{n}^{(3)}<\frac{1}{2^{2}} \quad, \quad, \quad c_{n}^{(3)}>2 c_{n}^{(2)} \\
& , \quad n \geqq n_{k}>n_{k-1} \quad, \quad r_{n}^{(k+1)}<\frac{1}{2^{k}} \quad, \quad, \quad c_{n}^{(k+1)}>2 c_{n}^{(k)}
\end{aligned}
$$

1) Pringsheim, a. a. O., S. 357.

${ }^{2}$ ) Fiir die logarithmische Skala hat dies $P$. $d u$ Bois-Reymond (J. f. d. reine u. angew. Math., Bd. 76 (1873), S. 88) gezeigt. Die obige allgemeinere Antwort riihrt von J.Hadamard (Acta math., Bd. 18, 1894, S. 325) her. 
ausfällt $\left.{ }^{1}\right)$. Dann ist die Reihe $\Sigma c_{n}$ sicher konvergent, denn die aufeinanderfolgenden Stücke derselben sind gewiß kleiner als die mit diesen Stücken beginnenden Reste der betreffenden Reihen, also für $k=2,3, \ldots$, stets $<\frac{1}{2^{k}}$. Es strebt aber auch für jedes feste $k$

$$
\frac{c_{n}}{c_{n}^{(k)}} \rightarrow+\infty
$$

denn für $n>n_{q}(q>k)$ ist ersichtlich $\frac{c_{n}}{c_{n}^{(k)}}>2^{q-k}$. Damit ist schon alles bewiesen. - Insbesondere gibt es also Reihen, die langsamer konvergieren als eine jede Reihe unserer logarithmischen Skala ${ }^{2}$ ).

8. Genau ebenso einfach zeigt man: Wenn $\Sigma d_{n}^{(k)}$ für $k=1,2, \ldots$ lauter divergente Reihen sind, von denen jede folgende in der Weise langsamer divergiert als die vorangehende, daß $d_{n}^{(k+1)}: d_{n}^{(k)} \rightarrow 0$ strebt, so gibt es immer noch divergente Reihen $\Sigma d_{n}$, die schwächer divergieren, als eine jede der Reihen $\Sigma d_{n}^{(k)}$.

Alle diese Bemerkungen führen näher an die Frage heran, ob und inwieweit sich die Glieder konvergenter Reihen von denen divergenter Reihen prinzipiell unterscheiden. Nach 7. wird die folgende von Stieltjes herrührende Bemerkung nicht mehr überraschen:

9. Wenn $\left(\varepsilon_{1}, \varepsilon_{2}, \ldots\right)$ eine beliebig langsam monoton gegen 0 fallende Zahlenfolge bedeutet, so kann man doch stets cine konvergente Reihe $\Sigma c_{n}$ und eine divergente Reihe $\sum d_{n}$ so angeben, da $\beta$ für sie $c_{n}=\varepsilon_{n} d_{n}$ ist, - was etwa besagt, daß sich die Glieder beider Reihen nur relativ wenig voneinander zu unterscheiden brauchen. - In der Tat, wenn $\varepsilon_{n}$ monoton $\rightarrow 0$ fällt, wird $p_{n}=\frac{1}{\varepsilon_{n}}$ monoton $\rightarrow+\infty$ wachsen. Die Reihe

$$
p_{1}+\left(p_{2}-p_{1}\right)+\ldots+\left(p_{n}-p_{n-1}\right)+\ldots,
$$

deren Teilsummen ersichtlich die $p_{n}$ sind, ist also 'divergent. Nach dem Abel-Dinischen Satze ist dann auch

$$
\sum_{n=1}^{\infty} d_{n} \equiv \sum_{n=1}^{\infty} \frac{p_{n+1}-p_{n}}{p_{n+1}}
$$

divergent. Aber die Reihe $\Sigma c_{n} \equiv \Sigma \varepsilon_{n} d_{n} \equiv \Sigma\left(\frac{1}{p_{n}}-\frac{1}{p_{n+1}}\right)$ ist nach

131 konvergent. - Wesentlich dasselbe besagt die weitere Bemerkung: 10. Wenn $p_{n}$ beliebig langsam monoton $\rightarrow+\infty$ wächst, so gibt es stets eine konvergente Reihe $\Sigma c_{n}$, und eine divergente Reihe $\Sigma d_{n}$, für die $d_{n}=p_{n} c_{n}$ ist.

1) Mit $r_{n}^{(k)}$ bezeichnen wir den hinter dem $n$ ten Gliede beginnenden Rest der Reihe $\Sigma c_{n}^{(k)}$.

2) Die fehlenden Anfangsglieder dieser Reihen mag man sich dabei durch lauter Einsen ersetzt denken. 
178. §41. Allgemeine Bemerkungen zur Konvergenztheorie.

Noch krasser erscheinen in dieser Hinsicht die beiden schon unter 5. und 6. gegebenen von Pringsheim herrührenden Bemerkungen, wenn man sie so formuliert:

11. Wie stark auch $\sum c_{n}$ konvergieren mag, es gibt stets divergente Reihen - und sogar solche mit monoton zu 0 abnehmenden Gliedern -, für die

$$
\underline{\lim } \frac{d_{n}}{c_{n}}=0
$$

ist, so daß also $\Sigma d_{n}$ unendlich viele Glieder haben muß, die wesentlich kleiner sind als die entsprechenden Glieder von $\Sigma c_{n}$. Und umgekehrt:

Wie stark auch $\Sigma d_{n}$ divergieren möge, es gibt, wofern nur $d_{n} \rightarrow 0$ strebt, stets konvergente Reihen $\Sigma c_{n}$, für die $\varlimsup_{\lim } \frac{c_{n}}{d_{n}}=+\infty$ ist. - Wir haben nur noch das erste zu beweisen, und da leistet schon eine Reihe $\Sigma d_{n}$ der Form

$$
\begin{aligned}
\sum_{n=0}^{\infty} d_{n} \equiv c_{1}+c_{1} & +\ldots+c_{1}+\frac{1}{2} c_{n_{1}}+\frac{1}{2} c_{n_{1}}+\ldots+\frac{1}{2} c_{n_{1}} \\
& +\frac{1}{3} c_{n_{2}}+\frac{1}{3} c_{n_{2}}+\ldots+\frac{1}{3} c_{n_{2}}+\frac{1}{4} c_{n_{3}}+\ldots
\end{aligned}
$$

das verlangte, wenn die wachsenden ganzen Zahlen $n_{1}, n_{\mathfrak{9}}, \ldots$ passend gewählt werden und die einzelnen Gruppen von Gliedern je $n_{1},\left(n_{2}-n_{1}\right)$ $\left(n_{3}-n_{2}\right), \ldots$, Summanden enthalten. Damit die Reihe divergiert, braucht man nämlich die Anzahl der Summanden jedesmal nur so groß zu wählen, daß die Summe der Glieder einer jeden Gruppe $>1$ ausfällt; und damit die Gliederfolge monoton fällt, braucht man nur $n_{k}>n_{k-1}$ so groß zu wählen, daß $c_{n_{k}}<c_{n_{k-1}}$ ausfällt $(k=1,2, \ldots)$, was wegen $c_{n} \rightarrow 0$ stets möglich ist. (Hierbei ist unter $n_{0}$ der Index 1 zu verstehen.) Daß hier nun wirklich der Quotient $d_{n} / c_{n}$ die untere Häufungsgrenze 0 hat, folgt ja sofort daraus, daß er für $n=n_{k}$ den Wert $\frac{1}{k+1}$ hat.

Bei den bisherigen Bemerkungen haben wir nur von Konvergenz und Divergenz schlechthin gesprochen. Man könnte hoffen, unter engeren Voraussetzungen z. B. der, daß die Glieder der benutzten Reihe monoton abnehmen, sich entsprechend mehr erweisen ließe. So hatten wir schon gefunden, daß für eine konvergente Reihe $\Sigma c_{n}$ mit monoton abnehmenden Gliedern stets $n c_{n} \rightarrow 0$ strebt. Läßt sich hier noch mehr aussagen? Die Antwort ist verneinend (vgl. Bem. 5):

12. Wie schwach auch die positiven Zahlen $p_{n} \rightarrow \infty$ wachsen mögen, es gibt stets konvergente Reihen mit monoton zu 0 abnehmenden Gliedern, für die

$$
n p_{n} c_{n}
$$


nicht nur nicht $\rightarrow 0$ strebt, sondern sogar $+\infty$ zur oberen Häufungsgrenze hat ${ }^{1}$ ).

Der Beweis ist wieder ganz leicht. Man wähle wachsende natürliche Zahlen $n_{1}, n_{2}, \ldots$ für die

ist und setze

$$
p_{n,}>4^{\prime \prime}
$$$$
(v=1,2, \ldots)
$$

$$
\begin{gathered}
c_{1}=c_{2}=\ldots=c_{n_{1}}=\frac{1}{n_{1} \sqrt{p_{n_{1}}}}, \\
c_{n_{1}+1}=c_{n_{1}+2}=\ldots=c_{n_{2}}=\frac{1}{n_{2} \sqrt{p_{n_{2}}}}, \\
\cdot \cdot \cdot \cdot \cdot \cdot \cdot \cdot \cdot \cdot \cdot \cdot \cdot \cdot, \\
c_{n_{v-1}+1}=\ldots=c_{n_{v}}=\frac{1}{n_{v} \sqrt{p_{n_{v}}}},
\end{gathered}
$$

Die hiermit angedeuteten Gruppen von Gliedern liefern nacheinander Beiträge zur Summe der Reihe $\Sigma c_{n}$, die $<\frac{1}{2},<\frac{1}{2^{2}}, \ldots, \frac{1}{2^{v}}, \ldots$ sind, so daß $\Sigma c_{n}$ konvergieren wird. Andrerseits ist für $n=n_{v}$, stets so daß in der Tat

$$
n p_{n} c_{n}=\sqrt{p_{n}} \text {. }
$$

$$
\lim n \cdot p_{n} \cdot c_{n}=+\sim
$$

sein muß.

13. Durch die vorstehenden Bemerkungen, die sich leicht nach den mannigfachsten Richtungen hin vermebren und vertiefen ließen ${ }^{2}$ ), ist wohl hinlänglich deutlich geworden, daß es ganz aussichtslos ist, so etwas wie eine Scheidelinie zwischen konvergenten und divergenten Reihen einzuführen, wie dies noch von $P$. $d u$ Bois-Reymond versucht worden ist. Wie man auch diesen zunächst ganz verschwommenen Begriff präzisieren mag, in keiner Form entspricht er den tatsächlichen Verhältnissen. Wir deuten dies nach der nächstliegenden Richtung hin an ${ }^{2}$ ).

a) Solange die Glieder der Reihen $2 c_{n}$ und $\Sigma d_{n}$ keiner Beschränkung (außer der, $>0$ zu sein) unterworfen werden, bleibt der

1) Pringsheim, a. a. O. - Speziell die Frage, ob bei konvergenten Reihen mit monoton abnehmenden Gliedern $n \log n \cdot c_{n} \rightarrow 0$ streben müsse, ist lange diskutiert worden; und von vielen wurde die Bedingung $n \log n \cdot c_{n} \rightarrow 0$ noch 1860 für notwendig zur Konvergenz gehalten.

?) Eine sehr eingehende und sorgfältige Diskussion aller hierher gehörigen Fragen findet sich in dem S. 2 genannten' Werke von Pringsheim sowie in dessen mehrfach genannten Arbeiten in den Math. Ann. Bd. 35 und in den Miinch. Ber., Bd. 26 (1896) und 27 (1897). 
Quotient $\frac{c_{n}}{d_{n}}$ aller nur denkbaren Werte fähig, da neben der sicher bestehenden Beziehung

$$
\underline{\lim } \frac{c_{n}}{d_{n}}=0 \quad \text { zugleich } \quad \varlimsup \frac{c_{n}}{d_{n}}=+\infty
$$

sein kann. Die gebrochenen Linienzüge, durch die nach $\boldsymbol{7}, 6$ die Zahlenfolgen $\left(c_{n}\right)$ und $\left(d_{n}\right)$ veranschaulicht werden, können sich also unbegrenzt oft (und beliebig stark) durchsetzen.

b) Nach Bem. 11 ist dies auch noch der Fall, wenn die Folgen $\left(c_{n}\right)$ und $\left(d_{n}\right)$ beide monoton sind, die obengenannten Linienzüge also beide monoton fallen. Es gibt also sicher keinen sich nach rechts erstreckenden Linienzug, der die Eigenschaft hätte, daß das Bild jeder Folge vom Typus $\left(c_{n}\right)$ niemals $\ddot{u} b e r$ ihm, und zugleich das Bild jeder Folge vom Typus $\left(d_{n}\right)$ niєmals unter $i h m$ gelegen wäre, - auch dann nicht, wenn die genannten Gebilde monoton sind und erst von einer hinreichend weit rechts gelegenen Stelle an betrachtet werden.

$\mathrm{Ob}$ und inwieweit diese negativen Feststellungen sich in etwas ändern, wenn man die Zahlenfolge $\left(c_{n}\right)$ und $\left(d_{n}\right)$ nicht nur, wie soeben einfach-monoton, sondern im Sinne der S. 256 aufgestellten Definition voll-monoton annimmt, ist zur Zeit noch nicht geklärt.

\section{\$ 42. Systematisierung der allgemeinen Konvergenztheorie.}

Der Charakter einer gewissen Zufälligkeit, der der bisher entwickelten Konvergenztheorie der unendlichen Reihen nicht abgesprochen werden kann, gab die Veranlassung zu verschiedenen Versuchen, die Kriterien nach umfassenderen Gesichtspunkten zu systematisieren. In größerem Umfange wurden solche Versuche zuerst von $P$. Du Bois-Reymond $d^{1}$ ) gemacht, der ihnen indessen noch keineswegs einen Abschluf zu geben vermochte. Das ist in theoretisch und praktisch befriedigender Weise erst durch die Arbeiten von A. Pringsheim ${ }^{2}$ ) geschehen. Wir wollen hier noch ganz kurz iiber die Hauptgedanken seiner Entwicklungen berichten ${ }^{3}$ ).

Alle bisher in diesen Kapiteln zusammengestellten Kriterien sind Vergleichskriterien, ihre gemeinsame Quelle die beiden Vergleichskriterien I. und II. Art 157 und 158. Während dasjenige I. Art, also das Kriterium

$$
a_{n} \leqq c_{n} \quad: \quad \mathcal{e}, \quad a_{n} \geqq d_{n}
$$

als das denkbar einfachste und natürlichste angesprochen werden muf, gilt nicht das gleiche von demjenigen II. Art, für das wir ursprünglich die Form

$$
\frac{a_{n+1}}{a_{n}} \leqq \frac{c_{n+1}}{c_{n}} \quad: \quad \mathfrak{C}, \quad \frac{a_{n+1}}{a_{n}} \geqq \frac{d_{n+1}}{d_{n}} \quad: \quad \mathfrak{T}
$$

1) J. f. d. reine u. angew. Math., Bd. 76 (1873), S. 61.

2) Math. Ann., Bd. 35 (1890), S. 297-394.

$\left.{ }^{3}\right)$ Ein Eingehen auf die Einzelheiten können wir hier um so eher unterlassen, als die Pringsheimschen Untersuchungen in mehreren leicht zugänglichen und ausführlichen Darstellungen von ihres Autors Hand vorliegen, und dies in Formen, denen nicht leicht etwas hinzuzufügen wäre. 
kennen lernten. Schon die Heranziehung des Quotienten zweier aufeinanderfolgender Glieder ist etwas durch die Sache selbst nicht Gefordertes. Man könnte daher zunächst versuchen, durch Heranziehung anderer Verbindungen von zwei oder mehr Reihengliedern noch weitere Kriterienarten aufzustellen; doch hat sich dadurch für die Untersuchung allgemeiner Reihen kein Kriterium von Belang ergeben.

Aber auch wenn man beim Quotienten stehen bleibt, so kann man dem Kriterium II. Art noch mannigfache andere Formen geben, da man z. B. die Ungleichungen mit den positiven Faktoren $a_{n}$ oder $c_{n}$ multiplizieren darf, ohne dafs sich ihre Bedeutung ändert, o. ä. Hierauf wollen wir weiter unten zurückkommen. Aber von diesen relativ unwesentlichen Umformungen abgesehen, haben wir in (I) und (II) die Grundformen aller Kriterien zu sehen ${ }^{1}$ ). Alle nur denkbaren speziellen Vergleichskriterien wird man daher erhalten, wenn man in (I) und (II) alle nur denkbaren konvergenten und divergenten Reihen sich eingesetzt denkt und evtl. Umformungen der eben angedeuteten Art vornimmt.

Eine Systematisierung der allgemeinen Konvergenztheorie wird also in erster Linie die Aufgabe haben, eine Übersicht über alle nur denkbaren konvergenten und divergenten Reihen zu geben.

Diese Aufgabe kann natürlich nicht im wörtlichen Sinne gelöst werden (da ja sonst von jeder Reihe schon das Konvergenzverhalten feststünde); sie kann nur auf andere zurückgeführt werden, die von sich aus mehr Übersichtlichkeit haben und daher das Bedürfnis nach weiterer Behandlung nicht so dringlich erscheinen lassen. Pringsherm zeigt nun - und das ist der wesentliche Ausgangspunkt seiner Untersuchungen -, daß eine Systematisierung der allgemeinen Konvergenztheorie vollständig durchgeführt werden kann, wenn man sich die Gesamtheit aller monoton ins Unendliche wachsenden (positiven) Zahlenfolgen gegeben denkt.

Bezeichnen wir eine solche Folge in diesem Paragraphen weiterhin mit $\left(p_{n}\right)$, so dafi also $0<p_{0} \leq p_{1} \leq p_{2} \leq \ldots$ ist und $p_{n} \rightarrow+\infty$ strebt, so ist diese Aufgabe im Prinzip schon durch die folgenden beiden einfachen Bemerkungen gelöst:

179. a) Jede divergente Reihe $\Sigma d_{n}$ läßt sich - und eine jede nur auf genau eine Weise - mittelst einer passenden Folge vom Typus $\left(p_{n}\right)$ in der Form

$$
\sum_{n=0}^{\infty} d_{n}=p_{0}+\left(p_{1}-p_{0}\right)+\ldots+\left(p_{n}-p_{n-1}\right)+\ldots
$$

darstellen, und jede Reihe dieser Form ist divergent.

b) Auch jede konvergente Reihe ${ }^{2}$ ) läßt sich - und eine jede nur auf genau eine Weise - mittelst einer passenden Folge vom Typus $\left(p_{n}\right)$ in der Form

$$
\sum_{n=0}^{\infty} c_{n}=\left(\frac{1}{p_{0}}-\frac{1}{p_{1}}\right)+\left(\frac{1}{p_{1}}-\frac{1}{p_{2}}\right)+\ldots+\left(\frac{1}{p_{n}}-\frac{1}{p_{n+1}}\right)+\ldots
$$

darstellen, und jede Reihe dieser Form ist konvergent ${ }^{3}$ ).

In der Tat braucht man, nachdem dies festgestellt ist, in die Vergleichskriterien (I) und (II) für die $c_{n}$ und $d_{n}$ nur

$$
\frac{p_{n+1}-p_{n}}{p_{n} \cdot p_{n+1}} \quad \text { bzw. } \quad\left(p_{n}-p_{n-1}\right)
$$

1) Also ist - da wir in 160, 1,2 sahen, daf II eine Folge von (I) ist letzten Endes (I) doch dasjenige Kriterium, aus dem alles übrige folgt.

2) Falls ihre Glieder von keiner Stelle an sämtlich $=0$ sind.

3) Die Beweise dieser beiden Behauptungen sind so einfach, daf 3 wir nicht weiter darauf einzugehen brauchen. 
mit allen nur denkbaren Folgen des Typus $\left(p_{n}\right)$ einzusetzen, um im Prinzip alle nur denkbaren Kriterien erster oder zweiter Art zu gewinnen: Alle besonderen Kriterien müssen aus den so erhaltenen durch mehr oder minder naheliegende Umformungen gewonnen werden können; diese werden eben darum theoretisch nie etwas prinzipiell Neues bieten, wohl aber werden sie und hierin ist der hauptsächliche Wert dieser ganzen Betrachtungsweise zu sehen - für den vertieften Einblick in die Zusammenhänge zwischen den verschiedenen Kriterien, für deren einheitliche Aufstellung und endlich für ihre praktische Verwendung von grofer Bedeutung. Darum lohnte es sich nun sehr, auf die Einzelheiten der speziellen Kriterienbildung genau einzugehen; trotzdem wollen wir uns dabei aus dem schon genannten Grunde ganz kurz fassen.

1. Neben die in a) und b) gegebenen typischen Formen für divergente 180. und konvergente Reihen, die wohl als die denkbar einfachsten angesehen werden miissen, lassen sich selbstverständlich noch viele andere stellen, wodurch die äufere Form der Kriterien in mannigfacher Weise verändert werden kann. So ist nach dem $A b e^{\prime}-D i n i$ schen Satze 173 mit $\Sigma\left(p_{n}-p_{n-1}\right)$ auch

$$
\sum \frac{p_{n}-p_{n-1}}{p_{n}} \text { und } \sum \frac{p_{n}-p_{n-1}}{p_{n-1}}
$$

divergent, während gleichzeitig nach dem Pringsheimschen Satze 174 die Reihen

$$
\sum \frac{p_{n}-p_{n-1}}{p_{n} \cdot p_{n-1}^{\varrho}} \text { und } \sum \frac{p_{n}-p_{n-1}}{p_{n}^{1+\varrho}}
$$

für $\varrho>0$ konvergieren. Und, von geringfügigen Einschränkungen abgesehen, kann auch jede divergente bzw. konvergente Reihe in diesen neuen Formen dargestellt werden.

2. Da bei den vorstehenden typischen Formen divergenter und konvergenter Reihen die $p_{n}$ lediglich der Bedingung unterworfen waren, monoton $\rightarrow+\infty$ zu streben, so darf man in ihnen $p_{n}$ durch $\log p_{n}, \log _{2} p_{n}, \ldots$ oder durch $F\left(p_{n}\right)$ ersetzen, wenn $F(x)$ irgendeine für $x>0$ definierte und zugleich mit $x$ (im engeren Sinne) monoton $\rightarrow+\infty$ wachsende Funktion bedeutet. Dies führt dann wieder zu Kriterien, die zwar nicht dem Wesen nach, wohl aber (bei der Wahl spezieller $p_{n}$ ) der Form nach neu sind. Da insbesondere, wie man sich leicht iberzeugt, die bisher genannten Reihentypen um so langsamer divergieren bzw. konvergieren, je langsamer $p_{n} \rightarrow+\infty$ strebt, so hat man, indem $p_{n}$ etwa durch $\log p_{n}, \log _{2} p_{n}, \ldots$ ersetzt wird, ein Mittel zur Bildung von Kriterienskalen vor $\operatorname{sich}^{1}$ ). Für den sich durch besondere Einfachheit aufdrängenden Fall $p_{n}=n$ ist die Ausführung der hiermit angedeuteten Gedanken der Hauptinhalt der $\S \S 37$ und 38.

3. Ein weiterer Vorteil dieser Betrachtungsweise besteht darin, daf mit Hilfe ein und derselben Folge $\left(p_{n}\right)$ sowohl eine divergente wie eine konvergente Reihe dargestellt werden kann. Darum bieten sich die Kriterien ganz natur-

1) Daß3 man hierbei von $p_{n}$ gewöhnlich gleich $\mathrm{zu} \log p_{n}, \log _{2} p_{n}, \ldots$ übergeht, ist natürlich wieder freie Willkür. Doch wird dies durch die Sätze $7 \boldsymbol{\gamma}$ und 175, 2 nahegelegt. Zwischen $p_{n}$ und $\log p_{n} z$. B. könnte man leicht noch viele weitere Stufen der Skala einführen, z. B. $e^{\sqrt{\log p_{n}}}$, das schwächer. als $p_{n}$ ja schwächer als jede noch so kleine aber feste positive Potenz von $p_{n}$ und dennoch stärker als jede noch so hohe (feste, positive) Potenz von $\log p_{n}$ anwächst, od. ähnl. 
gemäß immer paarweis dar. Z. B. kann jedes Vergleichskriterium erster Art aus dem Kriterienpaar

$$
a_{n}\left\{\begin{array}{l}
\leqq \frac{p_{n}-p_{n-1}}{p_{n} \cdot p_{n-1}} \\
\geqq \frac{p_{n}-p_{n-1}}{p_{n-1}} \quad:
\end{array}\right.
$$

hergeleitet werden, und entsprechend unter Benutzung der anderen typischen Reihenformen.

4. Durch eine leichte - wenn auch ihrem Wesen nach willkürliche, d. h. nicht aus dem allgemeinen Gedankengang mit Notwendigkeit sich ergebende Änderung kann man nun hier dem Ausdrucke auf der rechten Seite eine einheitliche Gestalt geben und damit das Kriterium in ein einziges sogenanntes disjunktives Kriterium verwandeln. So erkennt man z. B. sofort, dafi jede der Reihen

$$
\sum \frac{p_{n}-p_{n-1}}{p_{n}^{\alpha}} \text { und } \sum \frac{p_{n}-p_{n-1}}{\alpha^{p_{n}}}
$$

für $\alpha>1$ konvergiert, für $\alpha \leq 1$ divergiert. Das in 3. aufgestellte Kriterienpaar kann daher durch das folgende disjunktive Kriterium ersetzt werden:

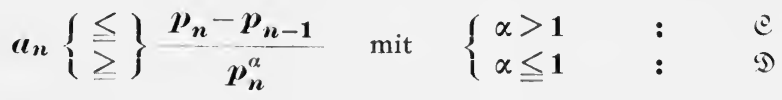

und im wesentlichen ${ }^{1}$ ) auch durch

$$
\boldsymbol{u}_{n}\left\{\frac{\leq}{\geq}\right\} \frac{\boldsymbol{p}_{n}-\boldsymbol{p}_{n-1}}{\alpha^{\boldsymbol{p}_{n}}} \quad \text { mit }\left\{\begin{array}{lll}
\alpha>1 & : & \mathfrak{C} \\
\alpha \leq 1 & : & \mathfrak{D} .
\end{array}\right.
$$

Bei den durch diese Umformung herausspringenden Konvergenzkriterien ist es bemerkenswert, dafs die Voraussetzung, es solle $p_{n} \rightarrow+\infty$ streben, gar nicht mehr notwendig ist! Es genügt, wenn $p_{n}$ monoton ist. Denn bleibt es beschränkt, so ist mit der Folge $\left(p_{n}\right)$ selber auch die Reihe $\Sigma\left(p_{n}-p_{n-1}\right)$ und folglich auch $\Sigma \frac{p_{n}-p_{n-1}}{p_{n}^{\alpha}}$ bzw. $\sum \frac{p_{n}-p_{n-1}}{\alpha^{p_{n}}}$ konvergent, da ja nun auch $\left(p_{n}^{-\alpha}\right)$ und $\left(\alpha^{-p_{n}}\right)$ beschränkte Folgen sind. Diese Konvergenzkriterien $\left.{ }^{2}\right)$ besitzen darum einen besonderen Grad von Allgemeinheiţ, ähnlich dem S. 302 genannten Kriterium II. Art von Kummer ${ }^{3}$.

5. Aus diesem disjunktiven Kriterium lassen sich nun wieder - und das gilt ganz allgemein von allen Kriterien - durch mannigfache Umformungen andere herleiten, die dann aber nur der Form nach neu sein können. Fiir solche Umformungen läßst sich natürlich kein allgemeines Schema angeben; Blick und Geschick werden hier stets neue Wege finden. Und gerade hierin liegt die Quelle für den grofen Reichtum an Kriterien, die letzten Endes jeder Systematisierung sich entziehen.

1) Der Ersatz ist kein vollständiger, d. h. unter Zugrundelegung derselben Folge $\left(p_{n}\right)$ leistet das neue Kriterium nicht soviel wie das alte, da z. B. die Divergenz der Reihe $\Sigma \frac{p_{n}-p_{n-1}}{p_{n}}$ zwar durch das alte, nicht aber durch das neue Kriterium erkannt werden kann.

2) Pringsheim, Math. Ann., Bd. 35 (1890), S. 342.

$\left.{ }^{3}\right)$ Journ. f. d. reine u. angew. Math., Bd. 13 (1835), S. 78. 
180. $§ 42$. Systematisierung der allgemeinen Konvergenztheorie.

Ganz naheliegend ist es, daf man jede Ungleichung mit irgendwelchen positiven Faktoren multiplizieren darf, ohne dafs sich ihre Bedeutung ändert; ebenso darf man von jeder Seite dieselbe Funktion $F(x)$ bilden, wenn $F(x)$ zugleich mit $x$ (im engeren Sinne) monoton wächst, insbesondere also darf man die beiden Seiten der Ungleichung eines Kriteriums logarithmieren, radizieren od. ähnl. So kann man z. B. dem letzten disjunktiven Kriterium die Form geben

oder

$$
\frac{\log \left(p_{n}-p_{n-1}\right)-\log a_{n}}{p_{n}}\left\{\begin{array}{lll}
\geq \beta>0 & : & \mathfrak{e} \\
\leqq 0 & : & \mathfrak{D}
\end{array}\right.
$$

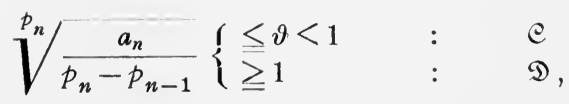

- und man erkennt nun mit einem Blick, dafs hiermit ein allgemeiner Rahmen für diejenigen Kriterien entstanden ist, die wir in den voraufgehenden Paragraphen durch die Annahme $p_{n} \equiv n$ oder $\equiv \log _{p} n$ erhalten hatten.

6. Wesentlich dieselben Bemerkungen bleiben nun auch in Kraft, wenn man in dem Grundkriterium zweiter Art (II) $c_{n}$ durch $\frac{p_{n}-p_{n-1}}{p_{n} \cdot p_{n-1}}$ und $d_{n}$ durch $p_{n}-p_{n-1}$, oder durch eine der anderen typischen Formen für die $c_{n}$ und $d_{n}$, ersetzt und dadurch die allgemeinste Form der Kriterien zweiter Art aufstellt.

7. Bemerkenswert ist hierbei wieder (vgl. Bem. 4), dafi das Konvergenzkriterium durch eine leichte Umformung so gestaltet werden kann, daf es mit dem Divergenzkriterium zusammen schlieflich ein einziges disjunktives Kriterium liefert. Das Konvergenzkriterium verlangt zunächst, dafi von einer Stelle $a b$

$$
\frac{c_{n+1}}{c_{n}}-\frac{a_{n+1}}{a_{n}} \geq 0 \quad \text { oder } \quad \frac{1}{c_{n}}-\frac{a_{n+1}}{a_{n}} \frac{1}{c_{n+1}} \geq 0
$$

bleibt. Ersetzt man hierin $c_{n}$ durch $\frac{p_{n}-p_{n-1}}{p_{n} \cdot p_{n-1}}$, so geht die erste Ungleichung über in

$$
\frac{p_{n+1}-p_{n}}{p_{n+1} \cdot p_{n}} \cdot \frac{p_{n} \cdot p_{n-1}}{p_{n}-p_{n-1}}-\frac{a_{n+1}}{a_{n}} \geq 0
$$

und da sich hier $p_{n}$ weghebt, so führen sich von selbst die typischen Glieder einer divergenten Reihe ein, und die Konvergenzbedingung lautet

oder

$$
d_{n+1}\left(\frac{1}{d_{n}}-1\right)-\frac{a_{n+1}}{a_{n}} \geq 0 \quad \therefore \quad e
$$

$$
\frac{d_{n+1}}{d_{n}}-\frac{a_{n+1}}{a_{n}} \geq d_{n+1} \quad: \quad \text { e. }
$$

Berücksichtigt man endlich, daß 3 mit $\Sigma d_{n}$ auch $\Sigma \varrho d_{n}$ divergiert $(\varrho>0)$, so erhält das Kriterium die Gestalt

$$
\frac{1}{d_{n}}-\frac{a_{n+1}}{a_{n}} \frac{1}{d_{n+1}} \geq \varrho>0 \quad: \quad e .
$$

Da nun dem ursprünglichen Kriterium sicher durch die Forderung

$$
\frac{1}{c_{n}}-\frac{a_{n+1}}{a_{n}} \cdot \frac{1}{c_{n+1}} \geq \varrho>0
$$


genügt wird, so zeigt es sich, dafi bei dieser letzten - seine Wirksamkeit ein klein wenig vermindernden - Gestalt es für das Kriterium ganz gleichguiltig ist, ob eine konvergente oder divergente Reihe zum Vergleich herangezogen wird. Oder also: Die $c_{n}$ sowohl wie die $d_{n}$ dürfen durch irgendwelche (positive). Zahlen ersetzt werden. Ist also $\left(b_{n}\right)$ irgendeine Folge positiver Zahlen, so liegt in

$$
b_{n}-\frac{a_{n+1}}{a_{n}} b_{n+1} \geq \varrho>0
$$

ein Konvergenzkriterium vor. Dieses Kriterium von besonderer Allgemeinheit. rürt von E. Kummer her ${ }^{1}$ ).

Andererseits hat man in

$$
\frac{1}{d_{n}}-\frac{a_{n+1}}{a_{n}} \cdot \frac{1}{d_{n+1}}\left\{\begin{array}{lll}
\geq 0>0 & : & \mathfrak{C} \\
\leqq 0 & : & \mathfrak{T}
\end{array}\right.
$$

ein disjunktives Kriterium zweiter Art gewonnen (da der auf die Divergenz bezügliche Teil ja eine unmittelbare Umformung von (II) ist.

Bezüglich aller weiteren Einzelheiten verweisen wir nochmals auf die Arbeiten und das Buch von A. Pringsheim. Aus den skizzierten Gedankengängen heraus können natürlich keineswegs alle Kriterien überhaupt, sondern nur diejenigen - diese aber sämtlich - entwickelt werden, die ihrem Charakter nach ein Vergleichskriterium I. oder II. Art sind. So konnte in den Betrachtungen dieses Paragraphen natürlich das Integralkriterium 176 und das Ermakoffsche Kriterium 177 nicht auftreten, da sie diesen Charakter nicht besitzen.

\section{Aufgaben zum IX. Kapitel.}

133. Man beweise bei den folgenden Reihen die Richtigkeit des angegebenen Konvergenzverhaltens:
a) $\leq \frac{1 \cdot 3 \ldots(2 n-1)}{2 \cdot 4 \ldots(2 n)} \frac{1}{2 n+1}:$ : ;

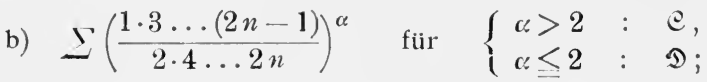
c) $\sum\left(\frac{1}{n}-\log \frac{n+1}{n}\right): \quad$;

$\left.{ }^{1}\right)$ Es wurde von Kummer schon 1835 gegeben (J. f. d. reine u. angew. Math. Bd. 13, S. 172), allerdings mit einer einschränkenden Bedingung, die erst U. Dini 1867 als überflüssig erkannte. Es wurde später mehrfach wiederentdeckt und gab noch 1888 Anla 3 zu heftigen Prioritätsstreitigkeiten. Erst O. Stolz (Vorlesungen. üb. allgem. Arithmetik Bd. 1, S. 259) fand den folgenden überaus einfachen Beweis, durch den das Kriterium erst voll verständlich wird:

Direkter Beweis: Das Kriterium besagt, dafs von einer Stelle an

$$
a_{n} b_{n}-a_{n+1} b_{n+1}>\varrho a_{n} .
$$

Dies hat einmal zur Folge, daf die Produkte $a_{n} b_{n}$ monoton fallen und also einem bestimmten Grenzwert $\gamma \geq 0$ zustreben. Nach 131 ist dann aber $\sum \frac{1}{\varrho}\left(a_{n} b_{n}-a_{n+1} b_{n+1}\right)$ eine konvergente Reihe mit positiven Gliedern. Und da $\Sigma a_{n}$ nicht größere Glieder hat, so ist diese Reihe gleichfalls konvergent. 
d) $\sum\left(\frac{1}{n \log n \log _{2} n}-\log \frac{\log _{2}(n+1)}{\log _{2} n}\right): \quad \mathfrak{e}$;

e) $\sum \frac{(x+1)(2 x+1) \ldots(n x+1)}{(y+1)(2 y+1) \ldots(n y+1)}$ für $\left\{\begin{array}{lll}y>x>0 & : & \mathbb{C}, \\ x \geq y>0: & :\end{array}\right.$

134. Für jedes feste $p$ hat bei $n \rightarrow+\infty$

$$
\left[\stackrel{n}{\Sigma} \frac{1}{v \log v \ldots \log _{p} v}-\log _{p+1} n\right]
$$

einen bestimmten Grenzwert $C_{p}$, wenn die Summation bei der ersten ganzen Zahl begonnen wird, für die $\log _{p} n>1$ ist.

135. Für jedes feste $\varrho$ in $0<\varrho<1$ hat bei $n \rightarrow \infty$

$$
\left[\sum_{v=1}^{n} \frac{1}{v^{1-\varrho}}-\frac{n^{\varrho}}{\varrho}\right]
$$

einen bestimmten Grenzwert $\gamma_{\varrho}$.

136. Aus $x_{n} \rightarrow \xi$ folgt

$$
\left[\frac{x_{p n+q}}{p n+q}+\frac{x_{p}(n+1)+q}{p(n+1)+q}+\cdots+\frac{x p p^{\prime} n+q}{p p^{\prime} n+q}\right] \rightarrow \frac{1}{p} \xi \log p^{\prime},
$$

wenn $p, p^{\prime}$ und $q$ gegebene natürliche Zahlen sind.

137. Ist $\Sigma d_{n}$ divergent, sind $D_{n}$ die Teilsummen und strebt $d_{n} \rightarrow 0$, so ist

$$
\sum_{v=1}^{n} d_{v} D_{v} \sim \frac{1}{2} D_{n}^{2}
$$

138. Hat $\sum a_{n}$ monoton abnehmende Glieder, so ist die Reihe sicher divergent, wenn für ein festes $p$ und alle $n$ von einer Stelle an $p \cdot a_{p n}-a_{n} \geqq 0$ ist.

139. Ist stets $0<d_{n}<1$, so sind die beiden Reihen

$$
\Sigma d_{n+1}\left[\left(1-d_{0}\right)\left(1-d_{1}\right) \ldots\left(1-d_{n}\right)\right]^{o}
$$

und

$$
\sum \frac{d_{n+1}}{\left[\left(1+d_{0}\right)\left(1+d_{1}\right) \ldots\left(1+d_{n}\right)\right]^{\prime \prime}}
$$

für jedes $\varrho>0$ konvergent.

140. Es soll das für Reihen mit monoton abnehmenden Gliedern gültige Konvergenzkriterium

$$
\varlimsup_{\lim } \frac{2^{n} a_{\mathbf{2}^{n}}}{a_{n}}\left\{\begin{array}{l}
<1: \mathfrak{C} \\
>2: \mathfrak{D}
\end{array}\right.
$$

ohne Benutzung des Ermakoffschen Kriteriums und ohne Benutzung der Integralrechnung direkt bewiesen werden.

141. Ist die Konvergenz einer Reihe $\sum a_{n}$ mit Hilfe eines Kriteriums der logarithmischen Skala 164, II festgestellt, so nimmt

$$
\left[n \log n \log _{2} n \ldots \log _{k} n\right] \cdot a_{n} \rightarrow 0
$$

ab, und zwar von einer Stelle an sogar monoton, welche natürliche Zahl auch $k$ bedeuten mag. 
X. Kapitel.

\section{Reihen mit beliebigen Gliedern.}

\section{$\S 43$. Konvergenzkriterien für Reihen mit beliebigen Gliedern.}

Während es bei den Reihen mit positiven Gliedern möglich war, die Untersuchung ihres Konvergenzverhaltens einigermaßen zu systematisieren, muß man bei Reihen mit beliebigen Gliedern fast ganz darauf verzichten. Der Grund hiervon ist weniger in einer ungenügenden Entwicklung der Theorie zu sehen, als in der Sache selbst. Denn eine Reihe mit beliebigen Gliedern kann konvergent sein, ohne absolut zu konvergieren ${ }^{1}$ ). Ja, dieser Fall wird hier sogar der fast allein interessierende, denn die Feststellung der etwaigen absoluten Konvergenz kommt nach $\mathbf{8 5}$ doch auf die Untersuchung einer Reihe mit positiven Gliedern zurück. Wir brauchen daher hier nur den Fall zu betrachten, daß die Reihe entweder tatsächlich nicht absolut konvergiert oder ihre absolute Konvergenz mit den bisherigen Mitteln nicht erkannt werden kann. Konvergiert aber die Reihe nur bedingt, so hängt die Konvergenz nicht nur von der Größe der einzelnen Glieder $a b$, sondern wesentlich noch von der Art ihrer Aufeinanderfolge. Etwaige Vergleichskriterien dürfen sich also nicht nur, wie früher, auf die einzelnen Glieder beziehen, sondern müssen im wesentlichen die ganze Reihe in Betracht ziehen. Das bedeutet aber letzten Endes, $\mathrm{da} ß$ jede Reihe für sich untersucht werden muß und sich also kein allgemeiner Zugang zu allen Reihen angeben läßt.

Wir müssen uns also hier mit Kriterien eines bescheideneren Wirkungskreises begnügen. Das wichtigste Hilfsmittel bei ihrer Aufstellung ist die sogenannte

182. ${ }^{\circ}$ Abelsche partielle Summation $\left.{ }^{2}\right)$. Sind $\left(a_{0}, a_{1}, \ldots\right)$ und $\left(b_{0}, b_{1}, \ldots\right)$ irgend zwai Zahlenfolgen und wird fïr $n \geqq 0$

$$
a_{0}+a_{1}+\ldots+a_{n}=A_{n}
$$

gesetzl, so ist für jedes $n \geqq 0$ und jedes $k \geqq 1$ stets

$$
\sum_{v=n+1}^{n+k} a_{v} b_{v}=\sum_{v=n+1}^{n+k} A_{v}\left(b_{v}-b_{v+1}\right)-A_{n} \cdot b_{n+1}+A_{n+k} \cdot b_{n+k+1} .
$$

1) Der Fall, daß̧ die Reihe durch „endlich viele Änderungen“ (s. 8:, 11) oder durch einen Vorzeichenwechsel aller Glieder in eine Reihe mit positiven Gliedern verwandelt werden kann, bedarf natürlich keiner besonderen Behandlung.

2) Journ. f. d. reine u. angew. Math., Bd. 1 (1826), S. 314. 
184. $\S 43$. Konvergenzkriterien für Reihen mit beliebigen Gliedern.

Beweis. Es ist

$$
\begin{gathered}
\sum_{v=n+1}^{n+k} a_{v} b_{v}=\left(A_{n+1}-A_{n}\right) b_{n+1}+\left(A_{n+2}-A_{n+1}\right) b_{n+2}+\ldots \\
\quad+\left(A_{n+k-1}-A_{n+k-2}\right) b_{n+k-1}+\left(A_{n+k}-A_{n+k-1}\right) b_{n+k} \\
=-A_{n} b_{n+1}+A_{n+1}\left(b_{n+1}-b_{n+2}\right)+A_{n+2}\left(b_{n+2}-b_{n+3}\right)+\ldots \\
\quad+A_{n+k-1}\left(b_{n+k-1}-b_{n+k}\right)+A_{n+k} \cdot b_{n+k} ;
\end{gathered}
$$

und fügt man hier rechts $0=-A_{n+k} b_{n+k+1}+A_{n+k} b_{n+k+1}$ hinzu, so ergibt sich genau die Behauptung $\left.{ }^{1}\right)$.

O Zusatz. Ist $c$ eine beliebige Konstante und setzt man $A_{v}+c=A_{\nu}^{\prime}, \mathbf{1 8 3}$. so ist auch

$$
\sum_{v=n+1}^{n+k} \boldsymbol{a}_{\nu} \boldsymbol{b}_{v}=\sum_{v=n+1}^{n+k} A_{n}^{\prime}\left(b_{v}-b_{v+1}\right)-A_{n}^{\prime} b_{n+1}+A_{n+k}^{\prime} \cdot b_{n+k+1},
$$

- denn es ist $a_{v}=A_{v}-A_{v-1}=A_{v}^{\prime}-A_{v-1}^{\prime}$

Man „darf“ also bei der Abelschen partiellen Summation die $A_{\nu}$ um eine beliebige Konstante vermehren oder vermindern.

Diese $A$ belsche partielle Summation gestattet nun fast unmittelbar eine Anzahl Konvergenzkriterien für Reihen der Form $\Sigma a_{v} b_{v}$ herzuleiten $^{2}$ ); sie liefert uns zunächst den folgenden sehr allgemeinen

- Satz. Die Reihe $\sum a_{v} b_{v}$ ist sicher konvergent, wenn

1) die Reihe $\sum A_{v}\left(b_{v}-b_{v+1}\right)$ konvergiert, und

2) wenn $\lim _{p \rightarrow+\infty} A_{p} \cdot b_{p+1}$ vorhanden ist.

Beweis. Die Formel 182 der Abelschen partiellen Summation zeigt sofort, daß - ohne Rücksicht auf den Wert von $k-$

$$
\lim _{n \rightarrow \infty} \sum_{\nu=n+1}^{n+k} a_{v} b_{v}=0
$$

ist. Denn wegen der ersten Voraussetzung ist (nach $\mathbf{8 1}, \mathbf{2}$ ) in demselben Sinne

$$
\lim _{n \rightarrow \infty} \sum_{v=n+1}^{n+k} A_{v} \cdot\left(b_{v}-b_{v+1}\right)=0
$$

1) Manchmal ist es vorteilhafter, die Formel in der zuerst erhaltenen Form

$$
\sum_{\nu=n+1}^{n+k} a_{\nu} b_{\nu}=\sum_{\nu=n+1}^{n+k-1} A_{\nu}\left(b_{\nu}-b_{\nu+1}\right)-A_{n} b_{n+1}+A_{n+k} b_{n+k}
$$

stehen $z u$ lassen.

$\left.{ }^{2}\right)$ Auf diese Form läßst sich natürlich jede Reihe bringen, da man ja jede Zahl als Produkt zweier anderer Zahlen schreiben kann. Der Erfolg in der Anwendung des obigen Satzes wird von dem Geschick in der Abspaltung der Faktoren abhängen. 
und wegen der zweiten Voraussetzung ist

$$
\lim _{n \rightarrow \infty}\left(-A_{n} b_{n+1}+A_{n+k} \cdot b_{n+k+1}\right)=0 .
$$

Nach $\mathbf{8 1}$ ist also $\Sigma_{a_{\boldsymbol{v}}} b_{\text {, }}$ wirklich konvergent.

Wir leiten nun aus diesem sehr weitgehenden Satze, durch den die Konvergenzfrage für $\sum a_{\boldsymbol{v}} b_{\text {, }}$ zwar nicht beantwortet, sondern nur auf zwei neue - aber eben in vielen Fällen einfachere - zurückgeführt wird, einige speziellere und leichter zu handhabende Kriterien her.

-1. Kriterium von $\left.\boldsymbol{A b e l}{ }^{1}\right) . \sum a_{v} b_{v}$ ist konvergent, wenn $\sum a_{v}$ konvergiert, und wenn die Folge $\left(b_{n}\right)$ monoton und beschränkt ist $\left.{ }^{2}\right)$.

Beweis. Nach Voraussetzung ist $\left(A_{n}\right)$, nach $\mathbf{4 6}\left(b_{n}\right)$ und somit auch $\left(A_{n} b_{n+1}\right)$ eine konvergente Folge. Nach 131 ist ferner $\Sigma\left(b_{v}-b_{v+1}\right)$ konvergent, und weil die Glieder dieser Reihe wegen der Monotonie der $b_{n}$ einerlei Vorzeichen haben, ist die Konvergenz sogar eine absolute. Folglich ist nach $\mathbf{5} \boldsymbol{\gamma}, 2$ auch die Reihe $\Sigma A_{v}\left(b_{v}-b_{v+1}\right)$ konvergent, denn die $A_{v}$, sind, da sie eine konvergente Folge bilden, sicher beschränkt. Die beiden Bedingungen des Satzes 184 sind also erfüllt und $\Sigma_{a} b_{v}$, somit konvergent.

○. Kriterium von Dirichlet $\left.{ }^{3}\right)$. $\Sigma a_{v} b_{v}$, ist konvergent, wenn $\sum a_{v}$, beschränkte Teilsummen hat $\left.{ }^{4}\right)$, und $\left(b_{n}\right)$ eine monotone Nullfolge ist.

Beweis. Aus denselben Gründen wie vorhin ist $\sum A_{v}\left(b_{v}-b_{v+1}\right)$ konvergent, und da mit $\left(b_{n}\right)$ wegen der Beschränktheit der $\left(A_{n}\right)$ auch $\left(A_{n} \cdot b_{n+1}\right)$ eine konvergente Folge ist, so sind wieder die Voraussetzungen des Satzes 184 erfültt.

\section{Kriterien von du Bois-Reymond $\left.{ }^{5}\right)$ und Dedekind ${ }^{6}$ ).}

a) $\Sigma a_{v} b_{v}$ ist konvergent, wenn $\Sigma\left(b_{v}-b_{v^{\prime}+1}\right)$ absolut und wenn $\Sigma a_{v}$ wenigstens bedingt konvergiert.

1) 1. c. - Das Abelsche Kriterium gibt eine hinreichende Bedingung für die $\left(b_{n}\right)$ an, damit aus der Konvergenz von $\Sigma a_{n}$ diejenige von $\sum a_{n} b_{n}$ folgt. J. Hadamard (Acta math., Bd. 27 (1903), S. 177) gibt die notwendigen und hinveichenden Bedingungen; vgl. auch E. B. Elliot (Quarterly Journ., Bd. 37 1906, S. 222), der die Fragestellung differenziert.

2) Oder auch: Eine konvergente Reihe „,darf" gliedweise mit monotonen und beschränkten Faktoren multipliziert werden. - Satz 184 und die daraus hergeleiteten Kriterien antworten alle auf die Frage: Mit was für Faktoren darf eine konvergente Reihe, bzw. muß eine divergente Reihe gliedweise multipliziert werden, damit wieder eine konvergente Reihe entsteht?

$\left.{ }^{3}\right)$ Vorlesungen iiber Zahlentheorie, 1. Aufl., Braunschweig 1863, § 101.

4) Oder - nach $69-$, wenn $\Sigma a_{n}$, zwischen endlichen Grenzen oszilliert".

5) Antrittsprogramm d. Univ. Freiburg, 1871. - Die oben benutzte Benennung der 3 Kriterien ist eine mehr konventionelle, da im Prinzip alle drei schon von Abel herrihren. Zur Geschichte dieser Kriterien vgl. A. Pringsheim, Math. Ann., Bd. 25 (1885), S. 423.

b) In $\S 143$ des in Fußnote 3 genannten Werkes. 
185. $\S 43$. Konvergenzkriterien für Reihen mit beliebigen Gliedern.

Beweis. Nach 87, 2 konvergiert auf Grund der Voraussetzungen auch $\sum A_{\nu} \cdot\left(b_{\nu}-b_{v+1}\right)$, da ja $\left(A_{n}\right)$ sicher beschränkt ist. Da ferner

$$
\left(b_{0}-b_{1}\right)+\left(b_{1}-b_{2}\right)+\cdots+\left(b_{n-1}-b_{n}\right)=b_{0}-b_{n}
$$

für $n \rightarrow+\infty$ gegen einen Grenzwert strebt, so ist $\lim b_{n}$ vorhanden; wegen der vorausgesetzten Existenz von $\lim A_{n}$ ist also auch $\lim A_{n} b_{n+1}$ vorhanden.

b) $\sum a_{v} b_{v}$ ist konvergent, wenn außer der absoluten Konergenz von $\Sigma\left(b_{\nu}-b_{\nu+1}\right)$ nur vorausgesetzt wird, daß $\sum a_{v}$, beschränkte Teilsummen hat, wofern dann $b_{n} \rightarrow 0$ strebt.

Beweis. Auch jetzt ist $\Sigma A_{v}\left(b_{v}-b_{v+1}\right)$ wieder konvergent und es strebt $A_{n} b_{n+1} \rightarrow 0$.

Beispiele und Anwendungen.

1. Mit $\Sigma a_{n}$ konvergiert nach dem Abelschen Kriterium auch $\sum \frac{a_{n}}{n}$, $\left.\sum \frac{a_{n}}{\log n}, \quad \sum \frac{a_{n}}{\log _{p} n}, \quad \sum^{n+1} a_{n}, \quad \sum \sqrt[n]{n} \cdot a_{n}^{1}\right) \quad \sum\left(1+\frac{1}{n}\right)^{n} \cdot a_{n}$, usw.

2. $\Sigma(-1)^{n}$ hat beschränkte Teilsummen. Ist also $\left(b_{n}\right)$ eine monotone Nullfolge, so ist

$$
\Sigma(-1)^{n} b_{n}
$$

nach dem Dirichletschen Kriterium konvergent. Wir haben so einen neuen Beweis für das Leibnizsche Kriterium bei alternierenden Reihen ( $\mathbf{( 2 ,} 10)$.

3 . Ist die Folge $\left(k_{0}, k_{1}, k_{2}, \ldots\right)$ von natürlichen Zahlen so beschaffen, daf $\Sigma(-1)^{k_{n}}$ beschränkte Teilsummen hat - bleibt also die Differenz zwischen der Anzahl der geraden und der der ungeraden unter den Zahlen $k_{0}, k_{1}, \ldots, k_{n}$ mit wachsendem $n$ beschränkt, so ist

$$
\Sigma(-1)^{k} b_{n}
$$

konvergent, wenn $\left(b_{n}\right)$ eine monotone Nullfolge bedeutet.

4. Ist $\Sigma a_{n}$ konvergent, so konvergiert für $0 \leqq x \leq+1$ die Potenzreihe $\Sigma a_{n} x^{n}$; denn die Faktoren $x^{n}$ bilden eine monotone Folge. - Hat $\Sigma a_{n}$ wenigstens beschränkte Teilsummen, so ist diese Potenzreihe jedenfalls für $0 \leqq x<1$ konvergent, weil dann $x^{n}$ monoton $\rightarrow 0$ strebt.

5. Die Reihe $\Sigma \sin n x$ hat für jedes (feste) reelle $x$ und die Reihe $\Sigma \cos n x$ hat für jedes von $2 k \pi$ verschiedene (feste) reelle $x$ beschränkte Teilsummen. Denn es gilt fuir jedes von $2 k \pi$ verschiedene $x$ die elementare, aber wichtige Formel

$$
\sin (\alpha+x)+\sin (\alpha+2 x)+\ldots+\sin (\alpha+n x)=\frac{\sin n \frac{x}{2} \cdot \sin \left(\alpha+(n+1) \frac{x}{2}\right)}{\sin \frac{x}{2}},
$$

für die S. 345 auch der Beweis angegeben wird. Sie liefert für $\alpha=0$ :

$$
\left.\sin x+\sin 2 x+\ldots+\sin n x=\frac{\sin n \frac{x}{2} \cdot \sin (n+1) \frac{x}{2}}{\sin \frac{x}{2}}, \quad\left(x \neq 2 k \pi^{2}\right)\right)
$$

1) Denn $\sqrt[n]{n}$ ist von $n=3$ an monoton fallend, aber $>1$.

2) Für $x=2 k \pi$ hat diese Summe natürlich für jedes $n$ den Wert 0 . 
und für $\alpha=\frac{\pi}{2}$ :

$$
\cos x+\cos 2 x+\ldots+\cos n x=\frac{\sin n \frac{x}{2} \cdot \cos (n+1) \frac{x}{2}}{\sin \frac{x}{2}}, \quad(x \neq 2 k \pi) .
$$

Aus ihnen kann die Beschränktheit der Teilsummen in dem behaupteten Umfange sofort abgelesen werden. Nach dem Kriterium $3 \mathrm{~b}$ ist also, wenn $\Sigma\left(b_{n}-b_{n+1}\right)$ absolut konvergiert und $b_{n} \rightarrow 0$ strebt,

die Reihe $\Sigma b_{n} \sin n x$ für jedes $x$

die Reihe $\Sigma b_{n} \cos n x$ für jedes von $2 k \pi$ verschiedene $x$

konvergent. Dies findet also insbesondere bei positiven monoton $z u \quad 0$ abnehmenden $b_{n}$ statt $^{1}$ ).

6. Sind die $b_{n}$ positiv und kann

$$
\frac{b_{n+1}}{b_{n}}=1-\frac{\alpha}{n}-\frac{\beta_{n}}{n^{1+\delta}}
$$

mit positivem $\delta$ und beschränkten $\beta_{n}$ gesetzt werden, so ist $\Sigma(-1)^{n} b_{n}$ dann und nur dann konvergent, wenn $\alpha>0$ ist. Denn ist $\alpha>0$, so folgt aus diesen Voraussetzungen zunächst, daß für alle $n$ von einer Stelle ab $b_{n+1} / b_{n}<1$, also die Folge $\left(b_{n}\right)$ monoton fallend ist. Die Konvergenz der Reihe wäre also nach 2. gesichert, wenn man noch zeigen könnte, daß $b_{n} \rightarrow 0$ strebt. Dies ergibt sich, ähnlich wie in 170, 1 , folgendermafien: Ist $0<\alpha^{\prime}<\alpha$, so ist von einer Stelle $a b$, etwa für alle $v>m$

$$
\frac{b_{v+1}}{b_{v}}<1-\frac{\alpha^{\prime}}{v} \text {. }
$$

Schreibt man diese Ungleichung für $y=m, m+1, \ldots, n-1$ an und multipliziert sie, so folgt

$$
b_{n}<b_{m} \cdot \prod_{\nu=m}^{n-1}\left(1-\frac{\alpha^{\prime}}{v}\right) .
$$

Wegen der Divergenz der harmonischen Reihe folgt hieraus wie in 170, 1 , daßs wirklich $b_{n} \rightarrow 0$ strebt.

Im Falle $\alpha<0$ nehmen aus entsprechenden Gründen die $b_{n}$ von einer Stelle an monoton $\mathrm{zu}$, so daßs $\Sigma(-1)^{n} b_{n}$ gewiß nicht konvergieren kann. Im Falle $\alpha=0$ endlich schliefst man, genau wie S. 281, Fufn. 3, daß auch jetzt $b_{n}$ nicht $\rightarrow 0$ strebt und also die Reihe wieder nicht konvergieren kann.

7. Wenn eine Reihe der Form $\Sigma \frac{a_{n}}{n^{x}}$ - man nennt sie Dirichletsche Reihe; wir werden sie später $(\S 58, \mathrm{~A})$ eingehender behandeln - für einen bestimmten Wert von $x$, etwa für $x=x_{0}$ konvergiert, so konvergiert sie auch für jedes $x>x_{0}$, denn $\frac{1}{n^{x-x_{0}}}$ bildet dann eine monotone Nullfolge. Aus dieser einfachen Anwendung des Abelschen Kriteriums ergibt sich, ganz ähnlich wie bei den Potenzreihen (93), der Satz: Jede Reihe der Form $\Sigma \frac{a_{n}}{n^{x}}$ besitzt eine wohl bestimmte Konvergenzabszisse $\lambda$ mit der Eigenschaft, daßs die Reihe für jedes $x>\lambda$ konvergiert, für jedes $x<\lambda$ divergiert. (Näheres s. §50.)

$\left.{ }^{1}\right)$ C. J. Malmstén, Nova acta Upsaliensis, Bd. (2) 12 (1844), S. 255. 
Allgemeine Bemerkungen.

1. Es war schon einleitend hervorgehoben worden, dafs bei beliebigen Reihen die Größe des einzelnen Gliedes nicht entscheidend für die Konvergenz ist. Insbesondere brauchen zwei Reihen $\sum a_{n}$ und $\Sigma b_{n}$, deren Glieder asymptotisch gleich sind, für die also $a_{n} / b_{n} \rightarrow 1$ strebt, nicht dasselbe Konvergenzverhalten aufzuweisen (vgl. dagegen $\mathbf{7 0}, 4)$.

Z. B. für

strebt

$$
a_{n}=\frac{(-1)^{n}}{n}+\frac{1}{n \log n} \quad \text { und } \quad b_{n}=\frac{(-1)^{n}}{n} \quad(n=2,3, \ldots)
$$

$$
\frac{a_{n}}{b_{n}}=1+\frac{(-1)^{n}}{\log n} \rightarrow 1
$$

Trotzdem ist $\Sigma b_{n}$ konvergent, $\Sigma a_{n}$ aber divergent, da ja $\Sigma\left(a_{n}-b_{n}\right)$ nach శ9, 2 divergiert.

2. Wenn eine Reihe $\Sigma a_{n}$ nicht absolut konvergiert (vgl. S. 130, Fußn. 1), so bilden ihre positiven und negativen Glieder fuir sich je eine divergente Reihe. Genauer: Es werde $p_{n}=a_{n}$ oder $=0$ gesetzt, je nachdem $a_{n}>0$ oder $\leqq 0$ ist; und ebenso werde $q_{n}=-a_{n}$ oder $=0$ gesetzt, je nachdem $a_{n}<0$ oder $\geqq 0$ ist $^{1}$ ). Dann sind $\Sigma p_{n}$ und $\Sigma q_{n}$ Reihen mit positiven Gliedern, deren erste nur die positiven und deren zweite nur die Beträge der negativen Glieder von $\Sigma a_{n}$ enthält (und zwar in unveränderter Stellung innerhalb der Reihe) und sonst nur Nullen. Diese beiden Reihen sind divergent. In der Tat, da jede Teilsumme von $\Sigma a_{n}$ die Differenz zweier passender Teilsummen von $\Sigma p_{n}$ und $\Sigma q_{n}$ ist, so folgt sofort: Wären $\Sigma p_{n}$ und $\Sigma q_{n}$ beide konvergent, so wäre $\Sigma\left|a_{n}\right|$ (nach 70) entgegen der Voraussetzung konvergent; wäre aber die eine Reihe konvergent, die andere divergent, so würden die Teilsummen von $\Sigma a_{n}$ wieder entgegen der Voraussetzung nach $+\infty$ oder $-\infty$ streben (je nachdem $\Sigma p_{n}$ oder $\Sigma q_{n}$ als divergent angenommen wird). Also müssen $\Sigma p_{n}$ und $\Sigma q_{n}$ beide divergieren.

3. Nach der vorigen Bemerkung erscheint also eine nur bedingt konvergente Reihe, d. h. also die Folge ihrer Teilsummen, als Differenz zweier monoton ins Unendliche wachsender Zahlenfolgen ${ }^{2}$ ). Über die Stärke des Anwachsens dieser beiden Folgen beweist man leicht den

Satz. Die Teilsummen von $\Sigma p_{n}$ und $\Sigma q_{n}$ sind asymptotisch gleich. - In der Tat ist

$$
\frac{p_{1}+p_{2}+\ldots+p_{n}}{q_{1}+q_{2}+\ldots+q_{n}}-1=\frac{a_{1}+a_{2}+\ldots+a_{n}}{q_{1}+q_{2}+\ldots+q_{n}}
$$

und da in diesem letzten Quotienten der Zähler beschränkt ìst, während der Nenner mit $n$ ins Unendliche wächst, so strebt dieser Quotient $\rightarrow 0$, - womit schon alles bewiesen ist.

4. Über die relative Häufigkeit positiver und negativer Glieder bei bedingt konvergenten Reihen $\Sigma a_{n}$, bei denen $\left|a_{n}\right|$ monoton fällt, hat $E$. Cesaro ${ }^{3}$ )

1) Es ist also $p_{n}=\frac{\left|a_{n}\right|+a_{n}}{2}$ und $q_{n}=\frac{\left|a_{n}\right|-a_{n}}{2}$; vgl. S. 133.

2) Die in manchen Darstellungen zu findende Ausdrucksweise, die Summe einer bedingt konvergenten Reihe sei in der Form $\infty-\infty$ gegeben, sollte als gar zu oberflächlich lieber vermieden werden.

$\left.{ }^{3}\right)$ Rom. Acc. Lincei Rend., Bd. (4) 4 (1888), S. 133. - Vgl. hierzu eine Note von G. H. Hardy, Messenger of Math., Bd. (2) 41 (1911), S. 17, und von H. Rademacher, Math. Zeitschrift, Bd. 11, 1921. 
den schönen Satz bewiesen, daf wenn $P_{n}$ die Anzahl der positiven und $Q_{n}$ die Anzahl der negtiven Glieder $a_{v}$, ist, für die $v \leqq n$ ist, so kann $\frac{P_{n}}{Q_{n}}$ keinen andern Grenzwert als 1 haben. (Doch braucht dieser Grenzwert nicht zu existieren.)

\section{$\$ 44$. Umordnung nur bedingt konvergenter Reihen.}

Schon in $\mathbf{8 9}, 2$ hatten wir den grundlegenden Unterschied zwischen absolut und nicht-absolut konvergenten Reihen hervorgehoben, der darin besteht, daß das Konvergenzverhalten der letzteren wesentlich von der Anordnung der Glieder der Reihe abhängt, daß also für diese Reihen das Kommutationsgesetz der Addition nicht gilt. Wir hatten dies dadurch bewiesen, daß wir zeigten, daß eine nicht-absolut konvergente Reihe lediglich durch Umordnung der Reihenfolge ihrer Glieder in eine divergente Reihe verwandelt werden kann. Dieser Satz läßt sich nun erheblich verfeinern. Es läßt sich nämlich genauer zeigen, daß sich durch eine passende Umordnung jedes nur denkbare vorgesshriebene Konvergenzverhalten bei der Reihe hervorrufen läßt. Es gilt also der folgende

187. Riemannsche Umordnungssatz. Ist $\Sigma a_{n}$ eine nur bedingt konvergente Reihe und ist $\Sigma a_{n}^{\prime}$ aus ihr durch Umordnung (s. 27, 3) entstanden, so kann man diese Umordnung so einrichten, daß die Reihe $\sum a_{n}^{\prime}$

a) mit einer willkürlich vorgeschriebenen Summe $s^{\prime}$ konvergiert $\left.^{1}\right)$;

b) nach $+\infty$ oder nach $-\infty$ divergiert;

c) beliebig vorgeschriebene Häufungsgrenzen $x$ und $\mu$ ihrer Teilsummen aufweist, - wosern nur $x \leqq \mu$ is:.

Beweis. Da die Behauptung c) offenbar die Behauptungen a) und b) als Spezialfälle enthält, nämlich a) für $x=\mu=s^{\prime}$ und b) für $\varkappa=\mu=+\infty$ bzw. $=-\infty$, so genügt es, c) zu beweisen.

Dazu wähle man irgendeine Zahlenfolge $\left(\varkappa_{n}\right)$, die $\rightarrow \varkappa$, und irgendeine Zahlenfolge $\left(\mu_{n}\right)$, die $\rightarrow, \mu$ strebt, für die jedoch stets $x_{n}<\mu_{n}$ und $\mu_{1}>0$ sein soll $^{2}$ ).

1) B. Riemann, Abh. d. Ges. z. Göttingen, Bd. 13 (1866/68), S. 97. - Die Behauptungen b) und c) sind naheliegende Ergänzungen.

2) Das ist offenbar in der mannigfachsten Weise möglich. Ist nämlich $\varkappa=\mu$ und der gemeinsame Wert endlich $\left(=s^{\prime}\right)$, so setze man $\varkappa_{n}=s^{\prime}-\frac{1}{n}$ und $\mu_{n}=s^{\prime}+\frac{1}{n}, \mu_{1}$ nötigenfalls noch größer; ist $\varkappa=\mu=+\infty(\mathrm{bzw} .=-\infty)$, so setze man $\varkappa_{n}=n$ (bzw. $=-n$ ) und $\mu_{n}=\varkappa_{n}+2$. Ist endlich $x<\mu$, so wähle man $\left(\varkappa_{n}\right)$ und $\left(\mu_{n}\right)$ ganz beliebig $\rightarrow \varkappa$ bzw. $\rightarrow \mu$ konvergierend; dann ist von einer Stelle an auch stets $\varkappa_{n}<\mu_{n}$, und man kann durch endlich viele Änderungen erreichen, dafis das von Anfang an der Fall und daf $\mu_{1}>0$ ist. 
In der Reihe $\Sigma a_{n} \equiv a_{1}+a_{2}+\ldots$ bezeichne man nun (etwas abweichend von 186, 2) diejenigen Glieder $a_{n}$, welche $\geqq 0$ sind in der Reihenfolge, wie sie auftreten, mit $p_{1}, p_{2}, \ldots$ und die Beträge derjenigen, die $<0$ sind, mit $q_{1}, q_{2}, \ldots$ Dann unterscheiden sich $\Sigma p_{n}$ und $\Sigma q_{n}$ von den damals ebenso bezeichneten Reihen nur durch das Fehlen vieler Nullen als Gliedern und es sind also wieder zwei divergente Reihen mit positiven Gliedern, welch letztere gegen 0 streben. Wir werden nun zeigen, daß schon eine Reihe vom Typus

$$
\begin{gathered}
p_{1}+p_{2}+\ldots+p_{m_{1}}-q_{1}-q_{2}-\ldots-q_{k_{1}}+p_{m_{1}+1}+\ldots+p_{m_{2}} \\
-q_{k_{1}+1}-\ldots-q_{k_{2}}+p_{m_{2}+1}+\ldots
\end{gathered}
$$

das Verlangte leistet. Eine solche Reihe ist offenbar eine Umordnung der gegebenen, und sogar eine solche, die die Reihenfolge der positiven Glieder unter sich und die der negativen unter sich ungeändert gelassen hat.

Wir wählen nun die beiden wachsenden Folgen der Indizes $m_{1}, m_{2}, \ldots$ und $k_{1}, k_{2}, \ldots$ so, daß diejenige Teilsumme der Reihe, die

1) mit $p_{m_{1}}$ abbricht, $>\mu_{1}$ ist, diejenige aber, die ein Glied früher abbricht, $\leqq \mu_{1}$ ist,

2) mit $q_{k_{1}}$ abbricht $<\varkappa_{1}$ ist, diejenige aber, die ein Glied früher abbricht, $\geqq \varkappa_{1}$ ist,

3) mit $p_{m_{2}}$ abbricht, $>\mu_{\mathrm{2}}$ ist, diejenige aber, die ein Glied früher abbricht, $\leqq \mu_{\mathrm{a}}$ ist,

4) mit $q_{k_{2}}$ abbricht, $<\varkappa_{2}$ ist, diejenige aber, die ein Glied früher abbricht, $\geqq \varkappa_{2}$ ist, usw.

Das kann stets erreicht werden; denn indem man hinreichend viele positive Glieder auftreten läßt, kann man die Teilsummen so groß machen, wie man will, und indem man hinreichend viele negative Glieder folgen läßt, kann man sie wieder so tief sinken lassen wie man will.

Ist nun $\Sigma a_{n}^{\prime}$ diese wohlbestimmte Umordnung von $\Sigma a_{n}$, so haben die Teilsummen dieser Reihe $\Sigma a_{n}^{\prime}$ die vorgeschriebenen Häufungsgrenzen. In der Tat, bezeichnen wir der Kürze halber die mit den Gliedern $p_{m_{1}}, p_{m_{2}}, \ldots$ abbrechenden Teilsummen mit $\tau_{1}, \tau_{2}, \ldots$, die mit $q_{k_{1}}, q_{k_{2}}, \ldots$ abbrechenden mit $\sigma_{1}, \sigma_{2}, \ldots$, so ist stets

$$
\left|\sigma_{v}-\varkappa_{\nu}\right|<q_{k,} \text { und } \mid \tau_{r}-\mu_{v},<p_{m_{v}} \text {. }
$$

Wegen $p_{n}>0$ und $q_{n}>0$ muß hiernach also $\sigma_{v}>\varkappa$ und $\tau_{n} \rightarrow \iota$ streben, d. h. $\varkappa$ und $\mu$ sind jedenfalls Häufungswerte der Teilsummen von $\sum a_{n}^{\prime}$. Daß sie nun auch deren Häufungsgrenzen darstellen, folgt einfach daraus, daß eine Teilsumme $s_{n}^{\prime}$ von $\Sigma a_{n}^{\prime}$, die weder einem $\sigma_{v}$ noch einem $\tau_{v}$ gleich ist, immer zwischen zwei aufeinanderfolgenden dieser besonderen Teilsummen gelegen ist. Die $s_{n}^{\prime}$ können also 
außerhalb des Intervalles $\varkappa \ldots \mu$ (bzw. außer dem gemeinsamen Wert beider) keine Häufungsstelle mehr besitzen.

Dieser Satz hat ähnliche Untersuchungen in mannigfachen Richtungen angeregt. Schon $M . \mathrm{Ohm}^{1}$ ) und $O$. Schlömilch ${ }^{2}$ ) haben die Wirkung verschiedener Umordnungen auf die spezielle Reihe $1-\frac{1}{2}+\frac{1}{3}-\frac{1}{4}+\ldots$ untersucht, insbesondere den Fall, dafs man auf je $p$ positive Glieder je $q$ negative folgen läßst. Aber erst $A$. Pringsheim ${ }^{3}$ ) hat allgemeinere Resultate für den Fall erzielt, daf3 in einer bedingt konvergenten Reihe die velative Häufigkeit der positiven und negativen Glieder nach bestimmten Vorschriften geändert wird. E. Borel ${ }^{4}$ ) umgekehrt hat die Frage untersucht, durch welche Umordnungen die Summe einer bedingt konvergenten Reihe nicht geändert wird. In neuerer Zeit hat $W$. Sierpinski ${ }^{6}$ ) gezeigt, daf , wenn $\Sigma a_{n}=s$ bedingt konvergiert und $s^{\prime}<\dot{s}$ ist, man durch alleinige Umordnurg der positiven Glieder, unter Belassung der negativen Glieder an ihrem Platze und in ihrer Anordnung, der Reihe die Summe $s^{\prime}$ geben kann, und analog jede Summe $s^{\prime \prime}>s$ durch alleinige Umordnung der negativen Glieder. (Der Beweis ist weniger einfach.)

\section{$\S$ 45. Multiplikation nur bedingt konvergenter Reihen.}

Im vorigen Paragraphen zeigten wir, in Ergänzung der Ausführungen von $\mathbf{8 9}, 2$, daß bei Reihen, die nur bedingt konvergieren, das Kommutationsgesetz der Addition nicht mehr gilt. Daß auch das Distributionsgesetz nicht allgemein weiterbesteht und daß also die Multiplikation zweier solcher Reihen $\Sigma a_{n}$ und $\Sigma b_{n}$ nicht mehr nach den elementaren Regeln ausgeführt werden darf, hatten wir gleichfalls schon ( $\$ 17$, Ende) an einem von Cauchy herrührenden Beispiel gesehen. Doch blieb dort die Frage noch unerledigt, ob die Produktreihe $\Sigma c_{n}$ (mit $\left.c_{n}=a_{0} b_{n}+a_{1} b_{n-1}+\ldots+a_{n} b_{0}\right)$ nicht schon bei weniger strengen Voraussetzungen über die Reihen $\Sigma a_{n}=A$ und $\Sigma b_{n}=B-$ wir verlangten in $\S 17$, da $\beta$ sie beide absolut konvergent seien - konvergieren und den Wert $A \cdot B$ zur Summe haben muß.

In dieser Richtung gilt zunächst der folgende

188. ${ }^{\circ}$ Satz von Mertens $\left.{ }^{6}\right)$. Wenn von den beiden Reihen $\Sigma a_{n}=A$ und $\Sigma b_{n}=B$ wenigstens eine - etwa die erste - absolut konvergiert, so ist $\Sigma c_{n}$ schon konvergent und $=A \cdot B$.

Beweis. Es ist nur zu zeigen, daß mit wachsendem $n$ die Teilsummen

$$
\begin{aligned}
C_{n} & =c_{0}+c_{1}+\ldots+c_{n} \\
& =a_{0} b_{0}+\left(a_{0} b_{1}+a_{1} b_{0}\right)+\ldots+\left(a_{0} b_{n}+a_{1} b_{n-1}+\ldots+a_{n} b_{0}\right)
\end{aligned}
$$

1) Antrittsprogramm Berlin, 1839.

${ }^{2}$ Zeitschr. f. Math. u. Phys., Bd. 18 (1873), S. 520.

3) Math. Ann., Bd. 23 (1883), S. 455

4) Bulletin des sciences mathém., Bd. (2) 14 (1890), S. 97.

$\left.{ }^{5}\right)$ Bull. internat. Ac. Sciences Cracovie, 1911, S. 149.

$\left.{ }^{6}\right)$ J. f. d. reine u. angew. Math., Bd. 79 (1875), S. 182. - Eine Erweiterung des Satzes gab T. J. Stieltjes (Nouv. Annales, Bd. (3) 6 (1887), S. 210.) 
gegen den Grenzwert $A \cdot B$ streben. Bezeichnen wir nun die Teilsummen von $\Sigma a_{n}$ mit $A_{n}$, die von $\Sigma b_{n}$ mit $B_{n}$, so ist zunächst

$$
C_{n}=a_{0} \cdot B_{n}+a_{1} B_{n-1}+\ldots+a_{n} B_{0}
$$

oder, wenn man $B_{n}=B+\beta_{n}$ setzt,

$$
=A_{n} \cdot B+\left(a_{0} \cdot \beta_{n}+a_{1} \beta_{n-1}+\ldots+a_{n} \beta_{0}\right) \text {. }
$$

Da hier $A_{n} \cdot B \rightarrow A \cdot B$ strebt, so ist einzig noch dies zu zeigen: Wenn $\sum a_{n}$ absolut konvergiert und $\beta_{n} \rightarrow 0$ strebt, so bildet auch der Ausdruck

$$
\omega_{n}=a_{n} \beta_{0}+a_{n-1} \beta_{1}+\ldots+a_{0} \beta_{n}
$$

eine Nullfolge. Das ist aber eine unmittelbare Folge von 43, $9^{\text {b }}$; man hat dort nur $x_{n}=\beta_{n}$ und $y_{n}=a_{n}$ zu setzen. Damit ist der Satz bewiesen.

Ehe wir weiter gehen, wollen wir die Frage beantworten, ob denn die Produktreihe $\Sigma c_{n}$, wenn sie konvergitrt, noiwendig den $W$ crt $A \cdot B$ zur Summe haben muß?

$\mathrm{Da} B$ diese Frage zu bejahen ist, besagt der folgende

- Satz von Abel $\left.^{1}\right)$. Wenn die Reihe $\Sigma c_{n}=\Sigma\left(a_{0} b_{n}+\ldots+a_{n} b_{0}\right) 189$. konvergiert, so ist ihre Summe stets $=A \cdot B$.

1. Beweis. Dieser Satz folgt ganz unmittelbar aus dem Abelschen Grenzwertsatz (100) und ist auch so zuerst von $A b e l$ bewiesen worden. Setzt man

$$
\sum a_{n} x^{n}=f_{1}(x), \quad \sum b_{n} x^{n}=f_{2}(x), \quad \sum c_{n} x^{n}=f_{3}(x),
$$

so sind diese drei Potenzreihen (vgl. 185, 4) sicher für $0 \leqq x<1$ absolut konvergent und für die dadurch definierten Funktionen gilt für diese Werte von $x$ die Beziehung

$$
f_{3}(x)=f_{1}(x) \cdot f_{2}(x) \text {. }
$$

Wegen der vorausgesetzten Konvergenz der drei Reihen $\Sigma a_{n}, \Sigma b_{n}$ und $\Sigma c_{n}$ strebt aber eine jede dieser Funktionen nach dem $A$ kelschen Grenzwertsatz einem Grenzwert zu, wenn $x$ von links her sich $\rightarrow+1$ bewegt; und zwar strebt dabei

$$
f_{1}(x) \rightarrow A=\Sigma a_{n}, \quad f_{2}(x) \rightarrow B=\Sigma b_{n}, \quad f_{3}(x) \rightarrow C=\Sigma c_{n} .
$$

Da nun für alle bei diesem Grenzübergang in Betracht kommenden Werte von $x$ die Gleichung (a) besteht, so folgt (nach $\S 19$, Satz 1) sofort, da $B$

$$
C=A \cdot B
$$

sein muß. - Ohne Verwendung von Funktionen gelangt der folgende

2. Beweis zum Ziel, der von Cesàro $^{2}$ ) herrührt: Schon oben sahen wir, daß

$$
C_{v}=a_{0} B_{v}+a_{1} B_{v-1}+\ldots+a_{v} B_{0}
$$

1) J. f. d. reine u. angew. Math., Bd. 1 (1826), S. 318.

2) Bull. des sciences math., Bd. (2) 14 (1890), S. 114. 
ist. Hieraus folgt, daß

$$
C_{0}+C_{1}+\ldots+C_{n}=A_{0} B_{n}+A_{1} B_{n-1}+\ldots+A_{n} B_{0}
$$

ist. Dividiert man beide Seiten dieser Gleichung durch $n+1$ und läßt $n \rightarrow+\infty$ streben, so strebt dabei (nach $\mathbf{4 3}, 2$ ) die linke Seite $\rightarrow C$, und (nach 44,9a) die rechte $\rightarrow A \cdot B$. Also ist $C=A \cdot B$, w. z. b. w.

Auf Grund dieses interessanten Satzes, der uns auch späterhin noch beschäftigen wird, brauchen sich die feineren Untersuchungen über die Multiplikation von Reihen nur noch mit der Frage zu beschäftigen, $o b$ die Produktreihe $\Sigma_{v_{n}}$ konvergiert oder nicht.

Auf diese Untersuchungen wollen wir hier indessen nicht eingehen $^{2}$ ).

\section{Aufgaben zum X. Kapitel.}

142. Man stelle das Konvergenzverhalten der folgenden Reihen fest:
a) $\sum_{n=1}^{\infty} \frac{(-1)^{[1 n]}}{n}$,
b) $\sum_{n=1}^{\infty} \frac{(-1)^{[\sqrt{n}]}}{n^{x}}$,
c) $\perp x\left(\frac{\sin n x}{n x}\right)^{2}$.
d) $\quad \leq \sin \frac{x}{n}$,
e) $\sum(-1)^{n} \sin \frac{x}{n}$,
f) $\leq \sin ^{2} \frac{x}{n}$,
g) $\Sigma \sin \left(n^{2} x\right)$,
i) $\quad \leq \frac{(-1)^{n}}{x+\log n}$,
h) $\Sigma \sin (n ! \pi x)$,
k) $\perp \frac{\sin ^{2} n x}{n}$,
1) $\leq\left(1+\frac{1}{2}+\ldots+\frac{1}{n}\right) \frac{\sin n x}{n}$,
m) $\Sigma \alpha_{n} \sin n x \cos ^{2} n x$.

Bei der letzten Reihe soll $\left(c_{n}\right)$ eine monotone Nullfolge sein. Die Reihe g) konvergiert fuir kein $x \neq k \pi$, die Reihe $\mathrm{h}$ ) konvergiert aufer fuir alle rationalen $x$ noch $z$. B. für $x=e,=(2 k+1) e,=\frac{2 k}{e},=\sin 1,=\cos 1$, ferner für

$$
x=\frac{1}{2} \frac{1}{4 !}-\frac{1}{5 !}+\frac{1}{2} \frac{1}{6 !}-\frac{1}{7 !}+\frac{1}{2} \frac{1}{8 !}-+\ldots
$$

und viele andere spezielle Werte von $x$. Man gebe Wérte von $x$ an, für die die Reihe sicher divergiert.

143.

$$
\sum_{n=1}^{\infty}\left[\frac{1}{x+2 n-1}+\frac{1}{x+2 n}-\frac{1}{x+n}\right]=\log 2
$$

und zwar für jedes $x>0$.

144. Wenn die Folge $\left(n a_{n}\right)$ und die Reihe $2 \sim n\left(a_{n}-a_{n+1}\right)$ konvergieren, so ist auch $\Sigma a_{n}$ konvergent.

2) Sätze der hier in Rede stehenden Art hat A. Pringsheim (Math. Ann. Bd. 21, 1883, S. 340) bewiesen und im Anschluf an dessen Arbeiten $A$. Vo $\beta$ (ebenda, Bd. 24, 1884, S. 42) und F. Cajori (Bull. of the Americ. Math. Soc., Bd. 8, 1901/2, S. 231 und Bd. 9, 1902/3, S. 188). - Vgl. auch $\$ 66$ des schon oft zitierten Werkes von A. Pringsheim, Vorlesungen über Zahlen- und Funktionenlehre (Leipzig 1916). Wesentlich tiefer liegt eine Gruppe hierher gehöriger Sätze, von denen G. H. Hardy (Proc. of the London Math. Soc. Bd. (2) 6, 1908 , S. 410) einen besonders schönen bewiesen hat. 
145. a) Wenn $\Sigma a_{n}$ und $\Sigma\left|b_{n}-b_{n+1}\right|$ beide konvergieren oder wenn b) $\sum a_{n}$ beschränkte Teilsummen hat, $\Sigma\left|b_{n}-b_{n+1}\right|$ konvergiert und $b_{n} \rightarrow 0$ strebt, so ist für jedes ganzzahlige $p \geq 1$ die Reihe $\sum a_{n} b_{n}{ }^{p}$ konvergent.

146. Die Bedingungen des Kriteriums 184, 3 sind in gewissem Sinne auch notwendig für die Konvergenz von $\Sigma a_{n} b_{n}$ : Soll die Faktorenfolge $\left(b_{n}\right)$ so beschaffen sein, daf für jede konvergente Reihe $\Sigma a_{n}$ auch $\Sigma a_{n} b_{n}$ konvergent ausfällt, so ist dazu notwendig und hinreichend, daf3 $\Sigma\left|b_{n}-b_{n+1}\right|$ konvergiert. - Man zeige noch, daß 3 es hierbei keinen wesentlichen Unterschied macht, ob man die Konvergenz von $\Sigma\left|b_{n}-b_{n+1}\right|$ verlangt oder nur fordert, daf die Folge $\left(b_{n}\right)$ monoton sei.

147. Ist $\Sigma a_{n}$ konvergent, und strebt $p_{n}$ monoton $\rightarrow+\infty$, doch so, daf $\Sigma p_{n}{ }^{-1}$ divergiert, so ist

$$
\varlimsup_{\lim } \frac{p_{1} a_{1}+p_{2} a_{2}+\ldots+p_{n} a_{n}}{n}\left\{\begin{array}{l}
\geqq 0 \\
\leq 0 .
\end{array}\right.
$$

148. Es strebe $a_{n}$ monoton $\rightarrow 0, \lim n a_{n}$ sei vorhanden, und es sei $\sum_{n=0}^{\infty}(-1)^{n} a_{n}=s$. Ordnet man diese Reihe so um, daß̧ stets auf $p$ positive Glieder $q$ negative folgen:

$$
a_{0}+a_{2}+\ldots+a_{2 p-2}-a_{1}-a_{3}-\ldots-a_{2 q-1}+a_{2 p}+\ldots
$$

so gilt für die Summe $s^{\prime}$ dieser neuen Reihe

$$
s^{\prime}=\dot{s}+\frac{1}{2} \lim \left(n a_{n}\right) \cdot \log \frac{p}{q} .
$$

149. Die notwendige und hinreichende Bedingung dafür, daf aus der Konvergen $z$ der Reihen $\Sigma a_{n}$ und $\Sigma b_{n}$ die der Produktreihe

$$
\Sigma c_{n}=\Sigma\left(a_{0} b_{n}+a_{1} b_{n-1}+\ldots+a_{n} b_{0}\right)
$$

folge, besteht darin, daf die Zahlen

$$
\varrho_{n}=\sum_{v=1}^{n} a_{v}\left(b_{n}+b_{n-1}+\ldots+b_{n-v+1}\right)
$$

eine Nullfolge bilden.

150. Streben die Folgen $\left(a_{n}\right)$ und $\left(b_{n}\right)$ monoton $\rightarrow 0$, so liefern die Reihen $\Sigma(-1)^{n} a_{n}$ und $\Sigma(-1)^{n} b_{n}$ dann und nur dann eine konvergente (Cauchysche) Produktreihe, wenn auch die Zahlen $\sigma_{n}=a_{n}\left(b_{0}+b_{1}+\ldots+b_{n}\right)$ und $\tau_{n}=b_{n}\left(a_{0}+a_{1}+\ldots+a_{n}\right)$ je eine Nullfolge bilden.

151. Die beiden Reihen $\Sigma \frac{(-1)^{n}}{n^{\alpha}}$ und $\Sigma \frac{(-1)^{n}}{n^{\beta}}, \alpha>0, \beta>0$, dürfen dann und nur dann nach der Cauchyschen Regel miteinander multipliziert werden, wenn $\alpha+\beta>1$ ist.

152. Sind $\left(a_{n}\right)$ und $\left(b_{n}\right)$ monotone Nullfolgen, so ist es für die Konvergenz des Cauchyschen Produktes der Reihen $\Sigma(-1)^{n} a_{n}$ und $\Sigma(-1)^{n} b_{n}$ hinreichend, daf $\Sigma a_{n} b_{n}$ konvergiert; und die notwendige und hinreichende Bedingung dafür besteht darin, daf $\Sigma\left(a_{n} b_{n}\right)^{1+\varrho}$ für jedes $\varrho>0$ konvergiert.

153. Ist von einer Stelle an

$$
\begin{aligned}
& a_{n}=n^{\alpha_{0}} \cdot \log ^{\alpha_{1}} n \cdot \log _{2}^{\alpha_{2}} n \ldots \log _{r}^{\alpha_{r}} n, \\
& b_{n}=n^{\beta_{0}} \cdot \log ^{\beta_{1}} n \cdot \log _{2}^{\beta_{2}} n \ldots \log _{s}^{\beta_{s}} n,
\end{aligned}
$$

ist $\Sigma b_{n}$ konvergent, aber $a_{n} \equiv \equiv b_{n}$, so ist

$$
\left(a_{0} b_{n}+a_{1} b_{n-1}+\ldots+a_{n} b_{0}\right) \simeq a_{n} \cdot\left(\sum_{v=0}^{\infty} b_{v}\right) .
$$


XI. Kapitel.

\section{Reihen mit veränderlichen Gliedern (Funktionenfolgen).}

\section{§ 46. Gleichmäßige Konvergenz.}

Bisher haben wir fast ausschließlich Reihen in den Kreis unserer Betrachtungen gezogen, deren Glieder bestimmt gegebene Zahlen (Konstante) waren. Nur in besonders durchsichtigen Fällen hing der Wert der Glieder noch von der Festlegung einer unbestimmten Größe, einer Variablen, ab. Dieser Fall lag z. B. vor, wenn wir die geometrische Reihe $\Sigma a^{n}$ oder die harmonische Reihe $\Sigma \frac{1}{n^{\alpha}}$ betrachteten, deren Konvergenzverhalten noch von der Wahl von $a$ bzw. $a$ abhängig war, - und er lag allgemeiner bei jeder Potenzreihe $\Sigma a_{n} x^{n}$ vor, bei der erst noch die Zahl $x$ fixiert werden mußte, ehe wir die Konvergenzfrage in Angriff nehmen konnten. Diese Situation soll jetzt in naheliegender Weise verallgemeinert werden: Wir wollen Reihen betrachten, deren Glieder in irgendeiner Weise von einer Variablen $x$ abhängen, die also Funktionen dieser Variablen sind. Wir bezeichnen diese Glieder darum mit $f_{n}(x)$ und betrachten also Reihen der Form

$$
\Sigma f_{n}(x) \text {. }
$$

Eine Funktion von $x$ ist nun im allgemeinen nur für gewisse Werte von $x$ definiert (s. $§ 19$, Def. 1) für unsere $Z$ wecke wird die Annahme genügen, daß sie in einem oder mehreren (offenen oder abgeschlossenen) Intervallen definiert sind. Dann wäre - wenn anders die hingeschriebene Reihe auch nur für einen einzigen Wert von $x$ einen Sinn haben soll - zunächst zu fordern, daß es wenigstens einen Punkt $x$ gibt, der den Definitionsintervallen sämtlicher Funktionen $f_{n}(x)$ angehört. Wir wollen aber sogleich fordern, daß es wenigstens ein Intervall gebe, in dem alle diese Funktionen definiert sind. Dann sind für jedes spezielle $x$ aus diesem Intervall jedenfalls die Glieder der Reihe $\Sigma f_{n}(x)$ bestimmte Zahlen, und es kann für jedes solche $x$ die Konvergenzfrage aufgeworfen werden. Wir wollen nun weiter annehmen, daß es ein (ev. kleineres) Intervall $J$ gäbe, für dessen sämtliche Punkte die Reihe $\Sigma f_{n}(x)$ sich nun als konvergent erweist.

190. Definition. Ist $J$ ein Intervall, für dessen sämtliche Punkte (mit Einschluß keines, eines oder beid̄r Enäpunkte) alle Furktionen $f_{n}(x)$ definiert sind und zugleich die Reike $\Sigma f_{n}(x)$ konvergitrt, so soll $J$ ein Konvergenzintervall dieser Reihe genannt werden. 
Beispiele und Erläuterungen.

1. Für die geometrische Reihe $\Sigma x^{n}$ ist das Intervall $-1<x<+1$ ein Konvergenzintervall, und es existiert kein außerhalb desselben gelegenes.

2. Für eine Potenzreihe $\Sigma a_{n}\left(x-x_{0}\right)^{n}$ existiert, wofern sie auch nur in einem einzigen von $x_{0}$ verschiedenen Punkte konvergiert, stets ein Konvergenzintervall der Form $x_{0}-r \ldots x_{0}+r$, mit Einschluf keines, eines oder beider Endpunkte. Und es existiert (bei richtiger Wahl von $r$ ) kein auferhalb desselben gelegenes Konvergenzintervall.

3. Für die harmonische Reihe $\sum \frac{1}{n^{x}}$ ist die Halbachse $x>1$ ein Konvergenzintervall und es existiert kein auferhalb derselben gelegenes.

4. Genau wie jede Reihe lediglich ein anderes Symbol für eine gewisse Folge war, so ist auch jetzt die Reihe $\Sigma f_{n}(x)$ lediglich ein anderes Symbol für eine Folge von Funktionen, nämlich für die Folge der Teilsummen

$$
s_{n}(x)=f_{0}(x)+f_{1}(x)+\ldots+f_{n}(x) \text {. }
$$

Es ist daher prinzipiell gleichgültig, ob man die Glieder der Reihe oder die Teilsummen derselben angibt; die einen sind durch die andern eindeutig bestimmt. Es ist also auch prinzipiell das gleiche, mag man von unendlichen Reihen mit veränderlichen Gliedern oder von Funktionenfolgen sprechen. Wir werden darum alle Definitionen und Sätze nur für die Reihen aussprechen und es dom Leser überlassen, sie sich auch für Funktionenfolgen zu formulieren ${ }^{1}$ ).

5. Ist für eine Reihe

$$
s_{n}(x)=\frac{\left(x^{2}\right)^{n}}{1+\left(x^{2}\right)^{n}}, \quad(n=1,2,3, \ldots),
$$

lautet also die Reihe

$$
\sum_{n=1}^{\infty} f_{n}(x)=\frac{x^{2}}{1+x^{2}}+\left(\frac{x^{4}}{1+x^{4}}-\frac{x^{2}}{1+x^{2}}\right)+\left(\frac{x^{6}}{1+x^{6}}-\frac{x^{4}}{1+x^{4}}\right)+\ldots
$$

so konvergiert die Reihe für jedes reelle $x$; denn es strebt ersichtlich

a) $s_{n}(x) \rightarrow 0$, falls $|x|<1$ ist,

b) $s_{n}(x) \rightarrow 1$, falls $|x|>1$ ist, und

c) $s_{n}(x) \rightarrow \frac{1}{2}$, falls $|x|=1$ ist.

6. Dagegen hat die Reihe, für die

$$
s_{n}(x)=(2 \sin x)^{n}
$$

ist, unendlich viele getrennte Konvergenzintervalle; denn $\lim s_{n}(x)$ ist offenbar dann und nur dann vorhanden, wenn $-\frac{1}{2}<\sin x \leqq \frac{1}{2}$ ist, also wenn

$$
-\frac{\pi}{6}<x \leqq \frac{\pi}{6} \quad \text { oder } \quad \frac{5 \pi}{6} \leqq x<\frac{7 \pi}{6}
$$

ist, oder wenn $x$ in einem der Intervalle liegt, die hieraus durch Verschiebung um ein ganzzahliges Vielfaches von $2 \pi$ entstehen. Die Summe der Reihe ist dann im Innern dieser Intervalle stets $=0$, und in dem einen $z u$ ihm gehörigen Endpunkt $=1$.

7. Die Reihe $\sin x+\frac{\sin 2 x}{2}+\frac{\sin 3 x}{3}+\ldots$ konvergiert nach 185, 5 für jedes reelle $x$; die Reihe $\cos x+\frac{\cos 2 x}{2}+\frac{\cos 3 x}{3}+\ldots$ für jedes von $2 k \pi$ verschiedene reelle $x$.

1) Trotzdem werden wir gelegentlich die Definitionen und Sätze auch ufür Funktionenfolgen benutzen. 
Ist nun eine vorgelegte Reihe der Form $\Sigma f_{n}(x)$ in einem bestimmten Intervall $J$ konvergent, so entspricht jedem Punkt $x$ von $J$ ein ganz bestimmter Summenwert der Reihe. Diese Summe ist dann also (gemäß $\S 19$, Def. 1) selbst eine Funktion von $x$, welche durch die Reihe definiert oder dargestellt wird. Man sagt auch, wenn man diese letztere Funktion als das wesentlichste ansieht, sie sei in die betreffende Reihe entwickelt. Wir schreiben in diesem Sinne

$$
F(x)=\sum_{n=0}^{\infty} f_{n}(x) \text {. }
$$

Für Potenzreihen und die durch sie dargestellten Funktionen (s. V. und VI. Kapitel) sind uns diese Begriffe ja schon geläufig.

Die wichtigste Frage, die wir in solchen Fällen zu beantworten haben, wird dann meist die sein, ob bzw. in welchem Maße sich die Eigenschaften, die allen als Reihenglieder auftretenden Funktionen $f_{n}(x)$ zukommen, sich auch auf die durch die Reihensumme definierte Funktion' $F(x)$ übertragen.

Schon die einfachen vorhin gegebenen Beispiele zeigen, daß dies für keine der bei den Funktionen als wertvoll geschätzten Eigenschaften der Fall zu sein braucht: Die geometrische Reihe zeigt, daß alle $f_{n}(x)$ beschränkt sein können, ohne daß $F(x)$ es ist; die Potenzreihe für $\sin x, x>0$, zeigt, da $\beta$ jedes $f_{n}(x)$ monoton sein kann, ohne daß $F(x)$ monoton ausfällt; das Beispiel 5. zeigt, daß jedes $f_{n}(x)$ stetig sein kann, ohne daß $F(x)$ es ist. Dasselbe Beispiel lehrt das entsprechende für die Differenzierbarkeit. Auch dafür, daß die Integrierbarkeit verloren gehen kann, ist es nicht schwer, ein Beispiel zu bilden.

Es sei etwa $s_{n}(x) \begin{cases}=1 & \text { für alle rationalen } x \text {, die mit einem (positiven) Nenner } \leqq n \text { ge- } \\ & \text { geschrieben werden können, } \\ =0 & \text { für alle andern } x .\end{cases}$ Dann ist $s_{n}(x)$ und folglich auch $f_{n}(x)$ über jedes beschränkte Intervall integrierbar, weil es dort nur endlich viele Unstetigkeiten aufweist (vgl. § 19, Satz 13). Auch ist $\lim s_{n}(x)=F(x)$ für jedes $x$ vorhanden. Ist nämlich $x$ rational, etwa $=\frac{p}{q}(q>0, p$ und $q$ teilerfremd $)$, so ist für $n>q$ stets $s_{n}(x)=1$ und also auch $F(x)=1$. Ist dagegen $x$ irrational, so ist stets $s_{n}(x)=0$ und also auch $F(x)=0$. Es ist somit die durch $\Sigma f_{n}(x)=\lim s_{n}(x)$ definierte Funktion

$$
F(x)= \begin{cases}1, & \text { falls } x \text { rational } \\ 0, & \text { falls } x \text { irrational }\end{cases}
$$

Diese Funktion ist aber nicht integrierbar, denn sie ist für jedes $x$ unstetig $^{1}$ ).

1) Definiert man $s_{n}(x)$ ein klein wenig abweichend so, daf $s_{n}(x)=1$ gesetzt wird für alle rationalen $x$, deren Nenner in $n$ ! aufgeht (es sind dies bei jedem $n$ aufier den eben gebrauchten natürlichen Zahlen $\leqq n$ noch eine bestimmte Anzahl weiterer), und $=0$ gesetzt wird für alle andern $x$, so erhält man als $\lim s_{n}(x)$ dieselbe Funktion $F(x)$, wie eben. Nun aber läfit sich $s_{n}(x)$, 
Diese Beispiele deuten schon an, daß bei den Reihen mit veränderlichen Gliedern nun eine ganz neue Kategorie von Problemen auftaucht und behandelt werden muß, nämlich zu untersuchen, unter welchen zusätzlichen Bedingungen nun doch diese oder jene Eigenschaft der Reihenglieder $f_{n}(x)$ sich auf die Reihensumme $F(x)$ überträgt. Nach den angeführten Beispielen ist es jedenfalls nicht die Tatsache der Konvergenz, die dies bewirkt, sondern der Grund muß in der Art der Konvergenz gesucht werden. Von der größten Bedeutung in dieser Hinsicht ist die sogenannte gleichmäßige Konvergenz einer Reihe $\Sigma f_{n}(x)$ in einem ihrer Konvergenzintervalle oder einem Teile derselben.

Der Begriff ist zwar leicht erklärt, aber sein tieferes Wesen nicht so schnell zu erfassen. Wir wollen uns daher, ehe wir zur begrifflichen Formulierung schreiten, den Sachverhalt zunächst einmal anschaulich klarmachen:

Wenn $\sum_{n=0}^{\infty} f_{n}(x)$ im Intervall $J$, etwa in $a \leqq x \leqq b$, konvergiert und $F(x)$ zur Summe hat, so wollen wir das Bild der Funktion $y=s_{n}(x)=f_{0}(x)+\ldots+f_{n}(x)$ die nte Approximationskurve und das Bild der Funktion $y=F(x)$ die Grenzkurve nennen. Die Tatsache der Konvergenz scheint dann zu besagen, dafo mit wachsendem $n$ sich die Approximationskurven mehr und mehr der Grenzkurve anschmiegen. Doch trifft dies den wahren Sachverhalt nur sehr unvollkommen. Denn die Konvergenz in $J$ bedeutet zunächst nur, daf in jedem einzelnen Punkte Konvergenz stattfindet; und wir können daher zunächst nur sagen, dafs bei jeder bestimmt ins Auge gefafiten (also festgehaltenen) Abszisse $x$ sich die zugehörigen Ordinaten der Approximationskurven mit wachsendem $n$ der an dieser Stelle stehenden Ordinate der Grenzkurve nähern. Was derweil die Kurven über einer andern Abszisse $x^{\prime}$ machen, steht noch völlig dahin: es braucht keineswegs die Approximationskurve $y=s_{n}(x)$ in ihrer ganzen Ausdehnung sich der Grenzkurve immer dichter anzuschmiegen. Diese etwas paradox klingende Behauptung werde zunächst an einem Beispiel erläutert.

Die Reihe, deren Teilsummen für $n=1,2, \ldots$ die Werte

$$
s_{n}(x)=\frac{n x}{1+n^{2} x^{2}}
$$

haben, ist gewif im Intervall $1 \leqq x \leqq 2$ konvergent. Denn es ist dort

$$
0<s_{n}(x)<\frac{n x}{n^{2} x^{2}} \leqq \frac{1}{n} \text {. }
$$

Die Grenzkurve ist also die Strecke $1 \leq x \leq 2$ der Abszissenachse; und die Approximationskurve, die oberhalb derselben verläuft, entfernt sich nach der

und $F(x)$ mit den üblichen Mitteln sogar durchaus geschlossen darstellen denn es ist $s_{n}(x)=\lim _{k \rightarrow \infty}\left(\cos ^{2} n ! \pi x\right)^{k}$ und also

$$
F(x)=\lim _{n \rightarrow \infty}\left[\lim _{k \rightarrow \infty}\left(\cos ^{2} n ! \pi x\right)^{k}\right] .
$$

Dieses merkwürdige Beispiel einer übevall unstetigen Funktion, die doch aus stetigen Funktionen mit Hilfe zweimaligen Grenzübergangs hergestellt werden kann, ruihrt schon von Dirichlet her. 
eben ausgeführten Abschätzung von ihr längs des ganzen Intervalles $1 \leq x \leq 2$ um weniger als $\frac{1}{n}$, also für grofe $n$ in ihrer ganzen Ausdehnung nur sehr wenig.

Hier lägen die Dinge also etwa so, wie man es erwarten mochte; ganz anders aber, wenn wir dieselbe Reihe im Intervall $0 \leq x \leq 1$ betrachten. Auch für jeden Punkt dieses Intervalles ist $\lim s_{n}(x)=0^{1}$ ), die Grenzkurve also das entsprechende Stück der $x$-Achse. Hier schmiegt sich aber für kcin (noch so großes) $n$ die Approximationskurve längs des ganzen Intervalles dicht an die Grenzkurve an, denn für $x=\frac{1}{n}$ ist stets $s_{n}(x)=\frac{1}{2}, \mathrm{~d}$. h. für jedes $n$ macht die Approximationskurve $z$ wischen 0 und 1 einen nach oben gehenden Buckel, der die Höhe ${ }_{2}^{1}$ hat!! Das Bild der Kurve $y=s_{4}(x)$ sieht ungefähr so aus:

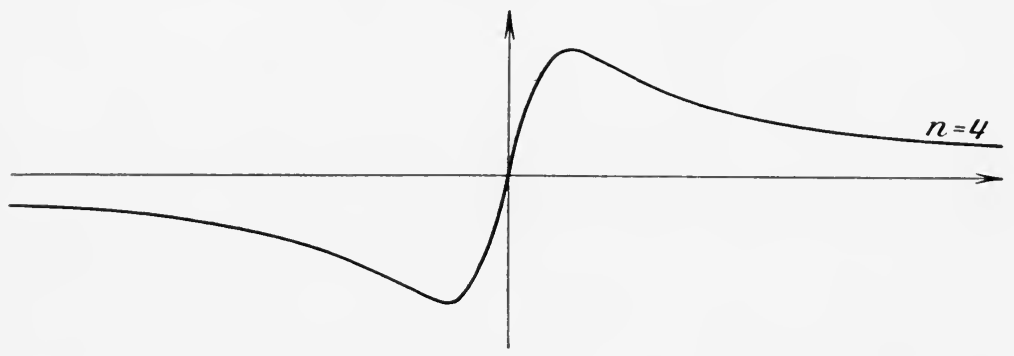

Fig. 4 .

Die Kurve $y=s_{40}(x)$ dagegen bietet schon etwa das folgende Bild:

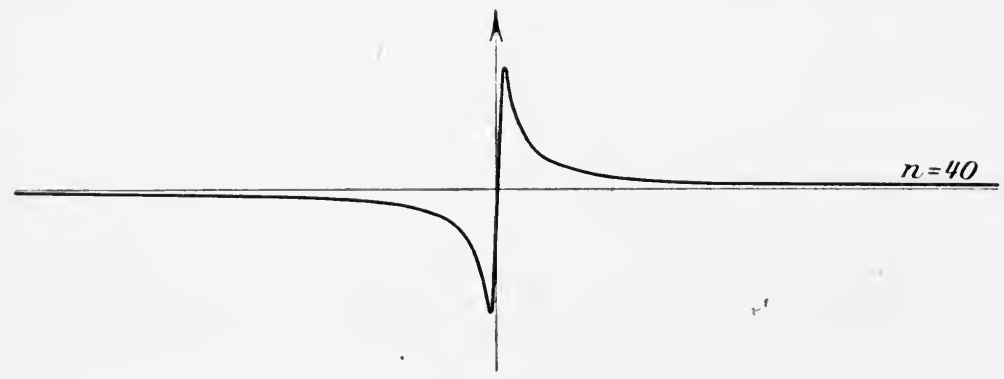

Fig. 5.

Und für die größseren $n$ wird der genannte Buckel - ohne dafs sich seine Höhe veränderte - immer dichter an die Ordinatenachse herangedrückt; die Approximationskurve schnellt vom Koordinatenanfangspunkt immer steiler herauf bis zur Höhe $\frac{1}{2}$, die schon für $x=\frac{1}{n}$ erreicht wird, um dann fast ebenso schnell wieder bis dicht an die Abszissenachse herabzufallen.

Der Anfänger, dem diese Erscheinung sehr befremdlich vorkommen wird, mache sich genau klar, daß trotzdem für jedes feste $x$ unseres Intervalles die Ordinaten der Approximationskurve sich schlieflich auf die Abszissenachse

1) Denn für $x>0$ ist wieder $0<s_{n}(x)=\frac{1}{n x}$, also $<\varepsilon$ für $n>\frac{1}{\varepsilon x}$; und für $x=0$ ist sogar dauernd $s_{n}(x)=0$. 
herabsenken, dafis also für jedes feste $x$ doch $\lim s_{n}(x)=0$ ist. Denn halten wir einen (wenn auch noch so kleinen) positiven Wert von $x$ fest, so wird für hinreichend hohe $n$ der störende Buckel der Kurve $y=s_{n}(x)$ doch ganz links von $x$ (aber noch rechts von der Ordinatenachse) liegen und an der Stelle $x$ selbst wird die Kurve sich schon wieder tief auf die Abszissenachse herabgesenkt haben ${ }^{1}$ ).

In dem Intervall $1 \leqq x \leqq 2$ werden wir die Konvergenz unserer Reihe eine gleichmäßige nennen, im Intervall $0 \leq x<1$ dagegen nicht.

Nachdem wir uns so mehr anschaulich mit dem Sachverhalt vertraut gemacht haben, schreiten wir zur begrifflichen Formulierung: Besitzt $\Sigma f_{n}(x)$ das Konvergenzintervall $J$, so ist die Reihe also für jedes einzelne $x$ desselben, etwa für $x=x_{0}$ konvergent, $\mathrm{d}$. h. wenn allgemein $F(x)=s_{n}(x)+r_{n}(x)$ gesetzt wird und $\varepsilon>0$ beliebig gegeben wird, so gibt es eine Zahl $n_{0}$ mit der Eigenschaft, daß nun für alle $n>n_{0}$ der Betrag des Restes

$$
\left|r_{n}\left(x_{0}\right)\right|<\varepsilon
$$

ausfällt. Wie schon früher betont (s. 10, Bem. 3) hängt $n_{\mathbf{0}}$ natürlich von der Wahl von $\varepsilon$ ab. Jetzt wird aber $n_{\mathbf{0}}$ auch noch von der Wahl von $x_{0}$ abhängen, denn für den einen Punkt aus $J$ wird die Reihe im allgemeinen schneller konvergieren als für den andern ${ }^{2}$ ). Wir werden daher, ähnlich wie in $\mathbf{1 0}, 3, n_{0}=n_{\mathbf{0}}\left(\varepsilon, x_{\mathbf{0}}\right)$ setzen, oder auch, indem wir uns den Index 0 sparen und die Abhängigkeit von $\varepsilon$ als selbstverständlich nicht mehr besonders betonen, sagen können: Bei gegebenen $\varepsilon>0$ und gegebenem $x$ aus $J$ läßt sich stets eine Zahl $n(x)$ so angeben, daß nun für alle $n>n(x)$ stets

$$
\left|r_{n}(x)\right|<\varepsilon
$$

ausfällt. Denkt man sich — immer bei einem bestimmt gegebenen $\varepsilon$ - die Zahl $n(x)$ hierbei - etwa als ganze Zahl - so klein wie möglich gewählt, so ist sie durch $x$ eindeutig bestimmt und ist also insofern eine Funktion von $x$. Ihr Wert kann in gewissem Sinne als ein Maß für die Güte der Konvergenz der Reihe an der Stelle $x$ angesehen werden. Wir definieren nun:

Definition der gleichmäßigen Konvergenz (1. Form). Die im Inter- 191. vall $J$ konvergente Reihe $\Sigma f_{n}(x)$ heißt in einem Teilintervall $J^{\prime}$ von $J$

1) Nimmt man etwa $x=\frac{1}{1000}$ und dafür $n=1000000$, so ist die Abszisse des höchsten Punktes des Buckels $=\frac{1}{1000000}$ und an unserer Stelle $x$ hat sich die Kurve schon wieder auf eine Höhe herabgesenkt, die $<\frac{1}{1000}$ ist.

2) Man vergegenwärtige sich etwa die Schnelligkeit der Konvergenz der geometrischen Reihe $\Sigma x^{n}$ (d. h. die Schnelligkeit, mit der ihr Rest bei wachsendem $n$ abnimmt) für $x=\frac{1}{100}$ und $x=\frac{99}{100}$ !

Knopp, Unendliche Reihen. 
gleichmäßig konvergent, wenn dic oben definierte Funktion $n(x)$ bei jeder Wahl von $\varepsilon$ in $J^{\prime}$ beschränkt ausfällt ${ }^{1}$ ). - Ist dann etwa stets $n(x)<N-$ und dies $N$ wird natürlich, wie die Zahlen $n(x)$ selber, immer noch von der Wahl von $\varepsilon$ abhängen, -- so können wir auch sagen:

2. (Haupt-)Form der Definition. Eine in $J$ konvergente Reihe $\sum f_{n}(x)$ heißt in einem Teilintervall $J^{\prime}$ von $J$ gleichmäßig konvergent, wenn nach $W a h l$ von $\varepsilon$ sich eine einzige $Z$ ahl $N=N(\varepsilon)$ so angeben läßt, daß der Betrag des Restes

$$
\left|\boldsymbol{r}_{n}(\boldsymbol{x})\right|<\varepsilon
$$

ausfällt, nicht nur (wie bisher) für jedes $n>N$, sondern gleichzeitig auch fïr jedes $x$ aus $J^{\prime}$.

\section{Erläuterungen und Beispiele.}

1. Die Gleichmäßigkeit der Konvergenz bezieht sich stets auf ein Intervall, niemals auf einzelne Punkte $^{2}$ ).

2. Eine in einem Intervall $J$ konvergente Reihe $\Sigma f_{n}(x)$ braucht in keinem Teilintervall desselben gleichmäßig zu konvergieren.

3. Hat die Potenzreihe $\Sigma a_{n}\left(x-x_{0}\right)^{n}$ den positiven Radius $r$ und ist $0<\varrho<r$, so ist, wenn das abgeschlossene Teilintervall $-\varrho \leq x-x_{0} \leqq+\varrho$ ihres Konvergenzintervalles mit $J^{\prime}$ bezeichnet wird, die Reilıe in $J^{\prime}$ gleichmäßig konvergent. Denn da der Punkt $x=x_{0}+\varrho$ im Innern des Konvergenzintervalles der Potenzreihe liegt, so ist diese dort absolut konvergent. Konvergiert aber $\Sigma a_{n} \varrho^{n}$ absolut, so kann man nach Wahl von $\varepsilon>0$ eine Zahl $N=N(\varepsilon)$ so bestimmen, daf für alle $n>N$ stets

$$
\left|a_{n+1}\right| \varrho^{n+1}+\left|a_{n+2}\right| \varrho^{n+2}+\ldots<\varepsilon
$$

ausfällt. Wegen $\left|x-x_{0}\right| \leq \varrho$ ist aber für alle in $J^{\prime}$ gelegenen $x$

$$
r_{n}(x)|\leqq| a_{n+1}\left|\cdot \varrho^{n+1}+\right| a_{n+2} \mid \cdot \varrho^{n+2}+\ldots
$$

Also ist fuir $n>N$ auch sicher $\left|v_{n}(x)\right|<\varepsilon$, wo auch $x$ in $J^{\prime}$ gelegen sein mag.

4. Nach diesem Beispiel wird es verständlich sein, wenn wir die Definition der gleichmäßigen Konvergenz etwas lockerer so formulleren: $\Sigma f_{n}(x)$ heißt in $J^{\prime}$ gleichmäßig konvergent, wenn dort eine für alle Lagen von $x$ gleichzeitig gültige Restabschätzung ," $v_{n}(x) !<\varepsilon$ "möglich ist.

5. Die Reihe $\underset{n=1}{x} \sin n x$ ist für alle $x$ gleichmäfig konvergent, denn $\in$ S ist - und $z$ war fuir jede Lage von $x-$

$$
\left|r_{n}(x)\right| \leq \frac{1}{(n+1)^{2}}+\frac{1}{(n+2)^{2}}+\ldots,
$$

woraus nach 4. das weitere zu ersehen ist.

1) Wenn also das oben erwähnte $M a \beta$ für die Güte der Konvergenz an den verschiedenen Stellen $x$ des Intervalles $J^{\prime}$ keine allzu beträchtlichen Ungleichmäfigkeiten aufzuweisen vermag. - In besonderen Fällen kann $J^{\prime}$ natürlich auch das ganze Intervall $J$ ausmachen.

2) Allgemeiner allenfalls auf Punktmengen, die dann aber notwendig unendliche sein miißsten. 
6. Die geometrische Reihe ist in ihrem ganzen Konvergenzintervall $-1<x<+1$ nicht gleichmäfig konvergent. Denn es ist dort

$$
r_{n}(x)=x^{n+1}+x^{n+2}+\ldots=\frac{x^{n+1}}{1-x}
$$

und wie grof jetzt auch $N$ genommen wird, man kann stets noch ein $v_{n}(x)$ mit $n>N$ und $0<x<1$ angeben, für das z. B. $r_{n}(x)>1$ ist.

Nehmen wir nämlich $n>4$ und $>N$ und zugleich so grof, daß $\left(1-\frac{1}{n}\right)^{n+1}>\frac{1}{4}$ ist - was wegen $\left(1-\frac{1}{n}\right)^{n+1} \rightarrow \frac{1}{e}>\frac{1}{3}$ sicher möglich ist so ist für ein solches $n$ und den Wert $x=\left(1-\frac{1}{n}\right)$ der Rest

$$
r_{n}(x)=n \cdot\left(1-\frac{1}{n}\right)^{n+1}>n \cdot \frac{1}{4}>1, \quad \text { w. z. b.w } .
$$

7. Der Unterschied zwischen gleichmäßiger und ungleichmäßiger Konvergènz und die grofe Bedeutung der ersteren für die Theorie der unendlichen Reihen ist zuerst (fast gleichzeitig) von Ph. L. v. Seidel (Abh. d. Münch. Akad., 1848 , S. 383) und G. G. Stokes (Transactions of the Cambridge philos. Soc., Bd. 8, 1848 , S. 533) erkannt worden. Doch muf $K$. Weierstraß, wie aus einer erst 1894 veröffentlichten Abhandlung (Werke, Bd. 1, S. 67) hervorgeht, diese Unterscheidung schon $1 \times 41$ gemacht haben. Allgemeingut der Mathematiker ist der Begriff der gleichmäßigen Konvergenz erst sehr viel später geworden.

\section{Andere Formen der Definition der gleichmäßigen Konvergenz.}

3. Form. $\Sigma f_{n}(x)$ heißt in $J^{\prime}$ gleichmäßig konvergent, wenn nach Wahl einer beliebigen dem Intervall $J^{\prime}$ entnommenen Zahlenfolge $\left.\left(x_{n}\right)^{1}\right)$ die zugehörigen Reste

\section{$r_{n}\left(x_{n}\right)$ \\ stets eine Nullfolge bilden. $\left.{ }^{2}\right)$

$$
\boldsymbol{r}_{n}\left(\boldsymbol{x}_{n}\right)
$$

$\mathrm{Da}$ diese Definition mit den vorigen äquivalent ist, erkennt man so:

a) Sind die Bedingungen der 2. Definitionsform erfüllt, ist also nach Wahl von $\varepsilon$ stets $N$ so bestimmbar, daß $\left|r_{n}(x)\right|<\varepsilon$ ist für alle $n>N$. und alle $x$ in $J^{\prime}$, so ist auch speziell

es strebt also stets $r_{n}\left(x_{n}\right) \rightarrow 0$.

$$
r_{n}\left(x_{n}\right) \mid<\varepsilon \text { für alle } n>N \text {; }
$$

b) Sind umgekehrt die Bedingungen der 3. Form erfüllt, strebt also für jede dem Intervall $J^{\prime}$ entnommene Zahlenfolge $\left(x_{n}\right)$ die zu-

1) Diese Folge braucht nicht zu konvergieren, sondern darf ganz beliebig in $J^{\prime}$ gelagert sein.

2) Besitzen die Funktionen $\left|r_{n}(x)\right|$ in $J^{\prime}$ ein Maximum, so kann man $x_{n}$ insbesondere so wählen, daf $\left|v_{n}\left(x_{n}\right)\right|=\operatorname{Max}\left|v_{n}(x)\right|$ ist, und unsere Definition erhält dann die

Form 3a. $\Sigma f_{n}(x)$ heißt in $J^{\prime}$ gleichmäßig konvergent, wenn die dortigen Maxima Max. $\left|r_{n}(x)\right|$ eine Nullfolge bilden.

Haben die Funktionen $\left|r_{n}(x)\right|$ in $J^{\prime}$ kein Maximum, so besitzen sie doch eine bestimmte obere Grenze $\mu_{n}$. Mit ihrer Hilfe können wir auch definieren:

Form 3 b. $\Sigma f_{n}(x)$ heißt in $J^{\prime}$ gleichmäßig konvergent, wenn $\mu_{n} \rightarrow 0$ strebt, (Beweis?) 
gehörige Folge der Reste $r_{n}\left(x_{n}\right) \rightarrow 0$, so müssen auch die Bedingungen der 2. Form erfüllbar sein. Denn entspräche nicht jedem $\varepsilon>0$ ein $N=N(\varepsilon)$ mit den dort formulierten Eigenschaften, so hieße dies: Bei einer speziellen Wahl von $\varepsilon$, etwa für $\varepsilon=\varepsilon_{0}$, hätte keine Zahl $N$ jene Eigenschaften, sondern es gäbe dann oberhalb jeder noch so großen Zahl $N$ immer noch wenigstens einen Index $n$, so daß für einen passenden Punkt $x=x_{n}$ aus $J^{\prime}$ der Betrag des Restes $\left|\boldsymbol{r}_{n}\left(x_{n}\right)\right| \geqq \varepsilon_{0}$ wäre (vgl. das letzte Beispiel, wo $\varepsilon_{0}=1$ und $x=x_{n}=1-\frac{1}{n}$ genommen wurde). Ist $n_{1}$ ein erster solcher Index, ist also $v_{n_{1}}\left(x_{n_{1}}\right) \mid \geqq \varepsilon_{0}$, so müßte es dann wieder oberhalb $n_{1}$ einen Index $n_{2}$ und einen zugehörigen Punkt $x_{n_{2}}$ geben, so daß auch $\left|\boldsymbol{r}_{n_{2}}\left(x_{n_{2}}\right)\right| \geqq \varepsilon_{0}$ wäre, oberhalb $n_{2}$ erneut, usw. Bilden wir nun eine Punktfolge $\left(x_{n}\right)$ aus $J^{\prime}$, von. der diese Punkte $x_{n_{1}}, x_{n_{2}}, \ldots$ eine Teilfolge sind, so wäre doch

$$
r_{n}\left(x_{n}\right)
$$

entgegen der Voraussetzung ersichtlich keine Nullfolge. Unsere Annahme, daß die Bedingungen der 2. Form nicht erfüllbar wären, ist unzulässig, und die 3. Form der Definition mit der 2. völlig äquivalent.

Bei den bisherigen Formen der Definition der gleichmäßigen Konvergenz haben wir stets die Reste der Reihe der Abschätzung unterworfen, die Reihe selbst also schon als konvergent vorausgesetzt. Benutzt man statt der ganzen Reste nur Teilstücke der Reihe (s. 82, 3), so kann man in die Definition der gleichmäßigen Konvergenz diejenige der Konvergenz selber mit hineinnehmen. Wir gelangen dann zu der folgenden Definition

4. Form. Eine Reihe $\Sigma f_{n}(x)$ heißt im Intervall $J^{\prime}$ gleichmäßig konvergent, wenn nach $W$ ahl von $\varepsilon>0$ eine Zahl $N=N(\varepsilon)$ so angegeben werden kann, daß nun

$$
\left|f_{n+1}(x)+f_{n+2}(x)+\ldots+f_{n+k}(x)\right|<\varepsilon
$$

ausfällt für jedes $n>N$, jedes $k \geqq 1$ und jedes $x$ aus $J^{\prime}$. Oder endlich:

5. Form. Eine Reihe $\Sigma f_{n}(x)$ heißt im Intervall $J^{\prime}$ gleichmäßig konvergent, wenn nach Wahl beliebiger natürlicher Zahlen $k_{1}, k_{2}, k_{3}, \ldots$ und beliebiger Punkte $x_{1}, x_{2}, x_{3}, \ldots$ aus $J$ die Werte

$$
\left[f_{n+1}\left(x_{n}\right)+f_{n+2}\left(x_{n}\right)+\ldots+f_{n+k_{n}}\left(x_{n}\right)\right]
$$

stets eine Nullfolge bilden $\left.{ }^{\mathbf{1}}\right)$.

Weitere Beispiele und Erläuterungen.

1. Da $r_{n}(x) \cdot F(x)-s_{n}(x)$, so kann die gleichmäßsige Konvergenz nun auch so interpretiert werden (vgl. die Vorbemerkungen S. 319): Für alle hin-

1) Nach 51 dürfte hier sogar $\left[f_{v_{n}+1}\left(x_{n}\right)+\ldots+f_{v_{n}+k_{n}}\left(x_{n}\right)\right]$ geschrieben werden, wenn die $v_{n}$ irgendwelche $\rightarrow+\infty$ strebende ganze Zahlen sind. 
reichend hohen Indizes schmiegt sich die Approximationskurve $y=s_{n}(x)$ während des ganzen Intervalles $J^{\prime}$ sehr nahe an die Grenzkurve $x=F(x)$ an.

2. Man studiere nun noch einmal das Verhalten der Reihe $\Sigma f_{n}(x)$ mit

$$
s_{n}(x)=\frac{n x}{1+n^{2} x^{2}}
$$

a) im Intervall $1 \leqq x \leqq 2$

b) im Intervall $0 \leqq x \leqq 1$. (Vgl. die Ausführungen S. 319/21.)

3. Für die Reihe

$$
1+(x-1)+\left(x^{2}-x\right)+\ldots+\left(x^{n}-x^{n-1}\right)+\ldots
$$

ist ersichtlich $s_{n}(x)=x^{n}$. Die Reihe konvergiert also im Intervall $J:-1<x \leq+1$, speziell also in dem Teilintervall $J^{\prime}: 0 \leq x \leq 1$. Dort ist

$$
F(x)\left\{\begin{array}{lll}
=0 & \text { für } & 0 \leqq x<1 \\
=1 & \text { für } & x=1
\end{array}\right.
$$

Die Konvergenz in diesem Intervall ist nicht gleichmäßig. Sie ist es nicht einmal in $J^{\prime \prime}: 0 \leqq x<1$; denn dort ist $r_{n}(x)=F(x)-s_{n}(x)=-x^{n}$. Wählt man nun aus $J^{\prime \prime}$ (also auch aus $J^{\prime}$ ) die Punktfolge

$$
x_{n}=1-\frac{1}{n} \quad(n=1,2, \ldots),
$$

so strebt $r_{n}\left(x_{n}\right)=-\left(1-\frac{1}{n}\right)^{n} \rightarrow-\frac{1}{e}$, so daß̧ die Reihe nicht gleichmäßig konvergieren kann ${ }^{1}$ ). - Geometrisch wird dies durch die in der Figur veranschaulichte Lage der Approximationskurven deutlich:

Für hohe $n$ läuft die Kurve $y=s_{n}(x)$ fast während des ganzen Intervalles ganz dicht an der Abszissenachse, die die Grenzkurve darstellt. Kurz vor +1 aber erhebt sie sich steil in die Höhe, um im Punkte $(1,1)$ zu enden. Wie grof man also auch $n$ nehmen mag, nie läuft die Kurve $y=s_{n}(x)$ während des ganzen Intervalles $J^{\prime \prime}$ (oder $J^{\prime}$ ) dicht an der Grenzkurve entlang ${ }^{2}$ ).

4. Bei dem vorigen Beispiel war die Ungleichmäßigkeit in der Approximation sozusagen $z u$ erwarten, weil $F(x)$ selbst im Endpunkt des Intervalles einen Sprung von der Höhe 1 macht. Anders lag es bei dem S. 319 behandelten Beispiel. Wir geben jetzt ein ähnliches, aber noch krasseres: Es sei die Reihe vorgelegt, für die

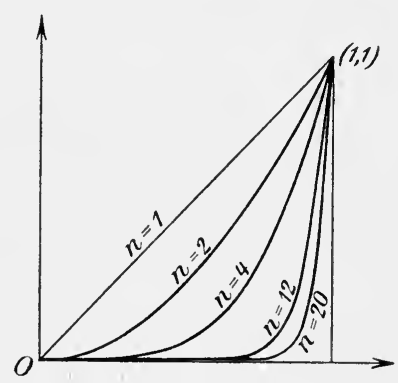

Fig. 6 .

$$
s_{n}(x)=n x e^{-\frac{1}{2} n x^{2}}
$$$$
(n=1,2, \ldots)
$$

ist. Für $x=0$ ist dauernd $s_{n}(0)=0$; für $x \neq 0$ ist $e^{-\frac{1}{2} x^{2}}$ ein positiver echter Bruch, so dafi (nach 38, 3) $s_{n}(x) \rightarrow 0$ strebt. Unsere Reihe ist also für jedes $x$

1) Für $x_{n}=\left(1-\frac{1}{n^{2}}\right)$ strebt sogar $v_{n}\left(x_{n}\right) \rightarrow-1$.

2) Trotzdem aber macht man sich wieder leicht klar, daß̧ bei jedem festen $x$ (aus $0<x<1$ ) die Werte $s_{n}(x)$ mit wachsendem $x$ auf 0 herabsinken, daß also das Hinaufschnellen zur Höhe 1 doch erst rechts von $x$ stattfindet, mag $x$ auch noch so dicht vor +1 liegen, - wenn nur $n$ grow genug genommen wird. 
konvergent und hat stets die Summe $F(x)=0$ : Die Grenzkurve ist mit der Abszissenachse identisch. Die Konvergenz ist aber durchaus keine gleichmäfige, wenn wir ein Intervall $J^{\prime}$ betrachten, das den Nullpunkt enthält. Denn für $x_{n}=\frac{1}{\sqrt{n}}$ ist

$$
r_{n}\left(x_{n}\right)=F\left(x_{n}\right)-s_{n}\left(x_{n}\right)-n x_{n} \cdot e^{-\frac{1}{2} n x_{n}^{2}}=-\sqrt{n} \cdot e^{-\frac{1}{2}}=-\sqrt{\frac{n}{e}},
$$

was gewif nicht $\rightarrow 0$ strebt. Die Approximationskurven verlaufen ähnlich wie in Fig. 4 und 5 , nur daß jetzt die Höhe der Buckel mit wachsendem $n$-sogar ohne Ende zunimmt, da ja

strebt $\left.{ }^{1}\right)$.

$$
s_{n}\left(\frac{1}{\sqrt{n}}\right)=\sqrt{\frac{n}{e}} \rightarrow+\infty
$$

\section{§ 4\%. Gliedweise Grenzübergänge.}

Sahen wir S. 318, daß die Grundeigenschaften der Funktionen $f_{n}(x)$ sich im allgemeinen nicht auf die durch die Reihe $\Sigma f_{n}(x)$ dargestellte Funktion $F(x)$ zu übertragen brauchen, so wollen wir nun zeigen, daß dies im großen und ganzen doch der Fall ist, wenn die Reihe gleichmäßig konvergiert ${ }^{2}$ ).

Wir beginnen mit dem einfachen, aber für die Anwendungen besonders wichtigen

193. Satz 1. Ist die Reihe $\Sigma f_{n}(x)$ im Intervall $a \leqq x \leqq b$ gleichmäßig konvergent und sind ihre Glieter $f_{n}(x)$ an einer Stelle $x_{0}$ dieses Intervalles stetig, so ist auch die dargestellte Funktion $F(x)$ an dieser Stelle stetig.

Bew eis. Bei gegebenem $\varepsilon>0$ ist (nach $\S 19$, Def. 6 b) die Existenz einer Zahl $\delta>0$ nachzuweisen, so daß

$$
F(x)-F\left(x_{0}\right) \mid<\varepsilon \text { bleibt für alle }\left|x-x_{0}\right|<\jmath,
$$

die dem Intervalle angehören. Nun kann

$$
F(x)-F\left(x_{0}\right)=s_{n}(x)-s_{n}\left(x_{0}\right)+r_{n}(x)-r_{n}\left(x_{0}\right)
$$

gesetzt werden. Und wählen wir hierin, was wegen der vorausgesetzten Gleichmäßigkeit der Konvergenz möglich ist, $n=m$ so groß, daß für alle $x$ des Intervalles stets $\left|r_{m}(x)\right|<\frac{\varepsilon}{3}$ ist, so ist

$$
F(x)-F\left(x_{0}\right) \leqq\left|s_{m}(x)-s_{m}\left(x_{0}\right)\right|+{ }_{3}^{2} \varepsilon .
$$

1) Die Stelle $x=\frac{1}{\sqrt{n}}$ ist für die Kurve $x=s_{n}(x)$ tatsächlich die Stelle des Maximums, wie man alls $s_{n}^{\prime}(x)=\left(n-n^{2} x^{2}\right) e^{-\frac{1}{2} n x^{2}}=0$ findet.

$\left.{ }^{2}\right)$ Doch sei gleich hier betont, dafi die Gleichmäfigkeit der Konvergenz immer nur eine hinveichende Bedingung in den folgenden Sätzen darstellt und im allgemeinen nicht notwendig ist. 
Nachdem $m$ so festgelegt ist, ist aber $s_{m}(x)$ als Summe einer festen Anzahl von in $x_{0}$ stetigen Funktionen (nach $\S 19$, Satz 3) dort selber stetig, und man kann daher $\delta$ so klein wählen, daß für alle $x$ des Intervalles, die der Bedingung $\left|x-x_{0}\right|<\delta$ genügen, stets

$$
\left|s_{m}(x)-s_{m}\left(x_{0}\right)\right|<\frac{\varepsilon}{3}
$$

bleibt. Für diese selben $x$ ist dann auch

$$
\left|F(x)-F\left(x_{0}\right)\right|<\varepsilon,
$$

womit die Stetigkeit von $F(x)$ in $x_{0}$ schon bewiesen ist.

Zusatz. Sind die $f_{n}(x)$ sämtlich in dem ganzen Intervall $a \ldots b$ stetig, so gilt das gleiche von $F(x)$.

In Verbindung mit dem Beispiel 191, $2^{3}$ haben wir hierin einen neuen Beweis für die Stetigkeit der durch eine Potenzreihe in ihrem Konvergenzintervall dargestellten Funktion.

Benutzen wir statt der $\varepsilon$-Definition der Stetigkeit die lim-Definition (s. $§ 19$, Def. 6), so kann man der Behauptung des Satzes auch die Form geben:

$$
\lim _{x \rightarrow x_{0}}\left(\sum_{n=0}^{\infty} f_{n}(x)\right)=\sum_{n=0}^{\infty}\left(\lim _{x \rightarrow x_{0}} f_{n}(x)\right)
$$

und in dieser Form erscheint er als ein Spezialfall des folgenden sehr viel feineren Satzes:

Satz 2. Es sei die Reihe $F(x)=\sum_{n=0}^{\infty} f_{n}(x)$ in dem offenen Inter-194. vall $x_{0} \ldots x_{1}$ gleichmäßig konvergent ${ }^{1}$ ), und es sei bei Annäherung aus dem Innern des Intervalles ${ }^{2}$ )

$$
\lim _{x \rightarrow x_{0}} f_{n}(x)=a_{n}
$$

für jedes einzelne $n$ vorhanden. Dann ist auch die Reihe $\sum_{n=0}^{\infty} a_{n}$ konvergent, und wenn ihre Summe mit $A$ bezeichnet wird, so ist

oder also

$$
\lim _{x \rightarrow x_{0}} F(x)=A \text {, }
$$

$$
\lim _{x \rightarrow x_{0}}\left(\sum_{n=0}^{\infty} f_{n}(x)\right)=\sum_{n=0}^{\infty}\left(\lim _{x \rightarrow x_{0}} f_{n}(x)\right) .
$$

(Wegen dieser zweiten Formulierung sagt man auch kurz: Im Falle gleichmäßiger Konvergenz darf man gliedweis zur Grenze übergehen.)

1) Es darf $x_{0}>$ oder auch $\left\langle x_{1}\right.$ sein. Ob die Reihe noch in $x_{0}$ konvergiert oder nicht, ja ob dort die Funktionen $f_{n}(x)$ überhaupt noch definiert sind oder nicht, ist für diesen Satz gleichgültig.

2) Es handelt sich also hier - und ebenso bei den beiden folgenden Behauptungen - um einen einseitigen Limes. 
Beweis. Ist $\varepsilon>0$ gegeben, so wählen wir zunächst $n_{1}$ so, daß (s. 4. Form der Definition 191) für $n>n_{1}$ alle $k \geqq 1$ und alle $x$ unseres Intervalles

$$
\left|f_{n+\mathbf{1}}(x)+\ldots+f_{n+k}(x)\right|<\varepsilon
$$

bleibt. Hält man hier für den Augenblick $n$ und $k$ fest und läßt $x \rightarrow x_{0}$ rücken, so folgt (nach $\S 19$, Satz $1 \mathrm{a}$ ), daß auch

$$
\left|a_{n+1}+a_{n+2}+\ldots+a_{n+k}\right| \leqq \varepsilon
$$

ist, und zwar für alle $n>n_{1}$ und alle $k \geqq 1$. Also ist $\Sigma a_{n}$ konvergent. Die Teilsummen dieser Reihe nennen wir $A_{n}$, die Summe $A$. Daß dann $F(x) \rightarrow A$ strebt, ist jetzt leicht zu sehen. Wird nämlich nach Wahl von $\varepsilon$ die Zahl $n_{0}$ so bestimmt, daß nicht nur für $n>n_{0}$ stets

$$
\left|r_{n}(x)\right|<\frac{\varepsilon}{3}, \quad \text { sondern auch } \quad A-A_{n}<\frac{\varepsilon}{3}
$$

ausfällt, so ist für ein (festes) $m>n_{0}$

$$
\begin{gathered}
\mid F(\dot{x})-A \\
=\left|\left(s_{m}(x)-A_{m}\right)-\left(A-A_{m}\right)+r_{m}(x)\right| \leqq\left|s_{m}(x)-A_{m}\right|+\frac{\varepsilon}{3}+\frac{\varepsilon}{3}
\end{gathered}
$$

und, weil für $x \rightarrow x_{0}$ nun $s_{m}(x) \rightarrow A_{m}$ strebt, kann man $\delta$ so bestimmen, daß

$$
\left|s_{m}(x)-A_{m}\right|<\frac{\varepsilon}{3} \text { ist für alle } 0<\left|x-x_{\mathbf{0}}\right|<\delta,
$$

die dem gegebenen Intervall angehören. Für diese $x$ ist dann auch

womit alles bewiesen ist.

$$
|F(x)-A|<\varepsilon,
$$

Ist $\left(x_{n}\right)$ eine ganz beliebige, dem Intervall gleichmä6iger Konvergenz entnommene Zahlenfolge, so hat wegen

$$
F\left(x_{n}\right)=s_{n}\left(x_{n}\right)+r_{n}\left(x_{n}\right)
$$

und wegen $r_{n}\left(x_{n}\right) \rightarrow 0$ (s. 191, 3. Form) die Folge der Zahlen $F\left(x_{n}\right)$ und die der Zahlen $s_{n}\left(x_{n}\right)$ stets das gleiche Konvergenzverhalten. Betrachten wir im Gegensatz dazu die als ungleichmäfig konvergent erkann̦te Reihe mit den Teilsummen $s_{n}(x)=\frac{n x}{1+n^{2} x^{2}}$, und nehmen wir $x_{n}=\frac{1}{n}$, so ist $F\left(x_{n}\right)=0$, also auch konvergent mit dem Grenzwert 0 , wïhrend $s_{n}\left(x_{n}\right)=\frac{1}{2}$ und also auch konvergent ist, aber mit dem Grenzwert $\frac{1}{2}$. Beide Folgen haben also verschiedenes Konvergenzverhalten.

195. Satz 3. Die Reihe $F(x)=\Sigma f_{u}(x)$ sei im Intervall $J$ gleichmäßig konvergent und in dem abgeschlossenen Teilintervall $J^{\prime}: a \leqq x \leqq b$ seien die Funktionen $f_{n}(x)$ sämtlich stetig, so daß auch $F(x)$ eine dort stetige Funktion ist. Dann kann das über das Intervall $J^{\prime}$ genommene Integral von $F(x)$ durch gliedweise Integration gewonnen werden, $d . h$. es ist

$$
\int_{a}^{b} F(x) d x \quad \text { oder also } \quad \int_{a}^{b}\left[\sum_{n=0}^{\infty} f_{n}(x)\right] \boldsymbol{d} \boldsymbol{x}=\sum_{n=0}^{\infty}\left[\int_{a}^{b} f_{n}(\boldsymbol{x}) \boldsymbol{d} \boldsymbol{x}\right] \text {. }
$$


(Genauer: Die letzte Reihe ist wieder konvergent und hat das gesuchte Integral von $F(x)$ zur Summe $\left.{ }^{1}\right)$.)

Beweis. Nach Wahl von $\varepsilon>0$ bestimmen wir $n_{0}$ so groß, daß für $n>n_{0}$ und alle $x$ des Intervalles $a \ldots b$ stets

$$
\left|r_{n}(x)\right|<\frac{\varepsilon}{b-a}
$$

bleibt. Dann ist wegen $F=s_{n}+r_{n}$ zunächst

$$
\left|\int_{a}^{b} F(x) d x-\int_{a}^{b} s_{n}(x) d x\right|=\left|\int_{a}^{b} r_{1 b}(x) d x\right|<\varepsilon,
$$

- das letzte wegen $\S 19$, Satz 21. Nun ist aber $s_{n}(x)$ eine Summe von endlich vielen Funktionen und die Anwendung von $\S 19$, Satz 22 liefert sofort, daß auch

$$
\left|\int_{a}^{b} F(x) d x-\sum_{\nu=1}^{n} \int_{a}^{b} f_{v}(x) d x\right|<\varepsilon .
$$

Dies besagt aber nach 69, daß die Reihe $\Sigma \int_{a}^{b} f_{v}(x) d x$ konvergiert und das entsprechende Integral von $F(x)$ zur Summe hat.

Nicht ganz so einfach liegen die Dinge für die gliedweise Differentiation.

In 190, 7 sahen wir z. B., dafs die Reihe

$$
\sum_{n=1}^{\infty} \frac{\sin n x}{n}
$$

für jedes $x$ konvergiert und also eine für alle reellen $x$ definierte Funktion $F(x)$ darstellt. Die Glieder dieser Reihe sind durchweg stetige und differenzierbare Funktionen. Nach gliedweiser Differentiation aber erhalten wir die Reihe

$$
\sum_{n=1}^{\infty} \cos n x,
$$

1) Es genuigt die $f_{n}(x)$ als integrierbar vorauszusetzen. Dann läßt sich zeigen, daß auch $F(x)$ integrierbar ausfällt und nun die gliedweise Integration gestattet ist. In der Tat, ist $\varepsilon>0$ gegeben, so können die etwaigen Unstetigkeitspunkte der integrierbaren Funktion $f_{0}(x)$ in Intervalle eingeschlossen werden, deren Gesamtlänge $<\frac{\varepsilon}{2}$ bleibt, ebenso die von $f_{1}(x)$ in Intervalle, deren Gesamtlänge $<\frac{\varepsilon}{4}$ bleibt, und allgemein diejenigen von $f_{n}(x)$ in Intervalle, deren Gesamtlänge $<\frac{\varepsilon}{2^{n+1}}$ bleibt. Alle Punkte unseres Intervalles, in denen auch nur eine einzige unserer Funktionen unstetig ist, liegen dann in Intervalle eingeschlossen, deren Gesamtlänge $<\varepsilon$ bleibt. Das gleiche gilt dann aber auch von den etwaigen Unstetigkeitsstellen von $F(x)$, da an den Stellen, an denen alle $f_{n}(x)$ stetig sind, nach Satz 1 auch $F(x)$ stetig sein muf. Also ist (nach $\$ 19$, Satz 14) auch $F(x)$ über $a \ldots b$ integrierbar. - Der uibrige Beweis verläıft dann wie oben für durchweg stetige Funktionen. 
die für jedes $x$ divergiert $\left.{ }^{1}\right)$. - Selbst wenn die Reihe für alle $x$ gleichmäßig konvergiert, wie $z$. B. die Reihe

$$
\sum_{n=1}^{\infty} \frac{\sin n x}{n^{2}}
$$

(vgl. 191, $2^{4}$ ), so liegen dic Dinge nicht günstiger, da wir durch gliedweise Differentiation die Reihe

$$
\sum_{n=1}^{\infty} \cos n x
$$

erhalten, die z. B. für $x=0$ divergiert.

Der Satz über gliedweise Differentiation wird daher ein etwas anderes Gepräge haben müssen, er lautet:

196. Satz 4. Liegt die Reihe $\sum_{n=0}^{\infty} f_{n}(x)$ vor $\left.{ }^{2}\right)$, deren. Glieder im Intervall $J-a \ldots b,(a<b)$, differenzierbare Funktionen sind, und ist die durch gliedweise Differentiation entstehende Reihe

$$
\sum_{n=0}^{x} f_{n}^{\prime}(x)
$$

in $J$ gleichmäßig konvergent, so gilt dasselbe von der gegebenen Reihe, falls diese in wenigstens einem Punkte von $J$ konvergiert. Und sind dann $F(x)$ und $p(x)$ die durch die beiden Reihen dargestellten Funktionen, so ist $F(x)$ in $I$ differenzierbar und es ist

$$
F^{\prime}(x)=p(x) \text {, }
$$

d. h. also: unter den gemachten Voraussetzungen darf die gegebene Reihe gliedweise diffrenziert werden.

Beweis. a) Ist $c$ ein (nach Voraussetzung vorhandener) Punkt aus $J$, für den $\Sigma f_{n}(c)$ konvergiert, so ist nach dem Mittelwertsatz der Differentialrechnung (s. $\$ 19$, Satz 8)

$$
\sum_{v=n+1}^{n+k}\left(f_{v}(x)-f_{v}(c)\right)=(x-c) \cdot \sum_{r=n+1}^{n+k} f_{n^{\prime}}^{\prime}(\xi) .
$$

wenn mit $\xi$ ein passender Punkt zwischen $x$ und $c$ bezeichnet wird. Nach Wahl von $\varepsilon>0$ kann man nun nach Voraussetzung $n_{0}$ so bestimmen, daß für $n>n_{0}, k \geqq 1$ und alle $x$ aus $J$

$$
\sum_{n=n+1}^{n+k} f_{v}^{\prime}(x) \mid \leqq \stackrel{\varepsilon}{\varepsilon-a}
$$

1) Nach den S. 345 bewiesenen Formeln ist fuir alle $x \neq 2 k \pi$

$$
\frac{1}{2}+\cos x+\cos 2 x+\ldots+\cos n x=\frac{\sin \left(n+\frac{1}{2}\right) x}{2 \sin \frac{x}{2}} .
$$

2) Über ihre Konvergenz wird zunïchst noch nichts vorausgesetzt. 
bleibt. Dann ist unter denselben Bedingungen

$$
\left|\sum_{v=n+1}^{n+k}\left(f_{v}(x)-f_{v}(c)\right)\right|<\varepsilon
$$

Und dies lehrt, daß $\Sigma\left(f_{n}(x)-f_{n}(c)\right)$ und folglich auch $\Sigma f_{n}(x)$ selbst in dem ganzen Intervall $J$ gleichmäßig konvergiert und somit eine bestimmte Funktion $F(x)$ darstellt.

b) Ist jetzt $x_{0}$ ein spezieller Punkt aus $J$, so werde

$$
\frac{f_{v}\left(x_{0}+h\right)-f_{v}\left(x_{0}\right)}{h}=g_{v}(h), \quad(v=0,1,2, \ldots),
$$

gesetzt, - Funktionen, die für alle $h \gtrless 0$ definiert sind, für die $x_{0}+h$ in $J$ liegt. Da nun, ähnlich wie eben

$$
\sum_{\nu=n+1}^{n+k} g_{\nu}(h)=\sum_{\nu=n+1}^{n+k} f_{v}^{\prime}\left(x_{0}+\vartheta h\right), \quad(0<\vartheta<1)
$$

ist, so erkennt man wie unter a), daß

$$
\sum_{n=0}^{\infty} g_{n}(h)
$$

für alle diese $h$ gleichmäßig konvergiert. Diese Reihe stellt die Funktion

$$
\frac{F\left(x_{0}+h\right)-F\left(x_{0}\right)}{h}
$$

dar. Nach Satz 2 können wir daher gliedweise $h \rightarrow 0$ streben lassen und finden, daß $F^{\prime}\left(x_{0}\right)$ existiert und

$$
=\sum_{n=0}^{\infty}\left(\lim _{h \rightarrow 0} g_{n}(h)\right)=\sum_{n=0}^{\infty} f_{n}^{\prime}\left(x_{0}\right)
$$

ist. Dies bedeutet aber, daß, wie behauptet, $F^{\prime}\left(x_{0}\right)=\varphi\left(x_{0}\right)$ ist.

\section{Beispiele und Bemerkungen.}

1. Hat $\Sigma a_{n}\left(x-x_{0}\right)^{n}$ den Radius $r>0$ und ist $0<\varrho<r$, so konvergiert $\Sigma n a_{n}\left(x-x_{0}\right)^{n-1}$ gleichmäßig für alle $\left|x-x_{0}\right| \leqq \varrho$. Die gegebene Potenzreihe stellt also nach Satz 4 eine Funktion dar, die für alle $\left|x-x_{0}\right| \leqq \varrho$ differenzierbar ist. Fafit man ein spezielles $x$ mit $\left|x-x_{0}\right|<r$ ins Auge, so kann man $\varrho<r$ stets so wählen, daßs auch $\left|x-x_{0}\right|<\varrho<r$ ist. Die Differenzierbarkeit der durch $\Sigma a_{n}\left(x-x_{0}\right)^{n}$ dargestellten Funktion besteht also sogar für jedes $x$ des offenen Intervalles $\left|x-x_{0}\right|<r$.

2. Die durch $\sum_{n=1}^{\infty} \frac{\sin n x}{n^{3}}$ dargestellte Funktion ist für jedes $x$ differenzierbar und hat die Ableitung $\sum_{n=1}^{\infty} \frac{\cos n x}{n^{2}} \cdot($ Vgl. 191, 25.)

3. Die Bedingung der gleichmäfigen Konvergenz ist bei allen vier Sätzen jedenfalls eine hinreichende. Doch bleibt es fraglich, ob sie auch notwendig ist.

a) Beim Stetigkeitssatz 1 oder seinem Zusatz ist das sicher nicht der Fall; denn die 192, 2 und 4 behandelten Reihen haben durchweg stetige Glieder und stellen auch durchweg stetige Funktionen dar. Die Konvergenz 
war aber keine gleichmäfige. Die notwendigen und hinreichenden Bedingungen zu ergriinden, ist nicht ganz leicht. In befriedigender Form hat dies zuerst S. Arzelà (Rendic. Accad. Bologna, Bd. (1) 19 (1883), S. 83) getan. Einen vereinfachten Beweis des von ihm ausgesprochenen Hauptsatzes findet man bei G. Vivanti (Rendiconti del circ. matem. di Palermo, Bd. 30 (1910), S. 85). - Sind die $f_{n}(x)$ positiv, so ist, wie $U$. Dini gezeigt hat, die gleichmä6ige Konvergenz auch notwendig für die Stetigkeit von $F(x)$. Vgl. Aufg. 158.

b) Dafs auch bei dem Satz $\mathbf{1 9 5}$ über die gliedweise Integration die Gleichmäßigkeit der Konvergenz der Reihe keine notwendige Bedingung für seine Gültigkeit ist, ist gleichfalls verschiedentlich durch Beispiele belegt worden. Für die in 192, 2 angegebene Reihe $\sum_{n=1}^{\infty} f_{n}(x)$, deren Teilsummen

$$
s_{n}(x)=\frac{n x}{1+n^{2} x^{2}}
$$

sind, und deren Summe $F(x)=0$ ist, findet man sofort

$$
\sum_{n=1}^{\infty} \int_{0}^{1} f_{n}(x) d x=\int_{0}^{1} F(x) d x=0 \text {. }
$$

Die gliedweise Integration führt also zum richtigen Ergebnis. Bei der Reihe 19:2, 4, bei der auch $\int_{u}^{1} F(x) d x=0$ ist, strebt aber

$$
\sum_{\nu=0}^{n} \int_{0}^{1} f_{v}(x) d x=\int_{0}^{1} s_{n}(x) d x=1-e^{-\frac{1}{2} n} \rightarrow 1
$$

und dies zeigt, daß die gliedweise Integration hier nicht erlaubt ist.

\section{$\$ 48$. Kriterien für gleichmäßige Konvergenz.}

Nachdem wir nun die Bedeutung des Begriffs der gleichmäßigen Konvergenz kennen gelernt haben, werden wir danach fragen, wie man bei einer vorgelegten Reihe feststellen kann, ob sie in ihrem Konvergenzintervall oder einem Teil desselben gleichmäßig konvergiert oder nicht. Ist schon, wie wir wissen, die Feststellung der Konvergenz nicht immer ganz einfach, so wird dies von der, der gleichmäßigen Konvergenz in noch höherem Maße gelten. Für die Anwendungen das wichtigste, weil am leichtesten zu handhabende, ist das folgende

197. Kriterium von Weierstraß. Sind die Funktionen $f_{n}(x)$ sämtlich im Intervall $J$ definiert und beschränkt und ist, wenn in $J$ etwa stets

$$
\left|f_{n}(x)\right| \leqq \gamma_{n}
$$

bleibt, die Reihe (mit positiven Gliedern) $\Sigma \gamma_{n}$ konvergent, so ist die Reihe $\Sigma f_{n}(x)$ in $J$ gleichmäßig konvergent.

Beweis. Ist $\left(x_{n}\right)$ eine beliebige Punktfolge aus $J$, so ist doch stets $\left|f_{n+1}\left(x_{n}\right)+f_{n+2}\left(x_{n}\right)+\ldots+f_{n+k_{n}}\left(x_{n}\right)\right| \leqq \gamma_{n+1}+\gamma_{n+2}+\ldots+\gamma_{n+k_{n}}$.

Nach 81, 2 strebt für $n \rightarrow \infty$ die rechte Seite $\rightarrow 0$; also tut dies (nach 26, 2) auch die linke Seite. Nach 191, 5. Form, ist daher $\Sigma f_{n}(x)$ in $J$ gleichmäßig konvergent. 


\section{Beispiele.}

1. In dem Beispiel 191, 3 hatten wir schon im Prinzip dieses Weierstra $\beta$ sche Kriterium benutzt.

2. Die harmonische Reihe $\Sigma \frac{1}{n^{x}}$, die für $x>1$ konvergiert, ist auf der Halbgeraden $x \geq 1+\delta$ gleichmäßig konvergent; hierbei darf $\delta$ irgendeine positive Zahl bedeuten. In der Tat ist für diese $x$ stets

$$
\left|\frac{1}{n^{x}}\right| \leqq \frac{1}{n^{1+\delta}}=\gamma_{n}
$$

mit konvergenter $\Sigma \gamma_{n}$. Damit ist die Behauptung schon bewiesen.

Die durch die harmonische Reihe dargestellte Funktion - man nennt sie die Riemannsche $\zeta$-Funktion und bezeichnet sie mit $\zeta(x)$ - ist also jedenfalls für $x>1$ stetig $^{1}$ ).

3. Durch gliedweise Differentiation der harmonischen Reihe entsteht die Reihe

$$
-\sum_{n=1}^{\infty} \frac{\log n}{n^{x}}
$$

Auch diese ist für alle $x \geq 1+\delta>1$ gleichmäßig konvergent. Denn für alle $n$ von einer Stelle ab ist (nach 38,5) $\frac{\log n}{n^{\delta / 2}}<1$; für diese $n$ und zugleich für alle $x \geq 1+\delta$ ist dann auch

$$
\left|\frac{\log n}{n^{x}}\right| \leqq \frac{1}{n^{1+\delta / 2}} \cdot \frac{\log n}{n^{\delta / 2}}<\frac{1}{n^{1+\delta / 2}}=\gamma_{n} .
$$

Die Riemannsche $\zeta$-Funktion ist also auch für alle $x>1$ differenzierbar.

4. Wenn $\sum a_{n}$ absolut konvergiert, so sind die Reihen

$$
\sum a_{n} \cos n x \text { und } \sum a_{n} \sin n x
$$

für alle $x$ gleichmäfig konvergent, denn es ist $z$. B. stets $\left|a_{n} \cos n x\right| \leq\left|a_{n}\right|=\gamma_{n}$. Diese Reihen definieren dann also durchweg stetige Funktionen.

Das Weierstraßsche Kriterium wird trotz seiner großen praktischen Bedeutung nur einen beschränkten Geltungsbereich haben, denn es verlangt ja insbesondere, daß die zu untersuchende Reihe absolut konvergiert. Wenn dies nicht der Fall ist, müssen feinere Kriterien einsetzen, die wir denen von $\S 43$ nachbilden. Das erfolgreichste Hilfsmittel $\mathrm{zu}$ ihrer Aufstellung ist auch hier wieder die Abelsche partielle Summation 182. Sie liefert uns, ganz ähnlich wie damals, zunächst den folgenden

Satz. Eine Reihe der Form $\sum_{n=0}^{\infty} a_{n}(x) \cdot b_{n}(x)$ ist sicher im Intervall $J \mathbf{1 9 S .}$ gleichmäßig konvergent, wenn dort

1) die Reihe $\sum_{v=0}^{\infty} A_{v} \cdot\left(b_{v}-b_{v+1}\right)$ gleichmäßig konvergent und

2) auch die Folge $A_{n} \cdot b_{n+1}$ gleichmäßig konvergent ist $\left.{ }^{2}\right)$.

1) Denn fafat man ein spezielles $x>1$ ins Auge, so kann man sich stets $\delta>0$ so gewählt denken, daß auch noch $x>1+\delta$ ist.

2) Die $a_{n}, b_{n}, A_{n}$ sind jetzt stets Funktionen von $x$, die in dem zugrunde gelegten Intervall $J$ definiert sind; doch lassen wir der Kürze halber das $x$ im folgenden häufig fort. - Beziiglich des Begriffs der gleichmäßigen Konvergenz einer Folge von Funktionen vgl. S. 317, Fufn. 1. 
Hierbei sollen die $A_{n}=A_{n}(x)$ die Teilsummen der Reihe $\Sigma a_{n}(x)$ bedeuten.

Beweis. Es ist genau wie dort - man hat nur in den $a_{v}, b_{v}$ und $A_{v}$ nicht Zahlen, sondern Funktionen von $x$ zu sehen - zunächst

$$
\sum_{v=n+1}^{n+k} a_{v} \cdot b_{v}=\left(\sum_{\nu=n+1}^{n+k} A_{v} \cdot\left(b_{v}-b_{v+1}\right)\right)+\left(A_{n+k} \cdot b_{n+k+1}-A_{n} \cdot b_{n+1}\right) .
$$

Machen wir hier $x$ und $k$ in beliebiger Weise von $n$ abhängig, so haben wir links eine Teilstückfolge

$$
\sum_{v=n+1}^{n+k_{n}} a_{v}\left(x_{n}\right) \cdot b_{v}\left(x_{n}\right)
$$

der Reihe $\Sigma a_{v}, b_{v}$, und rechts entsprechend eine solche der Reihe $\Sigma A_{v}\left(b_{v}-b_{v+1}\right)$ bzw. eine Differenzenfolge der Folge $A_{n} \cdot b_{n+1}$. Da nach Voraussetzung die letzteren beiden immer gegen 0 streben (s. 191, 5. Form), so ist dies auch mit der linksstehenden der Fall. Damit sind (wieder nach 191,5) die behaupteten Tatsachen schon bewiesen.

Genau wie in $\$ 43$ lassen sich aus diesem noch sehr allgemeinen Satze nun speziellere, aber leichter zu handhabende Kriterien ${ }^{1}$ ) herleiten:

1. Kriterium von Abel. $\Sigma a_{v}(x) \cdot b_{v}(x)$ ist in $J$ gleichmäßig konvergent, wenn dort $\sum a_{v}(x)$ gleichmäßig konvergiert, wenn überdies bei jedem festem $x$ die Zahlenfolge $b_{n}(x)$ monoton ausfällt und die Zahlen $b_{n}(x)$ für alle $n$ und alle $x$ aus $J$ ihrem Betrage nach unter ein und derselben Schranke $K$ verbleiben $\left.{ }^{2}\right)$.

Beweis. Bezeichnen wir die zu den Teilsummen $A_{n}(x)$ gehörigen Reste mit $\iota_{n}(x)$, setzen wir also $\sum_{n=0}^{\infty} a_{v}(x)=A_{n}(x)+\alpha_{n}(x)$, so dürfen wir in der Formel der Abelschen partiellen Summation (nach Zusatz 183) - $\iota_{v}$, statt $A_{v}$ setzen und haben dann

$$
\sum_{v=n+1}^{n+k} a_{v} \cdot b_{v}=-\sum_{v=n+1}^{n+k} a_{v} \cdot\left(b_{v}-b_{v+1}\right)-\left(a_{n+k} \cdot b_{n+k+1}-a_{n} \cdot b_{n+1}\right) \text {, }
$$

1) Wir benennen diese Kriterien der Einfachheit halber nach den entsprechenden Regeln mit konstanten Gliedern. - Vgl. S. 306, Fußn. 5.

2) Die $b_{n}(x)$ bilden bei festgehaltenem $x$ eine Zahlenfolge $b_{0}(x), b_{1}(x), \ldots$, bei festgehaltenem $n$ aber eine Funktion von $x$, die in $J$ definiert ist. Die obige Voraussetzung kann man dann so ausdrücken: Die sämtlichen (für die verschiedenen $x$ aus $J$ entstehenden) Zahlenfolgen sollen gleichmäßig in bezug auf alle diese $x$ beschränkt sein; d. h. eine jede ist beschränkt, und es gibt eine Zahl $K$, die für alle zugleich eine Schranke ist. Oder auch: Die sämtlichen (für die verschiedenen Werte von $n$ sich ergebenden) in $J$ definierten Funktionen sollen dort gleichmäbig (in bezug auf alle diese $n$ ) beschränkt sein; d. h. eine jede ist beschränkt, und es gibt eine $Z$ ahl $K$, die für alle zugleich eine Schranke ist. 
und es genügt wieder zu zeigen, daß sowohl die Reihe $\sum c_{v} \cdot\left(b_{v}-b_{v+1}\right)$ als auch die Folge $a_{n} \cdot b_{n+1}$ beide in $J$ gleichmäßig konvergieren. Da aber die $\alpha_{n}(x)$ als Reste einer gleichmäßig konvergenten Reihe gleichmäßig gegen 0 streben und die $b_{v}(x)$ ihrem Betrage nach stets $<K$ bleiben, so strebt auch die Folge $a_{n} \cdot b_{n+1}$ in $J$ gleichmäßig gegen 0 . Läßt man andrerseits bei der Summe $\sum_{v=k+1}^{n+k} c_{v} \cdot\left(b_{v}-b_{v+1}\right)$ die Variable $x$ und die Summandenanzahl $k$ in beliebiger Weise von $n$ abhängen, betrachtet man also die Größen

$$
T_{n}=\sum_{v=n+1}^{n+k_{n}} c_{v}\left(x_{n}\right) \cdot\left(b_{\nu}\left(x_{n}\right)-b_{v+1}\left(x_{n}\right)\right)
$$

so streben sie - und mit dem Nachweis hiervon wäre die gleichmäßige Konvergenz der in Rede stehenden Reihe im Intervall $J$ gesichert - wirklich immer $\rightarrow 0$. Denn ist $\bar{\alpha}_{n}$ der größte der unter den Zahlen $\left|\alpha_{\nu}\left(x_{n}\right)\right|,\left(v=n+1, n+2, \ldots, n+k_{n}\right)$, vorkommenden Werte, so strebt auch $\left.\bar{u}_{n} \rightarrow 0^{1}\right)$, und es ist

$$
T_{n}\left|\leqq \bar{a}_{n} \cdot \sum_{\nu=n+1}^{n+k_{n}}\right| b_{v}-b_{\nu+1}\left|\leqq \bar{a}_{n} \cdot\right| b_{n+1}-b_{n+k_{n}+1} \mid \leqq 2 K \cdot \bar{\iota}_{n},
$$

so daß in der Tat $T_{n} \rightarrow 0$ strebt.

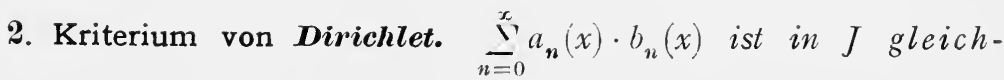
mäßig konvergent, wenn dort die Teilsummen der Reihe $\sum a_{n}(x)$ gleichmäßig beschränkt sind ${ }^{2}$ ), und wenn dort die Folge $b_{n}(x)$ gleichmäßig $\rightarrow 0$ konvergiert, und zwar für jedes feste $x$ monoton.

Beweis. Aus den Voraussetzungen folgt jetzt unmittelbar, daß die Folge $A_{n} \cdot b_{n+1}$ in $J$ gleichmäßig konvergiert (und zwar wieder $\rightarrow 0$ ). Ist dann weiter $K^{\prime}$ eine obere Schranke aller $\left|A_{n}(x)\right|$, so ist

$\left|\sum_{v=n+1}^{n+k} A_{v} \cdot\left(b_{\nu}-b_{v+1}\right)\right| \leqq K^{\prime} \cdot \sum_{v=n+1}^{n+k}\left|b_{v}-b_{n+1}\right| \leqq K^{\prime} \cdot\left|b_{n+l+1}-b_{n+1}\right| \cdot$

Wie aber auch $x$ und $k$ hier von $n$ abhängen mögen, die rechte Seite strebt infolge der Voraussetzungen immer gegen 0 , also tut es auch die Linke. Damit ist aber wieder die Gleichmäßigkeit der Konvergenz der in Rede stehenden Reihe für das Intervall $J$ bewiesen.

Die Monotonie im Verhalten der $b_{n}(x)$ wurde bei beiden Kriterien nur gebraucht, um eine bequeme Abschätzung der Teilstücke $\Sigma\left|b_{v}-b_{v+1}\right|$ $\mathrm{zu}$ ermöglichen. Modifiziert man zu diesem Zwecke ein klein wenig die Voraussetzungen, so erhält man

1) Man mache sich die Richtigkeit dieser Tatsache genau klar!

) Vgl. Fufn. 2 der vorigen Seite. 


\section{Zwei Kriterien von du Bois-Reymond und Dedekind.}

a) Die Reihe $\Sigma a_{v}(x) \cdot b_{v}(x)$ ist in $J$ gleichmäßig konvergent, wenn dort die beiden Reihen $\Sigma a_{r}$ und $\sum\left|b_{v}-b_{v+1}\right|$ gleichmäßig konvergieren und zugleich die Funktionenfolge der $b_{n}(x)$ dort gleichmäßig beschränkt ist.

Beweis. Wir benutzen wieder die Umformung

$$
\sum_{r=n+1}^{n+k} a_{r} \cdot b_{r}=-\sum_{r=n+1}^{n+k} c_{v} \cdot\left(b_{r}-b_{r+1}\right)-\left(c_{n+k} \cdot b_{n+k+1}-c_{n} \cdot b_{n+1}\right) \text {. }
$$

Da jetzt die Reste $\omega_{r}(x)$ gleichmäßig gegen 0 streben, so ist z. B. für $v>m$ stets $\left|\boldsymbol{c}_{r}(x)\right|<1$ für alle $x$ in $J$. Daher ist für $n \geqq m$

$$
\sum_{v=n+1}^{n+k} a_{v} \cdot\left(b_{v}-b_{v+1}\right) \mid \sum_{v=n+1}^{n+k} b_{v}-b_{v+1} ;
$$

und da nun - auch wenn $x$ und $k$ in beliebiger Weise von $n$ abhängen - die rechtsstehenden Größen mit wachsendem $n$ gegen 0 streben, so tun es auch die linken. Und daß auch $a_{n} \cdot b_{n+1}$ gleichmäßig in $J$ gegen 0 strebt, folgt daraus, daß die $c_{n}(x)$ dies tun und daß die $b_{r}(x)$ dort gleichmäßig beschränkt sind.

b) Die Reihe $\Sigma a_{r}(x) \cdot b_{v}(x)$ ist in $J$ gleichmäßig konvergent, wenn dort von den beiden Reihen $\Sigma a_{r}$ und $\Sigma b_{r}-b_{r+1}$ die zweite gleichmäßig konvergiert und die erste gleichmäßig beschränkte Teilsummen $h a t$, wofern dann noch die Funktionen $b_{n}(x)$ in $J$ gleichmäßig $\rightarrow 0$ streben.

Beweis. Aus den Voraussetzungen folgt zunächst wieder unmittelbar, daß $A_{n} b_{n+1}$ in $J$ gleichmäßig (gegen 0) konvergiert. Ferner ist, wenn $K^{\prime}$ dieselbe Bedeutung wie vorhin hat.

$$
\left.\right|_{v=n+1} ^{n+k} \sum_{v}^{\prime} A_{v} \cdot\left(b_{r}-b_{v+1}\right) \mid \leqq K^{\prime} \cdot \sum_{v=n+1}^{n+k} b_{v}-b_{r+1},
$$

woraus nun infolge der jetzigen V'oraussetzungen sofort die Gleichmäßigkeit der Konvergenz der neuen Reihe in $J$ abzulesen ist.

Beispiele und Erläuterungen.

199. 1. Bei den Anwendungen wird oft eine der beiden Funktionenfolgen, meist $a_{n}(x)$, sich auf eine Zahlenfolge reduzieren. Wenn dann $\Sigma a_{v}$ iberhaupt konvergiert, so ist diese Reihe natürlich als in jedem Intervall gleichmäßig konvergent anzusehen. Denn ihre Glieder und also auch ihre Teilstiicke hängen ja gar nicht mehr von $x$ ab und jede Abschätzung gilt also eo ipso für alle $x$. Ebenso gelten dann die Teilsummen von $\Sigma^{\prime} a_{v}$, falls iiberhaupt beschränkt, auch als gleichmäßig beschränkt in jedem Intervall.

2. Es sei $\left(a_{n}\right)$ eine Zahlenfolge, $\underset{*}{ } a_{n}$ konvergent und $b_{n}(x)=x^{n}$. Dann ist $\Sigma a_{n} x^{n}$ in $0<x \leq 1$ gleichmäßig konvergent. Denn dic Bedingungen des Abelschen Kriteriums sind in diesem Intervall erfüllt. In der Tat ist $\Sigma a_{n}$, wie unter 1. bemerkt, gleichmäßig konvergent; es ist ferner bei festem $x$ die Folge $\left(x^{n}\right)$ monoton und iiberdies ist für alle $n$ und alle $x$ des Intervalls stets 
$\left|x^{n}\right| \leqq 1$. - Nach dem Satz 194 vom gliedweisen Grenzübergang können wir also schliefenen, daßs

$$
\begin{aligned}
\lim _{x \rightarrow 1-0}\left(\Sigma a_{n} x^{n}\right) & =\Sigma\left(\lim _{x \rightarrow 1-0} a_{n} x^{n}\right), \quad \text { d. h. } \\
& =\Sigma a_{n}
\end{aligned}
$$

ist. Wir haben damit einen neuen Beweis des Abelschen Grenzwertsatzes 100.

3. Ebenso sind auch die Folgen $b_{n}(x)=\frac{1}{n^{x}}$ bei festem $x$ monoton und für alle $x \geq 0$ gleichmäßig beschränkt (nämlich wieder $\leqq 1$ ). Daher schliefit man ganz ähnlich wie eben, daf

$$
\lim _{x \rightarrow+0} \sum \frac{a_{n}}{n^{x}}=\sum a_{n}
$$

wenn $\Sigma a_{n}$ eine konvergente Reihe mit konstanten Gliedern bedeutet. ( $A$ belscher Grenzwertsatz für Divichletsche Reihen.)

4. Sei $a_{n}(x)=\cos n x$ oder $=\sin n x$ und $b_{n}(x)=\frac{1}{n^{\alpha}}, \alpha>0$. Dann erfiillt die Reihe

$$
\sum_{n=1}^{\infty} a_{n}(x) \cdot b_{n}(x)=\sum_{n=1}^{\infty} \frac{\cos n x}{n^{\alpha}} \quad \text { bzw. } \quad \sum_{n=1}^{\infty} \frac{\sin n x}{n^{\alpha}}, \quad(\alpha>0),
$$

die Bedingungen des Divichletschen Kriteriums in jedem Intervall der Form $\delta \leqq x \leqq 2 \pi-\delta^{1}$ ), bei der $\delta$ eine positive Zahl $<\pi$ bedeuten soll.

In der Tat sind nach 185, 5 die Teilsummen von $\Sigma a_{n}(x)$ dort gleichmäßig beschränkt $\left(K\right.$ kann $=\frac{1}{\sin \frac{1}{2} \delta}$ genommen werden $)$ und $b_{n}(x)$ strebt monoton $\rightarrow 0$, und zwar eo ipso gleichmäßig, weil die $b_{n}$ nicht von $x$ abhängen. Bedeuten die $b_{n}$ irgendeine monotone Nullfolge, so sind aus denselben Gründen und in denselben Intervallen auch die Reihen

$$
\Sigma b_{n} \cos n x \text { und } \Sigma b_{n} \sin n x
$$

gleichmäßig konvergent (vg1. dazu 185, 5). - Alle diese Reihen stellen also Funktionen dar, die für alle $x \neq 2 k \pi$ definiert und stetig sind ${ }^{2}$ ). Ob diese Stetigkeit sich auch noch auf die oben ausgeschlossenen Punkte $x=2 k \pi$ erstreckt, ist nicht $z u$ sehen, - auch bei der Reihe $\Sigma b_{n} \sin n x$ nicht, obwohl diese in diesen Punkten konvergiert (vgl. 216,4).

\section{$\$ 49$. Fouriersche Reihen.}

\section{A. Die Eulerschen Formeln.}

Eins der wichtigsten und auch in sich interessantesten Anwendungsfelder der allgemeinen Untersuchungen, die wir in den vorangegangenen Paragraphen entwickelt haben, bietet die Theorie der

1) Oder in denjenigen Intervallen, die aus dem obigen durch eine Verschiebung um ein ganzzahliges Vielfaches von $2 \pi$ entstehen.

$\left.{ }^{2}\right)$ Jedes feste $x \neq 2 k \pi$ kann in der Tat als in einem Intervall der obigen Form liegend angesehen werden, wenn $\delta$ passend gewählt wird. (Vgl. S. 331, Beisp. 1, und S. 333, Beisp. 2.) 
Fourierschen Reihen und allgemeiner die der trigonomeirischen Reihen, auf die wir nun eingehen wollen ${ }^{1}$ ).

Unter einer trigonometrischen Reihe soll jede Reihe der Form

$$
{ }_{2}^{1} a_{0}+\sum_{n=1}^{\infty}\left(a_{n} \cos n x+b_{n} \sin n x\right)
$$

mit konstanten $a_{n}$ und $b_{n}$ verstanden werden ${ }^{2}$ ). Wenn eine solche Reihe in einem Intervall der Form $c \leqq x<c+2 \pi$ konvergiert, so konvergiert sie wegen der Periodizität der trigonometrischen Funktionen für alle reellen $x$ und stellt also eine für alle $x$ definierte Funktion dar, die die Periode $2 \pi$ besitzt. Wir haben schon verschiedene durchweg konvergente trigonometrische Reihen kennen gelernt, so z. B. eben erst die Reihen

$$
\sum_{n=1}^{\infty} \frac{\sin n x}{n^{\alpha}}, \quad \alpha>0 ; \quad \sum_{n=1}^{\infty} \frac{\cos n x}{n^{\alpha}}, \quad \alpha>1 ; \quad \text { u. a. }
$$

Bei keiner von ihnen waren wir bisher in der Lage, die Summe für jedes $x$ anzugeben. Doch werden wir nun sehr bald sehen, daß solche trigonometrischen Reihen die merkwürdigsten Funktionen darzustellen vermögen, - Funktionen, denen man noch zu Eulers Zeiten gar nicht den Namen einer Funktion beizulegen gewagt hätte, da sie die mannigfachsten Unstetigkeiten und sonstigen Unregelmäßigkeiten aufweisen können und darum eher ein Flickwerk aus mehreren Funktionen als eine Funktion zu sein schienen.

So werden wir gleich nachher sehen, dafi z. B. die Summe der Reihe

$$
\sum_{n=1}^{\infty} \frac{\sin n x}{n}\left\{\begin{array}{l}
=0 \text { ist für } x=k \pi, \quad(k=0, \pm 1, \pm 2, \ldots), \text { dagegen } \\
=\frac{(2 k+1) \pi-x}{2} \text { ist für } 2 k \pi<x<2(k+1) \pi,
\end{array}\right.
$$

daf 3 also die durch diese Reihe dargestellte Funktion das folgende Bild bietet

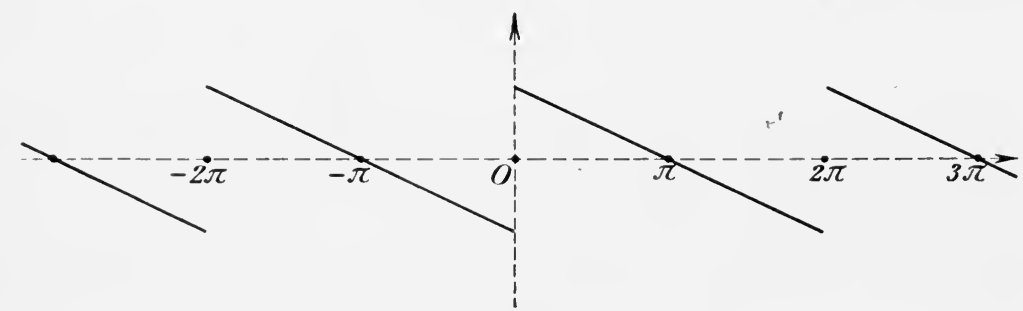

Fig. 7 .

$\left.{ }^{1}\right)$ Mehr oder weniger ausführliche Darstellungen der Theorie findet man in fast allen größeren Lehrbüchern der Differentialrechnung (insbesondere dem S. 2 genannten von H. v. Mangoldt, Bd. 3, 2. Aufl. (1920), 8. Abschn.). Als Einzeldarstellung sei auf $H$. Lebesgue, Leç. sur les séries trigonométriques, Paris 1906, und die besonders elementare Introduction to the theory of Fourier's series von $M$. Bôcher, Annals of Math., Bd. (2) 7 (1906), S. 81-152, hingewiesen. Besonders eingehend ist die Theorie dargestellt in E. W. Hobson, The theory of fonctions of a real variable and the theory of Fourier series, Cambridge 1907, und in H. Hahn, Theorie der reellen Funktionen, Berlin 1921. - 2) Dafs man $\frac{1}{2} a_{0}$ statt $a_{0}$ schreibt, geschieht lediglich aus Zweckmäßigkeitsgründen. 
199. $§ 49$. Fouriersche Reihen. - A. Die Eulerschen Formeln.

Und ebenso werden wir sehen, das

$$
\sum_{n=1}^{\infty} \frac{\sin (2 n+1) x}{2 n+1}\left\{\begin{array}{l}
=0 \text { ist für } x=k \pi, \text { dagegen } \\
=\frac{\pi}{4} \text { für } 2 k \pi<x<(2 k+1) \pi \text { und } \\
=-\frac{\pi}{4} \text { für }(2 k+1) \pi<x<2(k+1) \pi
\end{array}\right.
$$

und dafs die dargestellte Funktion also etwa das folgende Bild liefert:

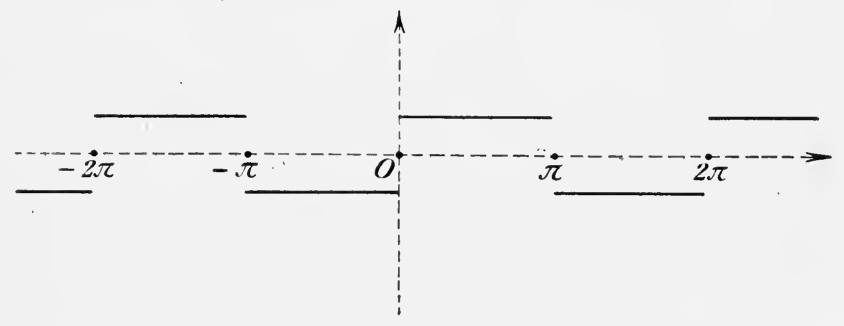

Fig. 8 .

Beidemal besteht das Funktionsbild aus getrennten (beiderseits nicht abgeschlossenen) Strecken und isolierten Punkten.

Aber gerade der Umstand, daß durch so einfache trigonometrische Reihen wie die eben genannten ganz unstetige und „zusammengestückte" Funktionen dargestellt werden können, hat im Anfang des 19. Jahrhunderts einen Hauptanlaß dazu gebildet, den Funktionsbegriff und damit überhaupt die Grundlagen der höheren Analysis einer genauen Revision zu unterziehen. Wir werden sehen, daß die trigonometrischen Reihen die meisten der sogenannten „willkürlichen“ Funktionen darzustellen vermögen ${ }^{\mathbf{1}}$ ), und daß sie also ein in dieser Hinsicht den Potenzreihen weit überlegenes Werkzeug der höheren Analysis bilden.

1) Der Begriff der "willkürlichen" Funktion ist natürlich kein fest umrissener. Man meint damit im allgemeinen Funktionen, die nicht durchweg durch ein und dieselbe geschlossene (d. h. die Anwendung von Grenzübergängen vermeidende) Formel gegeben werden können unter ausschlieflicher Benutzung der sogenannten elementaren Funktionen, - meint also insbesondere Funktionen damit, die aus verschiedenen Stiicken solcher einfacheren Funktionen zusammengesetzt erscheinen, wie die oben im Text als Beispiele gegebenen oder wie die folgenden fur alle reellen $x$ definierten Funktionen:

$$
\begin{aligned}
& \left.\begin{array}{l}
f(x)=k \\
f(x)=x^{2}-k^{2}+k \\
f(x)=x-k
\end{array}\right\} \begin{array}{l}
\text { in } k \leqq x<k+1 \\
(k=0, \pm 1, \pm 2, \ldots)
\end{array} \\
& f(x)\left\{\begin{array}{l}
=0 \text { für irrationale } x \\
=x \text { für rationale } x,
\end{array}\right.
\end{aligned}
$$

usw. - Vgl. jedoch dazu die S. 318, Fufnote, durch Grenziibergänge dargestellte "willkiirliche" Funktion. Erst seit man erkannt hat, daf auch eine solche ganz "willkiirliche" Funktion durch einen (noch relativ einfachen) Ausdruck, 
Nur beiläufig können wir erwähnen, daß das Anwendungsfeld dieses Werkzeugs keineswegs auf das Gebiet der reinen Mathematik beschränkt ist. Ganz im Gegenteil: In der theoretischen Physik bei der Untersuchung periodischer Bewegungen, also vor allem in der Akustik, Optik, Wärmelehre, wurde man zuerst auf solche Reihen geführt; und in seiner Théorie de la chaleur (1811) hat Fourier gewisse trigonometrische Reihen zum ersten Male etwas gründlicher untersucht, - freilich ohne daß er eins der grundlegenden Resultate ihrer Theorie schon gefördert hätte.

Welche Funktionen können nun durch trigonometrische Reihen dargestellt wcrden, und wie findet man die Darstellung einer gegebenen Funktion, falls sie ïberhaupt möglich ist?

Um zur Beantwortung dieser Frage einen Weg zu eröffnen, nehmen wir zunächst einmal an, wir hätten eine bestimmte Funktion $f(x)$ durch eine überall konvergente trigonometrische Reihe dargestellt:

$$
f(x)={ }_{2}^{1} a_{0}+\sum_{n=1}^{\infty}\left(a_{n} \cos n x+b_{n} \sin n x\right) .
$$

Wegen der Periodizität der trigonometrischen Funktionen ist dann auch $f(x)$ notwendig periodisch mit der Periode $2 \pi$, und wir können daher unsere Betrachtung auf irgendein Intervall der Länge $2 \pi$ beschränken. Wir wählen für alles folgende das Intervall $0 \leqq x \leqq 2 \pi$, von dem wir übrigens noch den einen Endpunkt weglassen dürften.

Die Funktion $f(x)$ wird dann in diesem Intervall durch eine konvergente Reihe stetiger Funktionen dargestellt. Wir wissen, daß trotzdem $f(x)$ unstetig sein kann, daß es aber gleichfalls stetig sein muß, wenn die Reihe in dem genannten Intervall sogar gleichmäßig konvergiert. Für den Augenblick wollen wir auch dies noch voraussetzen.

Es sei also die Reihe

$$
{ }_{2}^{1} a_{0}+\sum_{n=1}^{\infty}\left(a_{n} \cos n x+b_{n} \sin n x\right) \quad \text { in } \quad 0 \leqq x \leqq 2 \pi
$$

gleichmäßig konvergent ${ }^{1}$ ) und habe $f(x)$ zur Summe.

Unter diesen Voraussetzungen ergibt sich ein Zusammenhang zwischen $f(x)$ und den einzelnen Koeffizienten $a_{n}$ und $b_{n}$, den schon Euler vermutet hat. Es gilt darüber der folgende

wie cben jetzt unsere trigonometrischen Reihen oder andere Grenzübergänge, dargestellt werden kann, sah man sich gezwungen, in ihr wirklich eine Funktion und nicht ein Flickwerk aus mehreren anzuerkennen.

1) Wegen der Periodizität von $\cos x$ und $\sin x$ ist sie dann von selbst für alle $x$ gleichmäßig konvergent. 
200. $§ 49$. Fouriersche Reihen. - A. Die Eulerschen Formeln.

Satz 1. Unter den gemachten Voraussetzungen ist für $n=0,1,2, \ldots$

$$
\boldsymbol{r}_{n}=\frac{1}{\pi} \int_{0}^{\frac{2}{\pi}} f(x) \cos n x \boldsymbol{l}\left(x, \quad b_{n}=\frac{1}{\pi} \int_{0}^{2} f^{*}(x) \sin n x d x .\right.
$$

\section{(Eulersche Formeln) ${ }^{1}$ ).}

Beweis. Aus den Elementen her ist bekannt, daß für ganzzahlige $p$ und $q(\geqq 0)$ die folgenden Formeln gelten $\left.{ }^{2}\right)$ :

$$
\begin{aligned}
& \text { a) } \quad \int_{0}^{2 \pi} \cos p x \cdot \cos q x d x \begin{cases}=0, & \text { für } p \neq q \\
=\pi, & \text { für } p=q>0 \\
=2 \pi, & \text { für } p=q=0\end{cases} \\
& \text { b) } \quad \int_{0}^{2 \pi} \cos p x \cdot \sin q x d x=0 \\
& \text { c) } \quad \int_{0}^{2 \pi} \sin p x \cdot \sin q x d x\left\{\begin{array}{l}
=0 \text { für } p \neq q \text { und } p=q=0 \\
=\pi \text { für } p=q>0 .
\end{array}\right.
\end{aligned}
$$

Multipliziert man nun die nach Voraussetzung in $0 \leqq x \leqq 2 \pi$ gleichmäßig konvergente Reihen für $f(x)$ mit $\cos p x$, so wird dadurch die Gleichmäßigkeit der Konvergenz nicht gestört, da ja auch die Reste der Reihe nur mit $\cos p x$ multipliziert und also ihrem Betrage nach nicht vergrößert werden. Man darf daher (s. 195) nach dieser Multiplikation gliedweis von 0 bis $2 \pi$ integrieren und erhält dann sofort

$$
\int_{0}^{2 \pi} f(x) \cos p x d x\left\{\begin{array}{l}
=\frac{1}{2} a_{0} \cdot \int_{0}^{2 \pi} \cos p x d x \text { für } p=0, \\
=a_{p} \cdot \int^{2 \pi} \cos p x \cdot \cos p x d x \text { für } p>0 .
\end{array}\right.
$$

also in beiden Fällen

$$
a_{p}=\frac{1}{\pi} \int_{0}^{2 \pi} f(x) \cos p x d x
$$

denn alle übrigen Glieder der Reihe lieferten bei der Integration den Wert 0 . Ebenso liefert die Multiplikation der vorausgesetzten Reihendarstellung von $f(x)$ mit $\sin p x$ und die nachfolgende Integration sofort die zweite der Eulerschen Formeln

$$
b=\int_{\pi}^{1} \int_{0}^{2 \pi} f(x) \sin p x d x .
$$

1) Diese Bezeichnung ist eine mehr konventionelle; geschichtliche Bemerkungen gibt H. Lebesgue, a. a. O. S. 23, und in großer Ausführlichkeit A. Sachse, Versuch einer Geschichte der trigonometrischen Reihen, Inaug.-Diss. Göttingen 1879, sowie $P . d u$ Bois-Reymond in seiner Entgegnung (Tuibingen, 1880) auf die vorgenannte Schrift.

2) Nach den bekannten Additionstheoremen hat man nur das Produkt der beiden im Integranden stehenden Funktionen in eine Summe $z \mathbf{u}$ verwandeln (z. B. $\left.\cos p x \cdot \cos q x=\frac{1}{2}[\cos (p-q) x+\cos (p+q) x]\right)$, um dann unmittelbar integrieren zu können. 
Der Wert dieses Satz 1 ist zunächst noch durch die zu vielen Voraussetzungen beeinträchtigt, die wir zur Durchführung des Beweises machen mußten, und er sagt insbesondere noch nichts darüber aus, ob denn eine vorgelegte Funktion überhaupt in eine trigonometrische Reihe entwickelbar ist, und welchen Wert in diesem Falle die Koeffizienten haben.

Immerhin weist uns der Satz auf folgenden Weg: Es sei $f(x)$ eine ganz beliebige im Intervall $0 \leqq x \leqq 2 \pi$ definierte Funktion, die dort nur im Riemannschen Sinne integrierbar sein soll. Dann haben jedenfalls die in den Eulerschen Formeln auftretenden Integrale einen Sinn und liefern bestimmte Zahlen $a_{n}$ und $b_{n}$. Diese letzteren haben also unter der alleinigen Voraussetzung der Integrierbarkeit von $f(x)$ ganz bestimmte Werte. Wir nennen die so erhaltenen Zahlen $\frac{1}{2} a_{0}, a_{\mathbf{1}}, a_{\mathbf{2}}, \ldots$ und $b_{1}, b_{2}, \ldots$ die Fourierkonstanten oder Fourierkoeffizienten der Funktion $f(x)$. Mit ihrer Hilfe kann man nun jedenfalls die Reihe

$$
\frac{1}{2} a_{0}+\sum_{n=1}^{\infty}\left(a_{n} \cos n x+b_{n} \sin n x\right)
$$

ansetzen, d. h. hinschreiben, ohne damit schon etwas über ihr Konvergenzverhalten aussagen zu wollen. Diese Reihe nennen wir (also ohne Rücksicht auf ihr Konvergenzverhalten und auf ihre etwaige Summe) die durch $\boldsymbol{f}(\boldsymbol{x})$ erzeugte Fourierreihe oder kurz die Fourierreihe von $f(x)$ und schreiben in diesem Sinne

$$
\left.f(x) \sim \frac{1}{2} a_{0}+\sum_{n=1}^{\infty}\left(a_{n} \cos n x+b_{n} \sin n x\right)^{1}\right),
$$

was nur dahin zu verstehen ist, daß aus der lediglich als integrierbar vorausgesetzten Funktion $f(x)$ mit Hilfe der Eulerschen Formeln gewisse Konstante $a_{n}$ und $b_{n}$ gewonnen wurden und dann die obige Reihe hingeschrieben worden ist.

Die Herkunft der Reihe in Verbindung mit dem Satz 1 läßt nun allerdings die Hoffnung einigermaßen begründet 'erscheinen, daß sie konvergiert und die Funktion $f(x)$ zur Summe hat.

Das ist aber im allgemeinen leider nicht der Fall. (Beispiele werden wir sehr bald kennen lernen.) Vielmehr braucht die Reihe weder im ganzen Intervall noch auch nur an einzelnen Punkten zu konvergieren; und wenn sie es tut, so braucht ihre Summe nicht $=f(x)$ zu sein. Aber es ist nicht ohne weiteres zu sagen, wann das eine und wann das andere eintritt; und gerade dieser Umstand macht die Theorie der Fourierreihen auf der einen Seite zu einem nicht ganz einfachen Gegenstande, - macht sie auf der andern Seite aber auch außerordentlich reizvoll, weil hier ganz neue Probleme auftauchen und weil

1) Das Zeichen " $\sim$ " hat hier natürlich nichts mit dem 40, Definition 5, eingeftihrten Zeichen für "asymptotisch proportional" zu tun. Verwechslungen sind nicht $z u$ befürchten. 
wir auf eine, wie es scheint, wesentlich neue Fundamentaleigenschaft der Funktionen stoßen, nämlich die: eine konvergente Fouriem:ihe zu liefern, deren Summe die Funktion selber ist. Und es gilt dann weiter den Zusammenhang dieser neuen Eigenschaft mit den andern der Stetigkeit, der Monotonie, der Differenzierbarkeit, der Integrierbarkeit usw. - zu klären. Konkreter gefaßt tauchen also die folgenden Fragen auf:

1. Ist die Fourierreihe einer gegebenen (integrierbaren) Funktion $f(x)$ für gewisse oder für alle Werie von $x$ in $0 \leqq x \leqq 2 \pi k$ snvergent?

2. Hat die Fourierreihe von $f(x)$ im Falle der Konvergenz den Wert $f(x)$ der erzengenden Funktion zur Summe?

3. Ist die Konv.rgenz der Fourierrihe, falls sie in a'len Punk'en eines Intervalles $\alpha \leqq x \leqq \beta$ stattfindet, dor eine gleichmäßige?

Und da es weiter auch denkbar wäre, daß man auf einem andern Wege als über die Eulerschen Formeln gleichfalls zu einer trigonometrischen Entwicklung gegebener Funktionen gelangt, so werfen wir gleich noch die folgende weitere Frage auf:

4. Kann eine integrierbare Funktion, die überhaup: eine trigonometrische Entwicklung gestattet, deren mehrere besitzon, insbesondere a'so noch eine andere neben der eventuell durch die Eulerschen Formaln gelieferten Darstellung durch die Fourierreihe?

Auf alle diese Fragen ist es nicht ganz leicht, Antworten zu geben, und vollständig ist bis heute noch keine von ihnen beantwortet. Es würde uns zu weit führen, alle vier Fragen dem heutigen Stande der Wissenschaft entsprechend $\mathrm{zu}$ behandeln. Wir wollen uns vor allem den ersten beiden zuwenden, die dritte gelegentlich streifen und die vierte ganz bei Seite lassen ${ }^{\mathbf{1}}$ ).

Mit den nunmehr eingeführten Bezeichnungen können wir den Inhalt des Satz 1 auch so aussprechen:

Satz 1a. Wenn eine trigonometrische Reihe in $0 \leqq x \leqq 2 \pi$ gleich mäßig konvergier:, so ist sie die Fourierreihe der durch sie dargestellten Funktion; oder - was auf dasselbe hinauskommt - so sind ihre Koeffizienten die Fourierkonstanten ihrer Summe ${ }^{2}$ ).

Daß die Fourierreihen einer integrierbaren Funktion nicht zu konvergieren braucht, werden wir weiter unten sehen; und dafi sie auch im Falle

') Doch sei bemerkt, dafi die 4. Frage unter sehr allgemeinen Voraussetzungen dahin beantwortet ist, daf $z$ wei in $0 \leq x \leq 2 \pi$ konvergente trigonometrische Entwicklungen nicht dieselbe Funktion darstellen können, wenn sie nicht völlig miteinander identisch sind. Und ist dann die durch sie dargestellte Funktion $f(x)$ über $0 \ldots 2 \pi$ integrierbar, so sind ihre Koeffizienten die Fourierkoeffizienten von $f(x)$; vgl. G. Cantor (J. f. d. reine u. angew. Math., Bd. 72, 1870, S. 139) und P. du Bois-Reymond (Münch. Abh., Bd. 12, 1876, I. Abt., S. 117). Funktion.

2) Diese Summe ist dann eo ipso eine in $0 \leqq x \leq 2 \pi$, also überall, stetige 
der Konvergenz nicht den Wert von $f(x)$ zur Summe zu haben braucht, folgt ja schon daraus, daf zwei verschiedene Funktionen $f_{1}(x)$ und $f_{2}(x)$ sehr woh! genau dieselben Fourierkonstanten haben können; denn zwei integrierbare Funktionen liefern schon dann dieselben Integrale (und also auch dieselben Fourierkonstanten, und also auch dieselben Fourierreihen), wenn sie - ohne ganz übereinzustimmen -, z. B. nur für alle rationalen Werte von $x$ denselben Wert haben (s. $\$ 19$, Satz 18). Auch daf die Konvergenz in den Konvergenzintervallen keine gleichmäßige $z u$ sein braucht, können wir schon jetzt sehen, denn die Reihe $\sum \frac{\sin n x}{n}$ ist überall konvergent (s. 185, 5) und wiirde, falls die Konvergenz z. B. in $-1 \leqq x \leqq+1$ gleichmäfaig wäre, nach 193 dort eine stetige Funktion darstellen miissen. Das ist aber, wie wir schon S. 338 betonten und weiter unten S. 362 beweisen werden, nicht der Fall.

Diese wenigen Bemerkungen zeigen schon, daß die aufgeworfenen Fragen nicht ganz einfacher Natur sind. Wir folgen in ihrer Beantwortung zunächst dem Vorgange von G. Lejeune-Dirichlet, der in seiner Arbeit Sur la convərgence des séries trigonomélriques ${ }^{\mathbf{1}}$ ) den ersten wesentlichen Schritt zur Erledigung der aufgeworfenen Fragen getan hat.

\section{B. Das Dirichletsche Integral.}

Wir greifen zunächst die erste der aufgeworfenen Fragen, die Konvergenzfrage an: Soll die durch eine gegebene integrierbare Funktion $f(x)$ erzeugte Fourierreihe $\frac{1}{2} a_{0}+\underline{I}\left(a_{n} \cos n x+b_{n} \sin n x\right)$, in der also $a_{n}$ und $b_{n}$ die durch die Eulerschen Formeln gelieferten Zahlen bedeuten, für $x=x_{0}$ konvergieren, so muß die Folge ihrer Teilsummen

$$
s_{n}\left(x_{0}\right)=\frac{1}{2} a_{0}+\sum_{\nu=1}^{n}\left(a_{\nu} \cos \nu x_{0}+b_{\nu} \sin \nu x_{0}\right)
$$

für $n>+\infty$ einem Grenzwert zustreben. Die Feststellung nun, ob dies eintritt oder nicht, wird oft dadurch möglich, daß man $s_{n}\left(x_{0}\right)$ folgendermaßen durch ein bestimmtes Integral darstellt:

$$
\begin{aligned}
& \text { Für } v \geqq 1 \text { ist } a_{v}, \cos v x_{0}+b_{\nu} \sin v x_{0} \\
& \left.\frac{1}{\pi} \int_{0}^{2 \pi} f(t) \cos v t d t\right] \cos v x_{0}+\left[\int_{\pi}^{1} \int_{0}^{2 \pi} f(t) \sin v t d t_{1}\right] \sin v x_{0} \\
& \left.=\frac{1}{\pi} \int_{0}^{2 \pi} f(t) \cdot \cos v\left(t-x_{0}\right) d t^{2}\right)
\end{aligned}
$$

und also die Teilsumme

$$
\begin{aligned}
& s_{n}\left(x_{0}\right)=\frac{1}{2 \pi} \int_{0}^{2 \pi} f(t) d t+\int_{\pi}^{1} \int_{0}^{2 \pi} f(t) \cos \left(t-x_{0}\right) d t+\ldots \\
& \quad+\frac{1}{\pi} \int_{0}^{2 \pi} f(t) \cdot \cos n\left(t-x_{0}\right) d t \\
& =\frac{1}{\pi} \int_{0}^{2 \pi} f(t) \cdot\left[\frac{1}{2}+\cos \left(t-x_{0}\right)+\cos 2\left(t-x_{0}\right)+\ldots+\cos n\left(t-x_{0}\right)\right] d t . \\
& \text { 1) Journal f. d. reine u. angew. Math., Bd. 4 (1829), S. 157. } \\
& \text { 2) Zur besseren Unterscheidung von dem festgehaltenen Punkte } x_{0} \text { be- } \\
& \text { zeichnen wir von jetzt ab die Integrationsveränderliche mit } t \text {. }
\end{aligned}
$$


201. $\$ 49$. Fouriersche Reihen. - B. Das Dirichletsche Integral.

Das Entscheidende ist nun, daß man jetzt die in der eckigen Klammer stehende $(n+1)$-gliedrige Summe geschlossen angeben kann, denn es gilt für beliebige $z \neq 2 k \pi$, beliebige $\boldsymbol{c}$ und positiv-ganze $m$ die Formel

$$
\begin{gathered}
\cos (u+z)+\cos (u+2 z)+\cdots+\cos (\alpha+m z)= \\
\sin \left(u+\frac{\left.2 m+1 \frac{z}{2}\right)-\sin \left(\ell+\frac{z}{2}\right)}{2 \sin \frac{z}{2}}=\sin m \frac{z}{2} \cdot \cos \left(u+m+1 \frac{z}{2}\right)\right. \\
\sin \frac{z}{2}
\end{gathered}
$$

aus der sich durch Spezialisierung viele analoge herleiten lassen²). Nach ihr ist (für $c=0, \quad z=t-x_{0}, \quad m=n$ )

$$
\begin{gathered}
\frac{1}{2} \cos \left(t-x_{0}\right)+\ldots+\cos n\left(t-x_{0}\right) \\
=\frac{1}{2}+\frac{\sin (2 n+1) \frac{t-x_{0}}{2}-\sin \frac{t-x_{0}}{2}}{2 \sin \frac{t-x_{0}}{2}}=\frac{\sin (2 n+1)^{t-x_{0}}}{2} \\
2 \sin ^{t-x_{0}}
\end{gathered}
$$

1) Beweis. Bezeichnet man die linksstehende Summe mit $C_{m}$, so ist

$$
\begin{aligned}
& 2 \sin \underset{2}{z} \cdot C_{m}=\underline{\perp}_{v=1}^{m} \cdot 2 \sin \frac{\sim}{2} \cos (\epsilon-r) \\
& \left.=\sum_{v=1}^{m}-\sin \left(c+2 v-1 \frac{z}{2}\right)-\sin \left(c+2 v+1 \cdot \frac{z}{2}\right)\right] \\
& =-\sin \left(\begin{array}{ll}
a & z \\
2
\end{array}\right)+\sin \left(\begin{array}{llll}
c & 2 m & \cdots & z \\
z
\end{array}\right) \\
& =2 \sin m \frac{z}{2} \cdot \cos \left(\omega+\bar{m}+1 \frac{z}{2}\right) \text {. }
\end{aligned}
$$

Übrigens gilt die obige Formel auch noch für $z=2 k . \tau$, wenn man dann unter den rechtsstehenden Quotienten deren Grenzwert für $z \rightarrow 2 k_{\boldsymbol{\tau}}$, also den Wert $m \cos \alpha$, versteht.

) Wir nennen späteren Anwendungen zuliebe die folgenden:

1. $\frac{\pi}{2}+๘$ statt $\lll$ liefert: $\sin (\varkappa+z)+\sin (\omega+2 z)+\ldots+\sin (\kappa+m z)$

$$
=\frac{\sin m \frac{z}{2} \cdot \sin \left(c+m+1 \frac{z}{2}\right) ;}{\sin \frac{z}{2}}
$$

2. $\alpha=0$ liefert $: \cos z-\cos 2 z \ldots \ldots \cos m z=\sin _{2}^{z} \cos m+1 \frac{z}{2}$

$$
\begin{aligned}
& \text { ferner: }
\end{aligned}
$$

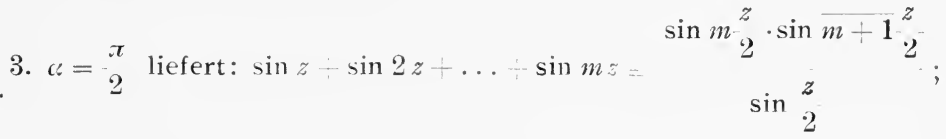$$
\sin \frac{z}{2}
$$ 
und folglich

$$
\left.s_{n}\left(x_{0}\right)=\frac{1}{2 \pi} \int_{0}^{2 \pi} f(t) \cdot \frac{\sin (2 n+1) \frac{t-x_{0}}{2}}{\sin \frac{t-x_{0}}{2}} d t^{1}\right) .
$$

Endlich kann man auch dies noch ein wenig umformen. Die Funktion $f(x)$ braucht nur in $0 \leqq x \leqq 2 \pi$ definiert und über dies Intervall integrierbar zu sein. Letzteres bleibt sie, wenn der Funktionswert $f(2 \pi)$ irgendwie abgeändert wird (vgl. § 19, Satz 17). Wir setzen ihn $=f(0)$ und dann allgemein für $2 k \pi \leqq x \leqq 2(k+1) \pi,(k= \pm 1, \pm 2, \ldots)$ stets

$$
f(x)=f(x-2 k \pi) .
$$

Dann ist $f(x)$ für alle reellen $x$ definiert und zu einer Funktion mit der Periode $2 \pi$ geworden. Für eine solche mit $2 \pi$ periodische Funktion $\varphi(x)$ ist aber (nach $\S 19$, Satz 19) bei beliebigem $c$ und $c^{\prime}$ stets $\int_{0}^{2 \pi} \varphi(t) d t=\int_{c}^{c+2 \pi} \varphi(t) d t=\int_{0}^{2 \pi} \varphi\left(c^{\prime}+t\right) d t \quad$ und $\quad \int_{\alpha}^{\beta} \varphi(t) d t=\int_{\alpha+2 \pi}^{\beta+2 \pi} \varphi(t) d t$, und da nun der Integrand in (a) eine solche Funktion ist, so hat man auch

$$
s_{n}\left(x_{0}\right)=\frac{1}{2 \pi} \int_{0}^{2 \pi} f\left(x_{0}+t\right) \cdot \frac{\sin (2 n+1)^{t}}{\sin _{2}^{t}} d t .
$$

Zerlegt man endlich dieses Integral in die beiden Teile von 0 bis $\pi$ und von $\pi$ bis $2 \pi$ und ersetzt im zweiten $t$ durch $-t$, so wird zunächst dieser zweite Teil (nach $\$ 19$, Satz 25 )

$$
=-\frac{1}{2 \pi} \int_{-\pi}^{-2 . \pi} f\left(x_{0}-t\right) \cdot \frac{\sin (2 n+1) \frac{t}{2}}{\sin \frac{t}{2}} d t .
$$

also gemäß der obigen Bemerkung über $\int_{a}^{\beta} \gamma(t) d t$ wieder

$$
=\frac{1}{2 \pi} \int_{0}^{+\pi} f\left(x_{0}-t\right) \cdot \sin (2 n+1) \frac{t}{2} d t .
$$

4. $z=2 x, \quad \alpha=y-x$ liefern:

$\cos (\gamma+x)+\cos (\gamma+3 x)+\ldots+\cos (\gamma+2 m-1 \cdot x)=\frac{\sin m x \cdot \cos (\gamma+m x)}{\sin x}$,

5. $z=2 x, \quad \Leftrightarrow=\frac{\pi}{2}+y-x$ liefern analog:

$\sin (y+x)+\sin (y+3 x)+\ldots+\sin (y+\overline{2 m-1} \cdot x)=\frac{\sin m x \cdot \sin (y+m x)}{\sin x}$.

1) Für $t=x_{0}$ ist, wie schon einmal betont, unter dem sin-Quotienten die Zahl $2 n+1$ zu verstehen, die man für $t \rightarrow x_{0}$ aus ihm erhält. 
202. §49. Fouriersche Reihen. - B. Das Dirichletsche Integral.

so $\mathrm{da}$ nun

$$
s_{n}\left(x_{0}\right)=\frac{1}{\pi} \int_{0}^{\pi} \frac{f\left(x_{0}+t\right)+f\left(x_{0}-t\right)}{2} \cdot \frac{\sin (2 n+1) \frac{t}{2}}{\sin \frac{t}{2}} d t
$$

wird, was endlich noch, wenn man $t$ durch $2 t$ ersetzt, auf die Form

$$
s_{n}\left(x_{0}\right)=\frac{2}{\pi} \int_{0}^{\frac{\pi}{2}} \frac{f\left(x_{0}+2 t\right)+f\left(x_{0}-2 t\right)}{2} \cdot \frac{\sin (2 n+1) t}{\sin t} d t
$$

gebracht werden kann. Dies ist das Dirichletsche Integral ${ }^{\mathbf{1}}$ ) für die Teilsummen der durch $f(x)$ erzeugten Fourierreihe. Diese letztere wird also dann und nur dann in $x_{0}$ konvergieren, wenn unser Dirichletsches Integral für $n \rightarrow \infty$ einem Grenzwert zustrebt.

Nehmen wir die zweite Frage (S. 343) nach der Summe der sich möglicherweise als konvergent erweisenden Fourierreihe hier gleich mit hinein, so können wir also sagen: Die durch $f(x)$ erzeugte Fourierreihe ist dann und nur dann an der Stelle $x_{0}$ konvergent mit der Summe $s\left(x_{0}\right)$, wenn für $n \rightarrow \infty$ die Differenz

$$
s_{n}\left(x_{0}\right)-s\left(x_{0}\right)
$$

gegen 0 strebt, in der $s_{n}\left(x_{0}\right)$ das oben aufgestellte Dirichletsche Integral bedeutet. Aber auch diesem Ergebnis kann man eine für das weitere noch günstigere Form geben, indem man die Zahl $s\left(x_{0}\right)$ auch in die Form eines Dirichletschen Integrals kleidet. Wegen

$$
\frac{1}{2}+\cos t+\cos 2 t+\ldots+\cos n t=\frac{\sin (2 n+1) \frac{t}{2}}{\sin \frac{t}{2}}
$$

ist nämlich

$$
\int_{0}^{2 \pi} \frac{\sin (2 n+1) \frac{t}{2}}{\sin \frac{t}{2}} d t=\pi,
$$

oder also, wenn wir dieselben Umformungen vornehmen, wie bei dem allgemeinen Integral:

$$
\frac{2}{\pi} \int_{0}^{\frac{\pi}{2}} \frac{\sin (2 n+1) t}{\sin t} d t=1 .
$$
Formen

1) Als Dirichletsches Integral bezeichnet man jedes Integral von einer der

$$
\int_{0}^{a} \varphi(t) \frac{\sin k t}{\sin t} d t \quad \text { oder } \quad \int_{0}^{a} \varphi(t) \frac{\sin k t}{t} d t
$$


Multipliziert man diese Gleichung mit der Zahl $s\left(x_{0}\right)$, so wird schließlich $s_{n}\left(x_{0}\right)-s\left(x_{0}\right)=\frac{2}{\pi} \int_{0}^{\frac{\pi}{2}}\left[\frac{f\left(x_{0}+2 t\right)+f\left(x_{0}-2 t\right)}{2}-s\left(x_{0}\right)\right] \frac{\sin (2 n+1) t}{\sin t} d t$

und wir können nun als erstes wichtiges Ergebnis den folgenden Satz aussprechen:

203. Satz 2. Dafür, daß die durch eine integrierbare (also beschränkte), mit $2 \pi$ periodische Funktion $f(x)$ erzeugte Fourierreihe im Punkte $x_{0}$ konvergiere und die Summe $s\left(x_{0}\right)$ habe, ist es notwendig und hinreichend, daß für $n \rightarrow+\infty$ das Dirichletsche Integral

$$
\frac{2}{\pi} \int_{0}^{\frac{\pi}{2}} p\left(t ; x_{0}\right) \frac{\sin (2 n+1) t}{\sin t} d t
$$

gegen 0 strebe, in welchem zur Abkürzung

$$
\left[\frac{f(x+2 t)+f(x-2 t)}{2}-s(x)\right]=p(t ; x)
$$

gesetzt worden ist.

Durch diesen Satz sind $z$ war die Fragen 1 und 2 noch keineswegs in einer Weise erledigt, die in konkret vorliegenden Fällen die Antwort lieferte; aber es ist jedenfalls eine wesentlich neue Angriffsmöglichkeit zu ihrer Erledigung geschaffen. Und dies gilt sogar noch für die dritte der S. 343 aufgeworfenen Fragen. Denn Satz 2 kann sofort $z \mathfrak{u}$ dem folgenden verschärft werden:

Satz 3. Die etwaige Konvergenz der Teilsummen $s_{n}(x)$ gegen $s(x)$ wird dann und nur dann für alle $x$ eines Intervalles $\alpha \leqq x \leqq \beta$ eine gleichmäßige sein, wenn nach Wahl von $\varepsilon>0$ sich ein $N=N(\varepsilon)$ so angeben läßt, daß

$$
\left|\frac{2}{\pi} \int_{0}^{\frac{\pi}{2}} \varphi(t ; x) \cdot \frac{\sin (2 n+1) t}{\sin t} d t\right|<\varepsilon
$$

ausfällt für alle $n>N$ und alle $x$ in $\alpha \leqq x \leqq \beta$.

Ehe wir nun den Satz 2 zur Aufstellung unmittelbarer Konvergenzkriterien für Fourierreihen ausnützen, wollen wir ihn noch in verschiedener Weise umformen und vereinfachen. Dazu beweisen wir zunächst einige scheinbar etwas abliegende Sätze, die aber auch an sich ein erhebliches Interesse beanspruchen:

Satz 4. Ist $f(x)$ integrierbar, so haben seine Fourierkonstanten eine konvergente Quadratsumme $\sum_{n=1}^{\infty}\left(\boldsymbol{a}_{n}^{2}+\boldsymbol{b}_{n}^{2}\right)$.

Beweis. Das Integral

$$
\int_{0}^{2 \pi}\left[f(t)-\sum_{v=1}^{n}\left(a_{v} \cos v t+b_{\nu} \sin v t\right)\right]^{2} d t
$$


203. $§ 49$. Fouriersche Reihen. - B. Das Dirichletsche Integral.

hat einen nie negativen Integranden und ist daher $\geqq 0$. Andererseits ist es

$$
\begin{aligned}
=\int_{0}^{t}[f(t)]^{2} d t & -2 \sum\left[a_{v} \int_{0}^{2 \pi} f(t) \cos v t d t\right]-2 \sum\left[b_{v} \int_{0}^{2 \pi} f(t) \sin v t d t\right] \\
& +\int_{0}^{2 \pi}\left[\Sigma\left(a_{v} \cos v t+b_{v} \sin v t\right)\right]^{2} d t \\
= & \int_{0}^{2 \pi}[f(t)]^{2} d t-2 \pi \Sigma a_{v}^{2}-2 \pi \Sigma b_{v}^{2}+\pi \Sigma a_{v}^{2}+\pi \Sigma b_{v}^{2} \\
= & \int_{0}^{2 \pi}[f(t)]^{2} d t-\pi \Sigma\left(a_{v}^{2}+b_{v}^{2}\right),
\end{aligned}
$$

wobei die Summen stets von $\boldsymbol{v}=1$ bis $\boldsymbol{v}=n \mathrm{zu}$ erstrecken sind. $\mathrm{Da}$ nun dieser Ausdruck nicht negativ ist, so ist

$$
\sum_{v=1}^{n}\left(a_{v}^{2}+b_{v}^{2}\right) \leqq \frac{1}{\pi} \int_{0}^{2 \pi}[f(t)]^{2} d t
$$

Und da hiernach die Teilsummen der in Rede stehenden Reihe (mit positiven Gliedern) beschränkt sind, so ist die Reihe, wie behauptet, konvergent.

Ganz speziell enthält dies den folgenden

Satz 5. Die Fourierkonstanten $\left(a_{n}\right)$ und $\left(b_{n}\right)$ einer integrierbaren Funktion bilden je eine Nullfolge. - Und hieraus folgt nun in einfacher Weise weiter der

Satz 6. Ist $\psi(t)$ im Intervall $a \leqq t \leqq b$ integrierbar, so strebt stets

$$
A_{n}=\int_{a}^{b} \psi(t) \cos n t d t \rightarrow 0
$$

und

$$
B_{n}=\int_{a}^{b} \psi(t) \sin n t d t \rightarrow 0 .
$$

Beweis. Liegen $a$ und $b$ beide nicht außerhalb eines Intervalles der Form $2 q \pi \leqq t \leqq 2(q+1) \pi$, so setze man $f(t)=\psi(t)$ in $a \leqq t \leqq b$ und $f(t)=0$ in den übrigen Punkten des erstgenannten Intervalles und definiere $f(t)$ für alle übrigen reellen $t$ durch die Bedingung, da $\beta$ es die Periode $2 \pi$ haben soll. Dann ist aber

$$
A_{n}=\int_{a}^{b} \psi(t) \cos n t d t=\int_{0}^{2 \pi} f(t) \cos n t d t=\pi a_{n}
$$

und ebenso $B_{n}=\pi b_{n}$, wenn $a_{n}$ und $b_{n}$ die Fourierkonstanten von von $f(t)$ bedeuten. Nach Satz 5 streben also die $A_{n}$ und $B_{n} \rightarrow 0$. Erfüllen $a$ und $b$ nicht die eben gemachte Annahme, so wird sich das Intervall $a \leqq t \leqq b$ in endlich viele Stücke derart zerlegen lassen, 
daß jedes einzelne dieser Stücke eine solche Annahme erfüllt. Demgemäß erscheinen $A_{n}$ und $B_{n}$ in endlich viele Summanden (von fester Anzahl) zerlegt, deren jeder mit $n \rightarrow \infty$ gegen 0 strebi. Also tun dies auch die $A_{n}$ und $B_{n}$ selber $^{\mathbf{1}}$ ).

Mit Hilfe dieses wichtigen Satzes $6^{2}$ ) kann man die Konvergenzfrage beim Dirichletschen Integral weiterhin vereinfachen.

Ist zunächst $0<\delta<\frac{\pi}{2}$ beliebig gewählt, so ist die Funktion

$$
\psi(t)=\frac{p\left(t ; x_{0}\right)}{\sin t}=\frac{1 \cdot\left[f\left(x_{0}+2 t\right)+f\left(x_{0}-2 t\right)\right]-s\left(x_{0}\right)}{\sin t}
$$

in $\delta \leqq t \leqq \frac{\pi}{2}$ integrierbar und es strebt also - bei festem $\delta-$

$$
\int_{\delta}^{\frac{\pi}{2}} y(t) \sin (2 n+1) t d t \rightarrow 0
$$

Daher hat das in Satz 2 genannte Dirichletsche Integral dann und nur dann für $n \rightarrow \infty$ den Grenzwert 0 , wenn - bei festem, aber an sich beliebigem $\delta>0-$ das neue Integral

$$
\frac{2}{\pi} \int_{0}^{\delta} p\left(t ; x_{0}\right) \frac{\sin (2 n+1) t}{\sin t} d t
$$

mit wachsendem $n$ gegen 0 strebt. Da in dies letzte Integral nur die Werte von $f^{\prime}\left(x_{0} \pm 2 t\right)$ in $0 \leqq t \leqq \delta$, also nur diejenigen von $f(x)$ in $x_{0}-2 \delta \leqq x \leqq x_{0}+2 \delta$ eingehen, und da $\delta>0$ beliebig klein gedacht werden kann, so enthält dies merkwürdige Ergebnis zugleich den folgenden

204. Satz von Riemann $\left.{ }^{3}\right)$. Das Konvergenzverhalten der Fourierreihe von $f(x)$ in $x_{0}$ hängt nur von der Natur von $f(x)$ in der Umgebung von $x_{0} a b$. Diese darf dabei beliebig klein gedacht werden.

Zur Erläuterung dieses besonders eigenartigen Satzes sei noch folgende Konsequenz desselben hervorgehoben: Man betrachte alle möglichen (in $0 \ldots 2 \pi$ integrierbaren) Funktionen $f(x)$, die in einem Punkte $x_{0}$ des Intervalles $0 \ldots 2 \pi$ und einer, wenn auch noch so kleinen und bei jeder dieser Funktionen auch verschieden kleinen Umgebung desselben ubereinstimmen. Dann sind die Fourierreihen aller dieser Funktionen - mögen sie außerhalb der genannten

1) Dieser wichtige Satz 6 wird anschaulich-plausibel, wenn man sich den Verlauf der Kurve $\psi(t) \cos n t$ für ein hohes $n$ gezeichnet denkt: Greift man ein kleines Teilintervall $\alpha \ldots \beta$ heraus, indem $\psi(t)$ nur eine geringe Schwankung hat (also annähernd konstant ist), und wählt man nun $n$ so grof, dafs $\cos n t$ in diesem Intervall schon viele Schwingungen macht, so wird die Kurve $\psi(t) \cos n t$ während des Intervalles $\alpha \ldots \beta$ ungefähr gleich viele und gleich große positive wie negative Flächenstücke umschliefien, das Integral also nahezu 0 sein.

2) Natürlich kann man Satz 6 auch ohne den Umweg uiber Satz 4 ganz direkt beweisen, doch ist Satz 4 ein gleichfalls sehr wichtiger Satz der Theorie, wenn wir ihn auch in der Folge zufällig nicht weiter anwenden werden.

3) Über die Darstellbarkeit einer Funktion durch eine trigonometrische Reihe, Hab.-Schrift, Göttingen 1854 (Werke, 2. Aufl., S. 227). 
Umgebungen von $x_{0}$ auch noch so verschiedenartig sein - entweder sämtlich konvergent oder sämtlich divergent und haben im ersten Falle sämtlich dieselbe Summe $s\left(x_{0}\right)$ (die dabei $=$ oder $\neq f\left(x_{0}\right)$ sein kann).

Nach diesen Zwischenbemerkungen formulieren wir noch einmal das Kriterium, das wir nunmehr an Stelle des Satzes 2 setzen können.

Satz 8. Die notwendige und hinreichende Bedingung dafür, daß die Fourierreihe von $f^{\prime}(x)$ in $x_{0}$ mit der Summe $s\left(x_{0}\right)$ konvergiere, besteht darin, daß nach $W$ ahl eines beliebigen positiven $\delta<\frac{\pi}{2}$ das Dirichletsche Integral

$$
\frac{2}{\pi} \int_{0}^{\delta} \varphi\left(t ; x_{0}\right) \frac{\sin (2 n+1) t}{\sin t} d t
$$

mit wachsendem $n$ gegen 0 strebt $\left.{ }^{\mathbf{1}}\right)$.

Endlich kann man noch leicht zeigen, daß in dem letzten Integranden der Nenner sin $t$ einfach durch $t$ ersetzt werden kann. Denn die Differenz des dadurch entstehenden Integrals vom alten, also das Integral

$$
\int_{\pi}^{2} \varphi\left(t ; x_{0}\right)\left[\frac{1}{\sin t}-\frac{1}{t}\right] \cdot \sin (2 n+1) t d t
$$

strebt nach Satz 6 mit wachsendem $n$ gegen 0 , weil $\frac{1}{\sin t}-\frac{1}{t}$ in $0<t \leqq \delta$ stetig und beschränkt ${ }^{2}$ ) und also integrierbar ist.

Wir können somit schließlich sagen:

Satz 9. Die notwendige und hinreichende Bedingung für die Kon- $\mathbf{2 0 5 .}$ vergenz der Fourierveihe einer mit $2 \pi$ periodischen und im Intervall $0 \ldots 2 \pi$ integrierbaren Funktion $f(x)$ im Punkte $x_{0}$ gegen die Summe s $\left(x_{0}\right)$ besteht darin, daß nach Wahl eins beliebigen positiven $\delta\left(<\frac{\pi}{2}\right)$ die Folge der Integralwerte

$$
\frac{2}{\pi} \int_{0}^{\delta} \varphi\left(t ; x_{0}\right) \frac{\sin (2 n+1) t}{t} d t
$$

eine Nullfolge bildet. Dabei hat $\varphi\left(t ; x_{0}\right)$ die in Satz 2 angegebene Be-

1) Die ev. Gleichmäßigkcit der Konvergenz können wir jetzt nicht mehr ohne weiteres iiberschauen, weil wir nicht wissen, ob das oben betrachtete Integral (c), welches bei jedem festen $x=x_{0}$ mit wachsendem $n$ gegen 0 strebt, dies auch gleichmäßig tut für alle $x$ eines bestimmten $x$-Intervalles. Tatsächlich ist dies für jedes $x$-Intervall der Fall, doch wollen wir hierauf nicht weiter eingehen.

2) Denn es ist dort $\frac{1}{\sin t}-\frac{1}{t}=\frac{1}{t} \cdot \frac{1}{1-\frac{t^{2}}{3 !}+\ldots}-\frac{1}{t}=\frac{t}{6}+\ldots$, was für $t \rightarrow 0$ selbst gegen 0 strebt. 
deutung. Oder auch darin, daßnach Wahl von $\varepsilon>7$ sich ein positives $\delta<\frac{\pi}{2}$ und cin $N>0$ so angeben lassen, daß für alle $n>N$ stets ausfällt $)^{1}$.

$$
\frac{2}{\pi} \int_{0}^{\delta} p\left(t ; x_{0}\right) \frac{\sin (2 n+1) t}{t} d t<\varepsilon
$$

\section{Konvergenzbedingungen.}

Nun sind die V'orbereitungen soweit gediehen, daß die Konvergenzfragen 1) und 2) von S. 343 selbst in Angriff genommen werden können. Sie sind durch die vorangehenden Untersuchungen vollständig auf das folgende Problem zurückgeführt:

Es liegt eine in $0 \leqq t \leqq \delta$ integrierbare Funktion $\varphi(t)$ vor; welche weiteren Voraussetzungen m'ß diese noch erfüllen, damit die Folge der Integrale

$$
\left.J_{k}=\frac{2}{\pi} \int_{u}^{\lambda} \varphi(t) \cdot{ }_{t}^{\sin k t} d t^{2}\right)
$$

bei wachsendem k einem Grenzwert zustrebt, und welches ist dann dieser Grenzwert ${ }^{3}$ )?

Da bei diesen Integralen $\partial$ einen zwar festen, aber an sich beliebig kleinen Wert haben darf, so ist das Verhalten von $\varphi(t)$ - vgl. den Satz von Riemann $\mathbf{2 0 4}$ - nur unmittelbar vechts von 0, also etwa in einem Intervall der Form $0<t<\delta_{1}(\leq \delta)$, für die Beantwortung dieser Frage maßgebend. Wir können also auch fragen: Welche Eigenschaften muß $\varphi(t)$ rechts von 0 besitzen, damit der fragliche Grenzwert existiert?

Unter den vielen Bedingungen, die man als hierzu ausreichend gefunden hat, sind es besonders zwei, die wegen ihrer großen Allgemeinheit für die meisten Zwecke ausreichend sind und mit deren Darlegung wir uns hier begnügen wollen. Das erste ist von Dirichlet in seiner oben (S. 344) genannten, für die 'Theorie der Fourierreihen durchaus grundlegenden Arbeit aufgestellt worden, und hat die erste exakte Konvergenzbedingung dieser Theorie geliefert. Das zweite rührt von $U$. Dini her und ist 1880 gefunden worden.

206. 1. Die Dirichletsche Regel. Wenn $\varphi(t)$ rechts von $0,-d$. $h$. also in einem Intervall der Form $0<t<\delta_{1}(\leqq \delta)$ - monoton ver. läuft, so ist der fragliche Grenzwert der Integrale vorhanden, und zwar ist

$$
\lim _{k \rightarrow+\infty} \cdot J_{k}=\lim _{k \rightarrow+\infty} \frac{2}{\pi} \int_{0}^{s} \%(t) \cdot \underset{t}{\sin k t} d t=\mathscr{T}_{0} .
$$

1) Man mache sich genau klar, dafi die zweite Formulierung wirklich dasselbe besagt wie die erste, obwohl das $\delta$ erst nach der Wahl von $\varepsilon$ bestimmt zu werden braucht.

2) Für $t-0$ soll im Integranden ${ }^{--} \sin ^{k} t$ die Zahl $k$ bedeuten.

$\left.{ }^{3}\right)$ Die Bemerkung, daßs es genuigen würde, wenn $k$ durch ungerade ganze Zahlen hindurch $\rightarrow \infty$ wächst, führt zu keiner Vereinfachung. 
206. $§ 49$. Fouriersche Reihen. - C. Konvergenzbedingungen.

wenn man mit $\varphi_{0}$ den unter den gemachten Annahmen sicher vorhandenen (rechtsseitigen) Grenzwert $\lim _{t \rightarrow+0} \varphi(t)$ bezeichnet $\left.{ }^{1}\right)$.

Beweis. 1) Es ist zunächst

$$
\lim _{x \rightarrow+\infty} \int_{0}^{x} \frac{\sin t}{t} d t=\int_{0}^{\infty} \frac{\sin t}{t} d t=\frac{\pi}{2} .
$$

Daß dieser Grenzwert vorhanden ist, daß also das an zweiter Stelle genannte Integral konvergiert, folgt einfach daraus, daß nach Wahl von $\varepsilon>0$ für irgend zwei Werte $x^{\prime}$ und $x^{\prime \prime}$, die beide $>\frac{4}{\varepsilon}$ sind, (nach $\S 19$, Satz 26)

und also

$$
\int_{x^{\prime}}^{x^{\prime \prime}} \frac{\sin t}{t} d t=\left[-\frac{\cos t}{t}\right]_{x^{\prime}}^{x^{\prime \prime}}-\int_{x^{\prime}}^{x^{\prime \prime}} \frac{\cos t}{t^{2}} d t
$$

$$
\left|\int_{x^{\prime}}^{x^{\prime \prime}} \frac{\sin t}{t} d t\right| \leqq \frac{1}{x^{\prime}}+\frac{1}{x^{\prime \prime}}+\int_{x^{\prime}}^{x^{\prime \prime}} \frac{d t}{t^{2}}<4 \cdot \frac{\varepsilon}{4}=\varepsilon
$$

bleibt. Nun sahen wir S. 347, Gleichung (b), daß die Integrale

$$
i_{n}=\int_{0}^{\pi} \frac{\sin (2 n+1) t}{\sin t} d t
$$

für $n=0,1,2, \ldots$ stets $=\frac{\pi}{2}$ sind. Es strebt also auch $i_{n} \rightarrow \frac{\pi}{2}$. Andrerseits bilden die Zahlen

$$
i_{n}{ }^{\prime}=\int_{0}^{\frac{\pi}{2}}\left(\frac{1}{\sin t}-\frac{1}{t}\right) \sin (2 n+1) t \cdot d t
$$

(vgl. die S. 351 durchgeführten Betrachtungen) nach Satz 6 eine Nullfolge. Also streben auch die Integrale

Da aber auch

$$
i_{n}{ }^{\prime \prime}=i_{n}-i_{n}{ }^{\prime}=\int_{0}^{\frac{\pi}{2}} \frac{\sin (2 n+1) t}{t} d t>\frac{\pi}{2} \text {. }
$$

$$
i_{n}^{\prime \prime}=\int_{0}^{(2 n+1)^{\pi}} \frac{2}{2} \frac{\sin t}{t} d t
$$

ist, so bedeutet dies gerade, daß der oben genannte Grenzwert den Wert $\frac{\pi}{2}$ hat. stets

2) Nach 1) gibt es also eine Konstante $K^{\prime}$, so daß für alle $x \geqq 0$

$$
\left|\int_{0}^{x} \frac{\sin t}{t} d t\right| \leqq K^{\prime}
$$

1) Denn $\varphi(t)$ ist, weil integrierbar, sicher beschränkt und nach Voraussetzung in $0<t<\delta_{1}$ monoton. - Übrigens braucht $\varphi_{0}$ nicht $=\varphi(0)$ zu sein. 
bleibt, - und folglich auch eine Konstante $K\left(=2 K^{\prime}\right)$, so daß für beliebige $0 \leqq a \leqq b$ stets

$$
\left|\int_{a}^{b} \frac{\sin t}{t} d t\right| \leqq K
$$

bleibt.

3. Ist nun $\varepsilon>0$ gegeben, so wähle man die positive Zahl $\delta^{\prime} \leqq \delta_{1}$ so, daß für $0<t \leqq \delta^{\prime}$ stets

$$
\left|\varphi(t)-\varphi_{0}\right|<\frac{\varepsilon}{3 K}
$$

bleibt. Setzen wir dann die Integrale

$$
\frac{2}{\pi} \int_{0}^{\delta^{\prime}} \varphi(t) \frac{\sin k t}{t} d t=J_{l^{\prime}},
$$

so strebt $J_{k}-J_{k}^{\prime}$ nach Satz 6 für $k \rightarrow+\infty$ gegen 0, und wir können $k^{\prime}$ so groß wählen, daß für $k>k^{\prime}$ stets $J_{k}-J_{k}{ }^{\prime} \mid<\frac{\varepsilon}{3}$ ausfällt. Weiter ist aber

$$
\text { (d) } J_{k}^{\prime}=\frac{2}{\pi} \int_{0}^{\delta^{\prime}}\left[\varphi(t)-\varphi_{0}\right] \cdot \frac{\sin k t}{t}-d t+{ }_{\pi}^{2} \varphi_{0} \cdot \int_{0}^{\delta^{\prime}} \frac{\sin k t}{t} d t=J_{k}{ }^{\prime \prime}+J_{k}^{\prime \prime \prime} \text {; }
$$

und da hierin der letzte Summand

$$
J_{k}^{\prime \prime \prime}=\frac{2}{\pi} \varphi_{0} \cdot \int_{0}^{k d^{\prime}} \frac{\sin t}{t} d t \quad \text { ist und also } \quad \rightarrow \frac{2}{\pi} \varphi_{0} \cdot \int_{0}^{\infty} \frac{\sin k t}{t} d t=\varphi_{0}
$$

strebt, so können wir $k_{0}>k^{\prime}$ so groß wählen, daß für $k>k_{0}$ stets

$$
J_{k}^{\prime \prime \prime}-\varphi_{0}|=| \frac{2}{\pi} \varphi_{0} \int_{0}^{d^{\prime}} \frac{\sin k t}{t} d t-\varphi_{0} \mid<\frac{\varepsilon}{3}
$$

bleibt. Auf den ersten Summanden $J_{k}^{\prime \prime}$ von (d) endlich wenden wir den zweiten Mittelwertsatz der Integralrechnung an (§ 19, Satz 27), welcher für ein passendes nicht negatives $\delta^{\prime \prime}<\delta^{\prime}$ liefert:

$$
J_{k}^{\prime \prime}=\frac{2}{\pi} \int_{0}^{\delta^{\prime}}\left[\varphi(t)-\varphi_{0}\right] \cdot{ }_{t}^{\sin k t} d t=\frac{2}{\pi}\left[\varphi\left(\delta^{\prime}\right)-\varphi_{0}\right] \cdot \int_{\delta^{\prime \prime}}^{\delta^{\prime}} \frac{\sin k t}{t} d t .
$$

Und da nun hier das letzte Integral $=\int_{k \delta^{\prime}}^{k d^{\prime \prime}} \frac{\sin t}{t} d t$ ist und also nach 2) seinem Betrage nach $<K$ bleibt, so gilt für den ganzen ersten Summanden $J_{k}^{\prime \prime}$ aus (d):

$$
\left|J_{k}^{\prime \prime}\right| \leqq \frac{2}{\pi} \cdot \frac{\varepsilon}{3 K} \cdot K<\frac{\varepsilon}{3} .
$$

Fügt man die drei Ergebnisse dieses Absatzes gemäß $J_{k}=\left(J_{k}-J_{k}{ }^{\prime}\right)$ $+J_{k}^{\prime \prime}+J_{k}^{\prime \prime \prime}$ zusammen, so lehren sie, daß nach Wahl von $\varepsilon>0$ stets $k_{0}$ so bestimmt werden kann, daß für alle $k>k_{0}$ stets

$$
J_{k}-\varphi_{0} \leqq\left|J_{k}-J_{k}{ }^{\prime}\right|+\left|J_{k}{ }^{\prime \prime}\right|+\left|J_{k}{ }^{\prime \prime \prime}-\varphi_{0}\right| \leqq 3 \cdot{ }_{3}^{\varepsilon}=\varepsilon
$$

bleibt. Damit ist die Behauptung in vollem Umfange bewiesen. 
206. §49. Fouriersche Reihen. - C. Konvergenzbedingungen.

355

2. Die Dinische Regel. Wenn $\lim _{t \rightarrow+0} \varphi(t)=\varphi_{0}$ existiert und wenn für alle positiven $\tau<\delta$ die Integralwerte

$$
\int_{\tau}^{\delta} \frac{\left|\varphi(t)-\varphi_{0}\right|}{t} d t
$$

unterhalb einer festen Schranke bleiben ${ }^{\mathbf{1}}$ ), so existiert $\lim _{k \rightarrow+\infty} J_{k}$ und ist $=\varphi_{0}$.

Beweis. Da das oben genannte Integral bei zu 0 abnehmendem $\tau$ monoton wächst, aber beschränkt bleibt, so hat es für $\tau \rightarrow 0$ einen Grenzwert, den man kurz durch

$$
\int_{0}^{\delta} \frac{\left|\varphi(t)-\varphi_{0}\right|}{t} d t
$$

bezeichnet. Ist dann $\varepsilon>0$ gegeben, so kann man ein positives $\delta^{\prime}<\delta$ so klein wählen, daß

$$
\int_{0}^{\delta^{\prime}} \frac{\left|\varphi(t)-\varphi_{0}\right|}{t} d t<\frac{\varepsilon}{3}
$$

ist. Setzen wir dann wieder, wie beim vorigen Beweise,

$$
J_{k}{ }^{\prime}=\frac{2}{\pi} \int_{0}^{\delta^{\prime}} \varphi(t) \frac{\sin k t}{t} d t \text { und } J_{k}{ }^{\prime}=J_{k}{ }^{\prime \prime}+J_{k}{ }^{\prime \prime \prime},
$$

so strebt zunächst $\left(J_{k}-J_{k}{ }^{\prime}\right)$ nach Satz 6 gegen 0 und es kann $k^{\prime}$ so groß gewählt werden, daß für $k>k^{\prime}$ stets $\left|J_{k}-J_{k}{ }^{\prime}\right|<\frac{\varepsilon}{3}$ bleibt. Weiter aber sahen wir vorhin, daß bei passender Wahl von $k_{0}>k^{\prime}$ auch

$$
\left|J_{k}^{\prime \prime \prime}-\varphi_{0}\right|=\left|\frac{2}{\pi} \varphi_{0} \cdot \int_{0}^{\delta^{\prime}} \frac{\sin k t}{t} d t-\varphi_{0}\right|<\frac{\varepsilon}{3}
$$

bleibt für alle $k>k_{0}$. Und da endlich

$$
\left|J_{k}^{\prime \prime}\right|=\left|\frac{2}{\pi} \int_{0}^{\delta^{\prime}}\left[\varphi(t)-\varphi_{0}\right] \cdot \frac{\sin k t}{t} d t\right|<\int_{0}^{\delta^{\prime}} \frac{\left|\varphi(t)-\varphi_{0}\right|}{t} d t
$$

ist und also gemäß der Wahl von $\delta^{\prime}$ gleichfalls $<_{3}^{\varepsilon}$ ausfällt, so ist genau wie beim vorigen Beweise für $k>k_{0}$ wieder

$$
\left|J_{k}-\varphi_{0}\right|<\varepsilon,
$$

womit auch die Richtigkeit der Dinischen Regel bewiesen ist.

Aus ihr lassen sich noch leicht die beiden folgenden Regeln ableiten: Sinn hat.

1) Kürzer: Wenn das (bei 0) uneigentliche Integral $\int_{0}^{\delta} \frac{\left|\varphi(t)-\varphi_{0}\right|}{t} d t$ einen 
3. Die Lipschitzsche Regel. Wenn zwsi positive Zahlen $A$ und a existieren, so daß rechts von $0-$ etwa in $0<t<\delta_{1}(\leqq \delta)-$ stets

$$
\varphi(t)-\varphi_{0}<A \cdot t^{a}
$$

ist, so streben die $\left.J_{k} \rightarrow \varphi_{0}{ }^{1}\right)$.

Beweis. Es ist

$$
\int_{\tau}^{\delta_{1}} \frac{\varphi(t)-\varphi_{0} \mid}{t} d t<A \int_{\tau}^{\delta_{1}} t^{\alpha-1} d t<A \cdot \frac{\delta_{1}^{a}}{\alpha^{\alpha}},
$$

also für alle positiven $\tau<\delta_{1}$ unterhalb einer festen Schranke gelegen, so daß infolge der Dinischen Regel in der Tat $J_{k} \rightarrow \varphi_{0}$ strebt.

4. Regel. Wenn $\varphi^{\prime}(0)$ existiert $\left.^{2}\right)$ und also $\lim _{t \rightarrow+0} \varphi(t)=\varphi_{0}=\varphi(0)$ vorhanden ist, so strebt wieder $J_{k} \rightarrow \varphi_{0}=\varphi(0)$.

Beweis. Wenn

$$
\lim _{t \rightarrow+0} \frac{\varphi(t)-\varphi(0)}{t}
$$

existiert, so bleibt dieser Quotient in einem Intervall der Form $0<t<\delta_{1}$ beschränkt, etwa seinem Betrage nach stets $<A$. Dann ist aber eine Lipschitz-Bedingung mit $\iota=1$ erfüllt und es strebt also $J_{k} \rightarrow \varphi_{0}$.

Ganz unmittelbar ergibt sich endlich noch der folgende

Zusatz. Läßt sich $\varphi(t)$ in zwei oder irgendeine feste Anzahl von Summanden so zerspalten, daß ein jeder von ihnen den Bedingungen einer der vorangehenden vier Regeln genügt, so ist wieder $\lim _{t \rightarrow+0} \varphi(t)=\varphi_{\mathbf{0}}$ vorhanden und es streben die mit der Funktion $\varphi(t)$ angesetzten Dirichletschen Integrale $J_{k} \rightarrow \varphi_{0}$.

Diese Regeln lassen sich ganz unmittelbar auf die Fourierreihe einer integrierbaren Funktion $f(x)$ übertragen, die wir uns von vornherein im Intervall $0 \leqq x<2 \pi$ gegeben und für alle übrigen reellen $x$ durch die Festsetzung

$$
f(x \pm 2 \pi)=f(x)
$$

erklärt denken. Soll nämlich die durch $f(x)$ erzeugte Fourierreihe in $x_{0}$ mit der Summe $s\left(x_{0}\right)$ konvergieren, so muß nach Satz $9(\mathbf{2 0 5})$ die Folge der Integrale

$$
J_{n}=\frac{2}{\pi} \int_{0}^{\delta} \varphi\left(t ; x_{0}\right) \frac{\sin (2 n+1) t}{t} d t
$$

eine Nullfolge bilden, wenn $\varphi\left(t ; x_{0}\right)$ die Bedeutung

$$
\varphi\left(t ; x_{0}\right)=\frac{1}{2}\left[f\left(x_{0}+2 t\right)+f\left(x_{0}-2 t\right)-s\left(x_{0}\right)\right]
$$

$\left.{ }^{1}\right)$ Die "Lipschitz-Bedingung", dafi $\left|\varphi(t)-\varphi_{0}\right|<A \cdot t^{\alpha}$ bleibt bei $t \rightarrow 0$, hat von selbst zur Folge, dafi $\lim _{t \rightarrow+0} \varphi(t)=\varphi_{0}$ existiert.

2) Es genuigt, wenn $\varphi^{\prime}(0)$ als rechtsseitige Ableitung $\varphi^{\prime}(+0)$ existiert, da es ja auf die etwaigen Werte von $\varphi(t)$ für $t \leqq 0$ gar nicht ankommt. 
hat. Diese Fassung des Kriteriums lehrt zunächst noch über den Riemannschen Satz $\mathbf{2 0 4}$ hinaus, daß das Verhalten von $f(x)$ weder unmittelbar rechts von $x_{0}$ noch dasjenige unmittelbar links von $x_{0}$ für sich allein irgendeinen Einfluß auf das Konvergenzverhalten der Fourierreihe von $f(x)$ in $x_{0}$ hat. Es kommit vielmehr nur darauf an, daß das Verhalten von $f(x)$ rechts von $x_{0}$ in einem gewissen Zusammenhange mit demjenigen links von $x_{0}$ stehe, derart nämlich, daß die Funktion

$$
\varphi(t)=\varphi\left(t ; x_{0}\right)=\frac{1}{2}\left[f\left(x_{0}+2 t\right)+f\left(x_{0}-2 t\right)\right]-s\left(x_{0}\right),
$$

die für die Existenz des Grenzwertes der damit angesetzten Dirichletschen Integrale $J_{k}(\mathbf{2 0 6})$ notwendigen und hinreichenden Eigenschaften besitzt $\left.{ }^{1}\right)$.

Welches diese Eigenschaften sind, weiß man nicht. Doch liefern uns die oben entwickelten vier Regeln für die Konvergenz Dirichletscher Integrale ebensoviele sehr weittragende hinreichende Bedingungen für die Konvergenz der Fourierveihe einer Funktion $f(x)$ in einem speziellen Punkte $x_{0}$. Alle jene Regeln verlangten zunächst, daß für $t \rightarrow+0$ die Funktion

$$
\varphi(t)=\varphi\left(t ; x_{0}\right)=\frac{1}{2}\left[f\left(x_{0}+2 t\right)+f\left(x_{0}-2 t\right)\right]-s\left(x_{0}\right)
$$

einem Grenzwert $\varphi_{0}$ zustrebe. Und wenn die Fourierreihe in $x_{0}$ konvergieren und dort die Summe $s\left(x_{0}\right)$ haben soll, so muß $\varphi_{0}=0$ sein. Da gegen diesen selben Grenzwert dann auch die Dirichletschen Integrale streben werden, so haben wir zunächst als gemeinsame Voraussetzung für alle jetzt aufzustellenden Regeln: Der Grenzwert

$$
\lim _{t \rightarrow+0} \frac{1}{2}\left[f\left(x_{0}+2 t\right)+f\left(x_{0}-2 t\right)\right]
$$

soll existieven. Sein Wert $s\left(x_{0}\right)$ wird im Falle der Konvergenz der Fourierreihe von $f(x)$ in $x_{0}$ dann auch die Summe dieser Reihe angeben. Deren Konvergenz wird nun gesichert sein, wenn die Funktion

$$
\varphi(t)=\varphi\left(t ; x_{0}\right)=\frac{1}{2}\left[f\left(x_{0}+2 t\right)+f\left(x_{0}-2 t\right)\right]-s\left(x_{0}\right)
$$

als Funktion von $t$ aufgefaßt die in einer der obigen vier Regeln genannten Bedingungen erfüllt. Wir nehmen demgemäß an, daß die folgenden beiden Voraussetzungen erfüllt sind:

1. Voraussetzung. Die Funktion $f(x)$ ist in $0 \leqq x<2 \pi$ definiert $\mathbf{2 0 7 .}$ und integrierbar, und für alle übrigen reellen $x$ durch die Festsetzung erklärt.

$$
f(x)=f(x+2 k \pi), \quad k= \pm 1, \pm 2, \ldots,
$$

\section{Voraussetzung. Der Grenzwert}

$$
\lim _{t \rightarrow+0} \frac{1}{2}\left[f\left(x_{0}+2 t\right)+f\left(x_{0}-2 t\right)\right]
$$

1) Man definiere z. B. $f(x)$ rechts von $x_{0}$ ganz beliebig (doch integrierbar in einem Intervall der Form $\left.x_{0}<x<x_{0}+\delta\right)$ und setze dann in $x_{0}-\delta<x<x_{0}$ etwa $f(x)=1-f\left(2 x_{0}-x\right)$, so erweist sich die Fourierreihe von $f(x)$ in $x_{0}$ konvergent mit der Summe $\frac{1}{2}$. (Beweis etwa mit Hilfe der nachfolgenden Dirichletschen Regel $\mathbf{2 0 8}, 1$.) 
bei dem $x_{0}$ eine beliebige, aber weiterhin feste reelle Zahl bedeutet, ist vorhanden und sein Wert mit $s\left(x_{0}\right)$ bezeichnet, so daß die Funktion

$$
\varphi(t)=\varphi\left(t ; x_{0}\right)=\frac{1}{2}\left[f\left(x_{0}+2 t\right)+f\left(x_{0}-2 t\right)\right]-s\left(x_{0}\right)
$$

den (rechtsseitigen Grenzwert) $\lim _{t \rightarrow+0} \varphi(t)=0$ besitzt.

Unter diesen gemeinsamen Voraussetzungen gelten die folgenden vier Kriterien für die Konvergenz der Fourierreihe von $f(x)$ im Punkte $x_{0}$ : 208. 1. Die Dirichletsche Regel Wenn $\varphi(t)$ in einem Intervall der Form $0<t<\delta_{1}$ monoton verläuft, so ist die Fourierreihe von $f(x)$ in $x_{0}$ konvergent und ihre Summe hat den Wert $\left.s\left(x_{0}\right)^{1}\right)$

2. Die Dinische Regel. Wenn nach Wahl einer festen (an sich beliebigen) positiven Zahl $\delta$ die Integralwerte

$$
\int_{\tau}^{\delta} \frac{|\varphi(t)|}{t} d t
$$

für alle $0<\tau<\delta$ unterhalb einer festen Schranke bleiben, so ist die Fourierreihe von $f(x)$ in $x_{0}$ konvergent, und ihre Summe hat den Wert $s\left(x_{0}\right)$.

3. Die Lipschitzsche Regel. Das gleiche gilt, wenn an Stelle der Beschränktheit der Integralwerte die Existenz zweier positiver Zahlen A und a gefordert wird, so daß für alle $0<t<\delta$

bleibt.

$$
|\varphi(t)|<A \cdot t^{a}
$$

4. Regel. Dasselbe gilt, wenn an Stelle der Lipschitz-Bedingung nur gefordert wird, daß $\varphi(t)$ in 0 eine rechtsseitige Ableitung besitzt.

Der Gebrauch dieser Regeln wird noch durch die folgenden $\mathrm{Zu}$ sätze beträchtlich erleichtert:

Zusatz 1. Läßt sich $f(x)$ in zwei oder irgendeine feste Anzahl von Summanden zerspalten, deren jeder die gemeinsamen beiden Voraussetzungen erfüllt und darüber hinaus in einer Umgebung eines Punktes $x_{0}$ den Bedingungen je einer der vorangehenden Regeln genügt, so erfüllt auch $f(x)$ die Voraussetzungen 1 und 2 und die Fourierreihe von $f(x)$ wird in $x_{0}$ mit der Summe $s\left(x_{0}\right)$ konvergieren.

Zusatz 2. Im besonderen genügt es, wenn $f(x)$ die Voraussetzungen 1 und 2 erfüllt und nun die beiden Funktionen

$$
\varphi_{1}(t)=f\left(x_{0}+2 t\right) \quad \text { und } \quad \varphi_{2}(t)=f\left(x_{0}-2 t\right)
$$

einzeln den Bedingungen je einer unserer vier Regeln genügen. Auch dann ist die Fourierreihe von $f(x)$ in $x_{0}$ konvergent und hat $s\left(x_{0}\right)$ zur Summe.

1) Im Falle ihrer Konvergenz in $x_{0}$ hat die Fourierreihe von $f(x)$ also dann und nur dann den dortigen Funktionswert $f\left(x_{0}\right)$ zur Summe, wenn der in der 2. Voraussetzung geforderte Grenzwert $s\left(x_{0}\right)=f\left(x_{0}\right)$ ist. Entsprechend bei den folgenden Regeln. 
Zwei ganz spezielle, aber für alle Anwendungen besonders wichtige Fälle merken wir noch in den beiden folgenden Zusätzen an:

Zusatz 3. Die von $f(x)$ erzengte Fourierreihe wird an jeder Stelle $x_{0}$ konvergieren und auch den dortigen Funktionswert $f\left(x_{0}\right)$ zur Summe haben, an der $f(x)$ stetig und in deren Umgebung $f(x)$ monoton ist $\left.{ }^{1}\right)$.

Zusatz 4. Die von $f(x)$ erzengte Fourierreihe wird an jeder Stelle $x_{0}$ konvergieren und auch den dortigen Funktionswert $f\left(x_{0}\right)$ zur Summe haben, an der $f(x)$ differenzierbar ist $\left.{ }^{2}\right)$.

\section{§50. Anwendungen der Theorie der Fourierschen Reihen.}

Die im vorangehenden entwickelten Konvergenzregeln zeigen, daßs sehr allgemeine Klassen von Funktionen durch ihre Fourierreihe dargestellt werden. Wir wollen dies nun durch eine Anzahl von Beispielen erläutern.

Die $z u$ entwickelnde Funktion $f(x)$ muß stets in $0 \leqq x<2 \pi$ gegeben sein und die Periode $2 \pi$ besitzen: $f(x+2 \pi)=f(x)$. Dannn erhält man im allgemeinen die zugehörige Fourierreihe in der Form

$$
\frac{1}{2} a_{0}+\sum_{n=1}^{\infty}\left(a_{n} \cos n x+b_{n} \sin n x\right) \text {. }
$$

In besonderen Fällen aber können die sin- oder cos-Glieder auch fehlen. Ist nämlich $f(x)$ eine gerade Funktion, ist also

$$
f(-x)=f(2 \pi-x)=f(x)
$$

(das Kurvenbild ist dann in bezug auf die Geraden $x=k \pi(k=0,+1, \perp 2, \ldots)$ symmetrisch), so ist

$$
b_{n}=\frac{1}{\pi} \int_{0}^{2 \pi} f(x) \sin n x d x=\frac{1}{\pi} \int_{0}^{\pi}+\frac{1}{\pi} \int_{\pi}^{2 \pi}=0,
$$

wie man sofort erkennt, wenn man im zweiten der beiden Teilintegrale $x$ durch $-x$ ersetzt. Die Fourierreihe von $f(x)$ reduziert sich dann also auf eine reine cos-Reihe. Ist andrerseits $f(x)$ eine ungerade Funktion, ist also

$$
f(-x)=f(2 \pi-x)=-f(x)
$$

1) Aber die Konvergenz findet nach Zusatz 2 auch schon statt, wenn $f(x)$ sowohl links von $x_{0}$ als auch rechts von $x_{0}$ monoton verläuft; und die Summe der Reihe ist dann das arithmetische Mittel aus den (sicher vorhandenen) rechtsund linksseitigen Grenzwerten von $f(x)$ bei $x_{0}$ :

$$
s\left(x_{0}\right)=\frac{1}{2}\left[f\left(x_{0}+0\right)+f\left(x_{0}-0\right)\right],
$$

wenn mit $f\left(x_{0}+0\right)$ und $f\left(x_{0}-0\right)$ die Grenzwerte von $f(x)$ für $x \rightarrow x_{0}+0$ bzw. $x \rightarrow x_{0}-0$ bezeichnet werden. Es ist also $s\left(x_{0}\right)$, kurz gesagt, gleich der Mitte des Sprunges von $f(x)$ an der Stelle $x_{0}$.

2) Aber auch hier genügt es nach Zusatz 2 wieder, wenn die rechts- und linksseitigen Ableitungen einzeln vorhanden sind. Ja es genügt schon, wenn die in der vorigen Fufnote genannten Grenzwerte $f\left(x_{0}+0\right)$ und $f\left(x_{0}-0\right)$ existieren und nun die (einseitigen) Ableitungen

$$
\lim _{h \rightarrow+0} \frac{f\left(x_{0}+h\right)-f\left(x_{0}+0\right)}{h} \quad \text { und } \quad \lim _{h \rightarrow+0} \frac{f\left(x_{0}-h\right)-f\left(x_{0}-0\right)}{-h}
$$

existieren. Auch dann konvergiert die Fourierreihe von $f(x)$ in $x_{0}$ und hat die in der vorigen Fußnote genannte Summe. 
(das Kurvenbild ist dann in bezug auf die Punkte $x=k \pi(k=0, \pm 1, \pm 2, \ldots)$ symmetrisch), so ist

$$
a_{n}=\frac{1}{\pi} \int_{0}^{2 \pi} f(x) \cos n x d \dot{x}=0,
$$

wie man ebenso leicht erkennt, und die Fourierreihe von $f(x)$. reduziert sich nun auf eine reine sin-Reihe.

Ist nun eine beliebige Funktion $F(x)$ in $a \leqq x \leqq b$ vorgelegt, so kann man sie demgemäß auf drei verschiedene Arten zur Entwicklung in eine trigonometrische Reihe zurechtmachen.

1. Art. Man schneidet aus dem Intervall $a \ldots b$, falls seine Länge $\geq 2 \pi$ ist, ein Stiick $\iota \leq x<\alpha+2 \pi$ von der Länge $2 \pi$ heraus, verschiebt den Nullpunkt der Abszissen nach $\alpha$ und erhält so eine in $0 \leq x<2 \pi$ definierte Funktion $f(x)$. Diese setze man durch die Bedingung $f(x \pm 2 \pi)=f(x)$ periodisch iiber die ganze $x$-Achse fort ${ }^{1}$ ). Ist $b-a<2 \pi$, so setze man $f(x)$ im Intervall $b \leq x<a+2 \pi$ konstant $=F(b)$ und verfahre dann wie soeben ${ }^{2}$ ).

2. Art. Man definiere genau wie eben mit Hilfe der gegebenen Funktion $F(x)$ eine Funktion $f(x)$ in $0 \leq x \leq \pi$ (nicht $2 \pi$ ), setze in $\pi \leq x \leq 2 \pi$ nun $f(x)=f(2 x-x)$ und definiere $\overline{f(x)}$ für alle übrigen $x$ durch die Periodizitätsbedingung.

3. Art. Man definiere $f(x)$ in $0 \leq x<\pi$ wie eben, setze $f(\pi)=0$, aber in $\pi<x \leqq 2 \pi$ jetzt $f(x)=-f(2 \pi-x)$ und definiere dann $f(x)$ für die tibrigen $x$ wieder durch die Periodizitätsbedingung.

Dic auf diese 3 Arten aus der gegebenen Funktion $F(x)$ hergestellten und nunmehr zur Erzeugung einer Fouvierreihe geeigneten Funktionen unterscheiden wir als $f_{1}(x), f_{2}(x)$ und $f_{3}(x)$. Während $f_{2}(x)$ sicher eine reine cosReihe, $f_{3}(x)$ sicher eine reine sin-Reihe liefert, wird $f_{1}(x)$ in der Regel eine Fourierreihe der allgemeinen Form liefern (nämlich nur dann nicht, wenn $f_{1}(x)$ von vornherein schon gerade oder ungerade ausgefallen ist).

Da unsere Konvergenzregeln die Konvergenz nur in solchen Punkten $x_{0}$ $z u$ erkennen gestatten, für die

$$
\lim _{t \rightarrow+0} \frac{1}{2}\left[f\left(x_{0}+2 t\right)+f\left(x_{0}-2 t\right)\right]
$$

existiert, so wird es ratsam sein, an den „Flickstellen“ $2 k \pi$-unserer drei Funktionen $f(x)$ den Funktionswert noch dahin abzuändérn, da 3 man

$$
f(0)=f(2 k \pi)=\lim _{x \rightarrow+0} \frac{1}{2}[f(x)+f(2 \pi-x)]
$$

setzt, falls dieser Grenzwert vorhanden ist. (Dies ist sicher bei $f_{3}(x)$ der Fall und liefert die neue Festsetzung $\left.f_{3}(0)=f_{3}(2 k \pi)=0\right)$. Ist er nicht vorhanden, so kommt es auf den Funktionswert $f(2 k \pi)$ gar nicht an, da wir ja dann die ev. Konvergenz der Fouvierreihe mit unsern Mitteln doch nicht erkennen können. - Aus einer entsprechenden Erwägung heraus hatten wir auch schon oben $f_{3}(\tau)=0$ gesetzt. -

1) War $b-a>2 x$, so gelangt hierbei ein Stuck der Kurve $y=F(x)$ gar nicht zur Darstellung. Will man diesen Übelstand vermeiden, so ändere man einfach den Maßsstab auf der $x$-Achse so, daf das Definitionsintervall von $F(x)$ nun die Länge $2 \pi$ bekommt, d. h. man ersetze $x \operatorname{durch} \frac{b-a}{2 \pi} x$.

2) Oder man gebe wieder durch Maßsstabsänderung dem Definitionsintervalle von $F(x)$ die genaue Länge $2 \pi$. 
209. $§ 50$. Anwendungen der Theorie der Fourierschen Reihen.

Wir gehen nunmehr zu konkreten Beispielen über.

1. Beispiel. $F(x) \equiv a \neq 0$. Hier wird

$$
\begin{aligned}
& f_{1}(x) \equiv f_{2}(x) \equiv a, \quad \text { während } \\
& f_{3}(x)\left\{\begin{array}{ccccc}
=0 & \text { zu } & \text { setzen ist für } & x=0 \text { und } \pi, \\
=a & " & " & " & \\
=-a & " & " & & 0<x<\pi,
\end{array}\right.
\end{aligned}
$$

Für alle drei Funktionen sind ersichtlich die Dirichletschen Bedingungen in jedem Punkte (einschließlich der Flickstellen) erfüllt. Die erhaltenen Entwicklungen müssen daher überall konvergieren und die Funktionen darstellen. Für $f_{1}(x)$ und $f_{2}(x)$ werden aber diese Entwicklungen trivial, weil sie sich auf das konstante Glied $\frac{1}{2} a_{0}=a$ reduzieren. Für $f_{3}(x)$ dagegen erhält man

$$
\begin{gathered}
b_{n}=\frac{1}{\pi} \int_{0}^{2 \pi} f_{3}(x) \sin n x d x=\frac{a}{\pi} \int_{0}^{\pi} \sin n x d x-\frac{a}{\pi} \int_{\pi}^{2 \pi} \sin n x d x=\frac{2}{\pi} \int_{0}^{\pi} \sin n x d x \\
\qquad \begin{array}{ll}
=0 & \text { für gerade } n, \\
=\frac{4 a}{n \pi} & \text { für ungerade } n .
\end{array}
\end{gathered}
$$

Die Entwicklung lautet also

$$
f_{3}(x)=\frac{4 a}{\pi}\left[\sin x+\frac{\sin 3 x}{3}+\frac{\sin 5 x}{5}+\ldots\right]
$$

oder

$$
\sin x+\frac{\sin 3 x}{3}+\frac{\sin 5 x}{5}+\ldots \begin{cases}=+\frac{\pi}{4} & \text { in } 0<x<\pi \\ 0 & \text { in } 0 \text { und } \pi \\ -\frac{\pi}{4} & \text { in } \pi<x<2 \pi\end{cases}
$$

Damit ist das zweite der S. 338/9 gegebenen Beispiele vollständig begründet und die Summe dieser merkwürdigen Reihe, deren Konvergenz uns nichts neues ist (s. 185, 5), ermittelt ${ }^{1}$ ). Für $x=\frac{\pi}{2}, \frac{\pi}{6}, \frac{\pi}{3}$ liefert sie die folgenden speziellen Reihen, von denen uns die erste schon aus einem ganz andern $Z_{u-}$ sammenhange heraus bekannt ist (s. 122):

$$
\begin{aligned}
1-\frac{1}{3}+\frac{1}{5}-\frac{1}{7}+-\ldots & =\frac{\pi}{4}, \\
1+\frac{1}{5}-\frac{1}{7}-\frac{1}{11}+\frac{1}{13}+\frac{1}{17}--++\ldots & =\frac{\pi}{3}, \\
1-\frac{1}{5}+\frac{1}{7}-\frac{1}{11}+\frac{1}{13}-+\ldots & =\frac{\pi}{2 \sqrt{3}} .
\end{aligned}
$$

1) Dieses und die folgenden Beispiele finden sich meist schon bei Euler. Viele andere sind von Fourier, Legendre, Cauchy, Frullani, Dirichlet u. a. gegeben worden. Man findet sie bequem zusammengestellt bei H. Burkhardt, Trigonometrische Reihen und Integrale, Enzyklopädie d. math. Wiss., Bd. IIA, S. 902-920. 
2. Beispiel. $F(x)=a x$. Hier ist

$$
\begin{aligned}
& f_{1}(x)\left\{\begin{array}{l}
-a x \text { in } 0<x-2 \pi, \\
-a \pi \text { in } 0 \text { und } 2 \pi,
\end{array}\right. \\
& f_{2}(x)\left\{\begin{array}{l}
-a x \text { in } 0 \leqq x \leq \pi, \\
-a(2 \pi-x) \text { in } \pi \leqq x<2 \pi
\end{array}\right. \\
& f_{3}(x) \begin{cases}a x \text { in } 0<x<\pi, \\
0 \text { in } 0 \text { und } \pi, \\
-a(2 \pi-x) \text { in } \pi \quad x \quad 2 \pi .\end{cases}
\end{aligned}
$$

Nach leichten Rechnungen liefert die Entwicklung von $f_{1}$

210. (a) $\sin x+\frac{\sin 2 x}{2}+\frac{\sin 3 x}{3}+\frac{\sin 4 x}{4}+\ldots\left\{\begin{array}{cl}\pi-x & \text { in } 0<x<2 \pi, \\ 2 & \text { in } 0 \text { und } 2 \pi,\end{array}\right.$

wodurch insbesondere das erste Beispiel von S. 338/9 vollständig begriindet ist. Und ganz ähnlich liefert die Entwicklung von $f_{3}$

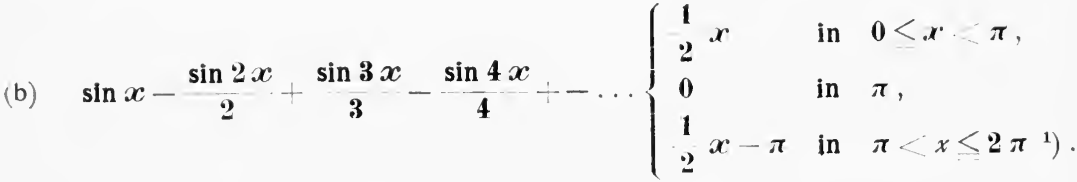

Dagegen liefert uns $f_{2}(x)$ die Entwicklung

$$
\frac{\cos x}{1^{2}}+{ }^{\cos 3 x}+3^{2}+5^{2}+\ldots\left\{\begin{array}{cc}
\frac{\pi^{2}}{8}-\frac{\pi x}{4} & \text { in } 0 \leq x \leq \pi \\
\frac{\pi \cdot x^{2}}{4}-\frac{3 \pi^{2}}{8} & \text { in } \pi \leq x<2 \pi .
\end{array}\right.
$$

Die erste dieser Entwicklungen liefert für $x \quad \frac{\pi}{2}$ wieder die bekannte Reihe für $\frac{\pi}{4}$, die letzte für $x-0$ die uns gleichfalls schon bekannte Reihe (135)

$$
1+\frac{1}{3^{2}}+\frac{1}{5^{2}}+\frac{1}{7^{2}} \ldots \ldots \boldsymbol{x}^{2}
$$

aus der man sofort die frïher (136) auf ganz anderem $W^{r+}$ ege bewiesene Gleichung

$$
1+\frac{1}{2^{2}}+\frac{1}{3^{2}}+\frac{1}{42}+\ldots \pi^{2}
$$

herleitet. - Der Vergleich beider Ergebnisse zeigt, dafi in $0<x \leqq \pi$ die Funktion $x$ die beiden Fourierentwicklungen gestattet

$$
x=\left\{\begin{array}{l}
\pi-2\left[\frac{\sin x}{1}+\frac{\sin 2 x}{2}+\frac{\sin 3 x}{3} \ldots \ldots\right] \\
\frac{\pi}{2}-\frac{4}{\pi}\left[\begin{array}{c}
\cos x \\
1^{2}
\end{array}+\frac{\cos 3 x}{3^{2}}+\cos ^{2} x\right.
\end{array}\right. \text { und }
$$

- ein besonders merkwürdiges Resultat.

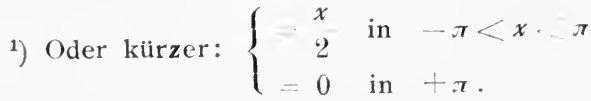


Um in die Bedeutung dieser Ergebnisse noch besser einzudringen, tut man gut, sich die Bilder der Funktionen $f(x)$ und einiger zugehöriger Approximationskurven zu skizzieren. Wir müssen das dem Leser iiberlassen und machen nur auf die folgende Erscheinung aufmerksam:

Die Konvergenz der Reihe $\mathbf{2 1 0} \mathrm{c}$ ist eine für alle $x$ gleichmäßige, nicht so dagegen bei den Reihen $\mathbf{2 1 0}$ a und b, da ja deren Summen bei 0 bzw. $\pi$ unstetig sind. Bei jener schmiegen sich also die Approximationskurven in ihrer ganzen Ausdehnung an die durch die Grenzkurve gelieferte Zickzacklinie an, während das entsprechende bei (a) und (b) nicht der Fall ist und nicht der Fall sein kann. (Vgl. hierzu 216, 4.)

3. Beispiel. $\left.F(x)=\cos \alpha x(\alpha \text { beliebig, doch } \neq 0, \pm 1, \pm 2, \ldots)^{1}\right)$.

a) Wir wollen hier zunächst die Funktion $f_{2}(x)$ bilden und setzen demgemäfs

$$
f_{2}(x)\left\{\begin{array}{l}
=\cos \alpha x \text { in } 0 \leqq x \leqq \pi \\
=\cos \omega(2 \pi-x) \text { in } \pi \leqq x \leqq 2 \pi,
\end{array}\right.
$$

so dafi wir eine durchweg stetige Funktion erhalten, die nach der Dirichletschen Regel dann auch eine durchweg konvergente Fourierreihe erzeugen wird, welche die gewählte Funktion darstellt und eine reine cos-Reihe sein muß. Hier ist

$$
\pi a_{n}=2 \int_{0}^{\pi} \cos \alpha x \cos n x d x=\int_{0}^{\pi}[\cos (\alpha+n) x+\cos (\alpha-n) x] d x,
$$

also, da $\alpha$ keine ganze Zahl sein sollte:

$$
\pi a_{n}=(-1)^{n} \frac{2 \omega \sin \alpha \pi}{\alpha^{2}-n^{2}} \text {. }
$$

Daher wird in $0 \leqq x \leqq 2 \pi$ die Funktion $f_{2}(x)$, oder also in $-\pi \leqq x \leqq+\pi$ die Funktion $\cos c x$ durch die Reihe dargestellt:

$$
\cos \alpha x=\frac{\sin \alpha \pi}{\pi}\left[\frac{1}{\alpha}-\frac{2 \alpha}{\alpha^{2}-1^{2}} \cos x+\frac{2 \alpha}{\alpha^{2}-2^{2}} \cos 2 x-+\ldots\right] .
$$

Für $x=\pi$ bekommen wir hieraus die früher aus ganz anderen Quellen hergeleitete Entwicklung 118

$$
\pi \frac{\cos \alpha \pi}{\sin \alpha \pi}=\pi \operatorname{ctg} \alpha \pi=\frac{1}{\alpha}+\frac{2 \alpha}{\alpha^{2}-1^{2}}+\frac{2 \alpha}{c^{2}-2^{2}}+\ldots
$$

Damit sind wir in den Kreis der Entwicklungen des $\S 24$ getreten. Natürlich kann man auch die andern dort hergeleiteten Reihendarstellungen aus unsern neuen Quellen direkt gewinnen. So liefert z. B. 212 für $x=0$ die Entwicklung

$$
\frac{\pi}{\sin \alpha \pi} \cdots \frac{1}{\alpha}-\frac{2 \alpha}{\alpha^{2}-1^{2}}+\frac{2 \alpha}{\alpha^{2}-2^{2}}-\frac{2 \alpha}{\alpha^{2}-3^{2}}+-\ldots,
$$

und wenn man hiervon die vorangehende ctg-Entwicklung abzieht, so erhält man

usw.

$$
\pi \frac{1-\cos \alpha \pi}{\sin \alpha \pi}=\pi \operatorname{tg} \frac{\alpha \pi}{2}=-\frac{4 \alpha}{\alpha^{2}-1^{2}}-\frac{4 \alpha}{\alpha^{2}-3^{2}}-\frac{4 \alpha}{\alpha^{2}-5^{2}}-\ldots,
$$

b) Machen wir uns ebenso aus $F(x)=-\cos \alpha x$ eine ungerade Funktion $f_{3}(x)$ zurecht, so ist

$$
f_{3}(x)\left\{\begin{array}{l}
=\cos \alpha x \text { in } 0<x<\pi \\
=0 \text { in } 0 \text { und } \pi, \\
=-\cos \alpha(2 \pi-x) \text { in } \pi<x<2 \pi
\end{array}\right.
$$

1) Weil sonst die cos-Entwicklung trivial wäre. 
zul setzen. Und hier darf $\alpha$ auch eine ganze Zahl sein, ohne dafi wir ein triviales Resultat erhielten. Die Koeffizienten $b_{n}$ erhält man durch leicht auszurechnende Integrale und mit ihnen schlieflich die in $0<x<\pi$ gültigen Entwicklungen:

213.

c) für $\iota \neq 0,+1, \quad 2, \ldots$

$$
\begin{aligned}
\cos \alpha x & =\frac{1+\cos \alpha \pi}{\pi}\left[\frac{2}{1-\alpha^{2}} \sin x+\frac{6}{3^{2}-\alpha^{2}} \sin 3 x+\frac{10}{5^{2}-\alpha^{2}} \sin 5 x+\ldots\right] \\
& +\frac{1-\cos \alpha \pi}{\pi}\left[\frac{4}{2^{2}-\alpha^{2}} \sin 2 x+\frac{8}{4^{2}-\alpha^{2}} \sin 4 x+\ldots\right]
\end{aligned}
$$

B) für $\alpha=+p=$ ganze Zahl

$\cos p x\left\{\begin{array}{l}\frac{4}{\pi}\left[\frac{1}{1-p^{2}} \sin x+\frac{3}{3^{2}-p^{2}} \sin 3 x+\ldots\right], \text { falls } p \text { gerade ist, } \\ 4\left[\frac{2}{\pi} \sin 2 x+\frac{4}{4^{2}-p^{2}} \sin 4 x+\ldots\right], \text { falls } p \text { ungerade ist. }\end{array}\right.$

Aus allen diesen Reihen kann man durch Spezialisierung von \& und $x$ ungezählte numerische Reihen herleiten.

4. Die Behandlung der Funktion $\boldsymbol{F}(\boldsymbol{x})=\sin \alpha \boldsymbol{x}$ führt zu ganz ähnlichen Entwicklungen.

5. Wird die Funktion $F(x)=-\log \left(2 \sin \frac{x}{2}\right)$ für die cos-Entwicklung zurechtgemacht, so findet man die in $0<x<\pi$ gültige Darstellung

21.

$$
\cos x+\frac{\cos 2 x}{2}+\frac{\cos 3 x}{3}+\ldots-\log \left(2 \sin \frac{x}{2}\right) \text {. }
$$

Hier muf jedoch durch eine besondere Untersuchung gezeigt werden, dafs das Ergebnis giiltig ist, obwohl die Funktion in der Umgebung der Punkte 0 und $2 \pi$ nicht beschränkt ist.

6. Beispiel. $F(x)=e^{a x}+e^{-a x}, \alpha \neq 0$, soll in eine cos-Reihe entwickelt werden. Es ist also zu setzen

$$
f_{2}(x)\left\{\begin{array}{l}
=F(x) \text { in } 0 \leqq x \leqq \pi \\
=F(2 \pi-x) \text { in } \pi \leqq x \leqq 2 \pi .
\end{array}\right.
$$

Nach Ausrechnung der sehr einfachen Integrale für dié'Koeffizienten $a_{n}$ erhält man, guiltig in $-\pi \leqq x \leqq+\pi$,

215.

$$
\begin{array}{ll}
\pi & e^{\alpha x}+e^{-\alpha x} \\
2 & e^{\alpha \pi}-e^{-\alpha \pi}
\end{array}=\frac{\alpha}{2 \alpha}-\frac{\alpha}{\alpha^{2}+1^{2}} \cos x+\frac{\alpha}{\alpha^{2}+2^{2}} \cos 2 x-+\ldots .
$$

Setzt man hierin z. B. $x=\pi$ und ersetzt dann $2 \alpha \pi$ einfacher durch $t$, so ergibt sich nach einfachen Umformungen - gültig für alle $t \neq 0-$

$$
\frac{1}{t}\left[\frac{1}{1-e^{-t}}-\frac{1}{t}-\frac{1}{2}\right]=2 \underset{n=1}{\sum^{\infty}} \frac{1}{(2 n \pi)^{2}+t^{2}},
$$

also eine „Partialbruch-Entwicklung" jener merkwürdigen Funktion, für die wir in $\S 24,4$ schon die Potenzreihenentwicklung gewonnen hatten.

\section{Verschiedene Bemerkungen.}

Gerade die Tatsache, daf die trigonometrischen Reihen auferordentlich umfangreiche Klassen von Funktionen darzustellen vermögen, macht die Frage nach den Grenzen dieser Leistungsfähigkeit doppelt interessant. Wie schon 
216. $\$ 50$. Anwendungen der Theorie der Fourierschen Reihen.

betont, kennt man die notwendigen und hinreichenden Bedingungen nicht, der eine Funktion genügen muf, wenn sie durch ihre Fourierreihe soll dargestellt werden können. Man ist vielmehr gezwungen, hierin eine wesentlich neue Grundeigenschaft der Funktionen $z u$ sehen, denn alle Versuche, sie direkt aus den andern Grundeigenschaften der Funktionen (Stetigkeit, Differenzierbarkeit, Integrierbarkeit usw.) aufzubauen, sind bisher gescheitert. Wir müssen es uns versagen, dies im einzelnen durch Ausführung entsprechender Beispiele zu belegen, möchten aber doch einige der hierhergehörigen Tatsachen kurz zur Sprache bringen.

1. Eine Vermutung, die man ganz naturgemä́s zuerst haben wird, ist die, dafs alle stetigen Funktionen durch ihre Fourierreihe dargestellt werden können. Dem ist nicht so, wie zuerst $d u$ Bois-Reymond an einem Beispiel gezeigt hat (Gött. Nachr. 1873, S. 571).
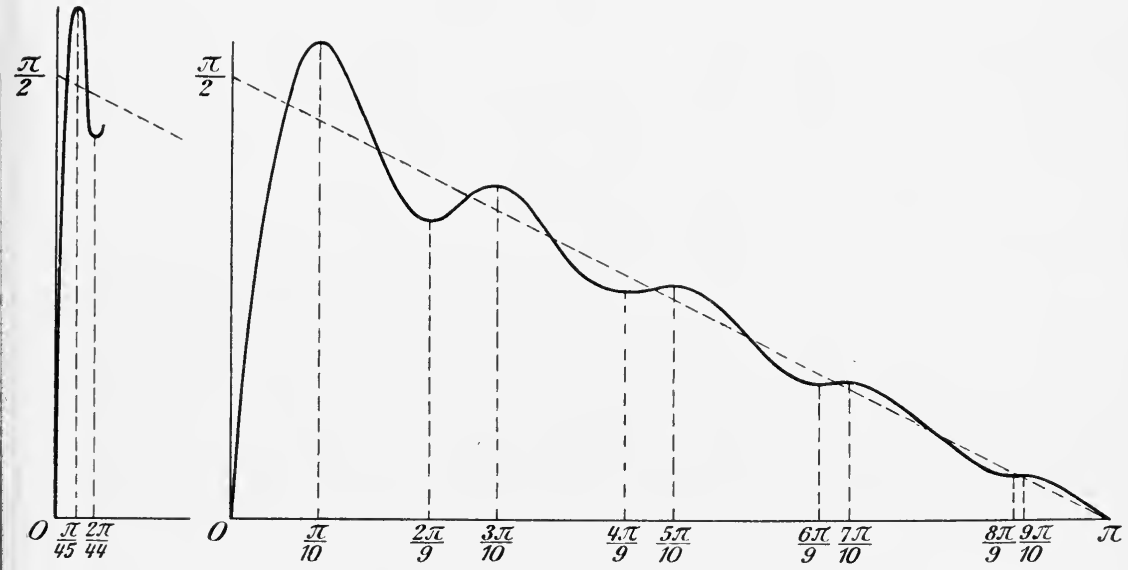

Fig. 9.

2. Dafi andrerseits zur Stetigkeit die Differenzierkeit nicht hinzuzukommen braucht, zeigt das Weierstraßsche ${ }^{1}$ ) Beispiel der gleichmäfig konvergenten trigonometrischen Reihe

$$
\sum_{n=1}^{\infty} a^{n} \cos b^{n} \pi x, \quad\left(0<a<1, b>0 \text { gan } z, a b>1+\frac{3}{2}_{2}^{3} \pi\right),
$$

die also die Fourierreihe ihrer Summe ist (s. 200,1a), welche eine stetige, aber nirgends differenzierbare Funktion darstellt.

3. Ob es stetige Funktionen gibt, deren Fourierreihe überall divergiert, ist zurzeit noch unbekannt.

4. Besonders bemerkenswert ist eine Erscheinung, die nach ihrem Entdecker als Gibbssche Erscheinung bezeichnet wird ${ }^{2}$ ) und bei der Reihe 210 a zuerst festgestellt worden ist: Die Approximationskurven $s_{n}(x)$ schiefen in der Nähe von $x=0$ sozusagen über das Ziel. Genauer: Bezeichnet man die Ab-

1) Abhandlungen zur Funktionenlehre, Werke Bd. 2, S. 223. (Zuerst bekannt geworden 1875.)

2) J.W. Gibbs in: Nature, Bd. 59 (London 1898/99), S. 606. - Vgl. auch T. H. Gronwall, Über die Gibbssche Erscheinung, Math. Annalen, Bd. 72 (1912), S. 228 . 
szisse des größten Maximums der Kurve $y=s_{n}(x)$, das zwischen 0 und $\pi \operatorname{liegt}^{1}$ ), mit $\xi_{n}$ und seine Ordinate mit $\eta_{n}$, so strebt $\xi_{n} \rightarrow 0$, aber $\eta_{n}$ nicht, wie man es erwarten sollte, $\rightarrow \frac{\pi}{2}$, sondern gegen einen Wert $g$, der $=\frac{\pi}{2} \cdot 1,089490 \ldots$ ist. Das Grenzgebilde, das die Kurven $s_{n}(x)$ approximieren, enthält also auf̉er der Funktion 210 a noch eine Strecke der Ordinatenachse zwischen den Punkten mit den Ordinaten $+g$, die um fast $9 \%$ länger ist als der Sprung der Funktion. In Fig. 9 ist die $n$ te Aproximationskurve für $n=9$ im Intervall $0 \ldots \pi$ ausgezeichnet und für $n \quad 44$ in ihrem Anfangsteil dargestellt.

\section{\$51. Produkte mit veränderlichen Gliedern.}

Liegt ein Produkt der Form

$$
\prod_{n=1}^{\infty}\left(1+f_{n}^{\prime}(x)\right)
$$

vor, dessen Glieder Funktionen von $x$ sind, so werden wir ganz entsprechend wie bei Reihen ein Intervall $J$ als ein Konvergenzintervall des Produktes bezeichnen, wenn für alle Punkte $x$ desselben nicht nur sämtliche Funktionen $f_{n}(x)$ definiert sind, sondern zugleich auch das Produkt selber konvergent ist.

So sind z. B. die Produkte

$$
\prod_{n=1}^{x}\left(1-\begin{array}{c}
x^{2} \\
n^{2}
\end{array}\right), \prod_{n=1}^{\infty}\left(1: \begin{array}{c}
x^{2} \\
n^{2}
\end{array}\right), \prod_{n=1}^{\infty}\left(1+(-1)^{n} \frac{x}{n}\right), \prod_{n=2}^{x}\left(1+\frac{x}{n \log ^{2} n}\right), \ldots
$$

für jedes reelle $x$ konvergent (s. 127, Satz 7), und dasselbe gilt von jedem Produkt der Form $\Pi\left(1+a_{n} x\right)$, wenn $\Sigma a_{n}$ entweder eine absolut konvergente oder eine solche nur bedingt konvergente Reihe ist, bei der $\Sigma a_{n}{ }^{2}$ absolut konvergiert (127, Satz 9).

Für jedes $x$ aus $J$ hat dann das Produkt einen ganz bestimmten Wert und es definiert dort also eine wohlbestimmte Funktion $F(x)$. Wir sagen wieder: das Produkt stelle in $J$ die Funktion $F(x)$ dar, oder $F(x)$ sei dort in das Produkt eniwickelt. Und die Hauptfrage ist wie damals: inwieweit übertragen sich die wesentlichen Eigenschaften (Stetigkeit, Differenzierbarkeit usw.) der Glieder $f_{n}(x)$ auf die dargestellte Funktion $F(x)$. Auch hier wird die Antwort lauten, daß dies in weitestgehendem Maße der Fall ist, solange die auftretenden Produkte gleichmäßig konvergieren.

Wie die Gleichmäßigkeit der Konvergenz bei Produkten zu definieren ist, ist ja im Anschluß an die entsprechende Definition bei Reihen fast selbstverständlich, da es sich ja in beiden Fällen im wesentlichen um Funktionenfolgen handelt (vgl. 190, 4). Doch wollen

1) Die dortigen Maxima liegen bei $x=\frac{\pi}{n+1}, \frac{3 \pi}{n+1}, \frac{5 \pi}{n+1}, \ldots$, und das erste von ihnen ist das gröbte. Die Minima liegen bei $x=\frac{2 \pi}{n}, \frac{4 \pi}{n}, \ldots$. 
wir die der damaligen 2. Form der Definition (191, 4) entsprechende hierher setzen:

Definition. Das Produkt $I\left(1+f_{n}(x)\right)$ heißt in einem Intervall $J \mathbf{2 1 7 .}$ gleichmäßig konvergent, wenn nach $W$ ahl von $\varepsilon>0$ sich eine Zahl $N$ so wählen läßt, daß nun für alle $n>N$, alle $k \geqq 1$ und alle $x$ aus $J$ stets

$$
\left|\left(1+f_{n+1}(x)\right)\left(1+f_{n+2}(x)\right) \ldots\left(1+f_{n+k}(x)\right)-1\right|<\varepsilon
$$

bleibt $\left.{ }^{1}\right)$.

Auf der Grundlage dieser Definition ist es nicht schwer zu zeigen, $\mathrm{daß}$ mutatis mutandis die Sätze des $\S 47$ auch für unendliche Produkte gelten ${ }^{2}$ ). Wir wollen die Ausführung der Einzelheiten hiervon indessen dem Leser überlassen und nur einige etwas weniger weitgehende Sätze beweisen, die aber für alle unsere Anwendungen vollkommen ausreichen werden und den Vorteil bieten, uns zugleich Kriterien für die Gleichmäßigkeit der Konvergenz eines Produktes zu liefern. Es gilt da zunächst der

Satz 1. Das Produkt $\Pi\left(1+f_{n}(x)\right)$ konvergiert gleichmäßig in $J \mathbf{2 1 8 .}$ und stellt eine dort stetige Funklion dar, wenn die Funktionen $f_{n}(x)$ in $J$ sämtlich stetig sind und dort die Reihe $\Sigma\left|f_{n}(x)\right|$ gleichmäßig konvergiert.

Beweis. Wenn $\Sigma^{\prime}\left|f_{n}(x)\right|$ in $J$ konvergiert, so ist nach 127, Satz 7 dasselbe mit dem Produkt $\Pi\left(1+f_{n}(x)\right)$ der Fall; es konvergiert dort sogar absolut. Die dargestellte Funktion heiße $F(x)$. Nun wählen wir $m$ so groß, daß für alle $x$ aus $J$ und alle $k \geqq 1$ stets

$$
\left|f_{m+1}(x)\right|+\left|f_{m+2}(x)\right|+\ldots+\left|f_{m+k}(x)\right|<1
$$

bleibt - was nach Voraussetzung möglich ist -, und betrachten das Produkt

$$
\prod_{n=m+1}^{\infty}\left(1+f_{n}(x)\right)
$$

1) Diese Definition schliefit die der Konvergenz mit ein. Setzt man die letztere voraus, so kann man vom "Rest" $r_{n}(x)=\left(1+f_{n+1}(x)\right)\left(1+f_{n+2}(x)\right) \ldots$ des Produktes sprechen und definieren: $I I\left(1+f_{n}(x)\right)$ soll in $J$ gleichmärig konvergent heißen, wenn für jede aus $J$ entnommene, sonst ganz beliebige Punktfolge $\left(x_{n}\right)$ stets $r_{n}\left(x_{n}\right) \rightarrow 1$ strebt.

2) Setzt $\operatorname{man} \prod_{v=1}^{m}\left(1+f_{v}(x)\right)=P_{m}(x)$ und $\prod_{v=m+1}^{\infty}\left(1+f_{v}(x)\right)=F_{m}(x)$, so ergibt sich z. B. die Stetigkeit von $F(x)$ in $x_{0}$ ganz leicht aus der Gleichung

$$
\begin{aligned}
F(x)-F\left(x_{0}\right) & =P_{m}(x) \cdot F_{m}(x)-P_{m}\left(x_{0}\right) F_{m}\left(x_{0}\right) \\
& =\left[P_{m}(x)-P_{m}\left(x_{0}\right)\right] F_{m}(x)+\left[F_{m}(x)-F_{m}\left(x_{0}\right)\right] P_{m}\left(x_{0}\right)
\end{aligned}
$$


dessen Teilprodukte wir mit $p_{n}(x), n>m$, bezeichnen. Die durch dasselbe dargestellte Funktion heiße $F_{m}(x)$. Nun ist aber (vgl. 190, 4)

$$
\begin{aligned}
F_{m} & =p_{m+1}+\left(p_{m+2}-p_{m+1}\right)+\ldots+\left(p_{n}-p_{n-1}\right)+\ldots \\
& =p_{m+1}+p_{m+1} \cdot f_{m+2}+p_{m+2} \cdot f_{m+3}+\ldots+p_{n-1} \cdot f_{n}+\ldots,
\end{aligned}
$$

d. h. $F_{m}(x)$ läßt sich auch - was ja nach $\S 30$ selbstverständlich ist -, durch eine unendliche Reihe darstellen. Diese ist aber in $J$ gleichmäßig konvergent. Denn für $n>m$ ist erstlich

$$
p_{n} \mid \leqq\left(1+f_{m+1} \mid\right) \cdot\left(1+\left|f_{m+2}\right|\right) \ldots\left(1+\left|f_{n}\right|\right)<e^{f_{m+1}+\left|f_{m+2}\right|+\ldots}<e<3,
$$

und andrerseits

$$
\sum_{n=m}^{\infty}\left|f_{n}(x)\right|
$$

nach Voraussetzung in $J$ gleichmäßig konvergent. Also strebt die Folge der Funktionen $p_{n}(x)$ gleichmäßig in $J$ gegen $F_{m}$, so daß damit das Produkt $\prod_{n=m+1}^{\infty}\left(\mathbf{1}+f_{n}(x)\right)$ als gleichmäßig konvergent erkannt ist, - eine Eigenschaft, die durch das Davortreten der ersten $m$ Faktoren nun nicht mehr gestört wird.

Nach 193 aber muß nun $F_{m}(x)$ eine in $J$ stetige Funktion sein, da ja die Glieder der sie darstellenden Reihe dort sämtlich stetig sind. Dasselbe gilt dann auch von der Funktion

$$
F(x)=\left(1+f_{1}(x)\right) \ldots\left(1+f_{m}(x)\right) F_{m}(x),
$$

w. z. b.w.

Ganz ähnlich beweist man den

Satz 2. Sind die Funktionen $f_{n}(x)$ sämtlich in $J$ differenzierbar und ist dort außer der Reihe $\Sigma\left|f_{n}(x)\right|$ auch noch die Reihe $\Sigma\left|f_{n}{ }^{\prime}(x)\right|$ gleichmäßig konvergent, so ist dort auch $F(x)$ differenzierbar; und für die Ableitung hat man in jedem Punkte $x$ aus $J_{\text {, in }} \operatorname{dem} F(x) \neq 0$ ist, die Reihentarstellung

$$
\left.\frac{F^{\prime}(x)}{F(x)}=\sum_{n=1}^{\infty}{\frac{f_{n}^{\prime}(x)}{1+f_{n}(x)}}^{1}\right) .
$$

Beweis. Man kann den Beweis ganz analog führen, wie den des vorigen Satzes; doch wollen wir hier, um auch andre Angriffs-

1) Ist $g(x)$ in einem speziellen Punkte $x$ differenzierbar und dort $g(x) \neq 0$, so nennt man den Quotienten $\frac{g^{\prime}(x)}{g(x)}$ die logarithmische Ableitung von $g(x)$, weil er $=\frac{d}{d x} \log g(x)$ ist. Ist $g(x)=g_{1}(x) \cdot g_{2}(x) \cdots g_{k}(x)$, so ist bekanntlich

$$
\frac{g^{\prime}(x)}{g(x)}=\frac{g_{1}{ }^{\prime}(x)}{g_{1}(x)}+\frac{g_{2}{ }^{\prime}(x)}{g_{2}(x)}+\ldots+\frac{g_{k}{ }^{\prime}(x)}{g_{k}(x)},
$$

falls die $g_{j}(x)$ sämtlich in dem betreffenden Punkte $x$ differenzierbar sind. 
methoden kennenzulernen, den Beweis mit Hilfe der log-Funktion führen. Wir wählen dazu $m$ jetzt so, daß für alle $x$ aus $J$

$$
\left|f_{m+\mathbf{1}}(x)\right|+\left|f_{m+2}(x)\right|+\ldots<\frac{1}{2}
$$

und also speziell für alle $n>n i$

$$
\left|f_{n}(x)\right|<\frac{1}{2}
$$

bleibt. Dann ist dort nach 127, Satz 8 auch die Reihe

$$
\sum_{n=m+1}^{\infty} \log \left(1+f_{n}(x)\right)
$$

absolut konvergent. Die daraus durch gliedweise Differentiation gewonnene Reihe

$$
\stackrel{\Perp}{n=m+1}_{n=\frac{f_{n}^{\prime}(x)}{1+f_{n}(x)}}
$$

ist dort sogar gleichmäßig (und absolut) konvergent. Denn weil $\left|f_{n}(x)\right|<\frac{1}{2}$ ist, bleibt $\left|1+f_{n}(x)\right|>\frac{1}{2}$ und also $\left|\frac{1}{1+f_{n}^{\prime}(x)}\right|<2$, so daß die Gleichmäßigkeit der Konvergenz von $\Sigma\left|f_{n}^{\prime}(x)\right|$ diejenige der letzten Reihe nach sich zieht. Also ist (nach 196)

$$
\frac{F_{m}{ }^{\prime}(x)}{F_{m}(x)}=\sum_{n=m+1}^{\infty} \frac{f_{n}^{\prime}(x)}{1+f_{n}(x)},
$$

wenn wie vorhin $\prod_{n=m+1}^{\infty}\left(1+f_{n}(x)\right)=F_{m}$ und also $\underset{n=m+1}{\stackrel{x}{x}} \log \left(1+f_{n}(x)\right)$ $=\log F_{m}(x)$ gesetzt wird. Da endlich

$$
F(x)=\left(1+f_{1}(x)\right) \ldots\left(1+f_{m}(x)\right) \cdot F_{m}(x)
$$

ist und der letzte Faktor rechterhand sich in $J$ nun als differenzierbar erwiesen hat, so ist dort auch $F(x)$ differenzierbar. Ist überdies $F(x) \neq 0$, so ergibt sich aus der letzten Gleichung nach der in der vorigen Fußnote genannten Differentiationsregel sofort die Behauptung.

1. Das Produkt

Anwendungen.

$$
F(x)=x \cdot \prod_{n=1}^{\infty}\left(1-\frac{x^{2}}{n^{2}}\right)
$$

ist in jedem beschränkten Intervall gleichmäßjig konvergent, weil

$$
\Sigma\left|\frac{x^{2}}{n^{2}}\right|=\left.x\right|^{2} \cdot \Sigma \frac{1}{n^{2}}
$$

eine dort ersichtlich gleichmäfig konvergente Reihe ist. Es definiert also eine durchweg stetige Funktion $F(x)$. Diese ist aber auch differenzierbar, denn auch $\Sigma\left|f_{n}^{\prime}(x)\right|=2|x| \Sigma \frac{1}{n^{2}}$ ist in jedem beschränkten Intervall gleichmäßig konvergent. Also ist für alle $x \neq 0, \pm 1,-2, \ldots$

Nach 117 bedeutet dies aber, daf

$$
\frac{F^{\prime}(x)}{F(x)}=\frac{1}{x}+\sum_{n=1}^{\infty} \frac{2 x}{x^{2}-n^{2}} .
$$

$$
\frac{F^{\prime}(x)}{F(x)}=\pi \operatorname{ctg} \pi x=\frac{(\sin \pi x)^{\prime}}{\sin \pi x}
$$

Knopp, Unendliche Reihen. 
ist. Aus

folgt aber, dafi auch

$$
\frac{F_{1}^{\prime}}{F_{1}}-\frac{F^{\prime}}{F}=\frac{F \cdot F_{1}^{\prime}-F_{1} \cdot F^{\prime}}{F \cdot F_{1}}=0
$$

d. h. hier

$$
\frac{d}{d x}\left(\frac{F_{1}}{F}\right)=0 \text { oder also } F_{1}=c \cdot F
$$

$$
\sin \pi x=c \cdot x \cdot \prod_{n=1}^{\infty}\left(1-\frac{x^{2}}{n^{2}}\right)
$$

ist, wenn unter $c$ eine passende Konstante verstanden wird. Diese bestimmt sich nun einfach dadurch, dafs man die letzte Gleichung durch $x$ dividiert und $x \rightarrow 0$ rücken läft. Dann strebt die linke Seite, wie die Potenzreihe für die sin-Funktion lehrt, $\rightarrow \pi$, die rechte aber $\rightarrow c$, weil das Produkt an der Stelle $x=0$ stetig ist. Also ist $c=\pi$ und

$$
\left.\sin \pi x=\pi x \cdot \prod_{n=1}^{x}\left(1-\frac{x^{2}}{n^{2}}\right)^{1}\right) .
$$

2. Für $\cos \pi x$ findet man nun ohne neue Rechnung

$$
\cos \pi x-\frac{\sin 2 \pi x}{2 \sin \pi x}=\frac{2 \pi x \cdot \Pi\left(1-\frac{4 x^{2}}{n^{2}}\right)}{2 \pi x \cdot \Pi\left(1-\frac{x^{2}}{n^{2}}\right)} \prod_{k=1}^{\infty}\left(1-\frac{4 x^{2}}{(2 k-1)^{2}}\right) .
$$

3. Das sin-Produkt liefert für spezielle $x$ wichtige numerische Produkte. Z. B. für $x=\frac{1}{2}$

oder also

$$
1-\frac{\pi}{2} \cdot \Pi\left(1-\frac{1}{4 n^{2}}\right)={ }_{2}^{\pi} \cdot \prod_{n=1}^{x}\left(\frac{(2 n-1)(2 n+1)}{2 n}\right)
$$

$$
\frac{\pi}{2}=\frac{2 \cdot 2 \cdot 4 \cdot 4 \cdot 6 \cdot 6 \cdot 8 \cdot 8 \ldots}{1 \cdot 3 \cdot 3 \cdot 5 \cdot 5 \cdot 7 \cdot 7 \cdot 9}
$$

\section{(Wallissches Produkt) ${ }^{2}$ ).}

Da hiernach auch

ist, oder also

$$
\lim _{k \rightarrow+\infty}\left(\frac{2}{1}\right)^{2} \cdot\left(\frac{4}{3}\right)^{2} \cdot \ldots \cdot\left(\begin{array}{cc}
2 k \\
2 k & 1
\end{array}\right)^{2} \cdot 2 k \frac{1}{2 k+1}=\begin{aligned}
& \pi \\
& 2
\end{aligned}
$$

$$
\frac{2}{1} \cdot \frac{4}{3} \cdot \frac{6}{5} \cdot \ldots \cdot 2 k-1 \cdot \frac{1}{\sqrt{k}}>\sqrt{\pi}
$$

strebt, so erhalten wir zugleich die merkwirdige Beziehung

$$
(1)^{n}\left(\begin{array}{c}
-\frac{1}{2} \\
n
\end{array}\right) \simeq \frac{1}{\sqrt{\pi n}}
$$

für den Koeffizienten von $x^{n}$ in der Entwicklung von $\frac{1}{1^{\prime} 1-x}$.

1) Dies Produkt, die gleich nachher in 2. und 4. besprochenen, ferner das merkwïrdige Produkt $\mathbf{2 5} 7$ und viele andere grundlegende Produktdarstellungen rühren von Euler her.

2) Arithmetica infinitorum, Oxford 1656. (Vgl. S. 212, Fufn.) 
4. Die Folge der Funktionen

$$
g_{n}(x)=\frac{x(x+1)(x+2) \ldots(x+n)}{n ! n^{x}}=\frac{1}{n^{x}} \cdot x\left(1+\frac{x}{1}\right)\left(1+\frac{x}{2}\right) \ldots\left(1+\frac{x}{n}\right),
$$

(s. 128, 4) vertritt nicht unmittelbar ein unendliches Produkt der Form $\Pi\left(1+f_{n}(x)\right)$, zumal $\Pi\left(1+\frac{x}{n}\right)$ nach 125, Satz 3 u. 4 divergiert. Aber nach 127, Satz 10 geschieht diese Divergenz in der Weise, daf

$$
\left(1+\frac{x}{1}\right)\left(1+\frac{x}{2}\right) \ldots\left(1+\frac{x}{n}\right) \sim e^{x\left(1+\frac{1}{2}+\ldots+\frac{1}{n}\right)}
$$

ist. Wegen 128, 2 bedeutet dies gerade, daf

$$
\frac{x \cdot\left(1+\frac{x}{1}\right)\left(1+\frac{x}{2}\right) \ldots\left(1+\frac{x}{n}\right)}{n^{x}}=g_{n}(x)
$$

für $n \rightarrow \infty$ einem wohlbestimmten endlichen und von 0 verschiedenen Grenzwert zustrebt; - letzteres natürlich nur, falls $x \neq 0,-1,-2, \ldots$ Hiernach ist auch

$$
\lim \frac{1}{g_{n}(x)}=\Gamma(x)
$$

für jedes $x \neq 0,-1,-2, \ldots$ eine wohlbestimmte Zahl. Man nennt die hierdurch definierte Funktion von $x$ die Gammafunktion. Sie ist von Euler (s. o.) in die Analysis eingeführt und gehört neben den elementaren Funktionen zu den wichtigsten. Die Untersuchung ihrer weiteren Eigenschaften liegt jedoch außerhalb des Rahmens dieses Buches. (Vgl. indessen noch S. 423/24.)

\section{Aufgaben zum XI. Kapitel.}

I. Beliebige Reihen mit veränderlichen Gliedern.

154. Es bedeute $(n x)$ die Differenz zwischen $n x$ und der zunächst bei $n x$ gelegenen ganzen Zahl, aber stets den Wert $+\frac{1}{2}$, wenn $n x$ gerade in der Mitte zwischen zwei ganzen Zahlen liegt. Dann ist die Reihe $\sum \frac{(n x)}{n^{2}}$ für alle $x$ gleichmäßig konvergent. Die dargestellte Funktion aber fïr $x=\frac{2 p}{2} \frac{1}{q}$, ( $p, q$ ganze Zahlen), unstetig, fuir irrationale $x$ stetig.

155. Wenn $a_{n} \rightarrow 0$ strebt, so konvergiert

$$
\sum a_{n} \cdot x\left(\frac{\sin n x}{n x}\right)^{2}
$$

gleichmäfig für alle $x$. Ist dies auch noch für $a_{n} \equiv 1$ der Fall?

156. Die Produkte
a) $\Pi\left(1+(-1)^{n} \frac{x}{n}\right)$,
b) $I I \cos \frac{x}{n}$,
c) $\Pi\left(1+\sin ^{2} \frac{x}{n}\right)$,
d) $\Pi\left(1+(-1)^{n} \sin \frac{x}{n}\right)$

konvergieren gleichmäfig in jedem beschränkten Intervall.

15\%. Die Reihe, deren Teilsummen die Werte $s_{n}(x)=\frac{x^{n}}{1+x^{2 n}}$ haben, konvergiert für jedes $x$. Ist die Konvergenz in jedem Intervall eine gleichmäßige? Man zeichne die Approximationskurven. 
158. Eine Reihe $\Sigma f_{n}(x)$ von stetigen pusitiven Funktionen ist sicher gleichmäßig konvergent, wenn sie eine stetige Funktion $F(x)$ darstellt. (Vgl. S. 332.)

159. Ist $\sum \frac{x}{n\left(1+n x^{2}\right)}$ in jedem Intervall gleichmäßig konvergent? Ist die dargestellte Funktion stetig?

160. Beim Beweise von 111 lag eine Situation folgender Art vor: In einem Ausdruck der Form

$$
F(n)=a_{0}(n)+a_{1}(n)+\cdots+a_{k}(n)+\cdots+a_{p_{n}}(n)
$$

strebt für jedes feste $k$ das Glied $a_{k}(n)$ mit wachsendem $n$ gegen einen Grenzwert $\alpha_{k}$. Gleichzeitig nimmt die Anzahl dieser Glieder zu: $p_{n} \rightarrow \infty$. Darf dann geschlossen werden, daf 3

$$
\lim _{n \rightarrow \infty} F(n)=\sum_{k=0}^{\infty} \kappa_{k}
$$

ist, falls die rechtsstehende Reihe konvergiert? Man zeige, daf dies sicher erlaubt ist, falls für jedes $k$ und alle $n$

$$
\left|a_{k}(n)\right|<\gamma_{k} \text { bleibt und } \Sigma \gamma_{k}
$$

konvergiert. - Man formuliere den entsprechenden Satz für unendliche Produkte. - (Vgl. hierzu Aufg. 15, wo solche gliedweisen Grenziibergänge nicht erlaubt waren.)

161. Die beiden Reihen

$$
\begin{aligned}
& x+\frac{x^{3}}{3}-\frac{x^{4}}{2}+\frac{x^{5}}{5}+\frac{x^{7}}{7}-\frac{x^{8}}{4}++--\cdots \\
& x+\frac{x^{3}}{3}-\frac{x^{2}}{2}+\frac{x^{5}}{5}+\frac{x^{7}}{7}-\frac{x^{4}}{4}++--\cdots
\end{aligned}
$$

sind beide für $0 \leqq x \leqq 1$ konvergent und haben für $x=+1$ dieselbe Summe $\frac{3}{2} \log 2$. Wie verhalten sie sich für $x \rightarrow 1-0$. Wie liegt es für $x \rightarrow+1+0$ bei den beiden für $x \geq 1$ konvergenten Reihen

$$
\begin{aligned}
& 1+\frac{1}{3^{x}}-\frac{2}{4^{x}}+\frac{1}{5^{x}}+\frac{1}{7^{x}}-\frac{2}{8^{x}}++\ldots \\
& 1+\frac{1}{3^{x}}-\frac{1}{2^{x}}+\frac{1}{5^{x}}+\frac{1}{7^{x}}-\frac{1}{4^{x}}++\ldots ?
\end{aligned}
$$

162. Die Reihe $\sum_{n=1}^{\infty}\left[\frac{x^{n}}{n}-\frac{x^{2 n-1}}{2 n-1}-\frac{x^{2 n}}{2 n}\right]$ ist in $0 \leqq x \leqq 1$ konvergent. Welche Summe hat sie? Ist die Konvergenz eine gleichmäßige?

163. Man zeige, daf 3 für $x \rightarrow 1+0$

a) $\lim (1-x) \sum_{n=1}^{\infty} \frac{1}{n^{x}}=1$,

b) $\lim \left[\sum_{n=1}^{\infty} \frac{1}{n^{x}}-\frac{1}{1-x}\right]=C \quad($ s. 176, 1$)$

ist.

164. Man zeige, daf für $x \rightarrow 1-0$

a) $\sum_{n=1}^{\infty} \frac{(-1)^{n-1}}{n} \cdot \frac{x^{n}}{1+x^{n}} \rightarrow \frac{1}{2} \log 2$,

b) $(1-x) \cdot \sum_{n=1}^{x}(-1)^{n-1} \frac{x^{n}}{1-x^{2 n}} \rightarrow \frac{1}{2} \log 2$,

c) $(1-x) \cdot \sum_{n=1}^{\infty}(-1)^{n-1} \frac{n x^{n}}{1-x^{2 n}} \rightarrow-\frac{1}{4}$

strebt. 
165. Die Reihe, deren Teilsummen $s_{n}(x)=\frac{n x}{1+n^{2} x^{4}}$ sind, gestattet über ein Intervall, das in 0 endet, keine gliedweise Integration. Man zeichne die Approximationskurven.

\section{Fourierreihen.}

166. Darf man von der Reihe $\mathbf{2 1 0}$ a durch gliedweise Integration zu den folgenden übergehen:
a) $\sum_{n=1}^{\infty} \frac{\cos 2 \pi n x}{n^{2}}=\left(x^{2}-x+\frac{1}{6}\right) \cdot \pi^{2}$,
b) $\sum_{n=1}^{\infty} \frac{\sin 2 \pi n x}{n^{3}}=\left(\frac{2}{3} x^{3}-x^{2}+\frac{1}{3} x\right) \cdot \pi^{3}$,
c) $\sum_{n=1}^{\infty} \frac{\cos 2 \pi n x}{n^{4}}=\left(-\frac{1}{3} x^{4}+\frac{2}{3} x^{3}-\frac{1}{3} x^{2}+\frac{1}{9} \overline{0}\right) \pi^{4} \quad$ usw.?

In welchem Intervall sind diese Gleichungen gültig?

167. Man leite ebenso aus $210 \mathrm{c}$ die Gleichungen

a) $\sum_{n=1}^{\infty} \frac{\sin (2 n-1) x}{(2 n-1)^{3}}=\frac{\pi x}{8}(\pi-x)$,

b) $\sum_{n=1}^{\infty} \frac{\cos (2 n-1) x}{(2 n-1)^{4}}=\frac{\pi}{48}\left(\frac{\pi}{2}-x\right)\left(\pi^{2}+2 \pi x-2 x^{2}\right)$.

Was würden die nächsten Integrationen ergeben? In welchen Intervallen gelten diese Darstellungen?

168. Man leite aus $\mathbf{2 0 9}, \mathbf{2 1 0}$ und den in den vorigen Aufgaben gegebenen Gleichungen noch die folgenden her und bestimme die genauen Gültigkeitsintervalle:
a) $\cos x-\frac{\cos 3 x}{3}+\frac{\cos 5 x}{5}-\ldots= \pm \frac{\pi}{4}$,
b) $\cos x-\frac{\cos 3 x}{3^{3}}+\frac{\cos 5 x}{5^{3}}-\ldots=\frac{\pi}{8}\left(\frac{\pi^{2}}{4}-x^{2}\right)$,
c) $\sin x-\frac{\sin 3 x}{3^{4}}+\frac{\sin 5 x}{5^{4}}-\ldots=\frac{\pi x}{8}\left(\frac{\pi^{2}}{4}-\frac{x^{2}}{3}\right)$,

usw.

169. Man leite aus $\mathbf{2 1 5}$ neue Darstellungen her, indem man $x$ durch $\pi-x$ ersetzt oder gliedweis differenziert. Ist diese letztere Operation erlaubt? Wie lauten die neuen Reihen?

170. Wie lautet die sin-Reihe, wie die $\cos -$ Reihe für $e^{a x}$ ? Wie lautet die vollständige Fourierentwicklung für $e^{\sin x}$ ? Man zeige, daßs die letztere die Form

$$
\frac{1}{2} a_{0}+b_{1} \sin x-a_{2} \cos 2 x-b_{3} \sin 3 x+a_{4} \cos 4 x+b_{5} \sin 5 x+\ldots
$$

hat mit positiven $a_{v}$ und $b_{v}$.

171. Sind $x$ und $y$ positiv aber $<\pi$, so ist

$$
\sum_{n=1}^{\infty} \frac{\sin n x \cos n y}{n}=\left\{\begin{array}{cll}
\frac{\pi}{2}-\frac{x}{2}, & \text { falls } x>y, \\
\frac{\pi}{4}-\frac{x}{2}, & \text { falls } x=y, \\
-\frac{x}{2}, & \text { falls } x<y .
\end{array}\right.
$$


172. Man berechne die Integrale

$$
\int_{0}^{\frac{\pi}{2}} \frac{\sin x}{x} d x \text { und } \int_{0}^{\pi} \frac{\sin x}{x} d x .
$$

(Das erste ist $=1,37498 \ldots$, das $z$ weite $=1,8519 \ldots$.)

173. Fiur alle $x$ und alle $n$ ist

$$
\left|\sin x+\frac{\sin 2 x}{2}+\ldots+\frac{\sin n x}{n}\right| \leqq \int_{0}^{\pi} \frac{\sin x}{x} d x,
$$

und die rechtsstehende Schranke kann dabei durch keine kleinere ersetzt werden. (Vgl. die vorige Aufgabe.)

(Weitere Aufgaben über spezielle Fourierreilen findet man noch beim nächsten Kapitel.)

XII. Kapitel.

\section{Reihen mit komplexen Gliedern.}

\section{§ 52. Komplexe Zahlen und Zahlenfolgen.}

Nachdem wir im 1. Kapitel alle für den Aufbau des Systems der reellen Zahlen wesentlichen Begriffsbildungen eingehend besprochen haben, bietet die Einführung weiterer Zahlenarten keine prinzipiellen Schwierigkeiten mehr. Und da die (gewöhnlichen) komplexen Zahlen und das Rechnen mit ihnen dem Leser bekannt sind, so können wir uns hier mit der kurzen Erwähnung einiger Hauptsachen begnügen.

1. In $\S 4$ hatten wir bewiesen, daf3 das System der reellen Zahlen keiner Erweiterung mehr fähig und überdies das einzige System von Zeichen sei, welches den an ein Zahlensystem von uns gestellten Forderungen genügt. Nun liegt in dem System der komplexen Zahlen trotzdem ein solches System von Zeichen vor, das als ein Zahlensystem angesprochen wird. Der Widerspruch, der hierin zu liegen scheint, ist leicht behoben. Denn die Charakterisierung des Zahlbegriffes war, wie wir schon auf S. 31, Fußn. 2, betonten, eine in gewissem Sinne willkürliche: Wir hatten eine Reihe von Eigenschaften, die uns an den rationalen Zahlen wesentlich erschienen wareh', zum Range charakteristischer Eigenschaften von Zahlen überhaupt erhoben, und der Erfolg gab uns insofern Recht, als wir wirklich ein - und im wesentlichen nur ein - System schaffen konnten, welches alle diese Eigenschaften besab.

Will man also noch anderen Systemen den Charakter eines Zahlsystems zusprechen, so mú man hiernach notwendig die Liste der in $\mathbf{4}, 1-4$ von uns aufgestellten charakteristischen Eigenschalten verkleinern. Da entsteht dann aber die Frage, welche dieser Eigenschaften wir am ersten fallen lassen könnten, welche also bei einem System von Zeichen allenfalls fehlen dürfte, ohne daf man diesem deswegen schon den Charakter eines Zahlensystems absprechen mïifte.

2. Unter den Eigenschaften 4 eines Systems von Zeichen wird man am ersten auf die Anordnungs- und Monotoniegesetze verzichten können, ohne befürchten $z$ u müssen, dafs es dadurch den Charakter eines Zahlensystems durchaus verliert. Diese haben nach 4, 1 ihre Quelle darin, daf von zwei verschiedenen Zahlen des Systems die eine stets kleiner als die andere, diese größer als jene, genannt werden kann. Läßt man diese Unterscheidung fallen, 
ersetzt vielmehr in 4 die Zeichen $<$ und $>$ sinngemäf beide durch $\neq$, so zeigt sich, daf den so geänderten Bedingungen 4 noch ein anderes, allgemeineres System von Zeichen, nämlich das System der gewöhnlichen komplexen Zahlen, genuigt, dafi ihnen aber kein anderes von jenem wesentlich verschiedenes System von Zeichen auch noch zu genügen vermöchte.

3. Demgemäf ist das System der (gewöhnlichen) komplexen Zahlen ein System von Zeichen - die man bekanntlich in der Form $x+y i$ annehmen kann, wenn hierin $x$ und $y$ reelle Zahlen sind und $i$ ein Zeichen ist, dessen Handhabung durch die eine Festsetzung $i^{2}=-1$ geregelt ist - , für deren Gebrauch die Grundgesetze der Arithmetik $\mathbf{2}$ ausnahmslos in Gültigkeit bleiben, wenn in ihnen die Zeichen $\langle$ und $>$ sinngemäfs durch $\neq$ ersetzt werden. Man sagt darum kurz: Mit den komplexen Zahlen kann (von der eben genannten Einschränkung abgesehen) formal ebenso gerechnet werden, wie mit den reellen Zahlen.

4. Die komplexen Zahlen können in bekannter Weise (vgl. S. 7) den Punkten einer Ebene umkehrbar eindeutig zugeordnet und so durch sie veranschaulicht werden: Der komplexen Zahl $x+y i$ ordnet man den Punkt $(x, y)$ einer $x y$-Ebene zu. Jede Rechnung läßi sich dann geometrisch verfolgen. Statt $x+y i$ durch den Punkt $(x, y)$ darzustellen, ist es oft vorteilhafter, sie durch eine gerichtete Strecke (den Vektor) zu veranschaulichen, die mit der von $(0,0)$ nach $(x, y)$ führenden Strecke gleichgerichtet und gleichlang ist.

5. Komplexe Zahlen bezeichnen wir weiterhin durch einen einzigen Buchstaben: $z, \zeta, a, b, \ldots ;$ und diese sollen, wenn das Gegenteil nicht ausdrücklich gesagt ist oder aus dem Zusammenhang unzweideutig hervorgeht, weiterhin stets komplexe Zahlen bedeuten dïrfen.

6. Unter dem absoluten Betrag $|z|$ der komplexen Zahl $z=x+y i$ versteht man den nicht-negativen, reellen Wert $\sqrt{x^{2}+y^{2}}$, unter ihrem Arcus (arc $z$ ) einen derjenigen Winkel $\varphi$, für die gleichzeitig $\cos \varphi=\frac{x}{|z|}$ und $\sin \varphi=\frac{y}{|z|}$ ist. Für das Rechnen mit den absoluten Beträgen gelten ungeändert die Regeln $\mathbf{3}, \mathrm{II}$, 1-4, während die 5. ihre Bedeutung verliert.

Da man hiernach mit den komplexen Zahlen im großen und ganzen genau so operieren kann wie mit den reellen Zahlen, so wird auch der überwiegende Teil aller bisherigen Entwicklungen sich in ganz analoger Weise im Bereich der komplexen Zahlen durchführen bzw. auf ihn übertragen lassen. Nur diejenigen Betrachtungen, bei denen die Zahlen selbst (nicht bloß ihre absoluten Beträge) durch < oder $>$ verbunden wurden, nur diese werden ausfallen oder sinngemäß geändert werden müssen.

Um uns trotz dieses parallelen Verlaufs nicht zu Wiederholungen zwingen zu lassen, haben wir vom 2. Kapitel an bei allen Definitionen und Sätzen, die wörtlich bestehen bleiben (und zwar, von einigen kleinen, gleich zu erläuternden Abweichungen abgesehen, einschließlich Beweis), wenn man die darin auftretenden beliebigen reellen Zahlen durch beliebige komplexe Zahlen ersetzt, das Zeichen $\circ$ herangesetzt, und wir können nun in einer kurzen Skizze alle vorangegangenen Entwicklungen noch einmal durchfliegen und die im Gebiet der komplexen Zahlen notwendigen Änderungen dabei erwähnen. Auch auf die etwas abweichende geometrische Veranschaulichung werden wir mit ein paar Worten eingehen. 
Die Definition 23 bleibt ungeändert. Eine Zahlenfolge wird jetzt durch eine Folge von (ein- oder mehrfach zählenden) Punkten in der Ebene veranschaulicht. Ist sie beschränkt $(\mathbf{2 4}, 1)$, so liegt keiner ihrer Punkte außerhalb des Kreises mit dem (passend gewählten) Radius $K$ um 0 .

Die Definition $\mathbf{2 5}$ der Nullfolge und die über solche Nullfolgen geltenden Sätze $\mathbf{2 6}, \mathbf{2 7}$ und $\mathbf{2 8}$ bleiben völlig ungeändert.

Die Zahlenfolgen $\left(z_{n}\right)$ mit

$$
z_{n}=\left(\frac{1+i}{2}\right)^{n}, \quad=\left(\frac{1+i \sqrt{3}}{3}\right)^{n}, \quad=\frac{\imath}{n}, \quad=\frac{(-i)^{n}}{n}, \quad(n=1,2, \ldots),
$$

sind Beispiele von Nullfolgen, deren Glieder nicht sämtlich reell sind. Man veranschauliche sich genau die Lage der entsprechenden Punktmengen und beweise, dafs es sich wirklich um Nullfolgen handelt.

Die in $\S 7$ gegebenen Definitionen der Wurzel, der allgemeinen Potenz und des Logarithmus machten wesentlich von den Anordnungssätzen der reellen Zahlen Gebrauch. Sie lassen sich daher in der vorliegenden Form nicht im Bereich der komplexen Zahlen durchführen. (Vgl. dazu später $§ 55$.)

Der grundlegende Begriff der Konvergenz und der der Divergenz einer Zahlenfolge (39 und $\mathbf{4 0}, 1)$ dagegen bleibt wieder ungeändert bestehen, die Veranschaulichung von $\left.z_{n} \rightarrow \zeta^{1}\right)$ ist jetzt aber diese: Wenn man um den Punkt $\zeta$ einen Kreis mit dem beliebigen (positiven) Radius $\varepsilon$ beschreibt, so läßt sich stets eine (positive) Zahl $n_{0}$ so angeben, daß alle Glieder der Folge $\left(z_{n}\right)$, deren Index $n>n_{0}$ ist, innerhalb jenes Kreises liegen. Es gilt also wörtlich die Bemerkung 39, 6 (1. Hälfte), wenn man als $\varepsilon$-Umgebung einer komplexen Zahl $\zeta$ den eben angegebenen Kreis versteht.

Bei der Aufstellung der Definitionen 40, 2, 3 fanden die Zeichen $<$ und > wesentliche Verwendung; sie können daher nicht unverändert beibehalten werden. Und obwohl es nicht schwer wäre, ihren Hauptinhalt ins Komplexe hinüber zu retten, lassen wir sie ganz fallen, bezeichnen also im Komplexen alle nicht konvergenten Folgen unterschiedslos als divergent ${ }^{2}$ ).

$\left.{ }^{1}\right)$ Für komplexe Zahlen und Zahlenfolgen bevorzugen wir weiterhin die Buchstaben $z, \zeta, Z, \ldots$

2) Man könnte dic Zahlenfolge $\left(z_{n}\right)$, falls nur $\left|z_{n}\right| \rightarrow+\infty$ strebt, als bestimmt divergent mit dem Grenzwert $\infty$ bezeichnen oder von ihr sagen, sie strebe oder divergiere (oder selbst: sie konvergiere) gegen $\infty$. Das wäre eine durchaus sinngemiife Festsetzung, wie sie auch in der Funktionentheorie tatsächlich üblich ist. Doch bedeutet es natürlich eine kleine Inkonsequenz gegen die im Reellen benutzte Ausdrucksweise, wenn z. B. die Folge der Zahlen $(-1)^{n} n$ als bestimmt oder unbestimmt divergent bezeichnet werden muf, je nachdem sie als komplexe oder als reelle Zahlenfolge angesprochen wird. Und obgleich dies bei einiger Aufmerksamkeit nicht stört, so wollen wir diese Definition hier doch nicht benutzen. 
Die Sätze 41, 1 bis 12 und die wichtige Gruppe $\mathbf{4 3}$ von Sätzen bleiben einschließlich aller Beweise wörtlich ungeändert.

Die wichtigsten dieser Sätze $-\mathbf{4 3}, 4$ und 5 - wollen wir nun, da wir inzwischen mit den unendlichen Reihen vertraut geworden sind, hier noch einmal in der schon 44, 10 angedeuteten Erweiterung und für komplexe Zahlenfolgen aussprechen:

Satz 1. Es sei $\left(z_{0}, z_{1}, \ldots\right)$ eine Nullfolge und die Koeffizienten 221. der Matrix

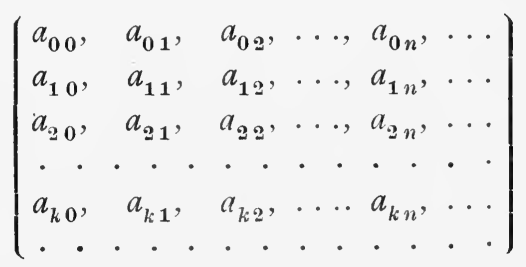

mögen den beiden Bedingungen genügen:

(a) In jeder Spalte stihen Nullfolgen, d. h. bei jedem festen $n \geqq 0$ strebt

$$
a_{k n} \rightarrow() \quad \text { für } k \rightarrow \infty .
$$

(b) Es gibt eine Konstante $K$, so daß die Summe der Beträge beliebig vieler Glieder einer jeden Zeile, also für jedes $k \geqq 0$ und jedes $n \geqq 0$ die Summe

$$
\left|a_{k 0}\right|+\left|a_{k 1}\right|+\ldots+\left|a_{k n}\right|<K
$$

bleibt. Dann ist auch die Folge der Zahlen

$$
z_{k}^{\prime}=a_{k 0} z_{0}+a_{k 1} z_{1}+\ldots=\sum_{n=0}^{\infty} a_{k n} z_{n}
$$

eine Nullfolge ${ }^{1}$ ).

Satz 2. Strebt $z_{n} \rightarrow \zeta$ und erfüllen die $a_{k n}$ außer den Bedingungen (a) und (b) des Satzes 1 noch die weitere, daß für $k \rightarrow \infty$

$$
A_{k}=\sum_{n=0}^{\infty} a_{k n} \rightarrow 1
$$

strebt $\left.{ }^{1}\right)$, so konvergiert auch die Folge der Zahlen

$$
z_{k}^{\prime}=a_{k 0} z_{0}+a_{k 1} z_{1}+\ldots \equiv \sum_{n=0}^{\infty} a_{k n} z_{n} \rightarrow \zeta .
$$

(Anwendungen dieses Satzes s. besonders bei $\mathbf{2 3 3}$ und in $\S 62$ ).

Von den beiden Hauptkriterien des $\S 9$ verlieren wir leider das 1., das uns bisher gerade die meisten Dienste geleistet hat. Auch von dem zweiten Hauptkriterium können wir den Beweis nicht auf

1) Wegen (b) ist $A_{k}=\sum_{n} a_{k n}$ absolut konvergent und folglich, da die $z_{n}$ eo ipso beschränkt sind, auch die Reihe $\sum_{n} a_{k n} z_{n}=z_{k^{\prime}}$ absolut konvergent. 
den Fall komplexer Zahlenfolgen übertragen, da in ihm dauernd von Anordnungssätzen Gebrauch gemacht wurde. Trotzdem werden wir sogleich sehen, daß das 2. Hauptkriterium selbst - und zwar in seinen sämtlichen Formen - ungeändert auch für komplexe Zahlenfolgen gïltig bleibt. Den Beweis kann man auf zweierlei Arten durchführen: Entweder man führt den neuen (komplexen) Satz auf den alten (reellen) zurück, oder man schafft sich für den Beweis des neuen Satzes die notwendigen neuen Grundlagen, indem man die Entwicklungen des $\S 10$ auf komplexe Zahlen überträgt. Beide Wege sind gleich leicht gangbar und sollen kurz angegeben werden:

1. Die Zurückfü'rrung der komp?exen Zahlenfolgen auf reelle geschieht in der einfachsten Weise durch Zerlegung ihrer Glieder in ihre reellen und imaginären Teile. Setzen wir $z_{n}=x_{n}+i y_{n}$ und $\zeta=\xi+i \eta$, so gilt der die Konvergenzfrage bei komplexen Zahlenfolgen vollständig auf das entsprechende reelle Problem zurückführende

222. Satz 1. Die Zahlenfolge $\left(z_{n}\right)=\left(x_{n}+i y_{n}\right)$ ist dann und nur dann gegen $\zeta=\xi+i \eta$ konvergent, wenn die reellen Teile $x_{n}>\xi$ und die imaginären Teile $y_{n} \rightarrow \eta$ konvergieren.

Beweis. a) Wenn $x_{n} \rightarrow \xi$ und $y_{n}>\eta$ strebt, so sind $\left(x_{n}-\xi\right)$ und $\left(y_{n}-\eta\right)$ Nullfolgen. Dasselbe gilt dann nach 26, 1 auch ron $i\left(y_{n}-\eta\right)$ und nach 28, 1 auch von der Folge

$$
\left(x_{n}-\xi\right)+i\left(y_{n}-\eta\right) \text {, d. h. von }\left(z_{n}-\zeta\right) \text {. }
$$

b) Wenn $z_{n} \rightarrow \zeta$ strebt, so ist $z_{n}-\zeta$ eine Nullfolge; da andererseits

$$
\left|x_{n}-\xi\right| \leqq \mid z_{n}-\zeta \quad \text { und } \quad y_{n}-\eta \leqq \mid z_{n}-\zeta
$$

ist $\left.^{1}\right)$, so sind nach $\mathbf{2 6}, 2$ auch $\left(x_{n}-\xi\right)$ und $\left(y_{n}-\eta\right)$ Nullfolgen, d. h. es strebt sowohl

Damit ist alles bewiesen.

$$
x_{n} \rightarrow \xi, \text { als } y_{n} \rightarrow \eta \text {. }
$$

Hieraus folgt schon unmittelbar der unser Ziel bildende

Satz 2. Auch für die Konvergenz einer komplexen Zahlenfolge $\left(z_{n}\right)$ sind die Bedingungen des 2. Hauptkriteriums 47 notwendig und hinreichend, - daß nämlich nach Wahl von $\varepsilon=0$ sich immer $n_{0}$ so angeben lasse, daß für irgend zwei Indizes $n$ und $n^{\prime}$, die beide $>n_{0}$ sind, stets

ausfällt.

$$
\left|z_{n^{\prime}}-z_{n}\right|<\varepsilon
$$

1) Der reelle Teil $x=\Re(z)$ einer komplexen $Z$ ahl $z$ hat nicht größeren absoluten Betrag als diese; ebenso der imaginäre Teil $y=\mathfrak{\Im}(z)$. Denn es ist

$$
\left.\left.\begin{array}{l}
x^{2} \\
y^{2}
\end{array}\right\} \leq x^{2}+y^{2}, \quad \text { also } \quad \begin{array}{l}
x \mid \\
y
\end{array}\right\} \leq \sqrt{ } x^{2}+y^{2}=\mid z .
$$


Beweis. a) Ist die Folge $\left(z_{n}\right)$ konvergent, so sind es nach dem vorigen Satz auch die (reellen) Folgen $\left(x_{n}\right)$ und $\left(y_{n}\right)$. Nach 47 wird man also, wenn $\varepsilon>0$ gegeben ist, $n_{1}$ und $n_{2}$ so wählen können, daß $\left|x_{n^{\prime}}-x_{n}\right|<\frac{\varepsilon}{2}$ ist, falls $n$ und $n^{\prime}$ beide $>n_{1}$ sind,

und $\quad\left|y_{n^{\prime}}-y_{n}\right|<\frac{\varepsilon}{2} \quad$ ist, falls $n$ und $n^{\prime}$ beide $>n_{2}$ sind.

Ist dann $n_{0}$ größer als $n_{1}$ und $n_{2}$, und sind $n$ und $n^{\prime}$ beide $>n_{0}$, so ist

$$
\begin{aligned}
\left|z_{n^{\prime}}-z_{n}\right| & =\left|\left(x_{n^{\prime}}-x_{n}\right)+i\left(y_{n^{\prime}}-y_{n}\right)\right| \\
& \leqq\left|x_{n^{\prime}}-x_{n}\right|+\left|y_{n^{\prime}}-y_{n}\right|<\frac{\varepsilon}{2}+\frac{\varepsilon}{2}=\varepsilon .
\end{aligned}
$$

Die Bedingungen unseres Satzes sind also notwendig.

b) Genügt umgekehrt $\left(z_{n}\right)$ den Bedingungen des Satzes, ist also bei gegebenen $\varepsilon>0$ immer $n_{0}$ so bestimmbar, daß $\left|z_{n^{\prime}}-z_{n}\right|<\varepsilon$ ausfällt, sobald $n$ und $n^{\prime}$ beide $>n_{0}$ sind, so ist für diese selben $n$ und $n^{\prime}$ (nach der letzten Fußnote) auch

$$
\left|x_{n^{\prime}}-x_{n}\right|<\varepsilon \quad \text { und } \quad\left|y_{n^{\prime}}-y_{n}\right|<\varepsilon .
$$

Nach 47 sind dann aber die beiden reellen Folgen $\left(x_{n}\right)$ und $\left(y_{n}\right)$ konvergent, so daß nach dem vorigen Satz auch $\left(z_{n}\right)$ konvergieren muß; die Bedingungen des Satzes sind auch hinreichend.

2. Direkte Behandlung der komplexen Zahlenfolgen. Bei reellen Zahlenfolgen bildeten die Intervallschachtelungen das häufigste Hilfsmittel für unsere Beweisführungen. Im Komplexen können uns die Quadratschachtelungen dieselben Dienste leisten:

Definition. $Q_{0}, Q_{1}, Q_{2}, \ldots$ sei eine Folge von Quadraten, deren $\mathbf{2 2 3 .}$ Seiten wir uns der Einfashheit halber sämtlich parallel zu den Achsen denken. Ist dann jedes von ihnen ganz im Vorangehenden enthalten, und bilden die Längen ihrer Seiten $l_{0}, l_{1}, \ldots$ eine Nullfolge, so sagen wir, es liege eine Quadratschachtelung vor. Von einer solchen gilt der

Satz. Es gibt ste's einen und n'r einen Punkt, der allen Quadraien einer gegebenen Quadra's;hashte'ung angehort. (Prinzip des innersten Punktes.)

Beweis. Der linke untere Eckpunkt von $Q_{n}$ heiße $a_{n}+i a_{n}{ }^{\prime}$ und der rechte obere Eckpunkt $b_{n}+i b_{n}^{\prime}$. Ein Punkt $z=x+i y$ gehört dann und nur dann dem Quadrat $Q_{n}$ an, wenn gleichzeitig

$$
a_{n} \leqq x \leqq b_{n} \quad \text { und } \quad a_{n}^{\prime} \leqq y \leqq b_{n}^{\prime}
$$

ist $^{1}$ ) Nun bilden aber infolge der Voraussetzungen die Intervalle

1) Durch diese Aussage sind zugleich diejenigen Größenbeziehungen rein arithmetisch festgelegt, die wir in Satz und Definition $\mathbf{2 3 6}$ in geometrischem Gewande aussprachen. 
$J_{n}=a_{n} \ldots b_{n}$ auf der Achse des Reellen, und ebenso auf der Achse des Imaginären die Intervalle $J_{n}^{\prime}=a_{n}^{\prime} i \ldots b_{n}^{\prime} i$ je eine Intervallschachtelung, und es gibt also auf jener nur genau einen Punkt $\xi$ und auf dieser nur genau einen Punkt $i \eta$, der allen diesen Intervallen angehört. Also gibt es auch nur genau einen Punkt $\zeta=\xi+i \eta$, der allen Quadraten $Q_{n}$ angehört.

Nunmehr können wir Definition $\mathbf{5 2}$ und Satz $\mathbf{5 4}$ ins Komplexe übertragen:

224. Definition. Ist $\left(z_{n}\right)$ eine beliebige Zahlenfolge, so heißt $\zeta$ ein Häufungswert derselben, wenn nach Wahl eines beliebigen $\varepsilon>0$ die Beziehung

$$
\left|z_{n}-\zeta\right|<\varepsilon
$$

fïr unendlich viele $n$ (insbesondere also: oberhalb jeder Zahl $n_{0}$ noch mindestens für ein n) erfüllt ist.

Satz. Jede beschränkte Zahlenfolge besitzt mindestens einen Häufungswert (Bolzano-Weierstraßscher Satz $\left.{ }^{1}\right)$ ).

Beweis. Ist etwa stets $|z|<K$, so zeichne man das Quadrat $Q_{0}$, dessen Seiten durch $\pm K$ und $\pm i K$ parallel zu den Achsen laufen. In ihm liegen alle $z_{n}$, also jedenfalls unendlich viele. $Q_{0}$ ist durch die Achsen in 4 kongruente Quadrate geteilt. In mindestens einem von ihnen müssen wieder unendlich viele $z_{n}$ liegen. (Denn lägen in jedem von ihnen nur endlich viele, so lägen auch im ganzen in $Q_{0}$ nur endlich viele, was doch nicht der Fall ist.) Das erste Viertel dieser Art, das wir antreffen ${ }^{2}$ ), nennen wir $Q_{1}$. Mit diesem verfahren wir ebenso, d. h. wir teilen es wieder in 4 kongruente Teile und nennen es das erste seiner Viertel ${ }^{2}$ ), in dem wir unendlich viele Glieder von $\left(z_{n}\right)$ antreffen, $Q_{2}$ usw. Diese wohlbestimmte Folge $Q_{0}$, $Q_{1}, Q_{2}, \ldots$ bildet eine $Q_{u}$ adratschachtelung, denn die $Q_{n}$ liegen ineinander und ihre Seiten bilden eine Nullfolge, weil die Seite von $Q_{n}$ gleich $2 K \cdot \frac{1}{2^{n}}$ ist. Ist nun $\zeta$ der innerste Punkt dieser Schachtelung ${ }^{3}$ ), so ist $\zeta$ ein Häufungspunkt von $\left(z_{n}\right)$. Denn wird $\varepsilon>0$ gegeben und $m$ so gewählt, daß die Seite von $Q_{m}$ kleiner als $\frac{\varepsilon}{2}$ ist, so liegt offenbar das ganze Quadrat $Q_{m}$ in der $\varepsilon$-Umgebung von $\zeta$. Mit ihm liegen dort also auch unendlich viele Glieder von $\left(z_{n}\right)$. Also ist $\zeta$ ein Häufungspunkt der Folge $\left(z_{n}\right)$, dessen Existenz somit bewiesen ist.

1) Vgl. $\mathbf{5 4}$.

2) Wir denken uns die 4 Viertel etwa so numeriert, wie man dies bei den 4 Quadranten der $x y$-Ebene gewöhnlich zu tun pflegt.

3) Das Verfahren zur Erfassung dieses Punktes entspricht genau der im Reellen oft benutzten Halbierungsmethode. 
Nunmehr können wir ganz analog wie unter $\mathbf{6 3}$ die Gültigkeit des 2. Hauptkriteriums für komplexe Zahlenfolgen noch einmal, jetzt aber ohne Benutzung der „reellen" Sätze erweisen:

Satz. Die Folge $\left(z_{n}\right)$ ist dann und nur dann konvergent, wenn 225. nach Wahl von $\varepsilon>0$ sich $n_{0}$ so angeben läßt, daß für alle Indizes $n$ und $n^{\prime}$, die beide $>n_{0}$ sind, stets $\left|\boldsymbol{*}_{n^{\prime}}-\boldsymbol{*}_{n}\right|<\varepsilon$ ausfällt.

Beweis. a) Wenn $z_{n} \rightarrow \zeta$ strebt, also $\left(z_{n}-\zeta\right)$ eine Nullfolge bildet, so kann $n_{0}$ so bestimmt werden, daß

$$
\left|z_{n}-\zeta\right|<\frac{\varepsilon}{2} \quad \text { und ebenso } \quad\left|z_{n^{\prime}}-\zeta\right|<\frac{\varepsilon}{2}
$$

ausfällt, sobald $n$ und $n^{\prime}>n_{0}$ sind (s. Teil a) des Beweises von 47). Dann ist für diese $n$ und $n^{\prime}$ auch

$$
\left|z_{n}-z_{n^{\prime}}\right| \leqq\left|z_{n^{\prime}}-\zeta\right|+\left|z_{n}-\zeta\right|<\varepsilon
$$

Die Bedingung ist also notwendig.

b) Ist umgekehrt die $\varepsilon$-Bedingung erfüllt, so ist $\left(z_{n}\right)$. sicher beschränkt. Denn ist $m>n_{0}$ und $n>m$, so ist doch

$$
\left|z_{n}-z_{m}\right|<\varepsilon
$$

d. h. alle $z_{n}$ mit $n>m$ liegen im Kreise mit $\varepsilon$ um $z_{m}$. Ist also $K$ größer als jede der Zahlen $\left|z_{1}\right|,\left|z_{2}\right|, \ldots,\left|z_{m-1}\right|,\left|z_{m}\right|+\varepsilon$, so ist für alle $n$ stets $\left|z_{n}\right|<K$.

Nach dem letzten Satz hat also $\left(z_{n}\right)$ mindestens eine Häufungsstelle $\zeta$. Gesetzt nun, es gäbe noch einen zweiten Häufungswert $\zeta^{\prime} \neq \zeta$, so wähle man

$$
\varepsilon=\frac{1}{3}\left|\zeta^{\prime}-\zeta\right|
$$

was $>0$ ist. Wie groß dann auch $n_{0}$ gewählt wird, es gibt - nach der Definition 224 des Häufungspunktes - stets ein $n>n_{0}$, so daß $\left|z_{n}-\zeta\right|<\varepsilon$, aber ebenso auch ein $n^{\prime}>n_{0}$, so daß $\left|z_{n^{\prime}}-\zeta^{\prime}\right|<\varepsilon$ ist. Es gibt dann also oberhalb jeder noch so großen Zahl $n_{0}$ ein Paar von Indizes $n$ und $n^{\prime}$, für die

$$
\left|z_{n^{\prime}}-z_{n}\right|>\varepsilon
$$

ist $^{1}$ ), entgegen der Annahme. Es muß also $\zeta$ der einzige Häufungspunkt sein, und es liegen außerhalb des Kreises mit $\varepsilon$ um $\zeta$ nur endlich viele $z_{n}$. Wird dann das $n_{0}$ passend gewählt, so ist für $n>n_{0}$ stets $\left|z_{n}-\zeta\right|<\varepsilon$ und es strebt also $z_{n} \rightarrow \zeta$. Die Bedingung des Satzes ist auch hinreichend ${ }^{2}$ ).

also

$$
\text { 1) }\left(z_{n^{\prime}}-z_{n}\right)=\left(\zeta^{\prime}-\zeta\right)+\left(z_{n^{\prime}}-\zeta^{\prime}\right)+\left(\zeta-z_{n}\right) \text {, }
$$

$$
\left|z_{n^{\prime}}-z_{n}\right| \geqq\left|\zeta^{\prime}-\zeta\right|-\left|z_{n^{\prime}}-\zeta^{\prime}\right|-\left|z_{n}-\zeta\right|>3 \varepsilon-\varepsilon-\varepsilon=\varepsilon
$$

2) Wir können also auch sagen: Eine Folge $\left(z_{n}\right)$ ist dann und nur dann konvergent, wenn sie beschränkt ist und nur genau einen Häufungswert besitzt. Dieser ist dann zugleich der Grenzwert der Folge. 


\section{$\S 5$ 53. Reihen mit komplexen Gliedern.}

Da eine Reihe $\Sigma a_{n}$ mit komplexen Gliedern selbstverständlich wieder nur die Folge ihrer Teilsummen bedeuten soll, so ist durch das Vorangehende die Grundlage für die Erweiterung unserer Theorie schon geschaffen.

Entsprechend 222, 1 haben wir also zunächst den

226. Satz. Eine Reihe $\sum a_{n}$ mit komplexen Gliedern ist dann und nur dann konvergent, wenn die Reihe $\Sigma \Re\left(a_{n}\right)$ und die Reihe $\Sigma \mathfrak{S}\left(a_{n}\right)$ der reellen und der imaginären Teile der Glieder für sich konvergieren. Und sind deren Summen bzw. $s^{\prime}$ und $s^{\prime \prime}$, so hat $\sum a_{n}$ die Summe. $s=s^{\prime}+i s^{\prime \prime}$.

Und gemäß 222, 2 und $\mathbf{2 2 5}$ bleibt das 2. Hauptkriterium $(\mathbf{8 1})$ für die Konvergenz unendlicher Reihen in all seinen Formen ungeändert bestehen, und mit ihm behalten auch die daraus hergeleiteten Sätze 83 über das Rechnen mit konvergenten Reihen ihre volle Gültigkeit.

Da sich ebenso an Satz $\mathbf{8 5}$ nichts ändert, werden wir auch bei Reihen mit komplexen Gliedern eine absolute und eine nicht absolute Konvergenz unterscheiden (Def. S6).

Hier gilt änlich wie oben der

227. Satz. Die Reihe $\Sigma a_{n}$ mit komplexen Gliedern ist dann und nur dann $a b$ solut konvergent, wenn die beiden Reihen $\mathfrak{Y} \mathfrak{R}\left(a_{n}\right)$ und $\Sigma \mathfrak{I}\left(a_{n}\right)$ ihrerseits absolut konvergieren.

Der Beweis folgt einfach daraus, daf fuir jede komplexe Zahl $z=x+i y$. die Abschätzung gilt

$$
\left.\mid \begin{array}{l}
x \mid \\
|y|
\end{array}\right\} \leqq|z| \leqq|x|+|y|
$$

Auf Grund dieses einfachen Satzes erkennt man weiter, daß es auch bei: Reihen mit komplexen Gliedern nicht auf die Reihenfolge dieser Glieder ankommt, wenn die Reihe absolut konvergiert (Satz S8, 1).

Ist aber die Reihe $\Sigma a_{n}$ nicht absolut konvergent, so muf entweder $\Sigma \Re\left(a_{n}\right)$. oder $\Sigma \mathfrak{s}\left(a_{n}\right)$ bedingt konvergieren. Durch eine geeignete Umordnung der Glieder kann dann jedenfalls die Konvergenz der Reihe zerstört werden, d. h.: Auch bei Reihen mit komplexen Gliedern hängt die Konvergenz, falls sie keine absolute ist, wesentlich noch von der Reihenfolge der Glieder ab. Wegen der Übertragung des Riemannschen Satzes $\S 44$ auf Reihen mit komplexen Gliedern vgl. die Bemerkungen auf der nächsten Seite.

Da nun die Erkennung der absoluten Konvergenz eine Frage über Reihen mit positiven Gliedern ist, so ist hiermit die gesamte Theorie der Reihen mit positiven Gliedern auch zur Untersuchung von Reihen mit komplexen Gliedern nutzbar gemacht: Alles was wir über absolut konvergente Reihen mit reellen Gliedern bewiesen haben, ist auch für absolut konvergente Reihen mit komplexen Gliedern brauchbar. 
Sehen wir dann beim Durchgehen der weiteren Paragraphen des II. Teils von den Potenzreihen zunächst noch ab (§ 18 bis $\S 27$ ), so kommen weiterhin erst wieder die Entwicklungen des X. Kapitels für eine Übertragung auf Reihen mit komplexen Gliedern in Frage.

Da hier die Abelsche partielle Summation 182 rein formaler Natur ist, gilt sie einschließlich des Zusatzes 183 natürlich auch für komplexe Zahlen, und mit ihr das unmittelbar darauf gegründete Konvergenzkriterium (184). Auch die spezielleren Fassungen dieses Kriteriums können wir sämtlich übernehmen, wenn wir nur an der 220, 5 getroffenen Übereinkunft festhalten, daß eine als monoton vorausgesetzte Zahlenfolge eo ipso reell ist. Bei den Kriterien von $d u$ Bois-Reymond und Dedekind fällt auch diese Vorsicht noch fort: sie gelten wörtlich und ohne jede Einschränkung auch für beliebige Reihen der Form $\sum a_{n} b_{n}$ mit komplexen $a_{n}$ und $b_{n}$.

Der Riemannsche Satz (\$44) dagegen ist ein spezifisch „reeller“ Satz. Denn wenn eine Reihe $\Sigma a_{n}$ mit komplexen Gliedern nicht absolut konvergiert, so ist dies nach $\mathbf{2 2 7}$ auch mit mindestens einer der beiden Reihen $\Sigma \Re\left(a_{n}\right)$ und $\Sigma \mathfrak{g}\left(a_{n}\right)$ der Fall. Durch eine passende Umordnung können wir also nach dem Riemannschen Satz zwar erreichen, daß eine dieser beiden Reihen ein vorgeschriebenes Konvergenzverhalten bekommt; da aber zugleich die andere Reihe in genau derselben Weise umgeordnet wird, so ist nicht ohne weiteres zu übersehen, welche Wirkung diese Umordnung auf diese andere Reihe und somit auf $\Sigma a_{n}$ selber hat. - In neuerer Zeit ist indessen gezeigt worden, daß wenn $\Sigma a_{n}$ nicht absolut konvergiert, sie durch eine passende Umordnung in eine wieder konvergente Reihe verwandelt werden kann, deren Summe je nach Lage des Falles entweder in der ganzen Ebene oder nur auf einer bestimmten dort gelegenen Geraden beliebig vorgeschrieben werden kann ${ }^{1}$ ).

Die Sätze 188 und 189 von Mertens und Abel über Reihenmultiplikation $(\S 45)$ bleiben wieder einschließlich Beweis wörtlich bestehen. Bei dem letzteren müssen wir uns allerdings - da wir die Potenzreihen noch übersprungen haben - vorläufig auf den 2. (Cesàroschen) Beweis allein stützen (vgl. später 232).

Damit sind wir aber auch schon im Besitze des ganzen Apparates,

1) Es gilt also der das Umordnungsproblem in gewissem Sinne abschliefende sehr schöne Satz: Der "Summenbereich" einer Reihe $\Sigma a_{n}$ mit komplexen Gliedern - d. h. die Menge der Zahlen, die man als Summen der wieder konvergent ausfallenden Umordnungen von $\Sigma a_{n}$ erhalten kann - ist entweder ein bestimmter Punkt oder eine bestimmte Gerade oder die ganze Ebene. Andere Fälle können nicht vorkommen. Ein Beweis findet sich wohl bei $P$. Levvy (Nouv. Annales, Bd. (4) 5 (1905), S. 506); in einwandfreier Darstellung aber erst bei E. Steinitz [Bedingt konvergente Reihen und konvexe Systeme, J. f. d. reine u. angew. Math., Bd. 143 (1913), Bd. 144 (1914), Bd. 146 (1915)]. 
der zur Beherrschung der Reihen mit komplexen Gliedern notwendig ist, und wir können sogleich zu seinen wichtigsten Anwendungen übergehen.

Vorher wollen wir nur noch als Anwendung und Ergänzung dieser Betrachtungen das folgende sehr weitreichende Kriterium herleiten. 228. Kriterium von Weierstra $\left.B^{1}\right)$. Ist die Reihe $\sum_{n=0}^{\infty} a_{n}$ mit komplexen Gliedern vor-

$$
\frac{a_{n+1}}{a_{n}}=1-\frac{\alpha}{n}-\frac{A_{n}}{n^{2}}
$$

mit beliebigem komplexem $a$, mit $\lambda>1$ und beschränkten $A_{n}$ gesetzt werden $\left.{ }^{2}\right)$, so ist sie dann und nur dann absolut konvergent, wenn $\Re(\alpha)>1$ ist. Für $\Re(\alpha) \leqq 0$ ist die Reihe stets divergent. Wenn $0<\Re(\alpha) \leq 1$ ist, so sind die beiden Reihen

konvergent $\left.{ }^{3}\right)$.

$$
\sum_{n=0}^{\infty}\left|a_{n}-a_{n+1}\right| \quad \text { und } \quad \sum_{n=0}^{\infty}(-1)^{n} a_{n}
$$

Beweis. 1. Es sei $\alpha=\beta+i \gamma$ und zunächst $\beta=\Re(\alpha)>1$. Dann ist wenn stets $\left|A_{n}\right|<K$ bleibt,

$$
\left|\frac{a_{n+1}}{a_{n}}\right| \leq\left|1-\frac{\beta+i \gamma}{n}\right|+\frac{K}{n^{\lambda}},
$$

also, wie man sofort nachrechnet, von einer Stelle ab sogar

$$
\leq 1-\frac{\beta^{\prime}}{n}
$$

wenn $1<\beta^{\prime}<\beta$ ist. Nach dem Raabeschen Kriterium ist hiernach die Reihe $\mathcal{Y}\left|a_{n}\right|$ konvergent.

2. Es sei jetzt $\Re(\alpha)=\beta \leqq 1$. Dann ist

$$
\left|\frac{a_{n+1}}{a_{n}}\right| \geq 1-\frac{\beta}{n}-\frac{K}{n^{\lambda}}
$$

und folglich die Reihe $\Sigma\left|a_{n}\right|$ nach dem Gaußischen Kriterium 172 divergent.

3 a. Ist sogar $\Re(\alpha)=\beta<0$, so lehrt die letzte Ungleichung, daß̧ von einer Stelle an

$$
\left|\frac{a_{n+1}}{a_{n}}\right|>1
$$

1) J. f. d. reine u. angew. Math., Bd. 51 (1856), S. 29; Werke I, S. 185.

2) Ein solcher Ansatz kann natürlich stets gemacht werden; man braucht ja nur $A_{n}=n^{i}\left(1-\frac{\alpha}{n}-\frac{a_{n+1}}{a_{n}}\right)$ zu setzen. Das Wesentliche an den Voraussetzungen ist auch hier wieder (vgl. Fufnote zu 166), dafs bei passender Wahl von $\lambda$ die $A_{n}$ beschränkt ausfallen. - Wesentlich dasselbe ist es, wenn

$$
\frac{a_{n}}{a_{n+1}}=1+\frac{\alpha}{n}+\frac{B_{n}}{n^{\lambda}}
$$

mit $\lambda>1$ und beschränkten $B_{n}$ gesetzt werden kann.

3) Bezüglich der Reihe $\Sigma a_{n}$ selber hat A.Pringsheim gezeigt (Archiv d. Math. und Phys. (3) 4,1902 , S. $1-19$, spez. S. $13-17$ ), daß sie für $\Re(\alpha) \leqq 1$ auch stets divergent ist. Der Beweis ist etwas mühsamer und die Tatsache selbst wird von uns nicht gebraucht. - Eine weitere genaue Untersuchung der Reihe $\Sigma a_{n}$ selber für $0 \leqq \Re(\alpha) \leq 1$ gibt $J$. A. Gmeiner (Monatshefte f. Math. u. Phys. Bd. 19, 1908, S. 149-163). 
ist, dak also die Glieder der Reihe $\ a_{n}$ ihrem Betrage nach niemals mehr abnehmen. Daher muf jetzt $\Sigma a_{n}$ divergieren.

3 b. Wenn $\Re(\alpha)=\beta=0$, also

$$
\frac{a_{n+1}}{a_{n}}=1-\frac{i \gamma}{n}-\frac{A_{n}}{n^{\hat{\lambda}}},
$$

ist, so rechnet man leicht nach, daf nun

$$
\left|\frac{a_{n+1}}{a_{n}}\right|=1-\frac{A_{n}^{\prime}}{n \hat{\imath}^{\prime}}
$$

gesetzt werden kann, wenn $\lambda^{\prime}>1$ die kleinere der Zahlen 2 und $\lambda$ ist und wenn die $A_{n}^{\prime}$ eine wieder beschränkte Zahlenfolge bedeuten. Es ist also, wenn $c$ eine passende positive Konstante bezeichnet,

$$
\left|\frac{a_{n+1}}{a_{n}}\right| \geq 1-\frac{c}{n n^{\prime}}>0
$$

etwa für alle $n \geq m$. Hieraus folgt durch Multiplikation

$$
\left|\frac{a_{n}}{a_{m}}\right|=\left|\frac{a_{m+1}}{a_{m}}\right| \cdots\left|\frac{a_{n}}{a_{n-1}}\right|>\prod_{\nu=m}^{n-1}\left(1-\frac{c}{\nu^{\prime}}\right)>\prod_{\nu=m}^{\infty}\left(1-\frac{c}{\nu^{\prime}}\right)=C_{m}>0 .
$$

Hiernach ist also für alle $n>m$ stets $\left|a_{n}\right|>C_{m} \cdot\left|a_{m}\right|$. Die $a_{n}$ streben also nicht nach 0 , so dafi $\Sigma a_{n}$ wieder divergieren muf (vgl. 170, 1).

4. Ist endlich $\Re(\alpha)=\beta>0$, so ist noch die Konvergenz der beiden Reihen

$$
\Sigma\left|a_{n}-a_{n+1}\right| \text { und } \Sigma(-1)^{n} a_{n}
$$

$z u$ beweisen. Jetzt ist aber wie bei 1. von einer Stelle an

$$
\left|\frac{a_{n+1}}{a_{n}}\right|<1-\frac{\beta^{\prime}}{n}, \text { mit } 0 \quad \beta^{\prime}<\beta,
$$

so dafs die $\left|a_{n}\right|$ von dieser Stelle an monoton abnehmen und demgemäß $\{$ gegen einen bestimmten Grenzwert $\geq 0$ streben. Folglich ist

a) nach 131 die Reihe $\Sigma\left(\left|a_{n}-a_{n+1}\right|\right)$ konvergent und hat iiberdies von einer Stelle an positive Glieder. Nun ist aber

$$
\frac{\left|a_{n}-a_{n+1}\right|}{\left|a_{n}\right|-\left|a_{n+1}\right|}=\frac{1-\frac{a_{n+1}}{a_{n}}}{1-\frac{a_{n+1}}{a_{n}} \mid} \leq \frac{\frac{c}{n}+\frac{A_{n}}{n^{2}}}{\frac{\beta^{\prime \prime}}{n}} ;
$$

und weil hier der letzte Bruch fuir $n>+\infty$ den positiven Grenzwert $\frac{|\alpha|}{\beta^{\prime}}$ hat, so bleibt der linksstehende Quotient von einer Stelle an $<$ als eine passende Konstante $A$. Nach $\boldsymbol{\sigma 0}, 4$ ist dann aber mit $\mathcal{Y}\left(\left|a_{n}\right|-\left|a_{n+1}\right|\right)$ auch die Reihe $\Sigma\left|a_{n}-a_{n+1}\right|$ konvergent. - Man kann aber

b) sogar genauer zeigen, daf $a_{n} \rightarrow 0$ strebt. Denn aus

$$
\left|\frac{a_{n+1}}{a_{n}}\right|<1-\frac{\beta^{\prime}}{n}, \quad(n>m),
$$

folgt wieder durch Multiplikation

$$
\left|\frac{a_{n}}{a_{m}}\right|<\left(1-\frac{\beta^{\prime}}{m}\right)\left(1-\frac{\beta^{\prime}}{m+1}\right) \ldots\left(1-\frac{\beta^{\prime}}{n-1}\right)
$$

Und da hier die rechte Seite für $n \rightarrow+\infty$ gegen 0 strebt, so mufi (vgl. 1\%0, 1) auch $a_{n} \rightarrow 0$ streben. Daher können wir in der Reihe

$$
\left(a_{0}-a_{1}\right)+\left(a_{2}-a_{3}\right)+\left(a_{4}-a_{5}\right)+\ldots-\sum_{k=0}^{\infty}\left(a_{2 k}-a_{2 k+1}\right)
$$


- eine Reihe, die als Teilreihe von $\Sigma\left(a_{n}-a_{n+1}\right)$ gleich dieser nach a) absolut konvergiert - die kleinen Kammern nach $\mathbf{8 3}$, Satz 2, Zusatz fortlassen, denn mit $a_{n}$ strebt auch $\left|a_{n}\right|+\left|a_{n+1}\right| \rightarrow 0$. Damit ist dann aber auch die Konvergenz von $\Sigma(-1)^{n} a_{n}$ vollständig bewiesen.

Mit Hilfe dieses Satzes kann man nun leicht noch den folgenden weiteren beweisen, der uns bald nuitzlich sein wird:

229.

Satz. Wenn wie im vorigen Satze

$$
\frac{a_{n+1}}{a_{n}}=1-\frac{\alpha}{n}-\frac{A_{n}}{n^{\lambda}}, \quad\left\{\begin{array}{l}
\alpha \text { beliebig, } \lambda>1, \\
\left(A_{n}\right) \text { beschränkt }
\end{array}\right.
$$

gesetzt werden kann, so ist die Reihe $\sum a_{n} z^{n}$ für $|z|<1$ absolut konvergent, für $|z|>1$ stets divergent und für die Punkte der Peripherie $|z|=1$ ist die Reihe

a) absolut konvergent, falls $\Re(\alpha)>1$ ist,

b) bedingt konvergent, falls $0<\Re(\alpha) \leq 1$ ist, 一 außer möglicherweise für den einen Punkt $z=+1^{1}$ ),

c) divergent, falls $\Re(\alpha) \leqq 0$ ist.

Beweis. Da

$$
\left|\frac{a_{n+1} z^{n+1}}{a_{n} z^{n}}\right| \rightarrow|z|
$$

strebt, so sind die auf $|z| \gtrless 1$ bezüglichen Behauptungen sofort als richtig erkannt. Für $|z|=1$ ist die Behauptung a) eine unmittelbare Folge der durch den vorigen Satz gesicherten Konvergenz von $\Sigma\left|a_{n}\right|$. Ebenso ist die Behauptung c) eine unmittelbare Folge der vorhin bewiesenen Tatsache, daf $\mathrm{s}$ in diesem Falle $\left|a_{n}\right|$ für alle $n$ von einer Stelle ab oberhalb einer positiven Schranke bleibt.

Ist endlich $0<\Re(\alpha) \leqq 1$ und $z \neq+1$, so ergibt sich die Konvergenz von $\sum a_{n} z^{n}$ nach dem Dedekindschen Kriterium 184,3. Denn wir hatten beim vorigen Satze bewiesen, daß $\Sigma\left|a_{n}-a_{n+1}\right|$ konvergiert und $a_{n} \rightarrow 0$ strebt; und daf die Teilsummen von $\Sigma z^{n}$ für jedes (feste) von +1 verschiedene $z$ auf der Peripherie $|z|=1$ beschränkt sind, folgt ja einfach daraus, daf für alle $n$

bleibt.

$$
\left|1+z+z^{2}+\ldots+z^{n}\right|=\left|\frac{1-z^{n+1}}{1-z}\right| \leqq \frac{2}{|1-z|}
$$

\section{§ 54. Potenzreihen. Analytische Funktionen.}

Unter einer Potenzreihe verstehen wir auch jetzt eine Reihe der Form $\sum a_{n} z^{n}$, oder allgemeiner der Form $\sum a_{n}\left(z-z_{0}\right)^{n}$, in der nun die Koeffizienten $a_{n}$ sowohl wie die Größen $z$ komplexe Zahlen sein dürfen.

Die in den $\S \S 18$ bis 21 entwickelte Theorie dieser Reihen bleibt dabei in allem wesentlichen ungeändert. Wir können uns daher bei der Übertragung der damaligen Entwicklungen ganz kurz fassen.

Da die Sätze 93, 1 und 2 völlig ungeändert ihre Gültigkeit behalten, so gilt zunächst das gleiche von dem Hauptsatz 93 selber, welcher über das Konvergenzverhalten der Potenzreihen im

1) Mit Hinzuziehung des in der vorigen Note erwähnten Pringsheimschen Resultates kann man hier genauer sagen: außer für $z=+1$. 
Reellen Aufschluß gab. Nur die geometrische Veranschaulichung ist hier eine etwas andre: Die Potenzreihe $\sum a_{n} z^{n}$ konvergiert - und sogar absolut - für alle $z$, die im Innern des Kreises mit $r$ um 0 liegen, und divergiert für alle außerhalb desselben gelegenen Punkte. Dieser Kreis wird dann kurz als der Konvergenzkreis der Potenzreihe bezeichnet, - und der Name Radius für die Zahl $r$ ist nun erst voll verständlich.

Über die Konvergenz auf der Peripherie des Konvergenzkreises läßt sich ebensowenig etwas Allgemeines aussagen, wie über die Konvergenz in den Endpunkten des Konvergenzintervalles im Falle reeller Potenzreihen. (Die sogleich folgenden Beispiele werden zeigen, daß das Verhalten ein sehr verschiedenartiges sein kann.)

Auch die übrigen Sätze des $\S 18$ behalten ihre unveränderte Gültigkeit.

\section{Beispiele.}

1. $\Sigma z^{n} ; r=1$. Im Innern des Einheitskreises konvergiert die Reihe und $\mathbf{2 3 0 .}$ ihre Summe ist dort $\frac{1}{1-z}$. Auf dem Rande, d. h. für $|z|=1$, ist sie überall divergent, denn $z^{n}$ strebt dort nicht $\rightarrow 0$.

2. $\left.\sum \frac{z^{n}}{n^{2}} ; r=1^{1}\right)$. Diese Reihe ist auch noch in allen Randpunkten $|z|=1$ (absolut) konvergent.

3. $\geq \frac{z^{n}}{n} ; r=1$. Die Reihe ist sicher nicht in allen Randpunkten konvergent, denn für $z=1$ bekommen wir die divergente Reihe $\sum \frac{1}{n}$. Sie ist aber auch nicht in allen Randpunkten divergent, denn für $z=-1$ bekommen wir eine konvergente Reihe. Der Satz $\mathbf{2 2 9}$ des letzten Paragraphen lehrt sogar genauer, daf 3 die Reihe in allen von +1 verschiedenen Punkten der Peripherie $|z|=1$ bedingt konvergiert, denn es ist hier

$$
\frac{a_{n}}{a_{n-1}}=\frac{n-1}{n}=1-\frac{1}{n} \text {. }
$$

Dasselbe Resultat erhält man auch unmittelbar aus dem Dirichletschen Kriterium $\mathbf{1 8 3}, \dot{2}$, denn $\Sigma z^{n}$ hat beschränkte Teilsummen, falls $z \neq+1$, aber $|z|=1$ ist (vgl. die letzte Formel des vorigen Paragraphen), und $\frac{1}{n}$ strebt monoton gegen $0^{2}$ ). Wegen $\Sigma\left|\frac{z^{n}}{n}\right|=\Sigma \frac{1}{n}$ ist die Konvergenz aber nur eine bedingte.

4. $\Sigma \frac{z^{4 n}}{4 n} ; r=1$. Diese Reihe ist in den 4 Randpunkten \pm 1 und $\pm i$ divergent, in allen andern Randpunkten bedingt konvergent.

1) Hat $\Sigma a_{n} z^{n}$ reelle Koeffizienten (wie meist im folgenden), so hat diese Potenzreihe natïrlich denselben Radius wie die reelle Potenzreihe $\Sigma a_{n} x^{n}$.

2) Endlich kann man diese Konvergenztatsachen auch aus 185, 5 entnehmen, indem man die Reihe in ihren reellen und imaginären Bestandteil zerlegt. 
5. Für $\sum \frac{z^{n}}{n !}$ ist $r=+\infty$. Für $\Sigma n ! z^{n}$ ist $r=0$; diese Reihe ist also nirgends konvergent aufer in $z=0$.

6. Die Reihen $\sum_{k=0}^{\infty}(-1)^{k} \frac{z^{2 k}}{(2 k) !}$ und $\sum_{k=0}^{\infty}(-1)^{k} \frac{z^{2 k+1}}{(2 k+1) !}$ sind beständig konvergent.

7. Eine Potenzreihe der allgemeineren Form $\Sigma a_{n}\left(z-z_{0}\right)^{n}$ konvergiert absolut in allen inneren Punkten des Kreises mit $r$ um $z_{0}$, und divergiert anferhalb desselben, wenn $r$ den Radius von $\sum a_{n} z^{n}$ bedeutet.

Ehe wir nun weiter auf die Eigenschaften der Potenzreihen eingehen, schalten wir ein paar Bemerkungen ein über

\section{Funktionen einer komplexen Veränderlichen.}

Wenn auf irgendeine Weise jedem Punkte $z$ eines Kreises $\Re$ ein Wert $w$ zugeordnet ist, so sagen wir, es sei uns in diesem Kreise eine Funktion $w=f(z)$ der komplexen Veränderlichen z gegeben. Die Zuordnung kann dabei in der mannigfachsten Weise geschehen (vgl. die entsprechende Bemerkung für den reellen Funktionsbegriff, $\S 19$, Def. 1); im folgenden wird aber der Funktionswert fast immer durch eine explizite Formel aus $z$ berechnet werden können, oder wird die Summe einer konvergenten Reihe sein, bei der nun die Glieder explizit gegeben sind. Zahlreiche Beispiele werden wir sehr bald kennen lernen; im Augenblick denke man etwa an den Wert $w$, der jedem $z$ im Innern des Konvergenzkreises einer Potenzreihe als deren Summe zugeordnet wird.

Die Begriffe des Grenzwertes, der Stetigkeit und der Differenzierbarkeit einer Funktion, die uns hier wieder vor allen andern interessieren, werden im Prinzip genau so festgelegt, wie im Reellen:

231. 1. Definition des Grenzwertes. Ist die Funktion $w=f(z)$ für alle Punkte $z$ einer Umgebung des festen Punktes $\zeta$ definiert $^{1}$ ), so sagt man, es sei

$$
\lim _{z \rightarrow \zeta} f(z)=\omega
$$

oder es strebe

$$
f(z) \rightarrow \omega \text { für } z \rightarrow \zeta,
$$

wenn nach Wahl eines beliebigen $\varepsilon>0$ ein $\delta=\delta(\varepsilon)>0$ so angegeben werden kann, daßs für alle $z$, die der Bedingung $0<|z-\zeta|<\delta^{\prime}$ genligen, stets

$$
|f(z)-\omega|<\varepsilon
$$

ist. Oder wenn - es kommt dies auf genau dasselbe hinaus ${ }^{2}$ ) - jede gegen $\zeta$ konvergierende Zahlenfolge $\left(z_{n}\right)$, deren Glieder in der genannten Umgebung von $\zeta$ liegen und von $\zeta$ selbst verschieden sind, eine gegen $\omega$ konvergierende Folge von Funktionswerten $w_{n}=f\left(z_{n}\right)$ liefert.

Wird $f(z)$ nicht in allen Punkten einer Umgebung von $\zeta$ betrachtet, sondern nur in denjenigen, die z. B. auf einem bestimmten in $\zeta$ endenden Kurvenstück oder einem Winkelraum mit der Spitze in $\zeta$ liegen $^{3}$ ), so sagt man, es sei $\lim f(z)=\omega$ oder es strebe $f(z) \rightarrow \omega$ bei Annäherung von $z \rightarrow \zeta$ längs jenes Kurven-

1) Im Punkte $\zeta$ selbst braucht $f(z)$ nicht definiert zu sein, sondern also nur für alle $z$, die einer Bedingung der Form $0<|z-\zeta|<\varrho$ genügen. Das $\delta$ der obigen Definition mufs dann natïrlich $<\varrho$ gedacht werden.

2) Beweis wie im Reellen.

3) Oder allgemeiner: die einer Punktmenge $M$ angehören, für die $\zeta$ ein Häufungspunkt ist. 
stücks oder innerhalb jenes Winkelraumes ${ }^{1}$ ), wenn die obigen Bedingungen wenigstens für alle nun noch dubei in Betracht kommenden $z$ erfüllt sind.

2. Definition der Stetigkeit. Ist die Funktion $w=f(z)$ in einer Umgebung von $\zeta$ und in $\zeta$ selbst definiert, so sagt man, es sei $f(z)$ in diesem Punkte $\zeta$ stetig, wenn

$$
\lim _{z \rightarrow \zeta} f(z)
$$

vorhanden ist und mit dem Funktionswerte $f(\zeta)$ in $\zeta$ übereinstimmt, wenn also $f(z) \rightarrow f(\zeta)$ strebt. (Was Stetigkeit von $f(z)$ in $\zeta$ bei Beschränkung von $z$ auf ein durch $\zeta$ gehendes Kurvenstiuck und auf einen Winkelraum mit seiner Spitze in $\zeta^{2}$ ) bedeutet, ist nach 1 . nun selbstverständlich.

3. Definition der Differenzierbarkeit. Ist die Funktion $w=f(z)$ in einer Umgebung von $\zeta$ und in $\zeta$ selbst definiert, so heift sie in $\zeta$ differenzierbar, wenn gemäßs 1. der Grenzwert

$$
\lim _{z \rightarrow \zeta} \frac{f(z)-f(\zeta)}{z-\zeta}
$$

existiert. Seinen Wert nennt man die Ableitung von $f(z)$ in $\zeta$ und bezeichnet sie mit $f^{\prime}(\zeta)$. (Auch hier können der Beweglichkeit von $z$ wieder Beschränkungen auferlegt werden)

Mit diesen wenigen Festsetzungen über die allgemeinen Funktionen einer komplexen Veränderlichen müssen wir uns begnügen. Das vertiefte Studium derselben bildet den Gegenstand der sogen. Funktionentheorie, eines der umfangreichsten Gebiete der neueren Mathematik, auf die hier des genaueren einzugehen natirlich nicht der Raum ist ${ }^{3}$ ).

Diese Erklärungen genügen nun vollauf, um die wichtigen Entwicklungen der $\S \S 20$ und 21 auf Potenzreihen mit komplexen Gliedern $\mathrm{zu}$ übertragen.

In der Tat gelten die dort gegebenen Entwicklungen ausnahmslos auch für unsern jetzigen allgemeineren Fall, wenn wir dort nur sinngemäß statt „Konvergenzintervall“ überall „Konvergenzkreis“ setzen. Nur dem Satz 5 (unter 99) können wir hier kein Analogon an die Seite stellen, weil wir den Integralbegriff für Funktionen komplexen Argumentes nicht eingeführt haben. Das alles ist so einfach, daß der Leser bei der nochmaligen Durchsicht dieser Paragraphen keine Mühe haben wird, sie so $z u$ lesen, als ob dort von vornherein von Potenzreihen mit komplexen Gliedern gehandelt würde.

Ein paar Bemerkungen sind höchstens bezüglich des Abelschen Grenzwertsatzes 100 und Satzes 107 von der Umkehrung einer Potenzreihe nötig. Bei dem letzteren wurde nämlich die Konvergenz der Reihe $y+\beta_{2} y^{2}+\ldots$ und damit auch die Konvergenz der den

1) Oder: auf der Punktmenge $M$.

2) Oder allgemein: auf eine Punktmenge $M$ mit Häufungspunkt in $\zeta$.

3) Zur kurzen Orientierung iber die wichtigsten Grundlagen der Funktionentheorie sei auf die kleinen Heftchen des Verf. „Funktionentheorie, I. Teil: Grundlagen der allgemeinen Theorie, 2. Aufl., Leipzig 1918; II. Teil: Anwendungen und Weiterführung der allgemeinen Theorie, 2. Aufl., Leipzig 1920 “ (Sammlung Göschen, Nr. 668 u. 703) hingewiesen. 
Bedingungen des Problems genügenden Reihe $y+b_{2} y^{2}+\ldots$ nur für relle $y$ erwiesen. Das ist aber ersichtlich ausreichend, denn damit ist ja dargetan, daß diese Potenzreihe einen positiven Konvergenzradius besitzt, - und das genügt.

Bezüglich des Abelschen Grenzwertsatzes aber läßt sich hier sogar - entsprechend der größeren Bewegungsfreiheit von $z$ - mehr beweisen als damals, und darum wollen wir noch einmal auf ihn eingehen:

Es sei $\sum a_{n} z^{n}$ eine gegebene nicht beständig konvergente Potenzreihe mit positivem Radius. Dann bedeutet es zunächst genau wie damals keine sachliche Einschränkung, wenn wir annehmen, daß dieser Radius gerade $=1$ ist. Auf dem Rande ihres Konvergenzkreises $|z|=1$ liege wenigstens ein Punkt $z_{0}$, in dem die Reihe noch konvergiert. Auch hier dürfen wir annehmen, daß $z_{0}$ den speziellen Punkt +1 bedeutet. Denn setzen wir, falls $z_{0} \neq+1$ ist

$$
a_{n} z_{0}^{n}=a_{n}^{\prime} \text {, }
$$

so ist $\sum a_{n}{ }^{\prime} z^{n}$ eine Potenzreihe, die gleichfalls den Radius 1 hat und die nun in dem Randpunkte +1 konvergiert.

Der damals gegebene Beweis, bei dem nun alles „komplex" gelesen werden kann, lehrt dann den

232. Satz. Hat die Potenzreihe $\Sigma a_{n} z^{n}$ den Radius 1 und ist sie in dem Randpunkte +1 des Einheitskreises noch konvergent und ist etwa $\Sigma a_{n}=s$, so ist auch

$$
\lim _{z \rightarrow+1}\left(\sum a_{n} z^{n}\right)=s,
$$

wenn sich z längs des positiv-reellen Radius von 0 her der Stelle + 1 nähert $^{1}$ ). - Wir können jetzt ohne Schwierigkeit mehr beweisen:

\section{3.}

Erweiterter Abelscher Grenzwertsatz. Unter den Vorausselzungen des vorigen Satzes bleibt die Beziehung

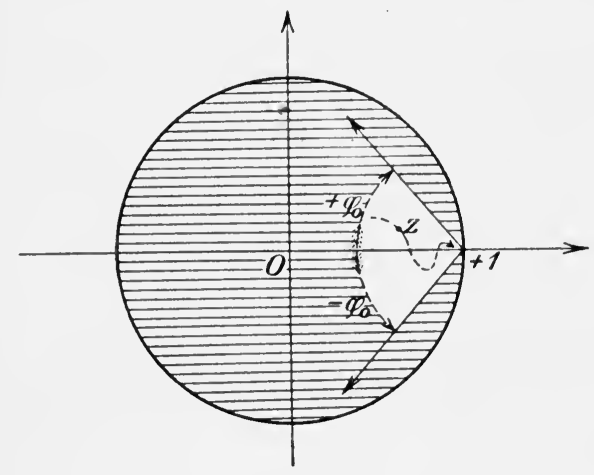

Fig. 10 .

$$
\lim _{z \rightarrow+1}^{r^{\prime}}\left(\sum a_{n} z^{n}\right)=s
$$

auch dann noch gültig, wenn $z$ bei der Annäherung an +1 nur auf einen Winkelraum beschräkt wird, der von irgend zwei (festen) Strahlen gebildet wird, die von +1 in das Innere des Einheitskreises dringen (s. Fig. 10).

Den Beweis wollen wir ganz unabhängig von den damaligen Betrachtungen lie-

1) Wir haben hier also einen Grenzwert der oben $\mathbf{2 4 4}, 1$ genannten spezielleren Art vor uns. 
fern, so daß wir hierdurch zugleich einen dritten Beweis des Abelschen Grenzwertsatzes bekommen: Ist $z_{0}, z_{1}, \ldots, z_{k}, \ldots$, eine beliebige Zahlenfolge, die in dem genannten Winkelraum liegt, und mit wachsendem $k$ gegen den Randpunkt +1 rückt, so ist $\mathrm{zu}$ beweisen, daß

$$
f\left(z_{k}\right) \rightarrow s
$$

strebt, wenn wie damals $\sum a_{n} z^{n}=f(z)$ gesetzt wird. Wählt man aber in dem Toeplitzschen Satze $\mathbf{2 2 1}, 2$ für die Zahlen $a_{k n}$ die Werte

$$
a_{k n}=\left(1-z_{k}\right) \cdot z_{k}^{n}
$$

und wendet ihn auf die nach Voraussetzung $\rightarrow s$ strebende Folge der Teilsummen $s_{n}=a_{0}+a_{1}+\ldots+a_{n}$ an, so liefert er unmittelbar, daß mit wachsendem $k$ auch

$$
\sum_{n=0}^{\infty}\left(1-z_{k}\right) \cdot z_{k}^{n} \cdot s_{n}=\left(1-z_{k}\right) \cdot \sum_{n=0}^{\infty} s_{n} z_{k}^{n}=\sum_{n=0}^{\infty} a_{n} z_{k}^{n}=f\left(z_{k}\right)
$$

$\rightarrow s$ strebt. Damit wäre schon alles bewiesen, sofern die gewählten Zahlen $a_{k n}$ die Voraussetzungen (a), (b) und (c) von 221 erfüllen. Daß aber (a) erfüllt ist, ist wegen $z_{k} \rightarrow 1$ evident, und die Zeilensummen sind $=A_{k}=\left(1-z_{k}\right) \sum_{n=0}^{\infty} z_{k}^{n}=1$, so daß auch (c) erfüllt ist. Die Bedingung (b) endlich verlangt, daß eine Konstante $K$ existiere, so daß für jeden Punkt $z=z_{k} \neq+1$ im Winkelraum (oder eines beliebigen sektorförmigen Teiles desselben, der seine Spitze in +1 hat)

$$
|1-z| \cdot \sum_{n=0}^{\infty}\left|z^{n}\right|=\frac{|1-z|}{1-|z|}<K
$$

bleibt, - was auch der Fall ist ${ }^{\mathbf{1}}$ ). Damit ist alles bewiesen. - Diese

1) Es wirdalso(s. Fig. 10) verlangt: Wenn $z=1-\varrho(\cos \varphi+i \sin \varphi)$ ist und hierin $\varphi \mid \leq \varphi_{0}<\frac{\pi}{2}$ und $0<\varrho \leqq \varrho_{0}<2 \cos \varphi_{0}$ bleibt, so gibt es eine nur von $\varphi_{0}$ und $\varrho_{0}$ abhängige Konstante $A=A\left(\varphi_{0}, \varrho_{0}\right)$, so daß für alle genannten $z$ stets

$$
\frac{1-z \mid}{1-|z|} \leqq A
$$

bleibt. - Beweis. Wir wählen, was zum Beweise ausreicht, $\varrho_{0}=\cos \varphi_{0}$ und werden zeigen, daß $A=\frac{2}{\cos \varphi_{0}}$ das verlangte leistet. In der Tat lautet dann die Behauptung, daßi für $0<\varrho<\cos \varphi_{0}$ und $|\varphi| \leq \varphi_{0}$

oder

$$
\frac{\varrho}{1-\sqrt{1-2 \varrho \cos \varphi+\varrho^{2}}}<\frac{2}{\cos \varphi_{0}}
$$

$$
-2 \varrho \cos \varphi+\varrho^{2} \leq-\varrho \cos \varphi_{0}+\frac{1}{4} \varrho^{2} \cos ^{2} \varphi_{0}
$$

ist. Vergrößert man aber hier die linke Seite, indem man $\varphi$ durch $\varphi_{0}$ und $\varrho^{2}$ durch $\varrho \cos \varphi_{0}$ ersetzt, so würde es genügen zu zeigen, dafi

$$
-\varrho \cos \varphi_{0}<-\varrho \cos \varphi_{0}+\frac{1}{4} \varrho^{2} \cos ^{2} \varphi_{0}
$$

ist, - was aber gewifi der Fall. 
Erweiterung des Abelschen Grenzwertsatzes auf „,komplexe Annäherung“ oder „Annäherung im Winkelraum“ rührt von $O$. Stolz ${ }^{\mathbf{1}}$ ) her.

Damit sind die sämtlichen Sätze der $§ \S 20$ und 21 - mit einziger Ausnahme des Satzes über die hier nicht definierte Integration auf den Fall komplexer Größen übertragen. Insbesondere gilt dadurch als festgestellt, daß eine Potenzreihe im Innern ihres Konvergenzkreises eine Funktion komplexen Argumentes definiert, die dort stetig und differenzierbar ist - und letzteres ,gliedweise" und beliebig oft -, und die somit diejenigen beiden Eigenschaften besitzt, die man in erster Linie für alle Anwendungszwecke von einer Funktion verlangt. Deswegen und wegen ihrer sonstigen großen Bedeutung für die weitere Entwicklung der Theorie hat man einer Funktion, die sich für die Umgebung eines Punkte $z_{0}$ durch eine Potenzreihe $\Sigma a_{n}\left(z-z_{0}\right)^{n}$ darstellen läßt, einen besonderen Namen gegeben. Man sagt, daß sie sich in $z_{0}$ analytisch oder regulär verhalte. Da sie sich dann nach 99 von selbst auch in jedem andern inneren Punkte des Konvergenzkreises analytisch verhält, so nennt man sie kurz eine in diesem Kreise analytische oder reguläre Funktion ${ }^{2}$ ).

Alle Sätze, die wir über Funktionen bewiesen haben, die durch Potenzreihen gegeben waren, sind Sätze über analytische Funktionen. Von diesen heben wir, weil für das Folgende besonders wichtig, nur die folgenden beiden noch einmal besonders hervor.

234. 1. Sind zwei Funktionen in ein und demselben Kreise analytisch, so ist es (nach \$ 21) auch ihre Summe, ihre Differenz, ihr Produkt und ihr Quotient; letzteres aber natürlich nur in den Punkten, in denen die im Nenner stehende Funktion nicht $=0$ ist.

2. Stimmen zwei in einem und demselben Kreise analytische Funktionen in einer wenn auch noch so kleinen Umgebung des Mittelpunktes überein, so sind sie dort völlig identisch (= Identitätssatz für Potenzreihen 97).

Außer diesen beiden, uns lediglich der Form nach neuen Sätzen wollen wir noch den folgenden wichtigen Satz beweisen, der über den Zusammenhang zwischen den Beträgen der Koeffizienten einer Potenzreihe und dem Betrag der durch sie dargestellten Funktion einige Auskunft gibt:

1) Zeitschrift f. Math. u. Phys., Bd. 20 (1875), S. 369. In neuerer Zeit hat die Frage nach der Umkehrbarkeit des Abelschen Grenzwertsatzes den Gegenstand zahlreicher Untersuchungen abgegeben, also die Frage, unter welchen (möglichst wenig verlangenden) Bedingungen für die $a_{n}$ aus der Existenz des Grenzwertes von $f(z)$ bei $z \rightarrow 1$ (im Winkelraum) auf die Konvergenz von $\Sigma a_{n}$ zurückgeschlossen werden kann.

2) "Analytisch" oder "regulär" in einem Kreise $\Omega$ heiłst hiernach eine Funktion einfach dann, wenn sie durch eine in diesem Kreise konvergente Potenzreihe dargestellt werden kann. 
Satz. Ist $f(z)=\sum_{n=0}^{\infty} a_{n}\left(z-z_{0}\right)^{n}$ für $\mid z-z_{0}<r$ konvergent, so ist $\mathbf{2 3 5 .}$

$$
\left|a_{p}\right| \leqq \frac{M}{\varrho^{p}}, \quad(p=0,1,2, \ldots)
$$

wenn $0<\varrho<r$ ist, und wenn $M$ eine Zahl bedeutet, die von $|f(z)|$ längs der Peripherie $\left|z-z_{0}\right|=\varrho$ nirgends übertroffen wird. (Cauchysche Abschätzungsformel.)

Beweis ${ }^{1}$ ). Wir wählen vorerst eine komplexe Zahl $\eta$, deren Betrag $=1$ ist, für die aber stets $\eta^{q} \neq 1$ ist, welchen ganzzahligen Exponenten auch $q \gtrless 0$ bedeuten möge ${ }^{2}$ ). Nun betrachten wir für einen bestimmten ganzzahligen Exponenten $k \gtrless 0$ die ganz spezielle Funktion

$$
g(z)=a \cdot\left(z-z_{0}\right)^{k}
$$

und bilden ihre Werte für $z=z_{0}+\varrho \cdot \eta^{\nu}, v=0,1,2, \ldots$ Bezeichnen wir diese kurz mit $g_{0}, g_{1}, g_{2}, \ldots$, so ist für $n \geqq 1$

und also

$$
g_{0}+g_{1}+\ldots+g_{n-1}=a \cdot \varrho^{k} \cdot \frac{1-\eta^{k n}}{1-\eta^{k}}
$$

$$
\left|\frac{g_{0}+g_{1}+\ldots+g_{n-1}}{n}\right| \leqq \frac{2}{n} \cdot \varrho^{k} \cdot\left|\frac{a}{1-\eta^{k}}\right|
$$

Und da hier rechterhand alles außer dem Nenner $n$ feste Werte hat, so streben mit wachsendem $n$ diese arithmetischen Mittel

$$
\frac{g_{0}+g_{1}+\cdots+g_{n-1}}{n} \rightarrow 0 .
$$

Für $k=0$ würde es sich um die identisch konstante Funktion $g(z) \equiv a$ handeln, für welche dann auch die Mittel

$$
\frac{g_{0}+g_{1}+\ldots+g_{n-1}}{n} \rightarrow a
$$

streben, denn der Quotient ist ja jetzt für jedes $n$ stets $=a$. Betrachtet man endlich die etwas allgemeinere Funktion

$$
\begin{gathered}
g(z)=\frac{b_{-l}}{\left(z-z_{0}\right)^{l}}+\frac{b_{-l+1}}{\left(z-z_{0}\right)^{l-1}}+\ldots+\frac{b_{-1}}{z-z_{0}}+b_{0}+b_{1}\left(z-z_{0}\right)+\ldots \\
\cdots+b_{m}\left(z-z_{0}\right)^{m}
\end{gathered}
$$

1) Der folgende sehr schöne Beweis rührt von Weierstraß her (Werke II, S. 224) und stammt schon aus dem Jahre 1841. Cauchy (Mémoire lithogr., Turin 1831) bewies die Formel auf dem Umweg über seine Integraldarstellung von $f(z)$. Daf uberhaupt eine Konstante $M$ mit der im Satze verlangten Eigenschaft existiert, ist ja fast selbstverständlich, da $\Sigma\left|a_{n}\right| \cdot \varrho^{n}$ konvergiert und also die Folge $\left|a_{n}\right| \cdot \varrho^{n}$ beschränkt ist.

2) Solche Zahlen $\eta$ gibt es natürlich, denn ist $\eta=\cos (\alpha \pi)+i \sin (c \pi)$, so ist $\eta^{q}=\cos (q \alpha \pi)+i \sin (q \propto \pi)$; und dies wird niemals $=1$, wenn man $\alpha$ irrational wählt. 
bei der $l$ und $m$ feste ganze Zahlen $\geqq 0$ bedeuten, und bildet man die analogen arithmetischen Mittel

$$
g_{0}+g_{1}+\ldots+g_{n-1}
$$

(bei denen also wieder $g_{v}=g\left(z_{0}+\varrho \eta^{\nu}\right), \nu=0,1, \ldots$, gesetzt ist), so streben diese nach den beiden vorweg behandelten Fällen ersichtlich $\rightarrow b_{0}$. Weiß man überdies, daß die Funktion $g(z)$ für alle $z$ des Kreises $\left|z-z_{0}\right|=\varrho$ ihrem Betrage nach niemals größer als die Konstante $K$ ist, so ist auch stets

$$
\left|g_{0}+g_{1}+\ldots+g_{n-1}\right| \leqq \frac{n K}{n}-K
$$

und folglich auch

$$
b_{0} \leqq K .
$$

Nach diesen Vorbemerkungen ist nun der Beweis des Satzes ganz einfach: Es sei $p$ eine bestimmte ganze Zahl $\geqq 0$. Da $\Sigma\left|a_{n}\right| \varrho^{n}$ konvergiert, kann nach Wahl von $\varepsilon>0$ die Zahl $q>p$ so bestimmt werden, daß

$$
\left|a_{q+1}\right| \varrho^{q+1}+\left|a_{q+2}\right| \varrho^{q+2}+\ldots<\varepsilon
$$

ist. Dann ist erst recht für alle $\left|z-z_{0}\right|=\varrho$

$$
\sum_{n=q+1}^{\infty} a_{n}\left(z-z_{0}\right)^{n} \mid<\varepsilon
$$

und also für dieselben $z$

$$
\sum_{n=0}^{q} a_{n}\left(z-z_{0}\right)^{n} \mid<M+\varepsilon
$$

wenn $M$ die im Satz genannte Bedeutung hat. Folglich ist auf dieser Peripherie $\left|z-z_{0}\right|=\varrho$ stets

$$
\begin{aligned}
\mid \frac{a_{0}}{\left(z-z_{0}\right)^{p}}+\ldots+\frac{a_{p-1}}{z-z_{0}}+a_{p} & +a_{p+1}\left(z-z_{0}\right)+\ldots+a_{q}\left(z-z_{0}\right)^{q-p} \mid \\
& \leqq \frac{M+\varepsilon}{\varrho^{p}} .
\end{aligned}
$$

Hier steht aber eine Funktion zwischen den Absolutzeichen, wie wir sie eben behandelt haben. Die dabei gewonnene Abschätzung $\left|b_{0}\right| \leqq K$ lautet jetzt

$$
\left|a_{p}\right| \leqq \frac{M+\varepsilon}{e^{p}},
$$

und da $\varepsilon>0$ beliebig war, so muß sogar (vgl. Fußnote zu 41, 1)

$$
\mid a_{p} \leqq \frac{M}{\varrho^{p}}
$$

sein, w. z. b. w. 


\section{$\S 55$. Die elementarèn analytischen Funktionen.}

\section{Die rationalen Funktionen.}

1. Die rationale Funkton $w=\frac{1}{1-z}$ ist für jeden von +1 verschiedenen Mittelpunkt $z_{0}$ in eine Potenzreihe entwickelbar:

$$
\frac{1}{1-z}=\frac{1}{1-z_{0}-\left(z-z_{0}\right)}=\frac{1}{1-z_{0}} \cdot \frac{1}{1-\frac{z-z_{0}}{1-z_{0}}}=\sum_{n=0}^{\infty} \frac{1}{\left(1-z_{0}\right)^{n+1}} \cdot\left(z-z_{0}\right)^{n}
$$

und diese Reihe ist konvergent, wenn $\left|z-z_{0}\right|<\left|1-z_{0}\right|$ ist, wenn also $z$ näher an $z_{0}$ liegt, als es der Punkt +1 tut; m. a. W. der Konvergenzkreis ist der durch den Punkt +1 gehende Kreis mit dem Mittelpunkt $z_{0}$. Die Funktion $\frac{1}{1-z}$ ist also in jedem von +1 verschiedenen Punkte der Ebene analytisch.

Bei diesem Beispiel machen wir noch flüchtig auf folgende Erscheinung aufmerksam, die in der Funktionentheorie prinzipielle Bedeutung gewinnt: Wenn die geometrische Reihe $\Sigma z^{n}$, deren Konvergenzkreis der Einheitskreis ist, nach dem Taylorschen Satz um einen im Innern des Einheitskreises gelegenen neuen Mittelpunkt $z_{1}$ entwickelt wird, so könnten wir nach jenem Satz mit Sicherheit nur behaupten, daf die neue Reihe mindestens in demjenigen Kreise konvergiert, der den Mittelpunkt $z_{1}$ hat und den Einheitskreis von innen berührt. Jetzt sehen wir, dafi der Konvergenzkreis der neuen Reihe sehr wohl über den alten hinausreichen kann. Dies wird nämlich immer eintreten, wenn $z_{1}$ nicht gerade positiv reell ist. Ist $z_{1}$ negativ reell, so wird der neue Kreis sogar den alten völlig einschliefien. (Vgl. Fufnote zu 99.)

2. Da eine ganze rationale Funktion

$$
a_{0}+a_{1} z+a_{\mathbf{2}} z^{2}+\ldots+a_{m} z^{m}
$$

als eine beständig konvergente Potenzreihe angesehen werden muß, so sind diese Funktionen in der ganzen Ebene analytisch. Also sind auch die gebrochenen rationalen Funktionen

$$
\frac{z_{0}+a_{1} z+\cdots+a_{m} a^{m}}{b_{0}+b_{1} z+\ldots+b_{m} b^{m}}
$$

in allen Punkten der Ebene analytisch, in denen der Nenner nicht $=0$ ist, - also überall mit Ausnahme von endlich vielen Punkten. Ihre Potenzreihenentwicklung in einem Punkte $z_{0}$, in dem der Nenner $\neq 0$ ist, ergibt sich folgendermaßen: Indem man in Zähler und Nenner $z$ durch $z_{0}+\left(z-z_{0}\right)$ ersetzt und nach Potenzen von $\left(z-z_{0}\right)$ umordnet, erhält die Funktion die Form

$$
\frac{a_{0}{ }^{\prime}+a_{1}{ }^{\prime}\left(z-z_{0}\right)+\cdots+a_{m}^{\prime}\left(z-z_{0}\right)^{m}}{b_{0}{ }^{\prime}+b_{1}{ }^{\prime}\left(z-z_{0}\right)+\ldots+b_{k}{ }^{\prime}\left(z-z_{0}\right)^{m}}
$$

in der unserer Annahme gemäß $b_{0}^{\prime} \neq 0$ ist. Nun kann nach 105, 4 
die Division ausgeführt und der Quotient in die verlangte Potenzreihe der Form $\sum c_{n}\left(z-z_{0}\right)^{n}$ entwickelt werden $\left.{ }^{\mathbf{1}}\right)$.

Die Reihe

\section{Die Exponentialfunktion.}

$$
1+\frac{z}{1 !}+\frac{z^{2}}{2 !}+\ldots+\frac{z^{n}}{n !}+\ldots
$$

ist eine beständig konvergente Potenzreihe und definiert also eine in der ganzen Ebene reguläre Funktion, oder wie man kurz sagt, eine ganze Funktion: jedem Punkte $z$ der Ebene ist eine wohlbestimmte Zahl w als Summe dieser Reihe zugeordnet.

Diese Funktion, die für reelle Werte von $z$ den gemäß Definition 33 erklärten Wert von $e^{z}$ zur Summe hat, benutzt man zweckmäßig dazu, um die Potenzen der Basis $e$ (und dann weiterhin auch gleich diejenigen einer beliebigen positiven Basis) für alle komplexen Exponenten zu definieren:

236. Definition. Für alle reellen oder komplexen Exponenten wird die Bedeutung der Potenz ez eindeutig durch die Gleichung

$$
\boldsymbol{e}^{z}=1+\frac{z}{1 !}+\frac{z^{2}}{2 !}+\ldots+\frac{z^{n}}{n !}+\ldots
$$

festgelegt. Ist dann weiter $p$ eine beliebige positive Zahl, so soll unter $p^{z}$ der eindeutig durch die Formel

$$
p^{z}=e^{z \log p}
$$

festgelegte Wert verstanden werden, wenn hierin $\log p$ den in $\mathbf{3 6}$ definierten (reellen) natürlichen Logarithmus von $p$ bedeutet ${ }^{2}$ ). (Für eine nicht positive, also negativ reelle oder nicht reelle Basis $b$ läßt sich die Potenz $b^{z}$ nicht mehr eindeutig definieren; doch vgl. dazu S. 407).

1) Ein anderer Weg ist der, daf3 man zunächst die gegebene rationale Funktion in Partialbrïche zerlegt. Sie erscheint dann als Summe endlich vieler Brüche der Form

$$
\frac{A}{(z-a)^{q}}=\frac{A}{(-a)^{q}} \cdot\left(\frac{1}{1-\frac{z}{a}}\right)^{q}
$$

die man nun, wenn $z_{0} \neq a$ ist, nach 1. einzeln in Potenzreihen der Form $\Sigma c_{n}\left(z-z_{0}\right)^{n}$ entwickelt. - Bei dieser Methode erkennt man noch, daß der Radius der entstehenden Entwicklung gleich dem Abstande sein wird, den $z_{0}$ von dem nächstgelegenen derjenigen Punkte hat, in denen der Nenner der gegebenen Funktion verschwindet.

2) Man beachte, wie weit man sich mit dieser Definition von der elementaren Definition $" x^{k}$ ist ein Produkt von $k$ Faktoren, die alle $=x$ sind", entfernt hat. - Im Augenblick ist noch gar nicht zu erkennen, welchen Wert z. B. $2^{i}$ hat; er ist aber jedenfalls durch die obige Definition eindeutig festgelegt. 
238. §55. Die elementaren Funktionen. - II. Exponentialfunktion.

Da eine Potenz mit komplexen Exponenten von sich aus gar keine Bedeutung hatte, so durfte man sie erklären wie man will. Maßgebend für die Wahl einer bestimmten Erklärung können dabei nur Zweckmäßigkeitsgründe sein. $\mathrm{Da}$ die eben gegebene Erklärung eine durchaus zweckmäßige ist, liegt in der Formel 91, Beispiel 3 begründet, die, weil auf Grund einer allgemein gültigen Reihenmultiplikation bewiesen, nun auch für beliebige komplexe Exponenten gelten muß:

und folglich auch

$$
\boldsymbol{e}^{z_{1}} \cdot \boldsymbol{e}^{z_{2}}=\boldsymbol{e}^{z_{1}+z_{2}}
$$

$$
p^{z_{1}} \cdot p^{z_{2}}=p^{z_{1}+z_{2}} .
$$

Dieses wichtige Grundgesetz für das Rechnen mit Potenzen bleibt also jedenfalls erhalten. Es liefert uns zugleich den Schlüssel zur weiteren Erforschung der Funktion $e^{z}$.

1. Berechnung von $e^{z}$. Für reelles $y$ ist

$$
\begin{aligned}
e^{i y} & =\sum_{n=0}^{\infty} \frac{(i y)^{n}}{n !}=\sum_{k=0}^{\infty}(-1)^{k} \frac{y^{2 k}}{(2 k !)}+i \sum_{k=0}^{\infty}(-1)^{k} \frac{y^{2 k+1}}{(2 k+1) !} \\
& =\cos y+i \sin y
\end{aligned}
$$

und folglich für $z=x+i y$

$$
\boldsymbol{e}^{z}=e^{x+i y}=e^{x} \cdot e^{i y}=\boldsymbol{e}^{x}(\cos \boldsymbol{y}+\boldsymbol{i} \sin \boldsymbol{y}),
$$

eine Formel $^{1}$ ), mit deren Hilfe man nun bequem den Wert von $e^{z}$ für jedes komplexe $z$ bequem berechnen kann.

Darüber hinaus aber gibt uns diese Formel eine bequeme und vollständige Einsicht in die Werte, die die Funktion $e^{z}$ in den verschiedenen Punkten der komplexen Ebene hat (kurz: in ihren Wertevorrat). Wir heben die folgenden Tatsachen hervor.

2. Es ist $\left|e^{z}\right|=e^{\Re(z)}=e^{x}$. In der Tat ist $e^{i y}|=| \cos y+i \sin y \mid$ $=\sqrt{\cos ^{2} y+\sin ^{2} y}=1$, also $\left|e^{z}\right|=\left|e^{x}\right| \cdot \mid e^{i y}=e^{x}$, weil $e^{x}>0$ und der zweite Faktor $=1$ ist. Ebenso ist

$$
\operatorname{arc} \epsilon^{z}=\mathfrak{\Im}(z)=y,
$$

wie gleichfalls aus der eben benutzten Formel 238, 1 abzulesen ist.

3. $e^{z}$ hat die Periode $2 \pi i$, d. h. es ist für alle $z$

$$
e^{z}=\epsilon^{z+2 \pi i}=\epsilon^{z+2 k \pi i}, \quad(k \gtreqless 0, \text { ganz }) ;
$$

denn wenn $z$ um $2 \pi i$ vermehrt wird, so vermehrt sich sein imaginärer Teil $y$ um $2 \pi$, was nach 1 . und $\S 24,2$ keine Änderung des Wertes von $e^{z}$ hervorbringt. Alle Werte also, die $e^{z}$ überhaupt anzunehmen vermag, nimmt es schon in dem Streifen $-\pi<\mathfrak{s}(z)=y \leqq \pi$ an

1) Euler, Intr. in Analysin inf., Bd. 1 (1748), § 138. 
oder in irgendeinem Streifen, der aus diesem durch eine Parallelverschiebung um $2 k \pi$ in der Richtung der $y$-Achse erhalten werden kann. Jeden dieser Streifen bezeichnet man als einen Periodensireifen; Fig. 11 veranschaulicht den zuerst genannten unter ihnen.

4. $e^{z}$ hat auch keine anderen Perioden, ja sogar genauer: wenn für zwei spezielle Zahlen $z_{1}$ und $z_{2}$ die Gleichung

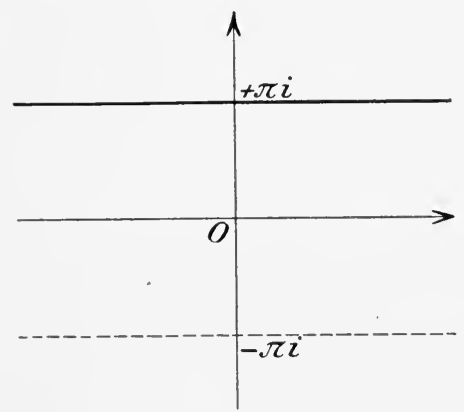

Fig. 11 .

$$
e^{z_{1}}=\epsilon^{z_{2}}
$$

besteht, so muß

$$
z_{2}=z_{1}+2 k \pi i
$$

sein. In der Tat folgt zunächst, daß. $e^{z_{2}-z_{1}}=1$ sein muß. Ist aber $e^{z}=e^{x+i y}=e^{x}(\cos y+i \sin y)=1$, so ist nach 2. zunächst $e^{x}=1$, also$x=0$. Dann ergibt sich weiter

$$
\cos y+i \sin y=1 \text {, }
$$

d. h. $\quad \cos y=1, \quad \sin y=0$,

also $y=2 k \pi$, und folglich

$$
z=z_{2}-z_{1}=2 k \pi i \text {, w. z. b. w. }
$$

5. $e^{z}$ nimmt im Periodenstreifen jeden Wert $w \neq 0$ ein und nur einmal an; oder: die Gleichung $e^{z}=w_{1}$ hat bei gegebenem $w_{1} \neq 0$ in jenem Streifen eine und nur eine Lösung.

Ist $w_{1}=R_{1}\left(\cos \Phi_{1}+i \sin \Phi_{1}\right)$ mit $R_{1}>0$, so ist

$$
z_{1}=\log R_{1}+i \Phi_{1}
$$

wegen $\epsilon^{z_{1}}=e^{\log R_{1}} \epsilon^{i \omega_{1}}=R_{1}\left(\cos \Phi_{1}+i \sin \Phi_{1}\right)=w_{1}$ jedenfalls eine Lösung von $e^{z_{1}}=w_{1}$. Nach 3. sind dann auch die, Zahlen

$$
z_{1}+2 k \pi i \quad(k=0, \pm 1, \pm 2, \ldots)
$$

Lösungen derselben Gleichung, und nach 4. kann es außer diesen keine andern geben. Hierin kann man nun aber $k$ stets auf eine und nur eine Weise so wählen, daß

wird, - w. z. b. w.

$$
-\pi<J\left(z_{1}+2 k \pi i\right) \leqq+\pi
$$

6. Den Wert 0 nimmt $e^{z}$ nirgends an; denn nach $\mathbf{2 3 7}$ ist

$$
e^{z} \cdot e^{-z}=1
$$

so da $B$ niemals $e^{z}=0$ sein kann.

7. Aus 238, 1 erhält man noch die besonderen Werte

$$
e^{2 \pi i}=1, \quad e^{\pi i}=-1, \quad e^{\frac{\pi i}{2}}=i, \quad e^{-\frac{\pi i}{2}}=-i .
$$


240. $§ 55$. Die elementaren Funktionen. - III. $\cos z$ und $\sin z$.

\section{Die Funktionen $\cos \approx$ und $\sin \approx$.}

Auch bei den trigonometrischen Funktionen kann man ihre beständig konvergenten Potenzreihenentwicklungen dazu benutzen, um sie für alle komplexen Werte des Argumentes zu erklären.

Definition. Die Summe der beständig konvergenten Potenzreihe

$$
1-\frac{z^{2}}{2 !}+\frac{z^{4}}{4 !}-+\cdots+(-1)^{k} \frac{z^{2}}{(2 k) !}+\ldots
$$

bezeichnet man mit $\cos \approx$, die der beständig konvergenten Reihe

$$
\frac{z}{1 !}-\frac{z^{3}}{3 !}+\frac{z^{5}}{5 !}-+\ldots+(-1)^{k} \frac{z^{2 k+1}}{(2 k+1) !}+\ldots
$$

mit $\sin z,-$ und zwar für jedes komplexe $z$.

Wir haben nur wieder zu prüfen, ob diese Definitionen zweckmäßig sind, d. h. ob die durch sie erklärten in der ganzen Ebene analytischen, also ganzen Funktionen dieselben Grundeigenschaften besitzen, wie die reellen Funktionen $\cos x$ und $\sin x$. Daß dies wieder in vollstem Maße der Fall ist, wird durch die folgende Zusammenstellung ihrer Haupteigenschaften belegt:

1. Es gelten für jedes komplexe $z$ die Formeln

$$
\cos \approx+i \sin \approx=e^{i z} \quad \cos \approx-i \sin \approx=e^{-i z},
$$

aus denen weiter

$$
\cos \approx=\frac{e^{i z}+e^{-i z}}{2} \quad \sin \approx=\frac{e^{i z}-e^{-i z}}{2 i}
$$

folgt (Eulersche Formeln). - Der Beweis ergibt sich unmittelbar, indem man beiderseits die Potenzreihen einsetzt, die die betreffenden Funktionen definieren.

2. Es gelten auch für komplexe $z$ die Additionstheoreme

$$
\begin{aligned}
& \cos \left(z_{1}+z_{2}\right)=\cos z_{1} \cos z_{2}-\sin z_{1} \sin z_{2}, \\
& \sin \left(z_{1}+z_{2}\right)=\cos z_{1} \sin z_{2}+\sin z_{1} \cos z_{2} ;
\end{aligned}
$$

denn nach $\mathbf{2 3 7}$ ist zunächst

also nach 1 .

$$
e^{i\left(z_{1}+z_{2}\right)}=e^{i z_{1}} \cdot e^{i z_{2}}
$$

$$
\begin{aligned}
\cos \left(z_{1}\right. & \left.+z_{2}\right)+i \sin \left(z_{1}+z_{2}\right) \\
& =\left(\cos z_{1}+i \sin z_{1}\right)\left(\cos z_{2}+i \sin z_{2}\right) \\
& =\left(\cos z_{1} \cos z_{2}-\sin z_{1} \cdot \sin z_{2}\right)+i\left(\cos z_{1} \sin z_{2}+\sin z_{1} \cos z_{2}\right) .
\end{aligned}
$$

Ersetzt man $z_{1}$ und $z_{2}$ durch $-z_{1}$ und $-z_{2}$ und beachtet, daß $\cos z$ eine gerade, $\sin z$ eine ungerade Funktion ist, so ergibt sich eine entsprechende Formel, bei der nur beiderseits der Faktor $i$ durch $-i$ ersetzt erscheint. Addition und Subtraktion beider liefert dann die Additionstheoreme. 
3. Dadurch, daß die Additionstheoreme für unsere beiden ganzen Funktionen formal die gleichen sind wie für die Funktionen $\cos x$ und $\sin x$ des reellen Argumentes $x$, ist nicht nur die Berechtigung für die Bezeichnung dieser Funktionen als $\cos z$ und $\sin z$ hinreichend erbracht, sondern zugleich gezeigt, daß der gesamte Formelapparat der sogenannten Goniometrie, der doch aus den Additionstheoremen hergeleitet wird, ungeändert weiter bestehen bleibt. Insbesondere gelten also die Formeln

$$
\begin{array}{ll}
\cos ^{2} z+\sin ^{2} z=1, & \cos 2 z=\cos ^{2} z-\sin ^{2} z, \\
\sin 2 z=2 \sin z \cos z, & \text { usw. }
\end{array}
$$

ungeändert auch für jedes komplexe z.

4. Auch die Periodizitätseigenschaften der Funktionen bleiben im Komplexen erilatten, denn auch sie sind ja nur eine Folge der Additionstheoreme:

$$
\begin{aligned}
& \cos (z+2 \pi)=\cos z \cdot \cos 2 \pi-\sin z \cdot \sin 2 \pi=\cos z \\
& \sin (z+2 \pi)=\cos z \cdot \sin 2 \pi+\sin z \cdot \cos 2 \pi=\sin z .
\end{aligned}
$$

5. Die aus dem Reellen her bekannten Nullstellen von $\cos z$ und $\sin z$ sind auch im Komplexen die einzigen ${ }^{1}$ ). In der Tat, soll $\cos z=0$ sein, so muß nach 1 . notwendig $e^{i z}=-e^{-i z}$, oder

und also

$$
e^{2 i z}=-1=e^{\pi i}
$$

$$
e^{2 i z-\pi i}=1
$$

sein. Nach 238. 4 findet dies dann und nur dann statt, wenn

$$
2 i z-\pi i=2 k \pi i \text { oder also } z=(2 k+1) \frac{\pi}{2}
$$

ist. Und soll $\sin z=0$ sein, so muß nach 1 . jetzt $e^{i z}=e^{-i z}$ oder $e^{2 i z}=1$, also $2 i z=2 k \pi i, z=k \pi$ sein, w. z. b. w.

6. Es ist dann und nur dann $\cos z_{1}=\cos z_{2}$, wenn $z_{2}= \pm z_{1}+2 k \pi$ ist, -- also unter denselben Bedingungen wie im Reellen. Ebenso ist dann und nur dann $\sin z_{1}=\sin z_{2}$, wenn $z_{2}=z_{1}+2 k \pi$ oder $=\pi-z_{1}+2 k \pi$. In der Tat folgt aus

$$
\cos z_{1}-\cos z_{2}=-2 \sin \frac{z_{1}+z_{2}}{2} \sin \frac{z_{1}-z_{2}}{2}=0
$$

1) Oder also: Die Summe der Potenzreihe $1-\frac{z^{2}}{2 !}+\ldots$ ist dann und nur dann $=0$, wenn $z$ einen der Werte $(2 k+1) \frac{\pi}{2}, k=0,+1,+2, \ldots$, bedeutet; — und analog für die sin-Reihe. 
240. $\$ 55$. Die elementaren Funktionen. - III. $\cos z$ und $\sin z$.

nach 5., daß entweder $\frac{z_{1}+z_{2}}{2}$ oder $\frac{z_{1}-z_{2}}{2}=k \pi$ sein muß; und ebenso folgt aus

$$
\sin z_{1}-\sin z_{2}=2 \cos \frac{z_{1}+z_{2}}{2} \sin \frac{z_{1}-z_{2}}{2}=0
$$

nach 5., daß entweder $\frac{z_{1}-z_{2}}{2}=k \pi$ oder $\frac{z_{1}+z_{2}}{2}=(2 k+1) \frac{\pi}{2}$ sein muß, w. z. b. w.

7. $\cos z$ und $\sin z$ nehmen im Periodenstreifen, d. h. in dem Streifen $-\pi<\Re(z) \leqq+\pi$, jeden komplexen Wert $w$ an, und zwar hat dort jede der Gleichungen $\cos z=w$ und $\sin z=w$ genau zwei Lösungen, wenn $w \neq \pm 1$ ist, dagegen nur genau eine Lösung, wenn $w= \pm 1$ ist.

Beweis. Soll $\cos z=w$ sein, so $\operatorname{muß} e^{i z}+e^{-i z}=2 w$ oder $e^{i z}=w+\sqrt{w^{2}-1}$ sein. (Hierbei soll $\sqrt{r(\cos \varphi+i \sin \varphi)}$ eine der Zahlen $r^{\frac{1}{2}}\left(\cos \frac{\varphi}{2}+i \sin \frac{\varphi}{2}\right)$ bedeuten, deren Quadrat den Radikanden ergibt.) Da nun sicher $w+\sqrt{w^{2}-1} \neq 0$ ist $\left.{ }^{1}\right)$, so gibt es eine komplexe Zahl $z^{\prime}$ mit $-\pi<\mathfrak{J}\left(z^{\prime}\right) \leqq+\pi$, für die $e^{z^{\prime}}=w+\sqrt{w^{2}-1}$. Setzt man $-i z^{\prime}=z$, so ist $z$ eine Zahl, für die

$$
-\pi<\Re(z) \leqq+\pi \text { und } e^{i z}=w+\sqrt{w^{2}-1} \text { oder } \cos z=w
$$

ist. Die vorgelegte Gleichung hat also unter allen Umständen mindestens eine Lösung im Periodenstreifen. Nach 6. gibt es aber dann und nur dann eine zweite davon verschiedene Lösung $\bar{z}$ im Periodenstreifen, wenn $z \neq 0$ und $\neq \pi$, also $w \neq \pm 1$ ist.

Ganz analog verfährt man mit der Gleichung $\sin z=w$. Bei dieser überzeugt man sich noch leicht, daß stets eine und nur eine Lösung dieser Gleichung in dem in Fig. 12 nicht schraffierten Teile des Pe-

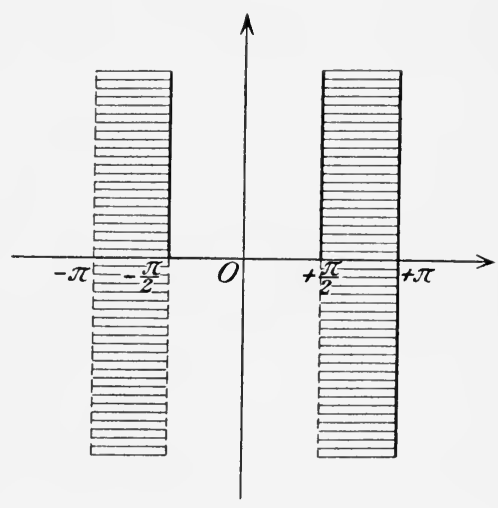

Fig. 12 . riodenstreifens liegt, wenn diesem die stark ausgezogenen, nicht aber die gestrichelten Teile des Randes zugerechnet werden (s. u. VI).

1) Denn da $w^{2}-1 \neq w^{2}$, so ist auch $\sqrt{w^{2}-1} \neq \pm w$. 


\section{Die Funktionen $\operatorname{ctg} \approx$ und $\operatorname{tg} \approx$.}

1. Da $\cos z$ und $\sin z$ in der ganzen Ebene analytisch sind, werden auch die Funktionen

$$
\operatorname{ctg} z=\frac{\cos z}{\sin z} \quad \text { und } \quad \operatorname{tg} z=\frac{\sin z}{\cos z}
$$

in der ganzen Ebene regulär sein, außer in den Punkten $k \pi$ bzw. $(2 k+1) \frac{\pi}{2}$, in denen $\sin z$ bzw. $\cos z$ verschwindet. Ihre Potenzreihenentwicklung könnte man durch Ausführung der Division der cos- und sin-Reihe gewinnen. Da diese Operation rein formaler Natur ist, muß sie jetzt dasselbe Ergebnis haben, wie die in $\S 24,4$ durchgeführte Division im Reellen. Es ist also (s. 115 und 116)

\section{1.}

$$
z \operatorname{ctg} z=\sum_{k=0}^{\infty}(-1)^{k} \frac{2^{2 k} B_{2 k}}{(2 k) !} z^{2 k}
$$

$$
\operatorname{tg} z=\sum_{k=1}^{\infty}(-1)^{k-1} \frac{2^{2 k}\left(2^{2 k}-1\right) B_{2 k}}{(2 k) !} z^{2 k-1}
$$

Auf Grund von $\mathbf{9 4}$ und 1336 sind wir jetzt auch in der Lage, den genauen Konvergenzradius dieser Reihen anzugeben: Der Betrag des Koeffizienten von $z^{2 k}$ in der ersten der beiden Reihen ist nach 136

$$
=(-1)^{k-1} \frac{2^{2 k} \cdot B_{2 k}}{(2 k) !}=\frac{2 \cdot 2^{2 k}}{(2 \pi)^{2 k}} \cdot \sum_{n=1}^{\infty} \frac{1}{n^{2 k}} .
$$

Die $(2 k)^{\text {te }}$ Wurzel hieraus ist

$$
=\frac{1}{\pi} \cdot \sqrt[2 k]{2} \cdot s_{2 k}
$$

wenn mit $s_{2 k}$ die Summe $\sum \frac{1}{n^{2 k}}$ bezeichnet wird. Da diese aber für alle $k=1,2, \ldots$ zwischen 1 und 2 gelegen ist (denn für $k=1$ ist sie $=\frac{\pi^{2}}{6}$, für alle andern $k$ kleiner, doch $\left.>1\right), r^{\prime}$ so strebt

$$
\sqrt[2 k]{(-1)^{k-1} \frac{2^{2 k} B_{2 k}}{(2 k) !}}, \frac{1}{\pi}
$$

so daß nach 94 der Radius der $\operatorname{ctg}$-Reihe $=\pi$ ist. Ähnlich ergibt sich der der tg-Reihe als $\frac{\pi}{2}$.

2. $\operatorname{ctg} z$ und $\operatorname{tg} z$ haben die Periode $\pi$, denn $\cos z$ und $\sin z$ wechseln beide nur das Vorzeichen, wenn $z$ um $\pi$ vermehrt wird. Auch hier kann genauer gezeigt werden, daß aus

stets

$$
\operatorname{ctg} z_{1}=\operatorname{ctg} z_{2} \quad \text { und ebenso aus } \operatorname{tg} z_{1}=\operatorname{tg} z_{2}
$$

folgt. In der Tat folgt aus

$$
z_{2}=z_{1}+k \pi(k=0, \pm 1, \ldots)
$$

$$
\operatorname{ctg} z_{1}-\operatorname{ctg} z_{2}=\frac{\cos z_{1}}{\sin z_{1}}-\frac{\cos z_{2}}{\sin z_{2}}=\frac{\sin \left(z_{2}-z_{1}\right)}{\sin z_{1} \cdot \sin z_{2}},
$$


241. $\$ 55$. Die elementaren Funktionen. - IV. $\operatorname{ctg} z$ und $\operatorname{tg} z$.

daß $\sin \left(z_{2}-z_{1}\right)=0$, also $z_{2}-z_{1}=k \pi$ sein muß; und ebenso ergibt sich die Behauptung bezüglich der zweiten Gleichung.

3. Im „Periodenstreifen", d.h. im Streifen $-\frac{\pi}{2}<\Re(z) \leqq+\frac{\pi}{2}$ nehmen $\operatorname{ctg} z$ und $\operatorname{tg} z$ jeden komplexen Wert $w \neq \pm i$ genau einmal $a n, w= \pm i$ werden gar nicht angenommen. Soll nämlich $\operatorname{ctg} z=w$ sein, so muß, wenn $e^{2 i z}=\zeta$ gesetzt wird,

$$
i \frac{\zeta+1}{\zeta-1}=w \quad \text { oder } \quad \zeta=\frac{w+i}{w-i} .
$$

Da nun $w \neq \pm i$ sein sollte, so ist $\zeta$ eine bestimmte von 0 verschiedene komplexe Zahl; es gibt daher (nach II, 5) ein $z^{\prime}$ mit $-\pi<\Im\left(z^{\prime}\right) \leqq \pi$, für das $e^{z^{\prime}}=\zeta$ ist. Für $z=-i \frac{z^{\prime}}{2}$ ist dann

$$
-\frac{\pi}{2}<\Re(z) \leqq \frac{\pi}{2} \quad \text { und } \quad \operatorname{ctg} z=w,
$$

d. h. $z$ ist eine Lösung der letzten Gleichung im vorgeschriebenen Streifen. Nach 2. kann dort keine zweite Lösung liegen. Daß endlich die Gleichungen $\operatorname{ctg} z= \pm i$ gar nicht lösbar sind, erkennt man daraus, da $\beta$ ihr Bestehen die Gleichung

$$
\operatorname{ctg}^{2} z+1=0
$$

zur Folge hätte, die wegen $\cos ^{2} z+\sin ^{2} z=1$ tatsächlich für kein $z$ gültig sein kann. - Für $\operatorname{tg} z$ verfährt man ganz analog.

4. Auch die in $\$ 24,4$ abgeleitete Partialbruchzerlegung für die ctg-Funktion bleibt ungeändert für alle komplexen $z$ bestehen, die von $0, \pm 1, \pm 2, \ldots$ verschieden sind (und ähnlich die Zerlegungen von $\operatorname{tg} z, \frac{1}{\sin z}$ usw.). In der Tat kann die ganze dortige Entwicklung, ohne auch nur ein Wort zu ändern, „komplex gelesen“ werden ${ }^{1}$ ). Es ist also insbesondere für alle obengenannten $z$

$$
\pi z \operatorname{ctg} \pi \cdot z=1+z \sum_{n=1}^{\infty}\left[\frac{1}{z-n}+\frac{1}{z+n}\right]=1+2 z^{2} \cdot \sum_{n=1}^{\infty} \frac{1}{z^{2}-n^{2}} .
$$

Da endlich

$$
\pi z \operatorname{ctg} \pi z=i \pi z \frac{e^{i \pi z}+e^{-i \pi z}}{e^{i \pi z}-e^{-i \pi z}}=i \pi z \frac{e^{2 i \pi z}+1}{e^{2 i \pi z}-1}
$$

ist, so erhält man hieraus, wenn man $2 i \pi z$ einfach durch $z$ ersetzt, noch die Entwicklung

$$
\frac{z e^{z}+1}{2}=1+\sum_{n=1}^{\infty} \frac{2 z^{2}}{e^{2}+4 \pi^{2} n^{2}}
$$

$\left.{ }^{1}\right)$ Gerade um dies zu ermöglichen, hatten wir damals einige Abschätzungen (z. B. die S. 198 und 199, Fußnote 1 erwähnte) etwas anders gemacht, als es in Reellen erforderlich war. 
oder also die Entwicklung

$$
\frac{1}{e^{2}-1}+\frac{1}{2}-\frac{1}{z}=\sum_{n=1}^{\infty} \frac{2 z}{z^{2}+4 \pi^{2} n^{2}}
$$

- gültig für alle komplexen $z$, die von $2 k \pi i,(k \gtreqless 0$, ganzzahlig), verschieden sind. Damit ist auch die Gültigkeit der S. 364 gewonnenen besonders merkwürdigen Partialbruchzerlegung auf komplexe $z$ erweitert und zugleich der wahre Zusammenhang zwischen dieser und der ctg-Zerlegung aufgedeckt, der bisher als ein mehr zufälliger erschien.

\section{Die logarithmische Reihe.}

In $\S 26$ sahen wir, daß für $|x|<1$ die Reihe

$$
y=\sum_{n=1}^{\infty} \frac{(-1)^{n-1}}{n} x^{n}
$$

die zur Exponentialfunktion $e^{y}-1$ inverse Funktion darstellt, d. h. wenn man $y$ in die Reihe

$$
y+\frac{y^{2}}{2 !}+\frac{y^{3}}{3 !}+\cdots
$$

einsetzt und die (sicher erlaubte) Umordnung nach Potenzen von $x$ vornimmt, so reduziert sich die neue Reihe einfach auf $x$. Diese Tatsache muß - weil rein formaler Natur - auch noch für komplexe Größen bestehen bleiben. Es ist daher auch für alle $|z|<1$

$$
e^{w}-1=z \quad \text { oder } \quad e^{w}=1+z \text {, }
$$

wenn w die Summe der Reihe

$$
w=\sum_{n=1}^{n} \frac{(-1)^{n-1}}{n} z^{n}
$$

bedeutet. Wir benutzen nun auch im Komplexen die folgende

242. Definition. Es soll a ein natürlicher Logärithmus von $c$, in Zeichen

$$
a=\log c
$$

genannt werden, wenn $e^{a}=c$ ist.

Nach II, 5 können wir dann zunächst sagen, daß jede von 0 verschiedene komplexe $\mathrm{Zahl} c$ einen und nur einen Logarithmus besitzt, dessen imaginärer Teil zwischen $-\pi$ ausschließlich und $+\pi$ einschließlich gelegen ist (der Zahl 0 aber kann nach II, 6 auf keine Weise ein Logarithmus zugesprochen werden). Diesen eindeutig bestimmten Wert wollen wir genauer den Hauptwert des natürlichen Logarithmus von $c$ nennen; denn neben ihm gibt es noch unendlich viele weitere Logarithmen, da neben $e^{a}=c$ auch $e^{a+2 k \pi i}=c$ ist. Ist also $a$ der Hauptwert des Logarithmus von $c$, so sind auch die Zahlen

$$
a+2 k \pi i
$$$$
(k \gtreqless 0 \text {, ganzzahlig) }
$$ 
als natürliche Logarithmen von $c$ zu bezeichnen. Man nennt sie genauer seine Nebenwerte ${ }^{1}$ ). Nach II, 4 kann es außer diesen Zahlen $a+2 k \pi i$ nun keine weiteren Logarithmen von $c$ mehr geben.

Nach diesen Festsetzungen können wir nun jedenfalls sagen: Die obige Reihe $(L)$ liefert im Einheitskreise einen Logarithmus von $(1+z)$. Wir beweisen nun aber gleich genauer den

Satz. Die logarithmische Reihe $w=\sum_{n=1}^{\infty} \frac{(-1)^{n-1}}{n} z^{n}$ liefert im Ein- $\mathbf{2 4 3}$. heitskreise einschließlich aller seiner von -1 verschiedenen Randpunkte den Hauptwert von $\log (1+z)$.

Beweis. Es ist

$$
\mathfrak{\Im}\left(\sum_{n=1}^{\infty} \frac{(-1)^{n-1}}{n} z^{n}\right)=\sum_{n=1}^{\infty}(-1)^{n-1} \cdot \frac{\sin n \varphi}{n} r^{n},
$$

wenn $z=r(\cos \varphi+i \sin \varphi)$ gesetzt wird. Man erkennt nun leicht, daß dieser imaginäre Teil bei konstant gehaltenem $\varphi^{2}$ ) von 0 bis zu dem Summenwerte der Reihe $\sin \varphi-\frac{\sin 2 \varphi}{2}+\frac{\sin 3 \varphi}{3}-+\ldots$ sich monoton ändert, wenn $r$ von 0 bis 1 (einschließlich) wächst. Setzt man ihn nämlich für den Augenblick $=f(\boldsymbol{r})$, so ist

$f^{\prime}(r)=\sum_{n=1}^{\infty}(-1)^{n-1} \sin n \varphi \cdot r^{n-1}=-\frac{1}{r} \mathfrak{\Im}\left(\frac{1}{1+z}\right)=\frac{\sin \varphi}{1+2 r \cos \varphi+r^{2}} ;$

und da hier der Nenner positiv ist, so hat $f^{\prime}(\boldsymbol{r})$ bei festem $\varphi \neq k \pi$ ein festes Vorzeichen für alle $0<r<1$, - womit unsere letzte Behauptung schon bewiesen ist. Unser imaginärer Teil erreicht also, da er für $r=0$ den Wert 0 hat, seine äußersten (positiven bzw. negativen) Werte für $r=1$, und es ist also dieser imaginäre Teil seinem Betrage nach nicht größer als der Betrag der Summe

$$
\sum_{n=1}^{\infty}(-1)^{n-1} \frac{\sin n \varphi}{n}
$$

und liegt also nach 210 zwischen $-\frac{\pi}{2}$ und $+\frac{\pi}{2}$ (mit Ausschluß beider Grenzen), so daß die logarithmische Reihe wirklich den Haupt. wert von $\log (1+z)$ zur Summe hat $\left.{ }^{3}\right)$.

1) Ist $c$ positiv reell, so stimmt der Hauptwert von $\log c$ mit dem früher (36, Def.) definierten (reellen) natürlichen Logarithmus überein.

2) Wir dürfen uns $\varphi \neq k \pi$ denken, da uns für die damit ausgeschlossenen reellen $z$ der Inhalt des Satzes schon von früher her bekannt ist (vgl. die vorige Fufnote).

3) Bei der nötigen Vorsicht kann man dies nach 98, 2 auch unmittelbar aus der logarithmischen Reihe selbst ablesen. 


\section{Die arc sin-Reihe.}

Wie wir unter III, 7 sahen, hat die Gleichung $\sin w=z$ bei gegebenem komplexen $z \neq \pm 1$ stets genau zwei, und für $z= \pm 1$ stets genau eine Lösung im Streifen $-\pi<\Re(w) \leq+\pi$. Da die beiden Lösungen (nach III, 6) entweder Spiegelbilder in bezug auf den Punkt $+\frac{\pi}{2}$ oder den Punkt $-\frac{\pi}{2}$ sind, so können wir genauer sagen: Die Gleichung $\sin w=z$ hat bei beliebig gegebenem komplexen $z$ (einschliefilich \pm 1 ) stets genau eine Lösung im Streifen

$$
-\frac{\pi}{2} \leqq \Re(w) \leqq+\frac{\pi}{2},
$$

wenn man von diesem Streifen noch die unterhalb der Achse des Reellen gelegenen Teile des Randes wegläßst (vgl. Fig. 12, wo die nicht zum Streifen zu zählenden Randteile gestrichelt, die andern stark gezogen sind). Diese für jedes komplexe $z$ eindeutig bestimmte Lösung der Gleichung $\sin w=z$ wird der Hauptwert der Funktion

$$
w=\arcsin z
$$

genannt. Alle iibrigen Werte sind nach III, 6 in den beiden Formeln

$$
\left\{\begin{array}{l}
\arcsin z+2 k \pi \\
\pi-\arcsin z+2 k \pi
\end{array}\right.
$$

enthalten. Man kann sie die Nebenwerte der Funktion nennen.

Für reelle $|x| \leq 1$ liefert die Reihe

$$
y=x+\frac{1}{2} \frac{x^{3}}{3}+\frac{1 \cdot 3}{2 \cdot 4} \frac{x^{5}}{5}+\ldots
$$

die Umkehrung der sin-Potenzreihe $y-\frac{y^{3}}{3 !}+\frac{y^{5}}{5 !}-\ldots$.

Genau dieselben Erwägungen wie unter V. bei der logarithmischen Reihe lehren nun, daf auch für komplexe $|z| \leq 1$ die Reihe

$$
w=z+\frac{1}{2} \frac{z^{3}}{3}+\frac{1 \cdot 3}{2 \cdot 4} \frac{z^{5}}{5}+\ldots
$$

die Umkehrung der sin-Potenzreihe $w-\frac{w^{3}}{3 !}+\ldots$ ist. Sie liefert daher jedenfalls einen der Werte von $\arcsin z$. Daß sie gerade den Hauptwert liefert, folgt hier einfach daraus, daf für $|z| \leq 1, z \neq \pm 1$,

$$
\begin{aligned}
|\Re(\arcsin z)| & \leqq|\arcsin z| \leqq|z|+\frac{1}{2} \frac{|z|^{3}}{3}+\frac{1 \cdot 3}{2 \cdot 4} \cdot \frac{|z|^{5}}{5}+\ldots \\
& =\arcsin |z| \leq \arcsin 1=\frac{\pi}{2}
\end{aligned}
$$

ist, - eine Bedingung, die eben nur von dem Hauptwert erfiullt wird.

\section{Die arctg-Reihe.}

Die Gleichung $\operatorname{tg} w=z$ hat, wie wir aus IV, 3 wissen, bei gegebenem $z \neq \pm i$ stets eine und nur eine Lösung im Streifen $-\frac{\pi}{2}<\Re(w) \leqq+\frac{\pi}{2}$. Man nennt sie den Hauptwert der Funktion

$$
w=\operatorname{arctg} z,
$$

deren sämtliche anderen Werte dann (nach IV, 2) durch die Formel $\operatorname{arctg} z+k \pi$ erhalten werden. Die Gleichungen $\operatorname{tg} z= \pm i$ aber haben überhaupt keine Lösung. 
244. §55. Die elementaren Funktionen. - VI. $\operatorname{arc} \sin z$. VII. $\operatorname{arctg} z . \quad 407$

Fast wörtlich dieselben Erwägungen wie soeben lehren nun wieder, daf für $|z|<1$ die Reihe

$$
w=z-\frac{z^{3}}{3}+\frac{z^{5}}{5}-\ldots
$$

eine der Lösungen der Gleichung tg $w=z$ liefert. Daß es gerade der Hauptwert von $\operatorname{arctg} z$ ist, dafs also der reelle Teil der Reihensumme zwischen $-\frac{\pi}{2}$ (ausschl.) und $+\frac{\pi}{2}$ (einschl.) liegt, und dafs dies auch noch fuir alle $|z|=1$ gilt, die von $\pm i$ verschieden sind, erkennt man auf Grund der beim Logarithmus durchgeführten Betrachtungen folgendermafien:

Die Summe $w$ der Reihe (A) ist, wie man durch Einsetzen der log-Reihen sofort erkennt, für alle von $\pm i$ verschiedenen $|z| \leqq 1$

$$
=w=\frac{1}{2 i} \log (1+i z)-\frac{1}{2 i} \log (1-i z),
$$

wobei unter den beiden Logarithmen deren Hauptwerte zu verstehen sind. Daher ist

$$
\Re(w)=\frac{1}{2} \mathfrak{I} \log (1+i z)-\frac{1}{2} \mathfrak{l} \log (1-i z) ;
$$

und da hier (nach $\mathbf{2 4 3}$ ) Minuend und Subtrahend zwischen $-\frac{\pi}{4}$ und $+\frac{\pi}{4}$ liegen, so liegt $\Re(w)$ zwischen $-\frac{\pi}{2}$ und $+\frac{\pi}{2}$, - jedesmal mit Ausschluf beider Grenzen. Die Reihe (A) stellt also sicher den Hauptwert von $\operatorname{arctg} z$ dar, solange $|z| \leqq 1$ und $z \neq \pm i$ ist, w. z. b.w.

\section{Die Binomialreihe.}

Von den im Reellen behandelten besonderen Potenzreihen bleibt nun allein noch die Binomialreihe

$$
(1+x)^{n}=\sum_{n=0}^{\infty}\left(\begin{array}{l}
\alpha \\
n
\end{array}\right) x^{n}
$$

für den Fall zı untersuchen, dą die auftretenden Gröfen - außer der Variablen $x$ also auch der Exponent $\alpha-$ komplexe Werte annehmen. Wir beginnen mit der

Definition: Unter dem Hauptwert der Potenz $\boldsymbol{b}^{\boldsymbol{a}}$, in der $a$ und $b$ be- $\mathbf{2 4 4}$. liebige komplexe Zahlen bedeuten, von denen nur $b \neq 0$ angenommen wird, soll der durch die Formel

$$
b^{a}=e^{a \log b}
$$

eindeutig festgelegte Wert verstanden worden, wenn hierin $\log b$ dessen Hauptwert bezeichnet. - Wählt man für $\log b$ einen seiner übrigen Werte, so erhält man auch weitere Werte der Potenz, die man als deren Nebenwerte bezeichnen kann. Ihre Gesamtheit wird durch die Formel

$$
b^{a}=e^{a\lfloor\log b+2 k \pi i]}
$$

gerade einmal geliefert, wenn hier $\log b$ seinen Hauptwert hat und $k$ alle ganzen Zahlen $\gtreqless 0$ durchläuft.

Bemerkungen und Beispiele.

1. Eine Potenz $b^{a}$ ist hiernach im allgemeinen unendlich vieldeutig, doch besitzt sie stets einen und nur einen Hauptwert. 
2. So bedeutet $z$. B. $i^{i}$ die unendlich vielen (übrigens sämtlich reellen) Zahlen

$$
e^{i(\log i+2 k x i)}=e^{i\left(\frac{\pi i}{2}+2 k \cdot \pi i\right)}=e^{-\frac{\pi}{2}-2 k \pi}, \quad(k=0, \pm 1, \pm 2, \ldots),
$$

unter denen $e^{-\frac{\pi}{2}}$ der Hauptwert der Potenz $i^{i}$ ist.

3. Die Poten $z b^{a}$ wird nur dann nicht unendlich viele Werte haben, wenn

$$
e^{a \cdot 2 k \pi i} \quad(k=0, \pm 1, \pm 2, \ldots)
$$

nur endlich viele Werte liefert; und dies wird dann und nur dann eintreten, wenn $k \cdot a$ für $k=0, \pm 1, \pm 2, \ldots$ nur endlich viele wesentlich verschiedene Zahlen liefert. Dabei sollen (nur für den Augenblick) zwei dieser Zahlen dann und nur dann wesentlich verschieden heißen, wenn sie sich nicht blof um eine ganze (reelle) Zahl unterscheiden. Das ist aber, wie man sofort sieht, dann und nur dann der Fall, wenn $a$ eine reelle rationale $Z$ ahl ist; und die Anzahl der "wesentlich verschiedenen" Werte, die $k \cdot a$ in diesem Falle annehmen kann, wird dann durch den (kleinsten) positiven Nenner angegeben, mit dem a geschrieben werden kann.

4. Speziell hat hiernach $b^{\frac{1}{p}}=\sqrt[p]{b}$ genau $p$ verschiedene Werte, von denen ein ganz bestimmter als Hauptwert ausgezeichnet ist, wenn $p$ eine ganze positive Zahl ist.

5. Die Anzahl der verschiedenen Werte von $b^{a}$ reduziert sich nach 3 . und 4. dann und nur dann auf eins, wenn $a$ eine reelle rationale Zahl mit dem Nenner 1, also eine reelle ganze Zahl ist. Für reelle ganzzahlige Exponenten (aber auch nur für diese) bleibt also die Potenz nach wie vor ein eindeutiges Symbol.

6. Ist $b$ positiv und $a$ reell, so ist der früher als Potenz $b^{a}$ definierte Wert jetzt der Hauptwert dieser Potenz.

7. Ebenso ist der durch $\mathbf{2 3 6}$ eindeutig festgelegte Wert von $e^{z}$ und $p^{z},(p>0)$, jetzt genauer der Hauptwert dieser Potenzen. An und für sich hätten beide Symbole nach unserer letzten Definition bei komplexen $z$ unendlich viele Werte. Nichtsdestoweniger soll an der Ubereinkunft festgehalten werden, daß unter $e^{z}$ und bei positivem $p$ unter $p^{z}$ immer der durch $\mathbf{2 3 6}$ erklärte Wert, also immer nur der Hauptwert verstanden werden soll.

8. Die folgenden Sätze werden uns lehren, dafis es sinngemäß ist, die Potenz $b^{a}$ auch dann noch zu definieren, wenn $b=0$, aber $\Re(a)>0$ ist. Man legt ihr dann (eindeutig) den Wert 0 bei.

Nach diesen Vorbereitungen beweisen wir nun den folgenden weitgehenden

245. Satz. Für beliebige komplexe Exponenten $\alpha$ und beliebige komplexe $|z|<1$ ist die Binomialreihe

$$
\sum_{n=0}^{\infty}\left(\begin{array}{l}
\alpha \\
n
\end{array}\right) z^{n}-1+\left(\begin{array}{l}
\alpha \\
1
\end{array}\right) z+\left(\begin{array}{l}
\alpha \\
2
\end{array}\right) z^{2}+\ldots+\left(\begin{array}{l}
\alpha \\
n
\end{array}\right) z^{n}+\ldots
$$

konvergent und hat stets den Hauptwert der Potenz $(1+z)^{\alpha}$ zur Summe $\left.{ }^{1}\right)$.

Beweis. Die Konvergenz ergibt sich wörtlich ebenso wie im Falle reeller $z$ und $\alpha$ (s. S. 201), so daf 3 wir nur die Behauptung uiber die Summe der Reihe zu beweisen haben. Wenn man aber für reelle $|x|<1$ und reelle $\alpha$ die Reihe

$$
\alpha \sum_{n=1}^{\infty} \frac{(-1)^{n-1}}{n} x^{n}=\alpha \log (1+x)
$$

1) Abel, J. f. d. reine u. angew. Math., Bd. 1 (1826), S. 311. 
246. $§ 55$. Die elementaren Funktionen. - VIII. Die Binomialreihe.

in die Exponentialreihe $e^{y}=1+y+\frac{y^{2}}{2 !}+\ldots$ einsetzt und die (nach 104) sicher erlaubte Umordnung nach Potenzen von $x$ vornimmt, so muß man die Potenzreihe für $e^{\alpha \log (1+x)}=(1+x)^{\alpha}$, also die Binomialreihe $\Sigma\left(\begin{array}{l}\alpha \\ n\end{array}\right) x^{n}$ erhalten. Tun wir, zunächst rein formal, dasselbe, indem wir uns $c$ komplex denken und $z$ statt $x$ schreiben, setzen wir also

$$
w=\alpha \cdot \sum_{n=1}^{\infty} \frac{(-1)^{n-1}}{n} z^{n} \quad \text { in } \quad e^{w}=\sum_{n=0}^{\infty} \frac{w^{n}}{n !}
$$

ein und ordnen wieder nach Potenzen von $z$ um, so erhalten wir - zunächst noch ohne Rücksicht auf die etwaige Konvergenz - notwendig wieder die Reihe

$$
\sum_{n=0}^{\infty}\left(\begin{array}{l}
\alpha \\
n
\end{array}\right) z^{n}
$$

Ihre Summe müßste also $=e^{\alpha \log (1+z)}=(1+z)^{\alpha}$ sein - und hier wären für den Logarithmus und also auch für die Potenz deren Hauptwert zu nehmen -, wenn wir noch nachweisen können, daßs die ausgeführte Umordnung erlaubt war. Das ist aber nach $\mathbf{1 0 4}$ sicher der Fall; denn die Exponentialreihe ist beständig konvergent und die Reihe $\alpha \underline{Y} \frac{(-1)^{n-1}}{n} z^{n}$ bleibt für $|z|<1$ konvergent, wenn wir $\alpha$ und alle Glieder der Reihe durch ihre absoluten Beträge ersetzen. Damit ist der Satz in seinem vollen Umfange bewiesen.

Bemerkung. Trennt man in $(1+z)^{\alpha}$ Reelles und Imaginäres, so gelangt man zu einer ziemlich kompliziert ausschauenden, von Abel herrührenden Formel, die immerhin zeigt, ein wie weitgehendes Resultat der eben bewiesene Satz ausspricht und der uns auch einen Weg zur Berechnung der Potenzen $(1+z)^{\alpha}$ liefert: Ist $z=r(\cos \varphi+i \sin \varphi)$ und $\alpha=\beta+i \gamma, 0<\gamma<1, \varphi, \beta, \gamma$ reell, so ist zunächst, wenn

gesetzt wird,

$$
1+z=R(\cos \Phi+i \sin \Phi)
$$

$$
\left.R=\sqrt{1+2 r \cos \varphi+r^{2}}, \Phi=\text { Hauptwert von } \operatorname{arctg} \frac{r \sin \varphi}{1+r \cos \varphi}{ }^{1}\right)
$$

und mit diesen Werten nun

$$
\begin{aligned}
(1+z)^{\alpha} & =e^{(\beta+\gamma i)[\log R+i \Phi]} \\
& =R^{\beta} \cdot e^{-\gamma \Phi} \cdot[\cos (\beta \Phi+\gamma \log R)+i \sin (\beta \Phi+\gamma \log R)] .
\end{aligned}
$$

Für den Fall $|z|<1$ ist durch den Satz $\mathbf{2 4 5}$ und die eben angefügte Bemerkung die Frage nach der Summe der Binomialreihe vollständig beantwortet. Es bleiben nur noch die Punkte der Peripherie $|z|=1$ zu betrachten. Aus dem Abelschen Grenzwertsatz in Verbindung mit der Stetigkeit des Hauptwertes von $\log (1+z)$ fuir alle von -1 verschiedenen $|z| \leqq 1$ und der durchgängigen Stetigkeit der Exponentialfunktion, ergibt sich da zunächst sofort der

Satz. Uberall, wo die Binomialveihe auf dem Rande $|z|=1$ des Einheitskreises noch konvergiert, außer möglicherweise in $z=-1$, ist ihre Summe nach wie vor der Hauptwert von $(1+z)^{a}$.

Die Feststellung, ob und für welche Werte von $c$ und $z$ die Binomialreihe auch noch auf dem Rande des Einheitskreises konvergiert, bereitet nach den Vorbereitungen, die wir in $\S 53$ (hauptsächlich zu dem jetzigen $Z$ weck)

1) $\Phi$ ist also $z$ wischen $-\frac{\pi}{2}$ und $+\frac{\pi}{2}$ zu wählen. 
getroffen haben, keinerlei Schwierigkeiten. Es gilt darüber der folgende, die ganze Frage noch einmal zusammenfassend beantwortende

Satz. Die Binomialreihe $\sum_{n=0}^{\infty}\left(\begin{array}{l}\alpha \\ n\end{array}\right) z^{n}$ reduziert sich für veelle ganzzahlige $\alpha \geqq 0$ auf eine endliche Summe und hat dann den (eo ipso eindeutigen) Wert $(1+z)^{\alpha}$, speziell den Wert 1 für $\alpha=0$, (auch für $z=-1$ ). Ist $\alpha$ von diesen speziellen Werten verschieden, so ist die Reihe für $|z|<1$ absolut konvergent und für $|z|>1$ divergent und weist in den Randpunkten $\mid z_{j}=1$ des Einheitskreises das folgende Verhalten aut:

a) für $\Re(\alpha)>0$ ist sie in allen diesen Randpunkten absolut konvergent;

b) für $\Re(\alpha) \leqq-1$ ist sie in allen jenen Randpunkten divergent;

c) für $-1<\Re(\alpha) \leqq 0$ ist sie in $z=-1$ divergent, in allen anderen Randpunkten des Einheitskreises dagegen bedingt konvergent.

Die Summe der Reihe ist hierbei im Falle der Konvergenz stets der Hauptwert von $(1+z)^{\alpha}$, speziell $=0$, wenn es sich um den Punkt $z=-1$ handelt.

Beweis. Setzen wir $(-1)^{n}\left(\begin{array}{l}\alpha \\ n\end{array}\right)=a_{n+1}$, so können wir unmittelbar den Satz 229 anwenden, denn es ist

$$
\frac{a_{n+1}}{a_{n}}=-\frac{\left(\begin{array}{l}
\alpha \\
n
\end{array}\right)}{\left(\begin{array}{c}
\alpha \\
n-1
\end{array}\right)}=\frac{n-(\alpha+1)}{n}=1-\frac{\omega+1}{n},
$$

und es kann somit die Richtigkeit der Behauptungen a), b), c) unmittelbar abgelesen werden. Nur der Punkt $z=-1$, also die Konvergenz der Reihe

$$
\sum_{n=0}^{\infty}(-1)^{n}\left(\begin{array}{l}
\alpha \\
n
\end{array}\right)
$$

bedarf einer besonderen Untersuchung. Hier ist aber einfach

$$
\begin{aligned}
1-\left(\begin{array}{c}
\alpha \\
1
\end{array}\right)+\left(\begin{array}{l}
\alpha \\
2
\end{array}\right) & =1-\alpha+\frac{\alpha(\alpha-1)}{1 \cdot 2}=(1-\alpha)\left(1-\frac{\alpha}{2}\right) \\
1-\left(\begin{array}{c}
\alpha \\
1
\end{array}\right)+\left(\begin{array}{l}
\alpha \\
2
\end{array}\right) & -\left(\begin{array}{l}
\alpha \\
3
\end{array}\right)=(1-\alpha)\left(1-\frac{\alpha}{2}\right)-\frac{\alpha}{3} \frac{(\alpha-1)(\alpha-2)}{1} \cdot \frac{\alpha}{2} \\
= & (1-\alpha)\left(1-\frac{\alpha}{2}\right)\left(1-\frac{\alpha}{3}\right),
\end{aligned}
$$

und allgemein, wie man durch Induktion sofort bestätigt:

$$
1-\left(\begin{array}{c}
\alpha \\
1
\end{array}\right)+\left(\begin{array}{l}
\alpha \\
2
\end{array}\right)-+\ldots+(-1)^{n}\left(\begin{array}{l}
\alpha \\
n
\end{array}\right)=(1-\alpha)\left(1-\frac{\alpha}{2}\right) \ldots\left(1-\frac{\alpha}{n}\right)
$$

d. h. die Teilsummen unserer Reihe sind den gleichstelligen Teilprodukten des Produktes $\prod_{n=1}^{\infty}\left(1-\frac{\alpha}{n}\right)$ gleich. Das Konvergenzverhalten dieses Produktes ist aber sofort $\mathrm{zu}$ überschauen. Denn ist

1. $\Re(\alpha)=\beta>0$, so wähle man $\beta^{\prime}$ gemäł $0<\beta^{\prime}<\beta$. Dann ist von einer Stelle ab, etwa für alle $n \geqq m$,

also

$$
\left|1-\frac{\alpha}{n}\right|<1-\frac{\beta^{\prime}}{n}
$$

$$
\left|\left(1-\frac{\alpha}{m}\right)\left(1-\frac{\alpha}{m+1}\right) \ldots\left(1-\frac{\alpha}{n}\right)\right|<\left(1-\frac{\beta^{\prime}}{m}\right)\left(1-\frac{\beta^{\prime}}{m+1}\right) \ldots\left(1-\frac{\beta^{\prime}}{n}\right),
$$


woraus nach 126, 2 abzulesen ist, dafi jetzt die Teilprodukte und folglich auch die Teilsummen unserer Reihe $\rightarrow 0$ streben. Die Reihe ist also konvergent mit der Summe $\mathbf{0}^{1}$ ). Ist aber

2. $\Re(\alpha)=-\beta<0$, so ist

$$
\left|1-\frac{\alpha}{n}\right|>1+\frac{\beta}{n},
$$

woraus wieder durch Multiplikation folgt, dafs.

$$
\left|\left(1-\frac{\alpha}{1}\right)\left(1-\frac{\alpha}{2}\right) \ldots\left(1-\frac{\alpha}{n}\right)\right|>\left(1+\frac{\beta}{1}\right)\left(1+\frac{\beta}{2}\right) \ldots\left(1+\frac{\beta}{n}\right)
$$

ist und also $\rightarrow+\infty$ strebt. Die Reihe ist jetzt also divergent. Ist endlich

3. $\Re(\alpha)=0$, etwa $\alpha=i \gamma$ mit $\gamma \gtrless 0$, so ist die $n^{\text {te }}$ Teilsumme unserer Reihe

$$
=(1+i \gamma)\left(1+\frac{i \gamma}{2}\right) \ldots\left(1+\frac{i \gamma}{n}\right)
$$

und dafi diese Werte für $n \rightarrow+\infty$ keinem Grenzwert zustreben, kann man in unserm Zusammenhange am schnellsten folgendermafen erkennen: Wegen der absoluten Konvergenz der Reihe $\Sigma\left(\frac{i \gamma}{n}\right)^{2}$ ist nach $\S 29$, Satz 10

$$
\left(1+\frac{i \gamma}{1}\right)\left(1+\frac{i \gamma}{2}\right) \ldots\left(1+\frac{i \gamma}{n}\right) \sim e^{i \gamma\left(1+\frac{1}{2}+\ldots+\frac{1}{n}\right)} .
$$

Wächst nun $n \rightarrow+\infty$, so strebt hier die rechte Seite ersichtlich keinem Grenzwert zu, sondern liefert eine Folge von Punkten, die um den Einheitskreis im mathematisch-positiven Sinne und in immer kleineren Schritten herumlaufen. ohne zur Ruhe zu kommen. Wegen des asymptotischen Zusammenhangs gilt dasselbe auch von der Folge der linkstehenden Teilprodukte. Also ist unsere. Reihe $\Sigma(-1)^{n}\left(\begin{array}{l}\alpha \\ n\end{array}\right)$ auch für $\Re(\alpha)=0$ divergent. Damit ist Satz $\mathbf{2 4 7}$ in allen Teilen bewiesen, das Konvergenzverhalten der Binomialreihe für alle $z$ und $\alpha$ ermittelt und ihre Summe in allen Konvergenzpunkten "geschlossen" angegeben.

\section{$\$$ 56. Reihen mit veränderlichen Gliedern. Gleichmäßige Konvergenz. Weierstraßscher Doppelreihensatz.}

Die grundsätzlichen Bemerkungen über Reihen mit veränderlichen Gliedern

$$
\sum_{n=0}^{\infty} f_{n}(z)
$$

sind wesentlich dieselben wie im Reellen (s. § 46), nur daß an die Stelle des gemeinsamen Definitionsintervalles jetzt ein Gebiet treten

1) Die Konvergenz der Reihe $\Sigma(-1)^{n}\left(\begin{array}{l}\alpha \\ n\end{array}\right)$ folgt ja schon aus 228 und ist für $\Re(\alpha)>0$ sogar eine absolute. Dafi ihre Summe dann aber $=0$ ist, ergibt sich erst wie oben. 
wird, als welches wir der Einfachheit halber - und auch für die meisten Zwecke ausreichend - einen Kreis wählen. Wir setzen demgemäß voraus:

1. Es gibt einen Kreis $\left|z-z_{0}\right|<r$, in dem alle unsere Funktionen $f(z)$ definiert sind.

2. Für jedes einzelne $z$ des Kreises $\left|z-z_{0}\right|<r$ ist die Reihe

konvergent.

$$
\sum_{n=0}^{\infty} f_{n}(z)
$$

Dann hat für jedes $z$ dieses Kreises die Reihe $\Sigma f_{n}(z)$ eine ganz bestimmte Summe, deren Wert also eine Funktion von $z$ (im Sinne der Definition von S. 388) sein wird. Wir schreiben dann etwa

$$
\sum_{n=0}^{\infty} f_{n}(z)=F(z)
$$

Bei solchen durch Reihen mit veränderlichen Gliedern dargestellten Funktionen treten nun genau dieselben Probleme auf wie in $\S 46$ und 47 für den Fall reeller Veränderlicher. Während es aber im Reellen für die Theorie und ihre Anwendungen von größter Bedeutung ist, den Funktionsbegriff in seiner vollen Allgemeinheit zu verwenden, hat sich dies im Komplexen nicht als wertvoll erwiesen. Man beschränkt sich hier vielmehr als für die meisten Zwecke ausreichend auf analytische Funktionen. Wir verlangen demgemäß noch:

3. Alle Funktionen $f_{n}(z)$ sollen in dem Kreise $\left|z-z_{0}\right|<r$ analytisch sein, sollen also durch je eine Polenzreike mit dtm Mittelpunkt $z_{0}$ darstellbar sein, deren Radius bei allen mindestens gleich der einen festen Zahl $r$ ist.

Wir sprechen dann kurz von Reihen analytissher Funhtionen ${ }^{1}$ ), und die Hauptfrage, um die es sich bei solchen Reihen handelt, ist jedesmal diese: Ist die dargestellte Funkticn $F(z)$ wieder eine im Kreise $\left|z-z_{0}\right|<r$ analytische Funktion oder nicht? Genau wie im Reellen läßt sich hier durch Beispiele belegen, daß dies ohne weitere Voraussetzungen noch nicht der Fall zu sein braucht. Dagegen läßt sich das gewünschte Verhalten von $F(z)$ erzwingen, wenn man wieder

1) Auch hier gilt (vgl. 190, Bem. 4) die prinzipielle Bemerkung: Es kommt sachlich auf dasselbe hinaus, ob man von Reihen mit veränderlichen Gliedern oder von Funktionenfolsen handelt. Die Reihe $\Sigma f_{n}(z)$ ist mit der Folge $s_{0}(z), s_{1}(z), \ldots$ ihrer Teilsummen gleichbedeutend, - und eine solche Folge von Funktionen ist umgekehrt gleichdeutend mit der Reihe $s_{0}(z)+\left(s_{1}(z)-s_{0}(z)\right)+\ldots$ Der Einfachheit halber sprechen wir im folgenden alle Definitionen und Sätze nur für Reihen aus; für Folgen wird sie sich jeder dann leicht selbst formulieren können. 
(vgl. § 47, 1. Absatz) voraussetzt, daß die Reihe gleichmäßig konvergiert. Wir stützen uns dabei, in fast wörtlicher Wiederholung von 191, auf die

Definition (2. Form) ${ }^{1}$ ). Eine Reihe $\Sigma f_{n}(z)$, deren Glieder sämtlich $\mathbf{2 4 8 .}$ im Kreise $\left|z-z_{0}\right|<r$ difinieri sind und die daselbst konvergiert, heißt in dem kleineren Kreise $\left|z-z_{0}\right| \leqq \varrho<r$ gleichmäßig konvergent, wenn es nach Wahl von $\varepsilon>0$ stets möglich ist, eine Zahl $N>0$ so zu bestimmen, daß für alle $n>N$ und alle $z$ im genannten Kreise $\left|z-z_{0}\right| \leqq \varrho$ stets

bleibt.

$$
\left|f_{n+1}(z)+f_{n+2}(z)+\ldots+=\right| r_{n}(z) \mid<\varepsilon
$$

Bemerkungen.

1. Die Gleichmäßigkeit der Konvergenz bezieht sich hiernach stets auf alle Punkte eines Kreises. Doch kann man selbstverständlich auch andre Gebiete, ja auch Linienzige oder irgendwelche unendliche Punktmengen $\mathfrak{M}$ zugrunde legen, die dem Kreise $\left|z-z_{0}\right|<v$ angehören. Die Definition bleibt sachlich dieselbe.

2. Hat die Potenzreihe $\sum a_{n}\left(z-z_{0}\right)^{n}$ den Radius $r$ und ist $0<\varrho<r$, so ist die Reihe in dem (abgeschlossenen) Kreise $\left|z-z_{0}\right| \leq \varrho$ gleichmäfig konvergent. Beweis: wörtlich wie S. 322 .

3. Ist $r$ der genaue Konvergenzradius von $\Sigma a_{n}\left(z-z_{0}\right)^{n}$, so braucht die Reihe in dem Kreise $\left|z-z_{0}\right|<r$ nicht gleichmäfig zu konvergieren. Beispiel: Die geometrische Reihe; Beweis: wie S. 323.

4. Genau wie damals erkennt man, dafs unsere Definition mit der folgenden völlig gleichen Inhalt hat:

3. Form. $\sum f_{n}(z)$ heißt in $\left|z-z_{0}\right| \leq \varrho$ (oder in der Punktmenge $\mathfrak{M}$ ) gleichmäßig konvergent, wenn nach Wahl einer ganz beliebigen, diesem Kreise (oder dieser Punktmenge) entnommenen Zahlenfolge $\left(z_{n}\right)$ die zugehörigen Reste $r_{n}\left(z_{n}\right)$ stets eine Nullfolge bilden.

Auch die 4. und 5. Form der Definition (S. 324) bleiben völlig ungeändert, und es erübrigt sich darum wohl, sie hier besonders $\mathrm{zu}$ formulieren.

Dagegen ist eine so eindrucksvolle Veranschaulichung der gleichmäßigen bzw. ungleichmäßigen Konvergenz einer Reihe wie im Reellen hier leider nicht möglich.

Nunmehr können wir schon den angedeuteten Hauptsatz formulieren und beweisen:

Weierstraßscher Doppelreihensatz $\left.{ }^{2}\right)$ : Es sei die Reihe

$$
\sum_{k=0}^{\infty} f_{k}(z)
$$

1) Diese Definition entspricht der damaligen 2. Form. Die 1. Form 191 kann hier forgelassen werden, da sie uns nur für die Einführung, nicht für den Gebrauch des Begriffs der gleichmäfigen Konvergenz wesentlich schien.

2) Werke, Bd. 1, S. 70. Der Beweis stammt aus dem Jahre 1841. 
vorgelegt. Jedes ihrer Glieder $f_{k}(z)$ sei mindestens für $\left|z-z_{0}\right|<r$ anulytisch, so daß die Entwicklungen ${ }^{1}$ )

$$
\begin{aligned}
& f_{0}(z)=a_{0}^{(0)}+a_{1}^{(0)}\left(z-z_{0}\right)+\ldots+a_{n}^{(0)}\left(z-z_{0}\right)^{n}+\ldots \\
& f_{1}(z)=a_{0}^{(1)}+a_{1}^{(1)}\left(z-z_{0}\right)+\ldots+a_{n}^{(1)}\left(z-z_{0}\right)^{n}+\ldots \\
& f_{k}(z)=a_{0}^{(k)}+a_{1}^{(k)}\left(z-z_{0}\right)+\ldots+a_{n}^{(k)}\left(z-z_{0}\right)^{n}+\ldots
\end{aligned}
$$

existieren und sämtlich mindestens für $\left|z-z_{0}\right|<r$ konvergieren. Endlich sei die Reihe $\Sigma f_{k}(z)$ gleichmäßig konvergent im Kreise $\left|z-z_{0}\right| \leqq \varrho$ und zwar für $j e d e s \varrho<r$, so da $\beta$ die Reihe inshesondere überall im Kreise $\left|z-z_{0}\right|<r$ konvergiert und dort eine wohlbestimmte Funktion $F(z)$ darstellt. Dann läßt sich zeigen:

1. Die spaltenweis untereinanderstehenden Koeffizienten liefern konvergente Reihen:

$$
\sum_{k=0}^{\infty} a_{n}^{(k)}=A_{n}, \quad(n \text { fest },=0,1,2 \ldots) .
$$

2. $\sum_{n=0}^{\infty} A_{n}\left(z-z_{0}\right)^{n}$ ist für $\left|z-z_{0}\right|<r$ konvergent.

3. Es ist für $\left|z-z_{0}\right|<r$

$$
\sum_{n=0}^{\infty} A_{n}\left(z-z_{0}\right)^{n}=\sum_{k=0}^{\infty} f_{k}(z)=F(z) \text {. }
$$

4. Für $\left|z-z_{0}\right|<r$ und für jedes $v=1,2, \ldots$ ist

$$
F^{(v)}(z)=\sum_{k=0}^{\infty} f_{k}^{(v)}(z) \text {. }
$$

\section{Bemerkungen.}

1. In etwas unschärfere Worte gekleidet besagt der Satz in erster Linie: Eine gleichmäßig konvergente Reihe analytischer Funktionen stellt wieder eine analytische Funktion dar. Sodann: Unter den im Satz genauer präzisierten Voraussetzungen "darf" man unendlich viele Potenzreihen gliedweis addieren. Endlich: Eine gleichmä6ig konvergente Reihe analytischer Funktionen "darf" man beliebig oft gliedweis differenzieren.

2. Die Voraussetzung, $\Sigma f_{k}(z)$ solle für jedes $\varrho<r$ in $\left|z-z_{0}\right|<r$ gleichmäbig konvergieren, ist z. B. für jede Potenzreihe $\Sigma c_{n}\left(z-z_{0}\right)^{n}$ erfüllt, die den Konvergenzradius $r$ hat. Ebenso auch z. B. für die Reihe $\sum \frac{z^{n}}{1-z^{n}}$ mit $r=1$; vgl. dazu $\$ 58, \mathrm{C}$.

3. Die erste unserer 4 Behauptungen zeigt, daf der jetzige Satz nicht etwa einfach als Anwendung der Markofschen Reihentransformationen bewiesen werden kann; denn bei dieser wurde die Konvergenz der Spalten vorausgesetzt, hier wird sie aus den übrigen Voraussetzungen gefolgert.

1) Der obere Index an den Koeffizienten $a_{n}^{(k)}$ gibt also die Nummer der Funktion in der vorgelegten Reihe, der untere seine Stellung in der Entwicklung derselben an. 
Beweis. 1. Es werde ein positives $\varrho<r$ festgelegt und $\varepsilon^{\prime}>0$ gewählt. Dann gibt es nach Voraussetzung ein $k_{0}$, so daß für alle $k^{\prime}>k>k_{0}$ stets

$$
\left|s_{k^{\prime}}-s_{k}\right|<\varepsilon^{\prime}
$$

bleibt. Hierbei soll $s_{k}=s_{k}(z)=f_{0}(z)+\ldots+f_{k}(z)$ gesetzt sein. Die Funktion $s_{k^{\prime}}(z)-s_{k}(z)$ ist aber eine bestimmte Potenzreihe, deren $n^{\text {ter }}$ Koeffizient

$$
=a_{n}^{(k+1)}+a_{n}^{(k+2)}+\ldots+a_{n}^{\left(k^{\prime}\right)}
$$

ist. Nach der Cauchyschen Abschätzungsformel $\mathbf{2 3 5}$ gilt für diesen die Abschätzung

$$
a_{n}^{(k+1)}+a_{n}^{(k+2)}+\ldots+a_{n}^{\left(k^{\prime}\right)} \mid<\frac{\varepsilon^{\prime}}{\varrho^{n}} \quad(n \text { fest }) .
$$

Da hierin $\varrho$ und $n$ fest gewählte Zahlen sind, so steht rechterhand eine Zahl $\varepsilon$, die durch passende Wahl von $\varepsilon^{\prime}$ jeder positiven Zahl gleich gemacht werden $k^{1} n^{1}$ ). Nach 81 ist also - immer bei festem $n$ - die Reihe

$$
a_{n}^{(0)}+a_{n}^{(1)}+\ldots+a_{n}^{(k)}+\ldots \equiv \sum_{k=0}^{\infty} a_{n}^{(k)}
$$

konvergent. Ihre Summe heiße $A_{n}$.

2. Es sei jetzt $M^{\prime}$ das Maximum von $\left|s_{k_{0}+1}(z)\right|$ längs der Peripherie $\left|z-z_{0}\right|=\varrho^{2}$ ). Dann ist für alle $k>k_{0}$ längs dieser selben Peripherie

$$
\left|s_{k}(z)\right| \leqq\left|s_{k_{0}+1}(z)\right|+\left|s_{k}(z)-s_{k_{0}+1}(z)\right| \leqq M^{\prime}+\varepsilon^{\prime}=M .
$$

Nach der Cauchyschen Abschätzungsformel ist also für jedes feste $n=0,1,2, \ldots$

$$
\left|a_{n}^{(0)}+a_{n}^{(1)}+\ldots+a_{n}^{(k)}\right| \leqq \frac{M}{\varrho^{n}},
$$

und dies ohne Rücksicht auf den Wert von $k$. Daher ist auch

$$
\left|A_{n}\right| \leqq \frac{M}{\varrho^{n}}
$$

und folglich $\sum_{n=0}^{\infty} A_{n}\left(z-z_{0}\right)^{n}$ für $\left|z-z_{0}\right|<\varrho$ konvergent. Da aber $\varrho$ nur der Beschränkung $<r$ zu sein unterworfen war, so muß diese Reihe sogar für $\left|z-z_{0}\right|<r$ konvergieren. (Denn wenn $z$ ein $b e$ -

1) Oder: Es sei $\varepsilon>0$ gegeben. Wird dann das positive $\varrho<r$ und die ganze Zahl $n \geq 0$ irgendwie gewählt und nun $\varepsilon^{\prime}=\varepsilon \cdot \varrho^{n}$ gesetzt, so kann $k_{0}$ wie oben gewählt werden, und es ist dann für $k^{\prime}>k>k_{0}$ stets $\left|a_{n}^{(k+1)}+\ldots+a_{n}^{\left(k^{\prime}\right)}\right|<\varepsilon$, was nach 81 die Konvergenz von $\Sigma a_{n}^{(k)}$ für das gewählte $n$ und somit, da dieses beliebig genommen werden durfte, für jedes feste $n \geqq 0$ zur Folge hat.

2) $\left|s_{k_{0}+1}(z)\right|$ ist längs der genannten Peripherie stetig und besitzt dort also ein wohlbestimmtes Maximum. 
stimmter der Bedingung $\left|z-z_{0}\right|<r$ genügender Punkt ist, so kann man sich das $\varrho$ stets so genommen denken, daß auch noch $\left|z-z_{0}\right|<\varrho<r$ ist). Die durch sie dargestellte Funktion heiße für den Augenblick $F_{1}(z)$; sie ist der Definition gemäß eine in $\left|z-z_{0}\right|<r$ analytische Funktion.

3. Daß nun $F_{1}(z) \equiv F(z)$ und insbesondere also auch $F(z)$ eine analytische Funktion ist, erkennt man so: Der $n^{\text {te }}$ Koeffizient der Potenzreihe $F_{1}(z)-\sum_{v=0}^{k} f_{p}(z)$ ist ersichtlich

$$
=A_{n}-\left(a_{n}^{(0)}+a_{n}^{(1)}+\ldots+a_{n}^{(k)}\right), \quad(n=0,1,2, \ldots),
$$

also nach der Abschätzung (a) für $k>k_{0}$ seinem Betrage nach $\leqq \frac{\varepsilon^{\prime}}{\varrho^{n}}$. Daher ist für $k>k_{0}$ und $\left|z-z_{0}\right|<\varrho$

$$
\left|F_{1}(z)-\sum_{v^{\prime}=0}^{k} f_{v}(z)\right| \leqq \varepsilon^{\prime} \cdot\left(1+\frac{\left|z-z_{0}\right|}{\varrho}+\frac{\left|z-z_{0}\right|^{2}}{\varrho^{2}}+\ldots\right) ;
$$

dies ist aber für alle $\left|z-z_{0}\right| \leqq \varrho^{\prime}<\varrho$ seinerseits

$$
\leqq \varepsilon^{\prime}\left(1+\frac{\varrho^{\prime}}{\varrho}+\frac{\varrho^{\prime 2}}{\varrho^{2}}+\ldots\right)=\varepsilon^{\prime} \cdot \frac{\varrho}{\varrho-\varrho^{\prime}} .
$$

Da dieser letzte Wert - bei festem $\varrho^{\prime}<\varrho<r$ - durch passende Annahme von $\varepsilon^{\prime}$ jeder positiven Zahl $\varepsilon$ gleich gemacht werden kann, so ist nach der letzten Abschätzung für alle $\left|z-z_{0}\right| \leqq \varrho^{\prime}$

$$
F_{1}(z)=\stackrel{\Perp}{r=0}_{r=0}^{\infty} f_{v}(z), \quad \text { also }=F(z),
$$

Da aber $\varrho^{\prime}$ und $\varrho$ nur der Bedingung $\varrho^{\prime}<\varrho<r$ unterworfen waren, so muß (s. o.) diese Gleichung für jedes $z$ des Kreises $\left|z-z_{0}\right|<r$ richtig sein.

4. Auch in dem Schema

$$
\begin{array}{r}
f_{0}^{\prime}(z)=a_{1}^{(0)}+2 a_{2}^{(0)}\left(z-z_{0}\right)+3 a_{3}^{(0)}\left(z-z_{0}\right)^{2}+\ldots \\
f_{1}^{\prime}(z)=a_{1}^{(1)}+2 a_{2}^{(1)}\left(z-z_{0}\right)+3 a_{3}^{(1)}\left(z-z_{0}\right)^{2}+\ldots \\
\cdot \cdot \cdot \cdot \cdot \cdot \cdot \cdot \cdot \cdot \cdot \cdot \cdot \cdot \cdot \cdot \cdot \cdot \cdot \cdot \\
A_{1}+2 A_{2}\left(z-z_{0}\right)+3 A_{3}\left(z-z_{0}\right)^{2}+\ldots
\end{array}
$$

konvergieren die spaltenweis untereinanderstehenden Koeffizienten gegen die darunterstehenden Werte und ganz ähnlich wie unter 3 . ist hiernach für $\left|z-z_{0}\right| \leqq \varrho^{\prime}<\varrho<r$ und alle $k>k_{0}$

$$
F^{\prime}(z)-\sum_{\nu=0}^{k} f_{\nu}^{\prime}(z) \leq \varepsilon^{\prime}\left[1+2 \frac{\varrho^{\prime}}{\varrho}+3 \frac{\varrho^{\prime 2}}{\varrho^{2}}+\ldots\right]=\varepsilon^{\prime} \cdot \frac{\varrho^{2}}{\left(\varrho-\varrho^{\prime}\right)^{2}} .
$$

Also ist dort - und aus denselben Gründen wie soeben sogar für alle $\left|z-z_{0}\right|<r-$

$$
F^{\prime}(z)=\sum_{k=0}^{x} f_{k}^{\prime}(z)
$$


Macht man den entsprechenden Ansatz mit den $\boldsymbol{v}^{\text {ten }}$ Ableitungen, so erhält man ganz ebenso, daß für $\left|z-z_{0}\right|<r$

$$
F^{(v)}(z)=\sum_{k=0}^{\infty} f_{k}^{(v)}(z) \quad(v=1,2, \ldots \text { fest })
$$

ist, d. h. daß die durch gliedweise $v$-malige Differentiation gewonnene Reihe $\sum f_{k}^{(\nu)}(z)$ in dem ganzen Kreise $\left|z-z_{0}\right|<r$ konvergiert und dort die $\nu^{\text {te }}$ Ableitung von $F(z)$ liefert.

\section{Bemerkungen.}

1. Der Ausführung einiger besonders wichtiger Beispiele ist der übernächste Paragraph gewidmet.

2. Daf die Gleichmäßjigkeit der Konvergenz gerade für ein Kreisgebiet vorausgesetzt wurde, ist für den wesentlichsten Teil des Satzes gleichgiiltig: Sind die Glieder der Reihe $\Sigma f_{k}(z)$ in einem irgendwie geformten Gebiete ${ }^{1}$ ) analytisch, und läßst sich um jeden Punlit $z_{0}$ desselben ein Kreis $\left|z-z_{0}\right| \leqq \varrho$ so angeben, dafi er auch noch zum Gebiete gehört und die Reihe in ihm gieichmäßig konvergiert, so stellt sie ebenfalls eine in dem betreffenden Gebiet analytische Funktion $F(z)$ dar, deren Ableitungen durch gliedweise Differentiation gewonnen werden können. - Auch hierfür werden in $\S 58$ Beispiele ausgefuihrt werden.

\section{§5\%. Produkte mit komplexen Gliedern.}

Wir haben die Ausführungen im VII. Kapitel so gehalten, daß alle Definitionen und Sätze, die sich auf Produkte mit „beliebigen“ Gliedern beziehen, unverändert ihre Gültigkeit behalten, wenn die Faktoren kmmp'exe Werte haben dürfen. Insbesondere bleiben also die Definition 125 der Konvergenz und die anschließenden Sätze 1, 2 und 5 einschließlich des Beweises völlig ungeändert bestehen. Auch bei der Definition 128 der absoluten Konvergenz und den anschließenden Sätzen 6 und 7 ist nichts zu ändern. Ein Zweifel bezüglich der wörtlichen Übernahme ins Komplexe könnte dagegen bei Satz 8 aufkommen. Doch kann auch hier alles „komplex gelesen“ werden, wenn wir nur übereinkommen, unter $\log \left(1+a_{n}\right)$ den Haupiwert des Logarithmus zu verstehen, sobald $n$ hinreichend groß 3 ist. Da man hier etwas vorsichtig schiießen muß, führen wir den Beweis aus:

Satz. Das Produkt II $\left(1+a_{n}\right)$ ist dann unt nur dann konvergent, $\mathbf{2 5 0}$. wenn die Glieder $a_{n}$ eine Nullfslge bilden und wenn die hinter einem passenden Index $m$ begonnene Reihe

$$
\stackrel{\infty}{n=m+1}_{n=0}^{\infty} \log \left(1+a_{n}\right)
$$

1) Auf eine strenge Definition eines Gebietes kommt es hier nicht an. Im folgenden handelt es sich dabei immer nur um das Innere von Flächenstücken, die von endlich vielen Geraden oder Kreisbögen begrenzt werden, insbesondere um Kreise und Halbebenen. 
deren Glieder die Hauptwerte von $\log \left(1+a_{n}\right)$ sein sollen, konvergiert. Hat sie die Summe L, so ist überdies

$$
\prod_{n=1}^{\infty}\left(1+a_{n}\right)=\left(1+a_{1}\right)\left(1+a_{2}\right) \ldots\left(1+a_{m}\right) \cdot e^{L} .
$$

Beweis. a) Die Bedingungen sind hinreichend. Denn ist für $n>m$ stets $\left|a_{n}\right|<\frac{1}{2}$ und ist die mit den Hauptwerten angesetzte Reihe $\sum_{n=m+1}^{\infty} \log \left(1+a_{n}\right)$ konvergent, so streben ihre Teilsummen $s_{n}$, $n>m$ ), gegen einen bestimmten Grenzwert $L$ und folglich (s. $\$ 55$, II und 42,1 )

$$
e^{s_{n}}=\left(1+a_{m+1}\right)\left(1+a_{m+2}\right) \ldots\left(1+a_{n}\right) \rightarrow e^{L},
$$

also insbesondere gegen einen Wert, der $\neq 0$ ist. Also ist das Produkt gemäß Definition 125 konvergent. Und daß sein Wert der angegebene ist, ist nun auch unmittelbar zu sehen.

b) Die Bedingungen sind notwendig. Denn ist das Produkt konvergent, so kann nach Wahl eines positiven $\varepsilon$, das wir uns $<1$ denken, $n_{0}$ so bestimmt werden, daß für alle $n \geqq n_{0}$ und alle $k \geqq 1$ stets

$$
\left(1+a_{n+1}\right)\left(1+a_{n+2}\right) \ldots\left(1+a_{n+k}\right)-1,<\frac{\varepsilon}{2}
$$

bleibt. Dann ist insbesondere für alle $n>n_{0}$ stets $\left|a_{n}\right|<\frac{\varepsilon}{2}<\frac{1}{2}$ und die Ungleichung $\left|a_{n}\right|<\frac{1}{2}$ also gewiß von einer passenden Stelle $m\left(\leqq n_{0}\right)$ an stets erfüllt. Weiter folgt aber, daß bei Benutzung der Hauptwerte des Logarithmus $^{1}$ ) für dieselben $n$ und $k$ stets

$$
\sum_{y=n+1}^{n+k} \log \left(1+a_{v}\right) \mid<\varepsilon
$$

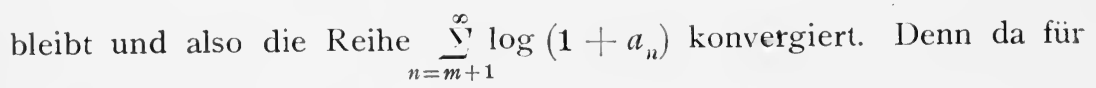
$v>n_{0}$ stets $\left|a_{v}\right|<\frac{\varepsilon}{2}$ war, so ist für diese $v$ auch

$$
\left.\log \left(1+a_{v}\right) \mid<\varepsilon^{2}\right)
$$

und ebenso für alle $n \geqq n_{0}$ und alle $k \geqq 1$

$$
\left|\log \left[\left(1+a_{n+1}\right) \ldots\left(1+a_{n+k}\right)\right]\right|<\varepsilon .
$$

Folglich ist für eine jedesmal passende ganze Zahl $q^{3}$ )

(c) $\left|\log \left(1+a_{n+1}\right)+\log \left(1+a_{n+2}\right)+\ldots+\log \left(1+a_{n+k}\right)+2 q \pi i\right|<\varepsilon$,

1) Die Logarithmen sollen im folgenden stets die Hauptwerte bedeuten.

2) Denn für $|z|<\frac{1}{2}$ ist

$|\log (1+z) \leqq| z\left|+\frac{|z|^{2}}{2} \cdots \leq z\right|+\left|z^{2}+\ldots=\frac{|z|}{1-|z|} \leq 2\right| z \mid$.

3) Denn der Hauptwert des Logarithmus eines Produktes braucht nicht gleich der Summe der Hauptwerte der Logarithmen der Faktoren zu sein, 
und es bleibt nur zu zeigen, daß $q$ immer $=0$ genommen werden muß. Halten wir ein bestimmtes $n \geqq n_{0}$ fest, so ist dies aber für $k=1$ nach (b) gewiß der Fall. Dann muß es aber auch für $k=2$ so sein. Denn da in

$$
\log \left(1+a_{n+1}\right)+\log \left(1+a_{n+2}\right)+2 q \pi i
$$

jeder der beiden ersten Summanden nach (b) seinem Betrage nach $<\varepsilon$ ist und nach (c) auch der Betrag der ganzen Summe $<\varepsilon$ sein soll, so kann $q$ nicht eine von 0 verschiedene ganze Zahl sein. Aus den entsprechenden Gründen folgt nun ebenso, da $\beta$ auch bei $k=3$ die ganze Zahl $q=0$ sein muß, und man bestätigt dies für jedes $k$ dann leicht durch Induktion. Damit ist alles bewiesen. daß also

Auch der auf die absolute Konvergenz bezügliche Teil des Satzes 8,

$$
\text { die Reihe } \sum_{n=m+1}^{\infty} \log \left(1+a_{n}\right) \text { und das Produkt } \prod_{n=m+1}^{\infty}\left(1+a_{n}\right)
$$

stets gleichzeitig absolut oder nicht absolut konvergieren, läßt sich ganz unmittelbar ins Komplexe übertragen und ebenso bleiben die Sätze $9-11$ in $\S 29$ und 30 gültig, denn auch für komplexe $a_{n}$, die ihrem Betrage nach $<\frac{1}{2}$ sind, bleibt die Tatsache bestehen, daß in

$$
\log \left(1+a_{n}\right)=a_{n}+\vartheta_{n} \cdot a_{n}{ }^{2}
$$

die $\vartheta_{n}$ eine beschränkte Zahlenfolge bilden, - denn es ist für $|z|<\frac{1}{2}$

$$
\log (1+z)=z+\left[-\frac{1}{2}+\frac{z}{3}-\frac{z^{2}}{4}+\ldots\right] \cdot z^{2},
$$

und hierin bleibt für die genannten $z$ der Betrag der eckigen Klammer ersichtlich $<1$.

Endlich bleiben auch die Bemerkungen über den allgemeinen Zusammenhang zwischen Reihen und Produkten — weil rein formaler Natur — ungeändert bestehen.

\section{Beispiele.}

1. $\Pi\left(1+\frac{i}{n}\right)$ ist divergent, denn da $\Sigma\left|a_{n}\right|^{2} \equiv \Sigma \frac{1}{n^{2}}$ konvergiert, so ver- $25 \mathbf{1}$. halten sich nach $\$ 29$, Satz 10 , die Teilprodukte

$$
p_{n}=\left(1+\frac{i}{1}\right)\left(1+\frac{i}{2}\right) \ldots\left(1+\frac{i}{n}\right) \sim e^{i\left(1+\frac{1}{2}+\ldots+\frac{1}{n}\right)}
$$

die rechte Seite liefert aber für $n=1,2, \ldots$ eine Punktfolge, die auf der Peripherie des Einheitskreises liegt und diese in immer kleineren Schritten unaufhörlich umkreist. $p_{n}$ strebt also keinem Grenzwert zu. (Vgl. S. 411.)

sondern diese kann sich um ein Vielfaches von $2 \pi i$ von jenem unterscheiden. So ist z. B. $\log i=\frac{\pi i}{2}$, aber $\log (i \cdot i \cdot i \cdot i)=\log 1=0$, wenn uberall die Hauptwerte genommen werden. 
2. $\prod_{n=0}^{\infty} \frac{n(n+1)+(1+i)}{n(n+1)+(1-i)}=-1$, denn man findet für das $n^{\text {te Teilprodukt }}$ sofort den Wert $\frac{1+(n+1) i}{1-(n+1) i}$, was $\rightarrow-1$ strebt.

3. Für ein $|z|<1$ ist $\prod_{n=0}^{\infty}\left(1+z^{2^{n}}\right)=\frac{1}{1-z}$, denn die (absolute) Konvergenz des Produktes steht nach 127, 7 außer Frage und für das $n^{\text {te }}$ Teilprodukt hat man

was $\rightarrow 1$ strebt.

$$
(1-z) \cdot(1+z)\left(1+z^{2}\right)\left(1+z^{4}\right) \ldots\left(1+z^{2^{n}}\right)=1-z^{2^{n+1}},
$$

Bei Produkten, deren Glieder Funktionen einer komplexen Veränderlichen sind und die also die Form

$$
\prod_{n=1}^{\infty}\left(1+f_{n}(z)\right)
$$

haben, beschränken wir uns, wie im vorigen Paragraphen bei den Reihen, wieder auf den einfachsten, aber auch wichtigsten Fall, daß alle $f_{n}(z)$ in ein und demselben Kreise $\left|z-z_{0}\right|<r$ analytisch sind (d. h. eine dort konvergente Potenzreihenentwicklung besitzen) und daß das Produkt in diesem Kreise konvergiert. Es stellt dann in demselben eine wohlbestimmte Funktion $F(z)$ dar, von der man auch umgekehrt sagt, sie sei in das Produk' entwrckelt worden.

Wir fragen nach brauchbaren Bedingungen, unter denen die durch ein solches Produkt dargestellte Funktion $F(z)$ in dem Kreise $\left|z-z_{0}\right|<r$ sich wieder analytisch verhält. Für die weitaus meisten Anwendungen genügt hier der folgende

252. Satz. Wenn die Funktionen $f_{1}(z), f_{2}(z), \ldots, f_{n}(z), \ldots$ sämtlich mindesiens in dem (fes.en) Kreise $\left|z-z_{0}\right|<r$ analytisch sind, wenn ferner die Reihe

$$
\sum_{n=1}^{\infty}\left|f_{n}(z)\right|
$$

für jetes pasitive $\varrho<r$ in dem k'eineren Krerse $z-z_{0} \mid \leqq \varrho$ gleichmäßig konvergier:, so is auch das Pruk: $\Pi\left(1+f_{n}(z)\right)$ in $\left|z-z_{0}\right|<r$ konvergen' unl s'ellt eine dort wie'er ana'y ische Funkti'n $F(z)$ dar.

Der Beweis geht fast wörtlich wie der Beweis des Stetigkeitssatzes 218, 1. Um nämlich die Konvergenz und den analytischen Charakter des Produktes in einem bestimmten Punkte $z_{1}$ des Kreises $\left|z-z_{0}\right|<r$ zu zeigen, greifen wir ein $\varrho$ heraus, für das $\left|z_{1}-z_{0}\right|<\varrho<r$ ist und zeigen beides gleich für alle $z$ des Kreises $\left|z-z_{0}\right|<\varrho$. Da aber $\Sigma\left|f_{n}(z)\right|$ für alle $\left|z-z_{0}\right| \leqq \varrho$ gleichmäßig konvergiert, so ist das Produkt $I\left(1+f_{n}(z)\right)$ dort gewiß auch konvergent (sogar absolut). Wir wählen nun $m$ so groß, daß für alle $n>m$ und alle $\left|z-z_{0}\right| \leqq \varrho$ stets

$$
\left|f_{m+1}(z)\right|+f_{m+2}(z)+\ldots+f_{n}(z) \mid<1
$$


und also

$$
\left|p_{n}(z)\right|=\left|\left(1+f_{m+1}(z)\right) \ldots\left(1+f_{n}(z)\right)\right| \leqq e^{\left|f_{m+1}(z)\right|+\ldots+\left|f_{n}(z)\right|}<3
$$

bleibt. Genau wie S. 368 ergibt sich nun, daß die Reihe

$$
p_{m+1}+\left(p_{m+2}-p_{m+1}\right)+\ldots+\left(p_{n}-p_{n-1}\right)+\ldots
$$

in $\left|z-z_{0}\right| \leqq \varrho$ g'eichmäßig konvergiert. Und da alle Glieder dieser Reihe in $\left|z-z_{0}\right|<r$ analytisch sind, so stellt sie selbst nach $\mathbf{2 4 9}$ eine in $\left|z-z_{0}\right|<\varrho$ analytische Funktion $F_{m}(z)$ dar. Dann ist aber auch

$$
F(z)=\prod_{n=1}^{\infty}\left(1+f_{n}(z)\right)=\left(1+f_{1}(z)\right) \ldots\left(1+f_{m}(z)\right) \cdot F_{m}(z)
$$

eine in diesem Kreise, also speziell in $z_{1}$ reguläre analytische Funktion. Da aber $z_{1}$ in $\left|z-z_{0}\right|<r$ beliebig gewählt war, so ist $F(z)$ eine in dem ganzen Kreise $\left|z-z_{0}\right|<r$ analytische Funktion, w. z. b. w.

Aus diesen Entwicklungen können wir noch zwei Sätze entnehmen, der uns ein Analogon zum Weierstraßschen Doppelreihensatz liefert:

Satz 1. Un'er den Voraussetzungen des vcrigen Satzes kann die $\mathbf{2 5 3 .}$ Potenzreikenentwicklvng von $F(z)$ durch g'iedweise Ausmultipitiation des Produk es gewnnen werden. Oder genauer: $D a f_{1}, f_{2}, \ldots$ sämtlich in Potenzreilen entwickeltar sind, die für $\left|z-z_{0}\right|<r$ konvergieren, so ist dasselbe auch mit dem (endlichen) Produkt

der Fall. Es sei dort etwa

$$
P_{k}(z)=\prod_{v=1}^{k}\left(1+f_{v}(z)\right)
$$

$P_{k}(z)=A_{0}^{(k)}+A_{1}^{(k)} \cdot\left(z-z_{0}\right)+A_{2}^{(k)} \cdot\left(z-z_{0}\right)^{2}+\ldots+A_{n}^{(k)} \cdot\left(z-z_{0}\right)^{n}+\ldots$

Dann existiert für jedes (festgehaltene) $n=0,1,2, \ldots$ der Grenzwert

und es ist

$$
\lim _{k \rightarrow+\infty} A_{n}^{(k)}=A_{n},
$$

$$
F(z)=\prod_{k=1}^{\infty}\left(1+f_{k}(z)\right)=\sum_{n=0}^{\infty} A_{n}\left(z-z_{0}\right)^{n}
$$

Beweis. Mit der beim vorigen Beweise gebrauchten Reihe

ist auch die Reihe

$$
p_{m+1}+\left(p_{m+2}-p_{m+1}\right)+\ldots
$$

$$
P_{1}(z)+\left[P_{2}(z)-P_{1}(z)\right]+\ldots+\left[P_{k}(z)-P_{k-1}(z)\right]+\ldots
$$

in $\left|z-z_{0}\right| \leqq \varrho$ gleichmäßig konvergent $\left.{ }^{1}\right)$. Die Anwendung des Weiers'raßschen Doppelreihensatzes auf diese Reihe gibt aber genau den obigen Satz.

1) Denn ihre Reste unterscheiden sich von denen der vorigen Reihe nur durch den gemeinsamen Faktor $P_{m}(z)$, der für alle $z$ des Kreises $\left|z-z_{0}\right| \leqq \varrho$, als eine in diesem abgeschlossenen Kreise stetige Funktion, beschränkte Werte hat. 
Endlich können wir hier auch einen ganz ähnlichen Satz, wie $\mathbf{2 1 8}, 2$, über die Ableitung von $F(z)$ beweisen:

Satz 2. Für jedes $z$ in $\left|z-z_{0}\right|<r$, für das $F(z) \neq 0$ ist, ist

$$
\frac{F^{\prime}(z)}{F(z)}=\sum_{n=1}^{\infty} \frac{f_{n}^{\prime \prime}(z)}{1+f_{n}^{\prime}(z)},
$$

$d$. $h$. die rechtsstehende Reihe ist für jedes solche $z$ konvergent und liefert den linksstehenden Quotienten, also die logarithmische Ableitung von $F(z)$.

Beweis. Es war die Darstellung

$$
F(z)=P_{1}(z)+\left(P_{2}(z)-P_{1}(z)\right)+\ldots
$$

im Kreise $\left|z-z_{0}\right| \leqq \varrho<r$ gleichmäßig konvergent. Nach 249 ist für alle $\left|z-z_{0}\right|<r$

$$
F^{\prime}(z)=P_{1}^{\prime}(z)+\left(P_{2}^{\prime}(z)-P_{1}^{\prime}(z)\right)+\ldots,
$$

d. h. aber es strebt dort

$$
P_{n}^{\prime}(z) \rightarrow F^{\prime}(z) .
$$

Ist nun in einem speziellen Punkte $F(z) \neq 0$, so sind dort auch alle $P_{n}(z) \neq 0$ und nach $\mathbf{4 1}, 11$ strebt daher

Wegen

$$
\frac{P_{n}{ }^{\prime}(z)}{P_{n}(z)} \rightarrow \frac{F^{\prime}(z)}{F(z)} \text {. }
$$

$$
\frac{P_{n}{ }^{\prime}(z)}{P_{n}(z)}=\frac{\sum_{v=1}^{n}}{1+f_{v}^{\prime}(z)}
$$

ist dies aber genau der Inhalt unserer Behauptung.

Beispiele.

254. 1. Ist $\Sigma a_{n}$ irgendeine absolut konvergente Reihe mit konstanten Gliedern, so stellt das Produkt

$$
\prod_{n=1}^{\infty}\left(1+a_{n} z\right)
$$

nach $\mathbf{2 5 2}$ eine in der ganzen Ebene reguläre Funktion dar. Ihre beständig konvergente Potenzreihenentwicklung lautet nach $\mathbf{2 5 3}$

wenn

$$
1+A_{1} z+A_{2} z^{2}+A_{3} z^{3}+\ldots+A_{k} z^{k}+\ldots
$$

$$
\begin{aligned}
& A_{1}=\sum_{\lambda=1}^{\infty} a_{\lambda_{1}}, \quad A_{2}=\sum_{1 \leqq \lambda_{1}<\lambda_{2}}^{\infty} a_{\lambda_{1}} \cdot a_{\lambda_{2}}, \quad A_{3}=\sum_{1 \leqq \lambda_{1}<\lambda_{2}<\lambda_{3}}^{\infty} a_{\lambda_{1}} \cdot a_{\lambda_{2}} \cdot a_{\lambda_{3}}, \ldots \\
& A_{k}=\sum_{1 \leq \lambda_{1}<\ldots<\lambda_{k}}^{\infty} a_{\lambda_{1}} \cdot a_{\lambda_{2}} \ldots a_{\lambda_{k}}, \ldots
\end{aligned}
$$

gesetzt wird. Hierbei sollen die Indizes $\lambda_{1}, \lambda_{2}, \ldots, \lambda_{k}$ unabhängig voneinander alle natürlichen Zahlen durchlaufen, nur der Bedingung gehorchend, daf $\lambda_{1}<\lambda_{2}<\ldots<\lambda_{k}$ bleibt. Die Existenz der mit $A_{1}, A_{2}, \ldots$ bezeichneten Reihenwerte ist dabei durch den Satz $\mathbf{2 5 3}$ selbst gesichert; auch überzengt man sich 
leicht, dafi es dabei auf die Reihenfolge der Summanden nicht ankommt. Die Anwendung dieses Satzes hat schon Euler ${ }^{1}$ ) und später C.G. J. Jacobi ${ }^{2}$ ) $z u$ einer Fiille der merkwürdigsten Formeln geführt.

2. Es ist

$$
\sin \pi z=\pi \approx \cdot \prod_{n=1}^{\infty}\left(1-\frac{z^{2}}{n^{2}}\right)
$$

und das rechtsstehende Produkt in der ganzen Ebene konvergent. Der Beweis ist wörtlich derselbe wie der $\mathbf{2 1 9}, 1$ für reelle Veränderliche gegebene.

3. Setzt man im vorigen sin-Produkt $z=i$, so erhält man

oder also

$$
\pi i \prod_{n=1}^{\infty}\left(1+\frac{1^{b}}{n^{2}}\right)=\sin \pi i=\frac{e^{-\pi}-e^{\pi}}{2 i},
$$

$$
\prod_{n=1}^{\infty}\left(1+\begin{array}{c}
1 \\
n^{2}
\end{array}\right)=\frac{e^{\pi}-\frac{e^{-\pi}}{2 \pi} .}{2}
$$

(Vgl. dagegen die sehr leichte Berechnung von $\left.\Pi\left(1-\frac{1}{n^{2}}\right), \mathbf{1 2 8}, 6.\right)$

4. Die Folge der Funktionen

$$
g_{n}(z)=\frac{z(z+1)(z+2) \ldots(z+n)}{n ! n^{z}}, \quad n=1,2, \ldots
$$

konvergiert fuir jedes $z$ der ganzen Ebene. In der Tat ist

$$
g_{n}(z)=z\left(1+\begin{array}{c}
z \\
1
\end{array}\right)\left(1+\begin{array}{l}
z \\
2
\end{array}\right) \ldots\left(1+\frac{z}{n}\right) \cdot n^{-z} .
$$

Da aber nach 128, Satz 10

$$
\left(1+\frac{z}{1}\right)\left(1+\frac{z}{2}\right) \ldots\left(1+\begin{array}{c}
z \\
n
\end{array}\right) \sim e^{z\left(1+\frac{1}{2}+\ldots+\frac{1}{n}\right)} \sim e^{z \log n}=n^{z}
$$

ist, so ist damit die Behauptung schon bewiesen. Der Grenzwert, wir wollen ihn $K(z)$ nennen, ist iiberdies dann und nur dann $=0$, wenn $z$ einen der Werte $0,-1,-2, \ldots$ hat. Schliefst man diese Werte aus, so ist für alle iibrigen $z$ auch

$$
\lim _{n \rightarrow+\infty} \frac{1}{g_{n}(z)}=\lim _{n \rightarrow+\infty} \frac{n ! n^{z}}{z(z+1)(z+2) \ldots(z+n)}=\frac{1}{K(z)}=\Gamma(z)
$$

vorhanden. Die durch diesen Grenzwert dargestellte Funktion der komplexen Veränderlichen $z$, welche nur $\neq 0,-1,-2, \ldots$ gehalten werden mufs, ist die sogen. $\Gamma$-Funktion, die wir S. 371 schon für reelle Argumente definiert haben.

Wir wollen noch zeigen, daf $K(z)$ eine in der ganzen Ebene analytische (also ganze) Funktion ist. Dazu genügt es, zu zeigen, daf die Reihe

$$
K(z)=g_{1}(z)+\left(g_{2}(z)-g_{1}(z)\right)+\ldots+\left(g_{n}(z)-g_{n-1}(z)\right)+\ldots
$$

in jedem Kreise $z<\varrho$ gleichmäßig konvergiert. Nun ist aber

$$
g_{n}(z)-g_{n-1}(z)=g_{n-1}(z) \cdot\left[\left(1+\frac{z}{n}\right)\left(1-\frac{1}{n}\right)^{z}-1\right]
$$

1) Introductio in analysin inf., Bd. 1 (1748), Kap. 15.

2) Fundamenta nova, Königsberg 1829. 
und da es eine Konstante $A$ geben muf́, so daf für alle $v=1,2,3, \ldots$ und alle $z \mid<\varrho$ stets $\left|g_{y}(z)\right| \leqq A$ bleibt ${ }^{1}$ ), da ferner (nach S. 275 Fufinote)

$$
\left(1-\frac{1}{n}\right)^{z}=1-\frac{z}{n}+\frac{\vartheta_{n}(z)}{n^{2}}
$$

gesetzt werden kann und hierbei $\vartheta_{n}(z)$ für alle genannten $z$ und alle $n=2,3, \ldots$ unterhalb einer festen Konstanten $B$ bleibt, so ist für alle diese $z$ und $n$

$$
\left|g_{n}(z)-g_{n-1}(z)\right| \leqq A \cdot\left|-\frac{z^{2}}{n^{2}}+\frac{\vartheta_{n}(z)}{n^{2}}+\frac{z \cdot \vartheta_{n}(z)}{n^{3}}\right| \leqq \frac{C}{n^{2}},
$$

wenn unter $C$ eine passende Konstante verstanden w rd. Nach 197 folgt hieraus aber die gleichmäßige Konvergenz der Reihe für $K(z)$ im Kreise $|z| \leqq \varrho$ und nach $\mathbf{2 4 9}$ auch der analytische Charakter von $K(z)$ in der ganzen Ebene.

1) Es sei $|z| \leq \varrho$ und $n>m \geq 2 \varrho$. Dann ist

$$
\begin{aligned}
g_{n}(z) & =z\left(1+\frac{z}{1}\right) \ldots\left(1+\frac{z}{m}\right) \cdot\left(1+\frac{z}{m+1}\right) \cdots\left(1+\frac{z}{n}\right) \cdot n^{-z} \\
& =z\left(1+\frac{z}{1}\right) \cdots\left(1+\frac{z}{m}\right) \cdot e^{z\left(\frac{1}{m+1}+\ldots+\frac{1}{n}-\log n\right)} \cdot e^{\frac{\eta m+1}{(m+1)^{2}}+\ldots+\frac{\eta_{n}}{n^{2}}},
\end{aligned}
$$

wenn $\log \left(1+\frac{z}{v}\right)=\frac{z}{v}+\frac{\eta_{v}}{v^{2}}$ gesetzt wird. Da nun hierin wegen $\left|\frac{z}{v}\right|<\frac{1}{2}$ (vgl. S. 419) stets $\left|\eta_{\boldsymbol{v}}\right| \leqq \mid z^{2} \leqq \varrho^{2}$ ist, so bleibt der letzte Faktor stets (d. h. für alle $|z| \leqq \varrho$ und alle $n>m)<e^{\varrho^{2} \cdot \frac{\pi^{2}}{6}}=A_{3}$, ebenso bleibt der zweite Faktor (s. S. 287) stets unter einer festen Schranke $A_{2}$. Und da auch der erste Faktor stets unterhalb einer festen Schranke $A_{1}$ bleibt, so bleibt für alle $|z| \leq \varrho$ und alle $n>m$ auch stets $\mid g_{n}(z) \leqq A_{1} \cdot A_{2} \cdot A_{3}$. Da nun die Funktionen $\left|g_{1}(z)\right|, \ldots$, $\left|g_{m}(z)\right|$ für alle $|z| \leqq \varrho$ ihrerseits beschränkt bleiben, so ist die oben behauptete Existenz von $A$ gesichert.

Wird $z$ auf einen Kreis $\Re$ beschränkt, in dessen Innerm und auf dessen Rande $z \neq 0,-1,-2, \ldots$ und überdies $|z| \leq \varrho$ ist, so ist für $n>m$

$$
\frac{1}{g_{n}(z)}=\frac{1}{z\left(1+\frac{z}{1}\right) \ldots\left(1+\frac{z}{m}\right)} \cdot e^{-z\left(\frac{1}{m+1}+\ldots+\frac{1}{n}-\log n\right)} \cdot e^{-\frac{\eta m+1}{(m+1)^{2}}-\ldots-\frac{\eta n}{n^{2}}} .
$$

Und hieraus liest man genau ebenso ab, daf auch eine Konstante $A^{\prime}$ existiert, so daf in $\Re$ stets $\left|\frac{1}{g_{n}(z)}\right|<A^{\prime}$ bleibt für alle $n=1,2, \ldots$ 


\section{$\S$ 58. Spezielle Klassen von Reihen analytischer Funktionen.}

\section{A. Dirichletsche Reihen.}

Unter einer Dirichletsch, $n$ Reihe versteht man eine Reihe der Form

$$
\left.\sum_{n=1}^{\infty} \frac{a_{n}}{n^{2}} 1\right)
$$

Hier sind die Reihenglieder - als Exponentialfunktionen - in der ganzen Ebene analytisch. Es wird sich also hauptsächlich darum handeln, festzustellen, ob und wo die Reihe konvergiert, bzw. gleichmäßig konvergiert. Hierüber gilt der

Satz 1. Jeder Dirichletsch $\in n$ Reihe entspricht tine reelle Zahl $\lambda$ - $\mathbf{2 5 5 .}$ die scgen. Konvergenzabszisse der Reihe - mit der Eigenschaft, daß die Reihe konvergiert oder divergiert, je nachdem

$$
\Re(z)=\lambda \text { oder } \Re(z)<\lambda
$$

ist. Dabei kann auch $\lambda=-\sim$ oder $=+\infty$ sein, in welchen Fällen die Rtihe beständig baw. nirgends kcnvergicrt. Licgt dsr letatere Fall nicht vor und ist $\lambda^{\prime}>\lambda$, so ist die Reihe scgar in jedem Kreise gleichmäßig kcnvergent, der in der Halbebene $\Re(z) \geqq \hat{\lambda}^{\prime}$ liegt, und sie stellt also nach dem Weiersiraßschtn Satze $\mathbf{2 4 9}$ eine in der ganzen Halbebene $\Re(z)>\lambda$ reguläre analylische Funktion dar $\left.{ }^{2}\right)$.

Der Beweis geht ähnlich vor wie bei den Potenzreihen (vgl. 93). Wir zeigen zunächst, daß, wenn die Reihe im Punkte $z_{0}$ konvergiert, sie auch in jedem Punkte konvergieren muß, für den $\Re(z)>\Re\left(z_{0}\right)$ ist. Da aber

$$
\stackrel{1}{a_{n}} n^{z}=\stackrel{a_{n}}{n^{z_{0}}} \cdot 1
$$

ist, so genügt es, nach 184, 3 zu zeigen, daß die Reihe

$$
\sum_{n=1}^{\infty}\left|\frac{1}{n^{z-z_{0}}}-\begin{array}{c}
1 \\
(n+1)^{z-z_{0}}
\end{array}\right|-\frac{\sum_{n=1}^{\infty}}{1}(n+1)^{3 i\left(z-z_{0}\right)} \cdots\left(1+\frac{1}{n}\right)^{z-z_{j}}-1
$$

konvergiert. Setzt man aber (bei festem Exponenten)

$$
\left(1+\frac{1}{n}\right)^{z-z_{0}}=1+\frac{\vartheta_{n}}{n}
$$

1) Oder allgemeiner eine Reihe der form $\sum_{p_{n}{ }^{2}}^{a_{n}}$ oder der Form $\sum a_{n} e^{-i_{n} z}$, in der die $p_{n}$ positive, die $\lambda_{n}$ beliebige reelle monoton gegen $+\infty$ wachsende Zahlen bedeuten.

$\left.{ }^{2}\right)$ Die Existenz der Konvergenzhalbebenen bewies J. L.W.V. Jensen (Tidskrift for Mathematik, Bd. (5) 2 (1884), S. 63); die Gleichmäß̈igkeit der Konvergenz und damit den analytischen Charakter der dargestellten Funktion zeigte E. Cahen (Annales Éc. Norm. sup., Bd. (3) 11 (1894), S. 75). 
so bedeuten die $\vartheta_{n}$ eine jedenfalls beschränkte Zahlenfolge, - denn sie streben, wie man sofort erkennt $\left.\rightarrow\left(z-z_{0}\right)^{1}\right)$. Ist etwa stets $\left|\vartheta_{n}\right|<A$, so sind die Glieder der letzten Reihe

$$
<\frac{A}{n^{1+\Re\left(z-z_{0}\right)}} \text {. }
$$

Wenn also $\Re\left(z-z_{\mathbf{0}}\right)>0$ ist, so ist sie tatsächlich konvergent.

Durch Umkehrung folgt aus diesem Satze: Wenn eine Dirichlet. sche Reihe für $z=z_{1}$ divergiert, so divergiert sie auch in jedem Punkte mit kleinerem reellen Teil. Und wenn nun die vorgelegte Dirichletsche Reihe weder überall noch nirgends konvergiert, so ergibt sich die Existenz der Grenzabszisse $\lambda$ (ähnlich wie bei 93) folgendermaßen: Ist $z^{\prime}$ ein Divergenzpunkt und $z^{\prime \prime}$ ein Konvergenzpunkt der Reihe, so wähle man eine reelle Zahl $x_{1}<\mathfrak{h}\left(z^{\prime}\right)$ und eine zweite reelle Zahl $y_{0}>\Re\left(z^{\prime \prime}\right)$. Dann wird die Reihe für $z=x_{0}$ wieder divergieren, für $z=y_{0}$ konvergieren. Auf das auf der reellen Achse gelegene Intervall $J_{0}=x_{0} \ldots y_{0}$ wende man nun wörtlich wie in $\mathbf{9 3}$ die Halbierungsmethode an, - der dadurch erfaßte innerste Wert $\lambda$ wird die gesuchte Grenzabszisse sein.

Ist nun weiter $\lambda^{\prime}>\lambda$ (für $\lambda=-\alpha$ darf also $\hat{\lambda}^{\prime}$ irgendeine reelle Zahl sein) und wird $z$ auf ein Gebiet $G$ beschränkt, in $\operatorname{dem} \Re(z) \geqq \lambda^{\prime}$ und $|z| \leqq R$ ist $-G$ wird also im allgemeinen die Form eines Kreissegmentes haben —, so ist unsere Reihe dort sogar gleichmäßig konvergent. Wählen wir nämlich, um dies zu zeigen, einen Punkt $z_{0}$, für den $\lambda<\Re\left(z_{0}\right)<\hat{\lambda}^{\prime}$ ist, so ist zunächst wieder

$$
\geq \frac{a_{n}}{n^{z}}=\geq \frac{a_{n}}{n^{z_{0}}} \cdot \frac{1}{n^{z-z_{0}}} \text {. }
$$

1) Allgemeiner bemerken wir gleich: Ist $|z| \leq \frac{1}{2}$ und $w^{w}<R$, und setzt man den Hauptwert von

$$
(1+z)^{w}=1+z w+\vartheta \cdot z^{2},
$$

so bleibt der Faktor $\vartheta$, welcher von $z$ und $w$ abhängt, für alle zugelassenen $z$ und $w$ unterhalb einer festen Konstanten. - Beweis. Es ist

$$
(1+z)^{w}=e^{w \log (1+z)}-e^{w\left(z+\eta \cdot z^{2} \downarrow\right.} \text { mit } \eta=-\frac{1}{2}+\frac{z}{3}-\frac{z^{2}}{4}+\ldots
$$

Für alle $|z| \leq_{2}^{1}$ ist also $|\eta| \leq 1$ und in

$$
\begin{gathered}
e^{w\left(z+\eta z^{2}\right)}=1+w z(1+\eta z)+\frac{w^{2} z^{2}(1+\eta z)^{2}}{2 !}+\ldots \\
=1+w z+\left[w \eta+\frac{w^{2}(1+\eta z)^{2}}{2 !}+\frac{z w^{3}(1+\eta z)^{3}}{3 !}+\ldots\right] \cdot z^{2}
\end{gathered}
$$

ist daher der Wert der vorhin mit $\vartheta$ bezeichneten eckigen Klammer

$$
|\vartheta| \leqq e^{2 R}
$$

wie man sofort sieht, wenn man in dieser eckigen Klammer alle Größsen durch ihre Beträge, sodann $|\eta|$ und $z \mid$ durch 1 und endlich $|w|$ durch $R$ ersetzt. 
255. §58. Reihen analytischer Funktionen. - A. Dirichletsche Reihen.

Da nun $\sum \frac{a_{n}}{n_{0}}$ eine konvergente Reihe mit konstanten Gliedern ist, so genügt es, nach 198,3 zu zeigen, daß

$$
\sum_{n=1}^{\infty}\left|\frac{1}{n^{z-z_{0}}}-\frac{1}{(n+1)^{z-z_{0}}}\right|
$$

für alle $z$ des genannten Gebietes gleichmäßig konvergiert. Es ist aber, wenn wir noch $\lambda^{\prime}-\mathfrak{R}\left(z_{0}\right)=\delta(>0)$ setzen,

$$
\left|\frac{1}{n^{z-z_{0}}}-\frac{1}{(n+1)^{z-z_{0}}}\right| \leqq \frac{1}{n^{\delta}} \cdot\left|\left(1+\frac{1}{n}\right)^{z-z_{0}}-1\right| .
$$

Auf Grund der in der letzten Fußnote gegebenen Abschätzung (oder auch direkt, indem man $\left(1+\frac{1}{n}\right)^{z-z_{0}}=e^{\left(z-z_{0}\right) \log \left(1+\frac{1}{n}\right)}$ nach Potenzen von $\left(z-z_{0}\right)$ entwickelt) erkennt man nun, daß es eine Konstante $A$ gibt, so daß für alle $z$ unseres Gebietes und alle $n=1,2,3, \ldots$ die rechterhand zwischen den Absolut-Zeichen stehende Differenz ihrem Betrage nach

$$
<\frac{A}{n}
$$

bleibt. Da dann die ganze rechte Seite der letzten Ungleichung

$$
<\frac{A}{n^{1+\delta}}
$$

bleibt, und da wegen $\left|\frac{1}{n^{z-z_{0}}}\right| \leqq \frac{1}{n^{\delta}}$ die Faktoren $\frac{1}{n^{z-z_{0}}}$ in $G$ gleichmäßig beschränkt bleiben, so ist damit nach 198,3 die gleichmäßige Konvergenz der Dirichletschen Reihe in dem behaupteten Umfange dargetan und also insbesondere allgemein bewiesen, daß die Dirichletschen Reihen innerhalb ihrer Konvergenzhalbebenen analytische Funktionen darstellen.

Aus

$$
\Sigma\left|\frac{a_{n}}{n^{z}}\right|=\Sigma\left|\frac{a_{n}}{a^{z_{0}}}\right| \cdot\left|\frac{1}{n^{z-z_{0}}}\right|
$$

ergibt sich sofort, wenn eine Dirichletsche Reihe im Punkte $z_{0}$ absolut konvergiert, so tut sie es auch in jedem Punkte $z$, für den $\Re(z)>\Re\left(z_{0}\right)$; und umgekehrt: wenn sie in $z_{0}$ nicht absolut konvergiert, so kann sie auch in keinem Punkte $z$ mit $\Re(z)<\Re\left(z_{0}\right)$ absolut konvergieren. Hieraus ergibt sich nun ganz ähnlich wieder der

Satz 2. Es gibt eine ganze bestimmte Zahl $l$ (die auch $=+\infty$ oder $=-\infty$ sein kann), so daß die Dirichletsche Reihe zwar für $\Re(z)>l$, aber nicht mehr für $\Re(z)<l$ absolut konvergiert.

Es ist selbstverständlich $\lambda \leqq l$; darüber hinaus gilt aber über die gegenseitige Lage der Geraden $\Re(z)=\lambda$ und $\Re(z)=l$ der 
Satz 3. Es ist stets $l-\lambda \leqq 1$.

Beweis. Ist $\sum \frac{a_{n}}{n^{z_{0}}}$ konvergent und $\Re(z)>\Re\left(z_{0}\right)+1$, so ist $\sum \frac{a_{n}}{n^{z}}$ absolut konvergent, da $\left|\frac{a_{n}}{n^{z}}\right|=\left|\frac{a_{n}}{n^{z_{0}}}\right| \cdot\left|\frac{1}{n^{\Re\left(z-z_{0}\right)}}\right|$ und $\Re\left(z-z_{0}\right)>1$ ist. Damit ist schon alles bewiesen.

Bemerkungen und Beispiele.

1. Wenn eine Dirichletsche Reihe weder überall noch nirgends konvergiert, so ist die Situation im allgemeinen die, dafs auf eine Halbebene $\Re(z)<\lambda$ der Divergenz ein Streifen $\lambda<\Re(z)<l$ bedingter Konvergen $z$ folgt. Die Breite dieses Streifes ist aber höchstens $=1$ und auf ihn folgt eine Halbebene $\Re(z)>l$, in der die Reihe absolut konvergiert.

2. Leichte Beispiele lehren, daf $l-\lambda$ jeden Wert zwischen 0 und 1 (beide einschliefilich) haben kann, und dafs das Konvergenzverhalten auf den Grenzgeraden $\Re(z)=\lambda$ und $\Re(z) \cdot l$ je nach Lage des Falles verschieden sein kann.

3. Die beiden Reihen $\sum \frac{1}{2^{n} \cdot n^{z}}$ und $\sum \frac{2^{n}}{n^{z}}$ bieten einfache Beispiele für eine beständig konvergente und eine nirgends konvergente Dirichletsche Reihe.

4. $\sum \frac{1}{n^{z}}$ hat die Konvergenzabszisse $\lambda=1$ und stellt also eine in der Halbebene $\Re(z)>1$ reguläre analytische Funktion dar. Sie wird als die Riemannsche $\zeta$-Funktion bezeichnet (s. 197, 2, 3) und in der analytischen Zahlentheorie wegen ihres Zusammenhanges mit dem Problem der Verteilung der Primzahlen (s. u. Bem. 9) verwendet ${ }^{\mathbf{1}}$ ).

5. Ahnlich wie sich bei den Potenzreihen der Radius aus den Koeffizienten ablesen läft (Satz 94), kann man auch bei den Dirichletschen Reihen die Lage der beiden Grenzgeraden aus den Koeffizienten ablesen. Es gilt darüber der

Satz. Die Konvergenzabzisse $\lambda$ der Grenzgtraden der Dirichletschen Rtihe $\perp \frac{a_{n}}{n^{z}}$ wird stets durch die Formel geliefert

$$
\lambda=\lim _{x \rightarrow+\infty} \frac{1}{x} \log \left|a_{u+1}+a_{u+2}+\ldots+a_{v}\right|,
$$

in der $x$ stetig über alle Greızen wachsen soll und zur Abkürzung

$$
e^{[x]}=u \quad \text { und } \quad\left[e^{x}\right]=v
$$

gesetzt ist. Ersetzt man die $a_{n}$ durch ihre Beträge $\left|a_{n}\right|$, so liefcrt dieselbe Formel die Grenzabszisse $l$ der absoluten Konvergenz ${ }^{2}$ ).

6. Eine gedrängte Ưbersicht iber die wichtigsten Ergebnisse der Theorie der Dirichletschen Reihen findet man bei G. H. Hardy und M. Riesz, Theory of Dirichlet's Series, Cambridge 1915.

1) Eine eingehende Untersuchung dieser merkwürdigen Funktion (wie auch beliebiger Dirichletschen Reihen) findet man in E. Landau, Handbuch der Lehre von der Verteilung der Primzahlen, Leipzig 1909, 2 Bände.

$\left.{ }^{2}\right)$ Bezüglich des Beweises muf auf die Note des Verfassers „Über die Abszisse der Grenzgeraden einer Dirichletschen Rtihe" in den Sitzungsberichten der Berliner Mathematischen Gesellschaft (X. Jahrgang, 1910, S.2) verwiesen werden. 
257. §58. Reihen analytischer Funktionen. - A. Dirichletsche Reihen.

7. Die durch gliedweise Differentiationen aus einer Dirichletschen Reihe $F(z)=\sum \frac{a_{n}}{n^{z}}$ entstehenden neuen Dirichletschen Reihen

$$
(-1)^{v} \sum_{n=1}^{\infty} \frac{a_{n} \log ^{v} n}{n^{2}}
$$

müssen - als unmittelbare Folge des Weierstraßschen Doppelreihensatzes dieselbe Konvergenzabszisse haben wie die ursprüngliche Reihe und in der Konvergenzhalbebene die Ableitung $F^{(\nu)}(z)$ darstellen.

8. Nach 255 kann die durch eine Dirichletsche Reihe dargestellte Funktion um jeden innerhalb der Konvergenzhalbebene gelegenen Punkt als Mittelpunkt in eine Potenzreihe entwickelt werden. Die Entwicklung selbst liefert der Weierstraßsche Doppelreihensatz. Soll z. B. $\zeta(z)=\sum_{k=1}^{\infty} \frac{1}{k^{z}}$ um $z_{0}=+2$ entwickelt werden, so hat man für $k=2,3, \ldots$

$$
\frac{1}{k^{z}}=\frac{1}{k^{2}} \cdot \frac{1}{k^{z-2}}=\frac{1}{k^{2}} \cdot e^{(z-2) \log k}=\frac{1}{k^{2}} \sum_{n=0}^{\infty} \frac{\log ^{n} k}{n !}(z-2)^{n} \quad \text { (k fest), }
$$

und dies gilt auch noch für $k=1$, falls man unter $\log ^{0} 1$ die Zah1 1 versteht. Daher ist für $n \geq 0$

$$
A_{n}=\frac{1}{n !} \sum_{k=1}^{\infty} \frac{\log ^{n} k}{k^{2}}
$$

was nun die gewünschte Entwicklung

liefert.

$$
\zeta(z)=\sum_{n=1}^{\infty}\left[\frac{1}{n !} \sum_{k=1}^{\infty} \frac{\log ^{n} k}{k^{2}}\right](z-2)^{n} \equiv \frac{\pi^{2}}{6}+\left(\sum_{k=1}^{\infty} \frac{\log k}{k^{2}}\right)(z-2)+\ldots
$$

9. Für $\Re(z)>1$ haben

$$
\text { die Reihe } \quad \sum_{n=1}^{\infty} \frac{1}{n^{z}} \quad \text { und das Produkt } \quad \prod_{1-p^{-z}}
$$

in welch letzterem $p$ die Folge der Primzahlen $2,3,5,7, \ldots$ durchlaufen soll, stets denselben Wert, stellen als o beide die Riemannsche $\zeta$-Funktion $\zeta(z)$ dar (Euler, 1737; s. Introd. in analysin, S. 225).

Beweis. Es sei $z$ ein bestimmter Punkt mit $\Re(z)=1+\delta>1$. Dann steht die absolute Konvergenz von Reihe und Produkt in diesem Punkte nach Bem. 4 und 127, 7 außer Frage. Es handelt sich nur darum, die Gleichheit ihrer Werte darzutun. Nun ist aber

$$
\frac{1}{1-p^{-z}}=1+\frac{1}{p^{z}}+\frac{1}{p^{2 z}}+\frac{1}{p^{3 z}}+\ldots
$$

und wenn man diese Entwicklungen für alle Primzahlen $p \leq N$ miteinander multpliziert - unter $N$ eine vorläufig feste natürliche Zalıl verstanden - , so ist dies (endliche) Produkt

$$
=\prod_{p \leq N} \frac{1}{1-p^{-z}}=\sum_{n=1}^{N} \frac{1}{n^{z}}+\sum_{n=N}^{\infty} \sum^{\prime} \frac{1}{n^{z}},
$$

wenn hier durch den Akzent am $\Sigma$ angedeutet werden soll, daßs von der hingeschriebenen Reihe nicht alle, sondern nur gewisse Glieder auftreten. Hierbei haben wir von dem elementaren Satz Gebrauch gemacht, dał sich jede na- 
türliche Zahl $\geqq 2$ auf eine und nur eine Art als Produkt von Potenzen verschiedener Primzahlen darstellen läßst (wofern als Exponenten nur ganze Zahlen len $>0$ zugelassen werden). Hiernach ist

$$
\left|\prod_{p \leq N} \frac{1}{1-p^{-z}}-\sum_{n=1}^{N} \frac{1}{n^{z}}\right| \leqq \sum_{n=N+1}^{\infty} \frac{1}{n^{1+\delta}} ;
$$

und da hier rechterhand der Rest einer konvergenten Reihe steht, so strebt dies $\rightarrow 0$ für $N \rightarrow+\infty$, womit die Gleichheit der Werte des unendlichen Produktes und der unendlichen Reihe schon bewiesen ist.

10. Nach der vorigen Nummer ist für $\Re(z)>1$

$$
\frac{1}{\zeta(z)}=\prod_{p}\left(1-p^{-z}\right)=\prod_{p}\left(1-\frac{1}{p^{z}}\right)=\sum_{n=1}^{\infty} \frac{\mu(n)}{n^{z}},
$$

wenn hierin $\mu(1)=1, \mu(2)=-1, \mu(3)=-1, \mu(4)=0, \mu(5)=-1, \mu(6)=+1$, $\ldots$ und allgemein $\mu(n)=0,+1$ oder -1 gesetzt wird, je nachdem $n$ durch das Quadrat einer Primzahl teilbar ist, oder nur aus einer geraden, oder nur aus einer ungeraden Anzahl verschiedener Primzahlen besteht. Diese Darstellung lehrt zugleich, dafs für $\Re(z)>1$ stets $\zeta(z) \neq 0$ ist. Die eigentümlichen Koeffizienten $\mu(n)$ heifen die Möbiusschen Koeffizienten. Die Aufeinanderfolge der Ziffern $0,+1,-1$ in der Folge dieser Zahlen $\mu(n)$ gehorcht keiner oberfläichlichen Gesetzmäßigkeit.

11. Da $\zeta(z)=\sum_{n^{z}}^{1}$ für $\Re(z)>1$ absolut konvergiert, so kann man durch Ausmultiplizieren $(\zeta(z))^{2}$ bilden. Ordnet man (was nach 91 erlaubt) die Produkte der Glieder wieder nach wachsenden Nennern an, so erhält man

$$
\zeta^{2} .(z)=\sum_{n=1}^{\infty} \frac{\tau_{n}}{n^{z}},
$$

wenn man mit $\tau_{n}$ die Anzahl der Teiler von $n$ bezeichnet. - Diese Beispiele mögen genügen, um die Bedeutung der $\zeta$-Funktion für zahlentheoretische Probleme erklärlich zı machen.

\section{B. Fakultätenreihen.}

Unter einer Fakultätenreihe (erster Art) versteht man eine Reihe der Form

$$
\sum_{n=1}^{\infty} \frac{n ! a_{n}}{z(z+1) \cdots(z+n)}
$$

die natürlich nur dann eine Bedeutung hat, wenn $z \neq 0,-1,-2, \ldots$ ist. Die Konvergenzfragen, zunächst geklärt von Jensen, werden vollständig erledigt durch den folgenden

258. Satz von Landau ${ }^{1}$ ). Die Fakultätenreihe (F) konvergiert - von den Punkten 0, - 1, - 2, .. abgesehen - überall da und nur da, wo die „zugeordnete" Dirichletsche Reihe

$$
\sum_{n=1}^{\infty} \frac{a_{n}}{n^{z}}
$$

1) Über die Grundlagen der Theorie der Fakultätenreihen, Münch. Ber., Bd. 36 (1906), S. 151-218. 
258. $§ 58$. Reihen analytischer Funktionen. - B. Fakultätenreihen.

konvergiert. Und sie konvergiert in jedem Kreise gleichmäßig und nur in solchen, in denen diese es tut, wofern weder im Innern noch auf dem Rande dieser Kreise einer der Punkte 0, - 1, - 2, .. gelegen ist.

Beweis. 1. Wir zeigen zunächst, daß aus der Konvergenz der Dirichletschen Reihe in einem bestimmten von $0,-1,-2, \ldots$ verschiedenen Punkte $z$ die Konvergenz der Fakultätenreihe in demselben Punkte folgt. Da aber

$$
\frac{n ! a_{n}}{z(z+1) \cdots(z+n)}=\frac{a_{n}}{n^{z}} \cdot \frac{1}{g_{n}(z)}
$$

ist, wenn $g_{n}(z)$ dieselbe Bedeutung hat wie in $\mathbf{2 5 4}$, Beisp. 4, so genügt es nach 184, 3 zu zeigen, daß die Reihe

$$
\sum_{n=1}^{\infty}\left|\frac{1}{g_{n}(z)}-\frac{1}{g_{n+1}}\right|=\sum_{n=1}^{\infty} \frac{g_{n+1}(z)-g_{n}(z) \mid}{\left|g_{n}(z) \cdot g_{n+1}(z)\right|}
$$

konvergent ist. Da aber $\frac{1}{g_{n}(z)}$ mit wachsendem $n$ einem endlichen Grenzwerte, nämlich dem Werte $\Gamma(z)$ zustrebt, so bleibt dieser Faktor (bei festem $z$ für alle $n$ ) beschränkt, und es genügt daher die Konvergenz der Reihe

$$
\sum_{n=1}^{\infty}\left|g_{n}(z)-g_{n+1}(z)\right|
$$

zu beweisen. Das ist aber schon in $\mathbf{2 5 4}$, Beisp. 4, geschehen.

2. Daß umgekehrt aus der Konvergenz der Fakultätenreihe die der Dirichletschen folgt, ergibt sich genau so, da es nach 184, 3 wieder nur auf die eben benutzte Konvergenz von $\Sigma\left|g_{n}(z)-g_{n+1}(z)\right|$ ankommt.

3. Es sei jetzt $\Omega$ ein Kreis, indem die Dirichletsche Reihe gleichmäßig konvergiert und der keinen der Punkte $0,-1,-2, \ldots$ in seinem Innern oder auf seinem Rande enthält. Dann soll gezeigt werden, daß dort auch die Fakultätenreihe gleichmäßig konvergiert. Nach 198, 3 kommt dies wieder darauf hinaus zu zeigen, daß

$$
\sum_{n=1}^{\infty}\left|\frac{g_{n+1}(z)-g_{n}(z)}{g_{n}(z) \cdot g_{n+1}(z)}\right|
$$

in $\Re$ gleichmäßig konvergiert. Von $\boldsymbol{\Sigma}\left|g_{n+\mathbf{1}}(z)-g_{n}(z)\right|$ ist uns dies nach 254, 4 schon bekannt. Und da in der dortigen Fußnote auch gezeigt ist, daß eine Konstante $A^{\prime}$ existiert, so da $\beta$ für alle $z$ in $\Re$ und alle $n$

$$
\left|\frac{1}{g_{n}(z)}\right|<A^{\prime}
$$

bleibt, so ist schon alles erforderliche dargetan.

4. Daß umgekehrt die Dirichletsche Reihe in einem Kreise $\Omega$ gleichmäßig konvergiert, in dem die Fakultätenreihe es tut, folgt nach 198, 3 sofort aus der daselbst gleichmäßigen Konvergenz der Reihe $\Sigma\left|g_{n+\mathbf{1}}(z)-g_{n}(z)\right|$, die in $\mathbf{2 5 4}, 4$ bewiesen wurde. 
1. Die Fakultiitenreihe

$$
\text { Beispiele. }
$$

$$
\sum_{n=1}^{x} 2^{n+1} \frac{n !}{z(z+1) \cdots(z+n)}
$$

konvergiert in allen (von $0, \ldots 1, \ldots$ verschiedenen) Punkten der ganzen Ebene, da die Divichletsche Reihe

$$
\sum_{n=1}^{x} 2^{n+1} \cdot n^{z}
$$

ersichtlich bestïndig konvergent ist.

wegen

$$
\begin{aligned}
& A_{x}^{1}-\frac{1}{x}-\frac{1}{x+1} \quad \begin{array}{c}
1 \\
x(x+1)
\end{array} \\
& \Lambda^{2} \frac{1}{x}=\begin{array}{c}
2 ! \\
x(x+1)(x+2)
\end{array}, \quad \cdots, \quad A^{k}{ }_{x}^{1} \quad x(x+1) \ldots(x+k)
\end{aligned}
$$

entsteht die vorgelegte Fakultätenreihe einfach durch die Eulersche Transformation 144 aus der Reihe

$$
\sum_{n=0}^{x} \frac{(-1)^{n}}{x+n} \quad \frac{1}{x} \quad \frac{1}{x+1}+\frac{1}{x+2}-\cdots
$$

2. Ganz leicht findet man auch (vgl. S. 258), daf

also

$$
\frac{1}{z^{2}} \quad \begin{gathered}
0 ! \\
z(z+1)
\end{gathered}+\begin{gathered}
1 ! \\
z(z+1)(z+2)
\end{gathered}+\ldots+\frac{(n-1) !}{z(z+1) \ldots(z+n)}+\ldots
$$

$$
z^{2}=\sum_{n=1}^{\infty} \frac{1}{n} \cdot z(z+1) \ldots(z+n) \cdot
$$

Zum Beweise hat man nur die Glieder der rechten Seite nacheinander von der linke Seite abzuziehen. Nach dem $n^{\text {ten }}$ Schritt erhält man

$$
\frac{n !}{z^{2}(z+1)(z+2) \ldots(z+n)} \frac{1}{z \cdot n^{z}} \quad z(z+1) \ldots(z \cdots n),
$$

was für $\Re(z), 0$ nach $\mathbf{2 5 4}$, Beisp. 4, gegen $\rightarrow 0$ strebt, falls $n \rightarrow \infty$ wächst. (Stirling, Methodus differentialis, London 1730, S. $6 \mathrm{ff}$.)

\section{Lambertsche Reihen.}

Als Lambertsche Reihe bezeichnet man cine Reihe der Form

$$
\left.\sum_{n=1}^{x} a_{n} \frac{z^{n}}{1-z^{n}} 1\right)
$$

Fragen wir hier wieder zunächst nach dem genauen Konvergenzbereich der Reihe, so ist dabei zu beachten, daß für jedes $z$. von dem eine positiv ganzzahlige Potenz - 1 ist, unendlich viele Glieder der Reihe sinnlos werden. Die Peripherie des Einheitskreises muß daher für die Konvergenzfrage ausscheiden und wir müssen getrennt die Punkte innerhalb und aụßerhalb des Einheitskreises untersuchen. Hier gilt nun der die Konvergenzfrage schon vollständig erledigende

$\left.{ }^{1}\right)$ Eine ausfiihrlichere Behandlung dieser Reihenart findet man in der Arbeit des Verfassers: Über Lambertsche Reihen, Journ. f. d. reine u. angew. Mathem., Bd. 142 (1913), S. 283-315. 
259. §58. Reihen analytischer Funktionen. - C. Lambertsche Reihen. 433

Satz. Wenn $\sum a_{n}$ konvergiert, so konvergiert die Lambertsche Reihe $\mathbf{2 5 9}$. für jedes $z$, dessen absoluter Betrag von 1 verschieden ist. Ist dagegen $\sum a_{n}$ nicht konvergent, so konvergiert die Lambertsche Reihe in genau denselben Punkten wie die ,zugehörige" Potenzreihe $\Sigma a_{n} z^{n}$ - wofern nach wie vor $|z| \gtrless 1$ gehalten wird.

In jedem Kreise $\Re$, der einschließlich seines Randes in einem der Konvergenzgebiete der Lambertschen Reihe liegt und keinen Punkt vom Betrage 1 enthält, ist die Konvergenz überdies eine gleichmäßige.

Beweis. 1. Es sei $\Sigma a_{n}$ divergent. Dann ist der Radius $r$ von $\sum a_{n} z^{n}$ notwendig $\leqq 1$ und wir haben zunächst zu zeigen, daß die Lambertsche Reihe und die zugehörige Potenzreihe für jedes $|z|<1$ gleichzeitig konvergieren oder divergieren, und daß auch die Lambertsche Reihe für $z>1$ divergiert. Nun ist

$$
\begin{aligned}
& \sum a_{n} z^{n}=\sum a_{n} \frac{z^{n}}{1-z^{n}} \cdot\left(1-z^{n}\right) \quad \text { und } \\
& \sum a_{n} \frac{z^{n}}{1-z^{n}} \equiv \sum a_{n} z^{n} \cdot \frac{1}{1-z^{n}} .
\end{aligned}
$$

Nach 184, 3 genügt es also, die Konvergenz der beiden Reihen

und

$$
\Sigma\left|\left(1-z^{n+1}\right)-\left(1-z^{n}\right)\right|=\Sigma\left|z^{n}-z^{n+1}\right|=|1-z| \cdot \Sigma\left|z^{n}\right|
$$

$$
\Sigma\left|\frac{1}{1-z^{n+1}}-\frac{1}{1-z^{n}}\right|=|1-z| \cdot \sum \frac{\mid z^{n}}{\left(1-z^{n}\right)\left(1-z^{n+1}\right) \mid}
$$

für $|z|<1$ zu beweisen. Hiervon ist aber das erste evident und das zweite eine Folge der Bemerkung, daß für $|z|<1$ von einer Stelle an $\left|1-z^{n}\right|>\frac{1}{2}$ ist.

Würde aber die Lambertsche Reihe für ein $z_{0}$ mit $\left|z_{0}\right|>1$ konvergieren, so hieße dies, daß die Potenzreihe

$$
\sum \frac{a_{n}}{1-z_{0}^{n}} z^{n}
$$

für $z=z_{0}$ konvergiert. Sie müßte dann nach 93, Satz 1 auch für $z=+1$ konvergieren, was dann auch die Konvergenz der Reihe

$$
\underline{\Sigma}_{1-z_{0}{ }^{n}}{ }^{a_{n}}-\Sigma^{\prime} a_{n} \frac{z_{0}^{n}}{1-z_{0}^{n}}=\Sigma a_{n}
$$

zur Folge hätte, die doch nach unserer jetzigen Annahme divergiert.

Daß endlich für alle $\mid z \leqq \varrho<r$ die Konvergenz der Lambertschen Reihe eine gleichmäßige ist, folgt sofort aus der Abschätzung

$$
\left|a_{n} \frac{z^{n}}{1-z^{n}}\right| \leqq \frac{1}{1-g} \mid a_{n} z^{n}
$$

und der entsprechenden Eigenschaft der Reihe $\Sigma\left|a_{n} z^{n}\right|$.

Damit ist für den Fall einer divergenten $\Sigma a_{n}$ schon alles bewiesen. 
2. Ist nun $\sum a_{n}$ konvergent, hat $\sum a_{n} z^{n}$ also einen Radius $r \geqq 1$ so ist die Lambertsche Reihe jedenfalls für jedes $|z|<1$ konvergent, und sogar gleichmäßig für alle $|z| \leqq \varrho<1$.

Ist aber $|z| \geqq \varrho^{\prime}>1$, so ist

$$
\Sigma a_{n} \frac{z^{n}}{1-z^{n}}=-\Sigma a_{n}-\sum a_{n} \frac{\left(\frac{1}{z}\right)^{n}}{1-\left(\frac{1}{z}\right)^{n}} ;
$$

und da $\left|\frac{1}{z}\right| \leqq \frac{1}{\varrho^{\prime}}<1$ ist, so sind die weiteren Behauptungen auf die vorigen zurückgeführt, und somit alle Teile des Satzes bewiesen.

Da hiernach im Falle der Konvergenz von $\Sigma a_{n}$ ein äußerst einfacher Zusammenhang zwischen der Reihensumme in einem Punkte $z$ außerhalb des Einheitskreises und derjenigen in dem Punkt $\frac{1}{z}$ derselben besteht, genügt es, wenn wir uns weiterhin mit dem innerhalb des Einheitskreises gelegenen Konvergenzgebiet beschäftigen. Dieses ist also, je nachdem $\Sigma a_{n} z^{n}$ einen Radius $r<1$ oder $\geqq 1$ besitzt, der Kreis $|z|<r$ oder der Einheitskreis $|z|<1$. Der Radius dieses wohlbestimmten Konvergenzkreises heiße $\boldsymbol{r}_{\mathbf{1}}$.

Da nun weiter die Glieder der Lambertschen Reihe für $|z|<r_{\mathbf{1}}$ reguläre analytische Funktionen sind und da für jedes positive $\varrho<\boldsymbol{r}_{1}$ die Reihe in $|z| \leqq \varrho$ gleichmäßig konvergiert, so können wir den Weierstraßschen Doppelreihensatz benutzen, um die Potenzreihenentwicklung. der durch die Lambertsche Reihe in $|z|<r_{1}$ definierten Funktionen $\mathrm{zu}$ bekommen. Es ist

$$
\begin{aligned}
& a_{1} \frac{z}{1-z}=a_{1} z+a_{1} z^{2}+a_{1} z^{3}+a_{1} z^{4}+a_{1} z^{5}+a_{1} z^{6}+a_{1} z^{7}+\ldots \\
& a_{2} \frac{z^{2}}{1-z^{2}}=\quad a_{2} z^{2} \quad+a_{2} z^{4} \quad+a_{2} z^{6} \quad+\ldots \\
& a_{3} \frac{z^{3}}{1-z^{3}}=\quad a_{3} z^{3} \quad+a_{3} z^{6} \quad+\ldots \\
& a_{4} \frac{z^{4}}{1-z^{4}}=\quad a_{4} z^{4}
\end{aligned}
$$

und alle diese Potenzreihen dürfen wir gliedweis addieren. Nun tritt eine bestimmte Potenz $z^{n}$ in der $k^{\text {ten }}$ Zeile dann und nur dann auf, wenn $n$ ein Vielfaches von $k$, oder also $k$ ein Teiler von $n$ ist. Daher wird der Koeffizient von $z^{n}$ gleich der Summe aller derjenigen $a_{v}$ sein, deren Index $v$ ein Teiler von $n$ ist (einschließlich 1 und $n$ selber). Wir drücken dies durch die Symbolik aus:

$$
\left.A_{n}=\sum_{d / n} a_{d}^{1}\right) \text {. }
$$

1) Sprich: Summe aller $a_{d}$, für die $d$ ein Teiler von $n$ ist. 
260. §58. Reihen analytischer Funktionen. - C. Lambertsche Reihen.

Beispiele.

1. $a_{n}=1$. Dann ist $A_{n}$ gleich der $A n z a h l$ der Teiler von $n$, die wir (wie $\mathbf{2 6 0}$. in $\mathbf{2 5 7}$, Beisp. 11) mit $\tau_{n}$ bezeichnen wollen, und

$$
\begin{aligned}
\sum_{n=1}^{\infty} \frac{z^{n}}{1-z^{n}} & \equiv \sum_{n=1}^{\infty} \tau_{n} z^{n} \quad \quad(|z|<1), \\
& =z+2 z^{2}+2 z^{3}+3 z^{4}+2 z^{5}+4 z^{6}+2 z^{7}+4 z^{8}+\ldots
\end{aligned}
$$

In dieser merkwürdigen Potenzreihe sind die Glieder $z^{n}$, deren Exponent eine Primzahl ist, dadurch ausgezeichnet, daß ihr Koeffizient $=2$ ist. Wegen dieser verführerisch nahen Beziehung zu den Primzahlproblemen hat diese spezielle Lambertsche Reihe (meist schlechtweg als die Lambertsche Reihe bezeichnet ${ }^{1}$ ) früher eine großse Rolle beim Angriff auf jene Probleme gespielt. Doch hat sich auf diesem Wege nichts wesentliches ergeben.

2. $a_{n} \equiv n$. Dann wird $A_{n}$ gleich der Summe aller Teiler von $n$, die wir mit $\tau_{n}^{\prime}$ bezeichnen wollen, so dafs also für $|z|,<1$

$$
\sum_{n=1}^{\infty} n \frac{z^{n}}{1-z^{n}}=\sum_{n=1}^{\infty} \tau_{n}{ }^{n} z^{n} \equiv z+3 z^{2}+4 z^{3}+7 z^{4}+6 z^{5}+12 z^{6}+\cdots
$$

3. Die Beziehung $A_{n}=\sum_{d / n} a_{d}$ ist eindeutig umkehrbar, d. h. bei gegebenen $A_{n}$ lassen sich die $a_{n}$ auf eine und nur eine Weise diesen Bedingungen gemä 3 bestimmen. Und zwar ist

$$
a_{n}=\sum_{d / n} \mu\left(\frac{n}{d}\right) \cdot A_{d}
$$

wenn $\mu(k)$ die in $\mathbf{2 5 7}$, Beisp. 10 definierten Möbiusschen Koeffizienten bedeutet, die nur die Werte $0,+1$ und -1 haben können. Diese Tatsache hat zur Folge, dafs nicht nur jede Lambertsche Reihe in eine Potenzreihe entwickelt werden kann, sondern daß auch das Umgekehrte stets auf eine und nur eine Weise möglich ist, wenn die Potenzreihe für $z=0$ verschwindet, wenn also $A_{0}=0$.

4. Ist z. B. $A_{1}=1$ und sind alle iibrigen $A_{n}=0$, so ist

$$
a_{n}=\mu(n)
$$

und man hat die merkwiirdige Identität

$$
z=\sum_{n=1}^{\infty} \mu(n) \frac{z^{n}}{1-z^{n}},
$$

5. Ebenso findet man die für $|z|<1$ gültige Darstellung

$$
\frac{z}{(1-z)^{2}}=\sum_{n=1}^{\infty} \varphi(n) \frac{z^{n}}{1-z^{n}}
$$

wenn $\varphi(n)$ die - von Euler eingeführte - Bedeutung hat, die Anzahl der zu $n$ teilerfremden Zahlen unter den Zahlen $1,2,3, \ldots, n-1$ anzugeben.

6. Wird $\sum_{n=1}^{\infty} a_{n} \frac{z^{n}}{1-z^{n}}=f^{\prime}(z)$ und $\sum_{n=1}^{\infty} a_{n} z^{n}=g(z)$ gesetzt, so wird, wenn man in dem Entwicklungsschema der Lambertschen Reihe auf S. 434 (was erlaubt ist) nach Schräglinien zusammenfafit:

$$
f(z)=g(z)+g\left(z^{2}\right)+\ldots=\sum_{m=1}^{\infty} g\left(z^{m}\right) .
$$

1) J. Lambert, Anlage zur Architektonik, Riga 1771, Bd. 2, S. 507. 
7. Für $a_{n}=(-1)^{n-1},=n,=(-1)^{n-1} n,=\frac{1}{n},=\frac{(-1)^{n-1}}{n},=\alpha^{n}, \ldots$ erhält man so der Reihe nach die folgenden für $|z|<1$ gültigen merkwürdigen Identitäten, bei denen die Summation stets von $n=1$ an $z u$ erstrecken ist.
a) $\Sigma(-1)^{n-1} \frac{z^{n}}{1-z^{n}} \equiv \Sigma \frac{z^{n}}{1+z^{n}}$
b) $\sum n \frac{z^{n}}{1-z^{n}} \quad \equiv \sum_{\frac{1}{\left(1-z^{n}\right)^{2}}}$
c) $\sum(-1)^{n-1} \cdot n \frac{z^{n}}{1-z^{n}}=\sum \frac{z^{n}}{\left(1+z^{n}\right)^{2}}$
d) $\sum \frac{1}{n} \frac{z^{n}}{1-z^{n}} \quad=\sum \log \frac{1}{1-z^{n}}$
e) $\sum \frac{(-1)^{n-1}}{n} \cdot \frac{z^{n}}{1-z^{n}}=\sum \log \left(1+z^{n}\right)$
f) $\perp \alpha^{n} \frac{z^{n}}{1-z^{n}} \equiv \sum \frac{\alpha}{1-\alpha z^{n}}$,
$(|\alpha|<1)$

usw.

8. Da bei den letzten Identitäten in d) und e) rechts eine Reihe von Logarithmen aufgetreten ist, so ergibt sich von hieraus ein leichter Zusammenhang zwischen gewissen Lambertschen Reihen und unendlichen Produkten. So ist

$$
\begin{array}{ll}
\Pi\left(1-z^{n}\right)=e^{w} \quad \text { mit } \quad w=-\sum \frac{1}{n} \frac{z^{n}}{1-z^{n}} \\
\Pi\left(1+z^{n}\right)=e^{w} \quad \text { mit } \quad w=\sum \frac{(-1)^{n-1}}{n} \frac{z^{n}}{1-z^{n}}
\end{array}
$$

9. Als interessantes numerisches Beispiel sei noch dieses erwähnt: Setzt man $u_{0}=0, u_{1}=1$ und für $n>1$ stets $u_{n}=u_{n-1}+u_{n-2}$, so erhält man die sogen. Fibonaccische Zahlenfolge (vgl. 6, 7)

$$
0,1,1,2,3,5,8,13,21,34,55, \ldots
$$

Es ist dann

$$
\sum_{k=1}^{\infty} \frac{1}{u_{2 k}}=1+\frac{1}{3}+\frac{1}{8}+\frac{1}{21}+\frac{1}{55}+\ldots=\sqrt{5}\left[L\left(\frac{3-\sqrt{5}}{2}\right)-L\left(\frac{7-3 \sqrt{5}}{2}\right)\right],
$$

wenn mit $L(x)$ die Summe der Lambertschen Reihe $\sum \frac{x^{n}}{1-x^{n}}$ bezeichnet wird ${ }^{1}$ ). Der Beweis beruht auf der leicht $z u$ beweisenden Tatsache, dafs

$$
u_{v}=\frac{\alpha^{v}-\beta^{v}}{\alpha-\beta} \quad(v=0,1,2, \ldots)
$$

gesetzt werden kann, wenn $\alpha$ und $\beta$ die Wurzeln der quadratischen Gleichung $x^{2}-x-1=0$ sind. (Vgl. Aufgabe 114.)

1) E. Landau, Bull. de la Soc. math. de France, Bd. 27 (1899), S. 298. 


\section{Aufgaben zum XII. Kapitel ${ }^{1}$ ).}

174. Es strebe $z_{n} \rightarrow \zeta$ und $b_{n} \rightarrow b \neq 0$. In welchem Ausmafe kann dann auf $b_{n}{ }^{z_{n}} \rightarrow b^{\zeta}$ geschlossen werden?

175. Es strebe $z_{n} \rightarrow \infty$ (d. h. also $\left|z_{n}\right| \rightarrow+\infty$ ). In welchem Ausmafie kann dann auf
a) $\left(1+\frac{z}{z_{n}}\right)^{z_{n}} \rightarrow e^{z}$
b) $z_{n} \cdot\left(z^{\frac{1}{z_{n}}}-1\right) \rightarrow \log z$

geschlossen werden?

176. Der Hauptwert von $z^{i}$ bleibt für alle $z$ beschränkt.

178. Ist

$$
z_{n}=\sum_{\nu=0}^{n}(-1)^{v}\left(\begin{array}{l}
z \\
v
\end{array}\right)
$$

so strebt $z_{n} \rightarrow 0$ oder $\frac{1}{z_{n}} \rightarrow 0$, je nachdem $\Re(z)>0$ oder $<0$ ist. Wie verhält $\operatorname{sich}\left(z_{n}\right)$ für $\Re(z)=0$ ?

178. Es seien $a, b, c, d$ vier Konstante, für die $a d-b c \neq 0$ ist, und es sei $z_{0}$ beliebig. Man untersuche die Zahlenfolge $\left(z_{0}, z_{1}, z_{2}, \ldots\right)$, die rekursiv durch die Formel

$$
z_{n+1}=\frac{a z_{n}+b}{c z_{n}+d}, \quad n=0,1,2, \ldots
$$

definiert ist. Welches sind die notwendigen und hinreichenden Bedingungen dafür, daßs $\left(z_{n}\right)$ oder $\left(\frac{1}{z_{n}}\right)$ konvergiert? Und wenn keine von beiden Folgen konvergiert, unter welchen Bedingungen kann für einen Index $p$ wieder $z_{p}=z_{0}$ sein? Wann ist die Folge identisch konstant?

179. Es sei $a \neq 0$ gegeben, $z_{0}$ beliebig gewählt und für $n>0$

$$
z_{n+1}=\frac{1}{2}\left(z_{n}+\frac{a}{z_{n}}\right)
$$

gesetzt. Dann konvergiert die Folge $\left(z_{n}\right)$ dann und nur dann, wenn $z_{0}$ nicht auf dem Mittellot derjenigen Strecke liegt, die die beiden Werte von $\sqrt{a}$ verbindet. Ist diese Bedingung erfüllt, so konvergiert $z_{n}$ gegen den nächstgelegenen dieser beiden Punkte. Welches Verhalten zeigt $\left(z_{n}\right)$, wenn $z_{0}$ auf dem genannten Mittellot liegt?

180. Die Reihe $\Sigma \frac{1}{n^{1+i \alpha}}$ konvergiert für kein reelles $\alpha$, die Reihe $\Sigma \frac{1}{n^{1+i \alpha} \cdot \log n}$ für jedes reelle $\alpha \neq 0$.

181. Hat bei festem $z$ und geeigneter Bestimmung des Logarithmus

einen Grenzwert?

$$
\left[\frac{1}{z+1}+\cdots+\frac{1}{z+n}-\log (z+n)\right]
$$

182. Für jedes feste $z$ mit $0<\Re(z)<1$ ist

$$
\lim \left[1+\frac{1}{2^{z}}+\frac{1}{3^{z}}+\cdots+\frac{1}{n^{z}}-\frac{n^{1-z}}{1-z}\right]
$$

vorhanden. (Vgl. Aufgabe 135.)

1) Hier sind, wenn das Gegenteil aus dem Zusammenhang nicht unzweideutig hervorgeht, alle Zahlen als komplex anzusehen. 
183. Die Funktion $(1-z) \cdot \sin \left(\log \frac{1}{1-z}\right)$ läf3t sich für $|z|<1$ in eine Potenzreihe $\Sigma a_{n} z^{n}$ entwickeln, wenn für den Logarithmus der Hauptwert genommen wird. Man zeige, dafs diese Reihe für $|z|=1$ noch absolut konvergiert.

184. Wenn $z$ aus dem Innern des Einheitskreises „im Winkelraum“ gegen $\rightarrow+1$ strebt, so strebt
a) $1-z+z^{4}-z^{9}+z^{16}-+\ldots \rightarrow \frac{1}{2}$;
b) $(1-z)\left[1+z+z^{4}+z^{9}+\ldots\right]^{2} \rightarrow \frac{\pi}{4}$;
c) $\frac{1}{\log \frac{1}{1-z}}\left[z+z^{p}+z^{p^{2}}+z^{p^{3}}+\ldots\right] \rightarrow \frac{1}{\log p}$ :
d) $(1-z)^{p+1}\left[z+2^{p} z^{2}+3^{p} z^{3}+\ldots\right] \rightarrow \Gamma(p+1)$;
e) $\frac{\Sigma a_{n} z^{n}}{\sum b_{n} z^{n}} \rightarrow \lim \frac{a_{n}}{b_{n}}$,

falls der rechtsstehende Grenzwert existiert, die $b_{n}$ positiv sind und $\Sigma b_{n}$ divergiert.

185. Man untersuche das Konvergenzverhalten der folgenden Potenzreihen auf dem Rande des Einheitskreises:
a) $\sum \frac{(-1)^{[\sqrt{n}]}}{n} z^{n}$
b) $\sum \frac{z^{n}}{n^{\alpha+i \beta}}, \quad \alpha>0$
c) $\sum \frac{z^{n}}{(n+a)^{\alpha+i \beta}}$;
d) $\sum \frac{z^{n}}{n \log n}$

e) $\sum \frac{\varepsilon_{n}}{n \log n} z^{n}$, wenn $\varepsilon_{n}$ dieselbe Bedeutung wie in Aufgabe 47 hat.

186. Ist $\Sigma a_{n} z^{n}$ für $|z|<1$ konvergent und ist für alle diese $z$ die Summe der Reihe ihrem Betrage nach $\leq 1$, so ist $\Sigma\left|a_{n}\right|^{2}$ konvergent und $<1$.

187. Die folgenden Potenzreihen
a) $\sum \frac{z^{n}}{n}$,
a) $\sum \frac{z^{2 k-1}}{2 k-1}$
c) $\Sigma(-1)^{k-1} \frac{z^{2 k-1}}{2 k-1}$,
d) $\Sigma(-1)^{n-1} \frac{z^{n}}{n}$
e) $\Sigma(-1)^{n} \frac{z^{n}}{(n+1)(n+2)}$,
f) $\sum(-1)^{n}\left(\begin{array}{c}-\frac{1}{2} \\ n\end{array}\right) z^{n}$
g) $\sum \frac{z^{n}}{(n-1) \cdot(n+1)}$,
h) $\sum \frac{z^{2 n}}{(2 n-1) \cdot 2 n}$

haben sämtlich den Einheitskreis zum Konvergenzkreis. Auf dem Rande derselben sina sie im allgemeinen, d. h. mit eventuellem Ausschluf einzelner Punkte, auch noch konvergent. Man suche ihre Summe mit Hilfe der elementaren Funktionen geschlossen darzustellen, trenne Reelles und Imaginäres, indem man $z=r(\cos x+i \sin x)$ setzt, und schreibe die gewonnenen trigonometrischen Entwicklungen nieder, und zwar getrennt für $r<1$ und $r=1$. Für welche $x$ sind diese konvergent? Welchen Wert hat ihre Summe? Sind es die Fourierreihen dieser ihrer Summe? 
188. Welche Summen haben hiernach die Reihen
a) $\sum \frac{\cos n x \cos n y}{n}$;
b) $\sum \frac{\cos n x \sin n y}{n}$;

c) $\sum \frac{\sin n x \sin n y}{n}$

und die weiteren 3 Reihen, die man erhält, wenn man den hingeschriebenen Reihengliedern das Vorzeichen $(-1)^{n}$ gibt.

189. Verfährt man mit der geometrischen Reihe $\Sigma z^{n}$ wie in Aufgabe 187, jedoch $r<1$ lassend, so erhält man die Darstellungen

a) $\sum_{n=0}^{\infty} r^{n} \cos n x=\frac{1-r \cos x}{1-2 r \cos x+r^{2}}$;

b) $\sum_{n=1}^{\infty} r^{n} \sin n x=\frac{r \sin x}{1-2 r \cos x+r^{2}}$.

Man leite aus ihnen die weiteren Entwicklungen her

c) $\sum_{n=1}^{\infty} \frac{\cos n x}{(2 \cos x)^{n}}=\cos 2 x$,

d) $\sum_{n=1}^{\infty} \frac{\sin x}{(2 \cos x)^{n}}=\sin 2 x$

und gebe die genauen Gültigkeitsintervalle derselben an.

190. Bei Aufgabe 187 a wird man u. a. die Darstellung

$$
\sum_{n=1}^{\infty} \frac{r^{n}}{n} \sin n x=\operatorname{arctg}\left(\frac{r \sin x}{1-r \cos x}\right)
$$

erhalten. Aus ihr leite man die Entwicklungen
a) $\sum_{n=1}^{\infty}(-1)^{n-1} r^{n} \sin ^{n} x \cdot \sin n x=\operatorname{arctg}(v+\operatorname{ctg} x)-\left(\frac{x}{2}-x\right)$,
b) $\sum_{n=1}^{\infty} \frac{\cos ^{n} x \sin n x}{n}=\frac{\pi}{2}-x$

her und bestimme die genauen Gültigkeitsintervalle.

191. Man stelle die genauen Konvergenzgebiete der folgenden Reihen fest :
a) $\sum \frac{(-1)^{n}}{z+n}$
b) $\sum_{1-z^{n}}$,
c) $\sum \frac{z^{n}}{p_{n}{ }^{n}}$
d) $\perp \frac{1}{p_{n}{ }^{2}}$,
e) $\Sigma\left[\frac{1}{z-p_{n}}+\frac{1}{p_{n}}+\frac{z}{p_{n}{ }^{2}}+\cdots+\frac{z^{n-1}}{p_{n}{ }^{n}}\right]$,
f) $\Sigma\left(\frac{z}{p_{n}}\right)^{[\log n]}$,
g) $\sum\left(\frac{z}{p_{n}}\right)^{[\log \log n]}$,

bei denen $\left(p_{n}\right)$ eine positive monoton ins Unendliche wachsende Zahlenfolge sein soll.

192. Man beweise die Gleichungen
a) $\sum \frac{z^{n}}{1-z^{n}}=\sum z^{n^{2}} \cdot \frac{1+z^{n}}{1-z^{n}}$
b) $\Sigma^{\prime}(-1)^{n-1} \frac{z^{2 n-1}}{1-z^{2 n-1}}=\sum \frac{z^{n}}{1+z^{2 n}}$,

bei denen die Summation mit $n=1$ beginnen soll. 
193. Dem Satz von Landau (271) entsprechend gilt der folgende: Die Dirichletsche Reihe $\Sigma(-1)^{n-1} \frac{a_{n}}{n^{z}}$ einerseits und die sogen. Binomialkoffizientenreihe $\Sigma a_{n}\left(\begin{array}{c}z-1 \\ n\end{array}\right)$ andrerseits sind stets gleichzeitig konvergent und gleichzeitig divergent.

194. Für welche $z$ gilt die Gleichung

$$
\sum_{n=0}^{\infty}(-1)^{n}\left(\begin{array}{c}
z-1 \\
n
\end{array}\right)=0 \text { ? }
$$

195. Man stelle das genane Konvergenzgebiet der folgenden Produkte fest:
a) $I I\left(1-\frac{1}{n^{z}}\right)$,
b) $\Pi\left(1-\frac{(-1)^{n}}{n^{z}}\right)$
c) $\Pi\left(1+z^{2 n+1}\right)$
d) $\Pi\left(1+n^{2} z^{n}\right)$;
e) $\Pi\left(1-\frac{z^{n}}{1-z^{n}}\right)$,
f) $\Pi\left[\left(1+\frac{z}{n}\right)\left(1-\frac{1}{n}\right)^{z}\right]$,
g) $\Pi\left[\left(1-\frac{z}{z_{n}}\right) e^{\frac{z}{z_{n}}+\frac{1}{2}\left(\frac{z}{z_{n}}\right)^{2}+\ldots+\frac{1}{n}\left(\frac{z}{z_{n}}\right)^{n}}\right.$, wenn $\left|z_{n}\right| \rightarrow+\infty$ strebt,
h) $\Pi\left(1-\frac{z}{n}\right)$,
i) $\Pi\left(1-\frac{(-1)^{n}}{n} z\right)$,
k) $\Pi\left(1-\frac{(-1)^{n}}{2 n+1} z\right)$.

196. Man bestimme mit Hilfe des sin-Produktes die Werte der Produkte
a) $I I\left(1+\frac{x^{2}}{n^{2}}\right)$
b) $\Pi\left(1+\frac{x^{4}}{n^{4}}\right)$,
c) $\Pi\left(1+\frac{x^{6}}{n^{6}}\right)$

für reelle $x$. Das zweite von ihnen hat den Wert

$$
2 \pi^{2} x^{2}[\operatorname{coshyp}(\pi x \sqrt{2})-\cos (\pi x \sqrt{2})] .
$$

Gilt dies auch noch für komplexe Werte von $x$ ?

197. Die Werte der Produkte 195 i) und k) lassen' sich mit Hilfe der $\Gamma$-Funktion geschlossen angeben.

19.9. Es ist

$$
(1-z)\left(1-\frac{1}{\left.z^{3}\right)\left(1-z^{5}\right) \ldots}=(1+z)\left(1+z^{2}\right)\left(1+z^{3}\right) \cdots\right.
$$

199. Mit Hilfe des sin-Produktes bzw. der ctg-Partialbruchzerlegung lassen sich die folgenden Reihen und Produkte geschlossen auswerten, in denen $x$ und $y$ reell sein sollen:
a) $\sum_{n=-\infty}^{+\infty} \frac{1}{(n+x)^{2}+y^{2}}$,
b) $\sum_{n=-\infty}^{+\infty} \frac{1}{n^{4}+x^{4}}$
c) $\sum_{n=-x}^{+\infty} \frac{1}{(x-n)^{3}}$
d) $\sum_{n=-\infty}^{+\infty}\left(1-\frac{4 x^{2}}{(n+x)^{2}}\right)$. 
XIII. Kapitel.

\section{Divergente Reihen.}

\section{§ 59. Allgemeine Bemerkungen über divergente Reihen.}

Die Auffassung von dem Wesen der unendlichen Reihen, wie wir sie in allem Vorangehenden, insbesondere in $\S 11$ dargelegt haben, ist vergleichsweise neuen Datums. Denn ein strenger und einwandfreier Aufbau der Theorie war erst möglich, nachdem der Begriff der reellen Zahl geklärt war. Aber selbst wenn man es gelten läßt, $d a ß$ dieser Begriff und mit ihm irgendein allgemeines Konvergenzkriterium für Zahlenfolgen, etwa unser 2. Hauptkriterium, als von fast axiomatischer Natur ohne Beweis einfach anerkannt wird, so ist immer noch die Theorie der unendlichen Reihen viel jüngeren Datums als ihr ausgiebiger Gebrauch und als die Entdeckung ihrer schönsten Resultate etwa durch Euler und seine Zeitgenossen, oder gar schon durch Leibniz und Newton und deren Zeitgenossen. Diesen boten sich die unendlichen Reihen in der natürlichsten Weise als Rechnungsergebnisse dar, drängten sich ihnen sozusagen auf, wie z. B. die geometrische Reihe $1+x+x^{2}+\ldots$ als nicht abbrechendes Divisionsergebnis von $1:(1-x)$, die Taylorsche Reihe und mit ihr fast alle Reihen des VI. Kapitels durch das Prinzip der Koeffizientenvergleichung oder aus geometrischen Erwägungen heraus. Es wurde also nicht, wie wir es in unserer Darstellung getan haben, das Symbol der unendlichen Reihen geschaffen und nun mit ihm gearbeitet, sondern die unendlichen Reihen waren $d a$, und es galt sich mit ihnen auseinanderzusetzen.

Daher lagen Konvergenzfragen im heutigen Sinn diesen Mathematikern zunächst noch ganz fern ${ }^{1}$ ). Und so ist es nicht zu verwundern, daß z. B. Euler die geometrische Reihe

$$
1+x+x^{2}+\ldots=\frac{1}{1-x}
$$

auch für $x=-1$ oder $x=-2$ noch gelten läßt und also unbedenklich

$$
\begin{aligned}
& \left.1-1+1-1+-\ldots=\frac{1}{2}^{2}\right) \\
& 1-2+2^{2}-2^{3}+-\ldots=\frac{1}{3}
\end{aligned}
$$

1) Vgl. die Bemerkungen S. 290.

2) Diese Gleichung tritt schon bei Jak. Bernoulli (Posit. arithm., 3. Teil, Basel 1696) auf und wird von ihm als "paradoxon non inelegans" bezeichnet. Näheres über die heftige Fehde, die sich hieran anschloß, findet man in dem S. 99 genannten Buche von $R$. Reiff. 
setzt, und entsprechend etwa aus $\left(\frac{1}{1-x}\right)^{2}=1+2 x+3 x^{2}+\ldots$ die Gleichung

$$
1-2+3-4+-\ldots=\frac{1}{4}
$$

herleitet und vieles andere mehr. Freilich hielt die meisten Mathematiker jener Zeiten eine instinktive Scheu von solchen Ergebnissen ab und lie $B$ sie nur solche Resultate anerkennen, die auch in unserm heutigen Sinn richtig sind. Aber eine klare Einsicht in die Gründe, warum das eine Ergebnis anerkannt wurde, das andere nicht, fehlte ihnen damals noch.

Es ist hier nicht der Raum, auf die sehr lehrreichen Auseinandersetzungen zwischen den Mathematikern des 17. und 18. Jahrhunderts über diesen Punkt einzugehen ${ }^{1}$ ). Wir müssen uns damit begnügen, festzustellen, daß Euler eine unendliche Reihe stets dann gelten ließ, wenn sie sich auf natürliche Weise durch Entwicklung eines analytischen Ausdrucks einstellte, der seinerseits einen bestimmten Wert besaß2). Dieser letztere wurde dann in jedem Falle als Summe der Reihe angesehen.

Es ist klar, daß diese Übereinkunft keine strenge Grundlage hat. Wenn z. B. die Reihe $1-1+1-1+-\ldots$ auch in der einfachsten Weise aus der Division von $1:(1-x)$ für $x=-1$ entsteht (s. o.), und also $=\frac{1}{2}$ zu setzen wäre, so ist doch nicht einzusehen, warum nicht dieselbe Reihe auch aus ganz andern analytischen Ausdrücken sollte entstehen können und auf Grund solcher anderweitigen Entstehung nun auch einen andern Wert erhalten müßte. In der Tat entsteht die obige Reihe auch aus der Funktion $f(x)$, die für $x>0$ durch die Dirichletsche Reihe

$$
f(x)=\sum_{n=1}^{\infty} \frac{(-1)^{n-1}}{n^{x}}=1-\frac{1}{2^{x}}+\frac{1}{3^{x}}-\frac{1}{4^{x}}+\ldots
$$

dargestellt wird, für $x=0$ oder aus

$$
\frac{1+x}{1+x+x^{2}}=\frac{1-x^{2}}{1-x^{3}}=1-x^{2}+x^{3}-x^{5}+x^{6}-x^{5}+\ldots
$$

für $x=1$. Auf Grund der letzteren Entstehung müßte $1-1+\ldots=\frac{2}{3}$ gesetzt werden und bei der andern ist nicht ohne weiteres zu sehen, was $f(0)$ für einen Wert haben mag. Er könnte jedenfalls von $+\frac{1}{2}$ verschieden sein.

Das Eulersche Prinzip ist also jedenfalls unsicher, und nur der ungewöhnliche Instinkt Eulers für das mathematisch Richtige hat ihn

1) Näheres in R. Reif, a. a. O.

$\left.{ }^{2}\right)$ In einem Briefe an Goldbach (7. VIII. 1745) sagt er geradezu: „... so habe ich diese neue Definition von der Summe einer jeglichen seriei gegeben: Summa cujusque seriei est valor expressionis illius finitae, ex cujus evolutione illa series oritur". 
trotz der ausgiebigen Benutzung solcher divergenten Reihen davor bewahrt, falsche Ergebnisse zu zeitigen ${ }^{1}$ ). Erst Cauchy und $A b e l$ klärten den Konvergenzbegriff und verwarfen alle nicht konvergenten Reihen; Cauchy in seiner Analyse algébrique (1821), Abel in seiner Arbeit über die Binomialreihe (1826), die sich schon ausdrücklich auf das Cauchysche Werk stützt. Beide haben sich erst zögernd zu diesem entscheidenden Schritte entschlossen ${ }^{2}$ ), doch erschien er schließlich beiden unvermeidlich, um ihre Schlüsse zu lückenlos scharfen zu machen.

Wir sind heute in der Lage, das Problem sozusagen von oben her zu überschauen; und da werden die Dinge sofort klar, wenn wir uns erinnern, daß das Symbol der unendlichen Reihen, wie oft betont, von sich aus keinerlei Bedeutung hat noch haben kann, sondern daß ihm eine solche erst von uns durch freie Übereinkunft gegeben wird. Und diese Verabredung besagte, daß die unendliche Reihe lediglich ein anderes Symbol für die Folge ihrer Teilsummen sein sollte. So naheliegend und naturgemäß nun auch diese Festsetzung ist und so eng sie sich auch an die Entstehung der unendlichen Reihen (etwa als Entwicklung eines Ausdrucks in eine Summe von immer mehr und mehr Summanden) anschließen mag, so ist die Festsetzung doch unter allen Umständen als eine willkürliche zu bezeichnen, und sie könnte auch durch ganz andere ersetzt werden. Nur die Zweckmäßigkeit und der Erfolg ist hier entscheidend, ob die eine oder die andere Festsetzung vorzuziehen ist; in der Natur der Sache selbst, d. h. in dem Symbol , $\Sigma a_{n}$ " einer unendlichen Reihe liegt kein bindender Anhalt dafür vor.

Daher ist die Frage sehr wohl berechtigt, ob die (wenigstens stellenweis beträchtliche) Kompliziertheit der Theorie der unendlichen Reihen nicht etwa darauf zurückzuführen ist, daß unsere -- wenn auch scheinbar noch so naheliegende - Deutung des Symbols " $\Sigma a_{n}{ }^{\prime \prime}$ doch eine ungünstige ist. Denn in der mannigfachsten Weise könnte man andere Festsetzungen treffen, unter denen dann - vielleicht wenigstens - sich auch zweckmäßigere fänden. Wir geben zunächst das folgende erste Beispiel für eine solche anders geartete Übereinkunft bezüglich der Deutung von " $\Sigma a_{n}{ }^{\prime \prime}$ : Wir setzen wie bisher

$$
a_{0}+a_{1}+\ldots+a_{n}=s_{n} \text {, }
$$

1) Vgl. dagegen S. 126, Fufin. 1.

2) Man vergl. hierzu beziiglich Cauchy das Vorwort zur Analyse algébrique, wo es u. a. heifst: Je me suis vu forcé d'admettre plusieurs propositions qui paraîtront peut-être un peu dures, par exemple qu'une serie divergente n'a pas de somme. Und bezüglich Abel seinen Brief an Holmboe (16. I. 1826), in dem es heißst: Les séries divergentes sont, en général, quelque chose de bien fatal, et c'est une honte qu'on ose $\mathrm{y}$ fonder aucune démonstration. - Übrigens hat sich auch schon $J$. d'Alembert 1768 in ähnlichem Sinne geäufert. 
betrachten aber nun die Zahlenfolge

$$
s_{n}^{\prime}=\frac{s_{0}+s_{1}+\ldots+s_{n}}{n+1} \quad(n=0,1,2, \ldots)
$$

und definieren: Dann und nur dann, wenn $s_{n}^{\prime}$ einem Grenzwert $s$ zustrebt, soll $\sum a_{n}$ "konvergent" mit der "Summe" $s$ heißen.

Wir prüfen ganz kurz die Zweckmäßigkeit dieser Festsetzung. Zunächst, wenn $s_{n} \rightarrow s$ strebt, wenn also die Reihe im alten Sinne konvergent ist $^{1}$ ) und die Summe $s$ hat, so strebt nach 43,2 auch $s_{n}^{\prime} \rightarrow s$. Dies besagt aber, daß der neue Konvergenzbegriff mit dem alten nicht nur nicht in Widerspruch steht, sondern ihn als speziellen Fall enthält, d. h. jede Reihe, die im alten Sinne konvergent war, würde es auch im neuen Sinne sein. Der neue Begriff leistet aber tatsächlich mehr. Denn ist $\Sigma a_{n}$ die oben mehrfach genannte Reihe

$$
\sum_{n=0}^{\infty}(-1)^{n} \equiv 1-1+1-1+\ldots
$$

so ist $s_{0}=1, s_{1}=0, s_{2}=1, \ldots, s_{n}=\frac{1+(-1)^{n}}{2}$ und also

$$
s_{n}^{\prime}=\frac{s_{0}+s_{1}+\ldots+s_{n}}{n+1}=\frac{(n+1)+\frac{1}{2}\left(1+(-1)^{n}\right)}{2(n+1)}=\frac{1}{2}+\frac{1+(-1)^{n}}{4(n+1)}
$$

Hier strebt also $s_{n}^{\prime} \rightarrow \frac{1}{2}$ und wir hätten durch diese Festsetzung die Reihe $1-1+1-+\ldots$ zu einer "im neuen Sinne konvergenten" Reihe mit der Summe $\frac{1}{2}$ gemacht. "Das wäre eine durchaus einwandfreie Festsetzung.

Durch diese beiden Feststellungen hätte dieser neue Konvergenzbegriff schon eine beträchtliche Zweckmäßigkeit bekundet, und man könnte auf ihm als Grundlage eine entsprechende neue Theorie der unendlichen Reihen aufbauen. Selbstverständlich könnte man noch in mannigfach andrer Weise einen „neuen Konvergenzbegriff“ einführen und ihn auf seine Zweckmäßigkeit hin prüfen. Wir werden das gleich nachher noch an einigen Beispielen erläutern. Doch wollen wir uns von vornherein klarmachen, welche Eigenschaften ein solch „neuer Konvergenzbegriff“" haben soll, damit wir seine Einführung als zweckmäßig anerkennen können.

1) Sobald man verschiedene Konvergenzbegriffe bei Reihen einführt, würde die Gefahr von Mißsverständnissen entstehen. Bei dem Begriff der Integrierbarkeit, wo etwas Ähnliches vorliegt, pflegt man durch Hinzufügung einer Initiale dieser Gefahr vorzubeugen. So spricht man von Funktionen, die integrierbar $(R)$ oder integrierbar $(L)$ sind, je nachdem man Integrierbarkeit im Riemannschen Sinne oder im Lebesgueschen Sinne meint. Ebenso könnte man hier eine Reihe $(A)$-konvergent oder $(N)$-konvergent nennen, je nachdem sie im alten oder in dem oben als Beispiel eingefuhrten neuen Sinne konvergent ist. Doch werden wir gleich sehen, dafs man diese Verwechselungsgefahr auf andere Weise vermeidet. 
$\mathrm{Da}$ wäre $\mathrm{zu}$ allererst $\mathrm{zu}$ verlangen, daß der neue Begriff mit dem alten nicht in Widerspruch steht. Denn unser bisheriger Konvergenzbegriff bei den unendlichen Reihen war ein viel zu naturgemäßer und hat sich in viel zu hervorragender Weise bewährt, als $\mathrm{da} B$ von einer Deutung des Symbols " $\Sigma a_{n}{ }^{\prime}$, die mit ihm im Widerspruch steht ${ }^{1}$ ), Nutzen zu erwarten wäre. Sondern: Eine Reihe $\Sigma a_{n}$, die im alten Sinne konvergent war, muß es auch - und zwar mit ungeänderter "Summe" - bleiben, wie immer man den Konvergenzbegriff etwa definieren mag. Das ist die Hauptforderung, die wir als Permanenzbedingung bezeichnen wollen. Doch müßte man weiter wohl verlangen, daß z. B. aus der „Konvergenz" von $\sum_{n=0}^{\infty} a_{n}$ auch diejenige von $\sum_{n=1}^{\infty} a_{n}$ folgte, und daß, wenn $s$ und $s^{\prime}$ ihre Summen sind, $s=a_{0}+s^{\prime}$ sei; müßte wohl auch verlangen, daß man „konvergente“ Reihen gliedweis addieren darf und einiges andere mehr ${ }^{2}$ ).

Solche Forderungen sind natürlich nicht völlig unerläßlich; aber je mehr von solchen naheliegenden Forderungen ein etwaiger neuer Konvergenzbegriff erfüllt, desto zweckmäßiger wird er uns erscheinen.

Endlich aber: damit die Einführung eines solchen neuen Begriffes sich lohnt, muß es Reihen geben, die im alten Sinne divergent waren, im neuen aber "konvergent" sind, - wie dies bei dem oben als Beispiel eingeführten neuen Konvergenzbegriff bezüglich der Reihe $\Sigma(-1)^{n}$ der Fall war. Und wir können dann gleich sagen: Je mehr (im alten Sinne) divergente Reihen bei der neuen Fassung des Begriffes zu konvergenten werden, desto vorteilhafter wird es sein, diesen neuen Begriff einzuführen.

Unter diesen Gesichtspunkten wollen wir nun einige neue Arten, dem Symbol $\sum a_{n}$ eine Bedeutung beizulegen, näher untersuchen. Doch sei im voraus schon betont, daß keiner dieser neuen Konvergenzbegriffe unserm bisherigen an Bedeutung gleichkommt. Um daher die Benennung der Begriffe zu vereinfachen, ist es vorteilhafter, unter einer konvergenten unendlichen Reihe $\Sigma a_{n}$ nach wie vor allein das $\mathrm{zu}$ verstehen, was wir bisher darunter verstanden haben, und für etwaige neue Festsetzungen auch neue Namen einzuführen. In dieser Beziehung setzen wir fest: Wenn dem Symbol. ", $\sum_{n=0}^{\infty} a_{n}$ "durch irgendein neues Verfahren (wie etwa das vorhin beschriebene) ein Sinn und ein

1) Die also z. B. einer im alten Sinne konvergenten Reihe mit der Summe $s$ eine davon verschiedene Summe $s^{\prime}$ zuspräche, oder sie als divergent bezeichnete.

2) Welche Forderungen der bezeichneten Art als zweckmäßig anzusehen sind, ist von G. Doetsch in seiner Inaug.-Diss. „Eine neue Verallgemeinerung der Borelschen Summabilitätstheorie“, Göttingen 1920, genauer diskutiert worden. 
Wert s beigelegt wird, so wollen wir die Reihe durch dieses Verfahren summierbar nennen mit dem Werte s, oder auch einfach weil gar kein Mißverständnis zu befürchten ist - mit der Summe s. So können wir auf Grund des vorhin beschriebenen Verfahrens sagen: Die Reihe $1-1+1-+\ldots$ ist summierbar "durch die Methode der arithmetischen Mittel erster Ordnung “ mit der Summe $\frac{1}{2}$, oder etwa kurz (s. S. 444, Fußn.): sie sei $(M 1)$-summierbar mit der Summe $\frac{1}{2}{ }^{1}$ ).

Wir gehen nun daran, weitere Beispiele für „Summierungsverfahren" zu geben, d. h. für anders geartete Methoden, dem (an sich - es sei dies erneut betont - bedeutungslosen) Symbol $\Sigma a_{n}$ einen Sinn beizulegen:

1. Art (Verfahren der arithmetischen Mittel). Wenn wir nach Bildung der Teilsummen $s_{n}$ und der arithmetischen Mittel 1. Ordnung

$$
s_{n}^{\prime}=\frac{s_{0}+s_{1}+\ldots+s_{n}}{n+1} \quad(n=0,1,2, \ldots)
$$

statt sogleich nach einem etwaigen Grenzwert der $s_{n}^{\prime}$ zu fragen, erst erneut die Mittel

$$
s_{n}^{\prime \prime}=\frac{s_{0}{ }^{\prime}+s_{1}{ }^{\prime}+\ldots+s_{n}{ }^{\prime}}{n+1} \quad(n=0,1,2, \ldots)
$$

bilden und nun erst nach dem Grenzwert der Folge $\left(s_{n}^{\prime \prime}\right)$ fragen, so kann man definieren: Die Reihe $\sum a_{n}$ heiße ( $M$ 2)-summierbar ${ }^{2}$ ) mit der Summe $s$, wenn $s_{n}^{\prime \prime} \rightarrow s$ strebt.

Man sieht sofort, daf diese Methode die vorhin beschriebene der ( $M 1)$ Summierbarkeit und damit die gewöhnliche Konvergenz als Spezialfall enthält, also die Permanenzbedingung erfullt, andererseits aber weiter reicht als jene. So ist z. B., wie man ziemlich leicht nachrechnet, die Reihe

$$
\sum_{n=0}^{\infty}(-1)^{n}(n+1) \equiv 1-2+3-4+5-+\cdots
$$

(M 2)-summierbar, - aber nicht ( $M$ 1)-summierbar oder gar konvergent mit der Summe $\left.\frac{1}{4}^{3}\right)$. Denn die $s_{n}^{\prime}$ sind für $n=0,1,2_{n-1}$. der Reihe nach $=1,0, \frac{2}{3}, 0, \frac{3}{5}, 0, \frac{4}{7}, \ldots$; die $s_{n}^{\prime} \rightarrow$ streben also keinem Grenzwert $z \mathrm{u}$, während nun $s_{n}^{\prime \prime} \rightarrow \frac{1}{4}$ strebt.

Geht man auch bei den $s_{n}^{\prime \prime}$ noch nicht zur Grenze über, sondern bildet erst

$$
s_{n}^{\prime \prime \prime}=\frac{s_{0}^{\prime \prime}+s_{1}^{\prime \prime}+\ldots+s_{n}^{\prime \prime}}{n+1}
$$

$$
(n=0,1,2, \ldots)
$$

1) Werden verśchiedene Verfahren zur Summierung benutzt, so unterscheidet man sie voneinander durch eine dem Worte summierbar beigefuigte Initiale.

2) Genauer also: Summierbar durch die Methode der zweimaligen Bildung arithmetischer Mittel.

$\left.{ }^{3}\right)$ Der S. 442 genannten paradoxen Eulerschen Gleichung liegt also doch ein tieferer (rein arithmetischer) Sinn zu Grunde. 
und allgemein

$$
s_{n}^{(r)}=\frac{s_{0}^{(r-1)}+s_{1}^{(r-1)}+\ldots+s_{n}^{(r-1)}}{n+1} \quad(n=0,1,2, \ldots)
$$

und es strebt nun (bei einem bestimmten $r$ ) $s_{n}^{(r)} \rightarrow s$, so können wir analog definieren: Die Reihe $\Sigma a_{n}$ heiße $(\boldsymbol{M} r)$-summierbar mit der Summe $s$, wenn $s_{n}^{(r)} \rightarrow s$ strebt $\left.{ }^{1}\right)$. Jedes dieser Verfahren $(\operatorname{mit} r=1,2, \ldots)$ enthält die vorangehenden und damit den alten Konvergenzbegriff, den man als dem Index $r=0$ entsprechend ansehen kann, als Spezialfall in sich und jede ist, wie einfache Beispiele lehren, umfassender als die vorangehende.

Die Prüfung, ob und inwieweit diese Summierungsmethode die übrigen oben aufgestellten $Z$ weckmäßigkeitsforderungen erfüllt, müssen wir dem Leser überlassen.

2. Art (Cesàrosches Verfahren $\left.)^{2}\right)$ : Wir bilden wieder die Teilsummen von $\Sigma a_{n}$, bezeichnen sie aber jetzt mit $S_{n}^{(0)}=s_{n}=a_{0}+a_{1}+\ldots+a_{n}$ und setzen dann weiter

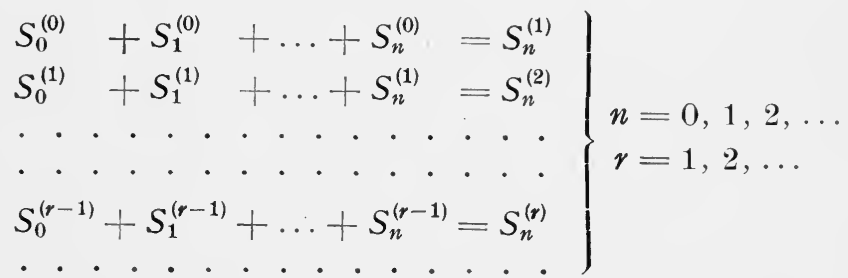

und untersuchen für ein bestimmtes $r$ das Konvergenzverhalten der Folge

$$
\sigma_{n}^{(r)}=\frac{S_{n}^{(r)}}{\left(\begin{array}{c}
n+r \\
r
\end{array}\right)} \quad n=0,1,2, \ldots
$$

und definieren nun: Die Reihe $\Sigma a_{n}$ heiße $(C r)$-summierbar mit der Summe $s$, wenn (bei festem $r$ ) die Folge $\sigma_{n}^{(r)} \rightarrow s$ strebt $^{3}$ ).

Daßs auch dieses Verfahren die Permanenzbedingung erfüllt, wird uns der Satz des $\S 60$ lehren und uiber das Verhältnis dieses Verfahrens zum voran-

1) Arithmetische Mittel dieser Art sind zum ersten Male von O. Hölder (Math. Annalen, Bd. 20 (1882), S. 535) für die Theorie der unendlichen Reihen nutzbar gemacht worden; man nennt sie darum auch Höldersche Mittel.

2) Bull. des sciences math., Bd. (2) 14 (1890), S. 114. - Verf. hat die Cesàrosche Methode in der Weise verallgemeinert, daf $r$ eine beliebige reelle Zahl $>-1$ sein darf (Inaug.-Diss., Berlin 1907, S. 46, und Sitzungsber. d. Berl. math. Ges., Bd. 7 (1907), S. 1).

$\left.{ }^{3}\right)$ Genauer: Summierbar mit der Summe $s$ nach der Methode der Cesàroschen Mittel $r$ ter Ordnung. (C 1)- und $(M 1)$-Summierbarkeit sind hiernach identisch. Ebenso $(C 0)$ - und $(M 0)$-Summierbarkeit, die beide als identisch mit der bisherigen Konvergenz anzusehen sind. 
gehenden, vgl. den dortigen Äquivalenzsatz. Beispiele für dieses Verfahren wenigstens für den Fall $r=1$, in welchem es mit dem vorangehenden identisch ist - werden wir in jenem Paragraphen genauer kennen lernen, so dafi sie sich im Augenblick erïbrigen.

3. Art (Abelsches Verfahren). Ist $\Sigma a_{n}$ vorgelegt, so betrachten wir die Potenzreihe $\Sigma a_{n} x^{n}$ und nennen die Reihe $\Sigma a_{n}$ nun (A)-summierbar mit der Summe s, wenn der Radius dieser Potenzreihe $\geqq 1$ ist und wenn

ist.

$$
\lim _{x \rightarrow 1-0}\left(\sum a_{n} x^{n}\right)=s
$$

Infolge des Abelschen Grenzwertsatzes erfüllt auch dieses Verfahren die Permanenzbedingung, - ist aber weiter als diese, da z. B.

$$
\sum_{n=0}^{\infty}(-1)^{n}=\lim _{x \rightarrow 1-0}\left(\sum(-1)^{n} x^{n}\right)=\lim _{x \rightarrow 1} \frac{1}{1+x}=\frac{1}{2}
$$

zu setzen ist. Ebenso wird nach dieser Methode

$$
1-\left(\begin{array}{c}
p+1 \\
p
\end{array}\right)+\left(\begin{array}{c}
p+2 \\
p
\end{array}\right)-\left(\begin{array}{c}
p+3 \\
p
\end{array}\right)+-\ldots=\frac{1}{2^{p+1}}
$$

$z u$ setzen sein, weil

$$
\sum_{n}(-1)^{n}\left(\begin{array}{c}
n+p \\
p
\end{array}\right) x^{n}=\left(\begin{array}{c}
1 \cdot \\
1+x
\end{array}\right)^{p+1} \rightarrow \frac{1}{2^{p+1}} \text { strebt für } x \rightarrow 1-0 .
$$

In $\$ 62$ werden wir auch sehen, dafi dieses $A$ belsche Verfahren ${ }^{1}$ ) das Höldersche und das Cesàrosche als Spezialfälle enthält und also das wirksamste aller bisher genannten Verfahren darstellt. Man kann es auch in folgende Form kleiden: Man bilde die Teilsummen $s_{n}$ von $\Sigma a_{n}$ und setze die Potenzreihe $\Sigma s_{n} x^{n}$ dadurch in Vergleich zur geometrtschen Reihe $\Sigma x^{n}$, daf man den Quotienten

$$
\begin{gathered}
\Sigma s_{n} x^{n} \\
\Sigma x^{n}
\end{gathered}
$$

für $x \rightarrow 1-0$ untersucht. Strebt er einem Grenzwert $s \quad z u$, so soll $\Sigma a_{n}$ nun $(A)$-summierbar heifen mit der Summe $s$. Das ist offenbar dasselbe, da der hingeschriebene Quotient $=\Sigma a_{n} x^{n}$ ist (vgl. 102). Diese Auffassung macht die Entstehung des folgenden, zunächst etwas fremdartig erscheinenden Verfahrens begreiflich:

4. Art (Borelsches Verfahren). E. Borel ${ }^{2}$ ) sucht dem Abelschen Verfahren, welches doch nur zum Ziele führen kann, wenn $\sum a_{n} x^{n}$ oder $\sum s_{n} x^{n}$ mindestens den Einheitskreis zum Konvergenzkreis hat, dadurch eine größere Wirksamkeit zu verleihen, daß er in ganz analoger Weise die Reihen

$$
\perp s_{n} \frac{x^{n}}{n !} \quad \text { und } \quad \leq \frac{x^{n}}{n !}
$$

1) Es ist natürlich nicht von $A b e l$ als solches geschaffen, stützt sich aber so unmittelbar auf den Abelschen Grenzwertsatz, dafis es gerechterweise nach ihm benannt werden muf.

2) Sur la sommation des séries divergentes, C. R. de l'Acc. Paris, Bd. 121 (1895), S. 1125 - und in vielen späteren Abhandlungen. Eine zusammenfassende Darstellung gab er in seinen Leçons sur les séries divergentes, Paris 1901. 
und ihren Quotienten - oder also das Produkt

$$
e^{-x} \cdot \sum_{n=0}^{\infty} s_{n}{ }^{x^{n}} n
$$

für $x \rightarrow+\infty$ untersucht. Strebt es dabei einem Grenzwert zu, so soll $\sum a_{n}$ nun (B)-summierbar heißen mit der Summes.

Nehmen wir etwa, um dieses Verfalıren ein wenig zu erläutern, zunächst wieder $\Sigma a_{n} \equiv \sum_{n=0}^{\infty}(-1)^{n}$, so ist $s_{n}=1$ oder $=0$, je nachdem $n$ gerade oder ungerade ist. Also wird

$$
\perp^{\prime} \frac{x^{n}}{n !}=1+\frac{x^{2}}{2 !}+\frac{x^{4}}{4 !}+\ldots=\frac{e^{x}+e^{-x}}{2},
$$

und es handelt sich um den Grenzwert

$$
\lim _{x \rightarrow+\infty} e^{-x} \cdot e^{x}+e^{-x}
$$

der offenbar $=\frac{1}{2}$ ist. $\stackrel{Y}{(-1)^{n}}$ ist also auch $(B)$-summierbar mit der Summe $\frac{1}{2}$.

Dies Verfahren erfüllt auch die Permanenzbedingung; denn ist $\Sigma a_{n}$ im alten Sinne konvergent, strebt also $s_{n} \rightarrow s$, so ist diese Reihe auch $(B)$-summierbar mit der Summe $s$; denn es ist

$$
\left(e^{-x} \cdot \sum_{n=0}^{\infty} x_{n} \frac{x^{n}}{n !}\right)-s=e^{-x} \cdot \sum_{n=0}^{\infty}\left(s_{n}-s\right) \cdot \frac{x^{n}}{n !}
$$

und wählt man bei gegebenem $\varepsilon>0$ ein $m$ so grof, dái für $n>m$ stets $\left|s_{n}-s\right|<\frac{\varepsilon}{2}$ bleibt, so ist der Betrag dieses Ausdrucks für positive $x$

$$
\leqq e^{-x} \cdot \sum_{n=0}^{\infty}\left|s_{n}-s\right| \frac{x^{n}}{n !} \leq e^{-x} \cdot \sum_{n=0}^{m}\left|s_{n}-s\right| \cdot \frac{x^{n}}{n !}+\frac{\varepsilon}{2} .
$$

Da aber für $x \rightarrow+\infty$ des Produkt von $e^{-x}$ mit einem Polynom $m$ ten Grades $\rightarrow 0$ strebt, so kann $\xi$ so grof gewählt werden, daß für $x>\xi$ dieses Produkt $<\frac{\varepsilon}{2}$ ist. Dann ist der Betrag des ganzen Ausdrucks $<\varepsilon$ und somit unsere Behauptung bewiesen ${ }^{1}$ ).

Ohne auf dieses Borelsche Verfahren näher einzugehen, wollen wir doch betonen, daß es wesentlich wirksamer ist als das Abelsche - und also auch als das Cesàrosche und Höldersche - ${ }^{2}$ ), und daßs es doch noch als ein ver-

1) Auch dieser Satz ist in dem S. 377 formulierten allgemeinen Satze als Spezialfall enthalten.

$\left.{ }^{2}\right)$ So ist $z$. B. die geometrische Reihe $\sum_{n=0}^{\infty}(-1)^{n} a^{n}$ für $0 \leqq a<1$ konvergent im alten Sinne; für $a=+1$ ist sie summierbar nach allen 4 bisher beschriebenen Verfahren und jedesmal mit der Summe $\frac{1}{2}$. Aber nach dem Borelschen Verfahren ist sie auch noch für alle $a>1$ summierbar und jedesmal mit der Summe $\frac{1}{1+a}$. In der Tat ist $s_{n}=1-a+a^{2}-+\cdots+(-1)^{n} a^{n}=\frac{1+(-1)^{n} a^{n+1}}{1+a}$ und für $x \rightarrow+\infty$ strebt nun

$$
e^{-x} \cdot \sum s_{n} \frac{x^{n}}{n !}=\frac{1}{1+a}+\frac{a}{1+a} e^{-(a+1) x} \rightarrow \frac{1}{1+a} \text {. }
$$

Man erkennt sogar, dafi alles dies schon gilt, wenn nur $\mathfrak{R}(a)>-1$ ist.

Knopp, Unendliche Reihen. 
hältnismäfig naheliegendes bezeichnet werden muß́. Auch ist sein Gebrauch noch vergleichsweise einfach.

5. Art (Erweitertes Borelsches Verfahren.) Statt wie eben die Exponentialreihe selbst zu nehmen, benutze man die wirksamere Reihe

$$
\sum_{n=0}^{\infty} \frac{x^{r n}}{(r n) !}
$$

in der $r$ eine feste ganze Zahl $>1$ bedeuten soll. Man untersuche also für $x \rightarrow+\infty$ den Quotienten der beiden Funktionen

$$
\sum_{n=0}^{\infty} s_{n} \frac{x^{r n}}{(r n) !} \quad \text { und } \quad \sum_{n=0}^{\infty} \frac{x^{r n}}{(r n) !}
$$

oder, was auf dasselbe hinauskommt, das Produkt

$$
r \cdot e^{-x} \cdot \sum_{n=0}^{\infty} s_{n} \frac{x^{r n}}{(r n) !}
$$

und nenne die Reihe $\Sigma a_{n}$ nun $(B r)$-summierbar mit der Summe $s$, wenn es dabei dem Grenzwert $s$ zustrebt.

Durch dies Verfahren (für $r=2$ ) ist z. B. sogar die Reihe $1 !-2 !+3 !-4$ ! $+5 !-+\ldots$ summierbar, trotzdem die zugehörige Potenzreihe $\Sigma(-1)^{n-1} n ! x^{n}$ nirgends konvergiert (s. 230,5). Ihre durch diese Methode bestimmte Summe ist $=0,40365 \ldots{ }^{1}$ ).

Der gemeinsame offene oder etwas versteckte Grundgedanke aller beschriebenen Verfahren ist der, dafi den Gliedern der Reihe $\Sigma a_{n}$ variable Faktoren zugeteilt werden, die die Reihe konvergent (im alten Sinne) machen und dadurch den Einfluf der fernen Gliedern zunächst herabdrücken, die aber allmählich gegen 1 steigen und so schlieflich doch alle Glieder zur vollen Wirksamkeit heranziehen. So ist z. B. bei der ( $M$ 1)- oder $(C 1)$-Summierbarkeit $s_{n}^{\prime}=\frac{s_{0}+s_{1}+\ldots+s_{n}}{n+1}=a_{0}+\left(1-\frac{1}{n+1}\right) a_{1}+\left(1-\frac{2}{n+1}\right) a_{2}+\ldots+\left(1-\frac{n}{n+1}\right) a_{n}$

und man sieht hier deutlich, wie die einzelnen Glieder immer stärker, bis zum vollen Gewicht, herangezogen werden, wenn $n$ wächst. Bei der $(A)$-Summierbarkeit sind es die Faktoren $x^{n}$, welche den Einfluf der fernen Glieder herabmindern (da $x<1$ ist), die aber diesen Einflufs immer stärker werden lassen, wenn $x \rightarrow 1$ wächst. Auch das Borelsche Verfahren läfst sich so interpretieren, nur da 6 bei ihr die Teilsummen, nicht die Glieder der Reihe, in Erscheinung treten. Am deutlichsten ist dieses Prinzip zur Grundlage der folgenden Methode gemacht:

6. Art (Le Roy's Verfahren ${ }^{2}$ ). Es sei $x$ ein positiver echter Bruch und

$$
\sum_{n=0}^{\infty} \frac{\Gamma(n x+1)}{n !} a_{n}
$$

sei konvergent für alle $0 \leqq x<1$. Die Summe dieser Reihe (im alten Sinne) ist dann eine Funktion von $x$ in dem genannten Intervall; strebt sie für $x \rightarrow 1-0$

1) Dieses merkwürdige Resultat findet sich schon - obwohl auf ganz anderen Wegen gefunden - bei Euler. (Opera posthuma Bd. 1, S. 547).

2) Sur les séries divergentes, Annales de la Fac. des sciences de Toulouse, Bd. (2) 2 (1900), S. 317. 
einem Grenzwert $s$ zu, so heiße $\Sigma a_{n}$ nun (R)-summierbar mit der Summe $s$. Die Methode ist analytisch weniger leicht zugänglich und darum von geringerer Bedeutung.

Von äußerlich ganz anderer Art ist das folgende Verfahren, das wir als letztes skizzieren wollen:

\%. Art (Eulersches Verfahren.) Wir sahen 144, daß, wenn von den beiden Reihen

$$
\sum_{n=0}^{\infty}(-1)^{n} a_{n} \quad \text { und } \quad \sum_{k=0}^{\infty} \frac{\Delta^{k} a_{0}}{2^{k+1}}
$$

die erste konvergiert, so konvergiert auch die zweite und liefert dieselbe Summe. Nun zeigen aber einfachste Beispiele, daß die zweite Reihe sehr wohl konvergieren kann, wenn die erste es nicht tut.

\section{Beispiele.} lauten also

1. Wenn $a_{n}=1$, so ist $a_{0}=1, \Delta^{k} a_{0}=0$ für $k \geq 1$. Die beiden Reihen

$$
1-1+1-1+-\ldots \quad \text { und } \frac{1}{2}+0+0+0+\ldots
$$

2. Ist für $n=0,1,2, \ldots$

so ist

$$
a_{n}=1, \quad 2, \quad 3, \quad 4, \ldots,
$$

und für $k \geq 2$.

$$
\Delta a_{n}=-1,-1,-1, \ldots
$$

Die beiden Reihen lauten also

$$
1-2+3-4+-\ldots \quad \text { und } \quad \frac{1}{2}-\frac{1}{4}+0+0+\ldots \text {. }
$$

3. Ebenso findet man aus $a_{n}=(n+1)^{3}$, dafi $\Delta a_{0}=-7, \quad \Delta^{2} a_{0}=12$, $\Delta^{3} a_{0}=-6$ und für $k>3$ stets $A^{k} a_{0}=0$ ist. Die beiden Reihen lauten also hier:

$$
1-8+27-64+-\ldots=\frac{1}{2}-\frac{7}{4}+\frac{12}{8}-\frac{6}{16}+0+0+\ldots .
$$

Daraufhin nennen wir eine Reihe $\sum_{n=0}^{\infty}(-1)^{n} a_{n}$ nun $(E)$-summierbar mit der Summe $s$, wenn $\sum_{k=0}^{\infty} \frac{\Delta^{k} a_{0}}{2^{k+1}}$ im alten Sinne konvergiert und die Summe $s$ hat.

Unser damaliger Satz 144 besagt dann jedenfalls, dáb dieses Verfahren die Permanenzbedingung erfüllt, und die eben gegebenen Beispiele zeigen andrerseits, dafs es bedeutend umfassender ist.

8. Allgemeinste Summation. Die gemeinsame Struktur aller beschriebenen Summationsverfahren ist diese: Ist die Reihe $\sum_{n=0}^{\infty} a_{n}$ vorgelegt und sind $s_{0}, s_{1}, s_{2}, \ldots$ ihre Teilsummen, so bilde man aus ihnen mit Hilfe der Matrix (vgl. den Satz von Toeplitz,

$$
(A)=\left(a_{k n}\right), \quad\left(k, n=\underset{29^{*}}{0,1,2,3, \ldots),}\right.
$$


von der wir voraussetzen, daß sie die Bedingungen (a), (b), (c) der Sätze 221 erfüllt, die neue Folge

$$
s_{k}{ }^{\prime}=a_{k 0} s_{0}+a_{k 1} s_{1}+a_{k 2} s_{2}+\ldots+a_{k n} s_{n}+\ldots \quad(k=0,1,2, \ldots)
$$

und untersuche deren Konvergenz ${ }^{1}$ ). Fällt sie konvergent aus und strebt etwa $s_{k}{ }^{\prime} \rightarrow s$, so nenne man die vorgelegte Reihe $\left(a_{k n}\right)$-summierbar $\left.^{2}\right)$ und sehe $s$ als ihre Summe an. Auf Grund des genannten Satzes erfüllt dies Verfahren jedenfalls die Permanenzbedingung. Sein „,Konvergenzfeld" aber, d. h. die Gesamtheit der Reihen $\Sigma a_{n}$, die auf eine konvergente Folge $\left(s_{k}{ }^{\prime}\right)$ führen, wird je nach Wahl der Matrix $\left(a_{k n}\right)$ verschieden ausfallen.

Mit der Aufzählung dieser Verfahren müssen wir uns begnügen ${ }^{3}$ ). Auch ist es nicht unsere Absicht, nun in ihr genaueres Studium einzutreten; wir müssen uns vielmehr mit der Bemerkung begnügen, daß sie alle schon eingehend nach verschiedenen Richtungen untersucht worden sind. (Literatur s. u.). Insbesondere ist - wenn auch noch keineswegs in allen Einzelheiten - festgestellt, inwieweit sie die oben S. 445 genannten Zweckmäßigkeitsforderungen erfüllen und inwieweit auch noch andere bei gewöhnlichen konvergenten Reihen gültige Sätze (z. B. solche über Multiplikation und Division von Reihen, über die Zulässigkeit von endlich vielen Änderungen, u. a.) ihre Gültigkeit behalten. Die Untersuchungen sind dann weiter auf divergente Reihen mit veränderlichen Gliedern ausgedehnt worden, in welchem Falle dann auch die "Summe“ von einer Veränderlichen abhängen und die Frage nach ihrer Stetigkeit, Differenzierbarkeit usw, auftauchen wird, deren Beantwortung dann genau wie im Falle der Konvergenz die Einführung des Begriffs der gleichmäßigen Summierbarkeit wünschenswert macht. Des weiteren haben sich die Untersuchungen darauf bezogen, welche von zwei Methoden die wirksamere ist, ob sich also ihre Konvergenzfelder ganz oder teilweise decken, und ob, falls zwei verschiedene Verfahren auf ein und dieselbe Reihe anwendbar sind, ihr durch beide dieselbe „Summe“ zuerteilt wird oder nicht.

Zum Schluß sei noch bemerkt, daß wir hier nur aus mehr äußerlichen Gründen von der Summierbarkeit von Reihen gesprochen haben. Ganz allgemein aber wird man eine Zahlenfolge $\left(x_{0}, x_{1}, \ldots\right)$, die im alten Sinne nicht konvergiert, in einem neuen Sinne konvergent nennen, wenn die Reihe $x_{0}+\left(x_{1}-x_{0}\right)+\left(x_{2}-x_{1}\right)+\ldots$, die ihr

1) Die Operation, durch die man aus der Folge $\left(s_{n}\right)$ und der in der $k^{\text {ten }}$ Zeile der Matrix (A) stehenden Folge $\left(a_{k 0}, a_{k 1}, \ldots\right)$ die mit $s_{k}{ }^{\prime}$ bezeichnete Größse herleitet, bezeichnet man kurz als Komposition der Folge $\left(s_{0}, s_{1}, \ldots\right)$ mit der $k^{\text {ten }}$ Zeile von (A).

2) D. h. summierbar bei Benutzung der Matrix $\left(a_{k n}\right)=(A)$.

$\left.{ }^{3}\right)$ Eine weitere sehr schöne Methode gab $M$. Riesz an: Une méthode de sommation, C. R. de 1'Acc. Paris, Bd. 152 (1911), S. 1651. 
nach 67, 7 entspricht, in dem betreffenden Sinne summierbar ist. Eine unendliche Reihe und die Folge ihrer Teilsummen und umgekehrt eine Folge und die unendliche Reihe aus ihren Differenzen sind eben sachlich ganz dasselbe.

Aus der schon sehr reichbaltigen Literatur über diesen Gegenstand seien folgende Werke und Arbeiten - aufer den im vorangehenden schon zitierten genannt:

T. J. I'a. Bromwich, An introduction to the theory of infinite Series, London 1908, Kap. 11. (Behandelt hauptsächlich das Borelsche und Cesàrosche Verfahren.)

G. H. Hardy, Theorems relating to the summability and convergence of slowly oscillating series, Proc. of the London Math. Soc., Bd. (2) 8 (1910), S. 301.

G. H. Hardy und S. Chapman, A general view of the theory of summable series, Quarterly Journ., Bd. 42 (1911), S. 181.

S. Chapman, On the general theory of summability, with applications to Fourier's and other series, ebenda, Bd. 43 (1911), S. 1.

G. H. Hardy und J. E. Littlewood, The relations between Borel's and Cesàro's methods of summation, Proc. London Math. Soc. Bd. (2) 11 (1912), S. 1.

E: Ricotti, Sulle serie divergenti sommabili, Giornale di matematiche, Bd. 48 (1910), S. 79.

B. Ottolenghi, Somma generalizzata, ebenda, Bd. 49 (1911), S. 233.

O. Perron, Beitrag zur Theorie der divergenten Reihen, Math. Zeitschr., Bd. 6 (1920), S. 286-310.

G. Doetsch, Eine neue Verallgemeinerung der Borelschen Summabilitätstheorie, Inaug.-Diss., Göttingen 1920.

F. Hausdorff, Summationsmethoden und Momentfolgen I und II, Mathemat. Zeitschrift, Bd. 9 (1921), S. 74 und S. 280.

Während der Drucklegung des vorliegenden Buches endlich erschien das Heft II, C 4 der Enzyklopädie der mathematischen Wissenschaften:

L. Bieberbach, Neuere Untersuchungen über Funktionen von komplexen Variablen, welches ein mit genauen Literaturangaben versehenes Referat über die Summierungsverfahren enthält.

\section{$\S 60$. Die Summierung durch arithmetische Mittel.}

Von allen Summierungsverfahren, die wir im vorangehenden kurz angegeben haben, sind die beiden ersten - und besonders die bei beiden identische Summierung durch arithmetische Mittel 1. Ordnung - durch ihre große Einfachheit besonders wertvoll und sie haben sich auch für die mannigfachsten Anwendungen als besonders zweckmäßig erwiesen. Wir wollen daher auf diese beiden Verfahren - und auf diese allein - noch etwas näher eingehen.

$\mathrm{Da}$ beide äußerlich eine gewisse Verwandtschaft aufweisen, so liegt die Frage hier besonders nahe, ob sie sich in ihrer Wirksamkeit voneinander unterscheiden oder nicht, ob also eine Reihe, die durch das eine Verfahren summierbar ist, es auch stets durch das andere 
sein muß oder nicht. Diese Frage entscheidet der folgende vom Verfasser $^{1}$ ) und von Schnee ${ }^{2}$ ) herrührende

Äquivalenzsatz. Ist eine Reihe $\sum a_{n}$ für ein bestimmtes $r(M r)$ summierbar, so ist sie für dieses $r$ auch $(C r)$ - summierbar und umgekehrt, und beide Verfahren liefern für die Reihe dieselbe Summe.

Auf den nicht ganz einfachen Beweis dieses Satzes wollen wir hier nicht eingehen ${ }^{3}$ ), betrachten es aber doch auf Grund dieses Satzes nicht als eine sachliche Einschränkung, wenn wir uns weiterhin nur mit der leichter $\mathrm{zu}$ handhabenden Summierbarkeit $(C, r)$ beschäftigen.

Hier gilt zunächst, ähnlich wie wir es schon bei der Hölderschen Methode zeigten, der

Satz. Ist eine Reihe $(C r)$-summierbar mit der Summe s, so ist sie auch $(C r+1)$-summierbar mit derselben Summe $\left.s^{4}\right)$

Beweis. Es ist

$$
\frac{S_{n}^{(r+1)}}{\left(\begin{array}{c}
n+r+1 \\
r+1
\end{array}\right)}=\frac{S_{0}^{(r)}+S_{1}^{(r)}+\ldots+S_{n}^{(r)}}{\left(\begin{array}{c}
r \\
r
\end{array}\right)+\left(\begin{array}{c}
1+r \\
r
\end{array}\right)+\ldots+\left(\begin{array}{c}
n+r \\
r
\end{array}\right)},
$$

und nach dem Cauchy-Toeplitzschen Satze (44, Bem. 4 und 5) folgt unmittelbar die Behauptung.

Ist nun $r$ die kleinste $Z$ ahl, für die $\sum a_{n}$ noch $(C r)$-summierbar ist, so sagt man auch, die Reihe sei genau durch die Mittel $\boldsymbol{r}^{\text {ter }}$ Ordnung summierbar $\left.{ }^{5}\right)$.

Beispiele für $(C r)$-Summierbarkeit.

1. $\sum_{n=0}^{\infty}(-1)^{n}$ ist $(C 1)$-summierbar; $s=\frac{1}{2}$. Beweis s. o. S. 444 .

2. $\sum_{n=0}^{\infty}(-1)^{n}\left(\begin{array}{c}n+p \\ p\end{array}\right)$ ist $(C p+1)$-summierbar; $s=\frac{1}{2^{p+1}}$. Bew e is durch direkte Ausrechnung der $\left.S_{n}^{(p+1)}{ }^{6}\right)$.

1) Inaug.-Diss., Berlin, 1907, S. 19.

2) W. Schnee, Math. Ann., Bd. 67, 1909, S. 110.

$\left.{ }^{3}\right)$ Einen besonders schönen Beweis dieses Aquivalenzsatzes gab I. Schur (Math. Ann., Bd. 74 (1913), S. 447); ein anderer findet sich in der am Schluf des vorigen Paragraphen genannten Arbeit von $F$. Hausdorff.

4) Dieser Satz lehrt speziell, daf die $(C r)$ - Summierbarkeit die Permanenzbedingung erfüllt (vgl. S. 447).

$\left.{ }^{5}\right)$ Auch nennt man die Reihe dann $r$-fach unbestimmt; doch rührt diese Bezeichnung von veralteten Anschauungen her.

क) Mit Benutzung der Ausfübrungen von S. 463 ergeben sich die $S_{n}^{(p)}$ in diesem Falle als Koeffizienten von $x^{n}$ in

$$
\left(\frac{1}{1-x}\right)^{p+1} \cdot\left(\frac{1}{1+x}\right)^{p+1}=\sum_{k=0}^{\infty}\left(\begin{array}{c}
k+p \\
p
\end{array}\right) x^{2 k}
$$

woraus dann alles weitere leicht folgt. 
3. Die Reihe $\sum_{n=0}^{\infty}(-1)^{n}(n+1)^{p-1} \equiv 1-2^{p-1}+3^{p-1}-4^{p-1}+\ldots$ ist $(C p)$-summierbar mit der Summe $s=\frac{2^{p}-1}{p} B_{p}$, falls $p>1$.

4. Die Reihe $\frac{1}{2}+\cos x+\cos 2 x+\ldots+\cos n x+\ldots$ ist (C 1$)$ - summierbar mit der Summe 0 , falls $x \neq 2 k \pi$ ist.

Beweis. Nach $\mathbf{2 0 1}$ ist für $n=0,1,2, \ldots$

$$
s_{n}=\frac{1}{2}+\cos x+\cos 2 x+\ldots+\cos n x=\frac{\sin \left(n+\frac{1}{2}\right) x}{2 \sin \frac{x}{2}}
$$

und also

$s_{0}+s_{1}+\ldots+s_{n}=\frac{1}{2 \sin \frac{x}{2}}\left(\sin \frac{x}{2}+\sin 3 \frac{x}{2}+\ldots+\sin (2 n+1) \frac{x}{2}\right)=\frac{\sin ^{2}(n+1) \frac{x}{2}}{2 \sin ^{2} \frac{x}{2}}$ und folglich

$$
\left|\frac{s_{0}+s_{1}+\ldots+s_{n}}{n+1}\right| \leqq \frac{1}{n+1} \cdot \frac{1}{2 \sin ^{2} \frac{x}{2}} .
$$

Bei festem $x \neq 2 k \pi$ strebt aber der letzte Quotient mit wachsendem $n$ gegen 0 , womit schon alles bewiesen ist. - Hier haben wir ein erstes Beispiel einer summierbaren Reihe mit veränderlichen Gliedern. Die durch ihre "Summe* dargestellte Funktion ist $\equiv 0$ in jedem Intervall, das keinen der Punkte $2 k \pi$ enthält. In den ausgeschlossenen Punkten ist die Reihe bestimmt divergent gegen $+\infty$ !

5. Die Reihe $\sin x+\sin 2 x+\sin 3 x+\ldots$ ist für $x=k \pi$ ersichtlich konvergent mit der Summe 0 . Für $x \neq k \pi$ ist sie nicht mehr konvergent, wohl aber (C 1)-summierbar und ihre "Summe" ist dann $\frac{1}{2} \operatorname{ctg} \frac{x}{2}^{1}$ ).

Beweis. Es ist $s_{n}=\sin x+\ldots+\sin n x=\frac{1}{2} \operatorname{ctg} \frac{x}{2}-\frac{\cos (2 n+1) \frac{x}{2}}{2 \sin \frac{x}{2}}$, woraus dann ähnlich wie in 4. der Beweis folgt.

6. $\cos x+\cos 3 x+\cos 5 x+\ldots$ ist (C 1)-summierbar mit der Summe 0 , falls $x \neq k \pi$ ist.

7. $\sin x+\sin 3 x+\sin 5 x+\ldots$ ist ebenfalls (C 1)-summierbar und hat die Summe $\frac{1}{2 \sin x}$, falls $x \neq k \pi$ ist.

8. $1+z+z^{2}+\ldots$ ist für $|z|=1$ noch $(C 1)$ - summierbar mit der Summe $\frac{1}{1-z}$, falls nur $z \neq+1$ ist. Die Beispiele 4 und 5 ergeben sich hieraus durch Trennung von Reellem und Imaginärem.

Daß durch gliedweise Addition oder Subtraktion zweier summierbarer Reihen wieder eine solche entsteht und daß sich dabei auch

1) Das Bild dieser Funktion weist also an den Stellen $2 k \pi$ „unendlich grofe Sprünge" auf. 
deren Summen addieren bzw. subtrahieren, ist so leicht zu sehen, daß wir es nicht weiter ausführen. Ebenso, daß ,endlich viele Änderungen" (s. 82,11) auf die Summierbarkeit keinen Einfluß haben, und $\mathrm{da} B$ sich dabei die Summe genau so verändert, als ob die Reihe konvergent wäre.

Die Multiplikation unendlicher Reihen, die bei der Forderung der Konvergenz der Reihen eine recht komplizierte Theorie erfordert, erledigt sich wesentlich einfacher, wenn man nur die Summierbarkeit der Reihen verlangt. So können wir das beim 2. Beweise des Satzes 189 gewonnene Resultat jetzt so aussprechen:

Satz. Das Cauchysche Produkt zweier konvergenter Reihen mit den Summen $A$ und $B$ ist stets summierbar mit der Summe $A \cdot B$.

Dieser schöne, von Cesàro herrührende Satz ist einer der Ausgangspunkte einer systematischen Theorie der divergenten Reihen, da er zum ersten Male an einem schlagenden Beispiele den Nutzen des Summierungsbegriffes zeigte: Ein Sachverhalt, der bei ängstlichem Festhalten des Konvergenzbegriffes sehr undurchsichtig und schwierig war, ließ sich durch Zulassung des Summierbarkeitsbegriffes in einheitlicher Weise erschöpfend erledigen.

\section{§ 61. Anwendung der (C 1)-Summierbarkeit auf die Theorie der Fourierschen Reihen.}

Abgesehen von dem greifbaren Vorteil aller Summierungsverfahren, der darin besteht, daß viele unendliche Reihen, die wir bisher als sinnlos verwerfen mußten, nunmehr einen brauchbaren Sinn bekommen und da $B$ dadurch also das Anwendungsfeld der unendlichen Reihen sehr erheblich erweitert wird, liegt das theoretisch außerordentlich befriedigende dieser Verfahren darin, daß verworrene und undurchsichtige Sachverhalte nun plötzlich von äußerster Einfachheit werden. So sahen wir schon, daß die schwierige und bis heute noch nicht völlig geklärte Frage nach der Gültigkeit der Cauchyschen Multiplikationsregel

$$
\left.\sum a_{n} \cdot \Sigma b_{n}=\Sigma\left(a_{0} b_{n}+a_{1} b_{n-1}+\ldots+a_{n} b_{0}\right)^{1}\right)
$$

eine äußerst befriedigende Antwort zuläßt, sobald man Summierbarkeit an die Stelle der Konvergenz zulässt.

Aber die in diesem Sinne vielleicht schönste und auch praktisch bedeutungsvollste Anwendung der (C 1)-Summierung ist diejenige,

1) D. h. die Frage, was für notwendige und hinreichende Bedingungen die linksstehenden Reihen (aufer ihrer Konvergenz) noch zu erfüllen haben, damit auch die rechtsstehende Reihe konvergiert und zwischen den Summen aller drei die obige Gleichung besteht. 
die L. Fejér von ihr auf die Theorie der Fourierreihen gemacht hat ${ }^{1}$ ). Hier sahen wir (S. 357), daß die Frage nach den notwendigèn und hinreichenden Bedingungen, unter denen die Fourierreihe einer integrierbaren Funktion konvergiert und die gegebene Funktion darstellt, äußerst schwierig ist. Insbesondere weiß man z. B. nicht, was für notwendige und hinreichende Bedingungen eine an einer Stelle $x_{\mathbf{0}}$ stetige Funktion dort noch zu erfüllen hat, damit ihre Fourierreihe in diesem Punkte konvergiert und den betreffenden Funktionswert darstellt. Wir haben in $\S 49$, C verschiedene Kriterien dafür kennen geernt; aber sie hatten alle nur den Charakter hinreichender Bedingungen. Lange vermutete man auch, daß jede in $x_{0}$ stetige Funktion $f(x)$ eine Fourierreihe besitzt, die dort konvergiert und die Summe $f\left(x_{0}\right)$ hat. Erst durch ein Beispiel von du Bois-Reymond (s. 216, 1) wurde diese Vermutung zuschanden. Die Fourierreihe einer in $x_{0}$ stetigen Funkticn kann dort tatsächlich divergieven ${ }^{2}$ ).

Noch schwieriger wird die Frage, wenn wir — als zunächst wohl geringstes $\mathrm{Ma} \beta$ an Voraussetzungen über $f(x)$ - nur verlangen, daß die (integrierbare) Funktion $f(x)$ an der Stelle $x_{0}$ je einen rechtsseitigen und einen linksseitigen Limes $f\left(x_{0}+0\right)$ und $f\left(x_{0}-0\right)$ besitzt. Was für notwendige und hinreichende Bedingungen muß die Funktion $f(x)$ darüber hinaus noch erfüllen, damit ihre Fourierreihe in $x_{0}$ konvergiert und die Summe $\frac{1}{2}\left[f\left(x_{0}+0\right)+f\left(x_{0}-0\right)\right]$ hat?

Wie betont, ist die Frage noch keineswegs beantwortet. Aber dieser verworrene und undurchsichtige Sachverhalt wird nun aufs befriedigendste geklärt, wenn man an Stelle der Konvergenz die Summierbarkeit - und zwar genügt schon die (C 1)-Summierbarkeit - der Fourierreihen in Betracht zieht. Es gilt nämlich (wie dort) der folgende schöne

Satz von Fejér. Besitzt die in $0 \leqq x \leqq 2 \pi$ integrierbare Funktion $f(x)$ an einer Stelle $x_{0}$ dieses Intervalles die beiden Grenzwerte $f\left(x_{0}+0\right)$ und $\left.f\left(x_{0}-0\right)^{3}\right)$, so ist ihre Fourierreihe dort stets (C 1)-summierbar mit der Summe $\left.\frac{1}{2}\left[f\left(x_{0}+0\right)+f\left(x_{0}-0\right)\right]^{4}\right)$.

1) L. Fejér, Untersuchungen uiber die Fourierschen Reihen, Math. Annalen, Bd. 58 (1903), S. 51.

2) Heute gibt es einfachere Beispiele als das genannte. Z. B. hat L. Fejerr ein sehr schönes und durchsichtiges Beispiel gegeben (J. f. d. reine u. angew. Math., Bd. 137 (1909), S. 1).

3) Nach unsern früheren Verabredungen war $f(2 \pi)=f(0)$ zu setzen und die Funktion nun periodisch in die sämtlichen Intervalle $2 k \pi \leq x \leq 2(k+1) \pi$ fortzusetzen. Es ist demnach $f(0+0)$ als rechtsseitiger Limes in den beiden Endpunkten 0 und $2 \pi$ anzusehen; ebenso $f(2 \pi-0)$ als der linksseitige Limes an diesen beiden Stellen.

4) Auch hier ist es wieder eigentlich gar nicht nötig, dafi die Grenzwerte

$$
\lim _{t \rightarrow+0} f\left(x_{0}+t\right)=f\left(x_{0}+0\right) \text { und } \lim _{t \rightarrow+0} f\left(x_{0}-t\right)=f\left(x_{0}-0\right)
$$


Beweis. Ist

$$
\frac{1}{2} a_{0}+\sum_{n=1}^{x}\left(a_{n} \cos n x_{0}+b_{n} \sin n x_{0}\right)
$$

die Fourierreihe von $f(x)$ an der Stelle $x_{0}$, so hatten wir S. $344 / 46$ für die $n^{\text {te }}$ Teilsumme derselben gefunden:

$$
\begin{aligned}
s_{n}=s_{n}\left(x_{0}\right)= & \frac{1}{\pi} \int_{0}^{2 \pi} f\left(x_{0}+t\right)\left[\frac{1}{2}+\cos t+\cos 2 t+\ldots+\cos n t\right] d t \\
= & \frac{2}{\pi} \int_{0}^{\frac{\pi}{2}} \frac{1}{2}\left[f\left(x_{0}+2 t\right)+f\left(x_{0}-2 t\right)\right] \frac{\sin (2 n+1) t}{\sin t} d t, \\
& (n=0,1, \ldots) .
\end{aligned}
$$

Folglich ist für $n=1,2, \ldots$

$$
\begin{aligned}
& s_{0}+s_{1}+\ldots+s_{n-1} \\
& =\frac{2}{\pi} \int_{0}^{\frac{\pi}{2}} \frac{1}{2}\left[f\left(x_{0}+2 t\right)+f\left(x_{0}-2 t\right)\right]^{\sin t+\frac{\sin 3 t+\ldots+\sin (2 n-1) t}{\sin t}} d t .
\end{aligned}
$$

Nun war nach 201, 5 für $t \neq k \pi$

$$
\sin t+\sin 3 t+\ldots+\sin (2 n-1) t=\frac{\sin ^{2} n t}{\sin t}
$$

und dies gilt auch noch für $t=k \pi$, wenn man dann unter dem rechterhand stehenden Quotienten dessen Grenzwert für $t \rightarrow k \pi$ versteht, welcher ersichtlich 0 ist. Folglich ist ${ }^{1}$ )

$$
\begin{aligned}
\sigma_{n-1} & =\frac{s_{0}+s_{1}+\ldots+s_{n-1}}{n} \\
& =\frac{2}{n \pi} \int_{0}^{2} \frac{1}{2}\left|f\left(x_{0}+2 t\right)+f\left(x_{0}-2 t\right)\right|\left(\frac{\sin n t}{\sin t}\right)^{2} d t .
\end{aligned}
$$

In der Tatsache, daß in diesem Integral - man nennt es kurz das Fejérsche Integral - im Gegensatz zum Dirichletschen Integral der kritische Faktor $\frac{\sin n t}{\sin t}$ im Quadrat auftritt und also nur einerlei Vorzeichen haben kann und daß überdies der Faktor $\frac{1}{n}$ davor steht, liegt das Gelingen des weiteren Beweises begründet. Sind nun die beiden Grenzwerte $f\left(x_{0}+0\right)$ und $f\left(x_{0}-0\right)$ oder auch nur (s. Fußnote 4 der vorigen Seite)

$$
\lim _{t \rightarrow+0} \frac{1}{2}\left[f\left(x_{0}+2 t\right)+f\left(x_{0}-2 t\right)\right]=s\left(x_{0}\right)=s
$$

vorhanden, so behauptet der Fejérsche Satz einfach, daß $\sigma_{n} \rightarrow s$ strebt.

einzeln existieren, sondern es wird schon genügen, wenn

$$
\lim _{t \rightarrow+0} \frac{1}{2}\left[f\left(x_{0}+t\right)+f\left(x_{0}-t\right)\right]=s\left(x_{0}\right)
$$

vorhanden ist. '(Vgl. hierzu S. 357, oben.)

1) Wir bezeichnen hier die arithmetischen Mittel der $s_{n}$ mit $\sigma_{n}=\sigma_{n}(x)$ statt mit $s_{n}^{\prime}=s_{n}^{\prime}\left(x_{1}\right)$, um Verwechslungen mit der Ableitung auszuschliefien. 
Dazu bemerken wir zunächst, daß

$$
\int_{0}^{\frac{\pi}{2}}\left(\frac{\sin n t}{\sin t}\right)^{2} d t=n \frac{\pi}{2}
$$

ist, denn der Integrand ist seiner Entstehung nach

$$
=\sum_{\nu=1}^{n} \frac{\sin (2 v-1) t}{\sin t}
$$

und jeder Summand dieser Summe liefert, wenn man ihn von 0 bis $\frac{\pi}{2}$ integriert, den Wert $\frac{\pi}{2}$, denn es war ja

$$
\left.\frac{\sin (2 v-1) t}{\sin t}=1+2 \cos 2 t+2 \cos 4 t+\ldots+2 \cos 2(v-1) t^{1}\right) \text {. }
$$

Daher kann

und also

$$
s=\frac{2}{n \pi} \int_{0}^{\frac{\pi}{2}} s \cdot\left(\frac{\sin n t}{\sin t}\right)^{2} d t
$$

$$
\sigma_{n-1}-s=\frac{2}{n \pi} \int_{0}^{\frac{\pi}{2}}\left[\frac{f\left(x_{0}+2 t\right)+f\left(x_{0}-2 t\right)}{2}-s\right] \cdot\left(\frac{\sin n t}{\sin t}\right)^{2} d t
$$

gesetzt werden. Nach Voraussetzung strebt hier der Ausdruck, der in der eckigen Klammer steht, $\rightarrow 0$ für $t \rightarrow+0$. Damit also $\sigma_{n-1}$ oder $\sigma_{n} \rightarrow s$ strebt, genügt es, dies zu zeigen:

Wenn $\varphi(t)$ in $0 \ldots \frac{\pi}{2}$ integrierbar ist und der Bedingung

$$
\lim _{t \rightarrow+0} \varphi(t)=0
$$

genügt, so strebt bei wachsendem $n$

$$
\frac{2}{n \pi} \int_{0}^{\frac{\pi}{2}} \varphi(t) \cdot\left(\frac{\sin n t}{\sin t}\right)^{2} d t \rightarrow 0 .
$$

Das ergibt sich nun tatsächlich durch allereinfachste Abschätzungen. Wegen $\varphi(t) \rightarrow 0$ kann man nämlich, wenn $\varepsilon>0$ gegeben wird, ein positives $\delta<\frac{\pi}{2}$ so bestimmen, daß für $0<t \leqq \delta$ stets $|\varphi(t)|<\frac{\varepsilon}{2}$ bleibt. Dann ist

$$
\left|\frac{2}{n \pi} \int_{0}^{\delta} \varphi(t) \cdot\left(\frac{\sin n t}{\sin t}\right)^{2} d t\right| \leqq \frac{\varepsilon}{2} \cdot \frac{2}{n \pi} \int_{0}^{\delta}\left(\frac{\sin n t}{\sin t}\right)^{2} d t<\frac{\varepsilon}{2},
$$

$\mathrm{da}$ ja das letzte Integral einen positiven Integranden hat und also kleiner bleibt, als das von 0 bis $\frac{\pi}{2}$ erstreckte Integral über denselben

1) Man kann diesen Integralwert auch aus dem Fejérschen Integral selbst ablesen, indem man es auf die Funktion $f(x)=1$ anwendet, für die $a_{0}=2$ ist und alle andern Fourierkonstanten $=0$ werden. 
Integranden. Andrerseits gibt es eine Konstante $M$, so daß in $0<t<\frac{\pi}{2}$ stets $|\varphi(t)|<M$ bleibt. Folglich ist

$$
\left|\frac{2}{n \pi} \int_{\delta}^{\frac{\pi}{2}} \varphi(t) \cdot\left(\frac{\sin n t}{\sin t}\right)^{2} d t\right| \leqq \frac{2 M}{n \pi} \cdot \frac{\pi}{2} \cdot \frac{1}{\sin ^{2} \delta} .
$$

Und da hier alles außer $n$ feste Werte hat, so kann man $n_{0}$ so groß nehmen, daß für $n>n_{0}$ dieser Ausdruck seinerseits $<\frac{\varepsilon}{2}$ bleibt. Dann ist aber für diese $n$ stets

$$
\left|\sigma_{n-1}-s\right|<\varepsilon
$$

und es strebt also $\sigma_{n} \rightarrow s$. Damit ist der Fejérsche Satz in vollem Umfange bewiesen ${ }^{1}$ ).

Zusatz 1. Ist $f(x)$ in dem abgeschlossenen Intervall $0 \leqq x \leqq 2 \pi$ stetig und ist überdies $f(0)=f(2 \pi)$, so ist die Fourierreihe von $f(x)$ für jedes $x$ stets $(C 1)$-summierbar mit der Summe $f(x)$. Denn nun sind die Voraussetzungen des Fejérschen Satzes gewiß für jedes $x$ erfüllt und man hat stets $\frac{1}{2}[f(x+0)+f(x-0)]=f(x)$. Dabei denken wir uns wie immer, die Funktion $f(x)$ in den Intervallen $2 k \pi \leqq x \leqq 2(k+1) \pi$, $k= \pm 1, \pm 2, \ldots$, durch die Forderung der Periodizität $f(x)=f(x-2 k \pi)$ festgelegt.

Wir behaupten nun weiter:

Zusatz 2. Unter den Bedingungen des vorigen Zusalzes ist die fïr alle $x$ bestehende (C 1)-Summierbarkeit sogar eine für alle diese $x$ gleichmäßige, d.h. die Folge der Funkicnen $\sigma_{n}(x)$ strebt für alle $x$ gleichmäßig gegєn $f(x)$, oder also: Nach Wahl von $\varepsilon>0$ läßt sich eine Zahl $N$ so angeben, daß für alle $n>N$ ohne Rücksicht auf die Lage $x$ stets

bleibt $\left.^{2}\right)$.

$$
\left|\sigma_{n}(x)-f(x)\right|<\varepsilon
$$

Beweis. Wir haben nur zu zeigen, daß man die Abschätzungen des vorigen Beweises so durchführen kann, daß sie für jede Lage von $x$ gelten. Nun ist aber

$$
\varphi(t)=\varphi(t, x)=\frac{1}{2}[f(x+2 t)-f(x)]+\frac{1}{2}[f(x-2 t)-f(x)] ;
$$

1) Beiläufig sei noch erwähnt, daß die Approximationskurven $y=\sigma_{n}(x)$ die Gibbssche Erscheinung (s. 216,4) nicht a ufweisen (L. Fejerr, Math. Annalen, Bd. 64 (1907), S. 273).

2) Das Entsprechende gilt übrigens auch in dem allgemeinen Satz von Fejer für jedes abgeschlossene Intervall, in dem $f(x)$ stetig ist. 
und da $f(x)$ als durchweg stetige und periodische Funktion auch für alle $x$ gleichmäßig stetig ist (vgl. $\S 19$, Satz 5), so kann man, nachdem $\varepsilon$ gegeben ist, ein $\delta>0$ so bestimmen, daß für alle $|t|<\delta$

$$
|f(x \pm 2 t)-f(x)|<\frac{\varepsilon}{2}
$$

bleibt, - und dies für jede Lage von $x$. Dann ist aber auch - ohne Rücksicht auf die Lage von $x$ - für diese $t$ stets

$$
|\varphi(t)|=|\varphi(t, x)|<\frac{\varepsilon}{2}
$$

und also wie vorhin

$$
\left|\frac{2}{n \pi} \int_{0}^{\delta} \varphi(t)\left(\frac{\sin n t}{\sin t}\right)^{2} d t\right|<\frac{\varepsilon}{2} .
$$

Ferner ist $f(x)$ als durchweg stetige und periodische Funktion auch beschränkt. Ist etwa stets $|f(x)|<K$, so ist, wie man sofort sieht, stets, d. h. für alle $t$ und alle $x$,

$$
|\varphi(t)|=|\varphi(t, x)|<2 K
$$

und also ganz ähnlich wie vorhin

$$
\left|\frac{2}{n \pi} \int_{\delta}^{\frac{\pi}{2}} p(t) \cdot\left(\frac{\sin n t}{\sin t}\right)^{2} d t\right| \leqq \frac{1}{n} \cdot \frac{2 K}{\sin ^{2} \delta} .
$$

Nun kann man wirklich eine Zahl $N$ so bestimmen, daß dieser letzte Ausdruck für alle $n \geqq N$ stets $<\frac{\varepsilon}{2}$ bleibt. Für diese $n$ ist dann also $\left|\sigma_{n-1}-s\right|<\varepsilon$, so daß sich tatsächlich, wie behauptet, dem gegebenen $\varepsilon$ eine Zahl $N$ so zuordnen läßt, daß für alle $n>N$ und ohne Rücksicht auf die Lage von $x$ stets

bleibt.

$$
\left|\sigma_{n}(x)-f(x)\right|<\varepsilon
$$

\section{$\S$ 62. Anwendungen der $(C r)$-Summierbarkeit.}

Noch an einem andern markanten Beispiele wollen wir die Bedeutung der $(C r)$-Summierbarkeit zeigen: Der Abelsche Grenzwertsatz $\left(100\right.$ u. 233) besagte, daß, wenn die Potenzreihe $\Sigma a_{n} x^{n}$ den Einheitskreis zum Konvergenzkreis hat und im Randpunkt +1 desselben mit der Summe $s$ noch konvergiert, auch der Grenzwert

$$
\lim _{x \rightarrow 1-0}\left(\sum a_{n} x^{n}\right) \text { existiert und }=s=\sum a_{n}
$$


ist $^{1}$ ). Nun lehren aber einfachste Beispiele, etwa die geometrische Reihe $\Sigma(-1) x^{n}=\frac{1}{1+x}$, daB der linksstehende Grenzwert existieren kann, ohne daß die rechtsstehende Reihe konvergiert. Gerade dies gab ja Veranlassung zur Definition der „(A)-Summierbarkeit“, die wir S. 448 besprochen haben. Es entsteht nun die Frage, ob man in solchen Fällen, in denen also $\sum a_{n}$ divergiert, die Existenz des genannten Grenzwertes doch irgendwie aus der divergenten Reihe unmittelbar erschließen kann. Einen ersten Schritt in dieser Beziehung bedeutet ein Satz von Frobenius ${ }^{2}$ ), welcher in unserer jetzigen Ausdrucksweise besagt, daß es schon genügt, wenn $\Sigma a_{n}$ nur $(C 1)$ oder ( $M$ 1)-summierbar ist, um auf die Existenz des Grenzwertes (a) schließen zu können. Sein Wert ist dann wieder gleich der "Summe“ von $\sum a_{n}$. Oder kürzer: Aus der (C 1)-Summierbarkeit mit der Summe $s$ folgt stets die (A)-Summierbarkeit mit derselben Summe. Gerade dieses Frobeniussche Resultat ist wohl der erste Anlaß gewesen, sich gründlicher mit den divergenten Reihen zu beschäftigen, und im Anschluß an Frobenius hat auch Hölder seine Mittel $s_{n}^{(r)}$ eingeführt und allgemeiner gezeigt, daß schon aus der $(M r)$-Summierbarkeit die (A)-Summierbarkeit - und wieder mit derselben Summe folgt.

Wir wollen im folgenden das Entsprechende und, indem wir auch die Variable komplex annehmen, noch ein wenig mehr, für die $(C r)$-Summierbarkeit zeigen. Auf Grund des zu Beginn des Paragraphen genannten Äquivalenzsatzes kommt tatsächlich beides auf dasselbe hinaus. Wir beweisen also den folgenden

Satz. Ist $\Sigma a_{n}$ eine $(C r)$-summierbare Reihe mit der Summe s, so ist $\sum a_{n} z^{n}$ mindestens für $|z|<1$ konvergent und es gilt für die dadurch in $|z|<1$ definicrte Funklion $f(z)$ die Beziehung

$$
\lim _{z \rightarrow+1} f(z)=s \text {, }
$$

d.h. der linksstehende Grenzwert ist vorhanden und hat den Wert s, und zwar nicht nur für positiv-reelle, gegen +1 wachsende Werte der Variablen, sondern bei beliebiger ,,im Winkelraum" (vgl. Fig. 10, S. 390) erfolgender Annäherung der komplexen Veränderlichen $z$ an +1 .

Der Btweis läßt sich, ebenso wie der des Satzes 233, mit Hilfe des Toeplitzschen Satzes 221 ganz unmittelbar erbringen. Wir wählen wie damals eine beliebige, im Winkelraum liegende, gegen +1 strebende Punktfolge $\left(z_{0}, z_{1}, \ldots, z_{k}, \ldots\right)$ und haben nur zu zeigen,

1) Entsprechend, wenn $\sum a_{n} x^{n}$ einen beliebigen (endlichen) Radius hat und in einem beliebigen Randpunkt des Konvergenzkreises konvergiert. Obige Formulierung bedeutet sachlich keine Einschränkung.

2) J. f. d. reine u. angew. Math., Bd. 89 (1880), S. 262. 
daß $f\left(z_{k}\right) \rightarrow s$ strebt. Wenden wir aber den genannten Toeplitzschen Satz auf die Folge der Zahlen $\sigma_{n}=S_{n}^{(r)}:\left(\begin{array}{c}n+r \\ r\end{array}\right)$ an, die ja nach Voraussetzung $\rightarrow s$ konvergieren, und benutzen die Matrix $\left(a_{k n}\right)$, für die

$$
a_{k n}=\left(\begin{array}{c}
r+n \\
r
\end{array}\right)\left(1-z_{k}\right)^{r+1} \cdot z_{k}^{n}
$$

ist, so liefert er sofort, daß auch die Folge der Zahlen

$$
\sigma_{k}=\sum_{n=0}^{\infty} a_{k n} \cdot \sigma_{n}=\left(1-z_{k}\right)^{r+1} \sum_{n=0}^{\infty} S_{n}^{(r)} \cdot z_{k}^{n} \rightarrow s
$$

strebt. Es ist aber (nach 102)

$$
\begin{aligned}
& \left(1-z_{k}\right)^{r+1} \sum_{n=0}^{\infty} S_{n}^{(r)} z_{k}^{n}=\left(1-z_{k}\right)^{r} \cdot \sum_{n} S_{n}^{(r-1)} \cdot z_{k}^{n}=\ldots \\
= & \left(1-z_{k}\right)^{2} \sum_{n} S_{n}^{(1)} z_{k}^{n}=\left(1-z_{k}\right) \frac{\searrow}{n} s_{n} z_{k}^{n}=\sum_{n} a_{n} z_{k}^{n}=f\left(z_{k}\right) .
\end{aligned}
$$

Also strebt $f\left(z_{k}\right) \rightarrow s$. Damit wäre also schon alles bewiesen, wenn wir noch zeigen, daß die gewählte Matrix die Voraussetzungen (a), (b) und (c) der Sätze 221 erfüllt. Wegen $z_{k} \rightarrow 1$ ist dies für die Voraussetzung (a) evident; und wegen

$$
\begin{aligned}
A_{k}=\sum_{n=0}^{\infty} a_{k n} & =\left(1-z_{k}\right)^{r+1} \cdot \sum_{n=0}^{\infty}\left(\begin{array}{c}
r+n \\
r
\end{array}\right) z_{k}^{n}=\left(1-z_{k}\right)^{r+1} \sum_{n=0}^{\infty}(-1)^{n}\left(\begin{array}{c}
-r-1 \\
n
\end{array}\right) z_{k}^{n} \\
= & \left(1-z_{k}\right)^{r+1}\left(1-z_{k}\right)^{-r-1}=1
\end{aligned}
$$

ist auch (c) erfüllt. Die Bedingung (b) endlich verlangt die Existenz einer Konstanten $K^{\prime}$, so daß für alle $k$

$$
\frac{\Sigma}{n}\left|a_{k n}\right|=\left(\frac{1-z_{k} \mid}{1-\left|z_{k}\right|}\right)^{r+1}<K^{\prime} \text {. }
$$

Nach den Erwägungen von S. 391 leistet dies aber ersichtlich die Konstante $K^{\prime}=K^{r+1}$, wenn $K$ die dort festgelegte Bedeutung hat.

Für $r=0$ haben wir hierin genau den S. 391 durchgeführten Beweis des Abelschen Grenzwertsatzes in der Stolzschen Verallgemeinerúng. Für $r=1$ ergibt sich die obengenannte Frobeniussche Verallgemeinerung dieses Grenzwertsatzes ${ }^{1}$ ), und für $\dot{r}=2,3, \ldots$ ergeben sich entsprechend weitere Stufen der Verallgemeinerung, wie sie im wesentlichen von $O$. Hölder herrühren $\left.{ }^{2}\right)-$ mit $(M r)$ - statt $(C r)$-Summierbarkeit und mit radialer Annäherung statt solcher im Winkelraum - und wie sie in der bewiesenen Form (wenn auch

1) Frobenius bewies sie 1. c. nur für radiale Annäherung.

?) Math. Ann., Bd. 20 (1882), S. 535. 
mit ganz anderem Beweise) zuerst ron E. Lasker ${ }^{1}$ ) und A.Pringsheim ${ }^{2}$ ) ausgesprochen worden sind.

Die weiteren Anwendungen der $(C r)$-Summierbarkeit führen meist zu tief in die Funktionentheorie hinein, als daß wir hier ausführlicher darauf eingehen könnten. Doch möchten wir es uns nicht versagen, zum Schluß noch, ohne auf die Beweise einzugehen, über eine Anwendung zu berichten, die zu besonders schönen Ergebnissen geführt hat. Es ist dies die Anwendung der $(C r)$-Summierbarkeit auf die Theorie der Dirichletschen Reihen.

Die Dirichletsche Reihe

$$
\left.f(z)=\sum_{n=1}^{\infty}{\frac{(-1)^{n-1}}{n^{2}}}^{3}\right)
$$

war für alle $z$ konvergent, für die $\Re(z)>0$ war, für alle andern divergent. Im Punkte 0 aber, wo es sich um die Reihe $\sum_{n=1}^{\infty}(-1)^{n-1}$ handelt, ist sie $(C 1)$-summierbar mit der Summe $\frac{1}{2}$; im Punkte -1 , wo es sich um die Reihe $\sum_{n=1}^{\infty}(-1)^{n-1} n$ handelt, ist sie (vgl. S. 446) (C 2)-summierbar mit der Summe $\frac{1}{4}$; und die S. 455, oben, gemachten Angaben lehren, daß die Reihe für $z=-(r-1)$ noch $(C r)$-summierbar ist mit der Summe $\frac{2^{r}-1}{r} B_{r}$, welchen ganzzahligen Wert $\geqq 2$ auch $r$ haben mag.

Diese Eigenschaft nun, außerhalb ihres Konvergenzgebietes $\Re(z)>0$ für ein passendes $r$ noch $(C r)$-summierbar zu sein, beschränkt sich nicht auf die genannten Punkte, sondern man kann mit verhältnismäßig, einfachen Mitteln zeigen, daß unsere Reihe für alle $z$, für die $\Re(z)>-r$ ist, noch $(C r)$-summierbar ist. Und zwar ist die Ordnung dieser Summierbarkeit genan $=r$ für'alle der Bedingung

$$
-r<\Re(z) \leqq-(r-1)
$$

genügenden Punkte $z$, die einen leicht zu erkennenden Streifen der $z$-Ebene erfüllen. Der Konvərgenzgrenzgeraden gesellen sich also Grenzgeraden für die Summierbarkeit der verschiedenen Ordnungen zu, und zwar ist hiernach das Gebiet, in dem die Reihen summierbar von höchstens $r^{\text {ter }}$ Ordnung sind, die Halbebene

$$
\Re(z)-r \quad(r=0,1,2, \ldots) \text {. }
$$

1) Philosoph. Transactions, London, Bd. 196 A (1901), S. 431.

2) Acta mathematica, Bd. 28 (1904), S. 1.

3) Es ist $f(z)=\left(1-\frac{2}{2^{z}}\right) \cdot \zeta(z)$, wenn $\zeta(z)-\sum_{n \equiv 1}^{\infty} n^{1}$ die Riemannsche $\zeta$-Funktion bezeichnet. (Vgl. 256, 4, 9, 10 u. 11.) 
Während also früher nur jedem Punkte der rechten Halbebene $\Re(z)>0$ eine „Summe“ der Reihe $\Sigma \frac{(-1)^{n-1}}{n^{z}}$ zugeordnet wurde, wird jetzt jedem Punkte der ganzen Ebene eine solche Summe zugeordnet, durch die Reihe also in der ganzen Ebene eine Funktion von $z$ definiert. Ganz ähnlich nun, wie im Konvergenzgebiet unserer Dirichletschen Reihe, läßt sich nun weiter zeigen, daß diese Funktionswerte auch in dem Summierbarkeitsgebiete - also in der ganzen Ebene - eine analytische Funktion definieren. Durch unsere Reihe wird also eine ganze Funktion definiert ${ }^{1}$ ).

Ganz ähnliche Summierbarkeitsverhältnisse weist nun im allgemeinen jede Dirichletsche Reihe

$$
\sum_{n=1}^{\infty} \frac{a_{n}}{n^{z}}
$$

auf. Neben die Konvergenzgerade $\Re(z)=\lambda$ oder $\lambda_{0}$, wie wir nun lieber schreiben wollen, weil Konvergenz mit $(C 0)$-Summierbarkeit gleichbedeutend ist, treten noch die Grenzgeraden $\Re(z)=\lambda_{r}$ für die $(C r)$-Summierbarkeit $(r=1,2, \ldots)$. Sie sind charakterisiert durch die Bedingung, daß die Reihe für $\Re(z)>\lambda_{r}$ von höchstens $r^{\text {ter }}$ Ordnung summierbar ist, während dies für kein $z$ mit $\Re(z)<\lambda_{r}$ mehr der Fall ist. Es ist natürlich $\lambda_{0} \geqq \lambda_{1} \geqq \lambda_{2} \geqq \ldots$, und die $\lambda_{r}$ streben daher entweder $\rightarrow-\infty$ oder gegen einen bestimmten endlichen Grenzwert. Bezeichnen wir diesen in beiden Fällen mit $\Lambda$, so ist die vorgelegte Dirichletsche Reihe für jedes $z$ mit $\Re(z)>\Lambda$ bei passender Wahl von $r$ noch $(C r)$-summierbar, und ihre Summe definiert eine in diesem Gebiete reguläre analytische Funktion. Ist $\Lambda$ endlich, so wird die Gerade $\Re(z)=\Lambda$ als die Summierbarkeits-Grenzgerade bezeichnet.

Diese Anwendungen der Summierbarkeitstheorie auf die Dirichlet schen Reihen rühren von $\left.H . B o h r^{2}\right)$ her. Wir müssen es uns leider versagen, weiter darauf einzugehen.

1) Hieraus folgt dann ziemlich leicht über die Riemannsche $\zeta$-Funktion das wichtige Ergebnis, daf auch die Funktion $\zeta(z)-\frac{1}{1-z}$ eine ganze Funktion ist.

2) UUber die Summabilität Dirichletscher Reihen, Gött. Nachr. 1909, S. 247 und Bidrag til de Dirichletske Räkkers Theori, Dissert., Kopenhagen 1910. 


\section{Aufgaben zum XIII. Kapitel.}

200. Man beweise die auf S. 455, oben, ausgesprochene Behauptung, daf $1-2^{p-1}+3^{p-1}-4^{p-1}+\ldots(C p)$-summierbar ist mit der Summe

$$
\frac{2^{p}-1}{p} B_{p}
$$

falls $p>1$ ist. (Anl.: Die Reihe $e^{t}-e^{2 t}+e^{3 t}-+\ldots=\frac{e^{t}}{e^{t}+1}$ ist für $t<0$ konvergent; ihre $(p-1)^{\text {te }}$ Ableitung liefert für $t \rightarrow 0$ den gesuchten Wert.)

201. Die Reihe $1+2 z+3 z^{2}+4 z^{3}+\ldots$ ist in allen Punkten der Peripherie des Einheitskreises, aufer in +1 , (C2)-summierbar mit der Summe $\frac{1}{(1-z)^{2}}$. Durch Trennung von Reellem und Imaginärem ergibt sich z. B.

$$
\begin{aligned}
1+2 \cos x+3 \cos 2 x+4 \cos 3 x+\ldots & =\frac{1}{2}-\frac{1}{4 \sin ^{2} \frac{x}{2}} \\
\cos x+2 \cos 2 x+3 \cos 3 x+\ldots & =-\frac{1}{4 \sin ^{2} \frac{x}{2}}, \quad \text { u. a. }
\end{aligned}
$$

202. Ist $\left(a_{n}\right)$ eine positive monotone Nullfolge und wird

gesetzt, so ist

$$
a_{0}+a_{1}+a_{2}+\ldots+a_{n}=b_{n}
$$

$$
b_{0}-b_{1}+b_{2}+b_{3}+-\ldots
$$

(C1)-summierbar mit der Summe $s=\frac{1}{2} \Sigma(-1)^{n} a_{n}$.

203. Setzt man $1+\frac{1}{2}+\frac{1}{3}+\ldots+\frac{1}{n}=h_{n}$, so ergibt sich nach der vorigen Angabe

und ähnlich

$$
h_{1}-h_{2}+h_{3}-h_{4}+\ldots-\frac{1}{2} \log 2
$$

$$
\log 2-\log 3+\log 4-+\cdots=\frac{1}{2} \log \frac{\pi^{r^{\prime}}}{2} \text {. }
$$

204. Ist $\Sigma a_{n}$ konvergent oder (Ci)-summierbar mit der Summe s, so ist die folgende Reihe stets konvergent mit der Summe $s$ :

$$
a_{0}+\sum_{n=1}^{\infty} \frac{a_{1}+2 a_{2}+\cdots+n a_{n}}{n(n+1)}=s .
$$

205. Ist $\Sigma a_{n}$ als $(C 1)$-summierbar bekannt und ist $\Sigma n a_{n}{ }^{2}$ konvergent, so ist auch $\Sigma a_{n}$ selber konvergent.

206. Von dem Frobeniusschen Satz gelten auch die folgenden Erweiterungen: Ist $\sum_{n=1}^{\infty} a_{n}(C 1)$-summierbar mit der Summe $s$, so strebt fuir $z \rightarrow+1$ (im Winkelraum) auch

$$
\sum_{n=0}^{\infty} a_{n} z^{n^{2}} \rightarrow s \quad \text { und } \quad \sum_{n=0}^{\infty} a_{n} z^{n^{3}} \rightarrow s
$$


und allgemein für festes ganzzahliges $p>1$

$$
\sum_{n=0}^{\infty} a_{n} z^{n^{p}} \rightarrow s .
$$

Dagegen braucht $\Sigma a_{n} z^{n !}$ nicht mehr $\rightarrow s$ zu streben, wie an dem Beispiel

$$
\sum_{n=0}^{\infty}(-1)^{n} x^{n !}
$$

für reelle $x \rightarrow 1-0$ gezeigt werden soll. (Anl.: Das Maximum von $t-t^{n}$ und der Wert von $t$, für den es eintritt, rücken mit wachsendem $n$ beide von links her $\rightarrow+1$.)

207. Ist eine reelle Reihe $\Sigma a_{n}$ nicht (C 1)-summierbar, aber $\Sigma a_{n} x^{n}$ für $0 \leqq x<1$ konvergent, so läßst sich nach Wahl von $\varepsilon>0$ ein $\delta>0$ sn angeben, daß für alle $x$ in $1-\delta<x<1$ die Summe von $\sum_{n=0}^{\infty} a_{n} x^{n}$ zwischen $x-\varepsilon$ und $\mu+\varepsilon$ liegt, wenn mit $\varkappa$ und $\mu$ die untere bzw. obere Häufungsgrenze von $\frac{s_{0}+s_{1}+\ldots+s_{n}}{n+1}$ bezeichnet wird.

208. In Anschluf an den Fejérschen Satz soll bewiesen werden, dafi bei den dort betrachteten arithmetischen Nitteln $\sigma_{n}(x)$ die Gibbssche Erscheinung nicht eintritt. (Vgl. S. 460, Fufinote 1.) 


\section{Literatur.}

(Einige grundlegende Abhandlungen, zusammenfassende Darstellungen und Lehrbücher.)

1. J. Newton, De analysi per aequationes numero terminorum infinitas, London 1711 (verfafit 1669).

2. John Wallis, Treatise of algebra both historical and practical with some additional treatises, London 1685 .

3. Jakob Bernoulli, Propositiones arithmeticae de seriebus infinitis earumque summa finita, mit 4 Fortsetzungen, Basel 1689-1704.

4. L. Euler, Introductio in analysin infinitorum, Lausanne 1748.

5. L. Euler, Institutiones calculi differentialis cum ejus usu in analysi infinitorum ac doctrina serierum, Berlin 1755.

6. L. Euler, Institutiones calculi integralis, Petersburg 1768/69.

7. C. F. Gau $\beta$, Disquisitiones generales circa seriem infinitam $1+\frac{\alpha \cdot \beta}{1 \cdot \gamma} x$ $+\frac{\alpha(\alpha+1) \cdot \beta(\beta+1)}{1 \cdot 2 \cdot \gamma(\gamma+1)} x^{2}+$ etc., Göttingen 1812 .

8. A. L. Cauchy, Cours d'analyse de l'école polytechnique. Ire Partie. Analyse algébrique, Paris 1821.

9. N. H. Abel, Untersuchungen iber die Reihe $1+\frac{m}{1} x+\frac{m(m-1)}{1 \cdot 2} x^{2}+\ldots$, Journal für die reine und angewandte Mathematik Bd. 1, 1826, S. $311-339$.

10. $P . d u$ Bois-Raymond, Eine neue Theorie der Konvergenz und Divergenz von Reihen mit positiven Gliedern, Journal für die reine und angewandte Mathematik $\mathrm{Bd}$. $76,1873, \mathrm{~S} .61-91$.

11. A. Pringsheim, Allgemeine Theorie der Divergenz und Konvergenz von Reihen mit positiven Gliedern, Mathematische Annalen Bd. 35, 1890, S. 297-394.

12. A. Pringsheim, Irrationalzahlen und Konvergenz unendlicher Prozesse, Enzyklopädie der mathemathischen Wissenschaften Bd. I, 1, 3, Leipzig 1899.

13. E. Borel, Leçons sur les séries à termes positifs, Pàris 1902.

14. C. Runge, Theorie und Praxis der Reihen, Leipzig 1904.

15. O. Stolz und A. Gmeiner, Einleitung in die Funktionentheorie, Leipzig 1905.

16. A. Pringsheim und J. Molk, Algorithmes illimités de nombres réels, Encyclopédie des Sciences Mathématiques Bd. I, 1, 4, Leipzig 1907.

17. T. J. I'A. Bromwich, An introduction to the theory of infinite series London 1908.

18. A. Pringsheim und G. Faber, Algebraische Analysis, Enzyklopädie der mathematischen Wissenschaften Bd. II, C, 1, Leipzig 1909.

19. N. Nielsen, Lehrbuch der unendlichen Reihen, Leipzig 1909.

20. E. Fabry, Théorie des séries à termes constants, Paris 1910.

21. A. Pringsheim, G. Faber und J. Molk, Analyse algébrique, Encyclopédie des Sciences Mathematiques Bd. II, 2, 7, Leipzig 1911.

22. O Stolz und A. Gmeiner, Theoretische Arithmetik Bd. II, 2. Aufl., Leipzig 1915.

23. A. Pringsheim, Vorlesungen ïber Zahlen- und Funktionenlehre Bd. I, 2, Leipzig 1916. 


\title{
Autoren- und Sachverzeichnis.
}

\author{
Die Zahlen geben die Seiten an.
}

Abbildung 32 .

Abbrechen (eines Dezimalbruches) 242. Abel 117, 121, 203, 205, 207, 274, 282 ff., $291,294,299,306,312,334,383$, $408 \mathrm{ff} ., 443,448$.

Abelsche partielle Summation 304, 383. Abelscher Grenzwertsatz 169f., 229, $337,390 \mathrm{ff}$., $461 \mathrm{ff}$.

Abgeschlossen 18, 155.

Ableitung $156 \mathrm{ff}$.

Abschätzung, der Reste, s. Restabschätzung.

Abschätzungsformel, Cauchysche 393.

Absolute Konvergenz einer Reihe 129 ff., 382.

— - eines Produktes 216 ff., 419.

Absoluter Betrag 6, 7, 375.

Adams, J. C., 176, 249.

Addition 5, 29, 31.

- gliedweise 44, 66, 128.

Additionstheorem der Exponentialfunktion 141, 183.

- der Binomialkoeffizienten 202.

- der trigonometrischen Funktionen 399.

Ähnlich 9.

Anderungen, endlich viele, bei Folgen $44,66,90$.

- bei Reihen 125.

d'Alembert, 443.

Alternierende Reihen 124, 243, 255, 307.

Ames, L. D. 238.

Analytische Funktionen 392.

Annäherung im Winkelraum $390 \mathrm{ff}$.

Anordnung nach Quadraten 86.

- nach Schräglinien 85.

Anordnungssätze $5,28$.

Approximationskurve 319 .

Aquivalenzsatz von $K$ nopp und Schnee 454.

Archimedes 6, 99.
Arcus 375.

arcsin-Funktion $205 \mathrm{ff}$., 406.

arctg-Funktion $205 \mathrm{ff}$., 406.

Arithmetische Mittel $68 \mathrm{f} ., 446$.

Arzela, S. 332.

Assoziationsgesetz 5 .

- bei Reihen 125.

Asymptotisch gleich 64 .

- proportional 64 .

Ausmultiplikation von unendlichen Produkten 421.

Auswertung der Reihensumme 223 bis 266.

- - geschlossene 226-234.

Bedingt konvergent 133, 220.

Berechnung, numerische 241-259.

- von $e 244$.

- von $\pi 245$.

— der Logarithmen $247 \mathrm{ff}$.

- der trigonometrischen Funktionen 251.

- der Wurzeln 250.

Bernoulli, Jak. und Joh, 16, 62, 98, 124. 231, 441.

Bernoullische Ungleichung 16.

- Zahlen 175f., 195, $231 \mathrm{ff}$.

Bertrand, J. 274.

Beschränkte Folgen 14, 42, 75 .

- Funktionen 152.

Beständig konvergent 146, 174, 428.

Bewegung von $x 153$.

Bieberbach, L. 453.

Bild 32.

Bildungsgesetz 13, 35.

Binomische Reihe 182, 407-411.

Binomischer Lehrsatz 47, 182.

Bôcher, M. 338.

Bogenmak 56.

Bohr, H. 465.

Bolzano, B. 82, 86 . 
Bolzano-Weierstraßscher Satz 380.

Bonnet, O. 274.

Boormann, J. M. 186.

Borel, E. 312, 445, $448 \mathrm{ff}$.

Brigg 250.

Bromwich, T. J. I'a. 453.

Brouncker, W. 99.

Burkhardt, H. 361.

Cahen, E. 282, 425.

Cajori, F. 314.

Cantor, G. 2, 343.

- M. 11.

--Dedekindsches Axiom 24, 32.

Catalan, E. 240.

Cauchy, A. L. 17, 68, 70, 82, 91, 99, $108,111,112,115,117,131,141$, $142,148,178,188,212,277,286$, $361,443$.

Cauchysche Abschätzungsformel 393.

Cauchyscher Grenzwertsatz 68.

Cauchysches Produkt 141, 171, 450.

Cauchy-Toeplitzscher Grenzwertsatz $70 \mathrm{ff}$.

Cesàro, E. 284, 309, 312, 383, $447 \mathrm{f}$.

Chapman, S. 453.

$\cos 190 \mathrm{ff} ., 370,399 \mathrm{ff}$.

ctg $194 \mathrm{ff} ., 402 \mathrm{ff}$.

Darstellung reeller Zahlen 224.

Dedekind, R. 2, 39, 306, 336, 383.

Dedekindscher Schnitt 38, 39.

Definitionsintervall 152.

Dezimalbruch 111, 142 (s. a. Systembruch).

Dicht 11.

Differenz 29, 237.

Differentiation, gliedweise 330 .

Differenzenfolge 83.

Differenzierbarkeit $156,3 \$ 9$.

- einer Potenzreihe 166.

Dini, U. 220, 274, $282 \mathrm{ff} ., 285,294$, $299,302,332,352,355,358$.

Dirichlet, G. Lejeune- 132, $306 \mathrm{ff} ., 319$, $335,344,352,358,361$.

Dirichletsche Integrale $344-352$.

- Definition 347.

- Reihen $308,337,425-430,442,464 \mathrm{f}$.

Disjunktive Kriterien 113, 300 .

Divergenz $62,96,153,376$.

- bestimmte $62,96,153,376$.

- unbestimmte 63, 96, 153.

- eigentliche 63.

Division 6, 30, 45 .
Division, gliedweise 66 .

- von Potenzreihen 172, $174 \mathrm{ff}$.

Doetsch, G. 445, 453.

Doppelreihensatz 413.

du Bois-Reymond 82, 91, 293, $296 \mathrm{f}$., $306,336,341,343,365,383,457$.

Duhamel, J. M. C. 277.

Dyadischer Bruch 37, 41.

e 78, $186 \mathrm{f.,} 244$.

Einheitskreis 387 .

Eins 9.

Einzigkeitssatz 21.

Eisenstein, G. 172.

Elliot, E. B. 306.

Endlich viele 13, 15.

- - Änderungen, s. Änderungen.

Entgegengesetzt 29.

Ermakoffsches Kriterium 288 ff., 302.

$\varepsilon$-Umgebung 18 .

Eudoxus 6, 9, 25, 32.

Euklid 6, 18, 65 .

Euler, L. 1, 99, 185, 196, 203, 212, 231, $237,259,287,290,370,423,429$, $436,441 \mathrm{f}$., $446,450 \mathrm{ff}$.

Eulersche Formeln 341, 399.

- Konstante 287.

- Reihentransformation $237 \mathrm{ff}$., 255.

- Zahlen 233.

Exhaustionsmethode 65 .

Exponentialfunktion und -reihe 113, $141,183-190,396 \mathrm{ff}$.

Fabry, E. 259.

Fakultätenreihen $430 \mathrm{ff}$.

Fehlerabschätzungen $242 \mathrm{ff}$.

Fejer, L. $457 \mathrm{ff}$.

Fibonnacci 436.

Fourier, J. B. 340, 361.

Fourierkoeffizienten, -konstanten 342, $348,349$.

Fouriersche Reihen 337-366, 342, $456-461$.

Frobenius, G, 176, $462 \mathrm{f}$.

Frullani 361.

Funktion 151, 388.

Funkiionen, analytische 392.

- einer komplexen Veränderlichen $388 \mathrm{f}$.

- einer reellen Veränderlichen 151 bis 162 .

- elementare 181-208.

- elementare analytische $395-411$.

- rationale $181 \mathrm{f} ., 395$.

- trigonometrische 190-200, 251. 
Funktionen, willkürliche 339.

- zyklometrische $205 \mathrm{ff}$.

Funktionenfolgen $317,412$.

Gammafunktion, -produkt 219, 371, 423.

Gauß, C. F. 1, 108, 169, $280 \mathrm{ff}$.

Geordnet 5, 28.

Gerade Funktion 164.

Geschichte der unendlichen Reihen 99.

Gibbssche Erscheinung 365, 460.

Glaisher, J. W. L. 172.

Gleichheit 26.

Gleichmäfig beschränkt 334 .

Gleichmäfige Konvergenz 316-326, (Definition) 321, 411-417.

- - von Dirichletschen Reihen 333, $426 \mathrm{f}$.

- - von Fakultätenreihen 431.

- - von Fourierreihen 333, 343, 348.

- - von Lambertschen Reihen $433 \mathrm{f}$.

- - von Potenzreihen 322.

- _ von unendlichen Produkten 367 ff.

- Stetigkeit 155.

- Summierbarkeit 452 .

Glieder eines Produktes 212.

- einer Reihe 94.

Gliedweise Grenzübergänge 326-332, 414 (s. a. Addition, Subtraktion, Multiplikation, Division, Differentiation, Integration).

Gmeiner, J. A. 4, 384.

Goldbach 442.

Grandi, G. 127.

Gregory, J. 62, 206.

Grenze (untere, obere) 90, 91, 152.

Grenzkurve 319.

Grenzübergänge, gliedweise (s. gliedw. Gr.).

Grenzwert einer Folge 60, 97, 388.

- einer Funktion 152.

Grenzwertsätze s. Abel, Cauchy, Toeplitz.

Gronwall, T. H. 365.

Grundgesetze der Anordnung 5, 28.

- der Arithmetik 5, 31.

Hadamard, J. 148, 291, 293, 306.

Hagen, J. 174.

Hahn, H. 2.

Halbierungsmethode 37.

Hansted, B. 172.

Hardy, G. H. 309, 314, 428, 433.

Häufungsgrenze 88.

Häufungspunkt, -stelle, -wert 84, 380.
Hïufungspunkt, kleinster und größster $87 \mathrm{ff}$.

Hauptkriterium, erstes (für Folgen) 75.

- - (für Reihen) 105.

— weites (für Folgen) 79 - 84,378, 381.

- - (fuir Reihen) $121 \mathrm{f}$.

- drittes (für Folgen) 92.

Harptwert 404, 407.

$H$ : usdorff, F. 453, 454.

Fiermann. J. 124.

Hilbert, D. 10.

Hölder, O. 4, $447 \mathrm{f} ., 462 \mathrm{f}$.

Holmboe 443.

Hypergeometrische Reihe 281.

Identisch gleich 13 .

Identitätssatz für Potenzreihen 163.

Induktionsgesetz 6 .

Infinitär 14, 44, 90, 98.

Inhalt 161 .

Innerster Punkt 21, 379.

Integral, bestimmtes, $158 \mathrm{ff}$.

- unbestimmtes 157.

Integralkriterium 286, 302.

Integration, gliedweise 168, 328.

Integrierbarkeit, Riemannsche $158 \mathrm{f}$.

Intervall 18.

Intervallschachtelung $19 \mathrm{ff}$.

Isomorph 9 .

Jacobi, C. G. J. 423.

Jacobsthal, E. 238, 256.

Jensen, J. L. W. V. 70, 72, 425.

Jones, W. 246.

Jordan, C. 14.

\section{Kennziffer 55 .}

Kettenbriiche 100 .

Knopp, K. 71, 238, 389, 432, 447, 454.

Kommutationsgesetz $5,10$.

- bei Reihen 131.

- bei Produkten 221.

Komplexe Zahlen s. Zahlen.

Komposition 452.

Konvergente Zahlenfolgen s. Zahlenfolgen.

Konvergenz 60, 96, 153.

- absolute $129 \mathrm{ff}$.

- bedingte und unbedingte 133 .

- bessere und schlechtere 255, $271 \mathrm{ff}$.

Konvergenzabszisse 425 .

Konvergenzbereich 146 .

Konvergenzhalbebene 425 .

Konvergenzintervall $146,316$. 
Konvergenzkreis 387 .

Konvergenzkriterien für Fouviersche Reihen 348, 351-359.

— f. Folgen $75,79 \mathrm{ff}$.

- f. gleichmäßige Konvergenz 332 bis 337 .

- f. Reihen 105-112, 121, 274-282, $286,288,305 \mathrm{f}$.

- f. R. mit beliebigen Gliedern 121,1? ?

- f. R. m. komplexen Gl. 384 .

- f. R. m. monoton abnehmenden G1. 115, 116, 286, 288.

- f. R. m. positiven G1. 111, 112.

Konvergenzradius 146 .

Konvergenztheorie, allgemeine Bemerkungen zur 290-297.

- Systematisierung der 297-302.

Kowalewski, G. 2.

Kreisfunktionen 56 ; s. a. trigonom. F. Kriterien s. Konvergenzkrit.

Kriterienpaare 300 .

Kronecker, L. 123.

Kummer, E. E. 240, 300, 302.

Kummersche Reihentransformation 240 , 253.

Lagrange, J. L. 290.

Lambert, J. H. $432 \mathrm{ff}$.

Lambertsche Reihen 432-436.

Landau, E. 428, 430, 436.

Länge 161 .

Lasker, E. 464.

Lebesgue, H. 160, 338, 341, 444.

Leclert 240.

Legendre, A. M. 361.

Leibniz, G. W. 1, 62, 98, 124, 185, 207, $307,441$.

Le Roy, E. 450.

Lévy, P. 383.

Limes 60 .

- unterer und oberer 88.

- inferior und superior 88.

Linksseitige Differenzierbarkeit 156 .

- Stetigkeit 154.

Linksseitiger Grenzwert 153.

Lipschitz, R. 356, 358.

Littlewood, J. E. 453.

Logarithmen $54 \mathrm{ff}$., 189, $203 \mathrm{ff}$., $404 \mathrm{ff}$.

— Berechnung der 22, 189, 247.

Logarithmische Kriterien 274-277.

- Vergleichsskalen 271.

Loewy, A. 2, 4, 10.

Lücken im System d. rat. Zahlen $3 \mathrm{ff}$. Lückenlosigkeit der Geraden 24.
Machin, J. 246.

Malmstén, C. J. 308.

Mangoldt, H. v. 2, 338.

Mantisse 55 .

Markoff, A. A. 235, 257.

Markoffsche Reihentransformation 236, $257,414$.

Mascheronische Konstante 287.

Mercator, G. 99.

Mertens, F. 312, 383.

Mefibar 161.

Mittag-Leffler, G. 1.

Mittelpunkt einer Potenzreihe 151.

Mittelwertsatz, 1., der Differentialrechnung 157.

- 1., der Integralrechnung 160.

- 2., der Differentialrechnung 161.

Möbiussche Koeffizienten 430, 435.

Monoton 15, 42, 75, 155, 256.

Monotoniegesetz 5 .

Morgan, A. de 274.

Multiplikation $6, .29,45$.

- gliedweise 66, 128.

- unendlicher Reihen $139 \mathrm{ff}$.

— von Potenzreihen 171.

Näherungswert 224 .

Napier, J. 55.

Natürliche Zahlen s. Zahlen.

Nebenwerte 404, 407.

Neumann, C. 15.

Newton, J. 1, 99, 185, 203, 441.

Nicht-absolut konvergent 130, 382, 419.

Nirgends konvergent 146, 428.

Null 9.

Nullfolgen $15 \mathrm{ff}$., $42 \mathrm{ff}$., $56-60,68,71$.

NumerischeBerechnungen 75,223-266, bes. 241-259.

() ffen 18 .

Ohm, M. 176, 312.

Oldenburg 203.

Olivier, L., 118.

Orstrand, C. E. van 179.

Oszillieren 96, 98.

Ottolenghi, B. 453.

Partialbruchzerlegungen elementarer Funktionen 196-200, 229, $363 \mathrm{f}$. $403 \mathrm{f}$.

Partielle Integration 161.

- Summation, Abelsche 304, 383.

Periodenstreifen 398, 401, 403.

Periodische Systembriche 36. 
Periodische Funktionen 193, $397 \mathrm{ff}$.

Permanenzbedingung 445 .

Perron, O. 100, 453.

$\pi 192 \mathrm{ff} ., 224$.

- Reihen für 207, 208.

- Berechnung von 245.

Postulat des Eudoxus 9.

Potenzen 46-54, 407.

Potenzreihen 146-151, 162-179, 386 bis 394.

Primitive Periode 193.

Primzahlen 13, 429 f., 435.

Pringsheim, A. 2, 4, 81, 91, 167, $282 \mathrm{ff}$., 290, 292f., 295-302, 306, 312, 314, $384,464$.

Problem A und B 74, 100, $223 \mathrm{ff}$.

Produkte 30 .

- unendliche 99, 211-221.

- mit beliebigen Gliedern 215-221.

- mit komplexen Gl. 417-424.

- mit positiven G1. 212-214.

- mit veränderlichen G1. 366, $420 \mathrm{ff}$. Punktfolge 13.

Pythagoras 11.

Quadrate (Anordnung nach -n) 86, 410 . Quadratschachtelung 379.

Quotientenkriterium 112.

Raabe, J. L. 277.

Rademacher, H. 309.

Rationale Funktionen 155, $181 \mathrm{f} ., 395$.

- Zahlen s. Zahlen.

Rationalitätsbereich 6 .

Rationalwertig 26.

Rechtseitige Differenzierbarkeit 156.

- Stetigkeit 154.

Rechtsseitiger Grenzwert 153.

Reelle Zahlen s. Zahlen.

Reguläre Funktionen 392.

Reiff, R. 99, 127, $441 \mathrm{f}$.

Reihen, alternierende 124, 243, '255.

- analytischer Funktionen 412.

- binomische 182, 200-203,407-411.

- divergente 441-465.

- geometrische 106.

- harmonische 77, 107, 110.

- hypergeometrische 281.

- logarithmische $203 \mathrm{ff}$., $404 \mathrm{ff}$.

- mit beliebigen Gliedern 121-142, $303-314$.

- m. komplexen Gl. 382-417.

- m. monoton abnehmenden Gl. 114 bis $119,286-290$.
Reihen mit positiven G1. 105-120, $267-303$.

- m. veränderlichen G1. 145, 316-366, $411-417$.

- trigonometrische $307,317$.

- unendliche 93.

(s. a. Dirichletsche R., Fakultätenr., Lambertsche R.)

Reihensumme s. Auswertung d. R.

Reihentransformationen 234-240, 253 bis 259.

Restabschätzungen $242 \mathrm{ff}$., 252.

Reziprok 30.

Riccotti, E. 453.

Riemann, B. 158 f., 310, 350, 357, 383, 444.

Riemannsche $\zeta$-Funktion 428, $464 \mathrm{f}$.

Riemannscher Umordnungssatz 310 .

Riesz, M. 428.

Saalschütz, L. 176.

Sachse, A. 341.

Scherk, H. F. 233.

Schlömilch, O. 116, 279, 312.

Schnee, W. 454.

Schnitt 38, 39.

Schräglinien (Anordnung nach Schr.) $75,85,140,435$.

Schranke 14, 152.

Schröter, H. 196.

Schur, J. 454.

Schwankung 152.

Seidel, Ph. L. v. 323.

Sierpinski, W. 312.

sin $190 \mathrm{ff}$., $370,399 \mathrm{ff}$.

sin-Produkt 370 .

Spaltensummen 137.

Steinitz, E. 383.

Stetigkeit 154 f., 389.

- einer Potenzreihe 163, 166.

Stieltjes, Th. J. 232, 294, 312.

Stivling, J. 234, 432.

Stokes, G. G. 323.

Stolz, O. 4, 37, 70, 72, 82, 302, 392, 463.

Streifen bedingter Konvergenz 428 .

Substraktion 5, 29.

- gliedweise 45, 66, 128.

Summationsbuchstabe 94 .

Summe 29.

- einer Reihe $96 \mathrm{f}$.

Summenbereich 383 .

Summierbar 446.

Summierbarkeits-Verfahren $446-452$.

- - Grenzgerade 465 . 
Summierung durch arithmet. Mittel 453-456, 461-465.

- von Dirichletschen Reihen $464 \mathrm{f}$.

- von Fourierreihen 455.

Sylvester, J. J. 172.

Symbolische Gleichung 175.

Systembrüche $35 \mathrm{ff}$.

'rangens $194 \mathrm{ff}$., $402 \mathrm{ff}$.

Taylor, B. 167.

Taylorsche Reihe 167.

Teiler, Anzahl der 430, 435.

- Summe der 435.

Teilfolge 43, 87 .

Teilprodukte 99, 212, 218.

Teilreihe 111, 135.

Teilstiick einer Reihe 12\%.

- folge 122 .

Teilsumme 94, 218.

Toeplitz, O. 70, 451, $462 \mathrm{f}$.

Toeplitzscher Grenzwertsatz 70, 377 .

Trigonometrische Funktionen 190-200, $251,399-404$.

Umkehrbar 156, 176.

Umkehrung von Potenzreihen 176, 389.

Umordnung von Folgen 44, 66.

- von Produkten 221.

- von Reihen $131 \mathrm{ff} ., 310,383$.

Umordnungssatz, grofer, 137, 230.

Unbedingt konvergent 133, 220.

Uneigentliches Integral $161 \mathrm{f}$.

Unendlich klein 17.

Unendlich viele 13 .

Ungerade Funktion 164.

Ungleichheit 27.

Ungleichungen 6,7 .

Unitätssatz 164 .
Veranschaulichung 18, 376 .

Verdichtungssatz, Cauchyscher 115, 289.

Vergleichskriterium 1. und 2. Art $108 \mathrm{f}$. $267 \mathrm{ff}$.

Vergleichsskalen, logarithmische $271 \mathrm{ff}$.

Vieta, F. 212.

Vivanti, G. 332.

Vollmonoton 256.

Vollständigkeitssatz 32.

Vollständigkeitspostulat 33 .

Voß, A. 314 .

Wallis, J. 18, 37, 212.

Wallissches Produkt 370.

Weierstraß, C. 1, 86, 323, 332, 384, 393.

Weierstraßscher Doppelreihensatz 413.

Wert einer Reihe 96.

Winkelraum, Annäherung im $390 \mathrm{ff}$.

Wurzelkriterium 111.

Wurzeln 47, 250.

Zahlbegriff 8, 31.

Zahlen (s. a. Bernouillische Z., Eulersche Z.).

- irrationale $21 \mathrm{ff}$., 34 .

- komplexe 374-381.

- natïrliche 3 .

- rationale $3 \mathrm{ff} ., 34$.

- reelle $26,31,34,39$.

Zahlenfolgen, komplexe, 374-381.

- konvergente $60 \mathrm{ff} ., 64-73$.

- rationale $12 \mathrm{ff}$.

- reelle 41.

Zahlengerade 7 .

Zahlensystem 8, 26, 31 .

Zahlkörper 6 .

Zehnteilung 22, 47.

Zeilensummen 187.

$\zeta$-Funktion, Riemannsche 428 ff., 464. 
Felix Klein, Gesammelte mathematische Abhandlungen.

(Von F. Klein mit ergänzenden Zusätzen versehen.) In vier Bänden. Herausgegeben von $\mathbf{R}$. Fricke und A. Ostrowski. Zuerst erschien: Band I: Liniengeometrie - Grundlegung der Geometrie - Zum Erlanger Programm. I921.

Preis M. $186,-$.

Theorie der reellen Funktionen. Von Dr. Hans Hahn, Professor der Mathematik an der Universität Bonn. Erster Band: Mit 18 Textfiguren. 1921 .

Preis M. $136,-$

\section{Darstellung und Begründung einiger neuerer Ergebnisse} der Funktionentheorie. Von Prof. Dr. E. Landau, Göttingen. Mit II Textfiguren. I9I6.

Preis M. 4,80 .

\section{Vorlesungen über die Zahlentheorie der Quaternionen.}

Von Dr. Adolf Hurwitz, Professor der höheren Mathematik an der Eidgenössischen Technischen Hochschule in Zürich. 1919. Preis M. 8,- .

Gesammelte mathematische Abhandlungen. Von Prof. H. A. Schwarz. In zwei Bänden. Mit 93 Textfiguren und 4 Figurentafeln. I890. Preis M. 25, - ; in 2 Bände gebunden M. 28,-.

Schwarz-Festschrift. Mathematische Abhandlungen, Hermann Amandus Schwarz zu seinem fünfzigjährigen Doktorjubiläum am 6. August I9I4 gewidmet von Freunden und Schülern. Mit dem Bildnis von H. A. Schwarz und 53 Figuren im Text. I9I4.

Preis M. 24,一.

Formeln und Lehrsätze zum Gebrauche der elliptischen

Funktionen. Nach Vorlesungen und Aufzeichnungen des Herrn K. Weierstrass bearbeitet und herausgegeben von H. A. Schwarz, Professor an der Universität Göttingen. Zweite Ausgabe. I893.

Preis M. 10,-

Felix Klein, zur Feier seines siebzigsten Geburtstages gewidmetes Heft der Naturwissenschaften. (Jahrgang 1919, Heft 17). Preis M. 6,-. schläge, über die die Buchhandlungen und der Verlag gern Auskunft erteilen. 
Lehrbuch der darstellenden Geometrie. In zwei Bänden. Von Dr. Georg Scheffers, o. Professor an der Technischen Hochschule Berlin. Erster Band: Mit 404 Figuren im Text. 1919.

Preis M. 26,-; gebunden M. 30,60. Zweiter Band: Mit 396 Figuren im Text. 1920.

Preis M. 52,-; gebunden M. 6o,- .

Koordinaten-Geometrie. Von Dr. Hans Beck, Professor an der Universität Bonn. Erster Band: Die Ebene. Mit 47 Textabbildungen. I919. Preis M. 28,-; gebunden M. 31, -

Lehrbuch der darstellenden Geometrie. Von Dr. W. Ludwig, o. Professor an der Technischen Hochschule Dresden. Erster Teil: Das rechtwinklige Zweitafelsystem. Vielflache, Kreis, Zylinder, Kugel. Mit 58 Textfiguren. 1919 .

Preis M. 8,- .

Die übrigen Teile in Arbeit.

Abhandlungen aus der reinen Mathematik. Von N. Vandermonde. In deutscher Sprache herausgegeben von Carl Itzigsohn. 1888 .

Preis M. 3,-

Abhandlungen aus der Funktionenlehre. Von Karl Weierstrass, Professor an der Universität Berlin. 1886.

Preis M. 12,-.

Vorlesungen über die Bernoulli'schen Zahlen, ihren Zusammenhang mit den Secanten-Coefficienten und ihre wichtigeren Anwendungen. Von Dr. Louis Saalschütz, a. o. Professor der Mathematik an der Universität Königsberg. 1893 .

Preis M. 5, -

Abhandlungen über die algebraische Auflösung der Gleichungen. Von N. H. Abel und E. Galois. Deutsch herausgegeben von H. Maser. I 889 .

Preis M. 4,- .

Archimedes Werke. Mit modernen Bezeichnungen herausgegeben und mit einer Einleitung versehen von Sir Thomas L. Heath. Deutsch von Dr. Fritz Kliem. 1914.

Preis M. 16,一.

$\mathrm{Zu}$ den angegebenen Preisen der angezeigten älteren Bücher treten Verlagsteuerungszu-• schläge, über die dic Buchhandlungen und der Verlag gern Auskunft erteilen. 
B. Riemann, Über die Hypothesen, welche der Geometrie zu Grunde liegen. Neu herausgegeben und erläutert von $\mathrm{H}$. Weyl. $\mathrm{Zweite}$ Auflage. 1921 .

Preis M. 12,-

Raum - Zeit - Materie. Vorlesungen über allgemeine Relativitätstheorie. Von Hermann Weyl. Vierte, erweiterte Auflage. Mit I5 Textfiguren. I92I.

Preis M. 48,-

Raum und Zeit in der gegenwärtigen Physik. Zur Einführung in das Verständnis der Relativitäts- und Gravitationstheorie. Von Moritz Schlick. Vierte, vermehrte und verbesserte Auflage. In Vorbereitung.

Die Grundlagen der Einsteinschen Gravitationstheorie. Von Erwin Freundlich. Mit einem Vorwort von Albert Einstein. Vierte, erweiterte und verbesserte Auflage. 1920.

Preis M. ro,-.

Die Relativitätstheorie Einsteins und ihre physikalischen Grundlagen. Elementar dargestellt. Von Max Born. Z weite, umgearbeitete Auflage. Mit 133 Textabbildungen. (Bildet Band III der "Naturwissenschaftlichen Monographien und Lehrbücher". Herausgegeben von der Schriftleitung der "Naturwissenschaften". I 92 I.

Preis M. 39,-; gebunden M. 52,-.

Vorzugspreis für die Bezieher der "Naturwissenschaften"

M. 35,-; gebunden M. 48 ,- .

\section{Relativitätstheorie und Erkenntnis a priori. Von Hans Reichenbach. 1920. \\ Preis M. I4,--.}

Die Quantentheorie, ihr Ursprung und ihre Entwicklung. Von Fritz Reiche. Mit 15 Textfiguren. I921.

Preis M. 34, .

Einleitung in die Mengenlehre. Eine gemeinverständliche Einführung in das Reich der unendlichen Größen. Von Dr. Adolf Fraenkel, Privatdozent an der Universität Marburg. Mit io Textabbildungen. Igr9. Preis M. I0,- 


\section{Mathematische Zeitschrift}

Unter ständiger Mitwirkung von

$\begin{array}{ccc}\text { K. Knopp } & \text { E. Schmidt } & \text { I. Schur } \\ \text { Königsberg } & \text { Berlin } & \text { Berlin }\end{array}$

Herausgegeben von

\section{Lichtenstein}

Münster

Wissenschaftlicher Beirat:

W. Blaschke, L. Fejér, E. Hecke, G. Herglotz, A. Kneser

E. Landau, O. Perron, F. Schur, E. Study, H. Weyl

Erscheint in zwangslosen Heften, deren vier in einem Band vereinigt werden. Der Preis des Bandes beträgt M. 96,--

\section{Mathematische Annalen}

Begründet 1868 durch

\section{Alfred Clebsch und Carl Neumann}

Unter Mitwirkung von

Ludwig Bieberbach, Harald Bohr, Max Born, L. E. J. Brouwer, Richard Courant, Constantin Carathéodory, Walther v. Dyck, Otto Hölder, Theodor v. Kármán, Carl Neumann, Max Noether, Arnold Sommerfeld

Herausgegeben von

Felix Klein

in Göttingen

David Hilbert

in Göttingen
Albert Einstein

in Berlin
Otto Blumenthal in Aachen

Erscheinen in Heften, von denen vier einen Band bilden.

Der Preis des Bandes beträgt M. 96,- .

\section{Zeitschrift für Physik}

Herausgegeben von der

Deutschen Physikalischen Gesellschaft

als Ergänzung zu ihren „Verhandlungen"

unter der Redaktion von

Karl Scheel

Erscheint in zwanglosen Heften, deren sechs zu einem Bande vereinigt werden.

Der Preis des Bandes beträgt M. 80,- 





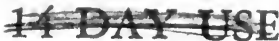 \\ RETURN TO IfESAFKO WIA BORROWED ASTRONOMY, PAPRAAICS.}

This book is dae on the last date stamped below, or on the date to which renewed.

Renewed books are subject to immediate recall.

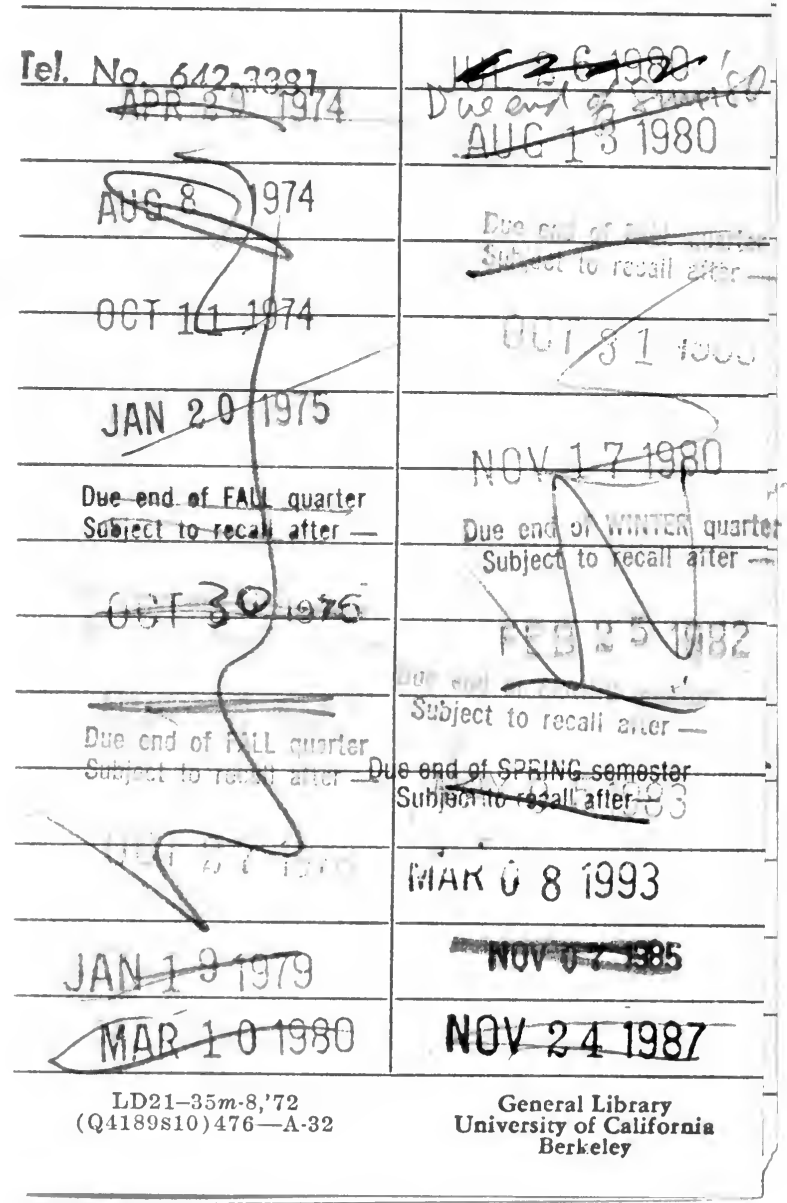




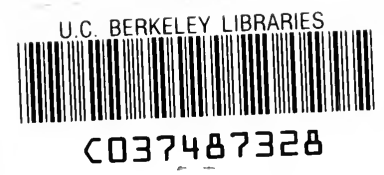

$$
\begin{aligned}
& \text { QA } 295 \\
& K 5
\end{aligned}
$$

C037487328

$$
739
$$

MATH.STAT.

LIBRARY 
